\title{
Statistical analysis of iron geochemical data suggests limited late Proterozoic oxygenation
}

\section{Citation}

Sperling, Erik A., Charles J. Wolock, Alex S. Morgan, Benjamin C. Gill, Marcus Kunzmann, Galen P. Halverson, Francis A. Macdonald, Andrew H. Knoll, and David T. Johnston. 2015. "Statistical Analysis of Iron Geochemical Data Suggests Limited Late Proterozoic Oxygenation." Nature 523 (7561) (July 22): 451-454. doi:10.1038/nature14589.

\section{Published Version}

doi:10.1038/nature14589

\section{Permanent link}

http://nrs.harvard.edu/urn-3:HUL.InstRepos:27716502

\section{Terms of Use}

This article was downloaded from Harvard University's DASH repository, and is made available under the terms and conditions applicable to Other Posted Material, as set forth at http:// nrs.harvard.edu/urn-3:HUL.InstRepos:dash.current.terms-of-use\#LAA

\section{Share Your Story}

The Harvard community has made this article openly available.

Please share how this access benefits you. Submit a story.

\section{Accessibility}




\title{
Nature Letter
}

\section{Statistical analysis of iron geochemical data suggests limited Late Proterozoic oxygenation}

\author{
Erik A. Sperling ${ }^{1 *+}$, Charles J. Wolock ${ }^{2}$, Alex S. Morgan ${ }^{1}$, Benjamin C. Gill ${ }^{3}$, \\ Marcus Kunzmann ${ }^{4}$, Galen P. Halverson ${ }^{4}$, Francis A. Macdonald ${ }^{1}$, Andrew H. \\ Knoll $^{1,2}$, David T. Johnston ${ }^{1^{*}}$
}

\author{
Affiliations: \\ ${ }^{1}$ Department of Earth and Planetary Sciences, Harvard University, Cambridge, MA 02138, USA \\ ${ }^{2}$ Department of Organismic and Evolutionary Biology, Harvard University, Cambridge, MA \\ 02138, USA \\ ${ }^{3}$ Department of Geosciences, Virginia Polytechnic Institute and State University, Blacksburg, \\ VA 24061, USA \\ ${ }^{4}$ Department of Earth and Planetary Sciences/GEOTOP, McGill University, Montreal, Quebec, \\ H3A 0E8 Canada \\ ${ }^{+}$Present address: Integrative Oceanography Division, Scripps Institution of Oceanography, La \\ Jolla, CA 90089, USA
}

*Correspondence to: sperling@fas.harvard.edu; johnston@eps.harvard.edu 
Sedimentary rocks deposited across the Proterozoic-Phanerozoic transition record extreme climate fluctuations, a potential rise in atmospheric oxygen or re-organization of the seafloor redox landscape, and the initial diversification of animals ${ }^{1,2}$. It is widely assumed that the inferred redox change facilitated the observed trends in biodiversity. Establishing this paleoenvironmental context, however, requires that changes in marine redox structure be tracked by means of geochemical proxies and translated into estimates of atmospheric oxygen. Iron-based proxies are among the most effective tools for tracking the redox chemistry of ancient oceans ${ }^{3,4}$. These proxies are inherently local, but gain global significance when analysed collectively and statistically. Here we analyse $\sim 4700$ iron speciation measurements from 2300-360 Ma shales. Our statistical analyses suggest that subsurface water masses in mid-Proterozoic oceans were predominantly anoxic and ferruginous, but with a tendency towards euxinia not observed in the Neoproterozoic Era. Analyses further indicate that early animals did not experience appreciable benthic sulfide stress. Finally, unlike proxies based on redox sensitive trace metal abundances ${ }^{1,5,6}$, iron geochemical data do not show a statistically significant change in oxygen content through the Ediacaran and Cambrian periods, sharply constraining the magnitude of the endProterozoic oxygen increase. Re-analysis of trace metal data is in fact consistent with oxygenation continuing well into the Paleozoic Era. Thus, if changing redox conditions facilitated animal diversification, it did so through a limited rise in oxygen past critical functional and ecological thresholds, as seen in modern Oxygen Minimum Zone benthos ${ }^{7-9}$. 
Proxies such as Fe-speciation chemistry record the redox state of local water masses immediately above accumulating sediments. Decades of work on the behavior of iron in marine sediments underpin the observation that enrichments in total (FeT) and highly reactive (FeHR) Fe phases track water column redox conditions (FeHR refers to iron in pyrite plus iron that is reactive to sulfide on early diagenetic timescales) $)^{3,4}$. This robust calibration permits the differentiation between oxic and anoxic water columns, as well as whether anoxic waters were iron- or sulfide-bearing [based on the proportion of highly-reactive iron that has been converted to pyrite $(\mathrm{FeP})]$.

Early studies of Fe-speciation in Proterozoic shales supported the prediction ${ }^{10}$ of euxinia in subsurface waters of Mesoproterozoic oceans and further suggested deep ocean oxygenation late in the Neoproterozoic Era ${ }^{11,12}$. However, and perhaps not surprisingly, a more complex and heterogeneous pattern of Earth surface evolution emerged as additional studies increased temporal and spatial coverage. For example, marine strata deposited ca. 1500 million years ago (Ma) from different localities show evidence of euxinic, ferruginous and oxic basins ${ }^{11,13-14}$. Similarly, Ediacaran deep-water sediments in Newfoundland indicate oxygenation at $580 \mathrm{Ma}^{12}$, yet coeval deep-water deposits in the Canadian Cordillera show an increasing prevalence of anoxia $^{15}$, or no change at all $^{16}$. Such regional heterogeneity is expected given local controls on water-column redox, and highlights the fact that Fe-speciation analyses of a single section or basin cannot be extrapolated to the global ocean.

Paleontologists have long contended with an analogous problem: how to infer global diversity through time from fossil assemblages in local stratigraphic sections. The solution was to treat tabulated data within a global statistical framework ${ }^{17}$. Following this template, we have 
developed a dataset of $\sim 4700$ new and published iron speciation measurements from fine-grained clastic rocks to test hypotheses of global redox change in Proterozoic/Paleozoic oceans and its potential links to animal evolution. Importantly, local proxy data in a global framework can track both the mean and variance of paleoenvironmental conditions through time. In addition to compiling data spanning the Great Oxidation Event (GOE, 2300 Ma) through the endDevonian, we provide 842 new analyses from Russia, northwestern Canada, Mongolia, Namibia, Svalbard, East Greenland and the western United States (Table S2), focusing on Neoproterozoic and Cambrian strata. Time-binned analysis of the entire dataset begins with the most basic distinctions: geographic region and depositional environment (inner shelf, outer shelf, and basinal; following refs. 121 15). Note that the 'basinal' environment does not represent true deep ocean depths in a modern oceanographic sense, but rather the deepest environments represented by sediments deposited during maximum flooding -- a recognizable and consistent sub-wave base environment that has been used to track deeper-water redox conditions through time (see SI). To test for significant differences, data were compared using ANOVA and Kruskal-Wallis (K-W) tests depending on normality of the data. Post hoc Tukey-Kramer tests $(\alpha=0.05)$, pairwise Wilcoxon tests and Steel-Dwass tests were applied to explore significant differences between time bins (See SI for binning rationale and sensitivity analyses).

We first investigated the proportion of anoxic water columns through time. It has been hypothesized that a major oxygenation event occurred around the Proterozoic-Phanerozoic transition, oxygenating the world's deep oceans and facilitating Cambrian animal diversification. This idea has been bolstered by redox-sensitive trace metal abundance data, which show evidence of increasing oxygen levels ${ }^{1,5,6}$, although the timing and magnitude remain poorly resolved $^{1,2}$. Aggregated iron-speciation data provide an informative complement to global trace 
metal data. Since the redox state of basinal water masses has traditionally been used as a proxy for the overall ocean-atmosphere system, and shallow-water samples are rare and heterogeneously distributed through time (Table S1), this analysis only includes samples from outer shelf and basinal environments. The proportion of samples likely deposited beneath an anoxic water column $(\mathrm{FeHR} / \mathrm{FeT}>0.38)^{1}$ was calculated for each region, and the mean and standard error were determined for each time bin. In contrast to trace metal data, analysis of Fespeciation data does not show significant change in the proportion of anoxic water columns from the Proterozoic into the early Paleozoic (ANOVA: $\mathrm{F}_{4},{ }_{52}=0.78, p=0.54$; K-W: Chi-Square $=$ $3.30 p=0.51)$ (Fig. 1A and Table S4), consistent with qualitative observations in a previous compilation ${ }^{15}$. Iron speciation more robustly identifies anoxia as opposed to oxic conditions, as FeHR enrichments can be muted during rapid deposition or in pervasively anoxic oceans where mass-balance requirements may not result in modern-style iron enrichment. Nonetheless, the proportion of oxic samples (using a conservative threshold of FeHR/FeT $<0.22)^{1}$ was tested, and again no significant differences were found (Table S4). This result raises a number of questions that we discuss below, ranging from diagnosing the nature of basinal anoxia to reconciling the seemingly divergent results between trace metal geochemistry and our database analysis.

To assess the nature of anoxic waters through time we focused on samples from deeperwater environments with FeHR/FeT $>0.38$. The average proportion of ferruginous samples between $2300-1000 \mathrm{Ma}$ is 0.59 (the balance being euxinic), consistent with recent arguments that basinal waters through the middle Proterozoic were predominantly ferruginous ${ }^{3,13}$ (the effect of subdividing the Proterozoic using a shorter 1600-1000 Ma bin was also tested; Table S4). In fact, anoxic waters throughout the Proterozoic and Paleozoic are more likely to be ferruginous than euxinic. However, real differences exist between time bins (K-W: Chi Square $=13.9, p=$ 
0.008). Specifically, the late Paleoproterozoic/Mesoproterozoic bin is more likely to capture euxinic conditions than the early Neoproterozoic, Ediacaran and Cambrian intervals, where the proportion of ferruginous samples approaches unity. The Ordovician-Devonian then marks a return to limited euxinia that is statistically distinct from the Neoproterozoic bins (Fig. 1B). Our analyses thus demonstrate that while a globally euxinic deep ocean ${ }^{10}$ did not exist, Mesoproterozoic oceans were statistically more prone to euxinia than those of the Neoproterozoic.

We further estimated sedimentary sulfide generation through Earth history. This property cannot be measured directly, but can be evaluated indirectly, as sulfide generated within sediments will bond with reactive iron to form pyrite. Hence, reactive iron acts as an effective sulfide sink, meaning that sulfide accumulation in pore waters and advective fluxes into marine waters - the free sulfide that would influence local animal ecology—will only occur in settings where most, if not all, highly reactive iron has been pyritized ${ }^{18}$. Thus, for shale deposited in oxic environments, pyrite contents broadly serve as a metric for total sulfide generation, and only environments with $\mathrm{FeP} / \mathrm{FeHR}>0.70$ could have contained high levels of pore water sulfide.

Analyses of the weight percent iron in pyrite from oxic sediments (Fig. 1C) show the inverse pattern from Fig. 1B, with higher pyrite contents in the late Paleoproterozoic/Mesoproterozoic bin, very low contents in the Neoproterozoic and Cambrian, and higher contents again in the Ordovician-Devonian (K-W: Chi Square $=25.44, p<0.0001$; Table S4), with the Neoproterozoic capturing a minimum in pyrite preservation at $~ 5 \mathrm{X}$ less than in modern oxic samples ${ }^{19}$. Similar results are seen for the proportion of oxic samples with inferred high levels of porewater sulfide (Table S4). It is worth emphasizing that the outlier is the 
Neoproterozoic — whether in the water column or the sediments, far more sulfide was generated in Mesoproterozoic and Paleozoic basins.

These results have important implications for the physiology and oxygen tolerance of early animals, which likely began to diverge $\sim 800 \mathrm{Ma}^{20}$. Based on observations from modern oxygen minimum zones (OMZs) $)^{21}$ and experiments on sponges ${ }^{22}$, it has been suggested that early animals would have tolerated the low-oxygen conditions believed to characterize the Neoproterozoic Era. With $\mathrm{O}_{2}$ partially removed as a handbrake on animal evolution, other inhibitors such as ambient sulfide ${ }^{23}$ should be considered. Sulfide is a synergistic stressor in low$\mathrm{O}_{2}$ conditions as it binds to cytochrome oxidase and consequently inhibits aerobic respiration, lowering survival times under hypoxia ${ }^{24}$. But in contrast to some modern OMZs where sulfide often reaches the sediment-water interface, Neoproterozoic animals would have experienced little, if any, benthic sulfide flux. In fact, out of 1,243 oxic Neoproterozoic samples analyzed, only $14(\sim 1.1 \%)$ show possible evidence of pore water sulfide. This bolsters suggestions that while earlier Neoproterozoic oceans may have prohibited large, metabolically active and carnivorous animals with higher oxygen demand, they could have accommodated early animals with small and thin body plans ${ }^{21-22}$. Continued research on other $\mathrm{pO}_{2}$ proxies will, in parallel, help place more precise constraints on early animal ecosystems ${ }^{25}$.

These results raise the question of whether observed trends reflect biases in the dataset, as there are known caveats when interpreting iron speciation data, most prominently including the effects of weathering and diagenesis ${ }^{3,4,15}$ (SI). However, as long as the data are sufficiently numerous and geological and analytical biases are randomly distributed with respect to time, these processes will not affect our results (e.g. ref. 26 regarding analogous errors in paleobiological data). The impact of random and systematic error can be tested with resampling 
and sensitivity analyses. Sensitivity analyses excluding possibly inappropriate samples and low data coverage regions, and a further analysis only using Mesoproterozoic (1600-1000 Ma) samples for time bin 1, are consistent with results on the entire dataset (Table S4). Further, in synthetically re-sampled datasets, the Cambrian distribution of anoxic samples is indistinguishable from the Ediacaran (Fig. S2). To test whether inappropriate binning may contribute to the invariance in Fig. 1A, data from each region from the 800-360 Ma interval were plotted individually with respect to time (Fig. 2). While there is clear spatial heterogeneity (as in the modern ocean), there are no apparent oxygenation 'events,' and a linear regression is not significant ( $p=0.45$; see also loess regression of geographically unbinned data, Fig. S1).

It has been argued that trace metals in anoxic shales capture the spatial contraction of basinal anoxia across the Ediacaran-Cambrian transition ${ }^{1,5,6}$, likely driven by increasing atmospheric $p \mathrm{O}_{2}$. To evaluate the consistency between iron speciation and trace metal results, we reanalyzed a well-vetted sedimentary uranium dataset ${ }^{6}$ using statistical methods similar to those employed in the Fe analyses, although lower data density precludes a basin-normalized approach. Maximum metal/total organic carbon (TOC) ratios are often taken as a guide to the metal inventory in ancient seawater; however, without a priori knowledge of basin restriction and secondary mineralization/local redistribution for each sample ${ }^{1,27}$, statistical approaches based on the entire population of data are appropriate.

When anoxic, organic-rich shales (TOC $>0.4 \%$ ) are binned into Neoproterozoic, Cambrian-Silurian and Devonian-Permian domains, U/TOC significantly increases with younger age (K-W: Chi Square $=75.53, p<0.0001$, all pairwise Wilcoxon tests $p<0.0001$; Table S5). The Devonian-Permian time bin contains a much higher number of enriched outlier values relative to the Cambrian-Silurian bin (Fig. S3). Thus, while the U/TOC record does show a 
punctuated increase at the Ediacaran-Cambrian boundary, it is also consistent with iron geochemical data (Fig. 1A) in suggesting full oxygenation of the oceans did not occur until later.

The question then becomes the magnitude of oxygenation implied by the Fe and trace metal datasets. Recent models indicate that relatively subtle changes in seafloor anoxia and the proportion of that seafloor that was ferruginous versus euxinic will lead to dramatic changes in seawater trace metal inventories, and by inference, trace metal enrichments in shales ${ }^{1,5,6,28}$. Trace metal enrichments, then, respond to the total size of anoxic sinks, whereas the binned iron data are tracking the percentage of sediments sampled in the stratigraphic record bathed by anoxic waters. As large changes in anoxic sink size can manifest as small shifts in the percentage of anoxic seafloor, we propose that trace metal abundances and the binned Fe-speciation records are complementary but with different thresholds; binned iron data require a larger change in global oxygen to record a significant signal.

Although absolute values of $\mathrm{pO}_{2}$ in the geological record are notoriously difficult to track, the Fe-speciation database results constrain the magnitude of the latest Proterozoic $\mathrm{pO}_{2}$ increase indicated by trace metal compilations. Canfield ${ }^{10}$ earlier posited that at atmospheric $p \mathrm{O}_{2}$ $<30-40 \%$ PAL (Present Atmospheric Levels), deeper water masses tend towards anoxia, albeit dependent upon phosphorous fluxes. While this was intended to constrain oxygen levels prior to Ediacaran oxygenation, it also provides an upper bound on Cambrian $\mathrm{pO}_{2}$ given the lack of statistical change through time. The distribution of animals in modern oceans ${ }^{8,29}$ suggest Cambrian metazoans recorded by fossils required oxygen levels above $~ 10 \%$ PAL, but not much more than that, as equally large, mobile and skeletonized animals live at and even below this level in the modern ocean ${ }^{8,29}$. The combined constraints from Fe-speciation and paleontological data are therefore consistent with Mo isotope data ${ }^{28}$, global sedimentary sulfate reduction rates ${ }^{30}$, 
$\mathrm{U} \mathrm{TOC}^{6}$ (Table S5) and some models of atmospheric $\mathrm{O}_{2}$ through time ${ }^{31}$. All offer evidence that oxygenation of the ocean/atmosphere system to essentially modern levels and a persistently oxygenated deep ocean is in large part a post-Cambrian phenomenon, as separately hypothesized on black shale distribution ${ }^{32}$. Overall, these analyses imply only a modest increase in $\mathrm{O}_{2}$ during the Ediacaran and Cambrian (Fig. 3).

This evolving picture of Earth's redox state would seemingly mute the impact of oxygen as a causal factor in Cambrian animal radiation. Observations from modern OMZs, however, suggest that a small increase in $p \mathrm{O}_{2}$ could still be a critical environmental trigger due to nonlinear threshold effects at very low oxygen levels. Many important ecological responses for macrofaunal organisms, including feeding efficiency ${ }^{9}$, species-level diversity ${ }^{8}$, and carnivore abundance and species richness ${ }^{7}$ exhibit threshold changes in the range of $5-20 \mu \mathrm{M} \mathrm{O}_{2}$, or $\sim 2-7 \%$ of modern surface ocean oxygen concentrations - strikingly similar to the changes accommodated by this analysis. Thus, a relatively small increase in $\mathrm{pO}_{2}$ could have reasonably moved animals past critical ecological thresholds, especially with respect to carnivory ${ }^{7}$, which might have driven Cambrian diversification. It remains possible though that sufficient oxygen for large, muscular carnivores existed prior to the Cambrian (Fig. 3). The critical question going forward is the availability of oxygen before the Ediacaran-Cambrian transition: were $p \mathrm{O}_{2}$ levels prior to the mid-Ediacaran in the $\sim 1-5 \%$ PAL range where modern animal ecology is severely limited, or higher?

Coupled with other geochemical data, our global database of Fe-speciation measurements provides an increasingly resolved and quantitative picture of redox evolution in Proterozoic and Paleozoic oceans. These data point to proportionally higher basinal euxinia in Mesoproterozoic and younger Paleozoic basins, with sediment and water-column sulfide generation reaching a 
minimum in the Neoproterozoic oceans. Ediacaran oxygenation was relatively modest, but may have been sufficient to remove environmental barriers to Cambrian animal evolution. Future sedimentary geochemical sampling of both iron and redox sensitive trace metal data will increase temporal resolution and the power of inference tests, with statistical analysis in a basinnormalized context providing more robust hypotheses of deep-time global change.

\section{References}

1. Lyons, T. W., Reinhard, C. T. \& Planavsky, N. J. The rise of oxygen in Earth's early ocean and atmosphere. Nature 506, 307-315 (2014).

2. Lenton, T. M., Boyle, R. A., Poulton, S. W., Shields-Zhou, G. A. \& Butterfield, N. J. Coevolution of eukaryotes and ocean oxygenation in the Neoproterozoic era. Nat. Geosci. 7, 257265 (2014).

3. Poulton, S. W. \& Canfield, D. E. Ferruginous conditions: A dominant feature of the ocean through Earth's history. Elements 7, 107-112 (2011).

4. Lyons, T. W. \& Severmann, S. A critical look at iron paleoredox proxies: new insights from modern euxinic marine basins. Geo. Cosm. Acta 70, 5698-5722 (2006).

5. Reinhard, C. T. et al. Proterozoic ocean redox and biogeochemical stasis. Proc. Nat. Acad. Sci. USA 110, 5357-5362 (2013).

6. Partin, C. A. et al. Large-scale fluctuations in Precambrian atmospheric and oceanic oxygen levels from the record of U in shales. Earth Plan. Sci. Lett. 369-370, 284-293 (2013).

7. Sperling, E. A. et al. Oxygen, ecology, and the Cambrian radiation of animals. Proc. Nat. Acad. Sci. USA 110, 13446-13451 (2013). 
8. Levin, L. A. Oxygen Minimum Zone benthos: adaptation and community response to hypoxia. Oceanogr. Mar. Biol.: Ann. Rev. 41, 1-45 (2003).

9. Woulds, C. et al. Oxygen as a control on seafloor biological communities and their roles in sedimentary carbon cycling. Limn. Oceanogr. 52, 1698-1709 (2007).

10. Canfield, D. E. A new model for Proterozoic ocean chemistry. Nature 396, 450-453 (1998).

11. Shen, Y., Knoll, A. H. \& Walter, M. R. Evidence for low sulphate and anoxia in a midProterozoic marine basin. Nature 423, 632-635 (2003).

12. Canfield, D. E., Poulton, S. W. \& Narbonne, G. M. Late-Neoproterozoic deep-ocean oxygenation and the rise of animal life. Science 315, 92-95 (2007).

13. Planavsky, N. J. et al. Widespread iron-rich conditions in the mid-Proterozoic ocean. Nature 477, 448-451 (2011).

14. Sperling, E. A. et al. Redox heterogeneity of subsurface waters in the Mesoproterozoic ocean. Geobiology 12, 373-386 (2014).

15. Canfield, D. E. et al. Ferruginous conditions dominated later Neoproterozoic deep-water chemistry. Science 321, 949-952 (2008).

16. Johnston, D. T. et al. Searching for an oxygenation event in the fossiliferous Ediacaran of northwestern Canada. Chem. Geol. 362, 273-286 (2013).

17. Alroy, J. et al. Phanerozoic trends in the global diversity of marine invertebrates. Science 321, 97-100 (2008).

18. Canfield, D. E. Reactive iron in marine sediments. Geo. Cosm. Acta 53, 619-632 (1989).

19. Raiswell, R. \& Canfield, D. E. Sources of iron for pyrite formation in marine sediments. Am. Jour. Sci. 298, 219-245 (1998). 
20. Erwin, D. H. et al. The Cambrian conundrum: Early divergence and later ecological success in the early history of animals. Science 334, 1091-1097 (2011).

21. Sperling, E. A., Halverson, G. P., Knoll, A. H., Macdonald, F. A. \& Johnston, D. T. A basin redox transect at the dawn of animal life. Earth Plan. Sci. Lett. 371, 143-155 (2013).

22. Mills, D. B. et al. Oxygen requirements of the earliest animals. Proc. Nat. Acad. Sci. USA 111, 4168-4172 (2014).

23. Martin, W. et al. Early cell evolution, eukaryotes, anoxia, sulfide, oxygen, fungi first(?), and a tree of genomes revisited. IUBMB Life 55, 193-204 (2003).

24. Vaquer-Sunyer, R. \& Duarte, C. M. Sulfide exposure accelerates hypoxia-driven mortality. Limn. Oceanogr. 55, 1075-1082 (2010).

25. Planavsky, N. J. et al. Low Mid-Proterozoic atmospheric oxygen levels and the delayed rise of animals. Science 346, 635-638 (2014).

26. Sepkoski Jr, J. J. Ten years in the library: new data confirm paleontological patterns. Paleobiology 19, 43-51 (1993).

27. Algeo, T. J. \& Rowe, H. Paleoceanographic applications of trace-metal concentration data. Chem. Geol. 324-325, 6-18 (2011).

28. Dahl, T. W. et al. Devonian rise in atmospheric oxygen correlated to the radiation of terrestrial plants and large predatory fish. Proc. Nat. Acad. Sci. USA 107, 17911-17915 (2010). 29. Rhoads, D. C. \& Morse, J. W. Evolutionary and ecologic significance of oxygendeficient marine basins. Lethaia 4, 413-428 (1971).

30. Leavitt, W. D., Halevy, I., Bradley, A. S. \& Johnston, D. T. Influence of sulfate reduction rates on the Phanerozoic sulfur isotope record. Proc. Nat. Acad. Sci. USA 110, 11244-11249 (2013). 
31. Bergman, N. M., Lenton, T. M. \& Watson, A. J. COPSE: a new model of biogeochemical cycling over Phanerozoic time. Am. Jour. Sci. 304, 397-437 (2004).

32. Berry, W. B. \& Wilde, P. Progressive ventilation of the oceans; an explanation for the distribution of the lower Paleozoic black shales. Am. Jour. Sci. 278, 257-275 (1978).

33. Belcher, C. M. \& McElwain, J. C. Limits for combustion in low $\mathrm{O}_{2}$ redefine paleoatmospheric predictions for the Mesozoic. Science 321, 1197-1200 (2008).

Supplementary Information is linked to the online version of the paper at www.nature.com/nature.

Acknowledgements- We thank D. Cole, A. Masterson, E. Beirne, G. Resendiz, A. Miller and W. Mai for lab assistance, E. Smith, T. Petach, M. Laflamme, S. Darroch, P. Myrow, J. Strauss, C. Carbone and G. Narbonne for field assistance, L. Levin, P. Girguis, C. Frieder, L. Duncan, C. Partin, S. Darroch and U. Farrell for helpful discussion, and E. Hammarlund, C. Scott, R. Raiswell, G. Gilleaudeau, S. Sahoo, Y. Shen, J. Creveling, T. Dahl, C. Partin and D. Stolper for providing raw data from published studies. E.A.S. is funded by Agouron Geobiology and NAI Postdoctoral Fellowships. This work is supported by NSF-EAR 1324095 to DTJ and the NASA Astrobiology Institute (AHK and FAM).

Author Contributions- E.A.S. and D.T.J. conceived the project, E.A.S., A.S.M., B.C.G., G.P.H., F.A.M. and D.T.J. collected samples, E.A.S., A.S.M., B.C.G. and M.K. completed 
geochemical measurements, E.A.S. and C.J.W. compiled data and analyzed the global dataset, and E.A.S. wrote the paper with input from all coauthors.

Reprints and permissions information is available at www.nature.com/reprints

The authors declare no competing financial interests.

Correspondence and requests for materials should be addressed to sperling@fas.harvard.edu

\section{Figures:}

Fig. 1- Iron geochemical data compared among five time bins (2300-1000 Ma, 1000-635 Ma, 635-542 Мa, 542-485 Ma, 485-360 Ma). Number of regions included in each bin shown in light grey across from (\#). In B) and C), light grey letters opposite W. represent results of pairwise Wilcoxon tests. Bins joined by the same lower-case letter are not statistically significant ( $p>$ 0.05). A) Proportion of samples deposited beneath anoxic water columns (FeHR/FeT > 0.38; refs. 3,19) from outer shelf and deep basin depositional environments. Circle represents average of regional proportions and whiskers represent standard error. No bins are statistically different, and the proportion of oxic samples using a conservative threshold of FeHR/FeT $<0.22$ is also not significant (Table S4). B) Proportion of samples deposited beneath ferruginous conditions from anoxic water columns (FeHR/FeT $>0.38$; FeP/FeHR $<0.70$; ref. 3) from outer shelf and deep basin depositional environments. Circle represents average of regional proportions and whiskers represent standard error. C) Weight percent iron in pyrite from samples deposited under oxic water columns from all depositional environments. Circle represents average of regional medians and whiskers represent standard error. Dashed line represents modern oxic average from ref. 19. GOE = Great Oxidation Event. Mesoprot. = Mesoproterozoic. Neoprot. = 
Neoproterozoic.

Fig. 2- Unbinned analysis of the proportion of anoxic samples from each region for the time period of 800-360 Ma. Ages for different regions based on best geological estimates; Neoproterozoic samples from the same region were separated based on the global Sturtian and Marinoan glaciations, the Gaskiers glaciation or mid-Ediacaran Shuram carbon isotope excursion and its equivalents, and the Ediacaran-Cambrian boundary. Grey bars represent 95\% binomial confidence intervals.

Fig. 3- Ocean/atmosphere oxygenation through the Proterozoic-Phanerozoic transition based on the combined absence of a statistically significant oxygenation event in iron speciation data and the presence of an oxygenation event in redox-sensitive trace metal inventories. Oxygen constraints include: 1 ) persistently anoxic subsurface waters requiring $<40 \% \mathrm{PAL}^{10}$ (iron speciation data for the Ordovician-Devonian is not statistically different than previous time bins, but data are sparse and may be subject to sampling biases—see SI), 2) minimal $\mathrm{O}_{2}$ of $\sim 0.5-1 \%$ PAL required for appearance of mass-dependent sulfur isotope fractionation, red beds, and the earliest animals $^{1,21}$; $\mathrm{O}_{2}$ levels prior to $\sim 810$ Ma may have been lower ${ }^{25}$, 3) $\mathrm{O}_{2}$ requirements of the Cambrian biota of $>10 \% \mathrm{PAL}^{8,29}$, and 4) $\mathrm{O}_{2}$ levels $>70 \%$ PAL in the latest Silurian based on the presence of fires ${ }^{33}$. Between these constraints, oxygenation could have followed many different paths, but full oxygenation of the ocean/atmosphere system is a Paleozoic phenomenon. Abbreviations: Ediac $=$ Ediacaran, Cryo $=$ Cryogenian, Cam $=$ Cambrian, Ord $=$ Ordovician, $\mathbf{S}=$ Silurian, Dev = Devonian. 
a. outer shelf and basinal environments $(n=3988)$

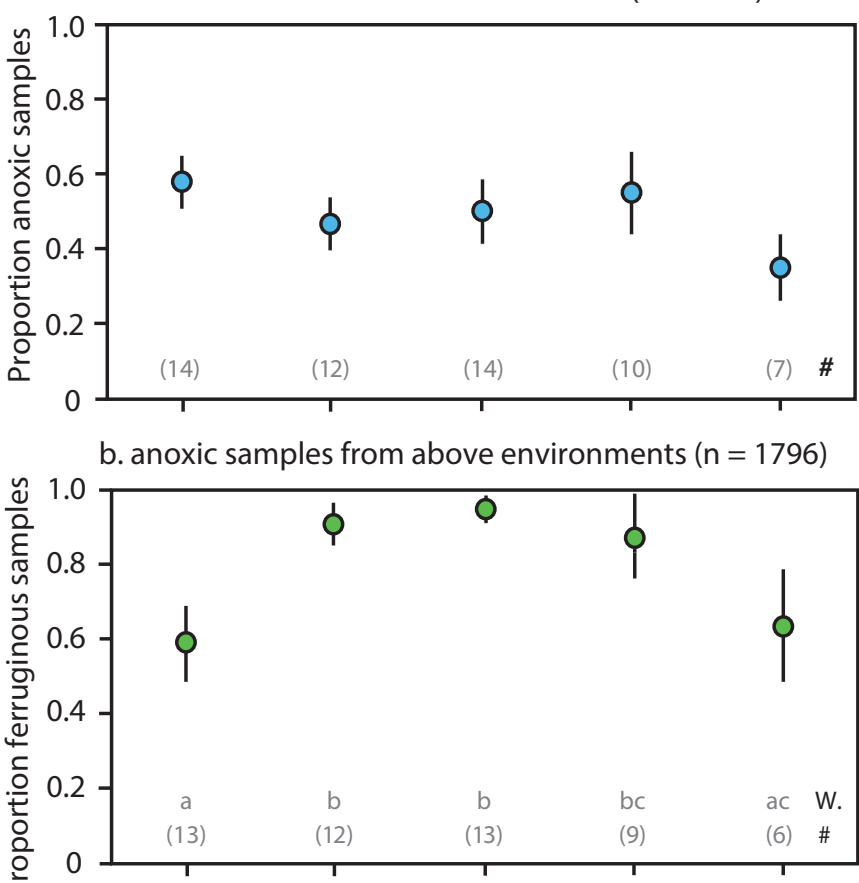

c. oxic samples from all environments $(n=2501)$

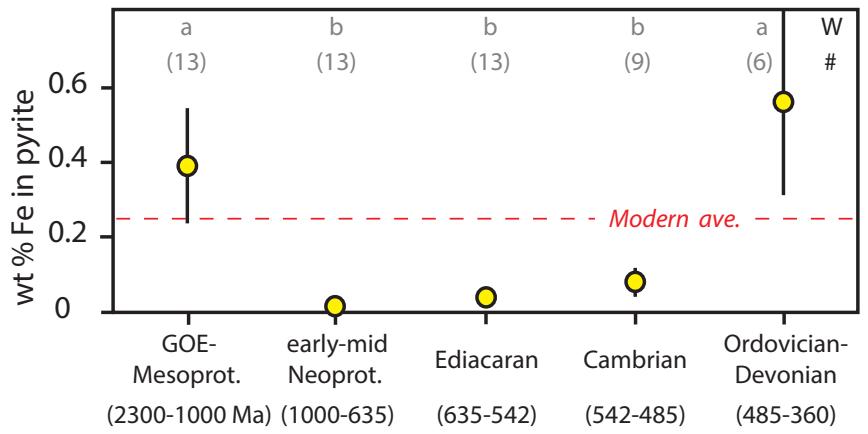




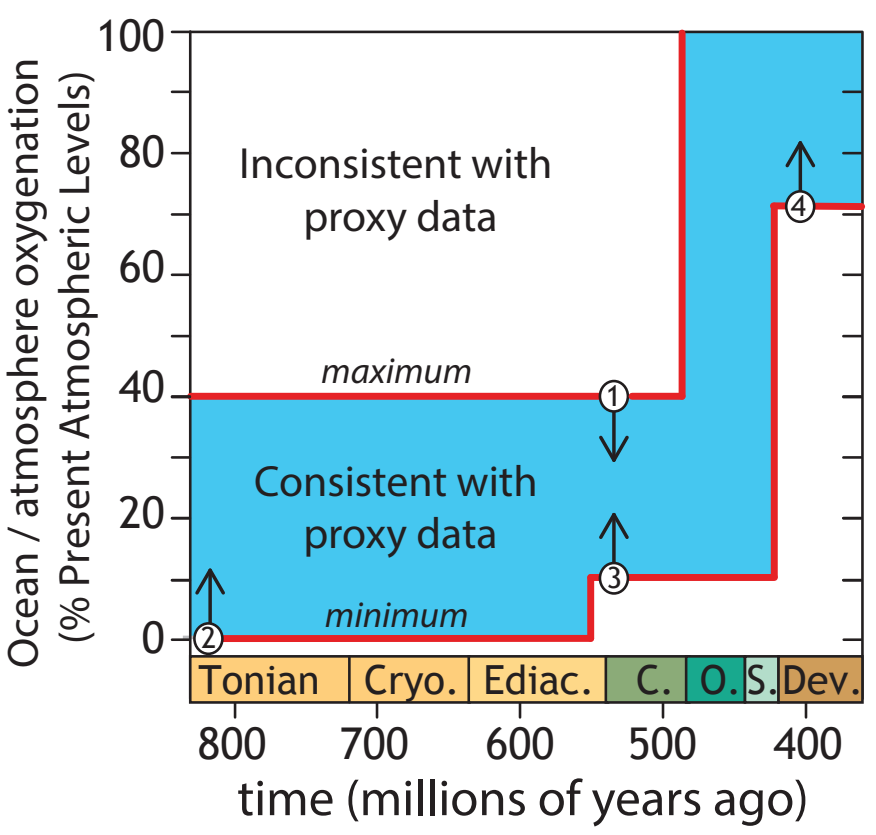




\title{
Supplementary Information
}

\section{Statistical analysis of iron geochemical data suggests limited Late Proterozoic oxygenation}

\author{
Erik A. Sperling ${ }^{1 *+}$, Charles J. Wolock², Alex S. Morgan, Benjamin C. Gill ${ }^{3}$, \\ Marcus Kunzmann ${ }^{4}$, Galen P. Halverson ${ }^{4}$, Francis A. Macdonald ${ }^{1}$, Andrew \\ H. Knoll ${ }^{1,2}$, David T. Johnston ${ }^{1 *}$
}

\author{
Affiliations: \\ ${ }^{1}$ Department of Earth and Planetary Sciences, Harvard University, Cambridge, MA \\ 02138, USA \\ 2 Department of Organismic and Evolutionary Biology, Harvard University, Cambridge, \\ MA 02138, USA \\ ${ }^{3}$ Department of Geosciences, Virginia Polytechnic Institute and State University, \\ Blacksburg, VA 24061, USA \\ ${ }^{4}$ Department of Earth and Planetary Sciences/GEOTOP, McGill University, Montreal, \\ Quebec, H3A 2A7 Canada \\ ${ }^{+}$Present address: Integrative Oceanography Division, Scripps Institution of \\ Oceanography, La Jolla, CA 90089, USA
}

*Correspondence to: sperling@fas.harvard.edu; johnston@eps.harvard.edu 


\section{Dataset compilation}

The complete dataset was assembled starting with the new analyses from this study and then compiling all published iron speciation analyses available at the time of submission. Data was available for 35 studies spanning the Proterozoic through Lower Paleozoic $^{11,12-16,21,28,34-60}$, as well as modern sediments ${ }^{19}$, and essentially all available measurements were included (Dataset S1 and Table S2). The only excluded samples at this stage were pure carbonates and Banded Iron Formations. This yielded a total of 4,706 samples, essentially all of which are fine-grained clastics (shale). All quantified iron pools and ratios were standardized to three decimal places, and published iron pools listed as $<0.01$ weight percent were converted to zero. Samples with quantification of some iron pools, but without the full set needed to calculate the FeHR/FeT ratio $(\mathrm{n}=103)$ were then excluded, as were samples with FeHR/FeT ratios $>1.05$ ( $n=68$, cutoff slightly greater than 1 chosen to allow for aggregate measurement errors - see Figure S4). This left a total of 4,535 samples (Dataset S1, total samples calculated including modern sediments ${ }^{19}$ ). While the vast majority of these samples are shales, there are some samples (marls, rather than true carbonates) with appreciable carbonate contents. Recently, Clarkson et al. (ref. 61) calibrated the iron speciation proxy in carbonate rocks and found it robustly recorded water-column redox conditions in samples with FeT $>0.5$ weight percent. In our dataset there are 96 samples (primarily from the Callison Lake Dolostone in the Ogilvie Mountains and Datangpo, Doushantou and Niutitang formations in South China) with less than $<0.5 \%$ total iron. As this represents a very low percentage of the total dataset (2.1\%) and the South China samples span the Cryogenian - Cambrian, the samples were included in the analyses.

Each sample was then coded for its age and depositional environment. Two ages were estimated for each sample. First, the sample was binned into five relatively longduration age bins, specifically 2300 - 1000 Ma, 1000 - 635 Ma, 635 - 542 Ma, 542 - 485 $\mathrm{Ma}$, and 485 - $360 \mathrm{Ma}$. In general, our binning strategy was based on attempting to include roughly equal levels of data in each bin, while also trying to use the geological time-scale when data density allowed (e.g. Ediacaran, Cambrian). The first time bin is notably long, and to test the influence of such a long bin we have conducted a sensitivity analysis only using samples from the Mesoproterozoic (Table S4). Unfortunately, such long time bins for the middle part of Earth history are generally the norm in deep-time sedimentary geochemical studies (e.g. ref. 6), and will remain so until there is a significant sampling effort in this interval. These ages were used in the binned analyses in Figure 1 of the main text. Second, a more precise age was estimated for each sample. These were based on age constraints within each individual section/basin, and in some cases the stratigraphic distance to age-constrained horizons and consideration of likely sedimentation rates. Thus, these estimates should be treated qualitatively, although the errors are likely not large with respect to the long time span of interest here. These estimates were used for the unbinned analysis in Fig. 2 of the main text and Figure S1. Coding of depositional environment followed the strategy of refs. (11) and (15), with samples coded as 1) inner shelf, 2) outer shelf, 3) basinal. It is acknowledged that the vast majority of sedimentary basins preserved in the geological record are not continental margins with a typical 'shelf.' Additionally, some samples (e.g. ref. 43) are from lacustrine settings. Thus, while many basins may not show a classic shelf-to-basin 
transition, the environmental distinctions can be more broadly considered as:

1) Environment 1/Inner Shelf: Shale interbedded with abundant shallowwater indicators. This includes clastic beds with wave-generated sedimentary structures as well as shallow-water carbonates such as stromatolites, oolites, and rip-up conglomerates. Evidence of exposure-i.e. mudcracks, karsting, teepee structures-are often in relatively close stratigraphic proximity on the meters to 10 s of meters scale.

2) Environment 2/Outer Shelf: Shale from sequences that generally show little wave activity, but with occasional evidence for storm and/or wave activity, such as hummocky cross-stratified sands encased in shales. Evidence for exposure is not in close stratigraphic proximity.

3) Environment 3/Basinal: Shale from successions with no evidence for any storm and/or wave activity for an appreciable (i.e. $>50 \mathrm{~m}$ ) stratigraphic distance. Generally located considerably basin-ward of shallower-water facies.

\begin{tabular}{|l|c|c|c|c|c|}
\hline & $\begin{array}{c}\text { Bin 1 } \\
2300-1000 \mathrm{Ma}\end{array}$ & $\begin{array}{c}\text { Bin 2 } \\
1000-635 \mathrm{Ma}\end{array}$ & $\begin{array}{c}\text { Bin 3 } \\
635-542 \mathrm{Ma}\end{array}$ & $\begin{array}{c}\text { Bin 4 } \\
542-485 \mathrm{Ma}\end{array}$ & $\begin{array}{c}\text { Bin 5 } \\
485-350 \mathrm{Ma}\end{array}$ \\
\hline Inner shelf & 76 & 339 & 97 & 35 & 0 \\
\hline Outer shelf & 246 & 244 & 316 & 127 & 144 \\
\hline Deep basin & 704 & 340 & 985 & 405 & 243 \\
\hline Total & 1026 & 923 & 1398 & 567 & 387 \\
\hline
\end{tabular}

Table S1: Distribution of samples by environment through time (modern samples ${ }^{19}$ were not coded by depth).

It is recognized that the absolute water depth of samples deposited beneath storm wave base is notoriously difficult to distinguish (see discussion in ref. 14). There are certainly differences in water depth between basinal samples in this study, but the differences are difficult to quantify. Nonetheless, the basinal environment represents an identifiable and continuous environment in the geological record. As long as the water depth of samples is not unevenly distributed with respect to time, these differences should not affect the overall statistical analyses. As more data becomes available, tests between different environments (for instance between distal basinal shales and turbidite basins) can be usefully compared.

\begin{tabular}{|c|c|l|l|c|c|c|}
\hline $\begin{array}{c}\text { Age } \\
\text { Bin }\end{array}$ & $\begin{array}{c}\text { Age } \\
\text { (Ma) }\end{array}$ & $\begin{array}{c}\text { Basin/Geographic } \\
\text { Region }\end{array}$ & \multicolumn{1}{|c|}{ Publication } & $\begin{array}{c}\text { Core/Out } \\
\text { crop }\end{array}$ & \#samples & Notes \\
\hline $\begin{array}{c}2300- \\
1000\end{array}$ & 1800 & Animikie Basin & $\begin{array}{l}\text { Poulton et al., 2010, Nature } \\
\text { Geoscience (34) }\end{array}$ & Core & 269 & 1,2 \\
\hline $2300-$ & 1460 & Belt Basin & Planavsky et al., 2011, Nature (13) & Core & 14 & \\
\hline
\end{tabular}




\begin{tabular}{|c|c|c|c|c|c|c|}
\hline 1000 & & & & & & \\
\hline $\begin{array}{c}2300- \\
1000\end{array}$ & 1200 & Borden Basin & Planavsky et al., 2011, Nature (13) & Outcrop & 6 & \\
\hline $\begin{array}{c}2300- \\
1000\end{array}$ & 2150 & Botswana & $\begin{array}{l}\text { Scott et al., 2014, Earth and } \\
\text { Planetary Science Letters (37) }\end{array}$ & Core & 15 & \\
\hline $\begin{array}{c}2300- \\
1000\end{array}$ & 1100 & Brasília Fold Belt & $\begin{array}{l}\text { Geboy et al., 2013, Precambrian } \\
\text { Research (42) }\end{array}$ & Core & 40 & \\
\hline $\begin{array}{c}2300- \\
1000\end{array}$ & 2000 & Fennoscandia & $\begin{array}{l}\text { Reuschel et al., 2012, Precambrian } \\
\text { Research (35) }\end{array}$ & Core & 36 & 1 \\
\hline $\begin{array}{c}2300- \\
1000\end{array}$ & 2100 & $\begin{array}{l}\text { Francevillian } \\
\text { Basin }\end{array}$ & Canfield et al., 2013, PNAS (38) & Both & 139 & \\
\hline $\begin{array}{c}2300- \\
1000 \\
\end{array}$ & 2100 & $\begin{array}{l}\text { Francevillian } \\
\text { Basin }\end{array}$ & El Albani et al., 2010, Nature (39) & Both & 24 & \\
\hline $\begin{array}{c}2300- \\
1000\end{array}$ & 1100 & Mauritania & $\begin{array}{l}\text { Gilleaudeau and Kah, 2013, } \\
\text { Chemical Geology (41) }\end{array}$ & Core & 128 & \\
\hline $\begin{array}{c}2300- \\
1000 \\
\end{array}$ & 1650 & McArthur Basin & $\begin{array}{l}\text { Shen et al., 2002, American } \\
\text { Journal of Science (40) }\end{array}$ & Core & 24 & 7 \\
\hline $\begin{array}{c}2300- \\
1000\end{array}$ & 1650 & McArthur Basin & Planavsky et al., 2011, Nature (13) & Core & 50 & \\
\hline $\begin{array}{c}2300- \\
1000\end{array}$ & 1080 & Midcontinent Rift & $\begin{array}{l}\text { Cumming et al., 2013, Geology } \\
\text { (43) }\end{array}$ & Both & 62 & 3 \\
\hline $\begin{array}{c}2300- \\
1000\end{array}$ & 1680 & North China & Planavsky et al., 2011, Nature (13) & Outcrop & 31 & \\
\hline $\begin{array}{c}2300- \\
1000\end{array}$ & 2000 & Onega Basin & $\begin{array}{l}\text { Scott et al., 2014, Earth and } \\
\text { Planetary Science Letters (37) }\end{array}$ & Core & 51 & \\
\hline $\begin{array}{c}2300- \\
1000\end{array}$ & 2000 & Onega Basin & $\begin{array}{l}\text { Asael et al., 2013, Chemical } \\
\text { Geology (36) }\end{array}$ & Core & 22 & 1 \\
\hline $\begin{array}{c}2300- \\
1000\end{array}$ & 1400 & Roper Basin & Shen et al., 2003, Nature (11) & Core & 117 & 7 \\
\hline $\begin{array}{c}2300- \\
1000 \\
\end{array}$ & 1440 & Southern Urals & $\begin{array}{l}\text { Sperling et al., 2014, Geobiology } \\
\text { (14) }\end{array}$ & Core & 43 & \\
\hline $\begin{array}{c}2300- \\
1000 \\
\end{array}$ & 1440 & Southern Urals & This study & Core & 7 & \\
\hline $\begin{array}{c}1000- \\
635\end{array}$ & $\begin{array}{l}660 \\
\text { and } \\
805\end{array}$ & $\begin{array}{l}\text { Centralian } \\
\text { Superbasin }\end{array}$ & Canfield et al., 2008, Science (15) & Core & 110 & 5 \\
\hline $\begin{array}{c}1000- \\
635\end{array}$ & 750 & Death Valley & This study & Outcrop & 104 & 4 \\
\hline $\begin{array}{c}1000- \\
635\end{array}$ & 660 & East Greenland & This study & Outcrop & 7 & \\
\hline $\begin{array}{c}1000- \\
635\end{array}$ & $\begin{array}{c}660 \\
\text { and } \\
800- \\
750 \\
\end{array}$ & East Greenland & Canfield et al., 2008, Science (15) & Outcrop & 44 & \\
\hline $\begin{array}{c}1000- \\
635 \\
\end{array}$ & 800 & $\begin{array}{l}\text { Eastern European } \\
\text { Platform }\end{array}$ & $\begin{array}{l}\text { Johnston et al., 2012, Earth and } \\
\text { Planetary Science Letters (44) }\end{array}$ & Core & 19 & \\
\hline $\begin{array}{c}1000- \\
635\end{array}$ & 750 & Grand Canyon & Canfield et al., 2008, Science (15) & Outcrop & 16 & 4 \\
\hline $\begin{array}{c}1000- \\
635\end{array}$ & 750 & Grand Canyon & $\begin{array}{l}\text { Johnston et al., 2010, Earth and } \\
\text { Planetary Science Letters (45) }\end{array}$ & Outcrop & 105 & 4 \\
\hline $\begin{array}{c}1000- \\
635\end{array}$ & 650 & $\begin{array}{l}\text { Mackenzie } \\
\text { Mountains }\end{array}$ & This study & Outcrop & 41 & \\
\hline $\begin{array}{c}1000- \\
635\end{array}$ & 660 & $\begin{array}{l}\text { Mackenzie } \\
\text { Mountains }\end{array}$ & Canfield et al., 2008, Science (15) & Outcrop & 19 & \\
\hline $\begin{array}{c}1000- \\
635\end{array}$ & 800 & Ogilvie Mountains & $\begin{array}{l}\text { Sperling et al., 2013, Earth and } \\
\text { Planetary Science Letters (21) }\end{array}$ & Outcrop & 234 & \\
\hline $\begin{array}{c}1000- \\
635\end{array}$ & 660 & South China & $\begin{array}{l}\text { Feng et al., 2010, Gondwana } \\
\text { Research (47) }\end{array}$ & Outcrop & 16 & 7 \\
\hline
\end{tabular}




\begin{tabular}{|c|c|c|c|c|c|c|}
\hline $\begin{array}{c}1000- \\
635 \\
\end{array}$ & 660 & South China & $\begin{array}{l}\text { Li et al., 2012, Earth and Planetary } \\
\text { Science Letters (48) }\end{array}$ & Outcrop & 27 & \\
\hline $\begin{array}{c}1000- \\
635\end{array}$ & 660 & Spitsbergen & Canfield et al., 2008, Science (15) & Outcrop & 9 & \\
\hline $\begin{array}{c}1000- \\
635\end{array}$ & $\begin{array}{c}650 \\
\text { and } \\
835- \\
740 \\
\end{array}$ & Svalbard & This study & Outcrop & 40 & \\
\hline $\begin{array}{c}1000- \\
635\end{array}$ & 750 & Uinta Mountains & Hayes, 2013 (46) & Outcrop & 73 & 4 \\
\hline $\begin{array}{c}1000- \\
635\end{array}$ & 650 & $\begin{array}{l}\text { Wernecke } \\
\text { Mountains }\end{array}$ & This study & Outcrop & 5 & \\
\hline $\begin{array}{c}1000- \\
635\end{array}$ & 650 & $\begin{array}{l}\text { Wernecke } \\
\text { Mountains }\end{array}$ & This study & Outcrop & 27 & \\
\hline $\begin{array}{c}635- \\
542 \\
\end{array}$ & $\begin{array}{c}590- \\
560 \\
\end{array}$ & $\begin{array}{l}\text { Avalon } \\
\text { (Newfoundland) }\end{array}$ & Canfield et al., 2007, Science (12) & Outcrop & 200 & 5 \\
\hline $\begin{array}{l}635- \\
542\end{array}$ & 610 & East Greenland & Canfield et al., 2008, Science (15) & Outcrop & 23 & 3 \\
\hline $\begin{array}{l}635- \\
542 \\
\end{array}$ & $\begin{array}{l}600- \\
550 \\
\end{array}$ & $\begin{array}{l}\text { Eastern European } \\
\text { Platform }\end{array}$ & $\begin{array}{l}\text { Johnston et al., 2012, Earth and } \\
\text { Planetary Science Letters (44) }\end{array}$ & Core & 84 & \\
\hline $\begin{array}{l}635- \\
542\end{array}$ & 620 & $\begin{array}{l}\text { Mackenzie } \\
\text { Mountains }\end{array}$ & Shen et al., 2008, PNAS (50) & Outcrop & 59 & 7 \\
\hline $\begin{array}{l}635- \\
542 \\
\end{array}$ & $\begin{array}{l}570- \\
542\end{array}$ & $\begin{array}{l}\text { Mackenzie } \\
\text { Mountains }\end{array}$ & This study & Outcrop & 183 & \\
\hline $\begin{array}{l}635- \\
542\end{array}$ & $\begin{array}{l}620 \\
\text { and } \\
570\end{array}$ & $\begin{array}{l}\text { Mackenzie } \\
\text { Mountains }\end{array}$ & Canfield et al., 2008, Science (15) & Outcrop & 27 & \\
\hline $\begin{array}{l}635- \\
542 \\
\end{array}$ & 544 & Mongolia & This study & Outcrop & 4 & \\
\hline $\begin{array}{l}635- \\
542 \\
\end{array}$ & $\begin{array}{c}548- \\
544\end{array}$ & Namibia & This study & Outcrop & 49 & \\
\hline $\begin{array}{c}635- \\
542 \\
\end{array}$ & 555 & Nico Perez Terrane & $\begin{array}{l}\text { Frei et al., 2013, Gondwana } \\
\text { Research (52) }\end{array}$ & Outcrop & 30 & \\
\hline $\begin{array}{l}635- \\
542\end{array}$ & $\begin{array}{c}620- \\
550\end{array}$ & Ogilvie Mountains & This study & Outcrop & 37 & \\
\hline $\begin{array}{l}635- \\
542 \\
\end{array}$ & 545 & Siberia & Canfield et al., 2008, Science (15) & Outcrop & 3 & \\
\hline $\begin{array}{l}635- \\
542\end{array}$ & 550 & South China & $\begin{array}{l}\text { Wang et al., 2012, Chemical } \\
\text { Geology (53) }\end{array}$ & Outcrop & 26 & \\
\hline $\begin{array}{c}635- \\
542 \\
\end{array}$ & 580 & South China & Li et al., 2010, Science (51) & Outcrop & 112 & \\
\hline $\begin{array}{l}635- \\
542 \\
\end{array}$ & 620 & South China & Shen et al., 2008, PNAS (50) & Outcrop & 34 & 7 \\
\hline $\begin{array}{l}635- \\
542 \\
\end{array}$ & 630 & South China & Sahoo et al., 2012, Nature (49) & Outcrop & 67 & \\
\hline $\begin{array}{l}635- \\
542\end{array}$ & $\begin{array}{l}550 \\
\text { and } \\
600 \\
\end{array}$ & South China & Canfield et al., 2008, Science (15) & Outcrop & 15 & \\
\hline $\begin{array}{c}635- \\
542 \\
\end{array}$ & $\begin{array}{c}620- \\
565 \\
\end{array}$ & $\begin{array}{l}\text { Southern Canadian } \\
\text { Cordillera } \\
\end{array}$ & Canfield et al., 2008, Science (15) & Outcrop & 191 & \\
\hline $\begin{array}{c}635- \\
542 \\
\end{array}$ & 620 & Spitsbergen & Canfield et al., 2008, Science (15) & Outcrop & 3 & \\
\hline $\begin{array}{l}635- \\
542 \\
\end{array}$ & $\begin{array}{c}625- \\
590 \\
\end{array}$ & $\begin{array}{l}\text { Stuart Shelf, South } \\
\text { Australia }\end{array}$ & Canfield et al., 2008, Science (15) & Core & 46 & \\
\hline $\begin{array}{l}635- \\
542 \\
\end{array}$ & $\begin{array}{c}630- \\
635 \\
\end{array}$ & Svalbard & This study & Outcrop & 17 & \\
\hline $\begin{array}{c}635- \\
542 \\
\end{array}$ & $\begin{array}{l}570- \\
545 \\
\end{array}$ & $\begin{array}{l}\text { Wernecke } \\
\text { Mountains }\end{array}$ & This study & Outcrop & 68 & \\
\hline $635-$ & $630-$ & Wernecke & Johnston et al., 2013, Chemical & Outcrop & 179 & \\
\hline
\end{tabular}




\begin{tabular}{|c|c|c|c|c|c|c|}
\hline 542 & 550 & Mountains & Geology (16) & & & \\
\hline $\begin{array}{c}542- \\
485\end{array}$ & 530 & East Greenland & Canfield et al., 2008, Science (15) & Outcrop & 5 & \\
\hline $\begin{array}{c}542- \\
485\end{array}$ & 510 & Georgina Basin & $\begin{array}{l}\text { Creveling et al., 2014, GSA } \\
\text { Bulletin (56) }\end{array}$ & Core & 34 & 6 \\
\hline $\begin{array}{c}542- \\
485\end{array}$ & 505 & House Embayment & This study & Outcrop & 138 & \\
\hline $\begin{array}{c}542- \\
485\end{array}$ & 535 & $\begin{array}{l}\text { Mackenzie } \\
\text { Mountains }\end{array}$ & This study & Outcrop & 51 & \\
\hline $\begin{array}{c}542- \\
485 \\
\end{array}$ & 538 & Mongolia & This study & Outcrop & 20 & \\
\hline $\begin{array}{l}542- \\
485 \\
\end{array}$ & 538 & Namibia & This study & Outcrop & 25 & \\
\hline $\begin{array}{c}542- \\
485 \\
\end{array}$ & 535 & Ogilvie Mountains & This study & Outcrop & 2 & \\
\hline $\begin{array}{c}542- \\
485 \\
\end{array}$ & 540 & Siberia & Canfield et al., 2008, Science (15) & Outcrop & 6 & \\
\hline $\begin{array}{c}542- \\
485\end{array}$ & 520 & South China & Dahl et al., 2010, PNAS (28) & Core & 6 & \\
\hline $\begin{array}{c}542- \\
485\end{array}$ & 525 & South China & Canfield et al., 2008, Science (15) & Outcrop & 40 & \\
\hline $\begin{array}{c}542- \\
485\end{array}$ & 525 & South China & $\begin{array}{l}\text { Feng et al., 2014, Precambrian } \\
\text { Research (54) }\end{array}$ & Outcrop & 30 & \\
\hline $\begin{array}{c}542- \\
485\end{array}$ & 525 & South China & $\begin{array}{l}\text { Och et al., 2013, Precambrian } \\
\text { Research (55) }\end{array}$ & Outcrop & 142 & \\
\hline $\begin{array}{c}542- \\
485\end{array}$ & 525 & South China & $\begin{array}{l}\text { Wang et al., 2012, Chemical } \\
\text { Geology (53) }\end{array}$ & Outcrop & 35 & \\
\hline $\begin{array}{c}542- \\
485 \\
\end{array}$ & 505 & $\begin{array}{l}\text { Southern Canadian } \\
\text { Cordillera }\end{array}$ & Dahl et al., 2010, PNAS (28) & Outcrop & 4 & \\
\hline $\begin{array}{c}542- \\
485\end{array}$ & 497 & Sweden & Gill et al., 2011, Nature (57) & Core & 30 & \\
\hline $\begin{array}{c}542- \\
485 \\
\end{array}$ & 535 & $\begin{array}{l}\text { Wernecke } \\
\text { Mountains } \\
\end{array}$ & This study & Outcrop & 19 & \\
\hline $\begin{array}{l}485- \\
359\end{array}$ & $\begin{array}{l}365 \\
\text { and } \\
367\end{array}$ & Acadian & Dahl et al., 2010, PNAS (28) & Outcrop & 4 & \\
\hline $\begin{array}{l}485- \\
359\end{array}$ & $\begin{array}{l}382 \\
\text { and } \\
387\end{array}$ & Acadian & $\begin{array}{l}\text { Boyer et al., 2011, Palaeogeog., } \\
\text { Palaeoclim., Palaeoecol. (58) }\end{array}$ & Outcrop & 101 & \\
\hline $\begin{array}{l}485- \\
359 \\
\end{array}$ & 445 & Carnic Alps & $\begin{array}{l}\text { Hammarlund et al., 2012, Earth } \\
\text { and Planetary Science Letters (60) }\end{array}$ & Outcrop & 39 & \\
\hline $\begin{array}{c}485- \\
359\end{array}$ & 443 & Denmark & Dahl et al., 2010, PNAS (10) & Core & 10 & \\
\hline $\begin{array}{c}485- \\
359\end{array}$ & 445 & Denmark & $\begin{array}{l}\text { Hammarlund et al., 2012, Earth } \\
\text { and Planetary Science Letters (60) }\end{array}$ & Core & 77 & \\
\hline $\begin{array}{c}485- \\
359\end{array}$ & 445 & Scotland & $\begin{array}{l}\text { Hammarlund et al., 2012, Earth } \\
\text { and Planetary Science Letters (60) }\end{array}$ & Outcrop & 46 & \\
\hline $\begin{array}{l}485- \\
359 \\
\end{array}$ & 435 & South Africa & Dahl et al., 2010, PNAS (28) & Core & 3 & \\
\hline $\begin{array}{l}485- \\
359\end{array}$ & $\begin{array}{r}435- \\
485 \\
\end{array}$ & Sweden & Dahl et al., 2010, PNAS (28) & Core & 18 & \\
\hline $\begin{array}{c}485- \\
359\end{array}$ & 480 & Taconic & $\begin{array}{l}\text { Farrell et al., 2013, American } \\
\text { Journal of Science (59) }\end{array}$ & Outcrop & 93 & \\
\hline Modern & 0 & Many sites & $\begin{array}{l}\text { Raiswell and Canfield, 1998, } \\
\text { American Journal of Science (19) }\end{array}$ & Sediment & 233 & \\
\hline
\end{tabular}

Table S2: Datasets used in this study. Number of samples from each study that were incorporated into this database may be slightly less than the published number if there were 
problems obtaining the data or converting it from published files. Reference numbers listed in parentheses following author names. Notes: 1- Some samples contain appreciable Fe-AVS (Acid Volatile Sulfides), 2- Only samples from the clastic Rove and Virginia Formations were included, 3- Lacustrine, 4- Some samples may represent nonmarine to marginal marine environments, 5Coarse-grained rocks present in tillite units, 6- Only samples with $>40 \%$ insoluble residue were included, 7- Samples analyzed under non-sequential, dithionite-only iron extraction protocol for phases other than pyrite.

\section{Time-binned geographic analyses}

Proportion of anoxic samples through time: 547 samples from inner shelf environments were removed, resulting in 3,988 samples from outer shelf and basinal environments (including modern samples ${ }^{19}$, as for all counts below). The proportion of anoxic samples was then calculated based on an anoxic threshold of FeHR/FeT ratio $>0.38$ (refs. 3 and 19).

Proportion of oxic samples through time: 547 samples from inner shelf environments were removed, resulting in 3,988 samples from outer shelf and basinal environments. The proportion of oxic samples was then calculated based on an oxic threshold of FeHR/FeT ratio $<0.22$ (refs. 3 and 62 ).

Proportion of ferruginous samples through time: From the 3,988 samples from outer shelf and basinal environments, 2,192 samples with FeHR/FeT $\leq 0.38$ were removed. This resulted in 1,796 samples from more distal environments that were likely deposited beneath anoxic water columns. Two thresholds (0.7 and 0.8$)$ currently exist for distinguishing between euxinic and ferruginous water columns ${ }^{3}$. Due to the high proportion of samples from outcrop in the database, which may have experienced minor to major oxidative weathering (converting FeP to other highly-reactive phases, specifically iron oxides ${ }^{15}$ ) the lower 0.7 threshold was used.

FeP contents of oxic sediments: From the 4,535 total samples, 2,034 samples from all three depositional environments were removed that had FeHR/FeT $>0.38$. This resulted in 2,501 samples likely deposited under an oxygenated water column.

Proportion of oxic sediments with high porewater sulfide levels: The same set of 2,501 samples likely deposited under an oxygenated water column as in the analyses of FeP contents was used. Samples with FeP/FeHR $>0.7$ were considered to be ancient sediments that potentially carried high levels of porewater sulfide beneath an oxygenated water column.

For ratios in all analyses, the proportion for each region was calculated, and the global average, standard deviation and standard error calculated for each time bin. For FeP contents, the median value was calculated for each region, and the global average, standard deviation and standard error calculated for each time bin based on the medians. The results of these calculations are shown in Table S3. 


\section{A. Proportion of anoxic samples (FeHR/FeT $>0.38)$ from outer shelf and deep basin environments}

\begin{tabular}{|l|c|}
\hline Time bin 1 (2300 - 1000 Ma) & Proportion anoxic \\
\hline Fennoscandia & 0.94 \\
North China & 0.42 \\
Belt Basin & 0.79 \\
Borden Basin & 0.67 \\
Mauritania & 0.63 \\
Francevillian Basin & 0.40 \\
Southern Urals & 0.00 \\
Roper Basin & 0.26 \\
McArthur Basin & 0.84 \\
Animikie Basin & 0.37 \\
Brasília Fold Belt & 0.62 \\
Onega Basin & 0.76 \\
Midcontinent Rift & 0.45 \\
Botswana & 0.93 \\
\hline
\end{tabular}

\begin{tabular}{|l|c|}
\hline 14 regions & $\mathrm{n}=$ \\
\hline Average & 0.58 \\
\hline Standard dev. & 0.27 \\
\hline Standard error & 0.07 \\
\hline
\end{tabular}

\begin{tabular}{|l|c|}
\hline Time bin 2 (1000 - 635 Ma) & Proportion anoxic \\
\hline South China & 0.56 \\
Wernecke Mountains & 0.30 \\
Death Valley & 0.38 \\
Grand Canyon & 0.55 \\
Centralian Superbasin & 0.64 \\
Spitsbergen & 0.78 \\
East Greenland & 0.13 \\
Ogilvie Mountains & 0.53 \\
Mackenzie Mountains & 0.14 \\
Uinta Mountains & 0.20 \\
Svalbard & 0.62 \\
Callison Lake & 0.86 \\
\hline
\end{tabular}

\begin{tabular}{|l|c|}
\hline & $\mathrm{n}=$ \\
12 regions & 584 \\
\hline Average & 0.47 \\
\hline Standard dev. & 0.24 \\
\hline Standard error & 0.07 \\
\hline
\end{tabular}

\begin{tabular}{|c|c|c|c|}
\hline Time bin 3 (635 - 542 Ma) & Proportion anoxic & & \\
\hline South China & 0.73 & 14 regions & $\begin{array}{c}\mathrm{n}= \\
1301\end{array}$ \\
\hline Wernecke Mountains & 0.49 & Average & 0.50 \\
\hline Eastern European Platform & 0.02 & Standard dev. & 0.33 \\
\hline Southern Canadian Cordillera & 0.24 & Standard error & 0.09 \\
\hline
\end{tabular}




\begin{tabular}{|l|l|} 
Avalon & 0.09 \\
Spitsbergen & 1.00 \\
East Greenland & 0.35 \\
Stuart Shelf & 0.81 \\
Ogilvie Mountains & 0.59 \\
Mackenzie Mountains & 0.55 \\
Nico Perez Terrane (Uruguay) & 0.60 \\
Mongolia & 1.00 \\
Svalbard & 0.47 \\
Namibia & 0.00 \\
\hline
\end{tabular}

\begin{tabular}{|c|c|c|c|}
\hline Time bin 4 (542 - 485 Ma) & Proportion anoxic & & \\
\hline South China & 0.81 & 10 regions & $\begin{array}{l}n= \\
532\end{array}$ \\
\hline Wernecke Mountains & 0.63 & Average & 0.55 \\
\hline House Embayment & 0.27 & Standard dev. & 0.35 \\
\hline Sweden & 1.00 & Standard error & 0.11 \\
\hline Southern Canadian Cordillera & 0.50 & & \\
\hline Ogilvie Mountains & 1.00 & & \\
\hline Mackenzie Mountains & 0.37 & & \\
\hline Georgina Basin & 0.72 & & \\
\hline Namibia & 0.00 & & \\
\hline Mongolia & 0.15 & & \\
\hline
\end{tabular}

\begin{tabular}{|c|c|c|c|}
\hline Time bin 5 (485 - 350 Ma) & Proportion anoxic & & \\
\hline Acadian & 0.06 & 7 regions & $\begin{array}{l}\mathrm{n}= \\
387\end{array}$ \\
\hline Taconic & 0.30 & Average & 0.35 \\
\hline Scotland & 0.40 & Standard dev. & 0.24 \\
\hline Denmark & 0.53 & Standard error & 0.09 \\
\hline South Africa & 0.00 & & \\
\hline Sweden & 0.59 & & \\
\hline Carnic Alps & 0.57 & & \\
\hline
\end{tabular}

B. Proportion of oxic samples (FeHR/FeT $<0.22)$ from outer shelf and deep basin environments

\begin{tabular}{|c|c|c|c|}
\hline Time bin 1 (2300 - 1000 Ma) & Proportion oxic & & \\
\hline Fennoscandia & 0.03 & 14 regions & $\begin{array}{l}\mathrm{n}= \\
952\end{array}$ \\
\hline North China & 0.29 & Average & 0.22 \\
\hline Belt Basin & 0.00 & Standard dev. & 0.27 \\
\hline Borden Basin & 0.17 & Standard error & 0.07 \\
\hline Mauritania & 0.21 & & \\
\hline
\end{tabular}




\begin{tabular}{|l|l|} 
Francevillian Basin & 0.35 \\
Southern Urals & 0.95 \\
Roper Basin & 0.64 \\
McArthur Basin & 0.08 \\
Animikie Basin & 0.18 \\
Brasília Fold Belt & 0.13 \\
Onega Basin & 0.07 \\
Midcontinent Rift & 0.00 \\
Botswana & 0.00 \\
\hline
\end{tabular}

\begin{tabular}{|l|c|}
\hline Time bin 2 (1000 - 635 Ma) & Proportion oxic \\
\hline South China & 0.38 \\
Wernecke Mountains & 0.22 \\
Death Valley & 0.36 \\
Grand Canyon & 0.29 \\
Centralian Superbasin & 0.04 \\
Spitsbergen & 0.00 \\
East Greenland & 0.26 \\
Ogilvie Mountains & 0.19 \\
Mackenzie Mountains & 0.41 \\
Uinta Mountains & 0.00 \\
Svalbard & 0.14 \\
Callison Lake & 0.00 \\
\hline
\end{tabular}

\begin{tabular}{|l|l|}
\hline & $\mathrm{n}=$ \\
12 regions & 584 \\
\hline Average & 0.19 \\
\hline Standard dev. & 0.15 \\
\hline Standard error & 0.04 \\
\hline
\end{tabular}

\begin{tabular}{|l|c|}
\hline Time bin 3 (635 - 542 Ma) & Proportion oxic \\
\hline South China & 0.16 \\
Wernecke Mountains & 0.09 \\
Eastern European Platform & 0.19 \\
Southern Canadian Cordillera & 0.52 \\
Avalon & 0.33 \\
Spitsbergen & 0.00 \\
East Greenland & 0.18 \\
Stuart Shelf & 0.00 \\
Ogilvie Mountains & 0.05 \\
Mackenzie Mountains & 0.26 \\
Nico Perez Terrane (Uruguay) & 0.20 \\
Mongolia & 0.00 \\
Svalbard & 0.17 \\
Namibia & 0.98 \\
\hline
\end{tabular}

\begin{tabular}{|l|c|}
\hline 14 regions & $\begin{array}{c}\mathrm{n}= \\
1301\end{array}$ \\
\hline Average & 0.22 \\
\hline Standard dev. & 0.26 \\
\hline Standard error & 0.07 \\
\hline
\end{tabular}

\begin{tabular}{|l|l|}
\hline Time bin 4 (542 - 485 Ma) & Proportion oxic \\
\hline
\end{tabular}




\begin{tabular}{|l|l|} 
& \\
Wouth China & 0.03 \\
House Embayment & 0.00 \\
Sweden & 0.36 \\
Southern Canadian Cordillera & 0.00 \\
Ogilvie Mountains & 0.00 \\
Mackenzie Mountains & 0.00 \\
Georgina Basin & 0.00 \\
Namibia & 0.00 \\
Mongolia & 0.96 \\
\hline
\end{tabular}

\begin{tabular}{|c|c|}
\hline 10 regions & $\begin{array}{l}\mathrm{n}= \\
532\end{array}$ \\
\hline Average & 0.21 \\
\hline Standard dev. & 0.36 \\
\hline Standard error & 0.11 \\
\hline
\end{tabular}

\begin{tabular}{|l|c|}
\hline Time bin 5 (485 - 350 Ma) & Proportion oxic \\
\hline Acadian & 0.74 \\
Taconic & 0.14 \\
Scotland & 0.22 \\
Denmark & 0.22 \\
South Africa & 0.00 \\
Sweden & 0.06 \\
Carnic Alps & 0.03 \\
\hline
\end{tabular}

\begin{tabular}{|l|l|}
\hline & $\mathrm{n}=$ \\
7 regions & 387 \\
\hline Average & 0.20 \\
\hline Standard dev. & 0.25 \\
\hline Standard error & 0.10 \\
\hline
\end{tabular}

\section{Proportion of ferruginous samples $(\mathrm{FeHR} / \mathrm{FeT}>0.38$; FeP/FeHR $<0.7$ )} from outer shelf and deep basin environments

\begin{tabular}{|l|c|}
\hline Time bin 1 (2300 - 1000 Ma) & Proportion ferruginous \\
\hline Fennoscandia & 0.93 \\
North China & 1.00 \\
Belt Basin & 0.73 \\
Borden Basin & 1.00 \\
Mauritania & 0.05 \\
Francevillian Basin & 0.69 \\
Roper Basin & 0.00 \\
McArthur Basin & 0.66 \\
Animikie Basin & 0.34 \\
Brasília Fold Belt & 0.71 \\
Onega Basin & 0.29 \\
Midcontinent Rift & 1.00 \\
Botswana & 0.29 \\
\hline
\end{tabular}

\begin{tabular}{|l|l|}
\hline 13 regions & $\begin{array}{c}\mathrm{n}= \\
468\end{array}$ \\
\hline Average & 0.59 \\
\hline Standard dev. & 0.36 \\
\hline Standard error & 0.10 \\
\hline
\end{tabular}

\begin{tabular}{|l|c|}
\hline Time bin 2 (1000 - 635 Ma) & Proportion ferruginous \\
\hline South China & 0.27 \\
\hline
\end{tabular}

\begin{tabular}{|l|l|}
\hline 12 regions & $\mathrm{n}=$ \\
\hline
\end{tabular}




\begin{tabular}{|l|l|} 
& \\
Wernecke Mountains & 1.00 \\
Grand Canyon & 1.00 \\
Centralian Superbasin & 0.65 \\
Spitsbergen & 1.00 \\
East Greenland & 1.00 \\
Ogilvie Mountains & 1.00 \\
Mackenzie Mountains & 0.98 \\
Uinta Mountains & 1.00 \\
Svalbard & 1.00 \\
Callison Lake & 1.00 \\
\hline
\end{tabular}

\begin{tabular}{|l|l|}
\hline & 286 \\
\hline Average & 0.91 \\
\hline Standard dev. & 0.22 \\
\hline Standard error & 0.06 \\
\hline
\end{tabular}

\begin{tabular}{|l|c|}
\hline Time bin 3 (635 - 542 Ma) & Proportion ferruginous \\
\hline South China & 0.68 \\
Wernecke Mountains & 0.98 \\
Eastern European Platform & 1.00 \\
Southern Canadian Cordillera & 0.96 \\
Avalon & 1.00 \\
Spitsbergen & 1.00 \\
East Greenland & 1.00 \\
Stuart Shelf & 0.76 \\
Ogilvie Mountains & 1.00 \\
Mackenzie Mountains & 0.99 \\
Mongolia & 1.00 \\
Svalbard & 1.00 \\
Nico Perez Terrane (Uruguay) & 1.00 \\
\hline
\end{tabular}

\begin{tabular}{|l|l|}
\hline 13 regions & $\begin{array}{l}\mathrm{n}= \\
491\end{array}$ \\
\hline Average & 0.95 \\
\hline Standard dev. & 0.10 \\
\hline Standard error & 0.03 \\
\hline
\end{tabular}

\begin{tabular}{|l|c|}
\hline Time bin 4 (542 - 485 Ma) & Proportion ferruginous \\
\hline South China & 0.86 \\
Wernecke Mountains & 1.00 \\
House Embayment & 1.00 \\
Sweden & 0.04 \\
Southern Canadian Cordillera & 1.00 \\
Ogilvie Mountains & 1.00 \\
Mackenzie Mountains & 1.00 \\
Georgina Basin & 0.95 \\
Mongolia & 1.00 \\
\hline
\end{tabular}

\begin{tabular}{|l|c|}
\hline & $\mathrm{n}=$ \\
9 regions & 309 \\
\hline Average & 0.87 \\
\hline Standard dev. & 0.32 \\
\hline Standard error & 0.11 \\
\hline
\end{tabular}

\section{\begin{tabular}{|l|l|}
\hline Time bin 5 (485 - $350 \mathrm{Ma})$ & Proportion ferruginous \\
\hline
\end{tabular}}




\begin{tabular}{|c|c|c|c|}
\hline Acadian & 0.17 & 6 regions & $\begin{array}{l}\mathrm{n}= \\
129\end{array}$ \\
\hline Taconic & 0.96 & Average & 0.63 \\
\hline Scotland & 0.94 & Standard dev. & 0.37 \\
\hline Denmark & 0.33 & Standard error & 0.15 \\
\hline Sweden & 0.40 & & \\
\hline Carnic Alps & 1.00 & & \\
\hline
\end{tabular}

D. Iron in pyrite (FeP) from oxic sediments (FeHR/FeT < 0.38) from all environments. Regional values are median of data from that region.

\begin{tabular}{|c|c|c|c|}
\hline Time bin 1 (2300 - 1000 Ma) & FeP & & \\
\hline Fennoscandia & 2.15 & 13 regions & $\mathrm{n}=450$ \\
\hline North China & 0.27 & Average & 0.392 \\
\hline Belt Basin & 0.17 & Standard dev. & 0.552 \\
\hline Borden Basin & 0.42 & Standard error & 0.153 \\
\hline Francevillian Basin & 0.09 & & \\
\hline Southern Urals & 0.06 & & \\
\hline Roper Basin & 0.06 & & \\
\hline McArthur Basin & 0.27 & & \\
\hline Animikie Basin & 0.26 & & \\
\hline Brasília Fold Belt & 0.42 & & \\
\hline Onega Basin & 0.32 & & \\
\hline Midcontinent Rift & 0.05 & & \\
\hline Botswana & 0.56 & & \\
\hline
\end{tabular}

\begin{tabular}{|c|c|c|c|}
\hline Time bin 2 (1000 - $635 \mathrm{Ma})$ & FeP & & \\
\hline South China & 0.01 & 13 regions & $\begin{array}{l}n= \\
488\end{array}$ \\
\hline Wernecke Mountains & 0.00 & Average & 0.0200 \\
\hline Death Valley & 0.00 & Standard dev. & 0.041 \\
\hline Grand Canyon & 0.00 & Standard error & 0.011 \\
\hline Eastern European Platform & 0.01 & & \\
\hline Centralian Superbasin & 0.08 & & \\
\hline Spitsbergen & 0.03 & & \\
\hline East Greenland & 0.14 & & \\
\hline Ogilvie Mountains & 0.00 & & \\
\hline Mackenzie Mountains & 0.01 & & \\
\hline Uinta Mountains & 0.00 & & \\
\hline Svalbard & 0.01 & & \\
\hline Callison Lake & 0.00 & & \\
\hline
\end{tabular}




\begin{tabular}{|c|c|c|c|}
\hline Time bin 3 (635 - 542 Ma) & FeP & & \\
\hline South China & 0.03 & 13 regions & $\begin{array}{l}n= \\
754\end{array}$ \\
\hline Wernecke Mountains & 0.05 & Average & 0.040 \\
\hline Eastern European Platform & 0.02 & Standard dev. & 0.033 \\
\hline Southern Canadian Cordillera & 0.09 & Standard error & 0.009 \\
\hline Avalon & 0.01 & & \\
\hline East Greenland & 0.04 & & \\
\hline Stuart Shelf & 0.09 & & \\
\hline Siberia & 0.07 & & \\
\hline Ogilvie Mountains & 0.00 & & \\
\hline Mackenzie Mountains & 0.01 & & \\
\hline Nico Perez Terrane (Uruguay) & 0.00 & & \\
\hline Svalbard & 0.06 & & \\
\hline Namibia & 0.00 & & \\
\hline
\end{tabular}

\begin{tabular}{|c|c|c|c|}
\hline Time bin 4 (542 - 485 Ma) & FeP & & \\
\hline South China & 0.15 & 9 regions & $\begin{array}{l}n= \\
245\end{array}$ \\
\hline Wernecke Mountains & 0.21 & Average & 0.078 \\
\hline House Embayment & 0.01 & Standard dev. & 0.110 \\
\hline East Greenland & 0.03 & Standard error & 0.037 \\
\hline Siberia & 0.01 & & \\
\hline Mackenzie Mountains & 0.00 & & \\
\hline Georgina Basin & 0.29 & & \\
\hline Mongolia & 0.00 & & \\
\hline Namibia & 0.00 & & \\
\hline
\end{tabular}

\begin{tabular}{|c|c|c|c|}
\hline Time bin 5 (485 - $350 \mathrm{Ma})$ & FeP & & \\
\hline Acadian & 0.64 & 6 regions & $\begin{array}{l}\mathrm{n}= \\
227\end{array}$ \\
\hline Taconic & 0.75 & Average & 0.560 \\
\hline Scotland & 0.04 & Standard dev. & 0.618 \\
\hline Denmark & 0.25 & Standard error & 0.252 \\
\hline Sweden & 1.66 & & \\
\hline Carnic Alps & 0.02 & & \\
\hline
\end{tabular}

E. Proportion of sediments deposited under oxic water columns with high levels of porewater sulfide (FeHR/FeT < 0.38 ; FeP/FeHR $>0.7$ ) from all environments

\begin{tabular}{|c|c|c|c|}
\hline Time bin 1 (2300 - 1000 Ma) & $\begin{array}{l}\text { Proportion with high } \\
\text { porewater sulfide }\end{array}$ & & \\
\hline Fennoscandia & 0.00 & 14 regions & $\mathrm{n}=$ \\
\hline
\end{tabular}




\begin{tabular}{|l|l|} 
& \\
North China & 0.00 \\
Bolt Basin & 0.33 \\
Mauritania & 0.50 \\
Francevillian Basin & 0.51 \\
Southern Urals & 0.01 \\
Roper Basin & 0.00 \\
McArthur Basin & 0.09 \\
Animikie Basin & 0.17 \\
Brasília Fold Belt & 0.04 \\
Onega Basin & 0.50 \\
Midcontinent Rift & 0.20 \\
Botswana & 0.00 \\
\hline
\end{tabular}

\begin{tabular}{|l|l|}
\hline & 534 \\
\hline Average & 0.17 \\
\hline Standard dev. & 0.21 \\
\hline Standard error & 0.06 \\
\hline
\end{tabular}

\begin{tabular}{|l|c|}
\hline Time bin 2 (1000 - 635 Ma) & $\begin{array}{c}\text { Proportion with high } \\
\text { porewater sulfide }\end{array}$ \\
\hline South China & 0.11 \\
Wernecke Mountains & 0.00 \\
Death Valley & 0.00 \\
Grand Canyon & 0.02 \\
Eastern European Platform & 0.00 \\
Centralian Superbasin & 0.00 \\
Spitsbergen & 0.00 \\
East Greenland & 0.00 \\
Ogilvie Mountains & 0.02 \\
Mackenzie Mountains & 0.00 \\
Uinta Mountains & 0.00 \\
Svalbard & 0.00 \\
Callison Lake & 0.00 \\
\hline
\end{tabular}

\begin{tabular}{|l|l|}
\hline 13 regions & $\mathrm{n}=$ \\
490
\end{tabular}

\begin{tabular}{|c|c|c|c|}
\hline Time bin 3 (635 - 542 Ma) & $\begin{array}{c}\text { Proportion with high } \\
\text { porewater sulfide }\end{array}$ & & \\
\hline South China & 0.09 & 13 regions & $\begin{array}{l}\mathrm{n}= \\
753\end{array}$ \\
\hline Wernecke Mountains & 0.00 & Average & 0.01 \\
\hline Eastern European Platform & 0.00 & Standard dev. & 0.03 \\
\hline Southern Canadian Cordillera & 0.03 & Standard error & 0.01 \\
\hline Avalon & 0.01 & & \\
\hline East Greenland & 0.00 & & \\
\hline Stuart Shelf & 0.00 & & \\
\hline Siberia & 0.00 & & \\
\hline Ogilvie Mountains & 0.00 & & \\
\hline
\end{tabular}




\begin{tabular}{|l|l|} 
Mackenzie Mountains & 0.00 \\
Nico Perez Terrane (Uruguay) & 0.00 \\
Svalbard & 0.00 \\
Namibia & 0.00 \\
\hline
\end{tabular}

\begin{tabular}{|l|c|}
\hline Time bin 4 (542 - 485 Ma) & $\begin{array}{c}\text { Proportion with high } \\
\text { porewater sulfide }\end{array}$ \\
\hline South China & 0.16 \\
Wernecke Mountains & 0.00 \\
House Embayment & 0.00 \\
Southern Canadian Cordillera & 0.00 \\
East Greenland & 0.00 \\
Siberia & 0.00 \\
Mackenzie Mountains & 0.00 \\
Georgina Basin & 0.00 \\
Mongolia & 0.00 \\
Namibia & 0.00 \\
\hline
\end{tabular}

\begin{tabular}{|l|c|}
\hline & $\mathrm{n}=$ \\
10 regions & 247 \\
\hline Average & 0.02 \\
\hline Standard dev. & 0.05 \\
\hline Standard error & 0.02 \\
\hline
\end{tabular}

\begin{tabular}{|l|c|}
\hline Time bin $\mathbf{5}$ (485 - 350 Ma) & $\begin{array}{c}\text { Proportion with high } \\
\text { porewater sulfide }\end{array}$ \\
\hline Acadian & 0.82 \\
Taconic & 0.08 \\
Scotland & 0.07 \\
Denmark & 0.15 \\
Sweden & 0.29 \\
South Africa & 0.00 \\
Carnic Alps & 0.00 \\
\hline
\end{tabular}

\begin{tabular}{|l|c|}
\hline & $\mathrm{n}=$ \\
7 regions & 255 \\
\hline Average & 0.20 \\
\hline Standard dev. & 0.29 \\
\hline Standard error & 0.11 \\
\hline
\end{tabular}

Table S3: Results from each region from different time bins for A) proportion of anoxic samples (FeHR/FeT > 0.38) from outer shelf and basinal depositional environments, B) proportion of oxic samples based on a conservative value of FeHR/FeT $<0.22$ from outer shelf and basinal depositional environments, $\mathrm{C}$ ) proportion of ferruginous samples from anoxic water columns (FeHR/FeT > 0.38; FeP/FeHR < 0.7) from outer shelf and basinal depositional environments, D) Iron in pyrite (FeP) contents of sediments deposited beneath oxic water columns (FeHR/FeT < 0.38) from all environments, E) proportion of sediments with high levels of porewater sulfide from sediments deposited beneath oxic water columns from all environments (FeHR/FeT < 0.38; FeP/FeHR > 0.7). $\mathrm{N}=$ number of samples in that time bin.

\section{$\underline{\text { 3. Statistical analyses of iron geochemical data }}$}

The normality of the data in each time bin was first checked with the ShapiroWilk W test. Bins with low p-values $(<0.05)$ can be considered to have a skewed or non- 
normal distribution. Due to the high number of regions in the early Neoproterozoic (bin 2) and Ediacaran (bin 3) with essentially no sulfide, all analyses related to sulfide contents had strongly skewed data. This skew remained after various transformations were applied, and so non-parametric tests were used. For the full dataset, data from the five time bins were compared using Analysis of Variance (ANOVA) and Kruskal-Wallis tests. Post hoc Tukey-Kramer HSD tests $(\alpha=0.05)$, pairwise Wilcoxon tests and SteelDwass tests were used to further explore significant differences between bins. Although all tests were applied to the full dataset in all instances, the results of the Kruskal-Wallis, Wilcoxon, and Steel-Dwass tests should be considered more reliable for significantly skewed data (essentially all analyses except the proportion of anoxic and oxic samples through time) as these non-parametric tests do not require the assumption of normality.

This analytical approach therefore begins with an omnibus test (ANOVA and Kruskal-Wallis) designed to test whether the data from different groups originate from the same underlying distribution. The omnibus tests do not indicate which groups are significantly different, which requires post-hoc pairwise testing. Conducting these multiple comparisons invites the possibility of Type I error, or incorrectly rejecting the null hypothesis. The multiple comparison problem can be illustrated using the following example-the probability of flipping any given coin as "heads" 9 times out of 10 is very low; however, if 1000 coins are each flipped 10 times, the probability that one coin will be "heads" 9 times of 10 becomes high. It would be incorrect to conclude that specific coin is different from the rest. This issue is not relevant for the consideration of anoxic/oxic water columns through time (Fig. 1A) as none of the omnibus test statistics are significant (Table S4), but is important for analyses of ferruginous water columns through time and pyrite contents in oxic sediments (Fig. 1B and C). The multiple comparison problem can be addressed by applying corrections that account for the increased family-wise error rate (such as the Bonferroni correction, which for the ten comparisons conducted here would be $p<0.005$ using an overall $\alpha=0.05$ ) and tests that directly account for multiple comparisons, such as Tukey's HSD or Steel-Dwass tests. The issue with such corrections is that they can be very conservative, and potentially result in Type II error-incorrectly accepting the null hypothesis. This is especially true considering some a priori hypotheses do exist, for instance that Neoproterozoic oceans were more ferruginous ${ }^{15}$, and thus a Bonferroni correction based on all pairwise comparisons is overly stringent. As this is the first statistical analysis of iron geochemical data, and the number of sampled regions is still relatively small in a statistical sense, we do not wish to incorrectly remove hypotheses from future consideration. Thus, we present and interpret nonparametric pairwise comparisons using uncorrected p-values from Wilcoxon tests, Bonferroni-corrected p-values, and analyses that account for multiple comparisons (Steel-Dwass). Significant results from these latter two categories should be considered most robust, and significant results from uncorrected p-values should be considered as possible but requiring more data for full resolution.

Four sensitivity analyses were then conducted. In these analyses, only the most appropriate tests were applied based on the normality of the data. In the first analysis, inappropriate or altered samples or those from sedimentary facies in which the iron speciation proxy is not calibrated were removed. This included lacustrine rocks (the Nonesuch Shale as studied by ref. (43) and Spiral Creek Formation as studied by ref. (15), very coarse-grained diamictites (the Sturtian Tillite as studied by ref. (15) and the 
Gaskiers Formation as studied by ref. (12)), and a mineralized Nickel-MolybdenumSulfide layer in the Lower Cambrian Niutitang Formation of South China as studied by ref. 55. Studies analyzed using an older dithionite-only extraction methodology rather than the modern sequential extraction methodology ${ }^{11,40,47,50}$ were also removed (see ref. 59 for further discussion of these methodological differences). Finally, three studies that identified very high abundances of acid-volatile sulfur (FeAVS) were removed ${ }^{34-36}$, as this indicates alteration of the original iron-sulfur systematics. When the removed samples represented a subset of the samples from a region, the value for that region was re-calculated from the remaining samples, whereas if they represent all samples the entire region was removed from the analysis. In the second sensitivity analysis, regions with low sample coverage — specifically five or fewer samples — were excluded from the analysis. This exclusion did not particularly affect the analysis of the proportion of anoxic or oxic samples through time as most studies have good sampling coverage. More regions were removed from the other analyses that required either oxic or anoxic conditions, as these consequently represent only a subset of available data. In the third sensitivity analyses, only samples from the Mesoproterozoic (1600-1000 Ma) were included in time bin 1 . For the fourth sensitivity analysis, iron in pyrite from oxic sediments was analyzed on samples from outer shelf and deep basin environments (rather than all environments) for direct comparison with other analyses. The results of all analyses are contained in Table S4.

\section{Full dataset}

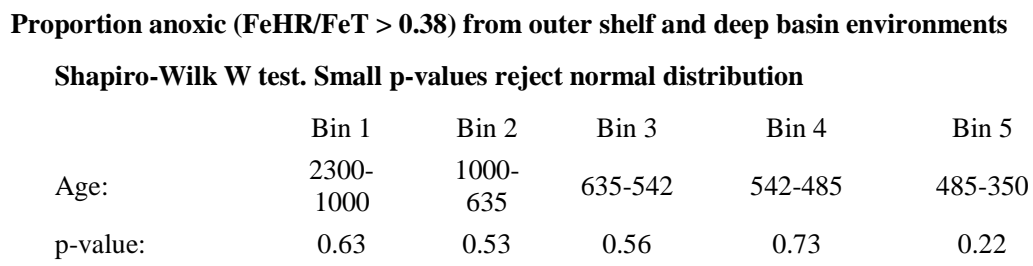

\section{ANOVA}

\begin{tabular}{|c|c|c|c|c|c|c|c|}
\hline Source & $\mathrm{DF}$ & SS & MS & F-ratio & $\mathbf{P}$ & & \\
\hline Model & 4 & 0.27 & 0.07 & 0.7844 & 0.5405 & \multirow{3}{*}{\multicolumn{2}{|c|}{$\begin{array}{l}\text { post-hoc Tukey HSD: } \\
\text { Not } \\
\text { significant }\end{array}$}} \\
\hline Error & 52 & 4.46 & \multirow[t]{3}{*}{0.09} & & & & \\
\hline Total & 56 & 4.73 & & & & & \\
\hline Kruskal-Wallis test & & & & Pairwise $\mathrm{V}$ & method & $\mathbf{P}$ & Steel-Dwass P \\
\hline Chi Square & DF & $\mathbf{P}$ & & No compar & re signific & & $\begin{array}{c}\text { None } \\
\text { significant }\end{array}$ \\
\hline 3.3 & 4 & 0.5094 & & & & & \\
\hline
\end{tabular}

Proportion oxic $(\mathrm{FeHR} / \mathrm{FeT}<0.22)$ from outer shelf and deep basin environments Shapiro-Wilk W test. Small p-values reject normal distribution

$\begin{array}{cccccc} & \text { Bin } 1 & \text { Bin 2 } & \text { Bin 3 } & \text { Bin 4 } & \text { Bin 5 } \\ \text { Age: } & 2300- & 1000- & 635-542 & 542-485 & 485-350\end{array}$


$\begin{array}{llllll}\text { p-value: } & 0.0003 & 0.18 & 0.002 & 0.0003 & 0.0169\end{array}$

ANOVA

\begin{tabular}{lccccc|l|} 
Source & DF & SS & MS & F-ratio & \multicolumn{2}{c}{ P } \\
\hline Model & 4 & 0.01 & 0.002 & 0.0328 & 0.9979 & post-hoc Tukey HSD: \\
Error & 52 & 3.67 & 0.07 & & Not \\
Total & 56 & 3.68 & & & & significant \\
\end{tabular}

Kruskal-Wallis test

Pairwise Wilcoxon method

No comparisons were significant
$\mathbf{P}$

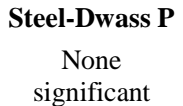

Chi Square

DF $\quad \mathbf{P}$

0.7483

Proportion ferruginous $(\mathrm{FeP} / \mathrm{FeHR}<0.7)$ of anoxic samples from outer shelf and deep basin environments

Shapiro-Wilk W test. Small p-values reject normal distribution

$\begin{array}{lccccc} & \text { Bin } 1 & \text { Bin 2 } & \text { Bin 3 } & \text { Bin 4 } & \text { Bin 5 } \\ \text { Age: } & 2300- & 1000- & 635-542 & 542-485 & 485-350 \\ \text { P } & 1000 & 635 & & & \\ & 0.11 & <0.0001 & <0.0001 & <0.0001 & 0.1\end{array}$

ANOVA

\begin{tabular}{lcccc|l|l|} 
Source & DF & SS & MS & F-ratio & \multicolumn{1}{c}{ P } & \\
\hline Model & 4 & 1.22 & 0.3 & 3.9149 & 0.0079 & post-hoc Tukey HSD: \\
Error & 48 & 3.73 & 0.08 & & & a, b, b, ab, ab \\
Total & 52 & 4.95 & & & & bins 1, 2, 3, 4, \\
5
\end{tabular}

Kruskal-Wallis test

Chi Square

13.91

\begin{tabular}{c|c} 
DF & $\mathbf{P}$ \\
4 & 0.0076
\end{tabular}

\begin{tabular}{l|c|c|}
\hline Pairwise Wilcoxon method & P & Steel-Dwass P \\
\cline { 2 - 3 } bin 1 and 3 & 0.0055 & 0.044 \\
\hline bin 1 and 2 & 0.0154 & 0.1094 \\
bin 1 and 4 & 0.0379 & 0.2307 \\
bin 3 and 5 & 0.0204 & 0.1387 \\
bin 2 and 5 & 0.0237 & 0.1573 \\
\hline
\end{tabular}

All other comparisons not significant

Iron in pyrite (FeP) from oxic sediments (FeHR/FeT < 0.38) in all environments

Shapiro-Wilk W test. Small p-values reject normal distribution

$\begin{array}{lccccc} & \text { Bin } 1 & \text { Bin 2 } & \text { Bin 3 } & \text { Bin } 4 & \text { Bin 5 } \\ \text { Age: } & 2300- & 1000- & 635-542 & 542-485 & 485-350 \\ \text { P } & 1000 & 635 & & & 0.22\end{array}$

ANOVA

$\begin{array}{llllll}\text { Source } & \text { DF } & \text { SS } & \text { MS } & \text { F-ratio } & \text { P }\end{array}$

\begin{tabular}{lllll|l|l} 
Model & 4 & 2.13 & 0.53 & 4.5868 & 0.0032 & post-hoc Tukey HSD:
\end{tabular}



Error
49
5.69
0.12
Total
53
7.82

Kruskal-Wallis test

Chi Square

25.44

$\begin{array}{cc}\text { DF } & \mathbf{P} \\ 4 & <0.0001\end{array}$

ab, b, b, ab, a

bins 1, 2, 3, 4,

Pairwise Wilcoxon method

bin 1 and 2

bin 1 and 3

bin 1 and 4

bin 5 and 2

bin 5 and 3

bin 5 and 4

All other comparisons not significant
5 , $2,3,4$,

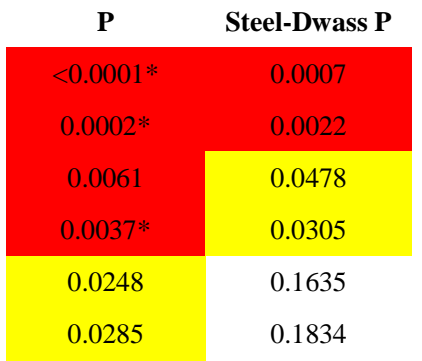

0.1834

Iron in pyrite (FeP) from oxic sediments (FeHR/FeT < 0.38) from outer shelf and deep-basin environments (sensitivity test)

Shapiro-Wilk W test. Small p-values reject normal distribution

$\begin{array}{lccccc} & \text { Bin } 1 & \text { Bin } 2 & \text { Bin 3 } & \text { Bin 4 } & \text { Bin 5 } \\ \text { Age: } & 2300- & 1000- & 635-542 & 542-485 & 485-350 \\ \text { P } & 1000 & 635 & & & 0.22\end{array}$

\section{ANOVA}

\begin{tabular}{|c|c|c|c|c|c|c|}
\hline Source & $\mathrm{DF}$ & SS & MS & F-ratio & $\mathbf{P}$ & \\
\hline Model & 4 & 2 & 0.5 & 3.9442 & 0.0079 & post-hoc Tukey HSD: \\
\hline Error & 45 & 5.71 & 0.13 & & & $a b, b, b, a b, a$ \\
\hline Total & 49 & 7.71 & & & & $\begin{array}{l}\text { bins } 1,2,3,4 \text {, } \\
5\end{array}$ \\
\hline
\end{tabular}

Kruskal-Wallis test

Chi Square

23.35
DF

4
Pairwise Wilcoxon method

bin 1 and 2

bin 1 and 3

bin 1 and 4

bin 5 and 2

bin 5 and 3

All other comparisons not significant

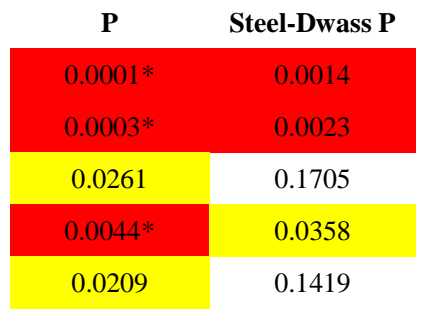

0.1419

Proportion of sediments deposited under oxic water columns with high levels of porewater sulfide from all environments

Shapiro-Wilk W test. Small p-values reject normal distribution

$\begin{array}{lccccc} & \text { Bin } 1 & \text { Bin 2 } & \text { Bin 3 } & \text { Bin 4 } & \text { Bin 5 } \\ \text { Age: } & 2300- & 1000- & 635-542 & 542-485 & 485-350 \\ \text { P } & 1000 & 635 & & & \\ & 0.0023 & <0.0001 & <0.0001 & <0.0001 & 0.0089\end{array}$

ANOVA

\begin{tabular}{cccccc|c|} 
Source & DF & SS & MS & F-ratio & P & \\
\hline Model & 4 & 0.37 & 0.09 & 4.4209 & 0.0038 & post-hoc Tukey HSD:
\end{tabular}




$\begin{array}{lrrr}\text { Error } & 52 & 1.1 & 0.02 \\ \text { Total } & 56 & 1.48 & \end{array}$

Not

significant

Kruskal-Wallis test

Chi Square

16.16
$\mathrm{DF}$

4
Pairwise Wilcoxon method

bin 1 and 2

bin 1 and 3

bin 1 and 4

bin 5 and 2

bin 5 and 3

bin 5 and 4

All other comparisons not significant
$\mathbf{P}$

0.0153

teel-Dwass $\mathbf{P}$

0.0132

0.1084

0.0952

0.0108

0.0799

0.111

0.1113

0.0157

0.1321

Sensitivity analyses- with removal of 1) lacustrine samples, 2) coarse-grained samples, 3) Samples from Lower Cambrian Ni-Mo layer, South China, 4) studies with high percentages of FeAVS, and 5) studies analyzed under the older non-sequential extraction methodology

Proportion anoxic (FeHR/FeT > 0.38) from outer shelf and deep basin environments

Shapiro-Wilk W test. Small p-values reject normal distribution

$\begin{array}{lccccc} & \text { Bin } 1 & \text { Bin 2 } & \text { Bin 3 } & \text { Bin 4 } & \text { Bin 5 } \\ \text { Age: } & 2300- & 1000- & 635-542 & 542-485 & 485-350 \\ \text { p-value: } & 1000 & 635 & & & 0.74\end{array}$

ANOVA

\begin{tabular}{lccccc|l} 
Source & DF & SS & MS & F-ratio & \multicolumn{2}{l}{ P } \\
\hline Model & 4 & 0.29 & 0.07 & 0.8491 & 0.5012 & post-hoc Tukey HSD: \\
Error & 48 & 4.16 & 0.09 & & & $\begin{array}{l}\text { Not } \\
\text { significant }\end{array}$ \\
Total & 52 & 4.54 & & & & \\
\hline
\end{tabular}

Proportion oxic $(\mathrm{FeHR} / \mathrm{FeT}<0.22)$ from outer shelf and deep basin environments

Shapiro-Wilk W test. Small p-values reject normal distribution

$\begin{array}{lccccc} & \text { Bin } 1 & \text { Bin 2 } & \text { Bin 3 } & \text { Bin 4 } & \text { Bin 5 } \\ \text { Age: } & 2300- & 1000- & 635-542 & 542-485 & 485-350 \\ \text { p-value: } & 1000 & 635 & & & \\ & 0.0029 & 0.3 & 0.0016 & 0.0003 & 0.0169\end{array}$

Kruskal-Wallis test

Chi Square

1.94

DF $\quad$ P

$4 \quad 0.7458$
Pairwise Wilcoxon method

No comparisons were significant

$\mathbf{P}$

Steel-Dwass P

None significant

Proportion ferruginous $(\mathrm{FeP} / \mathrm{FeHR}<0.7)$ of anoxic samples from outer shelf and deep basin environments Shapiro-Wilk W test. Small p-values reject normal distribution
Bin 1
Bin 2
Bin 3
Bin 4
Bin 5 


$\begin{array}{lccccc}\text { Age: } & 2300- & 1000- & 635-542 & 542-485 & 485-350 \\ \mathrm{P} & 1000 & 635 & & & \\ & 0.18 & <0.0001 & <0.0001 & <0.0001 & 0.1\end{array}$

Kruskal-Wallis test

Chi Square

11.51
DF

4
Pairwise Wilcoxon method

bin 1 and 3

bin 3 and 5

bin 1 and 2

bin 2 and 5

All other comparisons not significant
$\mathbf{P}$

0.0141

0.0204

0.0347

0.0308
Steel-Dwass P

0.1011

0.1387

0.2149

0.1954

Iron in pyrite ( $\mathrm{FeP}$ ) from oxic sediments in all environments

Shapiro-Wilk W test. Small p-values reject normal distribution

$\begin{array}{lccccc} & \text { Bin } 1 & \text { Bin 2 } & \text { Bin 3 } & \text { Bin 4 } & \text { Bin 5 } \\ \text { Age: } & 2300- & 1000- & & & \\ \text { P } & 1000 & 635 & 635-542 & 542-485 & 485-350 \\ & 0.85 & <0.0001 & 0.1 & 0.0056 & 0.22\end{array}$

ANOVA

\begin{tabular}{lcccc|l|l|} 
Source & DF & SS & MS & F-ratio & \multicolumn{1}{c}{ P } & \\
\hline Model & 4 & 1.6 & 0.4 & 7.9596 & $<0.0001$ & post-hoc Tukey HSD: \\
Error & 45 & 2.26 & 0.05 & & & ab, b, b, b, a \\
Total & 49 & 3.86 & & & & $\begin{array}{l}\text { bins 1, 2, 3, 4, } \\
5\end{array}$ \\
\hline
\end{tabular}

Kruskal-Wallis test

Chi Square

23.71

\begin{tabular}{c|c|} 
DF & P \\
\hline 4 & $<0.0001$ \\
\hline
\end{tabular}

Pairwise Wilcoxon method

bin 1 and 2

bin 1 and 3

bin 1 and 4

bin 5 and 2

bin 5 and 3

bin 5 and 4

All other comparisons not significant

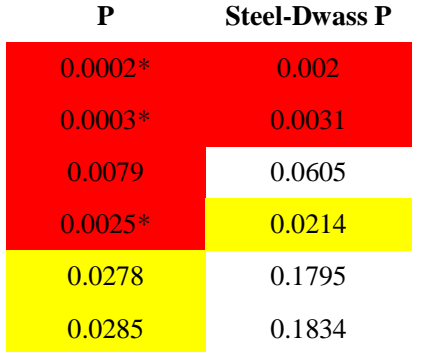

0.0285

0.1834

Proportion of sediments deposited under oxic water columns with high levels of porewater sulfide from all environments

Shapiro-Wilk $\mathrm{W}$ test. Small p-values reject normal distribution

$\begin{array}{lccccc} & \text { Bin } 1 & \text { Bin } 2 & \text { Bin 3 } & \text { Bin } 4 & \text { Bin 5 } \\ \text { Age: } & 2300- & 1000- & 635-542 & 542-485 & 485-350 \\ \text { P } & 1000 & 635 & & & \\ & 0.0013 & <0.0001 & <0.0001 & <0.0001 & 0.0089\end{array}$

Kruskal-Wallis test

Chi Square

13.61
DF

$4 \quad 0.0087$

Pairwise Wilcoxon method

bin 1 and 3

bin 1 and 4

bin 5 and 2

bin 5 and 3

\begin{tabular}{|c|c|}
\hline $\mathbf{P}$ & Steel-Dwass $\mathbf{P}$ \\
\hline 0.0296 & 0.1891 \\
\hline 0.0359 & 0.2207 \\
0.0177 & 0.1229 \\
\hline 0.0092 & 0.0697 \\
\hline
\end{tabular}


All other comparisons not significant

\section{Sensitivity analyses- with removal of regions with five or less samples}

Proportion anoxic $(\mathrm{FeHR} / \mathrm{FeT}>0.38)$ from outer shelf and deep basin environments

Shapiro-Wilk W test. Small p-values reject normal distribution

$\begin{array}{lccccc} & \text { Bin } 1 & \text { Bin } 2 & \text { Bin 3 } & \text { Bin 4 } & \text { Bin 5 } \\ \text { Age: } & 2300- & 1000- & 635-542 & 542-485 & 485-350 \\ \text { p-value: } & 1000 & 635 & & & 0.86 \\ & 0.57 & 0.6 & 0.47 & 0.29\end{array}$

ANOVA

\begin{tabular}{lccccc|l} 
Source & DF & SS & MS & F-ratio & \multicolumn{2}{l}{ P } \\
\hline Model & 4 & 0.23 & 0.06 & 0.7664 & 0.5526 & post-hoc Tukey HSD: \\
Error & 46 & 3.42 & 0.07 & & & Not \\
Total & 50 & 3.65 & & & & \\
& & & & & &
\end{tabular}

Proportion oxic $(\mathrm{FeHR} / \mathrm{FeT}<0.22)$ from outer shelf and deep basin environments

Shapiro-Wilk W test. Small p-values reject normal distribution

$\begin{array}{lccccc} & \text { Bin } 1 & \text { Bin } 2 & \text { Bin 3 } & \text { Bin 4 } & \text { Bin 5 } \\ \text { Age: } & 2300- & 1000- & 635-542 & 542-485 & 485-350 \\ \text { p-value: } & 1000 & 635 & & & \\ & 0.003 & 0.37 & 0.0045 & 0.0053 & 0.029\end{array}$

Kruskal-Wallis test

Chi Square

1.52
DF $\quad \mathbf{P}$

$4 \quad 0.8233$
Pairwise Wilcoxon method

No comparisons were significant

$\mathbf{P}$

Steel-Dwass $\mathbf{P}$

None significant

Proportion ferruginous of anoxic samples from outer shelf and deep basin environments

Shapiro-Wilk W test. Small p-values reject normal distribution

$\begin{array}{lccccc} & \text { Bin } 1 & \text { Bin 2 } & \text { Bin 3 } & \text { Bin 4 } & \text { Bin 5 } \\ \text { Age: } & 2300- & 1000- & 635-542 & 542-485 & 485-350 \\ \text { P } & 1000 & 635 & & & \\ & 0.23 & <0.0001 & <0.0001 & 0.0005 & 0.0984\end{array}$

Kruskal-Wallis test

Chi Square

12.48
$<0.0001$

\section{Pairwise Wilcoxon method}

bin 1 and 3

bin 1 and 2

bin 2 and 5

bin 3 and 5

All other comparisons not significant

\begin{tabular}{|c|c|}
\hline $\mathbf{P}$ & Steel-Dwass P \\
\hline 0.0062 & 0.0487 \\
\hline 0.0134 & 0.0968 \\
\hline 0.0338 & 0.2106 \\
\hline 0.0414 & 0.247 \\
\hline
\end{tabular}

Iron in pyrite (FeP) from oxic sediments in all environments 
Shapiro-Wilk W test. Small p-values reject normal distribution

$\begin{array}{lccccc} & \text { Bin } 1 & \text { Bin 2 } & \text { Bin 3 } & \text { Bin 4 } & \text { Bin 5 } \\ \text { Age: } & 2300- & 1000- & 635-542 & 542-485 & 485-350 \\ \text { P } & 1000 & 635 & & & \\ & 0.12 & <0.0001 & 0.07 & 0.0373 & 0.22\end{array}$

ANOVA

\begin{tabular}{|c|c|c|c|c|c|c|}
\hline Source & DF & SS & MS & F-ratio & $\mathbf{P}$ & \\
\hline Model & 4 & 1.39 & 0.35 & 6.3726 & 0.0005 & post-hoc Tukey HSD: \\
\hline Error & 40 & 2.18 & 0.05 & & & $\mathrm{a}, \mathrm{a}, \mathrm{a}, \mathrm{a}, \mathrm{b}$ \\
\hline Total & 44 & 3.57 & & & & bins $1,2,3,4$, \\
\hline
\end{tabular}

Kruskal-Wallis test

Chi Square

18.7

\begin{tabular}{c|c|}
\hline DF & P \\
\hline 4 & 0.0009 \\
\hline
\end{tabular}

Pairwise Wilcoxon method

bin 1 and 2

bin 1 and 3

bin 5 and 2

bin 5 and 3

All other comparisons not significant

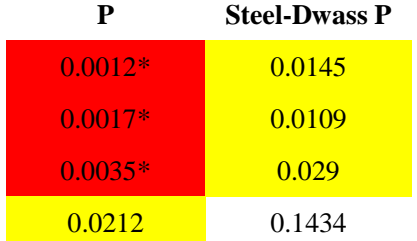

0.0212

0.1434

Proportion of sediments deposited under oxic water columns with high levels of porewater sulfide from all environments Shapiro-Wilk W test. Small p-values reject normal distribution

$\begin{array}{lccccc} & \text { Bin } 1 & \text { Bin 2 } & \text { Bin 3 } & \text { Bin 4 } & \text { Bin 5 } \\ \text { Age: } & 2300- & 1000- & 635-542 & 542-485 & 485-350 \\ \text { P } & 1000 & 635 & & & \\ & 0.0048 & <0.0001 & <0.0001 & <0.0001 & 0.0323\end{array}$

Kruskal-Wallis test

Chi Square

14.88
DF

4

Pairwise Wilcoxon method
bin 1 and 2
bin 1 and 3
bin 1 and 4
bin 5 and 2
bin 5 and 3
bin 5 and 4

All other comparisons not significan

\begin{tabular}{|c|c|}
\hline $\mathbf{P}$ & Steel-Dwass $\mathbf{P}$ \\
\hline 0.0301 & 0.1917 \\
0.0174 & 0.1213 \\
0.0393 & 0.2374 \\
0.0105 & 0.0781 \\
\hline 0.0075 & 0.058 \\
\hline 0.036 & 0.2214 \\
\hline
\end{tabular}

0.2214

\section{Sensitivity analyses- Bin 1 only containing Mesoproterozoic (1600-1000 Ma) samples}

Proportion anoxic (FeHR/FeT $>$ 0.38) from outer shelf and deep basin environments

Shapiro-Wilk $\mathrm{W}$ test. Small p-values reject normal distribution

$\begin{array}{lccccc} & \text { Bin } 1 & \operatorname{Bin} 2 & \operatorname{Bin} 3 & \operatorname{Bin} 4 & \operatorname{Bin} 5 \\ \text { Age: } & 2300- & 1000- & 635-542 & 542-485 & 485-350 \\ \text { p-value: } & 1000 & 635 & & & 0.22\end{array}$




\section{ANOVA}

\begin{tabular}{lcccc|l|} 
Source & DF & SS & MS & F-ratio & \multicolumn{2}{l}{ P } & 0.7575 & post-hoc Tukey HSD: \\
\hline Model & 4 & 0.16 & 0.04 & 0.4699 & $\begin{array}{l}\text { Not } \\
\text { significant }\end{array}$ \\
Error & 45 & 3.95 & 0.09 & & \\
Total & 49 & 4.11 & & & \\
\hline
\end{tabular}

Proportion oxic (FeHR/FeT < 0.22) from outer shelf and deep basin environments

Shapiro-Wilk W test. Small p-values reject normal distribution

$\begin{array}{lccccc} & \text { Bin 1 } & \text { Bin 2 } & \text { Bin 3 } & \text { Bin 4 } & \text { Bin 5 } \\ \text { Age: } & 2300- & 1000- & 635-542 & 542-485 & 485-350 \\ \text { p-value: } & 1000 & 635 & 0.002 & 0.003 & 0.0169\end{array}$

Kruskal-Wallis test

Chi Square

1.81

DF $\quad P$

$4 \quad 0.7706$
Pairwise Wilcoxon method

No comparisons were significant
$\mathbf{P}$

Steel-Dwass $\mathbf{P}$

None

significant

Proportion ferruginous of anoxic samples from outer shelf and deep basin environments

Shapiro-Wilk W test. Small p-values reject normal distribution

$\begin{array}{lccccc} & \text { Bin } 1 & \text { Bin } 2 & \text { Bin 3 } & \text { Bin } 4 & \text { Bin 5 } \\ \text { Age: } & 2300- & 1000- & 635-542 & 542-485 & 485-350 \\ \text { P } & 1000 & 635 & & & \\ & 0.09 & <0.0001 & <0.0001 & <0.0001 & 0.0984\end{array}$

Kruskal-Wallis test

Chi Square

9.3
DF

4

\section{Pairwise Wilcoxon method}

bin 5 and 3

bin 5 and 2

All other comparisons not significant

\section{$\mathbf{P}$}

0.0204

0.0237

0.054

Note: Bin 1 (Mesoproterozoic) is only left with 6 regions, although the percentage of ferruginous samples in bin 1 remains essentially unchanged (now 58\% versus 59\%). The drop in sampling intensity affects the power of the inference tests, and bin 1 is no longer statistically different from the Neoproterozoic at the $\mathrm{p}<0.05$ level. Nonetheless, the p-values of the pairwise Wilcoxon tests between bin 1 and bins 2, 3 and 4 remain relatively low ( $p=0.079,0.079$ and 0.14 respectively). More Mesoproterozoic sampling will help the statistical power of this Mesoproterozoic-Neoproterozoic comparison and reveal whether the change in significance is real (i.e. the signal in the full dataset is driven by Paleoproterozoic samples) or due to simple low sampling levels.

Iron in pyrite (FeP) from oxic sediments in all environments

Shapiro-Wilk $\mathrm{W}$ test. Small p-values reject normal distribution

$\begin{array}{lccccc} & \text { Bin } 1 & \text { Bin } 2 & \text { Bin } 3 & \text { Bin } 4 & \text { Bin 5 } \\ \text { Age: } & 2300- & 1000- & 635-542 & 542-485 & 485-350 \\ \text { P } & 1000 & 635 & & & \\ & 0.0266 & <0.0001 & 0.11 & 0.0056 & 0.22\end{array}$




\begin{tabular}{|c|c|c|c|c|c|}
\hline Kruskal-W & & & Pairwise Wilcoxon method & $\mathbf{P}$ & Steel-Dwass $\mathbf{P}$ \\
\hline Chi Square & $\mathrm{DF}$ & $\mathbf{P}$ & bin 1 and 2 & $0.0036 *$ & 0.0296 \\
\hline 16.73 & 4 & 0.0022 & bin 1 and 3 & 0.0173 & 0.1482 \\
\hline & & & bin 5 and 2 & $0.0027 *$ & 0.0228 \\
\hline & & & bin 5 and 3 & 0.0248 & 0.1635 \\
\hline & & & bin 5 and 4 & 0.0285 & 0.1834 \\
\hline
\end{tabular}

\begin{tabular}{|c|c|c|c|c|c|}
\hline \multicolumn{6}{|c|}{ Shapiro-Wilk W test. Small p-values reject normal distribution } \\
\hline & Bin 1 & Bin 2 & Bin 3 & Bin 4 & Bin 5 \\
\hline Age: & $\begin{array}{c}2300- \\
1000\end{array}$ & $\begin{array}{c}1000- \\
635\end{array}$ & $635-542$ & $542-485$ & 485 \\
\hline $\mathrm{P}$ & 0.0465 & $<0.0001$ & $<0.0001$ & $<0.0001$ & 0.0089 \\
\hline
\end{tabular}

\section{Kruskal-Wallis test}

Chi Square

16.35

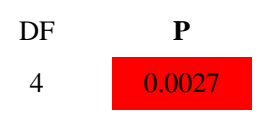

Pairwise Wilcoxon method

bin 1 and 2

bin 1 and 3

bin 1 and 4

bin 5 and 2

bin 5 and 3

bin 5 and 4

All other comparisons not significant

\begin{tabular}{|c|c|}
\hline $\mathbf{P}$ & Steel-Dwass $\mathbf{P}$ \\
\hline 0.0122 & 0.0891 \\
\hline 0.0107 & 0.0796 \\
0.0101 & 0.0757 \\
0.0157 & 0.111 \\
0.0157 & 0.1113 \\
0.0192 & 0.1321 \\
\hline
\end{tabular}

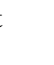

Table S4: Results of statistical analyses. Results for ANOVA, Kruskal-Wallis, Wilcoxon and Steel-Dwass tests that are significant at the $p<0.05$ level are colored in yellow, and results significant at the $p<0.01$ level are colored red. Both ANOVA and Kruskal-Wallis tests were performed for all analyses for the full dataset. The more reliable test should be considered based on normality of the data as assessed with the Shapiro-Wilk W test. For nonparametric analyses, significant differences between bins were analyzed using tests that only considered the bins in question (pairwise Wilcoxon tests) and using tests that correct for multiple comparisons (SteelDwass). Results of Steel-Dwass tests are shown to the right of each comparison found significant in Wilcoxon tests, and Wilcoxon tests significant after Bonferroni correction $(p<0.005)$ are indicated by an asterisk(*). Three sensitivity analyses were also performed. In the first, samples from lithologies/facies not calibrated for the iron speciation proxy, samples analyzed under an older dithionite-only extraction protocol, samples from a Lower Cambrian mineralized horizon in South China, and samples with high abundances of Acid Volatile Sulfide were removed. In the second, regions with low data coverage (5 or fewer samples) were removed. In the third, time bin 1 only contained data from the Mesoproterozoic (1600-1000 Ma). For the sensitivity analyses, only the most appropriate test was performed based on the normality of the data.

\title{
Loess regression-
}

To further explore changes in iron geochemical data through time, all data points were plotted without binning for time or geographic region and analyzed using a loess regression (Figure S1). Data are plotted in binary fashion as 1 (anoxic) and 0 (oxic) as 
FeHR/FeT ratios do not have meaning without reference to baseline values ${ }^{19}$. In other words, a ratio of 0.8 is not 'more anoxic' than a value of 0.6 , as anoxic implies zero oxygen. With respect to the oxic region of iron speciation space, the FeHR/FeT ratios of modern dysoxic samples covers the entire spectrum of FeHR/FeT ratios of fully oxygenated samples ${ }^{19}$ and therefore a sample cannot be interpreted as 'more oxic' based on a lower FeHR/FeT ratio.

The results of this analysis are instructive in demonstrating that completely unbinned data do not support an Ediacaran-Cambrian oxygenation event. However, such an approach also illustrates the difficulties of using unbinned data-specifically that a large number of data points from one region can unduly influence the signal. Most obviously, the large number of oxic samples in the Devonian of upstate New York are responsible for the impressive downturn in the loess regression for the proportion of anoxic samples at the youngest end of the graph (Fig. S1A and B versus C). Nonetheless, the loess regression is consistent with the geographically binned analysis and indicates that analysis of all data unbinned for time or geography does not reveal an obvious Ediacaran or Cambrian oxygenation event. 


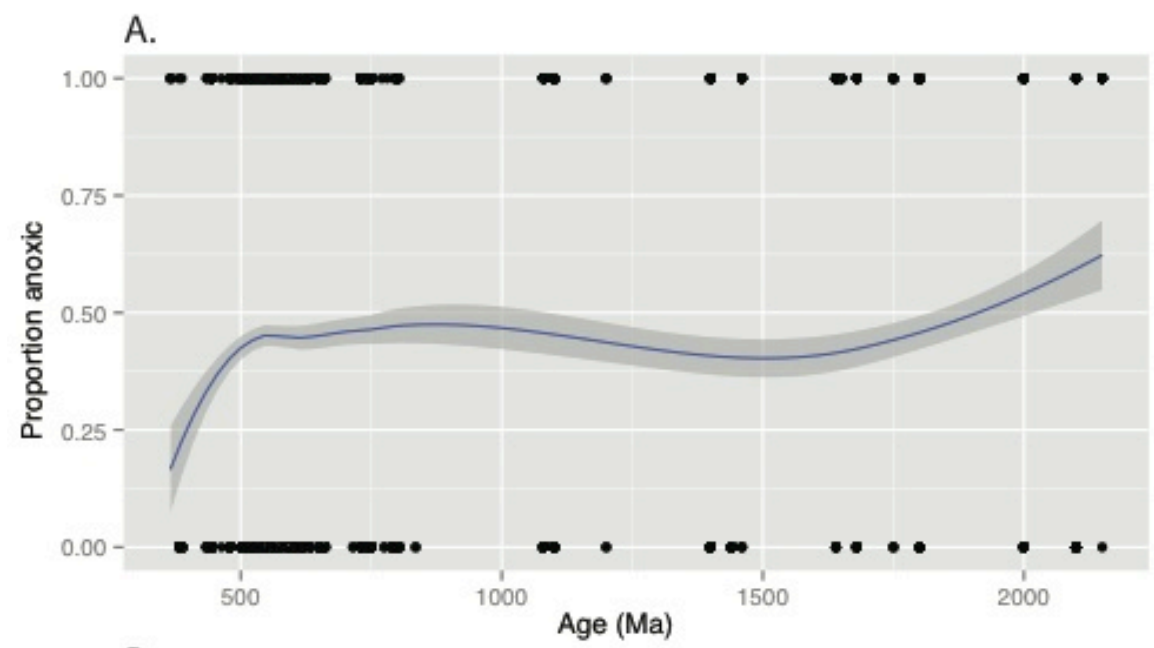

B.

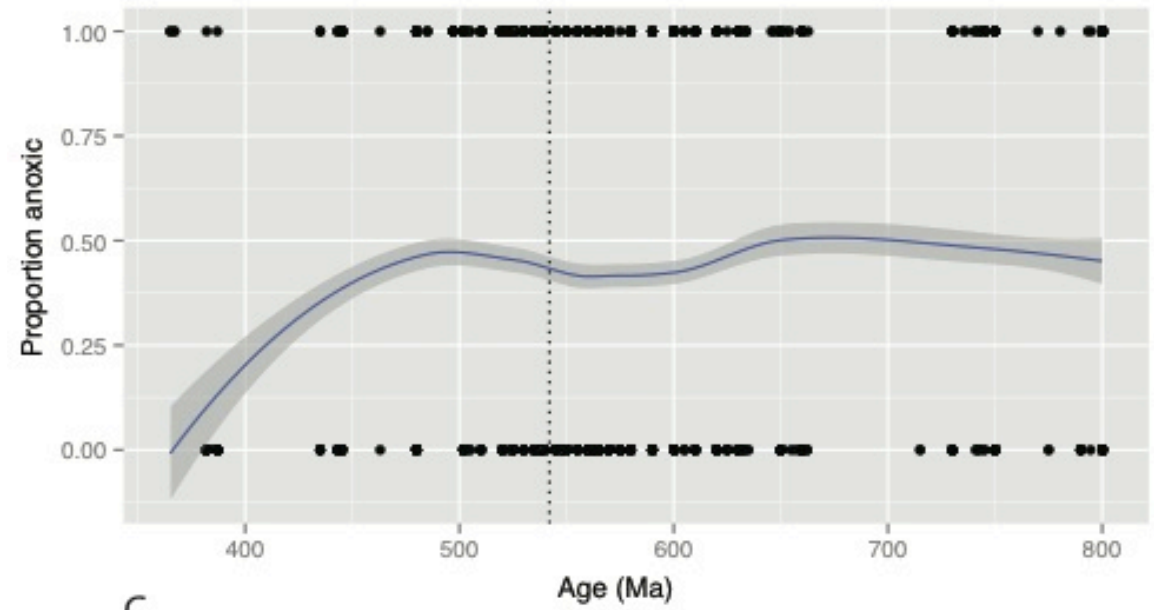

C.

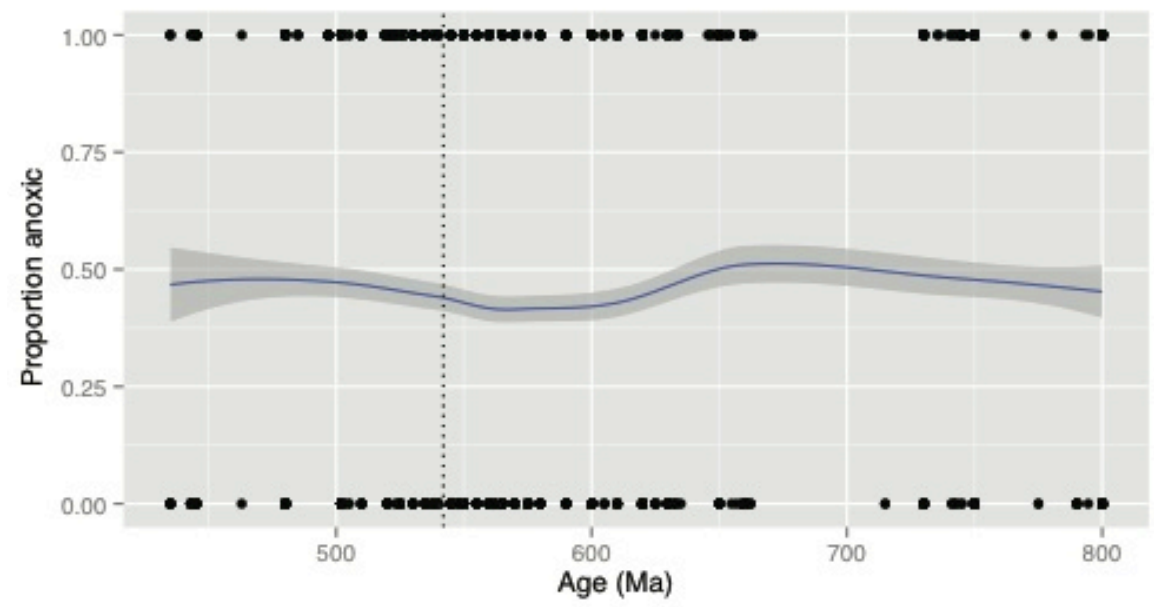

Figure S1: All data plotted without binning for time or geography and analyzed using a loess regression. $1=$ anoxic $(\mathrm{FeHR} / \mathrm{FeT}>0.38), 0=$ oxic $(\mathrm{FeHR} / \mathrm{FeT}<0.38)$. A) All data plotted. B) All data from 800 - 365 Ma plotted. C) All data from 800 Ma to end-Silurian plotted. This removes Devonian data points from Boyer et al., 2011 (ref. 58) and Dahl et al., 2010 (ref. 28; note only four samples), demonstrating that the dramatic decrease in the proportion of anoxic samples 
in A) and B) in the loess regression at the younger end of the plot is driven mainly by the large number of oxic samples in the single study by Boyer et al. 2011 (ref. 58). Vertical line in B) and C) represents Ediacaran - Cambrian boundary at $542 \mathrm{Ma}$.

\section{Resampling analyses-}

Finally, we probed the absence of significant change in Ediacaran-Cambrian Fespeciation data by creating 1000 synthetic replicates each containing ten randomly selected samples from each region in these bins (Fig. S2). From these synthetic datasets we observe that the distribution of anoxic samples in the Cambrian dataset is indistinguishable from the Ediacaran (Wilcoxon: Chi Square $=0.48, p=0.49$ ), consistent with the results in Fig. 1A in the main text.
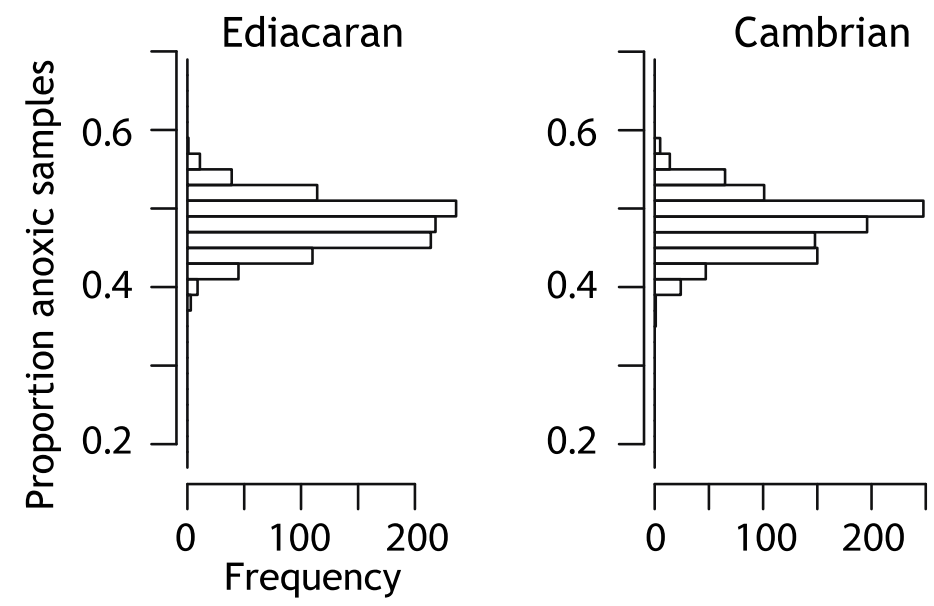

Figure S2: Histograms of sub-sampling analyses of the proportion of anoxic samples from the Ediacaran and Cambrian time bins. 1000 replicates from each bin contain ten randomlyselected samples from each region.

Some final thoughts on iron geochemical sampling through time-

One possible systematic effect that may affect these data is sampling differences between the Phanerozoic and Proterozoic. Without any body- or trace-fossil evidence constraining redox state, Proterozoic samples are generally collected at regular intervals through a stratigraphic succession. Phanerozoic sampling tends to focus on black shales, or intervals where the biota signals dysoxic or anoxic conditions; indeed, most of the data in the Ordovician-Devonian bin is of this nature (Table S2 and Database S1). It is possible that the lack of statistical change in the proportion of anoxic and oxic samples between the Ordovician-Devonian bin and the other bins is due to this systematic bias. Consequently, the $<40 \%$ PAL constraint in Figure 3 in the main text was not extended across the Ordovician. Much of the Cambrian data, however, specifically data from Namibia, Mongolia, northwest Canada, south China and the western United States, was collected in an analogous fashion to the Proterozoic, and where well-sampled regions can be compared across the Ediacaran-Cambrian transition they do not change in geochemical character ${ }^{16}$. Ultimately more data, and data collected to test specific biases, 
will be required to fully understand the influence of random and systematic error, but given current sampling, the absence of iron speciation evidence for Ediacaran or Cambrian oxygenation cannot be ascribed to any known bias.

\section{Statistical analysis of Uranium/TOC data}

To investigate the signal in Uranium/Total Organic Carbon (TOC) ratios as compared to iron speciation for the Neoproterozoic-Paleozoic interval, we analyzed the well-vetted sedimentary $U$ dataset of Partin et al. ${ }^{6}$. This dataset has been filtered to only contain shales that were likely deposited under anoxic condition (using $\mathrm{Fe} / \mathrm{Al}$ ratios and other independent proxy information) and hence can give insight into authigenic uranium enrichments through time. As these enrichments scale with uranium concentrations in seawater, which is itself controlled by the total size of the seafloor covered by reducing sinks, U/TOC ratios can track the global seafloor redox landscape.

The original study ${ }^{6}$ investigated how U/TOC ratios changed over $\sim 3.5$ billion years of Earth history, and hence the analyses used extremely long-duration age bins. The questions regarding oxygenation posed in this paper are focused on the NeoproterozoicPaleozoic, and so our re-analyses used Neoproterozoic, Cambrian-Silurian and DevonianPermian bins. Using finer bins increases age resolution but reduces the sample number and geographic representation of samples. While finer temporal binning is of course possible, even the binning scheme adopted here results in some time bins being dominated by particular geographic areas. For instance, in both the Neoproterozoic and Cambrian-Silurian bins, 56\% of samples come from the Neoproterozoic Doushantuo Formation in South China and the Upper Cambrian Alum Shale in Sweden. As there are clear basin-level influences of trace metal enrichments ${ }^{27}$, fine-scale temporal binning where each bin is dominated by one region leaves open the possibility of tracking local rather than global values. We note that these analyses were undertaken in order to test the possibility that there may some signal of continuing oxygenation throughout the Paleozoic, rather than a full interrogation of trace metal datasets, and view these bins as sufficient to address this question.

As in the original study ${ }^{6}$, we filtered samples based on independent evidence of anoxia and TOC $>0.4$ weight percent, which reduces the number of anomalously high U/TOC ratios in TOC-lean rocks. The U/TOC ratios in each bin were tested for normality, and appropriate statistical tests applied based on normality. Specifically, all bins were found to significantly reject normality $(p<0.0001)$ and so Kruskal-Wallis tests were used to test for significance and pairwise Wilcoxon tests were used to compare significant differences between bins. These analyses demonstrate statistically significant increases in U/TOC ratios between these time bins, and these differences remain significant after Bonferroni correction. The mean values for bins 2 and 3 are not dramatically different, indicating that the difference between bins is due to changes in the distribution rather than a simple increase to all values in the distribution (this can also be seen by the much larger standard deviation for bin 3). Standard boxplot analyses (Fig. S3) indicates this is due to a number of high U/TOC ratios in bin 3. Although inference tests incorporating sampling density are preferred for identifying differences between bins (see 
main text), qualitative observations such as Fig. S3 do indicate a higher number of enriched outlier values in bin 3 compared to bin 2 .

It is worth noting that some analytical choices made during analyses of the U/TOC dataset can affect these results. Most notable is the effect of removing the TOC > 0.4 weight percent filter (keeping the requirement for evidence of anoxia). In this case, the Neoproterozoic has significantly higher ratios than the Cambrian-Silurian (Wilcoxon test, $p=0.0025$ ). The cause of this is immediately clear when investigating the data table of Partin et al. (ref. 6) - many of the Neoproterozoic rocks have very low TOC contents, often $<0.05$ weight percent. Error on such small measurements will be high, and when dividing an $\sim$ crustal value by a potentially erroneous very small TOC value, the result is a likely erroneous and rather meaningless U/TOC ratio. For instance a sample from the 720 Ma Gongdong Fm. has 1.31 ppm U, 0.01 weight percent TOC, and a resulting U/TOC ratio of 131 - higher than essentially any Phanerozoic value. Thus, a TOC filter is reasonable, but given the relatively low TOC contents of Neoproterozoic shales in that database, a lower value may be more appropriate. Likewise, the Fe/Al cut-off of 0.5 used for detecting anoxia is well within the Phanerozoic 'normal' oxic shale value of $0.53 \pm$ 0.11 (ref. 63). We bring up these points not to disagree with the well-justified conclusions of Partin et al. regarding sedimentary U contents of anoxic shales through time, but rather to highlight that there is considerable texture in these records that should be considered as the field moves towards addressing finer-scale questions. Continued generation of both trace metal and iron speciation data and continued interrogation of these records in a statistical framework will lead to an increasingly sophisticated view of ancient redox landscapes as informed through the lens of different proxy records.

\begin{tabular}{ccccc}
\multicolumn{4}{l}{ U/TOC ratios of anoxic shales with TOC $>\mathbf{0 . 4} \%$} & \\
Bin & Age (Ma) & Samples in bin & Mean & Standard \\
Dev. \\
$\mathbf{1}$ & $1000-543$ & 97 & 3.68 & 4.39 \\
$\mathbf{2}$ & $542-419$ & 140 & 9.15 & 9.73 \\
$\mathbf{3}$ & $418-252$ & 443 & 9.86 & 17.81
\end{tabular}

\begin{tabular}{lccc}
\multicolumn{4}{l}{ Shapiro-Wilk W test. Small p-values reject normal distribution } \\
& Bin 1 & Bin 2 & Bin 3 \\
Age: & $1000-543$ & $542-419$ & $418-252$ \\
Samples in bin & 97 & 140 & 443 \\
P & $<0.0001$ & $<0.0001$ & $<0.0001$
\end{tabular}

Kruskal-Wallis test

Chi Square

75.53

$$
\text { DF }
$$

2
Pairwise Wilcoxon method

bin 1 and 2

bin 1 and 3

bin 2 and 3

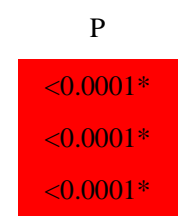

Table S5: Analysis of U/TOC dataset of Partin et al. ${ }^{6}$ binned into Neoproterozoic (1000$543 \mathrm{Ma}$ ), early Paleozoic (542-419 Ma) and late Paleozoic (418-252 Ma) bins. P-values in red for Kruskal-Wallis and Wilcoxon tests indicate values $<0.01$. Asterisks indicate significance after Bonferroni correction. 


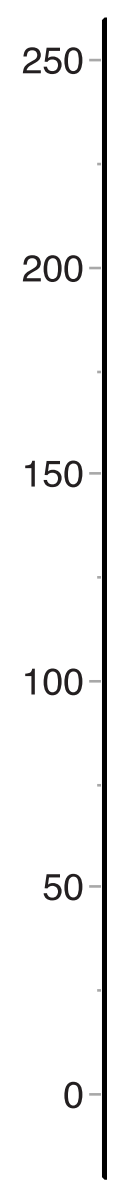

Figure S3: Standard box plot analyses of U/TOC dataset of Partin et al. ${ }^{6}$ binned into Neoproterozoic (1000-543 Ma), early Paleozoic (542-419 Ma) and late Paleozoic (418-252 Ma) bins. Red central line $=$ median; box $=$ interquartile range from first to third quartiles; whiskers = $1.5 \mathrm{x}$ interquartile range; jittered points = outliers above $1.5 \mathrm{x}$ interquartile range. Bin 3 has more enriched outlier values and higher overall values than bin 2 . Note that bin 3 has $\sim$ three times more samples than bin 2, though, and increased sampling density will result in more sampling of enriched values. Consequently, quantitative inference tests are preferable to qualitative plots, although in this case each returns similar results (Table S5).

\section{New analyses}

In this study, a total of 842 samples were analyzed for their iron speciation values. The location, age, and depositional environment of each sample set are described below. References to locality maps and sedimentological/stratigraphic information are given for sections that have been published in the literature. For new sections, the GPS coordinates 
for the base of the section and the meter height of a formational boundary or other reference point are given.

\section{Wernecke Mountains, northwestern Canada}

\section{F851 and W8 sections}

Locality Information- Dark grey to green shale and siltstone were sampled from the Mt. Profeit Dolostone and interfingering 'grit' units below the Goz A section in the Wernecke Mountains (see locality maps ${ }^{64-66}$ ). The W8 section consists of two thrust slices with meters 408 to 842 stratigraphically below and thrust over meters 0 to 408 . The F851 section is a parallel section to meters 408 to 842 of W8. Environment- Shale and silt were deposited in graded beds as turbidite flows on a northeast facing paleoslope (present coordinates) adjacent to the Mt. Profeit Dolostone, which formed on a paleohigh to the southwest. Paleoslope derived from slump folds was to the northeast, but paleocurrent flow derived from solemarks of turbidites was to the southeast ${ }^{66}$. These features suggest deposition in a southeasterly deepening basinal trough that was parallel to the Snake River Fault. Age- The Mt. Profeit Dolostone and grit rest unconformably on fanglomerate and diamicite of the Raptian Group. Stratigraphy equivalent with the 662.4 \pm 3.9 Ma basal Twitya cap carbonate ${ }^{68}$ is not present. The Mt. Profeit Dolostone and grit are unconformably overlain by the Ravensthroat basal Ediacaran cap dolostone, which has been dated globally at ca. $635 \mathrm{Ma}^{69-71}$. Thus, we infer that the Mt. Profeit Dolostone and grit are broadly correlative with the upper Twitya Formation and the Keele Formation in the Mackenzie Mountains and were deposited between 662 and 635 Ma.

\section{W-11 section}

Locality Information-Black to maroon colored shale were sampled from the Sheepbed Formation and June beds at the Goz B section in the Wernecke Mountains (see locality maps ${ }^{64-66}$ ). The base of the section starts with the Ravensthroat cap carbonate and 0 to $200 \mathrm{~m}$ are in the Sheepbed Formation, and 200 to $\sim 600 \mathrm{~m}$ are in the June beds. Environment- Section is plotted and described in ref. 45. These strata were deposited below wave base, predominantly as fissile black shale, but also including minor carbonate debrites and coarse siliciclastic grain flows. Age- The Sheepbed Formation was deposited during the earliest Ediacaran in the ca. $635 \mathrm{Ma}^{69-71}$ post-Marinoan glacioeustatic sea-level rise. The Sheepbed Formation is unconformably overlain by the June beds and it is unclear how much time is missing. Macdonald et al. (ref. 66) suggested that the unconformity could be related to the Gaskiers glaciation or late Ediacaran extension on the western margin of North America and that the June beds were deposited between ca. 580 and 560 Ma.

\section{W-15 section}

Locality Information-Dark grey to black shale and siltstone were sampled from the Blueflower Formation at the Goz B section (see locality maps ${ }^{65,66}$ ). Environment- Section is plotted and described in ref. 66. The Blueflower Formation in the Wernecke Mountains formed near storm wave base in two broad transgressive sequences sandwiched by highstand and low-stand systems tracts. Hummocky cross-stratification is present in the 
uppermost Blueflower Formation, and Planolites trace fossils are present in the lower and middle Blueflower Formation. Age- The Blueflower Formation contains Ediacaran fossils Aspidella and Beltanelliformis ${ }^{64}$ and was deposited above a carbon isotope anomaly in the Gametrail Formation that has been correlated with the Shuram carbon isotope excursion $^{66}$. These correlations suggest that the Blueflower Formation was deposited during the late Ediacaran between ca. 551 and 542 Ma.

\section{J1218 section}

Locality Information- Shales were sampled from the earliest Cambrian succession at the Goz D section (see locality maps ${ }^{64-66}$ ). The section begins in the upper Risky Formation, with the unconformable Risky-Ingta contact at 19.5 meters. The base of the section is located at N64' 33' 35.3”, W132 ${ }^{\circ}$ 58' 55.1”. Environment- Sampling was focused on the $\sim 40$ meter thick black shale above the Ingta phosphatic carbonates; these shales were deposited beneath storm wave-base. Age- The basal Ingta Formation at this locality includes a phosphatic horizon with earliest Cambrian small shelly fossils including Protohertzina and Anabarites ${ }^{65,72}$. In previous publications ${ }^{64-65,72}$ the black shale above the phosphatic horizon has been referred to the Vampire Formation. However, as the Vampire Formation is regarded as correlative with the Upper Member of the Backbone Ranges Formation ${ }^{73}$, and the dark shale at Goz D sits stratigraphically beneath a thick shallow-water sandstone-dominated unit reminiscent of the Backbone Ranges Formation elsewhere, this designation may not be correct. Regardless, sandstone beds at the top of this shale package include early Cambrian trace fossils such as Treptichnus, Rusophycus and Cruziana ${ }^{64-65,72}$, which along with the small shelly fossils establish it as Early Cambrian in age.

\section{S1203, S1206 and S1208}

Locality Information- Spot collections of shales were made from the Blueflower Formation at the Goz D locality (see locality maps ${ }^{64,65}$ ) where unfortunately exposure was too scarce for continuous sampling. A stratigraphic column for the Goz D section can be found in ref. 66. Section S1203 (2 samples) begins at the Gametrail-Blueflower contact, as does Section 1206 (3 samples), so both sample sets are from the basal Blueflower. Section S1208 (4 samples) was collected from the uppermost Blueflower ('Disc Member' of ref. 64) and is floating with respect to stratigraphic marker horizons as there is cover above and below. Environment- S1203 and S2106 are from the Yuletide member of the Blueflower as defined by ref. 64. These sections show abundant evidence for rip-ups and mud-cracks indicating very shallow water deposition. S1203 samples from the very base of the Blueflower do not show these indicators (although exposure is spotty) and so are classified as outer shelf, while S1206 is inner shelf. S1208 is located in the 'Disc member' and consists of dark shale and silt interbedded with rare, lenticular sands, and represents an outer shelf environment. Age- Based on carbon isotope stratigraphy ${ }^{66}$ and the presence of Ediacaran body fossils in the Disc member ${ }^{64}$ these samples likely span the mid-Ediacaran ( 570 Ma, S1203) to the late Ediacaran ( 550 Ma, S1208).

\section{Mackenzie Mountains, northwestern Canada}


F1161, F1162, F1163, F1164, F1165, J1128 sections

Locality Information- These sections are located at Sekwi Brook in the central Mackenzie Mountains. Locality maps and detailed information on the sedimentology and stratigraphy of these sections can be found in ref. 66. Environment- All sections record deep-water deposition beneath storm wave-base. Age- Section F1165 covers black shales and siltstones beneath an unconformity that has been tentatively correlated with the $\sim 580$ Ma Gaskiers glaciation (see discussion in ref. 66). Sections F1161 and F1162 cover the informally-named June beds, which sit above the unconformity surface and have yielded Ediacaran macroscopic fossils ${ }^{74}$. Sections F1163 and F1164 cover the transition from the Gametrail Formation into the overlying Blueflower Formation, which includes simple bilaterian traces ${ }^{75}$. Section J1128 includes the upper Blueflower Formation, which shows an increasing diversity and size of bilaterian traces ${ }^{75}$. Although direct age constraints are not present, based on stratigraphic position and body and trace fossil assemblages, the June beds are likely mid-Ediacaran and the Blueflower Formation is likely late Ediacaran in age.

\section{F1159 section}

Locality Information- This section is located at June Lake in the central Mackenzie Mountains. The section begins in the middle of the Blueflower Formation and ends at the apparent base of the Risky Formation (although the contact is gradational and conformable) at 946 meters. The top of the section is at N63 $28^{\prime} 14.23^{\prime \prime}$, W128 $41^{\prime}$ 08.40". A locality map (as section A) can be found in ref. 76 and a stratigraphic column for this section can be found in ref. 66. Although this section is nearly one kilometer thick, samples analyzed in this study come from the upper 200 meters below the Risky Formation. Environment- The Blueflower Formation gradually shoals from deep-water turbidite deposits to beds with indicators of wave-activity, such as hummocky crossstratified sands. Such wave indicators increase towards the base of the Risky but are relatively rare. The samples from this section are considered outer shelf. Age- Ediacaran body fossils are found in the Blueflower Formation elsewhere in the central Mackenzie Mountains $^{74}$ and in conjunction with carbon isotope stratigraphy ${ }^{66}$ and the stratigraphic position close to the Risky Formation the analyzed samples are likely late Ediacaran in age.

\section{J1125 section}

Locality Information- This section is located at June Lake in the central Mackenzie Mountains. A locality map (as section A) and general stratigraphic column can be found in ref. 76. The J1125 section begins in the very uppermost Blueflower Formation, with the base of the section at N63 28' 42.9”, W128 $31^{\prime}$ '08.5”. The contact with the Risky Formation is conformable and gradational, and the unconformable Risky-Ingta contact is at 83.1 meters. The ${ }^{13} \mathrm{C}$ isotope excursion recorded by ref. 77 is in intraformational conglomerates from 116.3 to 124.0 meters. Environment- The Ingta Formation at this locality is mainly dominated by shale and siltstones, with occasional beds of hummocky cross-stratified sandstone beds, suggesting deposition in an outer shelf environment. AgeBased on carbon isotope stratigraphy and trace fossil assemblages, the PrecambrianCambrian boundary on the June Lake panel has been suggested to lie low in the Ingta 
Formation, likely around parasequence 2 (refs. 75, 77). Thus this section spans the latest Ediacaran and earliest Cambrian.

S1305, S1306 and N13-6 sections

Locality Information- These sections are located at Ingta Ridge in the central Mackenzie Mountains, approximately 20 kilometers south of June Lake. This is the type section of the Ingta Formation ${ }^{78}$. Published stratigraphic sections for this area can be found in refs. 76 and 78. Because beds are overturned, all sections were measured stratigraphically downward. The base of S1306 is at the top of the Backbone Ranges carbonate at N63²0’38.1”, W128³8'09.2”. Section S1306 measured down-section through the lower Backbone Ranges Formation, and into the Ingta Formation, with the unconformity at the top of the Ingta Limestone at 67.1 meters. The top of Simple Sequence 1 of ref. 76, which is also the top of S1306, is at 268.7 meters. This is also the base of section S1305, which measures downward through Simple Sequence 1 of the Ingta Formation to the unconformable contact with the Risky Formation at 61.8 meters. Note that stratigraphic heights for the Ingta and Backbone Ranges are much thinner than in ref. 76 but correspond well to those in ref. 78. Section N13-6 measures downward from the base of the Risky Formation through the upper Blueflower Formation. Environment- The upper Ingta Formation (Simple Sequences 2-5 of ref. 76) shows abundant current-rippled sandstones and silts and other indicators of a shallow, inner shelf environment. These are lacking in Simple Sequence 1, which also contains rare slump folds ${ }^{78}$, suggesting a more distal, outer shelf environment for S1305. Similar to section F1159 at the June Lake section to the north, the uppermost Blueflower here is a shoaling sequence representing the transition from a basinal turbidite system to an outer shelf environment. Age- As at June Lake, the uppermost Blueflower is late Ediacaran in age, the Precambrian-Cambrian boundary likely lies near the Simple Sequence 1-Simple

Sequence 2 boundary in the Ingta Formation ${ }^{75}$, and the remainder of the Ingta Formation is earliest Cambrian in age.

\section{BFTW section}

Locality Information- Cryogenian (inter-glacial) shales of the Twitya and Ice Brook formations were sampled at the BFTW section near Bluefish Creek. The base of the section is at N64 $01^{\prime} 44.2^{\prime \prime}, \mathrm{W} 128^{\circ} 48^{\prime} 45.9^{\prime \prime} .25 .4$ meters in section corresponds to the contact between the Rapitan Group (Sturtian-age glacial deposits) and the Twitya Formation. The top of the section is at the base of the Stelfox Member of the Icebrook Formation (Marinoan-age glacial deposits). The macroscopic 'Twitya disc' fossils ${ }^{79}$ occur near the top of the section in what has been mapped as the lower members of the Ice Brook Formation $^{80}$. Environment- The base of the section consists $\sim 100 \mathrm{~m}$ of dark, fissile shale. The section then transitions to interbedded turbiditic shale, siltstone and fine sandstone for $\sim 400 \mathrm{~m}$. At $587 \mathrm{~m}$, the lithology becomes increasingly more coarse-grained with common load structures and convoluted bedding, and large grit channels are present, likely marking the base of the Ice Brook Formation. The grits dominate for $100 \mathrm{~m}$. The top $\sim 300 \mathrm{~m}$ of section consists of sandy turbidite beds with well-defined bases. There are no storm-wave indicators, and combined with the paleogeographic position towards the Selwyn Basin, all samples are interpreted as basinal. Age- The base of the Twitya Formation has been dated elsewhere in the Mackenzie Mountains using Re-Os dating at 
$662.4 \pm 3.9 \mathrm{Ma}^{68}$. The top of the section must be $<635 \mathrm{Ma}$ based on the presence of the presumed Marinoan-age Ravensthroat cap carbonate above the Icebrook diamictite. Additional notes- Some small faults with an estimated 5-10 meters of offset cut the upper part of the succession but are difficult to trace due to a lack of marker beds. Additionally, although the finest-grained lithologies available were sampled, the seven samples above the base of the grit channels at $\sim 590 \mathrm{~m}$ are coarser-grained (silt to fine sand) than those generally sampled for iron speciation studies.

\section{Ogilvie Mountains, northwestern Canada}

\section{F836, F1011, J1018 sections}

Locality information- Shales were sampled from the Callison Lake Dolostone at three sections. Locality maps for F836 and F1011 are found in ref. 81. The base of section J1018 is at N64 39' 47.5”, W139²3’ 50.7”. Environment- Despite a formational name indicating a dominantly carbonate composition, two shale intervals, approximately 10-30 meters thick, are present at the top and bottom of the Formation. The shales in the Callison Lake Dolostone are generally from relatively shallow-water environments, with the F1011 and most of F836 sections representing inner-shelf type environments, and J1018 and F836- $895 \mathrm{~m}$ and 898m samples representing outer shelf. Age- The age of the Callison Lake Dolostone is constrained by a Re-Os age of $739.9 \pm 6.1 \mathrm{Ma}$ in the upper shale horizon and the presence of Vase-Shaped Microfossils $^{82}$. Additional notes- As the Callison Lake Dolostone sits with angular unconformity on older units ${ }^{81}$, it represents a different basin-forming event, and is therefore considered separately from iron speciation data in older stratigraphy in the Ogilvie Mountains in the Fifteenmile Group (e.g. ref. 23).

\section{F838 and F842 sections}

Locality information- Locality maps and stratigraphic columns for the F838 and F842 sections can be found in ref. (81). Environment- The black shale in F838 shows no evidence for wave or current activity and was deposited beneath storm wave base. The F842 section has 3 samples at $135 \mathrm{~m}, 227 \mathrm{~m}$ and $229 \mathrm{~m}$. Sample 135 is a black shale interbedded with nodular limestone that was deposited below storm wavebase. Samples 227 and 229 were deposited in a sequence that includes cross-bedded sandstone that formed above wave base. Age- The F838 section begins above a white dolostone presumed to be a ca. 635 Ma Marinoan-aged cap carbonate and has been correlated with the Sheepbed Formation. The sampled black shale is unconformably overlain by a brecciated dolostone and a hummocky-cross stratified pink dolomite that records a largemagnitude negative carbon isotope excursion correlated with the mid-Ediacaran Shuram anomaly $^{66}$. Thus, the sampled black shales are early Ediacaran in age. The base of section F842 is the brecciated dolomite, which lies above the black shale that is correlated with the Sheepbed Formation. The sample at $135 \mathrm{~m}$ is from the shale directly above the Shuram anomaly and is tentatively correlated with the uppermost Gametrail Formation or the Blueflower Formation ${ }^{66}$, and is thus late Ediacaran in age. Samples 227 and 229 are from unit PH5, which contains Early Cambrian trace fossils ${ }^{83}$ and has been correlated with the Backbone Range Formation in the Mackenzie Mountains ${ }^{66}$. 


\title{
Svalbard and East Greenland
}

\author{
G155 section
}

Locality Information- The base of this section is at N79'58.0', E18 ${ }^{\circ} 37.7^{\prime}$, on the Krystalfjellet Peninsula in eastern Murchisonfjord, Nordaustlandet. This section is within an east-dipping panel of the Svanbergfjellet Formation and the collected samples are from the maximum flooding interval within a 13 m-thick shoaling upward sequence that marks the carbon isotope recovery from the Bitter Spring anomaly at the base of the informally defined lower limestone member (Svanberfjellet member 2). Here, 0.4-thick $m$ of black shale grade upwards into green, then tan and green shale, which is then overlain by $6.8 \mathrm{~m}$ of Conophyton stromatolites. This sequence overlies a succession of dominantly ribbon-bedded carbonates with minor grainstone. Environment- The black shales were deposited at or just below storm-wave base on a carbonate ramp during a transgression that followed subaerial exposure. Age- Based on correlation of the Bitter Springs anomaly in Svalbard with what is inferred to be the same anomaly in the Fifteenmile Group, Ogilvie Mountains, Yukon and an age model developed for that succession $^{81}$, these samples are estimated to be ca. $802 \mathrm{Ma}$.

\section{G306 section}

Locality Information- The base of this section is at N7905.0', E18 $26.6^{\prime}$, on the Dracoisen nunatak in Ny Friesland. It corresponds to section 4 in ref. 84. The section begins on a north-facing slope at the base of the Dracoisen Formation. All samples analyzed here are from the Member 2 of the Dracoisen Formation, which comprises a shoaling-upward shale-silt sequence above the basal Dracoisen cap dolostone and is capped by an exposure surface. Environment- Analyzed samples are from an interval of black to dark grey shales deposited below storm-weather wave base on what is interpreted to be a broad, thermally subsiding continental platform. Age- Assuming the Dracoisen Formation includes the basal Ediacaran cap carbonate, then the base of the Dracoisen is approximately $635 \mathrm{Ma}$. Dracoisen member 2 is interpreted to be between 635-630 Ma.

\section{G406 section}

Locality Information- The base of this section is at N79'56.2', E18 $19.7^{\circ}$ ', east of Sveanor on the south coast of Murchisonfjord in Nordaustlandet. This section is from the the Russøya member, which comprises the basal Elbobreen Formation of the Polarisbreen Group, corresponding to measured section 7 in ref. 84 and MS7 in ref. 85. The collected black shale samples are from an 85 m-thick interval of black limestone ribbonites with molar tooth structure and interbedded rhythmite and black shale within the middle of a 145 m-thick shoaling upward sequence. Sample heights are in m's above the $79 \mathrm{~m}$ level. This sequence occurs beneath the Sturtian-equivalent Petrovbreen Member glacial deposits. Environment- The black shale in this sequence was deposited at or just above storm wave base on a carbonate ramp. Age- These rocks are inferred to be $\sim 740 \mathrm{Ma}$ based on a thermal subsidence model calibrated with correlated ages.

\section{G407 section}


Locality Information- The base of this section is at N79'53.5', E18 29.3', southeast of Backaberget on the south side of Murchisonfjord in Nordaustlandet. It lies within a nearly complete west-dipping panel of Akademikerbreen-Polarisbreen strata. The samples are from the middle-lower Macdonaldryggen Formation, whose base is at $188 \mathrm{~m}$ in the section. Samples are black shale samples within an overall dark grey muddy siltstone. Environment- The shales were deposited at or below storm wave base on a broad mud-dominated platform. Age- Based on correlations of glacial successions, the age of the Cryogenian interglacial Macdonaldryggen Member is estimated to be ca. 660 to $645 \mathrm{Ma}$. These samples are estimated to be about $655 \mathrm{Ma}$.

G411 section

Locality Information- The base of this section is at N79'53.5', E18 29.3', aprroximately $0.5 \mathrm{~km}$ to the northwest of section G407, southeast of Backaberget on the south side of Murchisonfjord in Nordaustlandet. The samples are from the lower Dracoisen Formation, whose base is $0 \mathrm{~m}$ in the section. The Dracoisen Formation includes the cap carbonate sequence, which overlies the Marinoan Wilsonbreen Formation. The Dracoisen cap dolostone in this section is $6 \mathrm{~m}$-thick. Samples are black shale from Member 2 of the Dracoisen Formation. Environment- These shale samples were deposited at or below storm wave base on a broad mud-dominated platform, following the post-glacial transgression and maximum flooding. Age- Assuming the Dracoisen Formation includes the basal Ediacaran cap carbonate, then the base of the Dracoisen is approximately $635 \mathrm{Ma}$. Dracoisen member 2 is interpreted to be between 635-630 Ma.

\section{G419 section}

Locality Information- The base of this section is at N79'56.46', E18²17.73' near the south Murchisonfjord coastline at Sveanor. Samples are from the uppermost Macdonaldryggen Member, with the $0 \mathrm{~m}$ datum marking the base of the overlying Slangen Member and the section measured downward from that datum. This section is mixed black silty shale and grey siltstone. It grades upwards into muddy limestone ribbons. Environment- The upper Macdonaldryggen Member in this location was deposited above storm wave base on a thermally subsiding platform during a transition from a dominantly siliciclastic to carbonate environment. Age- the samples are estimated to be ca. $645 \mathrm{Ma}$ based on the fact that they occur just below the Slangen Member, which lies beneath the Marinoan Wilsonbreen Formation, but is presumably separated by a disconformity.

\section{G426 Section}

Locality Information- These samples were collected from an exposure of the Raudstupet-Sälodden Formation of the upper Veteranen Group to the southeast of Eltonbreen glacier on the north coast of Wahlenbergfjorden, Nordaustlandet at N79 $48.2^{\prime}, \mathrm{E}^{\circ} 8^{\circ} 34.6^{\prime}$. The samples are from a black pencil shale located $25 \mathrm{~m}$ below a quartzite in the upper Veternanen (exact stratigraphic height unknown). EnvironmentThis shale is inferred to have been deposited below storm wave base on a siliciclastic margin near the time of the rift-drift transition. Age- The Veteranen Group is constrainted to be younger than about $940 \mathrm{Ma}$ and older than $811 \mathrm{Ma}$ (based on correlation of the 
Bitter Springs anomaly in the overlying Grusdievebreen Formation) with the Fifteenmile Group in the Yukon Territory, Canada ${ }^{81}$. Thermal subsidence modeling suggests an age ca. 835 Ma for the upper Veteranen Group.

G435 section

Locality Information- The top of this section is at N79'48.8', E18 $36.2^{\prime}, 1.5 \mathrm{~km}$ south of the Forsiusbreen glacier near Gimleoden in western Nordaustlandet. It lies on the east side of a syncline cored by the Dracoisen Formation. Samples are from the middleupper Macdonaldryggen Member, with the $0 \mathrm{~m}$ datum marking the base of the overlying Slangen Member and the section measured downward from that datum. This section is mixed black silty shale and grey siltstone. Environment- The middle-upper Macdonaldryggen Member in this location was deposited below storm wave base on a thermally subsiding siliciclastic platform. Age- the samples are estimated to be ca. 650$645 \mathrm{Ma}$ based on the fact that they occur just below the Slangen Member, which lies beneath the Marinoan Wilsonbreen Formation, but is presumably separated by a disconformity.

G436 section

Locality Information- The base of this section is at N7948.6', E18 $34.6^{\prime}, 1.5 \mathrm{~km}$ south of the Forsiusbreen glacier near Gimleoden in western Nordaustlandet and $1 \mathrm{~km}$ west of section G435. Samples are from the lower Dracoisen member 2, with the $0 \mathrm{~m}$ datum marking the base Dracoisen Formation. The samples are from an interval of black shale grading up to silty black shale. Environment- The middle-upper Macdonaldryggen Member in this location was deposited below storm wave base on a thermally subsiding siliciclastic platform. Age- Assuming the Dracoisen Formation includes the basal Ediacaran cap carbonate, then the base of the Dracoisen is approximately $635 \mathrm{Ma}$. Dracoisen member 2 is interpreted to be between 635-630 Ma.

\section{G471 section}

Locality Information- The base of this section is at N80'09.1', E18 $20.1^{\prime}$ on the northern Storstein peninsula in northeastern Nordaustlandet. The section spans the sequence boundary that marks the end of the Bitter Springs negative carbon isotope anomaly, as in section G155. The black shale samples are from the lowermost part of a shoaling upward sequence that is $19.6 \mathrm{~m}$ thick and overlies an exposure surface. The black shales sit above $3.3 \mathrm{~m}$ of silty green shale and mark the maximum flooding interval of the sequence. Environment- The black shales were deposited at or just below storm-wave base on a carbonate ramp during a transgression following subaerial exposure. AgeBased on correlation of the Bitter Springs carbon isotope excursion in Svalbard with what is inferred to be the same anomaly in the Fifteenmile Group, Ogilvie Mountains, Yukon ${ }^{81}$ and an age model developed for that succession, these samples are estimated to be ca. 802 Ma.

\section{G512 section}

Locality Information- The base of this section is on a steep slope of shallowly dipping Svanberfjellet Formation on the western edge of Klofjellet nunatak in Olav V Land, Spitsbergen, at N7850’44.5”, E1806’24.2”. This section is entirely within 
Svanbergjellet member 3 (Upper Algal dolomite) and the base of the section is at the top of a prominent stromatolite biostrome. The black shales grade vertically into limestone rhythmites and dolomite ribbonites. Environment- Here the black shale occurs within the lower parts of poorly developed shale-to-carbonate parasequences, the lower part of which appears to have been deposited below storm wave base during a rare muddy interval in the otherwise carbonate-dominated Akademikerbreen Group. Age- Based on an age model developed for Svalbard, the upper Svanbergfjellet member 3 is ca. 795 Ma.

\section{G521 section}

Locality Information- This section was measured on the southwestern edge of the Backlundtoppen nunatak in Olav V Land, Spitsbergen at N78'42'48.3”, E18¹2'43.7”'. The section is of the lower-middle Russøya Member, where it overlies a karstified surface of the underlying 'Dartboard Dolomite,' which forms the top of the Akademikerbreen Group. The lowermost Russøya member here a thick-bedded grey limestone, which transitions upward to marly dolomitic shale, then black shale. The datum of the section is the top of the limestone. The black shale transitions upward into brown dolomitic silty shales. Environment- The lower-middle Russøya black shales occurs within the maximum flooding interval within a single, $\leq 150 \mathrm{~m}$-thick parasequence that marks the transition from the Akademikerbreen to Polarisbreen groups. The shale is inferred to have been deposited on a mixed carbonate-siliciclastic ramp below storm wave base. Age- Based on an age model developed for the Polarisbreen and Akademikerbeen using correlated ages, this part of the Russøya member is estimated to be ca. 743-740 Ma.

\section{East Greenland}

\section{Section GR12}

Locality Information- This section was measured on the northern part of Ella Island in the East Greenland Fjordland, at N72 ${ }^{\circ} 52^{\prime} 46^{\prime \prime}$, W25 $07^{\prime} 56^{\prime \prime}$. The section is of the interglacial Arena Formation, which is equivalent to the Macdonaldryggen Formation in East Greenland. Here, the Arena Formation is mostly covered, but exposed section includes fine sandstone, siltstone, and grey to black shale. The base of the section $(0 \mathrm{~m})$ is the base of the Arena Formation. The analyzed black shale samples are from the lower part of the formation, which contains minor calcite cement and grades upward into fine, green sand with mud chips. Environment- The lower Arena Formation was deposited below storm wave base on a broad, thermally subsiding platform. The uppermost analyzed sample (GR12-64.5) comes from an interval with interbedded ripple cross-laminated silts, suggesting deposition near or above storm wave base. Age- Similar to the Macdonaldryggen Member in Svalbard, the lower Arena Formation is estimated at ca. 660-655 Мa.

\section{House embayment, USA}

DM1, DM3, MP, SQ, UDQ sections

Locality information- Shales from the Wheeler Shale Formation were sampled from three stratigraphic sections in the Drum Mountains (DM1 and DM3) and House Range 
(MP, SQ, UDQ1 and UDQ2) of western Utah. DM1: base of section N39'30'48”/ W112 59'22”. DM3: base of section N39 30 '43”/ W112 59'57”. MP: base of section

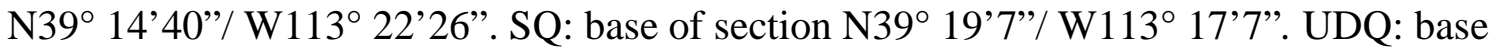
of section N39 21'15”/ W113 $16^{\circ}$ '43”. Environment - The Wheeler Shale represents initial deposition in an embayment that developed on the carbonate platform during late Stage 5 to Drumian Stages Cambrian ${ }^{86}$. The sections in the Drum Mountains represent distal carbonate ramp and deeper shale facies ${ }^{86-88}$. The House Range sections contain predominantly deeper water shale facies. Broadly the shale facies show little evidence for wave action and commonly contain wavy or parallel laminar fabrics ${ }^{89,90}$. Age - Drumian Stage of the Cambrian (504.5 to $500.5 \mathrm{Ma}$ ). The DM1 section contains the GSSP for the base of the Drumian Stage ${ }^{91}$.

\section{Death Valley, USA}

\section{E1101 and E1102 sections}

Locality Information- Shales were sampled from the E1101 and E1102 sections at Saratoga Spring in the southern Death Valley region. Detailed information on the geology of the region and these sections can be found in ref. (92) and references therein. A locality map of the E1101 section and a description of the stratigraphy can be found in ref. 92. E1102 is located 1 km to the south of E1101 (base of section N3540’54.2”, W116 ${ }^{\circ} 4^{\prime}$ 51.9”) and covers the transition from the Beck Spring Formation into the Kingston Peak Formation, with the Formation boundary at $59.3 \mathrm{~m}$. Environment- These sections span the Horse Thief Spring Formation, the Beck Spring Formation, and the Kingston Peak 1 (KP1) member. At Saratoga Spring, siliciclastic sediments in the Horse Thief Spring and Beck Spring formations represent very shallow water deposition and are interbedded with microbial carbonates including teepee structures and minor karsting. The top of the Beck Springs Formation is a gradual transition across a flooding surface to thin turbiditic sandstone with rare dolomite lenses of KP1. These are interpreted as deposited in a prodelta setting beneath storm wave base. Age- The Horse Thief Spring Formation contains $\sim 770$ Ma detrital zircons ${ }^{93}$, which provide a minimum age for the unit, and the Kingston Peak 1 member sits uncomformably beneath glaciogenic rocks correlated with the $\sim 720$ Ma Sturtian glaciation ${ }^{94}$. Additional notes- Although the finestgrained lithologies were always sampled, many of the samples in the E1101 section represent coarser-grained lithologies (silt to fine sand) than are generally analyzed in iron speciation studies.

\section{Urals Region, Russia}

\section{Vostok Askinsky-1 core (VA)}

Locality Information- This core was sampled at the core repository in Ufa, Russia. Detailed information on this core is found in ref. 95. Environment- The sediments sampled are relatively thin ( $<5$ meters stratigraphic thickness) black shales that are interbedded with shallow-water grainstones and intraclast conglomerates, and were likely deposited in very shallow lagoonal environments. Age- The sampled sediments are from an equivalent of the Satka Formation in the southern Urals outcrop belt, and the Kaltasy Formation in the Volgo-Ural region. These equivalent formations are bracketed by 1750 
Ma and 1380 Ma volcanic rocks in the Bashkirian megantclinorium in the southern Urals, and the Kaltasy Formation has been dated by Re-Os geochronology at $1414 \pm 40$ and $1427 \pm 43 \mathrm{Ma}$ (ref. 14, and references therein).

\section{Mongolia}

\section{E1216 section}

Locality Information- This section is in the upper Zuune Arts Formation in the Zavkhan Basin of Mongolia. The base of the section is located near N46 ${ }^{\circ} 41.863^{\prime} /$ E96 $^{\circ}$ 51.765'. Environment- The shales and carbonates in this succession are indicative of an outer shelf environment. Age- The location of these shales close beneath a large negative carbon isotope excursion interpreted to be the Precambrian-Cambrian boundary excursion suggest an age very late in the Ediacaran (E. Smith, pers. comm.).

\section{T1201 section}

Locality Information- This section is in the lower Bayan Gol Formation in the Zavkhan Basin of Mongolia. The base of the section is located near N46 $54.230^{\prime} /$ E95 $^{\circ}$ 15.208'. Environment- The shales and sandstones in this succession are indicative of an outer shelf environment. Age- The presence of small shelly fossils and carbon isotope chemostratigraphy suggest an age of $\sim 538$ Ma (E. Smith, pers. comm.).

\section{Nama Basin, Namibia}

\section{ZUB section}

Locality Information-Samples were collected from a roadcut of the Mara Member at Zuurburg Farm. The base of the section is located at S26 $15^{\prime} 40.6^{\prime \prime}$ / E16 $56^{\circ}$ 17.2”. The top of the section, at 11.2 meters, is a plateau that at other sections on Zuurburg Farm shows a karstic erosional surface. This is the sequence boundary at the top of the Mara Member $^{96}$. Environment- The collected shales were from the base of the section, which consists of interbedded micrite and shale. There is also a dark carbonate bed with a swaley/scoured appearance, indicating these sediments were within the reach of bottom currents. Thus these sediments are analogous to the "outer shelf” environmental category, albeit likely deposited on a carbonate ramp. The succession then shallows into carbonate grainstone and ultimately the sequence boundary. Age- The Mara Member in the southern Nama basin has not been dated directly, but the Kuibis Subgroup (to which the Mara Member belongs) has been directly dated in the northern Nama Basin at $548.1 \pm 1 \mathrm{Ma}^{97}$.

\section{ZBH section}

Locality Information- Three samples were taken from the Urikos Member in the Kuibis Subgroup at the Zebra River Farm. The samples were not collected from a measured stratigraphic column. Sample ZBH-1 was collected at S24³1.696’, E16 ${ }^{\circ} 18.627^{\prime}$; the other samples were stratigraphically below ZBH-1 within an 10 m interval. Environment- These samples are from green shale interbedded with grainstone and rare rippled sandstone that were deposited in an outer-shelf environment. Age- The Hoogland Member, which sits stratigraphically beneath the Urikos Member, has been dated in the northern Nama Basin at 548.1 $\pm 1 \mathrm{Ma}^{97}$. 


\section{SWP section}

Locality Information- Samples were collected from a measured section of the upper Spitskopf Member, Urusis Formation, at Farm Swartpunt. A locality map of the section can be found in ref. 98. The base of the section is at S27 $28.511^{\prime} / \mathrm{E} 16^{\circ} 41.587^{\prime}$. The zero mark of this stratigraphic section corresponds to 67.7 meters on the stratigraphic column in Narbonne et al. (ref. 98; their Figure 3). Environment- The clastic package measured here was described as a "mega-slump" 77 . While we recognize some slumping, overall the stratigraphy appears relatively coherent. Rather, we suggest that the broad, low-amplitude folding apparent in thick carbonate units resulted in folding and faulting within the clastic units that give the appearance of a mega-slump. The SWP section records a general coarsening upsection from shale to sandstone, with small scale Hummocky Cross-Stratification (HCS), parallel laminations, current ripple cross laminations, and combined flow ripples being frequent in the sandstones. The primary Pteridinium fossil bed is at $20 \mathrm{~m}^{98}$. Above this horizon is $\sim 15$ meters of green shale and cover, followed by partially- to wholly-dolomitized thrombolitic mounds and finelylaminated dolostone. The overall environment is regarded as outer shelf. Age- These samples are $~ 70$ meters above an ash bed dated at 543.1 $\pm 1 \mathrm{Ma}$, and Cambrian-aspect trace fossils appear above the thrombolitic carbonates at the top of the section ${ }^{97,98}$. Thus the samples are very latest Ediacaran in age.

\section{FSH section}

Locality Information- This section’s base was located at S $27^{\circ} 16^{\prime} 22.0^{\prime \prime}$ / E 17'37'59.4” on Sonntagsbrunn Farm. The section essentially corresponds to measured section 8 of ref. 99; see that publication for locality map and stratigraphic column. The section begins in the river valley and measures through the Feldschuhhorn Member of the Spitskopf Formation to the unconformably-overlying Lower Nomtsas Formation at 24.6m. Environment- Two $\sim 5$ meter thick relatively shaley intervals were sandwiched between more sandstone-dominated intervals, although silty and sandier units were present throughout the section. The unit had massive grey sandstone beds near the bottom with ripples, erosive bases, Hummocky Cross Stratification, and channelized flow, and is regarded as outer shelf. Age- Elsewhere in the Nama Basin the Feldschuhhorn Member is bracketed by radiometric ages of $548.8 \pm 1 \mathrm{Ma}$ and $545.1 \pm 1 \mathrm{Ma}^{97}$ and is stratigraphically closer to the younger age.

\section{VF1 section}

Locality Information- The base of this measured section was at S27 16' 46.9”, E17 38' 04.8” on Sonntagsbrunn Farm. This section corresponds essentially to measured section 3 and Figure 4 of ref. 99; see that publication for locality map and stratigraphic column. There are slight, meter-scale differences with respect to the stratigraphic column in that paper due to different starting points with respect to the irregular underlying unconformity surface. The section begins at the unconformity between the Feldschuhhorn Member of the Spitskopf Formation and Valley Fill 1 of the Nomtsas Formation, and extends to the erosive surface of the Upper Nomtsas Formation at $73.6 \mathrm{~m}$. EnvironmentThe sediments consist of shale, and rare beds of fine-grained sandstone. No evidence for

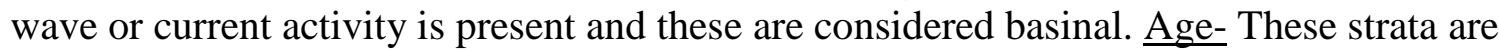
above an unconformity surface that elsewhere contains rocks dated to $539.4 \pm 1 \mathrm{Ma}$ and 
are considered younger than this age ${ }^{97,99}$. The overlying Valley Fill 2 sequence contains the earliest Cambrian trace fossil Treptichnus pedum, suggesting a likely age very close to the radiometric constraint.

\section{Analytical Methods}

Although all analyses in this study followed the same overall protocols, some minor differences between sample sets exist. These are detailed below and in Table S6.

\section{Crushing-}

Samples were crushed in either a hardened steel or tungsten carbide shatterbox puck/mill assembly. Tests of the same sample crushed in both assemblies revealed only a very minor iron contamination ( 0.015 weight percent) by the hardened steel shatterbox (see Supplemental Table 3 of ref. 21). This level is essentially at analytical error and negligible compared to an average shale iron weight percent of 4.72 (ref. 100). The grinding dish used in each sample set is detailed in Table S6.

\section{Total iron analyses-}

Total iron was analyzed via three separate methodologies. The bulk of the samples were digested by a standard four-acid (hydrofluoric, perchloric, hydrochloric and nitric) protocol and measured via ICP-AES at SGS Geochemical Services, Canada. The second highest quantity of samples were subjected to a three-acid digestion (hydroflouric, hydrochloric, nitric) and measured via ICP-OES at McGill University, Canada. One sample set (W8) was subjected to a three-acid digestion (hydrofluoric, hydrochloric and nitric) and analyzed via flame Atomic Absorption Spectroscopy (AAS) at Newcastle University. Each method has been used extensively and been demonstrated to carry sufficient precision for iron speciation applications.

\begin{tabular}{|l|c|l|l|}
\hline \multicolumn{1}{|c|}{ Location } & Section & \multicolumn{1}{c|}{ Grinding dish } & \multicolumn{1}{c|}{ Total iron } \\
\hline Southern Urals & VA-1 core & Tungsten carbide & 4 acid/ICP-AES (SGS) \\
\hline Death Valley & E1101 & Hardened steel & 4 acid/ICP-AES (SGS) \\
\hline Death Valley & E1102 & Hardened steel & 4 acid/ICP-AES (SGS) \\
\hline Mackenzie Mountains & F1159 & Tungsten carbide & 4 acid/ICP-AES (SGS) \\
\hline Mackenzie Mountains & F1161 & Tungsten carbide & 4 acid/ICP-AES (SGS) \\
\hline Mackenzie Mountains & F1162 & Tungsten carbide & 4 acid/ICP-AES (SGS) \\
\hline Mackenzie Mountains & F1163 & Tungsten carbide & 4 acid/ICP-AES (SGS) \\
\hline Mackenzie Mountains & F1164 & Tungsten carbide & 4 acid/ICP-AES (SGS) \\
\hline Mackenzie Mountains & F1165 & Tungsten carbide & 4 acid/ICP-AES (SGS) \\
\hline Mackenzie Mountains & J1128 & Tungsten carbide & 4 acid/ICP-AES (SGS) \\
\hline Mackenzie Mountains & J1125 & Tungsten carbide & 4 acid/ICP-AES (SGS) \\
\hline Mackenzie Mountains & BFTW & Tungsten carbide & 4 acid/ICP-AES (SGS) \\
\hline Mackenzie Mountains & S1305 & Tungsten carbide & 4 acid/ICP-AES (SGS) \\
\hline Mackenzie Mountains & S1306 & Tungsten carbide & 4 acid/ICP-AES (SGS) \\
\hline Mackenzie Mountains & N13 & Tungsten carbide & 4 acid/ICP-AES (SGS) \\
\hline
\end{tabular}




\begin{tabular}{|c|c|c|c|}
\hline Wernecke Mountains & S1203 & Tungsten carbide & 4 acid/ICP-AES (SGS) \\
\hline Wernecke Mountains & S1206 & Tungsten carbide & 4 acid/ICP-AES (SGS) \\
\hline Wernecke Mountains & S1208 & Tungsten carbide & 4 acid/ICP-AES (SGS) \\
\hline Wernecke Mountains & W8 & Hardened steel & 3 acid/Flame AA (Newcastle) \\
\hline Wernecke Mountains & W11 & Hardened steel & 3 acid/ICP-OES (McGill) \\
\hline Wernecke Mountains & W15 & Hardened steel & 3 acid/ICP-OES (McGill) \\
\hline Wernecke Mountains & F851 & Hardened steel & 4 acid/ICP-AES (SGS) \\
\hline Wernecke Mountains & J1218 & Tungsten carbide & 4 acid/ICP-AES (SGS) \\
\hline Ogilvie Mountains & F838 & Hardened steel & 3 acid/ICP-OES (McGill) \\
\hline Ogilvie Mountains & F842 & Hardened steel & 4 acid/ICP-AES (SGS) \\
\hline Namibia & ZUB & Tungsten carbide & 4 acid/ICP-AES (SGS) \\
\hline Namibia & ZBH & Tungsten carbide & 4 acid/ICP-AES (SGS) \\
\hline Namibia & SWP & Tungsten carbide & 4 acid/ICP-AES (SGS) \\
\hline Namibia & FSH & Tungsten carbide & 4 acid/ICP-AES (SGS) \\
\hline Namibia & VF1 & Tungsten carbide & 4 acid/ICP-AES (SGS) \\
\hline House Embayment & DM1 & Hardened steel & 4 acid/ICP-AES (SGS) \\
\hline House Embayment & DM3 & Hardened steel & 4 acid/ICP-AES (SGS) \\
\hline House Embayment & MP & Hardened steel & 4 acid/ICP-AES (SGS) \\
\hline House Embayment & SQ & Hardened steel & 4 acid/ICP-AES (SGS) \\
\hline House Embayment & UDQ & Hardened steel & 4 acid/ICP-AES (SGS) \\
\hline Mongolia & T1201 & Hardened steel & 3 acid/ICP-OES (McGill) \\
\hline Mongolia & E1216 & Tungsten carbide & 4 acid/ICP-AES (SGS) \\
\hline Svalbard & G155 & Hardened steel & 3 acid/ICP-OES (McGill) \\
\hline Svalbard & G306 & Hardened steel & 3 acid/ICP-OES (McGill) \\
\hline Svalbard & G406 & Hardened steel & 3 acid/ICP-OES (McGill) \\
\hline Svalbard & G407 & Hardened steel & 3 acid/ICP-OES (McGill) \\
\hline Svalbard & G411 & Hardened steel & 3 acid/ICP-OES (McGill) \\
\hline Svalbard & G419 & Hardened steel & 3 acid/ICP-OES (McGill) \\
\hline Svalbard & G426 & Hardened steel & 3 acid/ICP-OES (McGill) \\
\hline Svalbard & G435 & Hardened steel & 3 acid/ICP-OES (McGill) \\
\hline Svalbard & G436 & Hardened steel & 3 acid/ICP-OES (McGill) \\
\hline Svalbard & G471 & Hardened steel & 3 acid/ICP-OES (McGill) \\
\hline Svalbard & G512 & Hardened steel & 3 acid/ICP-OES (McGill) \\
\hline Svalbard & G521 & Hardened steel & 3 acid/ICP-OES (McGill) \\
\hline East Greenland & GR12 & Hardened steel & 3 acid/ICP-OES (McGill) \\
\hline
\end{tabular}

Table S6: Summary of analytical differences (grinding dish and total iron measurement) between sample sets for new iron speciation measurements.

\section{Sequential extractions}

Samples in this study were analyzed under the general protocols for iron sequential extraction ${ }^{101}$. Extended lab-specific practices as implemented in the 
Laboratory for Earth History and Isotope Geobiology at Harvard University can be found in the Supplementary Information of ref. 21. Samples from East Greenland and Svalbard were analyzed at McGill University under the same protocols.

As in ref. 21, extractions were run in batches of 48 samples including at least four internal replicates (of a set of six) to estimate precision. These replicate samples are distributed through the Neoproterozoic-Paleozoic time period of interest: (F1018- $54.5=$ Reefal Assemblage, Fifteenmile Group, Yukon, Canada, 820 Ma; F849- $112=$ Sheepbed Formation, Yukon, Canada, 620 Ma; F849- 225 = Sheepbed Formation, Yukon, Canada, 610 Ma; GO130- 286 = Chandindu Formation, Yukon, Canada, 840 Ma; MP- 67.5, Wheeler Formation, United States, 505 Ma; MP- 69.5, Wheeler Formation, United States, 505 Ma). Reagent blanks were also measured from each reaction solution and in all cases found to contain no measureable iron.

\begin{tabular}{|c|c|c|c|c|c|c|}
\hline Replicate & Extraction & $\begin{array}{c}\text { \# of } \\
\text { Analyses }\end{array}$ & $\begin{array}{c}\text { Average } \\
\text { weight \% }\end{array}$ & $\begin{array}{c}\text { Stan. } \\
\text { Dev. }\end{array}$ & $\begin{array}{c}\text { \% Stan. } \\
\text { Dev. }\end{array}$ & $\begin{array}{c}\text { \% Standard } \\
\text { Error }\end{array}$ \\
\hline F849-112 & Acetate & 17 & 0.775 & 0.029 & 3.68 & 0.89 \\
\hline F849-112 & Dithionite & 17 & 0.719 & 0.062 & 8.64 & 2.10 \\
\hline F849-112 & Oxalate & 16 & 0.035 & 0.008 & 23.78 & 5.94 \\
\hline F849- 225 & Acetate & 9 & 0.126 & 0.019 & 14.95 & 4.98 \\
\hline F849- 225 & Dithionite & 9 & 1.156 & 0.086 & 7.41 & 2.47 \\
\hline F849- 225 & Oxalate & 8 & 0.122 & 0.069 & 56.96 & 20.14 \\
\hline GO130-286 & Acetate & 17 & 0.065 & 0.008 & 11.62 & 2.82 \\
\hline GO130-286 & Dithionite & 17 & 1.491 & 0.096 & 6.46 & 1.57 \\
\hline GO130- 286 & Oxalate & 16 & 0.025 & 0.009 & 36.52 & 9.13 \\
\hline F1018-54.5 & Acetate & 18 & 0.047 & 0.009 & 18.80 & 4.43 \\
\hline F1018-54.5 & Dithionite & 18 & 0.458 & 0.070 & 15.35 & 3.62 \\
\hline F1018-54.5 & Oxalate & 16 & 0.080 & 0.024 & 30.00 & 7.50 \\
\hline MP-67.5 & Acetate & 10 & 0.440 & 0.011 & 2.48 & 0.79 \\
\hline MP- 67.5 & Dithionite & 10 & 0.146 & 0.027 & 18.38 & 5.81 \\
\hline MP- 67.5 & Oxalate & 9 & 0.008 & 0.003 & 31.26 & 10.42 \\
\hline MP- 69.5 & Acetate & 17 & 0.715 & 0.023 & 3.21 & 0.78 \\
\hline MP-69.5 & Dithionite & 17 & 0.144 & 0.022 & 15.42 & 3.74 \\
\hline MP-69.5 & Oxalate & 16 & 0.010 & 0.003 & 32.24 & 8.06 \\
\hline
\end{tabular}

Table S7: Summed results of internal replicates run alongside samples to estimate error in sequential reactions. One batch of oxalate replicates was lost during measurement and thus oxalate analyses have one fewer than acetate and dithionite. Samples from East Greenland and Svalbard were analyzed at McGill University alongside the same internal replicates and found to be consistent.

As found in ref. (21) for these internal replicates, precision is related to the weight percent iron in the extraction (Figure S4 and Table S7). Specifically, all extractions with greater than 0.3 weight percent iron had percent standard errors of $<5 \%$. Samples with 
low extractable iron for a specific pool are less precise, but given the low iron quantities involved, the low precision will not affect the overall iron speciation results.

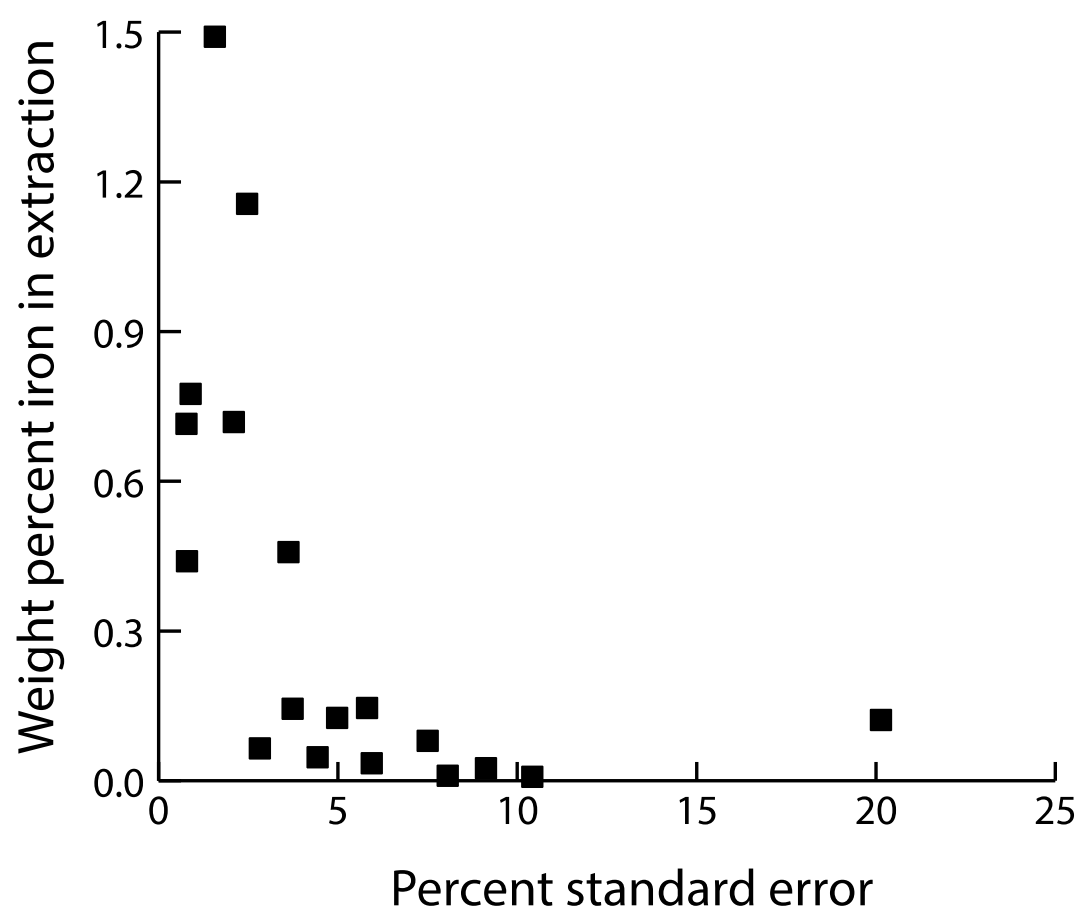

Figure S4: Average weight percent iron versus percent standard error on the three extractions (acetate, dithionite and oxalate) on six internal replicates used in this study.

Iron pyrite analyses

The amount of iron present in pyrite was quantified using the chromium-reducible sulfur (CRS) method ${ }^{102}$. The reaction vessels were purged of ambient atmosphere with pure nitrogen gas, and $20 \mathrm{~mL}$ of $1 \mathrm{M}$ chromous chloride, acidified to $0.5 \mathrm{~N} \mathrm{HCl}$, was added to the reaction vessels after $20 \mathrm{~mL}$ of $6 \mathrm{~N} \mathrm{HCl}$. The vessels were heated to nearboiling and the extraction allowed to proceed for at least two hours. Sulfur in pyrite (and other sulfur phases extracted by this solution-see ref. 102) is reduced to hydrogen sulfide, and the sulfide gas trapped in a $0.3 \mathrm{M}$ zinc acetate trapping solution. An appropriate amount of sediment was estimated for each sample set, and samples were rerun if more sulfide was present than the empirically determined maximum threshold for the volume of zinc acetate capture solution used. Zinc sulfide was then converted to silver sulfide by the addition of $0.3 \mathrm{M} \mathrm{AgNO}_{3}$. The resulting silver sulfide was vacuumfiltered onto a pre-weighed $0.2 \mu \mathrm{m}$ filter attached to a $15 \mathrm{~mL}$ chimney, washed with at least three volume-equivalents of DI water, one volume-equivalent of $1 \mathrm{M}$ ammonium hydroxide, and a final volume-equivalent of water. The filters were then air-dried overnight and re-weighed to obtain the mass of $\operatorname{Ag}_{2} \mathrm{~S}$. Weight percent iron in pyrite (FeP) was calculated stoichiometrically, assuming that all CRS-extractable sulfur was in the form of pyrite $\left(\mathrm{FeS}_{2}\right)$. Some samples may contain Acid Volatile Sulfide (AVS) including iron monosulfides such as pyrrhotite, which if present would result in an erroneous stoichiometric calculation. AVS is present in some studies in the overall database ${ }^{34-36}$, but 
is unlikely to form a significant component of the extractable iron pool in the new samples reported here. First, AVS is relatively rare in unmetamorphosed sedimentary rocks of any age. Second, and more important, the Neoproterozoic-Cambrian rocks that were the focus of new sampling are particularly pyrite-lean (Fig. 1B and 1C and Database S1), and the CRS extraction reduces sulfur in both pyrite and AVS. The iron in pyrite (FeP) estimated from the CRS extraction in the new samples average 0.034 weight percent; even under the extremely unrealistic assumption that this is entirely AVS with no pyrite present, such amounts would represent a relatively small percentage of the total highly reactive iron pool. CRS analyses in this study were conducted over a number of years, and not enough rock powder of internal replicates was available for the same replicate to be continually analyzed alongside all samples. Samples analyzed from 20102012 were run alongside replicate F845-184, which had a long-term standard deviation of $6.2 \%$ and standard error of 1.7\% (ref. 21) whereas samples analyzed from 2012-2014 were analyzed alongside replicate B203-4450, which had a long-term standard deviation of $7.8 \%$ and standard error of $2.8 \%$ (ref. 14 ).

\section{References}

34. Poulton, S. W., Fralick, P. W. \& Canfield, D. E. Spatial variability in oceanic redox structure 1.8 billion years ago. Nat. Geosci. 3, 486-490 (2010).

35. Reuschel, M., Melezhik, V. A. \& Strauss, H. Sulfur isotopic trends and iron speciation from the c. 2.0 Ga Pilgujarvi Sedimentary Formation, NW Russia. Precambrian Research 196, 193-203 (2012).

36. Asael, D. et al. Coupled molybdenum, iron and uranium stable isotopes as oceanic paleoredox proxies during the Paleoproterozoic Shunga Event. Chemical Geology 362, 193-210 (2013).

37. Scott, C. et al. Pyrite multiple-sulfur isotope evidence for rapid expansion and contraction of the early Paleoproterozoic seawater sulfate reservoir. Earth and Planetary Science Letters 389, 95-104 (2014).

38. Canfield, D. E. et al. Oxygen dynamics in the aftermath of the Great Oxidation of Earth's atmosphere. Proceedings of the National Academy of Sciences 110, 16736-16741 (2013).

39. El Albani, A. et al. Large colonial organisms with coordinated growth in oxygenated environments 2.1 Gyr ago. Nature 466, 100-104 (2010).

40. Shen, Y., Canfield, D. E. \& Knoll, A. H. Middle Proterozoic ocean chemistry: evidence from the McArthur Basin, northern Australia. American Journal of Science 302, 81-109 (2002).

41. Gilleaudeau, G. J. \& Kah, L. C. Oceanic molybdenum drawdown by epeiric sea expansion in the Mesoproterozoic. Chemical Geology 356, 21-37 (2013).

42. Geboy, N. J. et al. Re-Os age constraints and new observations of glacial deposits in the Mesoproterozoic Vazante Group, Brazil. Precambrian Research 238, 199-213 (2013).

43. Cumming, V. M., Poulton, S. W., Rooney, A. D. \& Selby, D. Anoxia in the terrestrial environment during the late Mesoproterozoic. Geology 41, 583-586 (2013).

44. Johnston, D. et al. Late Ediacaran redox stability and metazoan evolution. Earth and Planetary Science Letters 335, 25-35 (2012). 
45. Johnston, D. T. et al. An emerging picture of Neoproterozoic ocean chemistry: Insights from the Chuar Group, Grand Canyon, USA. Earth and Planetary Science Letters 290, 64-73 (2010).

46. Hayes, D. S. Two scenes from Utah's stratigraphic record: Neoproterozoic Snowball Earth, before and after. Ph.D. thesis. Utah State University (2013).

47. Feng, L.-J., Chu, X.-L., Huang, J., Zhang, Q.-R. \& Chang, H.-J. Reconstruction of paleo-redox conditions and early sulfur cycling during deposition of the Cryogenian Datangpo Formation in South China. Gondwana Research 18, 632-637 (2010). 48. Li, C. et al. Evidence for a redox stratified Cryogenian marine basin, Datangpo Formation, South China. Earth and Planetary Science Letters 331, 246-256 (2012).

49. Sahoo, S. K. et al. Ocean oxygenation in the wake of the Marinoan glaciation. Nature 489, 546-549 (2012).

50. Shen, Y., Zhang, T. \& Hoffman, P. F. On the coevolution of Ediacaran oceans and animals. Proceedings of the National Academy of Sciences, U.S.A. 105, 7376-7381 (2008).

51. Li, C. et al. A stratified redox model for the Ediacaran ocean. Science 328, 80-83 (2010).

52. Frei, R., Gaucher, C., Stolper, D. \& Canfield, D. E. Fluctuations in late Neoproterozoic atmospheric oxidation - Cr isotope chemostratigraphy and iron speciation of the late Ediacaran lower Arroyo del Soldado Group (Uruguay). Gondwana Research 23, 797-811 (2013).

53. Wang, J., Chen, D., Yan, D., Wei, H. \& Xiang, L. Evolution from an anoxic to oxic deep ocean during the Ediacaran-Cambrian transition and implications for bioradiation. Chemical Geology 306, 129-138 (2012).

54. Feng, L., Li, C., Huang, J., Chang, H. \& Chu, X. A sulfate control on marine middepth euxinia on the early Cambrian (ca. 529-521 Ma) Yangtze platform, South China Precambrian Research 246, 123-133 (2014).

55. Och, L. M. et al. Redox changes in Early Cambrian black shales at Xiaotan section, Yunnan Province, South China. Precambrian Research 225, 166-189 (2013).

56. Creveling, J. R., et al., Phosphorus sources for phosphatic Cambrian carbonates. Geological Society of America Bulletin 126, 145-163 (2014).

57. Gill, B. C. et al. Geochemical evidence for widespread euxinia in the later Cambrian ocean. Nature 469, 80-83 (2011).

58. Boyer, D. L., Owens, J. D., Lyons, T. W. \& Droser, M. L. Joining forces: Combined biological and geochemical proxies reveal a complex but refined highresolution palaeo-oxygen history in Devonian epeiric Seas. Palaeogeography, Palaeoclimatology, Palaeoecology 306, 134-146 (2011).

59. Farrell, U. C., Briggs, D. E. G., Hammarlund, E. U., Sperling, E. A. \& Gaines, R. R. Paleoredox and pyritization of soft-bodied fossils in the Ordovician Frankfort Shale of New York. American Journal of Science 313, 452-489 (2013).

60. Hammarlund, E. U. et al. A sulfidic driver for the end-Ordovician mass extinction. Earth and Planetary Science Letters 331, 128-139 (2012).

61. Clarkson M. O., Poulton S.W., Guilbaud R. \& Wood, R.A. Assessing the utility of $\mathrm{Fe} / \mathrm{Al}$ and $\mathrm{Fe}-$ speciation to record water column redox conditions in carbonate-rich sediments. Chemical Geology, 382,111-122. (2014) 
62. Poulton, S. W. \& Raiswell, R.. The low-temperature geochemical cycle of iron: from continental fluxes to marine sediment deposition. American Journal of Science, 302, 774-805 (2002).

63. Raiswell, R., et al. Turbidite depositional influences on the diagenesis of

Beecher's Trilobite Bed and the Hunsruck Slate; sites of soft tissue pyritization. American Journal of Science, 308,105-129 (2008).

64. Pyle, L. J., Narbonne, G. M., James, N. P., Dalrymple, R. W. \& Kaufman, A. J. Integrated Ediacaran chronostratigraphy, Wernecke Mountains, northwestern Canada. Precambrian Research 132, 1-27 (2004).

65. Pyle, L. J., Narbonne, G. M., Nowlan, G. S., Xiao, S. \& James, N. P. Early Cambrian metazoan eggs, embryos, and phosphatic microfossils from northwestern Canada. Journal of Paleontology 80, 811-825 (2006).

66. Macdonald, F. A., et al. The stratigraphic relationship between the Shuram carbon isotope excursion, the oxygenation of Neoproterozoic oceans, and the first appearance of the Ediacara biota and bilaterian trace fossils in northwestern Canada. Chemical Geology, 362, 250-272 (2013).

67. Eisbacher, G. H. Sedimentary tectonics and glacial record in the Windermere Supergroup, Mackenzie Mountains, northwestern Canada. Geological Survey of Canada Paper 80-27, 1-40 (1981).

68. Rooney, A. D., et al. Re-Os geochronology and coupled Os-Sr isotope constraints on the Sturtian snowball Earth. Proceedings of the National Academy of Sciences USA 111, 51-56 (2014).

69. Calver, C. R. et al. Globally synchronous Marinoan deglaciation indicated by U$\mathrm{Pb}$ geochronology of the Cottons Breccia, Tasmania, Australia. Geology 41, 1127-1130 (2013).

70. Condon, D. J. et al. U-Pb ages from the Neoproterozoic Doushantuo Formation, China. Science 308, 95-98 (2005).

71. Hoffmann, K.-H., Condon, D. J., Bowring, S. A. \& Crowley, J. L. U-Pb zircon date from the Neoproterozoic Ghaub Formation, Namibia: Constraints on Marinoan glaciation. Geology 32, 817-820 (2004).

72. Nowlan, G. S., Narbonne, G. M. \& Fritz, W. H. Small shelly fossils and trace fossils near the Precambrian-Cambrian boundary in the Yukon Territory, Canada. Lethaia 18, 233-256 (1985).

73. Fritz, W. H. Vampire Formation, a new upper Precambrian (?)/Lower Cambrian formation, Mackenzie Mountains, Yukon and Northwest Territories. Current Research, Part B; Geological Survey of Canada Paper 82-1B, 83-92 (1982).

74. Narbonne, G. M., Laflamme, M., Trusler, P. W., Dalrymple, R. W. \& Greentree, C. Deep-water Ediacaran fossils from northwestern Canada: Taphonomy, ecology, and evolution. Journal of Paleontology 88, 207-223 (2014).

75. Carbone, C. \& Narbonne, G. M. When life got smart: the evolution of behavioral complexity through the Ediacaran and early Cambrian of NW Canada. Journal of Paleontology 88, 309-330 (2014).

76. MacNaughton, R. B., Dalrymple, R. W. \& Narbonne, G. M. Multiple orders of relative sea-level change in an earliest Cambrian passive-margin succession, Mackenzie Mountains, Northwestern Canada. Journal of Sedimentary Research 67, 622-637 (1997). 
77. Kaufman, A. J., Knoll, A. H. \& Narbonne, G. M. Isotopes, ice ages, and terminal Proterozoic earth history. Proceedings of the National Academy of Sciences USA 94, 6600-6605 (1997).

78. Aitken, J. D. Uppermost Proterozoic formations in central Mackenzie Mountains, Northwest Territories. Geological Survey of Canada Bulletin 368, 1-26 (1989).

79. Hofmann, H. J., Narbonne, G. M. \& Aitken, J. D. Ediacaran remains from intertillite beds in northwestern Canada. Geology 18, 1199-1202 (1990).

80. Martel, E., Turner, E. C. \& Fischer, B. J. Geology of the central Mackenzie Mountains of the northern Canadian Cordillera, Sewki Mountain (105P), Mount Eduni (106A), and northwestern Wrigley Lake (95 M) map-areas, Northwest Territories. NWT Special Volume 1. NWT Geoscience Office, Yellowknife, CA.

81. Macdonald, F. A. et al. Early Neoproterozoic Basin Formation in Yukon, Canada: Implications for the make-up and break-up of Rodinia. Geoscience Canada 39, 77-99 (2012).

82. Strauss, J. V., Rooney, A. D., Macdonald, F. A., Brandon, A. D. \& Knoll, A. H. 740 Ma vase-shaped microfossils from Yukon, Canada: implications for Neoproterozoic chronology and biostratigraphy. Geology 42, 659-662 (2014).

83. Mustard, P. S., Donaldson, J. A. \& Thompson, R. I. Trace fossils and stratigraphy of the Precambrian-Cambrian boundary sequence, upper Harper group, Ogilvie Mountains, Yukon. Current Research, Part E: Geological Survey of Canada Paper 88-1E, 197-203 (1988).

84. Halverson, G. P., Maloof, A. C. \& Hoffman, P. F. The Marinoan glaciation (Neoproterozoic) in northeast Svalbard. Basin Research 16, 297-324 (2004).

85. Hoffman, P. F., et al. Cryogenian glaciation on the southern tropical paleomargin of Laurentia (NE Svalbard and East Greenland), and a primary origin for the upper Russøya (Islay) carbon isotope excursion. Precambrian Resarch 206, 137-158.

86. Rees, M. N. A fault-controlled trough through a carbonate platform: The Middle Cambrian House Range embayment. Geological Society of America Bulletin 97, 10541069 (1986).

87. Elrick, M. \& Snider, A. C. Deep-water stratigraphic cyclicity and carbonate mud mound development in the Middle Cambrian Marjum Formation, House Range, Utah, USA. Sedimentology 49, 1021-1047 (2002).

88. Langenburg, L. The Middle Cambrian Wheeler Formation: Sequence stratigraphy and geochemistry across a ramp-to-basin transition. M.S. Thesis. Utah State University (2003).

89. Gaines, R. R., Kennedy, M. J. \& Droser, M. L. A new hypothesis for organic preservation of Burgess Shale taxa in the Middle Cambrian Wheeler Formation, House Range, Utah. Palaeogeography, Palaeoclimatology, Palaeoecology 220, 193-205 (2005). 90. Brett, C. E., Allison, P. A., DeSantis, M. K., Liddell, W. D. \& Kramer, A. Sequence stratigraphy, cyclic facies, and lagerstätten in the Middle Cambrian Wheeler and Marjum Formations, Great Basin, Utah. Palaeogeography, Palaeoclimatology, Palaeoecology 277, 9-33 (2009).

91. Babcock, L. E., Robison, R. A., Rees, M. N., Peng, S. C., \& Saltzman, M.R. The global boundary stratotype section and point (GSSP) of the Drumian stage (Cambrian) in the Drum mountains, Utah, USA. Episodes 30, 85-95 (2007). 
92. Smith, E. F., Macdonald, F. A., Crowley, J. L., Hodgin, E. G. \& Schrag, D. P. Tectonostratigraphic evolution of the c. 780-730 Ma Beck Spring Dolomite: Basin formation in the core of Rodinia, In Li, Z. X., Evans, D. A. D. \& Murphy, J. B. (eds) Supercontinent cycles through Earth history. Geological Society, London, Special Publications 424 (in press).

93. Mahon, R. C., Dehler, C. M., Karlstrom, K. E., Link, P. K. \& Gehrels, G. E. Geochronologic and stratigraphic constraints on the Mesoproterozoic and Neoproterozoic Pahrump Group, Death Valley, California: A record of the assembly, stability, and breakup of Rodinia. Geological Society of America Bulletin 126, 652-664 (2014). 94. Macdonald, F. A. et al. The Laurentian record of Neoproterozoic glaciation, tectonism, and eukaryotic evolution in Death Valley, California. Geological Society of America Bulletin 125, 1203-1223 (2013).

95. Kozlov, V. I. et al. Riphean-Vendian deposits of the Kama-Belsky aulacogen penetrated by the well 1 East Askinsky. St. Petersburg: St. Petersburg Department of Printing "Nedra" [in Russian] (2007).

96. Saylor, B. Z., Grotzinger, J. P. \& Germs, G. J. B. Sequence stratigraphy and sedimentology of the Neoproterozoic Kuibis and Schwarzrand Subgroups (Nama Group), southwestern Namibia. Precambrian Research 73, 153-171 (1995).

97. Grotzinger, J. P., Bowring, S. A., Saylor, B. Z. \& Kaufman, A. J. Biostratigraphic and geochronologic constraints on early animal evolution. Science 270, 598-604 (1995).

98. Narbonne, G. M., Saylor, B. Z. \& Grotzinger, J. P. The youngest Ediacaran fossils from southern Africa. Journal of Paleontology 71, 953-967 (1997).

99. Wilson, J. P. et al. Deep-water incised valley deposits at the Ediacaran-Cambrian boundary in Southern Namibia contain abundant Treptichnus pedum. PALAIOS 27, 252273 (2012).

100. Turekian, K. K. \& Wedepohl, K. H. Distribution of the elements in some major units of the earth's crust. Geological Society of America Bulletin 72, 175-192 (1961). 101. Poulton, S. W. \& Canfield, D. E. Development of a sequential extraction procedure for iron: implications for iron partitioning in continentally derived particulates. Chemical Geology 214, 209-221 (2005).

102. Canfield, D. E., Raiswell, R., Westrich, J. T., Reaves, C. M. \& Berner, R. A. The use of chromium reduction in the analysis of reduced inorganic sulfur in sediments and shale. Chemical Geology 54, 149-155 (1986). 


\begin{tabular}{|c|c|c|c|c|}
\hline Age bin (Ma) & Age bin & Age & Geographic Area/Basin & Basin Number \\
\hline $2300-1000$ & 1 & 2000 & Fennoscandia & 2 \\
\hline $2300-1000$ & 1 & 2000 & Fennoscandia & 2 \\
\hline $2300-1000$ & 1 & 2000 & Fennoscandia & 2 \\
\hline $2300-1000$ & 1 & 2000 & Fennoscandia & 2 \\
\hline $2300-1000$ & 1 & 2000 & Fennoscandia & 2 \\
\hline $2300-1000$ & 1 & 2000 & Fennoscandia & 2 \\
\hline $2300-1000$ & 1 & 2000 & Fennoscandia & 2 \\
\hline $2300-1000$ & 1 & 2000 & Fennoscandia & 2 \\
\hline $2300-1000$ & 1 & 2000 & Fennoscandia & 2 \\
\hline $2300-1000$ & 1 & 2000 & Fennoscandia & 2 \\
\hline $2300-1000$ & 1 & 2000 & Fennoscandia & 2 \\
\hline $2300-1000$ & 1 & 2000 & Fennoscandia & 2 \\
\hline $2300-1000$ & 1 & 2000 & Fennoscandia & 2 \\
\hline $2300-1000$ & 1 & 2000 & Fennoscandia & 2 \\
\hline $2300-1000$ & 1 & 2000 & Fennoscandia & 2 \\
\hline $2300-1000$ & 1 & 2000 & Fennoscandia & 2 \\
\hline 2300-1000 & 1 & 2000 & Fennoscandia & 2 \\
\hline $2300-1000$ & 1 & 2000 & Fennoscandia & 2 \\
\hline $2300-1000$ & 1 & 2000 & Fennoscandia & 2 \\
\hline $2300-1000$ & 1 & 2000 & Fennoscandia & 2 \\
\hline $2300-1000$ & 1 & 2000 & Fennoscandia & 2 \\
\hline $2300-1000$ & 1 & 2000 & Fennoscandia & 2 \\
\hline $2300-1000$ & 1 & 2000 & Fennoscandia & 2 \\
\hline $2300-1000$ & 1 & 2000 & Fennoscandia & 2 \\
\hline $2300-1000$ & 1 & 2000 & Fennoscandia & 2 \\
\hline $2300-1000$ & 1 & 2000 & Fennoscandia & 2 \\
\hline $2300-1000$ & 1 & 2000 & Fennoscandia & 2 \\
\hline $2300-1000$ & 1 & 2000 & Fennoscandia & 2 \\
\hline $2300-1000$ & 1 & 2000 & Fennoscandia & 2 \\
\hline $2300-1000$ & 1 & 2000 & Fennoscandia & 2 \\
\hline $2300-1000$ & 1 & 2000 & Fennoscandia & 2 \\
\hline $2300-1000$ & 1 & 2000 & Fennoscandia & 2 \\
\hline $2300-1000$ & 1 & 1680 & North China & 6 \\
\hline $2300-1000$ & 1 & 1680 & North China & 6 \\
\hline $2300-1000$ & 1 & 1680 & North China & 6 \\
\hline 2300-1000 & 1 & 1680 & North China & 6 \\
\hline $2300-1000$ & 1 & 1680 & North China & 6 \\
\hline 2300-1000 & 1 & 1680 & North China & 6 \\
\hline 2300-1000 & 1 & 1680 & North China & 6 \\
\hline $2300-1000$ & 1 & 1680 & North China & 6 \\
\hline
\end{tabular}




\begin{tabular}{|c|c|c|c|c|}
\hline $2300-1000$ & 1 & 1680 & North China & 6 \\
\hline $2300-1000$ & 1 & 1680 & North China & 6 \\
\hline $2300-1000$ & 1 & 1680 & North China & 6 \\
\hline $2300-1000$ & 1 & 1680 & North China & 6 \\
\hline $2300-1000$ & 1 & 1680 & North China & 6 \\
\hline $2300-1000$ & 1 & 1680 & North China & 6 \\
\hline $2300-1000$ & 1 & 1680 & North China & 6 \\
\hline $2300-1000$ & 1 & 1680 & North China & 6 \\
\hline $2300-1000$ & 1 & 1680 & North China & 6 \\
\hline $2300-1000$ & 1 & 1680 & North China & 6 \\
\hline $2300-1000$ & 1 & 1680 & North China & 6 \\
\hline $2300-1000$ & 1 & 1680 & North China & 6 \\
\hline $2300-1000$ & 1 & 1680 & North China & 6 \\
\hline $2300-1000$ & 1 & 1680 & North China & 6 \\
\hline $2300-1000$ & 1 & 1680 & North China & 6 \\
\hline $2300-1000$ & 1 & 1680 & North China & 6 \\
\hline $2300-1000$ & 1 & 1680 & North China & 6 \\
\hline $2300-1000$ & 1 & 1680 & North China & 6 \\
\hline $2300-1000$ & 1 & 1680 & North China & 6 \\
\hline $2300-1000$ & 1 & 1680 & North China & 6 \\
\hline $2300-1000$ & 1 & 1680 & North China & 6 \\
\hline 2300-1000 & 1 & 1680 & North China & 6 \\
\hline $2300-1000$ & 1 & 1680 & North China & 6 \\
\hline $2300-1000$ & 1 & 1460 & Belt basin & 7 \\
\hline $2300-1000$ & 1 & 1460 & Belt basin & 7 \\
\hline $2300-1000$ & 1 & 1460 & Belt basin & 7 \\
\hline $2300-1000$ & 1 & 1460 & Belt basin & 7 \\
\hline $2300-1000$ & 1 & 1460 & Belt basin & 7 \\
\hline $2300-1000$ & 1 & 1460 & Belt basin & 7 \\
\hline $2300-1000$ & 1 & 1460 & Belt basin & 7 \\
\hline $2300-1000$ & 1 & 1460 & Belt basin & 7 \\
\hline $2300-1000$ & 1 & 1460 & Belt basin & 7 \\
\hline $2300-1000$ & 1 & 1460 & Belt basin & 7 \\
\hline 2300-1000 & 1 & 1460 & Belt basin & 7 \\
\hline $2300-1000$ & 1 & 1460 & Belt basin & 7 \\
\hline $2300-1000$ & 1 & 1460 & Belt basin & 7 \\
\hline $2300-1000$ & 1 & 1460 & Belt basin & 7 \\
\hline $2000-1200$ & 1 & 1200 & Borden Basin & 8 \\
\hline $2000-1200$ & 1 & 1200 & Borden Basin & 8 \\
\hline $2000-1200$ & 1 & 1200 & Borden Basin & 8 \\
\hline $2000-1200$ & 1 & 1200 & Borden Basin & 8 \\
\hline
\end{tabular}




\begin{tabular}{|c|c|c|c|c|}
\hline $2000-1200$ & 1 & 1200 & Borden Basin & 8 \\
\hline $2000-1200$ & 1 & 1200 & Borden Basin & 8 \\
\hline $2300-1000$ & 1 & 1100 & Mauritania & 25 \\
\hline $2300-1000$ & 1 & 1100 & Mauritania & 25 \\
\hline $2300-1000$ & 1 & 1100 & Mauritania & 25 \\
\hline $2300-1000$ & 1 & 1100 & Mauritania & 25 \\
\hline $2300-1000$ & 1 & 1100 & Mauritania & 25 \\
\hline $2300-1000$ & 1 & 1100 & Mauritania & 25 \\
\hline $2300-1000$ & 1 & 1100 & Mauritania & 25 \\
\hline $2300-1000$ & 1 & 1100 & Mauritania & 25 \\
\hline $2300-1000$ & 1 & 1100 & Mauritania & 25 \\
\hline $2300-1000$ & 1 & 1100 & Mauritania & 25 \\
\hline $2300-1000$ & 1 & 1100 & Mauritania & 25 \\
\hline $2300-1000$ & 1 & 1100 & Mauritania & 25 \\
\hline $2300-1000$ & 1 & 1100 & Mauritania & 25 \\
\hline $2300-1000$ & 1 & 1100 & Mauritania & 25 \\
\hline $2300-1000$ & 1 & 1100 & Mauritania & 25 \\
\hline $2300-1000$ & 1 & 1100 & Mauritania & 25 \\
\hline $2300-1000$ & 1 & 1100 & Mauritania & 25 \\
\hline $2300-1000$ & 1 & 1100 & Mauritania & 25 \\
\hline $2300-1000$ & 1 & 1100 & Mauritania & 25 \\
\hline $2300-1000$ & 1 & 1100 & Mauritania & 25 \\
\hline $2300-1000$ & 1 & 1100 & Mauritania & 25 \\
\hline $2300-1000$ & 1 & 1100 & Mauritania & 25 \\
\hline $2300-1000$ & 1 & 1100 & Mauritania & 25 \\
\hline $2300-1000$ & 1 & 1100 & Mauritania & 25 \\
\hline $2300-1000$ & 1 & 1100 & Mauritania & 25 \\
\hline $2300-1000$ & 1 & 1100 & Mauritania & 25 \\
\hline $2300-1000$ & 1 & 1100 & Mauritania & 25 \\
\hline $2300-1000$ & 1 & 1100 & Mauritania & 25 \\
\hline $2300-1000$ & 1 & 1100 & Mauritania & 25 \\
\hline $2300-1000$ & 1 & 1100 & Mauritania & 25 \\
\hline $2300-1000$ & 1 & 1100 & Mauritania & 25 \\
\hline $2300-1000$ & 1 & 1100 & Mauritania & 25 \\
\hline $2300-1000$ & 1 & 1100 & Mauritania & 25 \\
\hline $2300-1000$ & 1 & 1100 & Mauritania & 25 \\
\hline $2300-1000$ & 1 & 1100 & Mauritania & 25 \\
\hline $2300-1000$ & 1 & 1100 & Mauritania & 25 \\
\hline $2300-1000$ & 1 & 1100 & Mauritania & 25 \\
\hline $2300-1000$ & 1 & 1100 & Mauritania & 25 \\
\hline $2300-1000$ & 1 & 1100 & Mauritania & 25 \\
\hline
\end{tabular}




\begin{tabular}{|c|c|c|c|c|}
\hline $2300-1000$ & 1 & 1100 & Mauritania & 25 \\
\hline $2300-1000$ & 1 & 1100 & Mauritania & 25 \\
\hline $2300-1000$ & 1 & 1100 & Mauritania & 25 \\
\hline $2300-1000$ & 1 & 1100 & Mauritania & 25 \\
\hline $2300-1000$ & 1 & 1100 & Mauritania & 25 \\
\hline $2300-1000$ & 1 & 1100 & Mauritania & 25 \\
\hline $2300-1000$ & 1 & 1100 & Mauritania & 25 \\
\hline $2300-1000$ & 1 & 1100 & Mauritania & 25 \\
\hline $2300-1000$ & 1 & 1100 & Mauritania & 25 \\
\hline $2300-1000$ & 1 & 1100 & Mauritania & 25 \\
\hline $2300-1000$ & 1 & 1100 & Mauritania & 25 \\
\hline $2300-1000$ & 1 & 1100 & Mauritania & 25 \\
\hline $2300-1000$ & 1 & 1100 & Mauritania & 25 \\
\hline 2300-1000 & 1 & 1100 & Mauritania & 25 \\
\hline $2300-1000$ & 1 & 1100 & Mauritania & 25 \\
\hline 2300-1000 & 1 & 1100 & Mauritania & 25 \\
\hline $2300-1000$ & 1 & 1100 & Mauritania & 25 \\
\hline $2300-1000$ & 1 & 1100 & Mauritania & 25 \\
\hline $2300-1000$ & 1 & 1100 & Mauritania & 25 \\
\hline $2300-1000$ & 1 & 1100 & Mauritania & 25 \\
\hline 2300-1000 & 1 & 1100 & Mauritania & 25 \\
\hline $2300-1000$ & 1 & 1100 & Mauritania & 25 \\
\hline $2300-1000$ & 1 & 1100 & Mauritania & 25 \\
\hline 2300-1000 & 1 & 1100 & Mauritania & 25 \\
\hline $2300-1000$ & 1 & 1100 & Mauritania & 25 \\
\hline 2300-1000 & 1 & 1100 & Mauritania & 25 \\
\hline $2300-1000$ & 1 & 1100 & Mauritania & 25 \\
\hline $2300-1000$ & 1 & 1100 & Mauritania & 25 \\
\hline $2300-1000$ & 1 & 1100 & Mauritania & 25 \\
\hline 2300-1000 & 1 & 1100 & Mauritania & 25 \\
\hline 2300-1000 & 1 & 1100 & Mauritania & 25 \\
\hline 2300-1000 & 1 & 1100 & Mauritania & 25 \\
\hline 2300-1000 & 1 & 1100 & Mauritania & 25 \\
\hline 2300-1000 & 1 & 1100 & Mauritania & 25 \\
\hline 2300-1000 & 1 & 1100 & Mauritania & 25 \\
\hline $2300-1000$ & 1 & 1100 & Mauritania & 25 \\
\hline $2300-1000$ & 1 & 1100 & Mauritania & 25 \\
\hline $2300-1000$ & 1 & 1100 & Mauritania & 25 \\
\hline 2300-1000 & 1 & 1100 & Mauritania & 25 \\
\hline 2300-1000 & 1 & 1100 & Mauritania & 25 \\
\hline $2300-1000$ & 1 & 1100 & Mauritania & 25 \\
\hline
\end{tabular}




\begin{tabular}{|c|c|c|c|c|}
\hline $2300-1000$ & 1 & 1100 & Mauritania & 25 \\
\hline $2300-1000$ & 1 & 1100 & Mauritania & 25 \\
\hline $2300-1000$ & 1 & 1100 & Mauritania & 25 \\
\hline $2300-1000$ & 1 & 1100 & Mauritania & 25 \\
\hline $2300-1000$ & 1 & 1100 & Mauritania & 25 \\
\hline $2300-1000$ & 1 & 1100 & Mauritania & 25 \\
\hline $2300-1000$ & 1 & 1100 & Mauritania & 25 \\
\hline $2300-1000$ & 1 & 1100 & Mauritania & 25 \\
\hline $2300-1000$ & 1 & 1100 & Mauritania & 25 \\
\hline $2300-1000$ & 1 & 1100 & Mauritania & 25 \\
\hline $2300-1000$ & 1 & 1100 & Mauritania & 25 \\
\hline $2300-1000$ & 1 & 1100 & Mauritania & 25 \\
\hline $2300-1000$ & 1 & 1100 & Mauritania & 25 \\
\hline 2300-1000 & 1 & 1100 & Mauritania & 25 \\
\hline $2300-1000$ & 1 & 1100 & Mauritania & 25 \\
\hline 2300-1000 & 1 & 1100 & Mauritania & 25 \\
\hline $2300-1000$ & 1 & 1100 & Mauritania & 25 \\
\hline $2300-1000$ & 1 & 1100 & Mauritania & 25 \\
\hline $2300-1000$ & 1 & 1100 & Mauritania & 25 \\
\hline $2300-1000$ & 1 & 1100 & Mauritania & 25 \\
\hline 2300-1000 & 1 & 1100 & Mauritania & 25 \\
\hline $2300-1000$ & 1 & 1100 & Mauritania & 25 \\
\hline $2300-1000$ & 1 & 1100 & Mauritania & 25 \\
\hline 2300-1000 & 1 & 1100 & Mauritania & 25 \\
\hline $2300-1000$ & 1 & 1100 & Mauritania & 25 \\
\hline 2300-1000 & 1 & 1100 & Mauritania & 25 \\
\hline $2300-1000$ & 1 & 1100 & Mauritania & 25 \\
\hline $2300-1000$ & 1 & 1100 & Mauritania & 25 \\
\hline $2300-1000$ & 1 & 1100 & Mauritania & 25 \\
\hline 2300-1000 & 1 & 1100 & Mauritania & 25 \\
\hline 2300-1000 & 1 & 1100 & Mauritania & 25 \\
\hline 2300-1000 & 1 & 1100 & Mauritania & 25 \\
\hline 2300-1000 & 1 & 1100 & Mauritania & 25 \\
\hline 2300-1000 & 1 & 1100 & Mauritania & 25 \\
\hline 2300-1000 & 1 & 1100 & Mauritania & 25 \\
\hline $2300-1000$ & 1 & 1100 & Mauritania & 25 \\
\hline $2300-1000$ & 1 & 1100 & Mauritania & 25 \\
\hline $2300-1000$ & 1 & 1100 & Mauritania & 25 \\
\hline 2300-1000 & 1 & 1100 & Mauritania & 25 \\
\hline 2300-1000 & 1 & 1100 & Mauritania & 25 \\
\hline $2300-1000$ & 1 & 1100 & Mauritania & 25 \\
\hline
\end{tabular}




\begin{tabular}{|c|c|c|c|c|}
\hline $2300-1000$ & 1 & 1100 & Mauritania & 25 \\
\hline $2300-1000$ & 1 & 1100 & Mauritania & 25 \\
\hline $2300-1000$ & 1 & 1100 & Mauritania & 25 \\
\hline $2300-1000$ & 1 & 1100 & Mauritania & 25 \\
\hline $2300-1000$ & 1 & 1100 & Mauritania & 25 \\
\hline $2300-1000$ & 1 & 1100 & Mauritania & 25 \\
\hline $2300-1000$ & 1 & 2100 & Francevillian Basin & 33 \\
\hline $2300-1000$ & 1 & 2100 & Francevillian Basin & 33 \\
\hline $2300-1000$ & 1 & 2100 & Francevillian Basin & 33 \\
\hline $2300-1000$ & 1 & 2100 & Francevillian Basin & 33 \\
\hline $2300-1000$ & 1 & 2100 & Francevillian Basin & 33 \\
\hline $2300-1000$ & 1 & 2100 & Francevillian Basin & 33 \\
\hline $2300-1000$ & 1 & 2100 & Francevillian Basin & 33 \\
\hline $2300-1000$ & 1 & 2100 & Francevillian Basin & 33 \\
\hline $2300-1000$ & 1 & 2100 & Francevillian Basin & 33 \\
\hline $2300-1000$ & 1 & 2100 & Francevillian Basin & 33 \\
\hline $2300-1000$ & 1 & 2100 & Francevillian Basin & 33 \\
\hline $2300-1000$ & 1 & 2100 & Francevillian Basin & 33 \\
\hline $2300-1000$ & 1 & 2100 & Francevillian Basin & 33 \\
\hline $2300-1000$ & 1 & 2100 & Francevillian Basin & 33 \\
\hline $2300-1000$ & 1 & 2100 & Francevillian Basin & 33 \\
\hline $2300-1000$ & 1 & 2100 & Francevillian Basin & 33 \\
\hline $2300-1000$ & 1 & 2100 & Francevillian Basin & 33 \\
\hline $2300-1000$ & 1 & 2100 & Francevillian Basin & 33 \\
\hline $2300-1000$ & 1 & 2100 & Francevillian Basin & 33 \\
\hline $2300-1000$ & 1 & 2100 & Francevillian Basin & 33 \\
\hline $2300-1000$ & 1 & 2100 & Francevillian Basin & 33 \\
\hline $2300-1000$ & 1 & 2100 & Francevillian Basin & 33 \\
\hline $2300-1000$ & 1 & 2100 & Francevillian Basin & 33 \\
\hline $2300-1000$ & 1 & 2100 & Francevillian Basin & 33 \\
\hline $2300-1000$ & 1 & 2100 & Francevillian Basin & 33 \\
\hline $2300-1000$ & 1 & 2100 & Francevillian Basin & 33 \\
\hline $2300-1000$ & 1 & 2100 & Francevillian Basin & 33 \\
\hline $2300-1000$ & 1 & 2100 & Francevillian Basin & 33 \\
\hline $2300-1000$ & 1 & 2100 & Francevillian Basin & 33 \\
\hline $2300-1000$ & 1 & 2100 & Francevillian Basin & 33 \\
\hline $2300-1000$ & 1 & 2100 & Francevillian Basin & 33 \\
\hline $2300-1000$ & 1 & 2100 & Francevillian Basin & 33 \\
\hline $2300-1000$ & 1 & 2100 & Francevillian Basin & 33 \\
\hline $2300-1000$ & 1 & 2100 & Francevillian Basin & 33 \\
\hline $2300-1000$ & 1 & 2100 & Francevillian Basin & 33 \\
\hline
\end{tabular}




\begin{tabular}{|c|c|c|c|c|}
\hline $2300-1000$ & 1 & 2100 & Francevillian Basin & 33 \\
\hline $2300-1000$ & 1 & 2100 & Francevillian Basin & 33 \\
\hline $2300-1000$ & 1 & 2100 & Francevillian Basin & 33 \\
\hline $2300-1000$ & 1 & 2100 & Francevillian Basin & 33 \\
\hline $2300-1000$ & 1 & 2100 & Francevillian Basin & 33 \\
\hline $2300-1000$ & 1 & 2100 & Francevillian Basin & 33 \\
\hline $2300-1000$ & 1 & 2100 & Francevillian Basin & 33 \\
\hline $2300-1000$ & 1 & 2100 & Francevillian Basin & 33 \\
\hline $2300-1000$ & 1 & 2100 & Francevillian Basin & 33 \\
\hline $2300-1000$ & 1 & 2100 & Francevillian Basin & 33 \\
\hline $2300-1000$ & 1 & 2100 & Francevillian Basin & 33 \\
\hline $2300-1000$ & 1 & 2100 & Francevillian Basin & 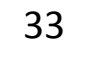 \\
\hline $2300-1000$ & 1 & 2100 & Francevillian Basin & 33 \\
\hline $2300-1000$ & 1 & 2100 & Francevillian Basin & 33 \\
\hline $2300-1000$ & 1 & 2100 & Francevillian Basin & 33 \\
\hline $2300-1000$ & 1 & 2100 & Francevillian Basin & 33 \\
\hline $2300-1000$ & 1 & 2100 & Francevillian Basin & 33 \\
\hline $2300-1000$ & 1 & 2100 & Francevillian Basin & 33 \\
\hline $2300-1000$ & 1 & 2100 & Francevillian Basin & 33 \\
\hline $2300-1000$ & 1 & 2100 & Francevillian Basin & 33 \\
\hline $2300-1000$ & 1 & 2100 & Francevillian Basin & 3 \\
\hline $2300-1000$ & 1 & 2100 & Francevillian Basin & 33 \\
\hline $2300-1000$ & 1 & 2100 & Francevillian Basin & 33 \\
\hline $2300-1000$ & 1 & 2100 & Francevillian Basin & 33 \\
\hline $2300-1000$ & 1 & 2100 & Francevillian Basin & 33 \\
\hline $2300-1000$ & 1 & 2100 & Francevillian Basin & 33 \\
\hline $2300-1000$ & 1 & 2100 & Francevillian Basin & 33 \\
\hline $2300-1000$ & 1 & 2100 & Francevillian Basin & 33 \\
\hline $2300-1000$ & 1 & 2100 & Francevillian Basin & 33 \\
\hline $2300-1000$ & 1 & 2100 & Francevillian Basin & 33 \\
\hline $2300-1000$ & 1 & 2100 & Francevillian Basin & 33 \\
\hline $2300-1000$ & 1 & 2100 & Francevillian Basin & 33 \\
\hline $2300-1000$ & 1 & 2100 & Francevillian Basin & 33 \\
\hline $2300-1000$ & 1 & 2100 & Francevillian Basin & 33 \\
\hline $2300-1000$ & 1 & 2100 & Francevillian Basin & 33 \\
\hline $2300-1000$ & 1 & 2100 & Francevillian Basin & 33 \\
\hline $2300-1000$ & 1 & 2100 & Francevillian Basin & 33 \\
\hline $2300-1000$ & 1 & 2100 & Francevillian Basin & 33 \\
\hline $2300-1000$ & 1 & 2100 & Francevillian Basin & 33 \\
\hline $2300-1000$ & 1 & 2100 & Francevillian Basin & 33 \\
\hline $2300-1000$ & 1 & 2100 & Francevillian Basin & 33 \\
\hline
\end{tabular}




\begin{tabular}{|c|c|c|c|c|}
\hline $2300-1000$ & 1 & 2100 & Francevillian Basin & 33 \\
\hline $2300-1000$ & 1 & 2100 & Francevillian Basin & 33 \\
\hline $2300-1000$ & 1 & 2100 & Francevillian Basin & 33 \\
\hline $2300-1000$ & 1 & 2100 & Francevillian Basin & 33 \\
\hline $2300-1000$ & 1 & 2100 & Francevillian Basin & 33 \\
\hline $2300-1000$ & 1 & 2100 & Francevillian Basin & 33 \\
\hline $2300-1000$ & 1 & 2100 & Francevillian Basin & 33 \\
\hline $2300-1000$ & 1 & 2100 & Francevillian Basin & 33 \\
\hline $2300-1000$ & 1 & 2100 & Francevillian Basin & 33 \\
\hline $2300-1000$ & 1 & 2100 & Francevillian Basin & 33 \\
\hline $2300-1000$ & 1 & 2100 & Francevillian Basin & 33 \\
\hline $2300-1000$ & 1 & 2100 & Francevillian Basin & 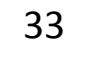 \\
\hline $2300-1000$ & 1 & 2100 & Francevillian Basin & 33 \\
\hline $2300-1000$ & 1 & 2100 & Francevillian Basin & 33 \\
\hline $2300-1000$ & 1 & 2100 & Francevillian Basin & 33 \\
\hline $2300-1000$ & 1 & 2100 & Francevillian Basin & 33 \\
\hline $2300-1000$ & 1 & 2100 & Francevillian Basin & 33 \\
\hline $2300-1000$ & 1 & 2100 & Francevillian Basin & 33 \\
\hline $2300-1000$ & 1 & 2100 & Francevillian Basin & 33 \\
\hline $2300-1000$ & 1 & 2100 & Francevillian Basin & 33 \\
\hline $2300-1000$ & 1 & 2100 & Francevillian Basin & 3 \\
\hline $2300-1000$ & 1 & 2100 & Francevillian Basin & 33 \\
\hline $2300-1000$ & 1 & 2100 & Francevillian Basin & 33 \\
\hline $2300-1000$ & 1 & 2100 & Francevillian Basin & 33 \\
\hline $2300-1000$ & 1 & 2100 & Francevillian Basin & 33 \\
\hline $2300-1000$ & 1 & 2100 & Francevillian Basin & 33 \\
\hline $2300-1000$ & 1 & 2100 & Francevillian Basin & 33 \\
\hline $2300-1000$ & 1 & 2100 & Francevillian Basin & 33 \\
\hline $2300-1000$ & 1 & 2100 & Francevillian Basin & 33 \\
\hline $2300-1000$ & 1 & 2100 & Francevillian Basin & 33 \\
\hline $2300-1000$ & 1 & 2100 & Francevillian Basin & 33 \\
\hline $2300-1000$ & 1 & 2100 & Francevillian Basin & 33 \\
\hline $2300-1000$ & 1 & 2100 & Francevillian Basin & 33 \\
\hline $2300-1000$ & 1 & 2100 & Francevillian Basin & 33 \\
\hline $2300-1000$ & 1 & 2100 & Francevillian Basin & 33 \\
\hline $2300-1000$ & 1 & 2100 & Francevillian Basin & 33 \\
\hline $2300-1000$ & 1 & 2100 & Francevillian Basin & 33 \\
\hline $2300-1000$ & 1 & 2100 & Francevillian Basin & 33 \\
\hline $2300-1000$ & 1 & 2100 & Francevillian Basin & 33 \\
\hline $2300-1000$ & 1 & 2100 & Francevillian Basin & 33 \\
\hline $2300-1000$ & 1 & 2100 & Francevillian Basin & 33 \\
\hline
\end{tabular}




\begin{tabular}{|c|c|c|c|c|}
\hline $2300-1000$ & 1 & 2100 & Francevillian Basin & 33 \\
\hline $2300-1000$ & 1 & 2100 & Francevillian Basin & 33 \\
\hline $2300-1000$ & 1 & 2100 & Francevillian Basin & 33 \\
\hline $2300-1000$ & 1 & 2100 & Francevillian Basin & 33 \\
\hline $2300-1000$ & 1 & 2100 & Francevillian Basin & 33 \\
\hline $2300-1000$ & 1 & 2100 & Francevillian Basin & 33 \\
\hline $2300-1000$ & 1 & 2100 & Francevillian Basin & 33 \\
\hline $2300-1000$ & 1 & 2100 & Francevillian Basin & 33 \\
\hline $2300-1000$ & 1 & 2100 & Francevillian Basin & 33 \\
\hline $2300-1000$ & 1 & 1440 & Southern Urals & 34 \\
\hline $2300-1000$ & 1 & 1440 & Southern Urals & 34 \\
\hline $2300-1000$ & 1 & 1440 & Southern Urals & 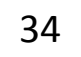 \\
\hline $2300-1000$ & 1 & 1440 & Southern Urals & 34 \\
\hline $2300-1000$ & 1 & 1440 & Southern Urals & 34 \\
\hline $2300-1000$ & 1 & 1440 & Southern Urals & 34 \\
\hline $2300-1000$ & 1 & 1440 & Southern Urals & 34 \\
\hline $2300-1000$ & 1 & 1440 & Southern Urals & 34 \\
\hline $2300-1000$ & 1 & 1440 & Southern Urals & 34 \\
\hline $2300-1000$ & 1 & 1440 & Southern Urals & 34 \\
\hline $2300-1000$ & 1 & 1440 & Southern Urals & 34 \\
\hline $2300-1000$ & 1 & 1440 & Southern Urals & דנו \\
\hline $2300-1000$ & 1 & 1440 & Southern Urals & 34 \\
\hline $2300-1000$ & 1 & 1440 & Southern Urals & 34 \\
\hline $2300-1000$ & 1 & 1440 & Southern Urals & 34 \\
\hline $2300-1000$ & 1 & 1440 & Southern Urals & 34 \\
\hline $2300-1000$ & 1 & 1440 & Southern Urals & 34 \\
\hline $2300-1000$ & 1 & 1440 & Southern Urals & 34 \\
\hline $2300-1000$ & 1 & 1440 & Southern Urals & 34 \\
\hline $2300-1000$ & 1 & 1440 & Southern Urals & 34 \\
\hline $2300-1000$ & 1 & 1440 & Southern Urals & 34 \\
\hline $2300-1000$ & 1 & 1440 & Southern Urals & 34 \\
\hline $2300-1000$ & 1 & 1440 & Southern Urals & 34 \\
\hline $2300-1000$ & 1 & 1440 & Southern Urals & 34 \\
\hline $2300-1000$ & 1 & 1440 & Southern Urals & 34 \\
\hline $2300-1000$ & 1 & 1440 & Southern Urals & 34 \\
\hline $2300-1000$ & 1 & 1440 & Southern Urals & 34 \\
\hline $2300-1000$ & 1 & 1440 & Southern Urals & 34 \\
\hline $2300-1000$ & 1 & 1440 & Southern Urals & 34 \\
\hline $2300-1000$ & 1 & 1440 & Southern Urals & 34 \\
\hline $2300-1000$ & 1 & 1440 & Southern Urals & 34 \\
\hline $2300-1000$ & 1 & 1440 & Southern Urals & $3<$ \\
\hline
\end{tabular}




\begin{tabular}{|c|c|c|c|c|}
\hline $2300-1000$ & 1 & 1440 & Southern Urals & 34 \\
\hline $2300-1000$ & 1 & 1440 & Southern Urals & 34 \\
\hline $2300-1000$ & 1 & 1440 & Southern Urals & 34 \\
\hline $2300-1000$ & 1 & 1440 & Southern Urals & 34 \\
\hline $2300-1000$ & 1 & 1440 & Southern Urals & 34 \\
\hline $2300-1000$ & 1 & 1440 & Southern Urals & 34 \\
\hline $2300-1000$ & 1 & 1440 & Southern Urals & 34 \\
\hline $2300-1000$ & 1 & 1440 & Southern Urals & 34 \\
\hline $2300-1000$ & 1 & 1440 & Southern Urals & 34 \\
\hline $2300-1000$ & 1 & 1440 & Southern Urals & 34 \\
\hline $2300-1000$ & 1 & 1440 & Southern Urals & \\
\hline $2300-1000$ & 1 & 1440 & Southern Urals & 34 \\
\hline $2300-1000$ & 1 & 1440 & Southern Urals & 34 \\
\hline $2300-1000$ & 1 & 1440 & Southern Urals & 34 \\
\hline $2300-1000$ & 1 & 1440 & Southern Urals & 34 \\
\hline $2300-1000$ & 1 & 1440 & Southern Urals & 34 \\
\hline $2300-1000$ & 1 & 1400 & Roper Basin & 35 \\
\hline $2300-1000$ & 1 & 1400 & Roper Basin & 35 \\
\hline $2300-1000$ & 1 & 1400 & Roper Basin & 35 \\
\hline $2300-1000$ & 1 & 1400 & Roper Basin & 35 \\
\hline 2300-1000 & 1 & 1400 & Roper Basin & 35 \\
\hline $2300-1000$ & 1 & 1400 & Roper Basin & 35 \\
\hline 2300-1000 & 1 & 1400 & Roper Basin & 35 \\
\hline $2300-1000$ & 1 & 1400 & Roper Basin & 35 \\
\hline $2300-1000$ & 1 & 1400 & Roper Basin & 35 \\
\hline $2300-1000$ & 1 & 1400 & Roper Basin & 35 \\
\hline $2300-1000$ & 1 & 1400 & Roper Basin & 35 \\
\hline $2300-1000$ & 1 & 1400 & Roper Basin & 35 \\
\hline $2300-1000$ & 1 & 1400 & Roper Basin & 35 \\
\hline 2300-1000 & 1 & 1400 & Roper Basin & 3 \\
\hline $2300-1000$ & 1 & 1400 & Roper Basin & 35 \\
\hline 2300-1000 & 1 & 1400 & Roper Basin & 35 \\
\hline $2300-1000$ & 1 & 1400 & Roper Basin & 35 \\
\hline 2300-1000 & 1 & 1400 & Roper Basin & 35 \\
\hline 2300-1000 & 1 & 1400 & Roper Basin & 35 \\
\hline $2300-1000$ & 1 & 1400 & Roper Basin & 35 \\
\hline $2300-1000$ & 1 & 1400 & Roper Basin & 35 \\
\hline $2300-1000$ & 1 & 1400 & Roper Basin & 35 \\
\hline 2300-1000 & 1 & 1400 & Roper Basin & 35 \\
\hline 2300-1000 & 1 & 1400 & Roper Basin & 35 \\
\hline $2300-1000$ & 1 & 1400 & Roper Basin & \\
\hline
\end{tabular}




\begin{tabular}{|c|c|c|c|c|}
\hline $2300-1000$ & 1 & 1400 & Roper Basin & 35 \\
\hline $2300-1000$ & 1 & 1400 & Roper Basin & 35 \\
\hline $2300-1000$ & 1 & 1400 & Roper Basin & 35 \\
\hline $2300-1000$ & 1 & 1400 & Roper Basin & 35 \\
\hline $2300-1000$ & 1 & 1400 & Roper Basin & 35 \\
\hline $2300-1000$ & 1 & 1400 & Roper Basin & 35 \\
\hline $2300-1000$ & 1 & 1400 & Roper Basin & 35 \\
\hline $2300-1000$ & 1 & 1400 & Roper Basin & 35 \\
\hline $2300-1000$ & 1 & 1400 & Roper Basin & 35 \\
\hline $2300-1000$ & 1 & 1400 & Roper Basin & 35 \\
\hline $2300-1000$ & 1 & 1400 & Roper Basin & 35 \\
\hline $2300-1000$ & 1 & 1400 & Roper Basin & 35 \\
\hline $2300-1000$ & 1 & 1400 & Roper Basin & 35 \\
\hline $2300-1000$ & 1 & 1400 & Roper Basin & 35 \\
\hline $2300-1000$ & 1 & 1400 & Roper Basin & 35 \\
\hline $2300-1000$ & 1 & 1400 & Roper Basin & 35 \\
\hline $2300-1000$ & 1 & 1400 & Roper Basin & 35 \\
\hline $2300-1000$ & 1 & 1400 & Roper Basin & 35 \\
\hline $2300-1000$ & 1 & 1400 & Roper Basin & 35 \\
\hline $2300-1000$ & 1 & 1400 & Roper Basin & 35 \\
\hline $2300-1000$ & 1 & 1400 & Roper Basin & 35 \\
\hline $2300-1000$ & 1 & 1400 & Roper Basin & 35 \\
\hline $2300-1000$ & 1 & 1400 & Roper Basin & 35 \\
\hline $2300-1000$ & 1 & 1400 & Roper Basin & 35 \\
\hline $2300-1000$ & 1 & 1400 & Roper Basin & 35 \\
\hline $2300-1000$ & 1 & 1400 & Roper Basin & 35 \\
\hline $2300-1000$ & 1 & 1400 & Roper Basin & 35 \\
\hline $2300-1000$ & 1 & 1400 & Roper Basin & 35 \\
\hline $2300-1000$ & 1 & 1400 & Roper Basin & 35 \\
\hline $2300-1000$ & 1 & 1400 & Roper Basin & 35 \\
\hline $2300-1000$ & 1 & 1400 & Roper Basin & 35 \\
\hline $2300-1000$ & 1 & 1400 & Roper Basin & 35 \\
\hline $2300-1000$ & 1 & 1400 & Roper Basin & 35 \\
\hline $2300-1000$ & 1 & 1400 & Roper Basin & 35 \\
\hline $2300-1000$ & 1 & 1400 & Roper Basin & 35 \\
\hline $2300-1000$ & 1 & 1400 & Roper Basin & 35 \\
\hline $2300-1000$ & 1 & 1400 & Roper Basin & 35 \\
\hline $2300-1000$ & 1 & 1400 & Roper Basin & 35 \\
\hline $2300-1000$ & 1 & 1400 & Roper Basin & 35 \\
\hline $2300-1000$ & 1 & 1400 & Roper Basin & 35 \\
\hline $2300-1000$ & 1 & 1400 & Roper Basin & 35 \\
\hline
\end{tabular}




\begin{tabular}{|c|c|c|c|c|}
\hline $2300-1000$ & 1 & 1400 & Roper Basin & 35 \\
\hline $2300-1000$ & 1 & 1400 & Roper Basin & 35 \\
\hline $2300-1000$ & 1 & 1400 & Roper Basin & 35 \\
\hline $2300-1000$ & 1 & 1400 & Roper Basin & 35 \\
\hline $2300-1000$ & 1 & 1400 & Roper Basin & 35 \\
\hline $2300-1000$ & 1 & 1400 & Roper Basin & 35 \\
\hline $2300-1000$ & 1 & 1400 & Roper Basin & 35 \\
\hline $2300-1000$ & 1 & 1400 & Roper Basin & 35 \\
\hline $2300-1000$ & 1 & 1400 & Roper Basin & 35 \\
\hline $2300-1000$ & 1 & 1400 & Roper Basin & 35 \\
\hline $2300-1000$ & 1 & 1400 & Roper Basin & 35 \\
\hline $2300-1000$ & 1 & 1400 & Roper Basin & 35 \\
\hline $2300-1000$ & 1 & 1400 & Roper Basin & 35 \\
\hline $2300-1000$ & 1 & 1400 & Roper Basin & 35 \\
\hline $2300-1000$ & 1 & 1400 & Roper Basin & 35 \\
\hline $2300-1000$ & 1 & 1400 & Roper Basin & 35 \\
\hline $2300-1000$ & 1 & 1400 & Roper Basin & 35 \\
\hline $2300-1000$ & 1 & 1400 & Roper Basin & 35 \\
\hline $2300-1000$ & 1 & 1400 & Roper Basin & 35 \\
\hline $2300-1000$ & 1 & 1400 & Roper Basin & 35 \\
\hline $2300-1000$ & 1 & 1400 & Roper Basin & 35 \\
\hline $2300-1000$ & 1 & 1400 & Roper Basin & 35 \\
\hline $2300-1000$ & 1 & 1400 & Roper Basin & 35 \\
\hline $2300-1000$ & 1 & 1400 & Roper Basin & 35 \\
\hline $2300-1000$ & 1 & 1400 & Roper Basin & 35 \\
\hline $2300-1000$ & 1 & 1400 & Roper Basin & 35 \\
\hline $2300-1000$ & 1 & 1400 & Roper Basin & 35 \\
\hline $2300-1000$ & 1 & 1400 & Roper Basin & 35 \\
\hline $2300-1000$ & 1 & 1400 & Roper Basin & 35 \\
\hline $2300-1000$ & 1 & 1400 & Roper Basin & 35 \\
\hline $2300-1000$ & 1 & 1400 & Roper Basin & 35 \\
\hline $2300-1000$ & 1 & 1400 & Roper Basin & 35 \\
\hline $2300-1000$ & 1 & 1400 & Roper Basin & 35 \\
\hline $2300-1000$ & 1 & 1400 & Roper Basin & 35 \\
\hline $2300-1000$ & 1 & 1400 & Roper Basin & 35 \\
\hline $2300-1000$ & 1 & 1400 & Roper Basin & 35 \\
\hline $2300-1000$ & 1 & 1400 & Roper Basin & 35 \\
\hline $2300-1000$ & 1 & 1400 & Roper Basin & 35 \\
\hline $2300-1000$ & 1 & 1400 & Roper Basin & 35 \\
\hline $2300-1000$ & 1 & 1400 & Roper Basin & 35 \\
\hline $2300-1000$ & 1 & 1400 & Roper Basin & 35 \\
\hline
\end{tabular}




\begin{tabular}{|c|c|c|c|c|}
\hline $2300-1000$ & 1 & 1400 & Roper Basin & 35 \\
\hline $2300-1000$ & 1 & 1400 & Roper Basin & 35 \\
\hline $2300-1000$ & 1 & 1400 & Roper Basin & 35 \\
\hline $2300-1000$ & 1 & 1400 & Roper Basin & 35 \\
\hline $2300-1000$ & 1 & 1400 & Roper Basin & 35 \\
\hline $2300-1000$ & 1 & 1400 & Roper Basin & 35 \\
\hline $2300-1000$ & 1 & 1400 & Roper Basin & 35 \\
\hline $2300-1000$ & 1 & 1400 & Roper Basin & 35 \\
\hline $2300-1000$ & 1 & 1400 & Roper Basin & 35 \\
\hline $2300-1000$ & 1 & 1400 & Roper Basin & 35 \\
\hline $2300-1000$ & 1 & 1640 & McArthur Basin & 38 \\
\hline $2300-1000$ & 1 & 1640 & McArthur Basin & 38 \\
\hline $2300-1000$ & 1 & 1640 & McArthur Basin & 38 \\
\hline $2300-1000$ & 1 & 1640 & McArthur Basin & 38 \\
\hline $2300-1000$ & 1 & 1640 & McArthur Basin & 38 \\
\hline $2300-1000$ & 1 & 1640 & McArthur Basin & 38 \\
\hline $2300-1000$ & 1 & 1640 & McArthur Basin & 38 \\
\hline $2300-1000$ & 1 & 1640 & McArthur Basin & 38 \\
\hline $2300-1000$ & 1 & 1640 & McArthur Basin & 38 \\
\hline $2300-1000$ & 1 & 1640 & McArthur Basin & 38 \\
\hline $2300-1000$ & 1 & 1640 & McArthur Basin & 38 \\
\hline $2300-1000$ & 1 & 1640 & McArthur Basin & 38 \\
\hline $2300-1000$ & 1 & 1640 & McArthur Basin & 38 \\
\hline $2300-1000$ & 1 & 1640 & McArthur Basin & 38 \\
\hline $2300-1000$ & 1 & 1640 & McArthur Basin & 38 \\
\hline $2300-1000$ & 1 & 1640 & McArthur Basin & 38 \\
\hline $2300-1000$ & 1 & 1640 & McArthur Basin & 38 \\
\hline $2300-1000$ & 1 & 1640 & McArthur Basin & 38 \\
\hline $2300-1000$ & 1 & 1640 & McArthur Basin & 38 \\
\hline $2300-1000$ & 1 & 1640 & McArthur Basin & 38 \\
\hline $2300-1000$ & 1 & 1640 & McArthur Basin & 38 \\
\hline $2300-1000$ & 1 & 1640 & McArthur Basin & 38 \\
\hline $2300-1000$ & 1 & 1640 & McArthur Basin & 38 \\
\hline $2300-1000$ & 1 & 1640 & McArthur Basin & 38 \\
\hline $2300-1000$ & 1 & 1640 & McArthur Basin & 38 \\
\hline $2300-1000$ & 1 & 1640 & McArthur Basin & 38 \\
\hline $2300-1000$ & 1 & 1640 & McArthur Basin & 38 \\
\hline $2300-1000$ & 1 & 1640 & McArthur Basin & 38 \\
\hline $2300-1000$ & 1 & 1640 & McArthur Basin & 38 \\
\hline $2300-1000$ & 1 & 1640 & McArthur Basin & 38 \\
\hline $2300-1000$ & 1 & 1640 & McArthur Basin & 38 \\
\hline
\end{tabular}




\begin{tabular}{|c|c|c|c|c|}
\hline $2300-1000$ & 1 & 1640 & McArthur Basin & 38 \\
\hline $2300-1000$ & 1 & 1640 & McArthur Basin & 38 \\
\hline $2300-1000$ & 1 & 1640 & McArthur Basin & 38 \\
\hline $2300-1000$ & 1 & 1640 & McArthur Basin & 38 \\
\hline $2300-1000$ & 1 & 1640 & McArthur Basin & 38 \\
\hline $2300-1000$ & 1 & 1640 & McArthur Basin & 38 \\
\hline $2300-1000$ & 1 & 1640 & McArthur Basin & 38 \\
\hline $2300-1000$ & 1 & 1640 & McArthur Basin & 38 \\
\hline $2300-1000$ & 1 & 1640 & McArthur Basin & 38 \\
\hline $2300-1000$ & 1 & 1640 & McArthur Basin & 38 \\
\hline $2300-1000$ & 1 & 1640 & McArthur Basin & 38 \\
\hline $2300-1000$ & 1 & 1640 & McArthur Basin & 38 \\
\hline $2300-1000$ & 1 & 1640 & McArthur Basin & 38 \\
\hline $2300-1000$ & 1 & 1640 & McArthur Basin & 38 \\
\hline $2300-1000$ & 1 & 1640 & McArthur Basin & 38 \\
\hline $2300-1000$ & 1 & 1640 & McArthur Basin & 38 \\
\hline $2300-1000$ & 1 & 1640 & McArthur Basin & 38 \\
\hline $2300-1000$ & 1 & 1640 & McArthur Basin & 38 \\
\hline $2300-1000$ & 1 & 1640 & McArthur Basin & 38 \\
\hline $2300-1000$ & 1 & 1650 & McArthur Basin & 38 \\
\hline $2300-1000$ & 1 & 1650 & McArthur Basin & 38 \\
\hline $2300-1000$ & 1 & 1650 & McArthur Basin & 38 \\
\hline $2300-1000$ & 1 & 1650 & McArthur Basin & 38 \\
\hline $2300-1000$ & 1 & 1650 & McArthur Basin & 38 \\
\hline $2300-1000$ & 1 & 1650 & McArthur Basin & 38 \\
\hline $2300-1000$ & 1 & 1650 & McArthur Basin & 38 \\
\hline $2300-1000$ & 1 & 1650 & McArthur Basin & 38 \\
\hline $2300-1000$ & 1 & 1650 & McArthur Basin & 38 \\
\hline $2300-1000$ & 1 & 1650 & McArthur Basin & 38 \\
\hline $2300-1000$ & 1 & 1750 & McArthur Basin & 38 \\
\hline $2300-1000$ & 1 & 1750 & McArthur Basin & 38 \\
\hline $2300-1000$ & 1 & 1750 & McArthur Basin & 38 \\
\hline $2300-1000$ & 1 & 1750 & McArthur Basin & 38 \\
\hline $2300-1000$ & 1 & 1750 & McArthur Basin & 38 \\
\hline $2300-1000$ & 1 & 1750 & McArthur Basin & 38 \\
\hline $2300-1000$ & 1 & 1750 & McArthur Basin & 38 \\
\hline $2300-1000$ & 1 & 1750 & McArthur Basin & 38 \\
\hline $2300-1000$ & 1 & 1750 & McArthur Basin & 38 \\
\hline $2300-1000$ & 1 & 1750 & McArthur Basin & 38 \\
\hline $2300-1000$ & 1 & 1750 & McArthur Basin & 38 \\
\hline $2300-1000$ & 1 & 1750 & McArthur Basin & 38 \\
\hline
\end{tabular}




\begin{tabular}{|c|c|c|c|c|}
\hline $2300-1000$ & 1 & 1750 & McArthur Basin & 38 \\
\hline $2300-1000$ & 1 & 1750 & McArthur Basin & 38 \\
\hline $2300-1000$ & 1 & 1800 & Animikie Basin & 39 \\
\hline $2300-1000$ & 1 & 1800 & Animikie Basin & 39 \\
\hline $2300-1000$ & 1 & 1800 & Animikie Basin & 39 \\
\hline $2300-1000$ & 1 & 1800 & Animikie Basin & 39 \\
\hline $2300-1000$ & 1 & 1800 & Animikie Basin & 39 \\
\hline $2300-1000$ & 1 & 1800 & Animikie Basin & 39 \\
\hline $2300-1000$ & 1 & 1800 & Animikie Basin & 39 \\
\hline $2300-1000$ & 1 & 1800 & Animikie Basin & 39 \\
\hline $2300-1000$ & 1 & 1800 & Animikie Basin & 39 \\
\hline $2300-1000$ & 1 & 1800 & Animikie Basin & 39 \\
\hline $2300-1000$ & 1 & 1800 & Animikie Basin & 39 \\
\hline $2300-1000$ & 1 & 1800 & Animikie Basin & 39 \\
\hline $2300-1000$ & 1 & 1800 & Animikie Basin & 39 \\
\hline $2300-1000$ & 1 & 1800 & Animikie Basin & 39 \\
\hline $2300-1000$ & 1 & 1800 & Animikie Basin & 39 \\
\hline $2300-1000$ & 1 & 1800 & Animikie Basin & 39 \\
\hline $2300-1000$ & 1 & 1800 & Animikie Basin & 39 \\
\hline $2300-1000$ & 1 & 1800 & Animikie Basin & 39 \\
\hline $2300-1000$ & 1 & 1800 & Animikie Basin & 39 \\
\hline $2300-1000$ & 1 & 1800 & Animikie Basin & 39 \\
\hline $2300-1000$ & 1 & 1800 & Animikie Basin & 39 \\
\hline $2300-1000$ & 1 & 1800 & Animikie Basin & 39 \\
\hline $2300-1000$ & 1 & 1800 & Animikie Basin & 39 \\
\hline $2300-1000$ & 1 & 1800 & Animikie Basin & 39 \\
\hline $2300-1000$ & 1 & 1800 & Animikie Basin & 39 \\
\hline $2300-1000$ & 1 & 1800 & Animikie Basin & 39 \\
\hline $2300-1000$ & 1 & 1800 & Animikie Basin & 39 \\
\hline $2300-1000$ & 1 & 1800 & Animikie Basin & 39 \\
\hline $2300-1000$ & 1 & 1800 & Animikie Basin & 39 \\
\hline $2300-1000$ & 1 & 1800 & Animikie Basin & 39 \\
\hline $2300-1000$ & 1 & 1800 & Animikie Basin & 39 \\
\hline $2300-1000$ & 1 & 1800 & Animikie Basin & 39 \\
\hline $2300-1000$ & 1 & 1800 & Animikie Basin & 39 \\
\hline $2300-1000$ & 1 & 1800 & Animikie Basin & 39 \\
\hline $2300-1000$ & 1 & 1800 & Animikie Basin & 39 \\
\hline $2300-1000$ & 1 & 1800 & Animikie Basin & 39 \\
\hline $2300-1000$ & 1 & 1800 & Animikie Basin & 39 \\
\hline $2300-1000$ & 1 & 1800 & Animikie Basin & 39 \\
\hline $2300-1000$ & 1 & 1800 & Animikie Basin & 39 \\
\hline
\end{tabular}




\begin{tabular}{|c|c|c|c|c|}
\hline $2300-1000$ & 1 & 1800 & Animikie Basin & 39 \\
\hline $2300-1000$ & 1 & 1800 & Animikie Basin & 39 \\
\hline $2300-1000$ & 1 & 1800 & Animikie Basin & 39 \\
\hline $2300-1000$ & 1 & 1800 & Animikie Basin & 39 \\
\hline $2300-1000$ & 1 & 1800 & Animikie Basin & 39 \\
\hline $2300-1000$ & 1 & 1800 & Animikie Basin & 39 \\
\hline $2300-1000$ & 1 & 1800 & Animikie Basin & 39 \\
\hline $2300-1000$ & 1 & 1800 & Animikie Basin & 39 \\
\hline $2300-1000$ & 1 & 1800 & Animikie Basin & 39 \\
\hline $2300-1000$ & 1 & 1800 & Animikie Basin & 39 \\
\hline $2300-1000$ & 1 & 1800 & Animikie Basin & 39 \\
\hline $2300-1000$ & 1 & 1800 & Animikie Basin & 39 \\
\hline $2300-1000$ & 1 & 1800 & Animikie Basin & 39 \\
\hline $2300-1000$ & 1 & 1800 & Animikie Basin & 39 \\
\hline $2300-1000$ & 1 & 1800 & Animikie Basin & 39 \\
\hline $2300-1000$ & 1 & 1800 & Animikie Basin & 39 \\
\hline $2300-1000$ & 1 & 1800 & Animikie Basin & 39 \\
\hline $2300-1000$ & 1 & 1800 & Animikie Basin & 39 \\
\hline $2300-1000$ & 1 & 1800 & Animikie Basin & 39 \\
\hline $2300-1000$ & 1 & 1800 & Animikie Basin & 39 \\
\hline $2300-1000$ & 1 & 1800 & Animikie Basin & 39 \\
\hline $2300-1000$ & 1 & 1800 & Animikie Basin & 39 \\
\hline $2300-1000$ & 1 & 1800 & Animikie Basin & 39 \\
\hline $2300-1000$ & 1 & 1800 & Animikie Basin & 39 \\
\hline $2300-1000$ & 1 & 1800 & Animikie Basin & 39 \\
\hline $2300-1000$ & 1 & 1800 & Animikie Basin & 39 \\
\hline $2300-1000$ & 1 & 1800 & Animikie Basin & 39 \\
\hline $2300-1000$ & 1 & 1800 & Animikie Basin & 39 \\
\hline $2300-1000$ & 1 & 1800 & Animikie Basin & 39 \\
\hline $2300-1000$ & 1 & 1800 & Animikie Basin & 39 \\
\hline $2300-1000$ & 1 & 1800 & Animikie Basin & 39 \\
\hline $2300-1000$ & 1 & 1800 & Animikie Basin & 39 \\
\hline $2300-1000$ & 1 & 1800 & Animikie Basin & 39 \\
\hline $2300-1000$ & 1 & 1800 & Animikie Basin & 39 \\
\hline $2300-1000$ & 1 & 1800 & Animikie Basin & 39 \\
\hline $2300-1000$ & 1 & 1800 & Animikie Basin & 39 \\
\hline $2300-1000$ & 1 & 1800 & Animikie Basin & 39 \\
\hline $2300-1000$ & 1 & 1800 & Animikie Basin & 39 \\
\hline $2300-1000$ & 1 & 1800 & Animikie Basin & 39 \\
\hline $2300-1000$ & 1 & 1800 & Animikie Basin & 39 \\
\hline $2300-1000$ & 1 & 1800 & Animikie Basin & 39 \\
\hline
\end{tabular}




\begin{tabular}{|c|c|c|c|c|}
\hline $2300-1000$ & 1 & 1800 & Animikie Basin & 39 \\
\hline $2300-1000$ & 1 & 1800 & Animikie Basin & 39 \\
\hline $2300-1000$ & 1 & 1800 & Animikie Basin & 39 \\
\hline $2300-1000$ & 1 & 1800 & Animikie Basin & 39 \\
\hline $2300-1000$ & 1 & 1800 & Animikie Basin & 39 \\
\hline $2300-1000$ & 1 & 1800 & Animikie Basin & 39 \\
\hline $2300-1000$ & 1 & 1800 & Animikie Basin & 39 \\
\hline $2300-1000$ & 1 & 1800 & Animikie Basin & 39 \\
\hline $2300-1000$ & 1 & 1800 & Animikie Basin & 39 \\
\hline $2300-1000$ & 1 & 1800 & Animikie Basin & 39 \\
\hline $2300-1000$ & 1 & 1800 & Animikie Basin & 39 \\
\hline $2300-1000$ & 1 & 1800 & Animikie Basin & 39 \\
\hline $2300-1000$ & 1 & 1800 & Animikie Basin & 39 \\
\hline $2300-1000$ & 1 & 1800 & Animikie Basin & 39 \\
\hline $2300-1000$ & 1 & 1800 & Animikie Basin & 39 \\
\hline $2300-1000$ & 1 & 1800 & Animikie Basin & 39 \\
\hline $2300-1000$ & 1 & 1800 & Animikie Basin & 39 \\
\hline $2300-1000$ & 1 & 1800 & Animikie Basin & 39 \\
\hline $2300-1000$ & 1 & 1800 & Animikie Basin & 39 \\
\hline $2300-1000$ & 1 & 1800 & Animikie Basin & 39 \\
\hline $2300-1000$ & 1 & 1800 & Animikie Basin & 39 \\
\hline $2300-1000$ & 1 & 1800 & Animikie Basin & 39 \\
\hline $2300-1000$ & 1 & 1800 & Animikie Basin & 39 \\
\hline $2300-1000$ & 1 & 1800 & Animikie Basin & 39 \\
\hline $2300-1000$ & 1 & 1800 & Animikie Basin & 39 \\
\hline $2300-1000$ & 1 & 1800 & Animikie Basin & 39 \\
\hline $2300-1000$ & 1 & 1800 & Animikie Basin & 39 \\
\hline $2300-1000$ & 1 & 1800 & Animikie Basin & 39 \\
\hline $2300-1000$ & 1 & 1800 & Animikie Basin & 39 \\
\hline $2300-1000$ & 1 & 1800 & Animikie Basin & 39 \\
\hline $2300-1000$ & 1 & 1800 & Animikie Basin & 39 \\
\hline $2300-1000$ & 1 & 1800 & Animikie Basin & 39 \\
\hline $2300-1000$ & 1 & 1800 & Animikie Basin & 39 \\
\hline $2300-1000$ & 1 & 1800 & Animikie Basin & 39 \\
\hline $2300-1000$ & 1 & 1800 & Animikie Basin & 39 \\
\hline $2300-1000$ & 1 & 1800 & Animikie Basin & 39 \\
\hline $2300-1000$ & 1 & 1800 & Animikie Basin & 39 \\
\hline $2300-1000$ & 1 & 1800 & Animikie Basin & 39 \\
\hline $2300-1000$ & 1 & 1800 & Animikie Basin & 39 \\
\hline $2300-1000$ & 1 & 1800 & Animikie Basin & 39 \\
\hline $2300-1000$ & 1 & 1800 & Animikie Basin & 39 \\
\hline
\end{tabular}




\begin{tabular}{|c|c|c|c|c|}
\hline $2300-1000$ & 1 & 1800 & Animikie Basin & 39 \\
\hline $2300-1000$ & 1 & 1800 & Animikie Basin & 39 \\
\hline $2300-1000$ & 1 & 1800 & Animikie Basin & 39 \\
\hline $2300-1000$ & 1 & 1800 & Animikie Basin & 39 \\
\hline $2300-1000$ & 1 & 1800 & Animikie Basin & 39 \\
\hline $2300-1000$ & 1 & 1800 & Animikie Basin & 39 \\
\hline $2300-1000$ & 1 & 1800 & Animikie Basin & 39 \\
\hline $2300-1000$ & 1 & 1800 & Animikie Basin & 39 \\
\hline $2300-1000$ & 1 & 1800 & Animikie Basin & 39 \\
\hline $2300-1000$ & 1 & 1800 & Animikie Basin & 39 \\
\hline $2300-1000$ & 1 & 1800 & Animikie Basin & 39 \\
\hline $2300-1000$ & 1 & 1800 & Animikie Basin & 39 \\
\hline $2300-1000$ & 1 & 1800 & Animikie Basin & 39 \\
\hline $2300-1000$ & 1 & 1800 & Animikie Basin & 39 \\
\hline $2300-1000$ & 1 & 1800 & Animikie Basin & 39 \\
\hline $2300-1000$ & 1 & 1800 & Animikie Basin & 39 \\
\hline $2300-1000$ & 1 & 1800 & Animikie Basin & 39 \\
\hline $2300-1000$ & 1 & 1800 & Animikie Basin & 39 \\
\hline $2300-1000$ & 1 & 1800 & Animikie Basin & 39 \\
\hline $2300-1000$ & 1 & 1800 & Animikie Basin & 39 \\
\hline $2300-1000$ & 1 & 1800 & Animikie Basin & 39 \\
\hline $2300-1000$ & 1 & 1800 & Animikie Basin & 39 \\
\hline $2300-1000$ & 1 & 1800 & Animikie Basin & 39 \\
\hline $2300-1000$ & 1 & 1800 & Animikie Basin & 39 \\
\hline $2300-1000$ & 1 & 1800 & Animikie Basin & 39 \\
\hline $2300-1000$ & 1 & 1800 & Animikie Basin & 39 \\
\hline $2300-1000$ & 1 & 1800 & Animikie Basin & 39 \\
\hline $2300-1000$ & 1 & 1800 & Animikie Basin & 39 \\
\hline $2300-1000$ & 1 & 1800 & Animikie Basin & 39 \\
\hline $2300-1000$ & 1 & 1800 & Animikie Basin & 39 \\
\hline $2300-1000$ & 1 & 1800 & Animikie Basin & 39 \\
\hline $2300-1000$ & 1 & 1800 & Animikie Basin & 39 \\
\hline $2300-1000$ & 1 & 1800 & Animikie Basin & 39 \\
\hline $2300-1000$ & 1 & 1800 & Animikie Basin & 39 \\
\hline $2300-1000$ & 1 & 1800 & Animikie Basin & 39 \\
\hline $2300-1000$ & 1 & 1800 & Animikie Basin & 39 \\
\hline $2300-1000$ & 1 & 1800 & Animikie Basin & 39 \\
\hline $2300-1000$ & 1 & 1800 & Animikie Basin & 39 \\
\hline $2300-1000$ & 1 & 1800 & Animikie Basin & 39 \\
\hline $2300-1000$ & 1 & 1800 & Animikie Basin & 39 \\
\hline $2300-1000$ & 1 & 1800 & Animikie Basin & 39 \\
\hline
\end{tabular}




\begin{tabular}{|c|c|c|c|c|}
\hline $2300-1000$ & 1 & 1800 & Animikie Basin & 39 \\
\hline $2300-1000$ & 1 & 1800 & Animikie Basin & 39 \\
\hline $2300-1000$ & 1 & 1800 & Animikie Basin & 39 \\
\hline $2300-1000$ & 1 & 1800 & Animikie Basin & 39 \\
\hline $2300-1000$ & 1 & 1800 & Animikie Basin & 39 \\
\hline $2300-1000$ & 1 & 1800 & Animikie Basin & 39 \\
\hline $2300-1000$ & 1 & 1800 & Animikie Basin & 39 \\
\hline $2300-1000$ & 1 & 1800 & Animikie Basin & 39 \\
\hline $2300-1000$ & 1 & 1800 & Animikie Basin & 39 \\
\hline $2300-1000$ & 1 & 1800 & Animikie Basin & 39 \\
\hline $2300-1000$ & 1 & 1800 & Animikie Basin & 39 \\
\hline $2300-1000$ & 1 & 1800 & Animikie Basin & 39 \\
\hline $2300-1000$ & 1 & 1800 & Animikie Basin & 39 \\
\hline $2300-1000$ & 1 & 1800 & Animikie Basin & 39 \\
\hline $2300-1000$ & 1 & 1800 & Animikie Basin & 39 \\
\hline $2300-1000$ & 1 & 1800 & Animikie Basin & 39 \\
\hline $2300-1000$ & 1 & 1800 & Animikie Basin & 39 \\
\hline $2300-1000$ & 1 & 1800 & Animikie Basin & 39 \\
\hline $2300-1000$ & 1 & 1800 & Animikie Basin & 39 \\
\hline $2300-1000$ & 1 & 1800 & Animikie Basin & 39 \\
\hline $2300-1000$ & 1 & 1800 & Animikie Basin & 39 \\
\hline $2300-1000$ & 1 & 1800 & Animikie Basin & 39 \\
\hline $2300-1000$ & 1 & 1800 & Animikie Basin & 39 \\
\hline $2300-1000$ & 1 & 1800 & Animikie Basin & 39 \\
\hline $2300-1000$ & 1 & 1800 & Animikie Basin & 39 \\
\hline $2300-1000$ & 1 & 1800 & Animikie Basin & 39 \\
\hline $2300-1000$ & 1 & 1800 & Animikie Basin & 39 \\
\hline $2300-1000$ & 1 & 1800 & Animikie Basin & 39 \\
\hline $2300-1000$ & 1 & 1800 & Animikie Basin & 39 \\
\hline $2300-1000$ & 1 & 1800 & Animikie Basin & 39 \\
\hline $2300-1000$ & 1 & 1800 & Animikie Basin & 39 \\
\hline $2300-1000$ & 1 & 1800 & Animikie Basin & 39 \\
\hline $2300-1000$ & 1 & 1800 & Animikie Basin & 39 \\
\hline $2300-1000$ & 1 & 1800 & Animikie Basin & 39 \\
\hline $2300-1000$ & 1 & 1800 & Animikie Basin & 39 \\
\hline $2300-1000$ & 1 & 1800 & Animikie Basin & 39 \\
\hline $2300-1000$ & 1 & 1800 & Animikie Basin & 39 \\
\hline $2300-1000$ & 1 & 1800 & Animikie Basin & 39 \\
\hline $2300-1000$ & 1 & 1800 & Animikie Basin & 39 \\
\hline $2300-1000$ & 1 & 1800 & Animikie Basin & 39 \\
\hline $2300-1000$ & 1 & 1800 & Animikie Basin & 39 \\
\hline
\end{tabular}




\begin{tabular}{|c|c|c|c|c|}
\hline $2300-1000$ & 1 & 1800 & Animikie Basin & 39 \\
\hline $2300-1000$ & 1 & 1800 & Animikie Basin & 39 \\
\hline $2300-1000$ & 1 & 1800 & Animikie Basin & 39 \\
\hline $2300-1000$ & 1 & 1800 & Animikie Basin & 39 \\
\hline $2300-1000$ & 1 & 1800 & Animikie Basin & 39 \\
\hline $2300-1000$ & 1 & 1800 & Animikie Basin & 39 \\
\hline $2300-1000$ & 1 & 1800 & Animikie Basin & 39 \\
\hline $2300-1000$ & 1 & 1800 & Animikie Basin & 39 \\
\hline $2300-1000$ & 1 & 1800 & Animikie Basin & 39 \\
\hline $2300-1000$ & 1 & 1800 & Animikie Basin & 39 \\
\hline $2300-1000$ & 1 & 1800 & Animikie Basin & 39 \\
\hline $2300-1000$ & 1 & 1800 & Animikie Basin & 39 \\
\hline $2300-1000$ & 1 & 1800 & Animikie Basin & 39 \\
\hline $2300-1000$ & 1 & 1800 & Animikie Basin & 39 \\
\hline $2300-1000$ & 1 & 1800 & Animikie Basin & 39 \\
\hline $2300-1000$ & 1 & 1800 & Animikie Basin & 39 \\
\hline $2300-1000$ & 1 & 1800 & Animikie Basin & 39 \\
\hline $2300-1000$ & 1 & 1800 & Animikie Basin & 39 \\
\hline $2300-1000$ & 1 & 1800 & Animikie Basin & 39 \\
\hline $2300-1000$ & 1 & 1800 & Animikie Basin & 39 \\
\hline $2300-1000$ & 1 & 1800 & Animikie Basin & 39 \\
\hline $2300-1000$ & 1 & 1800 & Animikie Basin & 39 \\
\hline $2300-1000$ & 1 & 1800 & Animikie Basin & 39 \\
\hline $2300-1000$ & 1 & 1800 & Animikie Basin & 39 \\
\hline $2300-1000$ & 1 & 1800 & Animikie Basin & 39 \\
\hline $2300-1000$ & 1 & 1800 & Animikie Basin & 39 \\
\hline $2300-1000$ & 1 & 1800 & Animikie Basin & 39 \\
\hline $2300-1000$ & 1 & 1800 & Animikie Basin & 39 \\
\hline $2300-1000$ & 1 & 1800 & Animikie Basin & 39 \\
\hline $2300-1000$ & 1 & 1800 & Animikie Basin & 39 \\
\hline $2300-1000$ & 1 & 1800 & Animikie Basin & 39 \\
\hline $2300-1000$ & 1 & 1800 & Animikie Basin & 39 \\
\hline $2300-1000$ & 1 & 1800 & Animikie Basin & 39 \\
\hline $2300-1000$ & 1 & 1800 & Animikie Basin & 39 \\
\hline $2300-1000$ & 1 & 1800 & Animikie Basin & 39 \\
\hline $2300-1000$ & 1 & 1800 & Animikie Basin & 39 \\
\hline $2300-1000$ & 1 & 1800 & Animikie Basin & 39 \\
\hline $2300-1000$ & 1 & 1800 & Animikie Basin & 39 \\
\hline $2300-1000$ & 1 & 1800 & Animikie Basin & 39 \\
\hline $2300-1000$ & 1 & 1800 & Animikie Basin & 39 \\
\hline $2300-1000$ & 1 & 1800 & Animikie Basin & 39 \\
\hline
\end{tabular}




\begin{tabular}{|c|c|c|c|c|}
\hline $2300-1000$ & 1 & 1800 & Animikie Basin & 39 \\
\hline $2300-1000$ & 1 & 1800 & Animikie Basin & 39 \\
\hline $2300-1000$ & 1 & 1800 & Animikie Basin & 39 \\
\hline $2300-1000$ & 1 & 1800 & Animikie Basin & 39 \\
\hline $2300-1000$ & 1 & 1800 & Animikie Basin & 39 \\
\hline $2300-1000$ & 1 & 1800 & Animikie Basin & 39 \\
\hline $2300-1000$ & 1 & 1800 & Animikie Basin & 39 \\
\hline $2300-1000$ & 1 & 1800 & Animikie Basin & 39 \\
\hline $2300-1000$ & 1 & 1800 & Animikie Basin & 39 \\
\hline $2300-1000$ & 1 & 1800 & Animikie Basin & 39 \\
\hline $2300-1000$ & 1 & 1800 & Animikie Basin & 39 \\
\hline $2300-1000$ & 1 & 1800 & Animikie Basin & 39 \\
\hline $2300-1000$ & 1 & 1800 & Animikie Basin & 39 \\
\hline $2300-1000$ & 1 & 1800 & Animikie Basin & 39 \\
\hline $2300-1000$ & 1 & 1800 & Animikie Basin & 39 \\
\hline $2300-1000$ & 1 & 1800 & Animikie Basin & 39 \\
\hline $2300-1000$ & 1 & 1100 & Animikie Basin & 39 \\
\hline $2300-1000$ & 1 & 1800 & Animikie Basin & 39 \\
\hline $2300-1000$ & 1 & 1800 & Animikie Basin & 39 \\
\hline $2300-1000$ & 1 & 1800 & Animikie Basin & 39 \\
\hline $2300-1000$ & 1 & 1800 & Animikie Basin & 39 \\
\hline $2300-1000$ & 1 & 1800 & Animikie Basin & 39 \\
\hline $2300-1000$ & 1 & 1800 & Animikie Basin & 39 \\
\hline $2300-1000$ & 1 & 1800 & Animikie Basin & 39 \\
\hline $2300-1000$ & 1 & 1100 & Brasília Fold Belt & 43 \\
\hline $2300-1000$ & 1 & 1100 & Brasília Fold Belt & 43 \\
\hline $2300-1000$ & 1 & 1100 & Brasília Fold Belt & 43 \\
\hline $2300-1000$ & 1 & 1100 & Brasília Fold Belt & 43 \\
\hline $2300-1000$ & 1 & 1100 & Brasília Fold Belt & 43 \\
\hline $2300-1000$ & 1 & 1100 & Brasília Fold Belt & 43 \\
\hline $2300-1000$ & 1 & 1100 & Brasília Fold Belt & 43 \\
\hline $2300-1000$ & 1 & 1100 & Brasília Fold Belt & 43 \\
\hline $2300-1000$ & 1 & 1100 & Brasília Fold Belt & 43 \\
\hline $2300-1000$ & 1 & 1100 & Brasília Fold Belt & 43 \\
\hline $2300-1000$ & 1 & 1100 & Brasília Fold Belt & 43 \\
\hline $2300-1000$ & 1 & 1100 & Brasília Fold Belt & 43 \\
\hline $2300-1000$ & 1 & 1100 & Brasília Fold Belt & 43 \\
\hline $2300-1000$ & 1 & 1100 & Brasília Fold Belt & 43 \\
\hline $2300-1000$ & 1 & 1100 & Brasília Fold Belt & 43 \\
\hline $2300-1000$ & 1 & 1100 & Brasília Fold Belt & 43 \\
\hline $2300-1000$ & 1 & 1100 & Brasília Fold Belt & 43 \\
\hline
\end{tabular}




\begin{tabular}{|c|c|c|c|c|}
\hline $2300-1000$ & 1 & 1100 & Brasília Fold Belt & 43 \\
\hline $2300-1000$ & 1 & 1100 & Brasília Fold Belt & 43 \\
\hline $2300-1000$ & 1 & 1100 & Brasília Fold Belt & 43 \\
\hline $2300-1000$ & 1 & 1100 & Brasília Fold Belt & 43 \\
\hline $2300-1000$ & 1 & 1100 & Brasília Fold Belt & 43 \\
\hline $2300-1000$ & 1 & 1100 & Brasília Fold Belt & 43 \\
\hline $2300-1000$ & 1 & 1100 & Brasília Fold Belt & 43 \\
\hline $2300-1000$ & 1 & 1100 & Brasília Fold Belt & 43 \\
\hline $2300-1000$ & 1 & 1100 & Brasília Fold Belt & 43 \\
\hline $2300-1000$ & 1 & 1100 & Brasília Fold Belt & 43 \\
\hline $2300-1000$ & 1 & 1100 & Brasília Fold Belt & 43 \\
\hline $2300-1000$ & 1 & 1100 & Brasília Fold Belt & 43 \\
\hline $2300-1000$ & 1 & 1100 & Brasília Fold Belt & 43 \\
\hline $2300-1000$ & 1 & 1100 & Brasília Fold Belt & 43 \\
\hline $2300-1000$ & 1 & 1100 & Brasília Fold Belt & 43 \\
\hline $2300-1000$ & 1 & 1100 & Brasília Fold Belt & 43 \\
\hline $2300-1000$ & 1 & 1100 & Brasília Fold Belt & 43 \\
\hline $2300-1000$ & 1 & 1100 & Brasília Fold Belt & 43 \\
\hline $2300-1000$ & 1 & 1100 & Brasília Fold Belt & 43 \\
\hline $2300-1000$ & 1 & 1100 & Brasília Fold Belt & 43 \\
\hline $2300-1000$ & 1 & 1100 & Brasília Fold Belt & 43 \\
\hline $2300-1000$ & 1 & 1100 & Brasília Fold Belt & 43 \\
\hline $2200-1000$ & 1 & 2000 & Onega Basin, Russia & 46 \\
\hline $2200-1000$ & 1 & 2000 & Onega Basin, Russia & 46 \\
\hline $2200-1000$ & 1 & 2000 & Onega Basin, Russia & 46 \\
\hline $2200-1000$ & 1 & 2000 & Onega Basin, Russia & 46 \\
\hline $2200-1000$ & 1 & 2000 & Onega Basin, Russia & 46 \\
\hline $2200-1000$ & 1 & 2000 & Onega Basin, Russia & 46 \\
\hline $2200-1000$ & 1 & 2000 & Onega Basin, Russia & 46 \\
\hline $2200-1000$ & 1 & 2000 & Onega Basin, Russia & 46 \\
\hline $2200-1000$ & 1 & 2000 & Onega Basin, Russia & 46 \\
\hline $2200-1000$ & 1 & 2000 & Onega Basin, Russia & 46 \\
\hline $2200-1000$ & 1 & 2000 & Onega Basin, Russia & 46 \\
\hline $2200-1000$ & 1 & 2000 & Onega Basin, Russia & 46 \\
\hline $2200-1000$ & 1 & 2000 & Onega Basin, Russia & 46 \\
\hline $2200-1000$ & 1 & 2000 & Onega Basin, Russia & 46 \\
\hline $2200-1000$ & 1 & 2000 & Onega Basin, Russia & 46 \\
\hline $2200-1000$ & 1 & 2000 & Onega Basin, Russia & 46 \\
\hline $2200-1000$ & 1 & 2000 & Onega Basin, Russia & 46 \\
\hline $2300-1000$ & 1 & 2000 & Onega Basin, Russia & 46 \\
\hline $2300-1000$ & 1 & 2000 & Onega Basin, Russia & 46 \\
\hline
\end{tabular}




\begin{tabular}{|c|c|c|c|c|}
\hline $2300-1000$ & 1 & 2000 & Onega Basin, Russia & 46 \\
\hline $2300-1000$ & 1 & 2000 & Onega Basin, Russia & 46 \\
\hline $2300-1000$ & 1 & 2000 & Onega Basin, Russia & 46 \\
\hline $2300-1000$ & 1 & 2000 & Onega Basin, Russia & 46 \\
\hline $2300-1000$ & 1 & 2000 & Onega Basin, Russia & 46 \\
\hline $2300-1000$ & 1 & 2000 & Onega Basin, Russia & 46 \\
\hline $2300-1000$ & 1 & 2000 & Onega Basin, Russia & 46 \\
\hline $2300-1000$ & 1 & 2000 & Onega Basin, Russia & 46 \\
\hline $2300-1000$ & 1 & 2000 & Onega Basin, Russia & 46 \\
\hline $2300-1000$ & 1 & 2000 & Onega Basin, Russia & 46 \\
\hline $2300-1000$ & 1 & 2000 & Onega Basin, Russia & 46 \\
\hline $2300-1000$ & 1 & 2000 & Onega Basin, Russia & 46 \\
\hline $2300-1000$ & 1 & 2000 & Onega Basin, Russia & 46 \\
\hline $2300-1000$ & 1 & 2000 & Onega Basin, Russia & 46 \\
\hline $2300-1000$ & 1 & 2000 & Onega Basin, Russia & 46 \\
\hline $2300-1000$ & 1 & 2000 & Onega Basin, Russia & 46 \\
\hline $2300-1000$ & 1 & 2000 & Onega Basin, Russia & 46 \\
\hline $2300-1000$ & 1 & 2000 & Onega Basin, Russia & 46 \\
\hline $2300-1000$ & 1 & 2000 & Onega Basin, Russia & 46 \\
\hline $2300-1000$ & 1 & 2000 & Onega Basin, Russia & 46 \\
\hline $2300-1000$ & 1 & 2000 & Onega Basin, Russia & 46 \\
\hline $2300-1000$ & 1 & 2000 & Onega Basin, Russia & 46 \\
\hline $2300-1000$ & 1 & 2000 & Onega Basin, Russia & 46 \\
\hline $2300-1000$ & 1 & 2000 & Onega Basin, Russia & 46 \\
\hline $2300-1000$ & 1 & 2000 & Onega Basin, Russia & 46 \\
\hline $2300-1000$ & 1 & 2000 & Onega Basin, Russia & 46 \\
\hline $2300-1000$ & 1 & 2000 & Onega Basin, Russia & 46 \\
\hline $2300-1000$ & 1 & 2000 & Onega Basin, Russia & 46 \\
\hline $2300-1000$ & 1 & 2000 & Onega Basin, Russia & 46 \\
\hline $2300-1000$ & 1 & 2000 & Onega Basin, Russia & 46 \\
\hline $2300-1000$ & 1 & 2000 & Onega Basin, Russia & 46 \\
\hline $2300-1000$ & 1 & 2000 & Onega Basin, Russia & 46 \\
\hline $2300-1000$ & 1 & 2000 & Onega Basin, Russia & 46 \\
\hline $2300-1000$ & 1 & 2000 & Onega Basin, Russia & 46 \\
\hline $2300-1000$ & 1 & 2000 & Onega Basin, Russia & 46 \\
\hline $2300-1000$ & 1 & 2000 & Onega Basin, Russia & 46 \\
\hline $2300-1000$ & 1 & 2000 & Onega Basin, Russia & 46 \\
\hline $2300-1000$ & 1 & 2000 & Onega Basin, Russia & 46 \\
\hline $2300-1000$ & 1 & 2000 & Onega Basin, Russia & 46 \\
\hline $2300-1000$ & 1 & 2000 & Onega Basin, Russia & 46 \\
\hline $2300-1000$ & 1 & 2000 & Onega Basin, Russia & 46 \\
\hline
\end{tabular}




\begin{tabular}{|c|c|c|c|c|}
\hline $2300-1000$ & 1 & 2000 & Onega Basin, Russia & 46 \\
\hline $2300-1000$ & 1 & 2000 & Onega Basin, Russia & 46 \\
\hline $2300-1000$ & 1 & 2000 & Onega Basin, Russia & 46 \\
\hline $2300-1000$ & 1 & 2000 & Onega Basin, Russia & 46 \\
\hline $2300-1000$ & 1 & 2000 & Onega Basin, Russia & 46 \\
\hline $2300-1000$ & 1 & 2000 & Onega Basin, Russia & 46 \\
\hline $2300-1000$ & 1 & 2000 & Onega Basin, Russia & 46 \\
\hline $2300-1000$ & 1 & 1080 & Midcontinent Rift & 47 \\
\hline $2300-1000$ & 1 & 1080 & Midcontinent Rift & 47 \\
\hline $2300-1000$ & 1 & 1080 & Midcontinent Rift & 47 \\
\hline $2300-1000$ & 1 & 1080 & Midcontinent Rift & 47 \\
\hline $2300-1000$ & 1 & 1080 & Midcontinent Rift & 47 \\
\hline $2300-1000$ & 1 & 1080 & Midcontinent Rift & 47 \\
\hline $2300-1000$ & 1 & 1080 & Midcontinent Rift & 47 \\
\hline $2300-1000$ & 1 & 1080 & Midcontinent Rift & 47 \\
\hline $2300-1000$ & 1 & 1080 & Midcontinent Rift & 47 \\
\hline $2300-1000$ & 1 & 1080 & Midcontinent Rift & 47 \\
\hline $2300-1000$ & 1 & 1080 & Midcontinent Rift & 47 \\
\hline $2300-1000$ & 1 & 1080 & Midcontinent Rift & 47 \\
\hline $2300-1000$ & 1 & 1080 & Midcontinent Rift & 47 \\
\hline $2300-1000$ & 1 & 1080 & Midcontinent Rift & 47 \\
\hline $2300-1000$ & 1 & 1080 & Midcontinent Rift & 47 \\
\hline $2300-1000$ & 1 & 1080 & Midcontinent Rift & 47 \\
\hline $2300-1000$ & 1 & 1080 & Midcontinent Rift & 47 \\
\hline $2300-1000$ & 1 & 1080 & Midcontinent Rift & 47 \\
\hline $2300-1000$ & 1 & 1080 & Midcontinent Rift & 47 \\
\hline $2300-1000$ & 1 & 1080 & Midcontinent Rift & 47 \\
\hline $2300-1000$ & 1 & 1080 & Midcontinent Rift & 47 \\
\hline $2300-1000$ & 1 & 1080 & Midcontinent Rift & 47 \\
\hline $2300-1000$ & 1 & 1080 & Midcontinent Rift & 47 \\
\hline $2300-1000$ & 1 & 1080 & Midcontinent Rift & 47 \\
\hline $2300-1000$ & 1 & 1080 & Midcontinent Rift & 47 \\
\hline $2300-1000$ & 1 & 1080 & Midcontinent Rift & 47 \\
\hline $2300-1000$ & 1 & 1080 & Midcontinent Rift & 47 \\
\hline $2300-1000$ & 1 & 1080 & Midcontinent Rift & 47 \\
\hline $2300-1000$ & 1 & 1080 & Midcontinent Rift & 47 \\
\hline $2300-1000$ & 1 & 1080 & Midcontinent Rift & 47 \\
\hline $2300-1000$ & 1 & 1080 & Midcontinent Rift & 47 \\
\hline $2300-1000$ & 1 & 1080 & Midcontinent Rift & 47 \\
\hline $2300-1000$ & 1 & 1080 & Midcontinent Rift & 47 \\
\hline $2300-1000$ & 1 & 1080 & Midcontinent Rift & 47 \\
\hline
\end{tabular}




\begin{tabular}{|c|c|c|c|c|}
\hline $2300-1000$ & 1 & 1080 & Midcontinent Rift & 47 \\
\hline $2300-1000$ & 1 & 1080 & Midcontinent Rift & 47 \\
\hline $2300-1000$ & 1 & 1080 & Midcontinent Rift & 47 \\
\hline $2300-1000$ & 1 & 1080 & Midcontinent Rift & 47 \\
\hline $2300-1000$ & 1 & 1080 & Midcontinent Rift & 47 \\
\hline $2300-1000$ & 1 & 1080 & Midcontinent Rift & 47 \\
\hline $2300-1000$ & 1 & 1080 & Midcontinent Rift & 47 \\
\hline $2300-1000$ & 1 & 1080 & Midcontinent Rift & 47 \\
\hline $2300-1000$ & 1 & 1080 & Midcontinent Rift & 47 \\
\hline $2300-1000$ & 1 & 1080 & Midcontinent Rift & 47 \\
\hline $2300-1000$ & 1 & 1080 & Midcontinent Rift & 47 \\
\hline $2300-1000$ & 1 & 1080 & Midcontinent Rift & 47 \\
\hline $2300-1000$ & 1 & 1080 & Midcontinent Rift & 47 \\
\hline $2300-1000$ & 1 & 1080 & Midcontinent Rift & 47 \\
\hline $2300-1000$ & 1 & 1080 & Midcontinent Rift & 47 \\
\hline $2300-1000$ & 1 & 1080 & Midcontinent Rift & 47 \\
\hline $2300-1000$ & 1 & 1080 & Midcontinent Rift & 47 \\
\hline $2300-1000$ & 1 & 1080 & Midcontinent Rift & 47 \\
\hline $2300-1000$ & 1 & 1080 & Midcontinent Rift & 47 \\
\hline $2300-1000$ & 1 & 1080 & Midcontinent Rift & 47 \\
\hline $2300-1000$ & 1 & 1080 & Midcontinent Rift & 47 \\
\hline $2300-1000$ & 1 & 1080 & Midcontinent Rift & 47 \\
\hline $2300-1000$ & 1 & 1080 & Midcontinent Rift & 47 \\
\hline $2300-1000$ & 1 & 1080 & Midcontinent Rift & 47 \\
\hline $2300-1000$ & 1 & 1080 & Midcontinent Rift & 47 \\
\hline $2300-1000$ & 1 & 1080 & Midcontinent Rift & 47 \\
\hline $2300-1000$ & 1 & 1080 & Midcontinent Rift & 47 \\
\hline $2300-1000$ & 1 & 1080 & Midcontinent Rift & 47 \\
\hline $2300-1000$ & 1 & 2150 & Botswana & 49 \\
\hline $2300-1000$ & 1 & 2150 & Botswana & 49 \\
\hline $2300-1000$ & 1 & 2150 & Botswana & 49 \\
\hline $2300-1000$ & 1 & 2150 & Botswana & 49 \\
\hline $2300-1000$ & 1 & 2150 & Botswana & 49 \\
\hline $2300-1000$ & 1 & 2150 & Botswana & 49 \\
\hline $2300-1000$ & 1 & 2150 & Botswana & 49 \\
\hline $2300-1000$ & 1 & 2150 & Botswana & 49 \\
\hline $2300-1000$ & 1 & 2150 & Botswana & 49 \\
\hline $2300-1000$ & 1 & 2150 & Botswana & 49 \\
\hline $2300-1000$ & 1 & 2150 & Botswana & 49 \\
\hline $2300-1000$ & 1 & 2150 & Botswana & 49 \\
\hline $2300-1000$ & 1 & 2150 & Botswana & 49 \\
\hline
\end{tabular}




\begin{tabular}{|c|c|c|c|c|}
\hline $2300-1000$ & 1 & 2150 & Botswana & 49 \\
\hline $2300-1000$ & 1 & 2150 & Botswana & \\
\hline $1000-635$ & 2 & 660 & South China & 1 \\
\hline $1000-635$ & 2 & 660 & South China & 1 \\
\hline $1000-635$ & 2 & 660 & South China & \\
\hline $1000-635$ & 2 & 660 & South China & $t$ \\
\hline $1000-635$ & 2 & 660 & South China & 1 \\
\hline $1000-635$ & 2 & 660 & South China & 1 \\
\hline $1000-635$ & 2 & 660 & South China & \\
\hline $1000-635$ & 2 & 660 & South China & \\
\hline $1000-635$ & 2 & 660 & South China & 1 \\
\hline $1000-635$ & 2 & 660 & South China & 1 \\
\hline $1000-635$ & 2 & 660 & South China & 1 \\
\hline $1000-635$ & 2 & 660 & South China & 1 \\
\hline $1000-635$ & 2 & 660 & South China & $\perp$ \\
\hline $1000-635$ & 2 & 660 & South China & 1 \\
\hline $1000-635$ & 2 & 660 & South China & 1 \\
\hline $1000-635$ & 2 & 660 & South China & 1 \\
\hline $1000-635$ & 2 & 660 & South China & 1 \\
\hline $1000-635$ & 2 & 660 & South China & 1 \\
\hline $1000-635$ & 2 & 660 & South China & 1 \\
\hline $1000-635$ & 2 & 660 & South China & 1 \\
\hline $1000-635$ & 2 & 660 & South China & 1 \\
\hline $1000-635$ & 2 & 660 & South China & 1 \\
\hline $1000-635$ & 2 & 660 & South China & 1 \\
\hline $1000-635$ & 2 & 660 & South China & 1 \\
\hline $1000-635$ & 2 & 660 & South China & $\perp$ \\
\hline $1000-635$ & 2 & 660 & South China & 1 \\
\hline $1000-635$ & 2 & 660 & South China & 1 \\
\hline $1000-635$ & 2 & 660 & South China & 1 \\
\hline $1000-635$ & 2 & 660 & South China & 1 \\
\hline $1000-635$ & 2 & 660 & South China & 1 \\
\hline $1000-635$ & 2 & 660 & South China & 1 \\
\hline $1000-635$ & 2 & 660 & South China & 1 \\
\hline $1000-635$ & 2 & 660 & South China & 1 \\
\hline $1000-635$ & 2 & 660 & South China & 1 \\
\hline $1000-635$ & 2 & 660 & South China & 1 \\
\hline $1000-635$ & 2 & 660 & South China & 1 \\
\hline $1000-635$ & 2 & 660 & South China & 1 \\
\hline $1000-635$ & 2 & 660 & South China & 1 \\
\hline $1000-635$ & 2 & 660 & South China & 1 \\
\hline
\end{tabular}




\begin{tabular}{|c|c|c|c|c|}
\hline $1000-635$ & 2 & 660 & South China & 1 \\
\hline $1000-635$ & 2 & 660 & South China & 1 \\
\hline $1000-635$ & 2 & 660 & South China & 1 \\
\hline $1000-635$ & 2 & 660 & South China & 1 \\
\hline $1000-635$ & 2 & 650 & Wernecke Mountains & 4 \\
\hline $1000-635$ & 2 & 650 & Wernecke Mountains & 4 \\
\hline $1000-635$ & 2 & 650 & Wernecke Mountains & 4 \\
\hline $1000-635$ & 2 & 650 & Wernecke Mountains & 4 \\
\hline $1000-635$ & 2 & 650 & Wernecke Mountains & 4 \\
\hline $1000-635$ & 2 & 650 & Wernecke Mountains & 4 \\
\hline $1000-635$ & 2 & 650 & Wernecke Mountains & 4 \\
\hline $1000-635$ & 2 & 650 & Wernecke Mountains & 4 \\
\hline $1000-635$ & 2 & 650 & Wernecke Mountains & 4 \\
\hline $1000-635$ & 2 & 650 & Wernecke Mountains & 4 \\
\hline $1000-635$ & 2 & 650 & Wernecke Mountains & 4 \\
\hline $1000-635$ & 2 & 650 & Wernecke Mountains & 4 \\
\hline $1000-635$ & 2 & 650 & Wernecke Mountains & 4 \\
\hline $1000-635$ & 2 & 650 & Wernecke Mountains & 4 \\
\hline $1000-635$ & 2 & 650 & Wernecke Mountains & 4 \\
\hline $1000-635$ & 2 & 650 & Wernecke Mountains & 4 \\
\hline $1000-635$ & 2 & 650 & Wernecke Mountains & 4 \\
\hline $1000-635$ & 2 & 650 & Wernecke Mountains & 4 \\
\hline $1000-635$ & 2 & 650 & Wernecke Mountains & 4 \\
\hline $1000-635$ & 2 & 650 & Wernecke Mountains & 4 \\
\hline $1000-635$ & 2 & 650 & Wernecke Mountains & 4 \\
\hline $1000-635$ & 2 & 650 & Wernecke Mountains & 4 \\
\hline $1000-635$ & 2 & 650 & Wernecke Mountains & 4 \\
\hline $1000-635$ & 2 & 650 & Wernecke Mountains & 4 \\
\hline $1000-635$ & 2 & 650 & Wernecke Mountains & 4 \\
\hline $1000-635$ & 2 & 650 & Wernecke Mountains & 4 \\
\hline $1000-635$ & 2 & 650 & Wernecke Mountains & 4 \\
\hline $1000-635$ & 2 & 750 & Death Valley & 11 \\
\hline $1000-635$ & 2 & 750 & Death Valley & 11 \\
\hline 1000-635 & 2 & 750 & Death Valley & 11 \\
\hline $1000-635$ & 2 & 750 & Death Valley & 11 \\
\hline 1000-635 & 2 & 750 & Death Valley & 11 \\
\hline $1000-635$ & 2 & 750 & Death Valley & 11 \\
\hline 1000-635 & 2 & 750 & Death Valley & 11 \\
\hline $1000-635$ & 2 & 750 & Death Valley & 11 \\
\hline 1000-635 & 2 & 750 & Death Valley & 11 \\
\hline $1000-635$ & 2 & 750 & Death Valley & 11 \\
\hline
\end{tabular}




\begin{tabular}{|c|c|c|c|c|}
\hline $1000-635$ & 2 & 750 & Death Valley & 11 \\
\hline $1000-635$ & 2 & 750 & Death Valley & 11 \\
\hline $1000-635$ & 2 & 750 & Death Valley & 11 \\
\hline $1000-635$ & 2 & 750 & Death Valley & 11 \\
\hline $1000-635$ & 2 & 750 & Death Valley & 11 \\
\hline $1000-635$ & 2 & 750 & Death Valley & 11 \\
\hline $1000-635$ & 2 & 750 & Death Valley & 11 \\
\hline $1000-635$ & 2 & 750 & Death Valley & 11 \\
\hline $1000-635$ & 2 & 750 & Death Valley & 11 \\
\hline $1000-635$ & 2 & 750 & Death Valley & 11 \\
\hline 1000-635 & 2 & 750 & Death Valley & 11 \\
\hline $1000-635$ & 2 & 750 & Death Valley & 11 \\
\hline $1000-635$ & 2 & 750 & Death Valley & 11 \\
\hline $1000-635$ & 2 & 750 & Death Valley & 11 \\
\hline $1000-635$ & 2 & 750 & Death Valley & 11 \\
\hline $1000-635$ & 2 & 750 & Death Valley & 11 \\
\hline $1000-635$ & 2 & 750 & Death Valley & 11 \\
\hline $1000-635$ & 2 & 750 & Death Valley & 11 \\
\hline $1000-635$ & 2 & 750 & Death Valley & 11 \\
\hline 1000-635 & 2 & 750 & Death Valley & 11 \\
\hline $1000-635$ & 2 & 750 & Death Valley & 11 \\
\hline 1000-635 & 2 & 750 & Death Valley & 11 \\
\hline $1000-635$ & 2 & 750 & Death Valley & 11 \\
\hline 1000-635 & 2 & 750 & Death Valley & 11 \\
\hline $1000-635$ & 2 & 750 & Death Valley & 11 \\
\hline $1000-635$ & 2 & 750 & Death Valley & 11 \\
\hline $1000-635$ & 2 & 750 & Death Valley & 11 \\
\hline $1000-635$ & 2 & 750 & Death Valley & 11 \\
\hline $1000-635$ & 2 & 750 & Death Valley & 11 \\
\hline $1000-635$ & 2 & 750 & Death Valley & 11 \\
\hline $1000-635$ & 2 & 750 & Death Valley & 11 \\
\hline $1000-635$ & 2 & 750 & Death Valley & 11 \\
\hline $1000-635$ & 2 & 750 & Death Valley & 11 \\
\hline $1000-635$ & 2 & 750 & Death Valley & 11 \\
\hline $1000-635$ & 2 & 750 & Death Valley & 11 \\
\hline $1000-635$ & 2 & 730 & Death Valley & 11 \\
\hline $1000-635$ & 2 & 730 & Death Valley & 11 \\
\hline 1000-635 & 2 & 730 & Death Valley & 11 \\
\hline $1000-635$ & 2 & 730 & Death Valley & 11 \\
\hline 1000-635 & 2 & 730 & Death Valley & 11 \\
\hline $1000-635$ & 2 & 730 & Death Valley & 11 \\
\hline
\end{tabular}




\begin{tabular}{|c|c|c|c|}
\hline $1000-635$ & 2 & 730 & Death Valley \\
\hline $1000-635$ & 2 & 730 & Death Valley \\
\hline $1000-635$ & 2 & 730 & Death Valley \\
\hline $1000-635$ & 2 & 730 & Death Valley \\
\hline $1000-635$ & 2 & 730 & Death Valley \\
\hline $1000-635$ & 2 & 730 & Death Valley \\
\hline $1000-635$ & 2 & 730 & Death Valley \\
\hline $1000-635$ & 2 & 730 & Death Valley \\
\hline $1000-635$ & 2 & 730 & Death Valley \\
\hline $1000-635$ & 2 & 730 & Death Valley \\
\hline $1000-635$ & 2 & 730 & Death Valley \\
\hline $1000-635$ & 2 & 730 & Death Valley \\
\hline $1000-635$ & 2 & 730 & Death Valley \\
\hline $1000-635$ & 2 & 730 & Death Valley \\
\hline $1000-635$ & 2 & 730 & Death Valley \\
\hline $1000-635$ & 2 & 730 & Death Valley \\
\hline $1000-635$ & 2 & 730 & Death Valley \\
\hline $1000-635$ & 2 & 730 & Death Valley \\
\hline $1000-635$ & 2 & 730 & Death Valley \\
\hline $1000-635$ & 2 & 730 & Death Valley \\
\hline $1000-635$ & 2 & 730 & Death Valley \\
\hline $1000-635$ & 2 & 730 & Death Valley \\
\hline $1000-635$ & 2 & 730 & Death Valley \\
\hline $1000-635$ & 2 & 730 & Death Valley \\
\hline $1000-635$ & 2 & 730 & Death Valley \\
\hline $1000-635$ & 2 & 730 & Death Valley \\
\hline $1000-635$ & 2 & 730 & Death Valley \\
\hline $1000-635$ & 2 & 730 & Death Valley \\
\hline $1000-635$ & 2 & 750 & Death Valley \\
\hline $1000-635$ & 2 & 750 & Death Valley \\
\hline $1000-635$ & 2 & 750 & Death Valley \\
\hline $1000-635$ & 2 & 750 & Death Valley \\
\hline $1000-635$ & 2 & 750 & Death Valley \\
\hline $1000-635$ & 2 & 750 & Death Valley \\
\hline $1000-635$ & 2 & 750 & Death Valley \\
\hline $1000-635$ & 2 & 750 & Death Valley \\
\hline 1000-635 & 2 & 750 & Death Valley \\
\hline $1000-635$ & 2 & 750 & Death Valley \\
\hline $1000-635$ & 2 & 750 & Death Valley \\
\hline $1000-635$ & 2 & 750 & Death Valley \\
\hline $1000-635$ & 2 & 750 & Death Valley \\
\hline
\end{tabular}




\begin{tabular}{|c|c|c|c|c|}
\hline $1000-635$ & 2 & 750 & Death Valley & 11 \\
\hline $1000-635$ & 2 & 750 & Death Valley & 11 \\
\hline $1000-635$ & 2 & 750 & Death Valley & 11 \\
\hline $1000-635$ & 2 & 730 & Death Valley & 11 \\
\hline $1000-635$ & 2 & 730 & Death Valley & 11 \\
\hline $1000-635$ & 2 & 730 & Death Valley & 11 \\
\hline $1000-635$ & 2 & 730 & Death Valley & 11 \\
\hline $1000-635$ & 2 & 730 & Death Valley & 11 \\
\hline $1000-635$ & 2 & 750 & Grand Canyon & 12 \\
\hline $1000-635$ & 2 & 750 & Grand Canyon & 12 \\
\hline 1000-635 & 2 & 750 & Grand Canyon & 12 \\
\hline $1000-635$ & 2 & 750 & Grand Canyon & 12 \\
\hline $1000-635$ & 2 & 750 & Grand Canyon & 12 \\
\hline $1000-635$ & 2 & 750 & Grand Canyon & 12 \\
\hline 1000-635 & 2 & 750 & Grand Canyon & 12 \\
\hline $1000-635$ & 2 & 750 & Grand Canyon & 12 \\
\hline $1000-635$ & 2 & 750 & Grand Canyon & 12 \\
\hline $1000-635$ & 2 & 750 & Grand Canyon & 12 \\
\hline $1000-635$ & 2 & 750 & Grand Canyon & 12 \\
\hline 1000-635 & 2 & 750 & Grand Canyon & 12 \\
\hline $1000-635$ & 2 & 750 & Grand Canyon & 12 \\
\hline 1000-635 & 2 & 750 & Grand Canyon & 12 \\
\hline $1000-635$ & 2 & 750 & Grand Canyon & 12 \\
\hline 1000-635 & 2 & 750 & Grand Canyon & 12 \\
\hline 1000-635 & 2 & 750 & Grand Canyon & 12 \\
\hline $1000-635$ & 2 & 750 & Grand Canyon & 12 \\
\hline $1000-635$ & 2 & 750 & Grand Canyon & 12 \\
\hline $1000-635$ & 2 & 750 & Grand Canyon & 12 \\
\hline $1000-635$ & 2 & 750 & Grand Canyon & 12 \\
\hline $1000-635$ & 2 & 750 & Grand Canyon & 12 \\
\hline $1000-635$ & 2 & 750 & Grand Canyon & 12 \\
\hline $1000-635$ & 2 & 750 & Grand Canyon & 12 \\
\hline $1000-635$ & 2 & 750 & Grand Canyon & 12 \\
\hline 1000-635 & 2 & 750 & Grand Canyon & 12 \\
\hline $1000-635$ & 2 & 750 & Grand Canyon & 12 \\
\hline $1000-635$ & 2 & 750 & Grand Canyon & 12 \\
\hline $1000-635$ & 2 & 750 & Grand Canyon & 12 \\
\hline 1000-635 & 2 & 750 & Grand Canyon & 12 \\
\hline $1000-635$ & 2 & 750 & Grand Canyon & 12 \\
\hline 1000-635 & 2 & 750 & Grand Canyon & 12 \\
\hline $1000-635$ & 2 & 750 & Grand Canyon & 12 \\
\hline
\end{tabular}




\begin{tabular}{|c|c|c|c|c|}
\hline $1000-635$ & 2 & 750 & Grand Canyon & 12 \\
\hline $1000-635$ & 2 & 750 & Grand Canyon & 12 \\
\hline $1000-635$ & 2 & 750 & Grand Canyon & 12 \\
\hline $1000-635$ & 2 & 750 & Grand Canyon & 12 \\
\hline $1000-635$ & 2 & 750 & Grand Canyon & 12 \\
\hline $1000-635$ & 2 & 750 & Grand Canyon & 12 \\
\hline $1000-635$ & 2 & 750 & Grand Canyon & 12 \\
\hline $1000-635$ & 2 & 750 & Grand Canyon & 12 \\
\hline $1000-635$ & 2 & 750 & Grand Canyon & 12 \\
\hline $1000-635$ & 2 & 750 & Grand Canyon & 12 \\
\hline 1000-635 & 2 & 750 & Grand Canyon & 12 \\
\hline $1000-635$ & 2 & 750 & Grand Canyon & 12 \\
\hline $1000-635$ & 2 & 750 & Grand Canyon & 12 \\
\hline $1000-635$ & 2 & 750 & Grand Canyon & 12 \\
\hline 1000-635 & 2 & 750 & Grand Canyon & 12 \\
\hline $1000-635$ & 2 & 750 & Grand Canyon & 12 \\
\hline $1000-635$ & 2 & 750 & Grand Canyon & 12 \\
\hline $1000-635$ & 2 & 750 & Grand Canyon & 12 \\
\hline $1000-635$ & 2 & 750 & Grand Canyon & 12 \\
\hline 1000-635 & 2 & 750 & Grand Canyon & 12 \\
\hline $1000-635$ & 2 & 750 & Grand Canyon & 12 \\
\hline 1000-635 & 2 & 750 & Grand Canyon & 12 \\
\hline $1000-635$ & 2 & 750 & Grand Canyon & 12 \\
\hline 1000-635 & 2 & 750 & Grand Canyon & 12 \\
\hline 1000-635 & 2 & 750 & Grand Canyon & 12 \\
\hline $1000-635$ & 2 & 750 & Grand Canyon & 12 \\
\hline $1000-635$ & 2 & 750 & Grand Canyon & 12 \\
\hline $1000-635$ & 2 & 750 & Grand Canyon & 12 \\
\hline $1000-635$ & 2 & 750 & Grand Canyon & 12 \\
\hline 1000-635 & 2 & 750 & Grand Canyon & 12 \\
\hline $1000-635$ & 2 & 750 & Grand Canyon & 12 \\
\hline $1000-635$ & 2 & 750 & Grand Canyon & 12 \\
\hline $1000-635$ & 2 & 750 & Grand Canyon & 12 \\
\hline 1000-635 & 2 & 750 & Grand Canyon & 12 \\
\hline $1000-635$ & 2 & 750 & Grand Canyon & 12 \\
\hline $1000-635$ & 2 & 750 & Grand Canyon & 12 \\
\hline $1000-635$ & 2 & 750 & Grand Canyon & 12 \\
\hline 1000-635 & 2 & 750 & Grand Canyon & 12 \\
\hline $1000-635$ & 2 & 750 & Grand Canyon & 12 \\
\hline 1000-635 & 2 & 750 & Grand Canyon & 12 \\
\hline $1000-635$ & 2 & 750 & Grand Canyon & 12 \\
\hline
\end{tabular}




\begin{tabular}{|c|c|c|c|c|}
\hline $1000-635$ & 2 & 750 & Grand Canyon & 12 \\
\hline $1000-635$ & 2 & 750 & Grand Canyon & 12 \\
\hline $1000-635$ & 2 & 750 & Grand Canyon & 12 \\
\hline $1000-635$ & 2 & 750 & Grand Canyon & 12 \\
\hline $1000-635$ & 2 & 750 & Grand Canyon & 12 \\
\hline $1000-635$ & 2 & 750 & Grand Canyon & 12 \\
\hline $1000-635$ & 2 & 750 & Grand Canyon & 12 \\
\hline $1000-635$ & 2 & 750 & Grand Canyon & 12 \\
\hline $1000-635$ & 2 & 750 & Grand Canyon & 12 \\
\hline $1000-635$ & 2 & 750 & Grand Canyon & 12 \\
\hline 1000-635 & 2 & 750 & Grand Canyon & 12 \\
\hline $1000-635$ & 2 & 750 & Grand Canyon & 12 \\
\hline $1000-635$ & 2 & 750 & Grand Canyon & 12 \\
\hline $1000-635$ & 2 & 750 & Grand Canyon & 12 \\
\hline 1000-635 & 2 & 750 & Grand Canyon & 12 \\
\hline $1000-635$ & 2 & 750 & Grand Canyon & 12 \\
\hline $1000-635$ & 2 & 750 & Grand Canyon & 12 \\
\hline $1000-635$ & 2 & 750 & Grand Canyon & 12 \\
\hline $1000-635$ & 2 & 750 & Grand Canyon & 12 \\
\hline 1000-635 & 2 & 750 & Grand Canyon & 12 \\
\hline $1000-635$ & 2 & 750 & Grand Canyon & 12 \\
\hline 1000-635 & 2 & 750 & Grand Canyon & 12 \\
\hline $1000-635$ & 2 & 750 & Grand Canyon & 12 \\
\hline 1000-635 & 2 & 750 & Grand Canyon & 12 \\
\hline 1000-635 & 2 & 750 & Grand Canyon & 12 \\
\hline $1000-635$ & 2 & 750 & Grand Canyon & 12 \\
\hline $1000-635$ & 2 & 750 & Grand Canyon & 12 \\
\hline $1000-635$ & 2 & 750 & Grand Canyon & 12 \\
\hline $1000-635$ & 2 & 750 & Grand Canyon & 12 \\
\hline 1000-635 & 2 & 750 & Grand Canyon & 12 \\
\hline $1000-635$ & 2 & 750 & Grand Canyon & 12 \\
\hline $1000-635$ & 2 & 750 & Grand Canyon & 12 \\
\hline $1000-635$ & 2 & 750 & Grand Canyon & 12 \\
\hline 1000-635 & 2 & 750 & Grand Canyon & 12 \\
\hline $1000-635$ & 2 & 750 & Grand Canyon & 12 \\
\hline $1000-635$ & 2 & 750 & Grand Canyon & 12 \\
\hline $1000-635$ & 2 & 750 & Grand Canyon & 12 \\
\hline 1000-635 & 2 & 750 & Grand Canyon & 12 \\
\hline $1000-635$ & 2 & 750 & Grand Canyon & 12 \\
\hline 1000-635 & 2 & 750 & Grand Canyon & 12 \\
\hline $1000-635$ & 2 & 750 & Grand Canyon & 12 \\
\hline
\end{tabular}




\begin{tabular}{|c|c|c|c|c|}
\hline $1000-635$ & 2 & 750 & Grand Canyon & 12 \\
\hline $1000-635$ & 2 & 750 & Grand Canyon & 12 \\
\hline $1000-635$ & 2 & 750 & Grand Canyon & 12 \\
\hline $1000-635$ & 2 & 750 & Grand Canyon & 12 \\
\hline $1000-635$ & 2 & 750 & Grand Canyon & 12 \\
\hline $1000-635$ & 2 & 750 & Grand Canyon & 12 \\
\hline $1000-635$ & 2 & 800 & Eastern European Platforr & 14 \\
\hline $1000-635$ & 2 & 800 & Eastern European Platforr & 14 \\
\hline $1000-635$ & 2 & 800 & Eastern European Platforr & 14 \\
\hline $1000-635$ & 2 & 800 & Eastern European Platforr & 14 \\
\hline $1000-635$ & 2 & 800 & Eastern European Platforr & 14 \\
\hline $1000-635$ & 2 & 800 & Eastern European Platforr & 14 \\
\hline $1000-635$ & 2 & 800 & Eastern European Platforr & 14 \\
\hline $1000-635$ & 2 & 800 & Eastern European Platforr & 14 \\
\hline $1000-635$ & 2 & 800 & Eastern European Platforr & 14 \\
\hline $1000-635$ & 2 & 800 & Eastern European Platforr & 14 \\
\hline $1000-635$ & 2 & 800 & Eastern European Platforr & 14 \\
\hline $1000-635$ & 2 & 800 & Eastern European Platforr & 14 \\
\hline $1000-635$ & 2 & 800 & Eastern European Platforr & 14 \\
\hline $1000-635$ & 2 & 800 & Eastern European Platforr & 14 \\
\hline $1000-635$ & 2 & 800 & Eastern European Platforr & 14 \\
\hline $1000-635$ & 2 & 800 & Eastern European Platforr & 14 \\
\hline $1000-635$ & 2 & 800 & Eastern European Platforr & 14 \\
\hline $1000-635$ & 2 & 800 & Eastern European Platforr & 14 \\
\hline $1000-635$ & 2 & 805 & Centralian Superbasin & 27 \\
\hline $1000-635$ & 2 & 805 & Centralian Superbasin & 27 \\
\hline $1000-635$ & 2 & 805 & Centralian Superbasin & 27 \\
\hline $1000-635$ & 2 & 660 & Centralian Superbasin & 27 \\
\hline $1000-635$ & 2 & 660 & Centralian Superbasin & 27 \\
\hline $1000-635$ & 2 & 660 & Centralian Superbasin & 27 \\
\hline $1000-635$ & 2 & 660 & Centralian Superbasin & 27 \\
\hline $1000-635$ & 2 & 660 & Centralian Superbasin & 27 \\
\hline $1000-635$ & 2 & 660 & Centralian Superbasin & 27 \\
\hline $1000-635$ & 2 & 660 & Centralian Superbasin & 27 \\
\hline $1000-635$ & 2 & 660 & Centralian Superbasin & 27 \\
\hline $1000-635$ & 2 & 660 & Centralian Superbasin & 27 \\
\hline $1000-635$ & 2 & 660 & Centralian Superbasin & 27 \\
\hline 1000-635 & 2 & 660 & Centralian Superbasin & 27 \\
\hline $1000-635$ & 2 & 660 & Centralian Superbasin & 27 \\
\hline 1000-635 & 2 & 660 & Centralian Superbasin & 27 \\
\hline $1000-635$ & 2 & 660 & Centralian Superbasin & 27 \\
\hline
\end{tabular}




\begin{tabular}{|c|c|c|c|c|}
\hline $1000-635$ & 2 & 660 & Centralian Superbasin & 27 \\
\hline $1000-635$ & 2 & 660 & Centralian Superbasin & 27 \\
\hline $1000-635$ & 2 & 660 & Centralian Superbasin & 27 \\
\hline $1000-635$ & 2 & 660 & Centralian Superbasin & 27 \\
\hline $1000-635$ & 2 & 660 & Centralian Superbasin & 27 \\
\hline $1000-635$ & 2 & 660 & Centralian Superbasin & 27 \\
\hline $1000-635$ & 2 & 660 & Centralian Superbasin & 27 \\
\hline $1000-635$ & 2 & 660 & Centralian Superbasin & 27 \\
\hline $1000-635$ & 2 & 660 & Centralian Superbasin & 27 \\
\hline $1000-635$ & 2 & 660 & Centralian Superbasin & 27 \\
\hline $1000-635$ & 2 & 660 & Centralian Superbasin & 27 \\
\hline $1000-635$ & 2 & 660 & Centralian Superbasin & 27 \\
\hline $1000-635$ & 2 & 660 & Centralian Superbasin & 27 \\
\hline $1000-635$ & 2 & 660 & Centralian Superbasin & 27 \\
\hline $1000-635$ & 2 & 660 & Centralian Superbasin & 27 \\
\hline $1000-635$ & 2 & 660 & Centralian Superbasin & 27 \\
\hline $1000-635$ & 2 & 660 & Centralian Superbasin & 27 \\
\hline $1000-635$ & 2 & 660 & Centralian Superbasin & 27 \\
\hline $1000-635$ & 2 & 660 & Centralian Superbasin & 27 \\
\hline $1000-635$ & 2 & 660 & Centralian Superbasin & 27 \\
\hline $1000-635$ & 2 & 660 & Centralian Superbasin & 27 \\
\hline $1000-635$ & 2 & 660 & Centralian Superbasin & 27 \\
\hline $1000-635$ & 2 & 660 & Centralian Superbasin & 27 \\
\hline $1000-635$ & 2 & 660 & Centralian Superbasin & 27 \\
\hline $1000-635$ & 2 & 660 & Centralian Superbasin & 27 \\
\hline $1000-635$ & 2 & 660 & Centralian Superbasin & 27 \\
\hline $1000-635$ & 2 & 660 & Centralian Superbasin & 27 \\
\hline $1000-635$ & 2 & 660 & Centralian Superbasin & 27 \\
\hline $1000-635$ & 2 & 660 & Centralian Superbasin & 27 \\
\hline $1000-635$ & 2 & 660 & Centralian Superbasin & 27 \\
\hline $1000-635$ & 2 & 660 & Centralian Superbasin & 27 \\
\hline $1000-635$ & 2 & 660 & Centralian Superbasin & 27 \\
\hline $1000-635$ & 2 & 660 & Centralian Superbasin & 27 \\
\hline $1000-635$ & 2 & 660 & Centralian Superbasin & 27 \\
\hline $1000-635$ & 2 & 660 & Centralian Superbasin & 27 \\
\hline $1000-635$ & 2 & 660 & Centralian Superbasin & 27 \\
\hline $1000-635$ & 2 & 660 & Centralian Superbasin & 27 \\
\hline $1000-635$ & 2 & 660 & Centralian Superbasin & 27 \\
\hline $1000-635$ & 2 & 660 & Centralian Superbasin & 27 \\
\hline $1000-635$ & 2 & 660 & Centralian Superbasin & 27 \\
\hline $1000-635$ & 2 & 660 & Centralian Superbasin & 27 \\
\hline
\end{tabular}




\begin{tabular}{|c|c|c|c|c|}
\hline $1000-635$ & 2 & 660 & Centralian Superbasin & 27 \\
\hline $1000-635$ & 2 & 660 & Centralian Superbasin & 27 \\
\hline $1000-635$ & 2 & 660 & Centralian Superbasin & 27 \\
\hline $1000-635$ & 2 & 660 & Centralian Superbasin & 27 \\
\hline $1000-635$ & 2 & 660 & Centralian Superbasin & 27 \\
\hline $1000-635$ & 2 & 660 & Centralian Superbasin & 27 \\
\hline $1000-635$ & 2 & 660 & Centralian Superbasin & 27 \\
\hline $1000-635$ & 2 & 660 & Centralian Superbasin & 27 \\
\hline $1000-635$ & 2 & 660 & Centralian Superbasin & 27 \\
\hline $1000-635$ & 2 & 660 & Centralian Superbasin & 27 \\
\hline $1000-635$ & 2 & 660 & Centralian Superbasin & 27 \\
\hline $1000-635$ & 2 & 660 & Centralian Superbasin & 27 \\
\hline $1000-635$ & 2 & 660 & Centralian Superbasin & 27 \\
\hline $1000-635$ & 2 & 660 & Centralian Superbasin & 27 \\
\hline $1000-635$ & 2 & 660 & Centralian Superbasin & 27 \\
\hline $1000-635$ & 2 & 660 & Centralian Superbasin & 27 \\
\hline $1000-635$ & 2 & 660 & Centralian Superbasin & 27 \\
\hline $1000-635$ & 2 & 660 & Centralian Superbasin & 27 \\
\hline $1000-635$ & 2 & 660 & Centralian Superbasin & 27 \\
\hline $1000-635$ & 2 & 660 & Centralian Superbasin & 27 \\
\hline $1000-635$ & 2 & 660 & Centralian Superbasin & 27 \\
\hline $1000-635$ & 2 & 660 & Centralian Superbasin & 27 \\
\hline $1000-635$ & 2 & 660 & Centralian Superbasin & 27 \\
\hline $1000-635$ & 2 & 660 & Centralian Superbasin & 27 \\
\hline $1000-635$ & 2 & 660 & Centralian Superbasin & 27 \\
\hline $1000-635$ & 2 & 660 & Centralian Superbasin & 27 \\
\hline $1000-635$ & 2 & 660 & Centralian Superbasin & 27 \\
\hline $1000-635$ & 2 & 660 & Centralian Superbasin & 27 \\
\hline $1000-635$ & 2 & 660 & Centralian Superbasin & 27 \\
\hline $1000-635$ & 2 & 660 & Centralian Superbasin & 27 \\
\hline $1000-635$ & 2 & 660 & Centralian Superbasin & 27 \\
\hline $1000-635$ & 2 & 660 & Centralian Superbasin & 27 \\
\hline $1000-635$ & 2 & 660 & Centralian Superbasin & 27 \\
\hline $1000-635$ & 2 & 660 & Centralian Superbasin & 27 \\
\hline $1000-635$ & 2 & 660 & Centralian Superbasin & 27 \\
\hline $1000-635$ & 2 & 660 & Centralian Superbasin & 27 \\
\hline $1000-635$ & 2 & 660 & Centralian Superbasin & 27 \\
\hline $1000-635$ & 2 & 660 & Centralian Superbasin & 27 \\
\hline $1000-635$ & 2 & 660 & Centralian Superbasin & 27 \\
\hline $1000-635$ & 2 & 660 & Centralian Superbasin & 27 \\
\hline $1000-635$ & 2 & 660 & Centralian Superbasin & 27 \\
\hline
\end{tabular}




\begin{tabular}{|c|c|c|c|c|}
\hline $1000-635$ & 2 & 660 & Centralian Superbasin & 27 \\
\hline $1000-635$ & 2 & 660 & Centralian Superbasin & 27 \\
\hline $1000-635$ & 2 & 660 & Centralian Superbasin & 27 \\
\hline $1000-635$ & 2 & 660 & Centralian Superbasin & 27 \\
\hline $1000-635$ & 2 & 660 & Centralian Superbasin & 27 \\
\hline $1000-635$ & 2 & 660 & Centralian Superbasin & 27 \\
\hline $1000-635$ & 2 & 660 & Centralian Superbasin & 27 \\
\hline $1000-635$ & 2 & 660 & Centralian Superbasin & 27 \\
\hline $1000-635$ & 2 & 660 & Centralian Superbasin & 27 \\
\hline $1000-635$ & 2 & 660 & Centralian Superbasin & 27 \\
\hline $1000-635$ & 2 & 650 & Spitsbergen & 29 \\
\hline $1000-635$ & 2 & 650 & Spitsbergen & 29 \\
\hline $1000-635$ & 2 & 650 & Spitsbergen & 29 \\
\hline $1000-635$ & 2 & 650 & Spitsbergen & 29 \\
\hline $1000-635$ & 2 & 650 & Spitsbergen & 29 \\
\hline $1000-635$ & 2 & 650 & Spitsbergen & 29 \\
\hline $1000-635$ & 2 & 650 & Spitsbergen & 29 \\
\hline $1000-635$ & 2 & 650 & Spitsbergen & 29 \\
\hline $1000-635$ & 2 & 650 & Spitsbergen & 29 \\
\hline $1000-635$ & 2 & 750 & East Greenland & 30 \\
\hline $1000-635$ & 2 & 750 & East Greenland & 30 \\
\hline $1000-635$ & 2 & 750 & East Greenland & 30 \\
\hline $1000-635$ & 2 & 750 & East Greenland & 30 \\
\hline $1000-635$ & 2 & 750 & East Greenland & 30 \\
\hline $1000-635$ & 2 & 750 & East Greenland & 30 \\
\hline $1000-635$ & 2 & 750 & East Greenland & 30 \\
\hline $1000-635$ & 2 & 750 & East Greenland & 30 \\
\hline $1000-635$ & 2 & 750 & East Greenland & 30 \\
\hline $1000-635$ & 2 & 750 & East Greenland & 30 \\
\hline $1000-635$ & 2 & 750 & East Greenland & 30 \\
\hline $1000-635$ & 2 & 750 & East Greenland & 30 \\
\hline $1000-635$ & 2 & 750 & East Greenland & 30 \\
\hline $1000-635$ & 2 & 750 & East Greenland & 30 \\
\hline $1000-635$ & 2 & 775 & East Greenland & 30 \\
\hline $1000-635$ & 2 & 775 & East Greenland & 30 \\
\hline $1000-635$ & 2 & 770 & East Greenland & 30 \\
\hline $1000-635$ & 2 & 790 & East Greenland & 30 \\
\hline $1000-635$ & 2 & 790 & East Greenland & 30 \\
\hline $1000-635$ & 2 & 790 & East Greenland & 30 \\
\hline 1000-635 & 2 & 790 & East Greenland & 30 \\
\hline $1000-635$ & 2 & 790 & East Greenland & 30 \\
\hline
\end{tabular}




\begin{tabular}{|c|c|c|c|c|}
\hline $1000-635$ & 2 & 790 & East Greenland & 30 \\
\hline $1000-635$ & 2 & 790 & East Greenland & 30 \\
\hline $1000-635$ & 2 & 790 & East Greenland & 30 \\
\hline $1000-635$ & 2 & 790 & East Greenland & 30 \\
\hline $1000-635$ & 2 & 790 & East Greenland & 30 \\
\hline $1000-635$ & 2 & 790 & East Greenland & 30 \\
\hline $1000-635$ & 2 & 660 & East Greenland & ט \\
\hline $1000-635$ & 2 & 660 & East Greenland & 30 \\
\hline $1000-635$ & 2 & 660 & East Greenland & 30 \\
\hline $1000-635$ & 2 & 660 & East Greenland & 30 \\
\hline $1000-635$ & 2 & 660 & East Greenland & 30 \\
\hline $1000-635$ & 2 & 660 & East Greenland & 30 \\
\hline $1000-635$ & 2 & 660 & East Greenland & 30 \\
\hline $1000-635$ & 2 & 660 & East Greenland & 30 \\
\hline $1000-635$ & 2 & 660 & East Greenland & 30 \\
\hline $1000-635$ & 2 & 660 & East Greenland & 0 \\
\hline $1000-635$ & 2 & 660 & East Greenland & 30 \\
\hline $1000-635$ & 2 & 660 & East Greenland & 30 \\
\hline $1000-635$ & 2 & 660 & East Greenland & 30 \\
\hline $1000-635$ & 2 & 660 & East Greenland & 30 \\
\hline $1000-635$ & 2 & 660 & East Greenland & 30 \\
\hline $1000-635$ & 2 & 660 & East Greenland & 30 \\
\hline $1000-635$ & 2 & 659.36 & East Greenland & 30 \\
\hline $1000-635$ & 2 & 659.39 & East Greenland & 30 \\
\hline $1000-635$ & 2 & 659.89 & East Greenland & 30 \\
\hline $1000-635$ & 2 & 661.86 & East Greenland & 30 \\
\hline $1000-635$ & 2 & 662.24 & East Greenland & 30 \\
\hline $1000-635$ & 2 & 662.39 & East Greenland & 30 \\
\hline $1000-635$ & 2 & 662.77 & East Greenland & 30 \\
\hline $1000-635$ & 2 & 800 & Ogilvie Mountains & 36 \\
\hline $1000-635$ & 2 & 800 & Ogilvie Mountains & 36 \\
\hline $1000-635$ & 2 & 800 & Ogilvie Mountains & 36 \\
\hline $1000-635$ & 2 & 800 & Ogilvie Mountains & 36 \\
\hline $1000-635$ & 2 & 800 & Ogilvie Mountains & 36 \\
\hline $1000-635$ & 2 & 800 & Ogilvie Mountains & 36 \\
\hline $1000-635$ & 2 & 800 & Ogilvie Mountains & 36 \\
\hline $1000-635$ & 2 & 800 & Ogilvie Mountains & 36 \\
\hline $1000-635$ & 2 & 800 & Ogilvie Mountains & 36 \\
\hline $1000-635$ & 2 & 800 & Ogilvie Mountains & 36 \\
\hline $1000-635$ & 2 & 800 & Ogilvie Mountains & 36 \\
\hline $1000-635$ & 2 & 800 & Ogilvie Mountains & 36 \\
\hline
\end{tabular}




\begin{tabular}{|c|c|c|c|c|}
\hline $1000-635$ & 2 & 800 & Ogilvie Mountains & 36 \\
\hline $1000-635$ & 2 & 800 & Ogilvie Mountains & 36 \\
\hline $1000-635$ & 2 & 800 & Ogilvie Mountains & 36 \\
\hline $1000-635$ & 2 & 800 & Ogilvie Mountains & 36 \\
\hline $1000-635$ & 2 & 800 & Ogilvie Mountains & 36 \\
\hline $1000-635$ & 2 & 800 & Ogilvie Mountains & 36 \\
\hline $1000-635$ & 2 & 800 & Ogilvie Mountains & 36 \\
\hline $1000-635$ & 2 & 800 & Ogilvie Mountains & 36 \\
\hline $1000-635$ & 2 & 800 & Ogilvie Mountains & 36 \\
\hline $1000-635$ & 2 & 800 & Ogilvie Mountains & 36 \\
\hline $1000-635$ & 2 & 800 & Ogilvie Mountains & 36 \\
\hline $1000-635$ & 2 & 800 & Ogilvie Mountains & 36 \\
\hline $1000-635$ & 2 & 800 & Ogilvie Mountains & 36 \\
\hline $1000-635$ & 2 & 800 & Ogilvie Mountains & 36 \\
\hline $1000-635$ & 2 & 800 & Ogilvie Mountains & 36 \\
\hline $1000-635$ & 2 & 800 & Ogilvie Mountains & 36 \\
\hline $1000-635$ & 2 & 800 & Ogilvie Mountains & 36 \\
\hline $1000-635$ & 2 & 800 & Ogilvie Mountains & 36 \\
\hline $1000-635$ & 2 & 800 & Ogilvie Mountains & 36 \\
\hline $1000-635$ & 2 & 800 & Ogilvie Mountains & 36 \\
\hline $1000-635$ & 2 & 800 & Ogilvie Mountains & 36 \\
\hline $1000-635$ & 2 & 800 & Ogilvie Mountains & 36 \\
\hline $1000-635$ & 2 & 800 & Ogilvie Mountains & 36 \\
\hline $1000-635$ & 2 & 800 & Ogilvie Mountains & 36 \\
\hline $1000-635$ & 2 & 800 & Ogilvie Mountains & 36 \\
\hline $1000-635$ & 2 & 800 & Ogilvie Mountains & 36 \\
\hline $1000-635$ & 2 & 800 & Ogilvie Mountains & 36 \\
\hline $1000-635$ & 2 & 800 & Ogilvie Mountains & 36 \\
\hline $1000-635$ & 2 & 800 & Ogilvie Mountains & 36 \\
\hline $1000-635$ & 2 & 800 & Ogilvie Mountains & 36 \\
\hline $1000-635$ & 2 & 800 & Ogilvie Mountains & 36 \\
\hline $1000-635$ & 2 & 800 & Ogilvie Mountains & 36 \\
\hline $1000-635$ & 2 & 800 & Ogilvie Mountains & 36 \\
\hline $1000-635$ & 2 & 800 & Ogilvie Mountains & 36 \\
\hline $1000-635$ & 2 & 800 & Ogilvie Mountains & 36 \\
\hline $1000-635$ & 2 & 800 & Ogilvie Mountains & 36 \\
\hline $1000-635$ & 2 & 800 & Ogilvie Mountains & 36 \\
\hline $1000-635$ & 2 & 800 & Ogilvie Mountains & 36 \\
\hline $1000-635$ & 2 & 800 & Ogilvie Mountains & 36 \\
\hline $1000-635$ & 2 & 800 & Ogilvie Mountains & 36 \\
\hline $1000-635$ & 2 & 800 & Ogilvie Mountains & 36 \\
\hline
\end{tabular}




\begin{tabular}{|c|c|c|c|c|}
\hline $1000-635$ & 2 & 800 & Ogilvie Mountains & 36 \\
\hline $1000-635$ & 2 & 800 & Ogilvie Mountains & 36 \\
\hline $1000-635$ & 2 & 800 & Ogilvie Mountains & 36 \\
\hline $1000-635$ & 2 & 800 & Ogilvie Mountains & 36 \\
\hline $1000-635$ & 2 & 800 & Ogilvie Mountains & 36 \\
\hline $1000-635$ & 2 & 800 & Ogilvie Mountains & 36 \\
\hline $1000-635$ & 2 & 800 & Ogilvie Mountains & 36 \\
\hline $1000-635$ & 2 & 800 & Ogilvie Mountains & 36 \\
\hline $1000-635$ & 2 & 800 & Ogilvie Mountains & 36 \\
\hline $1000-635$ & 2 & 800 & Ogilvie Mountains & 36 \\
\hline $1000-635$ & 2 & 800 & Ogilvie Mountains & 36 \\
\hline $1000-635$ & 2 & 800 & Ogilvie Mountains & 36 \\
\hline $1000-635$ & 2 & 800 & Ogilvie Mountains & 36 \\
\hline $1000-635$ & 2 & 800 & Ogilvie Mountains & 36 \\
\hline $1000-635$ & 2 & 800 & Ogilvie Mountains & 36 \\
\hline $1000-635$ & 2 & 800 & Ogilvie Mountains & 36 \\
\hline $1000-635$ & 2 & 800 & Ogilvie Mountains & 36 \\
\hline $1000-635$ & 2 & 800 & Ogilvie Mountains & 36 \\
\hline $1000-635$ & 2 & 800 & Ogilvie Mountains & 36 \\
\hline $1000-635$ & 2 & 800 & Ogilvie Mountains & 36 \\
\hline $1000-635$ & 2 & 800 & Ogilvie Mountains & 36 \\
\hline $1000-635$ & 2 & 800 & Ogilvie Mountains & 36 \\
\hline $1000-635$ & 2 & 800 & Ogilvie Mountains & 36 \\
\hline $1000-635$ & 2 & 800 & Ogilvie Mountains & 36 \\
\hline $1000-635$ & 2 & 800 & Ogilvie Mountains & 36 \\
\hline $1000-635$ & 2 & 800 & Ogilvie Mountains & 36 \\
\hline $1000-635$ & 2 & 800 & Ogilvie Mountains & 36 \\
\hline $1000-635$ & 2 & 800 & Ogilvie Mountains & 36 \\
\hline $1000-635$ & 2 & 800 & Ogilvie Mountains & 36 \\
\hline $1000-635$ & 2 & 800 & Ogilvie Mountains & 36 \\
\hline $1000-635$ & 2 & 800 & Ogilvie Mountains & 36 \\
\hline $1000-635$ & 2 & 800 & Ogilvie Mountains & 36 \\
\hline $1000-635$ & 2 & 800 & Ogilvie Mountains & 36 \\
\hline $1000-635$ & 2 & 800 & Ogilvie Mountains & 36 \\
\hline $1000-635$ & 2 & 800 & Ogilvie Mountains & 36 \\
\hline $1000-635$ & 2 & 800 & Ogilvie Mountains & 36 \\
\hline $1000-635$ & 2 & 800 & Ogilvie Mountains & 36 \\
\hline $1000-635$ & 2 & 800 & Ogilvie Mountains & 36 \\
\hline $1000-635$ & 2 & 800 & Ogilvie Mountains & 36 \\
\hline $1000-635$ & 2 & 800 & Ogilvie Mountains & 36 \\
\hline $1000-635$ & 2 & 800 & Ogilvie Mountains & 36 \\
\hline
\end{tabular}




\begin{tabular}{|c|c|c|c|c|}
\hline $1000-635$ & 2 & 800 & Ogilvie Mountains & 36 \\
\hline $1000-635$ & 2 & 800 & Ogilvie Mountains & 36 \\
\hline $1000-635$ & 2 & 800 & Ogilvie Mountains & 36 \\
\hline $1000-635$ & 2 & 800 & Ogilvie Mountains & 36 \\
\hline $1000-635$ & 2 & 800 & Ogilvie Mountains & 36 \\
\hline $1000-635$ & 2 & 800 & Ogilvie Mountains & 36 \\
\hline $1000-635$ & 2 & 800 & Ogilvie Mountains & 36 \\
\hline $1000-635$ & 2 & 800 & Ogilvie Mountains & 36 \\
\hline $1000-635$ & 2 & 800 & Ogilvie Mountains & 36 \\
\hline $1000-635$ & 2 & 800 & Ogilvie Mountains & 36 \\
\hline $1000-635$ & 2 & 800 & Ogilvie Mountains & 36 \\
\hline $1000-635$ & 2 & 800 & Ogilvie Mountains & 36 \\
\hline $1000-635$ & 2 & 800 & Ogilvie Mountains & 36 \\
\hline $1000-635$ & 2 & 800 & Ogilvie Mountains & 36 \\
\hline $1000-635$ & 2 & 800 & Ogilvie Mountains & 36 \\
\hline $1000-635$ & 2 & 800 & Ogilvie Mountains & 36 \\
\hline $1000-635$ & 2 & 800 & Ogilvie Mountains & 36 \\
\hline $1000-635$ & 2 & 800 & Ogilvie Mountains & 36 \\
\hline $1000-635$ & 2 & 800 & Ogilvie Mountains & 36 \\
\hline $1000-635$ & 2 & 800 & Ogilvie Mountains & 36 \\
\hline $1000-635$ & 2 & 800 & Ogilvie Mountains & 36 \\
\hline $1000-635$ & 2 & 800 & Ogilvie Mountains & 36 \\
\hline $1000-635$ & 2 & 800 & Ogilvie Mountains & 36 \\
\hline $1000-635$ & 2 & 800 & Ogilvie Mountains & 36 \\
\hline $1000-635$ & 2 & 800 & Ogilvie Mountains & 36 \\
\hline $1000-635$ & 2 & 800 & Ogilvie Mountains & 36 \\
\hline $1000-635$ & 2 & 800 & Ogilvie Mountains & 36 \\
\hline $1000-635$ & 2 & 800 & Ogilvie Mountains & 36 \\
\hline $1000-635$ & 2 & 800 & Ogilvie Mountains & 36 \\
\hline $1000-635$ & 2 & 800 & Ogilvie Mountains & 36 \\
\hline $1000-635$ & 2 & 800 & Ogilvie Mountains & 36 \\
\hline $1000-635$ & 2 & 800 & Ogilvie Mountains & 36 \\
\hline $1000-635$ & 2 & 800 & Ogilvie Mountains & 36 \\
\hline $1000-635$ & 2 & 800 & Ogilvie Mountains & 36 \\
\hline $1000-635$ & 2 & 800 & Ogilvie Mountains & 36 \\
\hline $1000-635$ & 2 & 800 & Ogilvie Mountains & 36 \\
\hline $1000-635$ & 2 & 800 & Ogilvie Mountains & 36 \\
\hline $1000-635$ & 2 & 800 & Ogilvie Mountains & 36 \\
\hline $1000-635$ & 2 & 800 & Ogilvie Mountains & 36 \\
\hline $1000-635$ & 2 & 800 & Ogilvie Mountains & 36 \\
\hline $1000-635$ & 2 & 800 & Ogilvie Mountains & 36 \\
\hline
\end{tabular}




\begin{tabular}{|c|c|c|c|c|}
\hline $1000-635$ & 2 & 800 & Ogilvie Mountains & 36 \\
\hline $1000-635$ & 2 & 800 & Ogilvie Mountains & 36 \\
\hline $1000-635$ & 2 & 800 & Ogilvie Mountains & 36 \\
\hline $1000-635$ & 2 & 800 & Ogilvie Mountains & 36 \\
\hline $1000-635$ & 2 & 800 & Ogilvie Mountains & 36 \\
\hline $1000-635$ & 2 & 800 & Ogilvie Mountains & 36 \\
\hline $1000-635$ & 2 & 800 & Ogilvie Mountains & 36 \\
\hline $1000-635$ & 2 & 800 & Ogilvie Mountains & 36 \\
\hline $1000-635$ & 2 & 800 & Ogilvie Mountains & 36 \\
\hline $1000-635$ & 2 & 800 & Ogilvie Mountains & 36 \\
\hline $1000-635$ & 2 & 800 & Ogilvie Mountains & 36 \\
\hline $1000-635$ & 2 & 800 & Ogilvie Mountains & 36 \\
\hline $1000-635$ & 2 & 800 & Ogilvie Mountains & 36 \\
\hline $1000-635$ & 2 & 800 & Ogilvie Mountains & 36 \\
\hline $1000-635$ & 2 & 800 & Ogilvie Mountains & 36 \\
\hline $1000-635$ & 2 & 800 & Ogilvie Mountains & 36 \\
\hline $1000-635$ & 2 & 800 & Ogilvie Mountains & 36 \\
\hline $1000-635$ & 2 & 800 & Ogilvie Mountains & 36 \\
\hline $1000-635$ & 2 & 800 & Ogilvie Mountains & 36 \\
\hline $1000-635$ & 2 & 800 & Ogilvie Mountains & 36 \\
\hline $1000-635$ & 2 & 800 & Ogilvie Mountains & 36 \\
\hline $1000-635$ & 2 & 800 & Ogilvie Mountains & 36 \\
\hline $1000-635$ & 2 & 800 & Ogilvie Mountains & 36 \\
\hline $1000-635$ & 2 & 800 & Ogilvie Mountains & 36 \\
\hline $1000-635$ & 2 & 800 & Ogilvie Mountains & 36 \\
\hline $1000-635$ & 2 & 800 & Ogilvie Mountains & 36 \\
\hline $1000-635$ & 2 & 800 & Ogilvie Mountains & 36 \\
\hline $1000-635$ & 2 & 800 & Ogilvie Mountains & 36 \\
\hline $1000-635$ & 2 & 800 & Ogilvie Mountains & 36 \\
\hline $1000-635$ & 2 & 800 & Ogilvie Mountains & 36 \\
\hline $1000-635$ & 2 & 800 & Ogilvie Mountains & 36 \\
\hline $1000-635$ & 2 & 800 & Ogilvie Mountains & 36 \\
\hline $1000-635$ & 2 & 800 & Ogilvie Mountains & 36 \\
\hline $1000-635$ & 2 & 800 & Ogilvie Mountains & 36 \\
\hline $1000-635$ & 2 & 800 & Ogilvie Mountains & 36 \\
\hline $1000-635$ & 2 & 800 & Ogilvie Mountains & 36 \\
\hline $1000-635$ & 2 & 800 & Ogilvie Mountains & 36 \\
\hline $1000-635$ & 2 & 800 & Ogilvie Mountains & 36 \\
\hline $1000-635$ & 2 & 800 & Ogilvie Mountains & 36 \\
\hline $1000-635$ & 2 & 800 & Ogilvie Mountains & 36 \\
\hline $1000-635$ & 2 & 800 & Ogilvie Mountains & 36 \\
\hline
\end{tabular}




\begin{tabular}{|c|c|c|c|c|}
\hline $1000-635$ & 2 & 800 & Ogilvie Mountains & 36 \\
\hline $1000-635$ & 2 & 800 & Ogilvie Mountains & 36 \\
\hline $1000-635$ & 2 & 800 & Ogilvie Mountains & 36 \\
\hline $1000-635$ & 2 & 800 & Ogilvie Mountains & 36 \\
\hline $1000-635$ & 2 & 800 & Ogilvie Mountains & 36 \\
\hline $1000-635$ & 2 & 800 & Ogilvie Mountains & 36 \\
\hline $1000-635$ & 2 & 800 & Ogilvie Mountains & 36 \\
\hline $1000-635$ & 2 & 800 & Ogilvie Mountains & 36 \\
\hline $1000-635$ & 2 & 800 & Ogilvie Mountains & 36 \\
\hline $1000-635$ & 2 & 800 & Ogilvie Mountains & 36 \\
\hline $1000-635$ & 2 & 800 & Ogilvie Mountains & 36 \\
\hline $1000-635$ & 2 & 800 & Ogilvie Mountains & 36 \\
\hline $1000-635$ & 2 & 800 & Ogilvie Mountains & 36 \\
\hline $1000-635$ & 2 & 800 & Ogilvie Mountains & 36 \\
\hline $1000-635$ & 2 & 800 & Ogilvie Mountains & 36 \\
\hline $1000-635$ & 2 & 800 & Ogilvie Mountains & 36 \\
\hline $1000-635$ & 2 & 800 & Ogilvie Mountains & 36 \\
\hline $1000-635$ & 2 & 800 & Ogilvie Mountains & 36 \\
\hline $1000-635$ & 2 & 800 & Ogilvie Mountains & 36 \\
\hline $1000-635$ & 2 & 800 & Ogilvie Mountains & 36 \\
\hline $1000-635$ & 2 & 800 & Ogilvie Mountains & 36 \\
\hline $1000-635$ & 2 & 800 & Ogilvie Mountains & 36 \\
\hline $1000-635$ & 2 & 800 & Ogilvie Mountains & 36 \\
\hline $1000-635$ & 2 & 800 & Ogilvie Mountains & 36 \\
\hline $1000-635$ & 2 & 800 & Ogilvie Mountains & 36 \\
\hline $1000-635$ & 2 & 800 & Ogilvie Mountains & 36 \\
\hline $1000-635$ & 2 & 800 & Ogilvie Mountains & 36 \\
\hline $1000-635$ & 2 & 800 & Ogilvie Mountains & 36 \\
\hline $1000-635$ & 2 & 800 & Ogilvie Mountains & 36 \\
\hline $1000-635$ & 2 & 800 & Ogilvie Mountains & 36 \\
\hline $1000-635$ & 2 & 800 & Ogilvie Mountains & 36 \\
\hline $1000-635$ & 2 & 800 & Ogilvie Mountains & 36 \\
\hline $1000-635$ & 2 & 800 & Ogilvie Mountains & 36 \\
\hline $1000-635$ & 2 & 800 & Ogilvie Mountains & 36 \\
\hline $1000-635$ & 2 & 800 & Ogilvie Mountains & 36 \\
\hline $1000-635$ & 2 & 800 & Ogilvie Mountains & 36 \\
\hline $1000-635$ & 2 & 800 & Ogilvie Mountains & 36 \\
\hline $1000-635$ & 2 & 800 & Ogilvie Mountains & 36 \\
\hline $1000-635$ & 2 & 800 & Ogilvie Mountains & 36 \\
\hline $1000-635$ & 2 & 800 & Ogilvie Mountains & 36 \\
\hline $1000-635$ & 2 & 800 & Ogilvie Mountains & 36 \\
\hline
\end{tabular}




\begin{tabular}{|c|c|c|c|c|}
\hline $1000-635$ & 2 & 800 & Ogilvie Mountains & 36 \\
\hline $1000-635$ & 2 & 800 & Ogilvie Mountains & 36 \\
\hline $1000-635$ & 2 & 800 & Ogilvie Mountains & 36 \\
\hline $1000-635$ & 2 & 800 & Ogilvie Mountains & 36 \\
\hline $1000-635$ & 2 & 800 & Ogilvie Mountains & 36 \\
\hline $1000-635$ & 2 & 800 & Ogilvie Mountains & 36 \\
\hline $1000-635$ & 2 & 800 & Ogilvie Mountains & 36 \\
\hline $1000-635$ & 2 & 800 & Ogilvie Mountains & 36 \\
\hline $1000-635$ & 2 & 800 & Ogilvie Mountains & 36 \\
\hline $1000-635$ & 2 & 800 & Ogilvie Mountains & 36 \\
\hline $1000-635$ & 2 & 800 & Ogilvie Mountains & 36 \\
\hline $1000-635$ & 2 & 800 & Ogilvie Mountains & 36 \\
\hline $1000-635$ & 2 & 800 & Ogilvie Mountains & 36 \\
\hline $1000-635$ & 2 & 800 & Ogilvie Mountains & 36 \\
\hline $1000-635$ & 2 & 800 & Ogilvie Mountains & 36 \\
\hline $1000-635$ & 2 & 800 & Ogilvie Mountains & 36 \\
\hline $1000-635$ & 2 & 660 & Mackenzie Mountains & 37 \\
\hline $1000-635$ & 2 & 660 & Mackenzie Mountains & 37 \\
\hline $1000-635$ & 2 & 660 & Mackenzie Mountains & 37 \\
\hline $1000-635$ & 2 & 660 & Mackenzie Mountains & 37 \\
\hline $1000-635$ & 2 & 660 & Mackenzie Mountains & 37 \\
\hline $1000-635$ & 2 & 660 & Mackenzie Mountains & 37 \\
\hline $1000-635$ & 2 & 660 & Mackenzie Mountains & 37 \\
\hline 1000-635 & 2 & 660 & Mackenzie Mountains & 37 \\
\hline $1000-635$ & 2 & 660 & Mackenzie Mountains & 37 \\
\hline $1000-635$ & 2 & 660 & Mackenzie Mountains & 37 \\
\hline $1000-635$ & 2 & 660 & Mackenzie Mountains & 37 \\
\hline $1000-635$ & 2 & 660 & Mackenzie Mountains & 37 \\
\hline 1000-635 & 2 & 660 & Mackenzie Mountains & 37 \\
\hline $1000-635$ & 2 & 660 & Mackenzie Mountains & 37 \\
\hline 1000-635 & 2 & 660 & Mackenzie Mountains & 37 \\
\hline $1000-635$ & 2 & 660 & Mackenzie Mountains & 37 \\
\hline 1000-635 & 2 & 660 & Mackenzie Mountains & 37 \\
\hline $1000-635$ & 2 & 660 & Mackenzie Mountains & 37 \\
\hline $1000-635$ & 2 & 660 & Mackenzie Mountains & 37 \\
\hline $1000-635$ & 2 & 650 & Mackenzie Mountains & 37 \\
\hline $1000-635$ & 2 & 650 & Mackenzie Mountains & 37 \\
\hline $1000-635$ & 2 & 650 & Mackenzie Mountains & 37 \\
\hline $1000-635$ & 2 & 650 & Mackenzie Mountains & 37 \\
\hline 1000-635 & 2 & 650 & Mackenzie Mountains & 37 \\
\hline $1000-635$ & 2 & 650 & Mackenzie Mountains & 37 \\
\hline
\end{tabular}




\begin{tabular}{|c|c|c|c|c|}
\hline $1000-635$ & 2 & 650 & Mackenzie Mountains & 37 \\
\hline $1000-635$ & 2 & 650 & Mackenzie Mountains & 37 \\
\hline $1000-635$ & 2 & 650 & Mackenzie Mountains & 37 \\
\hline $1000-635$ & 2 & 650 & Mackenzie Mountains & 37 \\
\hline $1000-635$ & 2 & 650 & Mackenzie Mountains & 37 \\
\hline $1000-635$ & 2 & 650 & Mackenzie Mountains & 37 \\
\hline $1000-635$ & 2 & 650 & Mackenzie Mountains & 37 \\
\hline $1000-635$ & 2 & 650 & Mackenzie Mountains & 37 \\
\hline $1000-635$ & 2 & 650 & Mackenzie Mountains & 37 \\
\hline $1000-635$ & 2 & 650 & Mackenzie Mountains & 37 \\
\hline $1000-635$ & 2 & 650 & Mackenzie Mountains & 37 \\
\hline $1000-635$ & 2 & 650 & Mackenzie Mountains & 37 \\
\hline $1000-635$ & 2 & 650 & Mackenzie Mountains & 37 \\
\hline $1000-635$ & 2 & 650 & Mackenzie Mountains & 37 \\
\hline $1000-635$ & 2 & 650 & Mackenzie Mountains & 37 \\
\hline $1000-635$ & 2 & 650 & Mackenzie Mountains & 37 \\
\hline $1000-635$ & 2 & 650 & Mackenzie Mountains & 37 \\
\hline $1000-635$ & 2 & 650 & Mackenzie Mountains & 37 \\
\hline $1000-635$ & 2 & 650 & Mackenzie Mountains & 37 \\
\hline $1000-635$ & 2 & 650 & Mackenzie Mountains & 37 \\
\hline $1000-635$ & 2 & 650 & Mackenzie Mountains & 37 \\
\hline $1000-635$ & 2 & 650 & Mackenzie Mountains & 37 \\
\hline $1000-635$ & 2 & 650 & Mackenzie Mountains & 37 \\
\hline $1000-635$ & 2 & 650 & Mackenzie Mountains & 37 \\
\hline $1000-635$ & 2 & 650 & Mackenzie Mountains & 37 \\
\hline $1000-635$ & 2 & 650 & Mackenzie Mountains & 37 \\
\hline $1000-635$ & 2 & 650 & Mackenzie Mountains & 37 \\
\hline $1000-635$ & 2 & 650 & Mackenzie Mountains & 37 \\
\hline $1000-635$ & 2 & 650 & Mackenzie Mountains & 37 \\
\hline $1000-635$ & 2 & 650 & Mackenzie Mountains & 37 \\
\hline $1000-635$ & 2 & 650 & Mackenzie Mountains & 37 \\
\hline $1000-635$ & 2 & 650 & Mackenzie Mountains & 37 \\
\hline $1000-635$ & 2 & 650 & Mackenzie Mountains & 37 \\
\hline $1000-635$ & 2 & 650 & Mackenzie Mountains & 37 \\
\hline $1000-635$ & 2 & 750 & Uinta Mountains & 40 \\
\hline $1000-635$ & 2 & 750 & Uinta Mountains & 40 \\
\hline $1000-635$ & 2 & 750 & Uinta Mountains & 40 \\
\hline $1000-635$ & 2 & 750 & Uinta Mountains & 40 \\
\hline $1000-635$ & 2 & 750 & Uinta Mountains & 40 \\
\hline $1000-635$ & 2 & 750 & Uinta Mountains & 40 \\
\hline $1000-635$ & 2 & 750 & Uinta Mountains & 40 \\
\hline
\end{tabular}




\begin{tabular}{|c|c|c|c|c|}
\hline $1000-635$ & 2 & 750 & Uinta Mountains & 40 \\
\hline $1000-635$ & 2 & 750 & Uinta Mountains & 40 \\
\hline $1000-635$ & 2 & 750 & Uinta Mountains & 40 \\
\hline $1000-635$ & 2 & 750 & Uinta Mountains & 40 \\
\hline $1000-635$ & 2 & 750 & Uinta Mountains & 40 \\
\hline $1000-635$ & 2 & 750 & Uinta Mountains & 40 \\
\hline $1000-635$ & 2 & 750 & Uinta Mountains & 40 \\
\hline $1000-635$ & 2 & 750 & Uinta Mountains & 40 \\
\hline $1000-635$ & 2 & 750 & Uinta Mountains & 40 \\
\hline $1000-635$ & 2 & 750 & Uinta Mountains & 40 \\
\hline $1000-635$ & 2 & 750 & Uinta Mountains & 40 \\
\hline $1000-635$ & 2 & 750 & Uinta Mountains & 40 \\
\hline $1000-635$ & 2 & 750 & Uinta Mountains & 40 \\
\hline $1000-635$ & 2 & 750 & Uinta Mountains & 40 \\
\hline $1000-635$ & 2 & 750 & Uinta Mountains & 40 \\
\hline $1000-635$ & 2 & 750 & Uinta Mountains & 40 \\
\hline $1000-635$ & 2 & 750 & Uinta Mountains & 40 \\
\hline $1000-635$ & 2 & 750 & Uinta Mountains & 40 \\
\hline $1000-635$ & 2 & 750 & Uinta Mountains & 40 \\
\hline $1000-635$ & 2 & 750 & Uinta Mountains & 40 \\
\hline $1000-635$ & 2 & 750 & Uinta Mountains & 40 \\
\hline $1000-635$ & 2 & 750 & Uinta Mountains & 40 \\
\hline $1000-635$ & 2 & 750 & Uinta Mountains & 40 \\
\hline $1000-635$ & 2 & 750 & Uinta Mountains & 40 \\
\hline $1000-635$ & 2 & 750 & Uinta Mountains & 40 \\
\hline $1000-635$ & 2 & 750 & Uinta Mountains & 40 \\
\hline $1000-635$ & 2 & 750 & Uinta Mountains & 40 \\
\hline $1000-635$ & 2 & 750 & Uinta Mountains & 40 \\
\hline $1000-635$ & 2 & 750 & Uinta Mountains & 40 \\
\hline $1000-635$ & 2 & 750 & Uinta Mountains & 40 \\
\hline $1000-635$ & 2 & 750 & Uinta Mountains & 40 \\
\hline $1000-635$ & 2 & 750 & Uinta Mountains & 40 \\
\hline $1000-635$ & 2 & 750 & Uinta Mountains & 40 \\
\hline $1000-635$ & 2 & 750 & Uinta Mountains & 40 \\
\hline $1000-635$ & 2 & 750 & Uinta Mountains & 40 \\
\hline $1000-635$ & 2 & 750 & Uinta Mountains & 40 \\
\hline $1000-635$ & 2 & 750 & Uinta Mountains & 40 \\
\hline $1000-635$ & 2 & 750 & Uinta Mountains & 40 \\
\hline $1000-635$ & 2 & 750 & Uinta Mountains & 40 \\
\hline $1000-635$ & 2 & 750 & Uinta Mountains & 40 \\
\hline $1000-635$ & 2 & 750 & Uinta Mountains & 40 \\
\hline
\end{tabular}




\begin{tabular}{|c|c|c|c|c|}
\hline $1000-635$ & 2 & 750 & Uinta Mountains & 40 \\
\hline $1000-635$ & 2 & 750 & Uinta Mountains & 40 \\
\hline $1000-635$ & 2 & 750 & Uinta Mountains & 40 \\
\hline $1000-635$ & 2 & 750 & Uinta Mountains & 40 \\
\hline $1000-635$ & 2 & 750 & Uinta Mountains & 40 \\
\hline $1000-635$ & 2 & 750 & Uinta Mountains & 40 \\
\hline $1000-635$ & 2 & 750 & Uinta Mountains & 40 \\
\hline $1000-635$ & 2 & 750 & Uinta Mountains & 40 \\
\hline $1000-635$ & 2 & 750 & Uinta Mountains & 40 \\
\hline $1000-635$ & 2 & 750 & Uinta Mountains & 40 \\
\hline $1000-635$ & 2 & 750 & Uinta Mountains & 40 \\
\hline $1000-635$ & 2 & 750 & Uinta Mountains & 40 \\
\hline $1000-635$ & 2 & 750 & Uinta Mountains & 40 \\
\hline $1000-635$ & 2 & 750 & Uinta Mountains & 40 \\
\hline $1000-635$ & 2 & 750 & Uinta Mountains & 40 \\
\hline $1000-635$ & 2 & 750 & Uinta Mountains & 40 \\
\hline $1000-635$ & 2 & 750 & Uinta Mountains & 40 \\
\hline $1000-635$ & 2 & 750 & Uinta Mountains & 40 \\
\hline $1000-635$ & 2 & 750 & Uinta Mountains & 40 \\
\hline $1000-635$ & 2 & 750 & Uinta Mountains & 40 \\
\hline $1000-635$ & 2 & 750 & Uinta Mountains & 40 \\
\hline $1000-635$ & 2 & 750 & Uinta Mountains & 40 \\
\hline $1000-635$ & 2 & 750 & Uinta Mountains & 40 \\
\hline $1000-635$ & 2 & 750 & Uinta Mountains & 40 \\
\hline $1000-635$ & 2 & 750 & Uinta Mountains & 40 \\
\hline $1000-635$ & 2 & 745 & Callison Lake Basin & 45 \\
\hline $1000-635$ & 2 & 745 & Callison Lake Basin & 45 \\
\hline $1000-635$ & 2 & 745 & Callison Lake Basin & 45 \\
\hline $1000-635$ & 2 & 745 & Callison Lake Basin & 45 \\
\hline $1000-635$ & 2 & 745 & Callison Lake Basin & 45 \\
\hline $1000-635$ & 2 & 745 & Callison Lake Basin & 45 \\
\hline $1000-635$ & 2 & 745 & Callison Lake Basin & 45 \\
\hline $1000-635$ & 2 & 745 & Callison Lake Basin & 45 \\
\hline $1000-635$ & 2 & 745 & Callison Lake Basin & 45 \\
\hline $1000-635$ & 2 & 745 & Callison Lake Basin & 45 \\
\hline $1000-635$ & 2 & 745 & Callison Lake Basin & 45 \\
\hline $1000-635$ & 2 & 745 & Callison Lake Basin & 45 \\
\hline $1000-635$ & 2 & 745 & Callison Lake Basin & 45 \\
\hline $1000-635$ & 2 & 745 & Callison Lake Basin & 45 \\
\hline $1000-635$ & 2 & 745 & Callison Lake Basin & 45 \\
\hline $1000-635$ & 2 & 745 & Callison Lake Basin & 45 \\
\hline
\end{tabular}




\begin{tabular}{|c|c|c|c|c|}
\hline $1000-635$ & 2 & 745 & Callison Lake Basin & 45 \\
\hline $1000-635$ & 2 & 745 & Callison Lake Basin & 45 \\
\hline $1000-635$ & 2 & 745 & Callison Lake Basin & 45 \\
\hline $1000-635$ & 2 & 745 & Callison Lake Basin & 45 \\
\hline $1000-635$ & 2 & 745 & Callison Lake Basin & 45 \\
\hline $1000-635$ & 2 & 745 & Callison Lake Basin & 45 \\
\hline $1000-635$ & 2 & 745 & Callison Lake Basin & 45 \\
\hline $1000-635$ & 2 & 745 & Callison Lake Basin & 45 \\
\hline $1000-635$ & 2 & 745 & Callison Lake Basin & 45 \\
\hline $1000-635$ & 2 & 745 & Callison Lake Basin & 45 \\
\hline $1000-635$ & 2 & 745 & Callison Lake Basin & 45 \\
\hline $1000-635$ & 2 & 730 & Callison Lake Basin & 45 \\
\hline $1000-635$ & 2 & 730 & Callison Lake Basin & 45 \\
\hline $1000-635$ & 2 & 730 & Callison Lake Basin & 45 \\
\hline $1000-635$ & 2 & 730 & Callison Lake Basin & 45 \\
\hline $1000-635$ & 2 & 730 & Callison Lake Basin & 45 \\
\hline $1000-635$ & 2 & 730 & Callison Lake Basin & 45 \\
\hline $1000-635$ & 2 & 730 & Callison Lake Basin & 45 \\
\hline $1000-635$ & 2 & 730 & Callison Lake Basin & 45 \\
\hline $1000-635$ & 2 & 730 & Callison Lake Basin & 45 \\
\hline $1000-635$ & 2 & 730 & Callison Lake Basin & 45 \\
\hline $1000-635$ & 2 & 730 & Callison Lake Basin & 45 \\
\hline $1000-635$ & 2 & 730 & Callison Lake Basin & 45 \\
\hline $1000-635$ & 2 & 730 & Callison Lake Basin & 45 \\
\hline $1000-635$ & 2 & 730 & Callison Lake Basin & 45 \\
\hline $1000-635$ & 2 & 645.59 & Svalbard & 51 \\
\hline $1000-635$ & 2 & 646.05 & Svalbard & 51 \\
\hline $1000-635$ & 2 & 646.68 & Svalbard & 51 \\
\hline $1000-635$ & 2 & 648.11 & Svalbard & 51 \\
\hline $1000-635$ & 2 & 649.24 & Svalbard & 51 \\
\hline $1000-635$ & 2 & 650.37 & Svalbard & 51 \\
\hline $1000-635$ & 2 & 651.6 & Svalbard & 51 \\
\hline $1000-635$ & 2 & 652.73 & Svalbard & 51 \\
\hline $1000-635$ & 2 & 653.86 & Svalbard & 51 \\
\hline $1000-635$ & 2 & 654.25 & Svalbard & 51 \\
\hline $1000-635$ & 2 & 654.74 & Svalbard & 51 \\
\hline $1000-635$ & 2 & 656.66 & Svalbard & 51 \\
\hline $1000-635$ & 2 & 658.46 & Svalbard & 51 \\
\hline $1000-635$ & 2 & 659.81 & Svalbard & 51 \\
\hline $1000-635$ & 2 & 715 & Svalbard & 51 \\
\hline $1000-635$ & 2 & 715 & Svalbard & 51 \\
\hline
\end{tabular}




\begin{tabular}{|c|c|c|c|c|}
\hline $1000-635$ & 2 & 735.41 & Svalbard & 51 \\
\hline $1000-635$ & 2 & 736.13 & Svalbard & \\
\hline $1000-635$ & 2 & 740.39 & Svalbard & \\
\hline $1000-635$ & 2 & 740.55 & Svalbard & \\
\hline $1000-635$ & 2 & 740.94 & Svalbard & \\
\hline $1000-635$ & 2 & 741.39 & Svalbard & \\
\hline $1000-635$ & 2 & 741.69 & Svalbard & \\
\hline $1000-635$ & 2 & 741.99 & Svalbard & \\
\hline $1000-635$ & 2 & 742.35 & Svalbard & \\
\hline $1000-635$ & 2 & 742.65 & Svalbard & \\
\hline $1000-635$ & 2 & 743.02 & Svalbard & \\
\hline $1000-635$ & 2 & 743.66 & Svalbard & \\
\hline $1000-635$ & 2 & 744.36 & Svalbard & \\
\hline $1000-635$ & 2 & 780.4 & Svalbard & \\
\hline $1000-635$ & 2 & 793.3 & Svalbard & \\
\hline $1000-635$ & 2 & 794.54 & Svalbard & \\
\hline $1000-635$ & 2 & 794.9 & Svalbard & \\
\hline $1000-635$ & 2 & 802.15 & Svalbard & \\
\hline $1000-635$ & 2 & 802.2 & Svalbard & \\
\hline $1000-635$ & 2 & 802.23 & Svalbard & \\
\hline $1000-635$ & 2 & 803.77 & Svalbard & \\
\hline $1000-635$ & 2 & 835 & Svalbard & \\
\hline $1000-635$ & 2 & 835 & Svalbard & \\
\hline $635-542$ & 3 & 600 & South China & \\
\hline $635-542$ & 3 & 600 & South China & 1 \\
\hline $635-542$ & 3 & 600 & South China & 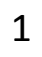 \\
\hline $635-542$ & 3 & 600 & South China & 1 \\
\hline $635-542$ & 3 & 550 & South China & 1 \\
\hline $635-542$ & 3 & 550 & South China & 2 \\
\hline $635-542$ & 3 & 550 & South China & $\perp$ \\
\hline $635-542$ & 3 & 550 & South China & 1 \\
\hline $635-542$ & 3 & 550 & South China & 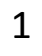 \\
\hline $635-542$ & 3 & 550 & South China & 2 \\
\hline $635-542$ & 3 & 550 & South China & ( \\
\hline $635-542$ & 3 & 550 & South China & - \\
\hline $635-542$ & 3 & 550 & South China & 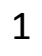 \\
\hline $635-542$ & 3 & 550 & South China & - \\
\hline $635-542$ & 3 & 550 & South China & 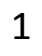 \\
\hline $635-542$ & 3 & 580 & South China & 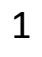 \\
\hline $635-542$ & 3 & 580 & South China & $\perp$ \\
\hline $635-542$ & 3 & 580 & South China & \\
\hline
\end{tabular}




\begin{tabular}{|c|c|c|c|c|}
\hline $635-542$ & 3 & 580 & South China & 1 \\
\hline $635-542$ & 3 & 580 & South China & 1 \\
\hline $635-542$ & 3 & 580 & South China & 1 \\
\hline $635-542$ & 3 & 580 & South China & 1 \\
\hline $635-542$ & 3 & 580 & South China & 1 \\
\hline $635-542$ & 3 & 580 & South China & 1 \\
\hline $635-542$ & 3 & 580 & South China & 1 \\
\hline $635-542$ & 3 & 580 & South China & 1 \\
\hline $635-542$ & 3 & 580 & South China & 1 \\
\hline $635-542$ & 3 & 580 & South China & 1 \\
\hline $635-542$ & 3 & 580 & South China & 1 \\
\hline $635-542$ & 3 & 580 & South China & 1 \\
\hline $635-542$ & 3 & 580 & South China & 1 \\
\hline $635-542$ & 3 & 580 & South China & 1 \\
\hline $635-542$ & 3 & 580 & South China & 1 \\
\hline $635-542$ & 3 & 580 & South China & 1 \\
\hline $635-542$ & 3 & 580 & South China & 1 \\
\hline $635-542$ & 3 & 580 & South China & 1 \\
\hline $635-542$ & 3 & 580 & South China & 1 \\
\hline $635-542$ & 3 & 580 & South China & 1 \\
\hline $635-542$ & 3 & 580 & South China & 1 \\
\hline $635-542$ & 3 & 580 & South China & 1 \\
\hline $635-542$ & 3 & 580 & South China & 1 \\
\hline $635-542$ & 3 & 580 & South China & 1 \\
\hline $635-542$ & 3 & 580 & South China & 1 \\
\hline $635-542$ & 3 & 580 & South China & 1 \\
\hline $635-542$ & 3 & 580 & South China & 1 \\
\hline $635-542$ & 3 & 580 & South China & 1 \\
\hline $635-542$ & 3 & 580 & South China & 1 \\
\hline $635-542$ & 3 & 580 & South China & 1 \\
\hline $635-542$ & 3 & 580 & South China & 1 \\
\hline $635-542$ & 3 & 580 & South China & 1 \\
\hline $635-542$ & 3 & 580 & South China & 1 \\
\hline $635-542$ & 3 & 580 & South China & 1 \\
\hline $635-542$ & 3 & 580 & South China & 1 \\
\hline $635-542$ & 3 & 580 & South China & 1 \\
\hline $635-542$ & 3 & 580 & South China & 1 \\
\hline $635-542$ & 3 & 580 & South China & 1 \\
\hline $635-542$ & 3 & 580 & South China & 1 \\
\hline $635-542$ & 3 & 580 & South China & 1 \\
\hline $635-542$ & 3 & 580 & South China & 1 \\
\hline
\end{tabular}




\begin{tabular}{|c|c|c|c|c|}
\hline $635-542$ & 3 & 580 & South China & 1 \\
\hline $635-542$ & 3 & 580 & South China & 1 \\
\hline $635-542$ & 3 & 580 & South China & 1 \\
\hline $635-542$ & 3 & 580 & South China & 1 \\
\hline $635-542$ & 3 & 580 & South China & 1 \\
\hline $635-542$ & 3 & 580 & South China & 1 \\
\hline $635-542$ & 3 & 580 & South China & 1 \\
\hline $635-542$ & 3 & 580 & South China & 1 \\
\hline $635-542$ & 3 & 580 & South China & 1 \\
\hline $635-542$ & 3 & 580 & South China & 1 \\
\hline $635-542$ & 3 & 580 & South China & 1 \\
\hline $635-542$ & 3 & 580 & South China & 1 \\
\hline $635-542$ & 3 & 580 & South China & 1 \\
\hline $635-542$ & 3 & 580 & South China & 1 \\
\hline $635-542$ & 3 & 580 & South China & 1 \\
\hline $635-542$ & 3 & 580 & South China & 1 \\
\hline $635-542$ & 3 & 580 & South China & 1 \\
\hline $635-542$ & 3 & 580 & South China & 1 \\
\hline $635-542$ & 3 & 580 & South China & 1 \\
\hline $635-542$ & 3 & 580 & South China & 1 \\
\hline $635-542$ & 3 & 580 & South China & 1 \\
\hline $635-542$ & 3 & 580 & South China & 1 \\
\hline $635-542$ & 3 & 580 & South China & 1 \\
\hline $635-542$ & 3 & 580 & South China & 1 \\
\hline $635-542$ & 3 & 580 & South China & 1 \\
\hline $635-542$ & 3 & 580 & South China & 1 \\
\hline $635-542$ & 3 & 580 & South China & 1 \\
\hline $635-542$ & 3 & 580 & South China & 1 \\
\hline $635-542$ & 3 & 580 & South China & 1 \\
\hline $635-542$ & 3 & 580 & South China & 1 \\
\hline $635-542$ & 3 & 580 & South China & 1 \\
\hline $635-542$ & 3 & 580 & South China & 1 \\
\hline $635-542$ & 3 & 580 & South China & 1 \\
\hline $635-542$ & 3 & 580 & South China & 1 \\
\hline $635-542$ & 3 & 580 & South China & 1 \\
\hline $635-542$ & 3 & 630 & South China & 1 \\
\hline $635-542$ & 3 & 630 & South China & 1 \\
\hline $635-542$ & 3 & 630 & South China & 1 \\
\hline $635-542$ & 3 & 630 & South China & 1 \\
\hline $635-542$ & 3 & 630 & South China & 1 \\
\hline $635-542$ & 3 & 630 & South China & 1 \\
\hline
\end{tabular}




\begin{tabular}{|c|c|c|c|c|}
\hline $635-542$ & 3 & 630 & South China & 1 \\
\hline $635-542$ & 3 & 630 & South China & 1 \\
\hline $635-542$ & 3 & 630 & South China & 1 \\
\hline $635-542$ & 3 & 630 & South China & 1 \\
\hline $635-542$ & 3 & 630 & South China & 1 \\
\hline $635-542$ & 3 & 630 & South China & 1 \\
\hline $635-542$ & 3 & 630 & South China & 1 \\
\hline $635-542$ & 3 & 630 & South China & 1 \\
\hline $635-542$ & 3 & 630 & South China & 1 \\
\hline $635-542$ & 3 & 630 & South China & 1 \\
\hline $635-542$ & 3 & 630 & South China & 1 \\
\hline $635-542$ & 3 & 630 & South China & 1 \\
\hline $635-542$ & 3 & 630 & South China & 1 \\
\hline $635-542$ & 3 & 630 & South China & 1 \\
\hline $635-542$ & 3 & 630 & South China & 1 \\
\hline $635-542$ & 3 & 630 & South China & 1 \\
\hline $635-542$ & 3 & 630 & South China & 1 \\
\hline $635-542$ & 3 & 630 & South China & 1 \\
\hline $635-542$ & 3 & 630 & South China & 1 \\
\hline $635-542$ & 3 & 630 & South China & 1 \\
\hline $635-542$ & 3 & 630 & South China & 1 \\
\hline $635-542$ & 3 & 630 & South China & 1 \\
\hline $635-542$ & 3 & 630 & South China & 1 \\
\hline $635-542$ & 3 & 630 & South China & 1 \\
\hline $635-542$ & 3 & 630 & South China & 1 \\
\hline $635-542$ & 3 & 630 & South China & 1 \\
\hline $635-542$ & 3 & 630 & South China & 1 \\
\hline $635-542$ & 3 & 630 & South China & 1 \\
\hline $635-542$ & 3 & 630 & South China & 1 \\
\hline $635-542$ & 3 & 630 & South China & 1 \\
\hline $635-542$ & 3 & 630 & South China & 1 \\
\hline $635-542$ & 3 & 630 & South China & 1 \\
\hline $635-542$ & 3 & 630 & South China & 1 \\
\hline $635-542$ & 3 & 630 & South China & 1 \\
\hline $635-542$ & 3 & 630 & South China & 1 \\
\hline $635-542$ & 3 & 630 & South China & 1 \\
\hline $635-542$ & 3 & 630 & South China & 1 \\
\hline $635-542$ & 3 & 630 & South China & 1 \\
\hline $635-542$ & 3 & 630 & South China & 1 \\
\hline $635-542$ & 3 & 630 & South China & 1 \\
\hline $635-542$ & 3 & 630 & South China & 1 \\
\hline
\end{tabular}




\begin{tabular}{|c|c|c|c|c|}
\hline $635-542$ & 3 & 630 & South China & 1 \\
\hline $635-542$ & 3 & 630 & South China & 1 \\
\hline $635-542$ & 3 & 630 & South China & 1 \\
\hline $635-542$ & 3 & 630 & South China & 1 \\
\hline $635-542$ & 3 & 630 & South China & 1 \\
\hline $635-542$ & 3 & 630 & South China & 1 \\
\hline $635-542$ & 3 & 630 & South China & 1 \\
\hline $635-542$ & 3 & 630 & South China & 1 \\
\hline $635-542$ & 3 & 630 & South China & 1 \\
\hline $635-542$ & 3 & 630 & South China & 1 \\
\hline $635-542$ & 3 & 630 & South China & 1 \\
\hline $635-542$ & 3 & 630 & South China & 1 \\
\hline $635-542$ & 3 & 630 & South China & 1 \\
\hline $635-542$ & 3 & 630 & South China & 1 \\
\hline $635-542$ & 3 & 630 & South China & 1 \\
\hline $635-542$ & 3 & 630 & South China & 1 \\
\hline $635-542$ & 3 & 630 & South China & 1 \\
\hline $635-542$ & 3 & 630 & South China & 1 \\
\hline $635-542$ & 3 & 620 & South China & 1 \\
\hline $635-542$ & 3 & 620 & South China & 1 \\
\hline $635-542$ & 3 & 620 & South China & 1 \\
\hline $635-542$ & 3 & 620 & South China & 1 \\
\hline $635-542$ & 3 & 620 & South China & 1 \\
\hline $635-542$ & 3 & 620 & South China & 1 \\
\hline $635-542$ & 3 & 620 & South China & 1 \\
\hline $635-542$ & 3 & 620 & South China & 1 \\
\hline $635-542$ & 3 & 620 & South China & 1 \\
\hline $635-542$ & 3 & 620 & South China & 1 \\
\hline $635-542$ & 3 & 620 & South China & 1 \\
\hline $635-542$ & 3 & 620 & South China & 1 \\
\hline $635-542$ & 3 & 620 & South China & 1 \\
\hline $635-542$ & 3 & 620 & South China & 1 \\
\hline $635-542$ & 3 & 620 & South China & 1 \\
\hline $635-542$ & 3 & 620 & South China & 1 \\
\hline $635-542$ & 3 & 620 & South China & 1 \\
\hline $635-542$ & 3 & 620 & South China & 1 \\
\hline $635-542$ & 3 & 620 & South China & 1 \\
\hline $635-542$ & 3 & 620 & South China & 1 \\
\hline $635-542$ & 3 & 620 & South China & 1 \\
\hline $635-542$ & 3 & 620 & South China & 1 \\
\hline $635-542$ & 3 & 620 & South China & 1 \\
\hline
\end{tabular}




\begin{tabular}{|c|c|c|c|}
\hline $635-542$ & 3 & 620 & South China \\
\hline $635-542$ & 3 & 620 & South China \\
\hline $635-542$ & 3 & 620 & South China \\
\hline $635-542$ & 3 & 620 & South China \\
\hline $635-542$ & 3 & 620 & South China \\
\hline $635-542$ & 3 & 620 & South China \\
\hline $635-542$ & 3 & 620 & South China \\
\hline $635-542$ & 3 & 620 & South China \\
\hline $635-542$ & 3 & 620 & South China \\
\hline $635-542$ & 3 & 620 & South China \\
\hline $635-542$ & 3 & 620 & South China \\
\hline $635-542$ & 3 & 550 & South China \\
\hline $635-542$ & 3 & 550 & South China \\
\hline $635-542$ & 3 & 550 & South China \\
\hline $635-542$ & 3 & 550 & South China \\
\hline $635-542$ & 3 & 550 & South China \\
\hline $635-542$ & 3 & 550 & South China \\
\hline $635-542$ & 3 & 550 & South China \\
\hline $635-542$ & 3 & 550 & South China \\
\hline $635-542$ & 3 & 550 & South China \\
\hline $635-542$ & 3 & 550 & South China \\
\hline $635-542$ & 3 & 550 & South China \\
\hline $635-542$ & 3 & 550 & South China \\
\hline $635-542$ & 3 & 550 & South China \\
\hline $635-542$ & 3 & 600 & Wernecke Mountains \\
\hline $635-542$ & 3 & 600 & Wernecke Mountains \\
\hline $635-542$ & 3 & 600 & Wernecke Mountains \\
\hline $635-542$ & 3 & 600 & Wernecke Mountains \\
\hline $635-542$ & 3 & 600 & Wernecke Mountains \\
\hline $635-542$ & 3 & 600 & Wernecke Mountains \\
\hline $635-542$ & 3 & 600 & Wernecke Mountains \\
\hline $635-542$ & 3 & 600 & Wernecke Mountains \\
\hline $635-542$ & 3 & 600 & Wernecke Mountains \\
\hline $635-542$ & 3 & 600 & Wernecke Mountains \\
\hline $635-542$ & 3 & 600 & Wernecke Mountains \\
\hline $635-542$ & 3 & 600 & Wernecke Mountains \\
\hline $635-542$ & 3 & 600 & Wernecke Mountains \\
\hline $635-542$ & 3 & 600 & Wernecke Mountains \\
\hline $635-542$ & 3 & 600 & Wernecke Mountains \\
\hline $635-542$ & 3 & 600 & Wernecke Mountains \\
\hline $635-542$ & 3 & 600 & Wernecke Mountains \\
\hline
\end{tabular}




\begin{tabular}{|c|c|c|c|}
\hline $635-542$ & 3 & 600 & Wernecke Mountains \\
\hline $635-542$ & 3 & 600 & Wernecke Mountains \\
\hline $635-542$ & 3 & 600 & Wernecke Mountains \\
\hline $635-542$ & 3 & 600 & Wernecke Mountains \\
\hline $635-542$ & 3 & 600 & Wernecke Mountains \\
\hline $635-542$ & 3 & 600 & Wernecke Mountains \\
\hline $635-542$ & 3 & 600 & Wernecke Mountains \\
\hline $635-542$ & 3 & 600 & Wernecke Mountains \\
\hline $635-542$ & 3 & 600 & Wernecke Mountains \\
\hline $635-542$ & 3 & 600 & Wernecke Mountains \\
\hline $635-542$ & 3 & 600 & Wernecke Mountains \\
\hline $635-542$ & 3 & 600 & Wernecke Mountains \\
\hline $635-542$ & 3 & 600 & Wernecke Mountains \\
\hline $635-542$ & 3 & 600 & Wernecke Mountains \\
\hline $635-542$ & 3 & 600 & Wernecke Mountains \\
\hline $635-542$ & 3 & 600 & Wernecke Mountains \\
\hline $635-542$ & 3 & 600 & Wernecke Mountains \\
\hline $635-542$ & 3 & 600 & Wernecke Mountains \\
\hline $635-542$ & 3 & 600 & Wernecke Mountains \\
\hline $635-542$ & 3 & 600 & Wernecke Mountains \\
\hline $635-542$ & 3 & 600 & Wernecke Mountains \\
\hline $635-542$ & 3 & 600 & Wernecke Mountains \\
\hline $635-542$ & 3 & 600 & Wernecke Mountains \\
\hline $635-542$ & 3 & 600 & Wernecke Mountains \\
\hline $635-542$ & 3 & 600 & Wernecke Mountains \\
\hline $635-542$ & 3 & 600 & Wernecke Mountains \\
\hline $635-542$ & 3 & 600 & Wernecke Mountains \\
\hline $635-542$ & 3 & 600 & Wernecke Mountains \\
\hline $635-542$ & 3 & 600 & Wernecke Mountains \\
\hline $635-542$ & 3 & 600 & Wernecke Mountains \\
\hline $635-542$ & 3 & 600 & Wernecke Mountains \\
\hline $635-542$ & 3 & 600 & Wernecke Mountains \\
\hline $635-542$ & 3 & 600 & Wernecke Mountains \\
\hline $635-542$ & 3 & 600 & Wernecke Mountains \\
\hline $635-542$ & 3 & 600 & Wernecke Mountains \\
\hline $635-542$ & 3 & 600 & Wernecke Mountains \\
\hline $635-542$ & 3 & 600 & Wernecke Mountains \\
\hline $635-542$ & 3 & 600 & Wernecke Mountains \\
\hline $635-542$ & 3 & 600 & Wernecke Mountains \\
\hline $635-542$ & 3 & 600 & Wernecke Mountains \\
\hline $635-542$ & 3 & 600 & Wernecke Mountains \\
\hline
\end{tabular}




\begin{tabular}{|c|c|c|c|}
\hline $635-542$ & 3 & 600 & Wernecke Mountains \\
\hline $635-542$ & 3 & 600 & Wernecke Mountains \\
\hline $635-542$ & 3 & 600 & Wernecke Mountains \\
\hline $635-542$ & 3 & 600 & Wernecke Mountains \\
\hline $635-542$ & 3 & 600 & Wernecke Mountains \\
\hline $635-542$ & 3 & 600 & Wernecke Mountains \\
\hline $635-542$ & 3 & 600 & Wernecke Mountains \\
\hline $635-542$ & 3 & 600 & Wernecke Mountains \\
\hline $635-542$ & 3 & 600 & Wernecke Mountains \\
\hline $635-542$ & 3 & 600 & Wernecke Mountains \\
\hline $635-542$ & 3 & 600 & Wernecke Mountains \\
\hline $635-542$ & 3 & 600 & Wernecke Mountains \\
\hline $635-542$ & 3 & 600 & Wernecke Mountains \\
\hline $635-542$ & 3 & 600 & Wernecke Mountains \\
\hline $635-542$ & 3 & 600 & Wernecke Mountains \\
\hline $635-542$ & 3 & 600 & Wernecke Mountains \\
\hline $635-542$ & 3 & 600 & Wernecke Mountains \\
\hline $635-542$ & 3 & 600 & Wernecke Mountains \\
\hline $635-542$ & 3 & 600 & Wernecke Mountains \\
\hline $635-542$ & 3 & 600 & Wernecke Mountains \\
\hline $635-542$ & 3 & 600 & Wernecke Mountains \\
\hline $635-542$ & 3 & 600 & Wernecke Mountains \\
\hline $635-542$ & 3 & 600 & Wernecke Mountains \\
\hline $635-542$ & 3 & 600 & Wernecke Mountains \\
\hline $635-542$ & 3 & 600 & Wernecke Mountains \\
\hline $635-542$ & 3 & 600 & Wernecke Mountains \\
\hline $635-542$ & 3 & 600 & Wernecke Mountains \\
\hline $635-542$ & 3 & 600 & Wernecke Mountains \\
\hline $635-542$ & 3 & 600 & Wernecke Mountains \\
\hline $635-542$ & 3 & 600 & Wernecke Mountains \\
\hline $635-542$ & 3 & 600 & Wernecke Mountains \\
\hline $635-542$ & 3 & 600 & Wernecke Mountains \\
\hline $635-542$ & 3 & 600 & Wernecke Mountains \\
\hline $635-542$ & 3 & 600 & Wernecke Mountains \\
\hline $635-542$ & 3 & 600 & Wernecke Mountains \\
\hline $635-542$ & 3 & 600 & Wernecke Mountains \\
\hline $635-542$ & 3 & 600 & Wernecke Mountains \\
\hline $635-542$ & 3 & 600 & Wernecke Mountains \\
\hline $635-542$ & 3 & 600 & Wernecke Mountains \\
\hline $635-542$ & 3 & 600 & Wernecke Mountains \\
\hline $635-542$ & 3 & 600 & Wernecke Mountains \\
\hline
\end{tabular}




\begin{tabular}{|c|c|c|c|}
\hline $635-542$ & 3 & 600 & Wernecke Mountains \\
\hline $635-542$ & 3 & 600 & Wernecke Mountains \\
\hline $635-542$ & 3 & 600 & Wernecke Mountains \\
\hline $635-542$ & 3 & 600 & Wernecke Mountains \\
\hline $635-542$ & 3 & 600 & Wernecke Mountains \\
\hline $635-542$ & 3 & 600 & Wernecke Mountains \\
\hline $635-542$ & 3 & 600 & Wernecke Mountains \\
\hline $635-542$ & 3 & 600 & Wernecke Mountains \\
\hline $635-542$ & 3 & 600 & Wernecke Mountains \\
\hline $635-542$ & 3 & 600 & Wernecke Mountains \\
\hline $635-542$ & 3 & 600 & Wernecke Mountains \\
\hline $635-542$ & 3 & 600 & Wernecke Mountains \\
\hline $635-542$ & 3 & 600 & Wernecke Mountains \\
\hline $635-542$ & 3 & 600 & Wernecke Mountains \\
\hline $635-542$ & 3 & 600 & Wernecke Mountains \\
\hline $635-542$ & 3 & 600 & Wernecke Mountains \\
\hline $635-542$ & 3 & 600 & Wernecke Mountains \\
\hline $635-542$ & 3 & 600 & Wernecke Mountains \\
\hline $635-542$ & 3 & 600 & Wernecke Mountains \\
\hline $635-542$ & 3 & 600 & Wernecke Mountains \\
\hline $635-542$ & 3 & 600 & Wernecke Mountains \\
\hline $635-542$ & 3 & 600 & Wernecke Mountains \\
\hline $635-542$ & 3 & 600 & Wernecke Mountains \\
\hline $635-542$ & 3 & 600 & Wernecke Mountains \\
\hline $635-542$ & 3 & 600 & Wernecke Mountains \\
\hline $635-542$ & 3 & 600 & Wernecke Mountains \\
\hline $635-542$ & 3 & 600 & Wernecke Mountains \\
\hline $635-542$ & 3 & 600 & Wernecke Mountains \\
\hline $635-542$ & 3 & 600 & Wernecke Mountains \\
\hline $635-542$ & 3 & 600 & Wernecke Mountains \\
\hline $635-542$ & 3 & 600 & Wernecke Mountains \\
\hline $635-542$ & 3 & 600 & Wernecke Mountains \\
\hline $635-542$ & 3 & 600 & Wernecke Mountains \\
\hline $635-542$ & 3 & 600 & Wernecke Mountains \\
\hline $635-542$ & 3 & 600 & Wernecke Mountains \\
\hline $635-542$ & 3 & 600 & Wernecke Mountains \\
\hline $635-542$ & 3 & 600 & Wernecke Mountains \\
\hline $635-542$ & 3 & 600 & Wernecke Mountains \\
\hline $635-542$ & 3 & 600 & Wernecke Mountains \\
\hline $635-542$ & 3 & 600 & Wernecke Mountains \\
\hline $635-542$ & 3 & 600 & Wernecke Mountains \\
\hline
\end{tabular}




\begin{tabular}{|c|c|c|c|}
\hline $635-542$ & 3 & 600 & Wernecke Mountains \\
\hline $635-542$ & 3 & 600 & Wernecke Mountains \\
\hline $635-542$ & 3 & 600 & Wernecke Mountains \\
\hline $635-542$ & 3 & 600 & Wernecke Mountains \\
\hline $635-542$ & 3 & 600 & Wernecke Mountains \\
\hline $635-542$ & 3 & 600 & Wernecke Mountains \\
\hline $635-542$ & 3 & 600 & Wernecke Mountains \\
\hline $635-542$ & 3 & 600 & Wernecke Mountains \\
\hline $635-542$ & 3 & 600 & Wernecke Mountains \\
\hline $635-542$ & 3 & 600 & Wernecke Mountains \\
\hline $635-542$ & 3 & 600 & Wernecke Mountains \\
\hline $635-542$ & 3 & 600 & Wernecke Mountains \\
\hline $635-542$ & 3 & 600 & Wernecke Mountains \\
\hline $635-542$ & 3 & 600 & Wernecke Mountains \\
\hline $635-542$ & 3 & 600 & Wernecke Mountains \\
\hline $635-542$ & 3 & 600 & Wernecke Mountains \\
\hline $635-542$ & 3 & 600 & Wernecke Mountains \\
\hline $635-542$ & 3 & 600 & Wernecke Mountains \\
\hline $635-542$ & 3 & 560 & Wernecke Mountains \\
\hline $635-542$ & 3 & 560 & Wernecke Mountains \\
\hline $635-542$ & 3 & 560 & Wernecke Mountains \\
\hline $635-542$ & 3 & 560 & Wernecke Mountains \\
\hline $635-542$ & 3 & 560 & Wernecke Mountains \\
\hline $635-542$ & 3 & 560 & Wernecke Mountains \\
\hline $635-542$ & 3 & 560 & Wernecke Mountains \\
\hline $635-542$ & 3 & 560 & Wernecke Mountains \\
\hline $635-542$ & 3 & 560 & Wernecke Mountains \\
\hline $635-542$ & 3 & 560 & Wernecke Mountains \\
\hline $635-542$ & 3 & 560 & Wernecke Mountains \\
\hline $635-542$ & 3 & 560 & Wernecke Mountains \\
\hline $635-542$ & 3 & 560 & Wernecke Mountains \\
\hline $635-542$ & 3 & 560 & Wernecke Mountains \\
\hline $635-542$ & 3 & 560 & Wernecke Mountains \\
\hline $635-542$ & 3 & 560 & Wernecke Mountains \\
\hline $635-542$ & 3 & 560 & Wernecke Mountains \\
\hline $635-542$ & 3 & 560 & Wernecke Mountains \\
\hline $635-542$ & 3 & 560 & Wernecke Mountains \\
\hline $635-542$ & 3 & 560 & Wernecke Mountains \\
\hline $635-542$ & 3 & 570 & Wernecke Mountains \\
\hline $635-542$ & 3 & 570 & Wernecke Mountains \\
\hline $635-542$ & 3 & 560 & Wernecke Mountains \\
\hline
\end{tabular}




\begin{tabular}{|c|c|c|c|}
\hline $635-542$ & 3 & 560 & Wernecke Mountains \\
\hline $635-542$ & 3 & 560 & Wernecke Mountains \\
\hline $635-542$ & 3 & 550 & Wernecke Mountains \\
\hline $635-542$ & 3 & 550 & Wernecke Mountains \\
\hline $635-542$ & 3 & 550 & Wernecke Mountains \\
\hline $635-542$ & 3 & 550 & Wernecke Mountains \\
\hline $635-542$ & 3 & 620 & Wernecke Mountains \\
\hline $635-542$ & 3 & 620 & Wernecke Mountains \\
\hline $635-542$ & 3 & 620 & Wernecke Mountains \\
\hline $635-542$ & 3 & 620 & Wernecke Mountains \\
\hline $635-542$ & 3 & 620 & Wernecke Mountains \\
\hline $635-542$ & 3 & 620 & Wernecke Mountains \\
\hline $635-542$ & 3 & 620 & Wernecke Mountains \\
\hline $635-542$ & 3 & 620 & Wernecke Mountains \\
\hline $635-542$ & 3 & 620 & Wernecke Mountains \\
\hline $635-542$ & 3 & 620 & Wernecke Mountains \\
\hline $635-542$ & 3 & 620 & Wernecke Mountains \\
\hline $635-542$ & 3 & 620 & Wernecke Mountains \\
\hline $635-542$ & 3 & 620 & Wernecke Mountains \\
\hline $635-542$ & 3 & 620 & Wernecke Mountains \\
\hline $635-542$ & 3 & 620 & Wernecke Mountains \\
\hline $635-542$ & 3 & 620 & Wernecke Mountains \\
\hline $635-542$ & 3 & 620 & Wernecke Mountains \\
\hline $635-542$ & 3 & 620 & Wernecke Mountains \\
\hline $635-542$ & 3 & 620 & Wernecke Mountains \\
\hline $635-542$ & 3 & 620 & Wernecke Mountains \\
\hline $635-542$ & 3 & 620 & Wernecke Mountains \\
\hline $635-542$ & 3 & 620 & Wernecke Mountains \\
\hline $635-542$ & 3 & 620 & Wernecke Mountains \\
\hline $635-542$ & 3 & 620 & Wernecke Mountains \\
\hline $635-542$ & 3 & 620 & Wernecke Mountains \\
\hline $635-542$ & 3 & 620 & Wernecke Mountains \\
\hline $635-542$ & 3 & 620 & Wernecke Mountains \\
\hline $635-542$ & 3 & 620 & Wernecke Mountains \\
\hline $635-542$ & 3 & 620 & Wernecke Mountains \\
\hline $635-542$ & 3 & 620 & Wernecke Mountains \\
\hline $635-542$ & 3 & 620 & Wernecke Mountains \\
\hline $635-542$ & 3 & 620 & Wernecke Mountains \\
\hline $635-542$ & 3 & 570 & Wernecke Mountains \\
\hline $635-542$ & 3 & 570 & Wernecke Mountains \\
\hline $635-542$ & 3 & 570 & Wernecke Mountains \\
\hline
\end{tabular}




\begin{tabular}{|c|c|c|c|c|}
\hline $635-542$ & 3 & 570 & Wernecke Mountains & 4 \\
\hline $635-542$ & 3 & 570 & Wernecke Mountains & 4 \\
\hline $635-542$ & 3 & 570 & Wernecke Mountains & 4 \\
\hline $635-542$ & 3 & 570 & Wernecke Mountains & 4 \\
\hline $635-542$ & 3 & 570 & Wernecke Mountains & 4 \\
\hline $635-542$ & 3 & 570 & Wernecke Mountains & 4 \\
\hline $635-542$ & 3 & 570 & Wernecke Mountains & 4 \\
\hline $635-542$ & 3 & 570 & Wernecke Mountains & 4 \\
\hline $635-542$ & 3 & 570 & Wernecke Mountains & 4 \\
\hline $635-542$ & 3 & 570 & Wernecke Mountains & 4 \\
\hline $635-542$ & 3 & 570 & Wernecke Mountains & 4 \\
\hline $635-542$ & 3 & 550 & Wernecke Mountains & 4 \\
\hline $635-542$ & 3 & 550 & Wernecke Mountains & 4 \\
\hline $635-542$ & 3 & 550 & Wernecke Mountains & 4 \\
\hline $635-542$ & 3 & 550 & Wernecke Mountains & 4 \\
\hline $635-542$ & 3 & 550 & Wernecke Mountains & 4 \\
\hline $635-542$ & 3 & 550 & Wernecke Mountains & 4 \\
\hline $635-542$ & 3 & 550 & Wernecke Mountains & 4 \\
\hline $635-542$ & 3 & 550 & Wernecke Mountains & 4 \\
\hline $635-542$ & 3 & 550 & Wernecke Mountains & 4 \\
\hline $635-542$ & 3 & 550 & Wernecke Mountains & 4 \\
\hline $635-542$ & 3 & 550 & Wernecke Mountains & 4 \\
\hline $635-542$ & 3 & 550 & Wernecke Mountains & 4 \\
\hline $635-542$ & 3 & 550 & Eastern European Platforr & 14 \\
\hline $635-542$ & 3 & 550 & Eastern European Platforr & 14 \\
\hline $635-542$ & 3 & 550 & Eastern European Platforr & 14 \\
\hline $635-542$ & 3 & 550 & Eastern European Platforr & 14 \\
\hline $635-542$ & 3 & 550 & Eastern European Platforr & 14 \\
\hline $635-542$ & 3 & 550 & Eastern European Platforr & 14 \\
\hline $635-542$ & 3 & 550 & Eastern European Platforr & 14 \\
\hline $635-542$ & 3 & 550 & Eastern European Platforr & 14 \\
\hline $635-542$ & 3 & 550 & Eastern European Platforr & 14 \\
\hline $635-542$ & 3 & 550 & Eastern European Platforr & 14 \\
\hline $635-542$ & 3 & 550 & Eastern European Platforr & 14 \\
\hline $635-542$ & 3 & 550 & Eastern European Platforr & 14 \\
\hline $635-542$ & 3 & 550 & Eastern European Platforr & 14 \\
\hline $635-542$ & 3 & 550 & Eastern European Platforr & 14 \\
\hline $635-542$ & 3 & 550 & Eastern European Platforr & 14 \\
\hline $635-542$ & 3 & 550 & Eastern European Platforr & 14 \\
\hline $635-542$ & 3 & 550 & Eastern European Platforr & 14 \\
\hline $635-542$ & 3 & 550 & Eastern European Platforr & 14 \\
\hline
\end{tabular}




\begin{tabular}{|c|c|c|c|}
\hline $635-542$ & 3 & 550 & Eastern European Platforr \\
\hline $635-542$ & 3 & 550 & Eastern European Platforr \\
\hline $635-542$ & 3 & 550 & Eastern European Platforr \\
\hline $635-542$ & 3 & 550 & Eastern European Platforr \\
\hline $635-542$ & 3 & 550 & Eastern European Platforr \\
\hline $635-542$ & 3 & 550 & Eastern European Platforr \\
\hline $635-542$ & 3 & 550 & Eastern European Platforr \\
\hline $635-542$ & 3 & 550 & Eastern European Platforr \\
\hline $635-542$ & 3 & 550 & Eastern European Platforr \\
\hline $635-542$ & 3 & 550 & Eastern European Platforr \\
\hline $635-542$ & 3 & 550 & Eastern European Platforr \\
\hline $635-542$ & 3 & 550 & Eastern European Platforr \\
\hline $635-542$ & 3 & 550 & Eastern European Platforr \\
\hline $635-542$ & 3 & 550 & Eastern European Platforr \\
\hline $635-542$ & 3 & 550 & Eastern European Platforr \\
\hline $635-542$ & 3 & 550 & Eastern European Platforr \\
\hline $635-542$ & 3 & 550 & Eastern European Platforr \\
\hline $635-542$ & 3 & 550 & Eastern European Platforr \\
\hline $635-542$ & 3 & 550 & Eastern European Platforr \\
\hline $635-542$ & 3 & 550 & Eastern European Platforr \\
\hline $635-542$ & 3 & 550 & Eastern European Platforr \\
\hline $635-542$ & 3 & 550 & Eastern European Platforr \\
\hline $635-542$ & 3 & 550 & Eastern European Platforr \\
\hline $635-542$ & 3 & 550 & Eastern European Platforr \\
\hline $635-542$ & 3 & 550 & Eastern European Platforr \\
\hline $635-542$ & 3 & 550 & Eastern European Platforr \\
\hline $635-542$ & 3 & 600 & Eastern European Platforr \\
\hline $635-542$ & 3 & 600 & Eastern European Platforr \\
\hline $635-542$ & 3 & 600 & Eastern European Platforr \\
\hline $635-542$ & 3 & 600 & Eastern European Platforr \\
\hline $635-542$ & 3 & 600 & Eastern European Platforr \\
\hline $635-542$ & 3 & 600 & Eastern European Platforr \\
\hline $635-542$ & 3 & 600 & Eastern European Platforr \\
\hline $635-542$ & 3 & 600 & Eastern European Platforr \\
\hline $635-542$ & 3 & 600 & Eastern European Platforr \\
\hline $635-542$ & 3 & 600 & Eastern European Platforr \\
\hline $635-542$ & 3 & 600 & Eastern European Platforr \\
\hline $635-542$ & 3 & 600 & Eastern European Platforr \\
\hline $635-542$ & 3 & 600 & Eastern European Platforr \\
\hline $635-542$ & 3 & 600 & Eastern European Platforr \\
\hline $635-542$ & 3 & 600 & Eastern European Platforr \\
\hline
\end{tabular}




\begin{tabular}{|c|c|c|c|}
\hline $635-542$ & 3 & 600 & Eastern European Platforr \\
\hline $635-542$ & 3 & 600 & Eastern European Platforr \\
\hline $635-542$ & 3 & 600 & Eastern European Platforr \\
\hline $635-542$ & 3 & 600 & Eastern European Platforr \\
\hline $635-542$ & 3 & 600 & Eastern European Platforr \\
\hline $635-542$ & 3 & 600 & Eastern European Platforr \\
\hline $635-542$ & 3 & 600 & Eastern European Platforr \\
\hline $635-542$ & 3 & 600 & Eastern European Platforr \\
\hline $635-542$ & 3 & 600 & Eastern European Platforr \\
\hline $635-542$ & 3 & 600 & Eastern European Platforr \\
\hline $635-542$ & 3 & 600 & Eastern European Platforr \\
\hline $635-542$ & 3 & 600 & Eastern European Platforr \\
\hline $635-542$ & 3 & 600 & Eastern European Platforr \\
\hline $635-542$ & 3 & 600 & Eastern European Platforr \\
\hline $635-542$ & 3 & 600 & Eastern European Platforr \\
\hline $635-542$ & 3 & 600 & Eastern European Platforr \\
\hline $635-542$ & 3 & 600 & Eastern European Platforr \\
\hline $635-542$ & 3 & 600 & Eastern European Platforr \\
\hline $635-542$ & 3 & 600 & Eastern European Platforr \\
\hline $635-542$ & 3 & 600 & Eastern European Platforr \\
\hline $635-542$ & 3 & 600 & Eastern European Platforr \\
\hline $635-542$ & 3 & 600 & Eastern European Platforr \\
\hline $635-542$ & 3 & 600 & Eastern European Platforr \\
\hline $635-542$ & 3 & 600 & Eastern European Platforr \\
\hline $635-542$ & 3 & 600 & Eastern European Platforr \\
\hline $635-542$ & 3 & 565 & Southern Canadian Cordill \\
\hline $635-542$ & 3 & 565 & Southern Canadian Cordill \\
\hline $635-542$ & 3 & 565 & Southern Canadian Cordill \\
\hline $635-542$ & 3 & 565 & Southern Canadian Cordill \\
\hline $635-542$ & 3 & 565 & Southern Canadian Cordill \\
\hline $635-542$ & 3 & 565 & Southern Canadian Cordill \\
\hline $635-542$ & 3 & 565 & Southern Canadian Cordill \\
\hline $635-542$ & 3 & 565 & Southern Canadian Cordill \\
\hline $635-542$ & 3 & 565 & Southern Canadian Cordill \\
\hline $635-542$ & 3 & 565 & Southern Canadian Cordill \\
\hline $635-542$ & 3 & 565 & Southern Canadian Cordill \\
\hline $635-542$ & 3 & 565 & Southern Canadian Cordill \\
\hline $635-542$ & 3 & 565 & Southern Canadian Cordill \\
\hline $635-542$ & 3 & 565 & Southern Canadian Cordill \\
\hline $635-542$ & 3 & 565 & Southern Canadian Cordill \\
\hline $635-542$ & 3 & 565 & Southern Canadian Cordill \\
\hline
\end{tabular}




\begin{tabular}{|c|c|c|c|}
\hline $635-542$ & 3 & 565 & Southern Canadian Cordill \\
\hline $635-542$ & 3 & 565 & Southern Canadian Cordill \\
\hline $635-542$ & 3 & 565 & Southern Canadian Cordill \\
\hline $635-542$ & 3 & 565 & Southern Canadian Cordill \\
\hline $635-542$ & 3 & 565 & Southern Canadian Cordill \\
\hline $635-542$ & 3 & 565 & Southern Canadian Cordill \\
\hline $635-542$ & 3 & 565 & Southern Canadian Cordill \\
\hline $635-542$ & 3 & 565 & Southern Canadian Cordill \\
\hline $635-542$ & 3 & 565 & Southern Canadian Cordill \\
\hline $635-542$ & 3 & 565 & Southern Canadian Cordill \\
\hline $635-542$ & 3 & 565 & Southern Canadian Cordill \\
\hline $635-542$ & 3 & 565 & Southern Canadian Cordill \\
\hline $635-542$ & 3 & 565 & Southern Canadian Cordill \\
\hline $635-542$ & 3 & 565 & Southern Canadian Cordill \\
\hline $635-542$ & 3 & 565 & Southern Canadian Cordill \\
\hline $635-542$ & 3 & 565 & Southern Canadian Cordill \\
\hline $635-542$ & 3 & 565 & Southern Canadian Cordill \\
\hline $635-542$ & 3 & 565 & Southern Canadian Cordill \\
\hline $635-542$ & 3 & 565 & Southern Canadian Cordill \\
\hline $635-542$ & 3 & 565 & Southern Canadian Cordill \\
\hline $635-542$ & 3 & 565 & Southern Canadian Cordill \\
\hline $635-542$ & 3 & 565 & Southern Canadian Cordill \\
\hline $635-542$ & 3 & 565 & Southern Canadian Cordill \\
\hline $635-542$ & 3 & 565 & Southern Canadian Cordill \\
\hline $635-542$ & 3 & 565 & Southern Canadian Cordill \\
\hline $635-542$ & 3 & 565 & Southern Canadian Cordill \\
\hline $635-542$ & 3 & 565 & Southern Canadian Cordill \\
\hline $635-542$ & 3 & 565 & Southern Canadian Cordill \\
\hline $635-542$ & 3 & 565 & Southern Canadian Cordill \\
\hline $635-542$ & 3 & 565 & Southern Canadian Cordill \\
\hline $635-542$ & 3 & 565 & Southern Canadian Cordill \\
\hline $635-542$ & 3 & 565 & Southern Canadian Cordill \\
\hline $635-542$ & 3 & 565 & Southern Canadian Cordill \\
\hline $635-542$ & 3 & 565 & Southern Canadian Cordill \\
\hline $635-542$ & 3 & 565 & Southern Canadian Cordill \\
\hline $635-542$ & 3 & 565 & Southern Canadian Cordill \\
\hline $635-542$ & 3 & 565 & Southern Canadian Cordill \\
\hline $635-542$ & 3 & 565 & Southern Canadian Cordill \\
\hline $635-542$ & 3 & 565 & Southern Canadian Cordill \\
\hline $635-542$ & 3 & 565 & Southern Canadian Cordill \\
\hline $635-542$ & 3 & 565 & Southern Canadian Cordill \\
\hline
\end{tabular}




\begin{tabular}{|c|c|c|c|}
\hline $635-542$ & 3 & 565 & Southern Canadian Cordill \\
\hline $635-542$ & 3 & 565 & Southern Canadian Cordill \\
\hline $635-542$ & 3 & 565 & Southern Canadian Cordill \\
\hline $635-542$ & 3 & 565 & Southern Canadian Cordill \\
\hline $635-542$ & 3 & 565 & Southern Canadian Cordill \\
\hline $635-542$ & 3 & 565 & Southern Canadian Cordill \\
\hline $635-542$ & 3 & 565 & Southern Canadian Cordill \\
\hline $635-542$ & 3 & 565 & Southern Canadian Cordill \\
\hline $635-542$ & 3 & 565 & Southern Canadian Cordill \\
\hline $635-542$ & 3 & 565 & Southern Canadian Cordill \\
\hline $635-542$ & 3 & 565 & Southern Canadian Cordill \\
\hline $635-542$ & 3 & 565 & Southern Canadian Cordill \\
\hline $635-542$ & 3 & 565 & Southern Canadian Cordill \\
\hline $635-542$ & 3 & 565 & Southern Canadian Cordill \\
\hline $635-542$ & 3 & 565 & Southern Canadian Cordill \\
\hline $635-542$ & 3 & 565 & Southern Canadian Cordill \\
\hline $635-542$ & 3 & 565 & Southern Canadian Cordill \\
\hline $635-542$ & 3 & 565 & Southern Canadian Cordill \\
\hline $635-542$ & 3 & 565 & Southern Canadian Cordill \\
\hline $635-542$ & 3 & 565 & Southern Canadian Cordill \\
\hline $635-542$ & 3 & 565 & Southern Canadian Cordill \\
\hline $635-542$ & 3 & 565 & Southern Canadian Cordill \\
\hline $635-542$ & 3 & 565 & Southern Canadian Cordill \\
\hline $635-542$ & 3 & 565 & Southern Canadian Cordill \\
\hline $635-542$ & 3 & 565 & Southern Canadian Cordill \\
\hline $635-542$ & 3 & 565 & Southern Canadian Cordill \\
\hline $635-542$ & 3 & 565 & Southern Canadian Cordill \\
\hline $635-542$ & 3 & 565 & Southern Canadian Cordill \\
\hline $635-542$ & 3 & 565 & Southern Canadian Cordill \\
\hline $635-542$ & 3 & 565 & Southern Canadian Cordill \\
\hline $635-542$ & 3 & 565 & Southern Canadian Cordill \\
\hline $635-542$ & 3 & 565 & Southern Canadian Cordill \\
\hline $635-542$ & 3 & 565 & Southern Canadian Cordill \\
\hline $635-542$ & 3 & 565 & Southern Canadian Cordill \\
\hline $635-542$ & 3 & 565 & Southern Canadian Cordill \\
\hline $635-542$ & 3 & 565 & Southern Canadian Cordill \\
\hline $635-542$ & 3 & 565 & Southern Canadian Cordill \\
\hline $635-542$ & 3 & 565 & Southern Canadian Cordill \\
\hline $635-542$ & 3 & 565 & Southern Canadian Cordill \\
\hline $635-542$ & 3 & 565 & Southern Canadian Cordill \\
\hline $635-542$ & 3 & 565 & Southern Canadian Cordill \\
\hline
\end{tabular}




\begin{tabular}{|c|c|c|c|}
\hline $635-542$ & 3 & 565 & Southern Canadian Cordill \\
\hline $635-542$ & 3 & 565 & Southern Canadian Cordill \\
\hline $635-542$ & 3 & 565 & Southern Canadian Cordill \\
\hline $635-542$ & 3 & 565 & Southern Canadian Cordill \\
\hline $635-542$ & 3 & 565 & Southern Canadian Cordill \\
\hline $635-542$ & 3 & 565 & Southern Canadian Cordill \\
\hline $635-542$ & 3 & 565 & Southern Canadian Cordill \\
\hline $635-542$ & 3 & 565 & Southern Canadian Cordill \\
\hline $635-542$ & 3 & 565 & Southern Canadian Cordill \\
\hline $635-542$ & 3 & 565 & Southern Canadian Cordill \\
\hline $635-542$ & 3 & 565 & Southern Canadian Cordill \\
\hline $635-542$ & 3 & 565 & Southern Canadian Cordill \\
\hline $635-542$ & 3 & 565 & Southern Canadian Cordill \\
\hline $635-542$ & 3 & 565 & Southern Canadian Cordill \\
\hline $635-542$ & 3 & 565 & Southern Canadian Cordill \\
\hline $635-542$ & 3 & 565 & Southern Canadian Cordill \\
\hline $635-542$ & 3 & 565 & Southern Canadian Cordill \\
\hline $635-542$ & 3 & 565 & Southern Canadian Cordill \\
\hline $635-542$ & 3 & 565 & Southern Canadian Cordill \\
\hline $635-542$ & 3 & 565 & Southern Canadian Cordill \\
\hline $635-542$ & 3 & 565 & Southern Canadian Cordill \\
\hline $635-542$ & 3 & 565 & Southern Canadian Cordill \\
\hline $635-542$ & 3 & 565 & Southern Canadian Cordill \\
\hline $635-542$ & 3 & 565 & Southern Canadian Cordill \\
\hline $635-542$ & 3 & 565 & Southern Canadian Cordill \\
\hline $635-542$ & 3 & 565 & Southern Canadian Cordill \\
\hline $635-542$ & 3 & 565 & Southern Canadian Cordill \\
\hline $635-542$ & 3 & 565 & Southern Canadian Cordill \\
\hline $635-542$ & 3 & 565 & Southern Canadian Cordill \\
\hline $635-542$ & 3 & 565 & Southern Canadian Cordill \\
\hline $635-542$ & 3 & 565 & Southern Canadian Cordill \\
\hline $635-542$ & 3 & 565 & Southern Canadian Cordill \\
\hline $635-542$ & 3 & 620 & Southern Canadian Cordill \\
\hline $635-542$ & 3 & 620 & Southern Canadian Cordill \\
\hline $635-542$ & 3 & 620 & Southern Canadian Cordill \\
\hline $635-542$ & 3 & 620 & Southern Canadian Cordill \\
\hline $635-542$ & 3 & 580 & Southern Canadian Cordill \\
\hline $635-542$ & 3 & 580 & Southern Canadian Cordill \\
\hline $635-542$ & 3 & 580 & Southern Canadian Cordill \\
\hline $635-542$ & 3 & 580 & Southern Canadian Cordill \\
\hline $635-542$ & 3 & 580 & Southern Canadian Cordill \\
\hline
\end{tabular}




\begin{tabular}{|c|c|c|c|}
\hline $635-542$ & 3 & 580 & Southern Canadian Cordill \\
\hline $635-542$ & 3 & 580 & Southern Canadian Cordill \\
\hline $635-542$ & 3 & 580 & Southern Canadian Cordill \\
\hline $635-542$ & 3 & 580 & Southern Canadian Cordill \\
\hline $635-542$ & 3 & 580 & Southern Canadian Cordill \\
\hline $635-542$ & 3 & 580 & Southern Canadian Cordill \\
\hline $635-542$ & 3 & 580 & Southern Canadian Cordill \\
\hline $635-542$ & 3 & 580 & Southern Canadian Cordill \\
\hline $635-542$ & 3 & 580 & Southern Canadian Cordill \\
\hline $635-542$ & 3 & 580 & Southern Canadian Cordill \\
\hline $635-542$ & 3 & 580 & Southern Canadian Cordill \\
\hline $635-542$ & 3 & 580 & Southern Canadian Cordill \\
\hline $635-542$ & 3 & 580 & Southern Canadian Cordill \\
\hline $635-542$ & 3 & 580 & Southern Canadian Cordill \\
\hline $635-542$ & 3 & 580 & Southern Canadian Cordill \\
\hline $635-542$ & 3 & 580 & Southern Canadian Cordill \\
\hline $635-542$ & 3 & 580 & Southern Canadian Cordill \\
\hline $635-542$ & 3 & 580 & Southern Canadian Cordill \\
\hline $635-542$ & 3 & 580 & Southern Canadian Cordill \\
\hline $635-542$ & 3 & 580 & Southern Canadian Cordill \\
\hline $635-542$ & 3 & 580 & Southern Canadian Cordill \\
\hline $635-542$ & 3 & 580 & Southern Canadian Cordill \\
\hline $635-542$ & 3 & 580 & Southern Canadian Cordill \\
\hline $635-542$ & 3 & 580 & Southern Canadian Cordill \\
\hline $635-542$ & 3 & 580 & Southern Canadian Cordill \\
\hline $635-542$ & 3 & 580 & Southern Canadian Cordill \\
\hline $635-542$ & 3 & 580 & Southern Canadian Cordill \\
\hline $635-542$ & 3 & 580 & Southern Canadian Cordill \\
\hline $635-542$ & 3 & 580 & Southern Canadian Cordill \\
\hline $635-542$ & 3 & 580 & Southern Canadian Cordill \\
\hline $635-542$ & 3 & 580 & Southern Canadian Cordill \\
\hline $635-542$ & 3 & 580 & Southern Canadian Cordill \\
\hline $635-542$ & 3 & 580 & Southern Canadian Cordill \\
\hline $635-542$ & 3 & 580 & Southern Canadian Cordill \\
\hline $635-542$ & 3 & 580 & Southern Canadian Cordill \\
\hline $635-542$ & 3 & 580 & Southern Canadian Cordill \\
\hline $635-542$ & 3 & 580 & Southern Canadian Cordill \\
\hline $635-542$ & 3 & 580 & Southern Canadian Cordill \\
\hline $635-542$ & 3 & 580 & Southern Canadian Cordill \\
\hline $635-542$ & 3 & 580 & Southern Canadian Cordill \\
\hline $635-542$ & 3 & 580 & Southern Canadian Cordill \\
\hline
\end{tabular}




\begin{tabular}{|c|c|c|c|}
\hline $635-542$ & 3 & 580 & Southern Canadian Cordill \\
\hline $635-542$ & 3 & 580 & Southern Canadian Cordill \\
\hline $635-542$ & 3 & 580 & Southern Canadian Cordill \\
\hline $635-542$ & 3 & 580 & Southern Canadian Cordill \\
\hline $635-542$ & 3 & 580 & Southern Canadian Cordill \\
\hline $635-542$ & 3 & 580 & Southern Canadian Cordill \\
\hline $635-542$ & 3 & 580 & Southern Canadian Cordill \\
\hline $635-542$ & 3 & 580 & Southern Canadian Cordill \\
\hline $635-542$ & 3 & 580 & Southern Canadian Cordill \\
\hline $635-542$ & 3 & 575 & Avalon (Newfoundland) \\
\hline $635-542$ & 3 & 560 & Avalon (Newfoundland) \\
\hline $635-542$ & 3 & 560 & Avalon (Newfoundland) \\
\hline $635-542$ & 3 & 560 & Avalon (Newfoundland) \\
\hline $635-542$ & 3 & 560 & Avalon (Newfoundland) \\
\hline $635-542$ & 3 & 560 & Avalon (Newfoundland) \\
\hline $635-542$ & 3 & 560 & Avalon (Newfoundland) \\
\hline $635-542$ & 3 & 560 & Avalon (Newfoundland) \\
\hline $635-542$ & 3 & 560 & Avalon (Newfoundland) \\
\hline $635-542$ & 3 & 560 & Avalon (Newfoundland) \\
\hline $635-542$ & 3 & 560 & Avalon (Newfoundland) \\
\hline $635-542$ & 3 & 560 & Avalon (Newfoundland) \\
\hline $635-542$ & 3 & 580 & Avalon (Newfoundland) \\
\hline $635-542$ & 3 & 580 & Avalon (Newfoundland) \\
\hline $635-542$ & 3 & 580 & Avalon (Newfoundland) \\
\hline $635-542$ & 3 & 580 & Avalon (Newfoundland) \\
\hline $635-542$ & 3 & 580 & Avalon (Newfoundland) \\
\hline $635-542$ & 3 & 580 & Avalon (Newfoundland) \\
\hline $635-542$ & 3 & 580 & Avalon (Newfoundland) \\
\hline $635-542$ & 3 & 580 & Avalon (Newfoundland) \\
\hline $635-542$ & 3 & 565 & Avalon (Newfoundland) \\
\hline $635-542$ & 3 & 565 & Avalon (Newfoundland) \\
\hline $635-542$ & 3 & 565 & Avalon (Newfoundland) \\
\hline $635-542$ & 3 & 565 & Avalon (Newfoundland) \\
\hline $635-542$ & 3 & 565 & Avalon (Newfoundland) \\
\hline $635-542$ & 3 & 565 & Avalon (Newfoundland) \\
\hline $635-542$ & 3 & 565 & Avalon (Newfoundland) \\
\hline $635-542$ & 3 & 565 & Avalon (Newfoundland) \\
\hline $635-542$ & 3 & 565 & Avalon (Newfoundland) \\
\hline $635-542$ & 3 & 575 & Avalon (Newfoundland) \\
\hline $635-542$ & 3 & 575 & Avalon (Newfoundland) \\
\hline $635-542$ & 3 & 575 & Avalon (Newfoundland) \\
\hline
\end{tabular}




\begin{tabular}{|c|c|c|c|}
\hline $635-542$ & 3 & 575 & Avalon (Newfoundland) \\
\hline $635-542$ & 3 & 575 & Avalon (Newfoundland) \\
\hline $635-542$ & 3 & 575 & Avalon (Newfoundland) \\
\hline $635-542$ & 3 & 575 & Avalon (Newfoundland) \\
\hline $635-542$ & 3 & 575 & Avalon (Newfoundland) \\
\hline $635-542$ & 3 & 575 & Avalon (Newfoundland) \\
\hline $635-542$ & 3 & 575 & Avalon (Newfoundland) \\
\hline $635-542$ & 3 & 575 & Avalon (Newfoundland) \\
\hline $635-542$ & 3 & 570 & Avalon (Newfoundland) \\
\hline $635-542$ & 3 & 570 & Avalon (Newfoundland) \\
\hline $635-542$ & 3 & 570 & Avalon (Newfoundland) \\
\hline $635-542$ & 3 & 570 & Avalon (Newfoundland) \\
\hline $635-542$ & 3 & 570 & Avalon (Newfoundland) \\
\hline $635-542$ & 3 & 570 & Avalon (Newfoundland) \\
\hline $635-542$ & 3 & 570 & Avalon (Newfoundland) \\
\hline $635-542$ & 3 & 570 & Avalon (Newfoundland) \\
\hline $635-542$ & 3 & 570 & Avalon (Newfoundland) \\
\hline $635-542$ & 3 & 570 & Avalon (Newfoundland) \\
\hline $635-542$ & 3 & 570 & Avalon (Newfoundland) \\
\hline $635-542$ & 3 & 570 & Avalon (Newfoundland) \\
\hline $635-542$ & 3 & 570 & Avalon (Newfoundland) \\
\hline $635-542$ & 3 & 570 & Avalon (Newfoundland) \\
\hline $635-542$ & 3 & 570 & Avalon (Newfoundland) \\
\hline $635-542$ & 3 & 575 & Avalon (Newfoundland) \\
\hline $635-542$ & 3 & 575 & Avalon (Newfoundland) \\
\hline $635-542$ & 3 & 575 & Avalon (Newfoundland) \\
\hline $635-542$ & 3 & 575 & Avalon (Newfoundland) \\
\hline $635-542$ & 3 & 575 & Avalon (Newfoundland) \\
\hline $635-542$ & 3 & 575 & Avalon (Newfoundland) \\
\hline $635-542$ & 3 & 575 & Avalon (Newfoundland) \\
\hline $635-542$ & 3 & 575 & Avalon (Newfoundland) \\
\hline $635-542$ & 3 & 575 & Avalon (Newfoundland) \\
\hline $635-542$ & 3 & 575 & Avalon (Newfoundland) \\
\hline $635-542$ & 3 & 580 & Avalon (Newfoundland) \\
\hline $635-542$ & 3 & 580 & Avalon (Newfoundland) \\
\hline $635-542$ & 3 & 580 & Avalon (Newfoundland) \\
\hline $635-542$ & 3 & 580 & Avalon (Newfoundland) \\
\hline $635-542$ & 3 & 580 & Avalon (Newfoundland) \\
\hline $635-542$ & 3 & 565 & Avalon (Newfoundland) \\
\hline $635-542$ & 3 & 565 & Avalon (Newfoundland) \\
\hline $635-542$ & 3 & 565 & Avalon (Newfoundland) \\
\hline
\end{tabular}




\begin{tabular}{|c|c|c|c|}
\hline $635-542$ & 3 & 565 & Avalon (Newfoundland) \\
\hline $635-542$ & 3 & 565 & Avalon (Newfoundland) \\
\hline $635-542$ & 3 & 565 & Avalon (Newfoundland) \\
\hline $635-542$ & 3 & 565 & Avalon (Newfoundland) \\
\hline $635-542$ & 3 & 565 & Avalon (Newfoundland) \\
\hline $635-542$ & 3 & 565 & Avalon (Newfoundland) \\
\hline $635-542$ & 3 & 565 & Avalon (Newfoundland) \\
\hline $635-542$ & 3 & 565 & Avalon (Newfoundland) \\
\hline $635-542$ & 3 & 565 & Avalon (Newfoundland) \\
\hline $635-542$ & 3 & 565 & Avalon (Newfoundland) \\
\hline $635-542$ & 3 & 565 & Avalon (Newfoundland) \\
\hline $635-542$ & 3 & 565 & Avalon (Newfoundland) \\
\hline $635-542$ & 3 & 560 & Avalon (Newfoundland) \\
\hline $635-542$ & 3 & 560 & Avalon (Newfoundland) \\
\hline $635-542$ & 3 & 560 & Avalon (Newfoundland) \\
\hline $635-542$ & 3 & 560 & Avalon (Newfoundland) \\
\hline $635-542$ & 3 & 560 & Avalon (Newfoundland) \\
\hline $635-542$ & 3 & 560 & Avalon (Newfoundland) \\
\hline $635-542$ & 3 & 560 & Avalon (Newfoundland) \\
\hline $635-542$ & 3 & 560 & Avalon (Newfoundland) \\
\hline $635-542$ & 3 & 560 & Avalon (Newfoundland) \\
\hline $635-542$ & 3 & 560 & Avalon (Newfoundland) \\
\hline $635-542$ & 3 & 560 & Avalon (Newfoundland) \\
\hline $635-542$ & 3 & 560 & Avalon (Newfoundland) \\
\hline $635-542$ & 3 & 560 & Avalon (Newfoundland) \\
\hline $635-542$ & 3 & 560 & Avalon (Newfoundland) \\
\hline $635-542$ & 3 & 560 & Avalon (Newfoundland) \\
\hline $635-542$ & 3 & 560 & Avalon (Newfoundland) \\
\hline $635-542$ & 3 & 560 & Avalon (Newfoundland) \\
\hline $635-542$ & 3 & 560 & Avalon (Newfoundland) \\
\hline $635-542$ & 3 & 562 & Avalon (Newfoundland) \\
\hline $635-542$ & 3 & 562 & Avalon (Newfoundland) \\
\hline $635-542$ & 3 & 562 & Avalon (Newfoundland) \\
\hline $635-542$ & 3 & 562 & Avalon (Newfoundland) \\
\hline $635-542$ & 3 & 562 & Avalon (Newfoundland) \\
\hline $635-542$ & 3 & 562 & Avalon (Newfoundland) \\
\hline $635-542$ & 3 & 562 & Avalon (Newfoundland) \\
\hline $635-542$ & 3 & 562 & Avalon (Newfoundland) \\
\hline $635-542$ & 3 & 562 & Avalon (Newfoundland) \\
\hline $635-542$ & 3 & 562 & Avalon (Newfoundland) \\
\hline $635-542$ & 3 & 562 & Avalon (Newfoundland) \\
\hline
\end{tabular}




\begin{tabular}{|c|c|c|c|}
\hline $635-542$ & 3 & 562 & Avalon (Newfoundland) \\
\hline $635-542$ & 3 & 562 & Avalon (Newfoundland) \\
\hline $635-542$ & 3 & 562 & Avalon (Newfoundland) \\
\hline $635-542$ & 3 & 562 & Avalon (Newfoundland) \\
\hline $635-542$ & 3 & 562 & Avalon (Newfoundland) \\
\hline $635-542$ & 3 & 562 & Avalon (Newfoundland) \\
\hline $635-542$ & 3 & 590 & Avalon (Newfoundland) \\
\hline $635-542$ & 3 & 590 & Avalon (Newfoundland) \\
\hline $635-542$ & 3 & 590 & Avalon (Newfoundland) \\
\hline $635-542$ & 3 & 590 & Avalon (Newfoundland) \\
\hline $635-542$ & 3 & 590 & Avalon (Newfoundland) \\
\hline $635-542$ & 3 & 590 & Avalon (Newfoundland) \\
\hline $635-542$ & 3 & 590 & Avalon (Newfoundland) \\
\hline $635-542$ & 3 & 590 & Avalon (Newfoundland) \\
\hline $635-542$ & 3 & 590 & Avalon (Newfoundland) \\
\hline $635-542$ & 3 & 590 & Avalon (Newfoundland) \\
\hline $635-542$ & 3 & 590 & Avalon (Newfoundland) \\
\hline $635-542$ & 3 & 590 & Avalon (Newfoundland) \\
\hline $635-542$ & 3 & 590 & Avalon (Newfoundland) \\
\hline $635-542$ & 3 & 590 & Avalon (Newfoundland) \\
\hline $635-542$ & 3 & 590 & Avalon (Newfoundland) \\
\hline $635-542$ & 3 & 590 & Avalon (Newfoundland) \\
\hline $635-542$ & 3 & 590 & Avalon (Newfoundland) \\
\hline $635-542$ & 3 & 590 & Avalon (Newfoundland) \\
\hline $635-542$ & 3 & 590 & Avalon (Newfoundland) \\
\hline $635-542$ & 3 & 590 & Avalon (Newfoundland) \\
\hline $635-542$ & 3 & 590 & Avalon (Newfoundland) \\
\hline $635-542$ & 3 & 590 & Avalon (Newfoundland) \\
\hline $635-542$ & 3 & 590 & Avalon (Newfoundland) \\
\hline $635-542$ & 3 & 590 & Avalon (Newfoundland) \\
\hline $635-542$ & 3 & 590 & Avalon (Newfoundland) \\
\hline $635-542$ & 3 & 590 & Avalon (Newfoundland) \\
\hline $635-542$ & 3 & 590 & Avalon (Newfoundland) \\
\hline $635-542$ & 3 & 590 & Avalon (Newfoundland) \\
\hline $635-542$ & 3 & 590 & Avalon (Newfoundland) \\
\hline $635-542$ & 3 & 575 & Avalon (Newfoundland) \\
\hline $635-542$ & 3 & 575 & Avalon (Newfoundland) \\
\hline $635-542$ & 3 & 575 & Avalon (Newfoundland) \\
\hline $635-542$ & 3 & 575 & Avalon (Newfoundland) \\
\hline $635-542$ & 3 & 575 & Avalon (Newfoundland) \\
\hline $635-542$ & 3 & 575 & Avalon (Newfoundland) \\
\hline
\end{tabular}




\begin{tabular}{|c|c|c|c|}
\hline $635-542$ & 3 & 575 & Avalon (Newfoundland) \\
\hline $635-542$ & 3 & 575 & Avalon (Newfoundland) \\
\hline $635-542$ & 3 & 575 & Avalon (Newfoundland) \\
\hline $635-542$ & 3 & 575 & Avalon (Newfoundland) \\
\hline $635-542$ & 3 & 575 & Avalon (Newfoundland) \\
\hline $635-542$ & 3 & 575 & Avalon (Newfoundland) \\
\hline $635-542$ & 3 & 575 & Avalon (Newfoundland) \\
\hline $635-542$ & 3 & 575 & Avalon (Newfoundland) \\
\hline $635-542$ & 3 & 575 & Avalon (Newfoundland) \\
\hline $635-542$ & 3 & 575 & Avalon (Newfoundland) \\
\hline $635-542$ & 3 & 575 & Avalon (Newfoundland) \\
\hline $635-542$ & 3 & 575 & Avalon (Newfoundland) \\
\hline $635-542$ & 3 & 575 & Avalon (Newfoundland) \\
\hline $635-542$ & 3 & 560 & Avalon (Newfoundland) \\
\hline $635-542$ & 3 & 560 & Avalon (Newfoundland) \\
\hline $635-542$ & 3 & 560 & Avalon (Newfoundland) \\
\hline $635-542$ & 3 & 560 & Avalon (Newfoundland) \\
\hline $635-542$ & 3 & 560 & Avalon (Newfoundland) \\
\hline $635-542$ & 3 & 560 & Avalon (Newfoundland) \\
\hline $635-542$ & 3 & 580 & Avalon (Newfoundland) \\
\hline $635-542$ & 3 & 580 & Avalon (Newfoundland) \\
\hline $635-542$ & 3 & 580 & Avalon (Newfoundland) \\
\hline $635-542$ & 3 & 580 & Avalon (Newfoundland) \\
\hline $635-542$ & 3 & 580 & Avalon (Newfoundland) \\
\hline $635-542$ & 3 & 580 & Avalon (Newfoundland) \\
\hline $635-542$ & 3 & 580 & Avalon (Newfoundland) \\
\hline $635-542$ & 3 & 580 & Avalon (Newfoundland) \\
\hline $635-542$ & 3 & 580 & Avalon (Newfoundland) \\
\hline $635-542$ & 3 & 580 & Avalon (Newfoundland) \\
\hline $635-542$ & 3 & 580 & Avalon (Newfoundland) \\
\hline $635-542$ & 3 & 580 & Avalon (Newfoundland) \\
\hline $635-542$ & 3 & 580 & Avalon (Newfoundland) \\
\hline $635-542$ & 3 & 580 & Avalon (Newfoundland) \\
\hline $635-542$ & 3 & 580 & Avalon (Newfoundland) \\
\hline $635-542$ & 3 & 580 & Avalon (Newfoundland) \\
\hline $635-542$ & 3 & 580 & Avalon (Newfoundland) \\
\hline $635-542$ & 3 & 580 & Avalon (Newfoundland) \\
\hline $635-542$ & 3 & 580 & Avalon (Newfoundland) \\
\hline $635-542$ & 3 & 565 & Avalon (Newfoundland) \\
\hline $635-542$ & 3 & 565 & Avalon (Newfoundland) \\
\hline $635-542$ & 3 & 565 & Avalon (Newfoundland) \\
\hline
\end{tabular}




\begin{tabular}{|c|c|c|c|c|}
\hline $635-542$ & 3 & 560 & Avalon (Newfoundland) & 28 \\
\hline $635-542$ & 3 & 620 & Spitsbergen & 29 \\
\hline $635-542$ & 3 & 620 & Spitsbergen & 29 \\
\hline $635-542$ & 3 & 620 & Spitsbergen & 29 \\
\hline $635-542$ & 3 & 610 & East Greenland & 30 \\
\hline $635-542$ & 3 & 610 & East Greenland & 30 \\
\hline $635-542$ & 3 & 610 & East Greenland & 30 \\
\hline $635-542$ & 3 & 610 & East Greenland & 30 \\
\hline $635-542$ & 3 & 610 & East Greenland & 30 \\
\hline $635-542$ & 3 & 610 & East Greenland & 30 \\
\hline $635-542$ & 3 & 610 & East Greenland & 30 \\
\hline $635-542$ & 3 & 610 & East Greenland & 30 \\
\hline $635-542$ & 3 & 610 & East Greenland & 30 \\
\hline $635-542$ & 3 & 610 & East Greenland & 30 \\
\hline $635-542$ & 3 & 610 & East Greenland & 30 \\
\hline $635-542$ & 3 & 610 & East Greenland & 30 \\
\hline $635-542$ & 3 & 610 & East Greenland & 30 \\
\hline $635-542$ & 3 & 610 & East Greenland & 30 \\
\hline $635-542$ & 3 & 610 & East Greenland & 30 \\
\hline $635-542$ & 3 & 610 & East Greenland & 30 \\
\hline $635-542$ & 3 & 610 & East Greenland & 30 \\
\hline $635-542$ & 3 & 605 & East Greenland & 30 \\
\hline $635-542$ & 3 & 605 & East Greenland & 30 \\
\hline $635-542$ & 3 & 605 & East Greenland & 30 \\
\hline $635-542$ & 3 & 605 & East Greenland & 30 \\
\hline $635-542$ & 3 & 605 & East Greenland & 30 \\
\hline $635-542$ & 3 & 605 & East Greenland & 30 \\
\hline $635-542$ & 3 & 620 & Stuart Shelf, South Austra & 31 \\
\hline $635-542$ & 3 & 620 & Stuart Shelf, South Austra & 31 \\
\hline $635-542$ & 3 & 620 & Stuart Shelf, South Austra & 31 \\
\hline $635-542$ & 3 & 620 & Stuart Shelf, South Austra & 31 \\
\hline $635-542$ & 3 & 620 & Stuart Shelf, South Austra & 31 \\
\hline $635-542$ & 3 & 620 & Stuart Shelf, South Austra & 31 \\
\hline $635-542$ & 3 & 620 & Stuart Shelf, South Austra & 31 \\
\hline $635-542$ & 3 & 610 & Stuart Shelf, South Austra & 31 \\
\hline $635-542$ & 3 & 610 & Stuart Shelf, South Austra & 31 \\
\hline $635-542$ & 3 & 610 & Stuart Shelf, South Austra & 31 \\
\hline $635-542$ & 3 & 610 & Stuart Shelf, South Austra & 31 \\
\hline $635-542$ & 3 & 610 & Stuart Shelf, South Austra & 31 \\
\hline $635-542$ & 3 & 610 & Stuart Shelf, South Austra & 31 \\
\hline $635-542$ & 3 & 610 & Stuart Shelf, South Austra & 31 \\
\hline
\end{tabular}




\begin{tabular}{|c|c|c|c|}
\hline $635-542$ & 3 & 610 & Stuart Shelf, South Austra \\
\hline $635-542$ & 3 & 610 & Stuart Shelf, South Austra \\
\hline $635-542$ & 3 & 610 & Stuart Shelf, South Austra \\
\hline $635-542$ & 3 & 610 & Stuart Shelf, South Austra \\
\hline $635-542$ & 3 & 610 & Stuart Shelf, South Austra \\
\hline $635-542$ & 3 & 610 & Stuart Shelf, South Austra \\
\hline $635-542$ & 3 & 610 & Stuart Shelf, South Austra \\
\hline $635-542$ & 3 & 610 & Stuart Shelf, South Austra \\
\hline $635-542$ & 3 & 610 & Stuart Shelf, South Austra \\
\hline $635-542$ & 3 & 610 & Stuart Shelf, South Austra \\
\hline $635-542$ & 3 & 610 & Stuart Shelf, South Austra \\
\hline $635-542$ & 3 & 610 & Stuart Shelf, South Austra \\
\hline $635-542$ & 3 & 610 & Stuart Shelf, South Austra \\
\hline $635-542$ & 3 & 610 & Stuart Shelf, South Austra \\
\hline $635-542$ & 3 & 590 & Stuart Shelf, South Austra \\
\hline $635-542$ & 3 & 590 & Stuart Shelf, South Austra \\
\hline $635-542$ & 3 & 590 & Stuart Shelf, South Austra \\
\hline $635-542$ & 3 & 590 & Stuart Shelf, South Austra \\
\hline $635-542$ & 3 & 590 & Stuart Shelf, South Austra \\
\hline $635-542$ & 3 & 590 & Stuart Shelf, South Austra \\
\hline $635-542$ & 3 & 590 & Stuart Shelf, South Austra \\
\hline $635-542$ & 3 & 590 & Stuart Shelf, South Austra \\
\hline $635-542$ & 3 & 590 & Stuart Shelf, South Austra \\
\hline $635-542$ & 3 & 590 & Stuart Shelf, South Austra \\
\hline $635-542$ & 3 & 625 & Stuart Shelf, South Austra \\
\hline $635-542$ & 3 & 625 & Stuart Shelf, South Austra \\
\hline $635-542$ & 3 & 625 & Stuart Shelf, South Austra \\
\hline $635-542$ & 3 & 625 & Stuart Shelf, South Austra \\
\hline $635-542$ & 3 & 625 & Stuart Shelf, South Austra \\
\hline $635-542$ & 3 & 625 & Stuart Shelf, South Austra \\
\hline $635-542$ & 3 & 625 & Stuart Shelf, South Austra \\
\hline $635-542$ & 3 & 625 & Stuart Shelf, South Austra \\
\hline $635-542$ & 3 & 545 & Siberia \\
\hline $635-542$ & 3 & 545 & Siberia \\
\hline $635-542$ & 3 & 545 & Siberia \\
\hline $635-542$ & 3 & 620 & Ogilvie Mountains \\
\hline $635-542$ & 3 & 620 & Ogilvie Mountains \\
\hline $635-542$ & 3 & 620 & Ogilvie Mountains \\
\hline $635-542$ & 3 & 620 & Ogilvie Mountains \\
\hline $635-542$ & 3 & 620 & Ogilvie Mountains \\
\hline $635-542$ & 3 & 620 & Ogilvie Mountains \\
\hline
\end{tabular}




\begin{tabular}{|c|c|c|c|c|}
\hline $635-542$ & 3 & 620 & Ogilvie Mountains & 36 \\
\hline $635-542$ & 3 & 620 & Ogilvie Mountains & 36 \\
\hline $635-542$ & 3 & 620 & Ogilvie Mountains & 36 \\
\hline $635-542$ & 3 & 620 & Ogilvie Mountains & 36 \\
\hline $635-542$ & 3 & 620 & Ogilvie Mountains & 36 \\
\hline $635-542$ & 3 & 620 & Ogilvie Mountains & 36 \\
\hline $635-542$ & 3 & 620 & Ogilvie Mountains & 36 \\
\hline $635-542$ & 3 & 620 & Ogilvie Mountains & 36 \\
\hline $635-542$ & 3 & 620 & Ogilvie Mountains & 36 \\
\hline $635-542$ & 3 & 620 & Ogilvie Mountains & 36 \\
\hline $635-542$ & 3 & 620 & Ogilvie Mountains & 36 \\
\hline $635-542$ & 3 & 620 & Ogilvie Mountains & 36 \\
\hline $635-542$ & 3 & 620 & Ogilvie Mountains & 36 \\
\hline $635-542$ & 3 & 620 & Ogilvie Mountains & 36 \\
\hline $635-542$ & 3 & 620 & Ogilvie Mountains & 36 \\
\hline $635-542$ & 3 & 620 & Ogilvie Mountains & 36 \\
\hline $635-542$ & 3 & 620 & Ogilvie Mountains & 36 \\
\hline $635-542$ & 3 & 620 & Ogilvie Mountains & 36 \\
\hline $635-542$ & 3 & 620 & Ogilvie Mountains & 36 \\
\hline $635-542$ & 3 & 620 & Ogilvie Mountains & 36 \\
\hline $635-542$ & 3 & 620 & Ogilvie Mountains & 36 \\
\hline $635-542$ & 3 & 620 & Ogilvie Mountains & 36 \\
\hline $635-542$ & 3 & 620 & Ogilvie Mountains & 36 \\
\hline $635-542$ & 3 & 620 & Ogilvie Mountains & 36 \\
\hline $635-542$ & 3 & 620 & Ogilvie Mountains & 36 \\
\hline $635-542$ & 3 & 620 & Ogilvie Mountains & 36 \\
\hline $635-542$ & 3 & 620 & Ogilvie Mountains & 36 \\
\hline $635-542$ & 3 & 620 & Ogilvie Mountains & 36 \\
\hline $635-542$ & 3 & 620 & Ogilvie Mountains & 36 \\
\hline $635-542$ & 3 & 620 & Ogilvie Mountains & 36 \\
\hline $635-542$ & 3 & 555 & Ogilvie Mountains & 36 \\
\hline $635-542$ & 3 & 570 & Mackenzie Mountains & 37 \\
\hline $635-542$ & 3 & 570 & Mackenzie Mountains & 37 \\
\hline $635-542$ & 3 & 570 & Mackenzie Mountains & 37 \\
\hline $635-542$ & 3 & 570 & Mackenzie Mountains & 37 \\
\hline $635-542$ & 3 & 570 & Mackenzie Mountains & 37 \\
\hline $635-542$ & 3 & 620 & Mackenzie Mountains & 37 \\
\hline $635-542$ & 3 & 620 & Mackenzie Mountains & 37 \\
\hline $635-542$ & 3 & 620 & Mackenzie Mountains & 37 \\
\hline $635-542$ & 3 & 620 & Mackenzie Mountains & 37 \\
\hline $635-542$ & 3 & 620 & Mackenzie Mountains & 37 \\
\hline
\end{tabular}




\begin{tabular}{|c|c|c|c|c|}
\hline $635-542$ & 3 & 620 & Mackenzie Mountains & 37 \\
\hline $635-542$ & 3 & 620 & Mackenzie Mountains & 37 \\
\hline $635-542$ & 3 & 620 & Mackenzie Mountains & 37 \\
\hline $635-542$ & 3 & 620 & Mackenzie Mountains & 37 \\
\hline $635-542$ & 3 & 620 & Mackenzie Mountains & 37 \\
\hline $635-542$ & 3 & 620 & Mackenzie Mountains & 37 \\
\hline $635-542$ & 3 & 620 & Mackenzie Mountains & 37 \\
\hline $635-542$ & 3 & 620 & Mackenzie Mountains & 37 \\
\hline $635-542$ & 3 & 620 & Mackenzie Mountains & 37 \\
\hline $635-542$ & 3 & 620 & Mackenzie Mountains & 37 \\
\hline $635-542$ & 3 & 620 & Mackenzie Mountains & 37 \\
\hline $635-542$ & 3 & 620 & Mackenzie Mountains & 37 \\
\hline $635-542$ & 3 & 620 & Mackenzie Mountains & 37 \\
\hline $635-542$ & 3 & 620 & Mackenzie Mountains & 37 \\
\hline $635-542$ & 3 & 620 & Mackenzie Mountains & 37 \\
\hline $635-542$ & 3 & 620 & Mackenzie Mountains & 37 \\
\hline $635-542$ & 3 & 620 & Mackenzie Mountains & 37 \\
\hline $635-542$ & 3 & 620 & Mackenzie Mountains & 37 \\
\hline $635-542$ & 3 & 620 & Mackenzie Mountains & 37 \\
\hline $635-542$ & 3 & 620 & Mackenzie Mountains & 37 \\
\hline $635-542$ & 3 & 620 & Mackenzie Mountains & 37 \\
\hline $635-542$ & 3 & 620 & Mackenzie Mountains & 37 \\
\hline $635-542$ & 3 & 620 & Mackenzie Mountains & 37 \\
\hline $635-542$ & 3 & 620 & Mackenzie Mountains & 37 \\
\hline $635-542$ & 3 & 620 & Mackenzie Mountains & 37 \\
\hline $635-542$ & 3 & 620 & Mackenzie Mountains & 37 \\
\hline $635-542$ & 3 & 620 & Mackenzie Mountains & 37 \\
\hline $635-542$ & 3 & 620 & Mackenzie Mountains & 37 \\
\hline $635-542$ & 3 & 620 & Mackenzie Mountains & 37 \\
\hline $635-542$ & 3 & 620 & Mackenzie Mountains & 37 \\
\hline $635-542$ & 3 & 620 & Mackenzie Mountains & 37 \\
\hline $635-542$ & 3 & 620 & Mackenzie Mountains & 37 \\
\hline $635-542$ & 3 & 620 & Mackenzie Mountains & 37 \\
\hline $635-542$ & 3 & 620 & Mackenzie Mountains & 37 \\
\hline $635-542$ & 3 & 620 & Mackenzie Mountains & 37 \\
\hline $635-542$ & 3 & 620 & Mackenzie Mountains & 37 \\
\hline $635-542$ & 3 & 620 & Mackenzie Mountains & 37 \\
\hline $635-542$ & 3 & 620 & Mackenzie Mountains & 37 \\
\hline $635-542$ & 3 & 620 & Mackenzie Mountains & 37 \\
\hline $635-542$ & 3 & 620 & Mackenzie Mountains & 37 \\
\hline $635-542$ & 3 & 620 & Mackenzie Mountains & 37 \\
\hline
\end{tabular}




\begin{tabular}{|c|c|c|c|c|}
\hline $635-542$ & 3 & 620 & Mackenzie Mountains & 37 \\
\hline $635-542$ & 3 & 620 & Mackenzie Mountains & 37 \\
\hline $635-542$ & 3 & 620 & Mackenzie Mountains & 37 \\
\hline $635-542$ & 3 & 620 & Mackenzie Mountains & 37 \\
\hline $635-542$ & 3 & 620 & Mackenzie Mountains & 37 \\
\hline $635-542$ & 3 & 620 & Mackenzie Mountains & 37 \\
\hline $635-542$ & 3 & 620 & Mackenzie Mountains & 37 \\
\hline $635-542$ & 3 & 620 & Mackenzie Mountains & 37 \\
\hline $635-542$ & 3 & 620 & Mackenzie Mountains & 37 \\
\hline $635-542$ & 3 & 620 & Mackenzie Mountains & 37 \\
\hline $635-542$ & 3 & 620 & Mackenzie Mountains & 37 \\
\hline $635-542$ & 3 & 620 & Mackenzie Mountains & 37 \\
\hline $635-542$ & 3 & 620 & Mackenzie Mountains & 37 \\
\hline $635-542$ & 3 & 620 & Mackenzie Mountains & 37 \\
\hline $635-542$ & 3 & 620 & Mackenzie Mountains & 37 \\
\hline $635-542$ & 3 & 620 & Mackenzie Mountains & 37 \\
\hline $635-542$ & 3 & 620 & Mackenzie Mountains & 37 \\
\hline $635-542$ & 3 & 620 & Mackenzie Mountains & 37 \\
\hline $635-542$ & 3 & 620 & Mackenzie Mountains & 37 \\
\hline $635-542$ & 3 & 620 & Mackenzie Mountains & 37 \\
\hline $635-542$ & 3 & 620 & Mackenzie Mountains & 37 \\
\hline $635-542$ & 3 & 620 & Mackenzie Mountains & 37 \\
\hline $635-542$ & 3 & 620 & Mackenzie Mountains & 37 \\
\hline $635-542$ & 3 & 620 & Mackenzie Mountains & 37 \\
\hline $635-542$ & 3 & 620 & Mackenzie Mountains & 37 \\
\hline $635-542$ & 3 & 620 & Mackenzie Mountains & 37 \\
\hline $635-542$ & 3 & 620 & Mackenzie Mountains & 37 \\
\hline $635-542$ & 3 & 620 & Mackenzie Mountains & 37 \\
\hline $635-542$ & 3 & 620 & Mackenzie Mountains & 37 \\
\hline $635-542$ & 3 & 620 & Mackenzie Mountains & 37 \\
\hline $635-542$ & 3 & 620 & Mackenzie Mountains & 37 \\
\hline $635-542$ & 3 & 620 & Mackenzie Mountains & 37 \\
\hline $635-542$ & 3 & 620 & Mackenzie Mountains & 37 \\
\hline $635-542$ & 3 & 620 & Mackenzie Mountains & 37 \\
\hline $635-542$ & 3 & 620 & Mackenzie Mountains & 37 \\
\hline $635-542$ & 3 & 550 & Mackenzie Mountains & 37 \\
\hline $635-542$ & 3 & 550 & Mackenzie Mountains & 37 \\
\hline $635-542$ & 3 & 550 & Mackenzie Mountains & 37 \\
\hline $635-542$ & 3 & 550 & Mackenzie Mountains & 37 \\
\hline $635-542$ & 3 & 550 & Mackenzie Mountains & 37 \\
\hline $635-542$ & 3 & 550 & Mackenzie Mountains & 37 \\
\hline
\end{tabular}




\begin{tabular}{|c|c|c|c|c|}
\hline $635-542$ & 3 & 550 & Mackenzie Mountains & 37 \\
\hline $635-542$ & 3 & 550 & Mackenzie Mountains & 37 \\
\hline $635-542$ & 3 & 550 & Mackenzie Mountains & 37 \\
\hline $635-542$ & 3 & 550 & Mackenzie Mountains & 37 \\
\hline $635-542$ & 3 & 550 & Mackenzie Mountains & 37 \\
\hline $635-542$ & 3 & 550 & Mackenzie Mountains & 37 \\
\hline $635-542$ & 3 & 550 & Mackenzie Mountains & 37 \\
\hline $635-542$ & 3 & 550 & Mackenzie Mountains & 37 \\
\hline $635-542$ & 3 & 550 & Mackenzie Mountains & 37 \\
\hline $635-542$ & 3 & 570 & Mackenzie Mountains & 37 \\
\hline $635-542$ & 3 & 570 & Mackenzie Mountains & 37 \\
\hline $635-542$ & 3 & 570 & Mackenzie Mountains & 37 \\
\hline $635-542$ & 3 & 570 & Mackenzie Mountains & 37 \\
\hline $635-542$ & 3 & 570 & Mackenzie Mountains & 37 \\
\hline $635-542$ & 3 & 570 & Mackenzie Mountains & 37 \\
\hline $635-542$ & 3 & 570 & Mackenzie Mountains & 37 \\
\hline $635-542$ & 3 & 570 & Mackenzie Mountains & 37 \\
\hline $635-542$ & 3 & 570 & Mackenzie Mountains & 37 \\
\hline $635-542$ & 3 & 570 & Mackenzie Mountains & 37 \\
\hline $635-542$ & 3 & 570 & Mackenzie Mountains & 37 \\
\hline $635-542$ & 3 & 570 & Mackenzie Mountains & 37 \\
\hline $635-542$ & 3 & 570 & Mackenzie Mountains & 37 \\
\hline $635-542$ & 3 & 570 & Mackenzie Mountains & 37 \\
\hline $635-542$ & 3 & 570 & Mackenzie Mountains & 37 \\
\hline $635-542$ & 3 & 570 & Mackenzie Mountains & 37 \\
\hline $635-542$ & 3 & 570 & Mackenzie Mountains & 37 \\
\hline $635-542$ & 3 & 570 & Mackenzie Mountains & 37 \\
\hline $635-542$ & 3 & 570 & Mackenzie Mountains & 37 \\
\hline $635-542$ & 3 & 570 & Mackenzie Mountains & 37 \\
\hline $635-542$ & 3 & 570 & Mackenzie Mountains & 37 \\
\hline $635-542$ & 3 & 570 & Mackenzie Mountains & 37 \\
\hline $635-542$ & 3 & 570 & Mackenzie Mountains & 37 \\
\hline $635-542$ & 3 & 570 & Mackenzie Mountains & 37 \\
\hline $635-542$ & 3 & 570 & Mackenzie Mountains & 37 \\
\hline $635-542$ & 3 & 570 & Mackenzie Mountains & 37 \\
\hline $635-542$ & 3 & 570 & Mackenzie Mountains & 37 \\
\hline $635-542$ & 3 & 570 & Mackenzie Mountains & 37 \\
\hline $635-542$ & 3 & 570 & Mackenzie Mountains & 37 \\
\hline $635-542$ & 3 & 570 & Mackenzie Mountains & 37 \\
\hline $635-542$ & 3 & 570 & Mackenzie Mountains & 37 \\
\hline $635-542$ & 3 & 570 & Mackenzie Mountains & 37 \\
\hline
\end{tabular}




\begin{tabular}{|c|c|c|c|c|}
\hline $635-542$ & 3 & 570 & Mackenzie Mountains & 37 \\
\hline $635-542$ & 3 & 570 & Mackenzie Mountains & 37 \\
\hline $635-542$ & 3 & 570 & Mackenzie Mountains & 37 \\
\hline $635-542$ & 3 & 570 & Mackenzie Mountains & 37 \\
\hline $635-542$ & 3 & 570 & Mackenzie Mountains & 37 \\
\hline $635-542$ & 3 & 570 & Mackenzie Mountains & 37 \\
\hline $635-542$ & 3 & 565 & Mackenzie Mountains & 37 \\
\hline $635-542$ & 3 & 565 & Mackenzie Mountains & 37 \\
\hline $635-542$ & 3 & 565 & Mackenzie Mountains & 37 \\
\hline $635-542$ & 3 & 565 & Mackenzie Mountains & 37 \\
\hline $635-542$ & 3 & 565 & Mackenzie Mountains & 37 \\
\hline $635-542$ & 3 & 565 & Mackenzie Mountains & 37 \\
\hline $635-542$ & 3 & 565 & Mackenzie Mountains & 37 \\
\hline $635-542$ & 3 & 565 & Mackenzie Mountains & 37 \\
\hline $635-542$ & 3 & 565 & Mackenzie Mountains & 37 \\
\hline $635-542$ & 3 & 565 & Mackenzie Mountains & 37 \\
\hline $635-542$ & 3 & 565 & Mackenzie Mountains & 37 \\
\hline $635-542$ & 3 & 565 & Mackenzie Mountains & 37 \\
\hline $635-542$ & 3 & 565 & Mackenzie Mountains & 37 \\
\hline $635-542$ & 3 & 565 & Mackenzie Mountains & 37 \\
\hline $635-542$ & 3 & 565 & Mackenzie Mountains & 37 \\
\hline $635-542$ & 3 & 565 & Mackenzie Mountains & 37 \\
\hline $635-542$ & 3 & 565 & Mackenzie Mountains & 37 \\
\hline $635-542$ & 3 & 565 & Mackenzie Mountains & 37 \\
\hline $635-542$ & 3 & 565 & Mackenzie Mountains & 37 \\
\hline $635-542$ & 3 & 565 & Mackenzie Mountains & 37 \\
\hline $635-542$ & 3 & 565 & Mackenzie Mountains & 37 \\
\hline $635-542$ & 3 & 565 & Mackenzie Mountains & 37 \\
\hline $635-542$ & 3 & 565 & Mackenzie Mountains & 37 \\
\hline $635-542$ & 3 & 565 & Mackenzie Mountains & 37 \\
\hline $635-542$ & 3 & 565 & Mackenzie Mountains & 37 \\
\hline $635-542$ & 3 & 565 & Mackenzie Mountains & 37 \\
\hline $635-542$ & 3 & 565 & Mackenzie Mountains & 37 \\
\hline $635-542$ & 3 & 565 & Mackenzie Mountains & 37 \\
\hline $635-542$ & 3 & 565 & Mackenzie Mountains & 37 \\
\hline $635-542$ & 3 & 565 & Mackenzie Mountains & 37 \\
\hline $635-542$ & 3 & 590 & Mackenzie Mountains & 37 \\
\hline $635-542$ & 3 & 590 & Mackenzie Mountains & 37 \\
\hline $635-542$ & 3 & 590 & Mackenzie Mountains & 37 \\
\hline $635-542$ & 3 & 590 & Mackenzie Mountains & 37 \\
\hline $635-542$ & 3 & 590 & Mackenzie Mountains & 37 \\
\hline
\end{tabular}




\begin{tabular}{|c|c|c|c|c|}
\hline $635-542$ & 3 & 590 & Mackenzie Mountains & 37 \\
\hline $635-542$ & 3 & 590 & Mackenzie Mountains & 37 \\
\hline $635-542$ & 3 & 590 & Mackenzie Mountains & 37 \\
\hline $635-542$ & 3 & 590 & Mackenzie Mountains & 37 \\
\hline $635-542$ & 3 & 590 & Mackenzie Mountains & 37 \\
\hline $635-542$ & 3 & 590 & Mackenzie Mountains & 37 \\
\hline $635-542$ & 3 & 590 & Mackenzie Mountains & 37 \\
\hline $635-542$ & 3 & 590 & Mackenzie Mountains & 37 \\
\hline $635-542$ & 3 & 590 & Mackenzie Mountains & 37 \\
\hline $635-542$ & 3 & 590 & Mackenzie Mountains & 37 \\
\hline $635-542$ & 3 & 590 & Mackenzie Mountains & 37 \\
\hline $635-542$ & 3 & 590 & Mackenzie Mountains & 37 \\
\hline $635-542$ & 3 & 590 & Mackenzie Mountains & 37 \\
\hline $635-542$ & 3 & 590 & Mackenzie Mountains & 37 \\
\hline $635-542$ & 3 & 590 & Mackenzie Mountains & 37 \\
\hline $635-542$ & 3 & 590 & Mackenzie Mountains & 37 \\
\hline $635-542$ & 3 & 590 & Mackenzie Mountains & 37 \\
\hline $635-542$ & 3 & 590 & Mackenzie Mountains & 37 \\
\hline $635-542$ & 3 & 590 & Mackenzie Mountains & 37 \\
\hline $635-542$ & 3 & 550 & Mackenzie Mountains & 37 \\
\hline $635-542$ & 3 & 550 & Mackenzie Mountains & 37 \\
\hline $635-542$ & 3 & 545 & Mackenzie Mountains & 37 \\
\hline $635-542$ & 3 & 545 & Mackenzie Mountains & 37 \\
\hline $635-542$ & 3 & 545 & Mackenzie Mountains & 37 \\
\hline $635-542$ & 3 & 545 & Mackenzie Mountains & 37 \\
\hline $635-542$ & 3 & 545 & Mackenzie Mountains & 37 \\
\hline $635-542$ & 3 & 545 & Mackenzie Mountains & 37 \\
\hline $635-542$ & 3 & 545 & Mackenzie Mountains & 37 \\
\hline $635-542$ & 3 & 550 & Mackenzie Mountains & 37 \\
\hline $635-542$ & 3 & 560 & Mackenzie Mountains & 37 \\
\hline $635-542$ & 3 & 550 & Mackenzie Mountains & 37 \\
\hline $635-542$ & 3 & 560 & Mackenzie Mountains & 37 \\
\hline $635-542$ & 3 & 560 & Mackenzie Mountains & 37 \\
\hline $635-542$ & 3 & 560 & Mackenzie Mountains & 37 \\
\hline $635-542$ & 3 & 560 & Mackenzie Mountains & 37 \\
\hline $635-542$ & 3 & 560 & Mackenzie Mountains & 37 \\
\hline $635-542$ & 3 & 560 & Mackenzie Mountains & 37 \\
\hline $635-542$ & 3 & 560 & Mackenzie Mountains & 37 \\
\hline $635-542$ & 3 & 560 & Mackenzie Mountains & 37 \\
\hline $635-542$ & 3 & 560 & Mackenzie Mountains & 37 \\
\hline $635-542$ & 3 & 560 & Mackenzie Mountains & 37 \\
\hline
\end{tabular}




\begin{tabular}{|c|c|c|c|c|}
\hline $635-542$ & 3 & 560 & Mackenzie Mountains & 37 \\
\hline $635-542$ & 3 & 560 & Mackenzie Mountains & 37 \\
\hline $635-542$ & 3 & 560 & Mackenzie Mountains & 37 \\
\hline $635-542$ & 3 & 560 & Mackenzie Mountains & 37 \\
\hline $635-542$ & 3 & 560 & Mackenzie Mountains & 37 \\
\hline $635-542$ & 3 & 560 & Mackenzie Mountains & 37 \\
\hline $635-542$ & 3 & 560 & Mackenzie Mountains & 37 \\
\hline $635-542$ & 3 & 560 & Mackenzie Mountains & 37 \\
\hline $635-542$ & 3 & 560 & Mackenzie Mountains & 37 \\
\hline $635-542$ & 3 & 560 & Mackenzie Mountains & 37 \\
\hline $635-542$ & 3 & 560 & Mackenzie Mountains & 37 \\
\hline $635-542$ & 3 & 560 & Mackenzie Mountains & 37 \\
\hline $635-542$ & 3 & 560 & Mackenzie Mountains & 37 \\
\hline $635-542$ & 3 & 560 & Mackenzie Mountains & 37 \\
\hline $635-542$ & 3 & 560 & Mackenzie Mountains & 37 \\
\hline $635-542$ & 3 & 560 & Mackenzie Mountains & 37 \\
\hline $635-542$ & 3 & 560 & Mackenzie Mountains & 37 \\
\hline $635-542$ & 3 & 560 & Mackenzie Mountains & 37 \\
\hline $635-542$ & 3 & 560 & Mackenzie Mountains & 37 \\
\hline $635-542$ & 3 & 550 & Mackenzie Mountains & 37 \\
\hline $635-542$ & 3 & 550 & Mackenzie Mountains & 37 \\
\hline $635-542$ & 3 & 550 & Mackenzie Mountains & 37 \\
\hline $635-542$ & 3 & 550 & Mackenzie Mountains & 37 \\
\hline $635-542$ & 3 & 550 & Mackenzie Mountains & 37 \\
\hline $635-542$ & 3 & 550 & Mackenzie Mountains & 37 \\
\hline $635-542$ & 3 & 550 & Mackenzie Mountains & 37 \\
\hline $635-542$ & 3 & 550 & Mackenzie Mountains & 37 \\
\hline $635-542$ & 3 & 550 & Mackenzie Mountains & 37 \\
\hline $635-542$ & 3 & 550 & Mackenzie Mountains & 37 \\
\hline $635-542$ & 3 & 550 & Mackenzie Mountains & 37 \\
\hline $635-542$ & 3 & 550 & Mackenzie Mountains & 37 \\
\hline $635-542$ & 3 & 550 & Mackenzie Mountains & 37 \\
\hline $635-542$ & 3 & 550 & Mackenzie Mountains & 37 \\
\hline $635-542$ & 3 & 550 & Mackenzie Mountains & 37 \\
\hline $635-542$ & 3 & 550 & Mackenzie Mountains & 37 \\
\hline $635-542$ & 3 & 550 & Mackenzie Mountains & 37 \\
\hline $635-542$ & 3 & 550 & Mackenzie Mountains & 37 \\
\hline $635-542$ & 3 & 550 & Mackenzie Mountains & 37 \\
\hline $635-542$ & 3 & 545 & Mackenzie Mountains & 37 \\
\hline $635-542$ & 3 & 545 & Mackenzie Mountains & 37 \\
\hline $635-542$ & 3 & 545 & Mackenzie Mountains & 37 \\
\hline
\end{tabular}




\begin{tabular}{|c|c|c|c|c|}
\hline $635-542$ & 3 & 545 & Mackenzie Mountains & 37 \\
\hline $635-542$ & 3 & 545 & Mackenzie Mountains & 37 \\
\hline $635-542$ & 3 & 545 & Mackenzie Mountains & 37 \\
\hline $635-542$ & 3 & 545 & Mackenzie Mountains & 37 \\
\hline $635-542$ & 3 & 545 & Mackenzie Mountains & 37 \\
\hline $635-542$ & 3 & 545 & Mackenzie Mountains & 37 \\
\hline $635-542$ & 3 & 545 & Mackenzie Mountains & 37 \\
\hline $635-542$ & 3 & 545 & Mackenzie Mountains & 37 \\
\hline $635-542$ & 3 & 545 & Mackenzie Mountains & 37 \\
\hline $635-542$ & 3 & 545 & Mackenzie Mountains & 37 \\
\hline $635-542$ & 3 & 555 & Nico Perez Terrane (Urugı & 42 \\
\hline $635-542$ & 3 & 555 & Nico Perez Terrane (Urugı & 42 \\
\hline $635-542$ & 3 & 555 & Nico Perez Terrane (Urugı & 42 \\
\hline $635-542$ & 3 & 555 & Nico Perez Terrane (Urugı & 42 \\
\hline $635-542$ & 3 & 555 & Nico Perez Terrane (Urugı & 42 \\
\hline $635-542$ & 3 & 555 & Nico Perez Terrane (Urugı & 42 \\
\hline $635-542$ & 3 & 555 & Nico Perez Terrane (Urugı & 42 \\
\hline $635-542$ & 3 & 555 & Nico Perez Terrane (Urugı & 42 \\
\hline $635-542$ & 3 & 555 & Nico Perez Terrane (Urugı & 42 \\
\hline $635-542$ & 3 & 555 & Nico Perez Terrane (Urugı & 42 \\
\hline $635-542$ & 3 & 555 & Nico Perez Terrane (Urugı & 42 \\
\hline $635-542$ & 3 & 555 & Nico Perez Terrane (Urugı & 42 \\
\hline $635-542$ & 3 & 555 & Nico Perez Terrane (Urugı & 42 \\
\hline $635-542$ & 3 & 555 & Nico Perez Terrane (Urugı & 42 \\
\hline $635-542$ & 3 & 555 & Nico Perez Terrane (Urugı & 42 \\
\hline $635-542$ & 3 & 555 & Nico Perez Terrane (Urugı & 42 \\
\hline $635-542$ & 3 & 555 & Nico Perez Terrane (Urugı & 42 \\
\hline $635-542$ & 3 & 555 & Nico Perez Terrane (Urugı & 42 \\
\hline $635-542$ & 3 & 555 & Nico Perez Terrane (Urugı & 42 \\
\hline $635-542$ & 3 & 555 & Nico Perez Terrane (Urugı & 42 \\
\hline $635-542$ & 3 & 555 & Nico Perez Terrane (Urugı & 42 \\
\hline $635-542$ & 3 & 555 & Nico Perez Terrane (Urugı & 42 \\
\hline $635-542$ & 3 & 555 & Nico Perez Terrane (Urugı & 42 \\
\hline $635-542$ & 3 & 555 & Nico Perez Terrane (Urugı & 42 \\
\hline $635-542$ & 3 & 555 & Nico Perez Terrane (Urugı & 42 \\
\hline $635-542$ & 3 & 555 & Nico Perez Terrane (Urugı & 42 \\
\hline $635-542$ & 3 & 555 & Nico Perez Terrane (Urugı & 42 \\
\hline $635-542$ & 3 & 555 & Nico Perez Terrane (Urugı & 42 \\
\hline $635-542$ & 3 & 555 & Nico Perez Terrane (Urugı & 42 \\
\hline $635-542$ & 3 & 555 & Nico Perez Terrane (Urugı & 42 \\
\hline $635-542$ & 3 & 548 & Namibia & 44 \\
\hline
\end{tabular}




\begin{tabular}{|c|c|c|c|c|}
\hline $635-542$ & 3 & 548 & Namibia & 44 \\
\hline $635-542$ & 3 & 548 & Namibia & 44 \\
\hline $635-542$ & 3 & 548 & Namibia & 44 \\
\hline $635-542$ & 3 & 548 & Namibia & 44 \\
\hline $635-542$ & 3 & 548 & Namibia & 44 \\
\hline $635-542$ & 3 & 548 & Namibia & 44 \\
\hline $635-542$ & 3 & 548 & Namibia & 44 \\
\hline $635-542$ & 3 & 548 & Namibia & 44 \\
\hline $635-542$ & 3 & 548 & Namibia & 44 \\
\hline $635-542$ & 3 & 548 & Namibia & 44 \\
\hline $635-542$ & 3 & 548 & Namibia & 44 \\
\hline $635-542$ & 3 & 548 & Namibia & 44 \\
\hline $635-542$ & 3 & 545 & Namibia & 44 \\
\hline $635-542$ & 3 & 545 & Namibia & 44 \\
\hline $635-542$ & 3 & 545 & Namibia & 44 \\
\hline $635-542$ & 3 & 545 & Namibia & 44 \\
\hline $635-542$ & 3 & 545 & Namibia & 44 \\
\hline $635-542$ & 3 & 545 & Namibia & 44 \\
\hline $635-542$ & 3 & 545 & Namibia & 44 \\
\hline $635-542$ & 3 & 545 & Namibia & 44 \\
\hline $635-542$ & 3 & 545 & Namibia & 44 \\
\hline $635-542$ & 3 & 545 & Namibia & 44 \\
\hline $635-542$ & 3 & 545 & Namibia & 44 \\
\hline $635-542$ & 3 & 545 & Namibia & 44 \\
\hline $635-542$ & 3 & 545 & Namibia & 44 \\
\hline $635-542$ & 3 & 545 & Namibia & 44 \\
\hline $635-542$ & 3 & 545 & Namibia & 44 \\
\hline $635-542$ & 3 & 545 & Namibia & 44 \\
\hline $635-542$ & 3 & 545 & Namibia & 44 \\
\hline $635-542$ & 3 & 545 & Namibia & 44 \\
\hline $635-542$ & 3 & 545 & Namibia & 44 \\
\hline $635-542$ & 3 & 545 & Namibia & 44 \\
\hline $635-542$ & 3 & 545 & Namibia & 44 \\
\hline $635-542$ & 3 & 545 & Namibia & 44 \\
\hline $635-542$ & 3 & 545 & Namibia & 44 \\
\hline $635-542$ & 3 & 545 & Namibia & 44 \\
\hline $635-542$ & 3 & 545 & Namibia & 44 \\
\hline $635-542$ & 3 & 545 & Namibia & 44 \\
\hline $635-542$ & 3 & 545 & Namibia & 44 \\
\hline $635-542$ & 3 & 548 & Namibia & 44 \\
\hline $635-542$ & 3 & 548 & Namibia & 44 \\
\hline
\end{tabular}




\begin{tabular}{|c|c|c|c|c|}
\hline $635-542$ & 3 & 548 & Namibia & 44 \\
\hline $635-542$ & 3 & 548 & Namibia & 44 \\
\hline $635-542$ & 3 & 548 & Namibia & 44 \\
\hline $635-542$ & 3 & 548 & Namibia & 44 \\
\hline $635-542$ & 3 & 548 & Namibia & 44 \\
\hline $635-542$ & 3 & 548 & Namibia & 44 \\
\hline $635-542$ & 3 & 548 & Namibia & 44 \\
\hline $635-542$ & 3 & 545 & Mongolia & 48 \\
\hline $635-542$ & 3 & 545 & Mongolia & 48 \\
\hline $635-542$ & 3 & 545 & Mongolia & 48 \\
\hline $635-542$ & 3 & 545 & Mongolia & 48 \\
\hline $635-542$ & 3 & 630.31 & Svalbard & 51 \\
\hline $635-542$ & 3 & 630.77 & Svalbard & 51 \\
\hline $635-542$ & 3 & 630.82 & Svalbard & 51 \\
\hline $635-542$ & 3 & 630.99 & Svalbard & 51 \\
\hline $635-542$ & 3 & 631.4 & Svalbard & 51 \\
\hline $635-542$ & 3 & 631.83 & Svalbard & 51 \\
\hline $635-542$ & 3 & 631.95 & Svalbard & 51 \\
\hline $635-542$ & 3 & 632.14 & Svalbard & 51 \\
\hline $635-542$ & 3 & 632.38 & Svalbard & 51 \\
\hline $635-542$ & 3 & 632.96 & Svalbard & 51 \\
\hline $635-542$ & 3 & 633.12 & Svalbard & 51 \\
\hline $635-542$ & 3 & 633.16 & Svalbard & 51 \\
\hline $635-542$ & 3 & 633.83 & Svalbard & 51 \\
\hline $635-542$ & 3 & 633.87 & Svalbard & 51 \\
\hline $635-542$ & 3 & 633.96 & Svalbard & 51 \\
\hline $635-542$ & 3 & 634.09 & Svalbard & 51 \\
\hline $635-542$ & 3 & 635 & Svalbard & 51 \\
\hline $542-490$ & 4 & 525 & South China & 1 \\
\hline $542-490$ & 4 & 525 & South China & 1 \\
\hline $542-490$ & 4 & 525 & South China & 1 \\
\hline $542-490$ & 4 & 525 & South China & 1 \\
\hline $542-490$ & 4 & 525 & South China & 1 \\
\hline $542-490$ & 4 & 525 & South China & 1 \\
\hline $542-490$ & 4 & 525 & South China & 1 \\
\hline $542-490$ & 4 & 525 & South China & 1 \\
\hline $542-490$ & 4 & 525 & South China & 1 \\
\hline $542-490$ & 4 & 525 & South China & 1 \\
\hline $542-490$ & 4 & 525 & South China & 1 \\
\hline $542-490$ & 4 & 525 & South China & 1 \\
\hline $542-490$ & 4 & 525 & South China & 1 \\
\hline
\end{tabular}




\begin{tabular}{|c|c|c|c|}
\hline $542-490$ & 4 & 525 & South China \\
\hline $542-490$ & 4 & 525 & South China \\
\hline $542-490$ & 4 & 525 & South China \\
\hline $542-490$ & 4 & 525 & South China \\
\hline $542-490$ & 4 & 525 & South China \\
\hline $542-490$ & 4 & 525 & South China \\
\hline $542-490$ & 4 & 525 & South China \\
\hline $542-490$ & 4 & 525 & South China \\
\hline $542-490$ & 4 & 525 & South China \\
\hline $542-490$ & 4 & 525 & South China \\
\hline $542-490$ & 4 & 525 & South China \\
\hline $542-490$ & 4 & 525 & South China \\
\hline $542-490$ & 4 & 525 & South China \\
\hline $542-490$ & 4 & 525 & South China \\
\hline $542-490$ & 4 & 525 & South China \\
\hline $542-490$ & 4 & 525 & South China \\
\hline $542-490$ & 4 & 525 & South China \\
\hline $542-490$ & 4 & 525 & South China \\
\hline $542-490$ & 4 & 525 & South China \\
\hline $542-490$ & 4 & 525 & South China \\
\hline $542-490$ & 4 & 525 & South China \\
\hline $542-490$ & 4 & 525 & South China \\
\hline $542-490$ & 4 & 525 & South China \\
\hline $542-490$ & 4 & 525 & South China \\
\hline $542-490$ & 4 & 525 & South China \\
\hline $542-490$ & 4 & 525 & South China \\
\hline $542-490$ & 4 & 525 & South China \\
\hline $542-490$ & 4 & 519 & South China \\
\hline $542-490$ & 4 & 519 & South China \\
\hline $542-490$ & 4 & 519 & South China \\
\hline $542-490$ & 4 & 519 & South China \\
\hline $542-490$ & 4 & 519 & South China \\
\hline $542-490$ & 4 & 525 & South China \\
\hline $542-490$ & 4 & 525 & South China \\
\hline $542-490$ & 4 & 525 & South China \\
\hline $542-490$ & 4 & 525 & South China \\
\hline $542-490$ & 4 & 525 & South China \\
\hline $542-490$ & 4 & 525 & South China \\
\hline $542-490$ & 4 & 525 & South China \\
\hline $542-490$ & 4 & 525 & South China \\
\hline $542-490$ & 4 & 525 & South China \\
\hline
\end{tabular}




\begin{tabular}{|c|c|c|c|}
\hline $542-490$ & 4 & 525 & South China \\
\hline $542-490$ & 4 & 525 & South China \\
\hline $542-490$ & 4 & 525 & South China \\
\hline $542-490$ & 4 & 525 & South China \\
\hline $542-490$ & 4 & 525 & South China \\
\hline $542-490$ & 4 & 525 & South China \\
\hline $542-490$ & 4 & 525 & South China \\
\hline $542-490$ & 4 & 525 & South China \\
\hline $542-490$ & 4 & 525 & South China \\
\hline $542-490$ & 4 & 525 & South China \\
\hline $542-490$ & 4 & 525 & South China \\
\hline $542-490$ & 4 & 525 & South China \\
\hline $542-490$ & 4 & 525 & South China \\
\hline $542-490$ & 4 & 525 & South China \\
\hline $542-490$ & 4 & 525 & South China \\
\hline $542-490$ & 4 & 525 & South China \\
\hline $542-490$ & 4 & 525 & South China \\
\hline $542-490$ & 4 & 525 & South China \\
\hline $542-490$ & 4 & 525 & South China \\
\hline $542-490$ & 4 & 525 & South China \\
\hline $542-490$ & 4 & 525 & South China \\
\hline $542-490$ & 4 & 525 & South China \\
\hline $542-490$ & 4 & 525 & South China \\
\hline $542-490$ & 4 & 525 & South China \\
\hline $542-490$ & 4 & 525 & South China \\
\hline $542-490$ & 4 & 525 & South China \\
\hline $542-490$ & 4 & 525 & South China \\
\hline $542-490$ & 4 & 525 & South China \\
\hline $542-490$ & 4 & 526 & South China \\
\hline $542-490$ & 4 & 526 & South China \\
\hline $542-490$ & 4 & 526 & South China \\
\hline $542-490$ & 4 & 526 & South China \\
\hline $542-490$ & 4 & 526 & South China \\
\hline $542-490$ & 4 & 526 & South China \\
\hline $542-490$ & 4 & 526 & South China \\
\hline $542-490$ & 4 & 526 & South China \\
\hline $542-490$ & 4 & 526 & South China \\
\hline $542-490$ & 4 & 526 & South China \\
\hline $542-490$ & 4 & 526 & South China \\
\hline $542-490$ & 4 & 526 & South China \\
\hline $542-490$ & 4 & 526 & South China \\
\hline
\end{tabular}




\begin{tabular}{|c|c|c|c|}
\hline $542-490$ & 4 & 526 & South China \\
\hline $542-490$ & 4 & 526 & South China \\
\hline $542-490$ & 4 & 526 & South China \\
\hline $542-490$ & 4 & 526 & South China \\
\hline $542-490$ & 4 & 526 & South China \\
\hline $542-490$ & 4 & 526 & South China \\
\hline $542-490$ & 4 & 526 & South China \\
\hline $542-490$ & 4 & 526 & South China \\
\hline $542-490$ & 4 & 526 & South China \\
\hline $542-490$ & 4 & 526 & South China \\
\hline $542-490$ & 4 & 526 & South China \\
\hline $542-490$ & 4 & 526 & South China \\
\hline $542-490$ & 4 & 526 & South China \\
\hline $542-490$ & 4 & 526 & South China \\
\hline $542-490$ & 4 & 526 & South China \\
\hline $542-490$ & 4 & 526 & South China \\
\hline $542-490$ & 4 & 526 & South China \\
\hline $542-490$ & 4 & 526 & South China \\
\hline $542-490$ & 4 & 526 & South China \\
\hline $542-490$ & 4 & 526 & South China \\
\hline $542-490$ & 4 & 526 & South China \\
\hline $542-490$ & 4 & 526 & South China \\
\hline $542-490$ & 4 & 526 & South China \\
\hline $542-490$ & 4 & 526 & South China \\
\hline $542-490$ & 4 & 526 & South China \\
\hline $542-490$ & 4 & 526 & South China \\
\hline $542-490$ & 4 & 526 & South China \\
\hline $542-490$ & 4 & 526 & South China \\
\hline $542-490$ & 4 & 526 & South China \\
\hline $542-490$ & 4 & 526 & South China \\
\hline $542-490$ & 4 & 523 & South China \\
\hline $542-490$ & 4 & 523 & South China \\
\hline $542-490$ & 4 & 523 & South China \\
\hline $542-490$ & 4 & 523 & South China \\
\hline $542-490$ & 4 & 523 & South China \\
\hline $542-490$ & 4 & 523 & South China \\
\hline $542-490$ & 4 & 523 & South China \\
\hline $542-490$ & 4 & 523 & South China \\
\hline $542-490$ & 4 & 523 & South China \\
\hline $542-490$ & 4 & 523 & South China \\
\hline $542-490$ & 4 & 523 & South China \\
\hline
\end{tabular}




\begin{tabular}{|c|c|c|c|}
\hline $542-490$ & 4 & 523 & South China \\
\hline $542-490$ & 4 & 523 & South China \\
\hline $542-490$ & 4 & 523 & South China \\
\hline $542-490$ & 4 & 523 & South China \\
\hline $542-490$ & 4 & 523 & South China \\
\hline $542-490$ & 4 & 523 & South China \\
\hline $542-490$ & 4 & 523 & South China \\
\hline $542-490$ & 4 & 523 & South China \\
\hline $542-490$ & 4 & 523 & South China \\
\hline $542-490$ & 4 & 523 & South China \\
\hline $542-490$ & 4 & 523 & South China \\
\hline $542-490$ & 4 & 523 & South China \\
\hline $542-490$ & 4 & 523 & South China \\
\hline $542-490$ & 4 & 523 & South China \\
\hline $542-490$ & 4 & 523 & South China \\
\hline $542-490$ & 4 & 523 & South China \\
\hline $542-490$ & 4 & 523 & South China \\
\hline $542-490$ & 4 & 523 & South China \\
\hline $542-490$ & 4 & 523 & South China \\
\hline $542-490$ & 4 & 523 & South China \\
\hline $542-490$ & 4 & 523 & South China \\
\hline $542-490$ & 4 & 523 & South China \\
\hline $542-490$ & 4 & 523 & South China \\
\hline $542-490$ & 4 & 523 & South China \\
\hline $542-490$ & 4 & 523 & South China \\
\hline $542-490$ & 4 & 523 & South China \\
\hline $542-490$ & 4 & 523 & South China \\
\hline $542-490$ & 4 & 523 & South China \\
\hline $542-490$ & 4 & 523 & South China \\
\hline $542-490$ & 4 & 523 & South China \\
\hline $542-490$ & 4 & 520 & South China \\
\hline $542-490$ & 4 & 520 & South China \\
\hline $542-490$ & 4 & 520 & South China \\
\hline $542-490$ & 4 & 520 & South China \\
\hline $542-490$ & 4 & 520 & South China \\
\hline $542-490$ & 4 & 523 & South China \\
\hline $542-490$ & 4 & 520 & South China \\
\hline $542-490$ & 4 & 520 & South China \\
\hline $542-490$ & 4 & 520 & South China \\
\hline $542-490$ & 4 & 520 & South China \\
\hline $542-490$ & 4 & 520 & South China \\
\hline
\end{tabular}




\begin{tabular}{|c|c|c|c|}
\hline $542-490$ & 4 & 520 & South China \\
\hline $542-490$ & 4 & 520 & South China \\
\hline $542-490$ & 4 & 520 & South China \\
\hline $542-490$ & 4 & 520 & South China \\
\hline $542-490$ & 4 & 520 & South China \\
\hline $542-490$ & 4 & 523 & South China \\
\hline $542-490$ & 4 & 520 & South China \\
\hline $542-490$ & 4 & 520 & South China \\
\hline $542-490$ & 4 & 520 & South China \\
\hline $542-490$ & 4 & 520 & South China \\
\hline $542-490$ & 4 & 520 & South China \\
\hline $542-490$ & 4 & 520 & South China \\
\hline $542-490$ & 4 & 520 & South China \\
\hline $542-490$ & 4 & 520 & South China \\
\hline $542-490$ & 4 & 520 & South China \\
\hline $542-490$ & 4 & 520 & South China \\
\hline $542-490$ & 4 & 523 & South China \\
\hline $542-490$ & 4 & 520 & South China \\
\hline $542-490$ & 4 & 520 & South China \\
\hline $542-490$ & 4 & 523 & South China \\
\hline $542-490$ & 4 & 523 & South China \\
\hline $542-490$ & 4 & 523 & South China \\
\hline $542-490$ & 4 & 520 & South China \\
\hline $542-490$ & 4 & 520 & South China \\
\hline $542-490$ & 4 & 520 & South China \\
\hline $542-490$ & 4 & 520 & South China \\
\hline $542-490$ & 4 & 520 & South China \\
\hline $542-490$ & 4 & 520 & South China \\
\hline $542-490$ & 4 & 520 & South China \\
\hline $542-490$ & 4 & 520 & South China \\
\hline $542-490$ & 4 & 520 & South China \\
\hline $542-490$ & 4 & 520 & South China \\
\hline $542-490$ & 4 & 520 & South China \\
\hline $542-490$ & 4 & 520 & South China \\
\hline $542-490$ & 4 & 520 & South China \\
\hline $542-490$ & 4 & 520 & South China \\
\hline $542-490$ & 4 & 520 & South China \\
\hline $542-490$ & 4 & 520 & South China \\
\hline $542-490$ & 4 & 530 & South China \\
\hline $542-490$ & 4 & 530 & South China \\
\hline $542-490$ & 4 & 530 & South China \\
\hline
\end{tabular}




\begin{tabular}{|c|c|c|c|}
\hline $542-490$ & 4 & 530 & South China \\
\hline $542-490$ & 4 & 540 & South China \\
\hline $635-542$ & 4 & 540 & South China \\
\hline $635-542$ & 4 & 540 & South China \\
\hline $635-542$ & 4 & 540 & South China \\
\hline $635-542$ & 4 & 540 & South China \\
\hline $542-490$ & 4 & 530 & South China \\
\hline $542-490$ & 4 & 530 & South China \\
\hline $542-490$ & 4 & 530 & South China \\
\hline $542-490$ & 4 & 530 & South China \\
\hline $542-490$ & 4 & 530 & South China \\
\hline $542-490$ & 4 & 530 & South China \\
\hline $542-490$ & 4 & 530 & South China \\
\hline $542-490$ & 4 & 530 & South China \\
\hline $542-490$ & 4 & 530 & South China \\
\hline $542-490$ & 4 & 530 & South China \\
\hline $542-490$ & 4 & 530 & South China \\
\hline $542-490$ & 4 & 530 & South China \\
\hline $542-490$ & 4 & 530 & South China \\
\hline $542-490$ & 4 & 530 & South China \\
\hline $542-490$ & 4 & 530 & South China \\
\hline $542-490$ & 4 & 530 & South China \\
\hline $542-490$ & 4 & 530 & South China \\
\hline $542-490$ & 4 & 530 & South China \\
\hline $542-490$ & 4 & 530 & South China \\
\hline $542-490$ & 4 & 535 & Wernecke Mountains \\
\hline $542-490$ & 4 & 535 & Wernecke Mountains \\
\hline $542-490$ & 4 & 535 & Wernecke Mountains \\
\hline $542-490$ & 4 & 535 & Wernecke Mountains \\
\hline $542-490$ & 4 & 535 & Wernecke Mountains \\
\hline $542-490$ & 4 & 535 & Wernecke Mountains \\
\hline $542-490$ & 4 & 535 & Wernecke Mountains \\
\hline $542-490$ & 4 & 535 & Wernecke Mountains \\
\hline $542-490$ & 4 & 535 & Wernecke Mountains \\
\hline $542-490$ & 4 & 535 & Wernecke Mountains \\
\hline $542-490$ & 4 & 535 & Wernecke Mountains \\
\hline $542-490$ & 4 & 535 & Wernecke Mountains \\
\hline $542-490$ & 4 & 535 & Wernecke Mountains \\
\hline $542-490$ & 4 & 535 & Wernecke Mountains \\
\hline $542-490$ & 4 & 535 & Wernecke Mountains \\
\hline $542-490$ & 4 & 535 & Wernecke Mountains \\
\hline
\end{tabular}




\begin{tabular}{|c|c|c|c|c|}
\hline $542-490$ & 4 & 535 & Wernecke Mountains & 4 \\
\hline $542-490$ & 4 & 535 & Wernecke Mountains & 4 \\
\hline $542-490$ & 4 & 535 & Wernecke Mountains & 4 \\
\hline $542-490$ & 4 & 502 & House Embayment & 18 \\
\hline $542-490$ & 4 & 502 & House Embayment & 18 \\
\hline $542-490$ & 4 & 502 & House Embayment & 18 \\
\hline $542-490$ & 4 & 502 & House Embayment & 18 \\
\hline $542-490$ & 4 & 502 & House Embayment & 18 \\
\hline $542-490$ & 4 & 502 & House Embayment & 18 \\
\hline $542-490$ & 4 & 502 & House Embayment & 18 \\
\hline $542-490$ & 4 & 502 & House Embayment & 18 \\
\hline $542-490$ & 4 & 502 & House Embayment & 18 \\
\hline $542-490$ & 4 & 502 & House Embayment & 18 \\
\hline $542-490$ & 4 & 502 & House Embayment & 18 \\
\hline $542-490$ & 4 & 502 & House Embayment & 18 \\
\hline $542-490$ & 4 & 502 & House Embayment & 18 \\
\hline $542-490$ & 4 & 502 & House Embayment & 18 \\
\hline $542-490$ & 4 & 502 & House Embayment & 18 \\
\hline $542-490$ & 4 & 502 & House Embayment & 18 \\
\hline $542-490$ & 4 & 502 & House Embayment & 18 \\
\hline $542-490$ & 4 & 502 & House Embayment & 18 \\
\hline $542-490$ & 4 & 502 & House Embayment & 18 \\
\hline $542-490$ & 4 & 502 & House Embayment & 18 \\
\hline $542-490$ & 4 & 502 & House Embayment & 18 \\
\hline $542-490$ & 4 & 502 & House Embayment & 18 \\
\hline $542-490$ & 4 & 502 & House Embayment & 18 \\
\hline $542-490$ & 4 & 502 & House Embayment & 18 \\
\hline $542-490$ & 4 & 502 & House Embayment & 18 \\
\hline $542-490$ & 4 & 502 & House Embayment & 18 \\
\hline $542-490$ & 4 & 502 & House Embayment & 18 \\
\hline $542-490$ & 4 & 502 & House Embayment & 18 \\
\hline $542-490$ & 4 & 502 & House Embayment & 18 \\
\hline $542-490$ & 4 & 502 & House Embayment & 18 \\
\hline $542-490$ & 4 & 502 & House Embayment & 18 \\
\hline $542-490$ & 4 & 502 & House Embayment & 18 \\
\hline $542-490$ & 4 & 502 & House Embayment & 18 \\
\hline $542-490$ & 4 & 502 & House Embayment & 18 \\
\hline $542-490$ & 4 & 502 & House Embayment & 18 \\
\hline $542-490$ & 4 & 502 & House Embayment & 18 \\
\hline $542-490$ & 4 & 502 & House Embayment & 18 \\
\hline $542-490$ & 4 & 502 & House Embayment & 18 \\
\hline
\end{tabular}




\begin{tabular}{|c|c|c|c|c|}
\hline $542-490$ & 4 & 502 & House Embayment & 18 \\
\hline $542-490$ & 4 & 502 & House Embayment & 18 \\
\hline $542-490$ & 4 & 502 & House Embayment & 18 \\
\hline $542-490$ & 4 & 502 & House Embayment & 18 \\
\hline $542-490$ & 4 & 502 & House Embayment & 18 \\
\hline $542-490$ & 4 & 502 & House Embayment & 18 \\
\hline $542-490$ & 4 & 502 & House Embayment & 18 \\
\hline $542-490$ & 4 & 502 & House Embayment & 18 \\
\hline $542-490$ & 4 & 502 & House Embayment & 18 \\
\hline $542-490$ & 4 & 502 & House Embayment & 18 \\
\hline $542-490$ & 4 & 502 & House Embayment & 18 \\
\hline $542-490$ & 4 & 502 & House Embayment & 18 \\
\hline $542-490$ & 4 & 502 & House Embayment & 18 \\
\hline $542-490$ & 4 & 502 & House Embayment & 18 \\
\hline $542-490$ & 4 & 502 & House Embayment & 18 \\
\hline $542-490$ & 4 & 502 & House Embayment & 18 \\
\hline $542-490$ & 4 & 502 & House Embayment & 18 \\
\hline $542-490$ & 4 & 502 & House Embayment & 18 \\
\hline $542-490$ & 4 & 502 & House Embayment & 18 \\
\hline $542-490$ & 4 & 502 & House Embayment & 18 \\
\hline $542-490$ & 4 & 502 & House Embayment & 18 \\
\hline $542-490$ & 4 & 502 & House Embayment & 18 \\
\hline $542-490$ & 4 & 502 & House Embayment & 18 \\
\hline $542-490$ & 4 & 502 & House Embayment & 18 \\
\hline $542-490$ & 4 & 502 & House Embayment & 18 \\
\hline $542-490$ & 4 & 502 & House Embayment & 18 \\
\hline $542-490$ & 4 & 502 & House Embayment & 18 \\
\hline $542-490$ & 4 & 502 & House Embayment & 18 \\
\hline $542-490$ & 4 & 502 & House Embayment & 18 \\
\hline $542-490$ & 4 & 502 & House Embayment & 18 \\
\hline $542-490$ & 4 & 502 & House Embayment & 18 \\
\hline $542-490$ & 4 & 502 & House Embayment & 18 \\
\hline $542-490$ & 4 & 502 & House Embayment & 18 \\
\hline $542-490$ & 4 & 502 & House Embayment & 18 \\
\hline $542-490$ & 4 & 502 & House Embayment & 18 \\
\hline $542-490$ & 4 & 502 & House Embayment & 18 \\
\hline $542-490$ & 4 & 502 & House Embayment & 18 \\
\hline $542-490$ & 4 & 502 & House Embayment & 18 \\
\hline $542-490$ & 4 & 502 & House Embayment & 18 \\
\hline $542-490$ & 4 & 502 & House Embayment & 18 \\
\hline $542-490$ & 4 & 502 & House Embayment & 18 \\
\hline
\end{tabular}




\begin{tabular}{|c|c|c|c|c|}
\hline $542-490$ & 4 & 502 & House Embayment & 18 \\
\hline $542-490$ & 4 & 502 & House Embayment & 18 \\
\hline $542-490$ & 4 & 502 & House Embayment & 18 \\
\hline $542-490$ & 4 & 502 & House Embayment & 18 \\
\hline $542-490$ & 4 & 502 & House Embayment & 18 \\
\hline $542-490$ & 4 & 502 & House Embayment & 18 \\
\hline $542-490$ & 4 & 502 & House Embayment & 18 \\
\hline $542-490$ & 4 & 502 & House Embayment & 18 \\
\hline $542-490$ & 4 & 502 & House Embayment & 18 \\
\hline $542-490$ & 4 & 502 & House Embayment & 18 \\
\hline $542-490$ & 4 & 502 & House Embayment & 18 \\
\hline $542-490$ & 4 & 502 & House Embayment & 18 \\
\hline $542-490$ & 4 & 502 & House Embayment & 18 \\
\hline $542-490$ & 4 & 502 & House Embayment & 18 \\
\hline $542-490$ & 4 & 502 & House Embayment & 18 \\
\hline $542-490$ & 4 & 502 & House Embayment & 18 \\
\hline $542-490$ & 4 & 502 & House Embayment & 18 \\
\hline $542-490$ & 4 & 502 & House Embayment & 18 \\
\hline $542-490$ & 4 & 502 & House Embayment & 18 \\
\hline $542-490$ & 4 & 502 & House Embayment & 18 \\
\hline $542-490$ & 4 & 502 & House Embayment & 18 \\
\hline $542-490$ & 4 & 502 & House Embayment & 18 \\
\hline $542-490$ & 4 & 502 & House Embayment & 18 \\
\hline $542-490$ & 4 & 502 & House Embayment & 18 \\
\hline $542-490$ & 4 & 502 & House Embayment & 18 \\
\hline $542-490$ & 4 & 502 & House Embayment & 18 \\
\hline $542-490$ & 4 & 502 & House Embayment & 18 \\
\hline $542-490$ & 4 & 502 & House Embayment & 18 \\
\hline $542-490$ & 4 & 502 & House Embayment & 18 \\
\hline $542-490$ & 4 & 502 & House Embayment & 18 \\
\hline $542-490$ & 4 & 502 & House Embayment & 18 \\
\hline $542-490$ & 4 & 502 & House Embayment & 18 \\
\hline $542-490$ & 4 & 502 & House Embayment & 18 \\
\hline $542-490$ & 4 & 502 & House Embayment & 18 \\
\hline $542-490$ & 4 & 502 & House Embayment & 18 \\
\hline $542-490$ & 4 & 502 & House Embayment & 18 \\
\hline $542-490$ & 4 & 502 & House Embayment & 18 \\
\hline $542-490$ & 4 & 502 & House Embayment & 18 \\
\hline $542-490$ & 4 & 502 & House Embayment & 18 \\
\hline $542-490$ & 4 & 502 & House Embayment & 18 \\
\hline $542-490$ & 4 & 502 & House Embayment & 18 \\
\hline
\end{tabular}




\begin{tabular}{|c|c|c|c|c|}
\hline $542-490$ & 4 & 502 & House Embayment & 18 \\
\hline $542-490$ & 4 & 502 & House Embayment & 18 \\
\hline $542-490$ & 4 & 502 & House Embayment & 18 \\
\hline $542-490$ & 4 & 502 & House Embayment & 18 \\
\hline $542-490$ & 4 & 502 & House Embayment & 18 \\
\hline $542-490$ & 4 & 502 & House Embayment & 18 \\
\hline $542-490$ & 4 & 502 & House Embayment & 18 \\
\hline $542-490$ & 4 & 502 & House Embayment & 18 \\
\hline $542-490$ & 4 & 502 & House Embayment & 18 \\
\hline $542-490$ & 4 & 502 & House Embayment & 18 \\
\hline $542-490$ & 4 & 502 & House Embayment & 18 \\
\hline $542-490$ & 4 & 502 & House Embayment & 18 \\
\hline $542-490$ & 4 & 502 & House Embayment & 18 \\
\hline $542-490$ & 4 & 502 & House Embayment & 18 \\
\hline $542-490$ & 4 & 502 & House Embayment & 18 \\
\hline $542-490$ & 4 & 502 & House Embayment & 18 \\
\hline $542-490$ & 4 & 502 & House Embayment & 18 \\
\hline $542-490$ & 4 & 497 & Sweden & 20 \\
\hline $542-490$ & 4 & 497 & Sweden & 20 \\
\hline $542-490$ & 4 & 497 & Sweden & 20 \\
\hline $542-490$ & 4 & 497 & Sweden & 20 \\
\hline $542-490$ & 4 & 497 & Sweden & 20 \\
\hline $542-490$ & 4 & 497 & Sweden & 20 \\
\hline $542-490$ & 4 & 497 & Sweden & 20 \\
\hline $542-490$ & 4 & 497 & Sweden & 20 \\
\hline $542-490$ & 4 & 497 & Sweden & 20 \\
\hline $542-490$ & 4 & 497 & Sweden & 20 \\
\hline $542-490$ & 4 & 497 & Sweden & 20 \\
\hline $542-490$ & 4 & 497 & Sweden & 20 \\
\hline $542-490$ & 4 & 497 & Sweden & 20 \\
\hline $542-490$ & 4 & 497 & Sweden & 20 \\
\hline $542-490$ & 4 & 497 & Sweden & 20 \\
\hline $542-490$ & 4 & 497 & Sweden & 20 \\
\hline $542-490$ & 4 & 497 & Sweden & 20 \\
\hline $542-490$ & 4 & 497 & Sweden & 20 \\
\hline $542-490$ & 4 & 497 & Sweden & 20 \\
\hline $542-490$ & 4 & 497 & Sweden & 20 \\
\hline $542-490$ & 4 & 497 & Sweden & 20 \\
\hline $542-490$ & 4 & 497 & Sweden & 20 \\
\hline $542-490$ & 4 & 497 & Sweden & 20 \\
\hline $542-490$ & 4 & 497 & Sweden & 20 \\
\hline
\end{tabular}




\begin{tabular}{|c|c|c|c|c|}
\hline $542-490$ & 4 & 497 & Sweden & 20 \\
\hline $542-490$ & 4 & 497 & Sweden & 20 \\
\hline $542-490$ & 4 & 505 & Southern Canadian Cordill & 26 \\
\hline $542-490$ & 4 & 505 & Southern Canadian Cordill & 26 \\
\hline $542-490$ & 4 & 505 & Southern Canadian Cordill & 26 \\
\hline $542-490$ & 4 & 505 & Southern Canadian Cordill & 26 \\
\hline $542-490$ & 4 & 530 & East Greenland & 30 \\
\hline $542-490$ & 4 & 530 & East Greenland & 30 \\
\hline $542-490$ & 4 & 530 & East Greenland & 30 \\
\hline $542-490$ & 4 & 530 & East Greenland & 30 \\
\hline $542-490$ & 4 & 530 & East Greenland & 30 \\
\hline $542-490$ & 4 & 540 & Siberia & 32 \\
\hline $542-490$ & 4 & 540 & Siberia & 32 \\
\hline $542-490$ & 4 & 540 & Siberia & 32 \\
\hline $542-490$ & 4 & 540 & Siberia & 32 \\
\hline $542-490$ & 4 & 540 & Siberia & 32 \\
\hline $542-490$ & 4 & 540 & Siberia & 32 \\
\hline $542-490$ & 4 & 530 & Ogilvie Mountains & 36 \\
\hline $542-490$ & 4 & 530 & Ogilvie Mountains & 36 \\
\hline $542-490$ & 4 & 535 & Mackenzie Mountains & 37 \\
\hline $542-490$ & 4 & 535 & Mackenzie Mountains & 37 \\
\hline $542-490$ & 4 & 535 & Mackenzie Mountains & 37 \\
\hline $542-490$ & 4 & 535 & Mackenzie Mountains & 37 \\
\hline $542-490$ & 4 & 535 & Mackenzie Mountains & 37 \\
\hline $542-490$ & 4 & 535 & Mackenzie Mountains & 37 \\
\hline $542-490$ & 4 & 535 & Mackenzie Mountains & 37 \\
\hline $542-490$ & 4 & 535 & Mackenzie Mountains & 37 \\
\hline $542-490$ & 4 & 535 & Mackenzie Mountains & 37 \\
\hline $542-490$ & 4 & 535 & Mackenzie Mountains & 37 \\
\hline $542-490$ & 4 & 535 & Mackenzie Mountains & 37 \\
\hline $542-490$ & 4 & 535 & Mackenzie Mountains & 37 \\
\hline $542-490$ & 4 & 535 & Mackenzie Mountains & 37 \\
\hline $542-490$ & 4 & 535 & Mackenzie Mountains & 37 \\
\hline $542-490$ & 4 & 535 & Mackenzie Mountains & 37 \\
\hline $542-490$ & 4 & 535 & Mackenzie Mountains & 37 \\
\hline $542-490$ & 4 & 535 & Mackenzie Mountains & 37 \\
\hline $542-490$ & 4 & 535 & Mackenzie Mountains & 37 \\
\hline $542-490$ & 4 & 535 & Mackenzie Mountains & 37 \\
\hline $542-490$ & 4 & 535 & Mackenzie Mountains & 37 \\
\hline $542-490$ & 4 & 535 & Mackenzie Mountains & 37 \\
\hline $542-490$ & 4 & 535 & Mackenzie Mountains & 37 \\
\hline
\end{tabular}




\begin{tabular}{|c|c|c|c|c|}
\hline $542-490$ & 4 & 535 & Mackenzie Mountains & 37 \\
\hline $542-490$ & 4 & 535 & Mackenzie Mountains & 37 \\
\hline $542-490$ & 4 & 535 & Mackenzie Mountains & 37 \\
\hline $542-490$ & 4 & 535 & Mackenzie Mountains & 37 \\
\hline $542-490$ & 4 & 535 & Mackenzie Mountains & 37 \\
\hline $542-490$ & 4 & 535 & Mackenzie Mountains & 37 \\
\hline $542-490$ & 4 & 535 & Mackenzie Mountains & 37 \\
\hline $542-490$ & 4 & 535 & Mackenzie Mountains & 37 \\
\hline $542-490$ & 4 & 535 & Mackenzie Mountains & 37 \\
\hline $542-490$ & 4 & 535 & Mackenzie Mountains & 37 \\
\hline $542-490$ & 4 & 535 & Mackenzie Mountains & 37 \\
\hline $542-490$ & 4 & 535 & Mackenzie Mountains & 37 \\
\hline $542-490$ & 4 & 535 & Mackenzie Mountains & 37 \\
\hline $542-490$ & 4 & 535 & Mackenzie Mountains & 37 \\
\hline $542-490$ & 4 & 535 & Mackenzie Mountains & 37 \\
\hline $542-490$ & 4 & 535 & Mackenzie Mountains & 37 \\
\hline $542-490$ & 4 & 535 & Mackenzie Mountains & 37 \\
\hline $542-490$ & 4 & 535 & Mackenzie Mountains & 37 \\
\hline $542-490$ & 4 & 535 & Mackenzie Mountains & 37 \\
\hline $542-490$ & 4 & 535 & Mackenzie Mountains & 37 \\
\hline $542-490$ & 4 & 535 & Mackenzie Mountains & 37 \\
\hline $542-490$ & 4 & 535 & Mackenzie Mountains & 37 \\
\hline $542-490$ & 4 & 535 & Mackenzie Mountains & 37 \\
\hline $542-490$ & 4 & 535 & Mackenzie Mountains & 37 \\
\hline $542-490$ & 4 & 535 & Mackenzie Mountains & 37 \\
\hline $542-490$ & 4 & 535 & Mackenzie Mountains & 37 \\
\hline $542-490$ & 4 & 535 & Mackenzie Mountains & 37 \\
\hline $542-490$ & 4 & 535 & Mackenzie Mountains & 37 \\
\hline $542-490$ & 4 & 535 & Mackenzie Mountains & 37 \\
\hline $542-490$ & 4 & 510 & Georgina Basin & 41 \\
\hline $542-490$ & 4 & 510 & Georgina Basin & 41 \\
\hline $542-490$ & 4 & 510 & Georgina Basin & 41 \\
\hline $542-490$ & 4 & 510 & Georgina Basin & 41 \\
\hline $542-490$ & 4 & 510 & Georgina Basin & 41 \\
\hline $542-490$ & 4 & 510 & Georgina Basin & 41 \\
\hline $542-490$ & 4 & 510 & Georgina Basin & 41 \\
\hline $542-490$ & 4 & 510 & Georgina Basin & 41 \\
\hline $542-490$ & 4 & 510 & Georgina Basin & 41 \\
\hline $542-490$ & 4 & 510 & Georgina Basin & 41 \\
\hline $542-490$ & 4 & 510 & Georgina Basin & 41 \\
\hline $542-490$ & 4 & 510 & Georgina Basin & 41 \\
\hline
\end{tabular}




\begin{tabular}{|c|c|c|c|c|}
\hline $542-490$ & 4 & 510 & Georgina Basin & 41 \\
\hline $542-490$ & 4 & 510 & Georgina Basin & 41 \\
\hline $542-490$ & 4 & 510 & Georgina Basin & 41 \\
\hline $542-490$ & 4 & 510 & Georgina Basin & 41 \\
\hline $542-490$ & 4 & 510 & Georgina Basin & 41 \\
\hline $542-490$ & 4 & 510 & Georgina Basin & 41 \\
\hline $542-490$ & 4 & 510 & Georgina Basin & 41 \\
\hline $542-490$ & 4 & 510 & Georgina Basin & 41 \\
\hline $542-490$ & 4 & 510 & Georgina Basin & 41 \\
\hline $542-490$ & 4 & 510 & Georgina Basin & 41 \\
\hline $542-490$ & 4 & 510 & Georgina Basin & 41 \\
\hline $542-490$ & 4 & 510 & Georgina Basin & 41 \\
\hline $542-490$ & 4 & 510 & Georgina Basin & 41 \\
\hline $542-490$ & 4 & 510 & Georgina Basin & 41 \\
\hline $542-490$ & 4 & 510 & Georgina Basin & 41 \\
\hline $542-490$ & 4 & 510 & Georgina Basin & 41 \\
\hline $542-490$ & 4 & 510 & Georgina Basin & 41 \\
\hline $542-490$ & 4 & 538 & Namibia & 44 \\
\hline $542-490$ & 4 & 538 & Namibia & 44 \\
\hline $542-490$ & 4 & 538 & Namibia & 44 \\
\hline $542-490$ & 4 & 538 & Namibia & 44 \\
\hline $542-490$ & 4 & 538 & Namibia & 44 \\
\hline $542-490$ & 4 & 538 & Namibia & 44 \\
\hline $542-490$ & 4 & 538 & Namibia & 44 \\
\hline $542-490$ & 4 & 538 & Namibia & 44 \\
\hline $542-490$ & 4 & 538 & Namibia & 44 \\
\hline $542-490$ & 4 & 538 & Namibia & 44 \\
\hline $542-490$ & 4 & 538 & Namibia & 44 \\
\hline $542-490$ & 4 & 538 & Namibia & 44 \\
\hline $542-490$ & 4 & 538 & Namibia & 44 \\
\hline $542-490$ & 4 & 538 & Namibia & 44 \\
\hline $542-490$ & 4 & 538 & Namibia & 44 \\
\hline $542-490$ & 4 & 538 & Namibia & 44 \\
\hline $542-490$ & 4 & 538 & Namibia & 44 \\
\hline $542-490$ & 4 & 538 & Namibia & 44 \\
\hline $542-490$ & 4 & 538 & Namibia & 44 \\
\hline $542-490$ & 4 & 538 & Namibia & 44 \\
\hline $542-490$ & 4 & 538 & Namibia & 44 \\
\hline $542-490$ & 4 & 538 & Namibia & 44 \\
\hline $542-490$ & 4 & 538 & Namibia & 44 \\
\hline $542-490$ & 4 & 538 & Namibia & 44 \\
\hline
\end{tabular}




\begin{tabular}{|c|c|c|c|c|}
\hline $542-490$ & 4 & 538 & Namibia & 44 \\
\hline $542-490$ & 4 & 538 & Mongolia & 48 \\
\hline $542-490$ & 4 & 538 & Mongolia & 48 \\
\hline $542-490$ & 4 & 538 & Mongolia & 48 \\
\hline $542-490$ & 4 & 538 & Mongolia & 48 \\
\hline $542-490$ & 4 & 538 & Mongolia & 48 \\
\hline $542-490$ & 4 & 538 & Mongolia & 48 \\
\hline $542-490$ & 4 & 538 & Mongolia & 48 \\
\hline $542-490$ & 4 & 538 & Mongolia & 48 \\
\hline $542-490$ & 4 & 538 & Mongolia & 48 \\
\hline $542-490$ & 4 & 538 & Mongolia & 48 \\
\hline $542-490$ & 4 & 538 & Mongolia & 48 \\
\hline $542-490$ & 4 & 538 & Mongolia & 48 \\
\hline $542-490$ & 4 & 538 & Mongolia & 48 \\
\hline $542-490$ & 4 & 538 & Mongolia & 48 \\
\hline $542-490$ & 4 & 538 & Mongolia & 48 \\
\hline $542-490$ & 4 & 538 & Mongolia & 48 \\
\hline $542-490$ & 4 & 538 & Mongolia & 48 \\
\hline $542-490$ & 4 & 538 & Mongolia & 48 \\
\hline $542-490$ & 4 & 538 & Mongolia & 48 \\
\hline $542-490$ & 4 & 538 & Mongolia & 48 \\
\hline $490-250$ & 5 & 382 & Acadian & 3 \\
\hline $490-250$ & 5 & 382 & Acadian & 3 \\
\hline $490-250$ & 5 & 382 & Acadian & 3 \\
\hline $490-250$ & 5 & 382 & Acadian & 3 \\
\hline $490-250$ & 5 & 382 & Acadian & 3 \\
\hline $490-250$ & 5 & 382 & Acadian & 3 \\
\hline $490-250$ & 5 & 382 & Acadian & 3 \\
\hline $490-250$ & 5 & 382 & Acadian & 3 \\
\hline $490-250$ & 5 & 382 & Acadian & 3 \\
\hline $490-250$ & 5 & 382 & Acadian & 3 \\
\hline $490-250$ & 5 & 382 & Acadian & 3 \\
\hline $490-250$ & 5 & 382 & Acadian & 3 \\
\hline $490-250$ & 5 & 382 & Acadian & 3 \\
\hline $490-250$ & 5 & 382 & Acadian & 3 \\
\hline $490-250$ & 5 & 382 & Acadian & 3 \\
\hline $490-250$ & 5 & 382 & Acadian & 3 \\
\hline $490-250$ & 5 & 382 & Acadian & 3 \\
\hline $490-250$ & 5 & 382 & Acadian & 3 \\
\hline $490-250$ & 5 & 382 & Acadian & 3 \\
\hline $490-250$ & 5 & 382 & Acadian & 3 \\
\hline
\end{tabular}




\begin{tabular}{|c|c|c|c|c|}
\hline $490-250$ & 5 & 382 & Acadian & 3 \\
\hline $490-250$ & 5 & 382 & Acadian & 3 \\
\hline $490-250$ & 5 & 382 & Acadian & 3 \\
\hline $490-250$ & 5 & 382 & Acadian & 3 \\
\hline $490-250$ & 5 & 382 & Acadian & 3 \\
\hline $490-250$ & 5 & 382 & Acadian & 3 \\
\hline $490-250$ & 5 & 382 & Acadian & 3 \\
\hline $490-250$ & 5 & 382 & Acadian & 3 \\
\hline $490-250$ & 5 & 382 & Acadian & 3 \\
\hline $490-250$ & 5 & 382 & Acadian & 3 \\
\hline $490-250$ & 5 & 382 & Acadian & 3 \\
\hline $490-250$ & 5 & 387 & Acadian & 3 \\
\hline $490-250$ & 5 & 387 & Acadian & 3 \\
\hline $490-250$ & 5 & 387 & Acadian & 3 \\
\hline $490-250$ & 5 & 387 & Acadian & 3 \\
\hline $490-250$ & 5 & 387 & Acadian & 3 \\
\hline $490-250$ & 5 & 387 & Acadian & 3 \\
\hline $490-250$ & 5 & 387 & Acadian & 3 \\
\hline $490-250$ & 5 & 387 & Acadian & 3 \\
\hline $490-250$ & 5 & 387 & Acadian & 3 \\
\hline $490-250$ & 5 & 387 & Acadian & 3 \\
\hline $490-250$ & 5 & 387 & Acadian & 3 \\
\hline $490-250$ & 5 & 387 & Acadian & 3 \\
\hline $490-250$ & 5 & 387 & Acadian & 3 \\
\hline $490-250$ & 5 & 387 & Acadian & 3 \\
\hline $490-250$ & 5 & 387 & Acadian & 3 \\
\hline $490-250$ & 5 & 387 & Acadian & 3 \\
\hline $490-250$ & 5 & 387 & Acadian & 3 \\
\hline $490-250$ & 5 & 387 & Acadian & 3 \\
\hline $490-250$ & 5 & 387 & Acadian & 3 \\
\hline $490-250$ & 5 & 387 & Acadian & 3 \\
\hline $490-250$ & 5 & 387 & Acadian & 3 \\
\hline $490-250$ & 5 & 387 & Acadian & 3 \\
\hline $490-250$ & 5 & 387 & Acadian & 3 \\
\hline $490-250$ & 5 & 387 & Acadian & 3 \\
\hline $490-250$ & 5 & 387 & Acadian & 3 \\
\hline $490-250$ & 5 & 387 & Acadian & 3 \\
\hline $490-250$ & 5 & 387 & Acadian & 3 \\
\hline $490-250$ & 5 & 387 & Acadian & 3 \\
\hline $490-250$ & 5 & 387 & Acadian & 3 \\
\hline $490-250$ & 5 & 387 & Acadian & 3 \\
\hline
\end{tabular}




\begin{tabular}{|c|c|c|c|c|}
\hline $490-250$ & 5 & 387 & Acadian & 3 \\
\hline $490-250$ & 5 & 387 & Acadian & 3 \\
\hline $490-250$ & 5 & 387 & Acadian & 3 \\
\hline $490-250$ & 5 & 387 & Acadian & 3 \\
\hline $490-250$ & 5 & 387 & Acadian & 3 \\
\hline $490-250$ & 5 & 387 & Acadian & 3 \\
\hline $490-250$ & 5 & 387 & Acadian & 3 \\
\hline $490-250$ & 5 & 387 & Acadian & 3 \\
\hline $490-250$ & 5 & 387 & Acadian & 3 \\
\hline $490-250$ & 5 & 387 & Acadian & 3 \\
\hline $490-250$ & 5 & 387 & Acadian & 3 \\
\hline $490-250$ & 5 & 387 & Acadian & 3 \\
\hline $490-250$ & 5 & 387 & Acadian & 3 \\
\hline $490-250$ & 5 & 387 & Acadian & 3 \\
\hline $490-250$ & 5 & 387 & Acadian & 3 \\
\hline $490-250$ & 5 & 387 & Acadian & 3 \\
\hline $490-250$ & 5 & 387 & Acadian & 3 \\
\hline $490-250$ & 5 & 387 & Acadian & 3 \\
\hline $490-250$ & 5 & 387 & Acadian & 3 \\
\hline $490-250$ & 5 & 387 & Acadian & 3 \\
\hline $490-250$ & 5 & 387 & Acadian & 3 \\
\hline $490-250$ & 5 & 387 & Acadian & 3 \\
\hline $490-250$ & 5 & 387 & Acadian & 3 \\
\hline $490-250$ & 5 & 387 & Acadian & 3 \\
\hline $490-250$ & 5 & 387 & Acadian & 3 \\
\hline $490-250$ & 5 & 387 & Acadian & 3 \\
\hline $490-250$ & 5 & 387 & Acadian & 3 \\
\hline $490-250$ & 5 & 387 & Acadian & 3 \\
\hline $490-250$ & 5 & 387 & Acadian & 3 \\
\hline $490-250$ & 5 & 387 & Acadian & 3 \\
\hline $490-250$ & 5 & 387 & Acadian & 3 \\
\hline $490-250$ & 5 & 387 & Acadian & 3 \\
\hline $490-250$ & 5 & 387 & Acadian & 3 \\
\hline $490-250$ & 5 & 387 & Acadian & 3 \\
\hline $490-250$ & 5 & 387 & Acadian & 3 \\
\hline $490-250$ & 5 & 387 & Acadian & 3 \\
\hline $490-250$ & 5 & 387 & Acadian & 3 \\
\hline $490-250$ & 5 & 387 & Acadian & 3 \\
\hline $490-250$ & 5 & 387 & Acadian & 3 \\
\hline $490-250$ & 5 & 387 & Acadian & 3 \\
\hline 490-365 & 5 & 367 & Acadian & 13 \\
\hline
\end{tabular}




\begin{tabular}{|c|c|c|c|c|}
\hline $490-365$ & 5 & 367 & Acadian & 13 \\
\hline $490-365$ & 5 & 365 & Acadian & 13 \\
\hline $490-365$ & 5 & 365 & Acadian & 13 \\
\hline $490-250$ & 5 & 480 & Taconic & 13 \\
\hline $490-250$ & 5 & 480 & Taconic & 13 \\
\hline $490-250$ & 5 & 480 & Taconic & 13 \\
\hline $490-250$ & 5 & 480 & Taconic & 13 \\
\hline $490-250$ & 5 & 480 & Taconic & 13 \\
\hline $490-250$ & 5 & 480 & Taconic & 13 \\
\hline $490-250$ & 5 & 480 & Taconic & 13 \\
\hline $490-250$ & 5 & 480 & Taconic & 13 \\
\hline $490-250$ & 5 & 480 & Taconic & 13 \\
\hline $490-250$ & 5 & 480 & Taconic & 13 \\
\hline $490-250$ & 5 & 480 & Taconic & 13 \\
\hline $490-250$ & 5 & 480 & Taconic & 13 \\
\hline $490-250$ & 5 & 480 & Taconic & 13 \\
\hline $490-250$ & 5 & 480 & Taconic & 13 \\
\hline $490-250$ & 5 & 480 & Taconic & 13 \\
\hline $490-250$ & 5 & 480 & Taconic & 13 \\
\hline $490-250$ & 5 & 480 & Taconic & 13 \\
\hline $490-250$ & 5 & 480 & Taconic & 13 \\
\hline $490-250$ & 5 & 480 & Taconic & 13 \\
\hline $490-250$ & 5 & 480 & Taconic & 13 \\
\hline $490-250$ & 5 & 480 & Taconic & 13 \\
\hline $490-250$ & 5 & 480 & Taconic & 13 \\
\hline $490-250$ & 5 & 480 & Taconic & 13 \\
\hline $490-250$ & 5 & 480 & Taconic & 13 \\
\hline $490-250$ & 5 & 480 & Taconic & 13 \\
\hline $490-250$ & 5 & 480 & Taconic & 13 \\
\hline $490-250$ & 5 & 480 & Taconic & 13 \\
\hline $490-250$ & 5 & 480 & Taconic & 13 \\
\hline $490-250$ & 5 & 480 & Taconic & 13 \\
\hline $490-250$ & 5 & 480 & Taconic & 13 \\
\hline $490-250$ & 5 & 480 & Taconic & 13 \\
\hline $490-250$ & 5 & 480 & Taconic & 13 \\
\hline $490-250$ & 5 & 480 & Taconic & 13 \\
\hline $490-250$ & 5 & 480 & Taconic & 13 \\
\hline $490-250$ & 5 & 480 & Taconic & 13 \\
\hline $490-250$ & 5 & 480 & Taconic & 13 \\
\hline $490-250$ & 5 & 480 & Taconic & 13 \\
\hline $490-250$ & 5 & 480 & Taconic & 13 \\
\hline
\end{tabular}




\begin{tabular}{|c|c|c|c|c|}
\hline $490-250$ & 5 & 480 & Taconic & 13 \\
\hline $490-250$ & 5 & 480 & Taconic & 13 \\
\hline $490-250$ & 5 & 480 & Taconic & 13 \\
\hline $490-250$ & 5 & 480 & Taconic & 13 \\
\hline $490-250$ & 5 & 480 & Taconic & 13 \\
\hline $490-250$ & 5 & 480 & Taconic & 13 \\
\hline $490-250$ & 5 & 480 & Taconic & 13 \\
\hline $490-250$ & 5 & 480 & Taconic & 13 \\
\hline $490-250$ & 5 & 480 & Taconic & 13 \\
\hline $490-250$ & 5 & 480 & Taconic & 13 \\
\hline $490-250$ & 5 & 480 & Taconic & 13 \\
\hline $490-250$ & 5 & 480 & Taconic & 13 \\
\hline $490-250$ & 5 & 480 & Taconic & 13 \\
\hline $490-250$ & 5 & 480 & Taconic & 13 \\
\hline $490-250$ & 5 & 480 & Taconic & 13 \\
\hline $490-250$ & 5 & 480 & Taconic & 13 \\
\hline $490-250$ & 5 & 480 & Taconic & 13 \\
\hline $490-250$ & 5 & 480 & Taconic & 13 \\
\hline $490-250$ & 5 & 480 & Taconic & 13 \\
\hline $490-250$ & 5 & 480 & Taconic & 13 \\
\hline $490-250$ & 5 & 480 & Taconic & 13 \\
\hline $490-250$ & 5 & 480 & Taconic & 13 \\
\hline $490-250$ & 5 & 480 & Taconic & 13 \\
\hline $490-250$ & 5 & 480 & Taconic & 13 \\
\hline $490-250$ & 5 & 480 & Taconic & 13 \\
\hline $490-250$ & 5 & 480 & Taconic & 13 \\
\hline $490-250$ & 5 & 480 & Taconic & 13 \\
\hline $490-250$ & 5 & 480 & Taconic & 13 \\
\hline $490-250$ & 5 & 480 & Taconic & 13 \\
\hline $490-250$ & 5 & 480 & Taconic & 13 \\
\hline $490-250$ & 5 & 480 & Taconic & 13 \\
\hline $490-250$ & 5 & 480 & Taconic & 13 \\
\hline $490-250$ & 5 & 480 & Taconic & 13 \\
\hline $490-250$ & 5 & 480 & Taconic & 13 \\
\hline $490-250$ & 5 & 480 & Taconic & 13 \\
\hline $490-250$ & 5 & 480 & Taconic & 13 \\
\hline $490-250$ & 5 & 480 & Taconic & 13 \\
\hline $490-250$ & 5 & 480 & Taconic & 13 \\
\hline $490-250$ & 5 & 480 & Taconic & 13 \\
\hline $490-250$ & 5 & 480 & Taconic & 13 \\
\hline $490-250$ & 5 & 480 & Taconic & 13 \\
\hline
\end{tabular}




\begin{tabular}{|c|c|c|c|c|}
\hline $490-250$ & 5 & 480 & Taconic & 13 \\
\hline $490-250$ & 5 & 480 & Taconic & 13 \\
\hline $490-250$ & 5 & 480 & Taconic & 13 \\
\hline $490-250$ & 5 & 480 & Taconic & 13 \\
\hline $490-250$ & 5 & 480 & Taconic & 13 \\
\hline $490-250$ & 5 & 480 & Taconic & 13 \\
\hline $490-250$ & 5 & 480 & Taconic & 13 \\
\hline $490-250$ & 5 & 480 & Taconic & 13 \\
\hline $490-250$ & 5 & 480 & Taconic & 13 \\
\hline $490-250$ & 5 & 480 & Taconic & 13 \\
\hline $490-250$ & 5 & 480 & Taconic & 13 \\
\hline $490-250$ & 5 & 480 & Taconic & 13 \\
\hline $490-250$ & 5 & 480 & Taconic & 13 \\
\hline $490-250$ & 5 & 480 & Taconic & 13 \\
\hline $490-250$ & 5 & 445 & Scotland & 15 \\
\hline $490-250$ & 5 & 445 & Scotland & 15 \\
\hline $490-250$ & 5 & 445 & Scotland & 15 \\
\hline $490-250$ & 5 & 445 & Scotland & 15 \\
\hline $490-250$ & 5 & 445 & Scotland & 15 \\
\hline $490-250$ & 5 & 445 & Scotland & 15 \\
\hline $490-250$ & 5 & 445 & Scotland & 15 \\
\hline $490-250$ & 5 & 445 & Scotland & 15 \\
\hline $490-250$ & 5 & 445 & Scotland & 15 \\
\hline $490-250$ & 5 & 445 & Scotland & 15 \\
\hline $490-250$ & 5 & 445 & Scotland & 15 \\
\hline $490-250$ & 5 & 445 & Scotland & 15 \\
\hline $490-250$ & 5 & 445 & Scotland & 15 \\
\hline $490-250$ & 5 & 445 & Scotland & 15 \\
\hline $490-250$ & 5 & 445 & Scotland & 15 \\
\hline $490-250$ & 5 & 445 & Scotland & 15 \\
\hline $490-250$ & 5 & 445 & Scotland & 15 \\
\hline $490-250$ & 5 & 445 & Scotland & 15 \\
\hline $490-250$ & 5 & 445 & Scotland & 15 \\
\hline $490-250$ & 5 & 445 & Scotland & 15 \\
\hline $490-250$ & 5 & 445 & Scotland & 15 \\
\hline $490-250$ & 5 & 445 & Scotland & 15 \\
\hline $490-250$ & 5 & 445 & Scotland & 15 \\
\hline $490-250$ & 5 & 445 & Scotland & 15 \\
\hline $490-250$ & 5 & 445 & Scotland & 15 \\
\hline $490-250$ & 5 & 445 & Scotland & 15 \\
\hline $490-250$ & 5 & 445 & Scotland & 15 \\
\hline
\end{tabular}




\begin{tabular}{|c|c|c|c|c|}
\hline $490-250$ & 5 & 445 & Scotland & 15 \\
\hline $490-250$ & 5 & 445 & Scotland & 15 \\
\hline $490-250$ & 5 & 445 & Scotland & 15 \\
\hline $490-250$ & 5 & 445 & Scotland & 15 \\
\hline $490-250$ & 5 & 445 & Scotland & 15 \\
\hline $490-250$ & 5 & 445 & Scotland & 15 \\
\hline $490-250$ & 5 & 445 & Scotland & 15 \\
\hline $490-250$ & 5 & 445 & Scotland & 15 \\
\hline $490-250$ & 5 & 445 & Scotland & 15 \\
\hline $490-250$ & 5 & 445 & Scotland & 15 \\
\hline $490-250$ & 5 & 445 & Scotland & 15 \\
\hline $490-250$ & 5 & 445 & Scotland & 15 \\
\hline $490-250$ & 5 & 445 & Scotland & 15 \\
\hline $490-250$ & 5 & 445 & Scotland & 15 \\
\hline $490-250$ & 5 & 445 & Scotland & 15 \\
\hline $490-250$ & 5 & 445 & Scotland & 15 \\
\hline $490-250$ & 5 & 445 & Scotland & 15 \\
\hline $490-250$ & 5 & 445 & Scotland & 15 \\
\hline $490-365$ & 5 & 443 & Denmark & 16 \\
\hline $490-365$ & 5 & 443 & Denmark & 16 \\
\hline $490-365$ & 5 & 443 & Denmark & 16 \\
\hline $490-365$ & 5 & 443 & Denmark & 16 \\
\hline $490-365$ & 5 & 443 & Denmark & 16 \\
\hline $490-365$ & 5 & 443 & Denmark & 16 \\
\hline $490-365$ & 5 & 443 & Denmark & 16 \\
\hline $490-365$ & 5 & 443 & Denmark & 16 \\
\hline $490-365$ & 5 & 443 & Denmark & 16 \\
\hline $490-365$ & 5 & 443 & Denmark & 16 \\
\hline $490-250$ & 5 & 445 & Denmark & 16 \\
\hline $490-250$ & 5 & 445 & Denmark & 16 \\
\hline $490-250$ & 5 & 445 & Denmark & 16 \\
\hline $490-250$ & 5 & 445 & Denmark & 16 \\
\hline $490-250$ & 5 & 445 & Denmark & 16 \\
\hline $490-250$ & 5 & 445 & Denmark & 16 \\
\hline $490-250$ & 5 & 445 & Denmark & 16 \\
\hline $490-250$ & 5 & 445 & Denmark & 16 \\
\hline $490-250$ & 5 & 445 & Denmark & 16 \\
\hline $490-250$ & 5 & 445 & Denmark & 16 \\
\hline $490-250$ & 5 & 445 & Denmark & 16 \\
\hline $490-250$ & 5 & 445 & Denmark & 16 \\
\hline $490-250$ & 5 & 445 & Denmark & 16 \\
\hline
\end{tabular}




\begin{tabular}{|c|c|c|c|c|}
\hline $490-250$ & 5 & 445 & Denmark & 16 \\
\hline $490-250$ & 5 & 445 & Denmark & 16 \\
\hline $490-250$ & 5 & 445 & Denmark & 16 \\
\hline $490-250$ & 5 & 445 & Denmark & 16 \\
\hline $490-250$ & 5 & 445 & Denmark & 16 \\
\hline $490-250$ & 5 & 445 & Denmark & 16 \\
\hline $490-250$ & 5 & 445 & Denmark & 16 \\
\hline $490-250$ & 5 & 445 & Denmark & 16 \\
\hline $490-250$ & 5 & 445 & Denmark & 16 \\
\hline $490-250$ & 5 & 445 & Denmark & 16 \\
\hline $490-250$ & 5 & 445 & Denmark & 16 \\
\hline $490-250$ & 5 & 445 & Denmark & 16 \\
\hline $490-250$ & 5 & 445 & Denmark & 16 \\
\hline $490-250$ & 5 & 445 & Denmark & 16 \\
\hline $490-250$ & 5 & 445 & Denmark & 16 \\
\hline $490-250$ & 5 & 445 & Denmark & 16 \\
\hline $490-250$ & 5 & 445 & Denmark & 16 \\
\hline $490-250$ & 5 & 445 & Denmark & 16 \\
\hline $490-250$ & 5 & 445 & Denmark & 16 \\
\hline $490-250$ & 5 & 445 & Denmark & 16 \\
\hline $490-250$ & 5 & 445 & Denmark & 16 \\
\hline $490-250$ & 5 & 445 & Denmark & 16 \\
\hline $490-250$ & 5 & 445 & Denmark & 16 \\
\hline $490-250$ & 5 & 445 & Denmark & 16 \\
\hline $490-250$ & 5 & 445 & Denmark & 16 \\
\hline $490-250$ & 5 & 445 & Denmark & 16 \\
\hline $490-250$ & 5 & 445 & Denmark & 16 \\
\hline $490-250$ & 5 & 445 & Denmark & 16 \\
\hline $490-250$ & 5 & 445 & Denmark & 16 \\
\hline $490-250$ & 5 & 445 & Denmark & 16 \\
\hline $490-250$ & 5 & 445 & Denmark & 16 \\
\hline $490-250$ & 5 & 445 & Denmark & 16 \\
\hline $490-250$ & 5 & 445 & Denmark & 16 \\
\hline $490-250$ & 5 & 445 & Denmark & 16 \\
\hline $490-250$ & 5 & 445 & Denmark & 16 \\
\hline $490-250$ & 5 & 445 & Denmark & 16 \\
\hline $490-250$ & 5 & 445 & Denmark & 16 \\
\hline $490-250$ & 5 & 445 & Denmark & 16 \\
\hline $490-250$ & 5 & 445 & Denmark & 16 \\
\hline $490-250$ & 5 & 445 & Denmark & 16 \\
\hline $490-250$ & 5 & 445 & Denmark & 16 \\
\hline
\end{tabular}




\begin{tabular}{|c|c|c|c|c|}
\hline $490-250$ & 5 & 445 & Denmark & 16 \\
\hline $490-250$ & 5 & 445 & Denmark & 16 \\
\hline $490-250$ & 5 & 445 & Denmark & 16 \\
\hline $490-250$ & 5 & 445 & Denmark & 16 \\
\hline $490-250$ & 5 & 445 & Denmark & 16 \\
\hline $490-250$ & 5 & 445 & Denmark & 16 \\
\hline $490-250$ & 5 & 445 & Denmark & 16 \\
\hline $490-250$ & 5 & 445 & Denmark & 16 \\
\hline $490-250$ & 5 & 445 & Denmark & 16 \\
\hline $490-250$ & 5 & 445 & Denmark & 16 \\
\hline $490-250$ & 5 & 445 & Denmark & 16 \\
\hline $490-250$ & 5 & 445 & Denmark & 16 \\
\hline $490-250$ & 5 & 445 & Denmark & 16 \\
\hline $490-250$ & 5 & 445 & Denmark & 16 \\
\hline $490-250$ & 5 & 445 & Denmark & 16 \\
\hline $490-250$ & 5 & 445 & Denmark & 16 \\
\hline $490-250$ & 5 & 445 & Denmark & 16 \\
\hline $490-250$ & 5 & 445 & Denmark & 16 \\
\hline $490-250$ & 5 & 445 & Denmark & 16 \\
\hline $490-250$ & 5 & 445 & Denmark & 16 \\
\hline $490-250$ & 5 & 445 & Denmark & 16 \\
\hline $490-250$ & 5 & 445 & Denmark & 16 \\
\hline $490-250$ & 5 & 445 & Denmark & 16 \\
\hline $490-250$ & 5 & 445 & Carnic Alps & 17 \\
\hline $490-250$ & 5 & 445 & Carnic Alps & 17 \\
\hline $490-250$ & 5 & 445 & Carnic Alps & 17 \\
\hline $490-250$ & 5 & 445 & Carnic Alps & 17 \\
\hline $490-250$ & 5 & 445 & Carnic Alps & 17 \\
\hline $490-250$ & 5 & 445 & Carnic Alps & 17 \\
\hline $490-250$ & 5 & 445 & Carnic Alps & 17 \\
\hline $490-250$ & 5 & 445 & Carnic Alps & 17 \\
\hline $490-250$ & 5 & 445 & Carnic Alps & 17 \\
\hline $490-250$ & 5 & 445 & Carnic Alps & 17 \\
\hline $490-250$ & 5 & 445 & Carnic Alps & 17 \\
\hline $490-250$ & 5 & 445 & Carnic Alps & 17 \\
\hline $490-250$ & 5 & 445 & Carnic Alps & 17 \\
\hline $490-250$ & 5 & 445 & Carnic Alps & 17 \\
\hline $490-250$ & 5 & 445 & Carnic Alps & 17 \\
\hline $490-250$ & 5 & 445 & Carnic Alps & 17 \\
\hline $490-250$ & 5 & 445 & Carnic Alps & 17 \\
\hline $490-250$ & 5 & 445 & Carnic Alps & 17 \\
\hline
\end{tabular}




\begin{tabular}{|c|c|c|c|c|}
\hline $490-250$ & 5 & 445 & Carnic Alps & 17 \\
\hline $490-250$ & 5 & 445 & Carnic Alps & 17 \\
\hline $490-250$ & 5 & 445 & Carnic Alps & 17 \\
\hline $490-250$ & 5 & 445 & Carnic Alps & 17 \\
\hline $490-250$ & 5 & 445 & Carnic Alps & 17 \\
\hline $490-250$ & 5 & 445 & Carnic Alps & 17 \\
\hline $490-250$ & 5 & 445 & Carnic Alps & 17 \\
\hline $490-250$ & 5 & 445 & Carnic Alps & 17 \\
\hline $490-250$ & 5 & 445 & Carnic Alps & 17 \\
\hline $490-250$ & 5 & 445 & Carnic Alps & 17 \\
\hline $490-250$ & 5 & 445 & Carnic Alps & 17 \\
\hline $490-250$ & 5 & 445 & Carnic Alps & 17 \\
\hline $490-250$ & 5 & 445 & Carnic Alps & 17 \\
\hline $490-250$ & 5 & 445 & Carnic Alps & 17 \\
\hline $490-250$ & 5 & 445 & Carnic Alps & 17 \\
\hline $490-250$ & 5 & 445 & Carnic Alps & 17 \\
\hline $490-250$ & 5 & 445 & Carnic Alps & 17 \\
\hline $490-250$ & 5 & 445 & Carnic Alps & 17 \\
\hline $490-250$ & 5 & 445 & Carnic Alps & 17 \\
\hline $490-365$ & 5 & 463 & Sweden & 20 \\
\hline $490-365$ & 5 & 463 & Sweden & 20 \\
\hline $490-365$ & 5 & 480 & Sweden & 20 \\
\hline $490-365$ & 5 & 480 & Sweden & 20 \\
\hline $490-365$ & 5 & 480 & Sweden & 20 \\
\hline $490-365$ & 5 & 485 & Sweden & 20 \\
\hline $490-365$ & 5 & 485 & Sweden & 20 \\
\hline $490-365$ & 5 & 485 & Sweden & 20 \\
\hline $490-365$ & 5 & 435 & Sweden & 20 \\
\hline $490-365$ & 5 & 435 & Sweden & 20 \\
\hline $490-365$ & 5 & 435 & Sweden & 20 \\
\hline $490-365$ & 5 & 435 & Sweden & 20 \\
\hline $490-365$ & 5 & 435 & Sweden & 20 \\
\hline $490-365$ & 5 & 435 & Sweden & 20 \\
\hline $490-365$ & 5 & 435 & Sweden & 20 \\
\hline $490-365$ & 5 & 435 & Sweden & 20 \\
\hline $490-365$ & 5 & 435 & Sweden & 20 \\
\hline $490-365$ & 5 & 435 & South Africa & 50 \\
\hline $490-365$ & 5 & 435 & South Africa & 50 \\
\hline $490-365$ & 5 & 435 & South Africa & 50 \\
\hline 0 & 7 & 0 & Modern continental marg & 21 \\
\hline 0 & 7 & 0 & Modern continental marg & 21 \\
\hline
\end{tabular}




\begin{tabular}{|c|c|c|c|c|}
\hline 0 & 7 & 0 & Modern continental marg & 21 \\
\hline 0 & 7 & 0 & Modern continental marg & 21 \\
\hline 0 & 7 & 0 & Modern continental marg & 21 \\
\hline 0 & 7 & 0 & Modern continental marg & 21 \\
\hline 0 & 7 & 0 & Modern continental marg & 21 \\
\hline 0 & 7 & 0 & Modern continental marg & 21 \\
\hline 0 & 7 & 0 & Modern continental marg & 21 \\
\hline 0 & 7 & 0 & Modern continental marg & 21 \\
\hline 0 & 7 & 0 & Modern continental marg & 21 \\
\hline 0 & 7 & 0 & Modern continental marg & 21 \\
\hline 0 & 7 & 0 & Modern continental marg & 21 \\
\hline 0 & 7 & 0 & Modern continental marg & 21 \\
\hline 0 & 7 & 0 & Modern continental marg & 21 \\
\hline 0 & 7 & 0 & Modern continental marg & 21 \\
\hline 0 & 7 & 0 & Modern continental marg & 21 \\
\hline 0 & 7 & 0 & Modern continental marg & 21 \\
\hline 0 & 7 & 0 & Modern continental marg & 21 \\
\hline 0 & 7 & 0 & Modern continental marg & 21 \\
\hline 0 & 7 & 0 & Modern continental marg & 21 \\
\hline 0 & 7 & 0 & Modern continental marg & 21 \\
\hline 0 & 7 & 0 & Modern continental marg & 21 \\
\hline 0 & 7 & 0 & Modern continental marg & 21 \\
\hline 0 & 7 & 0 & Modern continental marg & 21 \\
\hline 0 & 7 & 0 & Modern continental marg & 21 \\
\hline 0 & 7 & 0 & Modern continental marg & 21 \\
\hline 0 & 7 & 0 & Modern continental marg & 21 \\
\hline 0 & 7 & 0 & Modern continental marg & 21 \\
\hline 0 & 7 & 0 & Modern continental marg & 21 \\
\hline 0 & 7 & 0 & Modern continental marg & 21 \\
\hline 0 & 7 & 0 & Modern continental marg & 21 \\
\hline 0 & 7 & 0 & Modern continental marg & 21 \\
\hline 0 & 7 & 0 & Modern continental marg & 21 \\
\hline 0 & 7 & 0 & Modern continental marg & 21 \\
\hline 0 & 7 & 0 & Modern continental marg & 21 \\
\hline 0 & 7 & 0 & Modern continental marg & 21 \\
\hline 0 & 7 & 0 & Modern continental marg & 21 \\
\hline 0 & 7 & 0 & Modern continental marg & 21 \\
\hline 0 & 7 & 0 & Modern continental marg & 21 \\
\hline 0 & 7 & 0 & Modern continental marg & 21 \\
\hline 0 & 7 & 0 & Modern continental marg & 21 \\
\hline 0 & 7 & 0 & Modern continental marg & 21 \\
\hline
\end{tabular}




\begin{tabular}{|c|c|c|c|c|}
\hline 0 & 7 & 0 & Modern continental marg & 21 \\
\hline 0 & 7 & 0 & Modern continental marg & 21 \\
\hline 0 & 7 & 0 & Modern continental marg & 21 \\
\hline 0 & 7 & 0 & Modern continental marg & 21 \\
\hline 0 & 7 & 0 & Modern continental marg & 21 \\
\hline 0 & 7 & 0 & Modern continental marg & 21 \\
\hline 0 & 7 & 0 & Modern continental marg & 21 \\
\hline 0 & 7 & 0 & Modern continental marg & 21 \\
\hline 0 & 7 & 0 & Modern continental marg & 21 \\
\hline 0 & 7 & 0 & Modern continental marg & 21 \\
\hline 0 & 7 & 0 & Modern continental marg & 21 \\
\hline 0 & 7 & 0 & Modern continental marg & 21 \\
\hline 0 & 7 & 0 & Modern continental marg & 21 \\
\hline 0 & 7 & 0 & Modern continental marg & 21 \\
\hline 0 & 7 & 0 & Modern continental marg & 21 \\
\hline 0 & 7 & 0 & Modern deep sea & 22 \\
\hline 0 & 7 & 0 & Modern deep sea & 22 \\
\hline 0 & 7 & 0 & Modern deep sea & 22 \\
\hline 0 & 7 & 0 & Modern deep sea & 22 \\
\hline 0 & 7 & 0 & Modern deep sea & 22 \\
\hline 0 & 7 & 0 & Modern deep sea & 22 \\
\hline 0 & 7 & 0 & Modern deep sea & 22 \\
\hline 0 & 7 & 0 & Modern deep sea & 22 \\
\hline 0 & 7 & 0 & Modern deep sea & 22 \\
\hline 0 & 7 & 0 & Modern deep sea & 22 \\
\hline 0 & 7 & 0 & Modern deep sea & 22 \\
\hline 0 & 7 & 0 & Modern deep sea & 22 \\
\hline 0 & 7 & 0 & Modern deep sea & 22 \\
\hline 0 & 7 & 0 & Modern deep sea & 22 \\
\hline 0 & 7 & 0 & Modern deep sea & 22 \\
\hline 0 & 7 & 0 & Modern deep sea & 22 \\
\hline 0 & 7 & 0 & Modern deep sea & 22 \\
\hline 0 & 7 & 0 & Modern deep sea & 22 \\
\hline 0 & 7 & 0 & Modern deep sea & 22 \\
\hline 0 & 7 & 0 & Modern deep sea & 22 \\
\hline 0 & 7 & 0 & Modern deep sea & 22 \\
\hline 0 & 7 & 0 & Modern deep sea & 22 \\
\hline 0 & 7 & 0 & Modern deep sea & 22 \\
\hline 0 & 7 & 0 & Modern deep sea & 22 \\
\hline 0 & 7 & 0 & Modern deep sea & 22 \\
\hline 0 & 7 & 0 & Modern deep sea & 22 \\
\hline
\end{tabular}




\begin{tabular}{|c|c|c|c|c|}
\hline 0 & 7 & 0 & Modern deep sea & 22 \\
\hline 0 & 7 & 0 & Modern deep sea & 22 \\
\hline 0 & 7 & 0 & Modern deep sea & 22 \\
\hline 0 & 7 & 0 & Modern deep sea & 22 \\
\hline 0 & 7 & 0 & Modern deep sea & 22 \\
\hline 0 & 7 & 0 & Modern deep sea & 22 \\
\hline 0 & 7 & 0 & Modern deep sea & 22 \\
\hline 0 & 7 & 0 & Modern deep sea & 22 \\
\hline 0 & 7 & 0 & Modern deep sea & 22 \\
\hline 0 & 7 & 0 & Modern deep sea & 22 \\
\hline 0 & 7 & 0 & Modern deep sea & 22 \\
\hline 0 & 7 & 0 & Modern deep sea & 22 \\
\hline 0 & 7 & 0 & Modern deep sea & 22 \\
\hline 0 & 7 & 0 & Modern deep sea & 22 \\
\hline 0 & 7 & 0 & Modern deep sea & 22 \\
\hline 0 & 7 & 0 & Modern deep sea & 22 \\
\hline 0 & 7 & 0 & Modern deep sea & 22 \\
\hline 0 & 7 & 0 & Modern deep sea & 22 \\
\hline 0 & 7 & 0 & Modern deep sea & 22 \\
\hline 0 & 7 & 0 & Modern deep sea & 22 \\
\hline 0 & 7 & 0 & Modern deep sea & 22 \\
\hline 0 & 7 & 0 & Modern deep sea & 22 \\
\hline 0 & 7 & 0 & Modern deep sea & 22 \\
\hline 0 & 7 & 0 & Modern deep sea & 22 \\
\hline 0 & 7 & 0 & Modern deep sea & 22 \\
\hline 0 & 7 & 0 & Modern deep sea & 22 \\
\hline 0 & 7 & 0 & Modern deep sea & 22 \\
\hline 0 & 7 & 0 & Modern deep sea & 22 \\
\hline 0 & 7 & 0 & Modern deep sea & 22 \\
\hline 0 & 7 & 0 & Modern deep sea & 22 \\
\hline 0 & 7 & 0 & Modern deep sea & 22 \\
\hline 0 & 7 & 0 & Modern deep sea & 22 \\
\hline 0 & 7 & 0 & Modern deep sea & 22 \\
\hline 0 & 7 & 0 & Modern dysaerobic & 23 \\
\hline 0 & 7 & 0 & Modern dysaerobic & 23 \\
\hline 0 & 7 & 0 & Modern dysaerobic & 23 \\
\hline 0 & 7 & 0 & Modern dysaerobic & 23 \\
\hline 0 & 7 & 0 & Modern dysaerobic & 23 \\
\hline 0 & 7 & 0 & Modern dysaerobic & 23 \\
\hline 0 & 7 & 0 & Modern dysaerobic & 23 \\
\hline 0 & 7 & 0 & Modern dysaerobic & 23 \\
\hline
\end{tabular}




\begin{tabular}{|c|c|c|c|c|}
\hline 0 & 7 & 0 & Modern dysaerobic & 23 \\
\hline 0 & 7 & 0 & Modern dysaerobic & 23 \\
\hline 0 & 7 & 0 & Modern dysaerobic & 23 \\
\hline 0 & 7 & 0 & Modern dysaerobic & 23 \\
\hline 0 & 7 & 0 & Modern dysaerobic & 23 \\
\hline 0 & 7 & 0 & Modern dysaerobic & 23 \\
\hline 0 & 7 & 0 & Modern dysaerobic & 23 \\
\hline 0 & 7 & 0 & Modern dysaerobic & 23 \\
\hline 0 & 7 & 0 & Modern dysaerobic & 23 \\
\hline 0 & 7 & 0 & Modern dysaerobic & 23 \\
\hline 0 & 7 & 0 & Modern dysaerobic & 23 \\
\hline 0 & 7 & 0 & Modern dysaerobic & 23 \\
\hline 0 & 7 & 0 & Modern dysaerobic & 23 \\
\hline 0 & 7 & 0 & Modern dysaerobic & 23 \\
\hline 0 & 7 & 0 & Modern dysaerobic & 23 \\
\hline 0 & 7 & 0 & Modern dysaerobic & 23 \\
\hline 0 & 7 & 0 & Modern dysaerobic & 23 \\
\hline 0 & 7 & 0 & Modern dysaerobic & 23 \\
\hline 0 & 7 & 0 & Modern dysaerobic & 23 \\
\hline 0 & 7 & 0 & Modern dysaerobic & 23 \\
\hline 0 & 7 & 0 & Modern dysaerobic & 23 \\
\hline 0 & 7 & 0 & Modern anaerobic & 24 \\
\hline 0 & 7 & 0 & Modern anaerobic & 24 \\
\hline 0 & 7 & 0 & Modern anaerobic & 24 \\
\hline 0 & 7 & 0 & Modern anaerobic & 24 \\
\hline 0 & 7 & 0 & Modern anaerobic & 24 \\
\hline 0 & 7 & 0 & Modern anaerobic & 24 \\
\hline 0 & 7 & 0 & Modern anaerobic & 24 \\
\hline 0 & 7 & 0 & Modern anaerobic & 24 \\
\hline 0 & 7 & 0 & Modern anaerobic & 24 \\
\hline 0 & 7 & 0 & Modern anaerobic & 24 \\
\hline 0 & 7 & 0 & Modern anaerobic & 24 \\
\hline 0 & 7 & 0 & Modern anaerobic & 24 \\
\hline 0 & 7 & 0 & Modern anaerobic & 24 \\
\hline 0 & 7 & 0 & Modern anaerobic & 24 \\
\hline 0 & 7 & 0 & Modern anaerobic & 24 \\
\hline 0 & 7 & 0 & Modern anaerobic & 24 \\
\hline 0 & 7 & 0 & Modern anaerobic & 24 \\
\hline 0 & 7 & 0 & Modern anaerobic & 24 \\
\hline 0 & 7 & 0 & Modern anaerobic & 24 \\
\hline 0 & 7 & 0 & Modern anaerobic & 24 \\
\hline
\end{tabular}




\begin{tabular}{|c|c|c|c|c|}
\hline 0 & 7 & 0 & Modern anaerobic & 24 \\
\hline 0 & 7 & 0 & Modern anaerobic & 24 \\
\hline 0 & 7 & 0 & Modern anaerobic & 24 \\
\hline 0 & 7 & 0 & Modern anaerobic & 24 \\
\hline 0 & 7 & 0 & Modern anaerobic & 24 \\
\hline 0 & 7 & 0 & Modern anaerobic & 24 \\
\hline 0 & 7 & 0 & Modern anaerobic & 24 \\
\hline 0 & 7 & 0 & Modern anaerobic & 24 \\
\hline 0 & 7 & 0 & Modern anaerobic & 24 \\
\hline 0 & 7 & 0 & Modern anaerobic & 24 \\
\hline 0 & 7 & 0 & Modern anaerobic & 24 \\
\hline 0 & 7 & 0 & Modern anaerobic & 24 \\
\hline 0 & 7 & 0 & Modern anaerobic & 24 \\
\hline 0 & 7 & 0 & Modern anaerobic & 24 \\
\hline 0 & 7 & 0 & Modern anaerobic & 24 \\
\hline 0 & 7 & 0 & Modern anaerobic & 24 \\
\hline 0 & 7 & 0 & Modern anaerobic & 24 \\
\hline 0 & 7 & 0 & Modern anaerobic & 24 \\
\hline 0 & 7 & 0 & Modern anaerobic & 24 \\
\hline 0 & 7 & 0 & Modern anaerobic & 24 \\
\hline 0 & 7 & 0 & Modern anaerobic & 24 \\
\hline 0 & 7 & 0 & Modern anaerobic & 24 \\
\hline 0 & 7 & 0 & Modern anaerobic & 24 \\
\hline 0 & 7 & 0 & Modern anaerobic & 24 \\
\hline 0 & 7 & 0 & Modern anaerobic & 24 \\
\hline 0 & 7 & 0 & Modern anaerobic & 24 \\
\hline 0 & 7 & 0 & Modern anaerobic & 24 \\
\hline 0 & 7 & 0 & Modern anaerobic & 24 \\
\hline 0 & 7 & 0 & Modern anaerobic & 24 \\
\hline 0 & 7 & 0 & Modern anaerobic & 24 \\
\hline 0 & 7 & 0 & Modern anaerobic & 24 \\
\hline 0 & 7 & 0 & Modern anaerobic & 24 \\
\hline 0 & 7 & 0 & Modern anaerobic & 24 \\
\hline 0 & 7 & 0 & Modern anaerobic & 24 \\
\hline 0 & 7 & 0 & Modern anaerobic & 24 \\
\hline 0 & 7 & 0 & Modern anaerobic & 24 \\
\hline 0 & 7 & 0 & Modern anaerobic & 24 \\
\hline 0 & 7 & 0 & Modern anaerobic & 24 \\
\hline 0 & 7 & 0 & Modern anaerobic & 24 \\
\hline 0 & 7 & 0 & Modern anaerobic & 24 \\
\hline 0 & 7 & 0 & Modern anaerobic & 24 \\
\hline
\end{tabular}




\begin{tabular}{|c|c|c|c|c|}
\hline 0 & 7 & 0 & Modern anaerobic & 24 \\
\hline 0 & 7 & 0 & Modern anaerobic & 24 \\
\hline 0 & 7 & 0 & Modern anaerobic & 24 \\
\hline 0 & 7 & 0 & Modern anaerobic & 24 \\
\hline 0 & 7 & 0 & Modern anaerobic & 24 \\
\hline 0 & 7 & 0 & Modern anaerobic & 24 \\
\hline 0 & 7 & 0 & Modern anaerobic & 24 \\
\hline 0 & 7 & 0 & Modern anaerobic & 24 \\
\hline 0 & 7 & 0 & Modern anaerobic & 24 \\
\hline 0 & 7 & 0 & Modern anaerobic & 24 \\
\hline 0 & 7 & 0 & Modern anaerobic & 24 \\
\hline 0 & 7 & 0 & Modern anaerobic & 24 \\
\hline 0 & 7 & 0 & Modern anaerobic & 24 \\
\hline 0 & 7 & 0 & Modern anaerobic & 24 \\
\hline 0 & 7 & 0 & Modern anaerobic & 24 \\
\hline 0 & 7 & 0 & Modern anaerobic & 24 \\
\hline 0 & 7 & 0 & Modern anaerobic & 24 \\
\hline 0 & 7 & 0 & Modern anaerobic & 24 \\
\hline 0 & 7 & 0 & Modern anaerobic & 24 \\
\hline 0 & 7 & 0 & Modern anaerobic & 24 \\
\hline 0 & 7 & 0 & Modern anaerobic & 24 \\
\hline 0 & 7 & 0 & Modern anaerobic & 24 \\
\hline 0 & 7 & 0 & Modern anaerobic & 24 \\
\hline 0 & 7 & 0 & Modern anaerobic & 24 \\
\hline 0 & 7 & 0 & Modern anaerobic & 24 \\
\hline 0 & 7 & 0 & Modern anaerobic & 24 \\
\hline
\end{tabular}




\section{Publication}

Reuschel et al., 2012, Precambrian Research

Reuschel et al., 2012, Precambrian Research

Reuschel et al., 2012, Precambrian Research

Reuschel et al., 2012, Precambrian Research

Reuschel et al., 2012, Precambrian Research

Reuschel et al., 2012, Precambrian Research

Reuschel et al., 2012, Precambrian Research

Reuschel et al., 2012, Precambrian Research

Reuschel et al., 2012, Precambrian Research

Reuschel et al., 2012, Precambrian Research

Reuschel et al., 2012, Precambrian Research

Reuschel et al., 2012, Precambrian Research

Reuschel et al., 2012, Precambrian Research

Reuschel et al., 2012, Precambrian Research

Reuschel et al., 2012, Precambrian Research

Reuschel et al., 2012, Precambrian Research

Reuschel et al., 2012, Precambrian Research

Reuschel et al., 2012, Precambrian Research

Reuschel et al., 2012, Precambrian Research

Reuschel et al., 2012, Precambrian Research

Reuschel et al., 2012, Precambrian Research

Reuschel et al., 2012, Precambrian Research

Reuschel et al., 2012, Precambrian Research

Reuschel et al., 2012, Precambrian Research

Reuschel et al., 2012, Precambrian Research

Reuschel et al., 2012, Precambrian Research

Reuschel et al., 2012, Precambrian Research

Reuschel et al., 2012, Precambrian Research

Reuschel et al., 2012, Precambrian Research

Reuschel et al., 2012, Precambrian Research

Reuschel et al., 2012, Precambrian Research

Reuschel et al., 2012, Precambrian Research

Planavsky et al., 2011, Nature

Planavsky et al., 2011, Nature

Planavsky et al., 2011, Nature

Planavsky et al., 2011, Nature

Planavsky et al., 2011, Nature

Planavsky et al., 2011, Nature

Planavsky et al., 2011, Nature

Planavsky et al., 2011, Nature

\section{Core/Outcrop itional Enviro Notes}

\begin{tabular}{lll} 
Core & 3 & For these rocks, F \\
Core & 3 & For these rocks, F \\
Core & 3 & For these rocks, F \\
Core & 3 & For these rocks, F \\
Core & 3 & For these rocks, F \\
Core & 3 & For these rocks, F \\
Core & 3 & For these rocks, F \\
Core & 3 & For these rocks, F \\
Core & 3 & For these rocks, F \\
Core & 3 & For these rocks, F \\
Core & 3 & For these rocks, F \\
Core & 3 & For these rocks, F \\
Core & 3 & For these rocks, F \\
Core & 3 & For these rocks, F \\
Core & 3 & For these rocks, F \\
Core & 3 & For these rocks, F \\
Core & 3 & For these rocks, F \\
Core & 3 & For these rocks, F \\
Core & 3 & For these rocks, F \\
Core & 3 & For these rocks, F \\
Core & 3 & For these rocks, F \\
Core & 3 & For these rocks, F \\
Core & 3 & For these rocks, F \\
Core & 3 & For these rocks, F \\
Core & 3 & For these rocks, F \\
Core & 3 & For these rocks, F \\
Core & 3 & For these rocks, F \\
Core & 3 & For these rocks, F \\
Core & 3 & For these rocks, F \\
Core & 3 & For these rocks, F \\
Core & 3 & For these rocks, F \\
Core & 3 & For these rocks, F \\
Outcrop & 3 & \\
Outcrop & 3 & \\
Outcrop & 3 & \\
Outcrop & 3 & \\
Outcrop & \\
Outcrop & 3 & \\
Outcrop & \\
Outcrop & & \\
& 3 & \\
\hline
\end{tabular}


Planavsky et al., 2011, Nature

Planavsky et al., 2011, Nature

Planavsky et al., 2011, Nature

Planavsky et al., 2011, Nature

Planavsky et al., 2011, Nature

Planavsky et al., 2011, Nature

Planavsky et al., 2011, Nature

Planavsky et al., 2011, Nature

Planavsky et al., 2011, Nature

Planavsky et al., 2011, Nature

Planavsky et al., 2011, Nature

Planavsky et al., 2011, Nature

Planavsky et al., 2011, Nature

Planavsky et al., 2011, Nature

Planavsky et al., 2011, Nature

Planavsky et al., 2011, Nature

Planavsky et al., 2011, Nature

Planavsky et al., 2011, Nature

Planavsky et al., 2011, Nature

Planavsky et al., 2011, Nature

Planavsky et al., 2011, Nature

Planavsky et al., 2011, Nature

Planavsky et al., 2011, Nature

Planavsky et al., 2011, Nature

Planavsky et al., 2011, Nature

Planavsky et al., 2011, Nature

Planavsky et al., 2011, Nature

Planavsky et al., 2011, Nature

Planavsky et al., 2011, Nature

Planavsky et al., 2011, Nature

Planavsky et al., 2011, Nature

Planavsky et al., 2011, Nature

Planavsky et al., 2011, Nature

Planavsky et al., 2011, Nature

Planavsky et al., 2011, Nature

Planavsky et al., 2011, Nature

Planavsky et al., 2011, Nature

Planavsky et al., 2011, Nature

Planavsky et al., 2011, Nature

Planavsky et al., 2011, Nature

Planavsky et al., 2011, Nature

\begin{tabular}{|c|c|c|}
\hline Outcrop & 2 & \\
\hline Outcrop & 2 & \\
\hline Outcrop & 2 & \\
\hline Outcrop & 2 & \\
\hline Outcrop & 2 & \\
\hline Outcrop & 2 & \\
\hline Outcrop & 2 & \\
\hline Outcrop & 2 & \\
\hline Outcrop & 2 & \\
\hline Outcrop & 3 & \\
\hline Outcrop & 3 & \\
\hline Outcrop & 3 & \\
\hline Outcrop & 3 & \\
\hline Outcrop & 3 & \\
\hline Outcrop & 3 & \\
\hline Outcrop & 3 & \\
\hline Outcrop & 3 & \\
\hline Outcrop & 3 & \\
\hline Outcrop & 3 & \\
\hline Outcrop & 3 & \\
\hline Outcrop & 3 & \\
\hline Outcrop & 3 & \\
\hline Outcrop & 3 & \\
\hline Core & 3 & \\
\hline Core & 3 & \\
\hline Core & 3 & \\
\hline Core & 3 & \\
\hline Core & 3 & \\
\hline Core & 3 & \\
\hline Core & 3 & \\
\hline Core & 3 & \\
\hline Core & 3 & \\
\hline Core & 3 & \\
\hline Core & 3 & \\
\hline Core & 3 & \\
\hline Core & 3 & \\
\hline Core & 3 & \\
\hline Outcrop & 2 & Sample informatic \\
\hline Outcrop & 2 & Sample informatic \\
\hline Outcrop & 2 & Sample informatic \\
\hline Outcrop & 2 & Sample informatic \\
\hline
\end{tabular}




\begin{tabular}{|c|c|c|c|}
\hline Planavsky et al., 2011, Nature & Outcrop & 2 & Sample informatic \\
\hline Planavsky et al., 2011, Nature & Outcrop & 2 & Sample informatic \\
\hline Gilleaudeau and Kah, 2013, Chemical Geology & Core & 2 & \\
\hline Gilleaudeau and Kah, 2013, Chemical Geology & Core & 2 & \\
\hline Gilleaudeau and Kah, 2013, Chemical Geology & Core & 2 & \\
\hline Gilleaudeau and Kah, 2013, Chemical Geology & Core & 2 & \\
\hline Gilleaudeau and Kah, 2013, Chemical Geology & Core & 2 & \\
\hline Gilleaudeau and Kah, 2013, Chemical Geology & Core & 2 & \\
\hline Gilleaudeau and Kah, 2013, Chemical Geology & Core & 2 & \\
\hline Gilleaudeau and Kah, 2013, Chemical Geology & Core & 2 & \\
\hline Gilleaudeau and Kah, 2013, Chemical Geology & Core & 2 & \\
\hline Gilleaudeau and Kah, 2013, Chemical Geology & Core & 2 & \\
\hline Gilleaudeau and Kah, 2013, Chemical Geology & Core & 2 & \\
\hline Gilleaudeau and Kah, 2013, Chemical Geology & Core & 2 & \\
\hline Gilleaudeau and Kah, 2013, Chemical Geology & Core & 2 & \\
\hline Gilleaudeau and Kah, 2013, Chemical Geology & Core & 2 & \\
\hline Gilleaudeau and Kah, 2013, Chemical Geology & Core & 2 & \\
\hline Gilleaudeau and Kah, 2013, Chemical Geology & Core & 2 & \\
\hline Gilleaudeau and Kah, 2013, Chemical Geology & Core & 2 & \\
\hline Gilleaudeau and Kah, 2013, Chemical Geology & Core & 2 & \\
\hline Gilleaudeau and Kah, 2013, Chemical Geology & Core & 2 & \\
\hline Gilleaudeau and Kah, 2013, Chemical Geology & Core & 2 & \\
\hline Gilleaudeau and Kah, 2013, Chemical Geology & Core & 2 & \\
\hline Gilleaudeau and Kah, 2013, Chemical Geology & Core & 2 & \\
\hline Gilleaudeau and Kah, 2013, Chemical Geology & Core & 2 & \\
\hline Gilleaudeau and Kah, 2013, Chemical Geology & Core & 2 & \\
\hline Gilleaudeau and Kah, 2013, Chemical Geology & Core & 2 & \\
\hline Gilleaudeau and Kah, 2013, Chemical Geology & Core & 2 & \\
\hline Gilleaudeau and Kah, 2013, Chemical Geology & Core & 2 & \\
\hline Gilleaudeau and Kah, 2013, Chemical Geology & Core & 2 & \\
\hline Gilleaudeau and Kah, 2013, Chemical Geology & Core & 2 & \\
\hline Gilleaudeau and Kah, 2013, Chemical Geology & Core & 2 & \\
\hline Gilleaudeau and Kah, 2013, Chemical Geology & Core & 2 & \\
\hline Gilleaudeau and Kah, 2013, Chemical Geology & Core & 2 & \\
\hline Gilleaudeau and Kah, 2013, Chemical Geology & Core & 2 & \\
\hline Gilleaudeau and Kah, 2013, Chemical Geology & Core & 2 & \\
\hline Gilleaudeau and Kah, 2013, Chemical Geology & Core & 2 & \\
\hline Gilleaudeau and Kah, 2013, Chemical Geology & Core & 2 & \\
\hline Gilleaudeau and Kah, 2013, Chemical Geology & Core & 2 & \\
\hline Gilleaudeau and Kah, 2013, Chemical Geology & Core & 2 & \\
\hline Gilleaudeau and Kah, 2013, Chemical Geology & Core & 2 & \\
\hline
\end{tabular}




\begin{tabular}{|c|c|}
\hline Gilleaudeau and Kah, 2013, Chemical Geology & Core \\
\hline Gilleaudeau and Kah, 2013, Chemical Geology & Core \\
\hline Gilleaudeau and Kah, 2013, Chemical Geology & Core \\
\hline Gilleaudeau and Kah, 2013, Chemical Geology & Core \\
\hline Gilleaudeau and Kah, 2013, Chemical Geology & Core \\
\hline Gilleaudeau and Kah, 2013, Chemical Geology & Core \\
\hline Gilleaudeau and Kah, 2013, Chemical Geology & Core \\
\hline Gilleaudeau and Kah, 2013, Chemical Geology & Core \\
\hline Gilleaudeau and Kah, 2013, Chemical Geology & Core \\
\hline Gilleaudeau and Kah, 2013, Chemical Geology & Core \\
\hline Gilleaudeau and Kah, 2013, Chemical Geology & Core \\
\hline Gilleaudeau and Kah, 2013, Chemical Geology & Core \\
\hline Gilleaudeau and Kah, 2013, Chemical Geology & Core \\
\hline Gilleaudeau and Kah, 2013, Chemical Geology & Core \\
\hline Gilleaudeau and Kah, 2013, Chemical Geology & Core \\
\hline Gilleaudeau and Kah, 2013, Chemical Geology & Core \\
\hline Gilleaudeau and Kah, 2013, Chemical Geology & Core \\
\hline Gilleaudeau and Kah, 2013, Chemical Geology & Core \\
\hline Gilleaudeau and Kah, 2013, Chemical Geology & Core \\
\hline Gilleaudeau and Kah, 2013, Chemical Geology & Core \\
\hline Gilleaudeau and Kah, 2013, Chemical Geology & Core \\
\hline Gilleaudeau and Kah, 2013, Chemical Geology & Core \\
\hline Gilleaudeau and Kah, 2013, Chemical Geology & Core \\
\hline Gilleaudeau and Kah, 2013, Chemical Geology & Core \\
\hline Gilleaudeau and Kah, 2013, Chemical Geology & Core \\
\hline Gilleaudeau and Kah, 2013, Chemical Geology & Core \\
\hline Gilleaudeau and Kah, 2013, Chemical Geology & Core \\
\hline Gilleaudeau and Kah, 2013, Chemical Geology & Core \\
\hline Gilleaudeau and Kah, 2013, Chemical Geology & Core \\
\hline Gilleaudeau and Kah, 2013, Chemical Geology & Core \\
\hline Gilleaudeau and Kah, 2013, Chemical Geology & Core \\
\hline Gilleaudeau and Kah, 2013, Chemical Geology & Core \\
\hline Gilleaudeau and Kah, 2013, Chemical Geology & Core \\
\hline Gilleaudeau and Kah, 2013, Chemical Geology & Core \\
\hline Gilleaudeau and Kah, 2013, Chemical Geology & Core \\
\hline Gilleaudeau and Kah, 2013, Chemical Geology & Core \\
\hline Gilleaudeau and Kah, 2013, Chemical Geology & Core \\
\hline Gilleaudeau and Kah, 2013, Chemical Geology & Core \\
\hline Gilleaudeau and Kah, 2013, Chemical Geology & Core \\
\hline Gilleaudeau and Kah, 2013, Chemical Geology & Core \\
\hline Gilleaudeau and Kah, 2013, Chemical Geology & Core \\
\hline
\end{tabular}


Gilleaudeau and Kah, 2013, Chemical Geology Gilleaudeau and Kah, 2013, Chemical Geology Gilleaudeau and Kah, 2013, Chemical Geology Gilleaudeau and Kah, 2013, Chemical Geology Gilleaudeau and Kah, 2013, Chemical Geology Gilleaudeau and Kah, 2013, Chemical Geology Gilleaudeau and Kah, 2013, Chemical Geology Gilleaudeau and Kah, 2013, Chemical Geology Gilleaudeau and Kah, 2013, Chemical Geology Gilleaudeau and Kah, 2013, Chemical Geology Gilleaudeau and Kah, 2013, Chemical Geology Gilleaudeau and Kah, 2013, Chemical Geology Gilleaudeau and Kah, 2013, Chemical Geology Gilleaudeau and Kah, 2013, Chemical Geology Gilleaudeau and Kah, 2013, Chemical Geology Gilleaudeau and Kah, 2013, Chemical Geology Gilleaudeau and Kah, 2013, Chemical Geology Gilleaudeau and Kah, 2013, Chemical Geology Gilleaudeau and Kah, 2013, Chemical Geology Gilleaudeau and Kah, 2013, Chemical Geology Gilleaudeau and Kah, 2013, Chemical Geology Gilleaudeau and Kah, 2013, Chemical Geology Gilleaudeau and Kah, 2013, Chemical Geology Gilleaudeau and Kah, 2013, Chemical Geology Gilleaudeau and Kah, 2013, Chemical Geology Gilleaudeau and Kah, 2013, Chemical Geology Gilleaudeau and Kah, 2013, Chemical Geology Gilleaudeau and Kah, 2013, Chemical Geology Gilleaudeau and Kah, 2013, Chemical Geology Gilleaudeau and Kah, 2013, Chemical Geology Gilleaudeau and Kah, 2013, Chemical Geology Gilleaudeau and Kah, 2013, Chemical Geology Gilleaudeau and Kah, 2013, Chemical Geology Gilleaudeau and Kah, 2013, Chemical Geology Gilleaudeau and Kah, 2013, Chemical Geology Gilleaudeau and Kah, 2013, Chemical Geology Gilleaudeau and Kah, 2013, Chemical Geology Gilleaudeau and Kah, 2013, Chemical Geology Gilleaudeau and Kah, 2013, Chemical Geology Gilleaudeau and Kah, 2013, Chemical Geology Gilleaudeau and Kah, 2013, Chemical Geology
Core 2

Core 2

Core 2

Core 2

Core 2

Core 2

Core 2

Core 2

Core 2

Core 2

Core 2

Core 2

Core 2

Core 2

Core 2

Core 1

Core 1

Core 1

Core 1

Core 1

Core 1

Core 1

Core 1

Core 1

Core 1

Core 1

Core 1

Core 1

Core 1

Core 1

Core 1

Core 1

Core 1

Core 1

Core 1

Core 1

Core 1

Core 1

Core 1

Core 1

Core 1 
Gilleaudeau and Kah, 2013, Chemical Geology

Core $\quad 1$

Gilleaudeau and Kah, 2013, Chemical Geology Core 1

Gilleaudeau and Kah, 2013, Chemical Geology Core 1

Gilleaudeau and Kah, 2013, Chemical Geology Core 1

Gilleaudeau and Kah, 2013, Chemical Geology Core 1

Gilleaudeau and Kah, 2013, Chemical Geology Core 1

Canfield et al., 2013 PNAS Both 3

Canfield et al., 2013 PNAS Both 3

Canfield et al., 2013 PNAS Both 3

Canfield et al., 2013 PNAS Both 3

Canfield et al., 2013 PNAS Both 3

Canfield et al., 2013 PNAS Both 3

Canfield et al., 2013 PNAS Both 3

Canfield et al., 2013 PNAS Both 3

Canfield et al., 2013 PNAS Both 3

Canfield et al., 2013 PNAS Both 3

Canfield et al., 2013 PNAS Both 3

Canfield et al., 2013 PNAS Both 3

Canfield et al., 2013 PNAS Both 3

Canfield et al., 2013 PNAS Both 3

Canfield et al., 2013 PNAS Both 3

Canfield et al., 2013 PNAS Both 3

Canfield et al., 2013 PNAS Both 3

Canfield et al., 2013 PNAS Both 3

Canfield et al., 2013 PNAS Both 3

Canfield et al., 2013 PNAS Both 3

Canfield et al., 2013 PNAS Both 3

Canfield et al., 2013 PNAS Both 3

Canfield et al., 2013 PNAS Both 3

Canfield et al., 2013 PNAS Both 3

Canfield et al., 2013 PNAS Both 3

Canfield et al., 2013 PNAS Both 3

Canfield et al., 2013 PNAS Both 3

Canfield et al., 2013 PNAS Both 3

Canfield et al., 2013 PNAS Both 3

Canfield et al., 2013 PNAS Both 3

Canfield et al., 2013 PNAS Both 3

Canfield et al., 2013 PNAS Both 3

Canfield et al., 2013 PNAS Both 3

Canfield et al., 2013 PNAS Both 3

Canfield et al., 2013 PNAS Both 3 
Canfield et al., 2013 PNAS

Both 3

Canfield et al., 2013 PNAS

Both 3

Canfield et al., 2013 PNAS

Both 3

Canfield et al., 2013 PNAS

Both 3

Canfield et al., 2013 PNAS

Both 3

Canfield et al., 2013 PNAS

Both 3

Canfield et al., 2013 PNAS

Both 3

Canfield et al., 2013 PNAS

Both 3

Canfield et al., 2013 PNAS

Both 3

Canfield et al., 2013 PNAS

Both 3

Canfield et al., 2013 PNAS

Both 3

Canfield et al., 2013 PNAS

Both 3

Canfield et al., 2013 PNAS

Both 3

Canfield et al., 2013 PNAS

Both 3

Canfield et al., 2013 PNAS

Both 3

Canfield et al., 2013 PNAS

Both 3

Canfield et al., 2013 PNAS

Both 3

Canfield et al., 2013 PNAS

Both 3

Canfield et al., 2013 PNAS

Both 3

Canfield et al., 2013 PNAS

Both 3

Canfield et al., 2013 PNAS

Both 3

Canfield et al., 2013 PNAS

Both 3

Canfield et al., 2013 PNAS

Both 3

Canfield et al., 2013 PNAS

Both 3

Canfield et al., 2013 PNAS

Both 3

Canfield et al., 2013 PNAS

Both 3

Canfield et al., 2013 PNAS

Both 3

Canfield et al., 2013 PNAS

Both 3

Canfield et al., 2013 PNAS

Both 3

Canfield et al., 2013 PNAS

Both 3

Canfield et al., 2013 PNAS

Both 3

Canfield et al., 2013 PNAS

Canfield et al., 2013 PNAS

Both 3

Both 3

Canfield et al., 2013 PNAS

Both 3

Canfield et al., 2013 PNAS

Both 3

Canfield et al., 2013 PNAS

Canfield et al., 2013 PNAS

Both 3

Both 3

Canfield et al., 2013 PNAS

Canfield et al., 2013 PNAS

Both 3

Both 3

Canfield et al., 2013 PNAS

Both 3

Canfield et al., 2013 PNAS

Both 
Canfield et al., 2013 PNAS

Both 3

Canfield et al., 2013 PNAS

Both 3

Canfield et al., 2013 PNAS

Both 3

Canfield et al., 2013 PNAS

Both 3

Canfield et al., 2013 PNAS

Both 3

Canfield et al., 2013 PNAS

Both 3

Canfield et al., 2013 PNAS

Both 3

Canfield et al., 2013 PNAS

Both 3

Canfield et al., 2013 PNAS

Both 3

Canfield et al., 2013 PNAS

Both 3

Canfield et al., 2013 PNAS

Both 3

Canfield et al., 2013 PNAS

Both 3

Canfield et al., 2013 PNAS

Both 3

Canfield et al., 2013 PNAS

Both 3

Canfield et al., 2013 PNAS

Both 3

Canfield et al., 2013 PNAS

Both 3

Canfield et al., 2013 PNAS

Canfield et al., 2013 PNAS

Both 3

Both 3

Both 3

Canfield et al., 2013 PNAS

Both 3

Both 3

Canfield et al., 2013 PNAS

Both 3

Canfield et al., 2013 PNAS

Both 3

Canfield et al., 2013 PNAS

Both 3

Canfield et al., 2013 PNAS

Both 3

Canfield et al., 2013 PNAS

Both 3

El Albani et al., 2010, Nature

Core 2

El Albani et al., 2010, Nature

Core 2

El Albani et al., 2010, Nature

Core 2

El Albani et al., 2010, Nature

Core 2

El Albani et al., 2010, Nature

Core 2

El Albani et al., 2010, Nature

Core 2

El Albani et al., 2010, Nature

Core 2

El Albani et al., 2010, Nature

Core 2

El Albani et al., 2010, Nature

Outcrop 2

El Albani et al., 2010, Nature

Outcrop 2

El Albani et al., 2010, Nature

Outcrop 2

El Albani et al., 2010, Nature

Outcrop 2

El Albani et al., 2010, Nature

Outcrop 2

El Albani et al., 2010, Nature

Outcrop 2

El Albani et al., 2010, Nature

Outcrop 2


El Albani et al., 2010, Nature

El Albani et al., 2010, Nature

El Albani et al., 2010, Nature

El Albani et al., 2010, Nature

El Albani et al., 2010, Nature

El Albani et al., 2010, Nature

El Albani et al., 2010, Nature

El Albani et al., 2010, Nature

El Albani et al., 2010, Nature

Sperling et al., 2014, Geobiology

Sperling et al., 2014, Geobiology

Sperling et al., 2014, Geobiology

Sperling et al., 2014, Geobiology

Sperling et al., 2014, Geobiology

Sperling et al., 2014, Geobiology

Sperling et al., 2014, Geobiology

Sperling et al., 2014, Geobiology

Sperling et al., 2014, Geobiology

Sperling et al., 2014, Geobiology

Sperling et al., 2014, Geobiology

Sperling et al., 2014, Geobiology

Sperling et al., 2014, Geobiology

Sperling et al., 2014, Geobiology

Sperling et al., 2014, Geobiology

Sperling et al., 2014, Geobiology

Sperling et al., 2014, Geobiology

Sperling et al., 2014, Geobiology

Sperling et al., 2014, Geobiology

Sperling et al., 2014, Geobiology

Sperling et al., 2014, Geobiology

Sperling et al., 2014, Geobiology

Sperling et al., 2014, Geobiology

Sperling et al., 2014, Geobiology

Sperling et al., 2014, Geobiology

Sperling et al., 2014, Geobiology

Sperling et al., 2014, Geobiology

Sperling et al., 2014, Geobiology

Sperling et al., 2014, Geobiology

Sperling et al., 2014, Geobiology

Sperling et al., 2014, Geobiology

Sperling et al., 2014, Geobiology

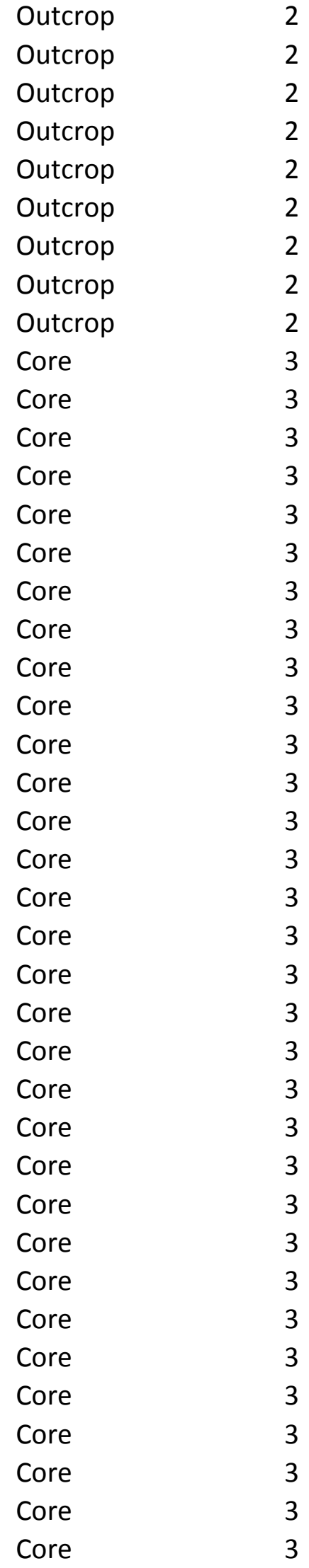


Sperling et al., 2014, Geobiology

Core 3

Sperling et al., 2014, Geobiology

Core 3

Sperling et al., 2014, Geobiology

Core 3

Sperling et al., 2014, Geobiology

Core 3

Sperling et al., 2014, Geobiology

Core 3

Sperling et al., 2014, Geobiology

Core 3

Sperling et al., 2014, Geobiology

Core 3

Sperling et al., 2014, Geobiology

Core 3

Sperling et al., 2014, Geobiology

Core 3

This Study

Core 1

This Study

Core 1

This Study

Core 1

This Study

Core 1

This Study

Core 1

This Study

Core 1

This Study

Shen et al., 2003, Nature

Core 1

Shen et al., 2003, Nature

Core 1

Shen et al., 2003, Nature

Core 1

Uses old extractio

Core 1

Uses old extractio

Shen et al., 2003, Nature

Core 1

Uses old extractio

Shen et al., 2003, Nature

Core 1

Shen et al., 2003, Nature

Core 1

Uses old extractio

Shen et al., 2003, Nature

Core 1

Shen et al., 2003, Nature

Core 1

Core 1

Shen et al., 2003, Nature

Core 1

Shen et al., 2003, Nature

Core 1

Shen et al., 2003, Nature

Core 1

Core 1

Core 1

Core 1

Core 1

Core 1

Core 1

Core 1

Core 3

Core 3

Core 3

Core 3

Core 2

Core 2

Uses old extractio

Uses old extractio

Uses old extractio

Uses old extractio

Uses old extractio

Uses old extractio

Uses old extractio

Uses old extractio

Uses old extractio

Uses old extractio

Uses old extractio

Uses old extractio

Uses old extractio

Uses old extractio

Uses old extractio

Uses old extractio

Uses old extractio

Uses old extractio Uses old extractio Uses old extractio Uses old extractio 
Shen et al., 2003, Nature

Shen et al., 2003, Nature

Shen et al., 2003, Nature

Shen et al., 2003, Nature

Shen et al., 2003, Nature

Shen et al., 2003, Nature

Shen et al., 2003, Nature

Shen et al., 2003, Nature

Shen et al., 2003, Nature

Shen et al., 2003, Nature

Shen et al., 2003, Nature

Shen et al., 2003, Nature

Shen et al., 2003, Nature

Shen et al., 2003, Nature

Shen et al., 2003, Nature

Shen et al., 2003, Nature

Shen et al., 2003, Nature

Shen et al., 2003, Nature

Shen et al., 2003, Nature

Shen et al., 2003, Nature

Shen et al., 2003, Nature

Shen et al., 2003, Nature

Shen et al., 2003, Nature

Shen et al., 2003, Nature

Shen et al., 2003, Nature

Shen et al., 2003, Nature

Shen et al., 2003, Nature

Shen et al., 2003, Nature

Shen et al., 2003, Nature

Shen et al., 2003, Nature

Shen et al., 2003, Nature

Shen et al., 2003, Nature

Shen et al., 2003, Nature

Shen et al., 2003, Nature

Shen et al., 2003, Nature

Shen et al., 2003, Nature

Shen et al., 2003, Nature

Shen et al., 2003, Nature

Shen et al., 2003, Nature

Shen et al., 2003, Nature

Shen et al., 2003, Nature

$\begin{array}{lll}\text { Core } & 2 & \text { Uses old extractio } \\ \text { Core } & 2 & \text { Uses old extractio } \\ \text { Core } & 2 & \text { Uses old extractio } \\ \text { Core } & 2 & \text { Uses old extractio } \\ \text { Core } & 2 & \text { Uses old extractio } \\ \text { Core } & 2 & \text { Uses old extractio } \\ \text { Core } & 3 & \text { Uses old extractio } \\ \text { Core } & 2 & \text { Uses old extractio } \\ \text { Core } & 2 & \text { Uses old extractio } \\ \text { Core } & 2 & \text { Uses old extractio } \\ \text { Core } & 3 & \text { Uses old extractio } \\ \text { Core } & 2 & \text { Uses old extractio } \\ \text { Core } & 3 & \text { Uses old extractio } \\ \text { Core } & 3 & \text { Uses old extractio } \\ \text { Core } & 3 & \text { Uses old extractio } \\ \text { Core } & 3 & \text { Uses old extractio } \\ \text { Core } & 3 & \text { Uses old extractio } \\ \text { Core } & 2 & \text { Uses old extractio } \\ \text { Core } & 3 & \text { Uses old extractio } \\ \text { Core } & 3 & \text { Uses old extractio } \\ \text { Core } & 3 & \text { Uses old extractio } \\ \text { Core } & 3 & \text { Uses old extractio } \\ \text { Core } & 3 & \text { Uses old extractio } \\ \text { Core } & 3 & \text { Uses old extractio } \\ \text { Core } & 3 & \text { Uses old extractio } \\ \text { Core } & 3 & \text { Uses old extractio } \\ \text { Core } & 3 & \text { Uses old extractio } \\ \text { Core } & 3 & \text { Uses old extractio } \\ \text { Core } & 3 & \text { Uses old extractio } \\ \text { Core } & 3 & \text { Uses old extractio } \\ \text { Core } & 3 & \text { Uses old extractio } \\ \text { Core } & 3 \text { Uses old extractio } \\ \text { Core } & 3 & \text { Uses old extractio } \\ \text { Core } & 3 & \text { Uses old extractio } \\ \text { Core } & 3 & \text { Uses old extractio } \\ \text { Core old extractio } \\ \text { Core }\end{array}$


Shen et al., 2003, Nature

Shen et al., 2003, Nature

Shen et al., 2003, Nature

Shen et al., 2003, Nature

Shen et al., 2003, Nature

Shen et al., 2003, Nature

Shen et al., 2003, Nature

Shen et al., 2003, Nature

Shen et al., 2003, Nature

Shen et al., 2003, Nature

Shen et al., 2003, Nature

Shen et al., 2003, Nature

Shen et al., 2003, Nature

Shen et al., 2003, Nature

Shen et al., 2003, Nature

Shen et al., 2003, Nature

Shen et al., 2003, Nature

Shen et al., 2003, Nature

Shen et al., 2003, Nature

Shen et al., 2003, Nature

Shen et al., 2003, Nature

Shen et al., 2003, Nature

Shen et al., 2003, Nature

Shen et al., 2003, Nature

Shen et al., 2003, Nature

Shen et al., 2003, Nature

Shen et al., 2003, Nature

Shen et al., 2003, Nature

Shen et al., 2003, Nature

Shen et al., 2003, Nature

Shen et al., 2003, Nature

Shen et al., 2003, Nature

Shen et al., 2003, Nature

Shen et al., 2003, Nature

Shen et al., 2003, Nature

Shen et al., 2003, Nature

Shen et al., 2003, Nature

Shen et al., 2003, Nature

Shen et al., 2003, Nature

Shen et al., 2003, Nature

Shen et al., 2003, Nature

\begin{tabular}{|c|c|c|}
\hline Core & 2 & Uses old extractio \\
\hline Core & 2 & Uses old extractio \\
\hline Core & 2 & Uses old extractio \\
\hline Core & 2 & Uses old extractio \\
\hline Core & 2 & Uses old extractio \\
\hline Core & 2 & Uses old extractio \\
\hline Core & 2 & Uses old extractio \\
\hline Core & 2 & Uses old extractio \\
\hline Core & 3 & Uses old extractio \\
\hline Core & 3 & Uses old extractio \\
\hline Core & 1 & Uses old extractio \\
\hline Core & 1 & Uses old extractio \\
\hline Core & 1 & Uses old extractio \\
\hline Core & 1 & Uses old extractio \\
\hline Core & 1 & Uses old extractio \\
\hline Core & 1 & Uses old extractio \\
\hline Core & 1 & Uses old extractio \\
\hline Core & 1 & Uses old extractio \\
\hline Core & 1 & Uses old extractio \\
\hline Core & 1 & Uses old extractio \\
\hline Core & 1 & Uses old extractio \\
\hline Core & 1 & Uses old extractio \\
\hline Core & 1 & Uses old extractio \\
\hline Core & 1 & Uses old extractio \\
\hline Core & 2 & Uses old extractio \\
\hline Core & 2 & Uses old extractio \\
\hline Core & 2 & Uses old extractio \\
\hline Core & 2 & Uses old extractio \\
\hline Core & 2 & Uses old extractio \\
\hline Core & 2 & Uses old extractio \\
\hline Core & 2 & Uses old extractio \\
\hline Core & 2 & Uses old extractio \\
\hline Core & 2 & Uses old extractio \\
\hline Core & 2 & Uses old extractio \\
\hline Core & 2 & Uses old extractio \\
\hline Core & 2 & Uses old extractio \\
\hline Core & 3 & Uses old extractio \\
\hline Core & 3 & Uses old extractio \\
\hline Core & 3 & Uses old extractio \\
\hline Core & 2 & Uses old extractio \\
\hline Core & 2 & Uses old extractio \\
\hline
\end{tabular}


Shen et al., 2003, Nature

Shen et al., 2003, Nature

Core

Core

Shen et al., 2003, Nature

Shen et al., 2003, Nature

Shen et al., 2003, Nature

Shen et al., 2003, Nature

Shen et al., 2003, Nature

Shen et al., 2003, Nature

Shen et al., 2003, Nature

Shen et al., 2003, Nature

Planavsky et al., 2011, Nature

Planavsky et al., 2011, Nature

Planavsky et al., 2011, Nature

Planavsky et al., 2011, Nature

Planavsky et al., 2011, Nature

Planavsky et al., 2011, Nature

Planavsky et al., 2011, Nature

Planavsky et al., 2011, Nature

Planavsky et al., 2011, Nature

Planavsky et al., 2011, Nature

Planavsky et al., 2011, Nature

Planavsky et al., 2011, Nature

Planavsky et al., 2011, Nature

Planavsky et al., 2011, Nature

Planavsky et al., 2011, Nature

Planavsky et al., 2011, Nature

Planavsky et al., 2011, Nature

Planavsky et al., 2011, Nature

Planavsky et al., 2011, Nature

Planavsky et al., 2011, Nature

Planavsky et al., 2011, Nature

Planavsky et al., 2011, Nature

Planavsky et al., 2011, Nature

Planavsky et al., 2011, Nature

Planavsky et al., 2011, Nature

Planavsky et al., 2011, Nature

Planavsky et al., 2011, Nature

Planavsky et al., 2011, Nature

Planavsky et al., 2011, Nature

Planavsky et al., 2011, Nature

Planavsky et al., 2011, Nature
Core

Core

Core

Core

Core

Core

Core

Core

Core

Core

Core

Core

Core

Core

Core

Core

Core

Core

Core

Core

Core

Core

Core

Core

Core

Core

Core

Core

Core

Core

Core

Core

Core

Core

Core

Core

Core

Core

Core
Uses old extractio Uses old extractio Uses old extractio Uses old extractio Uses old extractio Uses old extractio Uses old extractio Uses old extractio Uses old extractio Uses old extractio

\section{3}

3 
Planavsky et al., 2011, Nature

Planavsky et al., 2011, Nature

Planavsky et al., 2011, Nature

Planavsky et al., 2011, Nature

Planavsky et al., 2011, Nature

Planavsky et al., 2011, Nature

Planavsky et al., 2011, Nature

Planavsky et al., 2011, Nature

Planavsky et al., 2011, Nature

Planavsky et al., 2011, Nature

Planavsky et al., 2011, Nature

Planavsky et al., 2011, Nature

Planavsky et al., 2011, Nature

Planavsky et al., 2011, Nature

Planavsky et al., 2011, Nature

Planavsky et al., 2011, Nature

Planavsky et al., 2011, Nature

Planavsky et al., 2011, Nature

Planavsky et al., 2011, Nature

Shen et al., 2002, AJS

Shen et al., 2002, AJS

Shen et al., 2002, AJS

Shen et al., 2002, AJS

Shen et al., 2002, AJS

Shen et al., 2002, AJS

Shen et al., 2002, AJS

Shen et al., 2002, AJS

Shen et al., 2002, AJS

Shen et al., 2002, AJS

Shen et al., 2002, AJS

Shen et al., 2002, AJS

Shen et al., 2002, AJS

Shen et al., 2002, AJS

Shen et al., 2002, AJS

Shen et al., 2002, AJS

Shen et al., 2002, AJS

Shen et al., 2002, AJS

Shen et al., 2002, AJS

Shen et al., 2002, AJS

Shen et al., 2002, AJS

Shen et al., 2002, AJS
Core 3

Core 3

Core 3

Core 3

Core 3

Core 3

Core 3

Core 3

Core 3

Core 3

Core 3

Core 3

Core 3

Core 3

Core 3

Core 3

Core 3

Core 3

Core 3

Core 3

Core 3

Core 3

Core 3

Core 3

Core 3

Core 3

Core 3

Core 3

Core 3

Core 3

Core 3

Core 3

Core 3

Core 3

Core 3

Core 3

Core 3

Core 3

Core 3

Core 3

Core 3
Uses old extractic Uses old extractic Uses old extractic Uses old extractic Uses old extractic Uses old extractic Uses old extractic Uses old extractic Uses old extractic Uses old extractic Uses old extractic Uses old extractic Uses old extractic Uses old extractic Uses old extractic Uses old extractic Uses old extractic Uses old extractic Uses old extractic Uses old extractic Uses old extractic Uses old extractic 
Shen et al., 2002, AJS

Shen et al., 2002, AJS

Core

Core

Core

Core

Poulton et al., 2010, Nature Geoscience

Poulton et al., 2010, Nature Geoscience

Poulton et al., 2010, Nature Geoscience

Poulton et al., 2010, Nature Geoscience

Poulton et al., 2010, Nature Geoscience

Poulton et al., 2010, Nature Geoscience

Poulton et al., 2010, Nature Geoscience

Poulton et al., 2010, Nature Geoscience

Poulton et al., 2010, Nature Geoscience

Poulton et al., 2010, Nature Geoscience

Poulton et al., 2010, Nature Geoscience

Poulton et al., 2010, Nature Geoscience

Poulton et al., 2010, Nature Geoscience

Poulton et al., 2010, Nature Geoscience

Poulton et al., 2010, Nature Geoscience

Poulton et al., 2010, Nature Geoscience

Poulton et al., 2010, Nature Geoscience

Poulton et al., 2010, Nature Geoscience

Poulton et al., 2010, Nature Geoscience

Poulton et al., 2010, Nature Geoscience

Poulton et al., 2010, Nature Geoscience

Poulton et al., 2010, Nature Geoscience

Poulton et al., 2010, Nature Geoscience

Poulton et al., 2010, Nature Geoscience

Poulton et al., 2010, Nature Geoscience

Poulton et al., 2010, Nature Geoscience

Poulton et al., 2010, Nature Geoscience

Poulton et al., 2010, Nature Geoscience

Poulton et al., 2010, Nature Geoscience

Poulton et al., 2010, Nature Geoscience

Poulton et al., 2010, Nature Geoscience

Poulton et al., 2010, Nature Geoscience

Poulton et al., 2010, Nature Geoscience

Poulton et al., 2010, Nature Geoscience

Poulton et al., 2010, Nature Geoscience

Poulton et al., 2010, Nature Geoscience

Poulton et al., 2010, Nature Geoscience

Poulton et al., 2010, Nature Geoscience
Core 2

Core 2

Core 2

Core 2

Core 2

Core 2

Core 2

Core 2

Core 2

Core 2

Core 2

Core 2

Core 2

Core 2

Core 2

Core 2

Core 2

Core 2

Core 2

Core 2

Core 2

Core 2

Core 2

Core 2

Core 2

Core 2

Core 2

Core 2

Core 2

Core 2

Core 2

Core 2

Core 2

Core 2

Core 2

Core

Core
Uses old extractic

Uses old extractic

For these rocks, $\mathrm{F}$

For these rocks, $\mathrm{F}$

For these rocks, $\mathrm{F}$

For these rocks, $\mathrm{F}$

For these rocks, $\mathrm{F}$

For these rocks, $\mathrm{F}$

For these rocks, $\mathrm{F}$

For these rocks, $\mathrm{F}$

For these rocks, $\mathrm{F}$

For these rocks, $\mathrm{F}$

For these rocks, $\mathrm{F}$

For these rocks, $\mathrm{F}$

For these rocks, $\mathrm{F}$

For these rocks, $F$

For these rocks, $\mathrm{F}$

For these rocks, $\mathrm{F}$

For these rocks, $\mathrm{F}$

For these rocks, $\mathrm{F}$

For these rocks, $\mathrm{F}$

For these rocks, $F$

For these rocks, $\mathrm{F}$

For these rocks, $\mathrm{F}$

For these rocks, $\mathrm{F}$

For these rocks, $F$

For these rocks, $\mathrm{F}$

For these rocks, $F$

For these rocks, $\mathrm{F}$

For these rocks, $\mathrm{F}$

For these rocks, $\mathrm{F}$

For these rocks, $F$

For these rocks, $\mathrm{F}$

For these rocks, $\mathrm{F}$

For these rocks, $\mathrm{F}$

For these rocks, $\mathrm{F}$

For these rocks, $\mathrm{F}$

For these rocks, $\mathrm{F}$

For these rocks, $\mathrm{F}$

For these rocks, $\mathrm{F}$

For these rocks, $\mathrm{F}$ 
Poulton et al., 2010, Nature Geoscience

Poulton et al., 2010, Nature Geoscience

Poulton et al., 2010, Nature Geoscience

Poulton et al., 2010, Nature Geoscience

Poulton et al., 2010, Nature Geoscience

Poulton et al., 2010, Nature Geoscience

Poulton et al., 2010, Nature Geoscience

Poulton et al., 2010, Nature Geoscience

Poulton et al., 2010, Nature Geoscience

Poulton et al., 2010, Nature Geoscience

Poulton et al., 2010, Nature Geoscience

Poulton et al., 2010, Nature Geoscience

Poulton et al., 2010, Nature Geoscience

Poulton et al., 2010, Nature Geoscience

Poulton et al., 2010, Nature Geoscience

Poulton et al., 2010, Nature Geoscience

Poulton et al., 2010, Nature Geoscience

Poulton et al., 2010, Nature Geoscience

Poulton et al., 2010, Nature Geoscience

Poulton et al., 2010, Nature Geoscience

Poulton et al., 2010, Nature Geoscience

Poulton et al., 2010, Nature Geoscience

Poulton et al., 2010, Nature Geoscience

Poulton et al., 2010, Nature Geoscience

Poulton et al., 2010, Nature Geoscience

Poulton et al., 2010, Nature Geoscience

Poulton et al., 2010, Nature Geoscience

Poulton et al., 2010, Nature Geoscience

Poulton et al., 2010, Nature Geoscience

Poulton et al., 2010, Nature Geoscience

Poulton et al., 2010, Nature Geoscience

Poulton et al., 2010, Nature Geoscience

Poulton et al., 2010, Nature Geoscience

Poulton et al., 2010, Nature Geoscience

Poulton et al., 2010, Nature Geoscience

Poulton et al., 2010, Nature Geoscience

Poulton et al., 2010, Nature Geoscience

Poulton et al., 2010, Nature Geoscience

Poulton et al., 2010, Nature Geoscience

Poulton et al., 2010, Nature Geoscience

Poulton et al., 2010, Nature Geoscience

\begin{tabular}{|c|c|c|}
\hline Core & 2 & For these rocks, $\mathrm{F}$ \\
\hline Core & 2 & For these rocks, $\mathrm{F}$ \\
\hline Core & 2 & For these rocks, $\mathrm{F}$ \\
\hline Core & 2 & For these rocks, $\mathrm{F}$ \\
\hline Core & 2 & For these rocks, $\mathrm{F}$ \\
\hline Core & 2 & For these rocks, $\mathrm{F}$ \\
\hline Core & 2 & For these rocks, $\mathrm{F}$ \\
\hline Core & 2 & For these rocks, $\mathrm{F}$ \\
\hline Core & 2 & For these rocks, $\mathrm{F}$ \\
\hline Core & 2 & For these rocks, $\mathrm{F}$ \\
\hline Core & 2 & For these rocks, $\mathrm{F}$ \\
\hline Core & 2 & For these rocks, $\mathrm{F}$ \\
\hline Core & 2 & For these rocks, $\mathrm{F}$ \\
\hline Core & 2 & For these rocks, $\mathrm{F}$ \\
\hline Core & 2 & For these rocks, $\mathrm{F}$ \\
\hline Core & 2 & For these rocks, $\mathrm{F}$ \\
\hline Core & 2 & For these rocks, $\mathrm{F}$ \\
\hline Core & 2 & For these rocks, $\mathrm{F}$ \\
\hline Core & 2 & For these rocks, $\mathrm{F}$ \\
\hline Core & 2 & For these rocks, $\mathrm{F}$ \\
\hline Core & 2 & For these rocks, $\mathrm{F}$ \\
\hline Core & 2 & For these rocks, $\mathrm{F}$ \\
\hline Core & 2 & For these rocks, $\mathrm{F}$ \\
\hline Core & 2 & For these rocks, $\mathrm{F}$ \\
\hline Core & 2 & For these rocks, $\mathrm{F}$ \\
\hline Core & 2 & For these rocks, $\mathrm{F}$ \\
\hline Core & 2 & For these rocks, $\mathrm{F}$ \\
\hline Core & 2 & For these rocks, $\mathrm{F}$ \\
\hline Core & 2 & For these rocks, $\mathrm{F}$ \\
\hline Core & 2 & For these rocks, $\mathrm{F}$ \\
\hline Core & 2 & For these rocks, $\mathrm{F}$ \\
\hline Core & 2 & For these rocks, $\mathrm{F}$ \\
\hline Core & 2 & For these rocks, $\mathrm{F}$ \\
\hline Core & 2 & For these rocks, $\mathrm{F}$ \\
\hline Core & 3 & For these rocks, $\mathrm{F}$ \\
\hline Core & 3 & For these rocks, $\mathrm{F}$ \\
\hline Core & 3 & For these rocks, $\mathrm{F}$ \\
\hline Core & 3 & For these rocks, $\mathrm{F}$ \\
\hline Core & 3 & For these rocks, $\mathrm{F}$ \\
\hline Core & 3 & For these rocks, $\mathrm{F}$ \\
\hline Core & 3 & For these rocks, $\mathrm{F}$ \\
\hline
\end{tabular}


Poulton et al., 2010, Nature Geoscience

Poulton et al., 2010, Nature Geoscience

Poulton et al., 2010, Nature Geoscience

Poulton et al., 2010, Nature Geoscience

Poulton et al., 2010, Nature Geoscience

Poulton et al., 2010, Nature Geoscience

Poulton et al., 2010, Nature Geoscience

Poulton et al., 2010, Nature Geoscience

Poulton et al., 2010, Nature Geoscience

Poulton et al., 2010, Nature Geoscience

Poulton et al., 2010, Nature Geoscience

Poulton et al., 2010, Nature Geoscience

Poulton et al., 2010, Nature Geoscience

Poulton et al., 2010, Nature Geoscience

Poulton et al., 2010, Nature Geoscience

Poulton et al., 2010, Nature Geoscience

Poulton et al., 2010, Nature Geoscience

Poulton et al., 2010, Nature Geoscience

Poulton et al., 2010, Nature Geoscience

Poulton et al., 2010, Nature Geoscience

Poulton et al., 2010, Nature Geoscience

Poulton et al., 2010, Nature Geoscience

Poulton et al., 2010, Nature Geoscience

Poulton et al., 2010, Nature Geoscience

Poulton et al., 2010, Nature Geoscience

Poulton et al., 2010, Nature Geoscience

Poulton et al., 2010, Nature Geoscience

Poulton et al., 2010, Nature Geoscience

Poulton et al., 2010, Nature Geoscience

Poulton et al., 2010, Nature Geoscience

Poulton et al., 2010, Nature Geoscience

Poulton et al., 2010, Nature Geoscience

Poulton et al., 2010, Nature Geoscience

Poulton et al., 2010, Nature Geoscience

Poulton et al., 2010, Nature Geoscience

Poulton et al., 2010, Nature Geoscience

Poulton et al., 2010, Nature Geoscience

Poulton et al., 2010, Nature Geoscience

Poulton et al., 2010, Nature Geoscience

Poulton et al., 2010, Nature Geoscience

Poulton et al., 2010, Nature Geoscience

\begin{tabular}{|c|c|c|}
\hline Core & 3 & For these rocks, $\mathrm{F}$ \\
\hline Core & 3 & For these rocks, $\mathrm{F}$ \\
\hline Core & 3 & For these rocks, $\mathrm{F}$ \\
\hline Core & 3 & For these rocks, $\mathrm{F}$ \\
\hline Core & 3 & For these rocks, $\mathrm{F}$ \\
\hline Core & 3 & For these rocks, $\mathrm{F}$ \\
\hline Core & 3 & For these rocks, $\mathrm{F}$ \\
\hline Core & 3 & For these rocks, $\mathrm{F}$ \\
\hline Core & 3 & For these rocks, $\mathrm{F}$ \\
\hline Core & 3 & For these rocks, $\mathrm{F}$ \\
\hline Core & 3 & For these rocks, $\mathrm{F}$ \\
\hline Core & 3 & For these rocks, $\mathrm{F}$ \\
\hline Core & 3 & For these rocks, $\mathrm{F}$ \\
\hline Core & 3 & For these rocks, $\mathrm{F}$ \\
\hline Core & 3 & For these rocks, $\mathrm{F}$ \\
\hline Core & 3 & For these rocks, $\mathrm{F}$ \\
\hline Core & 3 & For these rocks, $\mathrm{F}$ \\
\hline Core & 3 & For these rocks, $\mathrm{F}$ \\
\hline Core & 3 & For these rocks, $\mathrm{F}$ \\
\hline Core & 3 & For these rocks, $\mathrm{F}$ \\
\hline Core & 3 & For these rocks, $\mathrm{F}$ \\
\hline Core & 3 & For these rocks, $\mathrm{F}$ \\
\hline Core & 3 & For these rocks, $\mathrm{F}$ \\
\hline Core & 3 & For these rocks, $\mathrm{F}$ \\
\hline Core & 3 & For these rocks, $\mathrm{F}$ \\
\hline Core & 3 & For these rocks, $\mathrm{F}$ \\
\hline Core & 3 & For these rocks, $\mathrm{F}$ \\
\hline Core & 3 & For these rocks, $\mathrm{F}$ \\
\hline Core & 3 & For these rocks, $\mathrm{F}$ \\
\hline Core & 3 & For these rocks, $\mathrm{F}$ \\
\hline Core & 3 & For these rocks, $\mathrm{F}$ \\
\hline Core & 3 & For these rocks, $\mathrm{F}$ \\
\hline Core & 3 & For these rocks, $\mathrm{F}$ \\
\hline Core & 3 & For these rocks, $\mathrm{F}$ \\
\hline Core & 3 & For these rocks, $\mathrm{F}$ \\
\hline Core & 3 & For these rocks, $\mathrm{F}$ \\
\hline Core & 3 & For these rocks, $\mathrm{F}$ \\
\hline Core & 3 & For these rocks, $\mathrm{F}$ \\
\hline Core & 3 & For these rocks, $\mathrm{F}$ \\
\hline Core & 3 & For these rocks, $\mathrm{F}$ \\
\hline Core & 3 & For these rocks, $\mathrm{F}$ \\
\hline
\end{tabular}


Poulton et al., 2010, Nature Geoscience

Poulton et al., 2010, Nature Geoscience

Poulton et al., 2010, Nature Geoscience

Poulton et al., 2010, Nature Geoscience

Poulton et al., 2010, Nature Geoscience

Poulton et al., 2010, Nature Geoscience

Poulton et al., 2010, Nature Geoscience

Poulton et al., 2010, Nature Geoscience

Poulton et al., 2010, Nature Geoscience

Poulton et al., 2010, Nature Geoscience

Poulton et al., 2010, Nature Geoscience

Poulton et al., 2010, Nature Geoscience

Poulton et al., 2010, Nature Geoscience

Poulton et al., 2010, Nature Geoscience

Poulton et al., 2010, Nature Geoscience

Poulton et al., 2010, Nature Geoscience

Poulton et al., 2010, Nature Geoscience

Poulton et al., 2010, Nature Geoscience

Poulton et al., 2010, Nature Geoscience

Poulton et al., 2010, Nature Geoscience

Poulton et al., 2010, Nature Geoscience

Poulton et al., 2010, Nature Geoscience

Poulton et al., 2010, Nature Geoscience

Poulton et al., 2010, Nature Geoscience

Poulton et al., 2010, Nature Geoscience

Poulton et al., 2010, Nature Geoscience

Poulton et al., 2010, Nature Geoscience

Poulton et al., 2010, Nature Geoscience

Poulton et al., 2010, Nature Geoscience

Poulton et al., 2010, Nature Geoscience

Poulton et al., 2010, Nature Geoscience

Poulton et al., 2010, Nature Geoscience

Poulton et al., 2010, Nature Geoscience

Poulton et al., 2010, Nature Geoscience

Poulton et al., 2010, Nature Geoscience

Poulton et al., 2010, Nature Geoscience

Poulton et al., 2010, Nature Geoscience

Poulton et al., 2010, Nature Geoscience

Poulton et al., 2010, Nature Geoscience

Poulton et al., 2010, Nature Geoscience

Poulton et al., 2010, Nature Geoscience

\begin{tabular}{|c|c|c|}
\hline Core & 3 & For these rocks, $\mathrm{F}$ \\
\hline Core & 3 & For these rocks, $\mathrm{F}$ \\
\hline Core & 3 & For these rocks, $\mathrm{F}$ \\
\hline Core & 3 & For these rocks, $\mathrm{F}$ \\
\hline Core & 3 & For these rocks, $\mathrm{F}$ \\
\hline Core & 3 & For these rocks, $\mathrm{F}$ \\
\hline Core & 3 & For these rocks, $\mathrm{F}$ \\
\hline Core & 3 & For these rocks, $\mathrm{F}$ \\
\hline Core & 3 & For these rocks, $\mathrm{F}$ \\
\hline Core & 3 & For these rocks, $\mathrm{F}$ \\
\hline Core & 3 & For these rocks, $\mathrm{F}$ \\
\hline Core & 3 & For these rocks, $\mathrm{F}$ \\
\hline Core & 3 & For these rocks, $\mathrm{F}$ \\
\hline Core & 3 & For these rocks, $\mathrm{F}$ \\
\hline Core & 3 & For these rocks, $\mathrm{F}$ \\
\hline Core & 3 & For these rocks, $\mathrm{F}$ \\
\hline Core & 3 & For these rocks, $\mathrm{F}$ \\
\hline Core & 3 & For these rocks, $\mathrm{F}$ \\
\hline Core & 3 & For these rocks, $\mathrm{F}$ \\
\hline Core & 3 & For these rocks, $\mathrm{F}$ \\
\hline Core & 3 & For these rocks, $\mathrm{F}$ \\
\hline Core & 3 & For these rocks, $\mathrm{F}$ \\
\hline Core & 3 & For these rocks, $\mathrm{F}$ \\
\hline Core & 3 & For these rocks, $\mathrm{F}$ \\
\hline Core & 3 & For these rocks, $\mathrm{F}$ \\
\hline Core & 3 & For these rocks, $\mathrm{F}$ \\
\hline Core & 3 & For these rocks, $\mathrm{F}$ \\
\hline Core & 3 & For these rocks, $\mathrm{F}$ \\
\hline Core & 3 & For these rocks, $\mathrm{F}$ \\
\hline Core & 3 & For these rocks, $\mathrm{F}$ \\
\hline Core & 3 & For these rocks, $\mathrm{F}$ \\
\hline Core & 3 & For these rocks, $\mathrm{F}$ \\
\hline Core & 3 & For these rocks, $\mathrm{F}$ \\
\hline Core & 3 & For these rocks, $\mathrm{F}$ \\
\hline Core & 3 & For these rocks, $\mathrm{F}$ \\
\hline Core & 3 & For these rocks, $\mathrm{F}$ \\
\hline Core & 3 & For these rocks, $\mathrm{F}$ \\
\hline Core & 3 & For these rocks, $\mathrm{F}$ \\
\hline Core & 3 & For these rocks, $\mathrm{F}$ \\
\hline Core & 3 & For these rocks, $\mathrm{F}$ \\
\hline Core & 3 & For these rocks, $\mathrm{F}$ \\
\hline
\end{tabular}


Poulton et al., 2010, Nature Geoscience

Poulton et al., 2010, Nature Geoscience

Poulton et al., 2010, Nature Geoscience

Poulton et al., 2010, Nature Geoscience

Poulton et al., 2010, Nature Geoscience

Poulton et al., 2010, Nature Geoscience

Poulton et al., 2010, Nature Geoscience

Poulton et al., 2010, Nature Geoscience

Poulton et al., 2010, Nature Geoscience

Poulton et al., 2010, Nature Geoscience

Poulton et al., 2010, Nature Geoscience

Poulton et al., 2010, Nature Geoscience

Poulton et al., 2010, Nature Geoscience

Poulton et al., 2010, Nature Geoscience

Poulton et al., 2010, Nature Geoscience

Poulton et al., 2010, Nature Geoscience

Poulton et al., 2010, Nature Geoscience

Poulton et al., 2010, Nature Geoscience

Poulton et al., 2010, Nature Geoscience

Poulton et al., 2010, Nature Geoscience

Poulton et al., 2010, Nature Geoscience

Poulton et al., 2010, Nature Geoscience

Poulton et al., 2010, Nature Geoscience

Poulton et al., 2010, Nature Geoscience

Poulton et al., 2010, Nature Geoscience

Poulton et al., 2010, Nature Geoscience

Poulton et al., 2010, Nature Geoscience

Poulton et al., 2010, Nature Geoscience

Poulton et al., 2010, Nature Geoscience

Poulton et al., 2010, Nature Geoscience

Poulton et al., 2010, Nature Geoscience

Poulton et al., 2010, Nature Geoscience

Poulton et al., 2010, Nature Geoscience

Poulton et al., 2010, Nature Geoscience

Poulton et al., 2010, Nature Geoscience

Poulton et al., 2010, Nature Geoscience

Poulton et al., 2010, Nature Geoscience

Poulton et al., 2010, Nature Geoscience

Poulton et al., 2010, Nature Geoscience

Poulton et al., 2010, Nature Geoscience

Poulton et al., 2010, Nature Geoscience

\begin{tabular}{|c|c|c|}
\hline Core & 3 & For these rocks, $\mathrm{F}$ \\
\hline Core & 3 & For these rocks, $\mathrm{F}$ \\
\hline Core & 3 & For these rocks, $\mathrm{F}$ \\
\hline Core & 3 & For these rocks, $\mathrm{F}$ \\
\hline Core & 3 & For these rocks, $\mathrm{F}$ \\
\hline Core & 3 & For these rocks, $\mathrm{F}$ \\
\hline Core & 3 & For these rocks, $\mathrm{F}$ \\
\hline Core & 3 & For these rocks, $\mathrm{F}$ \\
\hline Core & 3 & For these rocks, $\mathrm{F}$ \\
\hline Core & 3 & For these rocks, $\mathrm{F}$ \\
\hline Core & 3 & For these rocks, $\mathrm{F}$ \\
\hline Core & 3 & For these rocks, $\mathrm{F}$ \\
\hline Core & 3 & For these rocks, $\mathrm{F}$ \\
\hline Core & 3 & For these rocks, $\mathrm{F}$ \\
\hline Core & 3 & For these rocks, $\mathrm{F}$ \\
\hline Core & 3 & For these rocks, $\mathrm{F}$ \\
\hline Core & 3 & For these rocks, $\mathrm{F}$ \\
\hline Core & 3 & For these rocks, $\mathrm{F}$ \\
\hline Core & 3 & For these rocks, $\mathrm{F}$ \\
\hline Core & 3 & For these rocks, $\mathrm{F}$ \\
\hline Core & 3 & For these rocks, $\mathrm{F}$ \\
\hline Core & 3 & For these rocks, $\mathrm{F}$ \\
\hline Core & 3 & For these rocks, $\mathrm{F}$ \\
\hline Core & 3 & For these rocks, $\mathrm{F}$ \\
\hline Core & 3 & For these rocks, $\mathrm{F}$ \\
\hline Core & 3 & For these rocks, $\mathrm{F}$ \\
\hline Core & 3 & For these rocks, $\mathrm{F}$ \\
\hline Core & 3 & For these rocks, $\mathrm{F}$ \\
\hline Core & 3 & For these rocks, $\mathrm{F}$ \\
\hline Core & 3 & For these rocks, $\mathrm{F}$ \\
\hline Core & 3 & For these rocks, $\mathrm{F}$ \\
\hline Core & 3 & For these rocks, $\mathrm{F}$ \\
\hline Core & 3 & For these rocks, $\mathrm{F}$ \\
\hline Core & 3 & For these rocks, $\mathrm{F}$ \\
\hline Core & 3 & For these rocks, $\mathrm{F}$ \\
\hline Core & 3 & For these rocks, $\mathrm{F}$ \\
\hline Core & 3 & For these rocks, $\mathrm{F}$ \\
\hline Core & 3 & For these rocks, $\mathrm{F}$ \\
\hline Core & 3 & For these rocks, $\mathrm{F}$ \\
\hline Core & 3 & For these rocks, $\mathrm{F}$ \\
\hline Core & 3 & For these rocks, $\mathrm{F}$ \\
\hline
\end{tabular}


Poulton et al., 2010, Nature Geoscience

Poulton et al., 2010, Nature Geoscience

Poulton et al., 2010, Nature Geoscience

Poulton et al., 2010, Nature Geoscience

Poulton et al., 2010, Nature Geoscience

Poulton et al., 2010, Nature Geoscience

Poulton et al., 2010, Nature Geoscience

Poulton et al., 2010, Nature Geoscience

Poulton et al., 2010, Nature Geoscience

Poulton et al., 2010, Nature Geoscience

Poulton et al., 2010, Nature Geoscience

Poulton et al., 2010, Nature Geoscience

Poulton et al., 2010, Nature Geoscience

Poulton et al., 2010, Nature Geoscience

Poulton et al., 2010, Nature Geoscience

Poulton et al., 2010, Nature Geoscience

Poulton et al., 2010, Nature Geoscience

Poulton et al., 2010, Nature Geoscience

Poulton et al., 2010, Nature Geoscience

Poulton et al., 2010, Nature Geoscience

Poulton et al., 2010, Nature Geoscience

Poulton et al., 2010, Nature Geoscience

Poulton et al., 2010, Nature Geoscience

Poulton et al., 2010, Nature Geoscience

Poulton et al., 2010, Nature Geoscience

Poulton et al., 2010, Nature Geoscience

Poulton et al., 2010, Nature Geoscience

Poulton et al., 2010, Nature Geoscience

Poulton et al., 2010, Nature Geoscience

Poulton et al., 2010, Nature Geoscience

Poulton et al., 2010, Nature Geoscience

Poulton et al., 2010, Nature Geoscience

Poulton et al., 2010, Nature Geoscience

Poulton et al., 2010, Nature Geoscience

Poulton et al., 2010, Nature Geoscience

Poulton et al., 2010, Nature Geoscience

Poulton et al., 2010, Nature Geoscience

Poulton et al., 2010, Nature Geoscience

Poulton et al., 2010, Nature Geoscience

Poulton et al., 2010, Nature Geoscience

Poulton et al., 2010, Nature Geoscience

\begin{tabular}{|c|c|c|}
\hline Core & 3 & For these rocks, $\mathrm{F}$ \\
\hline Core & 3 & For these rocks, $\mathrm{F}$ \\
\hline Core & 3 & For these rocks, $\mathrm{F}$ \\
\hline Core & 3 & For these rocks, $\mathrm{F}$ \\
\hline Core & 3 & For these rocks, $\mathrm{F}$ \\
\hline Core & 3 & For these rocks, $\mathrm{F}$ \\
\hline Core & 3 & For these rocks, $\mathrm{F}$ \\
\hline Core & 3 & For these rocks, $\mathrm{F}$ \\
\hline Core & 3 & For these rocks, $\mathrm{F}$ \\
\hline Core & 3 & For these rocks, $\mathrm{F}$ \\
\hline Core & 3 & For these rocks, $\mathrm{F}$ \\
\hline Core & 3 & For these rocks, $\mathrm{F}$ \\
\hline Core & 3 & For these rocks, $\mathrm{F}$ \\
\hline Core & 3 & For these rocks, $\mathrm{F}$ \\
\hline Core & 3 & For these rocks, $\mathrm{F}$ \\
\hline Core & 3 & For these rocks, $\mathrm{F}$ \\
\hline Core & 3 & For these rocks, $\mathrm{F}$ \\
\hline Core & 3 & For these rocks, $\mathrm{F}$ \\
\hline Core & 3 & For these rocks, $\mathrm{F}$ \\
\hline Core & 3 & For these rocks, $\mathrm{F}$ \\
\hline Core & 3 & For these rocks, $\mathrm{F}$ \\
\hline Core & 3 & For these rocks, $\mathrm{F}$ \\
\hline Core & 3 & For these rocks, $\mathrm{F}$ \\
\hline Core & 3 & For these rocks, $\mathrm{F}$ \\
\hline Core & 3 & For these rocks, $\mathrm{F}$ \\
\hline Core & 3 & For these rocks, $\mathrm{F}$ \\
\hline Core & 3 & For these rocks, $\mathrm{F}$ \\
\hline Core & 3 & For these rocks, $\mathrm{F}$ \\
\hline Core & 3 & For these rocks, $\mathrm{F}$ \\
\hline Core & 3 & For these rocks, $\mathrm{F}$ \\
\hline Core & 3 & For these rocks, $\mathrm{F}$ \\
\hline Core & 3 & For these rocks, $\mathrm{F}$ \\
\hline Core & 3 & For these rocks, $\mathrm{F}$ \\
\hline Core & 3 & For these rocks, $\mathrm{F}$ \\
\hline Core & 3 & For these rocks, $\mathrm{F}$ \\
\hline Core & 3 & For these rocks, $\mathrm{F}$ \\
\hline Core & 3 & For these rocks, $\mathrm{F}$ \\
\hline Core & 3 & For these rocks, $\mathrm{F}$ \\
\hline Core & 3 & For these rocks, $\mathrm{F}$ \\
\hline Core & 3 & For these rocks, $\mathrm{F}$ \\
\hline Core & 3 & For these rocks, $\mathrm{F}$ \\
\hline
\end{tabular}


Poulton et al., 2010, Nature Geoscience

Poulton et al., 2010, Nature Geoscience

Poulton et al., 2010, Nature Geoscience

Poulton et al., 2010, Nature Geoscience

Poulton et al., 2010, Nature Geoscience

Poulton et al., 2010, Nature Geoscience

Poulton et al., 2010, Nature Geoscience

Poulton et al., 2010, Nature Geoscience

Poulton et al., 2010, Nature Geoscience

Poulton et al., 2010, Nature Geoscience

Poulton et al., 2010, Nature Geoscience

Poulton et al., 2010, Nature Geoscience

Poulton et al., 2010, Nature Geoscience

Poulton et al., 2010, Nature Geoscience

Poulton et al., 2010, Nature Geoscience

Poulton et al., 2010, Nature Geoscience

Poulton et al., 2010, Nature Geoscience

Poulton et al., 2010, Nature Geoscience

Poulton et al., 2010, Nature Geoscience

Poulton et al., 2010, Nature Geoscience

Poulton et al., 2010, Nature Geoscience

Poulton et al., 2010, Nature Geoscience

Poulton et al., 2010, Nature Geoscience

Poulton et al., 2010, Nature Geoscience

Geboy et al., 2013, Precambrian Research

Geboy et al., 2013, Precambrian Research

Geboy et al., 2013, Precambrian Research

Geboy et al., 2013, Precambrian Research

Geboy et al., 2013, Precambrian Research

Geboy et al., 2013, Precambrian Research

Geboy et al., 2013, Precambrian Research

Geboy et al., 2013, Precambrian Research

Geboy et al., 2013, Precambrian Research

Geboy et al., 2013, Precambrian Research

Geboy et al., 2013, Precambrian Research

Geboy et al., 2013, Precambrian Research

Geboy et al., 2013, Precambrian Research

Geboy et al., 2013, Precambrian Research

Geboy et al., 2013, Precambrian Research

Geboy et al., 2013, Precambrian Research

Geboy et al., 2013, Precambrian Research

\begin{tabular}{|c|c|c|}
\hline Core & 3 & For these rocks, $\mathrm{F}$ \\
\hline Core & 3 & For these rocks, $\mathrm{F}$ \\
\hline Core & 3 & For these rocks, $\mathrm{F}$ \\
\hline Core & 3 & For these rocks, $\mathrm{F}$ \\
\hline Core & 3 & For these rocks, $\mathrm{F}$ \\
\hline Core & 3 & For these rocks, $\mathrm{F}$ \\
\hline Core & 3 & For these rocks, $\mathrm{F}$ \\
\hline Core & 3 & For these rocks, $\mathrm{F}$ \\
\hline Core & 3 & For these rocks, $\mathrm{F}$ \\
\hline Core & 3 & For these rocks, F \\
\hline Core & 3 & For these rocks, $\mathrm{F}$ \\
\hline Core & 3 & For these rocks, $\mathrm{F}$ \\
\hline Core & 3 & For these rocks, $\mathrm{F}$ \\
\hline Core & 3 & For these rocks, $\mathrm{F}$ \\
\hline Core & 3 & For these rocks, $\mathrm{F}$ \\
\hline Core & 3 & For these rocks, $\mathrm{F}$ \\
\hline Core & 3 & For these rocks, $\mathrm{F}$ \\
\hline Core & 3 & For these rocks, $\mathrm{F}$ \\
\hline Core & 3 & For these rocks, $\mathrm{F}$ \\
\hline Core & 3 & For these rocks, $\mathrm{F}$ \\
\hline Core & 3 & For these rocks, $\mathrm{F}$ \\
\hline Core & 3 & For these rocks, $\mathrm{F}$ \\
\hline Core & 3 & For these rocks, $\mathrm{F}$ \\
\hline Core & 3 & For these rocks, $\mathrm{F}$ \\
\hline Core & 3 & \\
\hline Core & 3 & rock contains smé \\
\hline Core & 3 & \\
\hline Core & 3 & rock contains smé \\
\hline Core & 3 & \\
\hline Core & 3 & \\
\hline Core & 3 & \\
\hline Core & 3 & rock contains smé \\
\hline Core & 3 & \\
\hline Core & 3 & \\
\hline Core & 3 & \\
\hline Core & 3 & \\
\hline Core & 3 & \\
\hline Core & 3 & \\
\hline Core & 3 & \\
\hline Core & 3 & \\
\hline Core & 3 & \\
\hline
\end{tabular}


Geboy et al., 2013, Precambrian Research

Core 3

Geboy et al., 2013, Precambrian Research

Core 3

Geboy et al., 2013, Precambrian Research

Core 3

Geboy et al., 2013, Precambrian Research

Core 3

Geboy et al., 2013, Precambrian Research

Core 3

Geboy et al., 2013, Precambrian Research

Core 3

Geboy et al., 2013, Precambrian Research

Core 3

Geboy et al., 2013, Precambrian Research

Core 3

Geboy et al., 2013, Precambrian Research

Core 3

Geboy et al., 2013, Precambrian Research

Core 3

Geboy et al., 2013, Precambrian Research

Core 3

Geboy et al., 2013, Precambrian Research

Core 3

Geboy et al., 2013, Precambrian Research

Core 3

Geboy et al., 2013, Precambrian Research

Core 3

Geboy et al., 2013, Precambrian Research

Core 3

Geboy et al., 2013, Precambrian Research

Core 3

Geboy et al., 2013, Precambrian Research

Core 3

Geboy et al., 2013, Precambrian Research

Core 3

Geboy et al., 2013, Precambrian Research

Core 3

Geboy et al., 2013, Precambrian Research

Core 3

Geboy et al., 2013, Precambrian Research

Core 3

Geboy et al., 2013, Precambrian Research

Core 3

Asael et al., 2013, Chemical Geology

Core 3

Asael et al., 2013, Chemical Geology

Core 3

Asael et al., 2013, Chemical Geology

Core 3

Asael et al., 2013, Chemical Geology

Core

Core

Core

Core

Core

Core

Core

Core

Core

Core

Core

Core

Core

Core

Asael et al., 2013, Chemical Geology

Asael et al., 2013, Chemical Geology

Asael et al., 2013, Chemical Geology

Asael et al., 2013, Chemical Geology

3

Asael et al., 2013, Chemical Geology

3

3

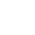

3

3

3

3

3

3

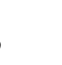

3

3

3

3

rock contains smé

3

rock contains smé

3

3

3

Many of these saı

Many of these sai

Many of these sai

Many of these sai

Many of these sai

Many of these saı

Many of these sai

Many of these sar

Many of these sal

Many of these sai

Many of these sai

Many of these sai

Many of these sai

Many of these sai

3 Many of these sai

3 Many of these saı

3 Many of these sai

Scott et al., 2014, Earth and Planetary Science Lı Core 3

Scott et al., 2014, Earth and Planetary Science Lı Core 3 
Scott et al., 2014, Earth and Planetary Science LıCore 3

Scott et al., 2014, Earth and Planetary Science LıCore 3

Scott et al., 2014, Earth and Planetary Science LıCore 3

Scott et al., 2014, Earth and Planetary Science LıCore 3

Scott et al., 2014, Earth and Planetary Science Li Core 3

Scott et al., 2014, Earth and Planetary Science Li Core 3

Scott et al., 2014, Earth and Planetary Science Li Core 3

Scott et al., 2014, Earth and Planetary Science LiCore 3

Scott et al., 2014, Earth and Planetary Science Lı Core 3

Scott et al., 2014, Earth and Planetary Science LıCore 3

Scott et al., 2014, Earth and Planetary Science Lı Core 3

Scott et al., 2014, Earth and Planetary Science Li Core 3

Scott et al., 2014, Earth and Planetary Science LıCore 3

Scott et al., 2014, Earth and Planetary Science Li Core 3

Scott et al., 2014, Earth and Planetary Science Li Core 3

Scott et al., 2014, Earth and Planetary Science Li Core 3

Scott et al., 2014, Earth and Planetary Science Li Core 3

Scott et al., 2014, Earth and Planetary Science Li Core 3

Scott et al., 2014, Earth and Planetary Science Lı Core 3

Scott et al., 2014, Earth and Planetary Science Li Core 3

Scott et al., 2014, Earth and Planetary Science Li Core 3

Scott et al., 2014, Earth and Planetary Science LıCore 3

Scott et al., 2014, Earth and Planetary Science Lı Core 3

Scott et al., 2014, Earth and Planetary Science LiCore 3

Scott et al., 2014, Earth and Planetary Science Li Core 3

Scott et al., 2014, Earth and Planetary Science LiCore 3

Scott et al., 2014, Earth and Planetary Science Li Core 3

Scott et al., 2014, Earth and Planetary Science LıCore 3

Scott et al., 2014, Earth and Planetary Science Li Core 3

Scott et al., 2014, Earth and Planetary Science Li Core 3

Scott et al., 2014, Earth and Planetary Science Li Core 3

Scott et al., 2014, Earth and Planetary Science LıCore 3

Scott et al., 2014, Earth and Planetary Science LiCore 3

Scott et al., 2014, Earth and Planetary Science LiCore 3

Scott et al., 2014, Earth and Planetary Science Lı Core 3

Scott et al., 2014, Earth and Planetary Science LıCore 3

Scott et al., 2014, Earth and Planetary Science LiCore 3

Scott et al., 2014, Earth and Planetary Science Li Core 3

Scott et al., 2014, Earth and Planetary Science Lı Core 3

Scott et al., 2014, Earth and Planetary Science LiCore 3

Scott et al., 2014, Earth and Planetary Science LıCore 3 
Scott et al., 2014, Earth and Planetary Science LıCore 3

Scott et al., 2014, Earth and Planetary Science Li Core 3

Scott et al., 2014, Earth and Planetary Science Li Core 3

Scott et al., 2014, Earth and Planetary Science Li Core 3

Scott et al., 2014, Earth and Planetary Science Li Core 3

Scott et al., 2014, Earth and Planetary Science LıCore 3

Scott et al., 2014, Earth and Planetary Science Lı Core 3

Cumming et al., 2013, Geology

Core 3 Lacustrine

Cumming et al., 2013, Geology

Outcrop 3

Cumming et al., 2013, Geology

Outcrop 3

Cumming et al., 2013, Geology

Outcrop 3

Cumming et al., 2013, Geology

Outcrop 3

Cumming et al., 2013, Geology

Outcrop

Cumming et al., 2013, Geology

Outcrop

Cumming et al., 2013, Geology

Outcrop

Cumming et al., 2013, Geology

Outcrop

Cumming et al., 2013, Geology

Outcrop

Cumming et al., 2013, Geology

Outcrop

Cumming et al., 2013, Geology

Outcrop

Cumming et al., 2013, Geology

Outcrop

Cumming et al., 2013, Geology

Outcrop

Cumming et al., 2013, Geology

Core

Cumming et al., 2013, Geology

Core

Cumming et al., 2013, Geology

Core

Cumming et al., 2013, Geology

Core

Cumming et al., 2013, Geology

Core

Cumming et al., 2013, Geology

Core

Cumming et al., 2013, Geology

Core

Cumming et al., 2013, Geology

Core

Cumming et al., 2013, Geology

Core

Cumming et al., 2013, Geology

Core

Cumming et al., 2013, Geology

Core

Cumming et al., 2013, Geology

Outcrop

Cumming et al., 2013, Geology

Outcrop

Cumming et al., 2013, Geology

Outcrop

Cumming et al., 2013, Geology

Core

Cumming et al., 2013, Geology

Core

Cumming et al., 2013, Geology

Core

Cumming et al., 2013, Geology

Core

Cumming et al., 2013, Geology

Core

Core

Lacustrine

3 Lacustrine

3 Lacustrine

3 Lacustrine

3 Lacustrine

3 Lacustrine

3 Lacustrine

3 Lacustrine

3 Lacustrine

3 Lacustrine

3 Lacustrine

3 Lacustrine

3 Lacustrine

3 Lacustrine

3 Lacustrine

3 Lacustrine

3 Lacustrine

3 Lacustrine

3 Lacustrine

3 Lacustrine

3 Lacustrine

3 Lacustrine

3 Lacustrine

3 Lacustrine

3 Lacustrine

3 Lacustrine

3 Lacustrine

3 Lacustrine

3 Lacustrine

3 Lacustrine

3 Lacustrine

Cumming et al., 2013, Geology

3 Lacustrine 
Cumming et al., 2013, Geology

Cumming et al., 2013, Geology

Cumming et al., 2013, Geology

Cumming et al., 2013, Geology

Cumming et al., 2013, Geology

Cumming et al., 2013, Geology

Cumming et al., 2013, Geology

Cumming et al., 2013, Geology

Cumming et al., 2013, Geology

Cumming et al., 2013, Geology

Cumming et al., 2013, Geology

Cumming et al., 2013, Geology

Cumming et al., 2013, Geology

Cumming et al., 2013, Geology

Cumming et al., 2013, Geology

Cumming et al., 2013, Geology

Cumming et al., 2013, Geology

Cumming et al., 2013, Geology

Cumming et al., 2013, Geology

Cumming et al., 2013, Geology

Cumming et al., 2013, Geology

Cumming et al., 2013, Geology

Cumming et al., 2013, Geology

Cumming et al., 2013, Geology

Cumming et al., 2013, Geology

Cumming et al., 2013, Geology

Cumming et al., 2013, Geology

Cumming et al., 2013, Geology
Core

Core

Core

Core

Core

Core

Core

Outcrop

Outcrop

Core

Core

Core

Core

Core

Core

Core

Core

Core

Core

Core

Core

Core

Core

Core

Core

Core

Core

Core

Scott et al., 2014, Earth and Planetary Science Lı Core

Scott et al., 2014, Earth and Planetary Science LiCore

Scott et al., 2014, Earth and Planetary Science Lı Core

Scott et al., 2014, Earth and Planetary Science Li Core

Scott et al., 2014, Earth and Planetary Science LıCore

Scott et al., 2014, Earth and Planetary Science Li Core

Scott et al., 2014, Earth and Planetary Science Lı Core

Scott et al., 2014, Earth and Planetary Science Lı Core

Scott et al., 2014, Earth and Planetary Science Lı Core

Scott et al., 2014, Earth and Planetary Science Li Core

Scott et al., 2014, Earth and Planetary Science Li Core

Scott et al., 2014, Earth and Planetary Science Lı Core

Scott et al., 2014, Earth and Planetary Science LıCore
3 Lacustrine

3 Lacustrine

3 Lacustrine

3 Lacustrine

3 Lacustrine

3 Lacustrine

3 Lacustrine

3 Lacustrine

3 Lacustrine

3 Lacustrine

3 Lacustrine

3 Lacustrine

3 Lacustrine

3 Lacustrine

3 Lacustrine

3 Lacustrine

3 Lacustrine

3 Lacustrine

3 Lacustrine

3 Lacustrine

3 Lacustrine

3 Lacustrine

3 Lacustrine

3 Lacustrine

3 Lacustrine

3 Lacustrine

3 Lacustrine

3 Lacustrine 
Scott et al., 2014, Earth and Planetary Science Lı Core 3

Scott et al., 2014, Earth and Planetary Science Lı Core 3

Feng et al., 2010, Gondwana Research Outcrop 3

Feng et al., 2010, Gondwana Research Outcrop 3

Feng et al., 2010, Gondwana Research

Outcrop 3

Feng et al., 2010, Gondwana Research

Outcrop

Feng et al., 2010, Gondwana Research

Outcrop

Outcrop

Feng et al., 2010, Gondwana Research

Outcrop

Outcrop

Feng et al., 2010, Gondwana Research

Outcrop

Outcrop

Feng et al., 2010, Gondwana Research

Outcrop

Outcrop

Feng et al., 2010, Gondwana Research

Outcrop

Outcrop

Feng et al., 2010, Gondwana Research

Outcrop

Outcrop

Feng et al., 2010, Gondwana Research

3

3

3

3

3

3
3 Uses old extractic

3 Uses old extractic

3 Uses old extractic

Li et al., 2012; Earth and Planetary Science Lette Outcrop 3

Li et al., 2012; Earth and Planetary Science Lette Outcrop 3

Li et al., 2012; Earth and Planetary Science Lette Outcrop 3

Li et al., 2012; Earth and Planetary Science Lette Outcrop 3

Li et al., 2012; Earth and Planetary Science Lette Outcrop 3

Li et al., 2012; Earth and Planetary Science Lette Outcrop 3

Li et al., 2012; Earth and Planetary Science Lette Outcrop 3

Li et al., 2012; Earth and Planetary Science Lette Outcrop 3

Li et al., 2012; Earth and Planetary Science Lette Outcrop 3

Li et al., 2012; Earth and Planetary Science Lette Outcrop 3

Li et al., 2012; Earth and Planetary Science Lette Outcrop 3

Li et al., 2012; Earth and Planetary Science Lette Outcrop 3

Li et al., 2012; Earth and Planetary Science Lette Outcrop 3

Li et al., 2012; Earth and Planetary Science Lette Outcrop 3

Li et al., 2012; Earth and Planetary Science Lette Outcrop 3

Li et al., 2012; Earth and Planetary Science Lette Outcrop 3

Li et al., 2012; Earth and Planetary Science Lette Outcrop 3

Li et al., 2012; Earth and Planetary Science Lette Outcrop 3

Li et al., 2012; Earth and Planetary Science Lette Outcrop 3

Li et al., 2012; Earth and Planetary Science Lette Outcrop 3

Li et al., 2012; Earth and Planetary Science Lette Outcrop 3

Li et al., 2012; Earth and Planetary Science Lette Outcrop 3
Uses old extractic

Uses old extractic

Uses old extractic

Uses old extractic

Uses old extractic

Uses old extractic

Uses old extractic

Uses old extractic

Uses old extractic

Uses old extractic

Uses old extractic

Uses old extractic

Uses old extractic 
Li et al., 2012; Earth and Planetary Science Lette Outcrop 1

Li et al., 2012; Earth and Planetary Science Lette Outcrop 1

Li et al., 2012; Earth and Planetary Science Lette Outcrop 1

Li et al., 2012; Earth and Planetary Science Lette Outcrop 1

This study Outcrop 3

This study Outcrop $\quad 3$

This study $\quad$ Outcrop 3

This study $\quad$ Outcrop 3

This study Outcrop 3

This study Outcrop $\quad 3$

This study Outcrop 3

This study Outcrop $\quad 3$

This study Outcrop 3

This study Outcrop $\quad 3$

This study Outcrop 3

This study $\quad$ Outcrop 3

This study Outcrop 3

This study Outcrop $\quad 3$

This study Outcrop 3

This study $\quad$ Outcrop 3

This study $\quad$ Outcrop 3

This study $\quad$ Outcrop 3

This study Outcrop $\quad 3$

This study $\quad$ Outcrop 3

This study $\quad$ Outcrop 3

This study $\quad$ Outcrop 3

This study Outcrop $\quad 3$

This study $\quad$ Outcrop 3

This study Outcrop 3

This study $\quad$ Outcrop 3

This study Outcrop 3

This study $\quad$ Outcrop 1

This study $\quad$ Outcrop 1

This study $\quad$ Outcrop 1

This study $\quad$ Outcrop 1

This study $\quad$ Outcrop 1

This study $\quad$ Outcrop 1

This study $\quad$ Outcrop 1

This study $\quad$ Outcrop 1

This study $\quad$ Outcrop 1

This study $\quad$ Outcrop 1 
This study

This study

This study

This study

This study

This study

This study

This study

This study

This study

This study

This study

This study

This study

This study

This study

This study

This study

This study

This study

This study

This study

This study

This study

This study

This study

This study

This study

This study

This study

This study

This study

This study

This study

This study

This study

This study

This study

This study

This study

This study
Outcrop 1

Outcrop 1

Outcrop 1

Outcrop 1

Outcrop 1

Outcrop 1

Outcrop 1

Outcrop 1

Outcrop 1

Outcrop 1

Outcrop 1

Outcrop 1

Outcrop 1

Outcrop 1

Outcrop 1

Outcrop 1

Outcrop 1

Outcrop 1

Outcrop 1

Outcrop 1

Outcrop 1

Outcrop 1

Outcrop 1

Outcrop 1

Outcrop 1

Outcrop 1

Outcrop 1

Outcrop 1

Outcrop 1

Outcrop 1

Outcrop 1

Outcrop 1

Outcrop 1

Outcrop 1

Outcrop 1

Outcrop 3

Outcrop 3

Outcrop 3

Outcrop 3

Outcrop 3

Outcrop 3 
This study

This study

This study

This study

This study

This study

This study

This study

This study

This study

This study

This study

This study

This study

This study

This study

This study

This study

This study

This study

This study

This study

This study

This study

This study

This study

This study

This study

This study

This study

This study

This study

This study

This study

This study

This study

This study

This study

This study

This study

This study
Outcrop 3

Outcrop 3

Outcrop 3

Outcrop 3

Outcrop 3

Outcrop 3

Outcrop 3

Outcrop 3

Outcrop 3

Outcrop 3

Outcrop 3

Outcrop 3

Outcrop 3

Outcrop 3

Outcrop 3

Outcrop 3

Outcrop 3

Outcrop 3

Outcrop 3

Outcrop 3

Outcrop 3

Outcrop 3

Outcrop 3

Outcrop 3

Outcrop 3

Outcrop 3

Outcrop 3

Outcrop 3

Outcrop 1

Outcrop 1

Outcrop 1

Outcrop 1

Outcrop 1

Outcrop 1

Outcrop 1

Outcrop 1

Outcrop 1

Outcrop 1

Outcrop 1

Outcrop 1

Outcrop 1 
This study

Outcrop 1

This study

Outcrop 1

This study

Outcrop 1

This study

Outcrop 3

This study

Outcrop 3

This study

Outcrop 3

This study

Outcrop 3

This study

Outcrop 3

Canfield et al., 2008, Science

Outcrop 2

Canfield et al., 2008, Science

Outcrop 1

Canfield et al., 2008, Science

Outcrop 1

Canfield et al., 2008, Science

Outcrop 1

Canfield et al., 2008, Science

Outcrop 1

Canfield et al., 2008, Science

Outcrop 3

Canfield et al., 2008, Science

Outcrop 3

Canfield et al., 2008, Science

Outcrop 3

Canfield et al., 2008, Science

Outcrop 3

Canfield et al., 2008, Science

Outcrop 3

Canfield et al., 2008, Science

Outcrop 3

Canfield et al., 2008, Science

Outcrop 3

Canfield et al., 2008, Science

Outcrop 3

Canfield et al., 2008, Science

Outcrop 3

Canfield et al., 2008, Science

Outcrop 3

Canfield et al., 2008, Science

Outcrop

Johnston et al., 2010, Earth and Planetary Scienı Outcrop

Johnston et al., 2010, Earth and Planetary Scienı Outcrop

Johnston et al., 2010, Earth and Planetary Scienı Outcrop

Johnston et al., 2010, Earth and Planetary Scienı Outcrop

Johnston et al., 2010, Earth and Planetary Scienı Outcrop

Johnston et al., 2010, Earth and Planetary Scienı Outcrop

Johnston et al., 2010, Earth and Planetary Scienı Outcrop

Johnston et al., 2010, Earth and Planetary Scienı Outcrop

Johnston et al., 2010, Earth and Planetary Scienı Outcrop

Johnston et al., 2010, Earth and Planetary Scienı Outcrop

Johnston et al., 2010, Earth and Planetary Scienı Outcrop

Johnston et al., 2010, Earth and Planetary Scienı Outcrop

Johnston et al., 2010, Earth and Planetary Scienı Outcrop

Johnston et al., 2010, Earth and Planetary Scienı Outcrop

Johnston et al., 2010, Earth and Planetary Scienı Outcrop

Johnston et al., 2010, Earth and Planetary Scienı Outcrop

Johnston et al., 2010, Earth and Planetary Scienı Outcrop 
Johnston et al., 2010, Earth and Planetary Scienı Outcrop 1

Johnston et al., 2010, Earth and Planetary Scienı Outcrop 2

Johnston et al., 2010, Earth and Planetary Scienı Outcrop 2

Johnston et al., 2010, Earth and Planetary Scienı Outcrop 2

Johnston et al., 2010, Earth and Planetary Scienı Outcrop 2

Johnston et al., 2010, Earth and Planetary Scienı Outcrop 2

Johnston et al., 2010, Earth and Planetary Scienı Outcrop 2

Johnston et al., 2010, Earth and Planetary Scienı Outcrop 2

Johnston et al., 2010, Earth and Planetary Scienı Outcrop 2

Johnston et al., 2010, Earth and Planetary Scienı Outcrop 2

Johnston et al., 2010, Earth and Planetary Scienı Outcrop 2

Johnston et al., 2010, Earth and Planetary Scienı Outcrop 1

Johnston et al., 2010, Earth and Planetary Scienı Outcrop 1

Johnston et al., 2010, Earth and Planetary Scienı Outcrop 1

Johnston et al., 2010, Earth and Planetary Scienı Outcrop 1

Johnston et al., 2010, Earth and Planetary Scienı Outcrop 1

Johnston et al., 2010, Earth and Planetary Scienı Outcrop 1

Johnston et al., 2010, Earth and Planetary Scienı Outcrop 1

Johnston et al., 2010, Earth and Planetary Scienı Outcrop 1

Johnston et al., 2010, Earth and Planetary Scienı Outcrop 1

Johnston et al., 2010, Earth and Planetary Scienı Outcrop 1

Johnston et al., 2010, Earth and Planetary Scienı Outcrop 1

Johnston et al., 2010, Earth and Planetary Scienı Outcrop 1

Johnston et al., 2010, Earth and Planetary Scienı Outcrop 1

Johnston et al., 2010, Earth and Planetary Scienı Outcrop 1

Johnston et al., 2010, Earth and Planetary Scienı Outcrop 1

Johnston et al., 2010, Earth and Planetary Scienı Outcrop 1

Johnston et al., 2010, Earth and Planetary Scienı Outcrop 1

Johnston et al., 2010, Earth and Planetary Scienı Outcrop 1

Johnston et al., 2010, Earth and Planetary Scienı Outcrop 1

Johnston et al., 2010, Earth and Planetary Scienı Outcrop 1

Johnston et al., 2010, Earth and Planetary Scienı Outcrop 1

Johnston et al., 2010, Earth and Planetary Scienı Outcrop 1

Johnston et al., 2010, Earth and Planetary Scienı Outcrop 1

Johnston et al., 2010, Earth and Planetary Scienı Outcrop 1

Johnston et al., 2010, Earth and Planetary Scienı Outcrop 1

Johnston et al., 2010, Earth and Planetary Scienı Outcrop 1

Johnston et al., 2010, Earth and Planetary Scienı Outcrop 1

Johnston et al., 2010, Earth and Planetary Scienı Outcrop 1

Johnston et al., 2010, Earth and Planetary Scienı Outcrop 1

Johnston et al., 2010, Earth and Planetary Scienı Outcrop 1 
Johnston et al., 2010, Earth and Planetary Scienı Outcrop 1

Johnston et al., 2010, Earth and Planetary Scienı Outcrop 1

Johnston et al., 2010, Earth and Planetary Scienı Outcrop 1

Johnston et al., 2010, Earth and Planetary Scienı Outcrop 1

Johnston et al., 2010, Earth and Planetary Scienı Outcrop 1

Johnston et al., 2010, Earth and Planetary Scienı Outcrop 1

Johnston et al., 2010, Earth and Planetary Scienı Outcrop 1

Johnston et al., 2010, Earth and Planetary Scienı Outcrop 1

Johnston et al., 2010, Earth and Planetary Scienı Outcrop 1

Johnston et al., 2010, Earth and Planetary Scienı Outcrop 1

Johnston et al., 2010, Earth and Planetary Scienı Outcrop 1

Johnston et al., 2010, Earth and Planetary Scienı Outcrop 1

Johnston et al., 2010, Earth and Planetary Scienı Outcrop 1

Johnston et al., 2010, Earth and Planetary Scienı Outcrop 1

Johnston et al., 2010, Earth and Planetary Scienı Outcrop 1

Johnston et al., 2010, Earth and Planetary Scienı Outcrop 1

Johnston et al., 2010, Earth and Planetary Scienı Outcrop 1

Johnston et al., 2010, Earth and Planetary Scienı Outcrop 1

Johnston et al., 2010, Earth and Planetary Scienı Outcrop 1

Johnston et al., 2010, Earth and Planetary Scienı Outcrop 1

Johnston et al., 2010, Earth and Planetary Scienı Outcrop 1

Johnston et al., 2010, Earth and Planetary Scienı Outcrop 1

Johnston et al., 2010, Earth and Planetary Scienı Outcrop 1

Johnston et al., 2010, Earth and Planetary Scienı Outcrop 1

Johnston et al., 2010, Earth and Planetary Scienı Outcrop 1

Johnston et al., 2010, Earth and Planetary Scienı Outcrop 1

Johnston et al., 2010, Earth and Planetary Scienı Outcrop 1

Johnston et al., 2010, Earth and Planetary Scienı Outcrop 1

Johnston et al., 2010, Earth and Planetary Scienı Outcrop 1

Johnston et al., 2010, Earth and Planetary Scienı Outcrop 1

Johnston et al., 2010, Earth and Planetary Scienı Outcrop 1

Johnston et al., 2010, Earth and Planetary Scienı Outcrop 1

Johnston et al., 2010, Earth and Planetary Scienı Outcrop 1

Johnston et al., 2010, Earth and Planetary Scienı Outcrop 1

Johnston et al., 2010, Earth and Planetary Scienı Outcrop 1

Johnston et al., 2010, Earth and Planetary Scienı Outcrop 1

Johnston et al., 2010, Earth and Planetary Scienı Outcrop 1

Johnston et al., 2010, Earth and Planetary Scienı Outcrop 1

Johnston et al., 2010, Earth and Planetary Scienı Outcrop 3

Johnston et al., 2010, Earth and Planetary Scienı Outcrop 3

Johnston et al., 2010, Earth and Planetary Scienı Outcrop 3 
Johnston et al., 2010, Earth and Planetary Scienı Outcrop 3

Johnston et al., 2010, Earth and Planetary Scienı Outcrop 3

Johnston et al., 2010, Earth and Planetary Scienı Outcrop 3

Johnston et al., 2010, Earth and Planetary Scienı Outcrop 3

Johnston et al., 2010, Earth and Planetary Scienı Outcrop 3

Johnston et al., 2010, Earth and Planetary Scienı Outcrop 3

Johnston et al., 2012, Earth and Planetary Scienı Core 1

Johnston et al., 2012, Earth and Planetary Scienı Core 1

Johnston et al., 2012, Earth and Planetary Scienı Core 1

Johnston et al., 2012, Earth and Planetary Scienı Core 1

Johnston et al., 2012, Earth and Planetary Scienı Core 1

Johnston et al., 2012, Earth and Planetary Scienı Core 1

Johnston et al., 2012, Earth and Planetary Scienı Core 1

Johnston et al., 2012, Earth and Planetary Scienı Core 1

Johnston et al., 2012, Earth and Planetary Scienı Core 1

Johnston et al., 2012, Earth and Planetary Scienı Core 1

Johnston et al., 2012, Earth and Planetary Scienı Core 1

Johnston et al., 2012, Earth and Planetary Scienı Core 1

Johnston et al., 2012, Earth and Planetary Scienı Core 1

Johnston et al., 2012, Earth and Planetary Scienı Core 1

Johnston et al., 2012, Earth and Planetary Scienı Core 1

Johnston et al., 2012, Earth and Planetary Scienı Core 1

Johnston et al., 2012, Earth and Planetary Scienı Core 1

Johnston et al., 2012, Earth and Planetary Scienı Core 1

Canfield et al., 2008 Science $\quad$ Outcrop 1

Canfield et al., 2008 Science $\quad$ Outcrop 1

Canfield et al., 2008 Science $\quad$ Outcrop 1

Canfield et al., 2008, Science $\quad$ Core 2

Canfield et al., 2008, Science Core 2

Canfield et al., 2008, Science $\quad$ Core 2

Canfield et al., 2008, Science $\quad$ Core 2

Canfield et al., 2008, Science Core $\quad 2$

Canfield et al., 2008, Science Core 2

Canfield et al., 2008, Science $\quad$ Core 2

Canfield et al., 2008, Science Core 2

Canfield et al., 2008, Science $\quad$ Core 2

Canfield et al., 2008, Science Core 2

Canfield et al., 2008, Science Core $\quad 2$

Canfield et al., 2008, Science Core 2

Canfield et al., 2008, Science Core $\quad 2$

Canfield et al., 2008, Science $\quad$ Core 2 
Canfield et al., 2008, Science

Core 2

Canfield et al., 2008, Science

Core 2

Canfield et al., 2008, Science

Core 2

Canfield et al., 2008, Science

Core 2

Canfield et al., 2008, Science

Core 2

Canfield et al., 2008, Science

Core 2

Canfield et al., 2008, Science

Core 2

Canfield et al., 2008, Science

Core 2

Canfield et al., 2008, Science

Core 2

Canfield et al., 2008, Science

Core 2

Canfield et al., 2008, Science

Core 2

Canfield et al., 2008, Science

Core 2

Canfield et al., 2008, Science

Core 2

Canfield et al., 2008, Science

Core 2

Canfield et al., 2008, Science

Core 2

Canfield et al., 2008, Science

Core 2

Canfield et al., 2008, Science

Core 2

Canfield et al., 2008, Science

Core 2

Canfield et al., 2008, Science

Core 2

Canfield et al., 2008, Science

Core 2

Canfield et al., 2008, Science

Core 2

Canfield et al., 2008, Science

Core 2

Canfield et al., 2008, Science

Core 2

Canfield et al., 2008, Science

Core 2

Canfield et al., 2008, Science

Core 2

Canfield et al., 2008, Science

Core 2

Canfield et al., 2008, Science

Core 2

Canfield et al., 2008, Science

Core 2

Canfield et al., 2008, Science

Core 2

Canfield et al., 2008, Science

Core 2

Canfield et al., 2008, Science

Core 2

Canfield et al., 2008, Science

Core 2

Canfield et al., 2008, Science

Core 2

Canfield et al., 2008, Science

Core 2

Canfield et al., 2008, Science

Core 2

Canfield et al., 2008, Science

Core 2

Canfield et al., 2008, Science

Core 2

Canfield et al., 2008, Science

Core 2

Canfield et al., 2008, Science

Core 2

Canfield et al., 2008, Science

Core 2

Canfield et al., 2008, Science

Core 2 
Canfield et al., 2008, Science

Core 2

Canfield et al., 2008, Science

Core 2

Canfield et al., 2008, Science

Core 2

Canfield et al., 2008, Science

Core 2

Canfield et al., 2008, Science

Core 2

Canfield et al., 2008, Science

Core 2

Canfield et al., 2008, Science

Core 2

Canfield et al., 2008, Science

Core 2

Canfield et al., 2008, Science

Core 2

Canfield et al., 2008, Science

Core 2

Canfield et al., 2008, Science

Core 2

Canfield et al., 2008, Science

Core 2

Canfield et al., 2008, Science

Core 2

Canfield et al., 2008, Science

Core 2

Canfield et al., 2008, Science

Core 2

Canfield et al., 2008, Science

Core 2

Canfield et al., 2008, Science

Core 2

Canfield et al., 2008, Science

Core 2

Canfield et al., 2008, Science

Core 2

Canfield et al., 2008, Science

Core 2

Canfield et al., 2008, Science

Core 2

Canfield et al., 2008, Science

Core 2

Canfield et al., 2008, Science

Core 2

Canfield et al., 2008, Science

Core 2

Canfield et al., 2008, Science

Core 2

Canfield et al., 2008, Science

Core 2

Canfield et al., 2008, Science

Core 2

Canfield et al., 2008, Science

Core 2

Canfield et al., 2008, Science

Core 2

Canfield et al., 2008, Science

Core 2

Canfield et al., 2008, Science

Core 2

Canfield et al., 2008, Science

Core 2

Canfield et al., 2008, Science

Core 2

Canfield et al., 2008, Science

Core 2

Canfield et al., 2008, Science

Core 2

Canfield et al., 2008, Science

Core 2

Canfield et al., 2008, Science

Core 2

Canfield et al., 2008, Science

Core 2

Canfield et al., 2008, Science

Core 2

Canfield et al., 2008, Science

Core 2

Canfield et al., 2008, Science

Core 2 
Canfield et al., 2008, Science

Core 2

Canfield et al., 2008, Science

Core 2

Canfield et al., 2008, Science

Core 2

Canfield et al., 2008, Science

Core 2

Canfield et al., 2008, Science

Core 2

Canfield et al., 2008, Science

Core 2

Canfield et al., 2008, Science

Core 2

Canfield et al., 2008, Science

Core 2

Canfield et al., 2008, Science

Core 2

Canfield et al., 2008, Science

Core 2

Canfield et al., 2008, Science

Outcrop 2

Canfield et al., 2008, Science

Outcrop 2

Canfield et al., 2008, Science

Outcrop 2

Canfield et al., 2008, Science

Outcrop 2

Canfield et al., 2008, Science

Outcrop 2

Canfield et al., 2008, Science

Outcrop 2

Canfield et al., 2008, Science

Outcrop 2

Canfield et al., 2008, Science

Outcrop 2

Canfield et al., 2008, Science

Outcrop 2

Canfield et al., 2008 Science

Outcrop 1

Canfield et al., 2008 Science

Outcrop 1

Canfield et al., 2008 Science

Outcrop 1

Canfield et al., 2008 Science

Outcrop 1

Canfield et al., 2008 Science

Outcrop 1

Canfield et al., 2008 Science

Outcrop 1

Canfield et al., 2008 Science

Outcrop 1

Outcrop 1

Canfield et al., 2008 Science

Outcrop 1

Outcrop 1

Canfield et al., 2008 Science

Outcrop 1

Outcrop 1

Canfield et al., 2008 Science

Outcrop 1

Outcrop 1

Outcrop 1

Outcrop 1

Outcrop 1

Outcrop 1

Outcrop 1

Outcrop 1

Outcrop 1

Outcrop 1

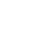

(1)

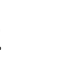

2

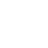

2

2

2

2

2

2

2

2

2

2

1

1

1

1

1

1

1

1

1

1

1

1

1

1

1

1

1

1

1

1

1

\footnotetext{
Canfield et al., 2008 Science
} 
Canfield et al., 2008 Science

Outcrop 1

Canfield et al., 2008 Science

Outcrop 1

Canfield et al., 2008 Science

Outcrop 1

Canfield et al., 2008 Science

Outcrop 1

Canfield et al., 2008 Science

Outcrop 1

Canfield et al., 2008 Science

Outcrop 1

Canfield et al., 2008, Science

Outcrop 2

Canfield et al., 2008, Science

Outcrop 2

Canfield et al., 2008, Science

Outcrop 2

Canfield et al., 2008, Science

Outcrop 2

Canfield et al., 2008, Science

Outcrop 2

Canfield et al., 2008, Science

Outcrop 2

Canfield et al., 2008, Science

Outcrop 2

Canfield et al., 2008, Science

Outcrop 2

Canfield et al., 2008, Science

Outcrop 2

Canfield et al., 2008, Science

Outcrop 2

Canfield et al., 2008, Science

Outcrop 2

Canfield et al., 2008, Science

Outcrop 2

Canfield et al., 2008, Science

Outcrop 2

Canfield et al., 2008, Science

Outcrop 2

Canfield et al., 2008, Science

Outcrop 2

Canfield et al., 2008, Science

Outcrop 2

This study

Outcrop 3

This study

Outcrop 3

This study

Outcrop 3

This study

Outcrop 3

This study

Outcrop 3

This study

Outcrop 3

This study

Outcrop

Sperling et al., 2013, Earth and Planetary Scienci Outcrop

Sperling et al., 2013, Earth and Planetary Scienci Outcrop

Sperling et al., 2013, Earth and Planetary Scienci Outcrop

Sperling et al., 2013, Earth and Planetary Sciencı Outcrop

Sperling et al., 2013, Earth and Planetary Scienci Outcrop

Sperling et al., 2013, Earth and Planetary Scienci Outcrop

Sperling et al., 2013, Earth and Planetary Scienci Outcrop

Sperling et al., 2013, Earth and Planetary Scienci Outcrop

Sperling et al., 2013, Earth and Planetary Scienci Outcrop

Sperling et al., 2013, Earth and Planetary Scienci Outcrop

Sperling et al., 2013, Earth and Planetary Scienci Outcrop

Sperling et al., 2013, Earth and Planetary Scienci Outcrop 
Sperling et al., 2013, Earth and Planetary Sciencı Outcrop 1

Sperling et al., 2013, Earth and Planetary Sciencı Outcrop 1

Sperling et al., 2013, Earth and Planetary Sciencı Outcrop 1

Sperling et al., 2013, Earth and Planetary Scienci Outcrop 1

Sperling et al., 2013, Earth and Planetary Sciencı Outcrop 1

Sperling et al., 2013, Earth and Planetary Scienci Outcrop 1

Sperling et al., 2013, Earth and Planetary Sciencı Outcrop 1

Sperling et al., 2013, Earth and Planetary Sciencı Outcrop 1

Sperling et al., 2013, Earth and Planetary Sciencı Outcrop 1

Sperling et al., 2013, Earth and Planetary Scienci Outcrop 1

Sperling et al., 2013, Earth and Planetary Scienci Outcrop 2

Sperling et al., 2013, Earth and Planetary Scienci Outcrop 2

Sperling et al., 2013, Earth and Planetary Sciencı Outcrop 2

Sperling et al., 2013, Earth and Planetary Scienci Outcrop 2

Sperling et al., 2013, Earth and Planetary Scienci Outcrop 2

Sperling et al., 2013, Earth and Planetary Scienci Outcrop 2

Sperling et al., 2013, Earth and Planetary Sciencı Outcrop 2

Sperling et al., 2013, Earth and Planetary Scienci Outcrop 2

Sperling et al., 2013, Earth and Planetary Scienci Outcrop 2

Sperling et al., 2013, Earth and Planetary Scienci Outcrop 2

Sperling et al., 2013, Earth and Planetary Sciencı Outcrop 2

Sperling et al., 2013, Earth and Planetary Scienci Outcrop 2

Sperling et al., 2013, Earth and Planetary Scienci Outcrop 2

Sperling et al., 2013, Earth and Planetary Scienci Outcrop 2

Sperling et al., 2013, Earth and Planetary Scienci Outcrop 2

Sperling et al., 2013, Earth and Planetary Scienci Outcrop 2

Sperling et al., 2013, Earth and Planetary Sciencı Outcrop 2

Sperling et al., 2013, Earth and Planetary Scienci Outcrop 2

Sperling et al., 2013, Earth and Planetary Scienci Outcrop 2

Sperling et al., 2013, Earth and Planetary Scienci Outcrop 2

Sperling et al., 2013, Earth and Planetary Sciencı Outcrop 2

Sperling et al., 2013, Earth and Planetary Sciencı Outcrop 2

Sperling et al., 2013, Earth and Planetary Scienci Outcrop 2

Sperling et al., 2013, Earth and Planetary Sciencı Outcrop 2

Sperling et al., 2013, Earth and Planetary Sciencı Outcrop 2

Sperling et al., 2013, Earth and Planetary Sciencı Outcrop 2

Sperling et al., 2013, Earth and Planetary Scienci Outcrop 2

Sperling et al., 2013, Earth and Planetary Sciencı Outcrop 2

Sperling et al., 2013, Earth and Planetary Sciencı Outcrop 1

Sperling et al., 2013, Earth and Planetary Sciencı Outcrop 1

Sperling et al., 2013, Earth and Planetary Sciencı Outcrop 1 
Sperling et al., 2013, Earth and Planetary Sciencı Outcrop 1

Sperling et al., 2013, Earth and Planetary Scienci Outcrop 1

Sperling et al., 2013, Earth and Planetary Sciencı Outcrop 1

Sperling et al., 2013, Earth and Planetary Scienci Outcrop 1

Sperling et al., 2013, Earth and Planetary Sciencı Outcrop 2

Sperling et al., 2013, Earth and Planetary Scienci Outcrop 2

Sperling et al., 2013, Earth and Planetary Scienci Outcrop 2

Sperling et al., 2013, Earth and Planetary Scienci Outcrop 2

Sperling et al., 2013, Earth and Planetary Sciencı Outcrop 2

Sperling et al., 2013, Earth and Planetary Scienci Outcrop 2

Sperling et al., 2013, Earth and Planetary Scienci Outcrop 2

Sperling et al., 2013, Earth and Planetary Scienci Outcrop 2

Sperling et al., 2013, Earth and Planetary Sciencı Outcrop 2

Sperling et al., 2013, Earth and Planetary Scienci Outcrop 2

Sperling et al., 2013, Earth and Planetary Scienci Outcrop 2

Sperling et al., 2013, Earth and Planetary Scienci Outcrop 2

Sperling et al., 2013, Earth and Planetary Sciencı Outcrop 2

Sperling et al., 2013, Earth and Planetary Scienci Outcrop 2

Sperling et al., 2013, Earth and Planetary Scienci Outcrop 2

Sperling et al., 2013, Earth and Planetary Scienci Outcrop 2

Sperling et al., 2013, Earth and Planetary Sciencı Outcrop 2

Sperling et al., 2013, Earth and Planetary Scienci Outcrop 2

Sperling et al., 2013, Earth and Planetary Scienci Outcrop 2

Sperling et al., 2013, Earth and Planetary Scienci Outcrop 2

Sperling et al., 2013, Earth and Planetary Scienci Outcrop 2

Sperling et al., 2013, Earth and Planetary Scienci Outcrop 2

Sperling et al., 2013, Earth and Planetary Scienci Outcrop 2

Sperling et al., 2013, Earth and Planetary Scienci Outcrop 2

Sperling et al., 2013, Earth and Planetary Scienci Outcrop 2

Sperling et al., 2013, Earth and Planetary Sciencı Outcrop 2

Sperling et al., 2013, Earth and Planetary Scienci Outcrop 2

Sperling et al., 2013, Earth and Planetary Scienci Outcrop 2

Sperling et al., 2013, Earth and Planetary Scienci Outcrop 2

Sperling et al., 2013, Earth and Planetary Scienci Outcrop 3

Sperling et al., 2013, Earth and Planetary Scienci Outcrop 3

Sperling et al., 2013, Earth and Planetary Scienci Outcrop 3

Sperling et al., 2013, Earth and Planetary Scienci Outcrop 3

Sperling et al., 2013, Earth and Planetary Scienci Outcrop 3

Sperling et al., 2013, Earth and Planetary Scienci Outcrop 3

Sperling et al., 2013, Earth and Planetary Scienci Outcrop 3

Sperling et al., 2013, Earth and Planetary Scienci Outcrop 3
FeHR does not inc FeHR does not inc FeHR does not inc 
Sperling et al., 2013, Earth and Planetary Sciencı Outcrop 3

Sperling et al., 2013, Earth and Planetary Sciencı Outcrop 3

Sperling et al., 2013, Earth and Planetary Sciencı Outcrop 3

Sperling et al., 2013, Earth and Planetary Sciencı Outcrop 3

Sperling et al., 2013, Earth and Planetary Sciencı Outcrop 3

Sperling et al., 2013, Earth and Planetary Sciencı Outcrop 3

Sperling et al., 2013, Earth and Planetary Sciencı Outcrop 3

Sperling et al., 2013, Earth and Planetary Sciencı Outcrop 3

Sperling et al., 2013, Earth and Planetary Sciencı Outcrop 3

Sperling et al., 2013, Earth and Planetary Sciencı Outcrop 3

Sperling et al., 2013, Earth and Planetary Sciencı Outcrop 3

Sperling et al., 2013, Earth and Planetary Sciencı Outcrop 3

Sperling et al., 2013, Earth and Planetary Sciencı Outcrop 3

Sperling et al., 2013, Earth and Planetary Sciencı Outcrop 3

Sperling et al., 2013, Earth and Planetary Sciencı Outcrop 3

Sperling et al., 2013, Earth and Planetary Sciencı Outcrop 3

Sperling et al., 2013, Earth and Planetary Sciencı Outcrop 3

Sperling et al., 2013, Earth and Planetary Sciencı Outcrop 3

Sperling et al., 2013, Earth and Planetary Sciencı Outcrop 3

Sperling et al., 2013, Earth and Planetary Sciencı Outcrop 3

Sperling et al., 2013, Earth and Planetary Sciencı Outcrop 3

Sperling et al., 2013, Earth and Planetary Sciencı Outcrop 3

Sperling et al., 2013, Earth and Planetary Sciencı Outcrop 3

Sperling et al., 2013, Earth and Planetary Sciencı Outcrop 3

Sperling et al., 2013, Earth and Planetary Sciencı Outcrop 3

Sperling et al., 2013, Earth and Planetary Sciencı Outcrop 3

Sperling et al., 2013, Earth and Planetary Sciencı Outcrop 3

Sperling et al., 2013, Earth and Planetary Sciencı Outcrop 3

Sperling et al., 2013, Earth and Planetary Sciencı Outcrop 3

Sperling et al., 2013, Earth and Planetary Sciencı Outcrop 3

Sperling et al., 2013, Earth and Planetary Sciencı Outcrop 3

Sperling et al., 2013, Earth and Planetary Sciencı Outcrop 3

Sperling et al., 2013, Earth and Planetary Scienci Outcrop 3

Sperling et al., 2013, Earth and Planetary Sciencı Outcrop 3

Sperling et al., 2013, Earth and Planetary Sciencı Outcrop 3

Sperling et al., 2013, Earth and Planetary Sciencı Outcrop 3

Sperling et al., 2013, Earth and Planetary Scienci Outcrop 3

Sperling et al., 2013, Earth and Planetary Sciencı Outcrop 3

Sperling et al., 2013, Earth and Planetary Sciencı Outcrop 3

Sperling et al., 2013, Earth and Planetary Sciencı Outcrop 3

Sperling et al., 2013, Earth and Planetary Sciencı Outcrop 3 
Sperling et al., 2013, Earth and Planetary Sciencı Outcrop 3

Sperling et al., 2013, Earth and Planetary Sciencı Outcrop 3

Sperling et al., 2013, Earth and Planetary Sciencı Outcrop 3

Sperling et al., 2013, Earth and Planetary Sciencı Outcrop 3

Sperling et al., 2013, Earth and Planetary Sciencı Outcrop 3

Sperling et al., 2013, Earth and Planetary Sciencı Outcrop 3

Sperling et al., 2013, Earth and Planetary Sciencı Outcrop 3

Sperling et al., 2013, Earth and Planetary Sciencı Outcrop 3

Sperling et al., 2013, Earth and Planetary Sciencı Outcrop 3

Sperling et al., 2013, Earth and Planetary Sciencı Outcrop 3

Sperling et al., 2013, Earth and Planetary Sciencı Outcrop 3

Sperling et al., 2013, Earth and Planetary Sciencı Outcrop 3

Sperling et al., 2013, Earth and Planetary Sciencı Outcrop 3

Sperling et al., 2013, Earth and Planetary Sciencı Outcrop 3

Sperling et al., 2013, Earth and Planetary Sciencı Outcrop 3

Sperling et al., 2013, Earth and Planetary Sciencı Outcrop 3

Sperling et al., 2013, Earth and Planetary Sciencı Outcrop 3

Sperling et al., 2013, Earth and Planetary Sciencı Outcrop 3

Sperling et al., 2013, Earth and Planetary Sciencı Outcrop 3

Sperling et al., 2013, Earth and Planetary Sciencı Outcrop 3

Sperling et al., 2013, Earth and Planetary Sciencı Outcrop 3

Sperling et al., 2013, Earth and Planetary Sciencı Outcrop 1

Sperling et al., 2013, Earth and Planetary Sciencı Outcrop 1

Sperling et al., 2013, Earth and Planetary Sciencı Outcrop 1

Sperling et al., 2013, Earth and Planetary Sciencı Outcrop 1

Sperling et al., 2013, Earth and Planetary Sciencı Outcrop 1

Sperling et al., 2013, Earth and Planetary Scienci Outcrop 1

Sperling et al., 2013, Earth and Planetary Sciencı Outcrop 1

Sperling et al., 2013, Earth and Planetary Sciencı Outcrop 1

Sperling et al., 2013, Earth and Planetary Sciencı Outcrop 3

Sperling et al., 2013, Earth and Planetary Sciencı Outcrop 3

Sperling et al., 2013, Earth and Planetary Sciencı Outcrop 3

Sperling et al., 2013, Earth and Planetary Scienci Outcrop 3

Sperling et al., 2013, Earth and Planetary Sciencı Outcrop 3

Sperling et al., 2013, Earth and Planetary Sciencı Outcrop 3

Sperling et al., 2013, Earth and Planetary Sciencı Outcrop 3

Sperling et al., 2013, Earth and Planetary Scienci Outcrop 3

Sperling et al., 2013, Earth and Planetary Sciencı Outcrop 3

Sperling et al., 2013, Earth and Planetary Sciencı Outcrop 3

Sperling et al., 2013, Earth and Planetary Sciencı Outcrop 3

Sperling et al., 2013, Earth and Planetary Sciencı Outcrop 3 
Sperling et al., 2013, Earth and Planetary Sciencı Outcrop 3

Sperling et al., 2013, Earth and Planetary Sciencı Outcrop 3

Sperling et al., 2013, Earth and Planetary Sciencı Outcrop 3

Sperling et al., 2013, Earth and Planetary Sciencı Outcrop 3

Sperling et al., 2013, Earth and Planetary Sciencı Outcrop 3

Sperling et al., 2013, Earth and Planetary Scienci Outcrop 2

Sperling et al., 2013, Earth and Planetary Scienci Outcrop 2

Sperling et al., 2013, Earth and Planetary Scienci Outcrop 2

Sperling et al., 2013, Earth and Planetary Sciencı Outcrop 2

Sperling et al., 2013, Earth and Planetary Scienci Outcrop 2

Sperling et al., 2013, Earth and Planetary Scienci Outcrop 2

Sperling et al., 2013, Earth and Planetary Scienci Outcrop 2

Sperling et al., 2013, Earth and Planetary Sciencı Outcrop 2

Sperling et al., 2013, Earth and Planetary Scienci Outcrop 2

Sperling et al., 2013, Earth and Planetary Scienci Outcrop 2

Sperling et al., 2013, Earth and Planetary Scienci Outcrop 2

Sperling et al., 2013, Earth and Planetary Sciencı Outcrop 2

Sperling et al., 2013, Earth and Planetary Scienci Outcrop 2

Sperling et al., 2013, Earth and Planetary Scienci Outcrop 2

Sperling et al., 2013, Earth and Planetary Scienci Outcrop 2

Sperling et al., 2013, Earth and Planetary Sciencı Outcrop 2

Sperling et al., 2013, Earth and Planetary Scienci Outcrop 2

Sperling et al., 2013, Earth and Planetary Scienci Outcrop 2

Sperling et al., 2013, Earth and Planetary Scienci Outcrop 2

Sperling et al., 2013, Earth and Planetary Scienci Outcrop 2

Sperling et al., 2013, Earth and Planetary Scienci Outcrop 2

Sperling et al., 2013, Earth and Planetary Sciencı Outcrop 2

Sperling et al., 2013, Earth and Planetary Sciencı Outcrop 2

Sperling et al., 2013, Earth and Planetary Scienci Outcrop 2

Sperling et al., 2013, Earth and Planetary Scienci Outcrop 2

Sperling et al., 2013, Earth and Planetary Sciencı Outcrop 2

Sperling et al., 2013, Earth and Planetary Sciencı Outcrop 3

Sperling et al., 2013, Earth and Planetary Scienci Outcrop 3

Sperling et al., 2013, Earth and Planetary Sciencı Outcrop 3

Sperling et al., 2013, Earth and Planetary Sciencı Outcrop 3

Sperling et al., 2013, Earth and Planetary Sciencı Outcrop 3

Sperling et al., 2013, Earth and Planetary Scienci Outcrop 3

Sperling et al., 2013, Earth and Planetary Sciencı Outcrop 3

Sperling et al., 2013, Earth and Planetary Sciencı Outcrop 3

Sperling et al., 2013, Earth and Planetary Sciencı Outcrop 3

Sperling et al., 2013, Earth and Planetary Sciencı Outcrop 3 
Sperling et al., 2013, Earth and Planetary Sciencı Outcrop 3

Sperling et al., 2013, Earth and Planetary Sciencı Outcrop 3

Sperling et al., 2013, Earth and Planetary Sciencı Outcrop 3

Sperling et al., 2013, Earth and Planetary Sciencı Outcrop 3

Sperling et al., 2013, Earth and Planetary Sciencı Outcrop 3

Sperling et al., 2013, Earth and Planetary Sciencı Outcrop 3

Sperling et al., 2013, Earth and Planetary Sciencı Outcrop 3

Sperling et al., 2013, Earth and Planetary Sciencı Outcrop 3

Sperling et al., 2013, Earth and Planetary Sciencı Outcrop 3

Sperling et al., 2013, Earth and Planetary Sciencı Outcrop 3

Sperling et al., 2013, Earth and Planetary Sciencı Outcrop 3

Sperling et al., 2013, Earth and Planetary Sciencı Outcrop 3

Sperling et al., 2013, Earth and Planetary Scienci Outcrop 3

Sperling et al., 2013, Earth and Planetary Sciencı Outcrop 3

Sperling et al., 2013, Earth and Planetary Sciencı Outcrop 3

Sperling et al., 2013, Earth and Planetary Sciencı Outcrop 3

Canfield et al., 2008, Science Outcrop 3

Canfield et al., 2008, Science Outcrop 3

Canfield et al., 2008, Science Outcrop 3

Canfield et al., 2008, Science $\quad$ Outcrop 3

Canfield et al., 2008, Science Outcrop 3

Canfield et al., 2008, Science $\quad$ Outcrop 3

Canfield et al., 2008, Science Outcrop 3

Canfield et al., 2008, Science $\quad$ Outcrop 3

Canfield et al., 2008, Science Outcrop 3

Canfield et al., 2008, Science Outcrop 3

Canfield et al., 2008, Science Outcrop 3

Canfield et al., 2008, Science $\quad$ Outcrop 3

Canfield et al., 2008, Science Outcrop 3

Canfield et al., 2008, Science Outcrop 3

Canfield et al., 2008, Science Outcrop 3

Canfield et al., 2008, Science Outcrop 3

Canfield et al., 2008, Science Outcrop 3

Canfield et al., 2008, Science Outcrop 3

Canfield et al., 2008, Science $\quad$ Outcrop 3

This study $\quad$ Outcrop 3

This study Outcrop 3

This study $\quad$ Outcrop 3

This study Outcrop 3

This study $\quad$ Outcrop 3

This study $\quad$ Outcrop 3 
This study

This study

This study

This study

This study

This study

This study

This study

This study

This study

This study

This study

This study

This study

This study

This study

This study

This study

This study

This study

This study

This study

This study

This study

This study

This study

This study

This study

This study

This study

This study

This study

This study

This study

Hayes, 2013

Hayes, 2013

Hayes, 2013

Hayes, 2013

Hayes, 2013

Hayes, 2013

Hayes, 2013
Outcrop 3

Outcrop 3

Outcrop 3

Outcrop 3

Outcrop 3

Outcrop 3

Outcrop 3

Outcrop 3

Outcrop 3

Outcrop 3

Outcrop 3

Outcrop 3

Outcrop 3

Outcrop 3

Outcrop 3

Outcrop 3

Outcrop 3

Outcrop 3

Outcrop 3

Outcrop 3

Outcrop 3

Outcrop 3

Outcrop 3

Outcrop 3

Outcrop 3

Outcrop 3

Outcrop 3

Outcrop 3

Outcrop 3

Outcrop 3

Outcrop 3

Outcrop 3

Outcrop 3

Outcrop 3

Outcrop 2

Outcrop 2

Outcrop 2

Outcrop 2

Outcrop 2

Outcrop 1

Outcrop 1 
Hayes, 2013

Outcrop 1

Hayes, 2013

Outcrop 1

Hayes, 2013

Outcrop 1

Hayes, 2013

Outcrop 1

Hayes, 2013

Outcrop 1

Hayes, 2013

Outcrop 1

Hayes, 2013

Outcrop 1

Hayes, 2013

Outcrop 1

Hayes, 2013

Outcrop 1

Hayes, 2013

Outcrop 1

Hayes, 2013

Outcrop 1

Hayes, 2013

Outcrop 1

Hayes, 2013

Outcrop 1

Hayes, 2013

Outcrop 1

Hayes, 2013

Outcrop 1

Hayes, 2013

Outcrop 1

Hayes, 2013

Outcrop 1

Hayes, 2013

Outcrop 1

Hayes, 2013

Outcrop 1

Hayes, 2013

Outcrop 1

Hayes, 2013

Outcrop 1

Hayes, 2013

Outcrop 1

Hayes, 2013

Outcrop 1

Hayes, 2013

Outcrop 1

Hayes, 2013

Outcrop 1

Hayes, 2013

Outcrop 1

Outcrop 1

Hayes, 2013

Outcrop 1

Outcrop 1

Hayes, 2013

Outcrop 1

Hayes, 2013

Outcrop 1

Hayes, 2013

Hayes, 2013

Outcrop 1

Outcrop 1

Hayes, 2013

Outcrop 1

Outcrop 1

Outcrop 1

Outcrop 1

Outcrop 1

Outcrop 1

Outcrop 1

Outcrop 1

Hayes, 2013

1


Hayes, 2013

Outcrop 1

Hayes, 2013

Outcrop 1

Hayes, 2013

Outcrop 1

Hayes, 2013

Outcrop 1

Hayes, 2013

Outcrop 1

Hayes, 2013

Outcrop 1

Hayes, 2013

Outcrop 1

Hayes, 2013

Outcrop 1

Hayes, 2013

Outcrop 1

Hayes, 2013

Outcrop 1

Hayes, 2013

Outcrop 1

Hayes, 2013

Outcrop 1

Hayes, 2013

Outcrop 1

Hayes, 2013

Outcrop 1

Hayes, 2013

Outcrop 1

Hayes, 2013

Outcrop 1

Hayes, 2013

Outcrop 1

Hayes, 2013

Outcrop 1

Hayes, 2013

Outcrop 1

Hayes, 2013

Outcrop 1

Hayes, 2013

Outcrop 1

Hayes, 2013

Outcrop 1

Hayes, 2013

Outcrop 1

Hayes, 2013

Outcrop 1

Hayes, 2013

Outcrop 1

This study

This study

Outcrop 1

Outcrop 1

This study

This study

Outcrop 1

Outcrop 1

This study

This study

Outcrop 1

Outcrop 1

This study

This study

This study

This study

This study

This study

This study

This study

This study

This study

Outcrop 1

Outcrop 1

Outcrop 1

Outcrop 1

Outcrop 1

Outcrop 1

Outcrop 1

Outcrop 1

Outcrop 1

Outcrop 1 
This study

This study

This study

This study

This study

This study

This study

This study

This study

This study

This study

This study

This study

This study

This study

This study

This study

This study

This study

This study

This study

This study

This study

This study

This study

This study

This study

This study

This study

This study

This study

This study

This study

This study

This study

This study

This study

This study

This study

This study

This study
Outcrop 1

Outcrop 1

Outcrop 1

Outcrop 1

Outcrop 1

Outcrop 1

Outcrop 1

Outcrop 1

Outcrop 1

Outcrop 1

Outcrop 1

Outcrop 2

Outcrop 2

Outcrop 2

Outcrop 2

Outcrop 2

Outcrop 2

Outcrop 2

Outcrop 2

Outcrop 2

Outcrop 2

Outcrop 2

Outcrop 2

Outcrop 2

Outcrop 2

Outcrop 3

Outcrop 3

Outcrop 3

Outcrop 3

Outcrop 3

Outcrop 3

Outcrop 3

Outcrop 3

Outcrop 3

Outcrop 3

Outcrop 3

Outcrop 3

Outcrop 3

Outcrop 3

Outcrop 3

Outcrop 3 
This study

This study

This study

This study

This study

This study

This study

This study

This study

This study

This study

This study

This study

This study

This study

This study

This study

This study

This study

This study

This study

This study

This study

Canfield et al., 2008, Science

Canfield et al., 2008, Science

Canfield et al., 2008, Science

Canfield et al., 2008, Science

Canfield et al., 2008, Science

Canfield et al., 2008, Science

Canfield et al., 2008, Science

Canfield et al., 2008, Science

Canfield et al., 2008, Science

Canfield et al., 2008, Science

Canfield et al., 2008, Science

Canfield et al., 2008, Science

Canfield et al., 2008, Science

Canfield et al., 2008, Science

Canfield et al., 2008, Science

Li et al., 2010, Science

Li et al., 2010, Science

Li et al., 2010, Science
Outcrop 3

Outcrop 3

Outcrop 3

Outcrop 3

Outcrop 3

Outcrop 3

Outcrop 3

Outcrop 3

Outcrop 3

Outcrop 3

Outcrop 3

Outcrop 3

Outcrop 3

Outcrop 3

Outcrop 3

Outcrop 1

Outcrop 3

Outcrop 1

Outcrop 1

Outcrop 1

Outcrop 1

Outcrop 3

Outcrop 3

Outcrop 3

Outcrop 3

Outcrop 3

Outcrop 3

Outcrop 3

Outcrop 3

Outcrop 3

Outcrop 3

Outcrop 3

Outcrop 3

Outcrop 3

Outcrop 3

Outcrop 3

Outcrop 3

Outcrop 3

Outcrop 1

Outcrop 1

Outcrop 1 


\begin{tabular}{|c|c|}
\hline Li et al., 2010, Science & Outcrop \\
\hline Li et al., 2010, Science & Outcrop \\
\hline Li et al., 2010, Science & Outcrop \\
\hline Li et al., 2010, Science & Outcrop \\
\hline Li et al., 2010, Science & Outcrop \\
\hline Li et al., 2010, Science & Outcrop \\
\hline Li et al., 2010, Science & Outcrop \\
\hline Li et al., 2010, Science & Outcrop \\
\hline Li et al., 2010, Science & Outcrop \\
\hline Li et al., 2010, Science & Outcrop \\
\hline Li et al., 2010, Science & Outcrop \\
\hline Li et al., 2010, Science & Outcrop \\
\hline Li et al., 2010, Science & Outcrop \\
\hline Li et al., 2010, Science & Outcrop \\
\hline Li et al., 2010, Science & Outcrop \\
\hline Li et al., 2010, Science & Outcrop \\
\hline Li et al., 2010, Science & Outcrop \\
\hline Li et al., 2010, Science & Outcrop \\
\hline Li et al., 2010, Science & Outcrop \\
\hline Li et al., 2010, Science & Outcrop \\
\hline Li et al., 2010, Science & Outcrop \\
\hline Li et al., 2010, Science & Outcrop \\
\hline Li et al., 2010, Science & Outcrop \\
\hline Li et al., 2010, Science & Outcrop \\
\hline Li et al., 2010, Science & Outcrop \\
\hline Li et al., 2010, Science & Outcrop \\
\hline Li et al., 2010, Science & Outcrop \\
\hline Li et al., 2010, Science & Outcrop \\
\hline Li et al., 2010, Science & Outcrop \\
\hline Li et al., 2010, Science & Outcrop \\
\hline Li et al., 2010, Science & Outcrop \\
\hline Li et al., 2010, Science & Outcrop \\
\hline Li et al., 2010, Science & Outcrop \\
\hline Li et al., 2010, Science & Outcrop \\
\hline Li et al., 2010, Science & Outcrop \\
\hline Li et al., 2010, Science & Outcrop \\
\hline Li et al., 2010, Science & Outcrop \\
\hline Li et al., 2010, Science & Outcrop \\
\hline Li et al., 2010, Science & Outcrop \\
\hline Li et al., 2010, Science & Outcrop \\
\hline Li et al., 2010, Science & Outcrop \\
\hline
\end{tabular}




\begin{tabular}{|c|c|}
\hline Li et al., 2010, Science & Outcrop \\
\hline Li et al., 2010, Science & Outcrop \\
\hline Li et al., 2010, Science & Outcrop \\
\hline Li et al., 2010, Science & Outcrop \\
\hline Li et al., 2010, Science & Outcrop \\
\hline Li et al., 2010, Science & Outcrop \\
\hline Li et al., 2010, Science & Outcrop \\
\hline Li et al., 2010, Science & Outcrop \\
\hline Li et al., 2010, Science & Outcrop \\
\hline Li et al., 2010, Science & Outcrop \\
\hline Li et al., 2010, Science & Outcrop \\
\hline Li et al., 2010, Science & Outcrop \\
\hline Li et al., 2010, Science & Outcrop \\
\hline Li et al., 2010, Science & Outcrop \\
\hline Li et al., 2010, Science & Outcrop \\
\hline Li et al., 2010, Science & Outcrop \\
\hline Li et al., 2010, Science & Outcrop \\
\hline Li et al., 2010, Science & Outcrop \\
\hline Li et al., 2010, Science & Outcrop \\
\hline Li et al., 2010, Science & Outcrop \\
\hline Li et al., 2010, Science & Outcrop \\
\hline Li et al., 2010, Science & Outcrop \\
\hline Li et al., 2010, Science & Outcrop \\
\hline Li et al., 2010, Science & Outcrop \\
\hline Li et al., 2010, Science & Outcrop \\
\hline Li et al., 2010, Science & Outcrop \\
\hline Li et al., 2010, Science & Outcrop \\
\hline Li et al., 2010, Science & Outcrop \\
\hline Li et al., 2010, Science & Outcrop \\
\hline Li et al., 2010, Science & Outcrop \\
\hline Li et al., 2010, Science & Outcrop \\
\hline Li et al., 2010, Science & Outcrop \\
\hline Li et al., 2010, Science & Outcrop \\
\hline Li et al., 2010, Science & Outcrop \\
\hline Li et al., 2010, Science & Outcrop \\
\hline Sahoo et al., 2012, Nature & Outcrop \\
\hline Sahoo et al., 2012, Nature & Outcrop \\
\hline Sahoo et al., 2012, Nature & Outcrop \\
\hline Sahoo et al., 2012, Nature & Outcrop \\
\hline Sahoo et al., 2012, Nature & Outcrop \\
\hline Sahoo et al., 2012, Nature & Outcrop \\
\hline
\end{tabular}


Sahoo et al., 2012, Nature

Sahoo et al., 2012, Nature

Sahoo et al., 2012, Nature

Sahoo et al., 2012, Nature

Sahoo et al., 2012, Nature

Sahoo et al., 2012, Nature

Sahoo et al., 2012, Nature

Sahoo et al., 2012, Nature

Sahoo et al., 2012, Nature

Sahoo et al., 2012, Nature

Sahoo et al., 2012, Nature

Sahoo et al., 2012, Nature

Sahoo et al., 2012, Nature

Sahoo et al., 2012, Nature

Sahoo et al., 2012, Nature

Sahoo et al., 2012, Nature

Sahoo et al., 2012, Nature

Sahoo et al., 2012, Nature

Sahoo et al., 2012, Nature

Sahoo et al., 2012, Nature

Sahoo et al., 2012, Nature

Sahoo et al., 2012, Nature

Sahoo et al., 2012, Nature

Sahoo et al., 2012, Nature

Sahoo et al., 2012, Nature

Sahoo et al., 2012, Nature

Sahoo et al., 2012, Nature

Sahoo et al., 2012, Nature

Sahoo et al., 2012, Nature

Sahoo et al., 2012, Nature

Sahoo et al., 2012, Nature

Sahoo et al., 2012, Nature

Sahoo et al., 2012, Nature

Sahoo et al., 2012, Nature

Sahoo et al., 2012, Nature

Sahoo et al., 2012, Nature

Sahoo et al., 2012, Nature

Sahoo et al., 2012, Nature

Sahoo et al., 2012, Nature

Sahoo et al., 2012, Nature

Sahoo et al., 2012, Nature
Outcrop 3

Outcrop 3

Outcrop 3

Outcrop 3

Outcrop 3

Outcrop 3

Outcrop 3

Outcrop 3

Outcrop 3

Outcrop 3

Outcrop 3

Outcrop 3

Outcrop 3

Outcrop 3

Outcrop 3

Outcrop 3

Outcrop 3

Outcrop 3

Outcrop 3

Outcrop 3

Outcrop 3

Outcrop 3

Outcrop 3

Outcrop 3

Outcrop 3

Outcrop 3

Outcrop 3

Outcrop 3

Outcrop 3

Outcrop 3

Outcrop 3

Outcrop 3

Outcrop 3

Outcrop 3

Outcrop 3

Outcrop 3

Outcrop 3

Outcrop 3

Outcrop 3

Outcrop 3

Outcrop 3 
Sahoo et al., 2012, Nature

Sahoo et al., 2012, Nature

Sahoo et al., 2012, Nature

Sahoo et al., 2012, Nature

Sahoo et al., 2012, Nature

Sahoo et al., 2012, Nature

Sahoo et al., 2012, Nature

Sahoo et al., 2012, Nature

Sahoo et al., 2012, Nature

Sahoo et al., 2012, Nature

Sahoo et al., 2012, Nature

Sahoo et al., 2012, Nature

Sahoo et al., 2012, Nature

Sahoo et al., 2012, Nature

Sahoo et al., 2012, Nature

Sahoo et al., 2012, Nature

Sahoo et al., 2012, Nature

Sahoo et al., 2012, Nature

Shen et al., 2008, PNAS

Shen et al., 2008, PNAS

Shen et al., 2008, PNAS

Shen et al., 2008, PNAS

Shen et al., 2008, PNAS

Shen et al., 2008, PNAS

Shen et al., 2008, PNAS

Shen et al., 2008, PNAS

Shen et al., 2008, PNAS

Shen et al., 2008, PNAS

Shen et al., 2008, PNAS

Shen et al., 2008, PNAS

Shen et al., 2008, PNAS

Shen et al., 2008, PNAS

Shen et al., 2008, PNAS

Shen et al., 2008, PNAS

Shen et al., 2008, PNAS

Shen et al., 2008, PNAS

Shen et al., 2008, PNAS

Shen et al., 2008, PNAS

Shen et al., 2008, PNAS

Shen et al., 2008, PNAS

Shen et al., 2008, PNAS
Outcrop 3

Outcrop 3

Outcrop 3

Outcrop 3

Outcrop 3

Outcrop 3

Outcrop 3

Outcrop 3

Outcrop 3

Outcrop 3

Outcrop 3

Outcrop 3

Outcrop 3

Outcrop 3

Outcrop 3

Outcrop 3

Outcrop 3

Outcrop 3

Outcrop 3 Uses old extractic

Outcrop 3 Uses old extractic

Outcrop $\quad 3$ Uses old extractic

Outcrop 3 Uses old extractic

Outcrop $\quad 3$ Uses old extractic

Outcrop $\quad 3$ Uses old extractic

Outcrop $\quad 3$ Uses old extractic

Outcrop 3 Uses old extractic

Outcrop $\quad 3$ Uses old extractic

Outcrop 3 Uses old extractic

Outcrop 3 Uses old extractic

Outcrop $\quad 3$ Uses old extractic

Outcrop 3 Uses old extractic

Outcrop 3 Uses old extractic

Outcrop $\quad 3$ Uses old extractic

Outcrop $\quad 3$ Uses old extractic

Outcrop 3 Uses old extractic

Outcrop 3 Uses old extractic

Outcrop $\quad 3$ Uses old extractic

Outcrop $\quad 3$ Uses old extractic

Outcrop $\quad 3$ Uses old extractic

Outcrop $\quad 3$ Uses old extractic

Outcrop $\quad 3$ Uses old extractic 
Shen et al., 2008, PNAS

Shen et al., 2008, PNAS

Shen et al., 2008, PNAS

Shen et al., 2008, PNAS

Shen et al., 2008, PNAS

Shen et al., 2008, PNAS

Shen et al., 2008, PNAS

Shen et al., 2008, PNAS

Shen et al., 2008, PNAS

Shen et al., 2008, PNAS

Shen et al., 2008, PNAS

Wang et al., 2012, Chemical Geology

Wang et al., 2012, Chemical Geology

Wang et al., 2012, Chemical Geology

Wang et al., 2012, Chemical Geology

Wang et al., 2012, Chemical Geology

Wang et al., 2012, Chemical Geology

Wang et al., 2012, Chemical Geology

Wang et al., 2012, Chemical Geology

Wang et al., 2012, Chemical Geology

Wang et al., 2012, Chemical Geology

Wang et al., 2012, Chemical Geology

Wang et al., 2012, Chemical Geology

Wang et al., 2012, Chemical Geology

Johnston et al., 2013 Chemical Geology

Johnston et al., 2013 Chemical Geology

Johnston et al., 2013 Chemical Geology

Johnston et al., 2013 Chemical Geology

Johnston et al., 2013 Chemical Geology

Johnston et al., 2013 Chemical Geology

Johnston et al., 2013 Chemical Geology

Johnston et al., 2013 Chemical Geology

Johnston et al., 2013 Chemical Geology

Johnston et al., 2013 Chemical Geology

Johnston et al., 2013 Chemical Geology

Johnston et al., 2013 Chemical Geology

Johnston et al., 2013 Chemical Geology

Johnston et al., 2013 Chemical Geology

Johnston et al., 2013 Chemical Geology

Johnston et al., 2013 Chemical Geology

Johnston et al., 2013 Chemical Geology

\begin{tabular}{|c|c|c|}
\hline Outcrop & 3 & Uses old extractic \\
\hline Outcrop & 3 & Uses old extractic \\
\hline Outcrop & 3 & Uses old extractic \\
\hline Outcrop & 3 & Uses old extractic \\
\hline Outcrop & 3 & Uses old extractic \\
\hline Outcrop & 3 & Uses old extract \\
\hline Outcrop & 3 & Uses old extractic \\
\hline Outcrop & 3 & Uses old extract \\
\hline Outcrop & 3 & Uses old extractic \\
\hline Outcrop & 3 & Uses old extractic \\
\hline Outcrop & 3 & Uses old extrac \\
\hline Outcrop & 3 & \\
\hline Outcrop & 3 & \\
\hline Outcrop & 3 & \\
\hline Outcrop & 3 & \\
\hline Outcrop & 3 & \\
\hline Outcrop & 3 & \\
\hline Outcrop & 3 & \\
\hline Outcrop & 3 & \\
\hline Outcrop & 3 & \\
\hline Outcrop & 3 & \\
\hline Outcrop & 3 & \\
\hline Outcrop & 3 & \\
\hline Outcrop & 3 & \\
\hline Outcrop & 3 & \\
\hline Outcrop & 3 & \\
\hline Outcrop & 3 & \\
\hline Outcrop & 3 & \\
\hline Outcrop & 3 & \\
\hline Outcrop & 3 & \\
\hline Outcrop & 3 & \\
\hline Outcrop & 3 & \\
\hline Outcrop & 3 & \\
\hline Outcrop & 3 & \\
\hline Outcrop & 3 & \\
\hline Outcrop & 3 & \\
\hline Outcrop & 3 & \\
\hline Outcrop & 3 & \\
\hline Outcrop & 3 & \\
\hline Outcrop & 3 & \\
\hline Outcrop & 3 & \\
\hline
\end{tabular}


Johnston et al., 2013 Chemical Geology

Johnston et al., 2013 Chemical Geology

Johnston et al., 2013 Chemical Geology

Johnston et al., 2013 Chemical Geology

Johnston et al., 2013 Chemical Geology

Johnston et al., 2013 Chemical Geology

Johnston et al., 2013 Chemical Geology

Johnston et al., 2013 Chemical Geology

Johnston et al., 2013 Chemical Geology

Johnston et al., 2013 Chemical Geology

Johnston et al., 2013 Chemical Geology

Johnston et al., 2013 Chemical Geology

Johnston et al., 2013 Chemical Geology

Johnston et al., 2013 Chemical Geology

Johnston et al., 2013 Chemical Geology

Johnston et al., 2013 Chemical Geology

Johnston et al., 2013 Chemical Geology

Johnston et al., 2013 Chemical Geology

Johnston et al., 2013 Chemical Geology

Johnston et al., 2013 Chemical Geology

Johnston et al., 2013 Chemical Geology

Johnston et al., 2013 Chemical Geology

Johnston et al., 2013 Chemical Geology

Johnston et al., 2013 Chemical Geology

Johnston et al., 2013 Chemical Geology

Johnston et al., 2013 Chemical Geology

Johnston et al., 2013 Chemical Geology

Johnston et al., 2013 Chemical Geology

Johnston et al., 2013 Chemical Geology

Johnston et al., 2013 Chemical Geology

Johnston et al., 2013 Chemical Geology

Johnston et al., 2013 Chemical Geology

Johnston et al., 2013 Chemical Geology

Johnston et al., 2013 Chemical Geology

Johnston et al., 2013 Chemical Geology

Johnston et al., 2013 Chemical Geology

Johnston et al., 2013 Chemical Geology

Johnston et al., 2013 Chemical Geology

Johnston et al., 2013 Chemical Geology

Johnston et al., 2013 Chemical Geology

Johnston et al., 2013 Chemical Geology
Outcrop 3

Outcrop 3

Outcrop 3

Outcrop 3

Outcrop 3

Outcrop 3

Outcrop 3

Outcrop 3

Outcrop 3

Outcrop 3

Outcrop 3

Outcrop 3

Outcrop 3

Outcrop 3

Outcrop 3

Outcrop 3

Outcrop 3

Outcrop 3

Outcrop 3

Outcrop 3

Outcrop 3

Outcrop 3

Outcrop 3

Outcrop 3

Outcrop 3

Outcrop 3

Outcrop 3

Outcrop 3

Outcrop 3

Outcrop 3

Outcrop 3

Outcrop 3

Outcrop 3

Outcrop 3

Outcrop 3

Outcrop 3

Outcrop 3

Outcrop 3

Outcrop 3

Outcrop 3

Outcrop 3 
Johnston et al., 2013 Chemical Geology

Johnston et al., 2013 Chemical Geology

Johnston et al., 2013 Chemical Geology

Johnston et al., 2013 Chemical Geology

Johnston et al., 2013 Chemical Geology

Johnston et al., 2013 Chemical Geology

Johnston et al., 2013 Chemical Geology

Johnston et al., 2013 Chemical Geology

Johnston et al., 2013 Chemical Geology

Johnston et al., 2013 Chemical Geology

Johnston et al., 2013 Chemical Geology

Johnston et al., 2013 Chemical Geology

Johnston et al., 2013 Chemical Geology

Johnston et al., 2013 Chemical Geology

Johnston et al., 2013 Chemical Geology

Johnston et al., 2013 Chemical Geology

Johnston et al., 2013 Chemical Geology

Johnston et al., 2013 Chemical Geology

Johnston et al., 2013 Chemical Geology

Johnston et al., 2013 Chemical Geology

Johnston et al., 2013 Chemical Geology

Johnston et al., 2013 Chemical Geology

Johnston et al., 2013 Chemical Geology

Johnston et al., 2013 Chemical Geology

Johnston et al., 2013 Chemical Geology

Johnston et al., 2013 Chemical Geology

Johnston et al., 2013 Chemical Geology

Johnston et al., 2013 Chemical Geology

Johnston et al., 2013 Chemical Geology

Johnston et al., 2013 Chemical Geology

Johnston et al., 2013 Chemical Geology

Johnston et al., 2013 Chemical Geology

Johnston et al., 2013 Chemical Geology

Johnston et al., 2013 Chemical Geology

Johnston et al., 2013 Chemical Geology

Johnston et al., 2013 Chemical Geology

Johnston et al., 2013 Chemical Geology

Johnston et al., 2013 Chemical Geology

Johnston et al., 2013 Chemical Geology

Johnston et al., 2013 Chemical Geology

Johnston et al., 2013 Chemical Geology
Outcrop 3

Outcrop 3

Outcrop 3

Outcrop 3

Outcrop 3

Outcrop 3

Outcrop 3

Outcrop 3

Outcrop 3

Outcrop 3

Outcrop 3

Outcrop 3

Outcrop 3

Outcrop 3

Outcrop 3

Outcrop 3

Outcrop 3

Outcrop 3

Outcrop 3

Outcrop 3

Outcrop 3

Outcrop 3

Outcrop 3

Outcrop 3

Outcrop 3

Outcrop 3

Outcrop 3

Outcrop 3

Outcrop 3

Outcrop 3

Outcrop 3

Outcrop 3

Outcrop 3

Outcrop 3

Outcrop 3

Outcrop 3

Outcrop 3

Outcrop 3

Outcrop 3

Outcrop 3

Outcrop 3 
Johnston et al., 2013 Chemical Geology

Johnston et al., 2013 Chemical Geology

Johnston et al., 2013 Chemical Geology

Johnston et al., 2013 Chemical Geology

Johnston et al., 2013 Chemical Geology

Johnston et al., 2013 Chemical Geology

Johnston et al., 2013 Chemical Geology

Johnston et al., 2013 Chemical Geology

Johnston et al., 2013 Chemical Geology

Johnston et al., 2013 Chemical Geology

Johnston et al., 2013 Chemical Geology

Johnston et al., 2013 Chemical Geology

Johnston et al., 2013 Chemical Geology

Johnston et al., 2013 Chemical Geology

Johnston et al., 2013 Chemical Geology

Johnston et al., 2013 Chemical Geology

Johnston et al., 2013 Chemical Geology

Johnston et al., 2013 Chemical Geology

Johnston et al., 2013 Chemical Geology

Johnston et al., 2013 Chemical Geology

Johnston et al., 2013 Chemical Geology

Johnston et al., 2013 Chemical Geology

Johnston et al., 2013 Chemical Geology

Johnston et al., 2013 Chemical Geology

Johnston et al., 2013 Chemical Geology

Johnston et al., 2013 Chemical Geology

Johnston et al., 2013 Chemical Geology

Johnston et al., 2013 Chemical Geology

Johnston et al., 2013 Chemical Geology

Johnston et al., 2013 Chemical Geology

Johnston et al., 2013 Chemical Geology

Johnston et al., 2013 Chemical Geology

Johnston et al., 2013 Chemical Geology

Johnston et al., 2013 Chemical Geology

Johnston et al., 2013 Chemical Geology

Johnston et al., 2013 Chemical Geology

Johnston et al., 2013 Chemical Geology

Johnston et al., 2013 Chemical Geology

Johnston et al., 2013 Chemical Geology

Johnston et al., 2013 Chemical Geology

Johnston et al., 2013 Chemical Geology
Outcrop 3

Outcrop 3

Outcrop 3

Outcrop 3

Outcrop 3

Outcrop 3

Outcrop 3

Outcrop 3

Outcrop 3

Outcrop 3

Outcrop 3

Outcrop 3

Outcrop 3

Outcrop 3

Outcrop 3

Outcrop 3

Outcrop 3

Outcrop 3

Outcrop 3

Outcrop 3

Outcrop 3

Outcrop 3

Outcrop 3

Outcrop 3

Outcrop 3

Outcrop 3

Outcrop 3

Outcrop 3

Outcrop 3

Outcrop 3

Outcrop 3

Outcrop 3

Outcrop 3

Outcrop 3

Outcrop 3

Outcrop 3

Outcrop 3

Outcrop 3

Outcrop 3

Outcrop 3

Outcrop 3 
Johnston et al., 2013 Chemical Geology

Johnston et al., 2013 Chemical Geology

Johnston et al., 2013 Chemical Geology

Johnston et al., 2013 Chemical Geology

Johnston et al., 2013 Chemical Geology

Johnston et al., 2013 Chemical Geology

Johnston et al., 2013 Chemical Geology

Johnston et al., 2013 Chemical Geology

Johnston et al., 2013 Chemical Geology

Johnston et al., 2013 Chemical Geology

Johnston et al., 2013 Chemical Geology

Johnston et al., 2013 Chemical Geology

Johnston et al., 2013 Chemical Geology

Johnston et al., 2013 Chemical Geology

Johnston et al., 2013 Chemical Geology

Johnston et al., 2013 Chemical Geology

Johnston et al., 2013 Chemical Geology

Johnston et al., 2013 Chemical Geology

Johnston et al., 2013 Chemical Geology

Johnston et al., 2013 Chemical Geology

Johnston et al., 2013 Chemical Geology

Johnston et al., 2013 Chemical Geology

Johnston et al., 2013 Chemical Geology

Johnston et al., 2013 Chemical Geology

Johnston et al., 2013 Chemical Geology

Johnston et al., 2013 Chemical Geology

Johnston et al., 2013 Chemical Geology

Johnston et al., 2013 Chemical Geology

Johnston et al., 2013 Chemical Geology

Johnston et al., 2013 Chemical Geology

Johnston et al., 2013 Chemical Geology

Johnston et al., 2013 Chemical Geology

Johnston et al., 2013 Chemical Geology

Johnston et al., 2013 Chemical Geology

Johnston et al., 2013 Chemical Geology

Johnston et al., 2013 Chemical Geology

Johnston et al., 2013 Chemical Geology

Johnston et al., 2013 Chemical Geology

This study

This study

This study
Outcrop 3

Outcrop 3

Outcrop 3

Outcrop 3

Outcrop 3

Outcrop 3

Outcrop 3

Outcrop 3

Outcrop 3

Outcrop 3

Outcrop 3

Outcrop 3

Outcrop 3

Outcrop 3

Outcrop 3

Outcrop 3

Outcrop 3

Outcrop 3

Outcrop 1

Outcrop 1

Outcrop 1

Outcrop 1

Outcrop 1

Outcrop 1

Outcrop 2

Outcrop 2

Outcrop 2

Outcrop 2

Outcrop 2

Outcrop 2

Outcrop 2

Outcrop 2

Outcrop 2

Outcrop 2

Outcrop 2

Outcrop 2

Outcrop 2

Outcrop 2

Outcrop 2

Outcrop 2

Outcrop 1 
This study

This study

This study

This study

This study

This study

This study

This study

This study

This study

This study

This study

This study

This study

This study

This study

This study

This study

This study

This study

This study

This study

This study

This study

This study

This study

This study

This study

This study

This study

This study

This study

This study

This study

This study

This study

This study

This study

This study

This study

This study
Outcrop 1

Outcrop 1

Outcrop 2

Outcrop 2

Outcrop 2

Outcrop 2

Outcrop 3

Outcrop 3

Outcrop 3

Outcrop 3

Outcrop 3

Outcrop 3

Outcrop 3

Outcrop 3

Outcrop 3

Outcrop 3

Outcrop 3

Outcrop 3

Outcrop 3

Outcrop 3

Outcrop 3

Outcrop 3

Outcrop 3

Outcrop 3

Outcrop 3

Outcrop 3

Outcrop 3

Outcrop 3

Outcrop 3

Outcrop 3

Outcrop 3

Outcrop 3

Outcrop 3

Outcrop 3

Outcrop 3

Outcrop 3

Outcrop 3

Outcrop 3

Outcrop 3

Outcrop 3

Outcrop 3 
This study

Outcrop 3

This study

Outcrop 3

This study

Outcrop 3

This study

Outcrop 3

This study

Outcrop 3

This study

Outcrop 3

This study

Outcrop 3

This study

Outcrop 3

This study

Outcrop 3

This study

Outcrop 3

This study

Outcrop 3

This study

Outcrop 2

This study

Outcrop 2

This study

Outcrop 2

This study

Outcrop 2

This study

Outcrop 2

This study

Outcrop 2

This study

Outcrop 2

This study

Outcrop 2

This study

Outcrop 2

This study

Outcrop 2

This study

Outcrop 2

This study

Outcrop 2

Johnston et al., 2012, Earth and Planetary Scienı Core 2

Johnston et al., 2012, Earth and Planetary Scienı Core 2

Johnston et al., 2012, Earth and Planetary Scienı Core 2

Johnston et al., 2012, Earth and Planetary Scienı Core 2

Johnston et al., 2012, Earth and Planetary Scienı Core 2

Johnston et al., 2012, Earth and Planetary Scienı Core 2

Johnston et al., 2012, Earth and Planetary Scienı Core 2

Johnston et al., 2012, Earth and Planetary Scienı Core 2

Johnston et al., 2012, Earth and Planetary Scienı Core 2

Johnston et al., 2012, Earth and Planetary Scienı Core 2

Johnston et al., 2012, Earth and Planetary Scienı Core 2

Johnston et al., 2012, Earth and Planetary Scienı Core 2

Johnston et al., 2012, Earth and Planetary Scienı Core 2

Johnston et al., 2012, Earth and Planetary ScienıCore 2

Johnston et al., 2012, Earth and Planetary Scienı Core 2

Johnston et al., 2012, Earth and Planetary Scienı Core 2

Johnston et al., 2012, Earth and Planetary Scienı Core 2

Johnston et al., 2012, Earth and Planetary ScienıCore 2 
Johnston et al., 2012, Earth and Planetary ScienıCore 2

Johnston et al., 2012, Earth and Planetary Scienı Core 2

Johnston et al., 2012, Earth and Planetary ScienıCore 2

Johnston et al., 2012, Earth and Planetary Scienı Core 2

Johnston et al., 2012, Earth and Planetary ScienıCore 2

Johnston et al., 2012, Earth and Planetary Scienı Core 2

Johnston et al., 2012, Earth and Planetary ScienıCore 2

Johnston et al., 2012, Earth and Planetary Scienı Core 2

Johnston et al., 2012, Earth and Planetary ScienıCore 2

Johnston et al., 2012, Earth and Planetary Scienı Core 1

Johnston et al., 2012, Earth and Planetary Scienı Core 1

Johnston et al., 2012, Earth and Planetary Scienı Core 1

Johnston et al., 2012, Earth and Planetary Scienı Core 1

Johnston et al., 2012, Earth and Planetary Scienı Core 1

Johnston et al., 2012, Earth and Planetary Scienı Core 1

Johnston et al., 2012, Earth and Planetary Scienı Core 1

Johnston et al., 2012, Earth and Planetary Scienı Core 1

Johnston et al., 2012, Earth and Planetary Scienı Core 1

Johnston et al., 2012, Earth and Planetary Scienı Core 1

Johnston et al., 2012, Earth and Planetary Scienı Core 1

Johnston et al., 2012, Earth and Planetary Scienı Core 1

Johnston et al., 2012, Earth and Planetary Scienı Core 1

Johnston et al., 2012, Earth and Planetary Scienı Core 1

Johnston et al., 2012, Earth and Planetary Scienı Core 1

Johnston et al., 2012, Earth and Planetary Scienı Core 1

Johnston et al., 2012, Earth and Planetary Scienı Core 1

Johnston et al., 2012, Earth and Planetary Scienı Core 2

Johnston et al., 2012, Earth and Planetary Scienı Core 2

Johnston et al., 2012, Earth and Planetary Scienı Core 2

Johnston et al., 2012, Earth and Planetary Scienı Core 2

Johnston et al., 2012, Earth and Planetary Scienı Core 2

Johnston et al., 2012, Earth and Planetary Scienı Core 2

Johnston et al., 2012, Earth and Planetary Scienı Core 2

Johnston et al., 2012, Earth and Planetary Scienı Core 2

Johnston et al., 2012, Earth and Planetary Scienı Core 2

Johnston et al., 2012, Earth and Planetary Scienı Core 2

Johnston et al., 2012, Earth and Planetary Scienı Core 2

Johnston et al., 2012, Earth and Planetary Scienı Core 2

Johnston et al., 2012, Earth and Planetary Scienı Core 2

Johnston et al., 2012, Earth and Planetary Scienı Core 2

Johnston et al., 2012, Earth and Planetary ScienıCore 2 
Johnston et al., 2012, Earth and Planetary Scienı Core 2

Johnston et al., 2012, Earth and Planetary Scienı Core 2

Johnston et al., 2012, Earth and Planetary Scienı Core 2

Johnston et al., 2012, Earth and Planetary Scienı Core 2

Johnston et al., 2012, Earth and Planetary Scienı Core 2

Johnston et al., 2012, Earth and Planetary Scienı Core 2

Johnston et al., 2012, Earth and Planetary Scienı Core 2

Johnston et al., 2012, Earth and Planetary Scienı Core 2

Johnston et al., 2012, Earth and Planetary Scienı Core 2

Johnston et al., 2012, Earth and Planetary Scienı Core 2

Johnston et al., 2012, Earth and Planetary Scienı Core 2

Johnston et al., 2012, Earth and Planetary Scienı Core 2

Johnston et al., 2012, Earth and Planetary Scienı Core 1

Johnston et al., 2012, Earth and Planetary Scienı Core 1

Johnston et al., 2012, Earth and Planetary Scienı Core 1

Johnston et al., 2012, Earth and Planetary Scienı Core 1

Johnston et al., 2012, Earth and Planetary Scienı Core 1

Johnston et al., 2012, Earth and Planetary Scienı Core 1

Johnston et al., 2012, Earth and Planetary Scienı Core 1

Johnston et al., 2012, Earth and Planetary Scienı Core 1

Johnston et al., 2012, Earth and Planetary Scienı Core 1

Johnston et al., 2012, Earth and Planetary Scienı Core 1

Johnston et al., 2012, Earth and Planetary Scienı Core 1

Johnston et al., 2012, Earth and Planetary Scienı Core 1

Johnston et al., 2012, Earth and Planetary Scienı Core 1

Canfield et al., 2008, Science Outcrop 3

Canfield et al., 2008, Science Outcrop 3

Canfield et al., 2008, Science Outcrop 3

Canfield et al., 2008, Science Outcrop 3

Canfield et al., 2008, Science Outcrop 3

Canfield et al., 2008, Science Outcrop 3

Canfield et al., 2008, Science Outcrop 3

Canfield et al., 2008, Science Outcrop 3

Canfield et al., 2008, Science Outcrop 3

Canfield et al., 2008, Science Outcrop 3

Canfield et al., 2008, Science Outcrop 3

Canfield et al., 2008, Science Outcrop 3

Canfield et al., 2008, Science Outcrop 3

Canfield et al., 2008, Science Outcrop 3

Canfield et al., 2008, Science Outcrop 3

Canfield et al., 2008, Science $\quad$ Outcrop 3 
Canfield et al., 2008, Science

Outcrop 3

Canfield et al., 2008, Science

Outcrop 3

Canfield et al., 2008, Science

Outcrop 3

Canfield et al., 2008, Science

Outcrop 3

Canfield et al., 2008, Science

Outcrop 3

Canfield et al., 2008, Science

Outcrop 3

Canfield et al., 2008, Science

Outcrop 3

Canfield et al., 2008, Science

Outcrop 3

Canfield et al., 2008, Science

Outcrop 3

Canfield et al., 2008, Science

Outcrop 3

Canfield et al., 2008, Science

Outcrop 3

Canfield et al., 2008, Science

Outcrop 3

Canfield et al., 2008, Science

Outcrop 3

Canfield et al., 2008, Science

Outcrop 3

Canfield et al., 2008, Science

Outcrop 3

Canfield et al., 2008, Science

Outcrop 3

Canfield et al., 2008, Science

Outcrop 3

Canfield et al., 2008, Science

Outcrop 3

Canfield et al., 2008, Science

Outcrop 3

Canfield et al., 2008, Science

Outcrop 3

Canfield et al., 2008, Science

Outcrop 3

Canfield et al., 2008, Science

Outcrop 3

Canfield et al., 2008, Science

Outcrop 3

Canfield et al., 2008, Science

Outcrop 3

Canfield et al., 2008, Science

Outcrop 3

Canfield et al., 2008, Science

Outcrop 3

Canfield et al., 2008, Science

Outcrop 3

Canfield et al., 2008, Science

Outcrop 3

Canfield et al., 2008, Science

Outcrop 3

Canfield et al., 2008, Science

Outcrop 3

Canfield et al., 2008, Science

Outcrop 3

Canfield et al., 2008, Science

Outcrop 3

Canfield et al., 2008, Science

Outcrop 3

Canfield et al., 2008, Science

Outcrop 3

Canfield et al., 2008, Science

Outcrop 3

Canfield et al., 2008, Science

Outcrop 3

Canfield et al., 2008, Science

Outcrop 3

Canfield et al., 2008, Science

Outcrop 3

Canfield et al., 2008, Science

Outcrop 3

Canfield et al., 2008, Science

Outcrop 3

Canfield et al., 2008, Science

Outcrop 
Canfield et al., 2008, Science

Outcrop 3

Canfield et al., 2008, Science

Outcrop 3

Canfield et al., 2008, Science

Outcrop 3

Canfield et al., 2008, Science

Outcrop 3

Canfield et al., 2008, Science

Outcrop 3

Canfield et al., 2008, Science

Outcrop 3

Canfield et al., 2008, Science

Outcrop 3

Canfield et al., 2008, Science

Outcrop 3

Canfield et al., 2008, Science

Outcrop 3

Canfield et al., 2008, Science

Outcrop 3

Canfield et al., 2008, Science

Outcrop 3

Canfield et al., 2008, Science

Outcrop 3

Canfield et al., 2008, Science

Outcrop 3

Canfield et al., 2008, Science

Outcrop 3

Canfield et al., 2008, Science

Outcrop 3

Canfield et al., 2008, Science

Outcrop 3

Canfield et al., 2008, Science

Outcrop 3

Canfield et al., 2008, Science

Outcrop 3

Canfield et al., 2008, Science

Outcrop 3

Canfield et al., 2008, Science

Outcrop 3

Canfield et al., 2008, Science

Outcrop 3

Canfield et al., 2008, Science

Outcrop 3

Canfield et al., 2008, Science

Outcrop 3

Canfield et al., 2008, Science

Outcrop 3

Canfield et al., 2008, Science

Outcrop 3

Canfield et al., 2008, Science

Outcrop 3

Canfield et al., 2008, Science

Outcrop 3

Canfield et al., 2008, Science

Outcrop 3

Canfield et al., 2008, Science

Outcrop 3

Canfield et al., 2008, Science

Outcrop 3

Canfield et al., 2008, Science

Outcrop 3

Canfield et al., 2008, Science

Outcrop 3

Canfield et al., 2008, Science

Outcrop 3

Canfield et al., 2008, Science

Outcrop 3

Canfield et al., 2008, Science

Outcrop 3

Canfield et al., 2008, Science

Outcrop 3

Canfield et al., 2008, Science

Outcrop 3

Canfield et al., 2008, Science

Outcrop 3

Canfield et al., 2008, Science

Outcrop 3

Canfield et al., 2008, Science

Outcrop 3

Canfield et al., 2008, Science

Outcrop 
Canfield et al., 2008, Science

Outcrop 3

Canfield et al., 2008, Science

Outcrop 3

Canfield et al., 2008, Science

Outcrop 3

Canfield et al., 2008, Science

Outcrop 3

Canfield et al., 2008, Science

Outcrop 3

Canfield et al., 2008, Science

Outcrop 3

Canfield et al., 2008, Science

Outcrop 3

Canfield et al., 2008, Science

Outcrop 3

Canfield et al., 2008, Science

Outcrop 3

Canfield et al., 2008, Science

Outcrop 3

Canfield et al., 2008, Science

Outcrop 3

Canfield et al., 2008, Science

Outcrop 3

Canfield et al., 2008, Science

Outcrop 3

Canfield et al., 2008, Science

Outcrop 3

Canfield et al., 2008, Science

Outcrop 3

Canfield et al., 2008, Science

Outcrop 3

Canfield et al., 2008, Science

Outcrop 3

Canfield et al., 2008, Science

Outcrop 3

Canfield et al., 2008, Science

Outcrop 3

Canfield et al., 2008, Science

Outcrop 3

Canfield et al., 2008, Science

Outcrop 3

Canfield et al., 2008, Science

Outcrop 3

Canfield et al., 2008, Science

Outcrop 3

Canfield et al., 2008, Science

Outcrop 3

Canfield et al., 2008, Science

Outcrop 3

Canfield et al., 2008, Science

Outcrop 3

Canfield et al., 2008, Science

Outcrop 3

Canfield et al., 2008, Science

Outcrop 3

Canfield et al., 2008, Science

Outcrop 3

Canfield et al., 2008, Science

Outcrop 3

Canfield et al., 2008, Science

Outcrop 3

Canfield et al., 2008, Science

Outcrop 3

Canfield et al., 2008, Science

Outcrop 3

Canfield et al., 2008, Science

Outcrop 3

Canfield et al., 2008, Science

Outcrop 3

Canfield et al., 2008, Science

Outcrop 3

Canfield et al., 2008, Science

Outcrop 3

Canfield et al., 2008, Science

Outcrop 3

Canfield et al., 2008, Science

Outcrop 3

Canfield et al., 2008, Science

Outcrop 3

Canfield et al., 2008, Science

Outcrop 
Canfield et al., 2008, Science

Outcrop 3

Canfield et al., 2008, Science

Outcrop 3

Canfield et al., 2008, Science

Outcrop 3

Canfield et al., 2008, Science

Outcrop 3

Canfield et al., 2008, Science

Outcrop 3

Canfield et al., 2008, Science

Outcrop 3

Canfield et al., 2008, Science

Outcrop 3

Canfield et al., 2008, Science

Outcrop 3

Canfield et al., 2008, Science

Outcrop 3

Canfield et al., 2008, Science

Outcrop 3

Canfield et al., 2008, Science

Outcrop 3

Canfield et al., 2008, Science

Outcrop 3

Canfield et al., 2008, Science

Outcrop 3

Canfield et al., 2008, Science

Outcrop 3

Canfield et al., 2008, Science

Outcrop 3

Canfield et al., 2008, Science

Outcrop 3

Canfield et al., 2008, Science

Outcrop 3

Canfield et al., 2008, Science

Outcrop 3

Canfield et al., 2008, Science

Outcrop 3

Canfield et al., 2008, Science

Outcrop 3

Canfield et al., 2008, Science

Outcrop 3

Canfield et al., 2008, Science

Outcrop 3

Canfield et al., 2008, Science

Outcrop 3

Canfield et al., 2008, Science

Outcrop 3

Canfield et al., 2008, Science

Outcrop 3

Canfield et al., 2008, Science

Outcrop 3

Canfield et al., 2008, Science

Outcrop 3

Canfield et al., 2008, Science

Outcrop 3

Canfield et al., 2008, Science

Outcrop 3

Canfield et al., 2008, Science

Outcrop 3

Canfield et al., 2008, Science

Outcrop 3

Canfield et al., 2008, Science

Outcrop 3

Canfield et al., 2008, Science

Outcrop 3

Canfield et al., 2008, Science

Outcrop 3

Canfield et al., 2008, Science

Outcrop 3

Canfield et al., 2008, Science

Outcrop 3

Canfield et al., 2008, Science

Outcrop 3

Canfield et al., 2008, Science

Outcrop 3

Canfield et al., 2008, Science

Outcrop 3

Canfield et al., 2008, Science

Outcrop 3

Canfield et al., 2008, Science

Outcrop 
Canfield et al., 2008, Science

Canfield et al., 2008, Science

Canfield et al., 2008, Science

Canfield et al., 2008, Science

Canfield et al., 2008, Science

Canfield et al., 2008, Science

Canfield et al., 2008, Science

Canfield et al., 2008, Science

Canfield et al., 2008, Science

Canfield et al., 2007, Science

Canfield et al., 2007, Science

Canfield et al., 2007, Science

Canfield et al., 2007, Science

Canfield et al., 2007, Science

Canfield et al., 2007, Science

Canfield et al., 2007, Science

Canfield et al., 2007, Science

Canfield et al., 2007, Science

Canfield et al., 2007, Science

Canfield et al., 2007, Science

Canfield et al., 2007, Science

Canfield et al., 2007, Science

Canfield et al., 2007, Science

Canfield et al., 2007, Science

Canfield et al., 2007, Science

Canfield et al., 2007, Science

Canfield et al., 2007, Science

Canfield et al., 2007, Science

Canfield et al., 2007, Science

Canfield et al., 2007, Science

Canfield et al., 2007, Science

Canfield et al., 2007, Science

Canfield et al., 2007, Science

Canfield et al., 2007, Science

Canfield et al., 2007, Science

Canfield et al., 2007, Science

Canfield et al., 2007, Science

Canfield et al., 2007, Science

Canfield et al., 2007, Science

Canfield et al., 2007, Science

Canfield et al., 2007, Science
Outcrop 3

Outcrop 3

Outcrop 3

Outcrop 3

Outcrop 3

Outcrop 3

Outcrop 3

Outcrop 3

Outcrop 3

Outcrop 3

Outcrop 3

Outcrop 3

Outcrop 3

Outcrop 3

Outcrop 3

Outcrop 3

Outcrop 3

Outcrop 3

Outcrop 3

Outcrop 3

Outcrop 3

Outcrop 3

Outcrop 3

Outcrop 3

Outcrop 3

Outcrop 3

Outcrop 3

Outcrop 3

Outcrop 3

Outcrop 3

Outcrop 3

Outcrop 3

Outcrop 3

Outcrop 3

Outcrop 3

Outcrop 3

Outcrop 3

Outcrop 3

Outcrop 3

Outcrop 3

Outcrop 3
Diamictite-not a $\xi$

Diamictite-not a $\varepsilon$

Diamictite-not a $\xi$

Diamictite-not a $\varepsilon$

Diamictite-not a $\varepsilon$

Diamictite-not a $\xi$

Diamictite-not a $\xi$

Diamictite-not a $\varepsilon$

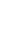

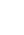

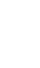

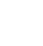

3

3

3

3

3

3
3


Canfield et al., 2007, Science

Canfield et al., 2007, Science

Canfield et al., 2007, Science

Canfield et al., 2007, Science

Canfield et al., 2007, Science

Canfield et al., 2007, Science

Canfield et al., 2007, Science

Canfield et al., 2007, Science

Canfield et al., 2007, Science

Canfield et al., 2007, Science

Canfield et al., 2007, Science

Canfield et al., 2007, Science

Canfield et al., 2007, Science

Canfield et al., 2007, Science

Canfield et al., 2007, Science

Canfield et al., 2007, Science

Canfield et al., 2007, Science

Canfield et al., 2007, Science

Canfield et al., 2007, Science

Canfield et al., 2007, Science

Canfield et al., 2007, Science

Canfield et al., 2007, Science

Canfield et al., 2007, Science

Canfield et al., 2007, Science

Canfield et al., 2007, Science

Canfield et al., 2007, Science

Canfield et al., 2007, Science

Canfield et al., 2007, Science

Canfield et al., 2007, Science

Canfield et al., 2007, Science

Canfield et al., 2007, Science

Canfield et al., 2007, Science

Canfield et al., 2007, Science

Canfield et al., 2007, Science

Canfield et al., 2007, Science

Canfield et al., 2007, Science

Canfield et al., 2007, Science

Canfield et al., 2007, Science

Canfield et al., 2007, Science

Canfield et al., 2007, Science

Canfield et al., 2007, Science
Outcrop 3

Outcrop 3

Outcrop 3

Outcrop 3

Outcrop 3

Outcrop 3

Outcrop 3

Outcrop 3

Outcrop 3

Outcrop 3

Outcrop 3

Outcrop 3

Outcrop 3

Outcrop 3

Outcrop 3

Outcrop 3

Outcrop 3

Outcrop 3

Outcrop 3

Outcrop 3

Outcrop 3

Outcrop 3

Outcrop 3

Outcrop 3

Outcrop 3

Outcrop 3

Outcrop 3

Outcrop 3

Outcrop 3

Outcrop 3

Outcrop 3

Outcrop 3

Outcrop 3

Outcrop 3

Outcrop 3

Outcrop 3

Outcrop 3

Outcrop 3

Outcrop 3

Outcrop 3

Outcrop 3

3

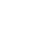

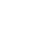

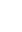

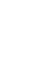

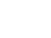

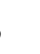

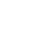

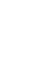

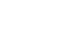

3

3

3


Canfield et al., 2007, Science

Outcrop 3

Canfield et al., 2007, Science

Outcrop 3

Canfield et al., 2007, Science

Outcrop 3

Canfield et al., 2007, Science

Outcrop 3

Canfield et al., 2007, Science

Outcrop 3

Canfield et al., 2007, Science

Outcrop 3

Canfield et al., 2007, Science

Outcrop 3

Canfield et al., 2007, Science

Outcrop 3

Canfield et al., 2007, Science

Outcrop 3

Canfield et al., 2007, Science

Outcrop 3

Canfield et al., 2007, Science

Outcrop 3

Canfield et al., 2007, Science

Outcrop 3

Canfield et al., 2007, Science

Outcrop 3

Canfield et al., 2007, Science

Outcrop 3

Canfield et al., 2007, Science

Outcrop 3

Canfield et al., 2007, Science

Outcrop 3

Canfield et al., 2007, Science

Outcrop 3

Canfield et al., 2007, Science

Outcrop 3

Canfield et al., 2007, Science

Outcrop 3

Canfield et al., 2007, Science

Outcrop 3

Canfield et al., 2007, Science

Outcrop 3

Canfield et al., 2007, Science

Outcrop 3

Canfield et al., 2007, Science

Outcrop 3

Canfield et al., 2007, Science

Outcrop 3

Canfield et al., 2007, Science

Outcrop 3

Canfield et al., 2007, Science

Outcrop 3

Canfield et al., 2007, Science

Outcrop 3

Canfield et al., 2007, Science

Outcrop 3

Canfield et al., 2007, Science

Outcrop 3

Canfield et al., 2007, Science

Outcrop 3

Canfield et al., 2007, Science

Outcrop 3

Canfield et al., 2007, Science

Outcrop 3

Canfield et al., 2007, Science

Outcrop 3

Canfield et al., 2007, Science

Outcrop 3

Canfield et al., 2007, Science

Outcrop 3

Canfield et al., 2007, Science

Outcrop 3

Canfield et al., 2007, Science

Outcrop 3

Canfield et al., 2007, Science

Outcrop 3

Canfield et al., 2007, Science

Outcrop 3

Canfield et al., 2007, Science

Outcrop 3

Canfield et al., 2007, Science

Outcrop 
Canfield et al., 2007, Science

Outcrop 3

Canfield et al., 2007, Science

Outcrop 3

Canfield et al., 2007, Science

Outcrop 3

Canfield et al., 2007, Science

Outcrop 3

Canfield et al., 2007, Science

Outcrop 3

Canfield et al., 2007, Science

Outcrop 3

Canfield et al., 2007, Science

Outcrop 3

Canfield et al., 2007, Science

Outcrop 3

Canfield et al., 2007, Science

Outcrop 3

Canfield et al., 2007, Science

Outcrop 3

Canfield et al., 2007, Science

Outcrop 3

Canfield et al., 2007, Science

Outcrop 3

Canfield et al., 2007, Science

Outcrop 3

Canfield et al., 2007, Science

Outcrop 3

Canfield et al., 2007, Science

Outcrop 3

Canfield et al., 2007, Science

Outcrop 3

Canfield et al., 2007, Science

Outcrop 3

Canfield et al., 2007, Science

Outcrop 3

Canfield et al., 2007, Science

Outcrop 3

Canfield et al., 2007, Science

Outcrop 3

Canfield et al., 2007, Science

Outcrop 3

Canfield et al., 2007, Science

Outcrop 3

Canfield et al., 2007, Science

Outcrop 3

Canfield et al., 2007, Science

Outcrop 3

Canfield et al., 2007, Science

Outcrop 3

Canfield et al., 2007, Science

Outcrop 3

Canfield et al., 2007, Science

Outcrop 3

Canfield et al., 2007, Science

Outcrop 3

Canfield et al., 2007, Science

Outcrop 3

Canfield et al., 2007, Science

Outcrop 3

Canfield et al., 2007, Science

Outcrop 3

Canfield et al., 2007, Science

Outcrop 3

Canfield et al., 2007, Science

Outcrop 3

Canfield et al., 2007, Science

Outcrop 3

Canfield et al., 2007, Science

Outcrop 3

Canfield et al., 2007, Science

Outcrop 3

Canfield et al., 2007, Science

Outcrop 3

Canfield et al., 2007, Science

Outcrop 3

Canfield et al., 2007, Science

Outcrop 3

Canfield et al., 2007, Science

Outcrop 3

Canfield et al., 2007, Science

Outcrop 
Canfield et al., 2007, Science

Canfield et al., 2007, Science

Canfield et al., 2007, Science

Canfield et al., 2007, Science

Canfield et al., 2007, Science

Canfield et al., 2007, Science

Canfield et al., 2007, Science

Canfield et al., 2007, Science

Canfield et al., 2007, Science

Canfield et al., 2007, Science

Canfield et al., 2007, Science

Canfield et al., 2007, Science

Canfield et al., 2007, Science

Canfield et al., 2007, Science

Canfield et al., 2007, Science

Canfield et al., 2007, Science

Canfield et al., 2007, Science

Canfield et al., 2007, Science

Canfield et al., 2007, Science

Canfield et al., 2007, Science

Canfield et al., 2007, Science

Canfield et al., 2007, Science

Canfield et al., 2007, Science

Canfield et al., 2007, Science

Canfield et al., 2007, Science

Canfield et al., 2007, Science

Canfield et al., 2007, Science

Canfield et al., 2007, Science

Canfield et al., 2007, Science

Canfield et al., 2007, Science

Canfield et al., 2007, Science

Canfield et al., 2007, Science

Canfield et al., 2007, Science

Canfield et al., 2007, Science

Canfield et al., 2007, Science

Canfield et al., 2007, Science

Canfield et al., 2007, Science

Canfield et al., 2007, Science

Canfield et al., 2007, Science

Canfield et al., 2007, Science

Canfield et al., 2007, Science
Outcrop 3

Outcrop 3

Outcrop 3

Outcrop 3

Outcrop 3

Outcrop 3

Outcrop 3

Outcrop 3

Outcrop 3

Outcrop 3

Outcrop 3

Outcrop 3

Outcrop 3

Outcrop 3

Outcrop 3

Outcrop 3

Outcrop 3

Outcrop 3

Outcrop 3

Outcrop 3

Outcrop 3

Outcrop 3

Outcrop 3

Outcrop 3

Outcrop 3

Outcrop 3

Outcrop 3

Outcrop 3

Outcrop 3

Outcrop 3

Outcrop 3

Outcrop 3

Outcrop 3

Outcrop 3

Outcrop 3

Outcrop 3

Outcrop 3

Outcrop 3

Outcrop 3

Outcrop 3

Outcrop 3
Diamictite-not a $\xi$

Diamictite-not a $\varepsilon$

Diamictite-not a $\varepsilon$

Diamictite-not a $\xi$

Diamictite-not a $\varepsilon$

Diamictite-not a $\varepsilon$

Diamictite-not a $\varepsilon$

Diamictite-not a $\varepsilon$

Diamictite-not a $\xi$

Diamictite-not a $\varepsilon$

Diamictite-not a $\varepsilon$

Diamictite-not a $\xi$

Diamictite-not a $\varepsilon$

Diamictite-not a $\xi$

Diamictite-not a $\varepsilon$

Diamictite-not a $\xi$

Diamictite-not a $\varepsilon$

Diamictite-not a $\xi$

Diamictite-not a $\varepsilon$

3

(1)


Canfield et al., 2008, Science

Canfield et al., 2008, Science

Canfield et al., 2008, Science

Canfield et al., 2008, Science

Canfield et al., 2008, Science

Canfield et al., 2008, Science

Canfield et al., 2008, Science

Canfield et al., 2008, Science

Canfield et al., 2008, Science

Canfield et al., 2008, Science

Canfield et al., 2008, Science

Canfield et al., 2008, Science

Canfield et al., 2008, Science

Canfield et al., 2008, Science

Canfield et al., 2008, Science

Canfield et al., 2008, Science

Canfield et al., 2008, Science

Canfield et al., 2008, Science

Canfield et al., 2008, Science

Canfield et al., 2008, Science

Canfield et al., 2008, Science

Canfield et al., 2008, Science

Canfield et al., 2008, Science

Canfield et al., 2008, Science

Canfield et al., 2008, Science

Canfield et al., 2008, Science

Canfield et al., 2008, Science

Canfield et al., 2008, Science

Canfield et al., 2008, Science

Canfield et al., 2008, Science

Canfield et al., 2008, Science

Canfield et al., 2008, Science

Canfield et al., 2008, Science

Canfield et al., 2008, Science

Canfield et al., 2008, Science

Canfield et al., 2008, Science

Canfield et al., 2008, Science

Canfield et al., 2008, Science

Canfield et al., 2008, Science

Canfield et al., 2008, Science

Canfield et al., 2008, Science

\begin{tabular}{|c|c|c|}
\hline Outcrop & 3 & \\
\hline Outcrop & 2 & \\
\hline Outcrop & 2 & \\
\hline Outcrop & 2 & \\
\hline Outcrop & 2 & \\
\hline Outcrop & 2 & \\
\hline Outcrop & 2 & \\
\hline Outcrop & 2 & \\
\hline Outcrop & 2 & \\
\hline Outcrop & 2 & \\
\hline Outcrop & 2 & \\
\hline Outcrop & 2 & \\
\hline Outcrop & 2 & \\
\hline Outcrop & 2 & \\
\hline Outcrop & 2 & \\
\hline Outcrop & 2 & \\
\hline Outcrop & 2 & \\
\hline Outcrop & 2 & \\
\hline Outcrop & 2 & \\
\hline Outcrop & 2 & \\
\hline Outcrop & 2 & \\
\hline Outcrop & 1 & Probable fresh wi \\
\hline Outcrop & 1 & Probable fresh wi \\
\hline Outcrop & 1 & Probable fresh wi \\
\hline Outcrop & 1 & Probable fresh wi \\
\hline Outcrop & 1 & Probable fresh wi \\
\hline Outcrop & 1 & Probable fresh wi \\
\hline Core & 1 & \\
\hline Core & 1 & \\
\hline Core & 1 & \\
\hline Core & 1 & \\
\hline Core & 1 & \\
\hline Core & 1 & \\
\hline Core & 1 & \\
\hline Core & 2 & \\
\hline Core & 2 & \\
\hline Core & 2 & \\
\hline Core & 2 & \\
\hline Core & 2 & \\
\hline Core & 2 & \\
\hline Core & 2 & \\
\hline
\end{tabular}


Canfield et al., 2008, Science

Core 2

Canfield et al., 2008, Science

Core 2

Canfield et al., 2008, Science

Core 2

Canfield et al., 2008, Science

Core 2

Canfield et al., 2008, Science

Core 2

Canfield et al., 2008, Science

Core 2

Canfield et al., 2008, Science

Core 2

Canfield et al., 2008, Science

Core 2

Canfield et al., 2008, Science

Core 2

Canfield et al., 2008, Science

Core 2

Canfield et al., 2008, Science

Core 2

Canfield et al., 2008, Science

Core 2

Canfield et al., 2008, Science

Core 2

Canfield et al., 2008, Science

Core 2

Canfield et al., 2008, Science

Core 2

Canfield et al., 2008, Science

Core 2

Canfield et al., 2008, Science

Core 2

Canfield et al., 2008, Science

Core 2

Canfield et al., 2008, Science

Core 2

Canfield et al., 2008, Science

Core 2

Canfield et al., 2008, Science

Core 2

Canfield et al., 2008, Science

Core 2

Canfield et al., 2008, Science

Core 2

Canfield et al., 2008, Science

Core 2

Canfield et al., 2008, Science

Core 1

Canfield et al., 2008, Science

Core 1

Canfield et al., 2008, Science

Core 1

Canfield et al., 2008, Science

Core 1

Canfield et al., 2008, Science

Core 1

Canfield et al., 2008, Science

Core 1

Core 1

Canfield et al., 2008, Science

Core 1

Outcrop 1

Outcrop 1

Outcrop 1

Outcrop 3

Outcrop 3

Outcrop 3

Outcrop 3

Outcrop 3

Outcrop 3

This study

3


This study

Outcrop 3

This study

Outcrop 3

This study

Outcrop 3

This study

Outcrop 3

This study

Outcrop 3

This study

Outcrop 3

This study

Outcrop 3

This study

Outcrop 3

This study

Outcrop 3

This study

Outcrop 3

This study

Outcrop 3

This study

Outcrop 3

Outcrop 3

This study

Outcrop 3

Outcrop 3

Outcrop 3

Outcrop 3

Outcrop 3

Outcrop 3

Outcrop 3

Outcrop 3

Outcrop 3

Outcrop 3

Outcrop 3

Outcrop 3

Outcrop 3

Outcrop 3

Outcrop 3

Outcrop 3

Outcrop 3

Outcrop 3

Outcrop 3

Outcrop 3

Outcrop 3

Outcrop 3

Outcrop 3

Outcrop 3

Outcrop 3

Outcrop 3

Outcrop 3

Outcrop 3

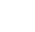

3

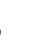

3

3

3

3

3

3

3

3

3

3

3

3

3

3

3

3

3

3

3

3

3

3

3

3

3

3

3

3

3

3

3

3

3

3

3

3

3


Canfield et al., 2008, Science

Outcrop 3

Canfield et al., 2008, Science

Outcrop 3

Canfield et al., 2008, Science

Outcrop 3

Canfield et al., 2008, Science

Outcrop 3

Canfield et al., 2008, Science

Outcrop 3

Canfield et al., 2008, Science

Outcrop 3

Canfield et al., 2008, Science

Outcrop 3

Canfield et al., 2008, Science

Outcrop 3

Canfield et al., 2008, Science

Outcrop 3

Canfield et al., 2008, Science

Outcrop 3

Canfield et al., 2008, Science

Outcrop 3

Canfield et al., 2008, Science

Outcrop 3

Canfield et al., 2008, Science

Outcrop 3

Canfield et al., 2008, Science

Outcrop 3

Canfield et al., 2008, Science

Outcrop 3

Canfield et al., 2008, Science

Outcrop 3

Canfield et al., 2008, Science

Outcrop 3

Shen et al., 2008, PNAS

Outcrop 3

Shen et al., 2008, PNAS

Shen et al., 2008, PNAS

Outcrop 3

Outcrop 3

Outcrop 3

Shen et al., 2008, PNAS

Outcrop 3

Outcrop 3

Outcrop 3

Outcrop 3

Outcrop 3

Outcrop 3

Outcrop 3

Outcrop 3

Outcrop 3

Outcrop 3

Outcrop 3

Outcrop 3

Outcrop 3

Outcrop 3

Outcrop 3

Outcrop 3

Outcrop 3

Outcrop 3

Outcrop 3

Outcrop 3

Uses old extractic

Uses old extractic

Uses old extractic

Uses old extractic

Uses old extractic

Uses old extractic

Uses old extractic

Uses old extractic

Uses old extractic

Uses old extractic

Uses old extractic

Uses old extractic

Uses old extractic

Uses old extractic

Uses old extractic

Uses old extractic

Uses old extractic

Uses old extractic

Uses old extractic Uses old extractic Uses old extractic Uses old extractic Uses old extractic

Shen et al., 2008, PNAS

Uses old extractic


Shen et al., 2008, PNAS

Shen et al., 2008, PNAS

Shen et al., 2008, PNAS

Shen et al., 2008, PNAS

Shen et al., 2008, PNAS

Shen et al., 2008, PNAS

Shen et al., 2008, PNAS

Shen et al., 2008, PNAS

Shen et al., 2008, PNAS

Shen et al., 2008, PNAS

Shen et al., 2008, PNAS

Shen et al., 2008, PNAS

Shen et al., 2008, PNAS

Shen et al., 2008, PNAS

Shen et al., 2008, PNAS

Shen et al., 2008, PNAS

Shen et al., 2008, PNAS

Shen et al., 2008, PNAS

Shen et al., 2008, PNAS

Shen et al., 2008, PNAS

Shen et al., 2008, PNAS

Shen et al., 2008, PNAS

Shen et al., 2008, PNAS

Shen et al., 2008, PNAS

Shen et al., 2008, PNAS

Shen et al., 2008, PNAS

Shen et al., 2008, PNAS

Shen et al., 2008, PNAS

Shen et al., 2008, PNAS

Shen et al., 2008, PNAS

Shen et al., 2008, PNAS

Shen et al., 2008, PNAS

Shen et al., 2008, PNAS

Shen et al., 2008, PNAS

Shen et al., 2008, PNAS

This study

This study

This study

This study

This study

This study

\begin{tabular}{|c|c|c|}
\hline Outcrop & 3 & Uses old extractic \\
\hline Outcrop & 3 & Uses old extractic \\
\hline Outcrop & 3 & Uses old extractic \\
\hline Outcrop & 3 & Uses old extractic \\
\hline Outcrop & 3 & Uses old extractic \\
\hline Outcrop & 3 & Uses old extractic \\
\hline Outcrop & 3 & Uses old extractic \\
\hline Outcrop & 3 & Uses old extractic \\
\hline Outcrop & 3 & Uses old extractic \\
\hline Outcrop & 3 & Uses old extractic \\
\hline Outcrop & 3 & Uses old extractic \\
\hline Outcrop & 3 & Uses old extractic \\
\hline Outcrop & 3 & Uses old extractic \\
\hline Outcrop & 3 & Uses old extractic \\
\hline Outcrop & 3 & Uses old extractic \\
\hline Outcrop & 3 & Uses old extractic \\
\hline Outcrop & 3 & Uses old extractic \\
\hline Outcrop & 3 & Uses old extractic \\
\hline Outcrop & 3 & Uses old extractic \\
\hline Outcrop & 3 & Uses old extractic \\
\hline Outcrop & 3 & Uses old extractic \\
\hline Outcrop & 3 & Uses old extractic \\
\hline Outcrop & 3 & Uses old extractic \\
\hline Outcrop & 3 & Uses old extractic \\
\hline Outcrop & 3 & Uses old extractic \\
\hline Outcrop & 3 & Uses old extractic \\
\hline Outcrop & 3 & Uses old extractic \\
\hline Outcrop & 3 & Uses old extractic \\
\hline Outcrop & 3 & Uses old extractic \\
\hline Outcrop & 3 & Uses old extractic \\
\hline Outcrop & 3 & Uses old extractic \\
\hline Outcrop & 3 & Uses old extractic \\
\hline Outcrop & 3 & Uses old extractic \\
\hline Outcrop & 3 & Uses old extractic \\
\hline Outcrop & 3 & Uses old extractic \\
\hline Outcrop & 2 & \\
\hline Outcrop & 2 & \\
\hline Outcrop & 2 & \\
\hline Outcrop & 2 & \\
\hline Outcrop & 2 & \\
\hline Outcrop & 2 & \\
\hline
\end{tabular}


This study

This study

This study

This study

This study

This study

This study

This study

This study

This study

This study

This study

This study

This study

This study

This study

This study

This study

This study

This study

This study

This study

This study

This study

This study

This study

This study

This study

This study

This study

This study

This study

This study

This study

This study

This study

This study

This study

This study

This study

This study
Outcrop 2

Outcrop 2

Outcrop 2

Outcrop 2

Outcrop 2

Outcrop 2

Outcrop 2

Outcrop 2

Outcrop 2

Outcrop 3

Outcrop 3

Outcrop 3

Outcrop 3

Outcrop 3

Outcrop 3

Outcrop 3

Outcrop 3

Outcrop 3

Outcrop 3

Outcrop 3

Outcrop 3

Outcrop 3

Outcrop 3

Outcrop 3

Outcrop 3

Outcrop 3

Outcrop 3

Outcrop 3

Outcrop 3

Outcrop 3

Outcrop 3

Outcrop 3

Outcrop 3

Outcrop 3

Outcrop 3

Outcrop 3

Outcrop 3

Outcrop 3

Outcrop 3

Outcrop 3

Outcrop 3 
This study

This study

This study

This study

This study

This study

This study

This study

This study

This study

This study

This study

This study

This study

This study

This study

This study

This study

This study

This study

This study

This study

This study

This study

This study

This study

This study

This study

This study

This study

This study

This study

This study

This study

This study

This study

This study

This study

This study

This study

This study
Outcrop 3

Outcrop 3

Outcrop 3

Outcrop 3

Outcrop 3

Outcrop 3

Outcrop 3

Outcrop 3

Outcrop 3

Outcrop 3

Outcrop 3

Outcrop 3

Outcrop 3

Outcrop 3

Outcrop 3

Outcrop 3

Outcrop 3

Outcrop 3

Outcrop 3

Outcrop 3

Outcrop 3

Outcrop 3

Outcrop 3

Outcrop 3

Outcrop 3

Outcrop 3

Outcrop 3

Outcrop 3

Outcrop 3

Outcrop 3

Outcrop 3

Outcrop 3

Outcrop 3

Outcrop 3

Outcrop 3

Outcrop 3

Outcrop 3 The exact stratigr

Outcrop 3 The exact stratigr

Outcrop $\quad 3$ The exact stratigr

Outcrop 3 The exact stratigr

Outcrop 3 The exact stratigr 
This study

This study

This study

This study

This study

This study

This study

This study

This study

This study

This study

This study

This study

This study

This study

This study

This study

This study

This study

This study

This study

This study

This study

This study

This study

This study

This study

This study

This study

This study

This study

This study

This study

This study

This study

This study

This study

This study

This study

This study

This study

\begin{tabular}{|c|c|c|}
\hline Outcrop & 3 & The exact stratigr \\
\hline Outcrop & 3 & The exact stratigr \\
\hline Outcrop & 3 & The exact stratigr \\
\hline Outcrop & 3 & The exact stratigr \\
\hline Outcrop & 3 & The exact stratigr \\
\hline Outcrop & 3 & The exact stratigr \\
\hline Outcrop & 3 & The exact stratigr \\
\hline Outcrop & 3 & The exact stratigr \\
\hline Outcrop & 3 & The exact stratigr \\
\hline Outcrop & 3 & The exact stratigr \\
\hline Outcrop & 3 & The exact stratigr \\
\hline Outcrop & 3 & The exact stratigr \\
\hline Outcrop & 3 & The exact stratigr \\
\hline Outcrop & 3 & The exact stratigr \\
\hline Outcrop & 3 & The exact stratigr \\
\hline Outcrop & 3 & The exact stratigr \\
\hline Outcrop & 3 & The exact stratigr \\
\hline Outcrop & 3 & The exact stratigr \\
\hline Outcrop & 3 & The exact stratigr \\
\hline Outcrop & 2 & \\
\hline Outcrop & 2 & \\
\hline Outcrop & 2 & \\
\hline Outcrop & 2 & \\
\hline Outcrop & 2 & \\
\hline Outcrop & 2 & \\
\hline Outcrop & 2 & \\
\hline Outcrop & 2 & \\
\hline Outcrop & 2 & \\
\hline Outcrop & 2 & \\
\hline Outcrop & 3 & \\
\hline Outcrop & 2 & \\
\hline Outcrop & 3 & \\
\hline Outcrop & 3 & \\
\hline Outcrop & 3 & \\
\hline Outcrop & 3 & \\
\hline Outcrop & 3 & \\
\hline Outcrop & 3 & \\
\hline Outcrop & 3 & \\
\hline Outcrop & 3 & \\
\hline Outcrop & 3 & \\
\hline Outcrop & 3 & \\
\hline
\end{tabular}


This study

This study

This study

This study

This study

This study

This study

This study

This study

This study

This study

This study

This study

This study

This study

This study

This study

This study

This study

This study

This study

This study

This study

This study

This study

This study

This study

This study

This study

This study

This study

This study

This study

This study

This study

This study

This study

This study

This study

This study

This study
Outcrop 3

Outcrop 3

Outcrop 3

Outcrop 3

Outcrop 3

Outcrop 3

Outcrop 3

Outcrop 3

Outcrop 3

Outcrop 3

Outcrop 3

Outcrop 3

Outcrop 3

Outcrop 3

Outcrop 3

Outcrop 3

Outcrop 3

Outcrop 3

Outcrop 3

Outcrop 2

Outcrop 2

Outcrop 2

Outcrop 2

Outcrop 2

Outcrop 2

Outcrop 2

Outcrop 2

Outcrop 2

Outcrop 2

Outcrop 2

Outcrop 2

Outcrop 2

Outcrop 2

Outcrop 2

Outcrop 2

Outcrop 2

Outcrop 2

Outcrop 2

Outcrop 2

Outcrop 2

Outcrop 2 
This study

Outcrop 2

This study

Outcrop 2

This study

Outcrop 2

This study

Outcrop 2

This study

Outcrop 2

This study

Outcrop 2

This study

Outcrop 2

This study

Outcrop 2

This study

Outcrop 2

This study

Outcrop 2

Frei et al., 2013

Outcrop 2

Frei et al., 2013

Outcrop 2

Frei et al., 2013

Outcrop 2

Frei et al., 2013

Outcrop 2

Frei et al., 2013

Outcrop 2

Frei et al., 2013

Outcrop 2

Frei et al., 2013

Outcrop 2

Frei et al., 2013

Outcrop 2

Frei et al., 2013

Outcrop 2

Frei et al., 2013

Outcrop 2

Frei et al., 2013

Outcrop 2

Frei et al., 2013

Outcrop 2

Frei et al., 2013

Outcrop 2

Frei et al., 2013

Outcrop 2

Frei et al., 2013

Outcrop 2

Frei et al., 2013

Outcrop 2

Frei et al., 2013

Frei et al., 2013

Outcrop 2

Outcrop 2

Frei et al., 2013

Frei et al., 2013

Outcrop 2

Outcrop 2

Frei et al., 2013

Frei et al., 2013

Outcrop 2

Outcrop 2

Frei et al., 2013

Frei et al., 2013

Outcrop 2

Outcrop 2

Frei et al., 2013

Frei et al., 2013

Outcrop 2

Outcrop 2

Outcrop 2

Outcrop 2

Frei et al., 2013

Frei et al., 2013

Frei et al., 2013

Outcrop 2

Outcrop 2

This study

Outcrop 2


This study

This study

This study

This study

This study

This study

This study

This study

This study

This study

This study

This study

This study

This study

This study

This study

This study

This study

This study

This study

This study

This study

This study

This study

This study

This study

This study

This study

This study

This study

This study

This study

This study

This study

This study

This study

This study

This study

This study

This study

This study
Outcrop 2

Outcrop 2

Outcrop 2

Outcrop 2

Outcrop 2

Outcrop 2

Outcrop 2

Outcrop 2

Outcrop 2

Outcrop 2

Outcrop 2

Outcrop 2

Outcrop 2

Outcrop 2

Outcrop 2

Outcrop 2

Outcrop 2

Outcrop 2

Outcrop 2

Outcrop 2

Outcrop 2

Outcrop 2

Outcrop 2

Outcrop 2

Outcrop 2

Outcrop 2

Outcrop 2

Outcrop 2

Outcrop 2

Outcrop 2

Outcrop 2

Outcrop 2

Outcrop 2

Outcrop 2

Outcrop 2

Outcrop 2

Outcrop 2

Outcrop 2

Outcrop 2

Outcrop 2

Outcrop 2 
This study

Outcrop 2

This study

Outcrop 2

This study

Outcrop 2

This study

Outcrop 2

This study

Outcrop 2

This study

Outcrop 2

This study

Outcrop 2

This study

Outcrop 2

This study

Outcrop 2

This study

Outcrop 2

This study

Outcrop 2

This study

Outcrop 3

Outcrop 3

Outcrop 3

Outcrop 3

Outcrop 3

Outcrop 3

Outcrop 3

Outcrop 3

Outcrop 3

Outcrop 3

Outcrop 3

Outcrop 3

Outcrop 3

Outcrop 3

Outcrop 3

Outcrop 3

Outcrop 3

Outcrop 3

Outcrop 3

Outcrop 3

Outcrop 3

Outcrop 3

Outcrop 3

Outcrop 3

Outcrop 3

Outcrop 3

Outcrop 3

Outcrop 3

Outcrop 3

Outcrop 3

2

2

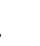

2

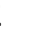

2

2

2

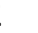

3

3

3

3

3

3

3

3

3

3

3

3

3

3

3

3

3

3

3

3

3

3

3

3

3

3

3

3

3

3


Canfield et al., 2008, Science

Outcrop 3

Canfield et al., 2008, Science

Outcrop 3

Canfield et al., 2008, Science

Outcrop 3

Canfield et al., 2008, Science

Outcrop 3

Canfield et al., 2008, Science

Outcrop 3

Canfield et al., 2008, Science

Outcrop 3

Canfield et al., 2008, Science

Outcrop 3

Canfield et al., 2008, Science

Outcrop 3

Canfield et al., 2008, Science

Outcrop 3

Canfield et al., 2008, Science

Outcrop 3

Canfield et al., 2008, Science

Outcrop 3

Canfield et al., 2008, Science

Outcrop 3

Canfield et al., 2008, Science

Outcrop 3

Canfield et al., 2008, Science

Outcrop 3

Canfield et al., 2008, Science

Outcrop 3

Canfield et al., 2008, Science

Outcrop 3

Canfield et al., 2008, Science

Outcrop 3

Canfield et al., 2008, Science

Outcrop 3

Canfield et al., 2008, Science

Outcrop 3

Canfield et al., 2008, Science

Outcrop 3

Canfield et al., 2008, Science

Outcrop 3

Canfield et al., 2008, Science

Outcrop 3

Canfield et al., 2008, Science

Outcrop 3

Canfield et al., 2008, Science

Outcrop 3

Canfield et al., 2008, Science

Outcrop 3

Canfield et al., 2008, Science

Outcrop 3

Canfield et al., 2008, Science

Outcrop 3

Dahl et al., 2010, PNAS

Dahl et al., 2010, PNAS

Core 3

Core 3

Dahl et al., 2010, PNAS

Dahl et al., 2010, PNAS

Core 3

Core 3

Dahl et al., 2010, PNAS

Core 3

Feng et al., 2014, Precambrian Research

Outcrop 2

Feng et al., 2014, Precambrian Research

Outcrop 2

Feng et al., 2014, Precambrian Research

Outcrop 2

Feng et al., 2014, Precambrian Research

Outcrop 2

Feng et al., 2014, Precambrian Research

Outcrop 2

Feng et al., 2014, Precambrian Research

Outcrop 2

Feng et al., 2014, Precambrian Research

Outcrop 2

Feng et al., 2014, Precambrian Research

Outcrop 2

Feng et al., 2014, Precambrian Research

Outcrop 
Feng et al., 2014, Precambrian Research

Feng et al., 2014, Precambrian Research

Feng et al., 2014, Precambrian Research

Feng et al., 2014, Precambrian Research

Feng et al., 2014, Precambrian Research

Feng et al., 2014, Precambrian Research

Feng et al., 2014, Precambrian Research

Feng et al., 2014, Precambrian Research

Feng et al., 2014, Precambrian Research

Feng et al., 2014, Precambrian Research

Feng et al., 2014, Precambrian Research

Feng et al., 2014, Precambrian Research

Feng et al., 2014, Precambrian Research

Feng et al., 2014, Precambrian Research

Feng et al., 2014, Precambrian Research

Feng et al., 2014, Precambrian Research

Feng et al., 2014, Precambrian Research

Feng et al., 2014, Precambrian Research

Feng et al., 2014, Precambrian Research

Feng et al., 2014, Precambrian Research

Feng et al., 2014, Precambrian Research

Och et al., 2013, Precambrian Research

Och et al., 2013, Precambrian Research

Och et al., 2013, Precambrian Research

Och et al., 2013, Precambrian Research

Och et al., 2013, Precambrian Research

Och et al., 2013, Precambrian Research

Och et al., 2013, Precambrian Research

Och et al., 2013, Precambrian Research

Och et al., 2013, Precambrian Research

Och et al., 2013, Precambrian Research

Och et al., 2013, Precambrian Research

Och et al., 2013, Precambrian Research

Och et al., 2013, Precambrian Research

Och et al., 2013, Precambrian Research

Och et al., 2013, Precambrian Research

Och et al., 2013, Precambrian Research

Och et al., 2013, Precambrian Research

Och et al., 2013, Precambrian Research

Och et al., 2013, Precambrian Research

Och et al., 2013, Precambrian Research

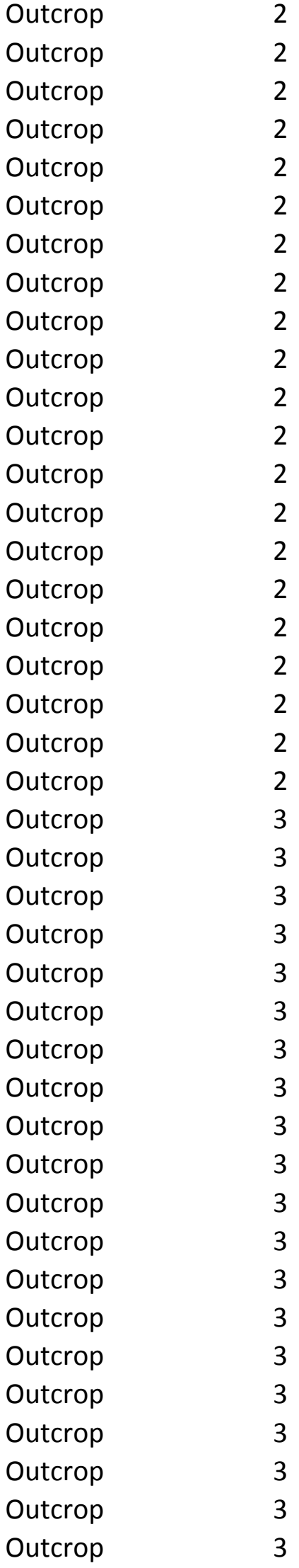


Och et al., 2013, Precambrian Research

Och et al., 2013, Precambrian Research

Och et al., 2013, Precambrian Research

Och et al., 2013, Precambrian Research

Och et al., 2013, Precambrian Research

Och et al., 2013, Precambrian Research

Och et al., 2013, Precambrian Research

Och et al., 2013, Precambrian Research

Och et al., 2013, Precambrian Research

Och et al., 2013, Precambrian Research

Och et al., 2013, Precambrian Research

Och et al., 2013, Precambrian Research

Och et al., 2013, Precambrian Research

Och et al., 2013, Precambrian Research

Och et al., 2013, Precambrian Research

Och et al., 2013, Precambrian Research

Och et al., 2013, Precambrian Research

Och et al., 2013, Precambrian Research

Och et al., 2013, Precambrian Research

Och et al., 2013, Precambrian Research

Och et al., 2013, Precambrian Research

Och et al., 2013, Precambrian Research

Och et al., 2013, Precambrian Research

Och et al., 2013, Precambrian Research

Och et al., 2013, Precambrian Research

Och et al., 2013, Precambrian Research

Och et al., 2013, Precambrian Research

Och et al., 2013, Precambrian Research

Och et al., 2013, Precambrian Research

Och et al., 2013, Precambrian Research

Och et al., 2013, Precambrian Research

Och et al., 2013, Precambrian Research

Och et al., 2013, Precambrian Research

Och et al., 2013, Precambrian Research

Och et al., 2013, Precambrian Research

Och et al., 2013, Precambrian Research

Och et al., 2013, Precambrian Research

Och et al., 2013, Precambrian Research

Och et al., 2013, Precambrian Research

Och et al., 2013, Precambrian Research

Och et al., 2013, Precambrian Research

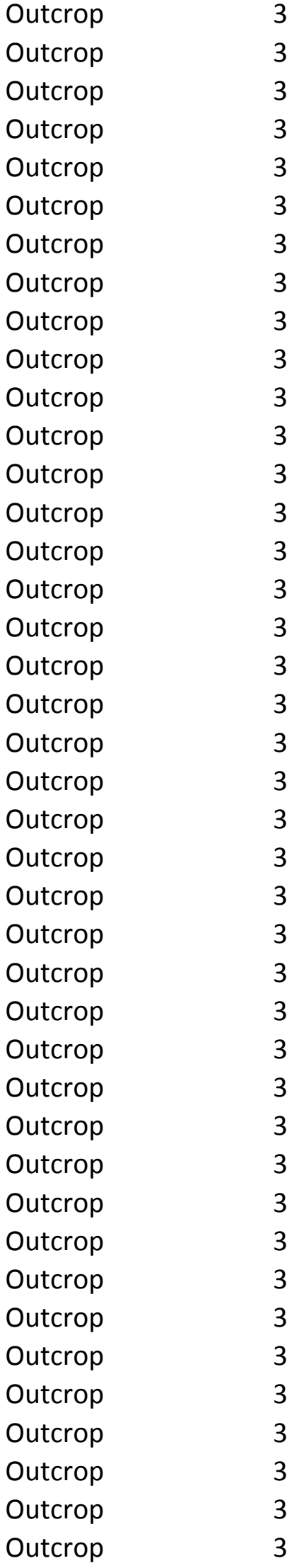


Och et al., 2013, Precambrian Research

Och et al., 2013, Precambrian Research

Och et al., 2013, Precambrian Research

Och et al., 2013, Precambrian Research

Och et al., 2013, Precambrian Research

Och et al., 2013, Precambrian Research

Och et al., 2013, Precambrian Research

Och et al., 2013, Precambrian Research

Och et al., 2013, Precambrian Research

Och et al., 2013, Precambrian Research

Och et al., 2013, Precambrian Research

Och et al., 2013, Precambrian Research

Och et al., 2013, Precambrian Research

Och et al., 2013, Precambrian Research

Och et al., 2013, Precambrian Research

Och et al., 2013, Precambrian Research

Och et al., 2013, Precambrian Research

Och et al., 2013, Precambrian Research

Och et al., 2013, Precambrian Research

Och et al., 2013, Precambrian Research

Och et al., 2013, Precambrian Research

Och et al., 2013, Precambrian Research

Och et al., 2013, Precambrian Research

Och et al., 2013, Precambrian Research

Och et al., 2013, Precambrian Research

Och et al., 2013, Precambrian Research

Och et al., 2013, Precambrian Research

Och et al., 2013, Precambrian Research

Och et al., 2013, Precambrian Research

Och et al., 2013, Precambrian Research

Och et al., 2013, Precambrian Research

Och et al., 2013, Precambrian Research

Och et al., 2013, Precambrian Research

Och et al., 2013, Precambrian Research

Och et al., 2013, Precambrian Research

Och et al., 2013, Precambrian Research

Och et al., 2013, Precambrian Research

Och et al., 2013, Precambrian Research

Och et al., 2013, Precambrian Research

Och et al., 2013, Precambrian Research

Och et al., 2013, Precambrian Research

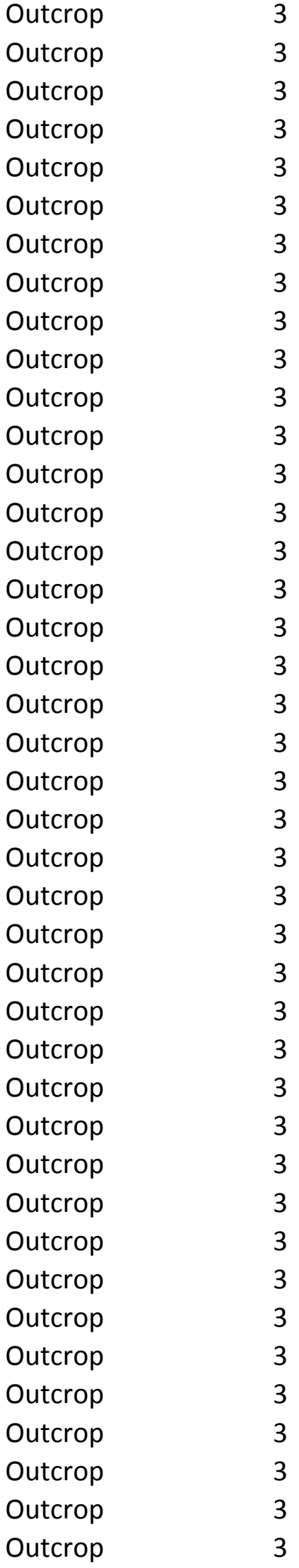


Och et al., 2013, Precambrian Research

Och et al., 2013, Precambrian Research

Och et al., 2013, Precambrian Research

Och et al., 2013, Precambrian Research

Och et al., 2013, Precambrian Research

Och et al., 2013, Precambrian Research

Och et al., 2013, Precambrian Research

Och et al., 2013, Precambrian Research

Och et al., 2013, Precambrian Research

Och et al., 2013, Precambrian Research

Och et al., 2013, Precambrian Research

Och et al., 2013, Precambrian Research

Och et al., 2013, Precambrian Research

Och et al., 2013, Precambrian Research

Och et al., 2013, Precambrian Research

Och et al., 2013, Precambrian Research

Och et al., 2013, Precambrian Research

Och et al., 2013, Precambrian Research

Och et al., 2013, Precambrian Research

Och et al., 2013, Precambrian Research

Och et al., 2013, Precambrian Research

Och et al., 2013, Precambrian Research

Och et al., 2013, Precambrian Research

Och et al., 2013, Precambrian Research

Och et al., 2013, Precambrian Research

Och et al., 2013, Precambrian Research

Och et al., 2013, Precambrian Research

Och et al., 2013, Precambrian Research

Och et al., 2013, Precambrian Research

Och et al., 2013, Precambrian Research

Och et al., 2013, Precambrian Research

Och et al., 2013, Precambrian Research

Och et al., 2013, Precambrian Research

Och et al., 2013, Precambrian Research

Och et al., 2013, Precambrian Research

Och et al., 2013, Precambrian Research

Och et al., 2013, Precambrian Research

Och et al., 2013, Precambrian Research

Wang et al., 2012, Chemical Geology

Wang et al., 2012, Chemical Geology

Wang et al., 2012, Chemical Geology

\begin{tabular}{|c|c|c|}
\hline Outcrop & 3 & \\
\hline Outcrop & 3 & \\
\hline Outcrop & 3 & \\
\hline Outcrop & 3 & \\
\hline Outcrop & 3 & \\
\hline Outcrop & 3 & \\
\hline Outcrop & 3 & \\
\hline Outcrop & 3 & \\
\hline Outcrop & 3 & \\
\hline Outcrop & 3 & \\
\hline Outcrop & 3 & \\
\hline Outcrop & 3 & \\
\hline Outcrop & 3 & \\
\hline Outcrop & 3 & \\
\hline Outcrop & 3 & \\
\hline Outcrop & 3 & \\
\hline Outcrop & 3 & \\
\hline Outcrop & 3 & \\
\hline Outcrop & 3 & \\
\hline Outcrop & 3 & \\
\hline Outcrop & 3 & \\
\hline Outcrop & 3 & \\
\hline Outcrop & 3 & This is the very " $n$ \\
\hline Outcrop & 3 & This is the very " $n$ \\
\hline Outcrop & 3 & This is the very " $n$ \\
\hline Outcrop & 3 & This is the very " $n$ \\
\hline Outcrop & 3 & This is the very " $n$ \\
\hline Outcrop & 3 & This is the very " $n$ \\
\hline Outcrop & 3 & This is the very " $n$ \\
\hline Outcrop & 3 & This is the very " $n$ \\
\hline Outcrop & 3 & This is the very " $n$ \\
\hline Outcrop & 3 & This is the very " $n$ \\
\hline Outcrop & 3 & This is the very " $n$ \\
\hline Outcrop & 3 & This is the very " $n$ \\
\hline Outcrop & 3 & This is the very " $n$ \\
\hline Outcrop & 3 & This is the very " $n$ \\
\hline Outcrop & 3 & This is the very " $n$ \\
\hline Outcrop & 3 & This is the very " $n$ \\
\hline Outcrop & 3 & \\
\hline Outcrop & 3 & \\
\hline Outcrop & 3 & \\
\hline
\end{tabular}


Wang et al., 2012, Chemical Geology

Outcrop 3

Wang et al., 2012, Chemical Geology

Outcrop 3

Wang et al., 2012, Chemical Geology

Outcrop 3

Wang et al., 2012, Chemical Geology

Outcrop 3

Wang et al., 2012, Chemical Geology

Outcrop 3

Wang et al., 2012, Chemical Geology

Outcrop 3

Wang et al., 2012, Chemical Geology

Outcrop 3

Wang et al., 2012, Chemical Geology

Outcrop 3

Wang et al., 2012, Chemical Geology

Outcrop 3

Wang et al., 2012, Chemical Geology

Outcrop 3

Wang et al., 2012, Chemical Geology

Outcrop 3

Wang et al., 2012, Chemical Geology

Outcrop 3

Wang et al., 2012, Chemical Geology

Outcrop 3

Wang et al., 2012, Chemical Geology

Outcrop 3

Wang et al., 2012, Chemical Geology

Outcrop 3

Wang et al., 2012, Chemical Geology

Outcrop 3

Wang et al., 2012, Chemical Geology

Outcrop 3

Wang et al., 2012, Chemical Geology

Outcrop 3

Wang et al., 2012, Chemical Geology

Outcrop 3

Wang et al., 2012, Chemical Geology

Outcrop 3

Wang et al., 2012, Chemical Geology

Outcrop 3

Wang et al., 2012, Chemical Geology

Outcrop 3

Wang et al., 2012, Chemical Geology

Outcrop 3

Wang et al., 2012, Chemical Geology

Outcrop 3

Wang et al., 2012, Chemical Geology

Outcrop 3

This study

Outcrop 3

This study

Outcrop 3

This study

Outcrop 3

This study

Outcrop 3

This study

Outcrop 3

This study

Outcrop 3

This study

Outcrop 3

This study

Outcrop 3

This study

Outcrop 3

This study

Outcrop 3

This study

Outcrop 3

This study

Outcrop 3

This study

Outcrop 3

This study

Outcrop 3

This study

Outcrop 3

This study

Outcrop 
This study

This study

This study

This study

This study

This study

This study

This study

This study

This study

This study

This study

This study

This study

This study

This study

This study

This study

This study

This study

This study

This study

This study

This study

This study

This study

This study

This study

This study

This study

This study

This study

This study

This study

This study

This study

This study

This study

This study

This study

This study
Outcrop 3

Outcrop 3

Outcrop 3

Outcrop 2

Outcrop 2

Outcrop 2

Outcrop 2

Outcrop 2

Outcrop 2

Outcrop 2

Outcrop 2

Outcrop 2

Outcrop 2

Outcrop 2

Outcrop 2

Outcrop 2

Outcrop 2

Outcrop 2

Outcrop 2

Outcrop 2

Outcrop 2

Outcrop 2

Outcrop 3

Outcrop 3

Outcrop 3

Outcrop 3

Outcrop 3

Outcrop 3

Outcrop 3

Outcrop 3

Outcrop 3

Outcrop 3

Outcrop 3

Outcrop 3

Outcrop 3

Outcrop 3

Outcrop 3

Outcrop 3

Outcrop 3

Outcrop 3

Outcrop 3 
This study

This study

This study

This study

This study

This study

This study

This study

This study

This study

This study

This study

This study

This study

This study

This study

This study

This study

This study

This study

This study

This study

This study

This study

This study

This study

This study

This study

This study

This study

This study

This study

This study

This study

This study

This study

This study

This study

This study

This study

This study
Outcrop 3

Outcrop 3

Outcrop 3

Outcrop 3

Outcrop 3

Outcrop 3

Outcrop 3

Outcrop 3

Outcrop 3

Outcrop 3

Outcrop 3

Outcrop 3

Outcrop 3

Outcrop 3

Outcrop 3

Outcrop 3

Outcrop 3

Outcrop 3

Outcrop 3

Outcrop 3

Outcrop 3

Outcrop 3

Outcrop 3

Outcrop 3

Outcrop 3

Outcrop 3

Outcrop 3

Outcrop 3

Outcrop 3

Outcrop 3

Outcrop 3

Outcrop 3

Outcrop 3

Outcrop 3

Outcrop 3

Outcrop 3

Outcrop 3

Outcrop 3

Outcrop 3

Outcrop 3

Outcrop 3 
This study

This study

This study

This study

This study

This study

This study

This study

This study

This study

This study

This study

This study

This study

This study

This study

This study

This study

This study

This study

This study

This study

This study

This study

This study

This study

This study

This study

This study

This study

This study

This study

This study

This study

This study

This study

This study

This study

This study

This study

This study
Outcrop 3

Outcrop 3

Outcrop 3

Outcrop 3

Outcrop 3

Outcrop 3

Outcrop 3

Outcrop 3

Outcrop 3

Outcrop 3

Outcrop 3

Outcrop 3

Outcrop 3

Outcrop 3

Outcrop 3

Outcrop 3

Outcrop 3

Outcrop 3

Outcrop 3

Outcrop 3

Outcrop 3

Outcrop 3

Outcrop 3

Outcrop 3

Outcrop 3

Outcrop 3

Outcrop 3

Outcrop 3

Outcrop 3

Outcrop 3

Outcrop 3

Outcrop 3

Outcrop 3

Outcrop 3

Outcrop 3

Outcrop 3

Outcrop 3

Outcrop 3

Outcrop 3

Outcrop 3

Outcrop 3 
This study

Outcrop 3

This study

Outcrop 3

This study

Outcrop 3

This study

Outcrop 3

This study

Outcrop 3

This study

Outcrop 3

This study

Outcrop 3

This study

Outcrop 3

This study

Outcrop 3

This study

Outcrop 3

This study

Outcrop 3

This study

Outcrop 3

This study

Outcrop 3

This study

Outcrop 3

This study

Outcrop 3

This study

Outcrop 3

This study

Outcrop 3

Gill et al., 2011, Nature

Core 3

Gill et al., 2011, Nature

Core 3

Gill et al., 2011, Nature

Core 3

Gill et al., 2011, Nature

Core 3

Gill et al., 2011, Nature

Core 3

Gill et al., 2011, Nature

Core 3

Gill et al., 2011, Nature

Core 3

Gill et al., 2011, Nature

Core 3

Gill et al., 2011, Nature

Core 3

Gill et al., 2011, Nature

Core 3

Gill et al., 2011, Nature

Core 3

Gill et al., 2011, Nature

Core 3

Gill et al., 2011, Nature

Core 3

Gill et al., 2011, Nature

Core 3

Gill et al., 2011, Nature

Core 3

Gill et al., 2011, Nature

Core 3

Gill et al., 2011, Nature

Core 3

Gill et al., 2011, Nature

Core 3

Gill et al., 2011, Nature

Core 3

Gill et al., 2011, Nature

Core 3

Gill et al., 2011, Nature

Core 3

Gill et al., 2011, Nature

Core 3

Gill et al., 2011, Nature

Core 3

Gill et al., 2011, Nature

Core 3


Gill et al., 2011, Nature

Core 3

Gill et al., 2011, Nature

Core 3

Dahl et al., 2010, PNAS

Outcrop 3

Dahl et al., 2010, PNAS

Outcrop 3

Dahl et al., 2010, PNAS

Outcrop 3

Dahl et al., 2010, PNAS

Outcrop 3

Canfield et al., 2008, Science

Outcrop 1

Canfield et al., 2008, Science

Outcrop 1

Canfield et al., 2008, Science

Outcrop 1

Canfield et al., 2008, Science

Outcrop 1

Canfield et al., 2008, Science

Outcrop 1

Canfield et al., 2008, Science

Outcrop 1

Canfield et al., 2008, Science

Outcrop 1

Canfield et al., 2008, Science

Outcrop 1

Canfield et al., 2008, Science

Outcrop 1

Canfield et al., 2008, Science

Outcrop 1

Canfield et al., 2008, Science

Outcrop 1

This study

Outcrop 2

This study

Outcrop 2

This study

Outcrop 2

This study

Outcrop 2

This study

Outcrop 2

This study

Outcrop 2

This study

Outcrop 2

This study

Outcrop 2

This study

Outcrop 2

This study

Outcrop 2

This study

Outcrop 2

This study

Outcrop 2

This study

Outcrop 2

This study

Outcrop 2

This study

This study

Outcrop 2

Outcrop 2

This study

This study

Outcrop 2

Outcrop 2

This study

This study

Outcrop 2

Outcrop 2

This study

This study

Outcrop 2

Outcrop 2

This study

Outcrop 2

This study

Outcrop 
This study

Outcrop 2

This study

Outcrop 2

This study

Outcrop 2

This study

Outcrop 2

This study

Outcrop 2

This study

Outcrop 1

This study

Outcrop 1

This study

Outcrop 1

This study

Outcrop 1

This study

Outcrop 1

This study

Outcrop 1

This study

Outcrop 1

This study

Outcrop 1

This study

Outcrop 1

This study

Outcrop 1

This study

Outcrop 1

This study

Outcrop 1

This study

Outcrop 1

This study

Outcrop 1

This study

Outcrop 1

This study

Outcrop 1

This study

Outcrop 1

This study

Outcrop 1

This study

Outcrop 1

This study

Outcrop 1

This study

Outcrop 1

This study

Outcrop 1

This study

This study

Creveling et al., 2014, GSA Bulletin

Outcrop 1

Outcrop 1

Creveling et al., 2014, GSA Bulletin

Core 2

Core 2

Creveling et al., 2014, GSA Bulletin

Core 2

Core 2

Core 2

Core 2

Core 2

Core 2

Core 2

Core 2

Core 2

Core 2

Only used sample Only used sample Only used sample Only used sample Only used sample Only used sample Only used sample Only used sample Only used sample Only used sample Only used sample Creveling et al., 2014, GSA Bulletin

2
Only used sample 
Creveling et al., 2014, GSA Bulletin

Creveling et al., 2014, GSA Bulletin

Creveling et al., 2014, GSA Bulletin

Creveling et al., 2014, GSA Bulletin

Creveling et al., 2014, GSA Bulletin

Creveling et al., 2014, GSA Bulletin

Creveling et al., 2014, GSA Bulletin

Creveling et al., 2014, GSA Bulletin

Creveling et al., 2014, GSA Bulletin

Creveling et al., 2014, GSA Bulletin

Creveling et al., 2014, GSA Bulletin

Creveling et al., 2014, GSA Bulletin

Creveling et al., 2014, GSA Bulletin

Creveling et al., 2014, GSA Bulletin

Creveling et al., 2014, GSA Bulletin

Creveling et al., 2014, GSA Bulletin

Creveling et al., 2014, GSA Bulletin

This study

This study

This study

This study

This study

This study

This study

This study

This study

This study

This study

This study

This study

This study

This study

This study

This study

This study

This study

This study

This study

This study

This study

This study

\begin{tabular}{|c|c|c|}
\hline Core & 2 & Only used sample \\
\hline Core & 2 & Only used sample \\
\hline Core & 2 & Only used sample \\
\hline Core & 2 & Only used sample \\
\hline Core & 2 & Only used sample \\
\hline Core & 2 & Only used sample \\
\hline Core & 2 & Only used sample \\
\hline Core & 2 & Only used sample \\
\hline Core & 2 & Only used sample \\
\hline Core & 2 & Only used sample \\
\hline Core & 2 & Only used sample \\
\hline Core & 2 & Only used sample \\
\hline Core & 2 & Only used sample \\
\hline Core & 2 & Only used sample \\
\hline Core & 2 & Only used sample \\
\hline Core & 2 & Only used sample \\
\hline Core & 2 & Only used sample \\
\hline Outcrop & 3 & \\
\hline Outcrop & 3 & \\
\hline Outcrop & 3 & \\
\hline Outcrop & 3 & \\
\hline Outcrop & 3 & \\
\hline Outcrop & 3 & \\
\hline Outcrop & 3 & \\
\hline Outcrop & 3 & \\
\hline Outcrop & 3 & \\
\hline Outcrop & 3 & \\
\hline Outcrop & 3 & \\
\hline Outcrop & 3 & \\
\hline Outcrop & 3 & \\
\hline Outcrop & 3 & \\
\hline Outcrop & 3 & \\
\hline Outcrop & 3 & \\
\hline Outcrop & 3 & \\
\hline Outcrop & 3 & \\
\hline Outcrop & 3 & \\
\hline Outcrop & 3 & \\
\hline Outcrop & 3 & \\
\hline Outcrop & 3 & \\
\hline Outcrop & 3 & \\
\hline Outcrop & 3 & \\
\hline
\end{tabular}


This study

Outcrop 3

This study

Outcrop 2

This study

Outcrop 2

This study

Outcrop 2

This study

Outcrop 2

This study

Outcrop 2

This study

Outcrop 2

This study

Outcrop 2

This study

Outcrop 2

This study

Outcrop 2

This study

Outcrop 2

This study

Outcrop 2

This study

Outcrop 2

This study

Outcrop 2

This study

Outcrop 2

This study

Outcrop 2

This study

Outcrop 2

This study

Outcrop 2

This study

Outcrop 2

This study

Outcrop 2

This study

Outcrop 2

Boyer et al., 2011, Palaeogeography, Palaeoclim Outcrop 3

Boyer et al., 2011, Palaeogeography, Palaeoclim Outcrop 3

Boyer et al., 2011, Palaeogeography, Palaeoclim Outcrop 3

Boyer et al., 2011, Palaeogeography, Palaeoclim Outcrop 3

Boyer et al., 2011, Palaeogeography, Palaeoclim Outcrop 3

Boyer et al., 2011, Palaeogeography, Palaeoclim Outcrop 3

Boyer et al., 2011, Palaeogeography, Palaeoclim Outcrop 3

Boyer et al., 2011, Palaeogeography, Palaeoclim Outcrop 3

Boyer et al., 2011, Palaeogeography, Palaeoclim Outcrop 3

Boyer et al., 2011, Palaeogeography, Palaeoclim Outcrop 3

Boyer et al., 2011, Palaeogeography, Palaeoclim Outcrop 3

Boyer et al., 2011, Palaeogeography, Palaeoclim Outcrop 3

Boyer et al., 2011, Palaeogeography, Palaeoclim Outcrop 3

Boyer et al., 2011, Palaeogeography, Palaeoclim Outcrop 3

Boyer et al., 2011, Palaeogeography, Palaeoclim Outcrop 3

Boyer et al., 2011, Palaeogeography, Palaeoclim Outcrop 3

Boyer et al., 2011, Palaeogeography, Palaeoclim Outcrop 3

Boyer et al., 2011, Palaeogeography, Palaeoclim Outcrop 3

Boyer et al., 2011, Palaeogeography, Palaeoclim Outcrop 3

Boyer et al., 2011, Palaeogeography, Palaeoclim Outcrop 3 
Boyer et al., 2011, Palaeogeography, Palaeoclim Outcrop 3

Boyer et al., 2011, Palaeogeography, Palaeoclim Outcrop 3

Boyer et al., 2011, Palaeogeography, Palaeoclim Outcrop 3

Boyer et al., 2011, Palaeogeography, Palaeoclim Outcrop 3

Boyer et al., 2011, Palaeogeography, Palaeoclim Outcrop 3

Boyer et al., 2011, Palaeogeography, Palaeoclim Outcrop 3

Boyer et al., 2011, Palaeogeography, Palaeoclim Outcrop 3

Boyer et al., 2011, Palaeogeography, Palaeoclim Outcrop 3

Boyer et al., 2011, Palaeogeography, Palaeoclim Outcrop 3

Boyer et al., 2011, Palaeogeography, Palaeoclim Outcrop 3

Boyer et al., 2011, Palaeogeography, Palaeoclim Outcrop 3

Boyer et al., 2011, Palaeogeography, Palaeoclim Outcrop 3

Boyer et al., 2011, Palaeogeography, Palaeoclim Outcrop 3

Boyer et al., 2011, Palaeogeography, Palaeoclim Outcrop 3

Boyer et al., 2011, Palaeogeography, Palaeoclim Outcrop 3

Boyer et al., 2011, Palaeogeography, Palaeoclim Outcrop 3

Boyer et al., 2011, Palaeogeography, Palaeoclim Outcrop 3

Boyer et al., 2011, Palaeogeography, Palaeoclim Outcrop 3

Boyer et al., 2011, Palaeogeography, Palaeoclim Outcrop 3

Boyer et al., 2011, Palaeogeography, Palaeoclim Outcrop 3

Boyer et al., 2011, Palaeogeography, Palaeoclim Outcrop 3

Boyer et al., 2011, Palaeogeography, Palaeoclim Outcrop 3

Boyer et al., 2011, Palaeogeography, Palaeoclim Outcrop 3

Boyer et al., 2011, Palaeogeography, Palaeoclim Outcrop 3

Boyer et al., 2011, Palaeogeography, Palaeoclim Outcrop 3

Boyer et al., 2011, Palaeogeography, Palaeoclim Outcrop 3

Boyer et al., 2011, Palaeogeography, Palaeoclim Outcrop 3

Boyer et al., 2011, Palaeogeography, Palaeoclim Outcrop 3

Boyer et al., 2011, Palaeogeography, Palaeoclim Outcrop 3

Boyer et al., 2011, Palaeogeography, Palaeoclim Outcrop 3

Boyer et al., 2011, Palaeogeography, Palaeoclim Outcrop 3

Boyer et al., 2011, Palaeogeography, Palaeoclim Outcrop 3

Boyer et al., 2011, Palaeogeography, Palaeoclim Outcrop 3

Boyer et al., 2011, Palaeogeography, Palaeoclim Outcrop 3

Boyer et al., 2011, Palaeogeography, Palaeoclim Outcrop 3

Boyer et al., 2011, Palaeogeography, Palaeoclim Outcrop 3

Boyer et al., 2011, Palaeogeography, Palaeoclim Outcrop 3

Boyer et al., 2011, Palaeogeography, Palaeoclim Outcrop 3

Boyer et al., 2011, Palaeogeography, Palaeoclim Outcrop 3

Boyer et al., 2011, Palaeogeography, Palaeoclim Outcrop 3

Boyer et al., 2011, Palaeogeography, Palaeoclim Outcrop 3 
Boyer et al., 2011, Palaeogeography, Palaeoclim Outcrop 3

Boyer et al., 2011, Palaeogeography, Palaeoclim Outcrop 3

Boyer et al., 2011, Palaeogeography, Palaeoclim Outcrop 3

Boyer et al., 2011, Palaeogeography, Palaeoclim Outcrop 3

Boyer et al., 2011, Palaeogeography, Palaeoclim Outcrop 3

Boyer et al., 2011, Palaeogeography, Palaeoclim Outcrop 3

Boyer et al., 2011, Palaeogeography, Palaeoclim Outcrop 3

Boyer et al., 2011, Palaeogeography, Palaeoclim Outcrop 3

Boyer et al., 2011, Palaeogeography, Palaeoclim Outcrop 3

Boyer et al., 2011, Palaeogeography, Palaeoclim Outcrop 3

Boyer et al., 2011, Palaeogeography, Palaeoclim Outcrop 3

Boyer et al., 2011, Palaeogeography, Palaeoclim Outcrop 3

Boyer et al., 2011, Palaeogeography, Palaeoclim Outcrop 3

Boyer et al., 2011, Palaeogeography, Palaeoclim Outcrop 3

Boyer et al., 2011, Palaeogeography, Palaeoclim Outcrop 3

Boyer et al., 2011, Palaeogeography, Palaeoclim Outcrop 3

Boyer et al., 2011, Palaeogeography, Palaeoclim Outcrop 3

Boyer et al., 2011, Palaeogeography, Palaeoclim Outcrop 3

Boyer et al., 2011, Palaeogeography, Palaeoclim Outcrop 3

Boyer et al., 2011, Palaeogeography, Palaeoclim Outcrop 3

Boyer et al., 2011, Palaeogeography, Palaeoclim Outcrop 3

Boyer et al., 2011, Palaeogeography, Palaeoclim Outcrop 3

Boyer et al., 2011, Palaeogeography, Palaeoclim Outcrop 3

Boyer et al., 2011, Palaeogeography, Palaeoclim Outcrop 3

Boyer et al., 2011, Palaeogeography, Palaeoclim Outcrop 3

Boyer et al., 2011, Palaeogeography, Palaeoclim Outcrop 3

Boyer et al., 2011, Palaeogeography, Palaeoclim Outcrop 3

Boyer et al., 2011, Palaeogeography, Palaeoclim Outcrop 3

Boyer et al., 2011, Palaeogeography, Palaeoclim Outcrop 3

Boyer et al., 2011, Palaeogeography, Palaeoclim Outcrop 3

Boyer et al., 2011, Palaeogeography, Palaeoclim Outcrop 3

Boyer et al., 2011, Palaeogeography, Palaeoclim Outcrop 3

Boyer et al., 2011, Palaeogeography, Palaeoclim Outcrop 3

Boyer et al., 2011, Palaeogeography, Palaeoclim Outcrop 3

Boyer et al., 2011, Palaeogeography, Palaeoclim Outcrop 3

Boyer et al., 2011, Palaeogeography, Palaeoclim Outcrop 3

Boyer et al., 2011, Palaeogeography, Palaeoclim Outcrop 3

Boyer et al., 2011, Palaeogeography, Palaeoclim Outcrop 3

Boyer et al., 2011, Palaeogeography, Palaeoclim Outcrop 3

Boyer et al., 2011, Palaeogeography, Palaeoclim Outcrop 3

Dahl et al., 2010, PNAS Outcrop 3 
Dahl et al., 2010, PNAS Outcrop 3

Dahl et al., 2010, PNAS Outcrop 3

Dahl et al., 2010, PNAS Outcrop 3

Farrell et al., 2013, American Journal of Science Outcrop 3

Farrell et al., 2013, American Journal of Science Outcrop 3

Farrell et al., 2013, American Journal of Science Outcrop 3

Farrell et al., 2013, American Journal of Science Outcrop 3

Farrell et al., 2013, American Journal of Science Outcrop 3

Farrell et al., 2013, American Journal of Science Outcrop 3

Farrell et al., 2013, American Journal of Science Outcrop 3

Farrell et al., 2013, American Journal of Science Outcrop 3

Farrell et al., 2013, American Journal of Science Outcrop 3

Farrell et al., 2013, American Journal of Science Outcrop 3

Farrell et al., 2013, American Journal of Science Outcrop 3

Farrell et al., 2013, American Journal of Science Outcrop 3

Farrell et al., 2013, American Journal of Science Outcrop 3

Farrell et al., 2013, American Journal of Science Outcrop 3

Farrell et al., 2013, American Journal of Science Outcrop 3

Farrell et al., 2013, American Journal of Science Outcrop 3

Farrell et al., 2013, American Journal of Science Outcrop 3

Farrell et al., 2013, American Journal of Science Outcrop 3

Farrell et al., 2013, American Journal of Science Outcrop 3

Farrell et al., 2013, American Journal of Science Outcrop 3

Farrell et al., 2013, American Journal of Science Outcrop 3

Farrell et al., 2013, American Journal of Science Outcrop 3

Farrell et al., 2013, American Journal of Science Outcrop 3

Farrell et al., 2013, American Journal of Science Outcrop 3

Farrell et al., 2013, American Journal of Science Outcrop 3

Farrell et al., 2013, American Journal of Science Outcrop 3

Farrell et al., 2013, American Journal of Science Outcrop 3

Farrell et al., 2013, American Journal of Science Outcrop 3

Farrell et al., 2013, American Journal of Science Outcrop 3

Farrell et al., 2013, American Journal of Science Outcrop 3

Farrell et al., 2013, American Journal of Science Outcrop 3

Farrell et al., 2013, American Journal of Science Outcrop 3

Farrell et al., 2013, American Journal of Science Outcrop 3

Farrell et al., 2013, American Journal of Science Outcrop 3

Farrell et al., 2013, American Journal of Science Outcrop 3

Farrell et al., 2013, American Journal of Science Outcrop 3

Farrell et al., 2013, American Journal of Science Outcrop 3

Farrell et al., 2013, American Journal of Science Outcrop 3 
Farrell et al., 2013, American Journal of Science Outcrop 3

Farrell et al., 2013, American Journal of Science Outcrop 3

Farrell et al., 2013, American Journal of Science Outcrop 3

Farrell et al., 2013, American Journal of Science Outcrop 3

Farrell et al., 2013, American Journal of Science Outcrop 3

Farrell et al., 2013, American Journal of Science Outcrop 3

Farrell et al., 2013, American Journal of Science Outcrop 3

Farrell et al., 2013, American Journal of Science Outcrop 3

Farrell et al., 2013, American Journal of Science Outcrop 3

Farrell et al., 2013, American Journal of Science Outcrop 3

Farrell et al., 2013, American Journal of Science Outcrop 3

Farrell et al., 2013, American Journal of Science Outcrop 3

Farrell et al., 2013, American Journal of Science Outcrop 3

Farrell et al., 2013, American Journal of Science Outcrop 3

Farrell et al., 2013, American Journal of Science Outcrop 3

Farrell et al., 2013, American Journal of Science Outcrop 3

Farrell et al., 2013, American Journal of Science Outcrop 3

Farrell et al., 2013, American Journal of Science Outcrop 3

Farrell et al., 2013, American Journal of Science Outcrop 3

Farrell et al., 2013, American Journal of Science Outcrop 3

Farrell et al., 2013, American Journal of Science Outcrop 3

Farrell et al., 2013, American Journal of Science Outcrop 3

Farrell et al., 2013, American Journal of Science Outcrop 3

Farrell et al., 2013, American Journal of Science Outcrop 3

Farrell et al., 2013, American Journal of Science Outcrop 3

Farrell et al., 2013, American Journal of Science Outcrop 3

Farrell et al., 2013, American Journal of Science Outcrop 3

Farrell et al., 2013, American Journal of Science Outcrop 3

Farrell et al., 2013, American Journal of Science Outcrop 3

Farrell et al., 2013, American Journal of Science Outcrop 3

Farrell et al., 2013, American Journal of Science Outcrop 3

Farrell et al., 2013, American Journal of Science Outcrop 3

Farrell et al., 2013, American Journal of Science Outcrop 3

Farrell et al., 2013, American Journal of Science Outcrop 3

Farrell et al., 2013, American Journal of Science Outcrop 3

Farrell et al., 2013, American Journal of Science Outcrop 3

Farrell et al., 2013, American Journal of Science Outcrop 3

Farrell et al., 2013, American Journal of Science Outcrop 3

Farrell et al., 2013, American Journal of Science Outcrop 3

Farrell et al., 2013, American Journal of Science Outcrop 3

Farrell et al., 2013, American Journal of Science Outcrop 3 
Farrell et al., 2013, American Journal of Science Outcrop 3

Farrell et al., 2013, American Journal of Science Outcrop 3

Farrell et al., 2013, American Journal of Science Outcrop 3

Farrell et al., 2013, American Journal of Science Outcrop 3

Farrell et al., 2013, American Journal of Science Outcrop 3

Farrell et al., 2013, American Journal of Science Outcrop 3

Farrell et al., 2013, American Journal of Science Outcrop 3

Farrell et al., 2013, American Journal of Science Outcrop 3

Farrell et al., 2013, American Journal of Science Outcrop 3

Farrell et al., 2013, American Journal of Science Outcrop 3

Farrell et al., 2013, American Journal of Science Outcrop 3

Farrell et al., 2013, American Journal of Science Outcrop 3

Farrell et al., 2013, American Journal of Science Outcrop 3

Farrell et al., 2013, American Journal of Science Outcrop 3

Hammarlund et al., 2012, Earth and Planetary Sı Outcrop 3

Hammarlund et al., 2012, Earth and Planetary SiOutcrop 3

Hammarlund et al., 2012, Earth and Planetary SiOutcrop 3

Hammarlund et al., 2012, Earth and Planetary Si Outcrop 3

Hammarlund et al., 2012, Earth and Planetary SiOutcrop 3

Hammarlund et al., 2012, Earth and Planetary Si Outcrop 3

Hammarlund et al., 2012, Earth and Planetary Sı Outcrop 3

Hammarlund et al., 2012, Earth and Planetary Si Outcrop 3

Hammarlund et al., 2012, Earth and Planetary SiOutcrop 3

Hammarlund et al., 2012, Earth and Planetary Si Outcrop 3

Hammarlund et al., 2012, Earth and Planetary Si Outcrop 3

Hammarlund et al., 2012, Earth and Planetary Si Outcrop 3

Hammarlund et al., 2012, Earth and Planetary SiOutcrop 3

Hammarlund et al., 2012, Earth and Planetary Si Outcrop 3

Hammarlund et al., 2012, Earth and Planetary Sı Outcrop 3

Hammarlund et al., 2012, Earth and Planetary Si Outcrop 3

Hammarlund et al., 2012, Earth and Planetary SiOutcrop 3

Hammarlund et al., 2012, Earth and Planetary SiOutcrop 3

Hammarlund et al., 2012, Earth and Planetary Si Outcrop 3

Hammarlund et al., 2012, Earth and Planetary SiOutcrop 3

Hammarlund et al., 2012, Earth and Planetary SiOutcrop 3

Hammarlund et al., 2012, Earth and Planetary Si Outcrop 3

Hammarlund et al., 2012, Earth and Planetary Si Outcrop 3

Hammarlund et al., 2012, Earth and Planetary SiOutcrop 3

Hammarlund et al., 2012, Earth and Planetary SiOutcrop 3

Hammarlund et al., 2012, Earth and Planetary SiOutcrop 3

Hammarlund et al., 2012, Earth and Planetary Sı Outcrop 3 
Hammarlund et al., 2012, Earth and Planetary Si Outcrop 3

Hammarlund et al., 2012, Earth and Planetary SiOutcrop 3

Hammarlund et al., 2012, Earth and Planetary SiOutcrop 3

Hammarlund et al., 2012, Earth and Planetary Si Outcrop 3

Hammarlund et al., 2012, Earth and Planetary SiOutcrop 3

Hammarlund et al., 2012, Earth and Planetary SiOutcrop 3

Hammarlund et al., 2012, Earth and Planetary Si Outcrop 3

Hammarlund et al., 2012, Earth and Planetary SiOutcrop 3

Hammarlund et al., 2012, Earth and Planetary SiOutcrop 3

Hammarlund et al., 2012, Earth and Planetary Si Outcrop 3

Hammarlund et al., 2012, Earth and Planetary Si Outcrop 3

Hammarlund et al., 2012, Earth and Planetary SiOutcrop 3

Hammarlund et al., 2012, Earth and Planetary SiOutcrop 3

Hammarlund et al., 2012, Earth and Planetary Si Outcrop 3

Hammarlund et al., 2012, Earth and Planetary Si Outcrop 3

Hammarlund et al., 2012, Earth and Planetary Si Outcrop 3

Hammarlund et al., 2012, Earth and Planetary SiOutcrop 3

Hammarlund et al., 2012, Earth and Planetary Sı Outcrop 3

Dahl et al., 2010, PNAS Core 2

Dahl et al., 2010, PNAS Core $\quad 2$

Dahl et al., 2010, PNAS Core 2

Dahl et al., 2010, PNAS Core 2

Dahl et al., 2010, PNAS Core 2

Dahl et al., 2010, PNAS Core 2

Dahl et al., 2010, PNAS Core 2

Dahl et al., 2010, PNAS Core 2

Dahl et al., 2010, PNAS Core 2

Dahl et al., 2010, PNAS Core 2

Hammarlund et al., 2012, Earth and Planetary SiCore 2

Hammarlund et al., 2012, Earth and Planetary SiCore 2

Hammarlund et al., 2012, Earth and Planetary SiCore 2

Hammarlund et al., 2012, Earth and Planetary SiCore 2

Hammarlund et al., 2012, Earth and Planetary SiCore 2

Hammarlund et al., 2012, Earth and Planetary SiCore 2

Hammarlund et al., 2012, Earth and Planetary SiCore 2

Hammarlund et al., 2012, Earth and Planetary SiCore 2

Hammarlund et al., 2012, Earth and Planetary SiCore 2

Hammarlund et al., 2012, Earth and Planetary SiCore 2

Hammarlund et al., 2012, Earth and Planetary SiCore 2

Hammarlund et al., 2012, Earth and Planetary SiCore 2

Hammarlund et al., 2012, Earth and Planetary SiCore 2 
Hammarlund et al., 2012, Earth and Planetary Sı Core 2

Hammarlund et al., 2012, Earth and Planetary Sı Core 2

Hammarlund et al., 2012, Earth and Planetary SiCore 2

Hammarlund et al., 2012, Earth and Planetary SiCore 2

Hammarlund et al., 2012, Earth and Planetary SiCore 2

Hammarlund et al., 2012, Earth and Planetary SıCore 2

Hammarlund et al., 2012, Earth and Planetary Sı Core 2

Hammarlund et al., 2012, Earth and Planetary SiCore 2

Hammarlund et al., 2012, Earth and Planetary SiCore 2

Hammarlund et al., 2012, Earth and Planetary SiCore 2

Hammarlund et al., 2012, Earth and Planetary SiCore 2

Hammarlund et al., 2012, Earth and Planetary SiCore 2

Hammarlund et al., 2012, Earth and Planetary SiCore 2

Hammarlund et al., 2012, Earth and Planetary SiCore 2

Hammarlund et al., 2012, Earth and Planetary Sı Core 2

Hammarlund et al., 2012, Earth and Planetary SiCore 2

Hammarlund et al., 2012, Earth and Planetary SiCore 2

Hammarlund et al., 2012, Earth and Planetary SiCore 2

Hammarlund et al., 2012, Earth and Planetary SiCore 2

Hammarlund et al., 2012, Earth and Planetary SiCore 2

Hammarlund et al., 2012, Earth and Planetary Sı Core 2

Hammarlund et al., 2012, Earth and Planetary SiCore 2

Hammarlund et al., 2012, Earth and Planetary Sı Core 2

Hammarlund et al., 2012, Earth and Planetary SiCore 2

Hammarlund et al., 2012, Earth and Planetary Sı Core 2

Hammarlund et al., 2012, Earth and Planetary SiCore 2

Hammarlund et al., 2012, Earth and Planetary Sı Core 2

Hammarlund et al., 2012, Earth and Planetary SiCore 2

Hammarlund et al., 2012, Earth and Planetary Sı Core 2

Hammarlund et al., 2012, Earth and Planetary SiCore 2

Hammarlund et al., 2012, Earth and Planetary Sı Core 2

Hammarlund et al., 2012, Earth and Planetary SiCore 2

Hammarlund et al., 2012, Earth and Planetary Sı Core 2

Hammarlund et al., 2012, Earth and Planetary SiCore 2

Hammarlund et al., 2012, Earth and Planetary Sı Core 2

Hammarlund et al., 2012, Earth and Planetary SiCore 2

Hammarlund et al., 2012, Earth and Planetary Sı Core 2

Hammarlund et al., 2012, Earth and Planetary SiCore 2

Hammarlund et al., 2012, Earth and Planetary Si Core 2

Hammarlund et al., 2012, Earth and Planetary SiCore 2

Hammarlund et al., 2012, Earth and Planetary Sı Core 2 
Hammarlund et al., 2012, Earth and Planetary Sı Core 2

Hammarlund et al., 2012, Earth and Planetary Sı Core 2

Hammarlund et al., 2012, Earth and Planetary SiCore 2

Hammarlund et al., 2012, Earth and Planetary SiCore 2

Hammarlund et al., 2012, Earth and Planetary Si Core 2

Hammarlund et al., 2012, Earth and Planetary SiCore 2

Hammarlund et al., 2012, Earth and Planetary Sı Core 2

Hammarlund et al., 2012, Earth and Planetary SiCore 2

Hammarlund et al., 2012, Earth and Planetary Si Core 2

Hammarlund et al., 2012, Earth and Planetary SiCore 2

Hammarlund et al., 2012, Earth and Planetary SiCore 2

Hammarlund et al., 2012, Earth and Planetary SiCore 2

Hammarlund et al., 2012, Earth and Planetary Si Core 2

Hammarlund et al., 2012, Earth and Planetary SiCore 2

Hammarlund et al., 2012, Earth and Planetary Sı Core 2

Hammarlund et al., 2012, Earth and Planetary SiCore 2

Hammarlund et al., 2012, Earth and Planetary Sı Core 2

Hammarlund et al., 2012, Earth and Planetary SiCore 2

Hammarlund et al., 2012, Earth and Planetary Si Core 2

Hammarlund et al., 2012, Earth and Planetary SiCore 2

Hammarlund et al., 2012, Earth and Planetary Sı Core 2

Hammarlund et al., 2012, Earth and Planetary SiCore 2

Hammarlund et al., 2012, Earth and Planetary Sı Core 2

Hammarlund et al., 2012, Earth and Planetary Si Outcrop 2

Hammarlund et al., 2012, Earth and Planetary Sı Outcrop 2

Hammarlund et al., 2012, Earth and Planetary Si Outcrop 2

Hammarlund et al., 2012, Earth and Planetary Sı Outcrop 2

Hammarlund et al., 2012, Earth and Planetary Sı Outcrop 2

Hammarlund et al., 2012, Earth and Planetary Sı Outcrop 2

Hammarlund et al., 2012, Earth and Planetary Sı Outcrop 2

Hammarlund et al., 2012, Earth and Planetary Sı Outcrop 2

Hammarlund et al., 2012, Earth and Planetary SiOutcrop 2

Hammarlund et al., 2012, Earth and Planetary Sı Outcrop 2

Hammarlund et al., 2012, Earth and Planetary Sı Outcrop 2

Hammarlund et al., 2012, Earth and Planetary Sı Outcrop 2

Hammarlund et al., 2012, Earth and Planetary Sı Outcrop 2

Hammarlund et al., 2012, Earth and Planetary Sı Outcrop 2

Hammarlund et al., 2012, Earth and Planetary Sı Outcrop 2

Hammarlund et al., 2012, Earth and Planetary Si Outcrop 2

Hammarlund et al., 2012, Earth and Planetary Si Outcrop 2

Hammarlund et al., 2012, Earth and Planetary Sı Outcrop 2 
Hammarlund et al., 2012, Earth and Planetary Si Outcrop 2

Hammarlund et al., 2012, Earth and Planetary SiOutcrop 2

Hammarlund et al., 2012, Earth and Planetary SiOutcrop 2

Hammarlund et al., 2012, Earth and Planetary SiOutcrop 2

Hammarlund et al., 2012, Earth and Planetary SiOutcrop 2

Hammarlund et al., 2012, Earth and Planetary SiOutcrop 2

Hammarlund et al., 2012, Earth and Planetary Si Outcrop 2

Hammarlund et al., 2012, Earth and Planetary SiOutcrop 2

Hammarlund et al., 2012, Earth and Planetary SiOutcrop 2

Hammarlund et al., 2012, Earth and Planetary Si Outcrop 2

Hammarlund et al., 2012, Earth and Planetary Si Outcrop 2

Hammarlund et al., 2012, Earth and Planetary SiOutcrop 2

Hammarlund et al., 2012, Earth and Planetary SiOutcrop 2

Hammarlund et al., 2012, Earth and Planetary Si Outcrop 2

Hammarlund et al., 2012, Earth and Planetary Si Outcrop 2

Hammarlund et al., 2012, Earth and Planetary Si Outcrop 2

Hammarlund et al., 2012, Earth and Planetary SiOutcrop 2

Hammarlund et al., 2012, Earth and Planetary SiOutcrop 2

Hammarlund et al., 2012, Earth and Planetary SiOutcrop 2

Dahl et al., 2010, PNAS Core 2

Dahl et al., 2010, PNAS Core 2

Dahl et al., 2010, PNAS Core 2

Dahl et al., 2010, PNAS Core 2

Dahl et al., 2010, PNAS Core 2

Dahl et al., 2010, PNAS Core 2

Dahl et al., 2010, PNAS Core 2

Dahl et al., 2010, PNAS Core 2

Dahl et al., 2010, PNAS Core 2

Dahl et al., 2010, PNAS Core 2

Dahl et al., 2010, PNAS Core 2

Dahl et al., 2010, PNAS Core 2

Dahl et al., 2010, PNAS Core 2

Dahl et al., 2010, PNAS Core 2

Dahl et al., 2010, PNAS Core 2

Dahl et al., 2010, PNAS Core 2

Dahl et al., 2010, PNAS Core 2

Dahl et al., 2010, PNAS Core $\quad 2$ Storm waves may

Dahl et al., 2010, PNAS Core $\quad 2$ Storm waves may

Dahl et al., 2010, PNAS Core 2 Storm waves may

Raiswell and Canfield, 1998, American Journal o Modern sediment

Raiswell and Canfield, 1998, American Journal o Modern sediment 
Raiswell and Canfield, 1998, American Journal o Modern sediment Raiswell and Canfield, 1998, American Journal o Modern sediment Raiswell and Canfield, 1998, American Journal o Modern sediment Raiswell and Canfield, 1998, American Journal o Modern sediment Raiswell and Canfield, 1998, American Journal o Modern sediment Raiswell and Canfield, 1998, American Journal o Modern sediment Raiswell and Canfield, 1998, American Journal o Modern sediment Raiswell and Canfield, 1998, American Journal o Modern sediment Raiswell and Canfield, 1998, American Journal o Modern sediment Raiswell and Canfield, 1998, American Journal o Modern sediment Raiswell and Canfield, 1998, American Journal o Modern sediment Raiswell and Canfield, 1998, American Journal o Modern sediment Raiswell and Canfield, 1998, American Journal o Modern sediment Raiswell and Canfield, 1998, American Journal o Modern sediment Raiswell and Canfield, 1998, American Journal o Modern sediment Raiswell and Canfield, 1998, American Journal o Modern sediment Raiswell and Canfield, 1998, American Journal o Modern sediment Raiswell and Canfield, 1998, American Journal o Modern sediment Raiswell and Canfield, 1998, American Journal o Modern sediment Raiswell and Canfield, 1998, American Journal o Modern sediment Raiswell and Canfield, 1998, American Journal o Modern sediment Raiswell and Canfield, 1998, American Journal o Modern sediment Raiswell and Canfield, 1998, American Journal o Modern sediment Raiswell and Canfield, 1998, American Journal o Modern sediment Raiswell and Canfield, 1998, American Journal o Modern sediment Raiswell and Canfield, 1998, American Journal o Modern sediment Raiswell and Canfield, 1998, American Journal o Modern sediment Raiswell and Canfield, 1998, American Journal o Modern sediment Raiswell and Canfield, 1998, American Journal o Modern sediment Raiswell and Canfield, 1998, American Journal o Modern sediment Raiswell and Canfield, 1998, American Journal o Modern sediment Raiswell and Canfield, 1998, American Journal o Modern sediment Raiswell and Canfield, 1998, American Journal o Modern sediment Raiswell and Canfield, 1998, American Journal o Modern sediment Raiswell and Canfield, 1998, American Journal o Modern sediment Raiswell and Canfield, 1998, American Journal o Modern sediment Raiswell and Canfield, 1998, American Journal o Modern sediment Raiswell and Canfield, 1998, American Journal o Modern sediment Raiswell and Canfield, 1998, American Journal o Modern sediment Raiswell and Canfield, 1998, American Journal o Modern sediment Raiswell and Canfield, 1998, American Journal o Modern sediment 
Raiswell and Canfield, 1998, American Journal o Modern sediment Raiswell and Canfield, 1998, American Journal o Modern sediment Raiswell and Canfield, 1998, American Journal o Modern sediment Raiswell and Canfield, 1998, American Journal o Modern sediment Raiswell and Canfield, 1998, American Journal o Modern sediment Raiswell and Canfield, 1998, American Journal o Modern sediment Raiswell and Canfield, 1998, American Journal o Modern sediment Raiswell and Canfield, 1998, American Journal o Modern sediment Raiswell and Canfield, 1998, American Journal o Modern sediment Raiswell and Canfield, 1998, American Journal o Modern sediment Raiswell and Canfield, 1998, American Journal o Modern sediment Raiswell and Canfield, 1998, American Journal o Modern sediment Raiswell and Canfield, 1998, American Journal o Modern sediment Raiswell and Canfield, 1998, American Journal o Modern sediment Raiswell and Canfield, 1998, American Journal o Modern sediment Raiswell and Canfield, 1998, American Journal o Modern sedime Raiswell and Canfield, 1998, American Journal o Modern sedime Raiswell and Canfield, 1998, American Journal o Modern sedime Raiswell and Canfield, 1998, American Journal o Modern sedime Raiswell and Canfield, 1998, American Journal o Modern sedime Raiswell and Canfield, 1998, American Journal o Modern sedime Raiswell and Canfield, 1998, American Journal o Modern sedime Raiswell and Canfield, 1998, American Journal o Modern sedime Raiswell and Canfield, 1998, American Journal o Modern sedime Raiswell and Canfield, 1998, American Journal o Modern sedime Raiswell and Canfield, 1998, American Journal o Modern sedime Raiswell and Canfield, 1998, American Journal o Modern sedime Raiswell and Canfield, 1998, American Journal o Modern sedime Raiswell and Canfield, 1998, American Journal o Modern sedime Raiswell and Canfield, 1998, American Journal o Modern sedime Raiswell and Canfield, 1998, American Journal o Modern sedime Raiswell and Canfield, 1998, American Journal o Modern sedime Raiswell and Canfield, 1998, American Journal o Modern sedime 3 Raiswell and Canfield, 1998, American Journal o Modern sedime 3 Raiswell and Canfield, 1998, American Journal o Modern sedime 3 Raiswell and Canfield, 1998, American Journal o Modern sedime 3 Raiswell and Canfield, 1998, American Journal o Modern sedime 3 Raiswell and Canfield, 1998, American Journal o Modern sedime 3 Raiswell and Canfield, 1998, American Journal o Modern sedime 3 Raiswell and Canfield, 1998, American Journal o Modern sedime 3 Raiswell and Canfield, 1998, American Journal o Modern sedime 3 
Raiswell and Canfield, 1998, American Journal o Modern sedime

Raiswell and Canfield, 1998, American Journal o Modern sedime 3

Raiswell and Canfield, 1998, American Journal o Modern sedime 3

Raiswell and Canfield, 1998, American Journal o Modern sedime 3

Raiswell and Canfield, 1998, American Journal o Modern sedime 3

Raiswell and Canfield, 1998, American Journal o Modern sedime 3

Raiswell and Canfield, 1998, American Journal o Modern sedime 3

Raiswell and Canfield, 1998, American Journal o Modern sedime 3

Raiswell and Canfield, 1998, American Journal o Modern sedime 3

Raiswell and Canfield, 1998, American Journal o Modern sedime 3

Raiswell and Canfield, 1998, American Journal o Modern sedime 3

Raiswell and Canfield, 1998, American Journal o Modern sedime 3

Raiswell and Canfield, 1998, American Journal o Modern sedime 3

Raiswell and Canfield, 1998, American Journal o Modern sedime 3

Raiswell and Canfield, 1998, American Journal o Modern sedime 3

Raiswell and Canfield, 1998, American Journal o Modern sedime 3

Raiswell and Canfield, 1998, American Journal o Modern sedime 3

Raiswell and Canfield, 1998, American Journal o Modern sedime 3

Raiswell and Canfield, 1998, American Journal o Modern sedime 3

Raiswell and Canfield, 1998, American Journal o Modern sedime 3

Raiswell and Canfield, 1998, American Journal o Modern sedime 3

Raiswell and Canfield, 1998, American Journal o Modern sedime 3

Raiswell and Canfield, 1998, American Journal o Modern sedime 3

Raiswell and Canfield, 1998, American Journal o Modern sedime 3

Raiswell and Canfield, 1998, American Journal o Modern sedime 3

Raiswell and Canfield, 1998, American Journal o Modern sedime 3

Raiswell and Canfield, 1998, American Journal o Modern sedime 3

Raiswell and Canfield, 1998, American Journal o Modern sedime 3

Raiswell and Canfield, 1998, American Journal o Modern sedime 3

Raiswell and Canfield, 1998, American Journal o Modern sedime 3

Raiswell and Canfield, 1998, American Journal o Modern sedime 3

Raiswell and Canfield, 1998, American Journal o Modern sedime 3

Raiswell and Canfield, 1998, American Journal o Modern sedime 3

Raiswell and Canfield, 1998, American Journal o Modern sediment

Raiswell and Canfield, 1998, American Journal o Modern sediment

Raiswell and Canfield, 1998, American Journal o Modern sediment

Raiswell and Canfield, 1998, American Journal o Modern sediment

Raiswell and Canfield, 1998, American Journal o Modern sediment

Raiswell and Canfield, 1998, American Journal o Modern sediment

Raiswell and Canfield, 1998, American Journal o Modern sediment

Raiswell and Canfield, 1998, American Journal o Modern sediment 
Raiswell and Canfield, 1998, American Journal o Modern sediment Raiswell and Canfield, 1998, American Journal o Modern sediment Raiswell and Canfield, 1998, American Journal o Modern sediment Raiswell and Canfield, 1998, American Journal o Modern sediment Raiswell and Canfield, 1998, American Journal o Modern sediment Raiswell and Canfield, 1998, American Journal o Modern sediment Raiswell and Canfield, 1998, American Journal o Modern sediment Raiswell and Canfield, 1998, American Journal o Modern sediment Raiswell and Canfield, 1998, American Journal o Modern sediment Raiswell and Canfield, 1998, American Journal o Modern sediment Raiswell and Canfield, 1998, American Journal o Modern sediment Raiswell and Canfield, 1998, American Journal o Modern sediment Raiswell and Canfield, 1998, American Journal o Modern sediment Raiswell and Canfield, 1998, American Journal o Modern sediment Raiswell and Canfield, 1998, American Journal o Modern sediment Raiswell and Canfield, 1998, American Journal o Modern sediment Raiswell and Canfield, 1998, American Journal o Modern sediment Raiswell and Canfield, 1998, American Journal o Modern sediment Raiswell and Canfield, 1998, American Journal o Modern sediment Raiswell and Canfield, 1998, American Journal o Modern sediment Raiswell and Canfield, 1998, American Journal o Modern sediment Raiswell and Canfield, 1998, American Journal o Modern sediment Raiswell and Canfield, 1998, American Journal o Modern sediment Raiswell and Canfield, 1998, American Journal o Modern sediment Raiswell and Canfield, 1998, American Journal o Modern sediment Raiswell and Canfield, 1998, American Journal o Modern sediment Raiswell and Canfield, 1998, American Journal o Modern sediment Raiswell and Canfield, 1998, American Journal o Modern sediment Raiswell and Canfield, 1998, American Journal o Modern sediment Raiswell and Canfield, 1998, American Journal o Modern sediment Raiswell and Canfield, 1998, American Journal o Modern sediment Raiswell and Canfield, 1998, American Journal o Modern sediment Raiswell and Canfield, 1998, American Journal o Modern sediment Raiswell and Canfield, 1998, American Journal o Modern sediment Raiswell and Canfield, 1998, American Journal o Modern sediment Raiswell and Canfield, 1998, American Journal o Modern sediment Raiswell and Canfield, 1998, American Journal o Modern sediment Raiswell and Canfield, 1998, American Journal o Modern sediment Raiswell and Canfield, 1998, American Journal o Modern sediment Raiswell and Canfield, 1998, American Journal o Modern sediment Raiswell and Canfield, 1998, American Journal o Modern sediment 
Raiswell and Canfield, 1998, American Journal o Modern sediment Raiswell and Canfield, 1998, American Journal o Modern sediment Raiswell and Canfield, 1998, American Journal o Modern sediment Raiswell and Canfield, 1998, American Journal o Modern sediment Raiswell and Canfield, 1998, American Journal o Modern sediment Raiswell and Canfield, 1998, American Journal o Modern sediment Raiswell and Canfield, 1998, American Journal o Modern sediment Raiswell and Canfield, 1998, American Journal o Modern sediment Raiswell and Canfield, 1998, American Journal o Modern sediment Raiswell and Canfield, 1998, American Journal o Modern sediment Raiswell and Canfield, 1998, American Journal o Modern sediment Raiswell and Canfield, 1998, American Journal o Modern sediment Raiswell and Canfield, 1998, American Journal o Modern sediment Raiswell and Canfield, 1998, American Journal o Modern sediment Raiswell and Canfield, 1998, American Journal o Modern sediment Raiswell and Canfield, 1998, American Journal o Modern sediment Raiswell and Canfield, 1998, American Journal o Modern sediment Raiswell and Canfield, 1998, American Journal o Modern sediment Raiswell and Canfield, 1998, American Journal o Modern sediment Raiswell and Canfield, 1998, American Journal o Modern sediment Raiswell and Canfield, 1998, American Journal o Modern sediment Raiswell and Canfield, 1998, American Journal o Modern sediment Raiswell and Canfield, 1998, American Journal o Modern sediment Raiswell and Canfield, 1998, American Journal o Modern sediment Raiswell and Canfield, 1998, American Journal o Modern sediment Raiswell and Canfield, 1998, American Journal o Modern sediment Raiswell and Canfield, 1998, American Journal o Modern sediment Raiswell and Canfield, 1998, American Journal o Modern sediment Raiswell and Canfield, 1998, American Journal o Modern sediment Raiswell and Canfield, 1998, American Journal o Modern sediment Raiswell and Canfield, 1998, American Journal o Modern sediment Raiswell and Canfield, 1998, American Journal o Modern sediment Raiswell and Canfield, 1998, American Journal o Modern sediment Raiswell and Canfield, 1998, American Journal o Modern sediment Raiswell and Canfield, 1998, American Journal o Modern sediment Raiswell and Canfield, 1998, American Journal o Modern sediment Raiswell and Canfield, 1998, American Journal o Modern sediment Raiswell and Canfield, 1998, American Journal o Modern sediment Raiswell and Canfield, 1998, American Journal o Modern sediment Raiswell and Canfield, 1998, American Journal o Modern sediment Raiswell and Canfield, 1998, American Journal o Modern sediment 
Raiswell and Canfield, 1998, American Journal o Modern sediment Raiswell and Canfield, 1998, American Journal o Modern sediment Raiswell and Canfield, 1998, American Journal o Modern sediment Raiswell and Canfield, 1998, American Journal o Modern sediment Raiswell and Canfield, 1998, American Journal o Modern sediment Raiswell and Canfield, 1998, American Journal o Modern sediment Raiswell and Canfield, 1998, American Journal o Modern sediment Raiswell and Canfield, 1998, American Journal o Modern sediment Raiswell and Canfield, 1998, American Journal o Modern sediment Raiswell and Canfield, 1998, American Journal o Modern sediment Raiswell and Canfield, 1998, American Journal o Modern sediment Raiswell and Canfield, 1998, American Journal o Modern sediment Raiswell and Canfield, 1998, American Journal o Modern sediment Raiswell and Canfield, 1998, American Journal o Modern sediment Raiswell and Canfield, 1998, American Journal o Modern sediment Raiswell and Canfield, 1998, American Journal o Modern sediment Raiswell and Canfield, 1998, American Journal o Modern sediment Raiswell and Canfield, 1998, American Journal o Modern sediment Raiswell and Canfield, 1998, American Journal o Modern sediment Raiswell and Canfield, 1998, American Journal o Modern sediment Raiswell and Canfield, 1998, American Journal o Modern sediment Raiswell and Canfield, 1998, American Journal o Modern sediment Raiswell and Canfield, 1998, American Journal o Modern sediment Raiswell and Canfield, 1998, American Journal o Modern sediment Raiswell and Canfield, 1998, American Journal o Modern sediment Raiswell and Canfield, 1998, American Journal o Modern sediment 


\begin{tabular}{|c|c|c|c|c|c|}
\hline \multicolumn{2}{|c|}{ Group/Formation Section } & \multirow{2}{*}{$\begin{array}{c}\text { Sample } \\
448\end{array}$} & \multirow{2}{*}{$\begin{array}{c}\text { FeP } \\
1.000\end{array}$} & \multirow{2}{*}{$\begin{array}{l}\text { FeHR } \\
6.700\end{array}$} & \multirow{2}{*}{$\begin{array}{c}\text { FeT } \\
6.300\end{array}$} \\
\hline Pilgujärvi & Drillhole 2900 & & & & \\
\hline Pilgujärvi & Drillhole 2900 & 453 & 1.400 & 9.100 & 25.700 \\
\hline Pilgujärvi & Drillhole 2900 & 467 & 3.200 & 7.900 & 31.900 \\
\hline Pilgujärvi & Drillhole 2900 & 490 & 1.700 & 10.100 & 28.100 \\
\hline Pilgujärvi & Drillhole 2900 & 508.5 & 1.100 & 6.300 & 19.200 \\
\hline Pilgujärvi & Drillhole 2900 & 517 & 1.900 & 12.700 & 13.100 \\
\hline Pilgujärvi & Drillhole 2900 & 524 & 1.700 & 10.600 & 11.500 \\
\hline Pilgujärvi & Drillhole 2900 & 536 & 0.800 & 11.100 & 12.200 \\
\hline Pilgujärvi & Drillhole 2900 & 549 & 0.600 & 5.900 & 6.600 \\
\hline Pilgujärvi & Drillhole 2900 & 561.8 & 0.400 & 6.500 & 11.800 \\
\hline Pilgujärvi & Drillhole 2900 & 562.9 & 0.400 & 13.400 & 13.400 \\
\hline Pilgujärvi & Drillhole 2900 & 578.2 & 0.400 & 7.900 & 13.200 \\
\hline Pilgujärvi & Drillhole 2900 & 590 & 0.600 & 13.600 & 11.500 \\
\hline Pilgujärvi & Drillhole 2900 & 656 & 1.000 & 15.800 & 14.100 \\
\hline Pilgujärvi & Drillhole 2900 & 666 & 1.400 & 8.300 & 11.600 \\
\hline Pilgujärvi & Drillhole 2900 & 677 & 0.800 & 9.900 & 12.800 \\
\hline Pilgujärvi & Drillhole 2900 & 687 & 1.300 & 9.200 & 12.700 \\
\hline Pilgujärvi & Drillhole 2900 & 705 & 1.200 & 10.600 & 12.400 \\
\hline Pilgujärvi & Drillhole 2900 & 743 & 0.400 & 6.700 & 12.900 \\
\hline Pilgujärvi & Drillhole 2900 & 763 & 0.300 & 6.500 & 7.400 \\
\hline Pilgujärvi & Drillhole 2900 & 788 & 12.500 & 20.900 & 20.200 \\
\hline Pilgujärvi & Drillhole 2900 & 815 & 2.100 & 8.300 & 9.400 \\
\hline Pilgujärvi & Drillhole 2900 & 822 & 4.800 & 11.400 & 11.600 \\
\hline Pilgujärvi & Drillhole 2900 & 833 & 3.400 & 8.300 & 10.600 \\
\hline Pilgujärvi & Drillhole 2900 & 849 & 1.600 & 6.800 & 8.400 \\
\hline Pilgujärvi & Drillhole 2900 & 894 & 2.400 & 7.200 & 8.000 \\
\hline Pilgujärvi & Drillhole 2900 & 933 & 1.700 & 9.200 & 7.500 \\
\hline Pilgujärvi & Drillhole 2900 & 940 & 1.600 & 10.400 & 9.800 \\
\hline Pilgujärvi & Drillhole 2900 & 956 & 0.400 & 6.800 & 10.800 \\
\hline Pilgujärvi & Drillhole 2900 & 996 & 2.200 & & 8.300 \\
\hline Pilgujärvi & Drillhole 2900 & 1011 & 2.500 & 4.500 & 4.900 \\
\hline Pilgujärvi & Drillhole 2900 & 1057 & 1.900 & 6.600 & 8.800 \\
\hline Chuanlinggou & CLG1-02 & 1.4 & 1.170 & 2.900 & 5.260 \\
\hline Chuanlinggou & CLG1-03 & 3.2 & 0.340 & 1.480 & 5.270 \\
\hline Chuanlinggou & CLG1-07 & 5.8 & 0.260 & 4.360 & 5.520 \\
\hline Chuanlinggou & CLG1-10 & 8.9 & 0.020 & 2.750 & 3.840 \\
\hline Chuanlinggou & CLG1-11 & 10.2 & 0.040 & 3.350 & 4.350 \\
\hline Chuanlinggou & CLG1-13 & 12.5 & b.d. & 2.720 & 3.800 \\
\hline Chuanlinggou & CLG2-01 & 0 & 0.350 & 0.690 & 3.450 \\
\hline Chuanlinggou & CLG2-02 & 0.7 & 0.410 & 1.630 & 5.250 \\
\hline
\end{tabular}




\begin{tabular}{|c|c|c|c|c|c|}
\hline Chuanlinggou & CLG2-03 & 1.3 & 0.350 & 0.650 & 3.630 \\
\hline Chuanlinggou & CLG2-04A & 1.8 & 0.370 & 1.380 & 5.260 \\
\hline Chuanlinggou & CLG2-04B & 1.6 & 0.170 & 0.510 & 4.240 \\
\hline Chuanlinggou & CLG2-05 & 2.7 & 0.230 & 0.780 & 4.230 \\
\hline Chuanlinggou & CLG2-06 & 3.2 & 0.200 & 0.630 & 4.250 \\
\hline Chuanlinggou & CLG2-08 & 3.8 & 0.240 & 1.150 & 4.810 \\
\hline Chuanlinggou & CLG2-09 & 40.5 & 0.260 & 0.510 & 3.450 \\
\hline Chuanlinggou & CLG2-10 & 41.1 & 0.090 & 0.550 & 3.610 \\
\hline Chuanlinggou & CLG2-12 & 42.4 & 0.360 & 0.730 & 2.990 \\
\hline Chuanlinggou & CLG4-03 & & 0.080 & 0.790 & 4.660 \\
\hline Chuanlinggou & CLG4-05 & & 0.060 & 3.080 & 14.610 \\
\hline Chuanlinggou & CLG5-31 & 41.2 & 0.450 & 1.350 & 2.400 \\
\hline Chuanlinggou & CLG5-32 & 40.1 & 0.150 & 1.670 & 2.390 \\
\hline Chuanlinggou & CLG5-35 & 36.7 & 0.230 & 1.390 & 2.460 \\
\hline Chuanlinggou & CLG5-37 & 33.3 & 0.560 & 3.440 & 4.520 \\
\hline Chuanlinggou & CLG5-43 & 23.9 & 0.410 & 1.580 & 2.790 \\
\hline Chuanlinggou & CLG5-44 & 23.2 & 0.390 & 1.730 & 2.860 \\
\hline Chuanlinggou & CLG5-45 & 20 & 0.510 & 1.530 & 3.280 \\
\hline Chuanlinggou & CLG5-53 & 10 & 0.440 & 1.160 & 2.170 \\
\hline Chuanlinggou & CLG7-01 & & 0.490 & 1.890 & 5.090 \\
\hline Chuanlinggou & CLG7-02 & & 0.130 & 2.180 & 6.750 \\
\hline Chuanlinggou & CLG7-03 & & 0.360 & 1.580 & 4.750 \\
\hline Chuanlinggou & CLG7-04 & & 0.270 & 1.420 & 4.570 \\
\hline Newland & M16 & 204.8 & 0.700 & 1.170 & 2.390 \\
\hline Newland & M16 & 204.85 & 0.720 & 1.380 & 2.640 \\
\hline Newland & M16 & 232.8 & 0.540 & 1.250 & 3.210 \\
\hline Newland & M16 & 313.6 & 0.990 & 1.590 & 2.940 \\
\hline Newland & M16 & 420.9 & 0.670 & 0.880 & 1.540 \\
\hline Newland & M16 & 495 & 0.760 & 1.850 & 2.450 \\
\hline Newland & M16 & 495.05 & 0.800 & 1.560 & 2.420 \\
\hline Newland & SC93 & 503.7 & 0.930 & 1.120 & 2.600 \\
\hline Newland & SC93 & 503.75 & 0.790 & 0.960 & 2.480 \\
\hline Newland & SC93 & 507.8 & 0.760 & 1.000 & 2.800 \\
\hline Newland & SC93 & 517.8 & 0.130 & 0.580 & 2.220 \\
\hline Newland & SC93 & 521.2 & 0.170 & 0.760 & 2.200 \\
\hline Newland & SC93 & 526.8 & 0.130 & 0.690 & 1.750 \\
\hline Newland & SC93 & 527 & 0.170 & 0.740 & 1.560 \\
\hline \multicolumn{3}{|c|}{ כn not given in this JD-77-70 E } & 0.510 & 0.980 & 2.520 \\
\hline \multicolumn{3}{|c|}{ on not given in this JD-79 $112 \mathrm{C}-1$} & 0.590 & 1.310 & 2.450 \\
\hline \multicolumn{3}{|c|}{ on not given in this JD-79-113-B } & 0.510 & 0.870 & 1.300 \\
\hline \multicolumn{3}{|c|}{ on not given in this JD-79-138D } & 0.420 & 0.530 & 2.770 \\
\hline
\end{tabular}


on not given in this JD-79-186K

on not given in this JD-79-I 112 C-2

Atar

Atar

Atar

Atar

Atar

Atar

Atar

Atar

Atar

Atar

Atar

Atar

Atar

Atar

Atar

Atar

Atar

Atar

Atar

Atar

Atar

Atar

Atar

Atar

Atar

Atar

Atar

Atar

Atar

Atar

Atar

Atar

Atar

Atar

Atar

Atar

Atar

Atar

Atar
Atar

Atar

Atar

Atar

Atar

Atar

Atar

Atar

Atar

Atar

Atar

Atar

Atar

Atar

Atar

Atar

Atar

Atar

Atar

Atar

Atar

Atar

Atar

Atar

Atar

Atar

Atar

Atar

Atar

Atar

Atar

Atar

Atar

Atar

Atar

Atar

Atar

Atar

Atar
0.420

0.790

2.520

1.260

2.220

2.840

R2-1

R2-12

R2-13

R2-14

R2-15A

R2-15B

R2-16

R2-17A

R2-17B

R2-18

R2-19

R2-2

R2-24

R2-25A

R2-25B

R2-27

R2-28

R2-29A

R2-3

R2-30

R2-31

R2-32

R2-33

R2-34

R2-35

R2-4

R2-40

R2-41

R2-42

R2-43A

R2-43B

R2-44

R2-45

R2-46

R2-47

R2-48

R2-6

R2-7

R2-8 


\begin{tabular}{|c|c|c|}
\hline Atar & Atar & $\mathrm{R} 4-12$ \\
\hline Atar & Atar & R4-16 \\
\hline Atar & Atar & R4-17 \\
\hline Atar & Atar & R4-18 \\
\hline Atar & Atar & R4-25 \\
\hline Atar & Atar & R4-26 \\
\hline Atar & Atar & R4-28 \\
\hline Atar & Atar & R4-31 \\
\hline Atar & Atar & R4-32 \\
\hline Atar & Atar & R4-33 \\
\hline Atar & Atar & R4-34 \\
\hline Atar & Atar & R4-35 \\
\hline Atar & Atar & R4-37 \\
\hline Atar & Atar & R4-38 \\
\hline Atar & Atar & R4-40 \\
\hline Atar & Atar & R4-43 \\
\hline Atar & Atar & R4-44 \\
\hline Atar & Atar & R4-45 \\
\hline Atar & Atar & R4-46 \\
\hline Atar & Atar & R4-47 \\
\hline Atar & Atar & R4-48 \\
\hline Atar & Atar & R4-49 \\
\hline Atar & Atar & R4-50 \\
\hline Atar & Atar & R4-53 \\
\hline Atar & Atar & R4-55 \\
\hline Atar & Atar & R4-56 \\
\hline Atar & Atar & R4-59 \\
\hline Atar & Atar & R4-6 \\
\hline Atar & Atar & R4-60 \\
\hline Atar & Atar & R4-61 \\
\hline Atar & Atar & R4-64 \\
\hline Atar & Atar & R4-65 \\
\hline Atar & Atar & R4-67 \\
\hline Atar & Atar & R4-69 \\
\hline Atar & Atar & R4-70 \\
\hline Atar & Atar & R4-73 \\
\hline Atar & Atar & R4-76 \\
\hline Atar & Atar & R4-79 \\
\hline Atar & Atar & R4-8 \\
\hline Atar & Atar & R4-80 \\
\hline Atar & Atar & R4-81 \\
\hline
\end{tabular}




\begin{tabular}{|c|c|c|}
\hline Atar & Atar & $\mathrm{R} 4-82$ \\
\hline Atar & Atar & R4-83 \\
\hline Atar & Atar & R4-84 \\
\hline Atar & Atar & R4-85 \\
\hline Atar & Atar & R4-86 \\
\hline Atar & Atar & R4-88 \\
\hline Atar & Atar & R4-89 \\
\hline Atar & Atar & R4-9 \\
\hline Atar & Atar & R4-90 \\
\hline Atar & Atar & R4-91 \\
\hline Atar & Atar & R4-92 \\
\hline Atar & Atar & R4-93 \\
\hline Atar & Atar & R4-94 \\
\hline Atar & Atar & R4-95 \\
\hline Atar & Atar & R4-97 \\
\hline El Mreiti & El Mreiti & F4-11 \\
\hline El Mreiti & El Mreiti & F4-12 \\
\hline El Mreiti & El Mreiti & F4-14 \\
\hline El Mreiti & El Mreiti & F4-24 \\
\hline El Mreiti & El Mreiti & F4-25 \\
\hline El Mreiti & El Mreiti & F4-27 \\
\hline El Mreiti & El Mreiti & F4-28 \\
\hline El Mreiti & El Mreiti & F4-29 \\
\hline El Mreiti & El Mreiti & F4-30 \\
\hline El Mreiti & El Mreiti & F4-31 \\
\hline El Mreiti & El Mreiti & F4-32 \\
\hline El Mreiti & El Mreiti & F4-34 \\
\hline El Mreiti & El Mreiti & F4-35 \\
\hline El Mreiti & El Mreiti & F4-36 \\
\hline El Mreiti & El Mreiti & F4-37 \\
\hline El Mreiti & El Mreiti & F4-44 \\
\hline El Mreiti & El Mreiti & F4-45 \\
\hline El Mreiti & El Mreiti & F4-46 \\
\hline El Mreiti & El Mreiti & F4-47 \\
\hline El Mreiti & El Mreiti & F4-51 \\
\hline El Mreiti & El Mreiti & F4-52 \\
\hline El Mreiti & El Mreiti & F4-55 \\
\hline El Mreiti & El Mreiti & F4-57 \\
\hline El Mreiti & El Mreiti & F4-59 \\
\hline El Mreiti & El Mreiti & F4-60 \\
\hline El Mreiti & El Mreiti & F4-61 \\
\hline
\end{tabular}




\begin{tabular}{|c|c|c|c|c|c|}
\hline El Mreiti & El Mreiti & F4-65 & & & \\
\hline El Mreiti & El Mreiti & F4-71 & & & \\
\hline El Mreiti & El Mreiti & F4-73 & & & \\
\hline El Mreiti & El Mreiti & F4-77 & & & \\
\hline El Mreiti & El Mreiti & F4-82 & & & \\
\hline El Mreiti & El Mreiti & F4-94 & & & \\
\hline FB1a & FB1a & & 0.362 & 0.647 & 4.310 \\
\hline FB1b & FB1b & & 0.004 & 0.066 & 2.190 \\
\hline FB1b & FB1b & & & & \\
\hline FB1b & FB1b & & & & \\
\hline FB1b & FB1b & & & & \\
\hline FB1b & FB1b & & & & \\
\hline FB1b & FB1b & & & & \\
\hline FB1b & FB1b & & & & \\
\hline FB1b & FB1b & & & & \\
\hline FB1b & FB1b & & & & \\
\hline FB1b & FB1b & & & & \\
\hline FB1b & FB1b & & & & \\
\hline FB1b & FB1b & & & & \\
\hline FB1b & FB1b & & 0.025 & 0.253 & 1.810 \\
\hline FB1b & FB1b & & & & \\
\hline FB1b & FB1b & & & & \\
\hline FB1b & FB1b & & 0.120 & 1.090 & 6.410 \\
\hline FB1b & FB1b & & & & \\
\hline FB1b & FB1b & & & & \\
\hline FB1b & FB1b & & 0.090 & 0.500 & 2.500 \\
\hline FB1b & FB1b & & & & \\
\hline FB1b & FB1b & & 0.049 & 0.290 & 1.320 \\
\hline FB1b & FB1b & & & & \\
\hline FB1b & FB1b & & & & \\
\hline FB1b & FB1b & & & & \\
\hline FB1b & FB1b & & 0.093 & 0.931 & 2.820 \\
\hline FB1b & FB1b & & & & \\
\hline FB1b & FB1b & & & & \\
\hline FB1b & FB1b & & 2.207 & 11.035 & 22.990 \\
\hline FB1b & FB1b & & 2.164 & 2.924 & 4.430 \\
\hline FB1c & FB1C & & 0.010 & 0.170 & 2.430 \\
\hline FB1C & FB1C & & & & \\
\hline FB1c & FB1c & & 0.078 & 0.652 & 5.430 \\
\hline FB1c & FB1C & & & & \\
\hline FB1C & FB1c & & 0.194 & 0.498 & 3.110 \\
\hline
\end{tabular}




\begin{tabular}{|c|c|c|c|c|}
\hline FB1C & FB1C & & & \\
\hline FB1C & FB1C & 0.053 & 0.529 & 3.110 \\
\hline FB1C & FB1C & 0.189 & 0.525 & 3.09 \\
\hline FB1C & FB1C & 0.295 & 0.374 & 1.780 \\
\hline FB1C & FB1C & & & \\
\hline FB1c & FB1C & & & \\
\hline FB1c & FB1c & & & \\
\hline FB1C & FB1c & & & \\
\hline FB1c & FB1c & & & \\
\hline FB1C & FB1C & 0.314 & 1.123 & 3.120 \\
\hline FB1C & FB1C & 0.131 & 0.385 & 1.070 \\
\hline FB1c & FB1c & & & \\
\hline FB1C & FB1C & & & \\
\hline FB1c & FB1c & & & \\
\hline FB1c & FB1c & 0.366 & 0.988 & 2.470 \\
\hline FB1c & FB1c & & & \\
\hline FB1c & FB1c & 0.392 & 0.933 & 2.170 \\
\hline FB1C & FB1C & & & \\
\hline FB1C & FB1C & & & \\
\hline FB1c & FB1C & 1.265 & 1.976 & 3.800 \\
\hline FB1c & FB1c & & & \\
\hline FB1C & FB1C & & & \\
\hline FB1c & FB1c & 0.049 & 4.902 & 8.600 \\
\hline FB1C & FB1c & 1.469 & 4.320 & 6.750 \\
\hline FB1c & FB1C & 0.988 & 2.059 & 3.120 \\
\hline FB1C & FB1c & & & \\
\hline FB1c & FB1c & & & \\
\hline FB1C & FB1C & & & \\
\hline FB1c & FB1C & & & \\
\hline FB1c & FB1c & & & \\
\hline FB1c & FB1c & & & \\
\hline FB1c & FB1c & 1.202 & 1.647 & 1.790 \\
\hline FB1C & FB1c & 1.331 & 2.421 & 2.470 \\
\hline FB1c & FB1c & 2.818 & 3.131 & 3.070 \\
\hline FB1c & FB1c & 1.704 & 1.893 & 1.820 \\
\hline FB2b & $\mathrm{FB} 2 \mathrm{~b}$ & 0.235 & 0.636 & 4.540 \\
\hline $\mathrm{FB} 2 \mathrm{~b}$ & $\mathrm{FB} 2 \mathrm{~b}$ & & & \\
\hline $\mathrm{FB} 2 \mathrm{~b}$ & $\mathrm{FB} 2 \mathrm{~b}$ & & & \\
\hline $\mathrm{FB} 2 \mathrm{~b}$ & $\mathrm{FB} 2 \mathrm{~b}$ & & & \\
\hline FB2b & $\mathrm{FB} 2 \mathrm{~b}$ & 0.110 & 0.844 & 2.410 \\
\hline $\mathrm{FB} 2 \mathrm{~b}$ & $\mathrm{FB} 2 \mathrm{~b}$ & & & \\
\hline
\end{tabular}




\begin{tabular}{|c|c|c|c|c|c|}
\hline $\mathrm{FB} 2 \mathrm{~b}$ & $\mathrm{FB} 2 \mathrm{~b}$ & & 1.332 & 2.184 & 2.800 \\
\hline $\mathrm{FB} 2 \mathrm{~b}$ & $\mathrm{FB} 2 \mathrm{~b}$ & & 0.475 & 1.696 & 2.120 \\
\hline $\mathrm{FB} 2 \mathrm{~b}$ & $\mathrm{FB} 2 \mathrm{~b}$ & & 1.062 & 1.831 & 2.180 \\
\hline $\mathrm{FB} 2 \mathrm{~b}$ & FB2b & & 0.702 & 1.404 & 1.560 \\
\hline $\mathrm{FB} 2 \mathrm{~b}$ & FB2b & & & & \\
\hline $\mathrm{FB} 2 \mathrm{~b}$ & $\mathrm{FB} 2 \mathrm{~b}$ & & 0.250 & 0.455 & 0.500 \\
\hline $\mathrm{FB} 2 \mathrm{~b}$ & FB2b & & 0.574 & 1.739 & 1.870 \\
\hline $\mathrm{FC}$ & $\mathrm{FC}$ & & 0.072 & 0.266 & 3.330 \\
\hline $\mathrm{FC}$ & $\mathrm{FC}$ & & 0.116 & 0.228 & 1.340 \\
\hline $\mathrm{FC}$ & $\mathrm{FC}$ & & 0.013 & 0.050 & 0.280 \\
\hline $\mathrm{FC}$ & $\mathrm{FC}$ & & 0.172 & 0.593 & 2.280 \\
\hline $\mathrm{FC}$ & $\mathrm{FC}$ & & 0.020 & 0.076 & 0.280 \\
\hline $\mathrm{FC}$ & $\mathrm{FC}$ & & 0.782 & 1.203 & 1.850 \\
\hline FD & FD & & 0.935 & 1.764 & 4.010 \\
\hline FD & FD & & 0.542 & 0.888 & 1.930 \\
\hline FD & FD & & 1.661 & 2.102 & 4.290 \\
\hline FD & FD & & 2.330 & 2.912 & 4.480 \\
\hline FD & FD & & 3.464 & 3.981 & 5.770 \\
\hline FD & FD & & 3.854 & 4.283 & 5.710 \\
\hline FD & FD & & 3.625 & 4.028 & 5.300 \\
\hline FD & FD & & 2.441 & 4.001 & 4.940 \\
\hline FD & FD & & 3.309 & 4.795 & 5.920 \\
\hline FD & FD & & 3.197 & 4.441 & 5.350 \\
\hline FD & FD & & 5.019 & 5.769 & 6.410 \\
\hline FD & FD & & & & \\
\hline \multirow[t]{16}{*}{ FD } & FD & & 1.978 & 2.223 & 2.39 \\
\hline & Bangombe & $.30 B-04(-193$ & 0.061 & 0.218 & 1.652 \\
\hline & Bangombe & $.30 \mathrm{~B}-07(-233$ & 0.148 & 0.337 & 2.633 \\
\hline & Bangombe & A.37-02 (-76n & 0.033 & 0.330 & 2.668 \\
\hline & Comilog & A.37-01 (-40n & 0.455 & 0.992 & 2.236 \\
\hline & Comilog & $12-B 4-06(-30 r$ & 0.077 & 0.688 & 1.077 \\
\hline & Comilog & Q4-B7-18 (-61 & 0.015 & 0.462 & 1.288 \\
\hline & Comilog & Q7-B2-15 (-34 & 0.042 & 0.363 & 1.522 \\
\hline & Comilog & 21-B3-09 (-57 & 0.007 & 0.607 & 3.549 \\
\hline & La Gare & omato. (base & 0.998 & 1.886 & 5.298 \\
\hline & La Gare & FBGM-04 & 0.429 & 1.318 & 6.914 \\
\hline & La Gare & FBGM-06 & 1.119 & 1.764 & 3.151 \\
\hline & La Gare & :BGM-10 (top & 0.224 & 0.737 & 1.863 \\
\hline & La Ville & FB1-03 (base) & 0.040 & 0.288 & 0.980 \\
\hline & La Ville & FB1-04 (top) & 1.125 & 1.810 & 8.340 \\
\hline & Socoba & 7 & 0.200 & 0.940 & 2.220 \\
\hline
\end{tabular}




\begin{tabular}{|c|c|c|c|c|c|}
\hline & Socoba & 8 & 0.830 & 1.030 & 2.570 \\
\hline & Socoba & 10 & 0.110 & 0.290 & 1.530 \\
\hline & Socoba & 12 & 0.110 & 0.430 & 2.410 \\
\hline & Socoba & 13 & 1.235 & 1.428 & 3.280 \\
\hline & Socoba & 14 & 0.317 & 0.741 & 2.584 \\
\hline & Socoba & 15 & 0.114 & 0.398 & 2.230 \\
\hline & Socoba & 18 & 0.024 & 0.676 & 2.063 \\
\hline & Socoba & rom fossil sur & 0.069 & 0.508 & 1.913 \\
\hline & Socoba & rom fossil sur & 0.093 & 0.740 & 2.142 \\
\hline Kaltasy & B203 & 3052 & 0.036 & 0.305 & 1.240 \\
\hline Kaltasy & B203 & 3263.2 & 0.196 & 0.612 & 3.550 \\
\hline Kaltasy & B203 & 3452 & 0.047 & 0.354 & 2.980 \\
\hline Kaltasy & B203 & 3505 & 0.045 & 0.396 & 3.253 \\
\hline Kaltasy & B203 & 3506.5 & 0.025 & 0.487 & 3.540 \\
\hline Kaltasy & B203 & 3559.5 & 0.127 & 0.454 & 2.930 \\
\hline Kaltasy & B203 & 3565 & 0.047 & 0.421 & 2.860 \\
\hline Kaltasy & B203 & 3653 & 0.010 & 0.540 & 2.550 \\
\hline Kaltasy & B203 & 3756.1 & 0.005 & 0.740 & 2.650 \\
\hline Kaltasy & B203 & 3853.3 & 0.009 & 0.264 & 2.220 \\
\hline Kaltasy & B203 & 3944.5 & 0.006 & 0.294 & 3.350 \\
\hline Kaltasy & B203 & 3949 & 0.012 & 0.501 & 3.240 \\
\hline Kaltasy & B203 & 4040 & 0.039 & 0.407 & 3.040 \\
\hline Kaltasy & B203 & 4050.7 & 0.011 & 0.390 & 2.670 \\
\hline Kaltasy & B203 & 4079.7 & 0.058 & 0.459 & 2.890 \\
\hline Kaltasy & B203 & 4081 & 0.042 & 0.368 & 2.810 \\
\hline Kaltasy & B203 & 4099 & 0.092 & 0.444 & 2.500 \\
\hline Kaltasy & B203 & 4101.9 & 0.013 & 0.452 & 3.950 \\
\hline Kaltasy & B203 & 4158.15 & 0.016 & 0.347 & 3.650 \\
\hline Kaltasy & B203 & 4169.7 & 0.035 & 0.454 & 3.730 \\
\hline Kaltasy & B203 & 4170.7 & 0.224 & 0.539 & 3.830 \\
\hline Kaltasy & B203 & 4195.5 & 0.078 & 0.552 & 3.790 \\
\hline Kaltasy & B203 & 4198.5 & 0.138 & 0.377 & 3.660 \\
\hline Kaltasy & B203 & 4201.5 & 0.056 & 0.397 & 4.260 \\
\hline Kaltasy & B203 & 4206 & 0.017 & 0.262 & 3.730 \\
\hline Kaltasy & B203 & 4207.3 & 0.189 & 0.513 & 3.630 \\
\hline Kaltasy & B203 & 4260.4 & 0.031 & 0.300 & 3.630 \\
\hline Kaltasy & B203 & 4264 & 0.019 & 0.531 & 4.220 \\
\hline Kaltasy & B203 & 4267 & 0.034 & 0.311 & 3.570 \\
\hline Kaltasy & B203 & 4270.4 & 0.127 & 0.561 & 3.700 \\
\hline Kaltasy & B203 & 4274.4 & 0.092 & 0.355 & 3.450 \\
\hline Kaltasy & B203 & 4284.5 & 0.340 & 0.602 & 3.960 \\
\hline
\end{tabular}




\begin{tabular}{|c|c|c|c|c|c|}
\hline Kaltasy & B203 & 4288.5 & 0.437 & 0.645 & 3.800 \\
\hline Kaltasy & B203 & 4349 & 0.208 & 0.503 & 3.460 \\
\hline Kaltasy & B203 & 4351 & 0.137 & 0.475 & 3.740 \\
\hline Kaltasy & B203 & 4353 & 0.127 & 0.483 & 3.740 \\
\hline Kaltasy & B203 & 4386.4 & 0.244 & 0.455 & 3.230 \\
\hline Kaltasy & B203 & 4391 & 0.270 & 0.516 & 3.170 \\
\hline Kaltasy & B203 & 4415 & 0.223 & 0.410 & 2.340 \\
\hline Kaltasy & B203 & 4450 & 0.192 & 0.465 & 2.410 \\
\hline Kaltasy & B203 & 4760.55 & 0.141 & 0.411 & 3.170 \\
\hline Satka equivalent & Vostok Askinsky-1 & 4111.1 & 0.034 & 0.329 & 2.570 \\
\hline Satka equivalent & Vostok Askinsky-1 & 4112 & 0.083 & 0.315 & 2.120 \\
\hline Satka equivalent & Vostok Askinsky-1 & 4113.5 & 0.050 & 0.685 & 2.790 \\
\hline Satka equivalent & Vostok Askinsky-1 & 4113.9 & 0.116 & 1.025 & 2.840 \\
\hline Satka equivalent & Vostok Askinsky-1 & 4114.7 & 0.033 & 0.189 & 2.280 \\
\hline Satka equivalent & Vostok Askinsky-1 & 4114.93 & 0.076 & 0.628 & 2.650 \\
\hline Satka equivalent & Vostok Askinsky-1 & 3824.8 coaly & 0.011 & 0.131 & 1.170 \\
\hline in method & Amoc 82/3 & $2-3-166.95$ & 0.040 & 0.200 & 2.840 \\
\hline in method & Amoc 82/3 & $2-3-328.35$ & 0.100 & 0.190 & 3.210 \\
\hline in method & Amoc 82/3 & $32-3-331.8$ & 0.080 & 0.230 & 6.050 \\
\hline in method & Amoc 82/3 & $2-3-345.8$ & 0.060 & 0.140 & 4.490 \\
\hline in method & Amoc 82/3 & $2-3-367.0$ & 0.070 & 0.160 & 2.640 \\
\hline in method & Amoc 82/3 & $2-3-379.1$ & 0.100 & 0.210 & 6.160 \\
\hline in method & Amoc 82/3 & $82-3-391.0$ & 0.130 & 0.270 & 2.950 \\
\hline in method & Amoc 82/3 & $2-3-407.65$ & 0.050 & 0.180 & 3.850 \\
\hline in method & Amoc $82 / 3$ & $2-3-439.9$ & 0.080 & 0.190 & 4.810 \\
\hline in method & Amoc 82/3 & $2-3-460.25$ & 0.040 & 0.160 & 4.330 \\
\hline in method & Amoc 82/3 & $2-3-465.5$ & 0.070 & 0.150 & 3.180 \\
\hline in method & Amoc 82/3 & $32-3-479.2$ & 0.240 & 0.690 & 4.010 \\
\hline in method & Amoc 82/3 & $32-3-521.45$ & 0.060 & 0.210 & 4.390 \\
\hline in method & Amoc 82/3 & $2-3-530.5$ & 0.080 & 0.270 & 4.380 \\
\hline in method & Amoc 82/3 & $2-3-544.1$ & 0.090 & 0.260 & 5.090 \\
\hline in method & Amoc 82/3 & $82-3-548.5$ & 0.040 & 0.340 & 4.330 \\
\hline in method & Amoc $82 / 3$ & $2-3-549.25$ & 0.060 & 0.260 & 4.480 \\
\hline in method & Amoc 82/3 & $2-3-561.05$ & 0.030 & 0.320 & 4.670 \\
\hline in method & Amoc $82 / 3$ & $2-3-587.05$ & 0.030 & 0.530 & 3.570 \\
\hline in method & Golden Grove 1 & j1-107.9 & 0.570 & 0.770 & 3.410 \\
\hline in method & Golden Grove 1 & $1-130.25$ & 0.030 & 0.180 & 4.850 \\
\hline in method & Golden Grove 1 & j1-131.1 & 0.050 & 0.150 & 3.570 \\
\hline in method & Golden Grove 1 & j1-171.5 & 0.180 & 0.520 & 4.310 \\
\hline in method & Golden Grove 1 & i1-206.75 & 0.070 & 0.530 & 3.940 \\
\hline in method & Golden Grove 1 & 31-229.5 & 0.040 & 0.130 & 4.130 \\
\hline
\end{tabular}




\begin{tabular}{|c|c|c|c|c|c|}
\hline in method & Golden Grove 1 & j1-231.1 & 0.060 & 0.210 & 3.740 \\
\hline in method & Golden Grove 1 & 31-266.2 & 0.050 & 0.180 & 4.590 \\
\hline in method & Golden Grove 1 & $31-274.8$ & 0.050 & 0.110 & 2.780 \\
\hline in method & Golden Grove 1 & $31-293.1$ & 0.100 & 0.300 & 4.300 \\
\hline in method & Golden Grove 1 & j1-299.1 & 0.040 & 0.120 & 6.590 \\
\hline in method & Golden Grove 1 & 31-304.9 & 0.000 & 0.080 & 2.970 \\
\hline in method & Golden Grove 1 & G1-31.5 & 0.040 & 0.140 & 3.360 \\
\hline in method & Golden Grove 1 & $31-316.5$ & 0.000 & 0.150 & 5.780 \\
\hline in method & Golden Grove 1 & j1-326.2 & 0.030 & 0.120 & 3.350 \\
\hline in method & Golden Grove 1 & j1-340.2 & 0.050 & 0.140 & 3.380 \\
\hline in method & Golden Grove 1 & G1-37.6 & 0.040 & 0.170 & 4.070 \\
\hline in method & Golden Grove 1 & j1-370.7 & 0.040 & 0.080 & 2.150 \\
\hline in method & Golden Grove 1 & $31-48.75$ & 0.040 & 0.140 & 3.110 \\
\hline in method & Golden Grove 1 & G1-53.2 & 0.030 & 0.130 & 3.460 \\
\hline in method & Urapung-4 & U4-110.6 & 1.270 & 1.500 & 3.440 \\
\hline in method & Urapung-4 & U4-117.0 & 1.590 & 1.730 & 3.100 \\
\hline in method & Urapung-4 & U4-124.0 & 1.430 & 1.580 & 2.730 \\
\hline in method & Urapung-4 & U4-135 & 1.770 & 1.960 & 6.670 \\
\hline in method & Urapung-4 & U4-140.1 & 1.050 & 1.280 & 4.450 \\
\hline in method & Urapung-4 & U4-156.1 & 1.230 & 1.270 & 2.460 \\
\hline in method & Urapung-4 & U4-164.7 & 1.480 & 1.620 & 5.180 \\
\hline in method & Urapung-4 & U4-173 & 0.890 & 0.940 & 1.850 \\
\hline in method & Urapung-4 & U4-183 & 1.240 & 1.330 & 2.130 \\
\hline in method & Urapung-4 & U4-189.7 & 1.850 & 1.930 & 2.650 \\
\hline in method & Urapung-4 & U4-196.0 & 1.550 & 1.650 & 2.430 \\
\hline in method & Urapung-4 & U4-204.1 & 2.590 & 2.670 & 3.370 \\
\hline in method & Urapung-4 & U4-214.1 & 2.660 & 2.960 & 5.490 \\
\hline in method & Urapung-4 & U4-225.6 & 0.020 & 0.120 & 2.000 \\
\hline in method & Urapung-4 & U4-24.7 & 0.000 & 0.370 & 9.290 \\
\hline in method & Urapung-4 & U4-249.5 & 0.010 & 0.180 & 4.530 \\
\hline in method & Urapung-4 & U4-26.4 & 0.240 & 0.600 & 7.660 \\
\hline in method & Urapung-4 & U4-29.0 & 0.490 & 0.990 & 9.880 \\
\hline in method & Urapung-4 & U4-315.0 & 0.540 & 0.740 & 4.470 \\
\hline in method & Urapung-4 & U4-33.2 & 0.180 & 1.430 & 16.210 \\
\hline in method & Urapung-4 & U4-330.1 & 0.260 & 0.380 & 3.320 \\
\hline in method & Urapung-4 & U4-335.2 & 0.250 & 0.400 & 4.300 \\
\hline in method & Urapung-4 & U4-343.6 & 0.030 & 0.190 & 4.530 \\
\hline in method & Urapung-4 & U4-355.4 & 1.690 & 1.790 & 4.160 \\
\hline in method & Urapung-4 & U4-363.0 & 2.030 & 2.170 & 4.380 \\
\hline in method & Urapung-4 & U4-417.2 & 0.140 & 0.240 & 3.240 \\
\hline in method & Urapung-4 & U4-420.0 & 0.270 & 0.440 & 4.290 \\
\hline
\end{tabular}




\begin{tabular}{|c|c|c|c|c|c|}
\hline in method & Urapung-4 & U4-421.5 & 0.150 & 0.260 & 3.490 \\
\hline in method & Urapung-4 & U4-424.5 & 0.110 & 0.290 & 5.160 \\
\hline in method & Urapung-4 & U4-430.1 & 0.200 & 0.330 & 3.110 \\
\hline in method & Urapung-4 & U4-431.0 & 0.720 & 0.890 & 4.040 \\
\hline in method & Urapung-4 & U4-432.1 & 0.670 & 0.920 & 4.190 \\
\hline in method & Urapung-4 & U4-432.25 & 0.590 & 0.820 & 3.850 \\
\hline in method & Urapung-4 & U4-440.95 & 0.150 & 0.260 & 2.890 \\
\hline in method & Urapung-4 & U4-446.85 & 0.300 & 0.430 & 3.360 \\
\hline in method & Urapung-4 & U4-89.6 & 1.380 & 1.500 & 3.190 \\
\hline in method & Urapung-4 & U4-98.5 & 1.670 & 1.840 & 4.500 \\
\hline in method & Urapung-5 & U5-113.9 & 0.050 & 0.130 & 0.690 \\
\hline in method & Urapung-5 & U5-120.2 & 0.210 & 0.330 & 3.120 \\
\hline in method & Urapung-5 & U5-125.1 & 0.040 & 0.140 & 3.400 \\
\hline in method & Urapung-5 & U5-130.5 & 0.070 & 0.220 & 1.220 \\
\hline in method & Urapung-5 & U5-140.25 & 0.050 & 0.110 & 1.460 \\
\hline in method & Urapung-5 & U5-140.7 & 0.000 & 0.070 & 1.390 \\
\hline in method & Urapung-5 & U5-151.3 & 0.040 & 0.140 & 1.810 \\
\hline in method & Urapung-5 & U5-325.1 & 0.050 & 0.160 & 2.730 \\
\hline in method & Urapung-5 & U5-326.2 & 0.070 & 0.240 & 4.550 \\
\hline in method & Urapung-5 & U5-334.3 & 0.120 & 0.260 & 5.590 \\
\hline in method & Urapung-5 & U5-355.1 & 0.080 & 0.190 & 3.710 \\
\hline in method & Urapung-5 & U5-366.6 & 0.080 & 0.200 & 5.090 \\
\hline in method & Urapung-5 & U5-383.7 & 0.290 & 0.600 & 5.320 \\
\hline in method & Urapung-5 & U5-398.95 & 0.030 & 0.260 & 5.120 \\
\hline in method & Urapung-5 & U5-414.7 & 0.020 & 0.130 & 4.330 \\
\hline in method & Urapung-5 & U5-424.0 & 0.090 & 0.250 & 4.950 \\
\hline in method & Urapung-5 & U5-425.1 & 0.040 & 0.310 & 4.270 \\
\hline in method & Urapung-5 & U5-436.7 & 0.040 & 0.260 & 5.510 \\
\hline in method & Urapung-5 & U5-437.1 & 0.010 & 0.220 & 4.030 \\
\hline in method & Urapung-5 & U5-440.0 & 0.010 & 0.290 & 4.380 \\
\hline in method & Urapung-5 & U5-448.5 & 0.070 & 0.250 & 4.530 \\
\hline in method & Urapung-5 & U5-450.0 & 0.030 & 0.290 & 4.310 \\
\hline in method & Urapung-5 & U5-459.6 & 0.110 & 0.340 & 4.950 \\
\hline in method & Urapung-5 & U5-465.1 & 0.040 & 0.310 & 4.890 \\
\hline in method & Urapung-5 & U5-470.25 & 0.070 & 0.420 & 4.470 \\
\hline in method & Urapung-5 & U5-479.6 & 0.050 & 0.310 & 5.360 \\
\hline in method & Urapung-5 & U5-505.1 & 0.060 & 0.500 & 5.200 \\
\hline in method & Urapung-5 & U5-508.6 & 0.050 & 0.440 & 7.520 \\
\hline in method & Urapung-5 & U5-510.1 & 0.010 & 0.310 & 4.640 \\
\hline in method & Urapung-5 & U5-518.9 & 0.060 & 0.330 & 4.630 \\
\hline in method & Urapung-5 & U5-526.2 & 0.050 & 0.350 & 5.400 \\
\hline
\end{tabular}




\begin{tabular}{|c|c|c|c|c|c|}
\hline in method & Urapung-5 & U5-536.6 & 0.160 & 0.350 & 4.810 \\
\hline in method & Urapung-5 & U5-547.75 & 2.310 & 2.480 & 4.460 \\
\hline in method & Urapung-5 & U5-561.4 & 1.330 & 1.410 & 3.380 \\
\hline in method & Urapung-5 & U5-569.1 & 1.570 & 1.720 & 4.580 \\
\hline in method & Urapung-5 & U5-570.1 & 1.650 & 1.750 & 3.250 \\
\hline in method & Urapung-5 & U5-580.1 & 2.270 & 2.400 & 4.290 \\
\hline in method & Urapung-5 & U5-581.9 & 1.580 & 1.770 & 4.330 \\
\hline in method & Urapung-5 & U5-592.0 & 1.350 & 1.460 & 3.430 \\
\hline in method & Urapung-5 & U5-593.1 & 1.580 & 1.810 & 4.890 \\
\hline \multirow[t]{6}{*}{ in method } & Urapung-5 & U5-603.9 & 0.630 & 0.810 & 1.230 \\
\hline & A83-4 & 223.9 & 0.210 & 1.470 & 2.270 \\
\hline & A83-4 & 236 & 0.600 & 1.220 & 2.920 \\
\hline & A83-4 & 247.3 & 0.050 & 0.390 & 2.620 \\
\hline & A83-4 & 271.1 & 0.030 & 0.350 & 2.850 \\
\hline & A83-4 & 289.95 & 1.110 & 1.590 & 2.070 \\
\hline Lady Loretta & LA64 & 116.8 & 0.750 & 1.420 & 2.920 \\
\hline Lady Loretta & LA64 & 133.6 & 0.510 & 1.240 & 2.840 \\
\hline Lady Loretta & LA64 & 146.5 & 0.560 & 1.170 & 2.870 \\
\hline Lady Loretta & LA64 & 162.5 & 0.540 & 1.760 & 3.300 \\
\hline Lady Loretta & LA64 & 210 & 0.210 & 1.440 & 2.130 \\
\hline Lady Loretta & LA64 & 221 & 0.190 & 1.650 & 1.870 \\
\hline Lady Loretta & LA64 & 261.8 & 0.280 & 0.870 & 2.700 \\
\hline Lady Loretta & LA64 & 282.5 & 0.620 & 1.640 & 3.570 \\
\hline Lady Loretta & LA64 & 289.8 & 0.400 & 1.350 & 3.970 \\
\hline Lady Loretta & LA64 & 301.8 & 0.750 & 1.530 & 3.410 \\
\hline Lady Loretta & LA64 & 334.5 & 0.550 & 1.310 & 3.180 \\
\hline Lady Loretta & LA64 & 361.5 & 0.370 & 0.780 & 3.500 \\
\hline Lady Loretta & LA64 & 429.2 & 0.260 & 1.040 & 3.920 \\
\hline Lady Loretta & LA64 & 451.5 & 0.510 & 1.750 & 3.750 \\
\hline Lady Loretta & LA64 & 471.4 & 0.590 & 1.030 & 2.460 \\
\hline Lady Loretta & LA64 & 485.8 & 0.390 & 2.240 & 4.180 \\
\hline Lady Loretta & LA64 & 543 & 0.280 & 2.540 & 2.780 \\
\hline Lady Loretta & LA64 & 564 & 0.480 & 1.770 & 2.450 \\
\hline Lady Loretta & LA64 & 585.5 & 0.160 & 2.540 & 2.540 \\
\hline Lady Loretta & LA64 & 600.5 & 1.030 & 1.530 & 2.540 \\
\hline Barney Creek & MY3 & 96.7 & 1.000 & 1.530 & 1.830 \\
\hline Barney Creek & MY3 & 100.6 & 0.490 & 0.960 & 1.770 \\
\hline Barney Creek & MY3 & 105.7 & 0.350 & 1.080 & 1.140 \\
\hline Barney Creek & MY3 & 125.2 & 0.550 & 0.960 & 1.020 \\
\hline Barney Creek & MY3 & 135 & 0.990 & 1.760 & 1.820 \\
\hline Barney Creek & MY3 & 145 & 2.180 & 2.680 & 2.750 \\
\hline
\end{tabular}




\begin{tabular}{|c|c|c|c|c|c|}
\hline Barney Creek & MY3 & 155 & 0.690 & 1.420 & 1.690 \\
\hline Barney Creek & MY3 & 175 & 0.400 & 0.850 & 0.910 \\
\hline Barney Creek & MY3 & 188 & 0.950 & 1.950 & 2.100 \\
\hline Barney Creek & MY3 & 198 & 1.100 & 1.770 & 2.030 \\
\hline Barney Creek & MY3 & 208 & 1.040 & 1.730 & 1.940 \\
\hline Barney Creek & MY3 & 218.2 & 1.100 & 1.950 & 2.260 \\
\hline Barney Creek & MY3 & 228.3 & 1.920 & 2.660 & 2.710 \\
\hline Barney Creek & MY3 & 233.8 & 0.380 & 0.660 & 0.770 \\
\hline \multirow[t]{5}{*}{ Teena } & MY3 & 249.5 & 0.140 & 0.350 & 0.590 \\
\hline & MY4 & 37 & 0.500 & 1.140 & 1.240 \\
\hline & MY4 & 51 & b.d. & 1.200 & 2.300 \\
\hline & MY4 & 61.5 & 0.880 & 1.430 & 2.500 \\
\hline & MY4 & 88.5 & 0.250 & 0.540 & 0.830 \\
\hline Mt. Les & WFDD84 & 70 & 0.160 & 0.830 & 5.340 \\
\hline Mt. Les & WFDD84 & 97.8 & 0.320 & 2.310 & 25.210 \\
\hline Mt. Les & WFDD84 & 103.5 & 0.550 & 1.420 & 2.600 \\
\hline Mt. Les & WFDD84 & 115.2 & 0.510 & 1.480 & 2.290 \\
\hline Mt. Les & WFDD84 & 132 & 0.050 & 0.330 & 3.010 \\
\hline Mt. Les & WFDD84 & 158.5 & 0.860 & 1.260 & 1.850 \\
\hline Reward & BMR McArthur Rive & 1 & 0.750 & 0.950 & 1.610 \\
\hline Reward & BMR McArthur Rive & 2 & 1.830 & 2.000 & 2.620 \\
\hline Reward & BMR McArthur Rive & 3 & 1.210 & 1.440 & 3.270 \\
\hline Reward & BMR McArthur Rive & 4 & 2.420 & 2.570 & 3.100 \\
\hline Reward & BMR McArthur Riv€ & 5 & 0.930 & 1.120 & 1.370 \\
\hline Reward & BMR McArthur Rive & 6 & 0.950 & 1.050 & 1.890 \\
\hline Reward & BMR McArthur Riv€ & 7 & 0.840 & 0.980 & 1.870 \\
\hline Reward & BMR McArthur Riv€ & 8 & 0.830 & 1.040 & 2.090 \\
\hline Reward & BMR McArthur Riv€ & 9 & 3.450 & 3.600 & 4.440 \\
\hline Reward & BMR McArthur Riv€ & 10 & 3.450 & 3.600 & 4.510 \\
\hline Wollogorang & BMR Mount Young & 1 & 1.350 & 1.550 & 4.570 \\
\hline Wollogorang & BMR Mount Young & 2 & 0.120 & 0.450 & 1.890 \\
\hline Wollogorang & BMR Mount Young & 3 & 0.840 & 1.050 & 1.870 \\
\hline Wollogorang & BMR Mount Young & 4 & 1.630 & 1.770 & 2.580 \\
\hline Wollogorang & BMR Mount Young & 5 & 2.390 & 2.760 & 3.660 \\
\hline Wollogorang & BMR Mount Young & 6 & 1.060 & 1.350 & 6.500 \\
\hline Wollogorang & BMR Mount Young & 7 & 1.180 & 1.380 & 2.240 \\
\hline Wollogorang & BMR Mount Young & 8 & 3.470 & 3.850 & 4.810 \\
\hline Wollogorang & BMR Mount Young & 9 & 1.220 & 1.300 & 1.700 \\
\hline Wollogorang & BMR Mount Young & 10 & 0.930 & 1.500 & 1.980 \\
\hline Wollogorang & BMR Mount Young & 11 & 1.660 & 1.760 & 2.770 \\
\hline Wollogorang & BMR Mount Young & 12 & 2.070 & 2.360 & 3.570 \\
\hline
\end{tabular}




\begin{tabular}{|c|c|c|c|c|c|}
\hline Wollogorang & BMR Mount Young & $9710828-0$ & 1.480 & 1.590 & 2.050 \\
\hline Wollogorang & BMR Mount Young & $9710828-1$ & 1.250 & 1.880 & 2.560 \\
\hline Rove & 89-MC-1 & 05-50? & 0.626 & 1.419 & 5.490 \\
\hline Rove & 89-MC-1 & 05-51? & 0.147 & 1.761 & 5.360 \\
\hline Rove & 89-MC-1 & 05-52? & 0.675 & 2.472 & 6.880 \\
\hline Rove & 89-MC-1 & 05-53? & 0.450 & 2.409 & 5.690 \\
\hline Rove & 89-MC-1 & 05-54? & 2.773 & 3.930 & 6.640 \\
\hline Rove & 89-MC-1 & 05-55? & 0.640 & 6.280 & 10.810 \\
\hline Rove & 89-MC-1 & 05-56? & 0.663 & 10.359 & 10.380 \\
\hline Rove & 89-MC-1 & 05-57? & 0.649 & 7.786 & 8.880 \\
\hline Rove & 89-MC-1 & 05-58? & 0.026 & 0.729 & 5.150 \\
\hline Rove & 89-MC-1 & 05-59? & 1.721 & 12.710 & 13.890 \\
\hline Rove & 89-MC-1 & 05-60? & 0.901 & 3.700 & 7.760 \\
\hline Rove & 89-MC-1 & 05-61? & 1.252 & 4.636 & 7.640 \\
\hline Rove & 89-MC-1 & 05-62? & 2.654 & 3.423 & 5.930 \\
\hline Rove & 89-MC-1 & 05-63? & 2.254 & 2.921 & 6.730 \\
\hline Rove & 89-MC-1 & 05-64? & 2.970 & 3.809 & 6.770 \\
\hline Rove & 89-MC-1 & 05-65? & 0.781 & 1.587 & 5.900 \\
\hline Rove & 89-MC-1 & 05-66? & 0.188 & 1.353 & 6.000 \\
\hline Rove & 89-MC-1 & R10? & 0.897 & 1.441 & 3.870 \\
\hline Rove & 89-MC-1 & R11? & 2.871 & 3.443 & 5.490 \\
\hline Rove & 89-MC-1 & R12?ㅜ & 0.444 & 0.875 & 3.140 \\
\hline Rove & 89-MC-1 & R13? & 0.635 & 1.007 & 3.030 \\
\hline Rove & 89-MC-1 & R14? & 1.966 & 2.535 & 4.850 \\
\hline Rove & 89-MC-1 & R15A? & 0.674 & 1.594 & 3.820 \\
\hline Rove & 89-MC-1 & R15B? & 1.225 & 1.859 & 4.340 \\
\hline Rove & 89-MC-1 & R16? & 0.661 & 2.164 & 3.550 \\
\hline Rove & 89-MC-1 & 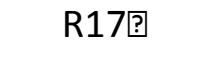 & 0.861 & 2.479 & 3.620 \\
\hline Rove & 89-MC-1 & R18? & 0.391 & 1.399 & 3.640 \\
\hline Rove & 89-MC-1 & R19? & 0.492 & 1.653 & 3.460 \\
\hline Rove & 89-MC-1 & R20? & 0.637 & 1.920 & 3.750 \\
\hline Rove & 89-MC-1 & R21? & 1.226 & 3.353 & 4.560 \\
\hline Rove & 89-MC-1 & R22? & 0.841 & 2.409 & 3.560 \\
\hline Rove & 89-MC-1 & R23? & 0.186 & 0.622 & 2.660 \\
\hline Rove & 89-MC-1 & 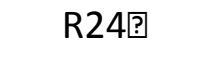 & 0.506 & 1.468 & 3.040 \\
\hline Rove & 89-MC-1 & R25? & 2.067 & 4.441 & 5.530 \\
\hline Rove & 89-MC-1 & R26A? & 1.621 & 2.486 & 4.210 \\
\hline Rove & 89-MC-1 & R26B? & 0.122 & 0.501 & 3.040 \\
\hline Rove & 89-MC-1 & R26C? & 2.855 & 3.785 & 5.800 \\
\hline Rove & 89-MC-1 & R26D? & 7.506 & 8.543 & 10.380 \\
\hline Rove & 89-MC-1 & R26F? & 0.875 & 2.546 & 3.980 \\
\hline
\end{tabular}




\begin{tabular}{|c|c|c|c|c|c|}
\hline Rove & 89-MC-1 & R26G? & 0.967 & 3.193 & 4.840 \\
\hline Rove & 89-MC-1 & R26H? & 1.027 & 3.181 & 4.740 \\
\hline Rove & 89-MC-1 & R261? & 0.913 & 1.900 & 3.460 \\
\hline Rove & 89-MC-1 & R26J? & 2.524 & 3.038 & 4.540 \\
\hline Rove & 89-MC-1 & 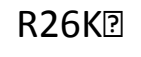 & 5.807 & 6.465 & 8.000 \\
\hline Rove & 89-MC-1 & R26L?? & 5.073 & 5.914 & 7.910 \\
\hline Rove & 89-MC-1 & R26M? & 6.505 & 7.473 & 9.860 \\
\hline Rove & 89-MC-1 & R26N? & 0.933 & 1.729 & 4.620 \\
\hline Rove & 89-MC-1 & R260? & 1.665 & 3.357 & 4.980 \\
\hline Rove & 89-MC-1 & 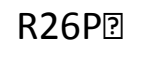 & 1.921 & 4.059 & 5.140 \\
\hline Rove & 89-MC-1 & R27? & 2.349 & 4.056 & 5.400 \\
\hline Rove & 89-MC-1 & R28? & 0.671 & 1.354 & 2.840 \\
\hline Rove & 89-MC-1 & R29? & 1.767 & 2.067 & 4.230 \\
\hline Rove & 89-MC-1 & R30? & 2.501 & 2.825 & 5.210 \\
\hline Rove & 89-MC-1 & R31? & 2.857 & 3.294 & 6.270 \\
\hline Rove & 89-MC-1 & R32? & 3.796 & 4.245 & 7.830 \\
\hline Rove & 89-MC-1 & R33? & 5.530 & 6.072 & 9.470 \\
\hline Rove & 89-MC-1 & R34? & 3.339 & 3.930 & 7.760 \\
\hline Rove & 89-MC-1 & R35? & 4.181 & 4.767 & 8.630 \\
\hline Rove & 89-MC-1 & R36? & 2.386 & 2.918 & 6.260 \\
\hline Rove & 89-MC-1 & R37? & 2.541 & 3.142 & 6.240 \\
\hline Rove & 89-MC-1 & R38? & 16.380 & 17.268 & 19.590 \\
\hline Rove & 89-MC-1 & R39? & 2.986 & 3.557 & 7.130 \\
\hline Rove & 89-MC-1 & R40? & 6.264 & 6.949 & 11.110 \\
\hline Rove & 89-MC-1 & R6? & 0.351 & 0.843 & 2.730 \\
\hline Rove & 89-MC-1 & R7? & 0.133 & 0.774 & 3.310 \\
\hline Rove & 89-MC-1 & R8? & 0.602 & 1.183 & 3.600 \\
\hline Rove & 89-MC-1 & R9? & 0.567 & 1.138 & 3.300 \\
\hline Rove & 89-MC-1 & T29? & 1.552 & 1.914 & 2.580 \\
\hline Rove & 89-MC-1 & T31? & 0.217 & 0.525 & 1.010 \\
\hline Rove & 89-MC-1 & 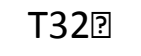 & 0.093 & 0.622 & 2.250 \\
\hline Rove & 89-MC-1 & T33? & 0.519 & 1.093 & 3.110 \\
\hline Rove & 89-MC-1 & T34? & 0.682 & 1.211 & 3.010 \\
\hline Rove & 89-MC-1 & T35回 & 0.450 & 1.102 & 3.400 \\
\hline Rove & $98-1$ & 5_1 & 0.291 & 1.070 & 1.740 \\
\hline Rove & $98-1$ & 5_10 & 1.920 & 2.427 & 4.950 \\
\hline Rove & $98-1$ & 5_11 & 2.930 & 3.315 & 5.430 \\
\hline Rove & $98-1$ & 5_12 & 2.071 & 2.579 & 4.920 \\
\hline Rove & $98-1$ & 5_13 & 1.906 & 3.072 & 6.460 \\
\hline Rove & $98-1$ & 5_14 & 0.072 & 4.361 & 6.660 \\
\hline Rove & $98-1$ & 5_15 & 0.027 & 0.621 & 4.780 \\
\hline
\end{tabular}




\begin{tabular}{|c|c|c|c|c|c|}
\hline Rove & $98-1$ & 5_16 & 0.043 & 1.082 & 5.290 \\
\hline Rove & $98-1$ & 5_17 & 0.937 & 3.593 & 5.960 \\
\hline Rove & $98-1$ & 5_18 & 0.137 & 1.080 & 5.300 \\
\hline Rove & $98-1$ & 5_19 & 0.038 & 1.152 & 5.220 \\
\hline Rove & $98-1$ & 5_2 & 0.229 & 1.022 & 3.270 \\
\hline Rove & $98-1$ & 5_20 & 0.016 & 0.800 & 4.930 \\
\hline Rove & $98-1$ & 5_21 & 0.044 & 0.662 & 5.120 \\
\hline Rove & $98-1$ & 5_22 & 1.479 & 1.979 & 5.080 \\
\hline Rove & $98-1$ & 5_23 & 1.283 & 2.092 & 5.230 \\
\hline Rove & $98-1$ & 5_24 & 2.679 & 8.071 & 9.770 \\
\hline Rove & $98-1$ & 5_25 & 0.086 & 0.508 & 5.240 \\
\hline Rove & $98-1$ & 5_26 & 0.068 & 0.579 & 4.780 \\
\hline Rove & $98-1$ & 5_27 & 0.236 & 2.173 & 5.740 \\
\hline Rove & $98-1$ & 5_28 & 1.326 & 4.496 & 6.020 \\
\hline Rove & $98-1$ & 5_29 & 0.035 & 0.646 & 5.270 \\
\hline Rove & $98-1$ & 5_3 & 0.237 & 1.026 & 2.940 \\
\hline Rove & $98-1$ & 5_30 & 1.123 & 2.458 & 5.440 \\
\hline Rove & $98-1$ & 5_31 & 0.236 & 2.497 & 5.810 \\
\hline Rove & $98-1$ & 5_32 & 0.065 & 0.543 & 5.220 \\
\hline Rove & $98-1$ & 5_33 & 1.275 & 4.063 & 5.470 \\
\hline Rove & $98-1$ & 5_34 & 0.022 & 0.517 & 5.110 \\
\hline Rove & $98-1$ & 5_35 & 0.267 & 1.478 & 5.410 \\
\hline Rove & $98-1$ & 5_36 & 0.015 & 0.582 & 4.710 \\
\hline Rove & $98-1$ & 5_4 & 0.587 & 1.457 & 2.640 \\
\hline Rove & $98-1$ & 5_5 & 0.109 & 0.462 & 1.450 \\
\hline Rove & $98-1$ & 5_6 & 0.023 & 0.297 & 1.320 \\
\hline Rove & $98-1$ & 5_7 & 0.018 & 0.534 & 3.750 \\
\hline Rove & $98-1$ & 5_8 & 0.613 & 2.350 & 3.550 \\
\hline Rove & $98-1$ & 5_9 & 0.658 & 1.019 & 2.770 \\
\hline Rove & GF-3 & GF3-01 & 1.296 & 1.694 & 4.040 \\
\hline Rove & GF-3 & GF3-02 & 0.072 & 1.614 & 3.710 \\
\hline Rove & GF-3 & GF3-03 & 0.080 & 1.628 & 4.400 \\
\hline Rove & GF-3 & GF3-04 & 0.111 & 1.390 & 4.170 \\
\hline Rove & GF-3 & GF3-05 & 0.091 & 1.246 & 3.630 \\
\hline Rove & GF-3 & GF3-06 & 0.023 & 0.540 & 2.250 \\
\hline Rove & GF-3 & GF3-07 & 0.062 & 1.073 & 3.520 \\
\hline Rove & GF-3 & GF3-08 & 0.007 & 0.583 & 5.470 \\
\hline Rove & GF-3 & GF3-09 & 0.028 & 0.842 & 5.360 \\
\hline Rove & GF-3 & GF3-10 & 0.061 & 1.172 & 3.910 \\
\hline Rove & GF-3 & GF3-11 & 0.122 & 1.946 & 4.270 \\
\hline Rove & GF-3 & GF3-12 & 0.052 & 1.395 & 4.180 \\
\hline
\end{tabular}




\begin{tabular}{|c|c|c|c|c|c|}
\hline Rove & GF-3 & GF3-13 & 1.235 & 1.876 & 4.160 \\
\hline Rove & GF-3 & GF3-14 & 1.100 & 2.102 & 5.020 \\
\hline Rove & GF-3 & GF3-15 & 0.083 & 1.577 & 4.220 \\
\hline Rove & GF-3 & GF3-16 & 0.298 & 1.422 & 3.710 \\
\hline Rove & GF-3 & GF3-17 & 0.035 & 0.750 & 3.970 \\
\hline Rove & GF-3 & GF3-18 & 0.044 & 1.541 & 4.320 \\
\hline Rove & GF-3 & GF3-19 & 0.057 & 2.311 & 5.360 \\
\hline Rove & GF-3 & GF3-20 & 0.075 & 2.117 & 5.340 \\
\hline Rove & GF-3 & GF3-21 & 0.173 & 2.272 & 4.900 \\
\hline Rove & GF-3 & GF3-22 & 0.158 & 2.004 & 5.110 \\
\hline Rove & GF-3 & GF3-23 & 0.113 & 2.303 & 5.270 \\
\hline Rove & GF-3 & GF3-24 & 0.087 & 1.937 & 4.090 \\
\hline Rove & GF-3 & GF3-25 & 0.219 & 2.210 & 2.460 \\
\hline Rove & GF-3 & GF3-26 & 2.131 & 2.825 & 4.860 \\
\hline Rove & GF-3 & GF3-27 & 0.089 & 1.409 & 11.210 \\
\hline Rove & GF-3 & GF3-28 & 0.088 & 1.785 & 4.560 \\
\hline Rove & GF-3 & GF3-29 & 0.067 & 1.061 & 4.040 \\
\hline Rove & GF-3 & GF3-30 & 0.039 & 0.938 & 4.050 \\
\hline Rove & GF-3 & GF3-31 & 0.035 & 0.502 & 3.310 \\
\hline Rove & GF-3 & GF3-32 & 0.469 & 1.819 & 3.740 \\
\hline Rove & GF-3 & GF3-33 & 2.247 & 3.078 & 5.320 \\
\hline Rove & GF-3 & GF3-34 & 4.439 & 6.284 & 7.210 \\
\hline Virginia & MGS-2 & BW01 & 0.055 & 1.343 & 5.310 \\
\hline Virginia & MGS-2 & BW02 & 0.254 & 1.307 & 5.180 \\
\hline Virginia & MGS-2 & BW03 & 0.196 & 1.496 & 5.300 \\
\hline Virginia & MGS-2 & BW04 & 0.143 & 1.526 & 5.470 \\
\hline Virginia & MGS-2 & BW05 & 0.388 & 1.611 & 4.970 \\
\hline Virginia & MGS-2 & BW06 & 0.051 & 1.384 & 5.390 \\
\hline Virginia & MGS-2 & BW07a & 0.797 & 1.791 & 4.690 \\
\hline Virginia & MGS-2 & BW07b & 0.358 & 1.499 & 4.860 \\
\hline Virginia & MGS-2 & BW07c & 0.464 & 1.717 & 5.240 \\
\hline Virginia & MGS-2 & BW08 & 0.080 & 1.268 & 5.130 \\
\hline Virginia & MGS-2 & BW09 & 0.483 & 1.669 & 5.150 \\
\hline Virginia & MGS-2 & BW10a & 0.609 & 1.583 & 4.480 \\
\hline Virginia & MGS-2 & BW10b & 0.215 & 1.457 & 5.270 \\
\hline Virginia & MGS-2 & BW11 & 0.326 & 1.517 & 5.320 \\
\hline Virginia & MGS-2 & BW12 & 0.136 & 1.202 & 4.730 \\
\hline Virginia & MGS-2 & BW13 & 0.319 & 1.398 & 4.520 \\
\hline Virginia & MGS-2 & BW14 & 0.265 & 1.533 & 5.400 \\
\hline Virginia & MGS-2 & BW15a & 0.051 & 1.351 & 4.990 \\
\hline Virginia & MGS-2 & BW15b & 0.177 & 1.319 & 4.820 \\
\hline
\end{tabular}




\begin{tabular}{|c|c|c|c|c|c|}
\hline Virginia & MGS-2 & BW15c & 0.090 & 1.295 & 4.680 \\
\hline Virginia & MGS-2 & BW16 & 0.076 & 1.318 & 5.100 \\
\hline Virginia & MGS-2 & BW17 & 0.496 & 1.496 & 4.560 \\
\hline Virginia & MGS-2 & BW18 & 0.792 & 1.908 & 5.850 \\
\hline Virginia & MGS-2 & BW19 & 0.151 & 1.391 & 4.730 \\
\hline Virginia & MGS-2 & MGS2-1 & 0.741 & 1.684 & 5.190 \\
\hline Virginia & MGS-2 & MGS2-10 & 1.427 & 2.308 & 6.100 \\
\hline Virginia & MGS-2 & MGS2-11 & 2.469 & 2.842 & 5.290 \\
\hline Virginia & MGS-2 & MGS2-12 & 2.124 & 2.708 & 5.940 \\
\hline Virginia & MGS-2 & MGS2-13 & 0.169 & 0.936 & 6.040 \\
\hline Virginia & MGS-2 & MGS2-14 & 0.886 & 1.416 & 4.550 \\
\hline Virginia & MGS-2 & MGS2-15 & 0.396 & 0.976 & 4.780 \\
\hline Virginia & MGS-2 & MGS2-16 & 0.199 & 0.751 & 4.560 \\
\hline Virginia & MGS-2 & MGS2-17 & 1.100 & 1.551 & 4.770 \\
\hline Virginia & MGS-2 & MGS2-18 & 0.107 & 0.687 & 5.570 \\
\hline Virginia & MGS-2 & MGS2-19 & 0.854 & 1.394 & 5.210 \\
\hline Virginia & MGS-2 & MGS2-2 & 0.155 & 0.859 & 4.620 \\
\hline Virginia & MGS-2 & MGS2-20 & 0.930 & 1.391 & 5.040 \\
\hline Virginia & MGS-2 & MGS2-21 & 1.746 & 2.386 & 4.960 \\
\hline Virginia & MGS-2 & MGS2-22 & 2.895 & 3.483 & 5.610 \\
\hline Virginia & MGS-2 & MGS2-23 & 1.224 & 1.800 & 4.780 \\
\hline Virginia & MGS-2 & MGS2-24 & 0.828 & 1.574 & 5.990 \\
\hline Virginia & MGS-2 & MGS2-25 & 0.608 & 1.371 & 5.580 \\
\hline Virginia & MGS-2 & MGS2-26 & 0.395 & 1.015 & 5.290 \\
\hline Virginia & MGS-2 & MGS2-27 & 0.306 & 0.967 & 5.790 \\
\hline Virginia & MGS-2 & MGS2-28 & 0.199 & 0.795 & 5.220 \\
\hline Virginia & MGS-2 & MGS2-29 & 0.980 & 1.616 & 4.750 \\
\hline Virginia & MGS-2 & MGS2-3 & 0.570 & 0.986 & 4.170 \\
\hline Virginia & MGS-2 & MGS2-30 & 0.240 & 0.970 & 6.230 \\
\hline Virginia & MGS-2 & MGS2-31 & 0.135 & 0.798 & 6.000 \\
\hline Virginia & MGS-2 & MGS2-32 & 0.252 & 0.972 & 6.010 \\
\hline Virginia & MGS-2 & MGS2-33 & 0.752 & 1.341 & 5.600 \\
\hline Virginia & MGS-2 & MGS2-34 & 0.855 & 1.439 & 5.470 \\
\hline Virginia & MGS-2 & MGS2-4 & 1.795 & 2.183 & 5.010 \\
\hline Virginia & MGS-2 & MGS2-5 & 0.191 & 0.785 & 2.760 \\
\hline Virginia & MGS-2 & MGS2-6 & 3.302 & 4.031 & 6.120 \\
\hline Virginia & MGS-2 & MGS2-7 & 3.282 & 4.173 & 6.870 \\
\hline Virginia & MGS-2 & MGS2-8 & 2.015 & 2.696 & 5.870 \\
\hline Virginia & MGS-2 & MGS2-9 & 1.383 & 2.172 & 5.740 \\
\hline Virginia & MGS-2 & V10 & 0.145 & 1.580 & 5.740 \\
\hline Virginia & MGS-2 & V11 & 0.222 & 0.451 & 2.220 \\
\hline
\end{tabular}




\begin{tabular}{|c|c|c|c|c|c|}
\hline Virginia & MGS-2 & V12 & 0.379 & 1.379 & 5.210 \\
\hline Virginia & MGS-2 & V13 & 0.600 & 1.588 & 5.330 \\
\hline Virginia & MGS-2 & V14 & 0.506 & 1.362 & 4.450 \\
\hline Virginia & MGS-2 & V15 & 0.597 & 1.735 & 5.240 \\
\hline Virginia & MGS-2 & V16a & 1.022 & 1.681 & 4.050 \\
\hline Virginia & MGS-2 & V16b & 0.185 & 0.784 & 2.950 \\
\hline Virginia & MGS-2 & V17a & 1.002 & 2.011 & 4.560 \\
\hline Virginia & MGS-2 & V17b & 0.355 & 1.156 & 4.010 \\
\hline Virginia & MGS-2 & V18 & 0.064 & 1.307 & 5.570 \\
\hline Virginia & MGS-2 & V1a & 0.031 & 1.029 & 4.920 \\
\hline Virginia & MGS-2 & V1b & 0.036 & 1.267 & 5.250 \\
\hline Virginia & MGS-2 & V2a & 0.070 & 1.050 & 4.780 \\
\hline Virginia & MGS-2 & $\mathrm{V} 2 \mathrm{~b}$ & 0.065 & 1.449 & 5.280 \\
\hline Virginia & MGS-2 & V3 & 0.269 & 1.335 & 5.160 \\
\hline Virginia & MGS-2 & V4a & 0.014 & 1.367 & 4.870 \\
\hline Virginia & MGS-2 & V4b & 0.016 & 1.044 & 4.820 \\
\hline Virginia & MGS-2 & V5 & 0.116 & 1.193 & 5.260 \\
\hline Virginia & MGS-2 & V6 & 0.186 & 1.485 & 5.500 \\
\hline Virginia & MGS-2 & V7 & 0.159 & 1.406 & 5.270 \\
\hline Virginia & MGS-2 & V8 & 0.075 & 1.683 & 6.400 \\
\hline Virginia & MGS-2 & V9 & 0.069 & 1.417 & 5.830 \\
\hline Virginia & MGS-7 & KV31 & 0.007 & 0.841 & 4.790 \\
\hline Virginia & MGS-7 & KV32 & 0.374 & 1.613 & 5.580 \\
\hline Virginia & MGS-7 & KV33 & 0.201 & 1.095 & 4.950 \\
\hline Virginia & MGS-7 & KV34 & 0.436 & 1.273 & 5.020 \\
\hline Virginia & MGS-7 & KV35 & 0.011 & 0.906 & 5.100 \\
\hline Virginia & MGS-7 & KV36 & 0.014 & 1.035 & 4.810 \\
\hline Virginia & MGS-7 & KV37 & 0.039 & 1.144 & 5.340 \\
\hline Virginia & MGS-7 & KV38 & 0.016 & 1.068 & 5.490 \\
\hline Virginia & MGS-7 & KV39 & 0.008 & 1.776 & 6.920 \\
\hline Virginia & MGS-7 & KV40 & 0.349 & 1.406 & 5.000 \\
\hline Virginia & MGS-7 & KV41 & 0.051 & 1.265 & 5.560 \\
\hline Virginia & MGS-7 & KV42 & 0.049 & 1.142 & 4.340 \\
\hline Virginia & MGS-7 & KV43 & 0.182 & 4.463 & 8.780 \\
\hline Virginia & MGS-7 & KV44 & 0.126 & 0.958 & 3.640 \\
\hline Virginia & MGS-7 & V19 & 0.145 & 1.874 & 6.020 \\
\hline Virginia & MGS-7 & V20 & 0.198 & 1.398 & 4.940 \\
\hline Virginia & MGS-8 & CV1 & 0.102 & 0.540 & 2.460 \\
\hline Virginia & MGS-8 & CV10? & 0.024 & 1.619 & 5.990 \\
\hline Virginia & MGS-8 & CV11? & 0.462 & 2.321 & 6.910 \\
\hline Virginia & MGS-8 & CV12? & 0.027 & 1.596 & 7.450 \\
\hline
\end{tabular}




\begin{tabular}{|c|c|c|c|c|c|}
\hline Virginia & MGS-8 & CV13? & 0.219 & 1.061 & 4.530 \\
\hline Virginia & MGS-8 & CV14? & 0.278 & 1.143 & 4.840 \\
\hline Virginia & MGS-8 & CV15? & 0.059 & 1.044 & 5.120 \\
\hline Virginia & MGS-8 & CV16? & 0.295 & 1.232 & 4.520 \\
\hline Virginia & MGS-8 & CV17? & 0.211 & 1.133 & 4.840 \\
\hline Virginia & MGS-8 & CV18? & 0.181 & 1.216 & 4.840 \\
\hline Virginia & MGS-8 & CV19? & 0.184 & 1.095 & 4.800 \\
\hline Virginia & MGS-8 & CV2 & 0.008 & 1.535 & 6.250 \\
\hline Virginia & MGS-8 & CV20? & 0.160 & 1.177 & 5.130 \\
\hline Virginia & MGS-8 & CV21? & 0.037 & 1.078 & 5.540 \\
\hline Virginia & MGS-8 & 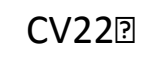 & 0.017 & 0.877 & 4.800 \\
\hline Virginia & MGS-8 & CV23? & 0.072 & 0.953 & 5.090 \\
\hline Virginia & MGS-8 & CV25? & 0.262 & 0.823 & 3.810 \\
\hline Virginia & MGS-8 & CV26a? & 0.426 & 0.871 & 3.220 \\
\hline Virginia & MGS-8 & CV26b? & 0.246 & 0.723 & 3.060 \\
\hline Virginia & MGS-8 & CV26c? & 0.054 & 0.651 & 3.910 \\
\hline Virginia & MGS-8 & CV26d? & 0.250 & 0.738 & 2.930 \\
\hline Virginia & MGS-8 & CV3? & 0.044 & 1.227 & 4.460 \\
\hline Virginia & MGS-8 & CV4? & 0.011 & 1.374 & 5.600 \\
\hline Virginia & MGS-8 & CV5? & 0.050 & 2.207 & 7.110 \\
\hline Virginia & MGS-8 & CV6? & 0.072 & 1.328 & 5.240 \\
\hline Virginia & MGS-8 & CV7? & 0.024 & 2.045 & 6.740 \\
\hline Virginia & MGS-8 & CV8? & 0.006 & 3.286 & 8.910 \\
\hline Virginia & MGS-8 & CV9? & 0.033 & 1.445 & 5.490 \\
\hline \multicolumn{2}{|c|}{ Morro do Calcário 42-88 } & 752.35 & 0.601 & 1.904 & 2.369 \\
\hline \multicolumn{2}{|c|}{ Morro do Calcário 42-88 } & 756.2 & 0.155 & 2.365 & 3.116 \\
\hline \multicolumn{2}{|c|}{ Morro do Calcário 42-88 } & 759.55 & 0.072 & 0.521 & 2.235 \\
\hline \multicolumn{2}{|c|}{ Morro do Calcário 42-88 } & 764.47 & 0.075 & 0.542 & 2.132 \\
\hline \multicolumn{2}{|c|}{ Morro do Calcário 42-88 } & 769.75 & 0.094 & 1.129 & 2.243 \\
\hline \multicolumn{2}{|c|}{ Morro do Calcário 42-88 } & 774.2 & 0.031 & 0.213 & 2.221 \\
\hline \multicolumn{2}{|c|}{ Morro do Calcário 42-88 } & 779.1 & 0.005 & 0.143 & 2.015 \\
\hline \multicolumn{2}{|c|}{ Morro do Calcário 42-88 } & 783.1 & 0.137 & 0.902 & 0.944 \\
\hline \multicolumn{2}{|c|}{ Morro do Calcário 42-88 } & 788.15 & 1.499 & 2.393 & 3.169 \\
\hline \multicolumn{2}{|c|}{ Morro do Calcário 42-88 } & 793.7 & 0.001 & 0.467 & 0.502 \\
\hline \multicolumn{2}{|c|}{ Morro do Calcário 42-88 } & 799.1 & 0.384 & 1.943 & 2.301 \\
\hline \multicolumn{2}{|c|}{ Morro do Calcário 42-88 } & 799.25 & 2.077 & 2.131 & 3.564 \\
\hline \multicolumn{2}{|c|}{ Morro do Calcário 42-88 } & 799.39 & 1.344 & 1.388 & 2.442 \\
\hline \multicolumn{2}{|c|}{ Morro do Calcário 42-88 } & 799.97 & 0.064 & 1.107 & 1.717 \\
\hline \multicolumn{2}{|c|}{ Morro do Calcário 42-88 } & 800.12 & 0.526 & 0.994 & 1.199 \\
\hline \multicolumn{2}{|c|}{ Morro do Calcário 42-88 } & 800.6 & 1.069 & 1.106 & 4.938 \\
\hline \multicolumn{2}{|c|}{ Morro do Calcário 42-88 } & 801.34 & 0.517 & 0.548 & 1.542 \\
\hline
\end{tabular}




\begin{tabular}{|c|c|c|c|c|c|}
\hline \multicolumn{2}{|c|}{ Morro do Calcário 42-88 } & 802.67 & 0.899 & 0.939 & 2.133 \\
\hline \multicolumn{2}{|c|}{ Morro do Calcário 42-88 } & 803.82 & 0.996 & 1.198 & 3.392 \\
\hline \multicolumn{2}{|c|}{ Morro do Calcário 42-88 } & 804.43 & 0.908 & 0.959 & 2.521 \\
\hline \multicolumn{2}{|c|}{ Morro do Calcário 42-88 } & 804.63 & 0.193 & 1.903 & 3.416 \\
\hline \multicolumn{2}{|c|}{ Morro do Calcário 42-88 } & 806.02 & 0.824 & 0.954 & 2.174 \\
\hline \multicolumn{2}{|c|}{ Morro do Calcário 42-88 } & 807.75 & 0.561 & 1.188 & 3.483 \\
\hline \multicolumn{2}{|c|}{ Morro do Calcário 42-88 } & 808.32 & 0.653 & 0.901 & 2.961 \\
\hline \multicolumn{2}{|c|}{ Morro do Calcário 42-88 } & 809.12 & 0.793 & 1.115 & 3.319 \\
\hline \multicolumn{2}{|c|}{ Morro do Calcário 42-88 } & 810.15 & 0.443 & 0.755 & 1.739 \\
\hline \multicolumn{2}{|c|}{ Morro do Calcário 42-88 } & 810.55 & 1.674 & 2.066 & 4.605 \\
\hline \multicolumn{2}{|c|}{ Morro do Calcário 42-88 } & 811.75 & 0.668 & 0.854 & 1.802 \\
\hline \multicolumn{2}{|c|}{ Morro do Calcário 42-88 } & 813.05 & 0.168 & 0.543 & 3.071 \\
\hline \multicolumn{2}{|c|}{ Morro do Calcário 42-88 } & 814.17 & 0.814 & 1.058 & 2.329 \\
\hline \multicolumn{2}{|c|}{ Morro do Calcário 42-88 } & 814.53 & 0.314 & 0.400 & 1.858 \\
\hline \multicolumn{2}{|c|}{ Morro do Calcário 42-88 } & 815.08 & 0.693 & 2.342 & 3.138 \\
\hline \multicolumn{2}{|c|}{ Morro do Calcário 42-88 } & 816.95 & 0.064 & 0.106 & 2.082 \\
\hline \multicolumn{2}{|c|}{ Morro do Calcário 42-88 } & 818 & 0.439 & 1.299 & 2.353 \\
\hline \multicolumn{2}{|c|}{ Morro do Calcário 42-88 } & 818.23 & 0.290 & 0.722 & 0.836 \\
\hline \multicolumn{2}{|c|}{ Morro do Calcário 42-88 } & 820.2 & 1.878 & 2.245 & 6.482 \\
\hline \multicolumn{2}{|c|}{ Morro do Calcário 42-88 } & 825.25 & 0.085 & 0.630 & 0.700 \\
\hline \multicolumn{2}{|c|}{ Morro do Calcário 42-88 } & 830 & 1.056 & 2.302 & 2.768 \\
\hline \multicolumn{2}{|c|}{ Morro do Calcário 42-88 } & 835.4 & 0.208 & 0.781 & 0.850 \\
\hline Zaonega & Core A-13 & 129.91 & & & 0.520 \\
\hline Zaonega & Core A-13 & 131.4 & 0.020 & & 0.930 \\
\hline Zaonega & Core A-13 & 133.98 & 1.110 & & 3.370 \\
\hline Zaonega & Core A-13 & 134.23 & 1.010 & & 2.710 \\
\hline Zaonega & Core A-13 & 134.97 & 0.860 & & 1.860 \\
\hline Zaonega & Core A-13 & 135.2 & 0.600 & & 1.760 \\
\hline Zaonega & Core A-13 & 135.56 & 0.680 & & 1.440 \\
\hline Zaonega & Core A-13 & 136.06 & 1.280 & & 1.740 \\
\hline Zaonega & Core A-13 & 137.01 & 1.170 & & 1.900 \\
\hline Zaonega & Core A-13 & 137.33 & 0.770 & & 2.130 \\
\hline Zaonega & Core A-13 & 137.77 & 0.410 & & 2.800 \\
\hline Zaonega & Core A-13 & 137.97 & 0.770 & & 2.750 \\
\hline Zaonega & Core A-13 & 138.12 & 0.640 & & 2.320 \\
\hline Zaonega & Core A-13 & 138.71 & 0.460 & & 2.520 \\
\hline Zaonega & Core A-13 & 140.29 & 1.980 & & 2.530 \\
\hline Zaonega & Core A-13 & 142.65 & 2.580 & & 3.330 \\
\hline Zaonega & Core A-13 & 145.53 & & & 2.010 \\
\hline Zaonega & C-175 & 16.6 & 1.600 & 1.930 & 3.350 \\
\hline Zaonega & C-175 & 30.2 & 1.490 & 1.760 & 3.530 \\
\hline
\end{tabular}




\begin{tabular}{|c|c|c|c|c|c|}
\hline Zaonega & $C-175$ & 33.7 & 4.170 & 4.510 & 5.000 \\
\hline Zaonega & $C-175$ & 36.5 & 2.320 & 2.480 & 2.580 \\
\hline Zaonega & $C-175$ & 36.9 & 2.950 & 3.320 & 3.180 \\
\hline Zaonega & $C-175$ & 38.4 & 5.970 & 6.060 & 5.830 \\
\hline Zaonega & $C-175$ & 54.9 & 7.490 & 7.730 & 7.940 \\
\hline Zaonega & $C-175$ & 57.3 & 6.440 & 6.610 & 6.440 \\
\hline Zaonega & C-175 & 70.6 & 0.510 & 0.690 & 2.580 \\
\hline Zaonega & $C-175$ & 72.6 & 1.590 & 1.610 & 1.550 \\
\hline Zaonega & $C-175$ & 75.2 & 0.920 & 0.940 & 1.050 \\
\hline Zaonega & $C-175$ & 80.3 & 0.830 & 0.840 & 0.870 \\
\hline Zaonega & C-175 & 81.8 & 0.580 & 0.600 & 0.730 \\
\hline Zaonega & C-175 & 84.5 & 0.730 & 0.750 & 1.690 \\
\hline Zaonega & $C-175$ & 86.9 & 0.780 & 0.930 & 1.380 \\
\hline Zaonega & C-175 & 95.3 & 0.880 & 0.980 & 1.070 \\
\hline Zaonega & C-175 & 96.9 & 1.560 & 1.620 & 1.570 \\
\hline Zaonega & C-175 & 97 & 2.120 & 2.160 & 2.470 \\
\hline Zaonega & $C-175$ & 98.8 & 1.220 & 1.320 & 1.270 \\
\hline Zaonega & C-175 & 101.5 & 1.130 & 1.200 & 1.350 \\
\hline Zaonega & C-175 & 175.2 & 1.610 & 2.380 & 4.630 \\
\hline Zaonega & C-175 & 176 & 1.590 & 2.300 & 3.390 \\
\hline Zaonega & C-175 & 179.4 & 1.910 & 2.800 & 3.990 \\
\hline Zaonega & C-175 & 180.7 & 2.360 & 3.330 & 5.030 \\
\hline Zaonega & C-175 & 194.8 & 1.660 & 2.030 & 3.250 \\
\hline Zaonega & $C-175$ & 204.3 & 1.710 & 1.790 & 2.980 \\
\hline Zaonega & C-175 & 206.1 & 0.950 & 1.050 & 1.860 \\
\hline Zaonega & $C-175$ & 217.2 & 1.510 & 1.590 & 3.740 \\
\hline Zaonega & C-175 & 218.6 & 0.950 & 1.140 & 1.090 \\
\hline Zaonega & C-5190 & 16 & 0.090 & 0.340 & 2.040 \\
\hline Zaonega & C-5190 & 83 & 1.310 & 2.550 & 3.620 \\
\hline Zaonega & C-5190 & 86.9 & 0.800 & 1.410 & 1.380 \\
\hline Zaonega & C-5190 & 92.5 & 0.090 & 0.680 & 1.340 \\
\hline Zaonega & C-5190 & 96 & 0.480 & 1.190 & 3.490 \\
\hline Zaonega & C-5190 & 100 & 0.460 & 1.000 & 2.320 \\
\hline Zaonega & C-5190 & 136.5 & 0.090 & 1.300 & 5.920 \\
\hline Zaonega & C-5190 & 146.6 & 0.360 & 0.660 & 1.720 \\
\hline Zaonega & C-5190 & 156 & 0.720 & 1.070 & 3.030 \\
\hline Zaonega & C-5190 & 184 & 0.120 & 0.290 & 1.190 \\
\hline Zaonega & C-5190 & 199 & 0.190 & 0.330 & 0.560 \\
\hline Zaonega & C-5190 & 204 & 0.680 & 0.740 & 2.350 \\
\hline Zaonega & C-5190 & 230 & 0.320 & 0.880 & 1.830 \\
\hline Zaonega & C-5190 & 234.5 & 0.230 & 0.690 & 2.780 \\
\hline
\end{tabular}




\begin{tabular}{|c|c|c|c|c|c|}
\hline Zaonega & C-5190 & 238.5 & 0.090 & 1.180 & 9.550 \\
\hline Zaonega & C-5190 & 245 & 0.720 & 1.500 & 3.720 \\
\hline Zaonega & C-5190 & 248 & 0.190 & 1.050 & 7.980 \\
\hline Zaonega & C-5190 & 287.5 & 0.780 & 1.790 & 9.280 \\
\hline Zaonega & C-5190 & 292.5 & 0.380 & 1.300 & 4.420 \\
\hline Zaonega & C-5190 & 293.2 & 0.240 & 0.430 & 0.660 \\
\hline Zaonega & C-5190 & 295.6 & 0.260 & 1.910 & 9.700 \\
\hline \multicolumn{2}{|c|}{ Noneshuch Formation } & NS1 & 0.009 & 3.240 & 7.840 \\
\hline \multicolumn{2}{|c|}{ Noneshuch Formation } & NS1-11 & 0.249 & 2.090 & 5.570 \\
\hline \multicolumn{2}{|c|}{ Noneshuch Formation } & NS1-1A & 0.809 & 1.330 & 2.880 \\
\hline \multicolumn{2}{|c|}{ Noneshuch Formation } & NS1-2A & 0.776 & 1.320 & 2.920 \\
\hline \multicolumn{2}{|c|}{ Noneshuch Formation } & NS1-2B & 0.765 & 1.360 & 3.430 \\
\hline \multicolumn{2}{|c|}{ Noneshuch Formation } & NS1-3B & 0.783 & 1.380 & 2.910 \\
\hline \multicolumn{2}{|c|}{ Noneshuch Formation } & NS1-4A & 0.648 & 1.13 & 2.29 \\
\hline \multicolumn{2}{|c|}{ Noneshuch Formation } & NS1-5B & 0.883 & 1.390 & 2.580 \\
\hline \multicolumn{2}{|c|}{ Noneshuch Formation } & NS1-6A & 0.747 & 1.220 & 2.820 \\
\hline \multicolumn{2}{|c|}{ Noneshuch Formation } & NS1-7A & 0.953 & 1.630 & 3.800 \\
\hline \multicolumn{2}{|c|}{ Noneshuch Formation } & NS1-7C & 0.947 & 1.680 & 3.810 \\
\hline \multicolumn{2}{|c|}{ Noneshuch Formation } & NS1-8A & 0.902 & 1.460 & 3.000 \\
\hline \multicolumn{2}{|c|}{ Noneshuch Formation } & NS1-8B & 0.727 & 1.260 & 2.990 \\
\hline \multicolumn{2}{|c|}{ Noneshuch Formation } & NS1-9A & 0.864 & 1.380 & 2.790 \\
\hline \multicolumn{2}{|c|}{ Noneshuch Formation } & NS10 & 0.016 & 1.680 & 5.970 \\
\hline \multicolumn{2}{|c|}{ Noneshuch Formation } & NS11 & 0.024 & 1.600 & 6.050 \\
\hline \multicolumn{2}{|c|}{ Noneshuch Formation } & NS12 & 0.082 & 1.930 & 5.480 \\
\hline \multicolumn{2}{|c|}{ Noneshuch Formation } & NS13 & 0.006 & 2.100 & 6.010 \\
\hline \multicolumn{2}{|c|}{ Noneshuch Formation } & NS14 & 0.012 & 1.830 & 5.270 \\
\hline \multicolumn{2}{|c|}{ Noneshuch Formation } & NS15 & 0.038 & 1.720 & 6.490 \\
\hline \multicolumn{2}{|c|}{ Noneshuch Formation } & NS16 & 0.011 & 2.000 & 6.750 \\
\hline \multicolumn{2}{|c|}{ Noneshuch Formation } & NS17 & 0.442 & 1.260 & 4.320 \\
\hline \multicolumn{2}{|c|}{ Noneshuch Formation } & NS18 & 0.401 & 1.620 & 4.450 \\
\hline \multicolumn{2}{|c|}{ Noneshuch Formation } & NS19 & 0.761 & 1.750 & 4.940 \\
\hline \multicolumn{2}{|c|}{ Noneshuch Formation } & NS2 & 0.038 & 2.720 & 7.760 \\
\hline \multicolumn{2}{|c|}{ Noneshuch Formation } & NS2-5A & 1.115 & 1.910 & 3.470 \\
\hline Noneshuc & ation & NS2-9A & 1.255 & 2.210 & 5.910 \\
\hline Noneshuc & ation & NS2-9B & 1.329 & 2.110 & 5.980 \\
\hline Noneshuc & ation & NS20 & 0.249 & 2.090 & 5.570 \\
\hline Noneshuc & ation & NS21 & 1.210 & 2.160 & 4.460 \\
\hline Noneshuc & ation & NS22 & 0.029 & 1.640 & 6.700 \\
\hline Noneshuc & ation & NS23 & 1.369 & 2.410 & 5.740 \\
\hline Noneshuc & ation & NS24 & 1.139 & 2.320 & 5.590 \\
\hline Noneshuc & ation & NS25 & 1.307 & 2.050 & 4.650 \\
\hline
\end{tabular}




\begin{tabular}{|c|c|c|c|c|}
\hline Noneshuch Formation & NS26 & 1.238 & 2.400 & 5.820 \\
\hline Noneshuch Formation & NS27 & 1.136 & 1.840 & 4.840 \\
\hline Noneshuch Formation & NS28 & 0.103 & 1.440 & 4.950 \\
\hline Noneshuch Formation & NS29 & 1.146 & 2.310 & 4.660 \\
\hline Noneshuch Formation & NS3 & 0.022 & 1.920 & 7.070 \\
\hline Noneshuch Formation & NS30 & 1.383 & 2.580 & 5.330 \\
\hline Noneshuch Formation & NS31 & 0.084 & 2.000 & 7.990 \\
\hline Noneshuch Formation & NS31 D & 0.030 & 3.289 & 9.180 \\
\hline Noneshuch Formation & NS31 L & 0.020 & 2.521 & 5.280 \\
\hline Noneshuch Formation & NS32 & 0.058 & 1.810 & 6.550 \\
\hline Noneshuch Formation & NS33 & 0.042 & 1.910 & 7.360 \\
\hline Noneshuch Formation & NS34 & 0.319 & 1.560 & 5.190 \\
\hline Noneshuch Formation & NS35 & 0.942 & 1.830 & 4.020 \\
\hline Noneshuch Formation & NS36 & 0.684 & 1.980 & 5.220 \\
\hline Noneshuch Formation & NS37 & 0.094 & 1.180 & 5.240 \\
\hline Noneshuch Formation & NS38 & 0.016 & 2.500 & 6.660 \\
\hline Noneshuch Formation & NS39 & 0.044 & 1.990 & 7.560 \\
\hline Noneshuch Formation & NS4 & 0.022 & 2.480 & 6.000 \\
\hline Noneshuch Formation & NS40 & 0.109 & 1.640 & 5.740 \\
\hline Noneshuch Formation & NS41 & 0.110 & 1.300 & 5.150 \\
\hline Noneshuch Formation & NS42 & 0.001 & 2.310 & 7.350 \\
\hline Noneshuch Formation & NS43 & 0.001 & 3.940 & 7.370 \\
\hline Noneshuch Formation & NS44 & 0.001 & 3.700 & 7.570 \\
\hline Noneshuch Formation & NS5 & 0.015 & 2.950 & 7.290 \\
\hline Noneshuch Formation & NS6 & 0.020 & 1.780 & 5.790 \\
\hline Noneshuch Formation & NS7 & 0.019 & 2.530 & 7.850 \\
\hline Noneshuch Formation & NS8 & 0.190 & 0.980 & 3.950 \\
\hline Noneshuch Formation & NS9 & 0.038 & 1.940 & 6.700 \\
\hline Sengoma Argillite Strat 2 & 142.8 & 0.500 & 1.450 & 3.350 \\
\hline Sengoma Argillite Strat 2 & 146.2 & 0.370 & 0.800 & 1.910 \\
\hline Sengoma Argillite Strat 2 & 171.5 & 2.010 & 2.410 & 2.680 \\
\hline Sengoma Argillite Strat 2 & 173.67 & 2.500 & 2.870 & 3.080 \\
\hline Sengoma Argillite Strat 2 & 177.7 & 1.900 & 2.280 & 2.660 \\
\hline Sengoma Argillite Strat 2 & 181.25 & 3.020 & 3.270 & 3.600 \\
\hline Sengoma Argillite Strat 2 & 184.5 & 2.440 & 3.450 & 3.720 \\
\hline Sengoma Argillite Strat 2 & 200.7 & 2.820 & 3.040 & 3.490 \\
\hline Sengoma Argillite Strat 2 & 202.5 & 2.730 & 2.970 & 3.240 \\
\hline Sengoma Argillite Strat 2 & 209 & 2.010 & 2.330 & 3.040 \\
\hline Sengoma Argillite Strat 2 & 212.7 & 2.100 & 2.470 & 2.710 \\
\hline Sengoma Argillite Strat 2 & 216.8 & 1.820 & 2.400 & 2.300 \\
\hline Sengoma Argillite Strat 2 & 219.1 & 1.120 & 1.850 & 2.460 \\
\hline
\end{tabular}




\begin{tabular}{|c|c|c|c|c|c|}
\hline Sengoma Argillite & Strat 2 & 224.87 & 0.910 & 1.310 & 1.730 \\
\hline Sengoma Argillite & Strat 2 & 286.6 & 0.560 & 0.890 & 3.430 \\
\hline Datangpo & Minle & ML56 & 0.010 & & 0.390 \\
\hline Datangpo & Minle & ML57 & 0.000 & & 0.360 \\
\hline Datangpo & Minle & ML60 & 0.300 & & 0.780 \\
\hline Datangpo & Minle & ML62 & 1.120 & & 3.050 \\
\hline Datangpo & Minle & ML64 & 0.010 & & 0.360 \\
\hline Datangpo & Minle & ML66 & 0.010 & & 0.390 \\
\hline Datangpo & Minle & ML68 & 1.910 & & 4.480 \\
\hline Datangpo & Minle & ML70 & 1.070 & & 2.320 \\
\hline Datangpo & Minle & ML72 & 0.680 & & 3.680 \\
\hline Datangpo & Minle & ML74 & 1.940 & & 4.390 \\
\hline Datangpo & Minle & ML77 & 2.110 & & 5.240 \\
\hline Datangpo & Minle & ML79 & 1.920 & & 4.160 \\
\hline Datangpo & Minle & ML80 & 0.010 & & 0.380 \\
\hline Datangpo & Minle & ML81 & 0.700 & & 1.830 \\
\hline Datangpo & Minle & ML82 & 1.640 & & 2.560 \\
\hline Datangpo & Minle & ML83 & 0.380 & & 0.600 \\
\hline Datangpo & Minle & ML-01 & 3.700 & 4.200 & 4.400 \\
\hline Datangpo & Minle & ML-02 & 1.900 & 2.300 & 2.600 \\
\hline Datangpo & Minle & ML-03 & 2.000 & 2.100 & 1.700 \\
\hline Datangpo & Minle & ML-04 & 2.300 & 2.400 & 2.400 \\
\hline Datangpo & Minle & ML-05 & 0.200 & 0.200 & 1.900 \\
\hline Datangpo & Minle & ML-06 & 0.200 & 0.200 & 0.400 \\
\hline Datangpo & Minle & ML-07 & 0.200 & 0.200 & 0.400 \\
\hline Datangpo & Minle & ML-08 & 0.200 & 0.200 & 0.300 \\
\hline Datangpo & Minle & ML-09 & 0.200 & 0.200 & 0.400 \\
\hline Datangpo & Minle & ML-10 & 0.200 & 0.200 & 0.300 \\
\hline Datangpo & Minle & ML-11 & 0.000 & 0.100 & 0.600 \\
\hline Datangpo & Minle & ML-12 & 0.000 & 0.000 & 0.400 \\
\hline Datangpo & Minle & ML-13 & 0.000 & 0.000 & 0.400 \\
\hline Datangpo & Minle & ML-14 & 1.200 & 1.600 & 2.700 \\
\hline Datangpo & Minle & ML-15 & 1.900 & 2.100 & 4.500 \\
\hline Datangpo & Minle & ML-16 & 1.800 & 2.000 & 4.200 \\
\hline Datangpo & Minle & ML-17 & 0.400 & 0.500 & 3.000 \\
\hline Datangpo & Minle & ML-24 & 0.100 & 0.400 & 3.800 \\
\hline Datangpo & Minle & ML-25 & 0.100 & 0.500 & 4.600 \\
\hline Datangpo & Minle & ML-26 & 0.000 & 0.700 & 4.400 \\
\hline Datangpo & Minle & ML-27 & 0.000 & 0.300 & 5.200 \\
\hline Datangpo & Minle & ML-28 & 0.000 & 0.800 & 4.500 \\
\hline Datangpo & Minle & ML-29 & 0.000 & 0.300 & 4.800 \\
\hline
\end{tabular}




\begin{tabular}{|c|c|c|c|c|c|}
\hline Datangpo & Yangjiaping & $\mathrm{SH}-03$ & 1.100 & 1.500 & 4.100 \\
\hline Datangpo & Yangjiaping & $\mathrm{SH}-04$ & 1.100 & 1.500 & 4.300 \\
\hline Datangpo & Yangjiaping & SH-06 & 1.000 & 1.300 & 5.300 \\
\hline Datangpo & Yangjiaping & $\mathrm{SH}-07$ & 0.000 & 0.200 & 4.200 \\
\hline \multicolumn{2}{|c|}{ Mt. Profeit DolostıF851 } & 27 & 0.005 & 1.767 & 4.550 \\
\hline \multicolumn{2}{|c|}{ Mt. Profeit DolostıF851 } & 39.5 & 0.004 & 1.818 & 3.820 \\
\hline \multicolumn{2}{|c|}{ Mt. Profeit DolostıF851 } & 55 & 0.004 & 0.941 & 3.170 \\
\hline \multicolumn{2}{|c|}{ Mt. Profeit DolostıF851 } & 67 & 0.007 & 1.140 & 4.710 \\
\hline \multicolumn{2}{|c|}{ Mt. Profeit DolostıF851 } & 82 & 0.007 & 1.893 & 6.680 \\
\hline \multicolumn{2}{|c|}{ Mt. Profeit DolostıW8 } & -1.4 & 0.114 & 1.529 & 3.524 \\
\hline \multicolumn{2}{|c|}{ Mt. Profeit DolostıW8 } & 44 & 0.003 & 2.318 & 6.920 \\
\hline \multicolumn{2}{|c|}{ Mt. Profeit DolostıW8 } & 104 & 0.378 & 2.872 & 9.100 \\
\hline \multicolumn{2}{|c|}{ Mt. Profeit DolostıW8 } & 122 & 0.110 & 1.893 & 6.831 \\
\hline \multicolumn{2}{|c|}{ Mt. Profeit DolostıW8 } & 140 & 0.037 & 1.642 & 6.389 \\
\hline \multicolumn{2}{|c|}{ Mt. Profeit DolostıW8 } & 218 & 0.008 & 2.060 & 7.372 \\
\hline \multicolumn{2}{|c|}{ Mt. Profeit DolostıW8 } & 242 & 0.009 & 1.817 & 6.485 \\
\hline \multicolumn{2}{|c|}{ Mt. Profeit DolostıW8 } & 333 & 0.002 & 1.612 & 4.871 \\
\hline \multicolumn{2}{|c|}{ Mt. Profeit DolostıW8 } & 380 & 0.005 & 0.904 & 4.408 \\
\hline \multicolumn{2}{|c|}{ Mt. Profeit DolostıW8 } & 561.4 & 0.006 & 2.121 & 3.754 \\
\hline \multicolumn{2}{|c|}{ Mt. Profeit DolostıW8 } & 573 & 0.037 & 2.484 & 4.890 \\
\hline \multicolumn{2}{|c|}{ Mt. Profeit DolostıW8 } & 582 & 0.011 & 2.318 & 4.890 \\
\hline \multicolumn{2}{|c|}{ Mt. Profeit DolostıW8 } & 627 & 0.005 & 2.491 & 4.890 \\
\hline \multicolumn{2}{|c|}{ Mt. Profeit DolostıW8 } & 643 & 0.002 & 2.311 & 8.866 \\
\hline \multicolumn{2}{|c|}{ Mt. Profeit DolostıW8 } & 684.5 & 0.002 & 1.797 & 3.865 \\
\hline \multicolumn{2}{|c|}{ Mt. Profeit DolostıW8 } & 689 & 0.001 & 1.425 & 4.134 \\
\hline \multicolumn{2}{|c|}{ Mt. Profeit DolostıW8 } & 692 & 0.004 & 1.291 & 7.055 \\
\hline \multicolumn{2}{|c|}{ Mt. Profeit DolostıW8 } & 695.5 & 0.001 & 1.279 & 7.745 \\
\hline \multicolumn{2}{|c|}{ Mt. Profeit DolostıW8 } & 698 & 0.000 & 1.164 & 6.544 \\
\hline \multicolumn{2}{|c|}{ Mt. Profeit DolostıW8 } & 700 & 0.001 & 1.085 & 5.682 \\
\hline \multicolumn{2}{|c|}{ Mt. Profeit DolostıW8 } & 705 & 0.004 & 1.139 & 5.186 \\
\hline Mt. Profeit D & W8 & 738 & 0.035 & 1.399 & 5.978 \\
\hline Beck Springs & E1101 & 45.7 & 0.000 & 0.221 & 1.400 \\
\hline Beck Springs & E1101 & 48.3 & 0.000 & 0.719 & 2.040 \\
\hline Beck Springs & E1101 & 51.7 & 0.000 & 0.132 & 1.100 \\
\hline Beck Springs & E1101 & 57 & 0.000 & 0.185 & 2.960 \\
\hline Beck Springs & E1101 & 60.9 & 0.000 & 0.258 & 2.850 \\
\hline Beck Springs & E1101 & 65 & 0.000 & 0.057 & 0.920 \\
\hline Beck Springs & E1101 & 67.45 & 0.000 & 0.395 & 1.320 \\
\hline Beck Springs & E1101 & 72.5 & 0.000 & 0.400 & 1.770 \\
\hline Beck Springs & E1101 & 76.4 & 0.000 & 1.088 & 3.060 \\
\hline Beck Springs & E1101 & 81.5 & 0.005 & 0.200 & 0.800 \\
\hline
\end{tabular}




\begin{tabular}{|c|c|c|c|c|c|}
\hline Beck Springs & E1101 & 85.6 & 0.002 & 2.969 & 4.260 \\
\hline Beck Springs & E1101 & 90.6 & 0.003 & 0.218 & 0.890 \\
\hline Beck Springs & E1101 & 101.7 & 0.003 & 0.099 & 0.840 \\
\hline Beck Springs & E1101 & 102.3 & 0.003 & 1.959 & 3.590 \\
\hline Beck Springs & E1101 & 106 & 0.003 & 0.182 & 0.910 \\
\hline Beck Springs & E1101 & 111.9 & 0.002 & 0.562 & 2.290 \\
\hline Beck Springs & E1101 & 134.8 & 0.001 & 0.493 & 2.550 \\
\hline Beck Springs & E1101 & 136.6 & 0.006 & 0.439 & 1.060 \\
\hline Beck Springs & E1101 & 142.7 & 0.002 & 0.132 & 1.240 \\
\hline Beck Springs & E1101 & 148.2 & 0.002 & 0.657 & 1.780 \\
\hline Beck Springs & E1101 & 153 & 0.008 & 0.418 & 0.570 \\
\hline Beck Springs & E1101 & 156.2 & 0.002 & 0.368 & 3.530 \\
\hline Beck Springs & E1101 & 164.6 & 0.002 & 0.642 & 1.940 \\
\hline Beck Springs & E1101 & 170.5 & 0.002 & 0.545 & 3.680 \\
\hline Beck Springs & E1101 & 175.4 & 0.002 & 0.264 & 2.770 \\
\hline Beck Springs & E1101 & 182.5 & 0.003 & 1.296 & 3.340 \\
\hline Beck Springs & E1101 & 205.4 & 0.002 & 0.465 & 3.710 \\
\hline Beck Springs & E1101 & 210.9 & 0.004 & 0.351 & 3.190 \\
\hline Beck Springs & E1101 & 216.2 & 0.002 & 0.111 & 3.130 \\
\hline Beck Springs & E1101 & 224.3 & 0.004 & 1.215 & 2.730 \\
\hline Beck Springs & E1101 & 226.3 & 0.000 & 0.104 & 2.180 \\
\hline Beck Springs & E1101 & 232 & 0.001 & 0.487 & 4.110 \\
\hline Beck Springs & E1101 & 235.3 & 0.001 & 0.580 & 2.150 \\
\hline Beck Springs & E1101 & 239.5 & 0.000 & 0.096 & 3.250 \\
\hline Beck Springs & E1101 & 248.1 & 0.001 & 0.095 & 1.640 \\
\hline Beck Springs & E1101 & 249.45 & 0.002 & 0.265 & 2.500 \\
\hline Beck Springs & E1101 & 263.9 & 0.001 & 2.006 & 3.140 \\
\hline Beck Springs & E1101 & 292.05 & 0.002 & 0.750 & 2.380 \\
\hline Beck Springs & E1101 & 292.4 & 0.002 & 0.549 & 2.950 \\
\hline Beck Springs & E1101 & 293.8 & 0.008 & 0.677 & 2.640 \\
\hline Beck Springs & E1101 & 297.3 & 0.001 & 1.118 & 2.580 \\
\hline Beck Springs & E1101 & 300.6 & 0.000 & 0.977 & 3.030 \\
\hline Beck Springs & E1101 & 302.9 & 0.002 & 0.710 & 2.680 \\
\hline Beck Springs & E1101 & 311.5 & 0.003 & 2.802 & 3.080 \\
\hline Beck Springs & E1101 & 316.5 & 0.002 & 1.869 & 2.880 \\
\hline Kingston Peak 1 & E1101 & 324.5 & 0.004 & 1.275 & 1.810 \\
\hline Kingston Peak 1 & E1101 & 329.5 & 0.003 & 0.980 & 2.460 \\
\hline Kingston Peak 1 & E1101 & 333.7 & 0.002 & 0.299 & 1.650 \\
\hline Kingston Peak 1 & E1101 & 337.6 & 0.002 & 0.885 & 2.660 \\
\hline Kingston Peak 1 & E1101 & 366.4 & 0.001 & 7.296 & 13.400 \\
\hline Kingston Peak 1 & E1101 & 369.6 & 0.002 & 0.385 & 0.650 \\
\hline
\end{tabular}




\begin{tabular}{|c|c|c|c|c|c|}
\hline Kingston Peak 1 & E1101 & 371.3 & 0.001 & 3.457 & 4.520 \\
\hline Kingston Peak 1 & E1101 & 375.9 & 0.002 & 1.306 & 3.110 \\
\hline Kingston Peak 1 & E1101 & 378 & 0.001 & 0.456 & 2.450 \\
\hline Kingston Peak 1 & E1101 & 390 & 0.000 & 0.685 & 4.260 \\
\hline Kingston Peak 1 & E1101 & 393 & 0.000 & 1.067 & 3.490 \\
\hline Kingston Peak 1 & E1101 & 396 & 0.000 & 2.327 & 4.790 \\
\hline Kingston Peak 1 & E1101 & 399 & 0.000 & 0.839 & 3.810 \\
\hline Kingston Peak 1 & E1101 & 402 & 0.000 & 0.582 & 4.140 \\
\hline Kingston Peak 1 & E1101 & 405 & 0.001 & 1.902 & 5.220 \\
\hline Kingston Peak 1 & E1101 & 415 & 0.002 & 1.256 & 4.030 \\
\hline Kingston Peak 1 & E1101 & 425 & 0.002 & 0.437 & 4.630 \\
\hline Kingston Peak 1 & E1101 & 435 & 0.002 & 0.268 & 4.160 \\
\hline Kingston Peak 1 & E1101 & 445 & 0.002 & 0.508 & 4.340 \\
\hline Kingston Peak 1 & E1101 & 455 & 0.002 & 0.374 & 3.870 \\
\hline Kingston Peak 1 & E1101 & 465 & 0.002 & 1.003 & 4.380 \\
\hline Kingston Peak 1 & E1101 & 475 & 0.002 & 0.941 & 4.030 \\
\hline Kingston Peak 1 & E1101 & 485 & 0.002 & 1.252 & 3.780 \\
\hline Kingston Peak 1 & E1101 & 495 & 0.002 & 0.305 & 3.650 \\
\hline Kingston Peak 1 & E1101 & 505 & 0.000 & 1.125 & 4.270 \\
\hline Kingston Peak 1 & E1101 & 515 & 0.000 & 0.705 & 3.620 \\
\hline Kingston Peak 1 & E1101 & 525 & 0.000 & 1.285 & 2.920 \\
\hline Kingston Peak 1 & E1101 & 535 & 0.000 & 0.600 & 3.950 \\
\hline Kingston Peak 1 & E1101 & 544 & 0.000 & 2.599 & 3.920 \\
\hline Kingston Peak 1 & E1101 & 555 & 0.000 & 0.887 & 3.310 \\
\hline Kingston Peak 1 & E1101 & 565 & 0.001 & 3.217 & 4.500 \\
\hline Kingston Peak 1 & E1101 & 575 & 0.001 & 3.983 & 5.580 \\
\hline Kingston Peak 1 & E1101 & 585 & 0.000 & 0.193 & 2.350 \\
\hline Kingston Peak 1 & E1101 & 591.7 & 0.001 & 1.619 & 2.170 \\
\hline Beck Springs & E1102 & 6.5 & 0.003 & 2.075 & 2.510 \\
\hline Beck Springs & E1102 & 6.75 & 0.002 & 0.841 & 1.580 \\
\hline Beck Springs & E1102 & 7.65 & 0.005 & 1.195 & 1.240 \\
\hline Beck Springs & E1102 & 12.4 & 0.001 & 7.067 & 16.000 \\
\hline Beck Springs & E1102 & 14.9 & 0.001 & 2.767 & 3.010 \\
\hline Beck Springs & E1102 & 19.2 & 0.001 & 1.548 & 2.090 \\
\hline Beck Springs & E1102 & 19.8 & 0.001 & 2.488 & 2.830 \\
\hline Beck Springs & E1102 & 21.85 & 0.003 & 2.702 & 3.290 \\
\hline Beck Springs & E1102 & 23.2 & 0.004 & 2.835 & 3.590 \\
\hline Beck Springs & E1102 & 29.7 & 0.002 & 2.063 & 2.490 \\
\hline Beck Springs & E1102 & 30.9 & 0.000 & 2.655 & 6.990 \\
\hline Beck Springs & E1102 & 32 & 0.000 & 2.054 & 3.190 \\
\hline Beck Springs & E1102 & 47.7 & 0.007 & 1.957 & 2.660 \\
\hline
\end{tabular}




\begin{tabular}{|c|c|c|c|c|c|}
\hline Beck Springs & E1102 & 52.9 & 0.001 & 0.158 & 0.420 \\
\hline Beck Springs & E1102 & 55.8 & 0.000 & 2.190 & 3.140 \\
\hline Beck Springs & E1102 & 56.3 & 0.000 & 2.198 & 3.660 \\
\hline Kingston Peak 1 & E1102 & 61 & 0.000 & 1.636 & 3.660 \\
\hline Kingston Peak 1 & E1102 & 65 & 0.000 & 1.398 & 2.200 \\
\hline Kingston Peak 1 & E1102 & 71 & 0.000 & 0.657 & 3.590 \\
\hline Kingston Peak 1 & E1102 & 78 & & 0.924 & 4.220 \\
\hline Kingston Peak 1 & E1102 & 80 & & 1.735 & 3.780 \\
\hline Kwagunt & Awatubi member & below Walcot & 0.000 & 0.130 & 1.370 \\
\hline Kwagunt & Kwagunt Formatio & ns below base & 0.020 & 1.100 & 1.340 \\
\hline Kwagunt & Kwagunt Formatio & rう̄ meters belo & 1.000 & 1.650 & 2.000 \\
\hline Kwagunt & Kwagunt Formatio & n7 meters belo & 0.040 & 2.290 & 2.830 \\
\hline Kwagunt & Kwagunt Formatio & n) meters belo & 0.060 & 0.440 & 1.540 \\
\hline Kwagunt & Walcott Member & 9 & 2.490 & 3.210 & 3.450 \\
\hline Kwagunt & Walcott Member & 145 & 1.800 & 2.310 & 2.790 \\
\hline Kwagunt & Walcott Member & 157 & 1.850 & 2.230 & 2.680 \\
\hline Kwagunt & Walcott Member & 175 & 0.240 & 0.760 & 0.740 \\
\hline Kwagunt & Walcott Member & 190 & 0.180 & 0.820 & 0.920 \\
\hline Kwagunt & Walcott Member & 200.2 & 2.350 & 2.670 & 3.080 \\
\hline Kwagunt & Walcott Member & 210 & 0.720 & 1.150 & 1.600 \\
\hline Kwagunt & Walcott Member & 215 & 3.180 & 3.790 & 4.720 \\
\hline Kwagunt & Walcott Member & 219.5 & 0.020 & 0.240 & 0.360 \\
\hline Kwagunt & Walcott Member & 230.5 & 2.380 & 2.770 & 3.470 \\
\hline Kwagunt & Walcott Member & s above Walc & 0.020 & 0.670 & 0.810 \\
\hline Kwagunt & Awatubi & 1151.5 & 0.001 & 1.120 & 2.737 \\
\hline Kwagunt & Awatubi & 1154.5 & 0.001 & 0.967 & 3.752 \\
\hline Kwagunt & Awatubi & 1157.5 & 0.003 & 1.393 & 2.457 \\
\hline Kwagunt & Awatubi & 1162 & 0.001 & 0.600 & 1.339 \\
\hline Kwagunt & Awatubi & 1167 & 0.001 & 0.361 & 1.006 \\
\hline Kwagunt & Awatubi & 1207 & 0.001 & 0.676 & 1.392 \\
\hline Kwagunt & Awatubi & 1226 & 0.001 & 0.075 & 0.817 \\
\hline Kwagunt & Awatubi & 1240.5 & 0.000 & 0.854 & 1.760 \\
\hline Kwagunt & Awatubi & 1259.5 & 0.001 & 0.633 & 1.491 \\
\hline Kwagunt & Awatubi & 1268.5 & 0.001 & 1.362 & 2.144 \\
\hline Kwagunt & Awatubi & 1271.5 & 0.024 & 0.249 & 1.199 \\
\hline Kwagunt & Awatubi & 1273 & 0.003 & 0.120 & 1.196 \\
\hline Kwagunt & Awatubi & 1273 & 0.001 & 0.349 & 1.233 \\
\hline Kwagunt & Awatubi & 1277.5 & 0.214 & 1.109 & 1.823 \\
\hline Kwagunt & Awatubi & 1280 & 0.105 & 0.965 & 1.912 \\
\hline Kwagunt & Awatubi & 1286.5 & 0.285 & 1.021 & 1.565 \\
\hline Kwagunt & Awatubi & 1295.5 & 0.002 & 1.552 & 2.355 \\
\hline
\end{tabular}




\begin{tabular}{|c|c|c|c|c|c|}
\hline Kwagunt & Awatubi & 1299.5 & 0.333 & 0.513 & 1.298 \\
\hline Kwagunt & Awatubi & 1301.5 & 0.001 & 0.459 & 1.657 \\
\hline Kwagunt & Awatubi & 1308 & 0.085 & 0.234 & 1.316 \\
\hline Kwagunt & Awatubi & 1311 & 0.130 & 0.232 & 1.052 \\
\hline Kwagunt & Awatubi & 1313.5 & 0.143 & 0.842 & 1.719 \\
\hline Kwagunt & Awatubi & 1318 & 0.014 & 2.253 & 3.009 \\
\hline Kwagunt & Awatubi & 1319.5 & 0.155 & 0.530 & 1.184 \\
\hline Kwagunt & Awatubi & 1323 & 0.022 & 0.203 & 1.267 \\
\hline Kwagunt & Awatubi & 1328 & 0.199 & 0.529 & 1.538 \\
\hline Kwagunt & Awatubi & 1331 & 0.004 & 0.873 & 1.801 \\
\hline Kwagunt & Awatubi & 1333 & 0.379 & 0.495 & 1.337 \\
\hline Galeros & Carbon Canyon & 451 & 0.000 & 0.359 & 1.347 \\
\hline Galeros & Carbon Canyon & 453 & 0.001 & 0.709 & 1.326 \\
\hline Galeros & Carbon Canyon & 453 & 0.001 & 3.146 & 3.932 \\
\hline Galeros & Carbon Canyon & 457.5 & 0.002 & 0.458 & 1.089 \\
\hline Galeros & Carbon Canyon & 469.5 & 0.009 & 8.633 & 11.200 \\
\hline Galeros & Carbon Canyon & 485.5 & 0.001 & 0.602 & 3.044 \\
\hline Galeros & Carbon Canyon & 495.5 & 0.002 & 0.339 & 1.554 \\
\hline Galeros & Carbon Canyon & 642 & 0.009 & 0.151 & 1.261 \\
\hline Galeros & Carbon Canyon & 672.5 & 0.005 & 0.637 & 1.166 \\
\hline Galeros & Carbon Canyon & 756 & 0.000 & 0.234 & 1.413 \\
\hline Galeros & Carbon Canyon & 801 & 0.004 & 0.211 & 1.600 \\
\hline Galeros & Carbon Canyon & 816 & 0.001 & 1.396 & 4.380 \\
\hline Galeros & Carbon Canyon & 816 & 0.000 & 0.428 & 1.186 \\
\hline Galeros & Carbon Canyon & 831 & 0.017 & 0.461 & 2.316 \\
\hline Galeros & Carbon Canyon & 842 & 0.027 & 0.573 & 1.999 \\
\hline Galeros & Carbon Canyon & 846.5 & 0.003 & 0.235 & 1.489 \\
\hline Galeros & Carbon Canyon & 860 & 0.001 & 0.241 & 1.986 \\
\hline Galeros & Carbon Canyon & 861 & 0.321 & 0.874 & 2.410 \\
\hline Galeros & Carbon Canyon & 866 & 0.006 & 1.843 & 3.176 \\
\hline Galeros & Carbon Canyon & 870 & 0.002 & 1.135 & 3.275 \\
\hline Galeros & Carbon Canyon & 871.5 & 0.000 & 0.519 & 3.173 \\
\hline Galeros & Carbon Canyon & 875.5 & 0.013 & 0.304 & 2.632 \\
\hline Galeros & Carbon Canyon & 893 & 0.006 & 0.193 & 1.506 \\
\hline Galeros & Carbon Canyon & 897 & 0.000 & 0.385 & 1.879 \\
\hline Galeros & Duppa & 951 & 0.001 & 1.317 & 3.099 \\
\hline Galeros & Duppa & 961 & 0.000 & 0.499 & 3.396 \\
\hline Galeros & Duppa & 962.5 & 0.000 & 1.944 & 4.084 \\
\hline Galeros & Duppa & 991.5 & 0.000 & 0.879 & 3.352 \\
\hline Galeros & Duppa & 997 & 0.004 & 0.637 & 1.508 \\
\hline Galeros & Duppa & 1012 & 0.001 & 0.586 & 1.884 \\
\hline
\end{tabular}




\begin{tabular}{|c|c|c|c|c|c|}
\hline Galeros & Jupiter & 204.5 & 0.001 & 0.998 & 3.260 \\
\hline Galeros & Jupiter & 230 & 0.000 & 0.783 & 1.997 \\
\hline Galeros & Jupiter & 248 & 0.001 & 0.347 & 1.217 \\
\hline Galeros & Jupiter & 315 & 0.000 & 2.088 & 3.928 \\
\hline Galeros & Jupiter & 370 & 0.000 & 1.208 & 2.115 \\
\hline Galeros & Jupiter & 408 & 0.000 & 0.203 & 1.755 \\
\hline Galeros & Jupiter & 412 & 0.000 & 0.123 & 1.665 \\
\hline Galeros & Tanner & 9 & 0.013 & 0.806 & 1.409 \\
\hline Galeros & Tanner & 12 & 0.071 & 1.190 & 2.013 \\
\hline Galeros & Tanner & 13.5 & 0.000 & 0.360 & 1.355 \\
\hline Galeros & Tanner & 16 & 0.051 & 2.273 & 4.117 \\
\hline Galeros & Tanner & 17.5 & 0.000 & 2.111 & 4.028 \\
\hline Galeros & Tanner & 20 & 0.000 & 1.702 & 7.111 \\
\hline Galeros & Tanner & 26 & 0.000 & 0.872 & 2.900 \\
\hline Galeros & Tanner & 31.5 & 0.022 & 1.071 & 2.936 \\
\hline Galeros & Tanner & 36 & 0.001 & 1.152 & 2.303 \\
\hline Galeros & Tanner & 38 & 0.000 & 0.348 & 1.925 \\
\hline Galeros & Tanner & 46 & 0.001 & 2.863 & 3.855 \\
\hline Galeros & Tanner & 48 & 0.001 & 0.736 & 1.918 \\
\hline Galeros & Tanner & 54 & 0.000 & 0.241 & 1.433 \\
\hline Galeros & Tanner & 55.5 & 0.001 & 0.201 & 0.968 \\
\hline Galeros & Tanner & 56 & 0.010 & 0.744 & 1.578 \\
\hline Galeros & Tanner & 57 & 0.027 & 0.245 & 1.092 \\
\hline Galeros & Tanner & 61.5 & 0.001 & 0.439 & 1.812 \\
\hline Galeros & Tanner & 63 & 0.001 & 0.720 & 1.854 \\
\hline Galeros & Tanner & 71 & 0.000 & 0.499 & 1.549 \\
\hline Galeros & Tanner & 76 & 0.004 & 0.369 & 1.234 \\
\hline Galeros & Tanner & 94 & 0.001 & 0.486 & 1.172 \\
\hline Galeros & Tanner & 100.5 & 0.001 & 0.171 & 0.805 \\
\hline Galeros & Tanner & 105 & 0.000 & 1.009 & 1.731 \\
\hline Galeros & Tanner & 111.5 & 0.004 & 0.269 & 1.108 \\
\hline Galeros & Tanner & 116 & 0.001 & 0.697 & 1.762 \\
\hline Galeros & Tanner & 120 & 0.000 & 0.681 & 1.372 \\
\hline Galeros & Tanner & 124.5 & 0.000 & 0.413 & 1.318 \\
\hline Galeros & Tanner & 129 & 0.004 & 0.549 & 1.297 \\
\hline Galeros & Tanner & 138.5 & 0.000 & 0.376 & 0.989 \\
\hline Galeros & Tanner & 153 & 0.017 & 0.957 & 1.887 \\
\hline Galeros & Tanner & 167 & 0.006 & 0.214 & 1.129 \\
\hline Kwagunt & Walcott & 1347.5 & 0.000 & 0.246 & 1.419 \\
\hline Kwagunt & Walcott & 1350 & 0.025 & 0.314 & 1.106 \\
\hline Kwagunt & Walcott & 1361 & 0.002 & 0.350 & 1.630 \\
\hline
\end{tabular}




\begin{tabular}{|c|c|c|c|c|c|}
\hline Kwagunt & Walcott & 1368 & 0.000 & 0.215 & 1.211 \\
\hline Kwagunt & Walcott & 1368.5 & 0.014 & 0.091 & 0.802 \\
\hline Kwagunt & Walcott & 1372.5 & 0.022 & 0.099 & 0.805 \\
\hline Kwagunt & Walcott & 1374.5 & 0.029 & 0.190 & 0.935 \\
\hline Kwagunt & Walcott & 1606.5 & 0.000 & 0.897 & 1.187 \\
\hline Kwagunt & Walcott & 1609 & 0.004 & 1.266 & 1.616 \\
\hline Valpol & Kel'tminskaya-1 & 2961 & 0.030 & 0.490 & 1.110 \\
\hline Valpol & Kel'tminskaya-1 & 3262 & 0.000 & 0.160 & 3.710 \\
\hline Valpol & Kel'tminskaya-1 & 3540 & 0.010 & 0.070 & 1.970 \\
\hline Valpol & Kel'tminskaya-1 & 3680 & 0.010 & 0.520 & 0.540 \\
\hline Yskemess & Kel'tminskaya-1 & 3946 & 0.160 & 0.800 & 1.380 \\
\hline Yskemess & Kel'tminskaya-1 & 3951.3 & 0.010 & 0.420 & 0.500 \\
\hline Yskemess & Kel'tminskaya-1 & 3994.5 & 0.180 & 0.700 & 1.330 \\
\hline Yskemess & Kel'tminskaya-1 & 4107 & 0.120 & 0.390 & 0.590 \\
\hline Yskemess & Kel'tminskaya-1 & 4307 & 0.100 & 1.200 & 1.770 \\
\hline Yskemess & Kel'tminskaya-1 & 4386 & 0.020 & 1.250 & 1.820 \\
\hline Yskemess & Kel'tminskaya-1 & 4387 & 0.000 & 0.200 & 2.490 \\
\hline Yskemess & Kel'tminskaya-1 & 4462 & 0.040 & 0.260 & 0.600 \\
\hline Yskemess & Kel'tminskaya-1 & 4502 & 0.010 & 0.220 & 0.770 \\
\hline Yskemess & Kel'tminskaya-1 & 4553.5 & 0.040 & 0.280 & 0.750 \\
\hline Yskemess & Kel'tminskaya-1 & 4640 & 0.040 & 0.270 & 0.440 \\
\hline Yskemess & Kel'tminskaya-1 & 4640.5 & 0.000 & 0.490 & 2.570 \\
\hline Yskemess & Kel'tminskaya-1 & 4852.5 & 0.010 & 0.490 & 1.090 \\
\hline Yskemess & Kel'tminskaya-1 & 4901 & 0.010 & 0.370 & 0.990 \\
\hline Bitter Springs & Bitter Springs & & 0.010 & 0.980 & 5.860 \\
\hline Bitter Springs & Bitter Springs & & 0.025 & 0.978 & 5.400 \\
\hline Bitter Springs & Bitter Springs & & 0.040 & 1.150 & 5.870 \\
\hline \multicolumn{2}{|c|}{ Brighton L.S./Why Brighton L.S./Whya } & 1150.2 & 0.000 & 0.430 & 2.170 \\
\hline \multicolumn{2}{|c|}{ Brighton L.S./Why Brighton L.S./Whya } & 1160.3 & 0.000 & 0.660 & 1.940 \\
\hline \multicolumn{2}{|c|}{ Brighton L.S./Why Brighton L.S./Whya } & 1170 & 0.030 & 1.060 & 1.830 \\
\hline \multicolumn{2}{|c|}{ Brighton L.S./Why Brighton L.S./Whya } & 1180 & 0.050 & 1.310 & 1.840 \\
\hline Sturt tillite & Sturt tillite & 690.2 & 0.010 & 3.190 & 3.670 \\
\hline Sturt tillite & Sturt tillite & 700.1 & 0.010 & 0.300 & 0.630 \\
\hline Sturt tillite & Sturt tillite & 710.7 & 0.050 & 2.070 & 2.380 \\
\hline Sturt tillite & Sturt tillite & 720.1 & 0.010 & 2.830 & 3.190 \\
\hline Sturt tillite & Sturt tillite & 730.3 & 0.020 & 1.120 & 1.630 \\
\hline Sturt tillite & Sturt tillite & 739.3 & 0.060 & 0.240 & 1.430 \\
\hline Sturt tillite & Sturt tillite & 752.2 & 0.010 & 1.260 & 1.910 \\
\hline Sturt tillite & Sturt tillite & 770.2 & 0.010 & 0.740 & 1.030 \\
\hline Sturt tillite & Sturt tillite & 779.7 & 0.000 & 0.030 & 0.620 \\
\hline Sturt tillite & Sturt tillite & 790 & 0.010 & 0.660 & 1.000 \\
\hline
\end{tabular}




\begin{tabular}{|c|c|c|c|c|c|}
\hline Sturt tillite & Sturt tillite & 800.6 & 0.270 & 0.690 & 1.480 \\
\hline Sturt tillite & Sturt tillite & 810 & 0.000 & 0.950 & 1.200 \\
\hline Sturt tillite & Sturt tillite & 820.2 & 0.000 & 1.040 & 1.230 \\
\hline Sturt tillite & Sturt tillite & 830 & 0.010 & 1.450 & 1.820 \\
\hline Sturt tillite & Sturt tillite & 840 & 0.000 & 0.900 & 1.160 \\
\hline Sturt tillite & Sturt tillite & 849.8 & 0.050 & 2.100 & 2.450 \\
\hline Sturt tillite & Sturt tillite & 860.2 & 0.000 & 2.740 & 3.130 \\
\hline Sturt tillite & Sturt tillite & 870.5 & 0.000 & 2.880 & 3.010 \\
\hline Sturt tillite & Sturt tillite & 880.1 & 0.070 & 1.010 & 1.400 \\
\hline Sturt Tillite & Sturt Tillite & 1380.2 & 0.130 & 0.800 & 1.950 \\
\hline Sturt Tillite & Sturt Tillite & 1380.2 & 0.190 & 0.940 & 1.810 \\
\hline Sturt Tillite & Sturt Tillite & 1389.9 & 0.100 & 0.890 & 1.650 \\
\hline Sturt Tillite & Sturt Tillite & 1400.3 & 0.030 & 0.750 & 0.950 \\
\hline Sturt Tillite & Sturt Tillite & 1410 & 0.100 & 0.940 & 1.890 \\
\hline Sturt Tillite & Sturt Tillite & 1420 & 0.070 & 0.880 & 2.050 \\
\hline Sturt Tillite & Sturt Tillite & 1430.2 & 0.100 & 1.070 & 2.200 \\
\hline Sturt Tillite & Sturt Tillite & 1440.2 & 0.010 & 0.750 & 2.200 \\
\hline Sturt Tillite & Sturt Tillite & 1450 & 0.020 & 0.630 & 1.810 \\
\hline Tapley Hill & Tapley Hill & 495 & 0.010 & 0.690 & 3.210 \\
\hline Tapley Hill & Tapley Hill & 500 & 0.130 & 1.090 & 3.060 \\
\hline Tapley Hill & Tapley Hill & 505 & 0.090 & 1.320 & 3.050 \\
\hline Tapley Hill & Tapley Hill & 510 & 0.090 & 0.500 & 0.990 \\
\hline Tapley Hill & Tapley Hill & 515.2 & 0.100 & 0.960 & 3.160 \\
\hline Tapley Hill & Tapley Hill & 520.7 & 0.160 & 1.580 & 3.880 \\
\hline Tapley Hill & Tapley Hill & 525.1 & 0.730 & 1.320 & 3.030 \\
\hline Tapley Hill & Tapley Hill & 530.6 & 0.200 & 2.140 & 3.730 \\
\hline Tapley Hill & Tapley Hill & 535.2 & 0.070 & 1.010 & 3.370 \\
\hline Tapley Hill & Tapley Hill & 539.9 & 0.210 & 1.250 & 3.540 \\
\hline Tapley Hill & Tapley Hill & 545.6 & 0.380 & 1.250 & 3.780 \\
\hline Tapley Hill & Tapley Hill & 550 & 0.330 & 1.720 & 4.310 \\
\hline Tapley Hill & Tapley Hill & 555.1 & 0.410 & 1.140 & 3.000 \\
\hline Tapley Hill & Tapley Hill & 560.8 & 0.480 & 2.730 & 4.060 \\
\hline Tapley Hill & Tapley Hill & 565.3 & 0.280 & 1.440 & 3.210 \\
\hline Tapley Hill & Tapley Hill & 570.5 & 0.220 & 2.070 & 2.330 \\
\hline Tapley Hill & Tapley Hill & 575 & 0.110 & 1.040 & 2.660 \\
\hline Tapley Hill & Tapley Hill & 581 & 0.240 & 2.920 & 3.950 \\
\hline Tapley Hill & Tapley Hill & 585.2 & 0.020 & 1.000 & 3.480 \\
\hline Tapley Hill & Tapley Hill & 589.5 & 0.620 & 1.980 & 4.270 \\
\hline Tapley Hill & Tapley Hill & 595.2 & 0.010 & 0.920 & 3.680 \\
\hline Tapley Hill & Tapley Hill & 600.8 & 0.900 & 1.690 & 3.070 \\
\hline Tapley Hill & Tapley Hill & 604.9 & 0.070 & 1.210 & 4.120 \\
\hline
\end{tabular}




\begin{tabular}{|c|c|c|c|c|c|}
\hline Tapley Hill & Tapley Hill & 610.2 & 0.720 & 2.160 & 2.290 \\
\hline Tapley Hill & Tapley Hill & 615 & 0.070 & 0.580 & 1.120 \\
\hline Tapley Hill & Tapley Hill & 625.4 & 0.010 & 1.080 & 4.210 \\
\hline Tapley Hill & Tapley Hill & 635.2 & 0.150 & 1.190 & 2.440 \\
\hline Tapley Hill & Tapley Hill & 645.8 & 0.140 & 1.170 & 3.620 \\
\hline Tapley Hill & Tapley Hill & 655.1 & 0.100 & 1.020 & 3.650 \\
\hline Tapley Hill & Tapley Hill & 666 & 0.010 & 1.230 & 1.640 \\
\hline Tapley Hill & Tapley Hill & 675 & 0.080 & 1.290 & 3.610 \\
\hline Tapley Hill & Tapley Hill & 685.2 & 0.080 & 1.210 & 3.930 \\
\hline Tapley Hill & Tapley Hill & 695.3 & 0.060 & 1.310 & 3.880 \\
\hline Tapley Hill & Tapley Hill & 705.7 & 0.080 & 1.340 & 3.980 \\
\hline Tapley Hill & Tapley Hill & 715.2 & 0.050 & 1.370 & 4.340 \\
\hline Tapley Hill & Tapley Hill & 725.4 & 0.060 & 1.290 & 3.980 \\
\hline Tapley Hill & Tapley Hill & 735.3 & 0.200 & 1.440 & 4.220 \\
\hline Tapley Hill & Tapley Hill & 744.9 & 0.040 & 1.110 & 3.650 \\
\hline Tapley Hill & Tapley Hill & 755.6 & 0.170 & 1.210 & 3.830 \\
\hline Tapley Hill & Tapley Hill & 765.6 & 0.430 & 1.880 & 4.070 \\
\hline Tapley Hill & Tapley Hill & 775.6 & 0.080 & 1.350 & 4.050 \\
\hline Tapley Hill & Tapley Hill & 785.7 & 0.120 & 1.810 & 3.810 \\
\hline Tapley Hill & Tapley Hill & 796.5 & 0.700 & 1.920 & 4.200 \\
\hline Tapley Hill & Tapley Hill & 805.4 & 0.730 & 1.900 & 4.130 \\
\hline Tapley Hill & Tapley Hill & 815.3 & 0.770 & 1.760 & 3.530 \\
\hline Tapley Hill & Tapley Hill & 825.3 & 1.350 & 2.260 & 4.190 \\
\hline Tapley Hill & Tapley Hill & 837 & 1.390 & 2.230 & 4.250 \\
\hline Tapley Hill & Tapley Hill & 845.6 & 1.070 & 2.250 & 4.650 \\
\hline Tapley Hill & Tapley Hill & 855.7 & 1.380 & 2.130 & 4.270 \\
\hline Tapley Hill & Tapley Hill & 865.5 & 1.120 & 1.950 & 3.900 \\
\hline Tapley Hill & Tapley Hill & 874.9 & 1.060 & 2.420 & 3.930 \\
\hline Tapley Hill & Tapley Hill & 885 & 0.880 & 2.440 & 3.700 \\
\hline Tapley Hill & Tapley Hill & 895.4 & 1.710 & 2.720 & 4.700 \\
\hline Tapley Hill & Tapley Hill & 905.5 & 1.140 & 3.030 & 3.820 \\
\hline Tapley Hill & Tapley Hill & 915.4 & 0.660 & 1.680 & 4.030 \\
\hline Tapley Hill & Tapley Hill & 1190 & 0.300 & 1.000 & 3.180 \\
\hline Tapley Hill & Tapley Hill & 1200 & 0.310 & 1.300 & 2.940 \\
\hline Tapley Hill & Tapley Hill & 1210 & 0.640 & 2.060 & 4.040 \\
\hline Tapley Hill & Tapley Hill & 1220.2 & 0.200 & 1.380 & 3.760 \\
\hline Tapley Hill & Tapley Hill & 1230.4 & 0.320 & 1.400 & 4.320 \\
\hline Tapley Hill & Tapley Hill & 1240 & 0.540 & 1.480 & 4.480 \\
\hline Tapley Hill & Tapley Hill & 1250 & 0.180 & 1.470 & 4.120 \\
\hline Tapley Hill & Tapley Hill & 1260 & 0.760 & 1.980 & 4.280 \\
\hline Tapley Hill & Tapley Hill & 1270 & 0.350 & 1.470 & 4.310 \\
\hline
\end{tabular}




\begin{tabular}{|c|c|c|c|c|c|}
\hline Tapley Hill & Tapley Hill & 1280.3 & 0.670 & 1.690 & 4.600 \\
\hline Tapley Hill & Tapley Hill & 1290.3 & 0.410 & 1.560 & 4.110 \\
\hline Tapley Hill & Tapley Hill & 1300 & 0.740 & 2.010 & 3.920 \\
\hline Tapley Hill & Tapley Hill & 1310.2 & 0.710 & 1.760 & 4.360 \\
\hline Tapley Hill & Tapley Hill & 1320.2 & 0.640 & 1.500 & 4.210 \\
\hline Tapley Hill & Tapley Hill & 1330 & 0.940 & 1.650 & 3.050 \\
\hline Tapley Hill & Tapley Hill & 1340 & 1.310 & 2.010 & 4.060 \\
\hline Tapley Hill & Tapley Hill & 1350 & 1.650 & 2.470 & 4.580 \\
\hline Tapley Hill & Tapley Hill & 1360 & 1.120 & 3.080 & 3.890 \\
\hline Tapley Hill & Tapley Hill & 1369.9 & 1.080 & 2.380 & 3.160 \\
\hline \multicolumn{3}{|c|}{ Elbobreen Fm, Spi Elbobreen Fm, Spitsbergen } & 0.007 & 1.162 & 5.065 \\
\hline \multicolumn{3}{|c|}{ Elbobreen Fm, Spi Elbobreen Fm, Spitsbergen } & 0.062 & 1.140 & 3.599 \\
\hline \multicolumn{3}{|c|}{ Elbobreen Fm, Spi Elbobreen Fm, Spitsbergen } & 0.454 & 1.538 & 3.688 \\
\hline \multicolumn{3}{|c|}{ Elbobreen Fm, Spi Elbobreen Fm, Spitsbergen } & 0.058 & 1.975 & 4.650 \\
\hline \multicolumn{3}{|c|}{ Elbobreen Fm, Spi Elbobreen Fm, Spitsbergen } & 0.107 & 1.489 & 3.258 \\
\hline \multicolumn{3}{|c|}{ Elbobreen Fm, Spi Elbobreen Fm, Spitsbergen } & 0.002 & 1.877 & 3.901 \\
\hline \multicolumn{3}{|c|}{ Elbobreen Fm, Spi Elbobreen Fm, Spitsbergen } & 0.032 & 2.351 & 4.066 \\
\hline \multicolumn{3}{|c|}{ Elbobreen Fm, Spi Elbobreen Fm, Spitsbergen } & 0.011 & 2.217 & 3.724 \\
\hline \multicolumn{3}{|c|}{ Elbobreen Fm, Spi Elbobreen Fm, Spitsbergen } & 0.707 & 2.231 & 3.387 \\
\hline Dolomite Series & Dolomite Series & 3700 & 0.510 & 0.700 & 0.950 \\
\hline Dolomite Series & Dolomite Series & 3800 & 0.330 & 0.580 & 0.800 \\
\hline Dolomite Series & Dolomite Series & 4000 & 0.140 & 1.650 & 11.300 \\
\hline Dolomite Series & Dolomite Series & 4000 & 0.100 & 1.420 & 4.580 \\
\hline Dolomite Series & Dolomite Series & 4050 & 0.430 & 3.190 & 10.100 \\
\hline Dolomite Series & Dolomite Series & 4050 & 0.030 & 2.740 & 8.030 \\
\hline Dolomite Series & Dolomite Series & 4060 & 0.020 & 1.580 & 5.140 \\
\hline Dolomite Series & Dolomite Series & 4070 & 0.280 & 3.370 & 9.220 \\
\hline Dolomite Series & Dolomite Series & 4080 & 0.170 & 1.780 & 6.400 \\
\hline Dolomite Series & Dolomite Series & 4080 & 0.490 & 3.110 & 9.780 \\
\hline Dolomite Series & Dolomite Series & 4090 & 0.240 & 1.950 & 7.560 \\
\hline Dolomite Series & Dolomite Series & 4090 & 0.190 & 1.860 & 6.840 \\
\hline Dolomite Series & Dolomite Series & 4100 & 0.190 & 2.440 & 8.000 \\
\hline Dolomite Series & Dolomite Series & 4100 & 0.140 & 2.160 & 7.020 \\
\hline Multicolor Series & Multicolor Series & 2550 & 0.000 & 0.480 & 4.200 \\
\hline Multicolor Series & Multicolor Series & 2600 & 0.000 & 0.120 & 1.870 \\
\hline Multicolor Series & Multicolor Series & 2900 & 0.010 & 1.020 & 1.230 \\
\hline Quartzite Series & Quartzite Series & 1000 & 0.000 & 0.630 & 4.410 \\
\hline Quartzite Series & Quartzite Series & 1150 & 0.000 & 0.490 & 3.170 \\
\hline Quartzite Series & Quartzite Series & 1250 & 0.110 & 0.900 & 4.280 \\
\hline Quartzite Series & Quartzite Series & 1300 & 0.010 & 0.560 & 2.340 \\
\hline Quartzite Series & Quartzite Series & 1400 & 0.010 & 0.420 & 2.880 \\
\hline
\end{tabular}




\begin{tabular}{|c|c|c|c|c|c|}
\hline Quartzite Series & Quartzite Series & 1400 & 0.000 & 0.600 & 3.300 \\
\hline Quartzite Series & Quartzite Series & 1500 & 0.010 & 0.730 & 2.090 \\
\hline Quartzite Series & Quartzite Series & 1600 & 0.000 & 0.720 & 5.250 \\
\hline Quartzite Series & Quartzite Series & 1600 & 0.040 & 0.410 & 2.180 \\
\hline Quartzite Series & Quartzite Series & 1700 & 0.000 & 0.630 & 3.200 \\
\hline Quartzite Series & Quartzite Series & 2000 & 0.000 & 0.590 & 3.360 \\
\hline \multicolumn{3}{|c|}{ Tillite Group East (Tillite Group East Greenland } & 0.009 & 0.168 & 2.746 \\
\hline \multicolumn{3}{|c|}{ Tillite Group East (Tillite Group East Greenland } & 0.005 & 0.196 & 1.932 \\
\hline \multicolumn{3}{|c|}{ Tillite Group East (Tillite Group East Greenland } & 0.041 & 1.397 & 8.167 \\
\hline \multicolumn{3}{|c|}{ Tillite Group East (Tillite Group East Greenland } & 0.078 & 1.670 & 8.072 \\
\hline \multicolumn{3}{|c|}{ Tillite Group East (Tillite Group East Greenland } & 0.331 & 1.805 & 8.233 \\
\hline \multicolumn{3}{|c|}{ Tillite Group East (Tillite Group East Greenland } & 0.057 & 2.115 & 8.528 \\
\hline \multicolumn{3}{|c|}{ Tillite Group East (Tillite Group East Greenland } & 1.016 & 2.041 & 7.653 \\
\hline \multicolumn{3}{|c|}{ Tillite Group East (Tillite Group East Greenland } & 0.295 & 2.471 & 9.189 \\
\hline \multicolumn{3}{|c|}{ Tillite Group East (Tillite Group East Greenland } & 0.515 & 2.174 & 7.653 \\
\hline \multicolumn{3}{|c|}{ Tillite Group East (Tillite Group East Greenland } & 0.033 & 2.468 & 8.305 \\
\hline \multicolumn{3}{|c|}{ Tillite Group East (Tillite Group East Greenland } & 0.180 & 2.516 & 8.433 \\
\hline \multicolumn{3}{|c|}{ Tillite Group East (Tillite Group East Greenland } & 0.682 & 2.968 & 9.469 \\
\hline \multicolumn{3}{|c|}{ Tillite Group East (Tillite Group East Greenland } & 1.360 & 2.617 & 7.118 \\
\hline \multicolumn{3}{|c|}{ Tillite Group East (Tillite Group East Greenland } & 0.224 & 3.014 & 8.186 \\
\hline \multicolumn{3}{|c|}{ Tillite Group East (Tillite Group East Greenland } & 1.381 & 3.383 & 6.431 \\
\hline \multicolumn{3}{|c|}{ Tillite Group East (Tillite Group East Greenland } & 0.114 & 3.578 & 6.783 \\
\hline Arena & GR12 & 64.5 & 0.610 & 1.830 & 8.168 \\
\hline Arena & GR12 & 78 & 0.145 & 1.126 & 7.146 \\
\hline Arena & GR12 & 71 & 0.725 & 1.926 & 7.260 \\
\hline Arena & GR12 & 15.9 & 0.826 & 2.111 & 7.704 \\
\hline Arena & GR12 & 10.5 & 1.098 & 2.059 & 7.337 \\
\hline Arena & GR12 & 8.5 & 1.566 & 2.418 & 6.685 \\
\hline Arena & GR12 & 3.3 & 0.428 & 2.581 & 3.392 \\
\hline Reefal Assembla & E1002 & 44 & 0.002 & 3.481 & 5.080 \\
\hline Reefal Assembla & E E1002 & 69.5 & 0.002 & 0.864 & 3.260 \\
\hline Reefal Assembla & E E1002 & 470.4 & 0.005 & 2.414 & 2.970 \\
\hline Reefal Assembla & EE1002 & 472.8 & 0.003 & 1.011 & 2.780 \\
\hline Reefal Assembla & EF1016 & 110.4 & 0.030 & 0.549 & 1.500 \\
\hline Reefal Assembla & EF1016 & 128.5 & 0.004 & 0.676 & 2.450 \\
\hline Reefal Assembla & EF1016 & 133 & 0.005 & 0.823 & 3.210 \\
\hline Reefal Assembla & EF1016 & 133.5 & 0.010 & 0.716 & 3.170 \\
\hline Reefal Assembla & EF1016 & 140.5 & 0.027 & 0.566 & 3.270 \\
\hline Reefal Assembla & EF1016 & 141 & 0.010 & 0.639 & 3.290 \\
\hline Reefal Assembla & EF1016 & 142 & 0.009 & 0.739 & 3.420 \\
\hline Reefal Assembla & EF1016 & 143 & 0.008 & 0.631 & 3.390 \\
\hline
\end{tabular}




\begin{tabular}{|c|c|c|c|c|}
\hline Reefal Assemblag $\epsilon$ F1016 & 144.5 & 0.021 & 0.523 & 3.290 \\
\hline Reefal Assemblag $\in$ F1016 & 150 & 0.004 & 0.479 & 2.870 \\
\hline Reefal Assemblag $\in$ F1016 & 157 & 0.005 & 1.035 & 3.790 \\
\hline Reefal Assemblag $\in$ F1016 & 164 & 0.035 & 0.461 & 3.390 \\
\hline Reefal Assemblag $\in$ F1016 & 170 & 0.001 & 0.520 & 3.260 \\
\hline Reefal Assemblag $\in$ F1016 & 251.8 & 0.060 & 0.846 & 1.660 \\
\hline Reefal Assemblag $\in$ F1016 & 254.5 & 0.009 & 0.606 & 0.690 \\
\hline Reefal Assemblag $\in$ F1016 & 290.8 & 0.002 & 0.126 & 1.100 \\
\hline Reefal Assemblag $\in$ F1016 & 292.3 & 0.008 & 0.179 & 1.200 \\
\hline Reefal Assemblag $\in$ F1016 & 293.8 & 0.000 & 0.101 & 1.590 \\
\hline Reefal Assemblag $\in$ F1018 & 40.5 & 0.000 & 0.253 & 1.670 \\
\hline Reefal Assemblag $\in$ F1018 & 50 & 0.009 & 0.513 & 3.170 \\
\hline Reefal Assemblag $\in$ F1018 & 54.5 & 0.000 & 0.349 & 2.500 \\
\hline Reefal Assemblag $\in$ F1018 & 64 & 0.000 & 0.954 & 4.200 \\
\hline Reefal Assemblag $\in$ F1018 & 70 & 0.000 & 0.981 & 3.780 \\
\hline Reefal AssemblagєF1018 & 72 & 0.000 & 0.863 & 3.650 \\
\hline Reefal Assemblag $\in$ F1018 & 81 & 0.000 & 1.816 & 4.430 \\
\hline Reefal Assemblag $\in$ F1018 & 87 & 0.000 & 0.617 & 2.730 \\
\hline Reefal Assemblag $\in$ F1018 & 106.5 & 0.000 & 1.006 & 3.600 \\
\hline Reefal Assemblag $\in$ F1018 & 115 & 0.002 & 0.671 & 1.570 \\
\hline Reefal Assemblag $\in$ F1018 & 127 & 0.000 & 0.694 & 3.030 \\
\hline Reefal Assemblag $\in$ F1018 & 141 & 0.000 & 1.288 & 6.020 \\
\hline Reefal Assemblag $\in$ F1018 & 147 & 0.000 & 1.262 & 4.130 \\
\hline Reefal Assemblag $\in$ F1018 & 162 & 0.001 & 1.570 & 3.230 \\
\hline Reefal Assemblag $\in$ F1018 & 170 & 0.000 & 0.774 & 3.320 \\
\hline Reefal Assemblag $\in$ F1018 & 178 & 0.000 & 0.847 & 2.340 \\
\hline Reefal Assemblag $\in$ F1018 & 185 & 0.000 & 0.753 & 3.290 \\
\hline Reefal Assemblag $\in$ F1018 & 195 & 0.000 & 0.801 & 3.820 \\
\hline Reefal Assemblag $\in$ F1018 & 205 & 0.000 & 0.405 & 2.470 \\
\hline Reefal Assemblag $\in$ F1018 & 215 & 0.000 & 3.213 & 6.820 \\
\hline Reefal Assemblag $\in$ F1018 & 225 & 0.000 & 0.489 & 2.720 \\
\hline Reefal Assemblag $\in$ F1018 & 235 & 0.000 & 0.452 & 1.550 \\
\hline Reefal Assemblag $\in$ F1018 & 282 & 0.124 & 3.286 & 4.710 \\
\hline Reefal Assemblag€F1018 & 289 & 0.010 & 0.315 & 1.790 \\
\hline Reefal Assemblag $\in$ F1018 & 294 & 0.002 & 1.622 & 2.940 \\
\hline Reefal Assemblag $€$ F1018 & 454 & 0.000 & 4.820 & 6.280 \\
\hline Reefal Assemblag $\in$ F1018 & 550.2 & 0.589 & 1.065 & 1.440 \\
\hline Reefal Assemblag $\in$ F1018 & 602 & 0.005 & 0.537 & 1.320 \\
\hline Chandindu & 4.3 & 0.002 & 0.685 & 2.960 \\
\hline Chandindu & 21.5 & 0.003 & 0.592 & 2.420 \\
\hline Chandindu & 31 & 0.005 & 0.578 & 2.800 \\
\hline
\end{tabular}




\begin{tabular}{|c|c|c|c|c|}
\hline Chandindu & 90 & 0.003 & 0.789 & 4.030 \\
\hline Chandindu & 262.5 & 0.002 & 0.565 & 4.830 \\
\hline Chandindu & 290 & 0.002 & 1.166 & 5.150 \\
\hline Chandindu & 302 & 0.000 & 0.309 & 1.890 \\
\hline Reefal Assemblag $\in$ F1157 & 20.5 & 0.002 & 0.264 & 2.160 \\
\hline Reefal Assemblag $\in$ F1157 & 29 & 0.002 & 0.770 & 2.920 \\
\hline Reefal Assemblag $\in$ F1157 & 41 & 0.001 & 0.917 & 2.900 \\
\hline Reefal Assemblag $\in$ F1157 & 46.8 & 0.004 & 0.638 & 2.710 \\
\hline Reefal Assemblag $\in$ F1157 & 54.2 & 0.067 & 1.080 & 2.940 \\
\hline Reefal Assemblag $\in$ F1157 & 64 & 0.010 & 0.770 & 2.480 \\
\hline Reefal Assemblag $\in$ F1157 & 72.5 & 0.002 & 0.365 & 2.590 \\
\hline Reefal Assemblag $\in$ F1157 & 89.5 & 0.006 & 0.709 & 1.520 \\
\hline Reefal Assemblag $€$ F1157 & 96 & 0.004 & 0.627 & 1.890 \\
\hline Reefal Assemblag $\in$ F1157 & 108 & 0.003 & 0.500 & 2.230 \\
\hline Reefal Assemblag $\in$ F1157 & 118 & 0.010 & 0.558 & 2.240 \\
\hline Reefal Assemblag $\in$ F1157 & 130.5 & 0.002 & 1.700 & 2.240 \\
\hline Reefal Assemblag $\in$ F1157 & 136 & 0.000 & 0.658 & 1.830 \\
\hline Reefal Assemblag $\in$ F1157 & 150.4 & 0.027 & 0.267 & 0.550 \\
\hline Reefal Assemblag $\in$ F1157 & 160.3 & 0.007 & 0.707 & 0.790 \\
\hline Reefal Assemblag $€$ F1157 & 172 & 0.001 & 0.566 & 1.560 \\
\hline Reefal Assemblag $\in$ F1157 & 202 & 0.002 & 0.591 & 2.230 \\
\hline Reefal Assemblag $\in$ F1157 & 207 & 0.001 & 0.327 & 1.790 \\
\hline Reefal Assemblag $\in$ F1157 & 213.5 & 0.000 & 2.040 & 4.000 \\
\hline Reefal Assemblag $\in$ F1157 & 223.3 & 0.001 & 0.504 & 2.420 \\
\hline Reefal Assemblag $\in$ F1157 & 226.2 & 0.000 & 0.467 & 2.520 \\
\hline Reefal Assemblag $\in$ F1157 & 245 & 0.001 & 0.646 & 2.220 \\
\hline Reefal Assemblag $\in$ F1157 & 252 & 0.001 & 1.881 & 2.930 \\
\hline Reefal Assemblag $\in$ F1157 & 260 & 0.000 & 1.622 & 2.510 \\
\hline Reefal Assemblag $€$ F1157 & 266 & 0.000 & 0.457 & 2.190 \\
\hline Reefal Assemblag $\in$ F1157 & 272 & 0.000 & 0.317 & 1.240 \\
\hline Reefal Assemblag $\in$ F1157 & 280 & N.D. & 0.220 & 0.740 \\
\hline Reefal Assemblag $\in$ F1157 & 300 & N.D. & 0.576 & 1.300 \\
\hline Reefal Assemblag $€$ F1157 & 326 & N.D. & 0.512 & 3.820 \\
\hline Reefal Assemblag $€$ F833 & 0 & 0.004 & 0.781 & 2.330 \\
\hline Reefal Assemblag $€$ F833 & 67 & 0.025 & 0.905 & 1.120 \\
\hline Reefal Assemblag€ F833 & 69 & 0.015 & 0.344 & 1.080 \\
\hline Reefal Assemblag $€$ F833 & 70.6 & 0.001 & 0.400 & 0.900 \\
\hline Reefal Assemblag€F833 & 74.5 & 0.014 & 1.123 & 1.660 \\
\hline Reefal Assemblag $€$ F833 & 91 & 0.022 & 1.161 & 1.440 \\
\hline Reefal Assemblag€F833 & 93.1 & 0.015 & 1.133 & 1.650 \\
\hline Reefal Assemblag€F833 & 246 & 0.011 & 0.099 & 0.130 \\
\hline
\end{tabular}


Reefal Assemblag $€$ F833

Reefal Assemblag€F833

Reefal Assemblag $€$ F833

Reefal Assemblag $€$ F833

Reefal Assemblag€F833

Reefal Assemblag $€$ F833

Reefal Assemblag $\epsilon$ F835

Reefal Assemblag $\epsilon$ F835

Reefal Assemblag $€$ F835

Reefal Assemblag $\epsilon$ F835

Reefal Assemblag $\epsilon 835$

Reefal Assemblag $€$ F835

Reefal Assemblag $\epsilon$ F835

Reefal Assemblag $\epsilon$ F835

Reefal Assemblag $€$ F835

Reefal Assemblag $€$ F835

Reefal Assemblag $\epsilon 835$

Reefal Assemblag $€$ F835

Reefal Assemblag $€$ F835

Reefal Assemblag $\epsilon$ F835

Reefal Assemblag $€$ F835

Reefal Assemblagє F835

Reefal Assemblag $\epsilon$ F835

Reefal Assemblag $€$ F835

Reefal Assemblag $€$ F835

Reefal Assemblagє F835

Reefal Assemblag $€$ F835

Reefal Assemblagє F835

Reefal Assemblage F845

Reefal Assemblag $\epsilon 845$

Reefal Assemblag $€$ F845

Reefal Assemblag $\epsilon 845$

Reefal Assemblag $\epsilon 845$

Reefal Assemblag $\epsilon 845$

Reefal Assemblag $€$ F845

Reefal Assemblag $\epsilon 845$

Reefal Assemblag $\epsilon 845$

Reefal Assemblag $€$ F845

Reefal Assemblag $\epsilon$ F845

Reefal Assemblag $\epsilon$ F845

Reefal AssemblagєF845

$\begin{array}{cccc}249.5 & 0.007 & 0.596 & 0.860 \\ 255 & 0.006 & 0.842 & 1.040 \\ 325 & 0.016 & 0.255 & 0.310 \\ 389.5 & 0.019 & 0.396 & 0.500 \\ 391 & 0.003 & 1.084 & 1.770 \\ 396 & 0.016 & 0.875 & 1.180 \\ 6.5 & 0.020 & 1.167 & 4.088 \\ 10.5 & 0.001 & 1.365 & 3.129 \\ 16.5 & 0.005 & 1.019 & 4.169 \\ 25 & 0.003 & 1.301 & 3.194 \\ 30.3 & 0.009 & 1.490 & 3.722 \\ 36.8 & 0.026 & 0.884 & 2.882 \\ 39 & 0.003 & 1.336 & 3.405 \\ 41 & 0.008 & 1.238 & 2.968 \\ 43 & 0.002 & 1.410 & 3.424 \\ 45.8 & 0.004 & 1.681 & 3.464 \\ 48 & 0.028 & 1.645 & 3.232 \\ 52 & 0.026 & 1.006 & 1.674 \\ 54 & 0.037 & 0.901 & 3.392 \\ 56 & 0.075 & 1.012 & 3.278 \\ 58.5 & 0.000 & 1.084 & 2.955 \\ 61 & 0.006 & 1.227 & 2.454 \\ 64 & 0.012 & 1.629 & 3.688 \\ 66 & 0.001 & 1.555 & 3.164 \\ 68 & 0.000 & 1.537 & 3.141 \\ 70 & 0.018 & 0.856 & 2.246 \\ 71 & 0.005 & 0.958 & 2.369 \\ 72.8 & 0.001 & 0.680 & 1.738 \\ 3 & 0.270 & 2.966 & 3.910 \\ 7.1 & 0.063 & 2.178 & 3.130 \\ 12 & 0.133 & 0.655 & 1.340 \\ 16.5 & 0.210 & 0.504 & 1.010 \\ 21 & 0.097 & 0.287 & 1.020 \\ 26 & 1.185 & 1.624 & 2.210 \\ 31 & 0.290 & 0.558 & 0.560 \\ 36 & 0.064 & 0.341 & 1.060 \\ 42 & 0.132 & 1.888 & 2.710 \\ 64 & 0.051 & 1.218 & 2.460 \\ 56 & 0.503 & 0.772 & 1.420 \\ 515 & 0.162 & 0.743 & 1.390 \\ 35 & 0.013 & 1.032 & 1.800\end{array}$




\begin{tabular}{|c|c|c|c|c|c|}
\hline \multicolumn{2}{|c|}{ Reefal Assemblag $€$ F845 } & 85 & 0.060 & 1.254 & 2.570 \\
\hline \multicolumn{2}{|c|}{ Reefal Assemblag $\in$ F845 } & 94 & 0.080 & 0.759 & 2.250 \\
\hline \multicolumn{2}{|c|}{ Reefal Assemblag $€$ F845 } & 98 & 0.080 & 0.400 & 2.000 \\
\hline \multicolumn{2}{|c|}{ Reefal Assemblag $\epsilon$ F845 } & 106 & 0.019 & 0.686 & 1.520 \\
\hline \multicolumn{2}{|c|}{ Reefal Assemblag $\epsilon$ F845 } & 111 & 0.080 & 0.704 & 1.520 \\
\hline \multicolumn{2}{|c|}{ Reefal Assemblag $\epsilon 845$} & 117 & 0.010 & 1.030 & 1.710 \\
\hline \multicolumn{2}{|c|}{ Reefal Assemblag $€$ F845 } & 139 & 0.020 & 1.880 & 2.280 \\
\hline \multicolumn{2}{|c|}{ Reefal Assemblag $\epsilon 845$} & 142 & 0.019 & 0.818 & 1.110 \\
\hline \multicolumn{2}{|c|}{ Reefal Assemblag $€$ F845 } & 171 & 0.023 & 0.264 & 0.540 \\
\hline \multicolumn{2}{|c|}{ Reefal Assemblag $€$ F845 } & 184 & 0.157 & 0.429 & 0.790 \\
\hline \multicolumn{2}{|c|}{ Reefal Assemblag $\in$ F845 } & 205.2 & 0.440 & 1.326 & 1.880 \\
\hline \multicolumn{2}{|c|}{ Reefal Assemblag $€$ F845 } & 281.5 & 0.025 & 0.481 & 0.610 \\
\hline \multicolumn{2}{|c|}{ Reefal Assemblag $€$ F845 } & 284.5 & 0.026 & 0.251 & 0.410 \\
\hline \multicolumn{2}{|c|}{ Reefal Assemblag $\epsilon$ F845 } & 327.5 & 0.010 & 3.240 & 3.920 \\
\hline \multicolumn{2}{|c|}{ Reefal Assemblag $€$ F845 } & 330 & 0.011 & 2.242 & 2.900 \\
\hline \multicolumn{2}{|c|}{ Reefal Assemblag $\epsilon$ F845 } & 331 & 0.002 & 3.617 & 4.520 \\
\hline \multicolumn{2}{|c|}{ Reefal Assemblag $€$ F845 } & 332 & 0.008 & 1.949 & 2.740 \\
\hline \multicolumn{2}{|c|}{ Reefal Assemblag $€$ F845 } & 334 & 0.023 & 2.087 & 2.660 \\
\hline \multicolumn{2}{|c|}{ Reefal Assemblag $€$ F845 } & 335 & 0.015 & 1.625 & 2.240 \\
\hline \multicolumn{2}{|c|}{ Reefal Assemblag $\in$ F845 } & 336 & 0.004 & 1.725 & 2.090 \\
\hline \multicolumn{2}{|c|}{ Reefal Assemblag $€$ F845 } & 361 & 0.047 & 0.456 & 1.680 \\
\hline Chandindu & G0130 & 242.7 & 0.000 & 1.102 & 5.000 \\
\hline Chandindu & G0130 & 260 & 0.000 & 1.024 & 4.190 \\
\hline Chandindu & G0130 & 267.5 & 0.000 & 1.180 & 4.170 \\
\hline Chandindu & G0130 & 275.4 & 0.000 & 1.595 & 3.940 \\
\hline Chandindu & G0130 & 277.8 & 0.000 & 0.979 & 3.370 \\
\hline Chandindu & G0130 & 286 & 0.000 & 1.507 & 2.710 \\
\hline Chandindu & G0130 & 330.3 & 0.000 & 0.881 & 3.960 \\
\hline Chandindu & G0130 & 333 & 0.000 & 1.465 & 6.680 \\
\hline Reefal Assen & $\in \mathrm{GO} 134$ & 0 & 0.041 & 0.185 & 0.880 \\
\hline Reefal Assen & $\in \mathrm{GO} 134$ & 5.6 & 0.075 & 0.186 & 0.850 \\
\hline Reefal Assen & $\in \mathrm{GO} 134$ & 10.6 & 0.030 & 0.357 & 1.160 \\
\hline Reefal Assen & $\in \mathrm{GO} 134$ & 15.6 & 0.053 & 0.150 & 0.780 \\
\hline Reefal Assen & $\in \mathrm{GO} 134$ & 22 & 0.046 & 0.181 & 0.850 \\
\hline Reefal Assen & $\in \mathrm{GO} 134$ & 30 & 0.045 & 0.220 & 0.970 \\
\hline Reefal Assen & $\in \mathrm{GO} 134$ & 35 & 0.093 & 0.478 & 1.220 \\
\hline Reefal Assen & $\in \mathrm{GO} 134$ & 40 & 0.041 & 0.172 & 0.850 \\
\hline Reefal Assen & $\in \mathrm{GO} 134$ & 45.5 & 0.235 & 0.384 & 0.890 \\
\hline Reefal Assen & $\in \mathrm{GO} 134$ & 50 & 0.229 & 0.478 & 0.910 \\
\hline Reefal Assen & $\in \mathrm{GO} 134$ & 57.8 & 0.008 & 0.123 & 0.890 \\
\hline Reefal Assen & $\in \mathrm{GO} 134$ & 102 & 0.001 & 0.644 & 4.550 \\
\hline
\end{tabular}


Reefal Assemblag€ G0134

Reefal Assemblag€ G0134

Reefal Assemblag $€ \mathrm{GO} 134$

Reefal Assemblag€ G0134

Reefal Assemblag $€ \mathrm{GO} 134$

Reefal Assemblag $€ \mathrm{GO} 21$

Reefal Assemblag $€ \mathrm{GO} 21$

Reefal Assemblag€ GO21

Reefal Assemblag $€$ GO21

Reefal Assemblag€ GO21

Reefal Assemblag $€$ GO21

Reefal Assemblag€ GO21

Reefal Assemblag $€$ GO21

Reefal Assemblag€ GO21

Reefal Assemblag€ GO21

Reefal Assemblag€ GO21

Reefal Assemblag $€$ GO21

Reefal Assemblag€ G021

Reefal Assemblag $€ \mathrm{GO} 22$

Reefal Assemblag€ GO22

Reefal Assemblag $€ \mathrm{GO} 22$

Reefal Assemblag€ GO22

Reefal Assemblag $€ \mathrm{GO} 22$

Reefal Assemblag€ GO22

Reefal Assemblag $€ \mathrm{GO} 22$

Reefal Assemblag€ GO22

Reefal Assemblag $€ \mathrm{GO} 22$

Reefal Assemblag€ GO22

Reefal Assemblag€ GO22

Reefal Assemblag€ GO22

Reefal Assemblag $€ \mathrm{GO} 22$

Reefal Assemblag€S1103

Reefal Assemblag€ $\mathrm{S} 1103$

Reefal Assemblag€S1103

Reefal Assemblag $€ \mathrm{~S} 1103$

Reefal Assemblag€S1103

Reefal Assemblag€ $\mathrm{S} 1103$

Reefal Assemblag€S1103

Reefal Assemblag $€ \mathrm{~S} 1103$

Reefal Assemblag€S1103

Reefal Assemblag€S1103

$\begin{array}{cccc}118 & 0.023 & 0.225 & 1.170 \\ 123 & 0.004 & 0.447 & 2.670 \\ 136.2 & 0.224 & 0.370 & 1.010 \\ 148 & 0.002 & 0.247 & 1.110 \\ 156 & 0.061 & 0.166 & 0.740 \\ 28.7 & 0.010 & 0.361 & 0.480 \\ 45.9 & 0.033 & 0.694 & 0.790 \\ 46.4 & 0.016 & 0.384 & 0.600 \\ 49.5 & 0.018 & 0.869 & 1.460 \\ 52 & 0.009 & 0.491 & 0.760 \\ 52.6 & 0.010 & 0.483 & 0.650 \\ 72.9 & 0.002 & 0.918 & 5.200 \\ 75.3 & 0.000 & 0.820 & 4.530 \\ 80.5 & 0.002 & 1.441 & 4.940 \\ 84.8 & 0.001 & 0.932 & 4.750 \\ 213.2 & 0.000 & 0.346 & 1.410 \\ 215.2 & 0.002 & 0.801 & 2.210 \\ 264.4 & 0.001 & 1.155 & 2.990 \\ 133 & 0.001 & 0.516 & 3.810 \\ 163 & 0.002 & 0.327 & 1.400 \\ 225.5 & 0.003 & 0.994 & 4.960 \\ 241 & 0.001 & 0.579 & 3.230 \\ 290 & 0.001 & 1.458 & 3.110 \\ 300.7 & 0.001 & 0.380 & 2.560 \\ 329.5 & 0.003 & 0.434 & 4.040 \\ 432.7 & 0.004 & 1.050 & 4.040 \\ 475.7 & 0.001 & 0.469 & 2.240 \\ 551.7 & 0.009 & 0.520 & 2.610 \\ 577.2 & 0.003 & 1.279 & 3.480 \\ 646 & 0.001 & 1.255 & 4.250 \\ 767 & 0.004 & 0.500 & 1.800 \\ 0 & 0.003 & 3.616 & 5.050 \\ 11 & 0.002 & 0.281 & 1.150 \\ 23.5 & 0.001 & 0.443 & 1.810 \\ 28 & 0.001 & 0.488 & 2.670 \\ 32 & 0.003 & 0.871 & 2.450 \\ 46.4 & 0.025 & 0.149 & 0.620 \\ 62.3 & 0.015 & 0.405 & 1.460 \\ 67 & 0.093 & 0.327 & 0.850 \\ 93.4 & 0.010 & 0.922 & 1.610 \\ 101 & 0.255 & 0.754 & 1.110\end{array}$




\begin{tabular}{|c|c|c|c|c|c|}
\hline \multicolumn{2}{|c|}{ Reefal Assemblag $€ \mathrm{~S} 1103$} & 111 & 0.111 & 0.273 & 0.820 \\
\hline \multicolumn{2}{|c|}{ Reefal Assemblag $€ \mathrm{~S} 1103$} & 130 & 0.148 & 0.470 & 1.020 \\
\hline \multicolumn{2}{|c|}{ Reefal Assemblag€ $€ 1103$} & 137.5 & 0.067 & 0.548 & 1.250 \\
\hline \multicolumn{2}{|c|}{ Reefal Assemblag $€ \mathrm{~S} 1103$} & 143 & 0.004 & 1.189 & 2.630 \\
\hline \multicolumn{2}{|c|}{ Reefal Assemblag $€ \mathrm{~S} 1103$} & 154 & 0.122 & 0.686 & 1.290 \\
\hline \multicolumn{2}{|c|}{ Reefal Assemblag $€ \mathrm{~S} 1103$} & 164 & 0.159 & 0.457 & 1.100 \\
\hline \multicolumn{2}{|c|}{ Reefal Assemblag $€ \mathrm{~S} 1103$} & 186 & 0.296 & 0.585 & 0.860 \\
\hline \multicolumn{2}{|c|}{ Reefal Assemblag $€ \mathrm{~S} 1103$} & 197.5 & 0.020 & 3.129 & 3.530 \\
\hline \multicolumn{2}{|c|}{ Reefal Assemblag $€ \mathrm{~S} 1103$} & 209 & 0.027 & 2.826 & 3.650 \\
\hline \multicolumn{2}{|c|}{ Reefal Assemblag $€ \mathrm{~S} 1103$} & 219 & 0.046 & 2.525 & 4.270 \\
\hline \multicolumn{2}{|c|}{ Reefal Assemblag $€ \mathrm{~S} 1103$} & 250.5 & 0.087 & 2.507 & 6.020 \\
\hline \multicolumn{2}{|c|}{ Reefal Assemblag $€$ S1107 } & 0 & 0.000 & 0.364 & 1.760 \\
\hline \multicolumn{2}{|c|}{ Reefal Assemblag $\in$ S1107 } & 1 & 0.000 & 0.626 & 2.720 \\
\hline \multicolumn{2}{|c|}{ Reefal Assemblag $\epsilon$ S1107 } & 2 & 0.000 & 4.291 & 5.690 \\
\hline \multicolumn{2}{|c|}{ Reefal Assemblag $\in$ S1107 } & 2.6 & 0.000 & 1.532 & 3.450 \\
\hline \multicolumn{2}{|c|}{ Reefal Assemblag $€$ S1107 } & 4 & 0.000 & 1.647 & 4.170 \\
\hline Twitya & Stoneknife Creek & 0 & 0.520 & 1.830 & 2.550 \\
\hline Twitya & Stoneknife Creek & 60 & 1.810 & 3.910 & 8.090 \\
\hline Twitya & Stoneknife Creek & 130 & 0.020 & 2.660 & 10.690 \\
\hline Twitya & Stoneknife Creek & 180 & 0.070 & 2.250 & 7.540 \\
\hline Twitya & Stoneknife Creek & 200 & 0.020 & 2.440 & 8.540 \\
\hline Twitya & Stoneknife Creek & 240 & 0.000 & 2.220 & 8.430 \\
\hline Twitya & Stoneknife Creek & 280 & 0.070 & 2.430 & 7.910 \\
\hline Twitya & Stoneknife Creek & 330 & 0.040 & 2.300 & 7.650 \\
\hline Twitya & Stoneknife Creek & 370 & 0.080 & 2.990 & 11.120 \\
\hline Twitya & Stoneknife Creek & 400 & 0.040 & 2.660 & 9.630 \\
\hline Twitya & Stoneknife Creek & 460 & 0.030 & 2.960 & 9.690 \\
\hline Twitya & Stoneknife Creek & 490 & 0.000 & 1.010 & 3.890 \\
\hline Twitya & Stoneknife Creek & 520 & 0.000 & 2.190 & 8.890 \\
\hline Twitya & Stoneknife Creek & 550 & 0.050 & 2.630 & 8.260 \\
\hline Twitya & Stoneknife Creek & 580 & 0.070 & 2.480 & 6.850 \\
\hline Twitya & Stoneknife Creek & 610 & 0.020 & 2.480 & 6.990 \\
\hline Twitya & Stoneknife Creek & 670 & 0.100 & 3.210 & 9.010 \\
\hline Twitya & Stoneknife Creek & 700 & 0.170 & 3.840 & 10.270 \\
\hline Twitya & Stoneknife Creek & 720 & 0.110 & 3.040 & 8.240 \\
\hline Twitya & BFTW & 34.9 & 0.162 & 1.002 & 1.270 \\
\hline Twitya & BFTW & 35.6 & 0.050 & 0.930 & 1.880 \\
\hline Twitya & BFTW & 43.9 & 0.038 & 0.092 & 1.220 \\
\hline Twitya & BFTW & 48.4 & 0.001 & 1.760 & 2.280 \\
\hline Twitya & BFTW & 55.5 & 0.002 & 1.438 & 3.420 \\
\hline Twitya & BFTW & 60.9 & 0.003 & 0.643 & 1.630 \\
\hline
\end{tabular}




\begin{tabular}{|c|c|c|c|c|c|}
\hline Twitya & BFTW & 71.9 & 0.025 & 1.432 & 2.600 \\
\hline Twitya & BFTW & 82.7 & 0.004 & 1.186 & 4.520 \\
\hline Twitya & BFTW & 92.9 & 0.007 & 0.736 & 4.970 \\
\hline Twitya & BFTW & 116.6 & 0.012 & 0.916 & 6.690 \\
\hline Twitya & BFTW & 136.3 & 0.061 & 0.473 & 6.610 \\
\hline Twitya & BFTW & 157.5 & 0.005 & 0.791 & 4.590 \\
\hline Twitya & BFTW & 179.1 & 0.004 & 0.588 & 5.950 \\
\hline Twitya & BFTW & 201 & 0.007 & 0.763 & 2.590 \\
\hline Twitya & BFTW & 222.5 & 0.004 & 0.416 & 4.650 \\
\hline Twitya & BFTW & 240.3 & 0.004 & 0.491 & 2.360 \\
\hline Twitya & BFTW & 261.8 & 0.002 & 0.318 & 2.060 \\
\hline Twitya & BFTW & 287.5 & 0.002 & 1.203 & 6.250 \\
\hline Twitya & BFTW & 299.3 & 0.001 & 0.193 & 2.390 \\
\hline Twitya & BFTW & 320.7 & 0.003 & 0.496 & 1.640 \\
\hline Twitya & BFTW & 340.2 & 0.001 & 0.948 & 3.170 \\
\hline Twitya & BFTW & 352.2 & 0.003 & 0.935 & 4.040 \\
\hline Twitya & BFTW & 369.2 & 0.003 & 0.477 & 3.520 \\
\hline Twitya & BFTW & 389.6 & 0.001 & 1.052 & 5.530 \\
\hline Twitya & BFTW & 411.8 & 0.002 & 0.205 & 3.850 \\
\hline Twitya & BFTW & 432.4 & 0.000 & 1.438 & 5.180 \\
\hline Twitya & BFTW & 449.3 & 0.009 & 0.916 & 6.650 \\
\hline Twitya & BFTW & 488.2 & 0.026 & 1.420 & 4.350 \\
\hline Twitya & BFTW & 508.7 & 0.014 & 0.776 & 5.270 \\
\hline Twitya & BFTW & 521.9 & 0.035 & 1.362 & 5.210 \\
\hline Twitya & BFTW & 536.8 & 0.046 & 0.892 & 5.690 \\
\hline Twitya & BFTW & 542.8 & 0.002 & 0.560 & 5.140 \\
\hline Twitya & BFTW & 565.9 & 0.007 & 0.363 & 6.790 \\
\hline Twitya & BFTW & 594 & 0.048 & 1.190 & 3.930 \\
\hline Twitya & BFTW & 617.3 & 0.066 & 0.669 & 3.990 \\
\hline Twitya & BFTW & 671.7 & 0.001 & 0.124 & 1.100 \\
\hline Twitya & BFTW & 724.4 & 0.009 & 0.460 & 3.970 \\
\hline Twitya & BFTW & 732.3 & 0.021 & 0.680 & 4.560 \\
\hline Twitya & BFTW & 746.7 & 0.001 & 0.915 & 4.280 \\
\hline Twitya & BFTW & 876.4 & 0.002 & 0.537 & 1.750 \\
\hline Red Pine Shale & Box Canyon & DHBCB1 & 0.000 & 1.012 & 4.370 \\
\hline Red Pine Shale & Box Canyon & DHBCC14 & 0.001 & 0.884 & 3.340 \\
\hline Red Pine Shale & Box Canyon & DHBCC21 & 0.001 & 0.691 & 2.610 \\
\hline Red Pine Shale & Box Canyon & DHBCC3 & 0.027 & 0.775 & 3.150 \\
\hline Red Pine Shale & Box Canyon & DHBCC6 & 0.000 & 1.864 & 3.360 \\
\hline Red Pine Shale & Fish Lake & 101DH12 & 0.001 & 0.870 & 2.550 \\
\hline Red Pine Shale & Fish Lake & 102DH12 & 0.001 & 0.908 & 2.660 \\
\hline
\end{tabular}




\begin{tabular}{|c|c|c|c|c|c|}
\hline Red Pine Shale & Fish Lake & $104 \mathrm{DH} 12$ & 0.012 & 0.770 & 2.420 \\
\hline Red Pine Shale & Fish Lake & 105DH12 & 0.000 & 0.811 & 2.100 \\
\hline Red Pine Shale & Fish Lake & 107DH12 & 0.000 & 1.061 & 2.270 \\
\hline Red Pine Shale & Fish Lake & 109DH12 & 0.001 & 0.881 & 2.020 \\
\hline Red Pine Shale & Fish Lake & 92DH12 & 0.000 & 1.710 & 4.490 \\
\hline Red Pine Shale & Fish Lake & 94DH12 & 0.004 & 0.878 & 3.320 \\
\hline Red Pine Shale & Fish Lake & 95DH12 & 0.004 & 1.363 & 3.090 \\
\hline Red Pine Shale & Fish Lake & 99DH12 & 0.001 & 1.668 & 3.300 \\
\hline Red Pine Shale & Leidy & DHLPA1 & 0.000 & 1.792 & 3.360 \\
\hline Red Pine Shale & Leidy & DHLPA13 & 0.001 & 1.250 & 3.950 \\
\hline Red Pine Shale & Leidy & DHLPA16 & 0.002 & 0.836 & 2.710 \\
\hline Red Pine Shale & Leidy & DHLPA4 & 0.000 & 0.891 & 2.900 \\
\hline Red Pine Shale & Leidy & DHLPA8 & 0.000 & 2.866 & 4.580 \\
\hline Red Pine Shale & Marsh Ridge 1 & 29DH11 & 0.001 & 0.129 & 2.130 \\
\hline Red Pine Shale & Marsh Ridge 1 & 31DH11 & 0.006 & 0.349 & 2.150 \\
\hline Red Pine Shale & Marsh Ridge 1 & $35 \mathrm{DH} 11 \mathrm{a}$ & 0.001 & 0.504 & 1.750 \\
\hline Red Pine Shale & Marsh Ridge 1 & 38DH11 & 0.001 & 0.294 & 2.380 \\
\hline Red Pine Shale & Marsh Ridge 1 & 41DH11 & 0.000 & 0.253 & 1.820 \\
\hline Red Pine Shale & Marsh Ridge 1 & 42DH11 & 0.000 & 0.332 & 2.340 \\
\hline Red Pine Shale & Marsh Ridge 1 & 44DH11 & 0.000 & 0.233 & 2.120 \\
\hline Red Pine Shale & Marsh Ridge 1 & 46DH11 & 0.001 & 0.348 & 1.970 \\
\hline Red Pine Shale & Marsh Ridge 1 & 47DH11 & 0.004 & 1.137 & 4.040 \\
\hline Red Pine Shale & Marsh Ridge 1 & 48DH11 & 0.001 & 0.131 & 1.490 \\
\hline Red Pine Shale & Marsh Ridge 1 & 50DH11 & 0.000 & 0.049 & 1.250 \\
\hline Red Pine Shale & Marsh Ridge 1 & 51DH11 & 0.000 & 0.488 & 2.020 \\
\hline Red Pine Shale & Marsh Ridge 1 & 52DH11 & 0.001 & 2.389 & 4.470 \\
\hline Red Pine Shale & Marsh Ridge 1 & 55DH11 & 0.001 & 1.700 & 2.930 \\
\hline Red Pine Shale & Marsh Ridge 1 & 55DH12 & 0.001 & 0.708 & 2.540 \\
\hline Red Pine Shale & Marsh Ridge 1 & 56DH12 & 0.001 & 2.242 & 4.440 \\
\hline Red Pine Shale & Marsh Ridge 1 & 57DH12 & 0.001 & 2.746 & 5.030 \\
\hline Red Pine Shale & Marsh Ridge 1 & 58DH11 & 0.003 & 2.029 & 4.070 \\
\hline Red Pine Shale & Marsh Ridge 1 & 59DH12 & 0.000 & 2.014 & 4.660 \\
\hline Red Pine Shale & Marsh Ridge 1 & 60DH11 & 0.001 & 1.910 & 4.530 \\
\hline Red Pine Shale & Marsh Ridge 1 & 61DH11 & 0.001 & 2.257 & 5.100 \\
\hline Red Pine Shale & Marsh Ridge 1 & $61 \mathrm{DH} 12 \mathrm{a}$ & 0.001 & 1.002 & 3.220 \\
\hline Red Pine Shale & Marsh Ridge 1 & 63DH11 & 0.001 & 1.103 & 3.080 \\
\hline Red Pine Shale & Marsh Ridge 1 & 63DH12 & 0.000 & 1.806 & 4.610 \\
\hline Red Pine Shale & Marsh Ridge 1 & 64DH11 & 0.002 & 1.752 & 5.770 \\
\hline Red Pine Shale & Marsh Ridge 1 & 64DH12 & 0.000 & 2.084 & 4.920 \\
\hline Red Pine Shale & Marsh Ridge 1 & 65DH11 & 0.010 & 0.766 & 3.400 \\
\hline Red Pine Shale & Marsh Ridge 1 & 65DH12 & 0.000 & 1.759 & 4.530 \\
\hline
\end{tabular}




\begin{tabular}{|c|c|c|c|c|c|}
\hline Red Pine Shale & Marsh Ridge 1 & 66DH11 & 0.000 & 0.866 & 3.210 \\
\hline Red Pine Shale & Marsh Ridge 1 & 66DH12 & 0.000 & 1.678 & 4.680 \\
\hline Red Pine Shale & Marsh Ridge 1 & 67DH11 & 0.000 & 1.066 & 3.100 \\
\hline Red Pine Shale & Marsh Ridge 1 & 67DH12 & 0.001 & 1.421 & 4.480 \\
\hline Red Pine Shale & Marsh Ridge 1 & 69DH12 & 0.001 & 2.931 & 5.950 \\
\hline Red Pine Shale & Marsh Ridge 1 & 70DH11 & 0.001 & 1.398 & 4.350 \\
\hline Red Pine Shale & Marsh Ridge 1 & 71DH12 & 0.000 & 1.948 & 5.280 \\
\hline Red Pine Shale & Marsh Ridge 1 & 72DH11 & 0.002 & 1.337 & 4.920 \\
\hline Red Pine Shale & Marsh Ridge 1 & 74DH11 & 0.001 & 1.049 & 4.870 \\
\hline Red Pine Shale & Marsh Ridge 1 & 74DH12 & 0.001 & 1.959 & 4.730 \\
\hline Red Pine Shale & Marsh Ridge 1 & 76DH11 & 0.000 & 0.696 & 1.950 \\
\hline Red Pine Shale & Marsh Ridge 2 & 77DH12 & 0.001 & 1.387 & 4.920 \\
\hline Red Pine Shale & Marsh Ridge 2 & 78DH11 & 0.000 & 1.263 & 3.330 \\
\hline Red Pine Shale & Marsh Ridge 2 & 79DH11 & 0.001 & 1.785 & 2.620 \\
\hline Red Pine Shale & Marsh Ridge 2 & 79DH12 & 0.000 & 0.898 & 4.460 \\
\hline Red Pine Shale & Marsh Ridge 2 & 81DH11 & 0.001 & 1.429 & 3.030 \\
\hline Red Pine Shale & Marsh Ridge 2 & 81DH12 & 0.000 & 1.438 & 4.470 \\
\hline Red Pine Shale & Marsh Ridge 2 & 82DH11 & 0.001 & 0.878 & 2.770 \\
\hline Red Pine Shale & Marsh Ridge 2 & 83DH11 & 0.002 & 0.929 & 2.500 \\
\hline Red Pine Shale & Marsh Ridge 2 & 85DH11 & 0.001 & 0.882 & 2.640 \\
\hline Red Pine Shale & Marsh Ridge 2 & 86DH11 & 0.000 & 1.024 & 3.000 \\
\hline Red Pine Shale & Marsh Ridge 2 & 87DH11 & 0.001 & 1.253 & 3.100 \\
\hline Red Pine Shale & Marsh Ridge 2 & 88DH11 & 0.001 & 1.993 & 4.260 \\
\hline Red Pine Shale & Marsh Ridge 2 & 90DH11 & 0.000 & 0.574 & 2.340 \\
\hline Red Pine Shale & Marsh Ridge 2 & 92DH11 & 0.001 & 0.690 & 4.580 \\
\hline \multicolumn{2}{|c|}{ Callison Lake Dolo: F1011 } & 4 & 0.000 & 2.561 & 4.730 \\
\hline \multicolumn{2}{|c|}{ Callison Lake Dolo: F1011 } & 5 & 0.001 & 4.184 & 8.650 \\
\hline \multicolumn{2}{|c|}{ Callison Lake Dolo: F1011 } & 6 & 0.000 & 0.958 & 3.390 \\
\hline \multicolumn{2}{|c|}{ Callison Lake Dolo: F1011 } & 7 & 0.000 & 8.394 & 13.300 \\
\hline \multicolumn{2}{|c|}{ Callison Lake Dolo: F1011 } & 7.7 & 0.000 & 1.886 & 3.570 \\
\hline \multicolumn{2}{|c|}{ Callison Lake Dolo:F1011 } & 14.3 & 0.005 & 0.758 & 1.370 \\
\hline \multicolumn{2}{|c|}{ Callison Lake Dolo: F1011 } & 15.2 & 0.026 & 0.669 & 1.120 \\
\hline \multicolumn{2}{|c|}{ Callison Lake Dolo: F1011 } & 18.8 & 0.001 & 0.864 & 1.500 \\
\hline \multicolumn{2}{|c|}{ Callison Lake Dolo: F1011 } & 20.2 & 0.003 & 0.997 & 1.470 \\
\hline \multicolumn{2}{|c|}{ Callison Lake Dolo: F1011 } & 24.8 & 0.010 & 0.343 & 0.620 \\
\hline \multicolumn{2}{|c|}{ Callison Lake Dolo: F1011 } & 28.5 & 0.018 & 0.312 & 0.390 \\
\hline \multicolumn{2}{|c|}{ Callison Lake Dolo: F1011 } & 29.5 & 0.011 & 0.064 & 0.120 \\
\hline \multicolumn{2}{|c|}{ Callison Lake Dolo: F1011 } & 30 & 0.002 & 0.036 & 0.090 \\
\hline \multicolumn{2}{|c|}{ Callison Lake Dolo: F1011 } & 30.4 & 0.009 & 0.023 & 0.060 \\
\hline \multicolumn{2}{|c|}{ Callison Lake Dolo:F1011 } & 33.5 & 0.006 & 0.032 & 0.070 \\
\hline \multicolumn{2}{|c|}{ Callison Lake Dolo: F1011 } & 34.5 & 0.003 & 0.093 & 0.180 \\
\hline
\end{tabular}


Callison Lake Dolo: F1011

Callison Lake Dolo: F1011

Callison Lake Dolo: F1011

Callison Lake Dolo: F1011

Callison Lake Dolo: F1011

Callison Lake Dolo: F1011

Callison Lake Dolo: F1011

Callison Lake Dolo: F836

Callison Lake Dolo: F836

Callison Lake Dolo: F836

Callison Lake Dolo: F836

Callison Lake Dolo: F836

Callison Lake Dolo: F836

Callison Lake Dolo:J1018

Callison Lake Dolo:J1018

Callison Lake Dolo:J1018

Callison Lake Dolo:J1018

Callison Lake Dolo:J1018

Callison Lake Dolo:J1018

Callison Lake Dolo:J1018

Callison Lake Dolo:J1018

Callison Lake Dolo:J1018

Callison Lake Dolo:J1018

Callison Lake Dolo:J1018

Callison Lake Dolo:J1018

Macdonaldryggen G419

Macdonaldryggen G419

Macdonaldryggen G419

Macdonaldryggen G435

Macdonaldryggen G435

Macdonaldryggen G435

Macdonaldryggen G435

Macdonaldryggen G435

Macdonaldryggen G435a

Macdonaldryggen G435

Macdonaldryggen G407

Macdonaldryggen G407

Macdonaldryggen G407

Macdonaldryggen G407

Russoya

G519

Russoya

G519

\begin{tabular}{|c|c|c|c|}
\hline 36.2 & 0.004 & 0.060 & 0.120 \\
\hline 62.7 & 0.027 & 0.093 & 0.220 \\
\hline 63 & 0.011 & 0.106 & 0.220 \\
\hline 63.5 & 0.011 & 0.093 & 0.170 \\
\hline 64.1 & 0.010 & 0.086 & 0.170 \\
\hline 64.7 & 0.017 & 0.086 & 0.150 \\
\hline 68.2 & 0.000 & 0.029 & 0.070 \\
\hline 292.5 & 0.003 & 1.688 & 3.860 \\
\hline 412 & 0.080 & 2.121 & 2.920 \\
\hline 413 & 0.112 & 0.594 & 0.880 \\
\hline 417 & 0.033 & 1.663 & 2.990 \\
\hline 895 & 0.005 & 0.657 & 2.270 \\
\hline 898 & & 0.798 & 2.300 \\
\hline 20.9 & 0.008 & 0.096 & 0.190 \\
\hline 21.3 & 0.003 & 0.332 & 0.820 \\
\hline 21.6 & 0.003 & 0.054 & 0.090 \\
\hline 22.3 & 0.016 & 0.274 & 0.370 \\
\hline 22.6 & 0.007 & 0.359 & 0.500 \\
\hline 25.4 & 0.008 & 0.763 & 0.980 \\
\hline 25.9 & 0.014 & 0.884 & 1.040 \\
\hline 26.5 & 0.005 & 0.641 & 0.800 \\
\hline 27.2 & 0.007 & 0.348 & 0.600 \\
\hline 27.8 & 0.005 & 0.603 & 0.800 \\
\hline 30.5 & 0.017 & 0.433 & 0.670 \\
\hline 34.2 & 0.032 & 0.433 & 0.750 \\
\hline 2.6 & 0.472 & 1.266 & 1.628 \\
\hline 6.7 & 0.723 & 1.649 & 2.233 \\
\hline 12.3 & 0.683 & 1.314 & 2.413 \\
\hline 25 & 0.289 & 1.358 & 2.483 \\
\hline 35 & 0.570 & 1.567 & 3.368 \\
\hline 45 & 0.653 & 1.775 & 4.178 \\
\hline 56 & 0.943 & 1.754 & 4.445 \\
\hline 66 & 1.050 & 2.228 & 4.325 \\
\hline 76 & 0.827 & 1.780 & 4.240 \\
\hline 79.5 & 0.727 & 1.670 & 4.219 \\
\hline 278 & 0.020 & 1.047 & 4.410 \\
\hline 261 & 0.011 & 1.287 & 4.598 \\
\hline 245 & 0.033 & 1.324 & 4.560 \\
\hline 233 & 0.015 & 1.245 & 4.399 \\
\hline a & 0.001 & 1.483 & 4.197 \\
\hline$b$ & 0.009 & 0.505 & 2.841 \\
\hline
\end{tabular}




\begin{tabular}{|c|c|c|c|c|c|}
\hline Russoya & G406 & 42 & 0.070 & 0.613 & 0.665 \\
\hline Russoya & G406 & 35 & 0.098 & 1.495 & 1.921 \\
\hline Russoya & G521 & 46 & 0.011 & 1.131 & 2.953 \\
\hline Russoya & G521 & 44.4 & 0.016 & 2.413 & 4.400 \\
\hline Russoya & G521 & 40.5 & 0.015 & 1.575 & 7.416 \\
\hline Russoya & G521 & 36 & 0.293 & 1.053 & 4.162 \\
\hline Russoya & G521 & 33 & 0.016 & 2.161 & 8.396 \\
\hline Russoya & G521 & 30 & 0.004 & 0.916 & 1.925 \\
\hline Russoya & G521 & 26.4 & 0.006 & 2.859 & 5.892 \\
\hline Russoya & G521 & 23.4 & 0.002 & 0.891 & 5.017 \\
\hline Russoya & G521 & 19.6 & 0.012 & 0.984 & 3.048 \\
\hline Russoya & G521 & 13.2 & 0.001 & 0.635 & 1.539 \\
\hline Russoya & G521 & 6.2 & 0.001 & 0.974 & 2.088 \\
\hline Backlundtoppen & G517 & & 0.024 & 0.313 & 0.715 \\
\hline Svanbergfjellet & G512 & 55 & 0.030 & 1.478 & 2.241 \\
\hline Svanbergfjellet & G512 & 40.6 & 0.000 & 1.265 & 3.356 \\
\hline Svanbergfjellet & G512 & 35.2 & 0.034 & 0.645 & 0.613 \\
\hline Svanbergfjellet & G155 & 149.3 & 0.012 & 0.891 & 3.670 \\
\hline Svanbergfjellet & G155 & 149.1 & 0.129 & 0.926 & 1.013 \\
\hline Svanbergfjellet & G471 & 6.1 & 0.002 & 0.813 & 3.161 \\
\hline Svanbergfjellet & G155 & 83.6 & 0.042 & 0.184 & 0.191 \\
\hline Veteranen & G426 & a & 0.012 & 1.101 & 8.737 \\
\hline Veteranen & G426 & $b$ & 0.004 & 0.928 & 5.573 \\
\hline Doushantuo & Doushantuo & 15 & 0.320 & 0.690 & 0.820 \\
\hline Doushantuo & Doushantuo & 22.2 & 0.300 & 0.930 & 0.970 \\
\hline Doushantuo & Doushantuo & 24.1 & 0.560 & 1.280 & 1.430 \\
\hline Doushantuo (Den & Doushantuo (Deng) & 25.1 & 0.100 & 0.590 & 0.620 \\
\hline Doushantuo (Den & Doushantuo (Deng) & 26.2 & 0.430 & 0.770 & 1.020 \\
\hline Liuchapo & Liuchapo & 37.05 & 0.180 & 0.880 & 0.970 \\
\hline Liuchapo & Liuchapo & 38.25 & 0.070 & 0.200 & 0.210 \\
\hline Liuchapo & Liuchapo & 38.6 & 0.090 & 0.170 & 0.270 \\
\hline Liuchapo & Liuchapo & 40.05 & 0.150 & 0.980 & 1.170 \\
\hline Liuchapo & Liuchapo & 41.65 & 0.070 & 0.600 & 0.730 \\
\hline Liuchapo & Liuchapo & 42.65 & 0.060 & 0.300 & 0.310 \\
\hline Liuchapo & Liuchapo & 45.1 & 0.100 & 0.210 & 0.260 \\
\hline Liuchapo & Liuchapo & 45.57 & 0.030 & 0.250 & 0.250 \\
\hline Liuchapo & Liuchapo & 45.65 & 0.020 & 0.050 & 0.060 \\
\hline Liuchapo & Liuchapo & 46.12 & 0.030 & 0.160 & 0.160 \\
\hline Doushantuo & Jiulongwan & HN-01? & 0.000 & 0.200 & 0.200 \\
\hline Doushantuo & Jiulongwan & HN-02? & 0.000 & 0.300 & 0.400 \\
\hline Doushantuo & Jiulongwan & HN-04? & 0.500 & 1.100 & 1.500 \\
\hline
\end{tabular}




\begin{tabular}{|c|c|c|c|c|c|}
\hline Doushantuo & Jiulongwan & HN-05? & 0.300 & 0.500 & 0.600 \\
\hline Doushantuo & Jiulongwan & HN-06? & 0.300 & 0.600 & 0.800 \\
\hline Doushantuo & Jiulongwan & HN-07? & 0.200 & 0.600 & 0.800 \\
\hline Doushantuo & Jiulongwan & HN-08? & 0.300 & 0.400 & 0.500 \\
\hline Doushantuo & Jiulongwan & HN-09? & 1.900 & 2.200 & 2.800 \\
\hline Doushantuo & Jiulongwan & HN-10? & 0.400 & 0.500 & 0.700 \\
\hline Doushantuo & Jiulongwan & HN-11? & 2.100 & 2.200 & 3.200 \\
\hline Doushantuo & Jiulongwan & HN-12? & 2.100 & 2.300 & 3.100 \\
\hline Doushantuo & Jiulongwan & $\mathrm{HN}-13$ ? & 1.000 & 1.100 & 2.900 \\
\hline Doushantuo & Jiulongwan & HN-15? & 1.400 & 1.500 & 2.700 \\
\hline Doushantuo & Jiulongwan & HN-18? & 1.000 & 1.100 & 2.300 \\
\hline Doushantuo & Jiulongwan & HN-21? & 1.000 & 1.100 & 2.700 \\
\hline Doushantuo & Jiulongwan & HN-23? & 1.900 & 2.300 & 3.300 \\
\hline Doushantuo & Jiulongwan & JS-17? & 1.500 & 1.800 & 2.300 \\
\hline Doushantuo & Jiulongwan & JS-18? & 1.200 & 1.500 & 2.400 \\
\hline Doushantuo & Jiulongwan & JS-21? & 0.900 & 1.100 & 1.900 \\
\hline Doushantuo & Jiulongwan & JS-24? & 1.100 & 1.300 & 2.500 \\
\hline Doushantuo & Jiulongwan & JS-26? & 0.200 & 0.600 & 1.200 \\
\hline Doushantuo & Jiulongwan & JS-29? & 0.000 & 1.100 & 1.900 \\
\hline Doushantuo & Jiulongwan & JS-34? & 0.000 & 0.200 & 0.200 \\
\hline Doushantuo & Jiulongwan & JS-37? & 0.000 & 1.000 & 1.600 \\
\hline Doushantuo & Jiulongwan & JS-40? & 0.000 & 1.400 & 2.500 \\
\hline Doushantuo & Jiulongwan & JS-42? & 0.800 & 1.200 & 2.300 \\
\hline Doushantuo & Jiulongwan & JS-45? & 0.400 & 0.700 & 1.100 \\
\hline Doushantuo & Jiulongwan & JS-48? & 0.500 & 0.800 & 1.700 \\
\hline Doushantuo & Jiulongwan & JS-51? & 0.500 & 0.800 & 1.400 \\
\hline Doushantuo & Jiulongwan & JS-56? & 1.000 & 1.200 & 2.400 \\
\hline Doushantuo & Jiulongwan & JS-60? & 0.700 & 0.900 & 1.800 \\
\hline Doushantuo & Jiulongwan & JS-64? & 0.500 & 0.800 & 1.500 \\
\hline Doushantuo & Jiulongwan & JS-681? & 0.600 & 0.800 & 1.600 \\
\hline Doushantuo & Jiulongwan & JS-680? & 0.700 & 0.900 & 1.700 \\
\hline Doushantuo & Longe & ZH07-23? & 0.000 & 0.000 & 0.300 \\
\hline Doushantuo & Longe & ZH07-26? & 0.000 & 0.100 & 0.400 \\
\hline Doushantuo & Longe & ZH07-28? & 0.000 & 0.100 & 0.300 \\
\hline Doushantuo & Longe & ZH07-30? & 0.000 & 0.100 & 0.200 \\
\hline Doushantuo & Minle & ML-19? & 0.000 & 0.700 & 3.000 \\
\hline Doushantuo & Minle & ML-20? & 0.100 & 1.400 & 4.200 \\
\hline Doushantuo & Minle & ML-21? & 0.000 & 0.500 & 2.500 \\
\hline Doushantuo & Zhongling & SH-13? & 1.700 & 2.300 & 2.600 \\
\hline Doushantuo & Zhongling & SH-15? & 0.200 & 1.600 & 2.400 \\
\hline Doushantuo & Zhongling & SH-16? & 0.000 & 0.600 & 1.700 \\
\hline
\end{tabular}




\begin{tabular}{|c|c|c|c|c|c|}
\hline Doushantuo & Zhongling & SH-18? & 2.900 & 3.300 & 3.900 \\
\hline Doushantuo & Zhongling & SH-20? & 1.900 & 3.100 & 4.100 \\
\hline Doushantuo & Zhongling & SH-23? & 2.000 & 2.700 & 3.700 \\
\hline Doushantuo & Zhongling & SH-24? & 2.500 & 3.400 & 4.100 \\
\hline Doushantuo & Zhongling & SH-26? & 1.300 & 1.900 & 2.800 \\
\hline Doushantuo & Zhongling & SH-28? & 0.000 & 0.400 & 1.100 \\
\hline Doushantuo & Zhongling & SH-31? & 0.000 & 1.000 & 5.100 \\
\hline Doushantuo & Zhongling & SH-34? & 0.100 & 2.400 & 4.300 \\
\hline Doushantuo & Zhongling & SH-35? & 0.000 & 0.800 & 5.200 \\
\hline Doushantuo & Zhongling & SH-37? & 0.000 & 0.500 & 7.700 \\
\hline Doushantuo & Zhongling & SH-38? & 0.000 & 0.700 & 6.700 \\
\hline Doushantuo & Zhongling & SH-40? & 0.100 & 0.200 & 0.200 \\
\hline Doushantuo & Zhongling & SH-42? & 0.800 & 0.800 & 0.900 \\
\hline Doushantuo & Zhongling & SH-44? & 0.100 & 0.100 & 0.100 \\
\hline Doushantuo & Zhongling & SH-47? & 0.100 & 1.100 & 1.400 \\
\hline Doushantuo & Zhongling & SH-48? & 0.100 & 1.900 & 2.500 \\
\hline Doushantuo & Zhongling & SH-50? & 0.000 & 0.100 & 0.100 \\
\hline Doushantuo & Zhongling & SH-51? & 0.000 & 0.400 & 0.400 \\
\hline Doushantuo & Zhongling & SH-53? & 0.000 & 0.200 & 0.200 \\
\hline Doushantuo & Zhongling & SH-55? & 0.100 & 0.500 & 0.500 \\
\hline Doushantuo & Zhongling & SH-56? & 0.000 & 0.400 & 0.400 \\
\hline Doushantuo & Zhongling & SH-57? & 0.000 & 0.300 & 0.400 \\
\hline Doushantuo & Zhongling & SH-59? & 0.100 & 0.300 & 0.300 \\
\hline Doushantuo & Zhongling & SH-61? & 0.100 & 0.300 & 0.300 \\
\hline Doushantuo & Zhongling & SH-62? & 0.100 & 0.500 & 0.600 \\
\hline Doushantuo & Zhongling & SH-63? & 0.000 & 0.700 & 0.900 \\
\hline Doushantuo & Zhongling & SH-65? & 0.100 & 0.400 & 0.500 \\
\hline Doushantuo & Zhongling & SH-67? & 0.000 & 0.400 & 0.400 \\
\hline Doushantuo & Zhongling & SH-69? & 0.000 & 0.900 & 1.200 \\
\hline Doushantuo & Zhongling & SH-71? & 0.000 & 0.900 & 1.100 \\
\hline Doushantuo & Zhongling & 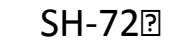 & 0.100 & 0.200 & 0.100 \\
\hline Doushantuo & Zhongling & SH-73? & 0.100 & 0.200 & 0.100 \\
\hline Doushantuo & Zhongling & SH-76? & 0.000 & 0.600 & 0.900 \\
\hline Doushantuo & Zhongling & SH-78? & 0.100 & 0.400 & 0.500 \\
\hline Doushantuo & Zhongling & SH-79? & 0.100 & 0.200 & 0.200 \\
\hline Doushantuo & Taoying & TY09-10 & 0.016 & 0.071 & 0.581 \\
\hline Doushantuo & Taoying & TY09-10.2 & 0.117 & 0.117 & 0.701 \\
\hline Doushantuo & Taoying & TY09-10.4 & 0.078 & 0.196 & 0.630 \\
\hline Doushantuo & Taoying & TY09-10.7 & 0.041 & 0.100 & 0.652 \\
\hline Doushantuo & Taoying & TY09-11 & 0.451 & 0.451 & 0.415 \\
\hline Doushantuo & Taoying & TY09-11.3 & 0.086 & 0.171 & 0.576 \\
\hline
\end{tabular}




\begin{tabular}{|c|c|c|c|c|c|}
\hline Doushantuo & Taoying & TY09-11.5 & 0.096 & 0.146 & 0.453 \\
\hline Doushantuo & Taoying & TY09-11.8 & 0.048 & 0.104 & 0.481 \\
\hline Doushantuo & Taoying & TY09-12 & 0.015 & 0.066 & 0.532 \\
\hline Doushantuo & Taoying & TY09-13.3 & 0.673 & 0.787 & 1.483 \\
\hline Doushantuo & Taoying & TY09-13.8 & 0.480 & 0.480 & 0.816 \\
\hline Doushantuo & Taoying & TY09-14 & 0.144 & 0.212 & 0.731 \\
\hline Doushantuo & Taoying & TY09-15.1 & 0.376 & 0.404 & 1.292 \\
\hline Doushantuo & Taoying & TY09-15.5 & 0.412 & 0.463 & 0.647 \\
\hline Doushantuo & Taoying & TY09-16.5 & 1.428 & 1.494 & 2.124 \\
\hline Doushantuo & Taoying & TY09-17.5 & 0.393 & 0.831 & 1.478 \\
\hline Doushantuo & Taoying & TY09-18.8 & 0.400 & 0.572 & 0.832 \\
\hline Doushantuo & Taoying & TY09-19.7 & 0.420 & 0.546 & 0.925 \\
\hline Doushantuo & Taoying & TY09-21.3 & 0.912 & 0.974 & 0.624 \\
\hline Doushantuo & Taoying & TY09-22.3 & 1.473 & 1.473 & 0.970 \\
\hline Doushantuo & Taoying & TY09-7.6 & 0.028 & 0.228 & 0.843 \\
\hline Doushantuo & Taoying & TY09-7.9 & 0.159 & 0.223 & 0.744 \\
\hline Doushantuo & Taoying & TY09-8 & 0.038 & 0.173 & 0.743 \\
\hline Doushantuo & Taoying & TY09-8.3 & 0.006 & 0.086 & 0.639 \\
\hline Doushantuo & Taoying & TY09-8.6 & 0.018 & 0.080 & 0.722 \\
\hline Doushantuo & Taoying & TY09-8.8 & 0.078 & 0.164 & 0.639 \\
\hline Doushantuo & Taoying & TY09-9 & 0.038 & 0.090 & 0.582 \\
\hline Doushantuo & Taoying & TY09-9.4 & 0.031 & 0.074 & 0.591 \\
\hline Doushantuo & Taoying & TY09-9.8 & 0.028 & 0.148 & 0.770 \\
\hline Doushantuo & Wuhe & WH09-11.6 & 1.896 & 1.987 & 2.981 \\
\hline Doushantuo & Wuhe & WH09-13.7 & 1.557 & 2.239 & 2.469 \\
\hline Doushantuo & Wuhe & WH09-2.4 & 0.153 & 0.372 & 2.186 \\
\hline Doushantuo & Wuhe & WH09-2.7 & 0.147 & 0.501 & 1.733 \\
\hline Doushantuo & Wuhe & WH09-3 & 0.552 & 0.757 & 1.926 \\
\hline Doushantuo & Wuhe & WH09-3.2 & 1.224 & 1.261 & 2.566 \\
\hline Doushantuo & Wuhe & WH09-3.5 & 0.185 & 0.821 & 2.060 \\
\hline Doushantuo & Wuhe & WH09-3.7 & 1.724 & 1.990 & 3.157 \\
\hline Doushantuo & Wuhe & WH09-4 & 0.746 & 0.875 & 2.282 \\
\hline Doushantuo & Wuhe & WH09-4.3 & 1.914 & 2.110 & 3.902 \\
\hline Doushantuo & Wuhe & WH09-4.6 & 1.120 & 1.965 & 3.180 \\
\hline Doushantuo & Wuhe & WH09-4.8 & 0.570 & 0.795 & 2.642 \\
\hline Doushantuo & Wuhe & WH09-5 & 1.269 & 3.691 & 5.267 \\
\hline Doushantuo & Wuhe & WH09-5.4 & 1.581 & 2.009 & 3.144 \\
\hline Doushantuo & Wuhe & WH09-5.6 & 1.441 & 2.477 & 3.959 \\
\hline Doushantuo & Wuhe & WH09-5.8 & 1.539 & 2.396 & 3.493 \\
\hline Doushantuo & Wuhe & WH09-6.0 & 2.589 & 2.599 & 3.791 \\
\hline Doushantuo & Wuhe & WH09-6.3 & 2.658 & 2.693 & 3.842 \\
\hline
\end{tabular}




\begin{tabular}{|c|c|c|c|c|c|}
\hline Doushantuo & Wuhe & WH09-6.5 & 0.033 & 0.722 & 2.461 \\
\hline Doushantuo & Wuhe & WH09-6.8 & 1.347 & 1.389 & 2.879 \\
\hline Doushantuo & Wuhe & WH09-7.0 & 1.948 & 2.129 & 3.429 \\
\hline Doushantuo & Wuhe & WH09-7.3 & 1.507 & 1.808 & 2.920 \\
\hline Doushantuo & Wuhe & WH09-8.6 & 2.182 & 2.509 & 3.759 \\
\hline Doushantuo & Wuhe & WH09-9.4 & 2.008 & 2.329 & 3.449 \\
\hline Doushantuo & Wuhe & WH09-9.7 & 1.909 & 2.107 & 2.975 \\
\hline Doushantuo & Wuhe & WHH-5.6 & 0.391 & 0.440 & 0.680 \\
\hline Doushantuo & Wuhe & WHH-5.7 & 1.635 & 1.744 & 2.437 \\
\hline Doushantuo & Wuhe & WHH-6.3 & 1.700 & 1.931 & 2.678 \\
\hline Doushantuo & Wuhe & WHH-6.4 & 2.124 & 2.285 & 3.145 \\
\hline Doushantuo & Wuhe & WHH-6.8 & 2.256 & 2.459 & 2.928 \\
\hline Doushantuo & Wuhe & WHH-6.9 & 2.777 & 2.909 & 4.208 \\
\hline Doushantuo & Wuhe & WHH-7.2 & 3.523 & 3.653 & 4.238 \\
\hline Doushantuo & Wuhe & WHH-7.4 & 3.544 & 3.668 & 4.528 \\
\hline Doushantuo & Wuhe & WHH-7.6 & 0.110 & 0.166 & 0.649 \\
\hline Doushantuo & Wuhe & WHH-9.1 & 0.013 & 0.066 & 0.609 \\
\hline Doushantuo & Wuhe & WHH-9.5 & 0.046 & 0.108 & 0.657 \\
\hline Lantian & Anhui- Lantian Fm. & 0 & & & \\
\hline Lantian & Anhui- Lantian Fm. & 5.4 & & & \\
\hline Lantian & Anhui- Lantian Fm. & 10 & & & \\
\hline Lantian & Anhui- Lantian Fm. & 12.6 & & & \\
\hline Lantian & Anhui- Lantian Fm. & 17 & & & \\
\hline Lantian & Anhui- Lantian Fm. & 20 & & & \\
\hline Lantian & Anhui- Lantian Fm. & 25 & & & \\
\hline Lantian & Anhui- Lantian Fm. & 40.9 & & & \\
\hline Lantian & Anhui- Lantian Fm. & 45.7 & & & \\
\hline Lantian & Anhui- Lantian Fm. & 50 & & & \\
\hline Lantian & Anhui- Lantian Fm. & 53.5 & & & \\
\hline Lantian & Anhui- Lantian Fm. & 58.5 & & & \\
\hline Lantian & Anhui- Lantian Fm. & 63.5 & & & \\
\hline Lantian & Anhui- Lantian Fm. & 66.7 & & & \\
\hline Lantian & Anhui- Lantian Fm. & 70 & & & \\
\hline Lantian & Anhui- Lantian Fm. & 73.3 & & & \\
\hline Lantian & Anhui- Lantian Fm. & 77.3 & & & \\
\hline Lantian & Anhui- Lantian Fm. & 81 & & & \\
\hline Lantian & Anhui- Lantian Fm. & 85.3 & & & \\
\hline Lantian & Anhui- Lantian Fm. & 87.8 & & & \\
\hline Lantian & Anhui- Lantian Fm. & 90 & & & \\
\hline Lantian & Anhui- Lantian Fm. & 92 & & & \\
\hline Lantian & Anhui- Lantian Fm. & 95 & & & \\
\hline
\end{tabular}




\begin{tabular}{|c|c|c|c|c|c|}
\hline Lantian & Anhui- Lantian Fm. & 98 & & & \\
\hline Lantian & Anhui- Lantian Fm. & 105 & & & \\
\hline Lantian & Anhui- Lantian Fm. & 108 & & & \\
\hline Lantian & Anhui- Lantian Fm. & 112 & & & \\
\hline Lantian & Anhui- Lantian Fm. & 114 & & & \\
\hline Lantian & Anhui- Lantian Fm. & 118 & & & \\
\hline Lantian & Anhui- Lantian Fm. & 121 & & & \\
\hline Lantian & Anhui- Lantian Fm. & 124.5 & & & \\
\hline Lantian & Anhui- Lantian Fm. & 127.4 & & & \\
\hline Lantian & Anhui- Lantian Fm. & 133.8 & & & \\
\hline Lantian & Anhui- Lantian Fm. & 136.2 & & & \\
\hline Liuchapo & Longbizui & LBZ-16 & 0.020 & 0.350 & 0.340 \\
\hline Liuchapo & Longbizui & LBZ-17 & 0.030 & 0.240 & 0.290 \\
\hline Liuchapo & Longbizui & LBZ-19 & 0.310 & 0.460 & 0.820 \\
\hline Liuchapo & Longbizui & LBZ-21 & 0.230 & 0.440 & 0.840 \\
\hline Liuchapo & Longbizui & LBZ-22 & 0.180 & 0.460 & 0.640 \\
\hline Liuchapo & Longbizui & LBZ-25 & 0.300 & 0.950 & 1.370 \\
\hline Liuchapo & Longbizui & LBZ-27 & 0.070 & 0.530 & 0.570 \\
\hline Liuchapo & Longbizui & LBZ-28 & 0.120 & 0.580 & 0.550 \\
\hline Liuchapo & Longbizui & LBZ-31 & 0.030 & 0.300 & 0.310 \\
\hline Liuchapo & Longbizui & LBZ-32 & 0.410 & 1.100 & 1.060 \\
\hline Liuchapo & Longbizui & LBZ-33 & 0.010 & 0.310 & 0.320 \\
\hline Liuchapo & Longbizui & LBZ-35 & 0.060 & 0.470 & 0.540 \\
\hline Liuchapo & Longbizui & LBZ-36 & 0.120 & 0.520 & 0.550 \\
\hline Sheepbed & F849 & 16 & 0.010 & 2.110 & 2.980 \\
\hline Sheepbed & F849 & 18 & 0.010 & 2.430 & 3.390 \\
\hline Sheepbed & F849 & 20 & 0.060 & 1.340 & 4.590 \\
\hline Sheepbed & F849 & 20 & 0.050 & 2.400 & 3.080 \\
\hline Sheepbed & F849 & 22 & 0.100 & 2.390 & 3.110 \\
\hline Sheepbed & F849 & 24 & 0.030 & 2.470 & 3.260 \\
\hline Sheepbed & F849 & 26 & 0.260 & 3.000 & 4.060 \\
\hline Sheepbed & F849 & 28 & 0.140 & 2.350 & 4.020 \\
\hline Sheepbed & F849 & 29.3 & 0.200 & 2.300 & 3.510 \\
\hline Sheepbed & F849 & 30.2 & 0.030 & 1.430 & 2.730 \\
\hline Sheepbed & F849 & 32 & 0.150 & 1.650 & 4.190 \\
\hline Sheepbed & F849 & 34 & 0.230 & 2.430 & 3.370 \\
\hline Sheepbed & F849 & 36 & 0.050 & 1.100 & 3.250 \\
\hline Sheepbed & F849 & 40 & 0.240 & 1.650 & 4.670 \\
\hline Sheepbed & F849 & 40 & 0.200 & 2.230 & 4.080 \\
\hline Sheepbed & F849 & 43.8 & 0.050 & 1.090 & 3.430 \\
\hline Sheepbed & F849 & 46 & 0.190 & 1.650 & 3.790 \\
\hline
\end{tabular}




\begin{tabular}{|c|c|c|c|c|c|}
\hline Sheepbed & F849 & 48.2 & 0.150 & 2.050 & 4.450 \\
\hline Sheepbed & F849 & 50 & 0.130 & 1.860 & 3.640 \\
\hline Sheepbed & F849 & 54 & 0.110 & 2.150 & 4.720 \\
\hline Sheepbed & F849 & 56 & 0.310 & 1.470 & 4.250 \\
\hline Sheepbed & F849 & 58 & 0.140 & 1.740 & 5.000 \\
\hline Sheepbed & F849 & 60 & 0.100 & 1.710 & 2.970 \\
\hline Sheepbed & F849 & 62.5 & 0.220 & 1.690 & 4.810 \\
\hline Sheepbed & F849 & 64 & 0.090 & 1.670 & 4.860 \\
\hline Sheepbed & F849 & 66 & 0.030 & 1.250 & 3.310 \\
\hline Sheepbed & F849 & 68 & 0.200 & 1.800 & 5.330 \\
\hline Sheepbed & F849 & 70 & 0.360 & 1.470 & 4.790 \\
\hline Sheepbed & F849 & 72 & 0.360 & 2.510 & 5.340 \\
\hline Sheepbed & F849 & 75 & 0.050 & 1.170 & 4.750 \\
\hline Sheepbed & F849 & 77 & 0.150 & 1.220 & 4.830 \\
\hline Sheepbed & F849 & 79 & 0.080 & 1.070 & 4.630 \\
\hline Sheepbed & F849 & 82 & 0.170 & 1.510 & 4.820 \\
\hline Sheepbed & F849 & 85 & 0.210 & 1.840 & 4.250 \\
\hline Sheepbed & F849 & 87 & 0.060 & 1.570 & 3.200 \\
\hline Sheepbed & F849 & 90 & 0.190 & 1.520 & 4.510 \\
\hline Sheepbed & F849 & 91 & 0.130 & 1.520 & 4.460 \\
\hline Sheepbed & F849 & 93 & 0.100 & 1.140 & 2.890 \\
\hline Sheepbed & F849 & 95 & 0.170 & 1.550 & 4.380 \\
\hline Sheepbed & F849 & 97 & 0.100 & 1.930 & 4.510 \\
\hline Sheepbed & F849 & 99 & 0.260 & 1.740 & 2.600 \\
\hline Sheepbed & F849 & 101 & 0.150 & 2.060 & 3.690 \\
\hline Sheepbed & F849 & 103 & 0.160 & 1.830 & 4.510 \\
\hline Sheepbed & F849 & 105 & 0.100 & 1.890 & 4.230 \\
\hline Sheepbed & F849 & 107 & 0.150 & 2.470 & 5.080 \\
\hline Sheepbed & F849 & 109 & 0.240 & 3.060 & 6.020 \\
\hline Sheepbed & F849 & 112 & 0.180 & 2.330 & 4.200 \\
\hline Sheepbed & F849 & 113 & 0.160 & 2.300 & 6.060 \\
\hline Sheepbed & F849 & 119 & 0.130 & 1.250 & 3.830 \\
\hline Sheepbed & F849 & 121 & 0.180 & 1.330 & 4.340 \\
\hline Sheepbed & F849 & 123 & 0.140 & 1.410 & 4.400 \\
\hline Sheepbed & F849 & 125 & 0.340 & 2.430 & 4.950 \\
\hline Sheepbed & F849 & 127 & 0.220 & 1.680 & 4.840 \\
\hline Sheepbed & F849 & 129 & 0.180 & 1.070 & 4.480 \\
\hline Sheepbed & F849 & 131 & 0.190 & 2.200 & 5.020 \\
\hline Sheepbed & F849 & 135 & 0.140 & 2.420 & 5.350 \\
\hline Sheepbed & F849 & 139 & 0.180 & 1.840 & 5.450 \\
\hline Sheepbed & F849 & 141 & 0.070 & 1.600 & 4.710 \\
\hline
\end{tabular}




\begin{tabular}{|c|c|c|c|c|c|}
\hline Sheepbed & F849 & 143 & 0.150 & 1.890 & 4.670 \\
\hline Sheepbed & F849 & 145 & 0.160 & 1.930 & 5.030 \\
\hline Sheepbed & F849 & 147 & 0.060 & 2.540 & 5.530 \\
\hline Sheepbed & F849 & 149 & 0.040 & 1.650 & 4.730 \\
\hline Sheepbed & F849 & 151 & 0.100 & 1.470 & 4.520 \\
\hline Sheepbed & F849 & 153 & 0.100 & 1.050 & 3.800 \\
\hline Sheepbed & F849 & 155 & 0.290 & 2.000 & 4.250 \\
\hline Sheepbed & F849 & 157 & 0.110 & 2.440 & 5.160 \\
\hline Sheepbed & F849 & 159 & 0.060 & 1.760 & 4.590 \\
\hline Sheepbed & F849 & 161 & 0.010 & 2.040 & 4.100 \\
\hline Sheepbed & F849 & 163 & 0.120 & 1.630 & 5.020 \\
\hline Sheepbed & F849 & 165 & 0.140 & 2.520 & 4.760 \\
\hline Sheepbed & F849 & 167 & 0.120 & 1.970 & 5.480 \\
\hline Sheepbed & F849 & 168 & 0.020 & 1.460 & 4.410 \\
\hline Sheepbed & F849 & 176 & 0.030 & 2.490 & 3.990 \\
\hline Sheepbed & F849 & 182 & 0.160 & 2.050 & 4.270 \\
\hline Sheepbed & F849 & 184 & 0.170 & 1.870 & 4.020 \\
\hline Sheepbed & F849 & 187 & 0.110 & 2.010 & 4.270 \\
\hline Sheepbed & F849 & 188.5 & 0.150 & 2.380 & 4.950 \\
\hline Sheepbed & F849 & 191 & 0.110 & 2.180 & 5.350 \\
\hline Sheepbed & F849 & 193.5 & 0.050 & 1.590 & 4.330 \\
\hline Sheepbed & F849 & 195 & 0.110 & 2.050 & 2.800 \\
\hline Sheepbed & F849 & 197 & 0.050 & 2.390 & 5.370 \\
\hline Sheepbed & F849 & 199 & 0.010 & 2.250 & 5.800 \\
\hline Sheepbed & F849 & 201 & 0.050 & 2.070 & 4.990 \\
\hline Sheepbed & F849 & 203 & 0.130 & 2.930 & 5.530 \\
\hline Sheepbed & F849 & 205.2 & 0.120 & 1.140 & 5.460 \\
\hline Sheepbed & F849 & 207 & 0.030 & 1.470 & 4.640 \\
\hline Sheepbed & F849 & 208.5 & 0.050 & 1.960 & 5.100 \\
\hline Sheepbed & F849 & 213 & 0.010 & 1.440 & 4.600 \\
\hline Sheepbed & F849 & 215 & 0.030 & 1.550 & 4.310 \\
\hline Sheepbed & F849 & 219 & 0.050 & 1.840 & 5.140 \\
\hline Sheepbed & F849 & 221 & 0.270 & 2.550 & 6.000 \\
\hline Sheepbed & F849 & 223 & 0.040 & 2.380 & 5.560 \\
\hline Sheepbed & F849 & 225 & 0.020 & 0.760 & 0.840 \\
\hline Sheepbed & F849 & 227 & 0.080 & 2.360 & 5.710 \\
\hline Sheepbed & F849 & 229 & 0.060 & 1.570 & 4.120 \\
\hline Sheepbed & F849 & 234 & 0.120 & 1.750 & 4.400 \\
\hline Sheepbed & F849 & 236 & 0.040 & 2.100 & 4.800 \\
\hline Sheepbed & F849 & 238 & 0.060 & 1.310 & 4.260 \\
\hline Sheepbed & F849 & 240 & 0.020 & 2.330 & 4.240 \\
\hline
\end{tabular}




\begin{tabular}{|c|c|c|c|c|c|}
\hline Sheepbed & F849 & 242 & 0.020 & 2.230 & 5.400 \\
\hline Sheepbed & F849 & 244 & 0.020 & 1.750 & 5.180 \\
\hline Sheepbed & F849 & 248 & 0.000 & 0.990 & 3.870 \\
\hline Sheepbed & F849 & 252 & 0.120 & 2.090 & 5.070 \\
\hline Sheepbed & F849 & 253 & 0.100 & 2.550 & 5.260 \\
\hline Sheepbed & F849 & 254 & 0.040 & 2.040 & 5.150 \\
\hline Sheepbed & F849 & 256 & 0.030 & 1.360 & 4.860 \\
\hline Sheepbed & F849 & 258 & 0.020 & 0.790 & 1.570 \\
\hline Sheepbed & F849 & 260 & 0.040 & 1.270 & 4.970 \\
\hline Sheepbed & F849 & 262 & 0.050 & 2.030 & 6.370 \\
\hline Sheepbed & F849 & 264 & 0.020 & 1.350 & 5.160 \\
\hline Sheepbed & F849 & 264 & 0.020 & 1.880 & 4.430 \\
\hline Sheepbed & F849 & 266 & 0.030 & 1.390 & 2.200 \\
\hline Sheepbed & F849 & 268 & 0.020 & 2.380 & 5.240 \\
\hline Sheepbed & F849 & 270 & 0.020 & 2.230 & 5.020 \\
\hline Sheepbed & F849 & 272 & 0.020 & 2.150 & 5.640 \\
\hline Sheepbed & F849 & 274 & 0.030 & 1.940 & 4.280 \\
\hline Sheepbed & F849 & 276 & 0.000 & 2.270 & 5.610 \\
\hline Sheepbed & F849 & 278 & 0.030 & 2.100 & 6.300 \\
\hline Sheepbed & F849 & 280 & 0.030 & 1.400 & 3.880 \\
\hline Sheepbed & F849 & 282 & 0.040 & 2.240 & 5.230 \\
\hline Sheepbed & F849 & 284 & 0.060 & 1.250 & 5.780 \\
\hline Sheepbed & F849 & 284 & 0.050 & 1.500 & 6.020 \\
\hline Sheepbed & F849 & 286 & 0.020 & 0.970 & 5.270 \\
\hline Sheepbed & F849 & 288 & 0.190 & 2.980 & 7.140 \\
\hline Sheepbed & F849 & 290 & 0.140 & 1.080 & 5.040 \\
\hline Sheepbed & F849 & 292 & 0.080 & 1.140 & 5.230 \\
\hline Sheepbed & F849 & 294 & 0.050 & 1.540 & 5.210 \\
\hline Sheepbed & F849 & 298 & 0.010 & 1.400 & 5.930 \\
\hline Sheepbed & F849 & 300 & 0.010 & 1.630 & 5.110 \\
\hline Sheepbed & F849 & 302 & 0.010 & 2.010 & 4.140 \\
\hline Sheepbed & F849 & 304 & 0.040 & 0.760 & 4.260 \\
\hline Sheepbed & F849 & 306 & 0.120 & 1.720 & 5.710 \\
\hline Sheepbed & F849 & 316 & 0.010 & 1.390 & 3.730 \\
\hline Sheepbed & F849 & 318 & 0.010 & 1.730 & 5.030 \\
\hline Sheepbed & F849 & 320 & 0.000 & 2.500 & 5.500 \\
\hline Sheepbed & F849 & 324 & 0.010 & 1.030 & 3.530 \\
\hline Sheepbed & F849 & 326 & 0.020 & 1.470 & 5.290 \\
\hline Sheepbed & F849 & 328 & 0.010 & 1.670 & 6.450 \\
\hline Sheepbed & F849 & 331 & 0.200 & 1.570 & 6.090 \\
\hline Sheepbed & F849 & 332 & 0.030 & 1.400 & 4.840 \\
\hline
\end{tabular}




\begin{tabular}{|c|c|c|c|c|c|}
\hline Sheepbed & F849 & 334 & 0.010 & 1.150 & 5.670 \\
\hline Sheepbed & F849 & 336 & 0.000 & 0.620 & 5.580 \\
\hline Sheepbed & F849 & 338 & 0.090 & 1.150 & 5.820 \\
\hline Sheepbed & F849 & 340 & 0.140 & 1.510 & 6.880 \\
\hline Sheepbed & F849 & 342 & 0.000 & 1.710 & 5.830 \\
\hline Sheepbed & F849 & 344 & 0.120 & 1.630 & 6.120 \\
\hline Sheepbed & F849 & 346 & 0.050 & 1.560 & 4.220 \\
\hline Sheepbed & F849 & 348.5 & 0.120 & 1.000 & 4.420 \\
\hline Sheepbed & F849 & 352 & 0.040 & 0.700 & 4.840 \\
\hline Sheepbed & F849 & 354 & 0.030 & 1.130 & 5.350 \\
\hline Sheepbed & F849 & 356 & 0.210 & 1.420 & 4.530 \\
\hline Sheepbed & F849 & 358 & 0.260 & 1.260 & 3.860 \\
\hline Sheepbed & F849 & 360 & 0.050 & 1.560 & 4.480 \\
\hline Sheepbed & F849 & 360 & 0.010 & 1.520 & 3.160 \\
\hline Sheepbed & F849 & 362 & 0.020 & 1.910 & 4.150 \\
\hline Sheepbed & F849 & 364 & 0.170 & 2.300 & 5.050 \\
\hline Sheepbed & F849 & 366 & 0.370 & 3.630 & 4.690 \\
\hline Sheepbed & F849 & 368 & 0.210 & 2.520 & 3.940 \\
\hline Blueflower & $\mathrm{F} 850$ & 262 & 0.000 & 0.640 & 1.410 \\
\hline Blueflower & $\mathrm{F} 850$ & 263.5 & 0.000 & 0.440 & 1.250 \\
\hline Blueflower & $\mathrm{F} 850$ & 266 & 0.000 & 2.800 & 6.300 \\
\hline Blueflower & $\mathrm{F} 850$ & 272.5 & 0.300 & 2.630 & 5.020 \\
\hline Blueflower & $\mathrm{F} 850$ & 273 & 0.190 & 2.840 & 5.090 \\
\hline Blueflower & $F 850$ & 276 & 0.060 & 2.330 & 5.610 \\
\hline Blueflower & F850 & 282 & 0.220 & 4.430 & 6.040 \\
\hline Blueflower & F850 & 287 & 0.000 & 0.400 & 1.210 \\
\hline Blueflower & F850 & 298 & 0.160 & 1.540 & 5.930 \\
\hline Blueflower & $F 850$ & 309.5 & 0.060 & 1.120 & 4.220 \\
\hline Blueflower & F850 & 311 & 0.190 & 2.520 & 4.640 \\
\hline Blueflower & F850 & 312 & 0.110 & 4.440 & 7.400 \\
\hline Blueflower & F850 & 332.5 & 0.000 & 0.530 & 1.450 \\
\hline Blueflower & F850 & 335 & 0.120 & 5.640 & 6.550 \\
\hline Blueflower & $\mathrm{F} 850$ & 337 & 0.140 & 2.110 & 2.960 \\
\hline Blueflower & F850 & 344.8 & 0.000 & 1.650 & 4.320 \\
\hline Blueflower & $\mathrm{F} 850$ & 388 & 0.030 & 1.580 & 4.050 \\
\hline Blueflower & $\mathrm{F} 850$ & 391 & 0.190 & 2.800 & 4.680 \\
\hline Blueflower & $\mathrm{F} 850$ & 393.5 & 0.010 & 2.590 & 4.320 \\
\hline Blueflower & $\mathrm{F} 850$ & 395 & 0.080 & 3.560 & 6.000 \\
\hline \multicolumn{2}{|c|}{ Blueflower FormatS1203 } & 0.7 & 0.425 & 0.557 & 0.860 \\
\hline \multicolumn{2}{|c|}{ Blueflower FormatS1203 } & 1 & 0.280 & 0.424 & 0.810 \\
\hline \multicolumn{2}{|c|}{ Blueflower FormatS1206 } & 36.8 & 0.047 & 0.408 & 3.460 \\
\hline
\end{tabular}




\begin{tabular}{|c|c|c|c|c|c|}
\hline \multicolumn{2}{|c|}{ Blueflower FormatS1206 } & 71.2 & 0.014 & 0.163 & 1.030 \\
\hline \multicolumn{2}{|c|}{ Blueflower FormatS1206 } & 72 & 0.011 & 0.335 & 1.260 \\
\hline \multicolumn{2}{|c|}{ Blueflower FormatS1208 } & 0 & 1.083 & 1.359 & 2.820 \\
\hline \multicolumn{2}{|c|}{ Blueflower FormatS1208 } & 8.5 & 0.000 & 0.380 & 2.340 \\
\hline \multicolumn{2}{|c|}{ Blueflower FormatS1208 } & 12.8 & 0.748 & 1.813 & 3.990 \\
\hline \multicolumn{2}{|c|}{ Blueflower FormatS1208 } & 20.8 & 0.529 & 2.505 & 3.710 \\
\hline Sheepbed & W11 & 1 & 0.042 & 1.594 & 4.410 \\
\hline Sheepbed & W11 & 28 & 0.057 & 1.095 & 3.279 \\
\hline Sheepbed & W11 & 32 & 0.033 & 1.045 & 2.843 \\
\hline Sheepbed & W11 & 37.5 & 0.083 & 1.266 & 3.335 \\
\hline Sheepbed & W11 & 43 & 0.043 & 1.060 & 3.030 \\
\hline Sheepbed & W11 & 47.5 & 0.145 & 1.455 & 3.825 \\
\hline Sheepbed & W11 & 49.5 & 0.103 & 1.737 & 3.699 \\
\hline Sheepbed & W11 & 55 & 0.083 & 1.173 & 3.939 \\
\hline Sheepbed & W11 & 60 & 0.109 & 1.562 & 3.439 \\
\hline Sheepbed & W11 & 65 & 0.113 & 1.335 & 4.175 \\
\hline Sheepbed & W11 & 70 & 0.081 & 1.870 & 5.074 \\
\hline Sheepbed & W11 & 75 & 0.046 & 1.746 & 2.809 \\
\hline Sheepbed & W11 & 80 & 0.153 & 1.731 & 6.232 \\
\hline Sheepbed & W11 & 86 & 0.059 & 1.524 & 3.514 \\
\hline Sheepbed & W11 & 90 & 0.082 & 1.195 & 2.915 \\
\hline Sheepbed & W11 & 95 & 0.030 & 1.521 & 3.469 \\
\hline Sheepbed & W11 & 100 & 0.028 & 1.479 & 4.793 \\
\hline Sheepbed & W11 & 105 & 0.110 & 1.221 & 2.762 \\
\hline Sheepbed & W11 & 110 & 0.078 & 1.318 & 2.796 \\
\hline Sheepbed & W11 & 115 & 0.071 & 0.987 & 3.482 \\
\hline Sheepbed & W11 & 120 & 0.069 & 1.301 & 2.874 \\
\hline Sheepbed & W11 & 125 & 0.108 & 1.523 & 2.183 \\
\hline Sheepbed & W11 & 131 & 0.063 & 1.022 & 3.231 \\
\hline Sheepbed & W11 & 135 & 0.112 & 1.502 & 3.797 \\
\hline Sheepbed & W11 & 140 & 0.009 & 0.766 & 2.427 \\
\hline Sheepbed & W11 & 145 & 0.127 & 1.130 & 2.794 \\
\hline Sheepbed & W11 & 150 & 0.040 & 1.601 & 3.500 \\
\hline Sheepbed & W11 & 154 & 0.006 & 0.737 & 3.281 \\
\hline Sheepbed & W11 & 160 & 0.043 & 1.010 & 3.787 \\
\hline Sheepbed & W11 & 165 & 0.010 & 0.424 & 5.589 \\
\hline Sheepbed & W11 & 170 & 0.007 & 0.410 & 5.088 \\
\hline Sheepbed & W11 & 175 & 0.015 & 1.361 & 3.377 \\
\hline June beds & W11 & 234 & 0.127 & 2.107 & 3.679 \\
\hline June beds & W11 & 236 & 0.072 & 0.756 & 1.437 \\
\hline June beds & W11 & 295 & 0.025 & 0.823 & 1.379 \\
\hline
\end{tabular}




\begin{tabular}{|c|c|c|c|c|c|}
\hline June beds & W11 & 398 & 0.000 & 0.979 & 3.551 \\
\hline June beds & W11 & 412 & 0.009 & 0.458 & 2.853 \\
\hline June beds & W11 & 432 & 0.114 & 0.486 & 3.327 \\
\hline June beds & W11 & 442 & 0.002 & 1.286 & 4.684 \\
\hline June beds & W11 & 458 & 0.000 & 0.991 & 4.921 \\
\hline June beds & W11 & 473 & 0.001 & 0.412 & 4.456 \\
\hline June beds & W11 & 501 & 0.001 & 2.158 & 4.247 \\
\hline June beds & W11 & 514 & 0.002 & 1.344 & 4.441 \\
\hline June beds & W11 & 528 & 0.001 & 0.435 & 3.644 \\
\hline June beds & W11 & 542 & 0.002 & 0.297 & 2.758 \\
\hline June beds & W11 & 570 & 0.005 & 0.651 & 3.743 \\
\hline Blueflower & W15 & 12.1 & 0.079 & 1.662 & 1.589 \\
\hline Blueflower & W15 & 90 & 0.084 & 3.579 & 6.033 \\
\hline Blueflower & W15 & 96 & 0.003 & 0.642 & 2.949 \\
\hline Blueflower & W15 & 100 & 0.204 & 1.296 & 3.796 \\
\hline Blueflower & W15 & 104 & 0.037 & 2.074 & 3.414 \\
\hline Blueflower & W15 & 105.4 & 0.073 & 3.214 & 4.726 \\
\hline Blueflower & W15 & 110 & 0.083 & 2.304 & 4.957 \\
\hline Blueflower & W15 & 117 & 0.574 & 1.981 & 5.775 \\
\hline Blueflower & W15 & 125.5 & 0.092 & 4.156 & 5.698 \\
\hline Blueflower & W15 & 129 & 0.003 & 0.585 & 3.476 \\
\hline Blueflower & W15 & 171 & 0.010 & 1.892 & 3.226 \\
\hline Blueflower & W15 & 197 & 0.024 & 0.314 & 0.997 \\
\hline Kotlin & Kel'tminskaya-1 & 1372 & 0.010 & 1.430 & 5.390 \\
\hline Kotlin & Kel'tminskaya-1 & 1374 & 0.000 & 1.330 & 4.980 \\
\hline Kotlin & Kel'tminskaya-1 & 1376 & 0.000 & 1.590 & 6.770 \\
\hline Kotlin & Kel'tminskaya-1 & 1412 & 0.000 & 1.080 & 5.360 \\
\hline Kotlin & Kel'tminskaya-1 & 1415.8 & 0.010 & 1.070 & 4.790 \\
\hline Kotlin & Kel'tminskaya-1 & 1417 & 0.010 & 1.860 & 6.520 \\
\hline Kotlin & Kel'tminskaya-1 & 1454.6 & 0.090 & 1.810 & 5.610 \\
\hline Kotlin & Kel'tminskaya-1 & 1457.3 & 0.010 & 1.450 & 6.020 \\
\hline Kotlin & Kel'tminskaya-1 & 1461.2 & 0.000 & 1.790 & 5.670 \\
\hline Kotlin & Kel'tminskaya-1 & 1497.3 & 0.000 & 1.240 & 5.690 \\
\hline Kotlin & Kel'tminskaya-1 & 1503.3 & 0.010 & 0.910 & 4.610 \\
\hline Kotlin & Kel'tminskaya-1 & 1542 & 0.000 & 1.240 & 5.190 \\
\hline Kotlin & Kel'tminskaya-1 & 1545.7 & 0.010 & 1.200 & 5.170 \\
\hline Kotlin & Kel'tminskaya-1 & 1581.3 & 0.010 & 0.780 & 4.640 \\
\hline Kotlin & Kel'tminskaya-1 & 1584 & 0.010 & 1.150 & 5.390 \\
\hline Kotlin & Kel'tminskaya-1 & 1587.3 & 0.000 & 1.300 & 5.030 \\
\hline Kotlin & Kel'tminskaya-1 & 1623.7 & 0.000 & 1.160 & 4.580 \\
\hline Kotlin & Kel'tminskaya-1 & 1629.2 & 0.000 & 1.380 & 4.450 \\
\hline
\end{tabular}




\begin{tabular}{|c|c|c|c|c|c|}
\hline Kotlin & Kel'tminskaya-1 & 1666.5 & 0.000 & 1.230 & 4.210 \\
\hline Kotlin & Kel'tminskaya-1 & 1670.7 & 0.000 & 1.190 & 4.000 \\
\hline Kotlin & Kel'tminskaya-1 & 1711 & 0.030 & 1.480 & 5.570 \\
\hline Kotlin & Kel'tminskaya-1 & 1751 & 0.040 & 1.460 & 4.920 \\
\hline Kotlin & Kel'tminskaya-1 & 1754.3 & 0.040 & 1.840 & 6.620 \\
\hline Kotlin & Kel'tminskaya-1 & 1783.7 & 0.040 & 1.620 & 5.460 \\
\hline Kotlin & Kel'tminskaya-1 & 1786.7 & 0.020 & 1.760 & 6.180 \\
\hline Kotlin & Kel'tminskaya-1 & 1829 & 0.010 & 1.770 & 6.490 \\
\hline Kotlin & Kel'tminskaya-1 & 1870 & 0.010 & 1.620 & 5.970 \\
\hline Redkino & Kel'tminskaya-1 & 1899 & 0.010 & 1.680 & 6.360 \\
\hline Redkino & Kel'tminskaya-1 & 1902.4 & 0.050 & 1.420 & 5.480 \\
\hline Redkino & Kel'tminskaya-1 & 1906 & 0.040 & 1.410 & 5.520 \\
\hline Redkino & Kel'tminskaya-1 & 1956.2 & 0.050 & 1.770 & 5.860 \\
\hline Redkino & Kel'tminskaya-1 & 1997 & 0.030 & 1.820 & 5.610 \\
\hline Redkino & Kel'tminskaya-1 & 2034 & 0.050 & 1.470 & 5.370 \\
\hline Redkino & Kel'tminskaya-1 & 2042 & 0.090 & 1.720 & 6.020 \\
\hline Redkino & Kel'tminskaya-1 & 2081 & 0.120 & 1.660 & 5.840 \\
\hline Redkino & Kel'tminskaya-1 & 2123 & 0.010 & 1.570 & 5.300 \\
\hline Redkino & Kel'tminskaya-1 & 2162 & 0.180 & 1.160 & 4.570 \\
\hline Redkino & Kel'tminskaya-1 & 2204 & 0.020 & 1.000 & 4.520 \\
\hline Redkino & Kel'tminskaya-1 & 2205 & 0.040 & 0.980 & 4.420 \\
\hline Redkino & Kel'tminskaya-1 & 2209 & 0.010 & 0.720 & 3.490 \\
\hline Redkino & Kel'tminskaya-1 & 2251 & 0.010 & 0.780 & 3.700 \\
\hline Redkino & Kel'tminskaya-1 & 2291 & 0.010 & 1.160 & 5.050 \\
\hline Redkino & Kel'tminskaya-1 & 2299 & 0.020 & 0.680 & 3.690 \\
\hline Redkino & Kel'tminskaya-1 & 2302.5 & 0.010 & 0.890 & 4.810 \\
\hline Vychegda & Kel'tminskaya-1 & 2348 & 0.010 & 0.890 & 3.950 \\
\hline Vychegda & Kel'tminskaya-1 & 2349 & 0.090 & 1.000 & 4.080 \\
\hline Vychegda & Kel'tminskaya-1 & 2350 & 0.070 & 1.100 & 4.200 \\
\hline Vychegda & Kel'tminskaya-1 & 2351 & 0.010 & 1.090 & 4.590 \\
\hline Vychegda & Kel'tminskaya-1 & 2351 & 0.020 & 1.640 & 4.930 \\
\hline Vychegda & Kel'tminskaya-1 & 2352 & 0.010 & 1.030 & 4.300 \\
\hline Vychegda & Kel'tminskaya-1 & 2394 & 0.030 & 1.110 & 8.510 \\
\hline Vychegda & Kel'tminskaya-1 & 2400 & 0.040 & 1.070 & 5.490 \\
\hline Vychegda & Kel'tminskaya-1 & 2445 & 0.050 & 0.470 & 2.210 \\
\hline Vychegda & Kel'tminskaya-1 & 2449 & 0.010 & 1.260 & 5.380 \\
\hline Vychegda & Kel'tminskaya-1 & 2450 & 0.050 & 1.280 & 1.540 \\
\hline Vychegda & Kel'tminskaya-1 & 2502 & 0.310 & 0.910 & 3.400 \\
\hline Vychegda & Kel'tminskaya-1 & 2504 & 0.120 & 0.600 & 2.170 \\
\hline Vychegda & Kel'tminskaya-1 & 2506 & 0.210 & 0.960 & 3.540 \\
\hline Vychegda & Kel'tminskaya-1 & 2508 & 0.320 & 0.950 & 2.990 \\
\hline
\end{tabular}




\begin{tabular}{|c|c|c|c|c|c|}
\hline Vychegda & Kel'tminskaya-1 & 2559 & 0.020 & 2.040 & 6.310 \\
\hline Vychegda & Kel'tminskaya-1 & 2563 & 0.140 & 1.870 & 6.230 \\
\hline Vychegda & Kel'tminskaya-1 & 2600 & 0.070 & 0.940 & 3.820 \\
\hline Vychegda & Kel'tminskaya-1 & 2601 & 0.100 & 0.590 & 3.410 \\
\hline Vychegda & Kel'tminskaya-1 & 2602 & 0.230 & 1.730 & 5.490 \\
\hline Vychegda & Kel'tminskaya-1 & 2603 & 0.070 & 0.550 & 4.410 \\
\hline Vychegda & Kel'tminskaya-1 & 2606 & 0.050 & 0.970 & 3.350 \\
\hline Vychegda & Kel'tminskaya-1 & 2688 & 0.000 & 1.560 & 4.890 \\
\hline Vychegda & Kel'tminskaya-1 & 2690 & 0.010 & 1.890 & 5.240 \\
\hline Vychegda & Kel'tminskaya-1 & 2691 & 0.010 & 1.420 & 5.780 \\
\hline Vychegda & Kel'tminskaya-1 & 2692 & 0.000 & 1.730 & 5.310 \\
\hline Vychegda & Kel'tminskaya-1 & 2728 & 0.020 & 0.340 & 2.380 \\
\hline Vychegda & Kel'tminskaya-1 & 2772 & 0.050 & 0.540 & 2.540 \\
\hline Vychegda & Kel'tminskaya-1 & 2772.5 & 0.010 & 0.480 & 1.230 \\
\hline Vychegda & Kel'tminskaya-1 & 2773.5 & 0.300 & 0.820 & 2.930 \\
\hline Vychegda & Kel'tminskaya-1 & 2774 & 0.040 & 0.570 & 3.160 \\
\hline Vychegda & Kel'tminskaya-1 & 2776 & 0.280 & 0.860 & 2.980 \\
\hline Vychegda & Kel'tminskaya-1 & 2777 & 0.060 & 0.580 & 2.870 \\
\hline Vychegda & Kel'tminskaya-1 & 2778 & 0.240 & 0.640 & 2.870 \\
\hline Vychegda & Kel'tminskaya-1 & 2779 & 0.010 & 0.410 & 4.350 \\
\hline Vychegda & Kel'tminskaya-1 & 2794 & 0.000 & 0.890 & 4.740 \\
\hline Vychegda & Kel'tminskaya-1 & 2797 & 0.010 & 1.840 & 8.030 \\
\hline Vychegda & Kel'tminskaya-1 & 2821 & 0.090 & 1.120 & 6.100 \\
\hline Vychegda & Kel'tminskaya-1 & 2858 & 0.060 & 0.760 & 5.720 \\
\hline Vychegda & Kel'tminskaya-1 & 2901 & 0.010 & 0.890 & 5.230 \\
\hline Isaac & Isaac & 790 & 0.100 & 0.360 & 6.000 \\
\hline Isaac & Isaac & 797.83 & 0.270 & 0.620 & 6.010 \\
\hline Isaac & Isaac & 805.66 & 0.300 & 0.850 & 5.120 \\
\hline Isaac & Isaac & 813.49 & 0.010 & 0.370 & 8.920 \\
\hline Isaac & Isaac & 821.32 & 0.260 & 0.610 & 7.230 \\
\hline Isaac & Isaac & 829.15 & 0.110 & 0.670 & 5.500 \\
\hline Isaac & Isaac & 836.98 & 0.000 & 0.260 & 5.260 \\
\hline Isaac & Isaac & 844.81 & 0.020 & 0.360 & 6.240 \\
\hline Isaac & Isaac & 852.64 & 0.160 & 0.760 & 7.920 \\
\hline Isaac & Isaac & 860.47 & 0.460 & 1.840 & 4.040 \\
\hline Isaac & Isaac & 868.3 & 0.040 & 0.570 & 1.400 \\
\hline Isaac & Isaac & 876.13 & 0.300 & 1.110 & 7.210 \\
\hline Isaac & Isaac & 883.96 & 0.240 & 0.970 & 8.180 \\
\hline Isaac & Isaac & 891.79 & 0.050 & 0.370 & 6.000 \\
\hline Isaac & Isaac & 899.62 & 0.010 & 0.470 & 6.880 \\
\hline Isaac & Isaac & 915.28 & 0.230 & 2.700 & 4.030 \\
\hline
\end{tabular}




\begin{tabular}{|c|c|c|c|c|c|}
\hline Isaac & Isaac & 923.11 & 0.170 & 0.610 & 6.030 \\
\hline Isaac & Isaac & 930.94 & 0.080 & 0.440 & 6.640 \\
\hline Isaac & Isaac & 938.77 & 0.070 & 0.470 & 5.840 \\
\hline Isaac & Isaac & 946.6 & 0.030 & 0.430 & 6.400 \\
\hline Isaac & Isaac & 946.6 & 0.090 & 5.810 & 6.450 \\
\hline Isaac & Isaac & 954.43 & 0.130 & 0.490 & 5.820 \\
\hline Isaac & Isaac & 962.2 & 0.030 & 0.530 & 5.540 \\
\hline Isaac & Isaac & 970 & 0.100 & 1.900 & 2.160 \\
\hline Isaac & Isaac & 980 & 0.080 & 1.100 & 1.960 \\
\hline Isaac & Isaac & 990 & 0.540 & 1.200 & 2.260 \\
\hline Isaac & Isaac & 1000 & 0.460 & 2.390 & 4.700 \\
\hline Isaac & Isaac & 1010 & 0.120 & 0.990 & 3.320 \\
\hline Isaac & Isaac & 1020 & 0.010 & 1.130 & 2.290 \\
\hline Isaac & Isaac & 1030 & 0.120 & 0.340 & 4.970 \\
\hline Isaac & Isaac & 1040 & 0.560 & 1.330 & 4.450 \\
\hline Isaac & Isaac & 1050 & 0.070 & 0.640 & 6.120 \\
\hline Isaac & Isaac & 1060 & 0.010 & 0.310 & 6.680 \\
\hline Isaac & Isaac & 1070 & 0.030 & 0.480 & 5.520 \\
\hline Isaac & Isaac & 1080 & 0.480 & 1.140 & 2.770 \\
\hline Isaac & Isaac & 1090 & 0.010 & 0.190 & 3.410 \\
\hline Isaac & Isaac & 1102.1 & 0.040 & 0.750 & 1.440 \\
\hline Isaac & Isaac & 1114.2 & 0.000 & 0.290 & 0.670 \\
\hline Isaac & Isaac & 1126.3 & 0.350 & 0.800 & 4.350 \\
\hline Isaac & Isaac & 1138.4 & 0.010 & 0.910 & 3.710 \\
\hline Isaac & Isaac & 1150.5 & 0.070 & 0.660 & 6.270 \\
\hline Isaac & Isaac & 1162.6 & 0.010 & 1.090 & 1.810 \\
\hline Isaac & Isaac & 1175 & 0.310 & 0.620 & 4.770 \\
\hline Isaac & Isaac & 1186.67 & 0.050 & 0.470 & 7.000 \\
\hline Isaac & Isaac & 1198.34 & 0.010 & 0.280 & 5.980 \\
\hline Isaac & Isaac & 1198.34 & 0.010 & 0.290 & 5.900 \\
\hline Isaac & Isaac & 1210.01 & 0.010 & 0.460 & 5.630 \\
\hline Isaac & Isaac & 1221.68 & 0.010 & 0.320 & 5.800 \\
\hline Isaac & Isaac & 1233.35 & 0.030 & 0.350 & 5.310 \\
\hline Isaac & Isaac & 1245.02 & 0.470 & 0.870 & 7.270 \\
\hline Isaac & Isaac & 1252.22 & 0.120 & 0.880 & 1.320 \\
\hline Isaac & Isaac & 1259.42 & 0.210 & 0.570 & 4.510 \\
\hline Isaac & Isaac & 1266.62 & 0.140 & 0.470 & 1.150 \\
\hline Isaac & Isaac & 1273.82 & 0.610 & 1.280 & 5.940 \\
\hline Isaac & Isaac & 1281.02 & 0.020 & 1.190 & 3.270 \\
\hline Isaac & Isaac & 1288.22 & 0.010 & 0.680 & 0.890 \\
\hline Isaac & Isaac & 1295.42 & 0.010 & 0.730 & 1.000 \\
\hline
\end{tabular}




\begin{tabular}{|c|c|c|c|c|c|}
\hline Isaac & Isaac & 1310 & 0.010 & 0.270 & 6.000 \\
\hline Isaac & Isaac & 1310 & 0.000 & 0.250 & 4.530 \\
\hline Isaac & Isaac & 1323.1 & 0.290 & 0.770 & 6.670 \\
\hline Isaac & Isaac & 1336.2 & 0.120 & 0.410 & 5.740 \\
\hline Isaac & Isaac & 1336.2 & 1.040 & 1.300 & 4.590 \\
\hline Isaac & Isaac & 1349.3 & 0.170 & 0.520 & 2.230 \\
\hline Isaac & Isaac & 1362.4 & 0.020 & 0.320 & 5.080 \\
\hline Isaac & Isaac & 1375.5 & 0.440 & 0.740 & 3.120 \\
\hline Isaac & Isaac & 1388.6 & 0.450 & 0.850 & 3.620 \\
\hline Isaac & Isaac & 1401.7 & 0.190 & 1.120 & 3.760 \\
\hline Isaac & Isaac & 1414.8 & 1.050 & 1.430 & 4.040 \\
\hline Isaac & Isaac & 1427.9 & 0.600 & 1.280 & 3.840 \\
\hline Isaac & Isaac & 1440 & 0.290 & 0.540 & 3.830 \\
\hline Isaac & Isaac & 1450.77 & 0.210 & 0.600 & 4.400 \\
\hline Isaac & Isaac & 1461.54 & 1.410 & 2.280 & 2.590 \\
\hline Isaac & Isaac & 1472.31 & 0.200 & 1.030 & 4.220 \\
\hline Isaac & Isaac & 1472.31 & 0.830 & 1.270 & 2.590 \\
\hline Isaac & Isaac & 1483.08 & 0.570 & 1.000 & 4.720 \\
\hline Isaac & Isaac & 1483.08 & 0.130 & 1.100 & 4.600 \\
\hline Isaac & Isaac & 1493.85 & 0.220 & 0.850 & 3.590 \\
\hline Isaac & Isaac & 1504.62 & 0.150 & 1.740 & 4.950 \\
\hline Isaac & Isaac & 1515.39 & 1.170 & 1.670 & 2.430 \\
\hline Isaac & Isaac & 1526.16 & 0.080 & 0.680 & 2.400 \\
\hline Isaac & Isaac & 1547.7 & 0.120 & 0.890 & 2.000 \\
\hline Isaac & Isaac & 1558.47 & 0.340 & 1.440 & 4.320 \\
\hline Isaac & Isaac & 1569.24 & 0.520 & 1.750 & 3.050 \\
\hline Isaac & Isaac & 1580.01 & 0.030 & 0.430 & 1.830 \\
\hline Isaac & Isaac & 1590 & 0.040 & 0.950 & 1.760 \\
\hline Isaac & Isaac & 1600 & 0.230 & 0.820 & 4.280 \\
\hline Isaac & Isaac & 1612 & 0.520 & 1.350 & 4.500 \\
\hline Isaac & Isaac & 1624 & 0.320 & 1.080 & 2.200 \\
\hline Isaac & Isaac & 1636 & 0.290 & 2.410 & 2.660 \\
\hline Isaac & Isaac & 1648 & 0.190 & 0.940 & 2.830 \\
\hline Isaac & Isaac & 1660 & 0.880 & 1.670 & 3.950 \\
\hline Isaac & Isaac & 1670 & 0.870 & 1.150 & 2.420 \\
\hline Isaac & Isaac & 1680 & 0.750 & 2.430 & 4.890 \\
\hline Isaac & Isaac & 1690 & 0.470 & 3.140 & 3.100 \\
\hline Isaac & Isaac & 1700 & 0.540 & 2.100 & 4.410 \\
\hline Isaac & Isaac & 1710 & 0.060 & 0.500 & 3.220 \\
\hline Isaac & Isaac & 1720 & 0.430 & 0.860 & 4.370 \\
\hline Isaac & Isaac & 1730 & 0.420 & 1.190 & 4.940 \\
\hline
\end{tabular}




\begin{tabular}{|c|c|c|c|c|c|}
\hline Isaac & Isaac & 1740 & 0.130 & 0.610 & 3.490 \\
\hline Isaac & Isaac & 1750 & 0.080 & 0.840 & 4.360 \\
\hline Isaac & Isaac & 1760 & 0.070 & 1.010 & 5.170 \\
\hline Isaac & Isaac & 1770 & 0.110 & 0.570 & 6.470 \\
\hline Isaac & Isaac & 1780 & 0.070 & 0.590 & 2.600 \\
\hline Isaac & Isaac & 1792.5 & 0.260 & 1.280 & 4.750 \\
\hline Isaac & Isaac & 1805 & 1.470 & 1.880 & 5.190 \\
\hline Isaac & Isaac & 1817.5 & 0.010 & 0.370 & 3.530 \\
\hline Isaac & Isaac & 1825 & 0.560 & 1.110 & 3.760 \\
\hline Isaac & Isaac & 1832.5 & 0.010 & 0.510 & 1.670 \\
\hline Isaac & Isaac & 1840 & 0.070 & 0.480 & 1.740 \\
\hline Isaac & Isaac & 1847.5 & 0.120 & 1.010 & 2.850 \\
\hline Isaac & Isaac & 1855 & 0.090 & 0.470 & 4.550 \\
\hline Isaac & Isaac & 1868.8 & 0.160 & 0.640 & 5.380 \\
\hline Isaac & Isaac & 1882.6 & 0.830 & 1.400 & 5.170 \\
\hline Isaac & Isaac & 1896.4 & 0.450 & 7.280 & 5.860 \\
\hline Isaac & Isaac & 1910.2 & 0.660 & 4.100 & 3.830 \\
\hline Isaac & Isaac & 1924 & 0.090 & 3.160 & 2.680 \\
\hline Isaac & Isaac & 1937.8 & 0.260 & 0.840 & 3.530 \\
\hline Isaac & Isaac & 1951.6 & 0.060 & 3.280 & 3.030 \\
\hline Isaac & Isaac & 1965.4 & 0.630 & 1.770 & 4.960 \\
\hline Isaac & Isaac & 1979.2 & 0.150 & 0.730 & 4.690 \\
\hline Isaac & Isaac & 1993 & 0.180 & 0.830 & 4.390 \\
\hline Isaac & Isaac & 2006.8 & 0.480 & 1.050 & 1.220 \\
\hline Isaac & Isaac & 2020.6 & 0.410 & 1.340 & 2.100 \\
\hline Isaac & Isaac & 2034.4 & 2.270 & 4.170 & 7.040 \\
\hline Isaac & Isaac & 2062 & 0.790 & 2.570 & 3.790 \\
\hline Isaac & Isaac & 2075.8 & 0.810 & 3.010 & 3.020 \\
\hline Isaac & Isaac & 2089.6 & 0.500 & 1.450 & 3.430 \\
\hline Isaac & Isaac & 2103.4 & 0.450 & 1.400 & 3.780 \\
\hline Isaac & Isaac & 2117.2 & 0.610 & 1.280 & 3.420 \\
\hline Isaac & Isaac & 2131 & 0.670 & 1.820 & 3.680 \\
\hline Old Fort Point & Old Fort Point & & 0.000 & 1.180 & 3.440 \\
\hline Old Fort Point & Old Fort Point & & 0.380 & 0.690 & 1.930 \\
\hline Old Fort Point & Old Fort Point & & 0.050 & 0.690 & 1.470 \\
\hline Old Fort Point & Old Fort Point & & 1.480 & 2.270 & 3.750 \\
\hline Upper Kaza & Upper Kaza & 380 & 0.000 & 0.280 & 4.230 \\
\hline Upper Kaza & Upper Kaza & 390 & 0.210 & 1.200 & 5.350 \\
\hline Upper Kaza & Upper Kaza & 400 & 0.030 & 0.250 & 4.620 \\
\hline Upper Kaza & Upper Kaza & 410 & 0.010 & 0.560 & 3.830 \\
\hline Upper Kaza & Upper Kaza & 420 & 0.000 & 0.550 & 3.440 \\
\hline
\end{tabular}




\begin{tabular}{|c|c|c|c|c|c|}
\hline Upper Kaza & Upper Kaza & 430 & 0.050 & 0.560 & 3.140 \\
\hline Upper Kaza & Upper Kaza & 440 & 0.180 & 0.470 & 5.020 \\
\hline Upper Kaza & Upper Kaza & 450 & 0.460 & 1.530 & 5.870 \\
\hline Upper Kaza & Upper Kaza & 460 & 0.090 & 0.650 & 3.860 \\
\hline Upper Kaza & Upper Kaza & 470 & 0.000 & 0.250 & 3.630 \\
\hline Upper Kaza & Upper Kaza & 480 & 0.000 & 0.570 & 3.930 \\
\hline Upper Kaza & Upper Kaza & 490 & 0.010 & 0.550 & 3.180 \\
\hline Upper Kaza & Upper Kaza & 500 & 0.030 & 0.660 & 3.980 \\
\hline Upper Kaza & Upper Kaza & 510 & 0.000 & 0.490 & 2.080 \\
\hline Upper Kaza & Upper Kaza & 520 & 0.000 & 0.320 & 6.590 \\
\hline Upper Kaza & Upper Kaza & 530 & 0.010 & 0.510 & 3.550 \\
\hline Upper Kaza & Upper Kaza & 540 & 0.000 & 0.550 & 4.920 \\
\hline Upper Kaza & Upper Kaza & 540 & 0.000 & 0.250 & 1.930 \\
\hline Upper Kaza & Upper Kaza & 550 & 0.280 & 0.890 & 4.920 \\
\hline Upper Kaza & Upper Kaza & 558.33 & 0.000 & 0.460 & 3.730 \\
\hline Upper Kaza & Upper Kaza & 566.66 & 0.120 & 0.570 & 4.470 \\
\hline Upper Kaza & Upper Kaza & 574.99 & 0.160 & 0.690 & 5.000 \\
\hline Upper Kaza & Upper Kaza & 583.32 & 0.290 & 0.740 & 5.560 \\
\hline Upper Kaza & Upper Kaza & 591.65 & 0.270 & 0.570 & 4.480 \\
\hline Upper Kaza & Upper Kaza & 599.98 & 0.000 & 0.590 & 3.950 \\
\hline Upper Kaza & Upper Kaza & 608.31 & 0.020 & 1.190 & 4.340 \\
\hline Upper Kaza & Upper Kaza & 616.64 & 0.030 & 0.580 & 8.530 \\
\hline Upper Kaza & Upper Kaza & 624.97 & 0.020 & 0.560 & 2.810 \\
\hline Upper Kaza & Upper Kaza & 633.3 & 0.010 & 0.910 & 4.000 \\
\hline Upper Kaza & Upper Kaza & 641.63 & 0.000 & 0.190 & 5.360 \\
\hline Upper Kaza & Upper Kaza & 649.96 & 0.180 & 0.520 & 5.670 \\
\hline Upper Kaza & Upper Kaza & 655.83 & 0.050 & 0.440 & 7.520 \\
\hline Upper Kaza & Upper Kaza & 655.83 & 0.100 & 0.500 & 5.040 \\
\hline Upper Kaza & Upper Kaza & 661.66 & 0.130 & 0.510 & 4.090 \\
\hline Upper Kaza & Upper Kaza & 667.49 & 0.000 & 0.320 & 2.910 \\
\hline Upper Kaza & Upper Kaza & 673.32 & 0.010 & 0.330 & 6.210 \\
\hline Upper Kaza & Upper Kaza & 679.15 & 0.000 & 0.430 & 2.300 \\
\hline Upper Kaza & Upper Kaza & 684.98 & 0.030 & 0.440 & 1.970 \\
\hline Upper Kaza & Upper Kaza & 690.81 & 0.030 & 0.580 & 7.920 \\
\hline Upper Kaza & Upper Kaza & 696.64 & 0.010 & 0.640 & 5.260 \\
\hline Upper Kaza & Upper Kaza & 702.47 & 0.000 & 0.460 & 4.530 \\
\hline Upper Kaza & Upper Kaza & 708.3 & 0.020 & 0.380 & 4.760 \\
\hline Upper Kaza & Upper Kaza & 714.13 & 0.010 & 0.450 & 7.140 \\
\hline Upper Kaza & Upper Kaza & 719.96 & 0.020 & 0.420 & 3.840 \\
\hline Upper Kaza & Upper Kaza & 725.79 & 0.170 & 0.630 & 11.610 \\
\hline Upper Kaza & Upper Kaza & 731.62 & 0.090 & 0.390 & 6.440 \\
\hline
\end{tabular}




\begin{tabular}{|c|c|c|c|c|c|}
\hline Upper Kaza & Upper Kaza & 737.45 & 0.020 & 0.380 & 1.620 \\
\hline Upper Kaza & Upper Kaza & 743.28 & 0.040 & 0.640 & 2.850 \\
\hline Upper Kaza & Upper Kaza & 749.11 & 0.460 & 0.640 & 2.980 \\
\hline Upper Kaza & Upper Kaza & 754.94 & 0.050 & 0.260 & 4.250 \\
\hline Upper Kaza & Upper Kaza & 760.77 & 0.460 & 0.930 & 6.150 \\
\hline Upper Kaza & Upper Kaza & 766.6 & 0.010 & 0.400 & 3.710 \\
\hline Upper Kaza & Upper Kaza & 772.43 & 0.010 & 0.790 & 1.970 \\
\hline Upper Kaza & Upper Kaza & 778.26 & 0.010 & 0.950 & 2.140 \\
\hline Upper Kaza & Upper Kaza & 784.09 & 0.020 & 0.520 & 0.970 \\
\hline \multicolumn{3}{|c|}{ Lower Drook @ HzLLower Drook @ Harwermost Droı } & 0.000 & 1.080 & 4.300 \\
\hline Lower Fermeuse & Lower Fermeuse & 2 & 0.090 & 0.730 & 4.710 \\
\hline Lower Fermeuse & Lower Fermeuse & 5 & 0.210 & 0.840 & 4.720 \\
\hline Lower Fermeuse & Lower Fermeuse & 8 & 0.120 & 0.780 & 4.760 \\
\hline Lower Fermeuse & Lower Fermeuse & 13 & 0.090 & 0.780 & 4.960 \\
\hline Lower Fermeuse & Lower Fermeuse & 18 & 0.010 & 0.780 & 4.460 \\
\hline Lower Fermeuse & Lower Fermeuse & 23 & 0.050 & 0.590 & 4.440 \\
\hline Lower Fermeuse & Lower Fermeuse & 28 & 0.170 & 0.920 & 4.270 \\
\hline Lower Fermeuse & Lower Fermeuse & 33 & 0.230 & 0.890 & 4.130 \\
\hline Lower Fermeuse & Lower Fermeuse & 38 & 0.020 & 0.610 & 3.150 \\
\hline Lower Fermeuse & Lower Fermeuse & 47 & 0.020 & 0.710 & 4.400 \\
\hline Lower Fermeuse & Lower Fermeuse & nning of sequ & 0.050 & 0.730 & 4.550 \\
\hline lower Gaskiers & lower Gaskiers & 0.5 & 0.080 & 1.520 & 3.510 \\
\hline lower Gaskiers & lower Gaskiers & 6.5 & 0.010 & 1.540 & 4.730 \\
\hline lower Gaskiers & lower Gaskiers & 7.5 & 0.010 & 1.000 & 3.020 \\
\hline lower Gaskiers & lower Gaskiers & 9.5 & 0.000 & 1.160 & 3.730 \\
\hline lower Gaskiers & lower Gaskiers & 11.5 & 0.010 & 1.100 & 4.070 \\
\hline lower Gaskiers & lower Gaskiers & 13.5 & 0.000 & 1.600 & 4.610 \\
\hline lower Gaskiers & lower Gaskiers & 16.5 & 0.010 & 1.540 & 4.450 \\
\hline lower Gaskiers & lower Gaskiers & rermost Gaski & 0.020 & 1.160 & 2.930 \\
\hline \multicolumn{2}{|c|}{ Lower Mistaken PcLower Mistaken Poi } & 2 & 0.080 & 1.070 & 4.280 \\
\hline \multicolumn{2}{|c|}{ Lower Mistaken PcLower Mistaken Poi } & 5 & 0.000 & 0.760 & 3.770 \\
\hline \multicolumn{2}{|c|}{ Lower Mistaken PcLower Mistaken Poi } & 8 & 0.000 & 0.540 & 3.760 \\
\hline \multicolumn{2}{|c|}{ Lower Mistaken PcLower Mistaken Poi } & 8.3 & 0.000 & 0.880 & 4.320 \\
\hline \multicolumn{2}{|c|}{ Lower Mistaken PıLower Mistaken Poi } & 13.3 & 0.010 & 0.420 & 2.520 \\
\hline \multicolumn{2}{|c|}{ Lower Mistaken PıLower Mistaken Poi } & 16.3 & 0.000 & 0.430 & 3.600 \\
\hline \multicolumn{2}{|c|}{ Lower Mistaken Pc Lower Mistaken Poi } & 19.3 & 0.000 & 0.370 & 1.870 \\
\hline \multicolumn{2}{|c|}{ Lower Mistaken PıLower Mistaken Poi } & 19.8 & 0.010 & 0.380 & 3.820 \\
\hline \multicolumn{3}{|c|}{ Lower Mistaken P(Lower Mistaken Poinning of sequ } & 0.000 & 0.750 & 4.040 \\
\hline \multicolumn{2}{|c|}{ Lowermost Drook Lowermost Drook a } & 2 & 0.010 & 0.410 & 2.180 \\
\hline \multicolumn{2}{|c|}{ Lowermost Drook Lowermost Drook a } & 2 & 0.000 & 0.810 & 3.330 \\
\hline \multicolumn{2}{|c|}{ Lowermost Drook Lowermost Drook a } & 2 & 0.000 & 0.640 & 2.650 \\
\hline
\end{tabular}




\begin{tabular}{|c|c|c|c|c|c|}
\hline \multicolumn{2}{|c|}{ Lowermost Drook Lowermost Drook a } & 2 & 0.000 & 0.710 & 2.670 \\
\hline \multicolumn{2}{|c|}{ Lowermost Drook Lowermost Drook a } & 3 & 0.000 & 1.240 & 4.160 \\
\hline \multicolumn{2}{|c|}{ Lowermost Drook Lowermost Drook a } & 5 & 0.000 & 0.540 & 2.770 \\
\hline \multicolumn{2}{|c|}{ Lowermost Drook Lowermost Drook a } & 9 & 0.010 & 0.610 & 2.830 \\
\hline \multicolumn{2}{|c|}{ Lowermost Drook Lowermost Drook a } & 13 & 0.070 & 0.930 & 3.530 \\
\hline \multicolumn{2}{|c|}{ Lowermost Drook Lowermost Drook a } & 18 & 0.010 & 0.680 & 3.620 \\
\hline \multicolumn{2}{|c|}{ Lowermost Drook Lowermost Drook a } & 28 & 0.010 & 0.620 & 3.340 \\
\hline \multicolumn{3}{|c|}{ Lowermost Drook Lowermost Drook awermost Droı } & 0.000 & 1.680 & 3.910 \\
\hline Middle Briscal & Middle Briscal & 2 & 0.000 & 1.160 & 4.520 \\
\hline Middle Briscal & Middle Briscal & 4 & 0.000 & 0.890 & 4.260 \\
\hline Middle Briscal & Middle Briscal & 6 & 0.040 & 0.940 & 3.820 \\
\hline Middle Briscal & Middle Briscal & 9 & 0.010 & 0.620 & 2.480 \\
\hline Middle Briscal & Middle Briscal & 12 & 0.000 & 0.820 & 3.470 \\
\hline Middle Briscal & Middle Briscal & 15 & 0.000 & 0.400 & 2.350 \\
\hline Middle Briscal & Middle Briscal & 18 & 0.010 & 0.580 & 2.510 \\
\hline Middle Briscal & Middle Briscal & 21 & 0.000 & 0.740 & 3.160 \\
\hline Middle Briscal & Middle Briscal & 24 & 0.000 & 0.760 & 3.580 \\
\hline Middle Briscal & Middle Briscal & 27 & 0.000 & 1.110 & 4.680 \\
\hline Middle Briscal & Middle Briscal & 30 & 0.000 & 0.950 & 3.620 \\
\hline Middle Briscal & Middle Briscal & 33 & 0.000 & 1.070 & 3.680 \\
\hline Middle Briscal & Middle Briscal & next contour & 0.000 & 1.090 & 4.320 \\
\hline Middle Briscal & Middle Briscal & next contour & 0.000 & 0.980 & 3.480 \\
\hline Middle Briscal & Middle Briscal & of sequence, & 0.000 & 1.130 & 4.090 \\
\hline Middle Drook Nev & Middle Drook New & 5 & 0.000 & 1.330 & 4.700 \\
\hline Middle Drook Nev & Middle Drook New & 10 & 0.080 & 0.630 & 1.860 \\
\hline Middle Drook Nev & Middle Drook New & 15 & 0.060 & 0.460 & 1.590 \\
\hline Middle Drook Nev & Middle Drook New & 20 & 0.030 & 0.750 & 3.250 \\
\hline Middle Drook Nev & Middle Drook New & 25 & 0.010 & 0.820 & 2.860 \\
\hline Middle Drook Nev & Middle Drook New & 35 & 0.000 & 0.540 & 2.190 \\
\hline Middle Drook Nev & Middle Drook New & 45 & 0.100 & 0.640 & 2.390 \\
\hline Middle Drook Nev & Middle Drook New & 55 & 0.070 & 0.590 & 2.150 \\
\hline Middle Drook Nev & Middle Drook New & 65 & 0.020 & 0.590 & 2.330 \\
\hline Middle Drook Nev & Middle Drook New & nning of sequ & 0.130 & 0.820 & 2.670 \\
\hline Middle Gaskiers & Middle Gaskiers & 31 & 0.060 & 1.170 & 3.970 \\
\hline Middle Gaskiers & Middle Gaskiers & 51 & 0.080 & 0.850 & 3.690 \\
\hline Middle Gaskiers & Middle Gaskiers & 81 & 0.020 & 1.060 & 3.840 \\
\hline Middle Gaskiers & Middle Gaskiers & ıbove Gaskier: & 0.140 & 0.830 & 3.130 \\
\hline Middle Gaskiers & Middle Gaskiers & elow top of $\mathrm{G}$ & 0.010 & 1.230 & 3.990 \\
\hline Middle Mistaken F & Middle Mistaken Pc & 2 & 0.000 & 1.380 & 4.520 \\
\hline Middle Mistaken F & Middle Mistaken Pc & 4 & 0.000 & 0.540 & 3.500 \\
\hline Middle Mistaken F & Middle Mistaken Pc & 5.3 & 0.000 & 0.630 & 2.950 \\
\hline
\end{tabular}




\begin{tabular}{|c|c|c|c|c|}
\hline Middle Mistaken F Middle Mistaken Pc & 5.5 & 0.000 & 0.990 & 5.790 \\
\hline Middle Mistaken FMiddle Mistaken Pc & 13 & 0.000 & 1.030 & 4.380 \\
\hline Middle Mistaken F Middle Mistaken Pc & 15 & 0.000 & 0.900 & 3.360 \\
\hline Middle Mistaken F Middle Mistaken Pc & 17 & 0.000 & 1.210 & 4.750 \\
\hline Middle Mistaken F Middle Mistaken Pc & 19 & 0.000 & 1.160 & 4.340 \\
\hline Middle Mistaken FMiddle Mistaken Pc & 20 & 0.000 & 0.940 & 5.230 \\
\hline Middle Mistaken F Middle Mistaken Pc & 22 & 0.000 & 0.820 & 4.250 \\
\hline Middle Mistaken FMiddle Mistaken Pc & 24 & 0.000 & 0.800 & 3.620 \\
\hline \multicolumn{2}{|c|}{ Middle Mistaken F Middle Mistaken Pcn below top $k$} & 0.000 & 1.040 & 4.230 \\
\hline \multicolumn{2}{|c|}{ Middle Mistaken F Middle Mistaken Pc1m above bec } & 0.000 & 0.920 & 4.030 \\
\hline \multicolumn{2}{|c|}{ Middle Mistaken F Middle Mistaken Pc1 m above be } & 0.000 & 1.050 & 4.410 \\
\hline \multicolumn{2}{|c|}{ Middle Mistaken F Middle Mistaken Pcnning of sequ } & 0.000 & 1.120 & 5.110 \\
\hline Middle to Upper F Middle to Upper Fe & 2 & 0.400 & 1.250 & 5.040 \\
\hline Middle to Upper F Middle to Upper Fe & 4 & 0.000 & 0.770 & 4.580 \\
\hline Middle to Upper F Middle to Upper Fe & 6 & 0.230 & 0.910 & 4.700 \\
\hline Middle to Upper F Middle to Upper Fe & 8 & 0.080 & 0.770 & 3.640 \\
\hline Middle to Upper F Middle to Upper Fe & 10 & 0.080 & 1.110 & 3.930 \\
\hline Middle to Upper F Middle to Upper Fe & 12 & 0.140 & 1.080 & 4.230 \\
\hline Middle to Upper F Middle to Upper Fe & 14 & 0.110 & 1.060 & 3.950 \\
\hline Middle to Upper F Middle to Upper Fe & 16 & 0.060 & 0.940 & 3.530 \\
\hline Middle to Upper F Middle to Upper Fe & 18 & 0.120 & 0.570 & 2.450 \\
\hline Middle to Upper F Middle to Upper Fe & 20 & 0.020 & 0.730 & 4.230 \\
\hline Middle to Upper F Middle to Upper Fe & 22 & 0.020 & 0.800 & 4.440 \\
\hline Middle to Upper F Middle to Upper Fe & 24 & 0.060 & 0.830 & 4.590 \\
\hline Middle to Upper F Middle to Upper Fe & 26 & 0.020 & 0.810 & 4.480 \\
\hline Middle to Upper F Middle to Upper Fe & 28 & 0.020 & 0.890 & 4.660 \\
\hline Middle to Upper F Middle to Upper Fe & 30 & 0.040 & 0.860 & 4.580 \\
\hline Middle to Upper F Middle to Upper Fe & 32 & 0.040 & 0.800 & 4.400 \\
\hline Middle to Upper F Middle to Upper Fe & 34 & 0.080 & 0.900 & 4.960 \\
\hline \multicolumn{2}{|c|}{ Middle to Upper F Middle to Upper Fenning of sequ } & 0.060 & 0.780 & 3.450 \\
\hline Middle to Upper T Middle to Upper Tr & 3 & 0.000 & 0.750 & 4.070 \\
\hline Middle to Upper T Middle to Upper Tr & 8 & 0.020 & 0.910 & 4.790 \\
\hline Middle to Upper T Middle to Upper Tr & 13 & 0.000 & 0.890 & 4.500 \\
\hline Middle to Upper T Middle to Upper Tr & 13 & 0.720 & 1.800 & 5.390 \\
\hline Middle to Upper T Middle to Upper Tr & 58 & 0.330 & 1.510 & 5.490 \\
\hline Middle to Upper T Middle to Upper Tr & 68 & 0.010 & 1.010 & 4.150 \\
\hline Middle to Upper T Middle to Upper Tr & 68 & 0.550 & 1.410 & 4.940 \\
\hline Middle to Upper T Middle to Upper Tr & 108 & 0.210 & 1.300 & 5.610 \\
\hline Middle to Upper T Middle to Upper Tr & 133 & 0.010 & 0.800 & 4.280 \\
\hline Middle to Upper T Middle to Upper Tr & 148 & 0.020 & 0.930 & 4.390 \\
\hline Middle to Upper T Middle to Upper Tr & 150 & 0.000 & 1.000 & 4.310 \\
\hline
\end{tabular}




\begin{tabular}{|c|c|c|c|c|c|}
\hline \multicolumn{2}{|c|}{ Middle to Upper T Middle to Upper Tr } & 170 & 0.140 & 1.020 & 3.770 \\
\hline \multicolumn{2}{|c|}{ Middle to Upper T Middle to Upper Tr } & 180 & 0.010 & 0.970 & 3.340 \\
\hline \multicolumn{2}{|c|}{ Middle to Upper T Middle to Upper Tr } & 200 & 0.000 & 0.820 & 3.300 \\
\hline \multicolumn{2}{|c|}{ Middle to Upper T Middle to Upper Tr } & 220 & 0.130 & 1.020 & 4.120 \\
\hline \multicolumn{3}{|c|}{ Middle to Upper T Middle to Upper Tr, top of Trepa } & 0.360 & 1.260 & 5.050 \\
\hline \multicolumn{3}{|c|}{ Middle to Upper T Middle to Upper Trnning of sequ } & 0.020 & 0.990 & 4.720 \\
\hline \multicolumn{2}{|c|}{ middle upper Mall middle upper Mall I } & 10 & 0.180 & 0.980 & 3.200 \\
\hline \multicolumn{2}{|c|}{ middle upper Mall middle upper Mall I } & 20 & 0.040 & 0.960 & 3.500 \\
\hline \multicolumn{2}{|c|}{ middle upper Mall middle upper Mall I } & 30 & 0.080 & 1.000 & 3.580 \\
\hline \multicolumn{2}{|c|}{ middle upper Mall middle upper Mall I } & 37 & 0.010 & 0.870 & 2.950 \\
\hline \multicolumn{2}{|c|}{ middle upper Mall middle upper Mall I } & 44 & 0.050 & 1.230 & 3.680 \\
\hline \multicolumn{2}{|c|}{ middle upper Mall middle upper Mall I } & 51 & 0.020 & 1.420 & 4.770 \\
\hline \multicolumn{2}{|c|}{ middle upper Mall middle upper Mall I } & 58 & 0.010 & 0.970 & 3.060 \\
\hline \multicolumn{2}{|c|}{ middle upper Mall middle upper Mall I } & 65 & 0.010 & 1.230 & 4.190 \\
\hline \multicolumn{2}{|c|}{ middle upper Mall middle upper Mall I } & 72 & 0.000 & 1.010 & 3.590 \\
\hline \multicolumn{2}{|c|}{ middle upper Mall middle upper Mall I } & 79 & 0.020 & 1.090 & 3.280 \\
\hline \multicolumn{2}{|c|}{ middle upper Mall middle upper Mall I } & 86 & 0.020 & 1.100 & 3.680 \\
\hline \multicolumn{2}{|c|}{ middle upper Mall middle upper Mall I } & 93 & 0.000 & 0.870 & 3.850 \\
\hline \multicolumn{2}{|c|}{ middle upper Mall middle upper Mall I } & 95 & 0.000 & 0.780 & 2.840 \\
\hline \multicolumn{2}{|c|}{ middle upper Mall middle upper Mall I } & 97 & 0.010 & 0.880 & 3.390 \\
\hline \multicolumn{2}{|c|}{ middle upper Mall middle upper Mall I } & 99 & 0.030 & 1.100 & 3.080 \\
\hline \multicolumn{2}{|c|}{ middle upper Mall middle upper Mall I } & 101 & 0.000 & 1.060 & 3.660 \\
\hline \multicolumn{2}{|c|}{ middle upper Mall middle upper Mall I } & 103 & 0.000 & 1.440 & 4.180 \\
\hline \multicolumn{2}{|c|}{ middle upper Mall middle upper Mall I } & 105 & 0.000 & 0.460 & 2.030 \\
\hline \multicolumn{2}{|c|}{ middle upper Mall middle upper Mall I } & 107 & 0.000 & 0.720 & 2.090 \\
\hline \multicolumn{2}{|c|}{ middle upper Mall middle upper Mall I } & 109 & 0.000 & 0.850 & 2.760 \\
\hline middle upper $\Lambda$ & I middle upper Mall I & 111 & 0.000 & 1.130 & 3.390 \\
\hline middle upper $\Lambda$ & I middle upper Mall I & 113 & 0.570 & 1.600 & 3.140 \\
\hline middle upper $I$ & I middle upper Mall I & 115 & 0.000 & 1.000 & 3.610 \\
\hline middle upper $\Lambda$ & I middle upper Mall I & 117 & 0.030 & 0.870 & 2.380 \\
\hline middle upper $I$ & I middle upper Mall I & 119 & 0.040 & 0.850 & 2.130 \\
\hline middle upper $I$ & I middle upper Mall I & 121 & 0.040 & 0.760 & 2.560 \\
\hline middle upper $I$ & I middle upper Mall I & 123 & 0.010 & 0.260 & 1.000 \\
\hline middle upper $n$ & I middle upper Mall I & ow Gaskiı & 0.170 & 0.560 & 1.310 \\
\hline middle upper $I$ & I middle upper Mall In & g of sequ & 0.070 & 1.000 & 2.790 \\
\hline Upper Drook & Upper Drook & 2 & 0.020 & 0.640 & 2.860 \\
\hline Upper Drook & Upper Drook & 5 & 0.000 & 0.760 & 3.910 \\
\hline Upper Drook & Upper Drook & 5 & 0.000 & 0.730 & 3.760 \\
\hline Upper Drook & Upper Drook & 12 & 0.240 & 0.710 & 2.480 \\
\hline Upper Drook & Upper Drook & 17 & 0.000 & 0.470 & 1.980 \\
\hline Upper Drook & Upper Drook & 17.5 & 0.010 & 0.530 & 2.740 \\
\hline
\end{tabular}




\begin{tabular}{|c|c|c|c|c|c|}
\hline Upper Drook & Upper Drook & 17.5 & 0.010 & 0.430 & 1.910 \\
\hline Upper Drook & Upper Drook & 26.5 & 0.000 & 0.620 & 2.300 \\
\hline Upper Drook & Upper Drook & 34.5 & 0.000 & 0.680 & 3.670 \\
\hline Upper Drook & Upper Drook & 41.5 & 0.000 & 0.510 & 3.060 \\
\hline Upper Drook & Upper Drook & 48.5 & 0.000 & 0.850 & 4.270 \\
\hline Upper Drook & Upper Drook & 52.5 & 0.000 & 0.810 & 4.810 \\
\hline Upper Drook & Upper Drook & 59.5 & 0.410 & 1.050 & 3.350 \\
\hline Upper Drook & Upper Drook & 66.5 & 0.000 & 0.690 & 5.140 \\
\hline Upper Drook & Upper Drook & 66.5 & 0.030 & 0.690 & 3.940 \\
\hline Upper Drook & Upper Drook & 73.5 & 0.510 & 1.180 & 3.910 \\
\hline Upper Drook & Upper Drook & ;0 $\mathrm{m}$ above $\mathrm{Br}$ & 0.020 & 0.800 & 4.710 \\
\hline Upper Drook & Upper Drook & low first Ives| & 0.530 & 1.230 & 3.970 \\
\hline Upper Drook & Upper Drook & in distance $a b$ & 0.000 & 0.830 & 4.450 \\
\hline Upper Fermeuse & Upper Fermeuse & 2 & 0.140 & 0.930 & 4.430 \\
\hline Upper Fermeuse & Upper Fermeuse & 8 & 0.080 & 0.860 & 4.660 \\
\hline Upper Fermeuse & Upper Fermeuse & 10 & 0.040 & 0.990 & 4.370 \\
\hline Upper Fermeuse & Upper Fermeuse & 15 & 0.120 & 0.870 & 4.240 \\
\hline Upper Fermeuse & Upper Fermeuse & 23 & 0.550 & 1.120 & 2.300 \\
\hline Upper Fermeuse & Upper Fermeuse & nning of sequ & 0.130 & 0.830 & 4.260 \\
\hline Upper Gaskiers Ha & Upper Gaskiers Har & 2 & 0.000 & 1.170 & 3.710 \\
\hline Upper Gaskiers Ha & Upper Gaskiers Har & 4 & 0.000 & 1.320 & 3.780 \\
\hline Upper Gaskiers Ha & Upper Gaskiers Har & 6 & 0.000 & 1.160 & 3.300 \\
\hline Upper Gaskiers Ha & Upper Gaskiers Har & 8 & 0.000 & 1.520 & 3.290 \\
\hline Upper Gaskiers Ha & Upper Gaskiers Har & 10 & 0.000 & 1.360 & 3.720 \\
\hline Upper Gaskiers Ha & Upper Gaskiers Har & 10.1 & 0.000 & 1.560 & 3.760 \\
\hline Upper Gaskiers Ha & Upper Gaskiers Har & 10.25 & 0.010 & 1.000 & 2.920 \\
\hline Upper Gaskiers Ha & Upper Gaskiers Har & 10.4 & 0.010 & 0.250 & 0.950 \\
\hline Upper Gaskiers Ha & Upper Gaskiers Har & 10.55 & 0.000 & 0.190 & 0.600 \\
\hline Upper Gaskiers Ha & Upper Gaskiers Har & 10.7 & 0.010 & 0.200 & 0.670 \\
\hline Upper Gaskiers Ha & Upper Gaskiers Har» & llow cap carb & 0.010 & 0.010 & 0.600 \\
\hline Upper Gaskiers Ha & Upper Gaskiers Harn & nning of sequ & 0.010 & 1.330 & 3.760 \\
\hline Upper Gaskiers Ha & Upper Gaskiers Hark & bour Main & 0.000 & 1.730 & 3.600 \\
\hline Upper Gaskiers St. & Upper Gaskiers St. I & 3 & 0.010 & 1.550 & 3.610 \\
\hline Upper Gaskiers St. & Upper Gaskiers St. I & 7 & 0.030 & 2.040 & 4.660 \\
\hline Upper Gaskiers St. & Upper Gaskiers St. I & 10 & 0.010 & 1.570 & 3.650 \\
\hline Upper Gaskiers St. & Upper Gaskiers St. I & 14 & 0.050 & 1.710 & 4.090 \\
\hline Upper Gaskiers St. & Upper Gaskiers St. In & Inning of sequ & 0.010 & 1.560 & 3.860 \\
\hline Upper Gaskiers St. & Upper Gaskiers St. I & top of tillite & 0.000 & 1.410 & 3.590 \\
\hline Upper Mistaken p & Upper Mistaken po & 1 & 0.010 & 0.820 & 4.880 \\
\hline Upper Mistaken p & Upper Mistaken po & 2 & 0.010 & 0.910 & 4.730 \\
\hline Upper Mistaken p & Upper Mistaken pon & nning of sequ & 0.010 & 1.100 & 5.360 \\
\hline
\end{tabular}




\begin{tabular}{|c|c|c|c|}
\hline uppermost Fermeı uppermost Fermeuse & 0.630 & 1.760 & 4.330 \\
\hline Dracoisan Formati Dracoisan Formation & 0.051 & 1.538 & 3.561 \\
\hline Dracoisan Formati Dracoisan Formation & 0.058 & 2.075 & 3.555 \\
\hline Dracoisan Formati Dracoisan Formation & 0.083 & 1.063 & 1.597 \\
\hline Canyon Fm East GıCanyon Fm East Greenland & 0.008 & 0.724 & 4.099 \\
\hline Canyon Fm East GıCanyon Fm East Greenland & 0.108 & 1.197 & 6.234 \\
\hline Canyon Fm East GıCanyon Fm East Greenland & 0.026 & 0.879 & 3.998 \\
\hline Canyon Fm East GıCanyon Fm East Greenland & 0.014 & 1.044 & 4.712 \\
\hline Canyon Fm East Gı Canyon Fm East Greenland & 0.307 & 0.750 & 3.382 \\
\hline Canyon Fm East GıCanyon Fm East Greenland & 0.052 & 0.862 & 3.710 \\
\hline Canyon Fm East GıCanyon Fm East Greenland & 0.053 & 1.076 & 4.598 \\
\hline Canyon Fm East GıCanyon Fm East Greenland & 0.006 & 0.867 & 3.617 \\
\hline Canyon Fm East GıCanyon Fm East Greenland & 0.140 & 1.035 & 4.235 \\
\hline Canyon Fm East GıCanyon Fm East Greenland & 0.413 & 1.395 & 4.229 \\
\hline Canyon Fm East GıCanyon Fm East Greenland & 0.022 & 1.595 & 4.379 \\
\hline Canyon Fm East GıCanyon Fm East Greenland & 0.027 & 0.550 & 1.344 \\
\hline Canyon Fm East GıCanyon Fm East Greenland & 0.162 & 1.406 & 2.895 \\
\hline Canyon Fm East Gı Canyon Fm East Greenland & 0.361 & 1.652 & 3.333 \\
\hline Canyon Fm East GıCanyon Fm East Greenland & 0.175 & 0.765 & 1.523 \\
\hline Canyon Fm East Gı Canyon Fm East Greenland & 0.028 & 1.083 & 1.808 \\
\hline Canyon Fm East Gı Canyon Fm East Greenland & 0.359 & 1.441 & 2.215 \\
\hline Spiral Creek Form`Spiral Creek Formation & 0.005 & 0.555 & 2.013 \\
\hline Spiral Creek Form` Spiral Creek Formation & 0.248 & 1.113 & 3.513 \\
\hline Spiral Creek Form`Spiral Creek Formation & 0.009 & 0.819 & 2.364 \\
\hline Spiral Creek Form``Spiral Creek Formation & 0.046 & 1.034 & 1.882 \\
\hline Spiral Creek Form`Spiral Creek Formation & 0.129 & 1.331 & 2.005 \\
\hline Spiral Creek Form` Spiral Creek Formation & 0.078 & 0.571 & 0.785 \\
\hline Arcoona $A B C$ Form Arcoona $A B C$ Formation & 0.098 & 0.517 & 3.406 \\
\hline Arcoona $A B C$ Form Arcoona $A B C$ Formation & 0.043 & 1.172 & 5.517 \\
\hline Arcoona $A B C$ Form Arcoona $A B C$ Formation & 0.036 & 1.736 & 6.319 \\
\hline Arcoona $A B C$ Form Arcoona $A B C$ Formation & 0.072 & 2.162 & 6.283 \\
\hline Arcoona $A B C$ Form Arcoona $A B C$ Formation & 0.217 & 1.713 & 4.697 \\
\hline Arcoona $A B C$ Form Arcoona $A B C$ Formation & 1.273 & 2.200 & 5.223 \\
\hline Arcoona $A B C$ Form Arcoona $A B C$ Formation & 1.265 & 2.743 & 6.140 \\
\hline Elyuah Formation Elyuah Formation & 0.010 & 1.069 & 4.178 \\
\hline Elyuah Formation Elyuah Formation & 0.022 & 1.061 & 3.712 \\
\hline Elyuah Formation Elyuah Formation & 0.080 & 1.417 & 4.710 \\
\hline Elyuah Formation Elyuah Formation & 0.434 & 1.054 & 3.424 \\
\hline Elyuah Formation Elyuah Formation & 0.075 & 1.026 & 2.520 \\
\hline Elyuah Formation Elyuah Formation & 1.355 & 2.413 & 5.675 \\
\hline Elyuah Formation Elyuah Formation & 1.758 & 2.271 & 5.104 \\
\hline
\end{tabular}




\begin{tabular}{|c|c|c|c|c|}
\hline Elyuah Formation Elyuah Formation & & 0.664 & 3.217 & 7.082 \\
\hline Elyuah Formation Elyuah Formation & & 1.007 & 2.296 & 4.989 \\
\hline Elyuah Formation Elyuah Formation & & 0.944 & 3.566 & 7.699 \\
\hline Elyuah Formation Elyuah Formation & & 0.969 & 2.238 & 4.831 \\
\hline Elyuah Formation Elyuah Formation & & 0.875 & 1.385 & 2.841 \\
\hline Elyuah Formation Elyuah Formation & & 1.120 & 2.106 & 4.278 \\
\hline Elyuah Formation Elyuah Formation & & 0.837 & 2.225 & 4.435 \\
\hline Elyuah Formation Elyuah Formation & & 1.640 & 2.426 & 4.812 \\
\hline Elyuah Formation Elyuah Formation & & 0.114 & 2.167 & 4.142 \\
\hline Elyuah Formation Elyuah Formation & & 1.848 & 2.899 & 5.177 \\
\hline Elyuah Formation Elyuah Formation & & 1.398 & 2.550 & 4.472 \\
\hline Elyuah Formation Elyuah Formation & & 1.505 & 2.700 & 4.340 \\
\hline Elyuah Formation Elyuah Formation & & 1.869 & 2.615 & 4.095 \\
\hline Elyuah Formation Elyuah Formation & & 2.748 & 3.840 & 5.185 \\
\hline Grants Bluff Form` Grants Bluff Formation & & 0.183 & 0.666 & 2.536 \\
\hline Grants Bluff Forma Grants Bluff Formation & & 0.338 & 1.216 & 4.531 \\
\hline Grants Bluff Form` Grants Bluff Formation & & 0.766 & 1.308 & 2.947 \\
\hline Grants Bluff Formi Grants Bluff Formation & & 0.952 & 1.587 & 3.555 \\
\hline Grants Bluff Forma Grants Bluff Formation & & 1.375 & 2.356 & 4.350 \\
\hline Grants Bluff Formi Grants Bluff Formation & & 1.389 & 2.353 & 4.186 \\
\hline Grants Bluff Form` Grants Bluff Formation & & 1.762 & 2.522 & 4.134 \\
\hline Grants Bluff Form` Grants Bluff Formation & & 1.847 & 2.618 & 4.140 \\
\hline Grants Bluff Form` Grants Bluff Formation & & 1.855 & 2.666 & 4.194 \\
\hline Grants Bluff Form` Grants Bluff Formation & & 1.549 & 2.334 & 3.469 \\
\hline Woomera Formati Woomera Formation & & 0.077 & 1.190 & 5.289 \\
\hline Woomera Formati Woomera Formation & & 0.045 & 0.894 & 3.693 \\
\hline Woomera Formati Woomera Formation & & 0.109 & 1.291 & 4.920 \\
\hline Woomera Formati Woomera Formation & & 0.205 & 1.375 & 4.423 \\
\hline Woomera Formati Woomera Formation & & 0.037 & 1.873 & 5.705 \\
\hline Woomera Formati Woomera Formation & & 0.657 & 1.626 & 4.790 \\
\hline Woomera Formati Woomera Formation & & 1.146 & 2.019 & 5.318 \\
\hline Woomera Formati Woomera Formation & & 1.020 & 1.968 & 4.871 \\
\hline Upper Staraya Fm Upper Staraya Fm & & 0.002 & 0.699 & 2.291 \\
\hline Upper Staraya Fm Upper Staraya Fm & & 0.147 & 1.023 & 2.992 \\
\hline Upper Staraya Fm Upper Staraya Fm & & 0.124 & 0.746 & 1.849 \\
\hline F838 & 31 & 0.074 & 0.781 & 1.246 \\
\hline F838 & 54 & 0.003 & 2.283 & 4.912 \\
\hline F838 & 58 & 0.000 & 2.416 & 4.812 \\
\hline F838 & 62 & 0.000 & 2.214 & 4.802 \\
\hline F838 & 70 & 0.000 & 1.622 & 4.657 \\
\hline F838 & 74 & 0.001 & 1.848 & 4.798 \\
\hline
\end{tabular}




\begin{tabular}{|c|c|c|c|c|c|}
\hline & F838 & 82 & 0.002 & 1.844 & 5.173 \\
\hline & F838 & 90 & 0.000 & 1.586 & 5.252 \\
\hline & F838 & 96 & 0.000 & 2.073 & 4.303 \\
\hline & F838 & 98 & 0.000 & 2.008 & 4.880 \\
\hline & F838 & 102 & 0.000 & 1.764 & 4.608 \\
\hline & F838 & 110 & 0.002 & 1.950 & 4.952 \\
\hline & F838 & 118 & 0.002 & 2.423 & 5.443 \\
\hline & F838 & 126 & 0.002 & 2.246 & 5.881 \\
\hline & F838 & 134 & 0.001 & 2.142 & 7.073 \\
\hline & F838 & 142 & 0.027 & 1.980 & 6.842 \\
\hline & F838 & 150 & 0.007 & 2.770 & 6.305 \\
\hline & F838 & 158 & 0.003 & 2.230 & 5.381 \\
\hline & F838 & 166 & 0.013 & 1.967 & 5.833 \\
\hline & F838 & 174 & 0.002 & 1.880 & 5.229 \\
\hline & F838 & 182 & 0.005 & 1.523 & 5.052 \\
\hline & F838 & 190 & 0.015 & 2.077 & 5.412 \\
\hline & F838 & 198 & 0.093 & 1.545 & 5.632 \\
\hline & F838 & 206 & 0.020 & 1.567 & 4.571 \\
\hline & F838 & 214 & 0.013 & 1.600 & 5.264 \\
\hline & F838 & 222 & 0.002 & 2.001 & 4.778 \\
\hline & F838 & 230 & 0.011 & 1.715 & 7.833 \\
\hline & F838 & 238 & 0.008 & 2.157 & 5.109 \\
\hline & F838 & 246 & 0.005 & 1.919 & 5.229 \\
\hline & F838 & 254 & 0.002 & 2.291 & 6.191 \\
\hline & F838 & 262 & 0.003 & 2.294 & 5.401 \\
\hline & F838 & 270 & 0.002 & 2.143 & 4.875 \\
\hline & F838 & 278 & 0.001 & 2.280 & 5.680 \\
\hline & F838 & 286 & 0.008 & 2.580 & 4.303 \\
\hline & F838 & 290 & 0.007 & 2.543 & 5.572 \\
\hline & F838 & 298 & 0.000 & 3.289 & 4.706 \\
\hline & F842 & 135 & 0.003 & 0.507 & 3.920 \\
\hline June beds & Sekwi Brook & & 0.160 & 2.150 & 2.940 \\
\hline June beds & Sekwi Brook & & 0.160 & 2.460 & 3.190 \\
\hline June beds & Sekwi Brook & & 0.210 & 2.370 & 3.040 \\
\hline June beds & Sekwi Brook & & 0.150 & 1.640 & 2.010 \\
\hline June beds & Sekwi Brook & & 0.390 & 3.640 & 4.260 \\
\hline Sheepbed & Shale Lake & 5 & 0.220 & 3.080 & 4.240 \\
\hline Sheepbed & Shale Lake & 10 & 0.300 & 2.730 & 4.280 \\
\hline Sheepbed & Shale Lake & 15 & 0.160 & 2.910 & 4.190 \\
\hline Sheepbed & Shale Lake & 21.5 & 0.150 & 1.650 & 3.460 \\
\hline Sheepbed & Shale Lake & 31 & 0.100 & 2.450 & 4.990 \\
\hline
\end{tabular}




\begin{tabular}{|c|c|c|c|c|c|}
\hline Sheepbed & Shale Lake & 43 & 0.050 & 5.030 & 6.820 \\
\hline Sheepbed & Shale Lake & 52 & 0.090 & 2.410 & 5.140 \\
\hline Sheepbed & Shale Lake & 60 & 0.060 & 2.760 & 5.790 \\
\hline Sheepbed & Shale Lake & 70 & 0.090 & 2.570 & 5.410 \\
\hline Sheepbed & Shale Lake & 87 & 0.050 & 0.750 & 2.610 \\
\hline Sheepbed & Shale Lake & 99 & 0.020 & 2.330 & 5.500 \\
\hline Sheepbed & Shale Lake & 146 & 0.030 & 2.500 & 6.270 \\
\hline Sheepbed & Shale Lake & 205 & 0.050 & 3.910 & 6.920 \\
\hline Sheepbed & Shale Lake & 240 & 0.050 & 3.250 & 6.080 \\
\hline Sheepbed & Shale Lake & 258 & 0.030 & 4.420 & 6.910 \\
\hline Sheepbed & Shale Lake & 307 & 0.020 & 2.340 & 5.040 \\
\hline Sheepbed & Shale Lake & 322 & 0.980 & 2.740 & 6.250 \\
\hline Sheepbed & Shale Lake & 375 & 0.060 & 4.060 & 7.030 \\
\hline Sheepbed & Shale Lake & 450 & 0.630 & 4.290 & 8.150 \\
\hline Sheepbed & Shale Lake & 468 & 1.160 & 2.310 & 4.490 \\
\hline Sheepbed & Shale Lake & 473 & 0.880 & 2.050 & 4.490 \\
\hline Sheepbed & Shale Lake & 483 & 0.200 & 4.570 & 6.980 \\
\hline Sheepbed & Section 1 & 4 & & & \\
\hline Sheepbed & Section 1 & 5 & & & \\
\hline Sheepbed & Section 1 & 5.5 & & & \\
\hline Sheepbed & Section 1 & 7 & & & \\
\hline Sheepbed & Section 1 & 8 & & & \\
\hline Sheepbed & Section 1 & 10 & & & \\
\hline Sheepbed & Section 1 & 12 & & & \\
\hline Sheepbed & Section 1 & 14 & & & \\
\hline Sheepbed & Section 1 & 16 & & & \\
\hline Sheepbed & Section 1 & 18 & & & \\
\hline Sheepbed & Section 1 & 23 & & & \\
\hline Sheepbed & Section 1 & 27 & & & \\
\hline Sheepbed & Section 1 & 29 & & & \\
\hline Sheepbed & Section 1 & 33 & & & \\
\hline Sheepbed & Section 1 & 35.5 & & & \\
\hline Sheepbed & Section 1 & 37.5 & & & \\
\hline Sheepbed & Section 1 & 39 & & & \\
\hline Sheepbed & Section 1 & 43 & & & \\
\hline Sheepbed & Section 1 & 44.5 & & & \\
\hline Sheepbed & Section 1 & 47 & & & \\
\hline Sheepbed & Section 1 & 50 & & & \\
\hline Sheepbed & Section 1 & 54 & & & \\
\hline Sheepbed & Section 1 & 57 & & & \\
\hline Sheepbed & Section 1 & 60 & & & \\
\hline
\end{tabular}




\begin{tabular}{|c|c|c|c|c|c|}
\hline Sheepbed & Section 2 & 34 & & & \\
\hline Sheepbed & Section 2 & 35.5 & & & \\
\hline Sheepbed & Section 2 & 39 & & & \\
\hline Sheepbed & Section 2 & 57.5 & & & \\
\hline Sheepbed & Section 2 & 74 & & & \\
\hline Sheepbed & Section 2 & 90 & & & \\
\hline Sheepbed & Section 2 & 118 & & & \\
\hline Sheepbed & Section 2 & 138 & & & \\
\hline Sheepbed & Section 2 & 157 & & & \\
\hline Sheepbed & Section 2 & 171 & & & \\
\hline Sheepbed & Section 2 & 186 & & & \\
\hline Sheepbed & Section 2 & 192 & & & \\
\hline Sheepbed & Section 2 & 195 & & & \\
\hline Sheepbed & Section 2 & 200 & & & \\
\hline Sheepbed & Section 2 & 209 & & & \\
\hline Sheepbed & Section 2 & 226 & & & \\
\hline Sheepbed & Section 2 & 244 & & & \\
\hline Sheepbed & Section 2 & 266 & & & \\
\hline Sheepbed & Section 2 & 276 & & & \\
\hline Sheepbed & Section 2 & 292 & & & \\
\hline Sheepbed & Section 2 & 304 & & & \\
\hline Sheepbed & Section 2 & 307 & & & \\
\hline Sheepbed & Section 2 & 313 & & & \\
\hline Sheepbed & Section 2 & 322 & & & \\
\hline Sheepbed & Section 2 & 331 & & & \\
\hline Sheepbed & Section 2 & 340 & & & \\
\hline Sheepbed & Section 2 & 345 & & & \\
\hline Sheepbed & Section 2 & 362 & & & \\
\hline Sheepbed & Section 2 & 373 & & & \\
\hline Sheepbed & Section 2 & 417 & & & \\
\hline Sheepbed & Section 2 & 446 & & & \\
\hline Sheepbed & Section 2 & 456 & & & \\
\hline Sheepbed & Section 2 & 476 & & & \\
\hline Sheepbed & Section 2 & 511 & & & \\
\hline Sheepbed & Section 2 & 520 & & & \\
\hline \multicolumn{2}{|c|}{ Blueflower FormatF1159 } & 732 & 0.003 & 1.951 & 4.260 \\
\hline \multicolumn{2}{|c|}{ Blueflower FormatF1159 } & 750 & 0.002 & 2.613 & 5.860 \\
\hline \multicolumn{2}{|c|}{ Blueflower FormatF1159 } & 766 & 0.003 & 1.085 & 4.970 \\
\hline \multicolumn{2}{|c|}{ Blueflower FormatF1159 } & 780 & 0.001 & 1.309 & 5.070 \\
\hline \multicolumn{2}{|c|}{ Blueflower FormatF1159 } & 796.5 & 0.000 & 0.381 & 4.320 \\
\hline \multicolumn{2}{|c|}{ Blueflower FormatF1159 } & 810 & 0.001 & 0.798 & 4.520 \\
\hline
\end{tabular}




\begin{tabular}{|c|c|c|c|c|c|}
\hline \multicolumn{2}{|c|}{ Blueflower FormatF1159 } & 828 & 0.002 & 0.554 & 3.910 \\
\hline \multicolumn{2}{|c|}{ Blueflower FormatF1159 } & 851.5 & 0.001 & 0.626 & 4.270 \\
\hline \multicolumn{2}{|c|}{ Blueflower FormatF1159 } & 852 & 0.000 & 1.679 & 4.370 \\
\hline \multicolumn{2}{|c|}{ Blueflower FormatF1159 } & 868 & 0.001 & 0.706 & 3.640 \\
\hline \multicolumn{2}{|c|}{ Blueflower FormatF1159 } & 883.5 & 0.002 & 3.110 & 5.750 \\
\hline \multicolumn{2}{|c|}{ Blueflower FormatF1159 } & 901 & 0.001 & 1.187 & 3.050 \\
\hline \multicolumn{2}{|c|}{ Blueflower FormatF1159 } & 916.5 & 0.001 & 1.174 & 3.960 \\
\hline \multicolumn{2}{|c|}{ Blueflower FormatF1159 } & 925 & 0.002 & 1.012 & 3.810 \\
\hline \multicolumn{2}{|c|}{ Blueflower FormatF1159 } & 936 & 0.003 & 0.833 & 2.070 \\
\hline June beds & F1161 & 28.5 & 0.528 & 2.137 & 3.720 \\
\hline June beds & F1161 & 31.2 & 0.211 & 1.981 & 2.960 \\
\hline June beds & F1161 & 33.75 & 0.090 & 1.324 & 2.820 \\
\hline June beds & F1161 & 38.6 & 0.182 & 1.304 & 3.480 \\
\hline June beds & F1161 & 53.3 & 0.176 & 1.728 & 3.970 \\
\hline June beds & F1161 & 66.5 & 0.117 & 1.517 & 2.610 \\
\hline June beds & F1161 & 70 & 0.124 & 1.933 & 3.000 \\
\hline June beds & F1161 & 76.9 & 0.018 & 1.771 & 3.270 \\
\hline June beds & F1161 & 86 & 0.109 & 2.220 & 4.030 \\
\hline June beds & F1161 & 92 & 0.027 & 1.475 & 4.120 \\
\hline June beds & F1161 & 103.5 & 0.149 & 1.895 & 2.600 \\
\hline June beds & F1161 & 116.5 & 0.086 & 1.704 & 2.990 \\
\hline June beds & F1161 & 126.8 & 0.009 & 0.801 & 1.420 \\
\hline June beds & F1161 & 141.3 & 0.002 & 0.304 & 3.350 \\
\hline June beds & F1161 & 159 & 0.059 & 0.541 & 4.230 \\
\hline June beds & F1161 & 181.9 & 0.019 & 0.460 & 4.330 \\
\hline June beds & F1161 & 196.2 & 0.060 & 1.510 & 3.170 \\
\hline June beds & F1161 & 209.2 & 0.037 & 1.889 & 3.880 \\
\hline June beds & F1161 & 217.5 & 0.088 & 2.104 & 4.140 \\
\hline June beds & F1161 & 223 & 0.084 & 2.164 & 3.620 \\
\hline June beds & F1161 & 232.5 & 0.159 & 1.501 & 2.590 \\
\hline June beds & F1161 & 253 & 0.049 & 1.823 & 3.130 \\
\hline June beds & F1161 & 278 & 0.121 & 2.234 & 3.760 \\
\hline June beds & F1161 & 289.5 & 0.111 & 2.610 & 4.010 \\
\hline June beds & F1161 & 303.4 & 0.140 & 2.448 & 3.020 \\
\hline June beds & F1162 & 51.3 & 0.167 & 2.720 & 3.220 \\
\hline June beds & F1162 & 56 & 0.159 & 1.107 & 1.750 \\
\hline June beds & F1162 & 122.5 & 0.075 & 2.591 & 2.890 \\
\hline June beds & F1162 & 135 & 0.102 & 1.219 & 1.630 \\
\hline June beds & F1162 & 145 & 0.169 & 2.258 & 2.730 \\
\hline June beds & F1162 & 165.2 & 0.086 & 2.083 & 2.480 \\
\hline June beds & F1162 & 176.5 & 0.077 & 3.179 & 4.100 \\
\hline
\end{tabular}




\begin{tabular}{|c|c|c|c|c|c|}
\hline June beds & F1162 & 179.5 & 0.154 & 3.406 & 3.830 \\
\hline June beds & F1162 & 194 & 0.034 & 1.939 & 2.870 \\
\hline June beds & F1162 & 203.4 & 0.012 & 0.422 & 1.110 \\
\hline June beds & F1162 & 212 & 0.107 & 0.297 & 2.700 \\
\hline June beds & F1162 & 226 & 0.026 & 0.975 & 1.510 \\
\hline June beds & F1162 & 250.5 & 0.078 & 1.188 & 1.260 \\
\hline Gametrail & F1162 & 445.7 & 0.043 & 1.689 & 1.880 \\
\hline Gametrail & F1162 & 496.4 & 0.028 & 1.852 & 2.040 \\
\hline Blueflower & F1162 & 542.3 & 0.004 & 2.109 & 2.690 \\
\hline Blueflower & F1162 & 542.7 & 0.003 & 0.954 & 1.610 \\
\hline Blueflower & F1162 & 547.8 & 0.003 & 0.438 & 1.140 \\
\hline Gametrail & F1162 & t19weatherec & 0.008 & 3.426 & 3.660 \\
\hline Blueflower & F1162 & 547.4silty & 0.009 & 0.914 & 1.440 \\
\hline Blueflower & F1163 & 22.5 & 0.088 & 1.691 & 2.170 \\
\hline Blueflower & F1163 & 28.6 & 0.206 & 1.200 & 1.430 \\
\hline Blueflower & F1163 & 46.4 & 0.200 & 1.510 & 3.740 \\
\hline Blueflower & F1163 & 50.3 & 0.143 & 1.209 & 1.500 \\
\hline Blueflower & F1163 & 61.3 & 0.194 & 1.830 & 2.660 \\
\hline Blueflower & F1163 & 71 & 0.057 & 0.188 & 0.590 \\
\hline Blueflower & F1163 & 90.7 & 0.010 & 0.745 & 2.410 \\
\hline Blueflower & F1163 & 103.5 & 0.017 & 0.463 & 2.510 \\
\hline Blueflower & F1163 & 113 & 0.034 & 2.472 & 3.680 \\
\hline Blueflower & F1163 & 128.2 & 0.019 & 1.953 & 4.020 \\
\hline Blueflower & F1163 & 134.6 & 0.038 & 2.344 & 3.510 \\
\hline Blueflower & F1163 & 147.1 & 0.097 & 2.667 & 3.500 \\
\hline Blueflower & F1163 & 80silty & 0.063 & 0.773 & 1.150 \\
\hline Blueflower & F1164 & 8.9 & 0.003 & 0.386 & 0.800 \\
\hline Blueflower & F1164 & 20.01 & 0.006 & 0.181 & 0.550 \\
\hline Blueflower & F1164 & 31.5 & 0.003 & 0.372 & 0.510 \\
\hline Blueflower & F1164 & 40.1 & 0.007 & 0.632 & 0.860 \\
\hline Blueflower & F1164 & 49.1 & 0.005 & 2.327 & 2.860 \\
\hline Blueflower & F1164 & 64 & 0.033 & 2.806 & 4.840 \\
\hline Blueflower & F1164 & 75.9 & 0.016 & 0.817 & 1.720 \\
\hline Blueflower & F1164 & 84.2 & 0.086 & 0.667 & 1.040 \\
\hline Blueflower & F1164 & 93.1 & 0.031 & 2.542 & 3.340 \\
\hline Blueflower & F1164 & 20.0neo & 0.014 & 0.258 & 0.650 \\
\hline Sheepbed? & F1165 & 70 & 0.003 & 0.477 & 3.640 \\
\hline Sheepbed? & F1165 & 78 & 0.007 & 0.319 & 3.240 \\
\hline Sheepbed? & F1165 & 88 & 0.004 & 1.071 & 3.400 \\
\hline Sheepbed? & F1165 & 99 & 0.052 & 1.406 & 2.380 \\
\hline Sheepbed? & F1165 & 105 & 0.020 & 0.931 & 1.580 \\
\hline
\end{tabular}




\begin{tabular}{|c|c|c|c|c|c|}
\hline Sheepbed? & F1165 & 109 & 0.070 & 0.418 & 0.710 \\
\hline Sheepbed? & F1165 & 114 & 0.063 & 0.539 & 4.260 \\
\hline Sheepbed? & F1165 & 118 & 0.077 & 0.651 & 1.130 \\
\hline Sheepbed? & F1165 & 124.3 & 0.008 & 0.253 & 2.740 \\
\hline Sheepbed? & F1165 & 128 & 0.115 & 1.043 & 3.080 \\
\hline Sheepbed? & F1165 & 132 & 1.041 & 1.458 & 3.440 \\
\hline Sheepbed? & F1165 & 139 & 0.185 & 0.836 & 1.070 \\
\hline Sheepbed? & F1165 & 146 & 0.155 & 1.912 & 2.400 \\
\hline Sheepbed? & F1165 & 156 & 0.076 & 0.780 & 1.350 \\
\hline Sheepbed? & F1165 & 166 & 0.128 & 1.132 & 3.400 \\
\hline Sheepbed? & F1165 & 175 & 0.064 & 2.031 & 3.600 \\
\hline Sheepbed? & F1165 & 205 & 0.003 & 1.109 & 5.030 \\
\hline Sheepbed? & F1165 & 216 & 0.013 & 0.737 & 4.530 \\
\hline Sheepbed? & F1165 & 228 & 0.010 & 0.794 & 4.490 \\
\hline Sheepbed? & F1165 & 237 & 0.002 & 0.694 & 6.510 \\
\hline Sheepbed? & F1165 & 243 & 0.002 & 0.323 & 3.250 \\
\hline Sheepbed? & F1165 & 253 & 0.022 & 1.987 & 2.910 \\
\hline Sheepbed? & F1165 & 263 & 0.067 & 2.427 & 3.660 \\
\hline Sheepbed? & F1165 & 119crumbly & 0.115 & 1.644 & 1.870 \\
\hline Blueflower & J1125 & J1125- 0.4 & 0.000 & 0.808 & 2.050 \\
\hline Blueflower & J1125 & J1125- 1.9 & 0.000 & 1.257 & 2.610 \\
\hline Ingta & J1125 & J1125- 102.7 & 0.000 & 1.555 & 3.070 \\
\hline Ingta & J1125 & J1125- 109.4 & 0.000 & 2.844 & 4.170 \\
\hline Ingta & J1125 & J1125- 112.4 & 0.000 & 1.793 & 3.680 \\
\hline Ingta & J1125 & J1125- 117.3 & 0.000 & 1.380 & 3.460 \\
\hline Ingta & $\mathrm{J} 1125$ & J1125- 120.2 & 0.000 & 1.116 & 4.450 \\
\hline Ingta & J1125 & J1125- 120.9 & 0.000 & 1.289 & 4.360 \\
\hline Ingta & J1125 & J1125- 9.7 & 0.000 & 0.715 & 1.970 \\
\hline Blueflower & J1128 & 4.7 & 0.016 & 0.293 & 1.380 \\
\hline Blueflower & J1128 & 7.7 & 0.172 & 1.757 & 3.250 \\
\hline Blueflower & J1128 & 10.2 & 0.009 & 0.409 & 1.160 \\
\hline Blueflower & J1128 & 18.3 & 0.024 & 1.821 & 4.150 \\
\hline Blueflower & J1128 & 28.3 & 0.010 & 1.678 & 2.400 \\
\hline Blueflower & J1128 & 47.9 & 0.007 & 0.427 & 0.990 \\
\hline Blueflower & J1128 & 55.4 & 0.010 & 1.366 & 3.640 \\
\hline Blueflower & J1128 & 61.9 & 0.001 & 0.518 & 1.930 \\
\hline Blueflower & J1128 & 74.3 & 0.008 & 0.181 & 0.860 \\
\hline Blueflower & J1128 & 82.8 & 0.038 & 1.060 & 3.160 \\
\hline Blueflower & J1128 & 92 & 0.034 & 1.670 & 3.190 \\
\hline Blueflower & J1128 & 104 & 0.005 & 1.108 & 2.330 \\
\hline Blueflower & J1128 & 108 & 0.003 & 0.953 & 2.550 \\
\hline
\end{tabular}




\begin{tabular}{|c|c|c|c|c|c|}
\hline Blueflower & $\mathrm{J} 1128$ & 117.4 & 0.003 & 0.282 & 0.980 \\
\hline Blueflower & J1128 & 127.5 & 0.013 & 1.521 & 3.670 \\
\hline Blueflower & $\mathrm{J} 1128$ & 136.2 & 0.025 & 1.436 & 3.170 \\
\hline Blueflower & $\mathrm{J} 1128$ & 145.9 & 0.014 & 0.877 & 3.120 \\
\hline Blueflower & $\mathrm{J} 1128$ & 157.9 & 0.006 & 0.889 & 1.910 \\
\hline Blueflower & J1128 & 168.7 & 0.001 & 1.745 & 2.740 \\
\hline Blueflower & $\mathrm{J} 1128$ & 177.3 & 0.027 & 0.942 & 3.440 \\
\hline Blueflower & J1128 & 187.2 & 0.077 & 1.663 & 4.920 \\
\hline Blueflower & $\mathrm{J} 1128$ & 197.8 & 0.054 & 2.328 & 4.690 \\
\hline Blueflower & $\mathrm{J} 1128$ & 207.5 & 0.201 & 2.153 & 5.870 \\
\hline Blueflower & $\mathrm{J} 1128$ & 215.6 & 0.024 & 1.275 & 4.180 \\
\hline Blueflower & $\mathrm{J} 1128$ & 225.8 & 0.028 & 1.515 & 4.460 \\
\hline Blueflower & $\mathrm{J} 1128$ & 234 & 0.032 & 1.608 & 4.560 \\
\hline Blueflower & J1128 & 243.1 & 0.003 & 0.294 & 1.570 \\
\hline Blueflower & $\mathrm{J} 1128$ & 252 & 0.023 & 1.475 & 4.030 \\
\hline Blueflower & $\mathrm{J} 1128$ & 262.8 & 0.030 & 1.246 & 2.560 \\
\hline Blueflower & $\mathrm{J} 1128$ & 272.2 & 0.128 & 1.758 & 2.490 \\
\hline Blueflower & J1128 & 279 & 0.069 & 0.296 & 0.830 \\
\hline Blueflower & $\mathrm{J} 1128$ & 281.8 & 0.009 & 1.018 & 2.050 \\
\hline \multicolumn{2}{|c|}{ Blueflower Format N13-6 } & 1.65 & 0.016 & 1.020 & 3.370 \\
\hline \multicolumn{2}{|c|}{ Blueflower Format N13-6 } & 2.75 & 0.005 & 0.760 & 3.430 \\
\hline \multicolumn{2}{|c|}{ Blueflower Format N13-6 } & 5.1 & 0.005 & 0.809 & 3.100 \\
\hline \multicolumn{2}{|c|}{ Blueflower Format N13-6 } & 6.5 & 0.002 & 0.594 & 3.760 \\
\hline \multicolumn{2}{|c|}{ Blueflower Format N13-6 } & 12.7 & 0.000 & 0.236 & 1.910 \\
\hline \multicolumn{2}{|c|}{ Blueflower Format N13-6 } & 17.4 & 0.012 & 0.324 & 3.380 \\
\hline \multicolumn{2}{|c|}{ Blueflower Format N13-6 } & 18 & 0.002 & 0.708 & 3.970 \\
\hline \multicolumn{2}{|c|}{ Blueflower Format N13-6 } & 20.8 & 0.005 & 0.599 & 2.880 \\
\hline \multicolumn{2}{|c|}{ Blueflower Format N13-6 } & 31.2 & 0.024 & 0.387 & 3.340 \\
\hline \multicolumn{2}{|c|}{ Blueflower Format N13-6 } & 34.1 & 0.005 & 0.992 & 3.740 \\
\hline \multicolumn{2}{|c|}{ Blueflower Format N13-6 } & 38.4 & 0.023 & 0.758 & 4.220 \\
\hline \multicolumn{2}{|c|}{ Blueflower Format N13-6 } & 42.5 & 0.023 & 0.691 & 4.490 \\
\hline \multicolumn{2}{|c|}{ Blueflower Format N13-6 } & 54.5 & 0.002 & 0.555 & 3.460 \\
\hline \multicolumn{2}{|c|}{ Blueflower Format N13-6 } & 57.4 & 0.002 & 0.524 & 4.430 \\
\hline \multicolumn{2}{|c|}{ Blueflower Format N13-6 } & 62.5 & 0.036 & 0.989 & 5.020 \\
\hline \multicolumn{2}{|c|}{ Blueflower Format N13-6 } & 66 & 0.002 & 1.564 & 5.390 \\
\hline \multicolumn{2}{|c|}{ Blueflower Format N13-6 } & 73.1 & 0.004 & 0.543 & 5.780 \\
\hline \multicolumn{2}{|c|}{ Blueflower Format N13-6 } & 97 & 0.005 & 1.579 & 4.900 \\
\hline \multicolumn{2}{|c|}{ Blueflower Format N13-6 } & 121.5 & 0.002 & 0.617 & 5.570 \\
\hline Ingta Forma & S1305 & 15.8 & 0.001 & 0.614 & 3.170 \\
\hline Ingta Forma & S1305 & 22.8 & 0.000 & 1.402 & 3.410 \\
\hline Ingta Forma & S1305 & 23.6 & 0.000 & 0.710 & 1.770 \\
\hline
\end{tabular}




\begin{tabular}{|c|c|c|c|c|c|}
\hline Ingta Formation & S1305 & 32.5 & 0.000 & 1.294 & 3.860 \\
\hline Ingta Formation & S1305 & 34.15 & 0.000 & 1.584 & 4.780 \\
\hline Ingta Formation & S1305 & 40.7 & 0.001 & 0.437 & 2.770 \\
\hline Ingta Formation & S1305 & 44.8 & 0.000 & 0.285 & 2.120 \\
\hline Ingta Formation & S1305 & 49.4 & 0.000 & 1.424 & 3.830 \\
\hline Ingta Formation & S1305 & 50.2 & 0.000 & 1.850 & 3.650 \\
\hline Ingta Formation & S1305 & 54 & 0.001 & 1.831 & 3.700 \\
\hline Ingta Formation & S1305 & 61.2 & 0.000 & 0.781 & 2.560 \\
\hline Ingta Formation & S1305 & 61.4 & 0.000 & 0.727 & 2.550 \\
\hline Ingta Formation & S1305 & 61.6 & 0.002 & 0.317 & 2.930 \\
\hline Yerbal & La Salvaje Farm & $1 \mathrm{AB}$ & 0.000 & 1.374 & 4.450 \\
\hline Yerbal & La Salvaje Farm & $1 A D$ & 0.000 & 2.122 & 5.906 \\
\hline Yerbal & La Salvaje Farm & $1 B A$ & 0.000 & 1.656 & 4.578 \\
\hline Yerbal & La Salvaje Farm & $1 \mathrm{~B} \mathrm{~B}$ & 0.008 & 3.273 & 7.650 \\
\hline Yerbal & La Salvaje Farm & $2 \mathrm{~A}$ & 0.001 & 1.261 & 1.484 \\
\hline Yerbal & La Salvaje Farm & $4 \mathrm{~A}$ & 0.002 & 1.370 & 2.693 \\
\hline Yerbal & La Salvaje Farm & $4 B$ & 0.002 & 1.411 & 2.590 \\
\hline Yerbal & La Salvaje Farm & $4 C$ & 0.002 & 1.410 & 2.542 \\
\hline Yerbal & La Salvaje Farm & 4D & 0.002 & 0.909 & 1.282 \\
\hline Yerbal & La Salvaje Farm & $4 \mathrm{E}$ & 0.006 & 3.142 & 4.855 \\
\hline Yerbal & La Salvaje Farm & $4 \mathrm{~F}$ & 0.008 & 4.385 & 7.803 \\
\hline Yerbal & La Salvaje Farm & $5 A$ & 0.003 & 3.960 & 6.032 \\
\hline Yerbal & La Salvaje Farm & $5 B$ & 0.002 & 5.174 & 7.619 \\
\hline Yerbal & La Salvaje Farm & $5 C$ & 0.002 & 3.726 & 6.860 \\
\hline Yerbal & La Salvaje Farm & $5 D$ & 0.003 & 3.950 & 6.921 \\
\hline Yerbal & La Salvaje Farm & $6 \mathrm{~A}$ & 0.727 & 4.401 & 6.096 \\
\hline Yerbal & La Salvaje Farm & $6 \mathrm{~B}$ & 0.002 & 2.480 & 7.464 \\
\hline Yerbal & La Salvaje Farm & $6 C$ & 0.015 & 6.673 & 15.186 \\
\hline Yerbal & La Salvaje Farm & $6 \mathrm{D}$ & 0.006 & 3.174 & 5.302 \\
\hline Yerbal & La Salvaje Farm & $7 \mathrm{AA}$ & 0.002 & 0.875 & 3.683 \\
\hline Yerbal & La Salvaje Farm & $7 A B$ & 0.002 & 1.584 & 3.975 \\
\hline Yerbal & La Salvaje Farm & 7BB A & 0.001 & 0.153 & 1.632 \\
\hline Yerbal & La Salvaje Farm & $7 B B$ B & 0.002 & 0.222 & 2.557 \\
\hline Yerbal & La Salvaje Farm & 7BB C & 0.002 & 0.301 & 3.457 \\
\hline Yerbal & La Salvaje Farm & 7BB D & 0.000 & 0.364 & 2.658 \\
\hline Yerbal & La Salvaje Farm & 7BB E & 0.001 & 0.419 & 2.542 \\
\hline Yerbal & La Salvaje Farm & $8 \mathrm{~A}$ & 0.001 & 1.046 & 5.010 \\
\hline Yerbal & La Salvaje Farm & $8 B$ & 0.001 & 4.373 & 9.501 \\
\hline Yerbal & La Salvaje Farm & $9 B \mathrm{~A}$ & 0.010 & 10.353 & 27.766 \\
\hline Yerbal & La Salvaje Farm & $9 B \mathrm{~B}$ & 0.007 & 14.532 & 36.244 \\
\hline \multicolumn{2}{|c|}{ Urusis (Feldschuht FSH-1 } & 0.5 & 0.000 & 0.330 & 5.010 \\
\hline
\end{tabular}




\begin{tabular}{|c|c|c|c|c|}
\hline Urusis (Feldschuht FSH-1 & 2.1 & 0.000 & 0.216 & 3.920 \\
\hline Urusis (Feldschuht FSH-1 & 4.5 & 0.000 & 0.412 & 4.680 \\
\hline Urusis (Feldschuhr FSH-1 & 5.5 & 0.000 & 0.357 & 4.790 \\
\hline Urusis (Feldschuhr FSH-1 & 7.4 & 0.000 & 0.291 & 4.710 \\
\hline Urusis (Feldschuht FSH-1 & 9 & 0.000 & 0.249 & 4.750 \\
\hline Urusis (Feldschuht FSH-1 & 13.2 & 0.000 & 0.255 & 4.270 \\
\hline Urusis (Feldschuht FSH-1 & 18.4 & 0.000 & 0.275 & 5.280 \\
\hline Urusis (Feldschuhr FSH-1 & 19.5 & 0.000 & 0.300 & 5.140 \\
\hline Urusis (Feldschuht FSH-1 & 20.5 & 0.001 & 0.350 & 5.120 \\
\hline Urusis (Feldschuht FSH-1 & 22.4 & 0.000 & 0.260 & 5.010 \\
\hline Urusis (Feldschuhr FSH-1 & 22.9 & 0.000 & 0.242 & 4.880 \\
\hline Urusis (Feldschuht FSH-1 & 23.6 & 0.000 & 0.277 & 4.940 \\
\hline Urusis (Spitskopf ^SWP & 1.7 & 0.000 & 0.278 & 4.770 \\
\hline Urusis (Spitskopf ^ SWP & 2.3 & 0.000 & 0.214 & 5.290 \\
\hline Urusis (Spitskopf $\wedge$ SWP & 3.05 & 0.000 & 0.247 & 5.040 \\
\hline Urusis (Spitskopf ^ SWP & 4.7 & 0.000 & 0.214 & 4.820 \\
\hline Urusis (Spitskopf ^ SWP & 5.6 & 0.000 & 0.217 & 4.390 \\
\hline Urusis (Spitskopf ^SWP & 6.6 & 0.000 & 0.269 & 5.030 \\
\hline Urusis (Spitskopf ^ SWP & 8.3 & 0.000 & 0.306 & 4.630 \\
\hline Urusis (Spitskopf ^SWP & 8.8 & 0.000 & 0.151 & 4.960 \\
\hline Urusis (Spitskopf ^ SWP & 10.2 & 0.000 & 0.198 & 4.540 \\
\hline Urusis (Spitskopf ^SWP & 10.8 & 0.000 & 0.189 & 4.430 \\
\hline Urusis (Spitskopf ^ SWP & 11.6 & 0.000 & 0.197 & 4.680 \\
\hline Urusis (Spitskopf ^SWP & 12.8 & 0.000 & 0.348 & 4.700 \\
\hline Urusis (Spitskopf ^ SWP & 14.3 & 0.000 & 0.358 & 4.790 \\
\hline Urusis (Spitskopf ^ SWP & 14.9 & 0.000 & 0.201 & 4.850 \\
\hline Urusis (Spitskopf ^SWP & 16 & 0.000 & 0.182 & 4.850 \\
\hline Urusis (Spitskopf ^SWP & 17 & 0.000 & 0.254 & 4.830 \\
\hline Urusis (Spitskopf ^SWP & 18.8 & 0.000 & 0.163 & 4.680 \\
\hline Urusis (Spitskopf ^ SWP & 19.2 & 0.000 & 0.280 & 4.510 \\
\hline Urusis (Spitskopf ^SWP & 21.9 & 0.000 & 0.207 & 4.460 \\
\hline Urusis (Spitskopf ^ SWP & 23.6 & 0.001 & 0.249 & 4.860 \\
\hline Urusis (Spitskopf ^SWP & 24 & 0.000 & 0.225 & 4.390 \\
\hline Urusis (Spitskopf ^ SWP & 27.7 & 0.002 & 0.303 & 4.300 \\
\hline Urusis (Spitskopf ^ SWP & 28.65 & 0.003 & 0.417 & 4.440 \\
\hline Urusis (Spitskopf ^SWP & 28.9 & 0.000 & 0.363 & 4.150 \\
\hline Urusis (Spitskopf ^SWP & 36.6 & 0.001 & 0.470 & 3.250 \\
\hline Urusis (Spitskopf ^SWP & 51.08 & 0.002 & 0.156 & 1.810 \\
\hline Urusis (Spitskopf ^ SWP & 51.18 & 0.000 & 0.152 & 1.600 \\
\hline Urikos Member (K ZBH (Zebra River) & 1 & 0.000 & 0.231 & 4.930 \\
\hline Urikos Member (K ZBH (Zebra River) & 2 & 0.000 & 0.201 & 5.770 \\
\hline
\end{tabular}




\begin{tabular}{|c|c|c|c|c|c|}
\hline \multicolumn{2}{|c|}{ Urikos Member (K ZBH (Zebra River) } & 4 & 0.000 & 0.369 & 6.010 \\
\hline \multicolumn{2}{|c|}{ Mara Member (KuZUB (Zuurburg Farn } & 2.25 & 0.000 & 0.867 & 4.080 \\
\hline \multicolumn{2}{|c|}{ Mara Member (KuZUB (Zuurburg Farn } & 2.4 & 0.002 & 0.469 & 2.880 \\
\hline \multicolumn{2}{|c|}{ Mara Member (KuZUB (Zuurburg Farn } & 3 & 0.000 & 0.694 & 3.890 \\
\hline \multicolumn{2}{|c|}{ Mara Member (KuZUB (Zuurburg Farn } & 3.7 & 0.000 & 0.684 & 3.710 \\
\hline \multicolumn{2}{|c|}{ Mara Member (KuZUB (Zuurburg Farn } & 3.95 & 0.000 & 0.614 & 3.680 \\
\hline \multicolumn{2}{|c|}{ Mara Member (Ku ZUB (Zuurburg Farn } & 4.45 & 0.000 & 1.285 & 4.280 \\
\hline \multicolumn{2}{|c|}{ Tsagaan Oloom (ZıE1216 } & 36.4 & 0.044 & 1.138 & 2.170 \\
\hline \multicolumn{2}{|c|}{ Tsagaan Oloom (ZıE1216 } & 36.8 & 0.444 & 1.254 & 2.140 \\
\hline \multicolumn{2}{|c|}{ Tsagaan Oloom (ZıE1216 } & 42.4 & 0.051 & 0.721 & 1.460 \\
\hline \multicolumn{2}{|c|}{ Tsagaan Oloom (ZıE1216 } & 43.2 & 0.627 & 1.528 & 2.150 \\
\hline Dracoisen & G306 & 159 & 0.349 & 1.169 & 1.430 \\
\hline Dracoisen & G436 & 138 & 0.036 & 0.944 & 2.631 \\
\hline Dracoisen & G306a & 143 & 0.984 & 2.811 & 3.965 \\
\hline Dracoisen & G306 & 131 & 0.688 & 1.841 & 3.529 \\
\hline Dracoisen & G436 & 120 & 0.022 & 0.719 & 4.262 \\
\hline Dracoisen & G436 & 105 & 0.548 & 1.170 & 5.192 \\
\hline Dracoisen & G436 & 101.5 & 0.716 & 1.318 & 3.313 \\
\hline Dracoisen & G436 & 95.5 & 0.182 & 0.822 & 3.825 \\
\hline Dracoisen & G436 & 88 & 0.062 & 0.456 & 3.612 \\
\hline Dracoisen & G411 & 72 & 0.059 & 1.029 & 3.813 \\
\hline Dracoisen & G411 & 67 & 0.003 & 0.953 & 4.324 \\
\hline Dracoisen & G436 & 64 & 0.660 & 1.567 & 3.464 \\
\hline Dracoisen & G411 & 45 & 0.023 & 2.062 & 3.381 \\
\hline Dracoisen & G436 & 42 & 0.282 & 1.260 & 3.037 \\
\hline Dracoisen & G411 & 41 & 0.271 & 2.757 & 4.369 \\
\hline Dracoisen & G411 & 37 & 0.037 & 1.541 & 4.420 \\
\hline Dracoisen & G436 & 10.5 & 0.480 & 1.110 & 3.706 \\
\hline above Niutitang & above Niutitang & 117.9 & 1.200 & 2.020 & 2.550 \\
\hline above Niutitang & above Niutitang & 118.6 & 1.420 & 2.830 & 3.320 \\
\hline Niutitang & Niutitang & 46.7 & 1.580 & 1.870 & 2.710 \\
\hline Niutitang & Niutitang & 46.85 & 0.050 & 0.080 & 0.110 \\
\hline Niutitang & Niutitang & 47.5 & 1.950 & 2.430 & 3.220 \\
\hline Niutitang & Niutitang & 48.5 & 1.380 & 1.690 & 2.400 \\
\hline Niutitang & Niutitang & 50.25 & 2.070 & 2.730 & 3.160 \\
\hline Niutitang & Niutitang & 50.45 & 0.300 & 0.470 & 0.480 \\
\hline Niutitang & Niutitang & 51.4 & 2.040 & 2.960 & 3.070 \\
\hline Niutitang & Niutitang & 52.15 & 2.340 & 2.600 & 3.640 \\
\hline Niutitang & Niutitang & 52.4 & 1.950 & 2.880 & 4.270 \\
\hline Niutitang & Niutitang & 53.3 & 0.630 & 0.750 & 0.760 \\
\hline Niutitang & Niutitang & 56.2 & 1.680 & 1.850 & 3.440 \\
\hline
\end{tabular}




\begin{tabular}{|c|c|c|c|c|c|}
\hline Niutitang & Niutitang & 57.7 & 1.760 & 2.920 & 3.010 \\
\hline Niutitang & Niutitang & 59.3 & 1.210 & 2.210 & 2.260 \\
\hline Niutitang & Niutitang & 60.3 & 0.240 & 0.590 & 0.620 \\
\hline Niutitang & Niutitang & 63.82 & 0.240 & 0.340 & 0.340 \\
\hline Niutitang & Niutitang & 64.92 & 0.010 & 0.080 & 0.230 \\
\hline Niutitang & Niutitang & 66.12 & 0.010 & 0.050 & 0.130 \\
\hline Niutitang & Niutitang & 70.12 & 0.820 & 1.320 & 1.440 \\
\hline Niutitang & Niutitang & 74.12 & 1.650 & 3.040 & 3.300 \\
\hline Niutitang & Niutitang & 77.62 & 2.730 & 3.640 & 3.720 \\
\hline Niutitang & Niutitang & 80.62 & 1.180 & 1.420 & 2.420 \\
\hline Niutitang & Niutitang & 82.92 & 0.030 & 0.260 & 0.390 \\
\hline Niutitang & Niutitang & 87.52 & 0.000 & 0.120 & 0.340 \\
\hline \multicolumn{2}{|c|}{ Niutitang (Xiaosi n Niutitang (Xiaosi mı } & 99.55 & 0.620 & 1.410 & 2.770 \\
\hline \multicolumn{2}{|c|}{ Niutitang (Xiaosi m Niutitang (Xiaosi mı } & 100.55 & 0.340 & 1.880 & 2.760 \\
\hline \multicolumn{2}{|c|}{ Niutitang (Xiaosi m Niutitang (Xiaosi mı } & 102.85 & 0.520 & 1.540 & 2.380 \\
\hline \multicolumn{2}{|c|}{ Niutitang (Xiaosi m Niutitang (Xiaosi mı } & 104.45 & 0.530 & 1.370 & 1.810 \\
\hline \multicolumn{2}{|c|}{ Niutitang (Xiaosi m Niutitang (Xiaosi mı } & 106.75 & 1.360 & 1.840 & 3.090 \\
\hline \multicolumn{2}{|c|}{ Niutitang (Xiaosi m Niutitang (Xiaosi mı } & 107.75 & 0.260 & 0.790 & 0.920 \\
\hline \multicolumn{2}{|c|}{ Niutitang (Xiaosi n Niutitang (Xiaosi mı } & 109.25 & 0.430 & 1.230 & 1.500 \\
\hline \multicolumn{2}{|c|}{ Niutitang (Xiaosi n Niutitang (Xiaosi mı } & 110.45 & 0.630 & 0.910 & 2.500 \\
\hline \multicolumn{2}{|c|}{ Niutitang (Xiaosi n Niutitang (Xiaosi mı } & 111.95 & 0.010 & 2.040 & 2.520 \\
\hline \multicolumn{2}{|c|}{ Niutitang (Xiaosi m Niutitang (Xiaosi mı } & 113.05 & 0.030 & 0.150 & 0.150 \\
\hline \multicolumn{2}{|c|}{ Niutitang (Xiaosi n Niutitang (Xiaosi mı } & 114.25 & 1.160 & 1.580 & 1.920 \\
\hline \multicolumn{2}{|c|}{ Niutitang (Xiaosi n Niutitang (Xiaosi mı } & 115.25 & 0.080 & 0.330 & 0.330 \\
\hline \multicolumn{2}{|c|}{ Niutitang (Xiaosi m Niutitang (Xiaosi mı } & 116.25 & 0.120 & 0.450 & 0.540 \\
\hline \multicolumn{2}{|c|}{ Niutitang (Xiaosi m Niutitang (Xiaosi mı } & 117.15 & 0.110 & 0.740 & 0.740 \\
\hline Niutitang? & Niutitang? & 46.65 & 0.050 & 0.150 & 0.160 \\
\hline Yu'anshan Shale & Ma'Feng core & Cheng438 & 2.104 & 2.473 & 4.994 \\
\hline Yu'anshan Shale & Ma'Feng core & Cheng442 & 2.691 & 3.037 & 5.499 \\
\hline Yu'anshan Shale & Ma'Feng core & Cheng456 & 1.493 & 1.795 & 4.363 \\
\hline Yu'anshan Shale & Ma'Feng core & Cheng476 & 1.679 & 1.889 & 4.321 \\
\hline Yu'anshan Shale & Ma'Feng core & Cheng485 & 1.932 & 2.264 & 4.455 \\
\hline Niutitang & Yangjiaping & 12.8 & 0.900 & 1.100 & 1.400 \\
\hline Niutitang & Yangjiaping & 13.8 & 1.100 & 1.100 & 1.500 \\
\hline Niutitang & Yangjiaping & 14.8 & 0.000 & 1.500 & 1.800 \\
\hline Niutitang & Yangjiaping & 15.3 & 0.200 & 0.200 & 0.400 \\
\hline Niutitang & Yangjiaping & 16.3 & 0.100 & 0.100 & 0.500 \\
\hline Niutitang & Yangjiaping & 24.3 & 0.900 & 1.100 & 2.000 \\
\hline Niutitang & Yangjiaping & 26.3 & 0.900 & 1.200 & 2.400 \\
\hline Niutitang & Yangjiaping & 29.3 & 0.000 & 0.200 & 1.400 \\
\hline Niutitang & Yangjiaping & 31.3 & 0.000 & 0.100 & 1.300 \\
\hline
\end{tabular}




\begin{tabular}{|c|c|c|c|c|c|}
\hline Niutitang & Yangjiaping & 33.3 & 0.000 & 0.200 & 2.000 \\
\hline Niutitang & Yangjiaping & 35.3 & 0.800 & 1.400 & 3.200 \\
\hline Niutitang & Yangjiaping & 37.3 & 0.600 & 0.900 & 2.500 \\
\hline Niutitang & Yangjiaping & 39.3 & 0.500 & 0.600 & 2.500 \\
\hline Niutitang & Yangjiaping & 41.3 & 0.400 & 0.800 & 2.500 \\
\hline Niutitang & Yangjiaping & 43.3 & 0.800 & 1.200 & 2.600 \\
\hline Niutitang & Yangjiaping & 45.3 & 1.000 & 1.500 & 3.200 \\
\hline Niutitang & Yangjiaping & 47.3 & 0.900 & 1.200 & 3.800 \\
\hline Niutitang & Yangjiaping & 49.3 & 1.100 & 1.300 & 4.100 \\
\hline Niutitang & Yangjiaping & 51.3 & 0.000 & 1.200 & 3.100 \\
\hline Niutitang & Yangjiaping & 53.3 & 0.200 & 0.700 & 1.500 \\
\hline Niutitang & Yangjiaping & 55.3 & 0.000 & 0.700 & 1.200 \\
\hline Niutitang & Yangjiaping & 57.3 & 0.100 & 0.300 & 2.400 \\
\hline Niutitang & Yangjiaping & 59.3 & 1.000 & 1.100 & 2.800 \\
\hline Niutitang & Yangjiaping & 63.3 & 0.600 & 0.800 & 2.600 \\
\hline Niutitang & Yangjiaping & 65.3 & 1.000 & 1.300 & 4.000 \\
\hline Niutitang & Yangjiaping & 69.3 & 1.000 & 1.300 & 3.900 \\
\hline Niutitang & Yangjiaping & 70.3 & 0.000 & 0.500 & 1.800 \\
\hline Niutitang & Yangjiaping & 73.3 & 0.000 & 0.700 & 2.200 \\
\hline Niutitang & Yangjiaping & 77.3 & 0.400 & 0.800 & 2.700 \\
\hline Niutitang & Yangjiaping & 79.3 & 0.000 & 1.500 & 3.200 \\
\hline Shiyantou & Kunyang Mine- Mei & KY2 & 1.350 & & 2.280 \\
\hline Shiyantou & Kunyang Mine- Mei & KY3 & 0.710 & & 2.170 \\
\hline Shiyantou & Kunyang Mine- Mei & KY4 & 1.150 & & 2.710 \\
\hline Shiyantou & Kunyang Mine- Mei & KY5 & 0.840 & & 2.750 \\
\hline Shiyantou & Kunyang Mine- Mei & KY6 & 1.320 & & 3.290 \\
\hline Shiyantou & Kunyang Mine- Mei & KY7 & 0.780 & & 2.680 \\
\hline Shiyantou & Kunyang Mine- Mei & KY8 & 0.610 & & 2.360 \\
\hline Shiyantou & Xiaotan & 0 & 0.010 & & 1.580 \\
\hline Shiyantou & Xiaotan & 0.3 & 0.010 & & 3.280 \\
\hline Shiyantou & Xiaotan & 1.3 & 0.000 & & 0.940 \\
\hline Shiyantou & Xiaotan & 2.3 & 0.000 & & 1.630 \\
\hline Shiyantou & Xiaotan & 3.6 & 0.000 & & 1.640 \\
\hline Shiyantou & Xiaotan & 5.1 & 0.050 & & 1.700 \\
\hline Shiyantou & Xiaotan & 5.9 & 0.000 & & 1.280 \\
\hline Shiyantou & Xiaotan & 6.07 & 0.010 & & 1.250 \\
\hline Shiyantou & Xiaotan & 6.1 & 0.000 & & 1.640 \\
\hline Shiyantou & Xiaotan & 6.17 & 0.010 & & 1.220 \\
\hline Shiyantou & Xiaotan & 6.28 & 0.000 & & 1.410 \\
\hline Shiyantou & Xiaotan & 6.43 & 0.000 & & 1.400 \\
\hline Shiyantou & Xiaotan & 6.53 & 0.000 & & 1.730 \\
\hline
\end{tabular}




\begin{tabular}{|c|c|c|c|c|}
\hline Shiyantou & Xiaotan & 6.55 & 0.000 & 2.010 \\
\hline Shiyantou & Xiaotan & 6.56 & 0.000 & 2.690 \\
\hline Shiyantou & Xiaotan & 6.58 & 0.010 & 1.940 \\
\hline Shiyantou & Xiaotan & 6.6 & 0.000 & 2.610 \\
\hline Shiyantou & Xiaotan & 6.7 & 0.010 & 1.560 \\
\hline Shiyantou & Xiaotan & 6.79 & 0.000 & 1.460 \\
\hline Shiyantou & Xiaotan & 6.91 & 0.010 & 0.810 \\
\hline Shiyantou & Xiaotan & 7.09 & 0.010 & 1.490 \\
\hline Shiyantou & Xiaotan & 7.1 & 0.010 & 1.510 \\
\hline Shiyantou & Xiaotan & 8.1 & 0.010 & 1.560 \\
\hline Shiyantou & Xiaotan & 9.6 & 0.010 & 1.500 \\
\hline Shiyantou & Xiaotan & 10.6 & 0.010 & 1.640 \\
\hline Shiyantou & Xiaotan & 12 & 0.010 & 1.190 \\
\hline Shiyantou & Xiaotan & 13.5 & 0.010 & 1.070 \\
\hline Shiyantou & Xiaotan & 14.5 & 0.010 & 1.230 \\
\hline Shiyantou & Xiaotan & 15.5 & 0.010 & 1.680 \\
\hline Shiyantou & Xiaotan & 16.5 & 0.010 & 1.540 \\
\hline Shiyantou & Xiaotan & 18.5 & 0.010 & 2.310 \\
\hline Shiyantou & Xiaotan & 20.3 & 0.010 & 1.420 \\
\hline Shiyantou & Xiaotan & 21.3 & 0.010 & 1.540 \\
\hline Shiyantou & Xiaotan & 22.6 & 0.010 & 1.720 \\
\hline Shiyantou & Xiaotan & 24.1 & 0.010 & 1.770 \\
\hline Shiyantou & Xiaotan & 25.1 & 0.010 & 1.990 \\
\hline Shiyantou & Xiaotan & 26.1 & 0.010 & 1.700 \\
\hline Shiyantou & Xiaotan & 27.9 & 0.010 & 2.290 \\
\hline Shiyantou & Xiaotan & 29.9 & 0.010 & 1.940 \\
\hline Shiyantou & Xiaotan & 30.9 & 0.010 & 1.630 \\
\hline Shiyantou & Xiaotan & 31.9 & 0.010 & 1.410 \\
\hline Shiyantou & Xiaotan & 33.9 & 0.010 & 3.500 \\
\hline Shiyantou & Xiaotan & 36.4 & 0.010 & 2.540 \\
\hline Shiyantou & Xiaotan & XTY1 & 0.010 & 1.600 \\
\hline Shiyantou & Xiaotan & XTY10 & 0.010 & 1.480 \\
\hline Shiyantou & Xiaotan & XTY11 & 0.010 & 1.560 \\
\hline Shiyantou & Xiaotan & XTY12 & 0.010 & 2.090 \\
\hline Shiyantou & Xiaotan & XTY13 & 0.010 & 2.450 \\
\hline Shiyantou & Xiaotan & XTY14 & 0.110 & 2.290 \\
\hline Shiyantou & Xiaotan & XTY15 & 0.170 & 2.240 \\
\hline Shiyantou & Xiaotan & XTY16 & 0.010 & 1.330 \\
\hline Shiyantou & Xiaotan & XTY17 & 0.010 & 2.650 \\
\hline Shiyantou & Xiaotan & XTY17a & 0.010 & 2.460 \\
\hline Shiyantou & Xiaotan & XTY17b & 0.000 & 2.160 \\
\hline
\end{tabular}




\begin{tabular}{|c|c|c|c|c|}
\hline Shiyantou & Xiaotan & XTY17c & 0.010 & 1.820 \\
\hline Shiyantou & Xiaotan & XTY17d & 0.030 & 2.130 \\
\hline Shiyantou & Xiaotan & XTY17e & 0.030 & 2.340 \\
\hline Shiyantou & Xiaotan & XTY17f & 0.020 & 1.740 \\
\hline Shiyantou & Xiaotan & XTY18 & 0.100 & 2.210 \\
\hline Shiyantou & Xiaotan & XTY18a & 0.000 & 3.140 \\
\hline Shiyantou & Xiaotan & XTY18b & 0.020 & 1.750 \\
\hline Shiyantou & Xiaotan & XTY18c & 0.010 & 1.750 \\
\hline Shiyantou & Xiaotan & XTY18d & 0.000 & 1.610 \\
\hline Shiyantou & Xiaotan & XTY18e & 0.000 & 2.240 \\
\hline Shiyantou & Xiaotan & XTY19 & 0.010 & 1.760 \\
\hline Shiyantou & Xiaotan & XTY19a & 0.070 & 1.630 \\
\hline Shiyantou & Xiaotan & XTY19b & 0.000 & 1.990 \\
\hline Shiyantou & Xiaotan & XTY19c & 0.070 & 1.900 \\
\hline Shiyantou & Xiaotan & XTY19d & 0.010 & 1.410 \\
\hline Shiyantou & Xiaotan & XTY19e & 0.020 & 2.820 \\
\hline Shiyantou & Xiaotan & XTY19f & 0.010 & 1.720 \\
\hline Shiyantou & Xiaotan & XTY2 & 0.000 & 2.650 \\
\hline Shiyantou & Xiaotan & XTY20 & 0.120 & 3.040 \\
\hline Shiyantou & Xiaotan & XTY21 & 0.000 & 1.790 \\
\hline Shiyantou & Xiaotan & XTY22 & 0.010 & 1.960 \\
\hline Shiyantou & Xiaotan & XTY23 & 0.070 & 3.260 \\
\hline Shiyantou & Xiaotan & XTY24 & 0.010 & 1.680 \\
\hline Shiyantou & Xiaotan & XTY25 & 0.010 & 2.440 \\
\hline Shiyantou & Xiaotan & XTY26 & 0.090 & 2.760 \\
\hline Shiyantou & Xiaotan & XTY27 & 0.000 & 4.440 \\
\hline Shiyantou & Xiaotan & XTY28 & 0.060 & 2.530 \\
\hline Shiyantou & Xiaotan & XTY29 & 0.000 & 2.950 \\
\hline Shiyantou & Xiaotan & XTY3 & 0.000 & 3.090 \\
\hline Shiyantou & Xiaotan & XTY30 & 0.000 & 4.270 \\
\hline Yuanshan & Xiaotan & XTY35 & 0.060 & 1.560 \\
\hline Yuanshan & Xiaotan & XTY36 & 0.010 & 3.100 \\
\hline Yuanshan & Xiaotan & XTY37 & 0.080 & 3.370 \\
\hline Yuanshan & Xiaotan & XTY38 & 0.010 & 3.330 \\
\hline Yuanshan & Xiaotan & XTY39 & 0.000 & 5.310 \\
\hline Shiyantou & Xiaotan & XTY4 & 0.010 & 2.640 \\
\hline Yuanshan & Xiaotan & XTY40 & 0.050 & 2.380 \\
\hline Yuanshan & Xiaotan & XTY41 & 0.150 & 4.520 \\
\hline Yuanshan & Xiaotan & XTY42 & 0.120 & 4.700 \\
\hline Yuanshan & Xiaotan & XTY43 & 0.090 & 3.830 \\
\hline Yuanshan & Xiaotan & XTY44 & 0.030 & 4.600 \\
\hline
\end{tabular}




\begin{tabular}{|c|c|c|c|c|c|}
\hline Yuanshan & Xiaotan & XTY45 & 0.200 & & 4.540 \\
\hline Yuanshan & Xiaotan & XTY46 & 0.160 & & 2.830 \\
\hline Yuanshan & Xiaotan & XTY47 & 0.080 & & 4.320 \\
\hline Yuanshan & Xiaotan & XTY48 & 0.190 & & 3.990 \\
\hline Yuanshan & Xiaotan & XTY49 & 0.180 & & 4.220 \\
\hline Shiyantou & Xiaotan & XTY5 & 0.010 & & 2.010 \\
\hline Yuanshan & Xiaotan & XTY50 & 0.170 & & 2.700 \\
\hline Yuanshan & Xiaotan & XTY51 & 0.730 & & 4.180 \\
\hline Yuanshan & Xiaotan & XTY52 & 0.730 & & 4.230 \\
\hline Yuanshan & Xiaotan & XTY53 & 0.800 & & 4.490 \\
\hline Yuanshan & Xiaotan & XTY54 & 0.590 & & 4.770 \\
\hline Yuanshan & Xiaotan & XTY55 & 0.300 & & 4.240 \\
\hline Yuanshan & Xiaotan & XTY56 & 0.340 & & 4.070 \\
\hline Yuanshan & Xiaotan & XTY57 & 0.020 & & 4.280 \\
\hline Yuanshan & Xiaotan & XTY58 & 0.330 & & 4.420 \\
\hline Yuanshan & Xiaotan & XTY59 & 0.010 & & 4.260 \\
\hline Shiyantou & Xiaotan & XTY6 & 0.010 & & 2.250 \\
\hline Yuanshan & Xiaotan & XTY60 & 0.100 & & 4.270 \\
\hline Yuanshan & Xiaotan & XTY61 & 0.020 & & 3.290 \\
\hline Shiyantou & Xiaotan & XTY7 & 0.010 & & 2.300 \\
\hline Shiyantou & Xiaotan & XTY8 & 0.010 & & 1.700 \\
\hline Shiyantou & Xiaotan & XTY9 & 0.020 & & 1.670 \\
\hline Niutitang & Zhongnan & ZNOA & 0.010 & & 0.720 \\
\hline Niutitang & Zhongnan & ZNOB & 0.020 & & 1.090 \\
\hline Niutitang & Zhongnan & ZNOC & 0.060 & & 1.800 \\
\hline Niutitang & Zhongnan & ZN1 & 0.020 & & 0.380 \\
\hline Niutitang & Zhongnan & ZN10 & 0.030 & & 0.590 \\
\hline Niutitang & Zhongnan & ZN11 & 0.020 & & 1.030 \\
\hline Niutitang & Zhongnan & ZN12 & 0.020 & & 0.890 \\
\hline Niutitang & Zhongnan & ZN13 & 0.010 & & 0.590 \\
\hline Niutitang & Zhongnan & ZN14 & 0.010 & & 0.450 \\
\hline Niutitang & Zhongnan & $\mathrm{ZN2}$ & 0.010 & & 1.920 \\
\hline Niutitang & Zhongnan & ZN3 & 0.860 & & 1.740 \\
\hline Niutitang & Zhongnan & ZN4 & 1.960 & & 13.010 \\
\hline Niutitang & Zhongnan & ZN5 & 8.150 & & 9.870 \\
\hline Niutitang & Zhongnan & ZN7 & 0.020 & & 1.080 \\
\hline Niutitang & Zhongnan & ZN8 & 0.030 & & 1.670 \\
\hline Niutitang & Zhongnan & ZN9 & 0.010 & & 0.430 \\
\hline Niutitang & Longbizui & LBZ-100 & 0.000 & 0.170 & 0.840 \\
\hline Niutitang & Longbizui & LBZ-101 & 0.040 & 0.230 & 0.970 \\
\hline Niutitang & Longbizui & LBZ-102 & 0.000 & 0.080 & 0.830 \\
\hline
\end{tabular}




\begin{tabular}{|c|c|c|c|c|c|}
\hline Niutitang & Longbizui & LBZ-103 & 0.850 & 1.570 & 4.660 \\
\hline Liuchapo & Longbizui & LBZ-37 & 0.070 & 1.040 & 1.700 \\
\hline Liuchapo & Longbizui & LBZ-38 & 0.060 & 0.310 & 0.310 \\
\hline Liuchapo & Longbizui & LBZ-39 & 0.050 & 0.400 & 0.400 \\
\hline Liuchapo & Longbizui & LBZ-40 & 0.100 & 0.510 & 0.600 \\
\hline Liuchapo & Longbizui & LBZ-41 & 0.080 & 0.670 & 0.670 \\
\hline Liuchapo & Longbizui & LBZ-42 & 0.080 & 0.420 & 0.470 \\
\hline Liuchapo & Longbizui & LBZ-43 & 0.090 & 0.410 & 0.460 \\
\hline Liuchapo & Longbizui & LBZ-44 & 0.130 & 0.380 & 0.440 \\
\hline Liuchapo & Longbizui & LBZ-45 & 0.070 & 0.350 & 0.370 \\
\hline Liuchapo & Longbizui & LBZ-46 & 0.080 & 0.320 & 0.340 \\
\hline Liuchapo & Longbizui & LBZ-54 & 0.030 & 0.260 & 0.270 \\
\hline Liuchapo & Longbizui & LBZ-57 & 1.850 & 2.400 & 2.930 \\
\hline Liuchapo & Longbizui & LBZ-59 & 0.070 & 0.280 & 0.290 \\
\hline Niutitang & Longbizui & LBZ-69 & 0.550 & 1.890 & 2.950 \\
\hline Niutitang & Longbizui & LBZ-73 & 0.770 & 1.470 & 1.510 \\
\hline Niutitang & Longbizui & LBZ-78 & 1.290 & 2.190 & 2.250 \\
\hline Niutitang & Longbizui & LBZ-80 & 0.420 & 0.670 & 0.730 \\
\hline Niutitang & Longbizui & LBZ-82 & 1.570 & 2.290 & 3.000 \\
\hline Niutitang & Longbizui & LBZ-88 & 0.230 & 0.860 & 0.900 \\
\hline Niutitang & Longbizui & LBZ-91 & 0.250 & 1.060 & 1.210 \\
\hline Niutitang & Longbizui & LBZ-94 & 0.580 & 2.740 & 2.730 \\
\hline Niutitang & Longbizui & LBZ-96 & 0.160 & 0.640 & 0.620 \\
\hline Niutitang & Longbizui & LBZ-98 & 0.370 & 1.400 & 1.410 \\
\hline \multirow[t]{17}{*}{ Niutitang } & Longbizui & LBZ-99 & 0.000 & 0.540 & 1.020 \\
\hline & J1218 & 41.7 & 0.003 & 0.657 & 1.530 \\
\hline & J1218 & 42 & 0.005 & 2.090 & 3.230 \\
\hline & J1218 & 42.9 & 0.588 & 2.527 & 3.470 \\
\hline & $\mathrm{J} 1218$ & 50.8 & 0.170 & 1.066 & 3.200 \\
\hline & $\mathrm{J} 1218$ & 52 & 0.380 & 1.761 & 3.270 \\
\hline & $\mathrm{J} 1218$ & 54 & 0.116 & 1.440 & 3.300 \\
\hline & J1218 & 56 & 0.671 & 1.511 & 3.720 \\
\hline & $\mathrm{J} 1218$ & 58 & 0.893 & 1.889 & 4.280 \\
\hline & J1218 & 60 & 0.666 & 1.832 & 3.670 \\
\hline & J1218 & 62 & 0.599 & 1.143 & 3.550 \\
\hline & J1218 & 63.3 & 0.314 & 0.812 & 3.390 \\
\hline & J1218 & 64 & 0.127 & 1.054 & 3.110 \\
\hline & J1218 & 66 & 0.374 & 1.003 & 3.090 \\
\hline & $\mathrm{J} 1218$ & 68 & 0.317 & 3.362 & 4.170 \\
\hline & J1218 & 74 & 0.650 & 1.494 & 2.840 \\
\hline & J1218 & 76 & 0.270 & 2.058 & 3.020 \\
\hline
\end{tabular}


J1218

J1218

J1218

Wheeler

Wheeler

Wheeler

Wheeler

Wheeler

Wheeler

Wheeler

Wheeler

Wheeler

Wheeler

Wheeler

Wheeler

Wheeler

Wheeler

Wheeler

Wheeler

Wheeler

Wheeler

Wheeler

Wheeler

Wheeler

Wheeler

Wheeler

Wheeler

Wheeler

Wheeler

Wheeler

Wheeler

Wheeler

Wheeler

Wheeler

Wheeler

Wheeler

Wheeler

Wheeler

Wheeler

Wheeler

Wheeler

\begin{tabular}{|c|c|c|c|}
\hline 78 & 0.006 & 0.773 & 2.180 \\
\hline 80 & 0.210 & 0.554 & 2.030 \\
\hline 90.3 & 0.087 & 1.371 & 1.840 \\
\hline DM30 & 0.000 & 1.221 & 2.770 \\
\hline DM310.5 & 0.180 & 0.592 & 4.140 \\
\hline DM313.5 & 0.050 & 0.752 & 3.420 \\
\hline DM315 & 0.040 & 0.751 & 4.010 \\
\hline DM319.5 & 0.070 & 1.039 & 3.610 \\
\hline DM321 & 0.070 & 1.056 & 4.180 \\
\hline DM322.5 & 0.110 & 1.054 & 4.480 \\
\hline DM324 & 0.010 & 0.649 & 3.680 \\
\hline DM325.5 & 0.130 & 1.210 & 3.270 \\
\hline DM327 & 0.060 & 0.665 & 3.890 \\
\hline DM328.5 & 0.060 & 0.702 & 3.920 \\
\hline DM330 & 0.070 & 0.662 & 3.230 \\
\hline DM331.5 & 0.020 & 0.942 & 2.820 \\
\hline DM334.5 & 0.010 & 0.655 & 2.830 \\
\hline DM336 & 0.010 & 0.770 & 2.710 \\
\hline DM337.5 & 0.040 & 0.578 & 2.680 \\
\hline DM339 & 0.040 & 0.895 & 2.530 \\
\hline DM34.5 & 0.060 & 1.648 & 4.950 \\
\hline DM39 & 0.010 & 0.400 & 5.040 \\
\hline MP10.5 & 0.000 & 0.834 & 2.870 \\
\hline MP100.5 & 0.020 & 0.491 & 3.500 \\
\hline MP102 & 0.040 & 0.845 & 2.420 \\
\hline MP103.5 & 0.000 & 1.384 & 2.360 \\
\hline MP105 & 0.010 & 0.573 & 3.200 \\
\hline MP106.5 & 0.010 & 1.204 & 2.180 \\
\hline MP108 & 0.000 & 1.050 & 2.590 \\
\hline MP109.5 & 0.010 & 1.465 & 2.240 \\
\hline MP111 & 0.010 & 1.004 & 3.050 \\
\hline MP112.5 & 0.010 & 0.925 & 2.840 \\
\hline MP114 & 0.010 & 0.880 & 2.150 \\
\hline MP115.5 & 0.010 & 0.981 & 1.830 \\
\hline MP117 & 0.000 & 0.721 & 1.910 \\
\hline MP118.5 & 0.000 & 0.647 & 2.000 \\
\hline MP12 & 0.010 & 1.135 & 3.090 \\
\hline MP120 & 0.010 & 0.840 & 2.420 \\
\hline MP121.5 & 0.060 & 0.866 & 2.450 \\
\hline MP123 & 0.040 & 0.741 & 1.970 \\
\hline MP124.5 & 0.020 & 0.832 & 2.140 \\
\hline
\end{tabular}




\begin{tabular}{|c|c|c|c|c|}
\hline Wheeler & MP126 & 0.000 & 0.818 & 2.060 \\
\hline Wheeler & MP128 & 0.020 & 0.779 & 1.630 \\
\hline Wheeler & MP136.25 & 0.000 & 0.746 & 1.500 \\
\hline Wheeler & MP137 & 0.000 & 0.717 & 2.850 \\
\hline Wheeler & MP15 & 0.000 & 0.738 & 3.810 \\
\hline Wheeler & MP16.5 & 0.010 & 0.654 & 4.310 \\
\hline Wheeler & MP18 & 0.020 & 0.674 & 4.140 \\
\hline Wheeler & MP19.5 & 0.020 & 0.241 & 0.340 \\
\hline Wheeler & MP2.5 & 0.000 & 0.427 & 0.590 \\
\hline Wheeler & MP25.5 & 0.000 & 0.737 & 1.210 \\
\hline Wheeler & MP30 & 0.000 & 0.619 & 2.510 \\
\hline Wheeler & MP31.5 & 0.010 & 0.565 & 2.740 \\
\hline Wheeler & MP33 & 0.010 & 0.485 & 3.780 \\
\hline Wheeler & MP34.5 & 0.020 & 0.485 & 3.400 \\
\hline Wheeler & MP36 & 0.030 & 0.415 & 3.050 \\
\hline Wheeler & MP37.5 & 0.010 & 0.380 & 2.540 \\
\hline Wheeler & MP39 & 0.010 & 0.973 & 3.330 \\
\hline Wheeler & MP42 & 0.000 & 0.529 & 4.790 \\
\hline Wheeler & MP45 & 0.020 & 0.489 & 4.760 \\
\hline Wheeler & MP46.5 & 0.060 & 0.569 & 4.170 \\
\hline Wheeler & MP50.25 & 0.000 & 0.442 & 3.210 \\
\hline Wheeler & MP51 & 0.010 & 0.702 & 2.260 \\
\hline Wheeler & MP52.5 & 0.000 & 0.810 & 2.070 \\
\hline Wheeler & MP55.5 & 0.000 & 0.829 & 2.680 \\
\hline Wheeler & MP57 & 0.000 & 0.753 & 2.830 \\
\hline Wheeler & MP58 & 0.000 & 0.552 & 1.790 \\
\hline Wheeler & MP6 & 0.000 & 0.687 & 4.280 \\
\hline Wheeler & MP62.5 & 0.000 & 0.512 & 2.890 \\
\hline Wheeler & MP64.5 & 0.000 & 0.700 & 2.280 \\
\hline Wheeler & MP65.5 & 0.030 & 0.635 & 1.790 \\
\hline Wheeler & MP67.5 & 0.000 & 0.589 & 1.820 \\
\hline Wheeler & MP69.5 & 0.000 & 0.866 & 2.230 \\
\hline Wheeler & MP71.75 & 0.000 & 0.642 & 2.720 \\
\hline Wheeler & MP76.5 & 0.010 & 1.890 & 3.060 \\
\hline Wheeler & MP78 & 0.000 & 1.190 & 3.060 \\
\hline Wheeler & MP79.5 & 0.010 & 0.782 & 2.240 \\
\hline Wheeler & MP81 & 0.010 & 0.992 & 2.510 \\
\hline Wheeler & MP82.5 & 0.010 & 0.660 & 2.800 \\
\hline Wheeler & MP84 & 0.010 & 1.446 & 2.100 \\
\hline Wheeler & MP87 & 0.000 & 0.576 & 3.020 \\
\hline Wheeler & MP88.5 & 0.010 & 1.063 & 2.940 \\
\hline
\end{tabular}




\begin{tabular}{|c|c|c|c|c|}
\hline Wheeler & MP90 & 0.010 & 1.226 & 3.220 \\
\hline Wheeler & MP91.5 & 0.010 & 1.213 & 2.330 \\
\hline Wheeler & MP95.25 & 0.000 & 1.237 & 2.530 \\
\hline Wheeler & MP99 & 0.000 & 0.469 & 2.460 \\
\hline Wheeler & SQO & 0.000 & 0.464 & 1.300 \\
\hline Wheeler & SQ0.25 & 0.010 & 0.570 & 1.900 \\
\hline Wheeler & SQ1 & 0.000 & 0.433 & 1.810 \\
\hline Wheeler & SQ1.75 & 0.000 & 0.368 & 1.960 \\
\hline Wheeler & SQ11.5 & 0.000 & 0.467 & 1.620 \\
\hline Wheeler & SQ15 & 0.000 & 0.535 & 2.160 \\
\hline Wheeler & SQ16 & 0.000 & 0.392 & 1.620 \\
\hline Wheeler & SQ25.5 & 0.010 & 0.406 & 1.980 \\
\hline Wheeler & SQ25.75 & 0.000 & 1.098 & 1.910 \\
\hline Wheeler & SQ26 & 0.000 & 0.770 & 1.860 \\
\hline Wheeler & SQ28.5 & 0.000 & 1.012 & 1.730 \\
\hline Wheeler & SQ30 & 0.010 & 1.596 & 1.600 \\
\hline Wheeler & SQ30.5 & 0.000 & 0.616 & 1.870 \\
\hline Wheeler & SQ31.75 & 0.000 & 0.486 & 1.670 \\
\hline Wheeler & SQ33.25 & 0.000 & 1.027 & 1.530 \\
\hline Wheeler & SQ35 & 0.000 & 0.812 & 1.660 \\
\hline Wheeler & SQ37.25 & 0.010 & 0.811 & 1.450 \\
\hline Wheeler & SQ39.25 & 0.000 & 0.667 & 1.370 \\
\hline Wheeler & SQ42.5 & 0.010 & 0.967 & 1.810 \\
\hline Wheeler & SQ44 & 0.000 & 0.888 & 1.360 \\
\hline Wheeler & SQ45.5 & 0.010 & 0.655 & 1.530 \\
\hline Wheeler & SQ50 & 0.000 & 0.602 & 1.510 \\
\hline Wheeler & SQ52 & 0.000 & 0.521 & 1.850 \\
\hline Wheeler & SQ6 & 0.000 & 0.524 & 2.160 \\
\hline Wheeler & SQ6.25 & 0.000 & 0.409 & 2.080 \\
\hline Wheeler & SQ7.5 & 0.030 & 0.461 & 1.860 \\
\hline Wheeler & SQ8.5 & 0.000 & 0.617 & 2.290 \\
\hline Wheeler & SQ9 & 0.000 & 0.460 & 1.940 \\
\hline Wheeler & SQ9.5 & 0.000 & 0.609 & 1.780 \\
\hline Wheeler & UDQO & 0.030 & 0.309 & 3.520 \\
\hline Wheeler & UDQO & 0.040 & 0.394 & 3.580 \\
\hline Wheeler & UDQ1 & 0.020 & 0.436 & 3.790 \\
\hline Wheeler & UDQ1.5 & 0.030 & 0.473 & 3.910 \\
\hline Wheeler & UDQ10 & 0.000 & 0.376 & 3.580 \\
\hline Wheeler & UDQ11 & 0.000 & 0.414 & 3.190 \\
\hline Wheeler & UDQ11 & 0.080 & 0.611 & 2.280 \\
\hline Wheeler & UDQ11-pyrite & 0.200 & 0.746 & 1.940 \\
\hline
\end{tabular}




\begin{tabular}{|c|c|c|c|c|c|}
\hline Wheeler & & UDQ12 & 0.000 & 0.313 & 3.820 \\
\hline Wheeler & & UDQ13 & 0.000 & 0.354 & 3.490 \\
\hline Wheeler & & UDQ15 & 0.010 & 0.407 & 4.330 \\
\hline Wheeler & & UDQ18 & 0.000 & 0.403 & 3.720 \\
\hline Wheeler & & UDQ19.5 & 0.000 & 0.437 & 3.150 \\
\hline Wheeler & & UDQ2 & 0.000 & 1.329 & 3.340 \\
\hline Wheeler & & UDQ22.5 & 0.010 & 0.482 & 2.960 \\
\hline Wheeler & & UDQ24 & 0.020 & 0.535 & 2.720 \\
\hline Wheeler & & UDQ25.5 & 0.040 & 0.363 & 2.280 \\
\hline Wheeler & & UDQ28 & 0.010 & 0.495 & 1.760 \\
\hline Wheeler & & UDQ3 & 0.010 & 0.400 & 3.360 \\
\hline Wheeler & & UDQ4 & 0.020 & 1.172 & 3.450 \\
\hline Wheeler & & UDQ5 & 0.010 & 0.383 & 3.040 \\
\hline Wheeler & & UDQ6 & 0.010 & 0.315 & 3.720 \\
\hline Wheeler & & UDQ6 & 0.000 & 0.391 & 1.180 \\
\hline Wheeler & & UDQ7 & 0.000 & 0.374 & 3.690 \\
\hline Wheeler & & UDQ8 & 0.000 & 0.415 & 3.180 \\
\hline Alum & Andrarum no. 3 Cor & 52 & 4.310 & 6.477 & 7.260 \\
\hline Alum & Andrarum no. 3 Cor & 53 & 3.660 & 5.112 & 5.750 \\
\hline Alum & Andrarum no. 3 Cor & 54 & 3.920 & 4.821 & 5.310 \\
\hline Alum & Andrarum no. 3 Cor & 56 & 3.390 & 4.574 & 5.430 \\
\hline Alum & Andrarum no. 3 Cor & 57 & 2.840 & 3.416 & 3.380 \\
\hline Alum & Andrarum no. 3 Cor & 58 & 3.340 & 4.257 & 4.830 \\
\hline Alum & Andrarum no. 3 Cor & 61 & 2.800 & 3.822 & 4.920 \\
\hline Alum & Andrarum no. 3 Cor & 62 & 3.770 & 4.728 & 5.210 \\
\hline Alum & Andrarum no. 3 Cor & 63 & 2.210 & 3.142 & 4.860 \\
\hline Alum & Andrarum no. 3 Cor & 64 & 3.920 & 4.716 & 5.750 \\
\hline Alum & Andrarum no. 3 Cor & 65 & 3.000 & 4.002 & 5.120 \\
\hline Alum & Andrarum no. 3 Cor & 66 & 4.440 & 5.813 & 6.080 \\
\hline Alum & Andrarum no. 3 Cor & 67 & 5.380 & 6.714 & 7.470 \\
\hline Alum & Andrarum no. 3 Cor & 68 & 5.370 & 6.673 & 6.900 \\
\hline Alum & Andrarum no. 3 Cor & 70 & 4.160 & 4.784 & 4.840 \\
\hline Alum & Andrarum no. 3 Cor & 71 & 4.240 & 5.039 & 5.370 \\
\hline Alum & Andrarum no. 3 Cor & 72 & 5.940 & 6.880 & 8.680 \\
\hline Alum & Andrarum no. 3 Cor & 73 & 4.420 & 5.114 & 6.010 \\
\hline Alum & Andrarum no. 3 Cor & 74 & 3.270 & 4.174 & 5.260 \\
\hline Alum & Andrarum no. 3 Cor & 75 & 5.700 & 6.559 & 8.790 \\
\hline Alum & Andrarum no. 3 Cor & 76 & 2.630 & 3.020 & 5.580 \\
\hline Alum & Andrarum no. 3 Cor & 77 & 3.890 & 4.754 & 6.090 \\
\hline Alum & Andrarum no. 3 Cor & 78 & 3.500 & 4.701 & 6.080 \\
\hline Alum & Andrarum no. 3 Cor & 79 & 3.110 & 3.869 & 4.710 \\
\hline
\end{tabular}




\begin{tabular}{|c|c|c|c|c|c|}
\hline Alum & Andrarum no. 3 Cor & 80 & 3.460 & 4.400 & 4.930 \\
\hline Alum & \multicolumn{2}{|c|}{ Andrarum no. 3 Cor } & 3.260 & 4.200 & 4.090 \\
\hline \multicolumn{2}{|c|}{ Stephen Formation } & WQ1 & & & \\
\hline \multicolumn{2}{|c|}{ Stephen Formation } & WQ2 & & & \\
\hline \multicolumn{2}{|c|}{ Stephen Formation } & WQ3 & & & \\
\hline \multicolumn{2}{|c|}{ Stephen Formation } & WQ4 & & & \\
\hline \multicolumn{3}{|c|}{ Koftelv Formation Koftelv Formation } & 0.104 & 0.228 & 2.426 \\
\hline \multicolumn{3}{|c|}{ Koftelv Formation Koftelv Formation } & 0.003 & 0.199 & 1.864 \\
\hline \multicolumn{3}{|c|}{ Koftelv Formation Koftelv Formation } & 0.002 & 0.149 & 1.240 \\
\hline \multicolumn{3}{|c|}{ Koftelv Formation Koftelv Formation } & 0.058 & 1.060 & 4.306 \\
\hline \multicolumn{3}{|c|}{ Koftelv Formation Koftelv Formation } & 0.026 & 1.607 & 3.340 \\
\hline \multicolumn{3}{|c|}{ Kessyusa Formatic Kessyusa Formation } & 0.022 & 2.324 & 7.679 \\
\hline \multicolumn{3}{|c|}{ Kessyusa Formatic Kessyusa Formation } & 0.005 & 2.351 & 7.061 \\
\hline \multicolumn{3}{|c|}{ Kessyusa Formatic Kessyusa Formation } & 0.002 & 2.464 & 7.388 \\
\hline \multicolumn{3}{|c|}{ Kessyusa Formatic Kessyusa Formation } & 0.006 & 4.463 & 12.100 \\
\hline \multicolumn{3}{|c|}{ Kessyusa Formatic Kessyusa Formation } & 0.167 & 4.756 & 12.840 \\
\hline \multicolumn{3}{|c|}{ Kessyusa Formatic Kessyusa Formation } & 0.013 & 0.756 & 1.417 \\
\hline F842 & F842 & 227 & 0.011 & 1.589 & 2.230 \\
\hline F842 & F842 & 229 & 0.006 & 0.535 & 1.020 \\
\hline Ingta & $\mathrm{J} 1125$ & i6-J1125-212. & 0.000 & 1.096 & 2.340 \\
\hline Ingta & $\mathrm{J} 1125$ & i7-J1125-216. & 0.000 & 1.054 & 3.280 \\
\hline Ingta & $\mathrm{J} 1125$ & i8-J1125-239. & 0.000 & 1.210 & 3.540 \\
\hline Ingta & $\mathrm{J} 1125$ & i9-J1125-241. & 0.000 & 0.909 & 2.710 \\
\hline Ingta & J1125 & '0-J1125-247.' & 0.000 & 2.143 & 3.550 \\
\hline Ingta & $\mathrm{J} 1125$ & '1-J1125-252. & 0.000 & 1.122 & 3.050 \\
\hline Ingta & $\mathrm{J} 1125$ & '2-J1125-258. & 0.000 & 2.253 & 3.560 \\
\hline Ingta & $\mathrm{J} 1125$ & '3-J1125-263. & 0.000 & 2.071 & 3.600 \\
\hline Ingta & J1125 & '4-J1125-268. & 0.000 & 0.960 & 3.050 \\
\hline Ingta & J1125 & '7-J1125-296. & 0.000 & 1.487 & 3.700 \\
\hline Ingta & $\mathrm{J} 1125$ & '8-J1125-313. & 0.000 & 1.071 & 2.980 \\
\hline Ingta & $\mathrm{J} 1125$ & '9-J1125-324.' & 0.000 & 1.452 & 3.840 \\
\hline Ingta & $\mathrm{J} 1125$ & J1125- 125.1 & 0.000 & 2.074 & 4.290 \\
\hline Ingta & $\mathrm{J} 1125$ & J1125- 129.6 & 0.000 & 1.248 & 3.060 \\
\hline Ingta & $\mathrm{J} 1125$ & J1125- 135.9 & 0.000 & 1.396 & 4.080 \\
\hline Ingta & $\mathrm{J} 1125$ & J1125- 142.3 & 0.000 & 1.525 & 4.790 \\
\hline Ingta & $\mathrm{J} 1125$ & J1125- 148.3 & 0.000 & 1.622 & 5.050 \\
\hline Ingta & J1125 & J1125- 155.1 & 0.000 & 2.728 & 6.170 \\
\hline Ingta & J1125 & J1125- 161.8 & 0.000 & 1.324 & 4.310 \\
\hline Ingta & $\mathrm{J} 1125$ & J1125- 167.4 & 0.000 & 1.325 & 3.870 \\
\hline Ingta & $\mathrm{J} 1125$ & J1125- 175.4 & 0.000 & 2.540 & 5.750 \\
\hline Ingta & $\mathrm{J} 1125$ & J1125- 182.1 & 0.000 & 1.821 & 3.830 \\
\hline
\end{tabular}




\begin{tabular}{|c|c|c|c|c|c|}
\hline Ingta & $\mathrm{J} 1125$ & J1125- 189.4 & 0.000 & 1.225 & 3.240 \\
\hline Ingta & $\mathrm{J} 1125$ & J1125- 194.8 & 0.000 & 1.540 & 4.730 \\
\hline Ingta & $\mathrm{J} 1125$ & J1125- 200 & 0.000 & 1.027 & 3.730 \\
\hline Ingta & $J 1125$ & J1125- 206.8 & 0.000 & 1.061 & 3.210 \\
\hline Ingta & $\mathrm{J} 1125$ & J1125- 209 & 0.000 & 0.760 & 3.400 \\
\hline Ingta Formation & S1306 & 62.5 & 0.001 & 1.321 & 2.330 \\
\hline Ingta Formation & S1306 & 89.3 & 0.000 & 1.369 & 3.230 \\
\hline Ingta Formation & S1306 & 91.5 & 0.000 & 2.549 & 4.110 \\
\hline Ingta Formation & S1306 & 98.4 & 0.008 & 1.990 & 4.840 \\
\hline Ingta Formation & S1306 & 98.7 & 0.000 & 2.141 & 5.210 \\
\hline Ingta Formation & S1306 & 118 & 0.000 & 0.662 & 5.060 \\
\hline Ingta Formation & S1306 & 139.5 & 0.000 & 0.795 & 5.350 \\
\hline Ingta Formation & S1306 & 154.7 & 0.000 & 1.011 & 4.230 \\
\hline Ingta Formation & S1306 & 172.1 & 0.000 & 0.400 & 4.110 \\
\hline Ingta Formation & S1306 & 174.8 & 0.000 & 0.838 & 6.430 \\
\hline Ingta Formation & S1306 & 178.1 & 0.000 & 0.700 & 5.760 \\
\hline Ingta Formation & S1306 & 179.4 & 0.000 & 0.646 & 6.160 \\
\hline Ingta Formation & S1306 & 179.9 & 0.000 & 0.584 & 5.650 \\
\hline Ingta Formation & S1306 & 197.2 & 0.000 & 1.730 & 4.680 \\
\hline Ingta Formation & S1306 & 202.3 & 0.000 & 0.445 & 4.620 \\
\hline Ingta Formation & S1306 & 203.2 & 0.000 & 0.352 & 5.310 \\
\hline Ingta Formation & S1306 & 211.5 & 0.000 & 0.399 & 6.030 \\
\hline Ingta Formation & S1306 & 216.3 & 0.000 & 0.790 & 3.930 \\
\hline Ingta Formation & S1306 & 223.4 & 0.000 & 0.903 & 4.630 \\
\hline Ingta Formation & S1306 & 233.6 & 0.001 & 3.387 & 6.600 \\
\hline Ingta Formation & S1306 & 235.6 & 0.000 & 0.259 & 5.520 \\
\hline Ingta Formation & S1306 & 240 & 0.000 & 0.589 & 5.160 \\
\hline Ingta Formation & S1306 & 241.2 & 0.000 & 0.487 & 3.760 \\
\hline Ingta Formation & S1306 & 267.5 & 0.000 & 1.482 & 3.840 \\
\hline Arthur Creek & LACF & 368.99 & 0.513 & 0.793 & 1.649 \\
\hline Arthur Creek & LACF & 379.01 & 0.260 & 0.575 & 2.106 \\
\hline Arthur Creek & LACF & 385 & 0.282 & 0.613 & 1.822 \\
\hline Arthur Creek & LACF & 388.95 & 0.321 & 0.615 & 1.954 \\
\hline Arthur Creek & LACF & 401 & 0.269 & 0.703 & 1.607 \\
\hline Arthur Creek & LACF & 405.02 & 0.254 & 0.559 & 1.790 \\
\hline Arthur Creek & LACF & 431 & 0.332 & 0.663 & 1.566 \\
\hline Arthur Creek & LACF & 440 & 0.289 & 0.565 & 1.678 \\
\hline Arthur Creek & LACF & 443 & 0.312 & 0.641 & 1.455 \\
\hline Arthur Creek & LACF & 449 & 0.433 & 0.763 & 1.864 \\
\hline Arthur Creek & LACF & 451.6 & 0.675 & 1.117 & 1.818 \\
\hline Arthur Creek & LACF & 455.48 & 0.473 & 0.788 & 2.224 \\
\hline
\end{tabular}




\begin{tabular}{|c|c|c|c|c|c|}
\hline Arthur Creek & LACF & 460 & 0.308 & 0.648 & 1.831 \\
\hline Arthur Creek & LACF & 464.5 & 0.277 & 0.619 & 1.854 \\
\hline Arthur Creek & LACF & 469.01 & 0.264 & 0.615 & 1.515 \\
\hline Arthur Creek & LACF & 471.55 & 0.521 & 0.874 & 2.006 \\
\hline Arthur Creek & LACF & 476 & 0.907 & 1.246 & 1.805 \\
\hline Arthur Creek & LACF & 480.5 & 0.386 & 0.710 & 1.523 \\
\hline Arthur Creek & LACF & 484 & 0.613 & 1.066 & 2.159 \\
\hline Arthur Creek & LACF & 487.5 & 0.321 & 0.722 & 1.487 \\
\hline Arthur Creek & LACF & 496 & 0.374 & 0.751 & 1.424 \\
\hline Arthur Creek & LACF & 505.32 & 0.609 & 0.973 & 2.040 \\
\hline Arthur Creek & LACF & 511.1 & 0.781 & 1.257 & 1.835 \\
\hline Arthur Creek & LACF & 545.75 & 1.081 & 1.712 & 2.594 \\
\hline Arthur Creek & LACF & 550.1 & 1.001 & 1.501 & 2.675 \\
\hline Arthur Creek & LACF & 553 & 0.808 & 1.461 & 1.911 \\
\hline Arthur Creek & LACF & 554 & 1.190 & 1.879 & 2.009 \\
\hline \multicolumn{2}{|c|}{ Thorntonia Limest MTL } & 563.06 & 0.690 & 1.116 & 1.099 \\
\hline \multicolumn{2}{|c|}{ Thorntonia Limest MTL } & 563.63 & 0.743 & 1.286 & 1.491 \\
\hline Nomtsas & VF1-S & 1 & 0.001 & 0.200 & 4.540 \\
\hline Nomtsas & VF1-S & 1.2 & 0.001 & 0.286 & 3.700 \\
\hline Nomtsas & VF1-S & 3 & 0.000 & 0.447 & 4.850 \\
\hline Nomtsas & VF1-S & 5.9 & 0.000 & 0.246 & 4.280 \\
\hline Nomtsas & VF1-S & 9.9 & 0.000 & 0.264 & 5.090 \\
\hline Nomtsas & VF1-S & 12.8 & 0.000 & 0.525 & 4.590 \\
\hline Nomtsas & VF1-S & 14.4 & 0.000 & 0.337 & 4.540 \\
\hline Nomtsas & VF1-S & 15.1 & 0.000 & 0.368 & 4.670 \\
\hline Nomtsas & VF1-S & 18.5 & 0.000 & 0.441 & 4.490 \\
\hline Nomtsas & VF1-S & 21.3 & 0.000 & 0.571 & 5.460 \\
\hline Nomtsas & VF1-S & 24.5 & 0.000 & 0.428 & 4.700 \\
\hline Nomtsas & VF1-S & 27.6 & 0.000 & 0.452 & 4.990 \\
\hline Nomtsas & VF1-S & 31 & 0.000 & 1.150 & 5.030 \\
\hline Nomtsas & VF1-S & 34 & 0.000 & 0.924 & 4.970 \\
\hline Nomtsas & VF1-S & 37 & 0.000 & 0.613 & 4.710 \\
\hline Nomtsas & VF1-S & 40 & 0.000 & 0.630 & 4.850 \\
\hline Nomtsas & VF1-S & 43 & 0.000 & 0.690 & 5.140 \\
\hline Nomtsas & VF1-S & 47.6 & 0.000 & 0.548 & 4.970 \\
\hline Nomtsas & VF1-S & 49.5 & 0.000 & 0.491 & 5.160 \\
\hline Nomtsas & VF1-S & 53.6 & 0.000 & 0.376 & 4.720 \\
\hline Nomtsas & VF1-S & 56.7 & 0.000 & 0.534 & 4.880 \\
\hline Nomtsas & VF1-S & 62.8 & 0.000 & 0.605 & 5.420 \\
\hline Nomtsas & VF1-S & 69 & 0.000 & 0.538 & 4.930 \\
\hline Nomtsas & VF1-S & 71.5 & 0.000 & 0.535 & 4.540 \\
\hline
\end{tabular}




\begin{tabular}{|c|c|c|c|c|c|}
\hline Nomtsas & VF1-S & 73 & 0.000 & 0.483 & 4.840 \\
\hline Bayan Gol & T1201 & 108.4 & 0.006 & 2.115 & 6.648 \\
\hline Bayan Gol & T1201 & 114 & 0.003 & 0.874 & 11.969 \\
\hline Bayan Gol & T1201 & 117.9 & 0.000 & 2.490 & 6.060 \\
\hline Bayan Gol & T1201 & 121 & 0.000 & 2.366 & 5.134 \\
\hline Bayan Gol & T1201 & 127.1 & 0.000 & 2.444 & 3.903 \\
\hline Bayan Gol & T1201 & 129.1 & 0.000 & 1.121 & 6.008 \\
\hline Bayan Gol & T1201 & 151.7 & 0.000 & 0.267 & 4.580 \\
\hline Bayan Gol & T1201 & 172 & 0.000 & 0.353 & 3.523 \\
\hline Bayan Gol & T1201 & 197.8 & 0.000 & 0.134 & 4.445 \\
\hline Bayan Gol & T1201 & 204 & 0.000 & 0.941 & 4.118 \\
\hline Bayan Gol & T1201 & 206.7 & 0.002 & 0.611 & 3.243 \\
\hline Bayan Gol & T1201 & 207.9 & 0.000 & 0.372 & 3.698 \\
\hline Bayan Gol & T1201 & 210.7 & 0.003 & 0.804 & 3.714 \\
\hline Bayan Gol & T1201 & 218.5 & 0.000 & 0.178 & 3.453 \\
\hline Bayan Gol & T1201 & 227.1 & 0.002 & 0.290 & 2.968 \\
\hline Bayan Gol & T1201 & 233 & 0.002 & 0.220 & 4.490 \\
\hline Bayan Gol & T1201 & 235.7 & 0.000 & 0.245 & 4.539 \\
\hline Bayan Gol & T1201 & 237.8 & 0.000 & 0.526 & 4.571 \\
\hline Bayan Gol & T1201 & 242.9 & 0.000 & 0.696 & 4.950 \\
\hline Bayan Gol & T1201 & 248.1 & 0.000 & 0.397 & 5.111 \\
\hline Penn Yan & Menteth Creek & 0 & 0.970 & 1.140 & 4.270 \\
\hline Penn Yan & Menteth Creek & 1 & 0.710 & 0.870 & 4.240 \\
\hline Penn Yan & Menteth Creek & 2 & 0.730 & 0.900 & 3.960 \\
\hline Penn Yan & Menteth Creek & 3 & 1.180 & 1.440 & 4.360 \\
\hline Penn Yan & Menteth Creek & 4 & 0.700 & 0.860 & 4.000 \\
\hline Penn Yan & Menteth Creek & 5 & 0.510 & 0.700 & 3.020 \\
\hline Penn Yan & Menteth Creek & 6 & 0.620 & 0.770 & 3.010 \\
\hline Penn Yan & Menteth Creek & 7 & 0.580 & 0.760 & 3.400 \\
\hline Penn Yan & Menteth Creek & 8 & 1.820 & 2.000 & 5.030 \\
\hline Penn Yan & Menteth Creek & 9 & 0.780 & 0.940 & 4.370 \\
\hline Penn Yan & Menteth Creek & 10 & 0.850 & 1.030 & 3.640 \\
\hline Penn Yan & Menteth Creek & 11 & 1.410 & 1.600 & 4.870 \\
\hline Penn Yan & Menteth Creek & 12 & 1.310 & 1.480 & 4.910 \\
\hline Penn Yan & Menteth Creek & 13 & 0.680 & 0.830 & 4.280 \\
\hline Penn Yan & Menteth Creek & 14 & 0.800 & 0.930 & 4.090 \\
\hline Penn Yan & Menteth Creek & 15 & 0.960 & 1.120 & 4.390 \\
\hline Penn Yan & Menteth Creek & 16 & 0.670 & 0.850 & 4.230 \\
\hline Penn Yan & Menteth Creek & 17 & 0.590 & 0.750 & 4.260 \\
\hline Penn Yan & Menteth Creek & 18 & 0.570 & 0.740 & 3.930 \\
\hline Penn Yan & Menteth Creek & 19 & 0.630 & 0.770 & 3.860 \\
\hline
\end{tabular}




\begin{tabular}{|c|c|c|c|c|c|}
\hline Penn Yan & Menteth Creek & 20 & 0.690 & 0.870 & 4.020 \\
\hline Penn Yan & Menteth Creek & 21 & 0.640 & 0.820 & 4.210 \\
\hline Penn Yan & Menteth Creek & 22 & 0.620 & 0.790 & 4.620 \\
\hline Penn Yan & Menteth Creek & 23 & 0.830 & 1.000 & 4.300 \\
\hline Penn Yan & Menteth Creek & 24 & 0.500 & 0.660 & 4.060 \\
\hline Penn Yan & Menteth Creek & 25 & 0.600 & 0.790 & 4.110 \\
\hline Penn Yan & Menteth Creek & 26 & 0.770 & 0.980 & 4.210 \\
\hline Penn Yan & Menteth Creek & 27 & 0.630 & 0.810 & 4.100 \\
\hline Penn Yan & Menteth Creek & 28 & 0.520 & 0.680 & 4.050 \\
\hline Penn Yan & Menteth Creek & 29 & 0.700 & 0.900 & 4.180 \\
\hline Penn Yan & Menteth Creek & 30 & 0.450 & 0.630 & 3.730 \\
\hline Ledyard & Paines Creek & 5 & 1.010 & 1.180 & 5.780 \\
\hline Ledyard & Paines Creek & 6 & 0.960 & 1.110 & 5.620 \\
\hline Ledyard & Paines Creek & 7 & 0.660 & 0.840 & 5.220 \\
\hline Ledyard & Paines Creek & 8 & 0.750 & 0.970 & 4.740 \\
\hline Ledyard & Paines Creek & 9 & 0.800 & 0.980 & 5.330 \\
\hline Ledyard & Paines Creek & 10 & 0.760 & 0.950 & 5.490 \\
\hline Ledyard & Paines Creek & 11 & 0.790 & 0.980 & 5.410 \\
\hline Ledyard & Paines Creek & 12 & 0.750 & 0.930 & 5.560 \\
\hline Ledyard & Paines Creek & 13 & 0.680 & 0.830 & 5.210 \\
\hline Ledyard & Paines Creek & 14 & 0.650 & 0.840 & 5.240 \\
\hline Ledyard & Paines Creek & 15 & 0.650 & 0.820 & 5.010 \\
\hline Ledyard & Paines Creek & 16 & 0.560 & 0.770 & 4.960 \\
\hline Ledyard & Paines Creek & 17 & 0.680 & 0.830 & 5.360 \\
\hline Ledyard & Paines Creek & 18 & 0.770 & 0.910 & 5.410 \\
\hline Ledyard & Paines Creek & 19 & 0.910 & 1.130 & 5.540 \\
\hline Ledyard & Paines Creek & 20 & 1.100 & 1.260 & 5.710 \\
\hline Ledyard & Paines Creek & 21 & 0.620 & 0.890 & 4.670 \\
\hline Ledyard & Paines Creek & 22 & 0.590 & 0.830 & 4.410 \\
\hline Ledyard & Paines Creek & 23 & 0.760 & 0.990 & 5.110 \\
\hline Ledyard & Paines Creek & 24 & 0.620 & 0.920 & 4.490 \\
\hline Ledyard & Paines Creek & 25 & 1.760 & 2.070 & 4.300 \\
\hline Ledyard & Paines Creek & 26 & 1.180 & 1.410 & 5.370 \\
\hline Ledyard & Paines Creek & 27 & 1.200 & 1.400 & 5.610 \\
\hline Ledyard & Paines Creek & 28 & 1.080 & 1.340 & 5.540 \\
\hline Ledyard & Paines Creek & 29 & 1.600 & 1.840 & 6.250 \\
\hline Ledyard & Paines Creek & 30 & 1.210 & 1.440 & 5.710 \\
\hline Ledyard & Paines Creek & 31 & 0.550 & 0.710 & 4.960 \\
\hline Ledyard & Paines Creek & 32 & 0.560 & 0.740 & 5.040 \\
\hline Ledyard & Paines Creek & 33 & 0.490 & 0.660 & 4.830 \\
\hline Ledyard & Paines Creek & 34 & 0.610 & 0.810 & 5.280 \\
\hline
\end{tabular}




\begin{tabular}{|c|c|c|c|c|c|}
\hline Ledyard & Paines Creek & 35 & 0.660 & 0.830 & 5.810 \\
\hline Ledyard & Paines Creek & 36 & 0.820 & 1.040 & 5.380 \\
\hline Ledyard & Paines Creek & 37 & 0.680 & 0.900 & 5.800 \\
\hline Ledyard & Paines Creek & 38 & 0.780 & 0.990 & 5.420 \\
\hline Ledyard & Paines Creek & 39 & 0.930 & 1.110 & 5.620 \\
\hline Ledyard & Paines Creek & 40 & 0.480 & 0.700 & 5.070 \\
\hline Ledyard & Paines Creek & 41 & 0.480 & 0.660 & 5.230 \\
\hline Ledyard & Paines Creek & 42 & 0.450 & 0.650 & 5.020 \\
\hline Ledyard & Paines Creek & 43 & 0.460 & 0.630 & 4.700 \\
\hline Ledyard & Paines Creek & 44 & 0.390 & 0.570 & 5.070 \\
\hline Ledyard & Paines Creek & 45 & 0.350 & 0.520 & 4.970 \\
\hline Ledyard & Paines Creek & 46 & 0.390 & 0.570 & 4.770 \\
\hline Ledyard & Paines Creek & 47 & 0.520 & 0.730 & 4.700 \\
\hline Ledyard & Paines Creek & 48 & 0.400 & 0.570 & 4.670 \\
\hline Ledyard & Paines Creek & 49 & 0.340 & 0.500 & 4.600 \\
\hline Ledyard & Paines Creek & 50 & 0.400 & 0.580 & 4.820 \\
\hline Ledyard & Paines Creek & 51 & 0.350 & 0.510 & 4.700 \\
\hline Ledyard & Paines Creek & 52 & 0.450 & 0.600 & 4.460 \\
\hline Ledyard & Paines Creek & 53 & 0.490 & 0.660 & 4.850 \\
\hline Ledyard & Paines Creek & 54 & 0.560 & 0.760 & 4.770 \\
\hline Ledyard & Paines Creek & 55 & 0.490 & 0.680 & 4.780 \\
\hline Ledyard & Paines Creek & 56 & 0.530 & 0.710 & 4.750 \\
\hline Ledyard & Paines Creek & 57 & 0.580 & 0.760 & 4.810 \\
\hline Ledyard & Paines Creek & 58 & 0.420 & 0.620 & 4.700 \\
\hline Ledyard & Paines Creek & 59 & 0.410 & 0.640 & 4.870 \\
\hline Ledyard & Paines Creek & 60 & 0.380 & 0.600 & 4.810 \\
\hline Ledyard & Paines Creek & 61 & 0.490 & 0.730 & 4.770 \\
\hline Ledyard & Paines Creek & 62 & 0.430 & 0.630 & 4.830 \\
\hline Ledyard & Paines Creek & 63 & 0.800 & 0.970 & 5.640 \\
\hline Ledyard & Paines Creek & 64 & 0.510 & 0.680 & 4.760 \\
\hline Ledyard & Paines Creek & 65 & 0.430 & 0.610 & 4.970 \\
\hline Ledyard & Paines Creek & 66 & 0.550 & 0.750 & 5.270 \\
\hline Ledyard & Paines Creek & 67 & 0.450 & 0.620 & 5.080 \\
\hline Ledyard & Paines Creek & 68 & 0.660 & 0.830 & 5.190 \\
\hline Ledyard & Paines Creek & 69 & 0.950 & 1.090 & 5.390 \\
\hline Ledyard & Paines Creek & 70 & 0.840 & 1.040 & 5.200 \\
\hline Ledyard & Paines Creek & 71 & 0.770 & 1.040 & 5.840 \\
\hline Ledyard & Paines Creek & 72 & 0.860 & 1.020 & 5.660 \\
\hline Ledyard & Paines Creek & 73 & 0.560 & 0.760 & 5.120 \\
\hline Ledyard & Paines Creek & 74 & 0.620 & 0.830 & 5.460 \\
\hline \multicolumn{2}{|c|}{ Chattanooga Shale } & Chattanoga $\mathrm{K} 8 / 7 / \subseteq$ & 5.040 & 6.278 & 8.881 \\
\hline
\end{tabular}




\begin{tabular}{|c|c|c|c|c|c|}
\hline \multicolumn{2}{|c|}{ Chattanooga Shale } & Chattanoga K8/7/؟ & 4.635 & 6.609 & 10.010 \\
\hline \multicolumn{2}{|c|}{ New Albany Shale } & Clegg 873-B10+ & 2.367 & 3.645 & 6.394 \\
\hline \multicolumn{2}{|c|}{ New Albany Shale } & Clegg 873-B8+2 & 3.416 & 4.357 & 6.379 \\
\hline Frankfort & Beecher & 2321 & 1.387 & 2.619 & 5.304 \\
\hline Frankfort & Beecher & 2322 & 1.439 & 2.261 & 5.310 \\
\hline Frankfort & Beecher & 2323 & 1.420 & 1.980 & 5.259 \\
\hline Frankfort & Beecher & 2324 & 1.712 & 2.221 & 5.610 \\
\hline Frankfort & Beecher & 2325 & 0.973 & 1.433 & 5.495 \\
\hline Frankfort & Beecher & 2326 & 1.247 & 1.863 & 5.488 \\
\hline Frankfort & Beecher & 2327 & 1.326 & 1.667 & 5.594 \\
\hline Frankfort & Beecher & 2328 & 1.478 & 2.033 & 5.830 \\
\hline Frankfort & Beecher & 2329 & 0.482 & 1.222 & 5.514 \\
\hline Frankfort & Beecher & 2330 & 0.410 & 0.752 & 4.684 \\
\hline Frankfort & Beecher & 2332 & 0.889 & 1.766 & 4.979 \\
\hline Frankfort & Beecher & 2333 & 1.261 & 1.794 & 5.419 \\
\hline Frankfort & Beecher & 2401 & 1.068 & 1.569 & 5.086 \\
\hline Frankfort & Beecher & 2402 & 0.506 & 1.082 & 4.891 \\
\hline Frankfort & Beecher & 2403 & 0.579 & 1.102 & 4.763 \\
\hline Frankfort & Beecher & 2409 & 0.871 & 1.570 & 5.143 \\
\hline Frankfort & Beecher & $2331 a$ & 0.892 & 1.388 & 5.005 \\
\hline Frankfort & Beecher & $2334 b(i)$ & 1.353 & 2.031 & 4.520 \\
\hline Frankfort & Beecher & $2334 b(\mathrm{ii})$ & 0.299 & 0.776 & 4.713 \\
\hline Frankfort & Beecher & 2408 base & 0.587 & 2.015 & 5.342 \\
\hline Frankfort & Beecher & 2408 top & 1.829 & 2.747 & 5.550 \\
\hline Frankfort & Corner & 1709 & 1.056 & 1.594 & 5.376 \\
\hline Frankfort & Corner & 1710 & 1.016 & 2.193 & 4.777 \\
\hline Frankfort & Corner & 1711 & 0.271 & 0.781 & 4.440 \\
\hline Frankfort & Corner & 1801 & 0.183 & 0.673 & 4.648 \\
\hline Frankfort & Corner & 1802 & 1.005 & 1.652 & 5.228 \\
\hline Frankfort & Corner & 1803 & 0.134 & 0.843 & 4.810 \\
\hline Frankfort & Corner & 1805 & 1.166 & 1.885 & 4.967 \\
\hline Frankfort & Corner & 1806 & 1.105 & 1.704 & 4.848 \\
\hline Frankfort & Corner & 1808 & 0.907 & 1.698 & 4.875 \\
\hline Frankfort & Corner & 1811 & 1.010 & 1.641 & 5.110 \\
\hline Frankfort & Corner & 1902 & 0.357 & 0.987 & 4.494 \\
\hline Frankfort & Corner & 1903 & 0.835 & 1.511 & 4.971 \\
\hline Frankfort & Corner & 1904 & 0.832 & 1.632 & 5.644 \\
\hline Frankfort & Corner & 1905 & 0.588 & 1.103 & 5.382 \\
\hline Frankfort & Corner & $1810 a$ & 0.885 & 1.530 & 5.351 \\
\hline Frankfort & Corner & $1810 c$ & 0.764 & 1.179 & 4.898 \\
\hline Frankfort & Corner & 5 (above nodı & 0.890 & 1.693 & 5.180 \\
\hline
\end{tabular}




\begin{tabular}{|c|c|c|c|c|c|}
\hline Frankfort & Corner & 1906 (1) & 0.410 & 1.094 & 4.913 \\
\hline Frankfort & Corner & 1907 (1) & 1.061 & 1.522 & 5.130 \\
\hline Frankfort & Walcott & 822 & 0.632 & 1.013 & 5.200 \\
\hline Frankfort & Walcott & 906 & 1.010 & 1.010 & 5.270 \\
\hline Frankfort & Walcott & 913 & 0.586 & 1.776 & 4.155 \\
\hline Frankfort & Walcott & 914 & 1.007 & 2.059 & 5.117 \\
\hline Frankfort & Walcott & 917 & 0.677 & 2.774 & 5.270 \\
\hline Frankfort & Walcott & 918 & 1.194 & 2.045 & 5.196 \\
\hline Frankfort & Walcott & 1001 & 0.752 & 1.476 & 4.170 \\
\hline Frankfort & Walcott & 1002 & 0.896 & 1.708 & 5.247 \\
\hline Frankfort & Walcott & 1003 & 1.039 & 2.080 & 4.635 \\
\hline Frankfort & Walcott & 1004 & 1.128 & 1.950 & 5.089 \\
\hline Frankfort & Walcott & 1005 & 1.116 & 1.890 & 4.193 \\
\hline Frankfort & Walcott & 1006 & 0.684 & 1.393 & 4.417 \\
\hline Frankfort & Walcott & 1007 & 0.176 & 0.850 & 4.476 \\
\hline Frankfort & Walcott & 1008 & 0.639 & 1.460 & 3.905 \\
\hline Frankfort & Walcott & 1009 & 0.974 & 1.932 & 4.663 \\
\hline Frankfort & Walcott & 1010 & 0.616 & 1.358 & 4.718 \\
\hline Frankfort & Walcott & 1011 & 0.747 & 1.620 & 4.577 \\
\hline Frankfort & Walcott & 1012 & 0.722 & 2.381 & 5.061 \\
\hline Frankfort & Walcott & 1013 & 1.168 & 2.037 & 5.063 \\
\hline Frankfort & Walcott & 1101 & 0.923 & 1.786 & 5.038 \\
\hline Frankfort & Walcott & 1102 & 1.080 & 1.777 & 4.994 \\
\hline Frankfort & Walcott & 1103 & 0.634 & 1.368 & 4.899 \\
\hline Frankfort & Walcott & 1104 & 0.772 & 2.134 & 4.793 \\
\hline Frankfort & Walcott & 1105 & 0.865 & 1.715 & 4.491 \\
\hline Frankfort & Walcott & 1106 & 0.758 & 2.963 & 5.088 \\
\hline Frankfort & Walcott & 1107 & 1.040 & 2.075 & 5.196 \\
\hline Frankfort & Walcott & 1108 & 0.643 & 1.388 & 4.454 \\
\hline Frankfort & Walcott & 1203 & 0.984 & 1.622 & 4.937 \\
\hline Frankfort & Walcott & 1204 & 0.224 & 0.940 & 4.527 \\
\hline Frankfort & Walcott & 1205 & 0.238 & 1.041 & 4.615 \\
\hline Frankfort & Walcott & 1206 & 0.599 & 1.252 & 4.702 \\
\hline Frankfort & Walcott & 1208 & 0.495 & 1.656 & 4.908 \\
\hline Frankfort & Walcott & 1210 & 0.700 & 1.494 & 4.514 \\
\hline Frankfort & Walcott & 1211 & 0.313 & 0.984 & 4.865 \\
\hline Frankfort & Walcott & 1214 & 0.677 & 1.497 & 5.223 \\
\hline Frankfort & Walcott & 1004 nodules & 0.869 & 2.595 & 4.777 \\
\hline Frankfort & Walcott & $1008 b$ & 0.884 & 1.515 & 4.446 \\
\hline Frankfort & Walcott & $1104 b$ & 0.311 & 2.226 & 4.620 \\
\hline Frankfort & Walcott & $1107 b$ & 1.102 & 1.834 & 4.711 \\
\hline
\end{tabular}




\begin{tabular}{|c|c|c|c|c|c|}
\hline Frankfort & Walcott & 8 (above nodı & 0.164 & 0.708 & 4.417 \\
\hline Frankfort & Walcott & 1215 base & 0.592 & 1.707 & 5.035 \\
\hline Frankfort & Walcott & $1215 \mathrm{c}$ & 0.350 & 1.134 & 4.605 \\
\hline Frankfort & Walcott & 1215 middle & 0.344 & 1.244 & 4.889 \\
\hline Frankfort & Walcott & 1216 top & 1.766 & 2.711 & 5.461 \\
\hline Frankfort & Walcott & 1218 base & 0.194 & 1.400 & 4.361 \\
\hline Frankfort & Walcott & 1218 middle & 0.350 & 1.574 & 4.660 \\
\hline Frankfort & Walcott & 1218 top & 1.351 & 2.473 & 5.637 \\
\hline Frankfort & Walcott & (around nodı & 0.962 & 1.496 & 5.458 \\
\hline Frankfort & Walcott & L5 (mixed bed & 1.187 & 2.433 & 4.487 \\
\hline Frankfort & Walcott & $916 a$ & 0.979 & 1.553 & 4.960 \\
\hline Frankfort & Walcott & $916 c$ & 1.550 & 2.247 & 5.130 \\
\hline Frankfort & Walcott & $916 d$ & 1.346 & 2.156 & 5.404 \\
\hline \multirow[t]{28}{*}{ Frankfort } & Walcott & Tril & 0.977 & 1.669 & 5.074 \\
\hline & DB 10 & 5.8 & 0.030 & & \\
\hline & DB1 & 8.4 & 1.140 & & \\
\hline & DB11 & 5.3 & 0.030 & & \\
\hline & DB12 & 5.2 & 0.100 & & \\
\hline & DB13 & 5.05 & 0.100 & & \\
\hline & DB14 & 4.95 & 0.020 & & \\
\hline & DB15 & 4.85 & 0.060 & & \\
\hline & DB16 & 4.7 & 0.040 & & \\
\hline & DB17 & 4.4 & 0.040 & & \\
\hline & DB18 & 4.25 & 0.870 & & \\
\hline & DB19 & 4.2 & 0.240 & & \\
\hline & DB2 & 8.2 & 0.020 & & \\
\hline & DB20 & 4.2 & 0.040 & & \\
\hline & DB21 & 4.15 & 0.020 & & \\
\hline & DB22 & 4.1 & 0.040 & & \\
\hline & DB23 & 3.85 & 0.020 & & \\
\hline & DB24 & 3.65 & 0.000 & & \\
\hline & DB25 & 3.25 & 0.020 & & \\
\hline & DB26A & 1.9 & 0.010 & & \\
\hline & DB26B & 0.95 & & & \\
\hline & DB28 & 8.9 & 0.700 & & \\
\hline & DB29 & 9.15 & 0.970 & & \\
\hline & DB3 & 8.05 & 0.020 & & \\
\hline & DB30 & 9.45 & 0.680 & & \\
\hline & DB31 & 9.7 & 0.490 & & \\
\hline & DB32 & 0.3 & 1.110 & & \\
\hline & DB33 & 0.1 & 0.040 & & \\
\hline
\end{tabular}




\begin{tabular}{|c|c|c|c|c|}
\hline DB34 & -1 & 0.030 & & \\
\hline DB4 & 7.85 & 0.030 & & \\
\hline DB5 & 7.65 & 0.130 & & \\
\hline DB6 & 7.1 & 1.100 & & \\
\hline DB7 & 6.85 & 0.060 & & \\
\hline DB8 & 6.35 & 0.030 & & \\
\hline DB9 & 6 & 0.060 & & \\
\hline DL1 & 8.3 & 0.070 & & \\
\hline DL10 & 17.3 & 0.130 & & \\
\hline DL12 & 17.8 & 0.120 & & \\
\hline DL2 & 9.3 & 1.630 & & \\
\hline DL3 & 10.3 & 1.210 & & \\
\hline DL4 & 11.3 & 1.710 & & \\
\hline DL5 & 12.3 & 1.020 & & \\
\hline DL6 & 13.3 & 1.330 & & \\
\hline DL7 & 14.3 & 1.160 & & \\
\hline DL8 & 15.3 & 1.560 & & \\
\hline DL9 & 16.3 & 1.500 & & \\
\hline Billegrav-1 & BG-1 & & & \\
\hline Billegrav-1 & BG-10 & & & \\
\hline Billegrav-1 & BG-2 & & & \\
\hline Billegrav-1 & BG-3 & & & \\
\hline Billegrav-1 & BG-4 & 2.337 & 3.005 & 6.728 \\
\hline Billegrav-1 & BG-5 & & & \\
\hline Billegrav-1 & BG-6 & & & \\
\hline Billegrav-1 & BG-7 & & & \\
\hline Billegrav-1 & BG-8 & & & \\
\hline Billegrav-1 & BG-9 & & & \\
\hline Billgrav 2 & 17.9 & 0.770 & & \\
\hline Billgrav 2 & 18.9 & 0.110 & & \\
\hline Billgrav 2 & 19.7 & 2.130 & & \\
\hline Billgrav 2 & 20.8 & 1.720 & & \\
\hline Billgrav 2 & 22.6 & 1.230 & & \\
\hline Billgrav 2 & 25.25 & 5.000 & & \\
\hline Billgrav 2 & 26.9 & 1.030 & & \\
\hline Billgrav 2 & 27.7 & 1.240 & & \\
\hline Billgrav 2 & 28.7 & 1.090 & & \\
\hline Billgrav 2 & 29.7 & 0.880 & & \\
\hline Billgrav 2 & 30.7 & 1.480 & & \\
\hline Billgrav 2 & 31.2 & 1.110 & & \\
\hline Billgrav 2 & 35.15 & 0.970 & & \\
\hline
\end{tabular}




\begin{tabular}{|c|c|c|}
\hline Billgrav 2 & 37.05 & 1.010 \\
\hline Billgrav 2 & 40.9 & 1.130 \\
\hline Billgrav 2 & 49.6 & 0.590 \\
\hline Billgrav 2 & 50.6 & 1.490 \\
\hline Billgrav 2 & 52.1 & 1.160 \\
\hline Billgrav 2 & 52.9 & 1.130 \\
\hline Billgrav 2 & 53.8 & 1.230 \\
\hline Billgrav 2 & 54.9 & 1.170 \\
\hline Billgrav 2 & 56.001 & 0.680 \\
\hline Billgrav 2 & 56.002 & 0.650 \\
\hline Billgrav 2 & 56.54 & 0.260 \\
\hline Billgrav 2 & 57.44 & 1.070 \\
\hline Billgrav 2 & 57.9 & 0.820 \\
\hline Billgrav 2 & 60 & \\
\hline Billgrav 2 & 60.09 & \\
\hline Billgrav 2 & 60.18 & \\
\hline Billgrav 2 & 60.33 & \\
\hline Billgrav 2 & 60.51 & \\
\hline Billgrav 2 & 60.64 & \\
\hline Billgrav 2 & 60.75 & 0.990 \\
\hline Billgrav 2 & 60.93 & \\
\hline Billgrav 2 & 61.05 & \\
\hline Billgrav 2 & 61.19 & \\
\hline Billgrav 2 & 61.61 & \\
\hline Billgrav 2 & 62.05 & \\
\hline Billgrav 2 & 62.2 & 3.740 \\
\hline Billgrav 2 & 62.56 & \\
\hline Billgrav 2 & 62.76 & \\
\hline Billgrav 2 & 62.95 & \\
\hline Billgrav 2 & 63.2 & 1.590 \\
\hline Billgrav 2 & 63.5 & \\
\hline Billgrav 2 & 63.72 & \\
\hline Billgrav 2 & 63.9 & \\
\hline Billgrav 2 & 63.94 & \\
\hline Billgrav 2 & 64.05 & 0.250 \\
\hline Billgrav 2 & 64.19 & \\
\hline Billgrav 2 & 65.8 & 0.160 \\
\hline Billgrav 2 & 65.9 & 0.250 \\
\hline Billgrav 2 & 66.9 & 0.000 \\
\hline Billgrav 2 & 67.3 & 0.000 \\
\hline Billgrav 2 & 67.95 & 0.050 \\
\hline
\end{tabular}




\begin{tabular}{|c|c|c|}
\hline Billgrav 2 & 68.9 & 0.000 \\
\hline Billgrav 2 & 69.9 & 0.030 \\
\hline Billgrav 2 & 71.15 & 0.020 \\
\hline Billgrav 2 & 71.85 & 0.020 \\
\hline Billgrav 2 & 73.1 & 0.140 \\
\hline Billgrav 2 & 73.35 & 0.060 \\
\hline Billgrav 2 & 73.9 & 0.180 \\
\hline Billgrav 2 & 74.35 & 0.020 \\
\hline Billgrav 2 & 75.35 & 2.500 \\
\hline Billgrav 2 & 76.1 & 0.870 \\
\hline Billgrav 2 & 76.7 & 1.020 \\
\hline Billgrav 2 & 77 & 0.950 \\
\hline Billgrav 2 & 78.2 & 1.350 \\
\hline Billgrav 2 & 79.2 & 1.030 \\
\hline Billgrav 2 & 80 & 0.650 \\
\hline Billgrav 2 & 80.45 & 0.800 \\
\hline Billgrav 2 & 81.15 & 0.750 \\
\hline Billgrav 2 & 81.85 & 0.720 \\
\hline Billgrav 2 & 82.9 & 1.160 \\
\hline Billgrav 2 & 83.4 & 0.510 \\
\hline Billgrav 2 & 84.4 & 2.940 \\
\hline Billgrav 2 & 85.3 & 0.250 \\
\hline \multirow[t]{19}{*}{ Billgrav 2} & 86.3 & 1.300 \\
\hline & 1 & 0.060 \\
\hline & 2 & 0.010 \\
\hline & 3 & 0.030 \\
\hline & 4 & 0.080 \\
\hline & 5 & 0.030 \\
\hline & 6 & 0.100 \\
\hline & 6 & 0.100 \\
\hline & 7 & 0.150 \\
\hline & 8 & 0.430 \\
\hline & $1-0,8$ & 0.120 \\
\hline & $4-1,10$ & 0.010 \\
\hline & $4-1,85$ & 0.090 \\
\hline & $4+0,2$ & 0.010 \\
\hline & $4+0,55$ & 0.000 \\
\hline & $4+0,8$ & 0.010 \\
\hline & $4+1,10$ & 0.100 \\
\hline & $4+1,35$ & 0.160 \\
\hline & $4+1.00$ & 0.010 \\
\hline
\end{tabular}




\begin{tabular}{|c|c|c|c|c|c|}
\hline & & $4+1.02$ & 0.010 & & \\
\hline & & $4+1.03$ & 0.010 & & \\
\hline & & $4+1.05$ & 0.040 & & \\
\hline & & $4+1.07$ & 0.050 & & \\
\hline & & $4+1.09$ & 0.040 & & \\
\hline & & $4+1.16$ & 0.070 & & \\
\hline & & $4+1.17$ & 0.080 & & \\
\hline & & $4+1.18$ & 0.030 & & \\
\hline & & $4+1.22$ & 0.030 & & \\
\hline & & $6+0,25$ & 0.100 & & \\
\hline & & $6+0,6$ & 0.330 & & \\
\hline & & $6+0,9$ & 0.230 & & \\
\hline & & $6+1,1$ & 0.500 & & \\
\hline & & $7-0,2$ & 0.600 & & \\
\hline & & $7-0,5$ & 0.240 & & \\
\hline & & $7+0,3$ & 0.640 & & \\
\hline & & $7+0,8$ & 0.260 & & \\
\hline & & $7+1,0$ & 0.240 & & \\
\hline & & $7+1,7$ & 0.940 & & \\
\hline & Albjära-1 & Alb79013 & 1.665 & 2.293 & 6.339 \\
\hline & Albjära-1 & Alb79016 & 4.277 & 6.057 & 6.729 \\
\hline & Albjära-1. & Alb97158 & 1.368 & 1.972 & 2.988 \\
\hline & Albjära-1. & Alb97160 & 2.707 & 3.720 & 4.014 \\
\hline & Albjära-1. & Alb97161 & & & \\
\hline & Gislövhammar-2 & Gis89931 & 1.091 & 1.562 & 2.871 \\
\hline & Gislövhammar-2 & Gis89933 & 1.479 & 2.011 & 3.664 \\
\hline & Gislövhammar-2 & Gis89934 & 0.946 & 1.339 & 3.020 \\
\hline & Lönstorp-1 & Lön79001 & & & \\
\hline & Lönstorp-1 & Lön79002 & 1.781 & 2.485 & 5.967 \\
\hline & Lönstorp-1 & Lön79003 & & & \\
\hline & Lönstorp-1 & Lön79004 & & & \\
\hline & Lönstorp-1 & Lön79005 & & & \\
\hline & Lönstorp-1 & Lön79006 & & & \\
\hline & Lönstorp-1 & Lön79007 & & & \\
\hline & Lönstorp-1 & Lön79008 & & & \\
\hline & Lönstorp-1 & Lön97154 & 2.619 & 3.544 & 6.859 \\
\hline Soom Shale & & K2.6 & & & \\
\hline Soom Shale & & $\mathrm{K} 2.6 \mathrm{~A}$ & & & \\
\hline Soom Shale & & K2.7 & & & \\
\hline & Modern Continenté & $250-260$ & 0.630 & 0.700 & 2.910 \\
\hline & Modern Continentē & $90-100$ & 0.370 & 0.460 & 2.130 \\
\hline
\end{tabular}




\begin{tabular}{|c|c|c|c|}
\hline Modern Continenté FOAM 0-2 & 0.130 & 0.360 & 2.220 \\
\hline Modern Continenté FOAM 10-20 & 0.670 & 0.740 & 3.060 \\
\hline Modern Continentż:OAM 140-151 & 0.680 & 0.750 & 3.160 \\
\hline Modern Continenté FOAM 2-4 & 0.360 & 0.550 & 2.790 \\
\hline Modern Continent $\bar{c}$ FOAM 4-6 & 0.510 & 0.650 & 3.170 \\
\hline Modern Continent $\bar{c}$ FOAM 6-9 & 0.440 & 0.510 & 3.180 \\
\hline Modern Continenté FOAM 60-70 & 0.580 & 0.650 & 2.720 \\
\hline Modern Continenté FOAM 9-12 & 0.610 & 0.670 & 3.250 \\
\hline Modern ContinentzFOAM 90-20C & 0.680 & 0.750 & 3.350 \\
\hline Modern Continent $\bar{c} \quad$ K1 20-24 & 0.080 & 0.550 & 2.000 \\
\hline Modern Continenté $\quad$ K3 0-0.5 & 0.230 & 0.870 & 2.840 \\
\hline Modern Continent $\bar{c} \quad$ K3 24-28 & 0.200 & 0.700 & 2.480 \\
\hline Modern Continentaxttegat K1 0-0 & 0.080 & 0.530 & 1.870 \\
\hline Modern ContinentżMed Sta 1 0-1 & 0.030 & 0.940 & 2.780 \\
\hline Modern Continentēs Delta Sta 18 & 0.000 & 1.320 & 4.440 \\
\hline Modern Continent $\bar{c} \quad$ NWC $0-2$ & 0.280 & 0.920 & 3.180 \\
\hline Modern Continenté NWC 30-40 & 0.610 & 0.750 & 3.170 \\
\hline Modern Continenté NWC 60-70 & 0.550 & 0.730 & 3.050 \\
\hline Modern Continenté $\mathbf{c}$ NWC 9-12 & 0.710 & 1.080 & 3.120 \\
\hline Modern Continentē S6 0-0.5 & 0.050 & 0.680 & 2.450 \\
\hline Modern Continent $\bar{c} \quad$ S6 24-28 & 0.160 & 0.760 & 3.210 \\
\hline Modern Continentē & 0.000 & 1.650 & 4.390 \\
\hline Modern Continent $\bar{c} \quad$ S7 30-35 & 0.230 & 1.060 & 4.080 \\
\hline Modern Continent=Sachem 20-25 & 0.500 & 0.800 & 3.450 \\
\hline Modern Continent $\bar{c}$ Sachem 6-9 & 0.430 & 1.020 & 3.490 \\
\hline Modern Continentägerrak S1 12 . & 0.000 & 0.310 & 1.020 \\
\hline Modern Continenté Sta 1 10-12 & 0.010 & 0.890 & 2.750 \\
\hline Modern Continentē sta $110-1$ & 0.000 & 0.740 & 1.950 \\
\hline Modern Continenté Sta 11 10-12 & 0.000 & 0.600 & 2.230 \\
\hline Modern Continenté $\bar{c}$ Sta $130-1$ & 0.000 & 0.740 & 2.430 \\
\hline Modern Continenté Sta 13 10-12 & 0.000 & 0.550 & 1.920 \\
\hline Modern Continenté $\bar{c}$ Sta $160-1$ & 0.000 & 0.730 & 2.530 \\
\hline Modern Continentē Sta 16 10-12 & 0.000 & 0.440 & 2.440 \\
\hline Modern Continentē sta $170-1$ & 0.000 & 1.030 & 3.090 \\
\hline Modern Continentē Sta 17 10-12 & 0.000 & 1.030 & 3.220 \\
\hline Modern Continenté Sta 18 30-35 & 0.420 & 1.270 & 3.910 \\
\hline Modern Continenté $\bar{c}$ Sta 19 0-3 & 0.000 & 1.480 & 4.260 \\
\hline Modern Continentē Sta 19 20-30 & 0.070 & 1.410 & 4.390 \\
\hline Modern Continenté sta $210-12$ & 0.110 & 1.050 & 3.120 \\
\hline Modern Continentzita 20 100-111 & 0.160 & 0.520 & 3.560 \\
\hline Modern Continentē Sta 20 40-50 & 0.180 & 0.640 & 3.65 \\
\hline
\end{tabular}




\begin{tabular}{|c|c|c|c|c|}
\hline \multicolumn{2}{|c|}{ Modern Continentż Sta 22 30-35 } & 0.000 & 0.620 & 3.720 \\
\hline \multicolumn{2}{|c|}{ Modern Continentaita 22 310-321 } & 0.010 & 0.330 & 3.440 \\
\hline \multicolumn{2}{|c|}{ Modern Continent=Sta 22 90-100 } & 0.000 & 0.610 & 3.540 \\
\hline Modern Continentē & ¿ Sta 4 0-1 & 0.000 & 0.940 & 2.880 \\
\hline Modern Continentē & c Sta 4 10-12 & 0.030 & 0.890 & 3.050 \\
\hline Modern Continentē & $\bar{c}$ Sta 5 0-1 & 0.030 & 0.730 & 2.460 \\
\hline Modern Continentē & c sta $510-12$ & 0.010 & 0.810 & 2.460 \\
\hline Modern Continentē & $\bar{c}$ Sta 6 0-1 & 0.000 & 0.980 & 3.260 \\
\hline Modern Continentē & c sta $610-12$ & 0.000 & 1.050 & 3.450 \\
\hline Modern Continentē & $\bar{c}$ Sta 7 0-1 & 0.000 & 0.600 & 1.910 \\
\hline Modern Continentē & c sta $710-12$ & 0.050 & 0.380 & 1.930 \\
\hline Modern Continentē & $\bar{c}$ sta $80-1$ & 0.000 & 0.990 & 3.220 \\
\hline Modern Continentē & c sta $810-12$ & 0.000 & 1.180 & 3.550 \\
\hline Modern Continentā & $\bar{c} \quad$ Sta $90-1$ & 0.000 & 0.790 & 2.440 \\
\hline Modern Continentē & c Sta 9 10-12 & 0.040 & 0.730 & 2.600 \\
\hline Deep Sea & alif Sta N 11-1 & 0.040 & 0.260 & 4.110 \\
\hline Deep Sea & akar Sta 10.5 & 0.000 & 0.650 & 2.630 \\
\hline Deep Sea & Domes A 6-7 & 0.000 & 0.600 & 4.130 \\
\hline Deep Sea & Domes B 9-1C & 0.000 & 0.650 & 4.710 \\
\hline Deep Sea & Jomes C 11-1: & 0.020 & 0.530 & 4.170 \\
\hline Deep Sea & t Med Sta 20 & 0.130 & 0.870 & 1.960 \\
\hline Deep Sea & N Med Sta 3 C & 0.000 & 0.910 & 2.710 \\
\hline Deep Sea & 3C 1354 35-41 & 0.060 & 0.830 & 4.400 \\
\hline Deep Sea & 3C 2007 40-51 & 0.020 & 0.300 & 2.900 \\
\hline Deep Sea & Sta 1 1-1.5 & 0.030 & 0.710 & 2.910 \\
\hline Deep Sea & Sta $12-3$ & 0.040 & 0.880 & 3.160 \\
\hline Deep Sea & Sta $14-5$ & 0.050 & 0.580 & 2.750 \\
\hline Deep Sea & Sta $100-1$ & 0.000 & 0.970 & 2.890 \\
\hline Deep Sea & Sta 10 10-12 & 0.000 & 0.970 & 2.920 \\
\hline Deep Sea & Sta 12 0-1 & 0.000 & 0.600 & 2.040 \\
\hline Deep Sea & Sta 12 10-12 & 0.000 & 0.460 & 2.060 \\
\hline Deep Sea & Sta 14 0-1 & 0.000 & 0.500 & 1.500 \\
\hline Deep Sea & sta $1410-12$ & 0.000 & 0.290 & 1.380 \\
\hline Deep Sea & Sta 15 0-1 & 0.000 & 0.890 & 2.900 \\
\hline Deep Sea & Sta 15 10-12 & 0.000 & 0.640 & 2.920 \\
\hline Deep Sea & Sta 2 0-1 & 0.000 & 0.670 & 2.250 \\
\hline Deep Sea & Sta 2 2-3 & 0.000 & 0.660 & 2.400 \\
\hline Deep Sea & Sta 2 4-5 & 0.000 & 0.640 & 2.420 \\
\hline Deep Sea & Sta 208 & 0.130 & 0.990 & 2.440 \\
\hline Deep Sea & Sta 220.5 & 0.000 & 0.890 & 2.970 \\
\hline Deep Sea & Sta 225 & 0.080 & 0.910 & 2.77 \\
\hline
\end{tabular}




\begin{tabular}{|c|c|c|c|c|}
\hline Deep Sea & Sta 230.5 & 0.070 & 1.240 & 3.360 \\
\hline Deep Sea & Sta 235 & 0.150 & 0.980 & 2.550 \\
\hline Deep Sea & Sta 3 1-2 & 0.110 & 0.870 & 3.640 \\
\hline Deep Sea & Sta $310-12$ & 0.000 & 0.760 & 2.710 \\
\hline Deep Sea & Sta 3 3-4 & 0.070 & 0.810 & 3.800 \\
\hline Deep Sea & Sta $34-5$ & 0.040 & 0.870 & 4.170 \\
\hline Deep Sea & Sta G 21-22 & 0.320 & 0.730 & 3.670 \\
\hline Deep Sea & Sta G 23-24 & 0.340 & 0.690 & 3.990 \\
\hline Deep Sea & Sta G5-6 & 0.110 & 0.680 & 3.850 \\
\hline Deep Sea & Sta M 0-1 & 0.110 & 0.720 & 3.600 \\
\hline Deep Sea & Sta M 21-22 & 0.160 & 0.420 & 3.420 \\
\hline Deep Sea & Sta M 29-30 & 0.400 & 0.630 & 3.600 \\
\hline Deep Sea & Sta M 5-6 & 0.100 & 0.590 & 3.680 \\
\hline Deep Sea & Sta N 0-1 & 0.120 & 0.170 & 4.090 \\
\hline Deep Sea & Sta N 21-22 & 0.020 & 0.300 & 4.080 \\
\hline Deep Sea & Sta N 39-40 & 0.010 & 0.490 & 4.830 \\
\hline Deep Sea & Sta UH 350.5 & & 0.920 & 2.280 \\
\hline Deep Sea & Sta UH 355.5 & & 0.890 & 2.240 \\
\hline Deep Sea & V 26103 54-! & 0.000 & 0.970 & 3.510 \\
\hline Deep Sea & V 27265 44-८ & 0.000 & 0.460 & 3.380 \\
\hline Deep Sea & V 28207 43-८ & 0.000 & 2.030 & 5.960 \\
\hline Deep Sea & 'M 2890 33-35 & & 0.300 & 2.990 \\
\hline Deep Sea & 'M 2898 45-4 & 0.000 & 0.270 & 0.980 \\
\hline Deep Sea & V 30089 24-i & 0.000 & 0.180 & 1.360 \\
\hline Deep Sea & V 30089 51-: & 0.003 & 0.243 & 1.410 \\
\hline Deep Sea & V 31152 52-! & 0.036 & 0.246 & 0.350 \\
\hline Deep Sea & V 31157 45-८ & 0.000 & 0.270 & 0.830 \\
\hline Deep Sea & V 32154 54-! & 0.120 & 0.430 & 2.410 \\
\hline Deep Sea & /M 32154 8- & 0.010 & 0.620 & 3.360 \\
\hline Deep Sea & V 32173 52-! & 0.020 & 0.130 & 0.890 \\
\hline Deep Sea & 'M 3264 42-4 & 0.000 & 0.970 & 4.260 \\
\hline Deep Sea & 'M 3271 34-3 & 0.000 & 0.900 & 4.940 \\
\hline Deep Sea & N 30021 54-! & 0.033 & 1.463 & 4.760 \\
\hline Dysaerobic & if Shelf Sta D & 0.044 & 1.014 & 5.230 \\
\hline Dysaerobic & JoC L121 5-11 & 0.770 & 0.840 & 2.720 \\
\hline Dysaerobic & L105 8-14 & 0.320 & 0.720 & 3.780 \\
\hline Dysaerobic & L105 84-90 & 0.270 & 1.220 & 3.650 \\
\hline Dysaerobic & L105 95-102 & 0.000 & 0.870 & 3.820 \\
\hline Dysaerobic & L121 52-57 & 0.580 & 0.680 & 1.880 \\
\hline Dysaerobic & L139 140-149 & 0.690 & 0.910 & 2.510 \\
\hline Dysaerobic & L139 16-33 & 0.770 & 0.920 & 2.600 \\
\hline
\end{tabular}




\begin{tabular}{|c|c|c|c|c|}
\hline Dysaerobic & L139 247-254 & 0.830 & 0.950 & 2.690 \\
\hline Dysaerobic & L154 35-42 & 0.450 & 0.560 & 1.680 \\
\hline Dysaerobic & L154 54-61 & 0.450 & 0.530 & 1.560 \\
\hline Dysaerobic & L42 29-38 & 0.820 & 0.880 & 2.550 \\
\hline Dysaerobic & L42 45-50 & 0.800 & 0.860 & 2.590 \\
\hline Dysaerobic & L62 38-46 & 0.800 & 0.870 & 2.310 \\
\hline Dysaerobic & L62 5-10 & 0.280 & 0.460 & 2.850 \\
\hline Dysaerobic & SBB 1 & 0.590 & 0.710 & 3.480 \\
\hline Dysaerobic & SBB 3 & 0.670 & 0.790 & 3.170 \\
\hline Dysaerobic & SBB 4 & 0.430 & 0.580 & 3.320 \\
\hline Dysaerobic & Sta D 0-1 & 0.200 & 1.240 & 4.410 \\
\hline Dysaerobic & Sta D 20-21 & 0.340 & 0.710 & 3.810 \\
\hline Dysaerobic & Sta D29-30 & 0.180 & 0.490 & 4.560 \\
\hline Dysaerobic & Sta J 0-1 & 0.260 & 0.860 & 3.210 \\
\hline Dysaerobic & Sta J 15-16 & 0.190 & 0.580 & 3.400 \\
\hline Dysaerobic & Sta J 25-26 & 0.200 & 0.430 & 3.280 \\
\hline Dysaerobic & Sta J 39-40 & 0.260 & 0.450 & 3.540 \\
\hline Dysaerobic & Sta K 0-1 & 0.190 & 0.830 & 3.370 \\
\hline Dysaerobic & Sta K 11-12 & 0.110 & 0.400 & 3.220 \\
\hline Dysaerobic & Sta K 29-30 & 0.430 & 0.660 & 3.730 \\
\hline Dysaerobic & Sta K 5-6 & 0.130 & 0.720 & 3.220 \\
\hline Anaerobic & 5 & 0.210 & 0.460 & 1.750 \\
\hline Anaerobic & 6 & 0.270 & 0.620 & 2.740 \\
\hline Anaerobic & 7 & 0.260 & 0.740 & 2.220 \\
\hline Anaerobic & 8 & 0.230 & 0.670 & 2.840 \\
\hline Anaerobic & 9 & 0.270 & 0.730 & 2.560 \\
\hline Anaerobic & 10 & 0.250 & 0.640 & 2.440 \\
\hline Anaerobic & 11 & 0.300 & 0.720 & 2.050 \\
\hline Anaerobic & 12 & 0.280 & 0.690 & 2.570 \\
\hline Anaerobic & B1 125-130 & 1.250 & 1.380 & 2.520 \\
\hline Anaerobic & B1 140-145 & 1.230 & 1.330 & 2.660 \\
\hline Anaerobic & B1 35-40 & 1.210 & 1.410 & 2.680 \\
\hline Anaerobic & B1 55-60 & 1.200 & 1.330 & 2.570 \\
\hline Anaerobic & B1 65-70 & 1.210 & 1.350 & 2.640 \\
\hline Anaerobic & B2 125-130 & 1.400 & 1.580 & 2.990 \\
\hline Anaerobic & B2 140-145 & 1.320 & 1.460 & 3.020 \\
\hline Anaerobic & B2 20-25 & 1.430 & 1.550 & 2.780 \\
\hline Anaerobic & B2 65-70 & 1.320 & 1.460 & 2.760 \\
\hline Anaerobic & B3 145-150 & 0.860 & 1.000 & 2.190 \\
\hline Anaerobic & B3 20-25 & 1.330 & 1.510 & 3.090 \\
\hline Anaerobic & B3 60-65 & 1.350 & 1.500 & 3.000 \\
\hline
\end{tabular}




\begin{tabular}{|c|c|c|c|c|}
\hline Anaerobic & B4 20-25 & 0.870 & 0.980 & 1.940 \\
\hline Anaerobic & B4 60-65 & 1.020 & 1.150 & 2.130 \\
\hline Anaerobic & B4 80-85 & 1.060 & 1.220 & 2.700 \\
\hline Anaerobic & z\&K BS Sta $9 \vdots$ & 1.340 & 1.600 & 2.270 \\
\hline Anaerobic & ariaco B1 15-ż & 1.110 & 1.320 & 2.700 \\
\hline Anaerobic & $\mathrm{F} 2$ & 1.070 & 3.860 & 4.000 \\
\hline Anaerobic & Framvaren F1 & 1.700 & 2.130 & 3.560 \\
\hline Anaerobic & $\mathrm{H} 2$ & 0.950 & 1.230 & 4.780 \\
\hline Anaerobic & L1-85471 44-L & 0.310 & 0.790 & 4.670 \\
\hline Anaerobic & « & 0.350 & 0.770 & 3.800 \\
\hline Anaerobic & K4-85077 6-7 & 0.470 & 1.050 & 4.100 \\
\hline Anaerobic & K4-85471 3-4 & 0.570 & 1.060 & 4.730 \\
\hline Anaerobic & K9 2-4 & 0.590 & 0.940 & 4.260 \\
\hline Anaerobic & Kau K3 2-3 & 0.780 & 1.360 & 5.320 \\
\hline Anaerobic & ons BS Sta $9 \epsilon$ & 1.610 & 1.760 & 2.440 \\
\hline Anaerobic & Orca 1 & 0.410 & 0.780 & 1.300 \\
\hline Anaerobic & v Deep Basin & 0.890 & 1.040 & 3.910 \\
\hline Anaerobic & Sat 1419 & 0.990 & 1.180 & 1.500 \\
\hline Anaerobic & Sta 14 10-12 & 0.680 & 0.820 & 1.230 \\
\hline Anaerobic & Sta 14 10-15 & 1.000 & 1.190 & 2.100 \\
\hline Anaerobic & Sta 1411 & 0.920 & 1.070 & 1.260 \\
\hline Anaerobic & Sta 1413 & 1.350 & 1.640 & 2.330 \\
\hline Anaerobic & Sta 1415 & 1.270 & 1.510 & 2.010 \\
\hline Anaerobic & Sta 14 15-20 & 0.540 & 0.760 & 1.550 \\
\hline Anaerobic & Sta 1417 & 1.500 & 1.760 & 2.550 \\
\hline Anaerobic & Sta 14 20-22 & 0.660 & 0.800 & 1.240 \\
\hline Anaerobic & L4 20-25 (bot1 & 1.040 & 1.460 & 1.540 \\
\hline Anaerobic & Sta 1421 & 1.150 & 1.320 & 1.560 \\
\hline Anaerobic & Sta 1423 & 1.210 & 1.460 & 2.150 \\
\hline Anaerobic & Sta 1425 & 1.420 & 1.710 & 2.260 \\
\hline Anaerobic & Sta 1427 & 1.360 & 1.700 & 2.530 \\
\hline Anaerobic & Sta 1429 & 1.590 & 2.020 & 3.100 \\
\hline Anaerobic & sta $145-10$ & 0.440 & 0.690 & 1.250 \\
\hline Anaerobic & Sta 147 & 1.400 & 1.770 & 2.580 \\
\hline Anaerobic & Sta 149 & 1.410 & 1.620 & 1.930 \\
\hline Anaerobic & 14 surface $(\mathrm{t}$ & 1.150 & 1.370 & 2.790 \\
\hline Anaerobic & Sta 214 & 1.130 & 1.130 & 2.700 \\
\hline Anaerobic & Sta 223 & 1.000 & 1.080 & 3.890 \\
\hline Anaerobic & Sta 223 & 0.790 & 0.950 & 3.280 \\
\hline Anaerobic & Sta 235 & 0.850 & 1.350 & 3.680 \\
\hline Anaerobic & Sta 243 & 0.860 & 1.010 & 4.580 \\
\hline
\end{tabular}




$\begin{array}{lclll}\text { Anaerobic } & \text { Sta } 245 & 0.540 & 0.860 & 4.090 \\ \text { Anaerobic } & \text { Sta } 254 & 0.740 & 0.880 & 1.670 \\ \text { Anaerobic } & \text { Sta } 255 & 1.060 & 1.060 & 2.230 \\ \text { Anaerobic } & \text { Sta } 256 & 1.020 & 1.020 & 3.650 \\ \text { Anaerobic } & \text { Sta } 257 & 0.860 & 0.860 & 3.710 \\ \text { Anaerobic } & \text { Sta } 278 & 1.150 & 1.400 & 3.620 \\ \text { Anaerobic } & \text { Sta } 287 & 0.960 & 0.960 & 3.720 \\ \text { Anaerobic } & \text { Sta } 288 & 0.810 & 0.810 & 3.630 \\ \text { Anaerobic } & \text { Sta } 289 & 0.920 & 0.920 & 3.100 \\ \text { Anaerobic } & \text { Sta } 4740 & 0.790 & 1.050 & 3.670 \\ \text { Anaerobic } & \text { Sta } 4751 & 0.690 & 0.820 & 4.010 \\ \text { Anaerobic } & \text { Sta } 9 & 1.340 & 1.630 & 4.170 \\ \text { Anaerobic } & \text { Sta } 912-14 & 0.890 & 1.010 & 1.250 \\ \text { Anaerobic } & \text { Sta } 914-16 & 0.610 & 0.710 & 0.820 \\ \text { Anaerobic } & \text { Sta } 915 & 1.420 & 1.560 & 1.690 \\ \text { Anaerobic } & \text { Sta } 917 & 1.450 & 1.570 & 1.760 \\ \text { Anaerobic } & \text { Sta } 919 & 0.840 & 0.920 & 1.010 \\ \text { Anaerobic } & \text { Sta } 920-22 & 1.410 & 1.550 & 1.990 \\ \text { Anaerobic } & \text { Sta } 921 & 1.340 & 1.430 & 1.500 \\ \text { Anaerobic } & \text { Sta 9 } 22-24 & 0.800 & 0.900 & 1.110 \\ \text { Anaerobic } & \text { Sta } 923 & 1.730 & 1.850 & 2.190 \\ \text { Anaerobic } & \text { Sta } 924-26 & 0.990 & 1.110 & 1.290 \\ \text { Anaerobic } & \text { Sta } 925 & 1.950 & 2.150 & 2.960 \\ \text { Anaerobic } & \text { Sta } 95 & 1.810 & 2.110 & 2.540 \\ \text { Anaerobic } & \text { Sta } 97 & 1.630 & 1.820 & 2.200 \\ \text { Anaerobic } & \text { Sta } 98-10 & 1.420 & 1.570 & 2.070\end{array}$




\begin{tabular}{|c|c|c|c|c|}
\hline FeHR/FeT & $\mathrm{FeP} / \mathrm{FeHR}$ & Fe-oxide & Fe-carb & Fe-mag \\
\hline 1.000 & 0.448 & 1.500 & & 2.2 \\
\hline 0.400 & 0.440 & 2.600 & & 2.5 \\
\hline 0.200 & 0.456 & 2.300 & & 2 \\
\hline 0.400 & 0.475 & 2.700 & & 2.6 \\
\hline 0.300 & 0.603 & 1.200 & & 1.3 \\
\hline 1.000 & 0.488 & 3.900 & & 2.6 \\
\hline 0.900 & 0.557 & 2.700 & & 2 \\
\hline 0.900 & 0.468 & 3.100 & & 2.8 \\
\hline 0.900 & 0.373 & 1.800 & & 1.9 \\
\hline 0.600 & 0.292 & 2.200 & & 2.4 \\
\hline 1.000 & 0.097 & 5.000 & & 7.1 \\
\hline 0.600 & 0.443 & 2.200 & & 2.2 \\
\hline 1.000 & 0.353 & 4.500 & & 4.3 \\
\hline 1.000 & 0.468 & 4.800 & & 3.6 \\
\hline 0.700 & 0.386 & 3.200 & & 1.9 \\
\hline 0.800 & 0.444 & 2.800 & & 2.7 \\
\hline 0.700 & 0.359 & 2.800 & & 3.1 \\
\hline 0.900 & 0.387 & 3.000 & & 3.5 \\
\hline 0.500 & 0.313 & 1.700 & & 2.9 \\
\hline 0.900 & 0.369 & 2.200 & & 1.9 \\
\hline 1.000 & 0.718 & 3.400 & & 2.5 \\
\hline 0.900 & 0.590 & 2.100 & & 1.3 \\
\hline 1.000 & 0.632 & 2.400 & & 1.8 \\
\hline 0.800 & 0.614 & 1.900 & & 1.3 \\
\hline 0.800 & 0.456 & 2.000 & & 1.7 \\
\hline 0.900 & 0.472 & 1.800 & & 2 \\
\hline 1.000 & 0.554 & 2.500 & & 1.6 \\
\hline 1.000 & 0.587 & 2.500 & & 1.8 \\
\hline 0.600 & 0.397 & 1.700 & & 2.4 \\
\hline 0.800 & & 2.200 & & 2.2 \\
\hline 0.900 & 0.756 & 0.600 & & 0.5 \\
\hline 0.700 & 0.545 & 1.600 & & 1.4 \\
\hline 0.551 & 0.403 & 0.740 & 0.180 & 0.8 \\
\hline 0.281 & 0.230 & 0.030 & 0.260 & 0.85 \\
\hline 0.790 & 0.060 & 0.100 & 3.680 & 0.32 \\
\hline 0.716 & 0.007 & 0.130 & 2.360 & 0.25 \\
\hline 0.770 & 0.012 & 0.120 & 3.010 & 0.18 \\
\hline 0.716 & 0.000 & 0.190 & 2.460 & 0.05 \\
\hline 0.200 & 0.507 & 0.060 & 0.210 & 0.07 \\
\hline 0.310 & 0.252 & 0.070 & 0.960 & 0.2 \\
\hline
\end{tabular}




$\begin{array}{lllll}0.179 & 0.538 & 0.020 & 0.120 & 0.16 \\ 0.262 & 0.268 & 0.050 & 0.660 & 0.3 \\ 0.120 & 0.333 & 0.090 & 0.110 & 0.15 \\ 0.184 & 0.295 & 0.020 & 0.350 & 0.19 \\ 0.148 & 0.317 & 0.030 & 0.210 & 0.19 \\ 0.239 & 0.209 & 0.050 & 0.640 & 0.22 \\ 0.148 & 0.510 & 0.030 & 0.100 & 0.12 \\ 0.152 & 0.164 & 0.010 & 0.350 & 0.11 \\ 0.244 & 0.493 & 0.130 & 0.190 & 0.05 \\ 0.170 & 0.101 & 0.250 & 0.170 & 0.3 \\ 0.211 & 0.019 & 0.180 & 1.310 & 1.52 \\ 0.563 & 0.333 & 0.160 & 0.700 & 0.04 \\ 0.699 & 0.090 & 0.730 & 0.700 & 0.08 \\ 0.565 & 0.165 & 0.400 & 0.710 & 0.06 \\ 0.761 & 0.163 & 0.660 & 1.850 & 0.36 \\ 0.566 & 0.259 & 0.560 & 0.560 & 0.06 \\ 0.605 & 0.225 & 0.240 & 1.050 & 0.05 \\ 0.466 & 0.333 & 0.080 & 0.880 & 0.05 \\ 0.535 & 0.379 & 0.020 & 0.640 & 0.06 \\ 0.371 & 0.259 & 0.020 & 0.200 & 1.17 \\ 0.323 & 0.060 & 0.030 & 0.400 & 1.62 \\ 0.333 & 0.228 & 0.010 & 0.140 & 1.08 \\ 0.311 & 0.190 & 0.020 & 0.130 & 1.01 \\ 0.490 & 0.598 & 0.130 & 0.060 & 0.28 \\ 0.523 & 0.522 & 0.220 & 0.110 & 0.32 \\ 0.389 & 0.432 & 0.200 & 0.080 & 0.43 \\ 0.541 & 0.623 & 0.150 & 0.130 & 0.32 \\ 0.571 & 0.761 & 0.030 & 0.070 & 0.1 \\ 0.755 & 0.411 & 0.180 & 0.400 & 0.51 \\ 0.645 & 0.513 & 0.200 & 0.060 & 0.49 \\ 0.431 & 0.830 & 0.040 & 0.090 & 0.06 \\ 0.387 & 0.823 & 0.050 & 0.060 & 0.06 \\ 0.357 & 0.760 & 0.060 & 0.110 & 0.08 \\ 0.261 & 0.224 & 0.060 & 0.260 & 0.13 \\ 0.345 & 0.224 & 0.060 & 0.410 & 0.13 \\ 0.394 & 0.188 & 0.050 & 0.340 & 0.17 \\ 0.474 & 0.230 & 0.050 & 0.390 & 0.13 \\ 0.389 & 0.520 & 0.050 & 0.240 & 0.18 \\ 0.535 & 0.450 & 0.270 & 0.350 & 0.1 \\ 0.669 & 0.586 & 0.000 & 0.220 & 0.15 \\ 0.191 & 0.792 & 0.020 & 0.070 & 0.02\end{array}$




$\begin{array}{llll}0.313 & 0.532 & 0.170 & 0.070 \\ 0.782 & 0.568 & 0.050 & 0.600 \\ 0.470 & 0.970 & & \\ 0.330 & 0.960 & & \\ 1.000 & 0.520 & \\ 0.540 & 0.980 & & \\ 0.990 & 0.950 & \\ 0.380 & 0.910 & \\ 0.970 & 0.940 & \\ 0.830 & 0.990 & \\ 0.960 & 0.990 & & \\ 0.890 & 0.990 & & \\ 0.970 & 0.970 & \\ 0.520 & 0.980 & \\ 0.890 & 0.970 & \\ 0.920 & 0.990 & \\ 1.000 & 0.990 & \\ 0.350 & 0.890 & \\ 0.690 & 0.970 & \\ 0.910 & 0.990 & \\ 0.690 & 0.980 & \\ 0.690 & 0.990 & \\ 1.000 & 0.990 & \\ 0.840 & 0.990 & \\ 0.650 & 0.990 & \\ 0.730 & 0.980 & \\ 0.740 & 0.970 & \\ 0.680 & 0.980 & \\ 0.820 & 0.990 & \\ 0.740 & 0.990 & & \\ 0.730 & 0.970 & & \\ 0.770 & 0.980 & & \\ 0.730 & 0.980 & & \\ 0.900 & 0.970 & & \\ 0.880 & 0.990 & & \\ 0.970 & 0.990 & & \\ 0.980 & & \end{array}$




$\begin{array}{ll}0.250 & 0.540 \\ 0.040 & 0.000 \\ 0.100 & 0.070 \\ 0.400 & 0.970 \\ 0.110 & 0.740 \\ 0.440 & 0.930 \\ 0.280 & 0.100 \\ 0.640 & 0.100 \\ 0.280 & 0.360 \\ 0.120 & 0.960 \\ 0.720 & 0.940 \\ 0.190 & 0.440 \\ 0.280 & 0.990 \\ 0.440 & 0.890 \\ 0.110 & 0.770 \\ 0.470 & 0.740 \\ 0.040 & 0.620 \\ 0.110 & 0.730 \\ 0.330 & 0.940 \\ 0.780 & 0.940 \\ 0.750 & 0.930 \\ 0.190 & 0.920 \\ 0.830 & 0.980 \\ 0.520 & 0.990 \\ 0.760 & 0.690 \\ 0.600 & 0.960 \\ 0.620 & 0.830 \\ 0.090 & 0.300 \\ 0.660 & 0.900 \\ 0.330 & 0.820 \\ 0.620 & 0.960 \\ 0.120 & 0.820 \\ 0.070 & 0.660 \\ 0.050 & 0.860 \\ 0.180 & 0.850 \\ 0.140 & 0.950 \\ 0.270 & 0.730 \\ 0.620 & 0.940 \\ 0.020 & 0.450 \\ 0.980 & 0.460\end{array}$




$\begin{array}{ll}0.310 & 0.910 \\ 0.340 & 0.530 \\ 0.350 & 0.880 \\ 0.180 & 0.960 \\ 0.630 & 0.960 \\ 0.580 & 1.000 \\ 0.350 & 1.000 \\ 0.030 & 0.530 \\ 0.400 & 0.930 \\ 0.770 & 0.870 \\ 0.510 & 0.910 \\ 0.040 & 0.740 \\ 0.370 & 0.360 \\ 0.660 & 0.760 \\ 0.530 & 0.960 \\ 0.230 & 0.850 \\ 0.100 & 0.300 \\ 0.240 & 0.750 \\ 0.600 & 0.950 \\ 0.420 & 0.950 \\ 0.290 & 0.870 \\ 0.380 & 0.890 \\ 0.680 & 0.910 \\ 0.490 & 0.870 \\ 0.380 & 0.910 \\ 0.550 & 0.850 \\ 0.560 & 0.960 \\ 0.440 & 0.950 \\ 0.070 & 0.000 \\ 0.120 & 0.310 \\ 0.920 & 0.840 \\ 0.470 & 0.960 \\ 0.480 & 0.820 \\ 0.160 & 0.810 \\ 0.550 & 0.980 \\ 0.140 & 0.680 \\ 0.560 & 0.830 \\ 0.130 & 0.700 \\ 0.200 & 0.810 \\ 0.760 & 0.960 \\ 0.960\end{array}$




\begin{tabular}{ll}
1.000 & 0.120 \\
0.140 & 0.000 \\
0.200 & 0.000 \\
0.160 & 0.000 \\
0.070 & 0.030 \\
0.090 & 0.210 \\
0.150 & 0.560 \\
0.030 & 0.060 \\
0.030 & 0.060 \\
0.060 & 0.150 \\
0.060 & 0.040 \\
0.070 & 0.080 \\
0.080 & 0.080 \\
0.090 & 0.150 \\
0.090 & 0.090 \\
0.090 & 0.040 \\
0.090 & 0.120 \\
0.100 & 0.140 \\
0.130 & 0.040 \\
0.140 & 0.100 \\
0.160 & 0.080 \\
0.160 & 0.340 \\
0.170 & 0.110 \\
0.170 & 0.100 \\
0.170 & 0.370 \\
0.200 & 0.180 \\
0.210 & 0.070 \\
0.220 & 0.170 \\
0.240 & 0.460 \\
0.300 & 0.490 \\
0.300 & 0.530 \\
0.330 & 0.100 \\
0.360 & 0.360 \\
0.370 & 0.580 \\
0.480 & 0.200 \\
0.660 & 0.740 \\
0.070 & 0.060 \\
0.110 & 0.080 \\
0.120 & 0.120 \\
0.120 & 0.200 \\
0.160 & 0.390 \\
\hline & \\
\hline
\end{tabular}




$\begin{array}{ll}0.170 & 0.100 \\ 0.170 & 0.100 \\ 0.170 & 0.360 \\ 0.210 & 0.790 \\ 0.280 & 0.290 \\ 0.310 & 0.100 \\ 0.310 & 0.200 \\ 0.350 & 0.440 \\ 0.360 & 0.400 \\ 0.360 & 0.280 \\ 0.360 & 0.340 \\ 0.370 & 0.020 \\ 0.370 & 0.020 \\ 0.370 & 0.360 \\ 0.400 & 0.370 \\ 0.420 & 0.470 \\ 0.430 & 0.420 \\ 0.430 & 0.480 \\ 0.460 & 0.480 \\ 0.520 & 0.640 \\ 0.520 & 0.690 \\ 0.540 & 0.010 \\ 0.570 & 0.010 \\ 0.640 & 0.340 \\ 0.660 & 0.480 \\ 0.670 & 0.230 \\ 0.690 & 0.560 \\ 0.710 & 0.390 \\ 0.760 & 0.340 \\ 0.780 & 0.250 \\ 0.780 & 0.790 \\ 0.920 & 0.730 \\ 0.980 & 0.550 \\ 1.020 & 0.900 \\ 1.040 & 0.900 \\ 0.140 & 0.370 \\ 0.140 & 0.320 \\ 0.370 & 0.140 \\ 0.350 & 0.040 \\ 0.130 \\ 0.240\end{array}$




\begin{tabular}{|c|c|c|c|c|}
\hline 0.780 & 0.610 & & & \\
\hline 0.800 & 0.280 & & & \\
\hline 0.840 & 0.580 & & & \\
\hline 0.900 & 0.500 & & & \\
\hline 0.900 & 0.620 & & & \\
\hline 0.910 & 0.550 & & & \\
\hline 0.930 & 0.330 & & & \\
\hline 0.080 & 0.270 & & & \\
\hline 0.170 & 0.510 & & & \\
\hline 0.180 & 0.250 & & & \\
\hline 0.260 & 0.290 & & & \\
\hline 0.270 & 0.270 & & & \\
\hline 0.650 & 0.650 & & & \\
\hline 0.440 & 0.530 & & & \\
\hline 0.460 & 0.610 & & & \\
\hline 0.490 & 0.790 & & & \\
\hline 0.650 & 0.800 & & & \\
\hline 0.690 & 0.870 & & & \\
\hline 0.750 & 0.900 & & & \\
\hline 0.760 & 0.900 & & & \\
\hline 0.810 & 0.610 & & & \\
\hline 0.810 & 0.690 & & & \\
\hline 0.830 & 0.720 & & & \\
\hline 0.900 & 0.870 & & & \\
\hline 0.930 & 0.850 & & & \\
\hline 0.930 & 0.890 & & & \\
\hline 0.132 & 0.279 & 0.031 & 0.127 & 0 \\
\hline 0.128 & 0.439 & 0.021 & 0.168 & 0 \\
\hline 0.124 & 0.101 & 0.137 & 0.115 & 0.04497215 \\
\hline 0.444 & 0.458 & 0.034 & 0.503 & 0 \\
\hline 0.639 & 0.113 & 0.008 & 0.603 & 0 \\
\hline 0.359 & 0.033 & 0.408 & 0.039 & 0 \\
\hline 0.239 & 0.116 & 0.262 & 0.059 & 0 \\
\hline 0.171 & 0.012 & 0.524 & 0.076 & 0 \\
\hline 0.356 & 0.529 & 0.050 & 0.838 & 0 \\
\hline 0.191 & 0.325 & 0.021 & 0.869 & 0 \\
\hline 0.560 & 0.634 & 0.034 & 0.610 & 0 \\
\hline 0.396 & 0.303 & 0.011 & 0.503 & 0 \\
\hline 0.294 & 0.140 & 0.210 & 0.030 & 0 \\
\hline 0.217 & 0.622 & 0.160 & 0.520 & 0 \\
\hline 0.423 & 0.211 & 0.010 & 0.740 & 0 \\
\hline
\end{tabular}




\begin{tabular}{|c|c|c|c|c|}
\hline 0.401 & 0.806 & 0.010 & 0.190 & 0 \\
\hline 0.188 & 0.392 & 0.020 & 0.160 & 0 \\
\hline 0.177 & 0.250 & 0.020 & 0.300 & 0 \\
\hline 0.435 & 0.865 & 0.021 & 0.172 & 0 \\
\hline 0.287 & 0.428 & 0.031 & 0.224 & 0.16980225 \\
\hline 0.178 & 0.286 & 0.050 & 0.234 & 0 \\
\hline 0.328 & 0.036 & 0.379 & 0.068 & 0.20469714 \\
\hline 0.266 & 0.135 & 0.152 & 0.195 & 0.0926728 \\
\hline 0.346 & 0.126 & 0.165 & 0.224 & 0.25763344 \\
\hline 0.246 & 0.119 & 0.040 & 0.205 & 0.02404309 \\
\hline 0.172 & 0.321 & 0.076 & 0.258 & 0.08173519 \\
\hline 0.119 & 0.133 & 0.075 & 0.153 & 0.07894951 \\
\hline 0.122 & 0.115 & 0.083 & 0.184 & 0.08362287 \\
\hline 0.137 & 0.052 & 0.093 & 0.276 & 0.09217505 \\
\hline 0.155 & 0.280 & 0.061 & 0.212 & 0.05391438 \\
\hline 0.147 & 0.112 & 0.060 & 0.260 & 0.05349139 \\
\hline 0.212 & 0.018 & 0.049 & 0.442 & 0.03925227 \\
\hline 0.279 & 0.006 & 0.253 & 0.440 & 0.04307399 \\
\hline 0.119 & 0.033 & 0.036 & 0.192 & 0.02767854 \\
\hline 0.088 & 0.022 & 0.061 & 0.152 & 0.07453556 \\
\hline 0.155 & 0.025 & 0.065 & 0.357 & 0.06679378 \\
\hline 0.134 & 0.095 & 0.049 & 0.267 & 0.05177467 \\
\hline 0.146 & 0.029 & 0.044 & 0.287 & 0.04680387 \\
\hline 0.159 & 0.126 & 0.051 & 0.311 & 0.03935602 \\
\hline 0.131 & 0.114 & 0.043 & 0.245 & 0.03785975 \\
\hline 0.178 & 0.206 & 0.039 & 0.279 & 0.03502507 \\
\hline 0.114 & 0.029 & 0.076 & 0.272 & 0.09204041 \\
\hline 0.095 & 0.046 & 0.055 & 0.205 & 0.07112602 \\
\hline 0.122 & 0.077 & 0.066 & 0.282 & 0.07063898 \\
\hline 0.141 & 0.417 & 0.058 & 0.188 & 0.06762044 \\
\hline 0.146 & 0.141 & 0.064 & 0.354 & 0.05662403 \\
\hline 0.103 & 0.367 & 0.052 & 0.120 & 0.06659617 \\
\hline 0.093 & 0.141 & 0.070 & 0.183 & 0.08773218 \\
\hline 0.070 & 0.066 & 0.052 & 0.129 & 0.06411325 \\
\hline 0.141 & 0.369 & 0.045 & 0.229 & 0.04998776 \\
\hline 0.083 & 0.103 & 0.056 & 0.140 & 0.07238918 \\
\hline 0.126 & 0.036 & 0.065 & 0.348 & 0.09878915 \\
\hline 0.087 & 0.109 & 0.058 & 0.149 & 0.07071954 \\
\hline 0.152 & 0.226 & 0.057 & 0.303 & 0.07362019 \\
\hline 0.103 & 0.258 & 0.053 & 0.141 & 0.06828643 \\
\hline 0.152 & 0.564 & 0.062 & 0.140 & 0.06099999 \\
\hline
\end{tabular}




\begin{tabular}{|c|c|c|c|c|}
\hline 0.170 & 0.677 & 0.045 & 0.121 & 0.04268577 \\
\hline 0.145 & 0.414 & 0.057 & 0.178 & 0.06012476 \\
\hline 0.127 & 0.287 & 0.054 & 0.229 & 0.05559126 \\
\hline 0.129 & 0.262 & 0.064 & 0.225 & 0.06654742 \\
\hline 0.141 & 0.537 & 0.045 & 0.124 & 0.04184856 \\
\hline 0.163 & 0.524 & 0.043 & 0.168 & 0.03478944 \\
\hline 0.175 & 0.544 & 0.032 & 0.132 & 0.02319293 \\
\hline 0.193 & 0.413 & 0.031 & 0.221 & 0.019959 \\
\hline 0.130 & 0.343 & 0.054 & 0.139 & 0.0769307 \\
\hline 0.128 & 0.104 & 0.054 & 0.214 & 0.02709177 \\
\hline 0.148 & 0.263 & 0.049 & 0.161 & 0.02188654 \\
\hline 0.246 & 0.073 & 0.050 & 0.555 & 0.03081753 \\
\hline 0.361 & 0.113 & 0.042 & 0.839 & 0.02805144 \\
\hline 0.083 & 0.174 & 0.049 & 0.084 & 0.02279929 \\
\hline 0.237 & 0.121 & 0.064 & 0.454 & 0.0332274 \\
\hline 0.112 & 0.082 & 0.026 & 0.079 & 0.01429201 \\
\hline 0.070 & 0.200 & 0.160 & & \\
\hline 0.060 & 0.526 & 0.090 & & \\
\hline 0.040 & 0.348 & 0.150 & & \\
\hline 0.030 & 0.429 & 0.080 & & \\
\hline 0.060 & 0.438 & 0.090 & & \\
\hline 0.030 & 0.476 & 0.110 & & \\
\hline 0.090 & 0.481 & 0.140 & & \\
\hline 0.050 & 0.278 & 0.130 & & \\
\hline 0.040 & 0.421 & 0.110 & & \\
\hline 0.040 & 0.250 & 0.120 & & \\
\hline 0.050 & 0.467 & 0.080 & & \\
\hline 0.170 & 0.348 & 0.450 & & \\
\hline 0.050 & 0.286 & 0.150 & & \\
\hline 0.060 & 0.296 & 0.190 & & \\
\hline 0.050 & 0.346 & 0.170 & & \\
\hline 0.080 & 0.118 & 0.300 & & \\
\hline 0.060 & 0.231 & 0.200 & & \\
\hline 0.070 & 0.094 & 0.290 & & \\
\hline 0.150 & 0.057 & 0.500 & & \\
\hline 0.230 & 0.740 & 0.200 & & \\
\hline 0.040 & 0.167 & 0.150 & & \\
\hline 0.040 & 0.333 & 0.100 & & \\
\hline 0.120 & 0.346 & 0.340 & & \\
\hline 0.140 & 0.132 & 0.460 & & \\
\hline 0.030 & 0.308 & 0.090 & & \\
\hline
\end{tabular}




$\begin{array}{lll}0.060 & 0.286 & 0.150 \\ 0.040 & 0.278 & 0.130 \\ 0.040 & 0.455 & 0.060 \\ 0.070 & 0.333 & 0.200 \\ 0.020 & 0.333 & 0.080 \\ 0.030 & 0.000 & 0.080 \\ 0.040 & 0.286 & 0.100 \\ 0.030 & 0.000 & 0.150 \\ 0.040 & 0.250 & 0.090 \\ 0.040 & 0.357 & 0.090 \\ 0.040 & 0.235 & 0.130 \\ 0.040 & 0.500 & 0.040 \\ 0.050 & 0.286 & 0.100 \\ 0.040 & 0.231 & 0.100 \\ 0.440 & 0.847 & 0.230 \\ 0.560 & 0.919 & 0.140 \\ 0.580 & 0.905 & 0.150 \\ 0.290 & 0.903 & 0.190 \\ 0.290 & 0.820 & 0.230 \\ 0.520 & 0.969 & 0.040 \\ 0.310 & 0.914 & 0.140 \\ 0.510 & 0.947 & 0.050 \\ 0.630 & 0.932 & 0.090 \\ 0.730 & 0.959 & 0.080 \\ 0.680 & 0.939 & 0.100 \\ 0.790 & 0.970 & 0.080 \\ 0.540 & 0.899 & 0.300 \\ 0.060 & 0.167 & 0.100 \\ 0.040 & 0.000 & 0.370 \\ 0.040 & 0.056 & 0.170 \\ 0.080 & 0.400 & 0.360 \\ 0.100 & 0.495 & 0.500 \\ 0.160 & 0.730 & 0.200 \\ 0.090 & 0.126 & 1.250 \\ 0.110 & 0.684 & 0.120 \\ 0.090 & 0.625 & 0.150 \\ 0.040 & 0.158 & 0.160 \\ 0.430 & 0.944 & 0.100 \\ 0.500 & 0.935 & 0.140 \\ 0.080 & 0.583 & 0.100 \\ 0.100 & 0.614 & 0.170\end{array}$




$\begin{array}{lll}0.070 & 0.577 & 0.110 \\ 0.060 & 0.379 & 0.180 \\ 0.110 & 0.606 & 0.130 \\ 0.220 & 0.809 & 0.170 \\ 0.220 & 0.728 & 0.250 \\ 0.210 & 0.720 & 0.230 \\ 0.090 & 0.577 & 0.110 \\ 0.130 & 0.698 & 0.130 \\ 0.470 & 0.920 & 0.120 \\ 0.410 & 0.908 & 0.170 \\ 0.180 & 0.385 & 0.080 \\ 0.100 & 0.636 & 0.120 \\ 0.040 & 0.286 & 0.100 \\ 0.180 & 0.318 & 0.150 \\ 0.070 & 0.455 & 0.060 \\ 0.050 & 0.000 & 0.070 \\ 0.080 & 0.286 & 0.100 \\ 0.060 & 0.313 & 0.110 \\ 0.050 & 0.292 & 0.170 \\ 0.050 & 0.462 & 0.140 \\ 0.050 & 0.421 & 0.110 \\ 0.040 & 0.400 & 0.120 \\ 0.110 & 0.483 & 0.310 \\ 0.050 & 0.115 & 0.230 \\ 0.030 & 0.154 & 0.110 \\ 0.050 & 0.360 & 0.160 \\ 0.070 & 0.129 & 0.270 \\ 0.050 & 0.154 & 0.220 \\ 0.050 & 0.045 & 0.210 \\ 0.070 & 0.034 & 0.280 \\ 0.060 & 0.280 & 0.180 \\ 0.070 & 0.103 & 0.260 \\ 0.070 & 0.324 & 0.230 \\ 0.060 & 0.129 & 0.270 \\ 0.090 & 0.167 & 0.350 \\ 0.060 & 0.161 & 0.260 \\ 0.100 & 0.120 & 0.440 \\ 0.060 & 0.114 & 0.390 \\ 0.070 & 0.032 & 0.300 \\ 0.070 & 0.182 & 0.270 \\ 0.070 & 0.143 & 0.300\end{array}$




\begin{tabular}{|c|c|c|c|c|}
\hline 0.070 & 0.457 & 0.190 & & \\
\hline 0.560 & 0.931 & 0.170 & & \\
\hline 0.420 & 0.943 & 0.080 & & \\
\hline 0.380 & 0.913 & 0.150 & & \\
\hline 0.540 & 0.943 & 0.100 & & \\
\hline 0.560 & 0.946 & 0.130 & & \\
\hline 0.410 & 0.893 & 0.190 & & \\
\hline 0.430 & 0.925 & 0.110 & & \\
\hline 0.370 & 0.873 & 0.230 & & \\
\hline 0.660 & 0.778 & 0.180 & & \\
\hline 0.648 & 0.143 & 0.100 & 1.070 & 0.09 \\
\hline 0.418 & 0.492 & 0.000 & 0.620 & 0 \\
\hline 0.149 & 0.128 & 0.160 & 0.020 & 0.16 \\
\hline 0.123 & 0.086 & 0.150 & 0.000 & 0.17 \\
\hline 0.768 & 0.698 & 0.000 & 0.480 & 0 \\
\hline 0.486 & 0.528 & 0.000 & 0.670 & 0 \\
\hline 0.437 & 0.411 & 0.130 & 0.510 & 0.1 \\
\hline 0.408 & 0.479 & 0.110 & 0.420 & 0.07 \\
\hline 0.533 & 0.307 & 0.000 & 1.220 & 0 \\
\hline 0.676 & 0.146 & 0.000 & 1.220 & 0 \\
\hline 0.882 & 0.115 & 0.000 & 1.470 & 0 \\
\hline 0.322 & 0.322 & 0.000 & 0.590 & 0 \\
\hline 0.459 & 0.378 & 0.130 & 0.780 & 0.11 \\
\hline 0.340 & 0.296 & 0.000 & 0.950 & 0 \\
\hline 0.449 & 0.490 & 0.120 & 0.560 & 0.09 \\
\hline 0.412 & 0.420 & 0.140 & 0.490 & 0.13 \\
\hline 0.223 & 0.474 & 0.000 & 0.410 & 0 \\
\hline 0.265 & 0.250 & 0.000 & 0.780 & 0 \\
\hline 0.467 & 0.291 & 0.130 & 1.000 & 0.11 \\
\hline 0.419 & 0.573 & 0.000 & 0.440 & 0 \\
\hline 0.536 & 0.174 & 0.010 & 1.650 & 0.19 \\
\hline 0.914 & 0.110 & 0.130 & 1.980 & 0.15 \\
\hline 0.722 & 0.271 & 0.000 & 1.120 & 0.17 \\
\hline 1.000 & 0.063 & 0.180 & 2.140 & 0.06 \\
\hline 0.602 & 0.673 & 0.000 & 0.500 & 0 \\
\hline 0.836 & 0.654 & 0.000 & 0.530 & 0 \\
\hline 0.542 & 0.510 & 0.310 & 0.150 & 0 \\
\hline 0.947 & 0.324 & 0.000 & 0.620 & 0.11 \\
\hline 0.941 & 0.573 & 0.000 & 0.420 & 0 \\
\hline 0.967 & 0.563 & 0.120 & 0.570 & 0.09 \\
\hline 0.975 & 0.813 & 0.000 & 0.500 & 0 \\
\hline
\end{tabular}




\begin{tabular}{|c|c|c|c|c|}
\hline 0.840 & 0.486 & 0.000 & 0.630 & 0.11 \\
\hline 0.934 & 0.471 & 0.000 & 0.440 & 0 \\
\hline 0.929 & 0.487 & 0.000 & 1.000 & 0 \\
\hline 0.872 & 0.621 & 0.000 & 0.670 & 0 \\
\hline 0.892 & 0.601 & 0.000 & 0.690 & 0 \\
\hline 0.863 & 0.564 & 0.000 & 0.850 & 0 \\
\hline 0.982 & 0.722 & 0.100 & 0.570 & 0.08 \\
\hline 0.857 & 0.576 & 0.000 & 0.280 & 0 \\
\hline 0.593 & 0.400 & 0.120 & 0.000 & 0.09 \\
\hline 0.919 & 0.439 & 0.210 & 0.340 & 0.1 \\
\hline 0.522 & 0.000 & 0.000 & 1.200 & 0 \\
\hline 0.572 & 0.615 & 0.000 & 0.550 & 0 \\
\hline 0.651 & 0.463 & 0.160 & 0.000 & 0.13 \\
\hline 0.155 & 0.193 & 0.210 & 0.070 & 0.38 \\
\hline 0.092 & 0.139 & 0.000 & 1.990 & 0 \\
\hline 0.546 & 0.387 & 0.490 & 0.260 & 0.12 \\
\hline 0.646 & 0.345 & 0.000 & 0.970 & 0 \\
\hline 0.110 & 0.152 & 0.150 & 0.020 & 0.12 \\
\hline 0.681 & 0.683 & 0.000 & 0.410 & 0 \\
\hline 0.590 & 0.789 & 0.200 & & \\
\hline 0.763 & 0.915 & 0.170 & & \\
\hline 0.440 & 0.840 & 0.230 & & \\
\hline 0.829 & 0.942 & 0.150 & & \\
\hline 0.818 & 0.830 & 0.190 & & \\
\hline 0.556 & 0.905 & 0.100 & & \\
\hline 0.524 & 0.857 & 0.140 & & \\
\hline 0.498 & 0.798 & 0.210 & & \\
\hline 0.811 & 0.958 & 0.150 & & \\
\hline 0.798 & 0.958 & 0.150 & & \\
\hline 0.339 & 0.871 & 0.200 & & \\
\hline 0.238 & 0.267 & 0.330 & & \\
\hline 0.561 & 0.800 & 0.210 & & \\
\hline 0.686 & 0.921 & 0.140 & & \\
\hline 0.754 & 0.866 & 0.370 & & \\
\hline 0.208 & 0.785 & 0.290 & & \\
\hline 0.616 & 0.855 & 0.200 & & \\
\hline 0.800 & 0.901 & 0.380 & & \\
\hline 0.765 & 0.938 & 0.080 & & \\
\hline 0.758 & 0.620 & 0.570 & & \\
\hline 0.635 & 0.943 & 0.100 & & \\
\hline 0.661 & 0.877 & 0.290 & & \\
\hline
\end{tabular}




$\begin{array}{lllll}0.776 & 0.931 & 0.110 & & \\ 0.734 & 0.665 & 0.630 & & \\ 0.258 & 0.450 & 0.126 & 0.461 & 0.206 \\ 0.329 & 0.346 & 0.868 & 0.550 & 0.196 \\ 0.359 & 0.362 & 1.010 & 0.588 & 0.199 \\ 0.423 & 0.793 & 0.926 & 0.876 & 0.157 \\ 0.592 & 0.805 & 0.385 & 0.585 & 0.187 \\ 0.581 & 0.994 & 4.449 & 1.008 & 0.183 \\ 0.998 & 0.839 & 7.980 & 1.571 & 0.145 \\ 0.877 & 0.957 & 2.692 & 4.065 & 0.38 \\ 0.142 & 0.257 & 0.169 & 0.374 & 0.16 \\ 0.915 & 0.882 & 2.345 & 8.175 & 0.469 \\ 0.477 & 0.510 & 1.734 & 0.952 & 0.113 \\ 0.607 & 0.491 & 1.972 & 1.210 & 0.202 \\ 0.577 & 0.775 & 0.251 & 0.368 & 0.15 \\ 0.434 & 0.772 & 0.175 & 0.385 & 0.107 \\ 0.563 & 0.780 & 0.266 & 0.465 & 0.108 \\ 0.269 & 0.492 & 0.117 & 0.477 & 0.212 \\ 0.226 & 0.191 & 0.270 & 0.523 & 0.372 \\ 0.372 & 0.622 & 0.031 & 0.368 & 0.145 \\ 0.627 & 0.834 & 0.026 & 0.425 & 0.121 \\ 0.279 & 0.507 & 0.022 & 0.308 & 0.101 \\ 0.332 & 0.631 & 0.026 & 0.227 & 0.119 \\ 0.523 & 0.776 & 0.031 & 0.382 & 0.156 \\ 0.417 & 0.423 & 0.129 & 0.644 & 0.147 \\ 0.428 & 0.659 & 0.036 & 0.432 & 0.166 \\ 0.610 & 0.305 & 0.172 & 1.219 & 0.112 \\ 0.685 & 0.347 & 0.153 & 1.347 & 0.118 \\ 0.384 & 0.279 & 0.123 & 0.755 & 0.13 \\ 0.478 & 0.298 & 0.108 & 0.972 & 0.081 \\ 0.512 & 0.332 & 0.150 & 1.018 & 0.115 \\ 0.735 & 0.366 & 0.550 & 1.325 & 0.252 \\ 0.677 & 0.349 & 0.185 & 1.301 & 0.082 \\ 0.234 & 0.299 & 0.102 & 0.264 & 0.07 \\ 0.483 & 0.345 & 0.218 & 0.644 & 0.1 \\ 0.803 & 0.465 & 0.156 & 2.038 & 0.18 \\ 0.590 & 0.652 & 0.070 & 0.736 & 0.059 \\ 0.165 & 0.244 & 0.022 & 0.273 & 0.084 \\ 0.653 & 0.754 & 0.056 & 0.771 & 0.103 \\ 0.823 & 0.879 & 0.055 & 0.872 & 0.11 \\ 0.640 & 0.344 & 0.254 & 1.191 & 0.226\end{array}$




$\begin{array}{llllc}0.660 & 0.303 & 0.468 & 1.391 & 0.367 \\ 0.671 & 0.323 & 0.494 & 1.354 & 0.306 \\ 0.549 & 0.481 & 0.113 & 0.744 & 0.13 \\ 0.669 & 0.831 & 0.021 & 0.435 & 0.058 \\ 0.808 & 0.898 & 0.024 & 0.578 & 0.056 \\ 0.748 & 0.858 & 0.041 & 0.714 & 0.086 \\ 0.758 & 0.870 & 0.055 & 0.811 & 0.102 \\ 0.374 & 0.540 & 0.056 & 0.622 & 0.118 \\ 0.674 & 0.496 & 0.485 & 0.817 & 0.39 \\ 0.790 & 0.473 & 0.447 & 1.264 & 0.427 \\ 0.751 & 0.579 & 0.313 & 0.814 & 0.58 \\ 0.477 & 0.496 & 0.216 & 0.392 & 0.075 \\ 0.489 & 0.855 & 0.015 & 0.191 & 0.094 \\ 0.542 & 0.885 & 0.018 & 0.207 & 0.099 \\ 0.525 & 0.867 & 0.019 & 0.267 & 0.151 \\ 0.542 & 0.894 & 0.023 & 0.277 & 0.149 \\ 0.641 & 0.911 & 0.034 & 0.342 & 0.166 \\ 0.506 & 0.850 & 0.042 & 0.314 & 0.235 \\ 0.552 & 0.877 & 0.039 & 0.310 & 0.237 \\ 0.466 & 0.818 & 0.041 & 0.258 & 0.233 \\ 0.504 & 0.809 & 0.045 & 0.295 & 0.261 \\ 0.881 & 0.949 & 0.030 & 0.787 & 0.071 \\ 0.499 & 0.839 & 0.040 & 0.305 & 0.226 \\ 0.625 & 0.901 & 0.039 & 0.424 & 0.222 \\ 0.309 & 0.416 & 0.016 & 0.358 & 0.118 \\ 0.234 & 0.172 & 0.043 & 0.414 & 0.184 \\ 0.329 & 0.509 & 0.039 & 0.379 & 0.163 \\ 0.345 & 0.498 & 0.037 & 0.372 & 0.162 \\ 0.742 & 0.811 & 0.019 & 0.305 & 0.038 \\ 0.520 & 0.413 & 0.017 & 0.242 & 0.049 \\ 0.276 & 0.150 & 0.031 & 0.346 & 0.152 \\ 0.351 & 0.475 & 0.029 & 0.307 & 0.238 \\ 0.402 & 0.563 & 0.026 & 0.290 & 0.213 \\ 0.324 & 0.408 & 0.031 & 0.343 & 0.278 \\ 0.615 & 0.295 & 0.630 & 0.113 & 0.036 \\ 0.490 & 0.834 & 0.406 & 0.076 & 0.025 \\ 0.610 & 0.907 & 0.276 & 0.089 & 0.02 \\ 0.524 & 0.807 & 0.310 & 0.180 & 0.018 \\ 0.476 & 0.802 & 0.131 & 0.887 & 0.148 \\ 0.655 & 0.811 & 4.173 & 0.096 & 0.02 \\ 0.130 & 0.258 & 0.209 & 0.217 & 0.168\end{array}$




$\begin{array}{lllll}0.205 & 0.311 & 0.468 & 0.325 & 0.246 \\ 0.603 & 0.370 & 2.040 & 0.491 & 0.125 \\ 0.204 & 0.272 & 0.543 & 0.243 & 0.157 \\ 0.221 & 0.557 & 0.691 & 0.275 & 0.148 \\ 0.313 & 0.444 & 0.500 & 0.214 & 0.079 \\ 0.162 & 0.551 & 0.384 & 0.247 & 0.153 \\ 0.129 & 0.770 & 0.345 & 0.173 & 0.1 \\ 0.390 & 0.813 & 0.221 & 0.196 & 0.083 \\ 0.400 & 0.826 & 0.440 & 0.236 & 0.133 \\ 0.826 & 0.989 & 4.382 & 0.838 & 0.172 \\ 0.097 & 0.270 & 0.115 & 0.203 & 0.104 \\ 0.121 & 0.482 & 0.197 & 0.193 & 0.121 \\ 0.379 & 0.872 & 1.462 & 0.378 & 0.097 \\ 0.747 & 0.308 & 2.522 & 0.557 & 0.091 \\ 0.123 & 0.180 & 0.229 & 0.209 & 0.173 \\ 0.349 & 0.435 & 0.492 & 0.203 & 0.094 \\ 0.452 & 0.823 & 0.997 & 0.240 & 0.098 \\ 0.430 & 0.960 & 1.829 & 0.324 & 0.108 \\ 0.104 & 0.140 & 0.114 & 0.201 & 0.163 \\ 0.743 & 0.340 & 2.325 & 0.368 & 0.095 \\ 0.101 & 0.043 & 0.097 & 0.213 & 0.185 \\ 0.273 & 0.248 & 0.867 & 0.186 & 0.158 \\ 0.124 & 0.153 & 0.198 & 0.198 & 0.171 \\ 0.552 & 0.420 & 0.520 & 0.283 & 0.067 \\ 0.319 & 0.361 & 0.243 & 0.073 & 0.037 \\ 0.225 & 0.101 & 0.220 & 0.041 & 0.013 \\ 0.142 & 0.081 & 0.146 & 0.240 & 0.13 \\ 0.662 & 0.437 & 1.061 & 0.509 & 0.167 \\ 0.368 & 0.835 & 0.122 & 0.192 & 0.047 \\ 0.419 & 0.824 & 0.223 & 0.126 & 0.049 \\ 0.435 & 0.983 & 1.254 & 0.206 & 0.082 \\ 0.370 & 0.818 & 1.254 & 0.206 & 0.088 \\ 0.333 & 0.468 & 0.853 & 0.271 & 0.155 \\ 0.343 & 0.579 & 0.838 & 0.206 & 0.111 \\ 0.240 & 0.089 & 0.224 & 0.187 & 0.106 \\ 0.305 & 0.591 & 0.706 & 0.192 & 0.113 \\ 0.107 & 0.019 & 0.234 & 0.179 & 0.163 \\ 0.157 & 0.309 & 0.447 & 0.213 & 0.154 \\ 0.300 & 0.375 & 0.721 & 0.238 & 0.152 \\ 0.456 & 0.830 & 1.444 & 0.236 & 0.144 \\ 0.334 & 0.246 & 0.718 & 0.340 & 0.285\end{array}$




$\begin{array}{llllc}0.451 & 0.818 & 0.370 & 0.206 & 0.065 \\ 0.419 & 0.985 & 0.249 & 0.543 & 0.21 \\ 0.374 & 0.434 & 0.993 & 0.286 & 0.215 \\ 0.383 & 0.840 & 0.735 & 0.294 & 0.095 \\ 0.189 & 0.068 & 0.297 & 0.198 & 0.22 \\ 0.357 & 0.526 & 0.994 & 0.297 & 0.206 \\ 0.431 & 0.886 & 1.822 & 0.280 & 0.152 \\ 0.396 & 0.991 & 1.728 & 0.228 & 0.086 \\ 0.464 & 0.808 & 1.490 & 0.373 & 0.236 \\ 0.392 & 0.854 & 1.398 & 0.271 & 0.177 \\ 0.437 & 0.813 & 1.700 & 0.295 & 0.195 \\ 0.474 & 0.949 & 1.523 & 0.234 & 0.093 \\ 0.898 & 0.952 & 1.615 & 0.264 & 0.112 \\ 0.581 & 0.807 & 0.427 & 0.206 & 0.061 \\ 0.126 & 0.202 & 0.560 & 0.326 & 0.434 \\ 0.391 & 0.955 & 1.353 & 0.236 & 0.108 \\ 0.263 & 0.767 & 0.618 & 0.221 & 0.155 \\ 0.232 & 0.793 & 0.635 & 0.173 & 0.091 \\ 0.152 & 0.490 & 0.267 & 0.131 & 0.069 \\ 0.486 & 0.805 & 0.898 & 0.277 & 0.175 \\ 0.579 & 0.862 & 0.528 & 0.238 & 0.065 \\ 0.872 & 0.997 & 1.385 & 0.367 & 0.093 \\ 0.253 & 0.041 & 0.115 & 1.019 & 0.154 \\ 0.252 & 0.194 & 0.130 & 0.786 & 0.137 \\ 0.282 & 0.131 & 0.140 & 1.017 & 0.143 \\ 0.279 & 0.094 & 0.116 & 1.123 & 0.144 \\ 0.324 & 0.241 & 0.142 & 0.956 & 0.125 \\ 0.257 & 0.037 & 0.127 & 1.053 & 0.153 \\ 0.382 & 0.445 & 0.143 & 0.750 & 0.101 \\ 0.308 & 0.239 & 0.135 & 0.876 & 0.13 \\ 0.328 & 0.270 & 0.166 & 0.975 & 0.112 \\ 0.247 & 0.063 & 0.127 & 0.957 & 0.104 \\ 0.324 & 0.289 & 0.140 & 0.885 & 0.161 \\ 0.353 & 0.385 & 0.144 & 0.738 & 0.092 \\ 0.276 & 0.148 & 0.144 & 0.964 & 0.134 \\ 0.285 & 0.215 & 0.151 & 0.911 & 0.129 \\ 0.254 & 0.113 & 0.103 & 0.852 & 0.111 \\ 0.309 & 0.228 & 0.142 & 0.832 & 0.105 \\ 0.284 & 0.173 & 0.162 & 0.991 & 0.115 \\ 0.271 & 0.038 & 0.098 & 1.085 & 0.117 \\ 0.274 & 0.134 & 0.103 & 0.927 & 0.112\end{array}$




$\begin{array}{llllc}0.277 & 0.069 & 0.107 & 0.983 & 0.115 \\ 0.258 & 0.058 & 0.140 & 0.982 & 0.12 \\ 0.328 & 0.332 & 0.126 & 0.778 & 0.096 \\ 0.326 & 0.415 & 0.138 & 0.852 & 0.126 \\ 0.294 & 0.109 & 0.111 & 1.024 & 0.105 \\ 0.324 & 0.440 & 0.568 & 0.229 & 0.146 \\ 0.378 & 0.618 & 0.347 & 0.285 & 0.249 \\ 0.537 & 0.869 & 0.112 & 0.156 & 0.105 \\ 0.456 & 0.784 & 0.246 & 0.212 & 0.126 \\ 0.155 & 0.181 & 0.363 & 0.308 & 0.096 \\ 0.311 & 0.626 & 0.183 & 0.227 & 0.12 \\ 0.204 & 0.406 & 0.232 & 0.233 & 0.115 \\ 0.165 & 0.265 & 0.229 & 0.211 & 0.112 \\ 0.325 & 0.709 & 0.146 & 0.188 & 0.117 \\ 0.123 & 0.156 & 0.232 & 0.233 & 0.115 \\ 0.268 & 0.613 & 0.256 & 0.179 & 0.105 \\ 0.186 & 0.180 & 0.295 & 0.247 & 0.162 \\ 0.276 & 0.669 & 0.164 & 0.186 & 0.111 \\ 0.481 & 0.732 & 0.228 & 0.283 & 0.129 \\ 0.621 & 0.831 & 0.262 & 0.192 & 0.134 \\ 0.377 & 0.680 & 0.249 & 0.235 & 0.092 \\ 0.263 & 0.526 & 0.316 & 0.269 & 0.161 \\ 0.246 & 0.443 & 0.365 & 0.294 & 0.104 \\ 0.192 & 0.389 & 0.268 & 0.240 & 0.112 \\ 0.167 & 0.316 & 0.259 & 0.272 & 0.13 \\ 0.152 & 0.250 & 0.254 & 0.241 & 0.101 \\ 0.340 & 0.606 & 0.247 & 0.236 & 0.153 \\ 0.236 & 0.578 & 0.140 & 0.174 & 0.102 \\ 0.156 & 0.247 & 0.295 & 0.310 & 0.125 \\ 0.133 & 0.169 & 0.251 & 0.268 & 0.144 \\ 0.162 & 0.259 & 0.305 & 0.272 & 0.143 \\ 0.239 & 0.561 & 0.209 & 0.243 & 0.137 \\ 0.263 & 0.594 & 0.205 & 0.225 & 0.154 \\ 0.436 & 0.822 & 0.164 & 0.149 & 0.075 \\ 0.284 & 0.243 & 0.121 & 0.118 & 0.355 \\ 0.659 & 0.819 & 0.205 & 0.225 & 0.299 \\ 0.607 & 0.786 & 0.300 & 0.258 & 0.333 \\ 0.459 & 0.747 & 0.200 & 0.212 & 0.269 \\ 0.378 & 0.637 & 0.274 & 0.228 & 0.287 \\ 0.275 & 0.092 & 0.045 & 0.993 & 0.397 \\ 0.203 & 0.492 & 0.004 & 0.163 & 0.062\end{array}$




$\begin{array}{llllc}0.265 & 0.275 & 0.028 & 0.709 & 0.263 \\ 0.298 & 0.378 & 0.024 & 0.689 & 0.275 \\ 0.306 & 0.372 & 0.025 & 0.609 & 0.222 \\ 0.331 & 0.344 & 0.033 & 0.828 & 0.277 \\ 0.415 & 0.608 & 0.005 & 0.504 & 0.15 \\ 0.266 & 0.236 & 0.011 & 0.472 & 0.116 \\ 0.441 & 0.498 & 0.040 & 0.746 & 0.223 \\ 0.288 & 0.307 & 0.034 & 0.587 & 0.18 \\ 0.235 & 0.049 & 0.049 & 0.900 & 0.294 \\ 0.209 & 0.030 & 0.026 & 0.723 & 0.249 \\ 0.241 & 0.028 & 0.028 & 0.916 & 0.287 \\ 0.220 & 0.067 & 0.034 & 0.677 & 0.269 \\ 0.274 & 0.045 & 0.036 & 1.015 & 0.333 \\ 0.259 & 0.201 & 0.034 & 0.733 & 0.299 \\ 0.281 & 0.010 & 0.039 & 0.959 & 0.355 \\ 0.217 & 0.015 & 0.025 & 0.714 & 0.289 \\ 0.227 & 0.097 & 0.024 & 0.746 & 0.307 \\ 0.270 & 0.125 & 0.039 & 0.908 & 0.352 \\ 0.267 & 0.113 & 0.021 & 0.900 & 0.326 \\ 0.263 & 0.045 & 0.048 & 1.115 & 0.445 \\ 0.243 & 0.049 & 0.042 & 0.917 & 0.389 \\ 0.176 & 0.008 & 0.038 & 0.464 & 0.332 \\ 0.289 & 0.232 & 0.059 & 0.687 & 0.493 \\ 0.221 & 0.184 & 0.037 & 0.529 & 0.328 \\ 0.254 & 0.342 & 0.039 & 0.486 & 0.312 \\ 0.178 & 0.012 & 0.028 & 0.557 & 0.31 \\ 0.215 & 0.014 & 0.033 & 0.636 & 0.352 \\ 0.214 & 0.034 & 0.031 & 0.728 & 0.346 \\ 0.195 & 0.015 & 0.039 & 0.662 & 0.351 \\ 0.257 & 0.005 & 0.067 & 1.081 & 0.62 \\ 0.281 & 0.248 & 0.040 & 0.644 & 0.373 \\ 0.228 & 0.040 & 0.038 & 0.729 & 0.447 \\ 0.263 & 0.043 & 0.041 & 0.661 & 0.391 \\ 0.508 & 0.041 & 0.086 & 3.290 & 0.905 \\ 0.263 & 0.132 & 0.019 & 0.559 & 0.254 \\ 0.311 & 0.077 & 0.072 & 1.112 & 0.545 \\ 0.283 & 0.142 & 0.044 & 0.853 & 0.303 \\ 0.220 & 0.189 & 0.008 & 0.301 & 0.129 \\ 0.270 & 0.015 & 0.033 & 1.043 & 0.519 \\ 0.336 & 0.199 & 0.057 & 1.275 & 0.527 \\ 0.214 & 0.017 & 0.049 & 1.019 & 0.501\end{array}$




$\begin{array}{llllc}0.234 & 0.206 & 0.018 & 0.541 & 0.283 \\ 0.236 & 0.243 & 0.005 & 0.572 & 0.288 \\ 0.204 & 0.057 & 0.014 & 0.664 & 0.307 \\ 0.273 & 0.239 & 0.010 & 0.627 & 0.3 \\ 0.234 & 0.186 & 0.011 & 0.594 & 0.317 \\ 0.251 & 0.149 & 0.040 & 0.710 & 0.285 \\ 0.228 & 0.168 & 0.043 & 0.585 & 0.283 \\ 0.246 & 0.005 & 0.050 & 0.983 & 0.494 \\ 0.229 & 0.136 & 0.047 & 0.653 & 0.317 \\ 0.195 & 0.034 & 0.042 & 0.704 & 0.295 \\ 0.183 & 0.019 & 0.036 & 0.590 & 0.234 \\ 0.187 & 0.076 & 0.040 & 0.582 & 0.259 \\ 0.216 & 0.318 & 0.027 & 0.371 & 0.163 \\ 0.270 & 0.489 & 0.019 & 0.338 & 0.088 \\ 0.236 & 0.340 & 0.019 & 0.362 & 0.096 \\ 0.166 & 0.083 & 0.013 & 0.490 & 0.094 \\ 0.252 & 0.339 & 0.020 & 0.372 & 0.096 \\ 0.275 & 0.036 & 0.036 & 0.739 & 0.408 \\ 0.245 & 0.008 & 0.036 & 0.863 & 0.464 \\ 0.310 & 0.023 & 0.048 & 1.625 & 0.484 \\ 0.253 & 0.054 & 0.033 & 0.806 & 0.417 \\ 0.303 & 0.012 & 0.053 & 1.207 & 0.761 \\ 0.369 & 0.002 & 0.066 & 2.501 & 0.713 \\ 0.263 & 0.023 & 0.031 & 0.959 & 0.422 \\ 0.804 & 0.316 & 0.268 & 0.856 & 0.179 \\ 0.759 & 0.066 & 0.463 & 1.442 & 0.305 \\ 0.233 & 0.138 & 0.063 & 0.324 & 0.062 \\ 0.254 & 0.138 & 0.104 & 0.302 & 0.061 \\ 0.503 & 0.083 & 0.194 & 0.725 & 0.116 \\ 0.096 & 0.146 & 0.075 & 0.077 & 0.03 \\ 0.071 & 0.035 & 0.038 & 0.077 & 0.023 \\ 0.956 & 0.152 & 0.145 & 0.527 & 0.093 \\ 0.755 & 0.626 & 0.129 & 0.608 & 0.157 \\ 0.930 & 0.002 & 0.082 & 0.359 & 0.025 \\ 0.844 & 0.198 & 0.220 & 1.032 & 0.307 \\ 0.598 & 0.975 & 0.031 & 0.012 & 0.011 \\ 0.568 & 0.968 & 0.025 & 0.011 & 0.008 \\ 0.645 & 0.058 & 0.178 & 0.600 & 0.265 \\ 0.829 & 0.529 & 0.079 & 0.322 & 0.067 \\ 0.224 & 0.967 & 0.023 & 0.007 & 0.007 \\ 0.355 & 0.943 & 0.020 & 0.004 & 0.007\end{array}$




\begin{tabular}{|c|c|c|c|c|}
\hline 0.440 & 0.957 & 0.027 & 0.008 & 0.005 \\
\hline 0.353 & 0.831 & 0.041 & 0.118 & 0.043 \\
\hline 0.380 & 0.947 & 0.027 & 0.023 & 0.001 \\
\hline 0.557 & 0.101 & 0.245 & 1.128 & 0.337 \\
\hline 0.439 & 0.864 & 0.036 & 0.068 & 0.026 \\
\hline 0.341 & 0.472 & 0.101 & 0.381 & 0.145 \\
\hline 0.304 & 0.725 & 0.040 & 0.160 & 0.048 \\
\hline 0.336 & 0.711 & 0.080 & 0.178 & 0.064 \\
\hline 0.434 & 0.587 & 0.069 & 0.184 & 0.059 \\
\hline 0.449 & 0.810 & 0.085 & 0.233 & 0.074 \\
\hline 0.474 & 0.782 & 0.054 & 0.088 & 0.044 \\
\hline 0.177 & 0.309 & 0.072 & 0.208 & 0.095 \\
\hline 0.454 & 0.769 & 0.063 & 0.148 & 0.033 \\
\hline 0.215 & 0.785 & 0.041 & 0.032 & 0.013 \\
\hline 0.746 & 0.296 & 0.291 & 1.102 & 0.256 \\
\hline 0.051 & 0.604 & 0.022 & 0.018 & 0.002 \\
\hline 0.552 & 0.338 & 0.128 & 0.563 & 0.169 \\
\hline 0.864 & 0.402 & 0.075 & 0.278 & 0.079 \\
\hline 0.346 & 0.837 & 0.076 & 0.247 & 0.044 \\
\hline 0.900 & 0.135 & 0.107 & 0.381 & 0.057 \\
\hline 0.832 & 0.459 & 0.188 & 0.862 & 0.196 \\
\hline 0.919 & 0.266 & 0.088 & 0.407 & 0.078 \\
\hline 0.630 & 0.600 & & & \\
\hline 0.670 & 0.310 & & & \\
\hline 0.440 & 0.870 & & & \\
\hline 0.570 & 0.790 & & & \\
\hline 0.780 & 0.780 & & & \\
\hline 0.680 & 0.820 & & & \\
\hline 0.780 & 0.820 & & & \\
\hline 0.910 & 0.910 & & & \\
\hline 0.860 & 0.880 & & & \\
\hline 0.550 & 0.880 & & & \\
\hline 0.300 & 0.860 & & & \\
\hline 0.440 & 0.900 & & & \\
\hline 0.390 & 0.850 & & & \\
\hline 0.390 & 0.770 & & & \\
\hline 0.910 & 0.960 & & & \\
\hline 0.910 & 0.910 & & & \\
\hline 0.430 & 0.540 & & & \\
\hline 0.580 & 0.830 & 0.120 & 0.100 & 0.11 \\
\hline 0.500 & 0.850 & 0.180 & 0.040 & 0.05 \\
\hline
\end{tabular}




$\begin{array}{llllc}0.900 & 0.920 & 0.240 & 0.050 & 0.06 \\ 0.960 & 0.930 & 0.110 & 0.030 & 0.02 \\ 1.040 & 0.590 & 0.240 & 0.090 & 0.04 \\ 1.040 & 0.980 & 0.010 & 0.050 & 0.05 \\ 0.970 & 0.970 & 0.030 & 0.170 & 0.04 \\ 1.030 & 0.980 & 0.010 & 0.140 & 0.01 \\ 0.270 & 0.740 & 0.010 & 0.160 & 0 \\ 1.040 & 0.990 & 0.020 & 0.000 & 0 \\ 0.890 & 0.980 & 0.010 & 0.000 & 0 \\ 0.960 & 0.990 & 0.010 & 0.000 & 0 \\ 0.830 & 0.960 & 0.010 & 0.010 & 0 \\ 0.450 & 0.970 & 0.020 & 0.000 & 0 \\ 0.670 & 0.840 & 0.120 & 0.020 & 0 \\ 0.910 & 0.900 & 0.040 & 0.050 & 0.01 \\ 1.030 & 0.960 & 0.020 & 0.040 & 0.01 \\ 0.870 & 0.980 & 0.010 & 0.020 & 0 \\ 1.040 & 0.920 & 0.070 & 0.010 & 0.02 \\ 0.890 & 0.940 & 0.020 & 0.030 & 0.02 \\ 0.510 & 0.680 & 0.240 & 0.520 & 0.01 \\ 0.680 & 0.690 & 0.270 & 0.430 & 0.01 \\ 0.700 & 0.680 & 0.330 & 0.540 & 0.01 \\ 0.660 & 0.710 & 0.410 & 0.540 & 0.02 \\ 0.630 & 0.820 & 0.030 & 0.300 & 0.05 \\ 0.600 & 0.950 & 0.020 & 0.030 & 0.03 \\ 0.570 & 0.900 & 0.030 & 0.040 & 0.04 \\ 0.420 & 0.950 & 0.020 & 0.060 & 0 \\ 1.050 & 0.830 & 0.150 & 0.020 & 0.03 \\ 0.170 & 0.270 & 0.080 & 0.010 & 0.16 \\ 0.700 & 0.510 & 0.700 & 0.160 & 0.38 \\ 1.020 & 0.570 & 0.120 & 0.110 & 0.36 \\ 0.510 & 0.130 & 0.210 & 0.100 & 0.29 \\ 0.340 & 0.400 & 0.290 & 0.120 & 0.3 \\ 0.430 & 0.460 & 0.250 & 0.050 & 0.25 \\ 0.220 & 0.070 & 0.180 & 0.260 & 0.77 \\ 0.380 & 0.540 & 0.030 & 0.200 & 0.08 \\ 0.350 & 0.670 & 0.100 & 0.090 & 0.15 \\ 0.240 & 0.410 & 0.070 & 0.020 & 0.09 \\ 0.580 & 0.590 & 0.020 & 0.030 & 0.08 \\ 0.310 & 0.920 & 0.020 & 0.010 & 0.04 \\ 0.480 & 0.370 & 0.060 & 0.290 & 0.21 \\ 0.250 & 0.330 & 0.150 & 0.150 & 0.16\end{array}$




$\begin{array}{lllll}0.120 & 0.080 & 0.250 & 0.460 & 0.38 \\ 0.400 & 0.480 & 0.210 & 0.200 & 0.38 \\ 0.130 & 0.180 & 0.240 & 0.250 & 0.38 \\ 0.190 & 0.440 & 0.290 & 0.260 & 0.47 \\ 0.290 & 0.290 & 0.190 & 0.360 & 0.36 \\ 0.660 & 0.550 & 0.070 & 0.030 & 0.09 \\ 0.200 & 0.140 & 0.420 & 0.550 & 0.68 \\ 0.413 & 0.003 & 0.514 & 1.116 & 1.598 \\ 0.375 & 0.119 & 0.185 & 0.784 & 0.871 \\ 0.462 & 0.608 & 0.049 & 0.257 & 0.211 \\ 0.452 & 0.588 & 0.070 & 0.265 & 0.215 \\ 0.397 & 0.563 & 0.089 & 0.287 & 0.215 \\ 0.474 & 0.567 & 0.062 & 0.303 & 0.234 \\ 0.493 & 0.573 & 0.044 & 0.276 & 0.162 \\ 0.539 & 0.635 & 0.047 & 0.273 & 0.191 \\ 0.433 & 0.612 & 0.046 & 0.227 & 0.201 \\ 0.429 & 0.585 & 0.071 & 0.380 & 0.231 \\ 0.441 & 0.564 & 0.077 & 0.403 & 0.257 \\ 0.487 & 0.618 & 0.064 & 0.271 & 0.219 \\ 0.421 & 0.577 & 0.071 & 0.307 & 0.155 \\ 0.495 & 0.626 & 0.044 & 0.276 & 0.192 \\ 0.281 & 0.010 & 0.321 & 0.401 & 0.938 \\ 0.264 & 0.015 & 0.153 & 0.556 & 0.869 \\ 0.352 & 0.042 & 0.181 & 0.712 & 0.955 \\ 0.349 & 0.003 & 0.241 & 0.766 & 1.091 \\ 0.347 & 0.007 & 0.262 & 0.644 & 0.908 \\ 0.265 & 0.022 & 0.320 & 0.551 & 0.81 \\ 0.296 & 0.006 & 0.411 & 0.434 & 1.149 \\ 0.292 & 0.351 & 0.088 & 0.326 & 0.406 \\ 0.364 & 0.248 & 0.123 & 0.517 & 0.575 \\ 0.354 & 0.435 & 0.108 & 0.433 & 0.451 \\ 0.351 & 0.014 & 0.422 & 0.771 & 1.494 \\ 0.550 & 0.584 & 0.069 & 0.428 & 0.295 \\ 0.374 & 0.568 & 0.106 & 0.509 & 0.338 \\ 0.353 & 0.630 & 0.100 & 0.394 & 0.283 \\ 0.375 & 0.119 & 0.185 & 0.784 & 0.871 \\ 0.484 & 0.560 & 0.100 & 0.432 & 0.417 \\ 0.245 & 0.018 & 0.151 & 0.569 & 0.896 \\ 0.420 & 0.568 & 0.108 & 0.436 & 0.495 \\ 0.441 & 0.491 & 0.136 & 0.494 & 0.555 \\ & 0.638 & 0.081 & 0.318 & 0.347\end{array}$




$\begin{array}{llllc}0.412 & 0.516 & 0.129 & 0.604 & 0.428 \\ 0.380 & 0.617 & 0.086 & 0.313 & 0.301 \\ 0.291 & 0.072 & 0.129 & 0.552 & 0.656 \\ 0.496 & 0.496 & 0.106 & 0.526 & 0.53 \\ 0.272 & 0.011 & 0.325 & 0.634 & 0.937 \\ 0.484 & 0.536 & 0.126 & 0.518 & 0.557 \\ 0.250 & 0.042 & 0.267 & 0.588 & 1.058 \\ 0.358 & 0.009 & 0.754 & 1.151 & 1.355 \\ 0.477 & 0.008 & 0.558 & 1.053 & 0.89 \\ 0.276 & 0.032 & 0.311 & 0.541 & 0.905 \\ 0.260 & 0.022 & 0.283 & 0.552 & 1.03 \\ 0.301 & 0.204 & 0.124 & 0.486 & 0.627 \\ 0.455 & 0.515 & 0.099 & 0.452 & 0.332 \\ 0.379 & 0.345 & 0.142 & 0.652 & 0.505 \\ 0.225 & 0.080 & 0.129 & 0.459 & 0.495 \\ 0.375 & 0.006 & 0.572 & 0.704 & 1.21 \\ 0.263 & 0.022 & 0.391 & 0.607 & 0.95 \\ 0.413 & 0.009 & 0.457 & 0.732 & 1.27 \\ 0.286 & 0.066 & 0.232 & 0.431 & 0.865 \\ 0.252 & 0.085 & 0.344 & 0.333 & 0.518 \\ 0.314 & 0.000 & 1.261 & 0.195 & 0.854 \\ 0.535 & 0.000 & 3.301 & 0.062 & 0.579 \\ 0.489 & 0.000 & 2.926 & 0.033 & 0.745 \\ 0.405 & 0.005 & 0.426 & 0.692 & 1.817 \\ 0.307 & 0.011 & 0.323 & 0.479 & 0.954 \\ 0.322 & 0.008 & 0.246 & 0.967 & 1.296 \\ 0.248 & 0.194 & 0.085 & 0.284 & 0.421 \\ 0.290 & 0.020 & 0.362 & 0.595 & 0.942 \\ 0.430 & 0.340 & 0.250 & 0.000 & 0.71 \\ 0.420 & 0.470 & 0.130 & 0.000 & 0.29 \\ 0.900 & 0.830 & 0.140 & 0.100 & 0.17 \\ 0.930 & 0.870 & 0.290 & 6.000 & 0.02 \\ 0.860 & 0.830 & 0.080 & 0.120 & 0.17 \\ 0.910 & 0.920 & 0.160 & 0.070 & 0.03 \\ 0.930 & 0.710 & 0.700 & 0.240 & 0.07 \\ 0.870 & 0.930 & 0.060 & 0.100 & 0.06 \\ 0.920 & 0.920 & 0.090 & 0.100 & 0.06 \\ 0.770 & 0.860 & 0.160 & 0.100 & 0.06 \\ 0.910 & 0.850 & 0.090 & 0.200 & 0.08 \\ 1.040 & 0.760 & 0.140 & 0.100 & 0.34 \\ 0.750 & 0.610 & 0.130 & 0.100 & 0.5\end{array}$




\begin{tabular}{|c|c|c|c|c|}
\hline 0.760 & 0.690 & 0.060 & 0.100 & 0.24 \\
\hline 0.260 & 0.630 & 0.030 & 0.080 & 0.23 \\
\hline 0.100 & 0.240 & & & \\
\hline 0.100 & 0.130 & & & \\
\hline 0.490 & 0.790 & & & \\
\hline 0.770 & 0.480 & & & \\
\hline 0.140 & 0.190 & & & \\
\hline 0.180 & 0.130 & & & \\
\hline 0.640 & 0.670 & & & \\
\hline 0.670 & 0.690 & & & \\
\hline 0.360 & 0.520 & & & \\
\hline 0.680 & 0.650 & & & \\
\hline 0.660 & 0.610 & & & \\
\hline 0.660 & 0.700 & & & \\
\hline 0.260 & 0.090 & & & \\
\hline 0.600 & 0.630 & & & \\
\hline 0.800 & 0.800 & & & \\
\hline 0.880 & 0.720 & & & \\
\hline 1.000 & 0.900 & 0.200 & 0.200 & 0.1 \\
\hline 0.900 & 0.800 & 0.100 & 0.100 & 0.2 \\
\hline 1.000 & 0.900 & 0.100 & 0.000 & 0 \\
\hline 1.000 & 0.900 & 0.100 & 0.000 & 0 \\
\hline 0.100 & 0.800 & 0.000 & 0.000 & 0 \\
\hline 0.400 & 1.000 & 0.000 & 0.000 & 0 \\
\hline 0.400 & 0.900 & 0.000 & 0.000 & 0 \\
\hline 0.500 & 1.000 & 0.000 & 0.000 & 0 \\
\hline 0.400 & 1.000 & 0.000 & 0.000 & 0 \\
\hline 0.700 & 0.900 & 0.000 & 0.000 & 0 \\
\hline 0.100 & 0.300 & 0.100 & 0.000 & 0 \\
\hline 0.000 & 0.500 & 0.000 & 0.000 & 0 \\
\hline 0.000 & 0.400 & 0.000 & 0.000 & 0 \\
\hline 0.600 & 0.700 & 0.100 & 0.200 & 0 \\
\hline 0.500 & 0.900 & 0.000 & 0.200 & 0 \\
\hline 0.500 & 0.900 & 0.000 & 0.200 & 0 \\
\hline 0.200 & 0.700 & 0.100 & 0.000 & 0 \\
\hline 0.100 & 0.300 & 0.100 & 0.100 & 0.1 \\
\hline 0.100 & 0.300 & 0.000 & 0.300 & 0.1 \\
\hline 0.200 & 0.000 & 0.500 & 0.000 & 0.2 \\
\hline 0.100 & 0.000 & 0.200 & 0.000 & 0.1 \\
\hline 0.200 & 0.000 & 0.200 & 0.400 & 0.2 \\
\hline 0.100 & 0.000 & 0.100 & 0.100 & 0.1 \\
\hline
\end{tabular}




$\begin{array}{llllc}0.400 & 0.700 & 0.200 & 0.100 & 0.1 \\ 0.400 & 0.700 & 0.200 & 0.200 & 0.1 \\ 0.200 & 0.800 & 0.100 & 0.100 & 0.1 \\ 0.100 & 0.000 & 0.100 & 0.100 & 0.1 \\ 0.388 & 0.003 & 1.164 & 0.208 & 0.3896661 \\ 0.476 & 0.002 & 1.348 & 0.261 & 0.20564847 \\ 0.297 & 0.004 & 0.732 & 0.091 & 0.11425892 \\ 0.242 & 0.006 & 0.825 & 0.143 & 0.16468875 \\ 0.283 & 0.004 & 1.072 & 0.545 & 0.26895796 \\ 0.434 & 0.075 & 0.395 & 0.893 & 0.12648336 \\ 0.335 & 0.001 & 0.635 & 1.171 & 0.50938053 \\ 0.316 & 0.132 & 0.631 & 0.770 & 1.09208054 \\ 0.277 & 0.058 & 0.429 & 0.567 & 0.78654504 \\ 0.257 & 0.023 & 0.358 & 0.518 & 0.72912705 \\ 0.279 & 0.004 & 0.350 & 0.842 & 0.85974499 \\ 0.280 & 0.005 & 0.319 & 0.684 & 0.80493671 \\ 0.331 & 0.001 & 0.226 & 1.061 & 0.32244389 \\ 0.205 & 0.005 & 0.311 & 0.292 & 0.29640553 \\ 0.565 & 0.003 & 0.175 & 1.485 & 0.45489007 \\ 0.508 & 0.015 & 0.303 & 1.177 & 0.96709402 \\ 0.474 & 0.005 & 0.276 & 1.205 & 0.82721617 \\ 0.509 & 0.002 & 0.323 & 1.202 & 0.96179183 \\ 0.261 & 0.001 & 0.272 & 1.204 & 0.83238547 \\ 0.465 & 0.001 & 0.259 & 0.874 & 0.66252822 \\ 0.345 & 0.001 & 0.294 & 0.682 & 0.4468651 \\ 0.183 & 0.003 & 0.318 & 0.448 & 0.52066574 \\ 0.165 & 0.000 & 0.316 & 0.440 & 0.52154696 \\ 0.178 & 0.000 & 0.293 & 0.355 & 0.51657397 \\ 0.191 & 0.001 & 0.288 & 0.285 & 0.51109123 \\ 0.220 & 0.003 & 0.292 & 0.306 & 0.53806328 \\ 0.234 & 0.025 & 0.408 & 0.408 & 0.54797017 \\ 0.158 & 0.000 & 0.213 & 0.004 & 0.00424104 \\ 0.353 & 0.000 & 0.687 & 0.005 & 0.02746615 \\ 0.120 & 0.000 & 0.115 & 0.010 & 0.00657417 \\ 0.062 & 0.000 & 0.088 & 0.059 & 0.03853913 \\ 0.091 & 0.000 & 0.193 & 0.041 & 0.02434639 \\ 0.062 & 0.000 & 0.043 & 0.006 & 0.00681388 \\ 0.299 & 0.000 & 0.373 & 0.004 & 0.01765384 \\ 0.226 & 0.000 & 0.366 & 0.006 & 0.02819525 \\ 0.249 & 0.023 & 0.180 & 0.010 & 0.00543132\end{array}$




\begin{tabular}{|c|c|c|c|c|}
\hline 0.697 & 0.001 & 2.632 & 0.005 & 0.33019471 \\
\hline 0.245 & 0.013 & 0.203 & 0.007 & 0.00482225 \\
\hline 0.118 & 0.030 & 0.080 & 0.008 & 0.00865563 \\
\hline 0.546 & 0.001 & 1.794 & 0.052 & 0.10922551 \\
\hline 0.200 & 0.016 & 0.165 & 0.006 & 0.00743425 \\
\hline 0.246 & 0.004 & 0.486 & 0.030 & 0.04366645 \\
\hline 0.193 & 0.003 & 0.424 & 0.041 & 0.02706419 \\
\hline 0.414 & 0.014 & 0.374 & 0.041 & 0.01818016 \\
\hline 0.106 & 0.017 & 0.078 & 0.040 & 0.01134718 \\
\hline 0.369 & 0.003 & 0.568 & 0.048 & 0.03914659 \\
\hline 0.734 & 0.020 & 0.126 & 0.281 & 0.00311704 \\
\hline 0.104 & 0.005 & 0.294 & 0.024 & 0.04746601 \\
\hline 0.331 & 0.004 & 0.489 & 0.108 & 0.04143268 \\
\hline 0.148 & 0.003 & 0.453 & 0.042 & 0.04839161 \\
\hline 0.095 & 0.007 & 0.161 & 0.074 & 0.02737327 \\
\hline 0.388 & 0.002 & 1.136 & 0.085 & 0.07256573 \\
\hline 0.125 & 0.004 & 0.372 & 0.057 & 0.03440962 \\
\hline 0.110 & 0.011 & 0.293 & 0.027 & 0.02695602 \\
\hline 0.036 & 0.018 & 0.058 & 0.038 & 0.01259478 \\
\hline 0.445 & 0.003 & 1.069 & 0.134 & 0.00812359 \\
\hline 0.048 & 0.000 & 0.028 & 0.061 & 0.01493258 \\
\hline 0.118 & 0.001 & 0.391 & 0.065 & 0.0304103 \\
\hline 0.270 & 0.001 & 0.522 & 0.037 & 0.01982783 \\
\hline 0.030 & 0.005 & 0.032 & 0.045 & 0.01783091 \\
\hline 0.058 & 0.014 & 0.028 & 0.057 & 0.00850706 \\
\hline 0.106 & 0.007 & 0.195 & 0.050 & 0.01776559 \\
\hline 0.639 & 0.001 & 1.856 & 0.013 & 0.13581004 \\
\hline 0.315 & 0.002 & 0.669 & 0.047 & 0.03245279 \\
\hline 0.186 & 0.003 & 0.427 & 0.070 & 0.05031677 \\
\hline 0.256 & 0.011 & 0.489 & 0.158 & 0.02147291 \\
\hline 0.433 & 0.001 & 0.987 & 0.047 & 0.08258699 \\
\hline 0.322 & 0.000 & 0.884 & 0.044 & 0.04878465 \\
\hline 0.265 & 0.003 & 0.611 & 0.055 & 0.04296273 \\
\hline 0.910 & 0.001 & 2.653 & 0.120 & 0.02650755 \\
\hline 0.649 & 0.001 & 1.817 & 0.036 & 0.01457712 \\
\hline 0.704 & 0.003 & 0.975 & 0.285 & 0.01082705 \\
\hline 0.398 & 0.003 & 0.882 & 0.044 & 0.0514519 \\
\hline 0.181 & 0.007 & 0.220 & 0.046 & 0.03084409 \\
\hline 0.333 & 0.002 & 0.811 & 0.026 & 0.04553234 \\
\hline 0.544 & 0.000 & 6.981 & 0.025 & 0.28930144 \\
\hline 0.592 & 0.006 & 0.354 & 0.026 & 0.00261376 \\
\hline
\end{tabular}




\begin{tabular}{lllll}
0.765 & 0.000 & 3.408 & 0.017 & 0.03146475 \\
0.420 & 0.001 & 1.206 & 0.033 & 0.06555644 \\
0.186 & 0.002 & 0.360 & 0.059 & 0.03659593 \\
0.161 & 0.000 & 0.612 & 0.037 & 0.03688686 \\
0.306 & 0.000 & 0.948 & 0.083 & 0.03580301 \\
0.486 & 0.000 & 2.170 & 0.081 & 0.07615137 \\
0.220 & 0.000 & 0.733 & 0.064 & 0.04169797 \\
0.141 & 0.000 & 0.472 & 0.066 & 0.04391909 \\
0.364 & 0.001 & 1.745 & 0.081 & 0.07490522 \\
0.312 & 0.001 & 1.094 & 0.073 & 0.08645567 \\
0.094 & 0.005 & 0.298 & 0.086 & 0.04990892 \\
0.064 & 0.007 & 0.138 & 0.094 & 0.03410917 \\
0.117 & 0.004 & 0.343 & 0.102 & 0.06007134 \\
0.097 & 0.005 & 0.233 & 0.096 & 0.0426011 \\
0.229 & 0.002 & 0.845 & 0.093 & 0.06314455 \\
0.233 & 0.002 & 0.802 & 0.085 & 0.05272843 \\
0.331 & 0.001 & 1.054 & 0.091 & 0.10529334 \\
0.084 & 0.007 & 0.226 & 0.049 & 0.02832642 \\
0.264 & 0.000 & 0.961 & 0.111 & 0.05341661 \\
0.195 & 0.000 & 0.601 & 0.054 & 0.05008104 \\
0.440 & 0.000 & 1.204 & 0.062 & 0.01935978 \\
0.152 & 0.000 & 0.428 & 0.145 & 0.02640843 \\
0.663 & 0.000 & 2.501 & 0.079 & 0.01866503 \\
0.268 & 0.000 & 0.789 & 0.080 & 0.01857988 \\
0.715 & 0.000 & 3.171 & 0.030 & 0.0151533 \\
0.714 & 0.000 & 3.887 & 0.017 & 0.07769112 \\
0.082 & 0.002 & 0.118 & 0.054 & 0.02116076 \\
0.746 & 0.001 & 1.507 & 0.083 & 0.02778207 \\
0.827 & 0.002 & 1.564 & 0.488 & 0.01931313 \\
0.532 & 0.002 & 0.669 & 0.122 & 0.04826522 \\
0.964 & 0.005 & 0.342 & 0.845 & 0.00267987 \\
0.442 & 0.000 & 6.445 & 0.007 & 0.6144484 \\
0.919 & 0.000 & 1.876 & 0.885 & 0.00574445 \\
0.741 & 0.001 & 1.239 & 0.278 & 0.02917976 \\
0.879 & 0.001 & 1.851 & 0.626 & 0.01014143 \\
0.821 & 0.001 & 2.574 & 0.076 & 0.04835509 \\
0.790 & 0.001 & 2.734 & 0.068 & 0.03023621 \\
0.829 & 0.001 & 1.663 & 0.382 & 0.01621975 \\
0.380 & 0.000 & 2.457 & 0.011 & 0.18732818 \\
0.736 & 0.000 & 1.746 & 0.287 & 0.02112968 \\
\hline & 0.004 & 1.892 & 0.035 & 0.02289442
\end{tabular}




\begin{tabular}{|c|c|c|c|c|}
\hline 0.377 & 0.006 & 0.135 & 0.021 & 0.00183378 \\
\hline 0.698 & 0.000 & 2.110 & 0.007 & 0.07365341 \\
\hline 0.600 & 0.000 & 2.097 & 0.012 & 0.08854419 \\
\hline 0.447 & 0.000 & 1.528 & 0.045 & 0.06274753 \\
\hline 0.636 & 0.000 & 1.217 & 0.124 & 0.05735044 \\
\hline 0.183 & 0.000 & 0.529 & 0.091 & 0.03792259 \\
\hline 0.219 & 0.000 & 0.756 & 0.091 & 0.07675744 \\
\hline 0.459 & 0.000 & 1.518 & 0.060 & 0.15726488 \\
\hline 0.100 & 0.010 & 0.090 & 0.020 & 0.02 \\
\hline 0.820 & 0.020 & 1.060 & 0.010 & 0.02 \\
\hline 0.820 & 0.610 & 0.140 & 0.500 & 0.01 \\
\hline 0.810 & 0.020 & 1.420 & 0.820 & 0.01 \\
\hline 0.280 & 0.140 & 0.290 & 0.080 & 0.01 \\
\hline 0.930 & 0.780 & 0.400 & 0.280 & 0.04 \\
\hline 0.830 & 0.780 & 0.360 & 0.120 & 0.04 \\
\hline 0.830 & 0.830 & 0.250 & 0.090 & 0.04 \\
\hline 1.020 & 0.320 & 0.210 & 0.300 & 0.01 \\
\hline 0.890 & 0.220 & 0.230 & 0.410 & 0.01 \\
\hline 0.870 & 0.880 & 0.150 & 0.140 & 0.03 \\
\hline 0.720 & 0.630 & 0.340 & 0.070 & 0.02 \\
\hline 0.800 & 0.840 & 0.270 & 0.290 & 0.05 \\
\hline 0.660 & 0.080 & 0.210 & 0.000 & 0.01 \\
\hline 0.800 & 0.860 & 0.240 & 0.120 & 0.03 \\
\hline 0.820 & 0.030 & 0.520 & 0.130 & 0.01 \\
\hline 0.409 & 0.001 & & & \\
\hline 0.258 & 0.001 & & & \\
\hline 0.567 & 0.002 & & & \\
\hline 0.448 & 0.002 & & & \\
\hline 0.359 & 0.002 & & & \\
\hline 0.486 & 0.002 & & & \\
\hline 0.092 & 0.015 & & & \\
\hline 0.485 & 0.000 & & & \\
\hline 0.425 & 0.002 & & & \\
\hline 0.635 & 0.001 & & & \\
\hline 0.208 & 0.098 & & & \\
\hline 0.100 & 0.027 & & & \\
\hline 0.283 & 0.002 & & & \\
\hline 0.608 & 0.193 & & & \\
\hline 0.505 & 0.109 & & & \\
\hline 0.653 & 0.279 & & & \\
\hline 0.659 & 0.001 & & & \\
\hline
\end{tabular}




$\begin{array}{ll}0.395 & 0.649 \\ 0.277 & 0.002 \\ 0.178 & 0.365 \\ 0.220 & 0.560 \\ 0.490 & 0.170 \\ 0.749 & 0.006 \\ 0.448 & 0.293 \\ 0.160 & 0.108 \\ 0.344 & 0.376 \\ 0.485 & 0.005 \\ 0.371 & 0.766 \\ 0.267 & 0.001 \\ 0.535 & 0.001 \\ 0.800 & 0.000 \\ 0.420 & 0.004 \\ 0.771 & 0.001 \\ 0.198 & 0.002 \\ 0.218 & 0.005 \\ 0.119 & 0.057 \\ 0.546 & 0.008 \\ 0.166 & 0.000 \\ 0.132 & 0.017 \\ 0.319 & 0.001 \\ 0.361 & 0.001 \\ 0.199 & 0.037 \\ 0.287 & 0.048 \\ 0.158 & 0.011 \\ 0.121 & 0.004 \\ 0.363 & 0.368 \\ 0.580 & 0.003 \\ 0.346 & 0.001 \\ 0.164 & 0.001 \\ 0.116 & 0.043 \\ 0.128 & 0.034 \\ 0.205 & 0.001 \\ 0.425 & 0.001 \\ 0.147 & 0.000 \\ 0.476 & 0.000 \\ 0.262 & 0.000 \\ 0.322 & 0.006 \\ & 0.002\end{array}$




$\begin{array}{ll}0.306 & 0.001 \\ 0.392 & 0.001 \\ 0.285 & 0.003 \\ 0.532 & 0.000 \\ 0.571 & 0.000 \\ 0.116 & 0.002 \\ 0.074 & 0.000 \\ 0.572 & 0.016 \\ 0.591 & 0.060 \\ 0.266 & 0.000 \\ 0.552 & 0.022 \\ 0.524 & 0.000 \\ 0.239 & 0.000 \\ 0.301 & 0.000 \\ 0.365 & 0.021 \\ 0.500 & 0.001 \\ 0.181 & 0.000 \\ 0.743 & 0.000 \\ 0.384 & 0.001 \\ 0.168 & 0.002 \\ 0.208 & 0.004 \\ 0.471 & 0.013 \\ 0.224 & 0.108 \\ 0.242 & 0.003 \\ 0.388 & 0.001 \\ 0.322 & 0.001 \\ 0.299 & 0.011 \\ 0.415 & 0.002 \\ 0.212 & 0.005 \\ 0.583 & 0.000 \\ 0.242 & 0.013 \\ 0.395 & 0.001 \\ 0.497 & 0.001 \\ 0.313 & 0.000 \\ 0.423 & 0.008 \\ 0.380 & 0.000 \\ 0.507 & 0.018 \\ 0.190 & 0.030 \\ 0.173 & 0.000 \\ 0.284 & 0.081 \\ 0.215 & 0.005\end{array}$




\begin{tabular}{|c|c|c|c|c|}
\hline 0.178 & 0.000 & & & \\
\hline 0.113 & 0.154 & & & \\
\hline 0.124 & 0.226 & & & \\
\hline 0.203 & 0.152 & & & \\
\hline 0.756 & 0.000 & & & \\
\hline 0.783 & 0.003 & & & \\
\hline 0.440 & 0.070 & & & \\
\hline 0.040 & 0.010 & & & \\
\hline 0.040 & 0.150 & & & \\
\hline 0.950 & 0.020 & & & \\
\hline 0.580 & 0.200 & & & \\
\hline 0.840 & 0.030 & & & \\
\hline 0.530 & 0.260 & & & \\
\hline 0.660 & 0.310 & & & \\
\hline 0.680 & 0.080 & & & \\
\hline 0.690 & 0.010 & & & \\
\hline 0.080 & 0.020 & & & \\
\hline 0.440 & 0.140 & & & \\
\hline 0.280 & 0.040 & & & \\
\hline 0.370 & 0.160 & & & \\
\hline 0.610 & 0.160 & & & \\
\hline 0.190 & 0.010 & & & \\
\hline 0.450 & 0.030 & & & \\
\hline 0.370 & 0.020 & & & \\
\hline 0.167 & 0.010 & 0.170 & 0.420 & 0.37 \\
\hline 0.181 & 0.030 & 0.170 & 0.410 & 0.37 \\
\hline 0.196 & 0.030 & 0.190 & 0.480 & 0.43 \\
\hline 0.200 & 0.000 & 0.390 & 0.000 & 0.04 \\
\hline 0.340 & 0.000 & 0.310 & 0.350 & 0 \\
\hline 0.580 & 0.030 & 0.270 & 0.750 & 0.01 \\
\hline 0.710 & 0.040 & 0.350 & 0.910 & 0 \\
\hline 0.870 & 0.000 & 0.480 & 2.640 & 0.07 \\
\hline 0.470 & 0.030 & 0.050 & 0.210 & 0.03 \\
\hline 0.870 & 0.030 & 0.320 & 1.660 & 0.03 \\
\hline 0.890 & 0.000 & 0.320 & 2.420 & 0.08 \\
\hline 0.690 & 0.020 & 0.280 & 0.800 & 0.02 \\
\hline 0.170 & 0.250 & 0.060 & 0.110 & 0.02 \\
\hline 0.660 & 0.010 & 0.170 & 1.050 & 0.04 \\
\hline 0.720 & 0.010 & 0.080 & 0.650 & 0 \\
\hline 0.060 & 0.140 & 0.000 & 0.030 & 0 \\
\hline 0.660 & 0.010 & 0.060 & 0.580 & 0.01 \\
\hline
\end{tabular}




$\begin{array}{llllc}0.470 & 0.390 & 0.100 & 0.320 & 0 \\ 0.790 & 0.000 & 0.020 & 0.920 & 0 \\ 0.850 & 0.000 & 0.110 & 0.920 & 0.01 \\ 0.800 & 0.010 & 0.190 & 1.230 & 0.01 \\ 0.780 & 0.000 & 0.170 & 0.730 & 0.01 \\ 0.860 & 0.030 & 0.320 & 1.680 & 0.04 \\ 0.870 & 0.000 & 0.870 & 1.840 & 0.03 \\ 0.960 & 0.000 & 0.460 & 2.410 & 0.01 \\ 0.720 & 0.070 & 0.170 & 0.760 & 0.01 \\ 0.410 & 0.160 & 0.160 & 0.480 & 0.03 \\ 0.520 & 0.200 & 0.170 & 0.540 & 0.04 \\ 0.540 & 0.120 & 0.210 & 0.550 & 0.02 \\ 0.790 & 0.040 & 0.160 & 0.550 & 0.01 \\ 0.500 & 0.110 & 0.210 & 0.600 & 0.03 \\ 0.430 & 0.090 & 0.200 & 0.560 & 0.05 \\ 0.490 & 0.090 & 0.300 & 0.630 & 0.05 \\ 0.340 & 0.010 & 0.270 & 0.410 & 0.06 \\ 0.350 & 0.030 & 0.240 & 0.330 & 0.03 \\ 0.210 & 0.010 & 0.190 & 0.420 & 0.07 \\ 0.360 & 0.120 & 0.210 & 0.630 & 0.14 \\ 0.430 & 0.070 & 0.260 & 0.900 & 0.07 \\ 0.500 & 0.180 & 0.050 & 0.300 & 0.06 \\ 0.300 & 0.110 & 0.120 & 0.650 & 0.1 \\ 0.410 & 0.100 & 0.290 & 0.890 & 0.25 \\ 0.440 & 0.560 & 0.120 & 0.350 & 0.12 \\ 0.570 & 0.090 & 0.560 & 1.280 & 0.1 \\ 0.300 & 0.070 & 0.250 & 0.590 & 0.11 \\ 0.350 & 0.160 & 0.300 & 0.650 & 0.1 \\ 0.330 & 0.300 & 0.220 & 0.520 & 0.14 \\ 0.400 & 0.190 & 0.330 & 0.770 & 0.3 \\ 0.380 & 0.360 & 0.210 & 0.390 & 0.13 \\ 0.670 & 0.170 & 0.560 & 1.530 & 0.17 \\ 0.450 & 0.200 & 0.340 & 0.680 & 0.14 \\ 0.890 & 0.110 & 0.150 & 1.580 & 0.12 \\ 0.390 & 0.110 & 0.330 & 0.500 & 0.1 \\ 0.740 & 0.080 & 0.770 & 1.790 & 0.12 \\ 0.290 & 0.020 & 0.310 & 0.540 & 0.13 \\ 0.460 & 0.310 & 0.290 & 0.800 & 0.27 \\ 0.250 & 0.010 & 0.330 & 0.490 & 0.09 \\ 0.550 & 0.530 & 0.170 & 0.520 & 0.1 \\ 0.290 & 0.060 & 0.360 & 0.620 & 0.16\end{array}$




$\begin{array}{lllll}0.940 & 0.330 & 0.340 & 1.070 & 0.03 \\ 0.520 & 0.120 & 0.280 & 0.190 & 0.04 \\ 0.260 & 0.010 & 0.360 & 0.560 & 0.15 \\ 0.490 & 0.130 & 0.410 & 0.520 & 0.1 \\ 0.320 & 0.120 & 0.270 & 0.580 & 0.18 \\ 0.280 & 0.100 & 0.260 & 0.490 & 0.17 \\ 0.750 & 0.000 & 0.480 & 0.720 & 0.02 \\ 0.360 & 0.060 & 0.280 & 0.750 & 0.19 \\ 0.310 & 0.060 & 0.320 & 0.540 & 0.28 \\ 0.340 & 0.050 & 0.290 & 0.670 & 0.28 \\ 0.340 & 0.060 & 0.350 & 0.680 & 0.24 \\ 0.320 & 0.040 & 0.280 & 0.790 & 0.26 \\ 0.320 & 0.040 & 0.290 & 0.640 & 0.3 \\ 0.340 & 0.140 & 0.340 & 0.630 & 0.28 \\ 0.300 & 0.030 & 0.290 & 0.540 & 0.24 \\ 0.320 & 0.140 & 0.300 & 0.500 & 0.24 \\ 0.460 & 0.230 & 0.390 & 0.850 & 0.21 \\ 0.330 & 0.060 & 0.380 & 0.720 & 0.16 \\ 0.470 & 0.070 & 0.400 & 1.050 & 0.24 \\ 0.460 & 0.360 & 0.260 & 0.730 & 0.23 \\ 0.460 & 0.380 & 0.220 & 0.740 & 0.21 \\ 0.500 & 0.440 & 0.250 & 0.590 & 0.16 \\ 0.540 & 0.600 & 0.250 & 0.540 & 0.12 \\ 0.520 & 0.620 & 0.190 & 0.510 & 0.14 \\ 0.480 & 0.480 & 0.310 & 0.670 & 0.2 \\ 0.500 & 0.650 & 0.190 & 0.420 & 0.14 \\ 0.500 & 0.570 & 0.210 & 0.510 & 0.11 \\ 0.620 & 0.440 & 0.400 & 0.860 & 0.09 \\ 0.660 & 0.360 & 0.440 & 1.000 & 0.12 \\ 0.580 & 0.630 & 0.240 & 0.630 & 0.13 \\ 0.790 & 0.370 & 0.520 & 1.300 & 0.08 \\ 0.420 & 0.390 & 0.240 & 0.780 & \\ 0.310 & 0.300 & 0.180 & 0.450 & 0.06 \\ 0.440 & 0.240 & 0.300 & 0.660 & 0.04 \\ 0.510 & 0.310 & 0.410 & 0.930 & 0.08 \\ 0.370 & 0.150 & 0.380 & 0.720 & 0.08 \\ 0.320 & 0.230 & 0.330 & 0.560 & 0.18 \\ 0.330 & 0.360 & 0.290 & 0.510 & 0.15 \\ 0.360 & 0.120 & 0.360 & 0.760 & 0.17 \\ 0.460 & 0.390 & 0.320 & 0.750 & 0.15 \\ 0.340 & 0.240 & 0.300 & 0.600 & 0.23\end{array}$




$\begin{array}{llllc}0.370 & 0.400 & 0.270 & 0.520 & 0.24 \\ 0.380 & 0.260 & 0.310 & 0.630 & 0.21 \\ 0.510 & 0.370 & 0.300 & 0.830 & 0.13 \\ 0.400 & 0.400 & 0.310 & 0.570 & 0.17 \\ 0.360 & 0.430 & 0.240 & 0.440 & 0.18 \\ 0.540 & 0.570 & 0.200 & 0.400 & 0.11 \\ 0.500 & 0.650 & 0.200 & 0.390 & 0.11 \\ 0.540 & 0.670 & 0.150 & 0.560 & 0.11 \\ 0.790 & 0.360 & 0.410 & 1.460 & 0.09 \\ 0.750 & 0.450 & 0.280 & 0.970 & 0.05 \\ 0.229 & 0.010 & 0.856 & 0.299 & 0 \\ 0.317 & 0.050 & 0.613 & 0.139 & 0.326 \\ 0.417 & 0.290 & 0.230 & 0.444 & 0.41 \\ 0.425 & 0.030 & 0.386 & 1.389 & 0.142 \\ 0.457 & 0.070 & 0.695 & 0.434 & 0.253 \\ 0.481 & 0.000 & 1.127 & 0.386 & 0.362 \\ 0.578 & 0.010 & 1.575 & 0.451 & 0.293 \\ 0.595 & 0.010 & 0.604 & 0.837 & 0.765 \\ 0.659 & 0.320 & 0.514 & 0.880 & 0.13 \\ 0.737 & 0.729 & 0.040 & 0.140 & 0 \\ 0.725 & 0.569 & 0.100 & 0.130 & 0 \\ 0.146 & 0.085 & 0.500 & 0.710 & 0.29 \\ 0.310 & 0.070 & 0.240 & 0.890 & 0.17 \\ 0.316 & 0.135 & 0.680 & 0.770 & 1.3 \\ 0.341 & 0.011 & 0.820 & 0.340 & 1.54 \\ 0.307 & 0.013 & 0.240 & 0.710 & 0.59 \\ 0.366 & 0.083 & 0.880 & 0.710 & 1.48 \\ 0.278 & 0.096 & 0.300 & 0.510 & 0.78 \\ 0.318 & 0.158 & 1.240 & 0.470 & 0.89 \\ 0.258 & 0.123 & 0.220 & 0.910 & 0.57 \\ 0.272 & 0.102 & 0.230 & 0.500 & 0.94 \\ 0.305 & 0.078 & 0.280 & 1.270 & 0.69 \\ 0.308 & 0.065 & 0.230 & 1.230 & 0.55 \\ 0.114 & 0.000 & 0.220 & 0.150 & 0.1 \\ 0.064 & 0.000 & 0.070 & 0.020 & 0.01 \\ 0.829 & 0.010 & 0.950 & 0.020 & 0.03 \\ 0.143 & 0.000 & 0.120 & 0.250 & 0.24 \\ 0.155 & 0.000 & 0.060 & 0.210 & 0.2 \\ 0.210 & 0.122 & 0.170 & 0.320 & 0.3 \\ 0.240 & 0.020 & 0.340 & 0.100 & 0.1 \\ 0.146 & 0.024 & 0.090 & 0.150 & 0.16\end{array}$




$\begin{array}{llllc}0.182 & 0.000 & 0.240 & 0.180 & 0.16 \\ 0.349 & 0.014 & 0.420 & 0.230 & 0.06 \\ 0.137 & 0.000 & 0.110 & 0.310 & 0.29 \\ 0.188 & 0.098 & 0.170 & 0.110 & 0.07 \\ 0.197 & 0.000 & 0.380 & 0.130 & 0.1 \\ 0.176 & 0.000 & 0.220 & 0.170 & 0.19 \\ 0.061 & 0.060 & -0.013 & 0.098 & 0.074 \\ 0.101 & 0.020 & 0.079 & 0.067 & 0.045 \\ 0.171 & 0.030 & 0.209 & 0.493 & 0.654 \\ 0.207 & 0.050 & 0.206 & 0.676 & 0.71 \\ 0.219 & 0.180 & 0.313 & 0.434 & 0.727 \\ 0.248 & 0.030 & 0.484 & 0.579 & 0.995 \\ 0.267 & 0.500 & 0.317 & 0.452 & 0.256 \\ 0.269 & 0.120 & 0.668 & 0.576 & 0.932 \\ 0.284 & 0.240 & 0.238 & 0.760 & 0.661 \\ 0.297 & 0.010 & 0.871 & 0.407 & 1.157 \\ 0.298 & 0.070 & 1.043 & & 1.293 \\ 0.314 & 0.230 & 0.542 & 0.721 & 1.023 \\ 0.368 & 0.520 & 0.380 & 0.566 & 0.311 \\ 0.368 & 0.070 & 1.261 & 0.547 & 0.982 \\ 0.526 & 0.410 & 0.827 & 0.658 & 0.517 \\ 0.528 & 0.030 & 2.243 & 0.671 & 0.55 \\ 0.224 & 0.333 & 0.202 & 0.570 & 0.44773734 \\ 0.158 & 0.129 & 0.192 & 0.465 & 0.32402795 \\ 0.265 & 0.376 & 0.350 & 0.463 & 0.38782002 \\ 0.274 & 0.391 & 0.267 & 0.553 & 0.46656461 \\ 0.281 & 0.533 & 0.167 & 0.499 & 0.29513504 \\ 0.362 & 0.648 & 0.189 & 0.456 & 0.20737529 \\ 0.761 & 0.166 & 0.166 & 1.926 & 0.06017646 \\ 0.685 & 0.001 & 3.365 & 0.049 & 0.06479611 \\ 0.265 & 0.002 & 0.804 & 0.031 & 0.0269004 \\ 0.813 & 0.002 & 2.324 & 0.063 & 0.02091328 \\ 0.364 & 0.003 & 0.958 & 0.028 & 0.02277646 \\ 0.366 & 0.054 & 0.470 & 0.029 & 0.01983014 \\ 0.276 & 0.005 & 0.588 & 0.037 & 0.04732432 \\ 0.256 & 0.007 & 0.547 & 0.122 & 0.14922865 \\ 0.226 & 0.014 & 0.459 & 0.124 & 0.12253183 \\ 0.173 & 0.048 & 0.288 & 0.153 & 0.09798687 \\ 0.194 & 0.015 & 0.399 & 0.128 & 0.10201442 \\ 0.216 & 0.012 & 0.470 & 0.141 & 0.11916305 \\ 0.186 & 0.012 & 0.376 & 0.147 & 0.09987131\end{array}$




$\begin{array}{lllll}0.159 & 0.040 & 0.265 & 0.159 & 0.07883441 \\ 0.167 & 0.008 & 0.333 & 0.077 & 0.06453526 \\ 0.273 & 0.005 & 0.631 & 0.162 & 0.23694098 \\ 0.136 & 0.077 & 0.187 & 0.152 & 0.08628889 \\ 0.159 & 0.002 & 0.297 & 0.129 & 0.09276898 \\ 0.509 & 0.072 & 0.673 & 0.096 & 0.01651511 \\ 0.879 & 0.015 & 0.350 & 0.246 & 0.00177477 \\ 0.114 & 0.015 & 0.095 & 0.006 & 0.02269034 \\ 0.149 & 0.047 & 0.143 & 0.009 & 0.018 \\ 0.064 & 0.000 & 0.059 & 0.008 & 0.034 \\ 0.152 & 0.000 & 0.235 & 0.015 & 0.00321213 \\ 0.162 & 0.018 & 0.383 & 0.060 & 0.06139507 \\ 0.140 & 0.000 & 0.267 & 0.037 & 0.09423412 \\ 0.227 & 0.000 & 0.497 & 0.115 & 0.34205477 \\ 0.259 & 0.000 & 0.587 & 0.061 & 0.33322473 \\ 0.236 & 0.000 & 0.541 & 0.053 & 0.26869447 \\ 0.410 & 0.000 & 1.344 & 0.092 & 0.38080745 \\ 0.226 & 0.000 & 0.355 & 0.083 & 0.17945883 \\ 0.279 & 0.000 & 0.570 & 0.159 & 0.27563119 \\ 0.427 & 0.003 & 0.505 & 0.082 & 0.08175592 \\ 0.229 & 0.000 & 0.503 & 0.042 & 0.14973952 \\ 0.214 & 0.000 & 0.756 & 0.070 & 0.46207835 \\ 0.306 & 0.000 & 0.949 & 0.081 & 0.23235784 \\ 0.486 & 0.001 & 1.243 & 0.138 & 0.18683932 \\ 0.233 & 0.000 & 0.423 & 0.063 & 0.28668378 \\ 0.362 & 0.000 & 0.692 & 0.068 & 0.08691797 \\ 0.229 & 0.000 & 0.374 & 0.105 & 0.2733657 \\ 0.210 & 0.000 & 0.455 & 0.079 & 0.26657156 \\ 0.164 & 0.000 & 0.222 & 0.074 & 0.10786436 \\ 0.471 & 0.000 & 2.740 & 0.057 & 0.41587459 \\ 0.180 & 0.000 & 0.336 & 0.060 & 0.0936029 \\ 0.292 & 0.000 & 0.406 & 0.022 & 0.02340961 \\ 0.698 & 0.038 & 3.010 & 0.056 & 0.09567419 \\ 0.176 & 0.032 & 0.279 & 0.011 & 0.01535835 \\ 0.552 & 0.001 & 1.556 & 0.036 & 0.0271345 \\ 0.768 & 0.000 & 4.680 & 0.011 & 0.12935716 \\ 0.740 & 0.553 & 0.285 & 0.172 & 0.01933866 \\ 0.407 & 0.008 & 0.488 & 0.023 & 0.02153906 \\ 0.232 & 0.001 & 0.569 & 0.071 & 0.04410754 \\ 0.245 & 0.001 & 0.475 & 0.084 & 0.03012459 \\ & 0.002 & 0.474 & 0.047 & 0.0521288\end{array}$




\begin{tabular}{|c|c|c|c|c|}
\hline 0.196 & 0.001 & 0.574 & 0.143 & 0.0706454 \\
\hline 0.117 & 0.000 & 0.245 & 0.141 & 0.17733271 \\
\hline 0.226 & 0.000 & 0.735 & 0.189 & 0.24101087 \\
\hline 0.164 & 0.000 & 0.164 & 0.117 & 0.02855782 \\
\hline 0.122 & 0.009 & 0.098 & 0.083 & 0.07998692 \\
\hline 0.264 & 0.002 & 0.549 & 0.050 & 0.16979207 \\
\hline 0.316 & 0.002 & 0.654 & 0.046 & 0.21586194 \\
\hline 0.235 & 0.006 & 0.421 & 0.084 & 0.1286372 \\
\hline 0.367 & 0.062 & 0.811 & 0.107 & 0.09467596 \\
\hline 0.311 & 0.012 & 0.459 & 0.113 & 0.18900625 \\
\hline 0.141 & 0.005 & 0.091 & 0.120 & 0.15169832 \\
\hline 0.466 & 0.009 & 0.576 & 0.052 & 0.07448483 \\
\hline 0.332 & 0.007 & 0.299 & 0.217 & 0.10605215 \\
\hline 0.224 & 0.006 & 0.424 & 0.021 & 0.05200568 \\
\hline 0.249 & 0.019 & 0.333 & 0.094 & 0.12021293 \\
\hline 0.759 & 0.001 & 1.440 & 0.126 & 0.13124223 \\
\hline 0.359 & 0.000 & 0.504 & 0.074 & 0.07990601 \\
\hline 0.485 & 0.100 & 0.122 & 0.116 & 0.00183915 \\
\hline 0.895 & 0.010 & 0.674 & 0.026 & 0.00105331 \\
\hline 0.363 & 0.002 & 0.521 & 0.011 & 0.0330488 \\
\hline 0.265 & 0.004 & 0.479 & 0.068 & 0.04160235 \\
\hline 0.182 & 0.004 & 0.254 & 0.034 & 0.03767832 \\
\hline 0.510 & 0.000 & 1.842 & 0.067 & 0.13081785 \\
\hline 0.208 & 0.002 & 0.331 & 0.089 & 0.08268633 \\
\hline 0.185 & 0.000 & 0.340 & 0.059 & 0.0683641 \\
\hline 0.291 & 0.001 & 0.419 & 0.158 & 0.0695502 \\
\hline 0.642 & 0.000 & 1.678 & 0.093 & 0.10980976 \\
\hline 0.646 & 0.000 & 1.540 & 0.064 & 0.01766553 \\
\hline 0.209 & -0.003 & 0.292 & 0.104 & 0.06252007 \\
\hline 0.255 & 0.000 & 0.264 & 0.040 & 0.01270792 \\
\hline 0.298 & N.D. & 0.153 & 0.063 & 0.004 \\
\hline 0.443 & N.D. & 0.547 & 0.029 & 0 \\
\hline 0.134 & N.D. & 0.483 & 0.223 & 0 \\
\hline 0.335 & 0.005 & 0.688 & 0.071 & 0.01790173 \\
\hline 0.808 & 0.027 & 0.821 & 0.048 & 0.01213492 \\
\hline 0.319 & 0.043 & 0.286 & 0.024 & 0.01848058 \\
\hline 0.444 & 0.003 & 0.355 & 0.042 & 0.00169724 \\
\hline 0.676 & 0.012 & 0.448 & 0.658 & 0.00257178 \\
\hline 0.806 & 0.019 & 1.032 & 0.104 & 0.00264363 \\
\hline 0.687 & 0.013 & 1.023 & 0.088 & 0.00740604 \\
\hline 0.765 & 0.111 & 0.083 & 0.006 & -0.0002779 \\
\hline
\end{tabular}




$\begin{array}{lllll}0.693 & 0.013 & 0.564 & 0.021 & 0.00356571 \\ 0.809 & 0.007 & 0.811 & 0.012 & 0.01293441 \\ 0.822 & 0.062 & 0.219 & 0.020 & -0.0002994 \\ 0.793 & 0.048 & 0.301 & 0.075 & 0.00110545 \\ 0.612 & 0.003 & 1.023 & 0.039 & 0.01782529 \\ 0.741 & 0.019 & 0.738 & 0.110 & 0.01000602 \\ 0.285 & 0.018 & 0.238 & 0.506 & 0.40234834 \\ 0.436 & 0.001 & 0.175 & 0.865 & 0.32338902 \\ 0.245 & 0.005 & 0.338 & 0.437 & 0.23937677 \\ 0.407 & 0.002 & 0.294 & 0.592 & 0.41182171 \\ 0.400 & 0.006 & 0.287 & 0.756 & 0.43819302 \\ 0.307 & 0.029 & 0.255 & 0.345 & 0.25738295 \\ 0.392 & 0.002 & 0.267 & 0.692 & 0.37329317 \\ 0.417 & 0.007 & 0.265 & 0.604 & 0.3609816 \\ 0.412 & 0.001 & 0.281 & 0.605 & 0.52154195 \\ 0.485 & 0.002 & 0.242 & 0.820 & 0.61470588 \\ 0.509 & 0.017 & 0.294 & 0.743 & 0.58019663 \\ 0.601 & 0.026 & 0.365 & 0.282 & 0.33308958 \\ 0.266 & 0.041 & 0.305 & 0.293 & 0.26598115 \\ 0.309 & 0.074 & 0.374 & 0.307 & 0.25575758 \\ 0.367 & 0.000 & 0.327 & 0.386 & 0.37104506 \\ 0.500 & 0.005 & 0.594 & 0.418 & 0.20969044 \\ 0.442 & 0.007 & 0.341 & 0.671 & 0.60553633 \\ 0.491 & 0.001 & 0.229 & 0.792 & 0.5326969 \\ 0.489 & 0.000 & 0.244 & 0.696 & 0.59674641 \\ 0.381 & 0.022 & 0.146 & 0.424 & 0.26767677 \\ 0.404 & 0.005 & 0.244 & 0.502 & 0.20708447 \\ 0.391 & 0.001 & 0.123 & 0.376 & 0.18105516 \\ 0.759 & 0.091 & 2.634 & 0.045 & 0.0176709 \\ 0.696 & 0.029 & 2.002 & 0.082 & 0.03203295 \\ 0.489 & 0.203 & 0.398 & 0.083 & 0.04105096 \\ 0.499 & 0.417 & 0.212 & 0.077 & 0.0045593 \\ 0.281 & 0.338 & 0.134 & 0.054 & 0.00221753 \\ 0.735 & 0.730 & 0.190 & 0.239 & 0.00908698 \\ 0.997 & 0.519 & 0.115 & 0.153 & 0.00088435 \\ 0.322 & 0.188 & 0.220 & 0.055 & 0.00223366 \\ 0.697 & 0.070 & 1.573 & 0.168 & 0.01414735 \\ 0.495 & 0.042 & 1.083 & 0.062 & 0.02178088 \\ 0.544 & 0.651 & 0.162 & 0.105 & 0.00229131 \\ 0.534 & 0.218 & 0.422 & 0.155 & 0.00391045 \\ 0.012 & 0.971 & 0.045 & 0.00290804\end{array}$




\begin{tabular}{|c|c|c|c|c|}
\hline 0.488 & 0.047 & 1.127 & 0.045 & 0.02199386 \\
\hline 0.337 & 0.105 & 0.612 & 0.052 & 0.01483794 \\
\hline 0.200 & 0.200 & 0.214 & 0.091 & 0.01520289 \\
\hline 0.451 & 0.028 & 0.585 & 0.079 & 0.00235516 \\
\hline 0.463 & 0.114 & 0.547 & 0.074 & 0.00258703 \\
\hline 0.603 & 0.009 & 0.951 & 0.068 & 0.00147674 \\
\hline 0.824 & 0.011 & 1.593 & 0.263 & 0.00286723 \\
\hline 0.737 & 0.023 & 0.312 & 0.486 & 0.00098405 \\
\hline 0.488 & 0.085 & 0.202 & 0.039 & 0.00052145 \\
\hline 0.543 & 0.366 & 0.224 & 0.047 & 0.0017625 \\
\hline 0.705 & 0.332 & 0.315 & 0.571 & $3.56 \mathrm{E}-05$ \\
\hline 0.789 & 0.053 & 0.410 & 0.046 & 0 \\
\hline 0.613 & 0.102 & 0.212 & 0.012 & 0.00131085 \\
\hline 0.826 & 0.003 & 3.135 & 0.082 & 0.01243074 \\
\hline 0.773 & 0.005 & 2.027 & 0.090 & 0.11306861 \\
\hline 0.800 & 0.000 & 3.437 & 0.143 & 0.03407913 \\
\hline 0.711 & 0.004 & 1.579 & 0.172 & 0.19028583 \\
\hline 0.784 & 0.011 & 1.805 & 0.236 & 0.0227028 \\
\hline 0.725 & 0.009 & 1.482 & 0.094 & 0.0341772 \\
\hline 0.825 & 0.003 & 1.632 & 0.074 & 0.01507142 \\
\hline 0.271 & 0.102 & 0.300 & 0.075 & 0.03484904 \\
\hline 0.220 & 0.000 & 0.770 & 0.079 & 0.25313966 \\
\hline 0.244 & 0.000 & 0.732 & 0.086 & 0.20590037 \\
\hline 0.283 & 0.000 & 0.972 & 0.053 & 0.15543488 \\
\hline 0.405 & 0.000 & 1.050 & 0.156 & 0.38914367 \\
\hline 0.290 & 0.000 & 0.823 & 0.048 & 0.10751303 \\
\hline 0.556 & 0.000 & 1.390 & 0.077 & 0.04041093 \\
\hline 0.222 & 0.000 & 0.480 & 0.154 & 0.24650035 \\
\hline 0.219 & 0.000 & 0.814 & 0.155 & 0.49489217 \\
\hline 0.211 & 0.220 & 0.094 & 0.044 & 0.00671336 \\
\hline 0.219 & 0.401 & 0.083 & 0.022 & 0.00617016 \\
\hline 0.308 & 0.084 & 0.261 & 0.043 & 0.02428988 \\
\hline 0.192 & 0.357 & 0.066 & 0.026 & 0.00373487 \\
\hline 0.213 & 0.256 & 0.086 & 0.036 & 0.0129853 \\
\hline 0.227 & 0.203 & 0.138 & 0.033 & 0.00423407 \\
\hline 0.392 & 0.195 & 0.325 & 0.053 & 0.00721445 \\
\hline 0.202 & 0.238 & 0.095 & 0.026 & 0.0095545 \\
\hline 0.431 & 0.613 & 0.111 & 0.032 & 0.00539782 \\
\hline 0.525 & 0.479 & 0.202 & 0.038 & 0.00856269 \\
\hline 0.138 & 0.067 & 0.089 & 0.012 & 0.01456959 \\
\hline 0.142 & 0.001 & 0.366 & 0.151 & 0.12551647 \\
\hline
\end{tabular}




\begin{tabular}{|c|c|c|c|c|}
\hline 0.192 & 0.100 & 0.138 & 0.055 & 0.00859256 \\
\hline 0.168 & 0.010 & 0.249 & 0.160 & 0.03442171 \\
\hline 0.366 & 0.605 & 0.106 & 0.026 & 0.01393281 \\
\hline 0.222 & 0.009 & 0.204 & 0.037 & 0.00328208 \\
\hline 0.225 & 0.369 & 0.065 & 0.035 & 0.00514808 \\
\hline 0.752 & 0.027 & 0.243 & 0.088 & 0.02096329 \\
\hline 0.879 & 0.047 & 0.540 & 0.075 & 0.0465305 \\
\hline 0.640 & 0.086 & 0.239 & 0.083 & 0.02937274 \\
\hline 0.595 & 0.021 & 0.710 & 0.103 & 0.03818482 \\
\hline 0.647 & 0.019 & 0.412 & 0.058 & 0.01177109 \\
\hline 0.743 & 0.020 & 0.365 & 0.103 & 0.00388878 \\
\hline 0.177 & 0.003 & 0.449 & 0.120 & 0.34575821 \\
\hline 0.181 & 0.001 & 0.342 & 0.148 & 0.32972611 \\
\hline 0.292 & 0.001 & 0.786 & 0.303 & 0.35034152 \\
\hline 0.196 & 0.001 & 0.438 & 0.263 & 0.22959783 \\
\hline 0.245 & 0.000 & 0.311 & 0.017 & 0.01822688 \\
\hline 0.363 & 0.002 & 0.681 & 0.042 & 0.07610279 \\
\hline 0.386 & 0.001 & 0.847 & 0.071 & 0.23557454 \\
\hline 0.135 & 0.002 & 0.193 & 0.102 & 0.22005236 \\
\hline 0.234 & 0.006 & 0.210 & 0.038 & 0.07713181 \\
\hline 0.200 & 0.003 & 0.466 & 0.148 & 0.37775451 \\
\hline 0.179 & 0.002 & 0.370 & 0.074 & 0.13386058 \\
\hline 0.469 & 0.001 & 1.246 & 0.126 & 0.08476112 \\
\hline 0.148 & 0.003 & 0.242 & 0.071 & 0.06542292 \\
\hline 0.107 & 0.006 & 0.237 & 0.079 & 0.11472586 \\
\hline 0.260 & 0.004 & 0.679 & 0.082 & 0.28590475 \\
\hline 0.209 & 0.002 & 0.297 & 0.106 & 0.06571926 \\
\hline 0.199 & 0.017 & 0.372 & 0.062 & 0.07809659 \\
\hline 0.368 & 0.002 & 1.048 & 0.070 & 0.15910262 \\
\hline 0.295 & 0.001 & 0.909 & 0.090 & 0.25505879 \\
\hline 0.278 & 0.008 & 0.412 & 0.048 & 0.03531957 \\
\hline 0.716 & 0.001 & 3.406 & 0.173 & 0.03476241 \\
\hline 0.244 & 0.006 & 0.199 & 0.066 & 0.01342698 \\
\hline 0.245 & 0.003 & 0.295 & 0.104 & 0.04282104 \\
\hline 0.183 & 0.003 & 0.347 & 0.080 & 0.05948453 \\
\hline 0.355 & 0.004 & 0.713 & 0.111 & 0.04400855 \\
\hline 0.241 & 0.169 & 0.108 & 0.015 & 0.00106918 \\
\hline 0.278 & 0.036 & 0.311 & 0.047 & 0.03229079 \\
\hline 0.385 & 0.287 & 0.203 & 0.021 & 0.00953212 \\
\hline 0.573 & 0.011 & 0.860 & 0.031 & 0.02145496 \\
\hline 0.679 & 0.339 & 0.459 & 0.032 & 0.00769959 \\
\hline
\end{tabular}




\begin{tabular}{|c|c|c|c|c|}
\hline 0.333 & 0.405 & 0.088 & 0.069 & 0.00582754 \\
\hline 0.461 & 0.316 & 0.286 & 0.025 & 0.01023092 \\
\hline 0.438 & 0.122 & 0.431 & 0.034 & 0.01619987 \\
\hline 0.452 & 0.003 & 0.653 & 0.424 & 0.10806035 \\
\hline 0.532 & 0.177 & 0.527 & 0.031 & 0.00630844 \\
\hline 0.415 & 0.349 & 0.246 & 0.045 & 0.00629869 \\
\hline 0.680 & 0.506 & 0.260 & 0.026 & 0.00267448 \\
\hline 0.886 & 0.006 & 3.051 & 0.031 & 0.02682745 \\
\hline 0.774 & 0.009 & 2.264 & 0.048 & 0.48663011 \\
\hline 0.591 & 0.018 & 2.189 & 0.130 & 0.15936132 \\
\hline 0.416 & 0.035 & 1.847 & 0.119 & 0.48424353 \\
\hline 0.207 & 0.000 & 0.341 & 0.007 & 0.01654711 \\
\hline 0.230 & 0.000 & 0.498 & 0.046 & 0.08232734 \\
\hline 0.754 & 0.000 & 4.173 & 0.015 & 0.1032057 \\
\hline 0.444 & 0.000 & 1.480 & 0.027 & 0.02576485 \\
\hline 0.395 & 0.000 & 1.562 & 0.015 & 0.07017769 \\
\hline 0.710 & 0.280 & 0.280 & 1.030 & 0 \\
\hline 0.480 & 0.460 & 0.360 & 1.620 & 0.12 \\
\hline 0.250 & 0.010 & 0.210 & 2.000 & 0.44 \\
\hline 0.300 & 0.030 & 0.110 & 1.670 & 0.41 \\
\hline 0.290 & 0.010 & 0.070 & 1.880 & 0.46 \\
\hline 0.260 & 0.000 & 0.090 & 1.710 & 0.42 \\
\hline 0.310 & 0.030 & 0.080 & 1.860 & 0.42 \\
\hline 0.300 & 0.020 & 0.070 & 1.770 & 0.42 \\
\hline 0.270 & 0.030 & 0.080 & 2.290 & 0.54 \\
\hline 0.280 & 0.010 & 0.240 & 1.930 & 0.45 \\
\hline 0.310 & 0.010 & 0.090 & 2.210 & 0.63 \\
\hline 0.260 & 0.000 & 0.020 & 0.890 & 0.1 \\
\hline 0.250 & 0.000 & 0.070 & 1.790 & 0.33 \\
\hline 0.320 & 0.020 & 0.070 & 2.100 & 0.42 \\
\hline 0.360 & 0.030 & 0.150 & 1.910 & 0.34 \\
\hline 0.350 & 0.010 & 0.080 & 1.950 & 0.44 \\
\hline 0.360 & 0.030 & 0.090 & 2.460 & 0.56 \\
\hline 0.370 & 0.050 & 0.090 & 2.800 & 0.77 \\
\hline 0.370 & 0.040 & 0.170 & 2.180 & 0.58 \\
\hline 0.789 & 0.162 & 0.815 & 0.019 & 0.00560541 \\
\hline 0.495 & 0.054 & 0.839 & 0.019 & 0.02202636 \\
\hline 0.076 & 0.413 & 0.022 & 0.020 & 0.0124425 \\
\hline 0.772 & 0.001 & 1.740 & 0.006 & 0.0120823 \\
\hline 0.421 & 0.001 & 1.323 & 0.078 & 0.03496138 \\
\hline 0.394 & 0.004 & 0.499 & 0.050 & 0.09117771 \\
\hline
\end{tabular}




\begin{tabular}{|c|c|c|c|c|}
\hline 0.551 & 0.018 & 1.340 & 0.022 & 0.0452888 \\
\hline 0.262 & 0.003 & 1.035 & 0.084 & 0.06334358 \\
\hline 0.148 & 0.009 & 0.551 & 0.118 & 0.05960178 \\
\hline 0.137 & 0.013 & 0.732 & 0.109 & 0.06254762 \\
\hline 0.072 & 0.130 & 0.135 & 0.197 & 0.07950784 \\
\hline 0.172 & 0.007 & 0.625 & 0.079 & 0.08165501 \\
\hline 0.099 & 0.007 & 0.350 & 0.154 & 0.08009402 \\
\hline 0.295 & 0.009 & 0.673 & 0.026 & 0.05774104 \\
\hline 0.090 & 0.010 & 0.288 & 0.077 & 0.04642994 \\
\hline 0.208 & 0.008 & 0.384 & 0.064 & 0.03920772 \\
\hline 0.154 & 0.007 & 0.265 & 0.021 & 0.02925627 \\
\hline 0.192 & 0.001 & 1.003 & 0.135 & 0.06305598 \\
\hline 0.081 & 0.003 & 0.144 & 0.023 & 0.02527092 \\
\hline 0.302 & 0.005 & 0.392 & 0.073 & 0.02850984 \\
\hline 0.299 & 0.001 & 0.871 & 0.034 & 0.04151911 \\
\hline 0.231 & 0.003 & 0.790 & 0.114 & 0.02845162 \\
\hline 0.135 & 0.006 & 0.389 & 0.039 & 0.04478603 \\
\hline 0.190 & 0.001 & 0.952 & 0.065 & 0.03352821 \\
\hline 0.053 & 0.009 & 0.113 & 0.059 & 0.03060199 \\
\hline 0.278 & 0.000 & 1.313 & 0.062 & 0.0628172 \\
\hline 0.138 & 0.010 & 0.751 & 0.100 & 0.05558835 \\
\hline 0.326 & 0.019 & 1.179 & 0.181 & 0.03425733 \\
\hline 0.147 & 0.019 & 0.259 & 0.453 & 0.05008354 \\
\hline 0.261 & 0.026 & 1.158 & 0.131 & 0.03830926 \\
\hline 0.157 & 0.051 & 0.562 & 0.233 & 0.05102368 \\
\hline 0.109 & 0.003 & 0.430 & 0.091 & 0.03706432 \\
\hline 0.053 & 0.019 & 0.185 & 0.115 & 0.05622804 \\
\hline 0.303 & 0.041 & 1.015 & 0.080 & 0.04659925 \\
\hline 0.168 & 0.098 & 0.478 & 0.083 & 0.04216075 \\
\hline 0.113 & 0.012 & 0.109 & 0.007 & 0.0057816 \\
\hline 0.116 & 0.019 & 0.219 & 0.115 & 0.11781844 \\
\hline 0.149 & 0.030 & 0.487 & 0.108 & 0.06492149 \\
\hline 0.214 & 0.001 & 0.742 & 0.101 & 0.0708137 \\
\hline 0.307 & 0.003 & 0.500 & 0.019 & 0.01605082 \\
\hline 0.232 & 0.000 & 0.525 & 0.185 & 0.30103448 \\
\hline 0.265 & 0.002 & 0.563 & 0.083 & 0.23637931 \\
\hline 0.265 & 0.002 & 0.584 & 0.041 & 0.06482759 \\
\hline 0.246 & 0.035 & 0.535 & 0.047 & 0.16655172 \\
\hline 0.555 & 0.000 & 1.585 & 0.043 & 0.23465517 \\
\hline 0.341 & 0.001 & 0.609 & 0.094 & 0.16568966 \\
\hline 0.341 & 0.001 & 0.616 & 0.061 & 0.22948276 \\
\hline
\end{tabular}




\begin{tabular}{|c|c|c|c|c|}
\hline 0.318 & 0.016 & 0.536 & 0.057 & 0.16568966 \\
\hline 0.386 & 0.000 & 0.635 & 0.045 & 0.1312069 \\
\hline 0.467 & 0.000 & 0.807 & 0.029 & 0.22431034 \\
\hline 0.436 & 0.001 & 0.734 & 0.125 & 0.02086207 \\
\hline 0.381 & 0.000 & 1.199 & 0.082 & 0.42948276 \\
\hline 0.265 & 0.004 & 0.582 & 0.060 & 0.23293103 \\
\hline 0.441 & 0.003 & 1.071 & 0.114 & 0.17344828 \\
\hline 0.505 & 0.000 & 1.420 & 0.072 & 0.17517241 \\
\hline 0.533 & 0.000 & 1.664 & 0.024 & 0.10362069 \\
\hline 0.316 & 0.001 & 0.808 & 0.051 & 0.39068966 \\
\hline 0.309 & 0.003 & 0.696 & 0.029 & 0.1087931 \\
\hline 0.307 & 0.000 & 0.718 & 0.034 & 0.13896552 \\
\hline 0.626 & 0.000 & 2.295 & 0.052 & 0.51913793 \\
\hline 0.061 & 0.005 & 0.037 & 0.017 & 0.07431034 \\
\hline 0.163 & 0.016 & 0.227 & 0.023 & 0.0937069 \\
\hline 0.288 & 0.001 & 0.424 & 0.019 & 0.05965517 \\
\hline 0.124 & 0.003 & 0.197 & 0.021 & 0.07517241 \\
\hline 0.139 & 0.000 & 0.147 & 0.030 & 0.07603448 \\
\hline 0.142 & 0.000 & 0.249 & 0.019 & 0.06396552 \\
\hline 0.110 & 0.000 & 0.150 & 0.021 & 0.06252874 \\
\hline 0.176 & 0.003 & 0.273 & 0.022 & 0.05189655 \\
\hline 0.281 & 0.004 & 0.824 & 0.047 & 0.26137931 \\
\hline 0.088 & 0.004 & 0.081 & 0.021 & 0.02862069 \\
\hline 0.039 & 0.000 & 0.035 & 0.014 & 0 \\
\hline 0.241 & 0.000 & 0.378 & 0.004 & 0.10534483 \\
\hline 0.534 & 0.000 & 1.609 & 0.013 & 0.76568966 \\
\hline 0.580 & 0.000 & 1.268 & 0.017 & 0.41482759 \\
\hline 0.279 & 0.002 & 0.556 & 0.042 & 0.10965517 \\
\hline 0.505 & 0.000 & 1.544 & 0.074 & 0.62344828 \\
\hline 0.546 & 0.000 & 2.062 & 0.038 & 0.64586207 \\
\hline 0.498 & 0.001 & 1.189 & 0.026 & 0.81051724 \\
\hline 0.432 & 0.000 & 1.368 & 0.033 & 0.61310345 \\
\hline 0.422 & 0.001 & 1.162 & 0.019 & 0.72689655 \\
\hline 0.443 & 0.001 & 1.421 & 0.020 & 0.81482759 \\
\hline 0.311 & 0.001 & 0.763 & 0.077 & 0.16051724 \\
\hline 0.358 & 0.001 & 0.715 & 0.029 & 0.35793103 \\
\hline 0.392 & 0.000 & 1.114 & 0.034 & 0.65706897 \\
\hline 0.304 & 0.001 & 1.237 & 0.059 & 0.45362069 \\
\hline 0.423 & 0.000 & 1.390 & 0.046 & 0.64758621 \\
\hline 0.225 & 0.013 & 0.395 & 0.155 & 0.2062069 \\
\hline 0.388 & 0.000 & 1.254 & 0.033 & 0.47258621 \\
\hline
\end{tabular}




\begin{tabular}{|c|c|c|c|c|}
\hline 0.270 & 0.000 & 0.475 & 0.059 & 0.33206897 \\
\hline 0.359 & 0.000 & 1.033 & 0.032 & 0.61310345 \\
\hline 0.344 & 0.000 & 0.734 & 0.053 & 0.27862069 \\
\hline 0.317 & 0.001 & 0.793 & 0.023 & 0.60448276 \\
\hline 0.493 & 0.000 & 2.319 & 0.033 & 0.57775862 \\
\hline 0.321 & 0.001 & 0.786 & 0.043 & 0.56741379 \\
\hline 0.369 & 0.000 & 1.226 & 0.073 & 0.64931034 \\
\hline 0.272 & 0.002 & 0.874 & 0.099 & 0.36224138 \\
\hline 0.215 & 0.001 & 0.656 & 0.062 & 0.32948276 \\
\hline 0.414 & 0.000 & 1.256 & 0.042 & 0.65965517 \\
\hline 0.357 & 0.000 & 0.570 & 0.023 & 0.10362069 \\
\hline 0.282 & 0.001 & 1.073 & 0.061 & 0.25189655 \\
\hline 0.379 & 0.000 & 0.935 & 0.020 & 0.30793103 \\
\hline 0.681 & 0.000 & 1.614 & 0.038 & 0.13293103 \\
\hline 0.201 & 0.000 & 0.606 & 0.097 & 0.19413793 \\
\hline 0.472 & 0.001 & 1.003 & 0.031 & 0.395 \\
\hline 0.322 & 0.000 & 1.153 & 0.084 & 0.20103448 \\
\hline 0.317 & 0.002 & 0.585 & 0.048 & 0.24327586 \\
\hline 0.372 & 0.002 & 0.641 & 0.062 & 0.22431034 \\
\hline 0.334 & 0.001 & 0.641 & 0.027 & 0.21396552 \\
\hline 0.341 & 0.000 & 0.621 & 0.030 & 0.37258621 \\
\hline 0.404 & 0.001 & 0.775 & 0.033 & 0.44413793 \\
\hline 0.468 & 0.000 & 1.361 & 0.088 & 0.54327586 \\
\hline 0.245 & 0.000 & 0.446 & 0.028 & 0.10017241 \\
\hline 0.151 & 0.001 & 0.368 & 0.062 & 0.2587931 \\
\hline 0.541 & 0.000 & 1.771 & 0.055 & 0.73570559 \\
\hline 0.484 & 0.000 & 1.912 & 0.166 & 2.10587887 \\
\hline 0.283 & 0.000 & 0.629 & 0.030 & 0.29846164 \\
\hline 0.631 & 0.000 & 3.936 & 0.177 & 4.28146423 \\
\hline 0.528 & 0.000 & 1.471 & 0.085 & 0.3298473 \\
\hline 0.553 & 0.000 & 0.630 & 0.101 & 0.02282065 \\
\hline 0.597 & 0.040 & 0.505 & 0.129 & 0.00819226 \\
\hline 0.576 & 0.001 & 0.840 & 0.021 & 0.00283992 \\
\hline 0.678 & 0.003 & 0.938 & 0.040 & 0.0154311 \\
\hline 0.554 & 0.029 & 0.214 & 0.110 & 0.00868654 \\
\hline 0.801 & 0.058 & 0.038 & 0.254 & 0.00205008 \\
\hline 0.534 & 0.172 & 0.043 & 0.003 & 0.00698537 \\
\hline 0.405 & 0.063 & 0.025 & 0.003 & 0.00566209 \\
\hline 0.378 & 0.387 & 0.009 & 0.004 & 0.00090101 \\
\hline 0.452 & 0.179 & 0.020 & 0.003 & 0.00284744 \\
\hline 0.515 & 0.032 & 0.074 & 0.004 & 0.01142239 \\
\hline
\end{tabular}




$\begin{array}{llllc}0.503 & 0.067 & 0.047 & 0.003 & 0.005753 \\ 0.421 & 0.295 & 0.047 & 0.008 & 0.01019946 \\ 0.480 & 0.108 & 0.075 & 0.008 & 0.01106168 \\ 0.548 & 0.115 & 0.069 & 0.004 & 0.00905648 \\ 0.504 & 0.114 & 0.061 & 0.004 & 0.010733 \\ 0.573 & 0.197 & 0.059 & 0.003 & 0.00732698 \\ 0.414 & 0.017 & 0.022 & 0.003 & 0.0030144 \\ 0.437 & 0.002 & 1.334 & 0.071 & 0.28031184 \\ 0.726 & 0.038 & 1.949 & 0.078 & 0.01369162 \\ 0.675 & 0.189 & 0.278 & 0.197 & 0.0070954 \\ 0.556 & 0.020 & 1.276 & 0.212 & 0.14157461 \\ 0.290 & 0.007 & 0.558 & 0.048 & 0.04679071 \\ 0.347 & 0.000 & 0.553 & 0.206 & 0.04011907 \\ 0.504 & 0.080 & 0.063 & 0.014 & 0.01057225 \\ 0.405 & 0.008 & 0.265 & 0.026 & 0.0387179 \\ 0.604 & 0.046 & 0.030 & 0.019 & 0.00276735 \\ 0.741 & 0.059 & 0.157 & 0.092 & 0.00847972 \\ 0.719 & 0.020 & 0.311 & 0.021 & 0.02031714 \\ 0.779 & 0.010 & 0.525 & 0.218 & 0.01367435 \\ 0.850 & 0.016 & 0.609 & 0.250 & 0.01149887 \\ 0.801 & 0.007 & 0.440 & 0.190 & 0.00619362 \\ 0.580 & 0.021 & 0.277 & 0.057 & 0.00632617 \\ 0.754 & 0.008 & 0.464 & 0.123 & 0.01133434 \\ 0.646 & 0.038 & 0.344 & 0.048 & 0.02455292 \\ 0.577 & 0.075 & 0.330 & 0.059 & 0.01185041 \\ 0.778 & 0.373 & 0.168 & 0.612 & 0.01389606 \\ 0.738 & 0.438 & 0.172 & 0.723 & 0.03034577 \\ 0.545 & 0.520 & 0.120 & 0.477 & 0.03453057 \\ 0.547 & 0.213 & 0.470 & 0.549 & 0.04956946 \\ 0.465 & 0.364 & 0.645 & 0.303 & 0.04899927 \\ 0.425 & 0.368 & 0.488 & 0.498 & 0.13605659 \\ 0.395 & 0.538 & 0.166 & 0.507 & 0.13813612 \\ 0.515 & 0.471 & 0.327 & 0.681 & 0.1697517 \\ 0.420 & 0.465 & 0.230 & 0.610 & 0.11275095 \\ 0.396 & 0.436 & 0.122 & 0.657 & 0.16323342 \\ 0.237 & 0.019 & 0.346 & 0.505 & 0.17597709 \\ 0.280 & 0.008 & 0.468 & 0.564 & 0.24429211 \\ 0.290 & 0.025 & 0.600 & 0.487 & 0.20435775 \\ 0.283 & 0.012 & 0.559 & 0.469 & 0.20261526 \\ 0.353 & 0.001 & 1.022 & 0.123 & 0.33734448 \\ 0.018 & 0.174 & 0.232 & 0.08954028\end{array}$




\begin{tabular}{|c|c|c|c|c|}
\hline 0.922 & 0.114 & 0.305 & 0.195 & 0.04251068 \\
\hline 0.778 & 0.066 & 1.167 & 0.178 & 0.05146135 \\
\hline 0.383 & 0.010 & 0.693 & 0.216 & 0.21045559 \\
\hline 0.548 & 0.007 & 1.966 & 0.178 & 0.25301677 \\
\hline 0.212 & 0.010 & 1.061 & 0.182 & 0.31629191 \\
\hline 0.253 & 0.279 & 0.352 & 0.227 & 0.18030736 \\
\hline 0.257 & 0.008 & 1.517 & 0.229 & 0.39781787 \\
\hline 0.476 & 0.004 & 0.510 & 0.204 & 0.19864614 \\
\hline 0.485 & 0.002 & 2.186 & 0.201 & 0.46528141 \\
\hline 0.178 & 0.003 & 0.392 & 0.274 & 0.22261734 \\
\hline 0.323 & 0.012 & 0.573 & 0.272 & 0.12621168 \\
\hline 0.413 & 0.002 & 0.472 & 0.122 & 0.03967364 \\
\hline 0.466 & 0.001 & 0.611 & 0.137 & 0.22508166 \\
\hline 0.438 & 0.077 & 0.184 & 0.064 & 0.04157189 \\
\hline 0.660 & 0.020 & 0.930 & 0.335 & 0.18281519 \\
\hline 0.377 & 0.000 & 0.873 & 0.106 & 0.28552655 \\
\hline 1.052 & 0.052 & 0.435 & 0.147 & 0.02909466 \\
\hline 0.243 & 0.014 & 0.491 & 0.229 & 0.15925577 \\
\hline 0.913 & 0.139 & 0.594 & 0.172 & 0.03010419 \\
\hline 0.257 & 0.003 & 0.569 & 0.084 & 0.15766195 \\
\hline 0.963 & 0.231 & 0.072 & 0.057 & 0.01286407 \\
\hline 0.126 & 0.011 & 0.334 & 0.266 & 0.48938466 \\
\hline 0.167 & 0.004 & 0.209 & 0.267 & 0.44839255 \\
\hline 0.840 & 0.460 & 0.020 & 0.350 & 0 \\
\hline 0.960 & 0.320 & 0.260 & 0.360 & 0.01 \\
\hline 0.900 & 0.440 & 0.370 & 0.360 & 0.01 \\
\hline 0.950 & 0.180 & 0.340 & 0.130 & 0.02 \\
\hline 0.760 & 0.560 & 0.230 & 0.110 & 0.01 \\
\hline 0.910 & 0.200 & 0.570 & 0.110 & 0.02 \\
\hline 0.950 & 0.370 & 0.070 & 0.050 & 0.01 \\
\hline 0.640 & 0.500 & 0.080 & 0.010 & 0 \\
\hline 0.830 & 0.150 & 0.680 & 0.130 & 0.02 \\
\hline 0.830 & 0.110 & 0.460 & 0.060 & 0.02 \\
\hline 0.960 & 0.190 & 0.110 & 0.140 & 0 \\
\hline 0.790 & 0.480 & 0.060 & 0.030 & 0.02 \\
\hline 0.990 & 0.100 & 0.170 & 0.050 & 0.01 \\
\hline 0.970 & 0.460 & 0.010 & 0.020 & 0 \\
\hline 0.980 & 0.180 & 0.090 & 0.050 & 0 \\
\hline 1.000 & 0.000 & 0.100 & 0.100 & 0 \\
\hline 0.900 & 0.000 & 0.200 & 0.100 & 0 \\
\hline 0.700 & 0.500 & 0.100 & 0.300 & 0.1 \\
\hline
\end{tabular}




$\begin{array}{llllc}0.800 & 0.600 & 0.000 & 0.100 & 0 \\ 0.700 & 0.500 & 0.000 & 0.200 & 0.1 \\ 0.700 & 0.400 & 0.000 & 0.200 & 0.1 \\ 0.700 & 0.700 & 0.000 & 0.100 & 0 \\ 0.800 & 0.900 & 0.100 & 0.100 & 0 \\ 0.800 & 0.700 & 0.000 & 0.100 & 0 \\ 0.700 & 0.900 & 0.100 & 0.100 & 0 \\ 0.800 & 0.900 & 0.100 & 0.100 & 0 \\ 0.400 & 0.900 & 0.000 & 0.100 & 0 \\ 0.500 & 0.900 & 0.000 & 0.100 & 0 \\ 0.500 & 0.900 & 0.000 & 0.100 & 0 \\ 0.400 & 0.900 & 0.000 & 0.100 & 0 \\ 0.700 & 0.800 & 0.100 & 0.200 & 0 \\ 0.800 & 0.800 & & 0.100 & 0 \\ 0.600 & 0.800 & 0.200 & 0.100 & 0.1 \\ 0.600 & 0.800 & 0.000 & 0.100 & 0 \\ 0.500 & 0.800 & 0.100 & 0.100 & 0.1 \\ 0.500 & 0.400 & 0.200 & 0.100 & 0 \\ 0.600 & 0.000 & 0.900 & 0.100 & 0.1 \\ 0.900 & 0.100 & 0.100 & 0.100 & 0 \\ 0.600 & 0.000 & 0.800 & 0.100 & 0 \\ 0.600 & 0.000 & 1.200 & 0.000 & 0.1 \\ 0.500 & 0.700 & 0.100 & 0.100 & 0.1 \\ 0.600 & 0.600 & 0.100 & 0.100 & 0.1 \\ 0.400 & 0.700 & 0.100 & 0.100 & 0.1 \\ 0.600 & 0.600 & 0.100 & 0.200 & 0 \\ 0.500 & 0.800 & 0.100 & 0.100 & 0.1 \\ 0.500 & 0.800 & 0.100 & 0.100 & 0.1 \\ 0.600 & 0.600 & 0.200 & 0.100 & 0.1 \\ 0.500 & 0.700 & 0.100 & 0.100 & 0.1 \\ 0.500 & 0.700 & 0.100 & 0.100 & 0.1 \\ 0.000 & & 0.000 & 0.000 & 0 \\ 0.200 & 0.100 & 0.100 & 0.000 & 0 \\ 0.200 & 0.100 & 0.100 & 0.000 & 0 \\ 0.400 & 0.200 & 0.000 & 0.000 & 0 \\ 0.200 & 0.000 & 0.100 & 0.400 & 0.1 \\ 0.300 & 0.000 & 0.900 & 0.300 & 0.1 \\ 0.200 & 0.000 & 0.400 & 0.000 & 0.1 \\ 0.900 & 0.700 & 0.100 & 0.400 & 0.1 \\ 0.700 & 0.100 & 1.300 & 0.000 & 0 \\ 0.300 & 0.000 & 0.500 & 0.000 & 0\end{array}$




$\begin{array}{llllc}0.800 & 0.900 & 0.200 & 0.200 & 0 \\ 0.700 & 0.600 & 0.800 & 0.300 & 0 \\ 0.700 & 0.800 & 0.200 & 0.400 & 0 \\ 0.800 & 0.700 & 0.500 & 0.100 & 0.2 \\ 0.700 & 0.700 & 0.200 & 0.200 & 0.1 \\ 0.400 & 0.100 & 0.300 & 0.100 & 0 \\ 0.200 & 0.000 & 0.900 & 0.000 & 0.1 \\ 0.600 & 0.000 & 0.500 & 0.900 & 0.9 \\ 0.200 & 0.000 & 0.500 & 0.300 & 0 \\ 0.100 & 0.100 & 0.100 & 0.200 & 0.2 \\ 0.100 & 0.000 & 0.300 & 0.400 & 0 \\ 1.000 & 0.300 & 0.000 & 0.100 & 0 \\ 0.800 & 1.000 & 0.000 & 0.000 & 0 \\ 1.000 & 0.700 & 0.000 & 0.000 & 0 \\ 0.800 & 0.100 & 1.000 & 0.000 & 0 \\ 0.800 & 0.100 & 1.800 & 0.000 & 0 \\ 1.000 & 0.500 & 0.000 & 0.000 & 0 \\ 1.000 & 0.100 & 0.200 & 0.100 & 0.1 \\ 1.000 & 0.200 & 0.100 & 0.000 & 0 \\ 0.900 & 0.100 & 0.400 & 0.000 & 0 \\ 0.900 & 0.100 & 0.300 & 0.000 & 0 \\ 0.900 & 0.100 & 0.300 & 0.100 & 0 \\ 0.900 & 0.300 & 0.200 & 0.100 & 0 \\ 1.000 & 0.200 & 0.200 & 0.100 & 0 \\ 0.900 & 0.100 & 0.300 & 0.100 & 0 \\ 0.700 & 0.000 & 0.400 & 0.200 & 0 \\ 0.800 & 0.200 & 0.200 & 0.100 & 0 \\ 0.900 & 0.100 & 0.200 & 0.100 & 0 \\ 0.800 & 0.000 & 0.600 & 0.200 & 0.1 \\ 0.800 & 0.000 & 0.700 & 0.100 & 0.1 \\ 1.000 & 0.400 & 0.100 & 0.000 & 0 \\ 1.000 & 0.700 & 0.000 & 0.000 & 0 \\ 0.700 & 0.100 & 0.500 & 0.100 & 0 \\ 0.900 & 0.200 & 0.300 & 0.000 & 0 \\ 1.000 & 0.800 & 0.000 & 0.000 & 0 \\ 0.122 & 0.233 & 0.047 & 0.000 & 0.00703207 \\ 0.167 & 1.000 & 0.000 & 0.000 & 0 \\ 0.311 & 0.400 & 0.104 & 0.000 & 0.01355223 \\ 0.154 & 0.412 & 0.044 & 0.004 & 0.01073281 \\ 1.000 & 1.000 & 0.000 & 0.000 & 0 \\ 0.297 & 0.505 & 0.074 & 0.000 & 0.01038405\end{array}$




\begin{tabular}{|c|c|c|c|c|}
\hline 0.324 & 0.658 & 0.041 & 0.000 & 0.0091709 \\
\hline 0.216 & 0.462 & 0.047 & 0.000 & 0.00880039 \\
\hline 0.124 & 0.230 & 0.042 & 0.000 & 0.00912455 \\
\hline 0.531 & 0.855 & 0.082 & 0.016 & 0.01587595 \\
\hline 0.588 & 1.000 & 0.000 & 0.000 & 0 \\
\hline 0.291 & 0.678 & 0.051 & 0.009 & 0.00873467 \\
\hline 0.313 & 0.929 & 0.029 & 0.000 & 0 \\
\hline 0.715 & 0.891 & 0.036 & 0.006 & 0.00816078 \\
\hline 0.703 & 0.956 & 0.066 & 0.000 & 0 \\
\hline 0.562 & 0.473 & 0.430 & 0.000 & 0.00760615 \\
\hline 0.688 & 0.698 & 0.173 & 0.000 & 0 \\
\hline 0.591 & 0.768 & 0.127 & 0.000 & 0 \\
\hline 1.000 & 0.936 & 0.062 & 0.000 & 0 \\
\hline 1.000 & 1.000 & 0.000 & 0.000 & 0 \\
\hline 0.270 & 0.123 & 0.150 & 0.000 & 0.04966725 \\
\hline 0.300 & 0.712 & 0.064 & 0.000 & 0 \\
\hline 0.232 & 0.220 & 0.128 & -0.001 & 0.00757028 \\
\hline 0.134 & 0.070 & 0.075 & 0.000 & 0.00507946 \\
\hline 0.111 & 0.220 & 0.050 & -0.003 & 0.01602647 \\
\hline 0.256 & 0.475 & 0.072 & 0.000 & 0.01413139 \\
\hline 0.154 & 0.425 & 0.041 & 0.000 & 0.00999517 \\
\hline 0.126 & 0.415 & 0.037 & 0.000 & 0.00615081 \\
\hline 0.192 & 0.192 & 0.097 & 0.000 & 0.02267551 \\
\hline 0.666 & 0.954 & 0.000 & 0.056 & 0.03495652 \\
\hline 0.907 & 0.696 & 0.008 & 0.673 & 0 \\
\hline 0.170 & 0.412 & 0.219 & 0.000 & 0 \\
\hline 0.289 & 0.293 & 0.257 & 0.035 & 0.0623215 \\
\hline 0.393 & 0.729 & 0.066 & 0.100 & 0.03862951 \\
\hline 0.491 & 0.971 & 0.000 & 0.024 & 0.01316346 \\
\hline 0.398 & 0.225 & 0.421 & 0.041 & 0.1744984 \\
\hline 0.630 & 0.866 & 0.097 & 0.087 & 0.08225643 \\
\hline 0.383 & 0.853 & 0.054 & 0.039 & 0.03515298 \\
\hline 0.541 & 0.907 & 0.042 & 0.113 & 0.04041151 \\
\hline 0.618 & 0.570 & 0.081 & 0.731 & 0.03247307 \\
\hline 0.301 & 0.717 & 0.056 & 0.130 & 0.03912226 \\
\hline 0.701 & 0.344 & 0.000 & 2.021 & 0.40009756 \\
\hline 0.639 & 0.787 & 0.018 & 0.410 & 0 \\
\hline 0.626 & 0.582 & 0.125 & 0.910 & 0 \\
\hline 0.686 & 0.642 & 0.072 & 0.785 & 0 \\
\hline 0.686 & 0.996 & 0.005 & 0.006 & 0 \\
\hline 0.701 & 0.987 & 0.008 & 0.027 & 0 \\
\hline
\end{tabular}




\begin{tabular}{|c|c|c|c|c|}
\hline 0.293 & 0.000 & 0.681 & 0.008 & 0 \\
\hline 0.482 & 0.970 & 0.042 & 0.000 & 0 \\
\hline 0.621 & 0.915 & 0.043 & 0.139 & 0 \\
\hline 0.619 & 0.833 & 0.000 & 0.275 & 0.02628571 \\
\hline 0.667 & 0.870 & 0.044 & 0.283 & 0 \\
\hline 0.675 & 0.862 & 0.053 & 0.238 & 0.02951774 \\
\hline 0.708 & 0.906 & 0.000 & 0.163 & 0.03509472 \\
\hline 0.647 & 0.888 & 0.035 & 0.000 & 0.01470219 \\
\hline 0.716 & 0.938 & 0.069 & 0.025 & 0.01430385 \\
\hline 0.721 & 0.880 & 0.069 & 0.153 & 0.00993257 \\
\hline 0.726 & 0.930 & 0.061 & 0.096 & 0.00354968 \\
\hline 0.840 & 0.917 & 0.037 & 0.164 & 0.00273982 \\
\hline 0.691 & 0.955 & 0.032 & 0.096 & 0.0035842 \\
\hline 0.862 & 0.965 & 0.041 & 0.083 & 0.00568897 \\
\hline 0.810 & 0.966 & 0.032 & 0.083 & 0.00865081 \\
\hline 0.255 & 0.666 & 0.051 & 0.000 & 0.00430319 \\
\hline 0.108 & 0.198 & 0.050 & 0.000 & 0.00267186 \\
\hline 0.165 & 0.425 & 0.057 & 0.000 & 0.00532836 \\
\hline \multicolumn{5}{|l|}{0.240} \\
\hline \multicolumn{5}{|l|}{0.450} \\
\hline \multicolumn{5}{|l|}{0.380} \\
\hline \multicolumn{5}{|l|}{0.560} \\
\hline \multicolumn{5}{|l|}{0.750} \\
\hline \multicolumn{5}{|l|}{0.820} \\
\hline \multicolumn{5}{|c|}{0.640} \\
\hline \multicolumn{5}{|c|}{0.630} \\
\hline \multicolumn{5}{|l|}{0.780} \\
\hline \multicolumn{5}{|c|}{0.210} \\
\hline \multicolumn{5}{|l|}{0.420} \\
\hline \multicolumn{5}{|l|}{0.460} \\
\hline \multicolumn{5}{|l|}{0.550} \\
\hline \multicolumn{5}{|c|}{0.200} \\
\hline \multicolumn{5}{|c|}{0.780} \\
\hline \multicolumn{5}{|c|}{0.510} \\
\hline \multicolumn{5}{|l|}{0.770} \\
\hline \multicolumn{5}{|l|}{0.770} \\
\hline \multicolumn{5}{|l|}{0.510} \\
\hline \multicolumn{5}{|l|}{0.510} \\
\hline \multicolumn{5}{|l|}{0.250} \\
\hline \multicolumn{5}{|l|}{0.210} \\
\hline 0.560 & & & & \\
\hline
\end{tabular}




\begin{tabular}{|c|c|c|c|c|}
\hline \multicolumn{5}{|l|}{0.210} \\
\hline 0.450 & & & & \\
\hline 0.510 & & & & \\
\hline 0.700 & & & & \\
\hline 0.220 & & & & \\
\hline 0.670 & & & & \\
\hline 0.190 & & & & \\
\hline 0.270 & & & & \\
\hline 0.410 & & & & \\
\hline 0.430 & & & & \\
\hline 0.410 & & & & \\
\hline 1.050 & 0.070 & 0.300 & 0.000 & 0.02 \\
\hline 0.840 & 0.130 & 0.190 & 0.000 & 0.02 \\
\hline 0.560 & 0.690 & 0.130 & 0.000 & 0.01 \\
\hline 0.520 & 0.540 & 0.190 & 0.000 & 0.02 \\
\hline 0.730 & 0.390 & 0.250 & 0.010 & 0.03 \\
\hline 0.690 & 0.310 & 0.610 & 0.000 & 0.05 \\
\hline 0.930 & 0.140 & 0.420 & 0.000 & 0.04 \\
\hline 1.050 & 0.200 & 0.420 & 0.000 & 0.03 \\
\hline 0.960 & 0.110 & 0.250 & 0.000 & 0.02 \\
\hline 1.040 & 0.370 & 0.640 & 0.000 & 0.05 \\
\hline 0.970 & 0.040 & 0.290 & 0.000 & 0.01 \\
\hline 0.880 & 0.120 & 0.380 & 0.000 & 0.04 \\
\hline 0.940 & 0.220 & 0.370 & 0.000 & 0.03 \\
\hline 0.710 & 0.000 & 0.800 & 1.310 & 0 \\
\hline 0.720 & 0.000 & 0.680 & 1.740 & 0 \\
\hline 0.290 & 0.050 & 0.810 & 0.360 & 0.12 \\
\hline 0.780 & 0.020 & 0.240 & 2.100 & 0 \\
\hline 0.770 & 0.040 & 0.650 & 1.630 & 0.01 \\
\hline 0.760 & 0.010 & 1.250 & 1.190 & 0 \\
\hline 0.740 & 0.090 & 1.070 & 1.660 & 0 \\
\hline 0.590 & 0.060 & 1.510 & 0.680 & 0.02 \\
\hline 0.660 & 0.090 & 1.510 & 0.550 & 0.04 \\
\hline 0.520 & 0.020 & 1.250 & 0.120 & 0.03 \\
\hline 0.390 & 0.090 & 0.690 & 0.760 & 0.05 \\
\hline 0.720 & 0.090 & 0.940 & 1.170 & 0.09 \\
\hline 0.340 & 0.040 & 0.380 & 0.620 & 0.06 \\
\hline 0.350 & 0.150 & 0.710 & 0.650 & 0.05 \\
\hline 0.550 & 0.090 & 0.980 & 0.950 & 0.1 \\
\hline 0.320 & 0.050 & 0.330 & 0.650 & 0.06 \\
\hline 0.440 & 0.120 & 0.480 & 0.870 & 0.11 \\
\hline
\end{tabular}




$\begin{array}{lllll}0.460 & 0.070 & 0.590 & 1.250 & 0.05 \\ 0.510 & 0.070 & 0.350 & 1.280 & 0.1 \\ 0.460 & 0.050 & 1.070 & 0.910 & 0.07 \\ 0.350 & 0.210 & 0.580 & 0.460 & 0.13 \\ 0.350 & 0.080 & 0.750 & 0.790 & 0.06 \\ 0.570 & 0.060 & 0.310 & 1.040 & 0.25 \\ 0.350 & 0.130 & 0.110 & 1.250 & 0.12 \\ 0.340 & 0.050 & 1.080 & 0.430 & 0.07 \\ 0.380 & 0.020 & 0.790 & 0.370 & 0.06 \\ 0.340 & 0.110 & 0.750 & 0.790 & 0.06 \\ 0.310 & 0.250 & 0.520 & 0.520 & 0.06 \\ 0.470 & 0.150 & 0.450 & 1.570 & 0.12 \\ 0.250 & 0.050 & 0.550 & 0.500 & 0.06 \\ 0.250 & 0.120 & 0.480 & 0.530 & 0.06 \\ 0.230 & 0.080 & 0.350 & 0.570 & 0.07 \\ 0.310 & 0.110 & 0.770 & 0.520 & 0.05 \\ 0.430 & 0.110 & 0.210 & 1.220 & 0.2 \\ 0.490 & 0.040 & 0.180 & 1.190 & 0.15 \\ 0.340 & 0.130 & 0.670 & 0.620 & 0.04 \\ 0.340 & 0.080 & 1.060 & 0.290 & 0.05 \\ 0.390 & 0.080 & 0.610 & 0.410 & 0.03 \\ 0.360 & 0.110 & 0.780 & 0.570 & 0.03 \\ 0.430 & 0.050 & 1.360 & 0.440 & 0.04 \\ 0.670 & 0.150 & 0.340 & 0.980 & 0.16 \\ 0.560 & 0.070 & 1.090 & 0.780 & 0.04 \\ 0.410 & 0.090 & 0.390 & 1.020 & 0.27 \\ 0.450 & 0.050 & 1.200 & 0.550 & 0.04 \\ 0.490 & 0.060 & 1.880 & 0.400 & 0.04 \\ 0.510 & 0.080 & 1.740 & 1.000 & 0.08 \\ 0.560 & 0.080 & 0.320 & 1.510 & 0.33 \\ 0.380 & 0.070 & 1.150 & 0.950 & 0.04 \\ 0.330 & 0.110 & 0.500 & 0.580 & 0.04 \\ 0.310 & 0.140 & 0.650 & 0.450 & 0.04 \\ 0.320 & 0.100 & 0.780 & 0.450 & 0.05 \\ 0.490 & 0.140 & 0.330 & 1.460 & 0.29 \\ 0.350 & 0.130 & 0.860 & 0.570 & 0.03 \\ 0.240 & 0.170 & 0.390 & 0.480 & 0.02 \\ 0.440 & 0.090 & 1.420 & 0.540 & 0.05 \\ 0.340 & 0.060 & 0.630 & 1.390 & 0.26 \\ 0.340 & 0.100 & 0.950 & 0.640 & 0.07 \\ 0.050 & 1.170 & 0.290 & 0.07\end{array}$




$\begin{array}{lllll}0.410 & 0.080 & 0.970 & 0.710 & 0.07 \\ 0.380 & 0.080 & 1.040 & 0.640 & 0.08 \\ 0.460 & 0.020 & 0.830 & 1.370 & 0.28 \\ 0.350 & 0.020 & 1.270 & 0.260 & 0.07 \\ 0.330 & 0.070 & 1.030 & 0.280 & 0.06 \\ 0.280 & 0.100 & 0.680 & 0.230 & 0.05 \\ 0.470 & 0.140 & 0.510 & 0.890 & 0.31 \\ 0.470 & 0.050 & 0.570 & 1.440 & 0.32 \\ 0.380 & 0.030 & 1.240 & 0.390 & 0.08 \\ 0.500 & 0.000 & 1.630 & 0.320 & 0.07 \\ 0.330 & 0.080 & 0.620 & 0.810 & 0.08 \\ 0.530 & 0.050 & 0.610 & 1.530 & 0.25 \\ 0.360 & 0.060 & 1.630 & 0.160 & 0.06 \\ 0.330 & 0.010 & 1.160 & 0.220 & 0.06 \\ 0.620 & 0.010 & 0.560 & 1.680 & 0.22 \\ 0.480 & 0.080 & 1.510 & 0.290 & 0.1 \\ 0.470 & 0.090 & 1.340 & 0.310 & 0.06 \\ 0.470 & 0.060 & 1.460 & 0.340 & 0.09 \\ 0.480 & 0.060 & 1.500 & 0.620 & 0.11 \\ 0.410 & 0.050 & 1.120 & 0.900 & 0.06 \\ 0.370 & 0.030 & 1.010 & 0.420 & 0.12 \\ 0.730 & 0.060 & 0.740 & 1.100 & 0.1 \\ 0.450 & 0.020 & 1.420 & 0.760 & 0.16 \\ 0.390 & 0.000 & 1.460 & 0.670 & 0.11 \\ 0.410 & 0.020 & 1.160 & 0.630 & 0.23 \\ 0.530 & 0.040 & 1.920 & 0.680 & 0.19 \\ 0.210 & 0.110 & 0.150 & 0.670 & 0.19 \\ 0.320 & 0.020 & 0.880 & 0.430 & 0.13 \\ 0.380 & 0.030 & 1.230 & 0.540 & 0.14 \\ 0.310 & 0.010 & 0.930 & 0.410 & 0.08 \\ 0.360 & 0.020 & 1.190 & 0.230 & 0.1 \\ 0.360 & 0.030 & 1.280 & 0.390 & 0.12 \\ 0.420 & 0.110 & 1.640 & 0.480 & 0.17 \\ 0.430 & 0.020 & 1.570 & 0.630 & 0.13 \\ 0.910 & 0.020 & 0.470 & 0.260 & 0.02 \\ 0.410 & 0.030 & 1.540 & 0.570 & 0.17 \\ 0.380 & 0.040 & 1.100 & 0.280 & 0.12 \\ 0.400 & 0.070 & 0.690 & 0.800 & 0.14 \\ 0.440 & 0.020 & 1.120 & 0.800 & 0.14 \\ 0.510 & 0.050 & 0.710 & 0.390 & 0.15 \\ 0.550 & 0.010 & 0.930 & 1.270 & 0.11\end{array}$




$\begin{array}{lllll}0.410 & 0.010 & 1.290 & 0.740 & 0.18 \\ 0.340 & 0.010 & 0.950 & 0.620 & 0.16 \\ 0.260 & 0.000 & 0.490 & 0.340 & 0.16 \\ 0.410 & 0.060 & 1.460 & 0.430 & 0.09 \\ 0.480 & 0.040 & 1.220 & 1.080 & 0.15 \\ 0.400 & 0.020 & 1.280 & 0.620 & 0.11 \\ 0.280 & 0.020 & 0.740 & 0.480 & 0.12 \\ 0.510 & 0.030 & 0.670 & 0.000 & 0.09 \\ 0.250 & 0.030 & 0.640 & 0.500 & 0.09 \\ 0.320 & 0.030 & 0.950 & 0.850 & 0.18 \\ 0.260 & 0.020 & 0.570 & 0.610 & 0.14 \\ 0.420 & 0.010 & 0.930 & 0.740 & 0.19 \\ 0.630 & 0.020 & 0.580 & 0.630 & 0.15 \\ 0.450 & 0.010 & 1.720 & 0.510 & 0.15 \\ 0.440 & 0.010 & 1.100 & 1.000 & 0.12 \\ 0.380 & 0.010 & 1.270 & 0.720 & 0.15 \\ 0.450 & 0.020 & 0.830 & 0.960 & 0.13 \\ 0.400 & 0.000 & 1.430 & 0.670 & 0.17 \\ 0.330 & 0.010 & 1.020 & 0.930 & 0.12 \\ 0.360 & 0.020 & 0.910 & 0.350 & 0.12 \\ 0.430 & 0.020 & 1.540 & 0.540 & 0.13 \\ 0.220 & 0.050 & 0.700 & 0.370 & 0.12 \\ 0.250 & 0.030 & 0.930 & 0.390 & 0.14 \\ 0.180 & 0.020 & 0.570 & 0.270 & 0.12 \\ 0.420 & 0.060 & 0.550 & 2.170 & 0.07 \\ 0.210 & 0.130 & 0.520 & 0.310 & 0.11 \\ 0.220 & 0.070 & 0.670 & 0.260 & 0.12 \\ 0.300 & 0.040 & 0.920 & 0.460 & 0.11 \\ 0.240 & 0.010 & 0.930 & 0.330 & 0.12 \\ 0.320 & 0.000 & 0.750 & 0.750 & 0.13 \\ 0.480 & 0.000 & 0.660 & 1.210 & 0.13 \\ 0.180 & 0.050 & 0.430 & 0.210 & 0.08 \\ 0.300 & 0.070 & 1.240 & 0.260 & 0.1 \\ 0.370 & 0.010 & 1.050 & 0.250 & 0.09 \\ 0.340 & 0.000 & 0.620 & 0.980 & 0.12 \\ 0.450 & 0.000 & 1.820 & 0.530 & 0.15 \\ 0.290 & 0.010 & 0.530 & 0.380 & 0.11 \\ 0.280 & 0.010 & 1.090 & 0.200 & 0.16 \\ 0.260 & 0.000 & 1.280 & 0.220 & 0.16 \\ 0.290 & 0.130 & 0.930 & 0.340 & 0.11 \\ 0.110 & 1.060 & 0.200 & 0.11\end{array}$




\begin{tabular}{|c|c|c|c|c|}
\hline 0.200 & 0.010 & 0.790 & 0.220 & 0.14 \\
\hline 0.110 & 0.000 & 0.120 & 0.340 & 0.15 \\
\hline 0.200 & 0.080 & 0.660 & 0.280 & 0.12 \\
\hline 0.220 & 0.090 & 0.940 & 0.280 & 0.16 \\
\hline 0.290 & 0.000 & 1.300 & 0.270 & 0.13 \\
\hline 0.270 & 0.080 & 0.980 & 0.410 & 0.12 \\
\hline 0.370 & 0.030 & 0.840 & 0.600 & 0.08 \\
\hline 0.230 & 0.120 & 0.650 & 0.160 & 0.08 \\
\hline 0.140 & 0.060 & 0.360 & 0.200 & 0.1 \\
\hline 0.210 & 0.030 & 0.780 & 0.210 & 0.1 \\
\hline 0.310 & 0.150 & 0.970 & 0.200 & 0.04 \\
\hline 0.330 & 0.200 & 0.710 & 0.250 & 0.05 \\
\hline 0.350 & 0.030 & 0.980 & 0.440 & 0.09 \\
\hline 0.480 & 0.010 & 0.730 & 0.700 & 0.07 \\
\hline 0.460 & 0.010 & 1.610 & 0.190 & 0.1 \\
\hline 0.460 & 0.070 & 1.870 & 0.180 & 0.09 \\
\hline 0.770 & 0.100 & 1.290 & 1.950 & 0.03 \\
\hline 0.640 & 0.080 & 1.430 & 0.870 & 0.01 \\
\hline 0.460 & 0.000 & 0.560 & 0.070 & 0.01 \\
\hline 0.360 & 0.000 & 0.370 & 0.060 & 0.01 \\
\hline 0.440 & 0.000 & 1.280 & 1.240 & 0.29 \\
\hline 0.520 & 0.120 & 0.580 & 1.700 & 0.06 \\
\hline 0.560 & 0.070 & 0.860 & 1.740 & 0.05 \\
\hline 0.420 & 0.030 & 1.430 & 0.670 & 0.17 \\
\hline 0.730 & 0.050 & 0.950 & 3.230 & 0.03 \\
\hline 0.330 & 0.000 & 0.340 & 0.050 & 0.01 \\
\hline 0.260 & 0.100 & 0.930 & 0.330 & 0.12 \\
\hline 0.270 & 0.050 & 0.720 & 0.200 & 0.14 \\
\hline 0.540 & 0.080 & 1.200 & 1.040 & 0.09 \\
\hline 0.600 & 0.020 & 3.030 & 1.060 & 0.24 \\
\hline 0.360 & 0.000 & 0.490 & 0.030 & 0 \\
\hline 0.860 & 0.020 & 3.020 & 2.450 & 0.04 \\
\hline 0.710 & 0.070 & 1.040 & 0.910 & 0.02 \\
\hline 0.380 & 0.000 & 1.050 & 0.560 & 0.04 \\
\hline 0.390 & 0.020 & 1.200 & 0.250 & 0.1 \\
\hline 0.600 & 0.070 & 1.920 & 0.630 & 0.06 \\
\hline 0.600 & 0.010 & 2.370 & 0.160 & 0.04 \\
\hline 0.590 & 0.020 & 3.290 & 0.110 & 0.09 \\
\hline 0.648 & 0.763 & 0.119 & 0.009 & 0.00381099 \\
\hline 0.523 & 0.661 & 0.126 & 0.012 & 0.00516212 \\
\hline 0.118 & 0.115 & 0.198 & 0.067 & 0.09588881 \\
\hline
\end{tabular}




\begin{tabular}{|c|c|c|c|c|}
\hline 0.158 & 0.087 & 0.137 & 0.005 & 0.00653011 \\
\hline 0.266 & 0.034 & 0.290 & 0.025 & 0.00767059 \\
\hline 0.482 & 0.797 & 0.141 & 0.103 & 0.0313547 \\
\hline 0.162 & 0.000 & 0.273 & 0.064 & 0.04284545 \\
\hline 0.454 & 0.413 & 0.704 & 0.304 & 0.05692151 \\
\hline 0.675 & 0.211 & 1.611 & 0.337 & 0.02842426 \\
\hline 0.361 & 0.027 & 1.217 & 0.220 & 0.11428334 \\
\hline 0.334 & 0.052 & 0.762 & 0.215 & 0.06054187 \\
\hline 0.368 & 0.032 & 0.765 & 0.195 & 0.05182996 \\
\hline 0.380 & 0.066 & 0.820 & 0.294 & 0.06917449 \\
\hline 0.350 & 0.040 & 0.753 & 0.181 & 0.08337715 \\
\hline 0.380 & 0.100 & 1.027 & 0.194 & 0.0886569 \\
\hline 0.470 & 0.059 & 1.240 & 0.303 & 0.09167658 \\
\hline 0.298 & 0.071 & 0.888 & 0.156 & 0.04658136 \\
\hline 0.454 & 0.070 & 1.017 & 0.289 & 0.14542628 \\
\hline 0.320 & 0.084 & 0.877 & 0.283 & 0.06154945 \\
\hline 0.369 & 0.043 & 1.442 & 0.230 & 0.11816207 \\
\hline 0.622 & 0.027 & 1.318 & 0.263 & 0.11928977 \\
\hline 0.278 & 0.088 & 1.145 & 0.324 & 0.1097269 \\
\hline 0.434 & 0.039 & 1.171 & 0.163 & 0.13146839 \\
\hline 0.410 & 0.068 & 0.750 & 0.207 & 0.15651054 \\
\hline 0.438 & 0.020 & 1.021 & 0.302 & 0.16772528 \\
\hline 0.308 & 0.019 & 1.156 & 0.170 & 0.12432003 \\
\hline 0.442 & 0.090 & 0.646 & 0.338 & 0.12736037 \\
\hline 0.471 & 0.059 & 0.887 & 0.236 & 0.11665717 \\
\hline 0.284 & 0.072 & 0.522 & 0.235 & 0.1601026 \\
\hline 0.453 & 0.053 & 0.865 & 0.266 & 0.10272025 \\
\hline 0.698 & 0.071 & 1.013 & 0.306 & 0.09538386 \\
\hline 0.316 & 0.062 & 0.563 & 0.284 & 0.11122722 \\
\hline 0.396 & 0.075 & 1.054 & 0.220 & 0.11519713 \\
\hline 0.316 & 0.012 & 0.507 & 0.135 & 0.11500906 \\
\hline 0.404 & 0.112 & 0.572 & 0.277 & 0.15442155 \\
\hline 0.457 & 0.025 & 1.246 & 0.260 & 0.05602382 \\
\hline 0.225 & 0.008 & 0.577 & 0.101 & 0.05342203 \\
\hline 0.267 & 0.043 & 0.653 & 0.236 & 0.07816838 \\
\hline 0.076 & 0.024 & 0.305 & 0.062 & 0.04660399 \\
\hline 0.081 & 0.017 & 0.192 & 0.121 & 0.08924221 \\
\hline 0.403 & 0.011 & 1.239 & 0.067 & 0.04022714 \\
\hline 0.573 & 0.060 & 1.813 & 0.138 & 0.0297192 \\
\hline 0.526 & 0.095 & 0.619 & 0.047 & 0.01777449 \\
\hline 0.596 & 0.030 & 0.716 & 0.076 & 0.00596134 \\
\hline
\end{tabular}




\begin{tabular}{|c|c|c|c|c|}
\hline 0.276 & 0.000 & 0.808 & 0.102 & 0.06915833 \\
\hline 0.161 & 0.020 & 0.266 & 0.120 & 0.06294911 \\
\hline 0.146 & 0.234 & 0.207 & 0.091 & 0.07353592 \\
\hline 0.275 & 0.001 & 1.101 & 0.111 & 0.07142619 \\
\hline 0.201 & 0.000 & 0.789 & 0.103 & 0.09851948 \\
\hline 0.092 & 0.002 & 0.201 & 0.115 & 0.09426204 \\
\hline 0.508 & 0.001 & 1.951 & 0.138 & 0.06712958 \\
\hline 0.303 & 0.002 & 1.160 & 0.095 & 0.08686099 \\
\hline 0.119 & 0.003 & 0.257 & 0.101 & 0.07547851 \\
\hline 0.108 & 0.007 & 0.143 & 0.090 & 0.06199606 \\
\hline 0.174 & 0.007 & 0.493 & 0.094 & 0.05914517 \\
\hline 1.046 & 0.048 & 0.906 & 0.673 & 0.00359777 \\
\hline 0.593 & 0.024 & 2.608 & 0.825 & 0.06117286 \\
\hline 0.218 & 0.004 & 0.405 & 0.162 & 0.07258208 \\
\hline 0.341 & 0.157 & 0.446 & 0.469 & 0.17568824 \\
\hline 0.608 & 0.018 & 1.504 & 0.500 & 0.03364261 \\
\hline 0.680 & 0.023 & 3.060 & 0.048 & 0.03187137 \\
\hline 0.465 & 0.036 & 2.045 & 0.124 & 0.05301145 \\
\hline 0.343 & 0.290 & 1.051 & 0.311 & 0.04462698 \\
\hline 0.729 & 0.022 & 2.497 & 1.494 & 0.07326601 \\
\hline 0.168 & 0.005 & 0.374 & 0.103 & 0.10419111 \\
\hline 0.586 & 0.005 & 1.806 & 0.052 & 0.02427949 \\
\hline 0.315 & 0.076 & 0.246 & 0.042 & 0.00302783 \\
\hline 0.260 & 0.010 & & & \\
\hline 0.270 & 0.000 & & & \\
\hline 0.230 & 0.000 & & & \\
\hline 0.200 & 0.000 & & & \\
\hline 0.220 & 0.010 & & & \\
\hline 0.280 & 0.000 & & & \\
\hline 0.320 & 0.050 & & & \\
\hline 0.240 & 0.010 & & & \\
\hline 0.320 & 0.000 & & & \\
\hline 0.220 & 0.000 & & & \\
\hline 0.200 & 0.010 & & & \\
\hline 0.240 & 0.000 & & & \\
\hline 0.230 & 0.010 & & & \\
\hline 0.170 & 0.020 & & & \\
\hline 0.210 & 0.010 & & & \\
\hline 0.260 & 0.000 & & & \\
\hline 0.250 & 0.000 & & & \\
\hline 0.310 & 0.000 & & & \\
\hline
\end{tabular}




$\begin{array}{ll}0.290 & 0.000 \\ 0.300 & 0.000 \\ 0.260 & 0.020 \\ 0.300 & 0.020 \\ 0.280 & 0.020 \\ 0.300 & 0.020 \\ 0.280 & 0.010 \\ 0.270 & 0.010 \\ 0.270 & 0.010 \\ 0.260 & 0.010 \\ 0.260 & 0.040 \\ 0.250 & 0.030 \\ 0.300 & 0.030 \\ 0.320 & 0.010 \\ 0.270 & 0.030 \\ 0.290 & 0.050 \\ 0.290 & 0.070 \\ 0.300 & 0.000 \\ 0.250 & 0.160 \\ 0.220 & 0.020 \\ 0.220 & 0.040 \\ 0.210 & 0.010 \\ 0.210 & 0.010 \\ 0.230 & 0.010 \\ 0.180 & 0.030 \\ 0.180 & 0.010 \\ 0.230 & 0.010 \\ 0.240 & 0.090 \\ 0.260 & 0.060 \\ 0.240 & 0.010 \\ 0.330 & 0.010 \\ 0.240 & 0.010 \\ 0.130 & 0.030 \\ 0.200 & 0.040 \\ 0.210 & 0.100 \\ 0.230 & 0.010 \\ 0.830 & 0.040 \\ 0.270 & 0.340 \\ 0.280 & 0.200 \\ 0.220 & 0.340\end{array}$




\begin{tabular}{|c|c|c|c|c|}
\hline 0.320 & 0.010 & & & \\
\hline 0.300 & 0.070 & & & \\
\hline 0.250 & 0.080 & & & \\
\hline 0.170 & 0.170 & & & \\
\hline 0.320 & 0.130 & & & \\
\hline 0.120 & 0.130 & & & \\
\hline 0.290 & 0.050 & & & \\
\hline 0.320 & 0.000 & & & \\
\hline 0.360 & 0.000 & & & \\
\hline 0.250 & 0.000 & & & \\
\hline 0.330 & 0.000 & & & \\
\hline 0.140 & 0.070 & & & \\
\hline 0.210 & 0.100 & & & \\
\hline 0.390 & 0.020 & & & \\
\hline 0.280 & 0.370 & & & \\
\hline 0.180 & 0.070 & & & \\
\hline 0.290 & 0.320 & & & \\
\hline 0.200 & 0.100 & & & \\
\hline 0.220 & 0.380 & & & \\
\hline 0.090 & 0.020 & & & \\
\hline 0.190 & 0.000 & & & \\
\hline 0.230 & 0.010 & & & \\
\hline 0.180 & 0.080 & & & \\
\hline 0.130 & 0.080 & & & \\
\hline 0.170 & 0.010 & & & \\
\hline 0.060 & 0.270 & 0.020 & 0.170 & 0.07 \\
\hline 0.100 & 0.430 & 0.030 & 0.270 & 0.07 \\
\hline 0.170 & 0.350 & 0.010 & 0.400 & 0.14 \\
\hline 0.040 & 0.030 & 0.010 & 0.240 & 0.11 \\
\hline 0.080 & 0.440 & 0.020 & 0.220 & 0.1 \\
\hline 0.120 & 0.160 & 0.090 & 0.320 & 0.15 \\
\hline 0.050 & 0.010 & 0.030 & 0.150 & 0.07 \\
\hline 0.060 & 0.070 & 0.030 & 0.210 & 0.1 \\
\hline 0.100 & 0.210 & 0.070 & 0.390 & 0.15 \\
\hline 0.460 & 0.250 & 0.020 & 1.190 & 0.18 \\
\hline 0.410 & 0.070 & 0.170 & 0.230 & 0.13 \\
\hline 0.150 & 0.270 & 0.390 & 0.300 & 0.12 \\
\hline 0.120 & 0.240 & 0.080 & 0.570 & 0.09 \\
\hline 0.060 & 0.130 & 0.000 & 0.250 & 0.07 \\
\hline 0.070 & 0.030 & 0.060 & 0.330 & 0.07 \\
\hline 0.670 & 0.090 & 0.280 & 2.130 & 0.07 \\
\hline
\end{tabular}




$\begin{array}{lllll}0.100 & 0.280 & 0.170 & 0.210 & 0.06 \\ 0.070 & 0.180 & 0.100 & 0.180 & 0.08 \\ 0.080 & 0.160 & 0.220 & 0.130 & 0.05 \\ 0.070 & 0.060 & 0.060 & 0.290 & 0.06 \\ 0.900 & 0.020 & 0.120 & 5.500 & 0.1 \\ 0.090 & 0.260 & 0.040 & 0.270 & 0.06 \\ 0.100 & 0.060 & 0.180 & 0.280 & 0.05 \\ 0.880 & 0.050 & 0.100 & 1.680 & 0.02 \\ 0.560 & 0.080 & 0.090 & 0.910 & 0.02 \\ 0.530 & 0.450 & 0.030 & 0.600 & 0.03 \\ 0.510 & 0.190 & 0.130 & 1.760 & 0.05 \\ 0.300 & 0.120 & 0.170 & 0.670 & 0.04 \\ 0.490 & 0.010 & 0.050 & 1.050 & 0.02 \\ 0.070 & 0.370 & 0.060 & 0.120 & 0.03 \\ 0.300 & 0.420 & 0.250 & 0.520 & \\ 0.100 & 0.110 & 0.080 & 0.440 & 0.06 \\ 0.050 & 0.030 & 0.070 & 0.160 & 0.08 \\ 0.090 & 0.060 & 0.250 & 0.140 & 0.06 \\ 0.410 & 0.420 & 0.140 & 0.470 & 0.04 \\ 0.050 & 0.080 & 0.020 & 0.090 & 0.06 \\ 0.520 & 0.050 & 0.440 & 0.150 & 0.12 \\ 0.440 & 0.010 & 0.100 & 0.100 & 0.1 \\ 0.180 & 0.440 & 0.190 & 0.170 & 0.09 \\ 0.240 & 0.010 & 0.430 & 0.300 & 0.17 \\ 0.110 & 0.100 & 0.370 & 0.150 & 0.08 \\ 0.600 & 0.010 & 0.700 & 0.160 & 0.23 \\ 0.130 & 0.500 & 0.050 & 0.180 & 0.09 \\ 0.070 & 0.120 & 0.180 & 0.150 & 0.08 \\ 0.050 & 0.030 & 0.050 & 0.150 & 0.07 \\ 0.050 & 0.020 & 0.030 & 0.180 & 0.07 \\ 0.080 & 0.020 & 0.220 & 0.160 & 0.07 \\ 0.060 & 0.020 & 0.030 & 0.220 & 0.08 \\ 0.070 & 0.080 & 0.120 & 0.150 & 0.06 \\ 0.120 & 0.540 & 0.040 & 0.290 & 0.08 \\ 0.670 & 0.140 & 0.040 & 0.630 & 0.09 \\ 0.130 & 0.370 & 0.040 & 0.210 & 0.1 \\ 0.410 & 0.310 & 0.090 & 0.130 & 0.11 \\ 0.220 & 0.480 & 0.080 & 0.470 & 0.12 \\ 0.370 & 0.010 & 0.150 & 0.790 & 0.24 \\ 0.760 & 0.020 & 0.320 & 0.160 & 0.19 \\ 0.730 & 0.020 & 0.050 & 0.490 & 0.18\end{array}$




$\begin{array}{llllc}0.050 & 0.030 & 0.060 & 0.130 & 0.07 \\ 0.060 & 0.020 & 0.030 & 0.150 & 0.07 \\ 0.110 & 0.370 & 0.040 & 0.270 & 0.17 \\ 0.070 & 0.300 & 0.050 & 0.160 & 0.08 \\ 0.280 & 0.800 & 0.060 & 0.130 & 0.06 \\ 0.230 & 0.320 & 0.110 & 0.120 & 0.13 \\ 0.060 & 0.070 & 0.070 & 0.180 & 0.05 \\ 0.240 & 0.600 & 0.130 & 0.130 & 0.04 \\ 0.230 & 0.530 & 0.050 & 0.290 & 0.06 \\ 0.300 & 0.170 & 0.150 & 0.640 & 0.14 \\ 0.350 & 0.740 & 0.060 & 0.210 & 0.1 \\ 0.330 & 0.470 & 0.080 & 0.520 & 0.08 \\ 0.140 & 0.540 & 0.030 & 0.160 & 0.06 \\ 0.140 & 0.350 & 0.110 & 0.190 & 0.09 \\ 0.880 & 0.620 & 0.050 & 0.760 & 0.07 \\ 0.240 & 0.190 & 0.320 & 0.420 & 0.08 \\ 0.490 & 0.650 & 0.290 & 0.110 & 0.05 \\ 0.210 & 0.580 & 0.080 & 0.250 & 0.1 \\ 0.240 & 0.120 & 0.180 & 0.670 & 0.12 \\ 0.240 & 0.260 & 0.160 & 0.380 & 0.09 \\ 0.350 & 0.090 & 0.930 & 0.520 & 0.13 \\ 0.690 & 0.700 & 0.150 & 0.220 & 0.13 \\ 0.280 & 0.110 & 0.050 & 0.350 & 0.2 \\ 0.450 & 0.130 & 0.150 & 0.370 & 0.26 \\ 0.330 & 0.240 & 0.160 & 0.760 & 0.19 \\ 0.570 & 0.300 & 0.070 & 1.030 & 0.13 \\ 0.230 & 0.060 & 0.060 & 0.200 & 0.15 \\ 0.540 & 0.040 & 0.050 & 0.640 & 0.22 \\ 0.190 & 0.280 & 0.010 & 0.450 & 0.12 \\ 0.300 & 0.390 & 0.190 & 0.530 & 0.1 \\ 0.490 & 0.290 & 0.140 & 0.480 & 0.16 \\ 0.910 & 0.120 & 0.470 & 1.460 & 0.19 \\ 0.330 & 0.210 & 0.040 & 0.470 & 0.24 \\ 0.420 & 0.530 & 0.510 & 0.180 & 0.09 \\ 0.470 & 0.760 & 0.030 & 0.160 & 0.09 \\ 0.500 & 0.310 & 0.000 & 1.550 & 0.13 \\ 1.010 & 0.150 & 0.060 & 2.440 & 0.17 \\ 0.480 & 0.260 & 0.140 & 1.220 & 0.2 \\ 0.160 & 0.130 & 0.170 & 0.190 & 0.08 \\ 0.200 & 0.500 & 0.040 & 0.270 & 0.12 \\ 0.240 & 0.360 & 0.090 & 0.520 & 0.16\end{array}$




$\begin{array}{lllll}0.180 & 0.210 & 0.050 & 0.330 & 0.11 \\ 0.190 & 0.090 & 0.270 & 0.360 & 0.13 \\ 0.200 & 0.070 & 0.050 & 0.710 & 0.18 \\ 0.090 & 0.190 & 0.090 & 0.270 & 0.1 \\ 0.230 & 0.120 & 0.050 & 0.300 & 0.18 \\ 0.270 & 0.200 & 0.070 & 0.810 & 0.14 \\ 0.360 & 0.780 & 0.040 & 0.260 & 0.12 \\ 0.110 & 0.020 & 0.120 & 0.160 & 0.09 \\ 0.290 & 0.510 & 0.040 & 0.390 & 0.12 \\ 0.300 & 0.020 & 0.080 & 0.240 & 0.18 \\ 0.280 & 0.140 & 0.010 & 0.260 & 0.14 \\ 0.360 & 0.120 & 0.040 & 0.700 & 0.16 \\ 0.100 & 0.190 & 0.120 & 0.180 & 0.08 \\ 0.120 & 0.250 & 0.100 & 0.270 & 0.11 \\ 0.270 & 0.590 & 0.180 & 0.270 & 0.12 \\ 1.000 & 0.060 & 0.180 & 6.500 & 0.15 \\ 1.000 & 0.160 & 0.130 & 3.130 & 0.19 \\ 1.000 & 0.030 & 0.070 & 2.900 & 0.11 \\ 0.240 & 0.310 & 0.130 & 0.360 & 0.09 \\ 1.000 & 0.020 & 0.030 & 3.060 & 0.13 \\ 0.360 & 0.360 & 0.100 & 0.830 & 0.21 \\ 0.150 & 0.210 & 0.070 & 0.320 & 0.19 \\ 0.190 & 0.220 & 0.270 & 0.260 & 0.13 \\ 0.860 & 0.450 & 0.040 & 0.390 & 0.13 \\ 0.640 & 0.300 & 0.090 & 0.760 & 0.09 \\ 0.590 & 0.540 & 0.210 & 1.340 & 0.35 \\ 0.680 & 0.310 & 0.190 & 1.410 & 0.18 \\ 1.000 & 0.270 & 0.180 & 1.830 & 0.19 \\ 0.420 & 0.350 & 0.140 & 0.650 & 0.15 \\ 0.370 & 0.320 & 0.120 & 0.690 & 0.15 \\ 0.380 & 0.480 & 0.120 & 0.390 & 0.17 \\ 0.490 & 0.370 & 0.130 & 0.880 & 0.14 \\ 0.340 & 0.000 & 0.160 & 0.680 & 0.33 \\ 0.360 & 0.540 & 0.000 & 0.310 & 0.01 \\ 0.470 & 0.080 & 0.000 & 0.610 & 0.02 \\ 0.610 & 0.650 & 0.020 & 0.740 & 0.03 \\ 0.070 & 0.000 & 0.020 & 0.160 & 0.1 \\ 0.220 & 0.170 & 0.100 & 0.590 & 0.31 \\ 0.050 & 0.100 & 0.040 & 0.140 & 0.05 \\ 0.150 & 0.020 & 0.070 & 0.320 & 0.17 \\ 0.160 & 0.000 & 0.070 & 0.290 & 0.19\end{array}$




$\begin{array}{lllll}0.180 & 0.090 & 0.050 & 0.280 & 0.18 \\ 0.090 & 0.370 & 0.030 & 0.170 & 0.09 \\ 0.260 & 0.300 & 0.280 & 0.530 & 0.26 \\ 0.170 & 0.140 & 0.110 & 0.290 & 0.16 \\ 0.070 & 0.020 & 0.100 & 0.100 & 0.05 \\ 0.140 & 0.000 & 0.050 & 0.320 & 0.19 \\ 0.170 & 0.020 & 0.030 & 0.330 & 0.18 \\ 0.170 & 0.040 & 0.060 & 0.400 & 0.18 \\ 0.230 & 0.010 & 0.040 & 0.280 & 0.16 \\ 0.050 & 0.000 & 0.060 & 0.170 & 0.09 \\ 0.140 & 0.010 & 0.020 & 0.330 & 0.16 \\ 0.110 & 0.000 & 0.030 & 0.320 & 0.2 \\ 0.130 & 0.010 & 0.030 & 0.220 & 0 \\ 0.180 & 0.320 & 0.080 & 0.270 & 0.25 \\ 0.120 & 0.000 & 0.040 & 0.280 & 0.14 \\ 0.130 & 0.210 & 0.060 & 0.250 & 0.15 \\ 0.140 & 0.240 & 0.270 & 0.210 & 0.05 \\ 0.130 & 0.400 & 0.040 & 0.300 & 0.1 \\ 0.130 & 0.480 & 0.010 & 0.200 & 0.09 \\ 0.150 & 0.000 & 0.080 & 0.350 & 0.16 \\ 0.270 & 0.010 & 0.040 & 0.950 & 0.19 \\ 0.070 & 0.040 & 0.000 & 0.380 & 0.18 \\ 0.200 & 0.040 & 0.020 & 0.340 & 0.18 \\ 0.230 & 0.010 & 0.030 & 0.700 & 0.18 \\ 0.030 & 0.010 & 0.000 & 0.130 & 0.05 \\ 0.090 & 0.360 & 0.030 & 0.230 & 0.08 \\ 0.060 & 0.110 & 0.030 & 0.240 & 0.11 \\ 0.100 & 0.210 & 0.020 & 0.240 & 0.14 \\ 0.120 & 0.270 & 0.040 & 0.220 & 0.11 \\ 0.110 & 0.000 & 0.210 & 0.080 & 0.03 \\ 0.050 & 0.040 & 0.100 & 0.170 & 0.05 \\ 0.180 & 0.000 & 0.060 & 0.230 & 0.14 \\ 0.220 & 0.060 & 0.000 & 0.250 & 0.16 \\ 0.070 & 0.050 & 0.010 & 0.350 & 0.19 \\ 0.120 & 0.010 & 0.020 & 0.410 & 0.21 \\ 0.100 & 0.000 & 0.040 & 0.300 & 0.12 \\ 0.080 & 0.050 & 0.160 & 0.140 & 0.05 \\ 0.060 & 0.020 & 0.030 & 0.290 & 0.13 \\ 0.110 & 0.050 & 0.010 & 0.250 & 0.15 \\ 0.050 & 0.270 & 0.000 & 0.320 & 0.14 \\ 0.060 & 0.240 & 0.070 & 0.170 & 0.06\end{array}$




$\begin{array}{lllll}0.230 & 0.050 & 0.020 & 0.170 & 0.17 \\ 0.220 & 0.060 & 0.010 & 0.420 & 0.17 \\ 0.210 & 0.720 & 0.000 & 0.110 & 0.07 \\ 0.060 & 0.190 & 0.080 & 0.100 & 0.04 \\ 0.150 & 0.500 & 0.020 & 0.300 & 0.14 \\ 0.110 & 0.010 & 0.000 & 0.230 & 0.16 \\ 0.400 & 0.020 & 0.010 & 0.670 & 0.1 \\ 0.450 & 0.010 & 0.550 & 0.230 & 0.17 \\ 0.540 & 0.030 & 0.060 & 0.360 & 0.09 \\ 0.250 & 0.000 & 0.360 & 0.380 & 0.34 \\ 0.160 & 0.120 & 0.200 & 0.240 & 0.21 \\ 0.180 & 0.250 & 0.230 & 0.210 & 0.2 \\ 0.160 & 0.150 & 0.210 & 0.220 & 0.23 \\ 0.160 & 0.120 & 0.230 & 0.220 & 0.23 \\ 0.180 & 0.010 & 0.320 & 0.230 & 0.21 \\ 0.130 & 0.080 & 0.160 & 0.210 & 0.18 \\ 0.220 & 0.180 & 0.280 & 0.260 & 0.21 \\ 0.220 & 0.250 & 0.200 & 0.250 & 0.21 \\ 0.190 & 0.030 & 0.210 & 0.200 & 0.18 \\ 0.160 & 0.030 & 0.250 & 0.230 & 0.21 \\ 0.160 & 0.070 & 0.260 & 0.220 & 0.2 \\ 0.430 & 0.050 & 0.440 & 0.410 & 0.58 \\ 0.320 & 0.010 & 0.410 & 0.490 & 0.62 \\ 0.330 & 0.010 & 0.240 & 0.440 & 0.3 \\ 0.310 & 0.000 & 0.450 & 0.380 & 0.33 \\ 0.270 & 0.010 & 0.600 & 0.170 & 0.32 \\ 0.350 & 0.000 & 0.780 & 0.290 & 0.52 \\ 0.350 & 0.010 & 0.710 & 0.310 & 0.51 \\ 0.400 & 0.020 & 0.360 & 0.440 & 0.33 \\ 0.250 & 0.080 & 0.560 & 0.220 & 0.21 \\ 0.200 & 0.000 & 0.350 & 0.240 & 0.16 \\ 0.140 & 0.000 & 0.160 & 0.180 & 0.2 \\ 0.200 & 0.000 & 0.430 & 0.240 & 0.21 \\ 0.170 & 0.010 & 0.160 & 0.150 & 0.11 \\ 0.120 & 0.010 & 0.050 & 0.230 & 0.15 \\ 0.200 & 0.010 & 0.290 & 0.060 & 0.02 \\ 0.100 & 0.020 & 0.090 & 0.150 & 0.13 \\ 0.190 & 0.000 & 0.210 & 0.300 & 0.24 \\ 0.190 & 0.020 & 0.130 & 0.160 & 0.11 \\ 0.240 & 0.000 & 0.400 & 0.180 & 0.22 \\ 0.240 & 0.010 & 0.270 & 0.170 & 0.19\end{array}$




$\begin{array}{lllll}0.270 & 0.000 & 0.340 & 0.160 & 0.21 \\ 0.300 & 0.000 & 0.820 & 0.040 & 0.37 \\ 0.200 & 0.010 & 0.200 & 0.180 & 0.16 \\ 0.220 & 0.010 & 0.210 & 0.210 & 0.18 \\ 0.260 & 0.070 & 0.310 & 0.280 & 0.27 \\ 0.190 & 0.010 & 0.200 & 0.250 & 0.23 \\ 0.190 & 0.010 & 0.170 & 0.240 & 0.21 \\ 0.430 & 0.000 & 1.160 & 0.170 & 0.35 \\ 0.260 & 0.000 & 0.500 & 0.340 & 0.32 \\ 0.210 & 0.000 & 0.350 & 0.280 & 0.26 \\ 0.250 & 0.040 & 0.330 & 0.300 & 0.27 \\ 0.250 & 0.020 & 0.170 & 0.250 & 0.18 \\ 0.240 & 0.000 & 0.180 & 0.390 & 0.25 \\ 0.170 & 0.000 & 0.130 & 0.160 & 0.11 \\ 0.230 & 0.010 & 0.160 & 0.240 & 0.17 \\ 0.230 & 0.000 & 0.320 & 0.200 & 0.23 \\ 0.210 & 0.000 & 0.290 & 0.240 & 0.23 \\ 0.240 & 0.000 & 0.410 & 0.350 & 0.36 \\ 0.260 & 0.000 & 0.370 & 0.290 & 0.3 \\ 0.290 & 0.000 & 0.500 & 0.280 & 0.29 \\ 0.250 & 0.000 & 0.470 & 0.320 & 0.29 \\ 0.280 & 0.000 & 0.470 & 0.260 & 0.24 \\ 0.280 & 0.000 & 0.470 & 0.360 & 0.3 \\ 0.280 & 0.000 & 0.300 & 0.600 & 0.42 \\ 0.340 & 0.130 & 0.160 & 0.250 & 0.14 \\ 0.290 & 0.130 & 0.130 & 0.180 & 0.09 \\ 0.230 & 0.040 & 0.220 & 0.290 & 0.21 \\ 0.290 & 0.020 & 0.190 & 0.270 & 0.36 \\ 0.250 & 0.000 & 0.150 & 0.230 & 0.17 \\ 0.270 & 0.150 & 0.160 & 0.220 & 0.17 \\ 0.280 & 0.110 & 0.140 & 0.220 & 0.16 \\ 0.250 & 0.040 & 0.150 & 0.240 & 0.17 \\ 0.310 & 0.160 & 0.200 & 0.270 & 0.21 \\ 0.300 & 0.050 & 0.410 & 0.280 & 0.43 \\ 0.230 & 0.090 & 0.160 & 0.290 & 0.33 \\ 0.280 & 0.020 & 0.240 & 0.310 & 0.48 \\ 0.270 & 0.160 & 0.170 & 0.250 & 0.28 \\ 0.310 & 0.000 & 0.420 & 0.270 & 0.53 \\ 0.310 & 0.000 & 1.000 & 0.150 & 0.23 \\ 0.2150 & 0.000 & 0.190 & 0.170 & 0.18 \\ 0.000 & 0.450 & 0.070 & 0.11\end{array}$




$\begin{array}{lllll}0.170 & 0.000 & 0.420 & 0.320 & 0.25 \\ 0.240 & 0.000 & 0.610 & 0.200 & 0.23 \\ 0.270 & 0.000 & 0.470 & 0.180 & 0.24 \\ 0.260 & 0.000 & 0.750 & 0.190 & 0.27 \\ 0.270 & 0.000 & 0.680 & 0.180 & 0.29 \\ 0.180 & 0.000 & 0.360 & 0.280 & 0.3 \\ 0.190 & 0.000 & 0.420 & 0.170 & 0.22 \\ 0.220 & 0.000 & 0.460 & 0.160 & 0.19 \\ 0.250 & 0.000 & 0.720 & 0.140 & 0.18 \\ 0.230 & 0.000 & 0.490 & 0.210 & 0.22 \\ 0.240 & 0.000 & 0.580 & 0.220 & 0.24 \\ 0.220 & 0.000 & 0.780 & 0.160 & 0.17 \\ 0.250 & 0.320 & 0.250 & 0.370 & 0.22 \\ 0.170 & 0.000 & 0.270 & 0.290 & 0.21 \\ 0.190 & 0.250 & 0.230 & 0.270 & 0.18 \\ 0.210 & 0.100 & 0.260 & 0.280 & 0.15 \\ 0.280 & 0.080 & 0.450 & 0.330 & 0.24 \\ 0.250 & 0.130 & 0.390 & 0.310 & 0.23 \\ 0.270 & 0.110 & 0.390 & 0.320 & 0.24 \\ 0.270 & 0.060 & 0.280 & 0.360 & 0.24 \\ 0.230 & 0.210 & 0.130 & 0.190 & 0.12 \\ 0.170 & 0.030 & 0.220 & 0.270 & 0.23 \\ 0.180 & 0.030 & 0.250 & 0.310 & 0.22 \\ 0.180 & 0.070 & 0.230 & 0.310 & 0.24 \\ 0.180 & 0.020 & 0.250 & 0.300 & 0.25 \\ 0.190 & 0.020 & 0.340 & 0.290 & 0.24 \\ 0.190 & 0.040 & 0.300 & 0.290 & 0.24 \\ 0.180 & 0.050 & 0.240 & 0.290 & 0.23 \\ 0.180 & 0.090 & 0.260 & 0.280 & 0.27 \\ 0.230 & 0.070 & 0.250 & 0.330 & 0.15 \\ 0.190 & 0.000 & 0.260 & 0.270 & 0.23 \\ 0.190 & 0.020 & 0.260 & 0.360 & 0.28 \\ 0.200 & 0.000 & 0.340 & 0.290 & 0.27 \\ 0.330 & 0.400 & 0.360 & 0.420 & 0.29 \\ 0.270 & 0.220 & 0.380 & 0.460 & 0.34 \\ 0.240 & 0.010 & 0.450 & 0.270 & 0.28 \\ 0.280 & 0.390 & 0.280 & 0.320 & 0.27 \\ 0.230 & 0.160 & 0.220 & 0.490 & 0.38 \\ 0.190 & 0.010 & 0.280 & 0.280 & 0.23 \\ 0.210 & 0.020 & 0.300 & 0.320 & 0.3 \\ 0.230 & 0.000 & 0.320 & 0.350 & 0.33\end{array}$




$\begin{array}{lllll}0.270 & 0.140 & 0.320 & 0.310 & 0.25 \\ 0.290 & 0.010 & 0.350 & 0.310 & 0.3 \\ 0.250 & 0.000 & 0.270 & 0.290 & 0.25 \\ 0.250 & 0.130 & 0.320 & 0.260 & 0.31 \\ 0.250 & 0.290 & 0.280 & 0.320 & 0.29 \\ 0.210 & 0.020 & 0.390 & 0.290 & 0.3 \\ 0.300 & 0.180 & 0.210 & 0.300 & 0.29 \\ 0.280 & 0.040 & 0.250 & 0.340 & 0.34 \\ 0.280 & 0.080 & 0.260 & 0.320 & 0.35 \\ 0.290 & 0.010 & 0.250 & 0.300 & 0.31 \\ 0.330 & 0.040 & 0.310 & 0.450 & 0.42 \\ 0.300 & 0.010 & 0.340 & 0.540 & 0.52 \\ 0.320 & 0.010 & 0.320 & 0.320 & 0.31 \\ 0.290 & 0.010 & 0.260 & 0.500 & 0.46 \\ 0.280 & 0.000 & 0.280 & 0.320 & 0.41 \\ 0.330 & 0.020 & 0.320 & 0.390 & 0.36 \\ 0.300 & 0.010 & 0.310 & 0.400 & 0.37 \\ 0.230 & 0.000 & 0.300 & 0.310 & 0.26 \\ 0.270 & 0.010 & 0.260 & 0.310 & 0.2 \\ 0.260 & 0.010 & 0.240 & 0.390 & 0.24 \\ 0.360 & 0.020 & 0.360 & 0.390 & 0.33 \\ 0.290 & 0.000 & 0.350 & 0.320 & 0.38 \\ 0.340 & 0.000 & 0.400 & 0.490 & 0.55 \\ 0.230 & 0.000 & 0.170 & 0.140 & 0.15 \\ 0.350 & 0.000 & 0.210 & 0.310 & 0.21 \\ 0.310 & 0.000 & 0.270 & 0.310 & 0.26 \\ 0.340 & 0.000 & 0.410 & 0.260 & 0.46 \\ 0.510 & 0.360 & 0.370 & 0.390 & 0.27 \\ 0.280 & 0.000 & 0.340 & 0.290 & 0.37 \\ 0.370 & 0.030 & 0.230 & 0.390 & 0.22 \\ 0.400 & 0.040 & 0.270 & 0.220 & 0.33 \\ 0.300 & 0.050 & 0.250 & 0.210 & 0.27 \\ 0.260 & 0.030 & 0.090 & 0.090 & 0.07 \\ 0.420 & 0.300 & 0.140 & 0.130 & 0.13 \\ 0.360 & 0.070 & 0.220 & 0.390 & 0.32 \\ 0.220 & 0.030 & 0.180 & 0.260 & 0.19 \\ 0.190 & 0.000 & 0.330 & 0.250 & 0.18 \\ 0.200 & 0.000 & 0.200 & 0.340 & 0.2 \\ 0.290 & 0.340 & 0.140 & 0.240 & 0.09 \\ 0.240 & 0.010 & 0.120 & 0.260 & 0.09 \\ 0.200 & 0.010 & 0.120 & 0.290 & 0.12\end{array}$




$\begin{array}{lllll}0.230 & 0.010 & 0.100 & 0.240 & 0.08 \\ 0.270 & 0.000 & 0.180 & 0.290 & 0.15 \\ 0.190 & 0.000 & 0.170 & 0.330 & 0.18 \\ 0.160 & 0.000 & 0.100 & 0.280 & 0.12 \\ 0.200 & 0.000 & 0.240 & 0.290 & 0.32 \\ 0.170 & 0.000 & 0.250 & 0.280 & 0.28 \\ 0.310 & 0.390 & 0.180 & 0.260 & 0.2 \\ 0.130 & 0.010 & 0.180 & 0.320 & 0.19 \\ 0.170 & 0.050 & 0.200 & 0.240 & 0.21 \\ 0.300 & 0.440 & 0.170 & 0.330 & 0.17 \\ 0.170 & 0.020 & 0.220 & 0.320 & 0.24 \\ 0.310 & 0.430 & 0.220 & 0.270 & 0.21 \\ 0.190 & 0.000 & 0.210 & 0.360 & 0.27 \\ 0.210 & 0.150 & 0.240 & 0.330 & 0.22 \\ 0.190 & 0.090 & 0.280 & 0.290 & 0.21 \\ 0.230 & 0.040 & 0.440 & 0.330 & 0.18 \\ 0.210 & 0.140 & 0.270 & 0.300 & 0.19 \\ 0.490 & 0.490 & 0.180 & 0.340 & 0.06 \\ 0.190 & 0.150 & 0.210 & 0.290 & 0.21 \\ 0.320 & 0.000 & 0.400 & 0.350 & 0.42 \\ 0.350 & 0.000 & 0.570 & 0.340 & 0.4 \\ 0.350 & 0.000 & 0.830 & 0.160 & 0.17 \\ 0.460 & 0.000 & 1.260 & 0.080 & 0.18 \\ 0.370 & 0.000 & 0.910 & 0.180 & 0.28 \\ 0.420 & 0.000 & 0.940 & 0.320 & 0.31 \\ 0.340 & 0.010 & 0.460 & 0.320 & 0.21 \\ 0.270 & 0.030 & 0.090 & 0.110 & 0.04 \\ 0.320 & 0.020 & 0.060 & 0.100 & 0.02 \\ 0.290 & 0.040 & 0.060 & 0.110 & 0.02 \\ 0.010 & 1.000 & 0.000 & & \\ 0.350 & 0.010 & 0.420 & 0.380 & 0.52 \\ 0.480 & 0.000 & 1.450 & 0.070 & 0.21 \\ 0.430 & 0.010 & 0.650 & 0.350 & 0.54 \\ 0.440 & 0.010 & 0.760 & 0.480 & 0.78 \\ 0.430 & 0.010 & 0.720 & 0.300 & 0.53 \\ 0.420 & 0.030 & 0.750 & 0.320 & 0.58 \\ 0.400 & 0.000 & 0.630 & 0.340 & 0.58 \\ 0.390 & 0.000 & 0.640 & 0.270 & 0.49 \\ 0.170 & 0.010 & 0.200 & 0.350 & 0.27 \\ 0.2190 & 0.010 & 0.180 & 0.440 & 0.28 \\ 0.000 & 0.000 & 0.260 & 0.510 & 0.33\end{array}$




$\begin{array}{llllc}0.410 & 0.360 & 0.350 & 0.430 & 0.34 \\ 0.432 & 0.030 & 1.083 & 0.186 & 0.218 \\ 0.584 & 0.030 & 1.490 & 0.271 & 0.256 \\ 0.666 & 0.080 & 0.871 & 0.045 & 0.064 \\ 0.177 & 0.010 & 0.116 & 0.340 & 0.26 \\ 0.192 & 0.090 & 0.287 & 0.658 & 0.144 \\ 0.220 & 0.030 & 0.474 & 0.193 & 0.186 \\ 0.222 & 0.010 & 0.215 & 0.426 & 0.389 \\ 0.222 & 0.410 & 0.127 & 0.217 & 0.099 \\ 0.232 & 0.060 & 0.180 & 0.397 & 0.233 \\ 0.234 & 0.050 & 0.535 & 0.249 & 0.239 \\ 0.240 & 0.010 & 0.188 & 0.592 & 0.081 \\ 0.244 & 0.140 & 0.648 & 0.155 & 0.092 \\ 0.330 & 0.300 & 0.642 & 0.142 & 0.198 \\ 0.364 & 0.010 & 1.169 & 0.216 & 0.188 \\ 0.409 & 0.050 & 0.411 & 0.106 & 0.006 \\ 0.486 & 0.120 & 1.039 & 0.171 & 0.034 \\ 0.496 & 0.220 & 0.909 & 0.333 & 0.049 \\ 0.502 & 0.230 & 0.072 & 0.517 & 0.001 \\ 0.599 & 0.030 & 0.810 & 0.221 & 0.024 \\ 0.651 & 0.250 & 0.435 & 0.613 & 0.034 \\ 0.276 & 0.010 & 0.211 & 0.196 & 0.143 \\ 0.317 & 0.220 & 0.450 & 0.300 & 0.115 \\ 0.346 & 0.010 & 0.193 & 0.363 & 0.254 \\ 0.550 & 0.040 & 0.584 & 0.366 & 0.038 \\ 0.664 & 0.100 & 0.478 & 0.695 & 0.029 \\ 0.727 & 0.140 & 0.229 & 0.259 & 0.005 \\ 0.152 & 0.190 & 0.064 & 0.187 & 0.168 \\ 0.213 & 0.040 & 0.165 & 0.522 & 0.442 \\ 0.275 & 0.020 & 0.267 & 0.839 & 0.594 \\ 0.344 & 0.030 & 0.149 & 1.273 & 0.668 \\ 0.365 & 0.130 & 0.410 & 0.792 & 0.294 \\ 0.421 & 0.580 & 0.195 & 0.481 & 0.251 \\ 0.447 & 0.460 & 0.481 & 0.641 & 0.356 \\ 0.256 & 0.010 & 0.120 & 0.472 & 0.467 \\ 0.286 & 0.020 & 0.147 & 0.437 & 0.455 \\ 0.301 & 0.060 & 0.104 & 0.774 & 0.459 \\ 0.308 & 0.410 & 0.084 & 0.304 & 0.232 \\ 0.407 & 0.070 & 0.050 & 0.638 & 0.263 \\ 0.425 & 0.560 & 0.147 & 0.656 & 0.255 \\ 0.445 & 0.770 & 0.068 & 0.242 & 0.203\end{array}$




$\begin{array}{lllll}0.454 & 0.210 & 0.232 & 0.938 & 1.383 \\ 0.460 & 0.440 & 0.294 & 0.507 & 0.488 \\ 0.463 & 0.260 & 0.278 & 0.875 & 1.469 \\ 0.463 & 0.430 & 0.233 & 0.574 & 0.462 \\ 0.488 & 0.630 & 0.045 & 0.294 & 0.171 \\ 0.492 & 0.530 & 0.084 & 0.577 & 0.325 \\ 0.502 & 0.380 & 0.101 & 0.863 & 0.424 \\ 0.504 & 0.680 & 0.069 & 0.485 & 0.232 \\ 0.523 & 0.050 & 0.089 & 1.367 & 0.597 \\ 0.560 & 0.640 & 0.273 & 0.580 & 0.198 \\ 0.570 & 0.550 & 0.072 & 0.768 & 0.312 \\ 0.622 & 0.560 & 0.262 & 0.660 & 0.273 \\ 0.639 & 0.710 & 0.169 & 0.461 & 0.116 \\ 0.741 & 0.720 & 0.173 & 0.618 & 0.301 \\ 0.263 & 0.270 & 0.030 & 0.408 & 0.045 \\ 0.268 & 0.280 & 0.110 & 0.367 & 0.401 \\ 0.444 & 0.590 & 0.087 & 0.313 & 0.142 \\ 0.446 & 0.600 & 0.111 & 0.455 & 0.069 \\ 0.542 & 0.580 & 0.097 & 0.613 & 0.271 \\ 0.562 & 0.590 & 0.278 & 0.515 & 0.171 \\ 0.610 & 0.700 & 0.112 & 0.492 & 0.156 \\ 0.632 & 0.710 & 0.099 & 0.512 & 0.16 \\ 0.636 & 0.700 & 0.191 & 0.468 & 0.152 \\ 0.673 & 0.660 & 0.169 & 0.467 & 0.149 \\ 0.225 & 0.060 & 0.422 & 0.316 & 0.375 \\ 0.242 & 0.050 & 0.115 & 0.465 & 0.269 \\ 0.263 & 0.080 & 0.530 & 0.304 & 0.348 \\ 0.311 & 0.150 & 0.258 & 0.536 & 0.376 \\ 0.328 & 0.020 & 0.964 & 0.316 & 0.556 \\ 0.340 & 0.400 & 0.161 & 0.522 & 0.286 \\ 0.380 & 0.570 & 0.209 & 0.426 & 0.238 \\ 0.404 & 0.520 & 0.142 & 0.507 & 0.299 \\ 0.305 & 0.000 & 0.453 & 0.151 & 0.093 \\ 0.342 & 0.140 & 0.649 & 0.212 & 0.015 \\ 0.403 & 0.170 & 0.385 & 0.221 & 0.016 \\ 0.627 & 0.094 & 0.664 & 0.030 & 0.01311763 \\ 0.465 & 0.001 & 2.038 & 0.083 & 0.15898438 \\ 0.502 & 0.000 & 2.235 & 0.084 & 0.09748037 \\ 0.461 & 0.000 & 2.034 & 0.063 & 0.11703635 \\ 0.385 & 0.000 & 1.323 & 0.145 & 0.15380759 \\ & 0.000 & 1.636 & 0.087 & 0.12426144\end{array}$




\begin{tabular}{|c|c|c|c|c|}
\hline 0.356 & 0.001 & 1.613 & 0.120 & 0.10801267 \\
\hline 0.302 & 0.000 & 1.392 & 0.082 & 0.11257015 \\
\hline 0.482 & 0.000 & 1.878 & 0.080 & 0.11444567 \\
\hline 0.411 & 0.000 & 1.751 & 0.148 & 0.10841788 \\
\hline 0.383 & 0.000 & 1.553 & 0.104 & 0.10658155 \\
\hline 0.394 & 0.001 & 1.697 & 0.106 & 0.14403862 \\
\hline 0.445 & 0.001 & 2.222 & 0.072 & 0.12681531 \\
\hline 0.382 & 0.001 & 2.033 & 0.091 & 0.12031079 \\
\hline 0.303 & 0.001 & 1.908 & 0.104 & 0.12848817 \\
\hline 0.289 & 0.014 & 1.539 & 0.262 & 0.15307118 \\
\hline 0.439 & 0.003 & 2.454 & 0.196 & 0.11287085 \\
\hline 0.414 & 0.001 & 1.969 & 0.118 & 0.13914601 \\
\hline 0.337 & 0.007 & 1.520 & 0.263 & 0.17151766 \\
\hline 0.360 & 0.001 & 1.554 & 0.172 & 0.1515213 \\
\hline 0.301 & 0.003 & 1.279 & 0.143 & 0.09612623 \\
\hline 0.384 & 0.007 & 1.699 & 0.223 & 0.14035476 \\
\hline 0.274 & 0.060 & 1.142 & 0.188 & 0.12235247 \\
\hline 0.343 & 0.013 & 1.016 & 0.263 & 0.26808071 \\
\hline 0.304 & 0.008 & 1.330 & 0.137 & 0.12087948 \\
\hline 0.419 & 0.001 & 1.654 & 0.131 & 0.21296251 \\
\hline 0.219 & 0.006 & 1.175 & 0.222 & 0.3070521 \\
\hline 0.422 & 0.004 & 1.871 & 0.146 & 0.13190202 \\
\hline 0.367 & 0.003 & 1.642 & 0.152 & 0.11937729 \\
\hline 0.370 & 0.001 & 1.534 & 0.293 & 0.46135365 \\
\hline 0.425 & 0.002 & 2.027 & 0.131 & 0.13349947 \\
\hline 0.440 & 0.001 & 1.806 & 0.231 & 0.10437083 \\
\hline 0.401 & 0.001 & 1.920 & 0.173 & 0.18651215 \\
\hline 0.599 & 0.003 & 2.184 & 0.254 & 0.13334416 \\
\hline 0.456 & 0.003 & 2.243 & 0.216 & 0.07662962 \\
\hline 0.699 & 0.000 & 3.045 & 0.194 & 0.04970197 \\
\hline 0.129 & 0.005 & 0.231 & 0.087 & 0.18714867 \\
\hline 0.730 & 0.070 & 1.930 & 0.050 & 0.01 \\
\hline 0.770 & 0.060 & 2.270 & 0.040 & 0.01 \\
\hline 0.780 & 0.090 & 2.110 & 0.040 & 0.01 \\
\hline 0.820 & 0.090 & 1.470 & 0.020 & 0 \\
\hline 0.860 & 0.110 & 3.220 & 0.030 & 0 \\
\hline 0.730 & 0.070 & 2.750 & 0.090 & 0.02 \\
\hline 0.640 & 0.110 & 2.270 & 0.120 & 0.04 \\
\hline 0.690 & 0.050 & 2.580 & 0.140 & 0.04 \\
\hline 0.480 & 0.090 & 1.140 & 0.290 & 0.07 \\
\hline 0.490 & 0.040 & 2.000 & 0.260 & 0.09 \\
\hline
\end{tabular}




\begin{tabular}{|c|c|c|c|c|}
\hline 0.740 & 0.010 & 4.550 & 0.330 & 0.1 \\
\hline 0.470 & 0.040 & 1.760 & 0.430 & 0.14 \\
\hline 0.480 & 0.020 & 2.150 & 0.420 & 0.14 \\
\hline 0.480 & 0.030 & 1.990 & 0.380 & 0.11 \\
\hline 0.290 & 0.070 & 0.390 & 0.240 & 0.07 \\
\hline 0.420 & 0.010 & 1.860 & 0.330 & 0.13 \\
\hline 0.400 & 0.010 & 1.830 & 0.500 & 0.15 \\
\hline 0.570 & 0.010 & 3.340 & 0.400 & 0.13 \\
\hline 0.530 & 0.010 & 2.700 & 0.380 & 0.12 \\
\hline 0.640 & 0.010 & 3.870 & 0.400 & 0.12 \\
\hline 0.460 & 0.010 & 1.920 & 0.310 & 0.09 \\
\hline 0.440 & 0.360 & 0.990 & 0.590 & 0.19 \\
\hline 0.580 & 0.010 & 3.450 & 0.410 & 0.14 \\
\hline 0.530 & 0.150 & 2.480 & 0.770 & 0.41 \\
\hline 0.520 & 0.500 & 0.600 & 0.480 & 0.07 \\
\hline 0.460 & 0.430 & 0.750 & 0.330 & 0.09 \\
\hline 0.650 & 0.040 & 3.800 & 0.450 & 0.12 \\
\hline \multicolumn{5}{|l|}{0.450} \\
\hline \multicolumn{5}{|l|}{0.400} \\
\hline \multicolumn{5}{|l|}{0.320} \\
\hline \multicolumn{5}{|l|}{0.310} \\
\hline \multicolumn{5}{|l|}{0.780} \\
\hline \multicolumn{5}{|l|}{0.540} \\
\hline \multicolumn{5}{|l|}{0.390} \\
\hline \multicolumn{5}{|c|}{0.710} \\
\hline \multicolumn{5}{|c|}{0.450} \\
\hline \multicolumn{5}{|l|}{0.370} \\
\hline \multicolumn{5}{|l|}{0.560} \\
\hline \multicolumn{5}{|l|}{0.260} \\
\hline \multicolumn{5}{|l|}{0.630} \\
\hline \multicolumn{5}{|l|}{0.640} \\
\hline \multicolumn{5}{|l|}{0.410} \\
\hline \multicolumn{5}{|c|}{0.650} \\
\hline \multicolumn{5}{|l|}{0.290} \\
\hline \multicolumn{5}{|l|}{0.380} \\
\hline \multicolumn{5}{|l|}{0.500} \\
\hline \multicolumn{5}{|l|}{0.410} \\
\hline \multicolumn{5}{|l|}{0.210} \\
\hline \multicolumn{5}{|l|}{0.600} \\
\hline \multicolumn{5}{|l|}{0.200} \\
\hline 0.590 & & & & \\
\hline
\end{tabular}




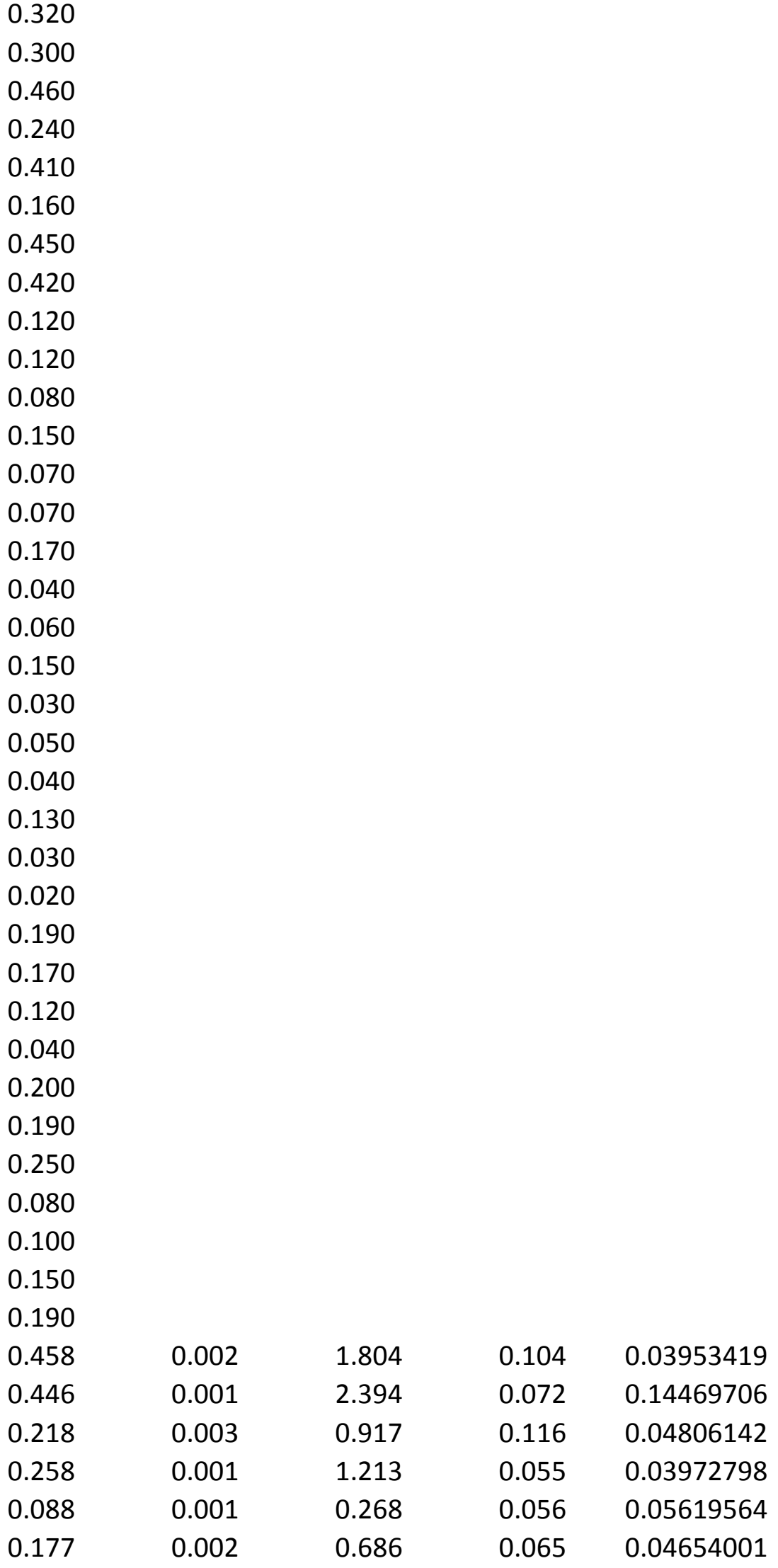




\begin{tabular}{|c|c|c|c|c|}
\hline 0.142 & 0.003 & 0.354 & 0.074 & 0.12370624 \\
\hline 0.147 & 0.002 & 0.463 & 0.084 & 0.07785007 \\
\hline 0.384 & 0.000 & 1.585 & 0.045 & 0.04910539 \\
\hline 0.194 & 0.002 & 0.556 & 0.058 & 0.09117904 \\
\hline 0.541 & 0.001 & 2.836 & 0.022 & 0.25014465 \\
\hline 0.389 & 0.001 & 1.076 & 0.037 & 0.07357508 \\
\hline 0.297 & 0.001 & 0.999 & 0.055 & 0.11842955 \\
\hline 0.266 & 0.002 & 0.865 & 0.050 & 0.09547272 \\
\hline 0.403 & 0.004 & 0.780 & 0.006 & 0.04408716 \\
\hline 0.575 & 0.247 & 1.344 & 0.238 & 0.02710287 \\
\hline 0.669 & 0.107 & 1.012 & 0.725 & 0.03326873 \\
\hline 0.469 & 0.068 & 1.056 & 0.157 & 0.02047853 \\
\hline 0.375 & 0.139 & 0.842 & 0.246 & 0.0336678 \\
\hline 0.435 & 0.102 & 1.235 & 0.282 & 0.03431959 \\
\hline 0.581 & 0.077 & 0.948 & 0.434 & 0.01760602 \\
\hline 0.644 & 0.064 & 1.397 & 0.388 & 0.02334613 \\
\hline 0.542 & 0.010 & 1.518 & 0.199 & 0.0359768 \\
\hline 0.551 & 0.049 & 1.984 & 0.091 & 0.0358871 \\
\hline 0.358 & 0.018 & 1.268 & 0.116 & 0.0643845 \\
\hline 0.729 & 0.078 & 1.364 & 0.360 & 0.02278016 \\
\hline 0.570 & 0.050 & 1.452 & 0.135 & 0.0303049 \\
\hline 0.564 & 0.011 & 0.749 & 0.025 & 0.0172884 \\
\hline 0.091 & 0.006 & 0.149 & 0.092 & 0.06127169 \\
\hline 0.128 & 0.110 & 0.291 & 0.123 & 0.06777896 \\
\hline 0.106 & 0.041 & 0.303 & 0.084 & 0.05390273 \\
\hline 0.476 & 0.040 & 1.315 & 0.086 & 0.04885759 \\
\hline 0.487 & 0.020 & 1.727 & 0.063 & 0.06209928 \\
\hline 0.508 & 0.042 & 1.756 & 0.164 & 0.09637391 \\
\hline 0.598 & 0.039 & 1.935 & 0.103 & 0.04212543 \\
\hline 0.579 & 0.106 & 1.175 & 0.113 & 0.05461689 \\
\hline 0.582 & 0.027 & 1.651 & 0.070 & 0.05287752 \\
\hline 0.594 & 0.054 & 1.829 & 0.198 & 0.08603652 \\
\hline 0.651 & 0.042 & 2.214 & 0.161 & 0.12404087 \\
\hline 0.811 & 0.057 & 2.188 & 0.095 & 0.02481443 \\
\hline 0.845 & 0.061 & 2.445 & 0.090 & 0.01826899 \\
\hline 0.632 & 0.144 & 0.871 & 0.070 & 0.00687564 \\
\hline 0.897 & 0.029 & 2.336 & 0.174 & 0.00659516 \\
\hline 0.748 & 0.084 & 1.036 & 0.076 & 0.00433123 \\
\hline 0.827 & 0.075 & 1.583 & 0.502 & 0.00387517 \\
\hline 0.840 & 0.041 & 1.853 & 0.138 & 0.00494859 \\
\hline 0.775 & 0.024 & 2.945 & 0.128 & 0.02895229 \\
\hline
\end{tabular}




\begin{tabular}{|c|c|c|c|c|}
\hline 0.889 & 0.045 & 3.170 & 0.068 & 0.01420186 \\
\hline 0.676 & 0.018 & 1.852 & 0.032 & 0.02135849 \\
\hline 0.380 & 0.027 & 0.384 & 0.022 & 0.00435574 \\
\hline 0.110 & 0.361 & 0.144 & 0.041 & 0.00478266 \\
\hline 0.646 & 0.027 & 0.893 & 0.052 & 0.00312576 \\
\hline 0.943 & 0.066 & 1.067 & 0.039 & 0.00431536 \\
\hline 0.898 & 0.026 & 1.469 & 0.174 & 0.00302642 \\
\hline 0.908 & 0.015 & 1.700 & 0.122 & 0.00224805 \\
\hline 0.784 & 0.002 & 2.074 & 0.024 & 0.00588546 \\
\hline 0.592 & 0.003 & 0.932 & 0.016 & 0.00299792 \\
\hline 0.384 & 0.006 & 0.427 & 0.009 & 0 \\
\hline 0.936 & 0.002 & 3.391 & 0.021 & 0.00648345 \\
\hline 0.635 & 0.009 & 0.878 & 0.019 & 0.00750529 \\
\hline 0.779 & 0.052 & 1.402 & 0.183 & 0.01725175 \\
\hline 0.839 & 0.172 & 0.861 & 0.126 & 0.0065235 \\
\hline 0.404 & 0.132 & 1.109 & 0.192 & 0.00949902 \\
\hline 0.806 & 0.119 & 0.988 & 0.077 & 0.00061901 \\
\hline 0.688 & 0.106 & 1.519 & 0.096 & 0.02070343 \\
\hline 0.319 & 0.305 & 0.109 & 0.021 & 0.00057127 \\
\hline 0.309 & 0.014 & 0.620 & 0.072 & 0.04143149 \\
\hline 0.184 & 0.037 & 0.334 & 0.060 & 0.0516718 \\
\hline 0.672 & 0.014 & 2.230 & 0.144 & 0.06387745 \\
\hline 0.486 & 0.010 & 1.811 & 0.076 & 0.04649527 \\
\hline 0.668 & 0.016 & 2.180 & 0.100 & 0.02590642 \\
\hline 0.762 & 0.036 & 2.382 & 0.166 & 0.02150609 \\
\hline 0.672 & 0.081 & 0.609 & 0.093 & 0.00745837 \\
\hline 0.482 & 0.008 & 0.346 & 0.036 & 0.00116444 \\
\hline 0.329 & 0.033 & 0.155 & 0.017 & 0.00208313 \\
\hline 0.730 & 0.008 & 0.280 & 0.091 & 0 \\
\hline 0.734 & 0.012 & 0.547 & 0.077 & 0 \\
\hline 0.814 & 0.002 & 2.192 & 0.125 & 0.00521224 \\
\hline 0.580 & 0.012 & 2.645 & 0.063 & 0.06588402 \\
\hline 0.475 & 0.020 & 0.675 & 0.112 & 0.0140398 \\
\hline 0.641 & 0.128 & 0.233 & 0.342 & 0.00608562 \\
\hline 0.761 & 0.012 & 2.272 & 0.212 & 0.02742723 \\
\hline 0.396 & 0.055 & 0.220 & 0.023 & 0.00060578 \\
\hline 0.131 & 0.006 & 0.339 & 0.095 & 0.0400659 \\
\hline 0.098 & 0.023 & 0.217 & 0.062 & 0.03267581 \\
\hline 0.315 & 0.004 & 0.932 & 0.100 & 0.03564034 \\
\hline 0.591 & 0.037 & 1.295 & 0.033 & 0.02600187 \\
\hline 0.589 & 0.022 & 0.856 & 0.031 & 0.02371558 \\
\hline
\end{tabular}




\begin{tabular}{|c|c|c|c|c|}
\hline 0.589 & 0.168 & 0.325 & 0.021 & 0.00217346 \\
\hline 0.126 & 0.117 & 0.279 & 0.150 & 0.04714227 \\
\hline 0.576 & 0.119 & 0.547 & 0.016 & 0.01017237 \\
\hline 0.092 & 0.032 & 0.167 & 0.054 & 0.02369744 \\
\hline 0.339 & 0.110 & 0.800 & 0.096 & 0.03208983 \\
\hline 0.424 & 0.714 & 0.141 & 0.255 & 0.0205908 \\
\hline 0.781 & 0.221 & 0.591 & 0.015 & 0.04589333 \\
\hline 0.797 & 0.081 & 1.699 & 0.024 & 0.03393359 \\
\hline 0.578 & 0.097 & 0.630 & 0.068 & 0.00738615 \\
\hline 0.333 & 0.113 & 0.751 & 0.221 & 0.03296788 \\
\hline 0.564 & 0.031 & 1.815 & 0.107 & 0.04494916 \\
\hline 0.220 & 0.003 & 0.951 & 0.102 & 0.05276024 \\
\hline 0.163 & 0.018 & 0.542 & 0.122 & 0.05949255 \\
\hline 0.177 & 0.012 & 0.593 & 0.143 & 0.04870762 \\
\hline 0.107 & 0.003 & 0.312 & 0.189 & 0.19066496 \\
\hline 0.099 & 0.007 & 0.228 & 0.064 & 0.02909111 \\
\hline 0.683 & 0.011 & 1.896 & 0.038 & 0.0314656 \\
\hline 0.663 & 0.028 & 2.129 & 0.195 & 0.03668135 \\
\hline 0.879 & 0.070 & 1.225 & 0.301 & 0.00277995 \\
\hline 0.394 & 0.000 & 0.764 & 0.025 & 0.01843426 \\
\hline 0.482 & 0.000 & 1.231 & 0.015 & 0.01041313 \\
\hline 0.506 & 0.000 & 1.517 & 0.018 & 0.02000683 \\
\hline 0.682 & 0.000 & 2.817 & 0.016 & 0.01115906 \\
\hline 0.487 & 0.000 & 1.739 & 0.018 & 0.03512465 \\
\hline 0.399 & 0.000 & 1.332 & 0.025 & 0.02217234 \\
\hline 0.251 & 0.000 & 0.933 & 0.050 & 0.13317007 \\
\hline 0.296 & 0.000 & 1.215 & 0.026 & 0.0474756 \\
\hline 0.363 & 0.000 & 0.691 & 0.010 & 0.01427943 \\
\hline 0.212 & 0.054 & 0.192 & 0.066 & 0.01801637 \\
\hline 0.541 & 0.098 & 1.422 & 0.115 & 0.04833514 \\
\hline 0.353 & 0.023 & 0.363 & 0.030 & 0.00723459 \\
\hline 0.439 & 0.013 & 1.661 & 0.071 & 0.06512337 \\
\hline 0.699 & 0.006 & 1.635 & 0.019 & 0.01521116 \\
\hline 0.431 & 0.016 & 0.397 & 0.021 & 0.00216216 \\
\hline 0.375 & 0.007 & 1.072 & 0.139 & 0.14538553 \\
\hline 0.268 & 0.002 & 0.324 & 0.157 & 0.03539944 \\
\hline 0.211 & 0.044 & 0.144 & 0.025 & 0.00382642 \\
\hline 0.335 & 0.036 & 0.845 & 0.097 & 0.08001967 \\
\hline 0.524 & 0.020 & 1.496 & 0.076 & 0.06345522 \\
\hline 0.476 & 0.004 & 0.980 & 0.094 & 0.03010862 \\
\hline 0.374 & 0.003 & 0.718 & 0.168 & 0.06491108 \\
\hline
\end{tabular}




\begin{tabular}{|c|c|c|c|c|}
\hline 0.287 & 0.011 & 0.251 & 0.017 & 0.01042835 \\
\hline 0.415 & 0.009 & 1.373 & 0.047 & 0.088116 \\
\hline 0.453 & 0.017 & 1.280 & 0.057 & 0.07424228 \\
\hline 0.281 & 0.016 & 0.732 & 0.053 & 0.07783024 \\
\hline 0.466 & 0.006 & 0.768 & 0.089 & 0.02665415 \\
\hline 0.637 & 0.001 & 1.533 & 0.186 & 0.02508162 \\
\hline 0.274 & 0.028 & 0.766 & 0.075 & 0.0748206 \\
\hline 0.338 & 0.046 & 1.340 & 0.126 & 0.11900233 \\
\hline 0.496 & 0.023 & 2.089 & 0.092 & 0.09352706 \\
\hline 0.367 & 0.093 & 1.579 & 0.194 & 0.17828547 \\
\hline 0.305 & 0.019 & 1.028 & 0.098 & 0.12448752 \\
\hline 0.340 & 0.018 & 1.266 & 0.095 & 0.12579529 \\
\hline 0.353 & 0.020 & 1.301 & 0.121 & 0.15399676 \\
\hline 0.188 & 0.011 & 0.231 & 0.029 & 0.03076796 \\
\hline 0.366 & 0.016 & 1.198 & 0.112 & 0.14164457 \\
\hline 0.487 & 0.024 & 1.148 & 0.038 & 0.0296215 \\
\hline 0.706 & 0.073 & 1.578 & 0.043 & 0.00871388 \\
\hline 0.356 & 0.234 & 0.209 & 0.016 & 0.00190757 \\
\hline 0.497 & 0.008 & 0.943 & 0.033 & 0.03259682 \\
\hline 0.303 & 0.016 & 0.869 & 0.066 & 0.06929395 \\
\hline 0.222 & 0.007 & 0.605 & 0.085 & 0.06543477 \\
\hline 0.261 & 0.006 & 0.643 & 0.093 & 0.06828396 \\
\hline 0.158 & 0.003 & 0.440 & 0.094 & 0.05893105 \\
\hline 0.124 & 0.000 & 0.191 & 0.017 & 0.02782127 \\
\hline 0.096 & 0.038 & 0.177 & 0.073 & 0.06229966 \\
\hline 0.178 & 0.002 & 0.613 & 0.033 & 0.06010495 \\
\hline 0.208 & 0.008 & 0.486 & 0.046 & 0.06251992 \\
\hline 0.116 & 0.063 & 0.285 & 0.040 & 0.03753956 \\
\hline 0.265 & 0.005 & 0.870 & 0.063 & 0.05333859 \\
\hline 0.180 & 0.030 & 0.554 & 0.109 & 0.07157926 \\
\hline 0.154 & 0.034 & 0.539 & 0.063 & 0.06509428 \\
\hline 0.160 & 0.003 & 0.459 & 0.058 & 0.0350185 \\
\hline 0.118 & 0.003 & 0.391 & 0.073 & 0.05868903 \\
\hline 0.197 & 0.037 & 0.830 & 0.069 & 0.05403094 \\
\hline 0.290 & 0.002 & 1.408 & 0.097 & 0.05681317 \\
\hline 0.094 & 0.007 & 0.395 & 0.089 & 0.05537825 \\
\hline 0.322 & 0.003 & 1.444 & 0.079 & 0.05169086 \\
\hline 0.111 & 0.004 & 0.501 & 0.063 & 0.05078718 \\
\hline 0.194 & 0.001 & 0.530 & 0.031 & 0.05264012 \\
\hline 0.411 & 0.000 & 1.317 & 0.025 & 0.05917397 \\
\hline 0.401 & 0.000 & 0.682 & 0.010 & 0.0169465 \\
\hline
\end{tabular}




\begin{tabular}{|c|c|c|c|c|}
\hline 0.335 & 0.000 & 1.217 & 0.030 & 0.04625244 \\
\hline 0.331 & 0.000 & 1.484 & 0.036 & 0.06403949 \\
\hline 0.158 & 0.003 & 0.238 & 0.029 & 0.16793474 \\
\hline 0.134 & 0.002 & 0.236 & 0.026 & 0.02213711 \\
\hline 0.372 & 0.000 & 1.305 & 0.021 & 0.09808017 \\
\hline 0.507 & 0.000 & 1.774 & 0.032 & 0.04412287 \\
\hline 0.495 & 0.000 & 1.774 & 0.020 & 0.03645804 \\
\hline 0.305 & 0.000 & 0.688 & 0.063 & 0.02978042 \\
\hline 0.285 & 0.000 & 0.675 & 0.020 & 0.03232905 \\
\hline 0.108 & 0.005 & 0.275 & 0.015 & 0.02615093 \\
\hline 0.309 & 0.000 & 1.345 & 0.006 & 0.02315789 \\
\hline 0.359 & 0.000 & 2.065 & 0.019 & 0.03821138 \\
\hline 0.362 & 0.000 & 1.630 & 0.003 & 0.02371795 \\
\hline 0.428 & 0.003 & 3.218 & 0.010 & 0.03705104 \\
\hline 0.850 & 0.001 & 0.048 & 1.212 & 0 \\
\hline 0.509 & 0.001 & 1.285 & 0.043 & 0.03984674 \\
\hline 0.545 & 0.001 & 1.340 & 0.008 & 0.06070039 \\
\hline 0.555 & 0.001 & 1.334 & 0.014 & 0.06072106 \\
\hline 0.709 & 0.003 & 0.853 & 0.033 & 0.02057613 \\
\hline 0.647 & 0.002 & 2.990 & 0.053 & 0.09365079 \\
\hline 0.562 & 0.002 & 4.233 & 0.044 & 0.09982548 \\
\hline 0.656 & 0.001 & 3.488 & 0.027 & 0.44232082 \\
\hline 0.679 & 0.000 & 4.781 & 0.054 & 0.33752535 \\
\hline 0.543 & 0.001 & 3.438 & 0.031 & 0.25424431 \\
\hline 0.571 & 0.001 & 3.530 & 0.050 & 0.36672566 \\
\hline 0.722 & 0.165 & 3.546 & 0.052 & 0.07480315 \\
\hline 0.332 & 0.001 & 2.353 & 0.011 & 0.11352254 \\
\hline 0.439 & 0.002 & 6.515 & 0.007 & 0.13493014 \\
\hline 0.599 & 0.002 & 3.164 & 0.000 & 0.00371747 \\
\hline 0.237 & 0.003 & 0.835 & 0.032 & 0.0053068 \\
\hline 0.398 & 0.001 & 1.507 & 0.054 & 0.02023346 \\
\hline 0.094 & 0.004 & 0.142 & 0.011 & 0 \\
\hline 0.087 & 0.010 & 0.214 & 0.006 & 0 \\
\hline 0.087 & 0.008 & 0.274 & 0.024 & 0 \\
\hline 0.137 & 0.000 & 0.332 & 0.030 & 0.00246407 \\
\hline 0.165 & 0.001 & 0.401 & 0.018 & 0 \\
\hline 0.209 & 0.001 & 1.023 & 0.023 & 0 \\
\hline 0.460 & 0.000 & 4.086 & 0.027 & 0.25901639 \\
\hline 0.373 & 0.001 & 10.116 & 0.031 & 0.19634888 \\
\hline 0.401 & 0.000 & 14.017 & 0.052 & 0.45583174 \\
\hline 0.066 & 0.000 & 0.200 & 0.067 & 0.06286517 \\
\hline
\end{tabular}




\begin{tabular}{|c|c|c|c|c|}
\hline 0.055 & 0.000 & 0.107 & 0.059 & 0.04970999 \\
\hline 0.088 & 0.001 & 0.294 & 0.057 & 0.06062286 \\
\hline 0.074 & 0.000 & 0.207 & 0.081 & 0.06850642 \\
\hline 0.062 & 0.000 & 0.162 & 0.065 & 0.06392091 \\
\hline 0.052 & 0.000 & 0.116 & 0.074 & 0.05978736 \\
\hline 0.060 & 0.000 & 0.091 & 0.092 & 0.07196429 \\
\hline 0.052 & 0.000 & 0.146 & 0.075 & 0.0547389 \\
\hline 0.058 & 0.000 & 0.161 & 0.075 & 0.06445044 \\
\hline 0.068 & 0.002 & 0.133 & 0.126 & 0.09135 \\
\hline 0.052 & 0.000 & 0.127 & 0.073 & 0.05908639 \\
\hline 0.050 & 0.000 & 0.088 & 0.085 & 0.0690295 \\
\hline 0.056 & 0.000 & 0.164 & 0.057 & 0.05522756 \\
\hline 0.058 & 0.001 & 0.157 & 0.073 & 0.04774383 \\
\hline 0.040 & 0.000 & 0.126 & 0.052 & 0.03561651 \\
\hline 0.049 & 0.000 & 0.128 & 0.063 & 0.05597572 \\
\hline 0.044 & 0.000 & 0.093 & 0.071 & 0.05056654 \\
\hline 0.049 & 0.001 & 0.124 & 0.055 & 0.03782261 \\
\hline 0.053 & 0.000 & 0.161 & 0.068 & 0.03932846 \\
\hline 0.066 & 0.000 & 0.197 & 0.065 & 0.045039 \\
\hline 0.030 & 0.000 & 0.071 & 0.050 & 0.02959877 \\
\hline 0.044 & 0.000 & 0.114 & 0.053 & 0.03018487 \\
\hline 0.043 & 0.000 & 0.115 & 0.043 & 0.03124509 \\
\hline 0.042 & 0.000 & 0.103 & 0.063 & 0.03127048 \\
\hline 0.074 & 0.000 & 0.247 & 0.059 & 0.04282206 \\
\hline 0.075 & 0.000 & 0.267 & 0.056 & 0.03560959 \\
\hline 0.041 & 0.000 & 0.102 & 0.057 & 0.0415081 \\
\hline 0.037 & 0.000 & 0.085 & 0.058 & 0.03833753 \\
\hline 0.053 & 0.000 & 0.140 & 0.068 & 0.04588698 \\
\hline 0.035 & 0.000 & 0.059 & 0.065 & 0.03959237 \\
\hline 0.062 & 0.000 & 0.181 & 0.062 & 0.03635501 \\
\hline 0.046 & 0.000 & 0.071 & 0.078 & 0.05812738 \\
\hline 0.051 & 0.003 & 0.088 & 0.093 & 0.06690223 \\
\hline 0.051 & 0.000 & 0.127 & 0.050 & 0.04877474 \\
\hline 0.071 & 0.006 & 0.201 & 0.059 & 0.04167863 \\
\hline 0.094 & 0.006 & 0.221 & 0.157 & 0.03578816 \\
\hline 0.087 & 0.001 & 0.254 & 0.058 & 0.05088677 \\
\hline 0.145 & 0.002 & 0.403 & 0.041 & 0.02568381 \\
\hline 0.086 & 0.010 & 0.103 & 0.039 & 0.0120617 \\
\hline 0.095 & 0.000 & 0.103 & 0.038 & 0.01031467 \\
\hline 0.047 & 0.000 & 0.095 & 0.085 & 0.05041167 \\
\hline 0.035 & 0.000 & 0.097 & 0.063 & 0.04010323 \\
\hline
\end{tabular}




\begin{tabular}{|c|c|c|c|c|}
\hline 0.061 & 0.000 & 0.211 & 0.076 & 0.08191117 \\
\hline 0.213 & 0.000 & 0.743 & 0.024 & 0.1003883 \\
\hline 0.163 & 0.005 & 0.362 & 0.047 & 0.05713177 \\
\hline 0.178 & 0.000 & 0.532 & 0.022 & 0.14008994 \\
\hline 0.184 & 0.000 & 0.535 & 0.024 & 0.12486907 \\
\hline 0.167 & 0.000 & 0.490 & 0.020 & 0.10330778 \\
\hline 0.300 & 0.000 & 1.033 & 0.020 & 0.23104542 \\
\hline 0.524 & 0.039 & 0.943 & 0.104 & 0.04633279 \\
\hline 0.586 & 0.354 & 0.521 & 0.208 & 0.08101419 \\
\hline 0.494 & 0.071 & 0.548 & 0.079 & 0.0434849 \\
\hline 0.711 & 0.411 & 0.422 & 0.376 & 0.10165704 \\
\hline 0.817 & 0.299 & 0.577 & 0.209 & 0.03413077 \\
\hline 0.359 & 0.038 & 0.120 & 0.729 & 0.05929831 \\
\hline 0.709 & 0.350 & 0.626 & 1.145 & 0.0550012 \\
\hline 0.522 & 0.374 & 0.851 & 0.236 & 0.06669555 \\
\hline 0.169 & 0.031 & 0.129 & 0.380 & 0.18819447 \\
\hline 0.225 & 0.468 & 0.129 & 0.367 & 0.12613424 \\
\hline 0.398 & 0.544 & 0.149 & 0.338 & 0.11428258 \\
\hline 0.215 & 0.221 & 0.135 & 0.310 & 0.19464811 \\
\hline 0.126 & 0.137 & 0.099 & 0.182 & 0.11211033 \\
\hline 0.270 & 0.057 & 0.530 & 0.251 & 0.19015911 \\
\hline 0.220 & 0.003 & 0.619 & 0.128 & 0.20358032 \\
\hline 0.452 & 0.421 & 0.240 & 0.556 & 0.11078224 \\
\hline 0.610 & 0.011 & 0.663 & 1.301 & 0.07592765 \\
\hline 0.415 & 0.224 & 0.638 & 0.228 & 0.11201757 \\
\hline 0.631 & 0.098 & 0.709 & 1.706 & 0.07185328 \\
\hline 0.349 & 0.024 & 0.770 & 0.542 & 0.19158617 \\
\hline 0.300 & 0.430 & 0.135 & 0.377 & 0.11443017 \\
\hline 0.790 & 0.590 & 0.140 & 0.660 & 0.02 \\
\hline 0.850 & 0.500 & 0.800 & 0.520 & 0.09 \\
\hline 0.690 & 0.850 & 0.060 & 0.220 & 0 \\
\hline 0.760 & 0.670 & 0.010 & 0.010 & 0 \\
\hline 0.750 & 0.800 & 0.200 & 0.130 & 0.16 \\
\hline 0.710 & 0.810 & 0.010 & 0.300 & 0.01 \\
\hline 0.860 & 0.760 & 0.010 & 0.650 & 0 \\
\hline 0.990 & 0.630 & 0.010 & 0.170 & 0 \\
\hline 0.960 & 0.690 & 0.030 & 0.890 & 0.01 \\
\hline 0.710 & 0.900 & 0.050 & 0.080 & 0.13 \\
\hline 0.670 & 0.680 & 0.030 & 0.890 & 0.01 \\
\hline 0.990 & 0.840 & 0.000 & 0.120 & 0 \\
\hline 0.540 & 0.910 & 0.040 & 0.110 & 0.02 \\
\hline
\end{tabular}




\begin{tabular}{|c|c|c|c|c|}
\hline 0.970 & 0.600 & 0.060 & 1.100 & 0 \\
\hline 0.980 & 0.550 & 0.060 & 0.920 & 0 \\
\hline 0.950 & 0.400 & 0.110 & 0.240 & 0 \\
\hline 0.990 & 0.700 & 0.040 & 0.060 & 0.01 \\
\hline 0.360 & 0.070 & 0.050 & 0.020 & 0.01 \\
\hline 0.360 & 0.230 & 0.020 & 0.020 & 0.01 \\
\hline 0.910 & 0.620 & 0.040 & 0.460 & 0 \\
\hline 0.920 & 0.540 & 0.050 & 1.330 & 0.01 \\
\hline 0.980 & 0.750 & 0.050 & 0.850 & 0.01 \\
\hline 0.590 & 0.830 & 0.140 & 0.100 & 0 \\
\hline 0.660 & 0.120 & 0.160 & 0.060 & 0.01 \\
\hline 0.340 & 0.010 & 0.090 & 0.020 & 0 \\
\hline 0.510 & 0.440 & 0.200 & 0.500 & 0.09 \\
\hline 0.680 & 0.180 & 0.900 & 0.580 & 0.06 \\
\hline 0.650 & 0.340 & 0.500 & 0.480 & 0.05 \\
\hline 0.750 & 0.390 & 0.010 & 0.820 & 0.01 \\
\hline 0.600 & 0.740 & 0.030 & 0.410 & 0.04 \\
\hline 0.860 & 0.320 & 0.010 & 0.520 & 0 \\
\hline 0.820 & 0.350 & 0.350 & 0.440 & 0.02 \\
\hline 0.370 & 0.690 & 0.010 & 0.260 & 0.02 \\
\hline 0.810 & 0.010 & 1.750 & 0.220 & 0.06 \\
\hline 0.990 & 0.230 & 0.010 & 0.100 & 0 \\
\hline 0.820 & 0.740 & 0.080 & 0.320 & 0.01 \\
\hline 1.000 & 0.250 & 0.010 & 0.240 & 0 \\
\hline 0.850 & 0.260 & 0.040 & 0.300 & 0 \\
\hline 1.000 & 0.150 & 0.010 & 0.620 & 0 \\
\hline 0.930 & 0.370 & 0.050 & 0.040 & 0 \\
\hline 0.495 & 0.851 & & & \\
\hline 0.552 & 0.886 & & & \\
\hline 0.411 & 0.832 & & & \\
\hline 0.437 & 0.889 & & & \\
\hline 0.508 & 0.854 & & & \\
\hline 0.800 & 0.830 & 0.200 & 0.000 & 0 \\
\hline 0.740 & 0.970 & 0.000 & 0.000 & 0 \\
\hline 0.880 & 0.010 & 1.300 & 0.000 & 0.2 \\
\hline 0.550 & 1.000 & 0.000 & 0.000 & 0 \\
\hline 0.240 & 0.990 & 0.000 & 0.000 & 0 \\
\hline 0.560 & 0.770 & 0.200 & 0.000 & 0 \\
\hline 0.510 & 0.710 & 0.300 & 0.000 & 0.1 \\
\hline 0.180 & 0.000 & 0.100 & 0.000 & 0.1 \\
\hline 0.090 & 0.000 & 0.000 & 0.000 & 0.1 \\
\hline
\end{tabular}




$\begin{array}{lllll}0.100 & 0.000 & 0.100 & 0.000 & 0.1 \\ 0.430 & 0.590 & 0.400 & 0.000 & 0.1 \\ 0.360 & 0.670 & 0.200 & 0.000 & 0.1 \\ 0.260 & 0.800 & 0.000 & 0.000 & 0.1 \\ 0.330 & 0.480 & 0.300 & 0.000 & 0.1 \\ 0.440 & 0.730 & 0.200 & 0.000 & 0.1 \\ 0.480 & 0.670 & 0.400 & 0.000 & 0.1 \\ 0.330 & 0.750 & 0.200 & 0.000 & 0.1 \\ 0.320 & 0.850 & 0.100 & 0.000 & 0.1 \\ 0.380 & 0.020 & 0.700 & 0.000 & 0.4 \\ 0.480 & 0.260 & 0.400 & 0.000 & 0.2 \\ 0.540 & 0.030 & 0.400 & 0.000 & 0.2 \\ 0.140 & 0.240 & 0.100 & 0.000 & 0.1 \\ 0.390 & 0.880 & 0.000 & 0.000 & 0.1 \\ 0.320 & 0.730 & 0.100 & 0.000 & 0.2 \\ 0.320 & 0.770 & 0.200 & 0.000 & 0.1 \\ 0.320 & 0.800 & 0.100 & 0.000 & 0.1 \\ 0.260 & 0.040 & 0.200 & 0.000 & 0.2 \\ 0.320 & 0.010 & 0.400 & 0.000 & 0.3 \\ 0.290 & 0.530 & 0.200 & 0.000 & 0.1 \\ 0.470 & 0.010 & 1.100 & 0.000 & 0.4 \\ 0.850 & 0.700 & 0.050 & 0.510 & 0.03 \\ 0.600 & 0.550 & 0.050 & 0.480 & 0.06 \\ 0.640 & 0.670 & 0.050 & 0.450 & 0.08 \\ 0.560 & 0.550 & 0.050 & 0.560 & 0.08 \\ 0.560 & 0.720 & 0.050 & 0.360 & 0.11 \\ 0.530 & 0.550 & 0.050 & 0.470 & 0.11 \\ 0.540 & 0.480 & 0.050 & 0.510 & 0.11 \\ 0.920 & 0.010 & 1.200 & 0.160 & 0.1 \\ 0.810 & 0.000 & 2.430 & 0.120 & 0.1 \\ 0.650 & 0.000 & 0.370 & 0.220 & 0.03 \\ 0.590 & 0.000 & 0.740 & 0.130 & 0.09 \\ 0.750 & 0.000 & 0.890 & 0.280 & 0.06 \\ 0.610 & 0.040 & 0.770 & 0.150 & 0.07 \\ 0.670 & 0.000 & 0.460 & 0.340 & 0.05 \\ 0.680 & 0.010 & 0.510 & 0.280 & 0.05 \\ 0.720 & 0.000 & 0.840 & 0.230 & 0.12 \\ 0.700 & 0.010 & 0.350 & 0.490 & 0.01 \\ 0.540 & 0.000 & 0.490 & 0.220 & 0.05 \\ 0.580 & 0.000 & 0.440 & 0.290 & 0.07 \\ 0.710 & 0.000 & 0.830 & 0.330 & 0.07\end{array}$




$\begin{array}{lllll}0.730 & 0.000 & 1.000 & 0.390 & 0.07 \\ 0.930 & 0.000 & 1.540 & 0.620 & 0.34 \\ 0.780 & 0.000 & 1.060 & 0.400 & 0.05 \\ 0.830 & 0.000 & 1.610 & 0.480 & 0.07 \\ 0.830 & 0.000 & 0.930 & 0.340 & 0.02 \\ 0.700 & 0.000 & 0.640 & 0.320 & 0.07 \\ 0.500 & 0.010 & 0.190 & 0.190 & 0.02 \\ 0.780 & 0.010 & 0.660 & 0.440 & 0.05 \\ 0.690 & 0.010 & 0.600 & 0.330 & 0.11 \\ 0.710 & 0.010 & 0.770 & 0.260 & 0.08 \\ 0.760 & 0.010 & 0.760 & 0.340 & 0.03 \\ 0.790 & 0.010 & 1.010 & 0.240 & 0.04 \\ 0.480 & 0.010 & 0.340 & 0.150 & 0.08 \\ 0.620 & 0.020 & 0.470 & 0.140 & 0.04 \\ 0.570 & 0.010 & 0.460 & 0.170 & 0.07 \\ 0.610 & 0.010 & 0.680 & 0.200 & 0.14 \\ 0.450 & 0.010 & 0.410 & 0.140 & 0.15 \\ 0.510 & 0.010 & 0.810 & 0.190 & 0.19 \\ 0.470 & 0.010 & 0.360 & 0.190 & 0.13 \\ 0.560 & 0.010 & 0.550 & 0.160 & 0.14 \\ 0.490 & 0.010 & 0.530 & 0.190 & 0.11 \\ 0.530 & 0.010 & 0.510 & 0.200 & 0.21 \\ 0.630 & 0.010 & 0.890 & 0.230 & 0.12 \\ 0.480 & 0.020 & 0.410 & 0.200 & 0.2 \\ 0.520 & 0.010 & 0.710 & 0.280 & 0.2 \\ 0.510 & 0.010 & 0.600 & 0.240 & 0.15 \\ 0.560 & 0.010 & 0.470 & 0.320 & 0.12 \\ 0.460 & 0.010 & 0.290 & 0.190 & 0.16 \\ 0.410 & 0.010 & 0.850 & 0.330 & 0.25 \\ 0.520 & 0.000 & 0.600 & 0.310 & 0.41 \\ 0.540 & 0.010 & 0.280 & 0.330 & 0.25 \\ 0.550 & 0.010 & 0.170 & 0.390 & 0.25 \\ 0.610 & 0.010 & 0.260 & 0.460 & 0.23 \\ 0.630 & 0.010 & 0.520 & 0.430 & 0.36 \\ 0.490 & 0.010 & 0.530 & 0.500 & 0.16 \\ 0.540 & 0.090 & 0.300 & 0.650 & 0.19 \\ 0.430 & 0.180 & 0.230 & 0.410 & 0.14 \\ 0.560 & 0.010 & 0.220 & 0.310 & 0.2 \\ 0.550 & 0.000 & 0.770 & 0.430 & 0.25 \\ 0.520 & 0.010 & 0.490 & 0.500 & 0.27 \\ 0.460 & 0.000 & 0.250 & 0.530 & 0.22\end{array}$




$\begin{array}{lllll}0.540 & 0.010 & 0.250 & 0.440 & 0.28 \\ 0.620 & 0.020 & 0.470 & 0.580 & 0.24 \\ 0.530 & 0.020 & 0.430 & 0.550 & 0.23 \\ 0.580 & 0.020 & 0.350 & 0.440 & 0.2 \\ 0.490 & 0.090 & 0.280 & 0.490 & 0.21 \\ 0.560 & 0.000 & 0.970 & 0.560 & 0.25 \\ 0.610 & 0.020 & 0.430 & 0.440 & 0.18 \\ 0.590 & 0.010 & 0.510 & 0.370 & 0.16 \\ 0.720 & 0.000 & 0.450 & 0.490 & 0.22 \\ 0.570 & 0.000 & 0.400 & 0.590 & 0.28 \\ 0.590 & 0.010 & 0.380 & 0.440 & 0.21 \\ 0.560 & 0.080 & 0.220 & 0.390 & 0.24 \\ 0.510 & 0.000 & 0.330 & 0.440 & 0.24 \\ 0.520 & 0.070 & 0.270 & 0.430 & 0.23 \\ 0.510 & 0.010 & 0.160 & 0.370 & 0.18 \\ 0.540 & 0.010 & 0.620 & 0.700 & 0.19 \\ 0.580 & 0.010 & 0.450 & 0.390 & 0.15 \\ 0.480 & 0.000 & 0.350 & 0.540 & 0.37 \\ 0.570 & 0.070 & 0.860 & 0.640 & 0.13 \\ 0.600 & 0.000 & 0.400 & 0.480 & 0.18 \\ 0.510 & 0.010 & 0.370 & 0.430 & 0.2 \\ 0.520 & 0.040 & 0.680 & 0.820 & 0.15 \\ 0.550 & 0.010 & 0.330 & 0.410 & 0.18 \\ 0.580 & 0.010 & 0.540 & 0.620 & 0.24 \\ 0.360 & 0.090 & 0.340 & 0.480 & 0.09 \\ 0.590 & 0.000 & 1.240 & 1.180 & 0.21 \\ 0.550 & 0.050 & 0.350 & 0.770 & 0.22 \\ 0.500 & 0.000 & 0.580 & 0.570 & 0.32 \\ 0.410 & 0.000 & 0.340 & 0.600 & 0.33 \\ 0.510 & 0.000 & 1.160 & 0.800 & 0.21 \\ 0.500 & 0.080 & 0.400 & 0.290 & 0.04 \\ 0.410 & 0.010 & 0.450 & 0.580 & 0.22 \\ 0.490 & 0.050 & 0.710 & 0.620 & 0.25 \\ 0.470 & 0.010 & 0.660 & 0.570 & 0.32 \\ 0.400 & 0.000 & 1.310 & 0.610 & 0.18 \\ 0.430 & 0.010 & 0.290 & 0.530 & 0.32 \\ 0.460 & 0.050 & 0.470 & 0.500 & 0.08 \\ 0.360 & 0.090 & 0.600 & 0.670 & 0.18 \\ 0.330 & 0.070 & 0.730 & 0.550 & 0.16 \\ 0.410 & 0.060 & 0.730 & 0.580 & 0.16 \\ 0.020 & 0.790 & 0.560 & 0.17\end{array}$




$\begin{array}{lllll}0.350 & 0.130 & 0.630 & 0.600 & 0.15 \\ 0.350 & 0.170 & 0.070 & 0.690 & 0.07 \\ 0.230 & 0.080 & 0.160 & 0.630 & 0.11 \\ 0.300 & 0.160 & 0.240 & 0.630 & 0.14 \\ 0.280 & 0.150 & 0.250 & 0.640 & 0.13 \\ 0.470 & 0.010 & 0.160 & 0.480 & 0.3 \\ 0.350 & 0.180 & 0.090 & 0.620 & 0.06 \\ 0.370 & 0.470 & 0.110 & 0.570 & 0.14 \\ 0.350 & 0.500 & 0.100 & 0.500 & 0.13 \\ 0.390 & 0.460 & 0.140 & 0.660 & 0.15 \\ 0.300 & 0.420 & 0.120 & 0.560 & 0.15 \\ 0.300 & 0.240 & 0.120 & 0.660 & 0.18 \\ 0.380 & 0.220 & 0.290 & 0.760 & 0.18 \\ 0.300 & 0.020 & 0.300 & 0.760 & 0.21 \\ 0.340 & 0.220 & 0.210 & 0.750 & 0.2 \\ 0.220 & 0.010 & 0.140 & 0.630 & 0.17 \\ 0.520 & 0.010 & 0.320 & 0.550 & 0.29 \\ 0.210 & 0.110 & 0.040 & 0.660 & 0.12 \\ 0.330 & 0.020 & 0.170 & 0.760 & 0.14 \\ 0.410 & 0.010 & 0.310 & 0.440 & 0.18 \\ 0.530 & 0.010 & 0.160 & 0.440 & 0.29 \\ 0.540 & 0.020 & 0.220 & 0.480 & 0.18 \\ 0.930 & 0.020 & 0.580 & 0.050 & 0.03 \\ 0.920 & 0.020 & 0.900 & 0.070 & 0.02 \\ 0.950 & 0.030 & 1.520 & 0.100 & 0.03 \\ 0.920 & 0.060 & 0.260 & 0.060 & 0.01 \\ 0.960 & 0.060 & 0.300 & 0.230 & 0 \\ 0.760 & 0.020 & 0.460 & 0.270 & 0.03 \\ 0.680 & 0.020 & 0.470 & 0.110 & 0.01 \\ 0.550 & 0.020 & 0.240 & 0.080 & 0 \\ 0.330 & 0.070 & 0.080 & 0.060 & 0 \\ 0.910 & 0.010 & 1.560 & 0.140 & 0.04 \\ 0.930 & 0.530 & 0.590 & 0.140 & 0.04 \\ 0.370 & 0.410 & 0.880 & 1.790 & 0.14 \\ 0.960 & 0.860 & 0.800 & 0.470 & 0.03 \\ 0.720 & 0.030 & 0.460 & 0.270 & 0.03 \\ 0.900 & 0.020 & 1.020 & 0.420 & 0.03 \\ 0.670 & 0.050 & 0.210 & 0.050 & 0.01 \\ 0.210 & 0.000 & 0.170 & 0.000 & 0.01 \\ 0.240 & 0.160 & 0.170 & 0.010 & 0.01 \\ 0.010 & 0.070 & 0.000 & 0.01\end{array}$




$\begin{array}{llllc}0.340 & 0.540 & 0.560 & 0.090 & 0.07 \\ 0.610 & 0.060 & 0.790 & 0.110 & 0.07 \\ 1.010 & 0.180 & 0.250 & 0.000 & 0.01 \\ 1.010 & 0.120 & 0.330 & 0.000 & 0.03 \\ 0.860 & 0.190 & 0.390 & 0.000 & 0.02 \\ 1.000 & 0.110 & 0.530 & 0.020 & 0.04 \\ 0.890 & 0.200 & 0.320 & 0.010 & 0.01 \\ 0.910 & 0.220 & 0.290 & 0.010 & 0.02 \\ 0.850 & 0.350 & 0.210 & 0.010 & 0.02 \\ 0.950 & 0.200 & 0.270 & 0.000 & 0.01 \\ 0.950 & 0.250 & 0.220 & 0.000 & 0.02 \\ 0.970 & 0.130 & 0.210 & 0.000 & 0.02 \\ 0.790 & 0.800 & 0.440 & 0.020 & 0.01 \\ 0.990 & 0.230 & 0.200 & 0.000 & 0.01 \\ 0.640 & 0.290 & 1.070 & 0.160 & 0.11 \\ 0.970 & 0.520 & 0.650 & 0.010 & 0.03 \\ 0.980 & 0.590 & 0.760 & 0.080 & 0.06 \\ 0.910 & 0.630 & 0.220 & 0.010 & 0.02 \\ 0.760 & 0.690 & 0.550 & 0.130 & 0.05 \\ 0.950 & 0.270 & 0.510 & 0.060 & 0.06 \\ 0.880 & 0.230 & 0.700 & 0.080 & 0.03 \\ 1.000 & 0.210 & 1.410 & 0.650 & 0.11 \\ 1.040 & 0.260 & 0.450 & 0.000 & 0.02 \\ 0.990 & 0.260 & 1.020 & 0.000 & 0.02 \\ 0.530 & 0.000 & 0.530 & 0.000 & 0.01 \\ 0.429 & 0.005 & 0.605 & 0.046 & 0.00227818 \\ 0.647 & 0.002 & 2.034 & 0.035 & 0.0160588 \\ 0.728 & 0.233 & 1.174 & 0.755 & 0.00948427 \\ 0.333 & 0.160 & 0.693 & 0.127 & 0.07593816 \\ 0.538 & 0.216 & 0.918 & 0.402 & 0.06016083 \\ 0.436 & 0.081 & 1.074 & 0.172 & 0.07697139 \\ 0.406 & 0.444 & 0.484 & 0.277 & 0.07891843 \\ 0.441 & 0.472 & 0.393 & 0.517 & 0.0872721 \\ 0.499 & 0.364 & 0.692 & 0.393 & 0.0807536 \\ 0.322 & 0.524 & 0.194 & 0.253 & 0.09744896 \\ 0.239 & 0.387 & 0.225 & 0.197 & 0.07564014 \\ 0.339 & 0.121 & 0.529 & 0.319 & 0.0784626 \\ 0.324 & 0.373 & 0.354 & 0.193 & 0.08097336 \\ 0.806 & 0.094 & 0.623 & 2.401 & 0.02046771 \\ 0.526 & 0.435 & 0.160 & 0.663 & 0.02083629 \\ 0.682 & 0.131 & 0.740 & 1.028 & 0.0196105\end{array}$




\begin{tabular}{|c|c|c|c|c|}
\hline 0.355 & 0.008 & 0.669 & 0.075 & 0.02389267 \\
\hline 0.273 & 0.378 & 0.237 & 0.089 & 0.01871744 \\
\hline 0.745 & 0.064 & 1.243 & 0.031 & 0.00948466 \\
\hline 0.441 & 0.003 & 0.482 & 0.628 & 0.10753704 \\
\hline 0.143 & 0.298 & 0.023 & 0.347 & 0.04652508 \\
\hline 0.220 & 0.068 & 0.023 & 0.606 & 0.07175751 \\
\hline 0.187 & 0.051 & 0.023 & 0.635 & 0.05427204 \\
\hline 0.288 & 0.067 & 0.026 & 0.836 & 0.10847048 \\
\hline 0.253 & 0.062 & 0.025 & 0.882 & 0.08392487 \\
\hline 0.235 & 0.104 & 0.037 & 0.752 & 0.15402392 \\
\hline 0.176 & 0.018 & 0.117 & 0.428 & 0.09177467 \\
\hline 0.370 & 0.109 & 0.035 & 0.946 & 0.09848863 \\
\hline 0.171 & 0.092 & 0.032 & 0.519 & 0.05199023 \\
\hline 0.179 & 0.080 & 0.025 & 0.572 & 0.04821526 \\
\hline 0.205 & 0.108 & 0.052 & 0.502 & 0.03587869 \\
\hline 0.334 & 0.019 & 0.068 & 0.764 & 0.0914178 \\
\hline 0.231 & 0.018 & 0.052 & 0.537 & 0.05383345 \\
\hline 0.284 & 0.015 & 0.116 & 0.584 & 0.05895953 \\
\hline 0.216 & 0.061 & 0.061 & 0.438 & 0.04390125 \\
\hline 0.354 & 0.046 & 0.059 & 0.732 & 0.06365927 \\
\hline 0.333 & 0.034 & 0.066 & 1.399 & 0.12649363 \\
\hline 0.079 & 0.032 & 0.204 & 0.130 & 0.05366287 \\
\hline 0.291 & 0.003 & 0.284 & 0.437 & 0.11014103 \\
\hline 0.140 & 0.039 & 0.113 & 0.342 & 0.01789258 \\
\hline 0.349 & 0.043 & 0.051 & 0.737 & 0.01966359 \\
\hline 0.586 & 0.001 & 0.245 & 1.126 & 0.01180199 \\
\hline 0.179 & 0.025 & 0.075 & 0.472 & 0.01191288 \\
\hline 0.552 & 0.007 & 0.108 & 1.065 & 0.02263182 \\
\hline 0.406 & 0.004 & 0.151 & 0.883 & 0.01246449 \\
\hline 0.654 & 0.005 & 0.257 & 1.187 & 0.01321391 \\
\hline 0.329 & 0.007 & 0.156 & 0.801 & 0.03947693 \\
\hline 0.326 & 0.009 & 0.214 & 0.682 & 0.02121109 \\
\hline 0.409 & 0.007 & 0.170 & 0.680 & 0.02422535 \\
\hline 0.536 & 0.006 & 0.149 & 0.798 & 0.02872253 \\
\hline 0.377 & 0.006 & 0.270 & 0.426 & 0.02064323 \\
\hline 0.324 & 0.006 & 0.225 & 0.372 & 0.04591957 \\
\hline 0.367 & 0.012 & 0.721 & 0.379 & 0.02131375 \\
\hline 0.347 & 0.010 & 0.249 & 0.550 & 0.03280081 \\
\hline 0.354 & 0.074 & 0.153 & 0.634 & 0.01476741 \\
\hline 0.376 & 0.054 & 0.126 & 0.564 & 0.01098599 \\
\hline 0.389 & 0.018 & 0.135 & 0.662 & 0.01968872 \\
\hline
\end{tabular}




\begin{tabular}{|c|c|c|c|c|}
\hline 0.397 & 0.005 & 0.135 & 0.661 & 0.01838365 \\
\hline 0.478 & 0.019 & 0.144 & 0.599 & 0.02001018 \\
\hline 0.498 & 0.005 & 0.293 & 0.254 & 0.19551922 \\
\hline 0.251 & 0.004 & 0.255 & 0.340 & 0.11872705 \\
\hline 0.194 & 0.003 & 0.290 & 0.413 & 0.0329596 \\
\hline 0.152 & 0.008 & 0.296 & 0.284 & 0.06904422 \\
\hline 0.163 & 0.023 & 0.281 & 0.341 & 0.03638425 \\
\hline 0.710 & 0.071 & 0.086 & 0.139 & 0 \\
\hline 0.724 & 0.002 & 0.153 & 0.216 & 0.05662621 \\
\hline 0.609 & 0.004 & 0.404 & 0.327 & 0.0031825 \\
\hline 0.246 & 0.007 & 0.278 & 0.311 & 0.02540886 \\
\hline 0.206 & 0.018 & 0.244 & 0.250 & 0.06098969 \\
\hline 0.128 & 0.014 & 0.259 & 0.194 & 0.02518905 \\
\hline 0.143 & 0.051 & 0.157 & 0.276 & 0.02667467 \\
\hline 0.136 & 0.075 & 0.093 & 0.250 & 0.04154935 \\
\hline 0.150 & 0.015 & 0.162 & 0.190 & 0.02265648 \\
\hline 0.292 & 0.005 & 0.574 & 0.361 & 0.0331358 \\
\hline 0.110 & 0.003 & 0.351 & 0.089 & 0.08757126 \\
\hline 0.103 & 0.044 & 0.217 & 0.211 & 0.03884144 \\
\hline 0.136 & 0.101 & 0.124 & 0.322 & 0.0653883 \\
\hline 0.138 & 0.005 & 0.226 & 0.180 & 0.03353922 \\
\hline 0.310 & 0.010 & 0.128 & 0.552 & 0.01523622 \\
\hline 0.391 & 0.006 & 0.198 & 0.595 & 0.01184021 \\
\hline 0.309 & 0.002 & 0.217 & 0.574 & 0.03662348 \\
\hline 0.266 & 0.003 & 0.136 & 0.589 & 0.02642069 \\
\hline 0.308 & 0.005 & 0.133 & 0.396 & 0.02054027 \\
\hline 0.160 & 0.000 & 0.598 & 0.038 & 0.05067667 \\
\hline 0.177 & 0.003 & 0.211 & 0.275 & 0.02452784 \\
\hline 0.307 & 0.000 & 0.118 & 0.564 & 0.01788452 \\
\hline 0.355 & 0.054 & 0.038 & 0.550 & 0.01338557 \\
\hline 0.324 & 0.006 & 0.123 & 0.450 & 0.0124331 \\
\hline 0.388 & 0.003 & 0.121 & 0.718 & 0.02445998 \\
\hline 0.236 & 0.005 & 0.148 & 0.465 & 0.02622045 \\
\hline 0.618 & 0.003 & 0.100 & 1.772 & 0.01258657 \\
\hline 0.389 & 0.004 & 0.163 & 1.009 & 0.0138241 \\
\hline 0.349 & 0.007 & 0.147 & 0.601 & 0.02804318 \\
\hline 0.395 & 0.006 & 0.159 & 0.816 & 0.01134887 \\
\hline 0.236 & 0.009 & 0.122 & 0.505 & 0.02742543 \\
\hline 0.689 & 0.007 & 0.133 & 1.286 & 0.01691715 \\
\hline 0.191 & 0.007 & 0.179 & 0.365 & 0.02742543 \\
\hline 0.362 & 0.007 & 0.130 & 0.896 & 0.02989615 \\
\hline
\end{tabular}




\begin{tabular}{|c|c|c|c|c|}
\hline 0.381 & 0.005 & 0.098 & 1.106 & 0.01506145 \\
\hline 0.521 & 0.008 & 0.071 & 1.118 & 0.01506145 \\
\hline 0.489 & 0.003 & 0.137 & 1.071 & 0.02480196 \\
\hline 0.190 & 0.007 & 0.252 & 0.178 & 0.03553833 \\
\hline 0.357 & 0.007 & 0.108 & 0.351 & 0.00197614 \\
\hline 0.300 & 0.010 & 0.221 & 0.215 & 0.12851949 \\
\hline 0.239 & 0.002 & 0.106 & 0.310 & 0.01592304 \\
\hline 0.188 & 0.003 & 0.131 & 0.206 & 0.0294656 \\
\hline 0.289 & 0.003 & 0.117 & 0.334 & 0.01499693 \\
\hline 0.248 & 0.006 & 0.218 & 0.221 & 0.09185202 \\
\hline 0.242 & 0.005 & 0.162 & 0.219 & 0.00890719 \\
\hline 0.205 & 0.015 & 0.197 & 0.176 & 0.02769872 \\
\hline 0.575 & 0.004 & 0.109 & 0.921 & 0.06419099 \\
\hline 0.414 & 0.006 & 0.213 & 0.512 & 0.0400248 \\
\hline 0.585 & 0.005 & 0.161 & 0.693 & 0.1526571 \\
\hline 0.997 & 0.004 & 0.281 & 1.282 & 0.02584604 \\
\hline 0.329 & 0.008 & 0.198 & 0.373 & 0.03962138 \\
\hline 0.291 & 0.010 & 0.160 & 0.267 & 0.05398728 \\
\hline 0.671 & 0.004 & 0.250 & 0.732 & 0.04128258 \\
\hline 0.489 & 0.005 & 0.145 & 0.637 & 0.02571822 \\
\hline 0.559 & 0.009 & 0.154 & 0.592 & 0.05753759 \\
\hline 0.487 & 0.005 & 0.289 & 0.359 & 0.01588333 \\
\hline 0.534 & 0.005 & 0.178 & 0.689 & 0.09492237 \\
\hline 0.653 & 0.004 & 0.228 & 0.572 & 0.08430029 \\
\hline 0.428 & 0.009 & 0.306 & 0.320 & 0.02244354 \\
\hline 0.399 & 0.005 & 0.353 & 0.223 & 0.02278359 \\
\hline 0.282 & 0.005 & 0.271 & 0.190 & 0.05765181 \\
\hline 0.243 & 0.002 & 0.147 & 0.332 & 0.04355249 \\
\hline 0.196 & 0.007 & 0.160 & 0.203 & 0.04199609 \\
\hline 0.248 & 0.063 & 0.042 & 0.366 & 0.02392007 \\
\hline 0.269 & 0.007 & 0.144 & 0.437 & 0.03096385 \\
\hline 0.237 & 0.006 & 0.182 & 0.263 & 0.01242689 \\
\hline 0.342 & 0.008 & 0.174 & 0.404 & 0.02601037 \\
\hline 0.088 & 0.088 & 0.039 & 0.204 & 0.03904159 \\
\hline 0.110 & 0.114 & 0.059 & 0.251 & 0.03947877 \\
\hline 0.115 & 0.054 & 0.082 & 0.267 & 0.06315277 \\
\hline 0.121 & 0.068 & 0.056 & 0.314 & 0.07031713 \\
\hline 0.105 & 0.008 & 0.150 & 0.175 & 0.04782219 \\
\hline 0.130 & 0.002 & 0.178 & 0.156 & 0.07901631 \\
\hline 0.268 & 0.131 & 0.092 & 0.415 & 0.02357309 \\
\hline 0.384 & 0.271 & 0.094 & 0.409 & 0.04095074 \\
\hline
\end{tabular}




\begin{tabular}{|c|c|c|c|c|}
\hline 0.082 & 0.011 & 0.110 & 0.128 & $0.0715164 \epsilon$ \\
\hline 0.102 & 0.005 & 0.122 & 0.158 & 0.07271192 \\
\hline 0.094 & 0.014 & 0.185 & 0.155 & 0.0615648 \\
\hline 0.108 & 0.010 & 0.171 & 0.134 & 0.09414819 \\
\hline 0.139 & 0.008 & 0.200 & 0.167 & 0.06722547 \\
\hline 0.398 & 0.001 & 0.100 & 1.213 & 0.01509817 \\
\hline 0.163 & 0.013 & 0.214 & 0.188 & 0.07330815 \\
\hline 0.197 & 0.039 & 0.151 & 0.323 & 0.04018591 \\
\hline 0.159 & 0.104 & 0.027 & 0.276 & $0.0226490 €$ \\
\hline 0.281 & 0.011 & 0.073 & 0.235 & 0.18048902 \\
\hline 0.119 & 0.017 & 0.101 & 0.243 & 0.04938886 \\
\hline 0.340 & 0.016 & 0.107 & 0.936 & 0.11020888 \\
\hline 0.126 & 0.016 & 0.133 & 0.163 & 0.08059074 \\
\hline 0.085 & 0.021 & 0.136 & 0.112 & 0.06003496 \\
\hline 0.332 & 0.008 & 0.138 & 0.249 & 0.00177105 \\
\hline 0.101 & 0.013 & 0.160 & 0.138 & 0.07124087 \\
\hline 0.131 & 0.010 & 0.173 & 0.140 & 0.09860341 \\
\hline 0.893 & 0.666 & & & \\
\hline 0.889 & 0.716 & & & \\
\hline 0.908 & 0.813 & & & \\
\hline 0.842 & 0.741 & & & \\
\hline 1.010 & 0.831 & & & \\
\hline 0.881 & 0.784 & & & \\
\hline 0.777 & 0.734 & & & \\
\hline 0.908 & 0.798 & & & \\
\hline 0.646 & 0.704 & & & \\
\hline 0.820 & 0.831 & & & \\
\hline 0.781 & 0.751 & & & \\
\hline 0.956 & 0.764 & & & \\
\hline 0.899 & 0.802 & & & \\
\hline 0.967 & 0.804 & & & \\
\hline 0.988 & 0.870 & & & \\
\hline 0.939 & 0.842 & & & \\
\hline 0.793 & 0.863 & & & \\
\hline 0.850 & 0.864 & & & \\
\hline 0.793 & 0.782 & & & \\
\hline 0.747 & 0.870 & & & \\
\hline 0.542 & 0.871 & & & \\
\hline 0.780 & 0.819 & & & \\
\hline 0.773 & 0.745 & & & \\
\hline 0.822 & 0.804 & & & \\
\hline
\end{tabular}




$\begin{array}{lllll}0.892 & 0.786 & & & \\ 1.026 & 0.776 & & & \\ 0.528 & 0.016 & & & \\ 0.647 & 0.056 & & & \\ 0.274 & 0.262 & & & \\ 0.246 & 0.492 & & & \\ 0.094 & 0.460 & 0.084 & 0.021 & 0.019 \\ 0.107 & 0.020 & 0.121 & 0.036 & 0.039 \\ 0.120 & 0.010 & 0.111 & 0.033 & 0.003 \\ 0.246 & 0.050 & 0.292 & 0.388 & 0.322 \\ 0.481 & 0.020 & 1.386 & 0.143 & 0.052 \\ 0.303 & 0.010 & 0.996 & 0.249 & 1.057 \\ 0.333 & 0.000 & 1.615 & 0.120 & 0.611 \\ 0.334 & 0.000 & 1.250 & 0.206 & 1.006 \\ 0.369 & 0.000 & 2.205 & 0.236 & 2.016 \\ 0.370 & 0.040 & 2.048 & 0.396 & 2.145 \\ 0.533 & 0.020 & 0.720 & 0.010 & 0.013 \\ 0.713 & 0.007 & 1.520 & 0.050 & 0.00762378 \\ 0.524 & 0.010 & 0.501 & 0.024 & 0.00330384 \\ 0.468 & 0.000 & 1.084 & 0.008 & 0.0035495 \\ 0.321 & 0.000 & 1.014 & 0.020 & 0.01987379 \\ 0.342 & 0.000 & 1.167 & 0.020 & 0.02282325 \\ 0.335 & 0.000 & 0.861 & 0.020 & 0.02814765 \\ 0.604 & 0.000 & 2.118 & 0.012 & 0.013139 \\ 0.368 & 0.000 & 1.092 & 0.013 & 0.01746865 \\ 0.633 & 0.000 & 2.230 & 0.012 & 0.01138841 \\ 0.575 & 0.000 & 2.028 & 0.021 & 0.02197871 \\ 0.315 & 0.000 & 0.932 & 0.012 & 0.01622863 \\ 0.402 & 0.000 & 1.437 & 0.024 & 0.02560577 \\ 0.359 & 0.000 & 1.018 & 0.036 & 0.0177508 \\ 0.378 & 0.000 & 1.377 & 0.031 & 0.04434149 \\ 0.483 & 0.000 & 1.996 & 0.033 & 0.0446548 \\ 0.408 & 0.000 & 1.185 & 0.034 & 0.02893688 \\ 0.342 & 0.000 & 1.289 & 0.040 & 0.06781 \\ 0.318 & 0.000 & 1.319 & 0.089 & 0.11736052 \\ 0.321 & 0.000 & 1.476 & 0.058 & 0.08730796 \\ 0.442 & 0.000 & 2.615 & 0.044 & 0.06914308 \\ 0.307 & 0.000 & 1.237 & 0.037 & 0.05000251 \\ 0.476 & 0.000 & 1.264 & 0.027 & 0.03382064 \\ 0.442 & 0.000 & 2.473 & 0.026 & 0.04033504 \\ 0.000 & 1.786 & 0.019 & 0.01723406\end{array}$




\begin{tabular}{|c|c|c|c|c|}
\hline 0.378 & 0.000 & 1.181 & 0.026 & 0.01914988 \\
\hline 0.326 & 0.000 & 1.432 & 0.047 & 0.06070209 \\
\hline 0.275 & 0.000 & 0.979 & 0.019 & 0.02951154 \\
\hline 0.331 & 0.000 & 0.995 & 0.037 & 0.02981223 \\
\hline 0.224 & 0.000 & 0.725 & 0.017 & 0.01809389 \\
\hline 0.567 & 0.000 & 1.247 & 0.019 & 0.05445209 \\
\hline 0.424 & 0.000 & 1.273 & 0.023 & 0.07310124 \\
\hline 0.620 & 0.000 & 2.455 & 0.018 & 0.0762597 \\
\hline 0.411 & 0.004 & 1.885 & 0.029 & 0.06853414 \\
\hline 0.411 & 0.000 & 2.028 & 0.034 & 0.07893594 \\
\hline 0.131 & 0.000 & 0.520 & 0.039 & 0.10255144 \\
\hline 0.149 & 0.000 & 0.702 & 0.028 & 0.06530733 \\
\hline 0.239 & 0.000 & 0.941 & 0.036 & 0.03410419 \\
\hline 0.097 & 0.000 & 0.327 & 0.036 & 0.03764429 \\
\hline 0.130 & 0.000 & 0.705 & 0.040 & 0.09306915 \\
\hline 0.122 & 0.000 & 0.615 & 0.021 & 0.0640033 \\
\hline 0.105 & 0.000 & 0.555 & 0.027 & 0.06411576 \\
\hline 0.103 & 0.000 & 0.409 & 0.027 & 0.14802764 \\
\hline 0.370 & 0.000 & 1.626 & 0.056 & 0.04790672 \\
\hline 0.096 & 0.000 & 0.283 & 0.114 & 0.04815213 \\
\hline 0.066 & 0.000 & 0.201 & 0.079 & 0.07205567 \\
\hline 0.066 & 0.000 & 0.197 & 0.119 & 0.08350834 \\
\hline 0.201 & 0.000 & 0.698 & 0.056 & 0.03657267 \\
\hline 0.195 & 0.000 & 0.789 & 0.061 & 0.0524974 \\
\hline 0.513 & 0.000 & 3.159 & 0.029 & 0.19846077 \\
\hline 0.047 & 0.000 & 0.066 & 0.102 & 0.09125906 \\
\hline 0.114 & 0.000 & 0.447 & 0.078 & 0.06415317 \\
\hline 0.129 & 0.000 & 0.350 & 0.075 & 0.06241543 \\
\hline 0.386 & 0.000 & 1.407 & 0.042 & 0.03334193 \\
\hline 0.481 & 0.647 & 0.016 & 0.231 & 0.033069 \\
\hline 0.273 & 0.453 & 0.018 & 0.249 & 0.04838 \\
\hline 0.336 & 0.460 & 0.024 & 0.271 & 0.036066 \\
\hline 0.315 & 0.522 & 0.025 & 0.224 & 0.0447 \\
\hline 0.437 & 0.383 & 0.022 & 0.383 & 0.028199 \\
\hline 0.313 & 0.454 & 0.025 & 0.242 & 0.039278 \\
\hline 0.424 & 0.501 & 0.015 & 0.289 & 0.027361 \\
\hline 0.336 & 0.511 & 0.019 & 0.223 & 0.034238 \\
\hline 0.440 & 0.487 & 0.020 & 0.277 & 0.031325 \\
\hline 0.410 & 0.567 & 0.021 & 0.278 & 0.032275 \\
\hline 0.614 & 0.604 & 0.012 & 0.402 & 0.029024 \\
\hline 0.354 & 0.600 & 0.029 & 0.248 & 0.038144 \\
\hline
\end{tabular}




$\begin{array}{llllc}0.354 & 0.474 & 0.021 & 0.284 & 0.036364 \\ 0.334 & 0.447 & 0.023 & 0.276 & 0.043467 \\ 0.406 & 0.429 & 0.019 & 0.306 & 0.026498 \\ 0.436 & 0.596 & 0.022 & 0.294 & 0.03727 \\ 0.690 & 0.728 & 0.020 & 0.277 & 0.042233 \\ 0.466 & 0.545 & 0.016 & 0.279 & 0.028601 \\ 0.494 & 0.575 & 0.027 & 0.386 & 0.040097 \\ 0.486 & 0.444 & 0.016 & 0.355 & 0.030387 \\ 0.527 & 0.499 & 0.016 & 0.333 & 0.027368 \\ 0.477 & 0.626 & 0.015 & 0.318 & 0.03007 \\ 0.685 & 0.622 & 0.019 & 0.432 & 0.024498 \\ 0.660 & 0.631 & 0.022 & 0.572 & 0.036765 \\ 0.561 & 0.667 & 0.019 & 0.451 & 0.02863 \\ 0.764 & 0.554 & 0.016 & 0.600 & 0.036213 \\ 0.935 & 0.633 & 0.019 & 0.644 & 0.025619 \\ 1.015 & 0.618 & 0.037 & 0.376 & 0.01393 \\ 0.862 & 0.578 & 0.103 & 0.427 & 0.012136 \\ 0.044 & 0.005 & 0.095 & 0.047 & 0.05698943 \\ 0.077 & 0.002 & 0.157 & 0.065 & 0.06421251 \\ 0.092 & 0.000 & 0.336 & 0.055 & 0.05606762 \\ 0.057 & 0.000 & 0.086 & 0.094 & 0.0656421 \\ 0.052 & 0.000 & 0.127 & 0.072 & 0.0647386 \\ 0.114 & 0.000 & 0.325 & 0.114 & 0.08586273 \\ 0.074 & 0.000 & 0.165 & 0.097 & 0.07528009 \\ 0.079 & 0.000 & 0.223 & 0.076 & 0.06864805 \\ 0.098 & 0.000 & 0.314 & 0.067 & 0.0593811 \\ 0.105 & 0.000 & 0.445 & 0.067 & 0.05938104 \\ 0.091 & 0.000 & 0.258 & 0.088 & 0.08204149 \\ 0.091 & 0.000 & 0.328 & 0.065 & 0.05880016 \\ 0.229 & 0.000 & 1.002 & 0.055 & 0.09230483 \\ 0.186 & 0.000 & 0.781 & 0.054 & 0.08954742 \\ 0.130 & 0.000 & 0.480 & 0.074 & 0.05816956 \\ 0.130 & 0.000 & 0.513 & 0.056 & 0.06150546 \\ 0.134 & 0.000 & 0.566 & 0.058 & 0.06552151 \\ 0.110 & 0.000 & 0.387 & 0.087 & 0.0736757 \\ 0.095 & 0.000 & 0.336 & 0.082 & 0.07306936 \\ 0.080 & 0.000 & 0.229 & 0.081 & 0.06483139 \\ 0.109 & 0.000 & 0.365 & 0.097 & 0.07258279 \\ 0.112 & 0.000 & 0.472 & 0.061 & 0.07229715 \\ 0.118 & 0.000 & 0.366 & 0.086 & 0.0835964\end{array}$




\begin{tabular}{|c|c|c|c|c|}
\hline 0.100 & 0.000 & 0.291 & 0.097 & 0.09419582 \\
\hline 0.318 & 0.003 & 1.910 & 0.135 & 0.06376564 \\
\hline 0.073 & 0.004 & 0.750 & 0.076 & 0.04481173 \\
\hline 0.411 & 0.000 & 2.401 & 0.050 & 0.03889876 \\
\hline 0.461 & 0.000 & 2.249 & 0.069 & 0.04806552 \\
\hline 0.626 & 0.000 & 2.382 & 0.032 & 0.0302053 \\
\hline 0.187 & 0.000 & 1.002 & 0.084 & 0.03536752 \\
\hline 0.058 & 0.000 & 0.205 & 0.050 & 0.01276794 \\
\hline 0.100 & 0.000 & 0.273 & 0.062 & 0.01777845 \\
\hline 0.030 & 0.000 & 0.055 & 0.060 & 0.01948171 \\
\hline 0.228 & 0.000 & 0.883 & 0.038 & 0.01953169 \\
\hline 0.189 & 0.003 & 0.537 & 0.056 & 0.0163252 \\
\hline 0.101 & 0.000 & 0.301 & 0.055 & 0.01603143 \\
\hline 0.217 & 0.003 & 0.730 & 0.057 & 0.01490588 \\
\hline 0.051 & 0.000 & 0.111 & 0.050 & 0.01695268 \\
\hline 0.098 & 0.008 & 0.208 & 0.063 & 0.01599553 \\
\hline 0.049 & 0.009 & 0.101 & 0.089 & 0.02748051 \\
\hline 0.054 & 0.000 & 0.126 & 0.087 & 0.03103097 \\
\hline 0.115 & 0.000 & 0.458 & 0.050 & 0.01861168 \\
\hline 0.141 & 0.000 & 0.624 & 0.050 & 0.02230467 \\
\hline 0.078 & 0.000 & 0.286 & 0.084 & 0.02705137 \\
\hline 0.270 & 0.840 & & & \\
\hline 0.210 & 0.810 & & & \\
\hline 0.230 & 0.810 & & & \\
\hline 0.330 & 0.820 & & & \\
\hline 0.210 & 0.820 & & & \\
\hline 0.230 & 0.730 & & & \\
\hline 0.260 & 0.800 & & & \\
\hline 0.220 & 0.760 & & & \\
\hline 0.400 & 0.910 & & & \\
\hline 0.210 & 0.830 & & & \\
\hline 0.280 & 0.820 & & & \\
\hline 0.330 & 0.880 & & & \\
\hline 0.300 & 0.880 & & & \\
\hline 0.190 & 0.820 & & & \\
\hline 0.230 & 0.860 & & & \\
\hline 0.250 & 0.860 & & & \\
\hline 0.200 & 0.790 & & & \\
\hline 0.180 & 0.790 & & & \\
\hline 0.190 & 0.760 & & & \\
\hline 0.200 & 0.820 & & & \\
\hline
\end{tabular}




$\begin{array}{ll}0.220 & 0.800 \\ 0.190 & 0.790 \\ 0.170 & 0.780 \\ 0.230 & 0.820 \\ 0.160 & 0.760 \\ 0.190 & 0.770 \\ 0.230 & 0.790 \\ 0.200 & 0.780 \\ 0.170 & 0.760 \\ 0.220 & 0.780 \\ 0.170 & 0.710 \\ 0.200 & 0.850 \\ 0.200 & 0.870 \\ 0.160 & 0.790 \\ 0.200 & 0.780 \\ 0.180 & 0.820 \\ 0.170 & 0.810 \\ 0.180 & 0.820 \\ 0.170 & 0.810 \\ 0.160 & 0.820 \\ 0.160 & 0.770 \\ 0.160 & 0.790 \\ 0.160 & 0.730 \\ 0.160 & 0.810 \\ 0.170 & 0.840 \\ 0.200 & 0.800 \\ 0.220 & 0.870 \\ 0.190 & 0.700 \\ 0.190 & 0.700 \\ 0.190 & 0.770 \\ 0.210 & 0.680 \\ 0.480 & 0.850 \\ 0.260 & 0.840 \\ 0.250 & 0.850 \\ 0.240 & 0.810 \\ 0.290 & 0.870 \\ 0.250 & 0.850 \\ 0.140 & 0.780 \\ 0.150 & 0.760 \\ 0.750 & 0.750 \\ 0.750\end{array}$




\begin{tabular}{ll}
0.140 & 0.800 \\
0.190 & 0.790 \\
0.160 & 0.760 \\
0.180 & 0.790 \\
0.200 & 0.840 \\
0.140 & 0.690 \\
0.130 & 0.730 \\
0.130 & 0.690 \\
0.130 & 0.740 \\
0.110 & 0.690 \\
0.100 & 0.680 \\
0.120 & 0.680 \\
0.160 & 0.710 \\
0.120 & 0.690 \\
0.110 & 0.670 \\
0.120 & 0.690 \\
0.110 & 0.690 \\
0.130 & 0.750 \\
0.140 & 0.740 \\
0.160 & 0.730 \\
0.140 & 0.710 \\
0.150 & 0.750 \\
0.160 & 0.770 \\
0.130 & 0.680 \\
0.130 & 0.630 \\
0.130 & 0.640 \\
0.150 & 0.670 \\
0.130 & 0.690 \\
0.170 & 0.830 \\
0.140 & 0.750 \\
0.120 & 0.690 \\
0.140 & 0.730 \\
0.120 & 0.710 \\
0.160 & 0.790 \\
0.200 & 0.870 \\
0.200 & 0.810 \\
0.180 & 0.740 \\
0.180 & 0.850 \\
0.150 & 0.730 \\
0.150 & 0.740 \\
0.803 \\
\hline
\end{tabular}




$\begin{array}{lcccc}0.660 & 0.701 & & & \\ 0.570 & 0.649 & & & \\ 0.683 & 0.784 & & & \\ 0.494 & 0.530 & 0.266 & 0.629 & 0.33662 \\ 0.426 & 0.636 & 0.149 & 0.525 & 0.14839616 \\ 0.376 & 0.717 & 0.081 & 0.439 & 0.039874 \\ 0.396 & 0.771 & 0.064 & 0.400 & 0.043946 \\ 0.261 & 0.679 & 0.056 & 0.310 & 0.094408 \\ 0.339 & 0.669 & 0.064 & 0.466 & 0.086285 \\ 0.298 & 0.795 & 0.064 & 0.261 & 0.016266 \\ 0.349 & 0.727 & 0.116 & 0.334 & 0.105278 \\ 0.222 & 0.394 & 0.286 & 0.242 & 0.212182 \\ 0.160 & 0.546 & 0.082 & 0.186 & 0.073252 \\ 0.355 & 0.504 & 0.074 & 0.722 & 0.080268 \\ 0.331 & 0.703 & 0.072 & 0.384 & 0.077653 \\ 0.309 & 0.681 & 0.044 & 0.350 & 0.106924 \\ 0.221 & 0.468 & 0.056 & 0.368 & 0.152064 \\ 0.231 & 0.525 & 0.041 & 0.400 & 0.082769 \\ 0.305 & 0.555 & 0.108 & 0.486 & 0.1050585 \\ 0.277 & 0.642 & 0.082 & 0.329 & 0.085483 \\ 0.449 & 0.666 & & 0.678 & \\ 0.165 & 0.386 & 0.075 & 0.298 & 0.103113 \\ 0.377 & 0.291 & 0.112 & 1.233 & 0.083432 \\ 0.495 & 0.666 & & 0.918 & \\ 0.297 & 0.663 & 0.057 & 0.383 & 0.09727925 \\ 0.459 & 0.463 & 0.052 & 1.065 & 0.059725 \\ 0.176 & 0.347 & 0.049 & 0.374 & 0.08764231 \\ 0.145 & 0.273 & 0.085 & 0.309 & 0.095516 \\ 0.316 & 0.609 & 0.126 & 0.420 & 0.10105619 \\ 0.175 & 0.159 & 0.122 & 0.448 & 0.1381632 \\ 0.380 & 0.618 & 0.088 & 0.506 & 0.1256775 \\ 0.352 & 0.649 & 0.058 & 0.453 & 0.0884512 \\ 0.348 & 0.534 & 0.079 & 0.606 & 0.10550667 \\ 0.321 & 0.615 & 0.048 & 0.534 & 0.04794138 \\ 0.220 & 0.362 & 0.054 & 0.458 & 0.11746615 \\ 0.304 & 0.553 & 0.061 & 0.491 & 0.12381574 \\ 0.289 & 0.510 & 0.094 & 0.474 & 0.232 \\ 0.205 & 0.533 & 0.040 & 0.401 & 0.0739832 \\ 0.327 & 0.578 & 0.093 & 0.381 & 0.1712336 \\ 0.526 & 0.648 & 0.032 & 0.346 & 0.03653282 \\ 0.56 & 0.091 & 0.522 & 0.18984936\end{array}$




$\begin{array}{lcccc}0.223 & 0.375 & 0.067 & 0.429 & 0.18913959 \\ 0.297 & 0.697 & 0.033 & 0.348 & 0.08047862 \\ 0.195 & 0.624 & 0.066 & 0.249 & 0.065981 \\ 0.192 & 1.000 & & & \\ 0.427 & 0.330 & 0.084 & 0.982 & 0.12400885 \\ 0.402 & 0.489 & & 0.812 & 0.24 \\ 0.526 & 0.244 & 0.063 & 1.825 & 0.209 \\ 0.393 & 0.584 & 0.064 & 0.575 & 0.21165083 \\ 0.354 & 0.509 & 0.038 & 0.575 & 0.112 \\ 0.325 & 0.525 & 0.089 & 0.500 & 0.223 \\ 0.449 & 0.500 & 0.069 & 0.672 & 0.3 \\ 0.383 & 0.578 & 0.066 & 0.501 & 0.256 \\ 0.451 & 0.591 & 0.059 & 0.536 & 0.179 \\ 0.315 & 0.491 & 0.053 & 0.457 & 0.199 \\ 0.190 & 0.207 & 0.049 & 0.355 & 0.2695 \\ 0.374 & 0.438 & 0.051 & 0.617 & 0.153 \\ 0.414 & 0.504 & 0.062 & 0.658 & 0.238 \\ 0.288 & 0.454 & 0.070 & 0.576 & 0.096 \\ 0.354 & 0.461 & 0.072 & 0.529 & 0.272 \\ 0.470 & 0.303 & 0.069 & 1.415 & 0.17552932 \\ 0.402 & 0.573 & 0.091 & 0.517 & 0.261 \\ 0.355 & 0.517 & 0.071 & 0.557 & 0.235 \\ 0.356 & 0.608 & 0.065 & 0.430 & 0.202 \\ 0.279 & 0.463 & 0.069 & 0.441 & 0.224 \\ 0.445 & 0.362 & 0.080 & 1.073 & 0.209 \\ 0.382 & 0.504 & 0.078 & 0.591 & 0.181 \\ 0.582 & 0.256 & 0.075 & 1.954 & 0.1765 \\ 0.399 & 0.501 & 0.078 & 0.674 & 0.283 \\ 0.312 & 0.463 & 0.089 & 0.403 & 0.253 \\ 0.329 & 0.607 & 0.076 & 0.389 & 0.173 \\ 0.208 & 0.238 & 0.076 & 0.362 & 0.278 \\ 0.226 & 0.229 & 0.099 & 0.453 & 0.25067814 \\ 0.266 & 0.478 & 0.076 & 0.366 & 0.211 \\ 0.337 & 0.299 & 0.069 & 0.941 & 0.14975248 \\ 0.331 & 0.468 & 0.075 & 0.548 & 0.171 \\ 0.202 & 0.318 & 0.064 & 0.388 & 0.21928394 \\ 0.287 & 0.452 & 0.063 & 0.581 & 0.17634889 \\ 0.543 & 0.335 & 0.091 & 1.533 & 0.102 \\ 0.341 & 0.584 & 0.056 & 0.390 & 0.185 \\ 0.389 & 0.601 & 0.085 & 0.433 & 0.214\end{array}$




\begin{tabular}{|c|c|c|c|c|}
\hline 0.160 & 0.231 & 0.061 & 0.364 & 0.12018682 \\
\hline 0.339 & 0.347 & 0.087 & 0.829 & 0.19882732 \\
\hline 0.246 & 0.308 & 0.239 & 0.402 & 0.14379786 \\
\hline 0.254 & 0.277 & 0.083 & 0.569 & 0.24743565 \\
\hline 0.496 & 0.652 & 0.077 & 0.667 & 0.19998164 \\
\hline 0.321 & 0.139 & 0.296 & 0.538 & 0.37312082 \\
\hline 0.338 & 0.222 & 0.312 & 0.514 & 0.39808174 \\
\hline 0.439 & 0.546 & 0.245 & 0.560 & 0.3165322 \\
\hline 0.274 & 0.643 & 0.061 & 0.379 & 0.09501563 \\
\hline 0.542 & 0.488 & 0.063 & 1.027 & 0.1556416 \\
\hline 0.313 & 0.631 & 0.043 & 0.405 & 0.12648738 \\
\hline 0.438 & 0.690 & 0.095 & 0.503 & 0.099196 \\
\hline 0.399 & 0.624 & 0.087 & 0.571 & 0.15129958 \\
\hline 0.329 & 0.585 & 0.062 & 0.385 & 0.246 \\
\hline 0.170 & 0.040 & & & \\
\hline 0.930 & 0.390 & & & \\
\hline 0.300 & 0.050 & & & \\
\hline 0.420 & 0.070 & & & \\
\hline 0.290 & 0.070 & & & \\
\hline 0.160 & 0.030 & & & \\
\hline 0.280 & 0.060 & & & \\
\hline 0.190 & 0.070 & & & \\
\hline 0.400 & 0.020 & & & \\
\hline 0.830 & 0.290 & & & \\
\hline 0.410 & 0.130 & & & \\
\hline 0.330 & 0.020 & & & \\
\hline 0.300 & 0.030 & & & \\
\hline 0.460 & 0.020 & & & \\
\hline 0.140 & 0.060 & & & \\
\hline 0.120 & 0.030 & & & \\
\hline 0.100 & 0.000 & & & \\
\hline 0.100 & 0.030 & & & \\
\hline 0.210 & 0.010 & & & \\
\hline 0.150 & 0.010 & & & \\
\hline 0.280 & 0.420 & & & \\
\hline 0.540 & 0.490 & & & \\
\hline 0.180 & 0.020 & & & \\
\hline 0.390 & 0.630 & & & \\
\hline 0.320 & 0.600 & & & \\
\hline 0.340 & 0.740 & & & \\
\hline 0.590 & 0.210 & & & \\
\hline
\end{tabular}




\begin{tabular}{ll}
0.300 & 0.250 \\
0.450 & 0.020 \\
0.710 & 0.060 \\
0.370 & 0.570 \\
0.270 & 0.060 \\
0.310 & 0.030 \\
0.350 & 0.040 \\
0.440 & 0.070 \\
0.380 & 0.140 \\
0.230 & 0.180 \\
0.630 & 0.740 \\
0.380 & 0.620 \\
0.540 & 0.690 \\
0.510 & 0.670 \\
0.310 & 0.760 \\
0.760 & 0.670 \\
0.990 & 0.650 \\
0.480 & 0.690 \\
0.365 & 0.677 \\
0.307 & 0.672 \\
0.398 & 0.716 \\
0.349 & 0.714 \\
0.447 & 0.778 \\
0.316 & 0.727 \\
0.344 & 0.722 \\
0.323 & 0.696 \\
0.338 & 0.693 \\
0.282 & 0.676 \\
0.140 & 0.240 \\
0.640 & 0.750 \\
0.640 & 0.720 \\
0.740 & 0.710 \\
0.610 & 0.790 \\
0.450 & 0.700 \\
0.750 & 0.720 \\
0.470 & 0.750 \\
0.410 & 0.750 \\
0.470 & 0.810 \\
0.450 & 0.730 \\
0.410 & 0.690 \\
\hline & 0.710
\end{tabular}




\begin{tabular}{ll}
0.540 & 0.710 \\
0.410 & 0.620 \\
0.570 & 0.710 \\
0.470 & 0.700 \\
0.440 & 0.690 \\
0.430 & 0.740 \\
0.410 & 0.700 \\
0.370 & 0.700 \\
0.290 & 0.660 \\
0.190 & 0.410 \\
0.400 & 0.700 \\
0.340 & 0.670 \\
0.350 & 0.680 \\
0.520 & 0.640 \\
0.510 & 0.680 \\
0.400 & 0.630 \\
0.480 & 0.710 \\
0.520 & 0.810 \\
0.440 & 0.790 \\
0.590 & 0.840 \\
0.230 & 0.520 \\
0.350 & 0.490 \\
0.310 & 0.710 \\
0.150 & 0.550 \\
0.390 & 0.610 \\
0.420 & 0.780 \\
0.270 & 0.440 \\
0.240 & 0.630 \\
0.170 & 0.430 \\
0.260 & 0.380 \\
0.590 & 0.780 \\
0.150 & 0.400 \\
0.160 & 0.510 \\
0.540 & 0.770 \\
0.270 & 0.580 \\
0.160 & 0.340 \\
0.130 & 0.460 \\
0.070 & 0.220 \\
0.100 & 0.230 \\
0.080 & 0.250 \\
\hline & 0.110
\end{tabular}




\begin{tabular}{ll}
0.110 & 0.060 \\
0.070 & 0.070 \\
0.110 & 0.030 \\
0.100 & 0.160 \\
0.130 & 0.090 \\
0.110 & 0.390 \\
0.370 & 0.740 \\
0.590 & 0.800 \\
0.420 & 0.700 \\
0.680 & 0.690 \\
0.610 & 0.650 \\
0.860 & 0.720 \\
0.760 & 0.690 \\
0.470 & 0.560 \\
0.520 & 0.660 \\
0.450 & 0.650 \\
0.520 & 0.730 \\
0.520 & 0.740 \\
0.250 & 0.550 \\
0.610 & 0.840 \\
0.180 & 0.410 \\
0.460 & 0.690 \\
0.460 & 0.620 \\
0.300 & 0.110 \\
0.360 & 0.030 \\
0.350 & 0.050 \\
0.390 & 0.140 \\
0.390 & 0.020 \\
0.620 & 0.100 \\
0.780 & 0.100 \\
0.700 & 0.260 \\
0.720 & 0.370 \\
0.310 & 0.120 \\
0.200 & 0.010 \\
0.330 & 0.190 \\
0.310 & 0.020 \\
0.340 & 0.010 \\
0.340 & 0.000 \\
0.310 & 0.100 \\
\hline & 0.010
\end{tabular}




$\begin{array}{ll}0.280 & 0.010 \\ 0.240 & 0.000 \\ 0.690 & 0.010 \\ 0.280 & 0.040 \\ 0.300 & 0.030 \\ 0.400 & 0.050 \\ 0.330 & 0.060 \\ 0.420 & 0.020 \\ 0.490 & 0.010 \\ 0.930 & 0.080 \\ 0.530 & 0.250 \\ 0.670 & 0.280 \\ 0.670 & 0.360 \\ 0.440 & 0.390 \\ 0.410 & 0.310 \\ 0.590 & 0.370 \\ 0.630 & 0.140 \\ 0.720 & 0.280 \\ 0.490 & 0.480 \\ 0.362 & 0.726 \\ 0.900 & 0.706 \\ 0.660 & 0.694 \\ 0.927 & 0.728 \\ 0.670 & 0.637 \\ 0.544 & 0.699 \\ 0.549 & 0.735 \\ 0.443 & 0.707 \\ 0.293 & 0.240 \\ 0.416 & 0.717 \\ 0.283 & 0.780 \\ 0.358 & 0.537 \\ 0.438 & 0.601 \\ 0.244 & 0.437 \\ 0.226 & 0.165 \\ 0.305 & 0.491 \\ 0.517 & 0.739 \\ 0.301 & 0.304 \\ 0.353 & 0.278 \\ 0.290 & 0.295 \\ 0.241 & 0.900 \\ 0.804\end{array}$




$\begin{array}{ll}0.162 & 0.361 \\ 0.242 & 0.905 \\ 0.237 & 0.907 \\ 0.197 & 0.655 \\ 0.205 & 0.785 \\ 0.160 & 0.863 \\ 0.239 & 0.892 \\ 0.206 & 0.910 \\ 0.224 & 0.907 \\ 0.275 & 0.145 \\ 0.306 & 0.264 \\ 0.282 & 0.286 \\ 0.283 & 0.151 \\ 0.338 & 0.032 \\ 0.297 & 0.000 \\ 0.289 & 0.304 \\ 0.237 & 0.813 \\ 0.239 & 0.753 \\ 0.346 & 0.657 \\ 0.278 & 0.074 \\ 0.237 & 0.211 \\ 0.376 & 0.000 \\ 0.260 & 0.217 \\ 0.232 & 0.625 \\ 0.292 & 0.422 \\ 0.304 & 0.000 \\ 0.324 & 0.011 \\ 0.379 & 0.000 \\ 0.269 & 0.000 \\ 0.305 & 0.000 \\ 0.286 & 0.000 \\ 0.289 & 0.000 \\ 0.180 & 0.000 \\ 0.333 & 0.000 \\ 0.320 & 0.000 \\ 0.325 & 0.331 \\ 0.347 & 0.000 \\ 0.321 & 0.050 \\ 0.337 & 0.105 \\ 0.146 & 0.308 \\ 0.175 & 0.281\end{array}$




$\begin{array}{ll}0.167 & 0.000 \\ 0.096 & 0.030 \\ 0.172 & 0.000 \\ 0.326 & 0.000 \\ 0.292 & 0.034 \\ 0.297 & 0.041 \\ 0.329 & 0.012 \\ 0.301 & 0.000 \\ 0.304 & 0.000 \\ 0.314 & 0.000 \\ 0.197 & 0.132 \\ 0.307 & 0.000 \\ 0.332 & 0.000 \\ 0.324 & 0.000 \\ 0.281 & 0.055 \\ 0.063 & 0.154 \\ 0.247 & 0.000 \\ 0.145 & 0.000 \\ 0.138 & 0.000 \\ 0.127 & 0.038 \\ 0.444 & 0.149 \\ 0.336 & 0.000 \\ 0.189 & 0.072 \\ 0.103 & 0.067 \\ 0.244 & 0.042 \\ 0.278 & 0.045 \\ 0.211 & 0.086 \\ 0.336 & 0.000 \\ 0.332 & 0.000 \\ 0.294 & 0.000 \\ 0.223 & 0.000 \\ 0.333 & 0.000 \\ 0.210 & 0.000 \\ 0.307 & 0.000 \\ 0.219 & 0.000 \\ 0.298 & 0.000 \\ 0.275 & 0.000 \\ 0.264 & 0.000 \\ 0.406 & 0.131 \\ 0.300 & 0.000 \\ 0.329 & 0.088\end{array}$




$\begin{array}{ll}0.369 & 0.056 \\ 0.384 & 0.153 \\ 0.239 & 0.126 \\ 0.280 & 0.000 \\ 0.213 & 0.086 \\ 0.209 & 0.046 \\ 0.199 & 0.438 \\ 0.173 & 0.493 \\ 0.177 & 0.162 \\ 0.200 & 0.153 \\ 0.123 & 0.381 \\ 0.175 & 0.635 \\ 0.160 & 0.169 \\ 0.042 & 0.706 \\ 0.074 & 0.067 \\ 0.101 & 0.020 \\ 0.404 & 0.000 \\ 0.397 & 0.000 \\ 0.276 & 0.000 \\ 0.136 & 0.000 \\ 0.341 & 0.000 \\ 0.100 & 0.000 \\ 0.276 & 0.000 \\ 0.132 & 0.000 \\ 0.172 & 0.012 \\ 0.703 & 0.146 \\ 0.325 & 0.000 \\ 0.178 & 0.279 \\ 0.185 & 0.016 \\ 0.146 & 0.154 \\ 0.228 & 0.000 \\ 0.182 & 0.000 \\ 0.307 & 0.023 \\ 0.194 & 0.043 \\ 0.309 & 0.917 \\ 0.190 & 0.444 \\ 0.334 & 0.221 \\ 0.228 & 0.000 \\ 0.362 & 0.853 \\ 0.363 & 0.758 \\ 0.354 & 0.837\end{array}$




$\begin{array}{ll}0.353 & 0.874 \\ 0.333 & 0.804 \\ 0.340 & 0.849 \\ 0.345 & 0.932 \\ 0.332 & 0.930 \\ 0.377 & 0.920 \\ 0.161 & 0.609 \\ 0.204 & 0.831 \\ 0.249 & 0.848 \\ 0.175 & 0.741 \\ 0.281 & 0.161 \\ 0.186 & 0.479 \\ 0.107 & 0.367 \\ 0.268 & 0.302 \\ 0.171 & 0.328 \\ 0.131 & 0.465 \\ 0.127 & 0.578 \\ 0.246 & 0.229 \\ 0.124 & 0.275 \\ 0.177 & 0.652 \\ 0.224 & 0.181 \\ 0.263 & 0.457 \\ 0.226 & 0.435 \\ 0.333 & 0.351 \\ 0.236 & 0.343 \\ 0.285 & 0.370 \\ 0.262 & 0.391 \\ 0.351 & 0.417 \\ 0.268 & 0.406 \\ 0.548 & 0.906 \\ 0.500 & 0.925 \\ 0.526 & 0.858 \\ 0.518 & 0.902 \\ 0.511 & 0.896 \\ 0.528 & 0.886 \\ 0.483 & 0.904 \\ 0.558 & 0.923 \\ 0.529 & 0.904 \\ 0.457 & 0.860 \\ 0.489 & 0.881 \\ 0.500 & 0.900\end{array}$




$\begin{array}{ll}0.505 & 0.888 \\ 0.540 & 0.887 \\ 0.452 & 0.869 \\ 0.705 & 0.838 \\ 0.489 & 0.841 \\ 0.965 & 0.277 \\ 0.598 & 0.798 \\ 0.257 & 0.772 \\ 0.169 & 0.392 \\ 0.203 & 0.455 \\ 0.256 & 0.448 \\ 0.224 & 0.538 \\ 0.221 & 0.628 \\ 0.256 & 0.574 \\ 0.721 & 0.915 \\ 0.600 & 0.526 \\ 0.266 & 0.856 \\ 0.787 & 0.839 \\ 0.667 & 0.829 \\ 0.567 & 0.840 \\ 0.849 & 0.860 \\ 0.704 & 0.823 \\ 0.751 & 0.841 \\ 0.490 & 0.711 \\ 0.690 & 0.852 \\ 0.645 & 0.825 \\ 0.948 & 0.712 \\ 0.846 & 0.871 \\ 0.679 & 0.829 \\ 0.757 & 0.830 \\ 0.672 & 0.800 \\ 0.652 & 0.787 \\ 0.552 & 0.638 \\ 0.686 & 0.791 \\ 0.839 & 0.870 \\ 0.491 & 0.839 \\ 0.419 & 1.000 \\ 0.278 & 0.926 \\ 0.367 & 0.832 \\ 0.221 & 0.851\end{array}$




$\begin{array}{ll}0.210 & 0.628 \\ 0.527 & 0.841 \\ 0.475 & 1.000 \\ 0.279 & 1.000 \\ 0.232 & 1.000 \\ 0.387 & 0.821 \\ 0.258 & 1.000 \\ 0.223 & 1.000 \\ 0.297 & 1.000 \\ 0.286 & 0.752 \\ 0.204 & 0.841 \\ 0.391 & 0.822 \\ 0.808 & 0.881 \\ 0.866 & 0.859 \\ 0.923 & 0.910 \\ 0.892 & 0.924 \\ 0.911 & 0.913 \\ 0.779 & 0.910 \\ 0.953 & 0.937 \\ 0.811 & 0.889 \\ 0.845 & 0.935 \\ 0.860 & 0.892 \\ 0.726 & 0.907 \\ 0.831 & 0.858 \\ 0.827 & 0.896 \\ 0.758 & 0.904\end{array}$

